\title{
$6^{\text {me }}$ CONGRES INTERNATIONAL
}

$$
\text { ZOOIOGIE }
$$

\section{COMPTE RENDU DBS SEAANCES}

\section{4}

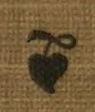

BERNE 

Gorit s Hiller fi. Gely 2, 1955- 



\section{CONPTE-RENDU DES SÉANCES $\mathrm{DU}$}

SIXIĖUE CONGRÉS INTERNATIONAL DE

ZOOLOGIE 

International Congress of 200logy. 633 Bern. 1904

\title{
COMPTE-RENDU
}

\author{
DES SEANCES
}

DU

\section{SIXIËUE CONGRĖS INTERIATIONAL \\ DE}

ZOOLOGIE

tenu à

BERNE DU 14 AU 16 AOUT 1904

\author{
Publié \\ par \\ M. BEDOT \\ Secrétaire général du Congrès
}

A vec 33 planches et 51 figures dans le texte.

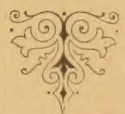

GENEVE

IMPRIMERIE W. KÜNDIG \& FILS, 4, VIEUX-COLLĖGE.

1905 


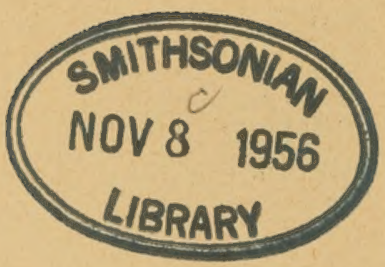




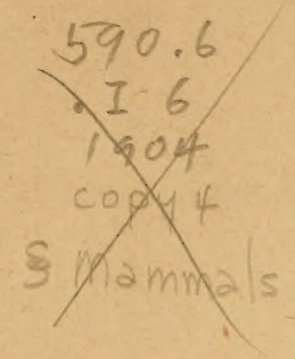

Je tiens à exprimer ici ma vive reconnaissance aux deux secrétaires du Congrès, MM. les Drs J. Carl et W. Volz, qui ont bien voulu me prêter leur précieux concours pour l'organisation du Secrétariat, et pour la publication de ce volume.

Genère, le 15 Mai 1905.

M. BEDOT

Secrétaire général. 



\title{
CONGRES INTERNATIONAL
}

\author{
DE \\ ZOOLOGIE
}

COMITÉ PERMANENT

Institué en 1892 par le Congrès de Moscou.

Voir: C. R. Congr. int. de Zool. Moscou, P. 2, p. LI. Hoscou 1892.

\section{Membres perpétuels ${ }^{1}$.}

MM. F. Perrter (Paris). MI. F. Président.

Th. Studer (Berne), M. F. A. P. Vice-Président.

L. Vaillant (Paris), M. F. Vice-Président.

R. Blanchard (Paris), M. F. Secrétaire géméral.

J. de Guerne (Paris), M. F. Secrétaire.

C. Schlumberger (Paris), M. F.

P. Kíapist (Moscou), A. P.

F. A. Jextink (Leyde), A. P.

J. LubBock (London), A. P.

K. Moebus (Berlin), A. P.

A. Agassiz (Cambridge), Président du prochain Congrès.

Membre temporaire.

11. M. Benot (Genève), Secrétaire général du bo Congrès.

${ }^{1}$ M. F. = Membre fondateur du Congrès.

A. $\mathrm{P} .=$ Ancien président du Congrès. 


\section{GOMMISSION INTERNATIONALE DES PRIX}

Instituée en 1892 par le Congrès de Moscou.

Voir: C. R. Congr. int. de Zool. Moscou, P. 2, pp. XLVIII à L. Moscou 1892; et: C. R. Congr. int. de Zool. Leyde, 1). 72. Leyde 1896.

\section{Membres de la Commission:}

Mil. E. Perrier (Paris), Président.

R. Branchard (Paris), Secrétaire.

F. A. Jentink (Leyde).

H. Ludwig (Bonn).

R. B. Sharpe (Londles).

Th. Studer (Berne).

N. de Zograf (Moscou).

Toì le Règlement des Prix à l'annexe. 


\section{COMMISSION INTERNATIONALE DE NOMENGLATURE}

Instituée en 1895 par le Congrès de Leyde.

Voir : C. R. Congr. int. de Zool. Leyde, p. 9 3̈. Leyde 1896 ; et: C. R. Congr. int. de Zool. Berne, p. 137. Gencre $1905 \%$.

\section{Membres de la Commission.}

Série sortant en 1907.

MI. R. Horst (Leyde).

F. A. Jentink (Leyde).

D. S. Jordan (Palo Alto).

F. E. Schelze (Berlin).

L. Stejneger (Washington).

Série sortant en 1910.

R. Blanchard (Paris), Président.

L. Joubin (Paris).

C. W. Stiles (Washington), Secrétaire.

'Th. Studer (Bel'ne).

R. Wright ('T'olonto).

Série sortant en 1913.

Ph. Dautzenber: (Paris).

W. E. Hoyse (Manchester).

L. von GrafF (Graz).

F. C. von M aeranthac (Berlin), Secrétaire.

H. F. Osborn (New-York). 


\section{COMMISSION INTERNATIONALE DU CONCILIUM BIBLIOGRAPHICUM}

Instituée en 1895 par le Congrès de Leyde.

Voir: C. R. Congr. int. de Zonl. Lerde, 1).93. Lerde 1896.

Membres de la Commission.

MIM. A. Lang (Zürich), Président.

R. Branchard (Paris), Secrétaire.

S. Hicksox (Manchester).

P. P. C. Hozk (Copenhague).

IV. Schimk Ewitcu (St-Pétersbourg).

IV. B. Scotт (Princeton).

J. IV. Spengel (Giessen). 


\section{LISTE DES LAURĖATS DES CONGRĖS INTERNATIONAUX DE ZOOLOGIE}

\section{Prix de S. M. l'Empereur Alexandre III.}

1898. M. E. De Pousareues (Paris).

1904. M. le Prof. R. Hexsons (Hannover-Münden).

\section{Prix de S. M. l'Empereur Nicolas II.}

1895. M. le Dr Scharffe (Dublin).

1898. M. le Dr E. Hecht (Nancy).

1901. M. le Dr J. Th. Oudemans (Amsterdam).

1904. M. le Dr R. von Stuminer-T'Taunfelds (Graz). 


\section{LISTE DES SESSIONS DU GONGRESS INTERNATIONAL} DE ZOOLOGIE

$\begin{array}{lrcl}\text { Paris } & 1859 & \text { Sous la présidence de } & \text { A. Mrdne-Edwards. † } \\ \text { Moscou } & 1892 & " & \text { P. Kapnist. } \\ \text { Leyde } & 1895 & " & \text { F.-A. Jentink. } \\ \text { Cambridge } 1898 & " & \text { J. Lubrock. } \\ \text { Berlin } & 1901 & " & \text { K. MöbuUs. } \\ \text { Berne } & 1904 & " & \text { Tr. Studer. }\end{array}$




\section{VI ${ }^{\text {ine }}$ CONGRES INTERNATIONAL}

DE

\section{ZOOLOGIE}

Berne, du 14 au 16 août 1904

Sous la Présidence de M. le Professeur Th. Stuner.

\section{COMITÉ SUISSE D'ORGANISATION}

\section{Bureau.}

Th. Studer, président.

E. BéRANECK, vice-président.

11. BLANC,

Y. Fatio.

I. Kathariver,

E. Yung,

F. ZSCHOKKF.

R. Blanchand, secrétaire ggénéral permanent.

II. ВEnot, secrétaire. Délégué à la Commission des publications.

J. CARL,

IV. VoLz.

E. von Bühen, trésorier. Délégué à la Commission des finances.

A. Pictet, 》

H. Stiasser. Délégué à la Commission des travaux scientifiques.

0 . RUBELI

des fètes.

E. Hess

des logements.

M. BÜHLEI de la presse.

H. KRONECKER

J.-H. GraF \# des réceptions.

des subsistances. 


\section{Membres du Comité d'organisation.}

E. Axpné, (Genève).

L. Asher, (Berne).

H. Bachuax, (Lucerne).

S. Breler, (Lausanne).

A. Blocir, (Soleure).

F. Bцосн, (Soleure).

E. Bugriox, (Lausamne).

R. Burchiardt, (Bale).

R. Buri, (Berne).

H. Corning, (Bâle).

U. Duenst, (Zurich).

A. Eтепxod, (Genève).

H. FIELD, (Zurich).

H. Fischer-Sigwart, (Zofingue).

A. Forel, (Chigny).

F.-A. Fonel, (Horges).

o. Fummans, (Neuchatel).

P. Godet, (Neuchâtel).

A. Gurwitsch, (Berne).

F. KonY, (Porrentruy).

J. Kollmaxx, (Bâle).
A. LAtg, (Zurich).

S. LAskowski, (Genève).

P. De Loriol-Le Fort, (Genève).

R. Martin, (Zurich).

M. Musr, (Fribourg).

J. Nuesch, (Schaffhouse).

E. Pexaiti, (Genève).

H. Rothenbühler, (Berne).

J. Roux, (Bale).

C. Sarasix, (Genève).

F. SARAsin, (Bâle).

P. Sarasin, (Bâle).

H. de Saussure, (Genève).

A. Schulthess-Rechberg, (Zurich).

II. Standfuss, (Zurich).

T. STEck, (Berne).

H. Stehlis, (Bâle).

T. Stingelis, (Olten).

E. Weber, (Genève).

K. Zimmermanx, (Berne).

\section{Commission des travaux scientifiques.}

H. STRAsser, président.

E. Béraneck, H. Blanc, E. Bugriox, R. Burckhardt, H. Consixg, H. Duenst, A. Forel, L. Kathariner, A. Lang, F. Sarasix, P. Salasis, H. Stehlin, Th. Studel, E. YuNG, F. ZsсHокке.

\section{Commission des publications.}

M. BEDot, président.

J. Cari, H. Sthasser. W. Volz.

COMITÉ LOCAI

Th. STunelk, président.

W. Volz, secrétaire.

\section{Commission des finances.}

E. vox Bürex, président.

O. Brechet, H. Lindt, A. Pigtet, Th. Steck, E. Stettlen, P. Thonmany. 


\section{Commission des fêtes.}

O. Rubeli, président.

H. Bloesch, E. Brückner, C. Daut, E. Davinet, R. A. Gullebelu, E. Noyer. J. Schapino, M. Walthard, K. W. Zimmenmaxy.

E. Hess, président.

G. Guggisberig, vice-président.

G. Rотнехвӥ̈неr, secrétaire.

J. Basler, P. Deugher, R. Mosmax, W. Ochsexbein, A. Sessler, E. Wagner.

\section{Commission de réception.}

H. KRONEcker, président.

F. von Ernst, R. A. Gullebenu, E. Röthlisberger, L. vox Tschanner, A. Vhlextix, J. von Wattexwyl.

\section{Commission des subsistances.}

J. H. GraF, président.

R. Dıck, vice-président.

F. Krumbein, secrétaire.

A. Badertscier, P. Kinebs, R. Li Nicca, K. Oswald, R. Walkeh.

\section{Commission de la presse.}

II. BüriLer, président.

G. Beck, H. Blösch, F. Burres, F.-A. Forel, R. Lüd, D. Ochsexbers, J. Repond, A. WeLti.

\section{Commission d'ordre.}

R. Guggisberg, président.

J. Mloos, F. Roth, H. Scheqchzer, G. G. Stucki.

\section{COMITÉ DE RÉCEPTION DES DAMES}

Wlle G. Auen, Mme Graf, Mme Gulldebeau, IIme Isenschnidt, Mme Jent, Ime Kronecker. Mme Strasser, Mme Studer, in ${ }^{\text {me }}$ Valentin. 


\section{REGLEMENT}

Artucle Premier. La sixième session du Congrès international de Zoologie s'ouvrira à Berne le 14 août 1904. La clôture du Congrès aura lieu le 19 août.

Art. 2. 'Tous les zoologistes et toutes les personnes s'intéressant aux sciences biologiques peuvent faire partie du Congrès sous les conditions suivantes :

a) Adresser au Président du Congrès une demande d'admission.

b) Verser entre les mains du trésorier du Congrès une somme de 25 frs. comme droit d'admission.

Ar's. 3. Chaque membre du Congres recevra un exemplaire du Compte rendu de la session.

ArT. 4. Les dames peuvent faire partie du Congrès aux mêmes conditions, ou en prenant me carte de dame, du prix de 10 frs.s, qui leur permet d'assister aux séances et réceptions, mais qui ne donne pas droit au volume du Compte rendu de la session.

ArT. 5̃. Les séances du Congrès ne sont pas publiques.

Ar't. 6. La session du Congrès comprendra 4 assemblées généralens et des séances de sections.

Axт. 7. Inans la première assemblée génélale, le Congrès nommera son bureau, ainsi que les présidents, vice-présidents et secrétaires des sections.

Arт. 8. Les ordres du jour seront établis par le bureau.

Arт. 9. Quand l'ordre du jour d'une séance n'aura pas été épuisé, la suite en sera reportée au commencement de la séance suivante.

Art. 10. Les membres du Congrès qui auront l'intention de faire des communications non annoncées au prosramme imprimé devront en faire la demande par écrit au président. Ils ne pourront prendre la parole qu'à la suite des orateurs inscrits. 
Ar'r. 11. Dans les séances de sections les orateurs ne pourront pas parler pendant plus de 15 minutes, à moins que la section n'en décido autrement. Pour les assemblées générales, la durée des discours est laissée à l'appréciation du Bur'eau.

ArT. 12. Les membres du Congrès devront remettre au secrétaire de la section, immédiatement après chaque séance, un résumé succinct de leurs communications. Ce résumé sera publié dans le "Bulletin du Congrès " qui paraîtra chaque jour pendant toute la durée de la session et contiendra le procés-verbal des séances de la veille et les annonces relatives au Congrès.

ArT. 13. Les discussions qui suivront les communications ne figureront pas dans le compte rendu des séances à moins que les personnes qui ont pris la parole n'en fassent la demande au secrétaire général en lui remettant le manuscrit de la note qu'ils desirent faire insérer au procès-verbal.

ArT. 14. Les manuscrits des discours et communications devront être remis par les auteur's au secrétaire général, si possible pendant la durée de la session et, au plus tard, le 15 septembie.

Art. 15. Lorsque les mémoires seront accompagnés de planches, les dessins devront être faits sans couleur's et de manière à pouvoir être reproduits par un procérlé à base photographique. Les dessins qui nu peuvent être reproduits que par la cravure ou la lithographie ne soront pas acceptés, à moins que les auteur's ne s'engagent à en supporter les firais.

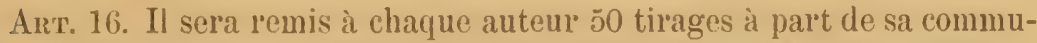
nication. Le's auteurs qui désileraient avoir des tirages à part supplémentaires pourront les obtenir au prix de revient.

ArT. 17. Les tirages à part devront porter la mention: Extrait des Comptes rendus du $6^{\mathrm{e}}$ Congrès international de Zoologie. Session de Berne 1904. 


\section{PROGRA II IIE}

\section{Dintanche 14 Aout.}

S I. du soir. liéception des membres du Congrès au Kormhauskeller. Discours de II. A. von Strerger, Président de la ville de Berne.

\section{Lundr 15 Aout.}

$S$ h. $1 / 2$ du matin. Séance du Comité permanent des Congrès internationaux de zoologie et des délégués des gouvernements dans la Salle de lecture du Palais du Parlement.

9 heures. Premiere assemblée générale au Palais du Parlement dans la Sialle du Conscil national. ()uverture du Congrès. Discour's de M. STuIER. Président du Congrès. Discour's des délégués. Election des présidents, vico-présidents et secrétaires des assemblées générales et des séances de sections. Choix du lieu de réunion de la prochaine session du Congrex.s. Conférences de MII. R. Blanchard (Paris) et A. Laxi: (Zurich).

3 heures après-midi. Séances des sections à l'Université.

sheures du soir. Réunion au Schänzli. Représentation théâtrale, illıminations.

\section{Mardi 16 Aou'r.}

9) hemes du mutiu. I) enxienne assemblè générale à l'Aula do l'Universite. (onferences de MII. H.-F. Osmond (New-York), W. Salensky (St-Pétersbourg) et C. Cuun (Leipzig).

3 heures après-midi. Séances des sections à l'Univer'sité.

i) heures $1 / 2$. Réunion au Gurten.

\section{Mercredi 17 AouT.}

9) heures du matin. Séances des sections à l'Université.

3 heures après midi. Séances des sections à l'Université.

$8 \%$ heures du soir. Concert d'orgues à la Cathédrale. 


\section{JEUd 18 Aout.}

g heures du mutin. Troisiène assembléc générale au Palais du Parlement. Confélences de Mal. H. von Berrerscil (Cassel), F. Sarasix (Bâle), C. Eusry (Bologne) et L. Joubin (Paris).

3 heures après midi. Séances des sections à l'Univer'sité.

כ heures. Banquet à l'Innere Enge. Concert.

\section{VENIREDI 19 AOUT.}

8 heures 5̃o du matin. Départ pour Interlaken.

Midi. Quatrième assemhlée générale an Kursaal d'Interlaken. Conférences de MIM. A. (irano (Paris) et P. P. C. Hak (Copenhague). ('loture du Congrès.

2 heures ${ }^{3} / 4$. Déjeuner à l'Hôtel Victoria.

8 heures du soir. Concert et feu d'artifices au Kursaal.

10 heures. Retoul à Berne.

SAMEDI 20 AOU'T.

Excursion à Genève.

8 h. 35 du matin. Dépal't de Berne par train spécial pour Genève.

Midi ${ }^{1} / 2$. Téjeuner au Fover clu Théâtre offert par l'Etat et la Ville de Genève.

2 heures. Visite aux Musées et Instituts scientifiques de la ville.

$6 \frac{1}{2}$ heures. Départ en bateau à vapeur spécial pour Genthod.

$i$ henres. Réception offerte par M. Henri de Salsssre et le Comité genevois au Creux de Genthod.

9 heures. Retour à Genève.

$9 \frac{1}{2}$ heures. Fête vénitienne offerte par la Société des Intérêts de Genève. Illumination de la rade. Feu d'artifices.

11 heures. Départ du train spécial pour Berne. 


\section{DÉLËGUÉS OFFICIEIS DES GOUVERNEMENTS}

\section{Autriche.}

L. vox GrafF, Gra\%.

\section{Espagne.}

I. BOLIYAR, Madrid.

\section{Belgique.}

P. Pelsenefr. Gand.

\section{Etats-Unis d'Amérique.}

G. S. Mrller jr., Washington; L. Stejneger, Washington; H. F. Osbonn, NewYork; C. S. Мinot, Boston; C. II. Stiles, Washington.

\section{France.}

E. Pennell, Paris (Président de la délégation); Y. Delage, Paris; A. Giand, Paris; E. L. Bouvier, Paris; L. Vaillant, Paris; L. Joubin, Paris; Ch. Grayer, Paris; R. Blanchand, Paris; A. Ratulet, Alfort près Paris; J. De Guelne, Paris; Ch. Schlumbergen, Paris; A. Jaxet, Paris; A. Pizon, Paris.

\section{Hollande.}

J. W. VAN WIJHE, Groningen:

G. Horvath, Budapest.

\section{Hongrie.}

\section{Italie.}

G. B. Grassi, Rome; L. Enery, Bologna; S. F. Monticeldi, Naples.

K. FuıI, Tokio.

Japon.

\section{Mexique.}

J. Sanchez, Mexico.

\section{Suède.}

E. Lösxberg, Stockholm.

A. Laxg. Ziirich.
Suivse. 
DÉLÉGUÉS DES UNIVERSITES, ACADÉMIES, MUSEEES
ET SOCIÉTÉS SAVANTES

\section{Allemagne.}

Deutsche Zoologische Gesellschaft:

J. W. Spengel, Giessen; E. Konschelt, Marburg.

Hauptstation des forstlichen Versuchswesens in Preussen:

K. Eckstern. Eherswalde.

Herzog1. Naturwissenschaftliche Sammlungen in Coburg:

R. Fischer, Veste Coburg.

Naturwissenschaftliches Museum der Stadt Magdeburg:

A. Mertens, Magdeburg.

Société d'histoire naturelle de Colmar:

J. Bourgeors. Ste-Marie-aux-Mines.

Tierschutzverein Meissen :

C. Schuufuss, Meissen.

University of Adelaide:

Austrenlio.

E. A. Johnson. London.

Autriche.

$\mathrm{Kgl}$. böhmische Akademie der Wissenschaften :

F. VejDovskT, Prag.

Museu Goeldi, Parà:

Brésil.

E. A. Goetdi, Parì.

Etats-Unis d'Amérique.

American Museum of Natural History, New-York:

0. P. H.r, New-York.

American Philosophical Society, Philadelphia:

W. B. Scott, Princeton.

New-York Zoological Society:

H. F. Osbons, New-York. 
United States National Museum, Washington, D. C.:

G. S. Mrcleir jr., Washington, D.C.; L. Stejnegen, Washington, J).C.

United States Public Health and Marine Hospital Service:

Ch. W. Strues, Washington, D. C.

University of California :

J. C. Merriam, San-Francisco.

University of Nebraska:

H. B. WAlI), Lincoln.

\section{France.}

Société française d'Entomologie:

A. Fauvel, Caen.

Société zoologique de France:

E. Hénouard, Paris; L. Joubin, Paris; J. Gulant, Paris.

Université de Bordeaux:

CH. PŕRez. Bordeaux.

\section{Grande Bretagne et Iriande.}

Free Public Museums to the Corporation of Liverpool:

H. O. Forbes, Liverpool.

Royal Zoological Society of Ireland:

R. F. SchanfF, Dublin.

University of Glasgow:

J. E. BLEs, Glasgow.

\section{Hollande.}

Nederlandsche Dierkundige Vereeniging:

P. P. C. Hoek, Copenhague; F. A. Jentink, Leiden.

Société royale de zoologie , Natura Artis Magistra“:

C. Кенвент, Amsterdam.

\section{Hongrie.}

Académie des Sciences de Hongrie :

G. ENTz, Budapest; G. Honvath, Budapest.

Musée national hongrois :

G. Honviti, Budapest; L. vox MÉnecr, Budapest. 


\section{Italie.}

Accademia dei Lincei, Roma :

G.-B. Grassi, Roma.

R. Accademia delle Scienze, Bologna :

C. Emerr, Bologna.

Unione Zoologica Italiana :

G. B. Grassi, Rome; C. Emery, Bologna ; S. F. Monticelit, Naples.

Societå Zoologica italiana, Roma :

R. BlanchaRd, Paris.

\section{Russie.}

Académie Impériale des Sciences de $\mathrm{S}^{\mathrm{t}}$-Pétersbourg :

IV. SAlEnsky, St-Pétersbourg.

\section{Suĩte.}

Académie royale des Sciences de Suède:

E. LönnBerg, Stockholm.

\section{Suisse.}

Société Helvétique des Sciences naturelles:

A. Ling, Zurich; F. Sarasin, Basel. 


\section{MEMBRES D'HONNEUR DU CONGRÈS}

Mir. R. Compesse, Président du Conseil fédéral.

E. Brenner,

A. Deucher,

L. Forrer,

E. MÜller,

M. RuChET,

J. ZEMP,

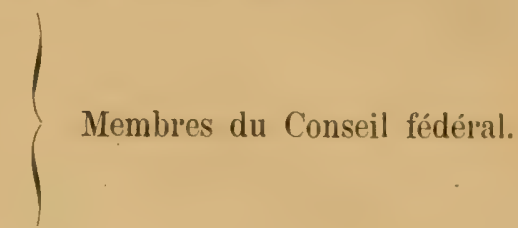

A. Gobat,

A. KílaY,

J. MINDER,

il. Morgenthaler,

J. Ritschard,

Membres du Conseil d'Etat du canton

E. von STEIGER, de Berne.

F. von WaTTENIVYL,

(i. Kínz,

A. von Steiger, Président du Conseil communal.

(i. Mứler, Membre du Conseil communal.

B. Studer,

K. Reisinger,

Membres du Conseil de la Bourgeoisie.

A. Guillebeau, Recteur de l'Université.

C. Friedneim, Doyen de la Faculté de Philosophie de l'Université. 


\title{
LISTE DES MEMBRES DU CONGRES
}

\author{
PAR ORDRE ALPHABÉTIQUE 1
}

* Agram, Zoologisches Nationalmuseum.

Allis, Edw.-L.-L. Dr. Menton, Palais Carnolis.

* Anderson, R.-J., Prof. Galway, Connaught (Ireland), Queens Collegre.

André, E., Dr. Privat-Docent. Genève, Délices, 10.

Andreae, F.-E., Mile, Stud. phil. Zuidhorn (Hollande).

Anthony, R., Dr. Préparateur au Muséum d'histoire naturelle, Paris. -

* Arechavaleta, J., Director general del Museo Nacional. Montevideo.

Arnold, J., Ichthyologiste du Ministère d'Agriculture et des Domaines.

St. Petersburg, Fontanka, 119,4.

Baer, G.-A., Naturaliste voyageur. Paris, me des Messageries, 8.

Baer (von), W.-B. Tübingen.

Bayern (von), Prinzessin Therese. München, Kgl. Residenz.

Beauclair, Médecin-vétérinaire, La Ferté-Bernard (Sarthe).

Beaufort (de), L.-F., Zool. cand. Leusden bei Amersfoort, (Hollande).

Beck, G., Dr. Bern, Bubenbergstrasse 33.

Bedot, M., Prof. Dr. Genève, Musée d'histoire naturelle.

Béraneck, E., Prof. Dr. Neuchàtel, Académie.

* Berlepsch (von), H. Freiherr. Cassel, Landaustrasse, 22.

Bieler, S., Prof. Dr. Lausanne, Champ de l'air.

Bigelow, R.-P., Dr. Boston, Institut of Technology.

Bignon, F., Dr, Mlle Paris $\mathrm{X}^{\mathrm{e}}$, Faubourg Poissonnière, 162.

Blane, H., Prof. Dr. Lausanne, Institut de Zoologie.

Blanchard, R., Prof. Dr. Paris, Boulevard Saint-Germain, 226.

*Blasius, W., Prof. Dr. Braunschweig, Gausstrasse, 17.

Bles, E.-J., Senior Assistant of Zoology. Glasgow, University.

Bloch, J., Prof. Dr. Solothurn.

Bloch, L., Dr. Solothurn, Museum.

${ }^{1}$ Les noms précédés d'un * sont ceux des personnes qui n’ont pas assisté au Congrès. 
Blunischli, H., Dr. Assistent. Zürich, Anatomisches Institut.

* Böhme, R., Dr. Posen, Langestrasse, 4.

Bolivar, I., Prof. Dr. Madrid, Jorge Juan, 17.

Bolsius, H., Prof. Collegium S. J., Oudenbosch (Hollande).

* B on a parte, Prince Roland. Paris, Avenue d'Jéna, 10.

* Borre (de), Preudhomme-A. Entomologiste. Genève, Petit-Saconnex.

Borodine, N. St. Pétersburg, Wass. Ostr. 14, linie 33.

Bourgeois, J., Entomologiste. Sainte-Marie-aux-Mines (Alsace).

Bourquin, J., Genève, Institut zoologique.

Bouvier, E.-L., Prof. Dr. Paris, rue Claude-Bernard, 39.

Bow ditsch, H.-P. Boston.

* Brian, A., Dr. Genova, Corso Carbonara, 10.

Brockmeier, H., Dr, M. Gladbach (Deutschland), Wallstr., 24.

Brolemann, H.-W. Cannes (Alp.-Mar.). Comptoir Nat. d'Escompte de Paris.

*Brunelli, G. Roma, Via Principe Amedeo, 76.

*Buenos-Ayres, Museo Nazional.

Bugnion, E., Prof. Dr. Lausanne, Mont Olivet.

Bïhler, M., Dr Jur. Bern, Bundesgasse, 40.

Bund, J.-Willis. London W. C., Old Square, 5̃, Lincoln's Inn.

Burckhardt, R., Prof. Dr. Basel, Elisabethenstrasse, 20.

Büren-von Salis (von), E. Bern, Käfiggässchen.

Buttel-Reepen (von), H. Dr. Oldenburg, Bismarkstrasse, 32.

Büttik ofer, J., Dr. Direktor des Zool. Gartens, Rotterdam.

Carl, J., Dr. Genève, Musée d'Histoire naturelle.

* Carruccio, A., Prof. Roma.

Caullery, M., Prof. à la Sorbonne. Paris.

* Chevreux, E., Correspondant du Museum. Bône (Algérie).

Chun C., Prof. Dr. Leipzig.

Clarck, H.-L., Dr, Olint. Mich. (U. S. A.).

* Colmar, Société d'Histoire naturelle.

Cori, C.-I., Prof. Triest.

Corning, H.-K., Prof. Dr. Basel, Vesalianum.

* Cosmovici, L.-L., Prof. Dr. Jassy (Roumanie).

Daiber, Marie, I) ${ }^{r}$, Frl. Zürich, Zoologisches Institut.

*Darboux, J.-G., Prof. Marseille, Boul ${ }^{\mathrm{d}}$ Périer, 13.

Dasen, H., Cand. phil. Bern, Schwarztorstrasse, 61.

D autzenberg, P. Paris, rue de l'Université, 209.

Da vid off, M., Dr. Villefranche-sur-Her (Alp.-Har.).

Davinet, E., Architekt. Bern, Waisenhausstrasse, 12.

Dean, Bashford, $)^{r}$. New-York, Columbia University, $437 \mathrm{~W} .59^{\text {th }}$ Street.

Debreuil, Ch. Paris, rue de Chateaudun, 25. 
*Deegener, P., Dr. Nieder-Schönhausen bei Berlin, Lindenstrasse, 20.

*Delage, Y., Prof. Dr. Sceaux, près Paris, Villa de Nice.

Delessert-de Mollins, Eug., Prol. Lutry, près Lausanne.

Deucher, P., Dr, Bern, Marktgasse, 63.

Dick, R., Dr. Bern, Zeughausgasse.

Dragnewitsch, Stud. phil. Bern, Eigerweg, 7.

Driesch, H., Dr. Heidelherg, Philosophenweg, 3.

Duerst, J.-Ulrich, Dr Dozent. Zürich, Englisch Viertel, :34.

Eckstein, K., Prol. Dr. Elerswalde, Nene Schweizerstr. 34.

Ehlers, E., Prol. Dr'. Göttingen, Rosdorferweg, 't.

Emery, C., Prof. Dr. Bologna.

Entz, G., Prof. I)r. Budapest, Esterhazystrasse, 1.

Entz, G.. jun., Dr. Assistent. Budapest, Kgl. Ung. Josephs-Polylechnikum.

Escherich, K., Dr. Privat-1)ozent. Strassburg i. E., Glacisstrasse, 1.

Eternod, A., Prol. Dr. Geneve, Villa Grands Acacias.

Fatio, V.. $\mathrm{D}^{\mathrm{r}}$. Genìve, rue Bellot, 1 .

Fauvel, P.. Prof. D ${ }^{r}$. Angers, Villa Cecilia, rue du Pin, 12.

Fernandez, M., Ziirich, Zoologisches Institut.

Field, H.-H., Dr, Direktor des Concilium bibliographicum. Ziiriclı, Neumünster, Eidmattstrasse, 38.

Fischer. R. Stalısarzt. Veste Coburg.

Flückiger, E., Präparator. Diirrenroth (Ǩt. Bern).

F'lückiger. E., Bern, Schwarzthorstrasse, 38.

Forel, Aug., Prol., Dr. Chign!, pris Horges.

Forel, A.-F., Prol'. Dr. Morges.

Forhes, H.-0., Dr. Liverpool, William Browne Streel.

Franȩois, Ph., Chef des travaux pratiques à la Sorhoune. Paris, rue des Fossés-Saint-Jacques, 20.

Fuhrmann, 0., Prof. Dr. Neuchatel, Académic.

Fujii. K., Prof. 1)r. Tokio, 1). a. München. Theresienstr, z2 II.

*Gadeau de Kerville, H. Rouen, rue Dupont, 7.

*Gaudry, A., Prol. Dr. Paris, rue des St-Pères, 7 bis.

Gadzikiewicz, W. Zürich, Zoologisches Institut.

Ghigi, A., Prof. Dr. Bologna, Via d'Azeglio, 44.

*Giard, A., Prof, Dr. Paris, rue Stanislas, 14.

Gisi, Julie, Frl., Stud. phil. Basel, Thiersteinerallee, 38.

Godel, P., Prol. Neuchâtel, Faubourg du Crêt, 10.

Godlewski, E., Dr. Krakau, Anatomisches Institut.

Gœldi, E.-A., Prof. Dr. Parà (Brésil), Museu Goeldi.

Goll, H., Zoologue. Lausanne. 
Golowine, E., Drivat-Docent. Kasan, Universität.

Gough, L.-H., Dr. Basel, Therwilerstrasse, 36.

Gra effe, E. Dr. Triest. Zoologische Station.

Graff (ron), L., Prof. Dr. Graz, Zoologisches Institut.

Grassi, G.-B., Prof. Dr. Roma, Via Manin, כ̈3.

iravier, Ch., Assistant. Paris, Museum d'histoire naturelle.

Gross, 0., Cand. phil. Basel, Peterskirchplatz, 10.

Gruber. A., Prof. Dr. Freiburg i. Breisgau, Stadtstrasse, 3.

Guerne (de), J. Baron. Paris, rue de Tournon, 6.

Guiart, J., Prol.' Dr. Paris, rue de l'Ecole-de-Médecine. Iö.

Guillebeau, Frau. Bern, Hirschengraben.

Gurwitseh, A., Dr. Privat-Dozent. Bern, Wallgasse, 4 .

II secker, Val., Prof. Dr. Stuttgart, Rheinburgstr. 42.

*Hamm, H., 1) ${ }^{r}$. Osnabrïck, Lortzingstrasse, 4 .

Harrison, R.-G., Prof. Baltimore (U. S. A.).

Haustein. Berlin, Gross Lichterfelde:

Hay, 0.-P. Dr. New-York. American Museum of Natural History.

Heck, L., Dr, IDirektor des Zoolog. Gartens. Berlin W., 62.

Il eilborn, El., Tierarzt. Bern, Kramgasse, 25.

Helbing, H., Dr. Basel, Rosengartenweg, 1.

If ennings, C., Dr-med. u. phil. Charlottenburg.

Herbst, K., Dr. Heidelberg, Blumenstrasse, 9.

ll crouard, E., Maître de conférences à la Sorbonne, Paris.

Hérubel, A.-H. Paris, rue Monge, 112.

*Hescheler, K., Prof. Dr. Zïrich, Zoologisches Institut.

Hess, E., Prof. Dr. Bern, Engestrasse, 10.

*Hetscher, W.-A.-B. Bognor (Sussex), England. Aldwick Manor.

He ymons, R., Prof. Dr. Hannover-Müntlen, Kgl. Forstakademie.

Hilzheimer, M., Dr. Strassburg i. E.. Zoologisches Museum.

Hoek, P.-P.-C., Dr. Copenhague.

Horváth, G., Dr. Budapest, Musée national Hongrois.

Huene (von), F. Dr. Privatdozent. Tüilingen.

I mh of, O.-E., Dr. Windisch. (Kit. Aargau).

I sensehmid, M.. Dr. Bern, Schänzlistrasse, $\mathbf{4} 7$.

*Issakowitsch. A. Orlessa.

*Issakowitsch. S. Odessa.

*Issel, R., 1)r. Libero docent di Zoologia in Modena.

I wanow, P.-1P. St-Petershury, Guttenhergstr., 12.

Jacobi, A., Prof. Dr. Tharandt (Saxe), Kgl. Forstakademie.

Janet, A., Ingénieur de la marine, Paris XVe, rue des Volontaires, 2!).

J anet, Ch.. J)r, Beauvais (Oise), rue de Paris, 71 . 
Janet, H., Etudiant, Paris XVe, rue des Volontaires, 29.

Janicki (von), C., Cand. phil. Basel, Leimenstrasse, 60.

Jentink, F.-A., Dr ${ }^{r}$, Direktor des Rijks-Museums. Leiden.

* Jjima, J., Prof. Dr. Tokio, Sci. Coll. Imp. University.

* Jverus, Jedo, Dr. Lovisa (Finlande).

Kallenberger, W., Dr.-Méd. Cannstadt.

Kampen (Van), P. N., Conservator am Zoologisches Huseum. Amsterdam, Singel 330.

* Kathariner, L., Prof. Dr. Freiburg, Universität.

Keller, C., Prof. Dr. Ziïrich. Polytechnicum.

Kempe, H.-A.-E., Dr. Bern, Bühlstrasse, 37.

*Kempen (van), Ch. St-Omer (Pas-de-Calais), rue St-Bertin, 12.

Kennel.(von), J., Prof. Dr. Dorpat, Loologisches Huseum, K. Universität.

Kerbert, C., Dr, Directeur de la Societe Rovale "Natura Artis Magistra ". Amsterdam.

Kirkaldy, J.-W. Oxford, Banbury Road, 12.

Kle inschmidt, O., Pastor. Volkmaritz, Bezirk Halle a. S.

Kohler, R., Prof. Dr. Lyon, rue Guillard, 29.

* Kolbe, H. J.. Prof. Gross Lichterfelde bei Berlin, Steinackerstrasse, 12.

Kollibay, P., Rechtsanwalt, Neisse (Schlesien), Victoriastrasse, $\mathbf{I}^{\mathrm{I}}$.

Kölliker (von), R.-A., Prof. Dr. Würzburg, Anatomisches Institut.

Kollmann, J.. Prof. Dr. Basel. St-Johann. 88.

Korolneff., A.-L., Prof. Dr. Kieff.

Kronecker, H., Prof. Dr. Bern. Erlachstrasse, 23.

Kupelwiese, H., Dr. Wien, Weigburgg, 32.

Lameere, A. Prol. Bruxelles, avenue du Haut Pont, 10.

Lang, A., Prol.' Dr. Zürich IV, Rigistrasse, 50.

Lan g, Th., I ${ }^{\mathrm{r}}$-merl. Bern, Christoflelgasse, 4 .

Langhans, Th., Prof. Dr. Bern, Rabbenthalstrasse, 38.

*La Roche, R., Stud. phil. Bern, Zähringerstrasse, 1/4.

Laskowski, S., Prof. Dr. Genève, rue de Carouge, 110.

Lehrs, Ph., Stud, phil. Dresden.

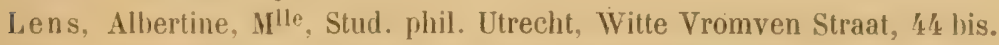

*Lenz, H., Prof. Dr. Lübeck, Naturhistorisches Museum.

Lessert (de), R., $\mathrm{D}^{\mathrm{r}}$. Genève.

Levien, M., Stıd. tech. pract. Winterthur, Bankstrasse, ə̃.

* Li esegang, E., Fabrikant. Düsseldorf.

*Lilchrist, J.-D.-F. Cape Town (South Africa), Agriculture Department.

Linais, II., Etudiant. La Ferté-Bernard. (Sarthe).

Linden (von), Maria, Grüfin, Dr. Bonn, Zoologisches Institut.

Linder, Ch., Dr. Lausanne, avenue de Montagibert; 10.

VIo CONGR. INT. ZoOL., 1904. 
*Loisel, G., Dr. Préparateur ì li Faculté des Sciences. Paris. Pue de l'Ecole de Médecine, 6.

Lönnberg, E., Prof. Dr. Stockholm, Vetenskap Akademia.

Loos, A., Prof. Dr. Le Caire, School of Medecine.

Lotma r, F., Dr-méd. Bern, Feldeggweg, 3.

*Lucanus (von), Fr., Oberleutnant. Berlin, Werftstrasse, 1 '4.

Liihe, M., Dr. Privat-docent. Königsberg i. Pr., Tragheimer Pulverstrasse, 4 a.

Ma as. 0., Prof. Dr. Mïnchen, Zoologisches Institut, Neuhauserstrasse.

Maehrenthal (von), Fr. C., Prof. Dr. Berlin, N. W. Invalidenstrasse, 43.

* Magretti, P., Dr. Milano, Foro Bonaparte, 76.

Malaquin, A., Prof. Dr. Lille, rue Brûle-Maison, 159.

II Isen (von), H., Freiherr. Lindau, Villa Amsee.

Matschie, P., Prof. Dr. Kustos am Zoolog. Museum. Berlin.

* Irarassowitsch, Dr. Skoardin-Skardona (Dalmatien).

Marval (de), L., I)r. Voens, près Saint-Blaise (Neuchâtel).

Ma yer-Eymar, C., Prof. I) . Zürich.

Méhely (von), L., Prof. Budapest, National Juseum.

* Meissner, M., Dr. Berlin, Invalidenstrasse, 43.

Ménégaux, H.-A. Prof. Dr. Assistant au Museum d'histoire naturelle, Paris.

Menneking, F., Tierarzt. Oldenburg, Marienstrasse, 14.

Merian, P., Stud. phil. Basel, Albananlage, כ̆4.

Merriam, J.-C. Berkeley. University of California.

Mertens, A., Dr. Magdeburg, Mittelstrasse, 49.

* Merton, H., Stud. zool. Heidelherg, Zoologisches Instilut.

Mesnil, M. Prof. Paris, Institut Pasteur, rue Dutot.

Metalnikoff, Sergino. St. Petersburg.

Meyer, A., Prof., Dr. Essen (Ruhr), Akazienallee.

Meyer, E., Prof. Dr. Kasan, Université.

* Millet, F.-E.-S. Eniscœ-Briaham, Devon (England).

Miller, Gerrit-S. Washington D. C.

Minot, Ch.-S., Prof. Dr, Boston (Mass), Harvard Medical School.

*Möbius, K., Prof. Dr. Berlin, N. Invalidenstrasse, 43.

* Monaco (de), Prince Albert I. Monaco.

Monti, A., Prof. Dr. Pavia

Monti, R., MIle, Dr, Privat-docent, Pavia.

Monticelli, E.-S., Prof. Dr. Napoli, R. Università.

* Morton, W. Naturaliste, Lausanne, Villa Collonges.

Mottaz, Ch., Zoologiste. Genève, Grand Pré, 39.

Miiller, P., Prof. Dr. Bern, Schanzenstrasse.

Muri i ier, P., Assistant. Lausanne, Place du Tunnel, 11.

Musỵ, II., Prof. Fribourg. Musée d'Histoire naturelle 
* Navas, L.-S.-J. Zaragoza, Collegio del Salvadore.

Neresheimer, E., Dr. Assistent. Minchen, Zoolog. Institut, Alte Akademie. * Newton, A., Prof. Cambridge, Magdalene College.

Nibelle, M.. Avocat. Rouen, rue des Arsins, 9.

Nierstrasz, H.-Fr., Dr. Utrecht. Willem Barentz Street, 73.

Nopesà, F., Dr. Szacsal 1. p. Hatszeg (Ungarn).

No yer, E., Prof. Dr. Bern, Engestrasse, 10.

* Obst, P., Dr. Berlin.

* Odier, H. Bern, Sulgenauweg, 24.

Olivier, E. Moulins (Allier), Cours de la Préfecture, 10.

Osborn, H.-F., Prof. New-York. American Museum of Nat. Ilist.

* Oudemans, J.-Th., Dr. Amsterlam, Paulus Potterstraat, 12.

Oxner, Mieczyslaw. Zürich. Zoologisehes Institut.

* Oye (van), S., Prof. Dr. Lille. rue de Toule. 11.

Paeske, E. Berlin I, Besselstrasse, 12.

Palacky, J. Prof. Dr, Prag, Weinberge, 114.

Peaumier, J. La Ferté-Bernarl (Sarthe).

Pellegrin. J., Dr. Paris, I'ue de Rennes, 143.

Pelseneer, P., Prof. Dr. Gand.

Penard, E., Dr. Genève, avenue Harc-Monnier, 7.

*Perez. Ch., Prof. Dr. Bordeaux, Université. Institut de Zoologie, Cours St-Jean.

Perrier, E., Prof. Dr. Paris, Jardin des Plantes, rue Cuvier, 57.

Petersen, W., Direktor der Realschule. Reval (Esthonie).

Pictet, Arnold. Genève, rue Petitot, 12.

Piepers, M.-C. La Haye, Noorleinde, $10^{\mathrm{a}}$.

Piéron, H., Préparateur à l'Ecole des Hautes Etudes. Paris.

Pizon, A., Prof. Dr. Paris, rue de la P'ompe, 92.

Pierantoni, U., Prof. Napoli.

Plate, L., Prof. Dr. Berlin, Institut fïr Meereskunde, Georgenstr. 34.

Popoli, N., Assistant au Laboratoire d'Embryologie. Lausanne. Université.

Popta, C -M.-L., Mlle, Dr,. Leiden, Nonnesteeg, 5.

* Porter, C.-k., Prol. Directeur du Musée, Valparaiso.

Pruvot. G., Prof., Dr. Prris, Laborat. d'Anatomie comparée, Sorbonne.

* Racovitza, E.-G., Sous-directeur du Laboratoire Arago. Banyuls-sur-Mier, (Pyr.-Orient.).

Railliet, A., Prof. Dr. Alfort, près Paris, Ecole vétérinaire.

* Raspail, X., Vice-prés. de la Soc. Zool, de France. Gouvieux (Oise).

Reitzenstein (von), Frl. Dr. Freiburg i. Br. Dreikönigstrasse, 46.

Rengel, C., Dr. Schöneberg hei Berlin, Beckerstrasse, 2.

Revilliod. P. Genève. 
*Richard, J., Dr, Directeur du Musée Océanographique. Monaco.

Ritter zu Grünstein (von), Freiherr, K. bayerischer Ministerresident. Bern, Kirchenfeldstrasse, 90.

*Rivera, M. J., Prof., Santiago (Chili). Istituto Agricola.

*Rodzianko, W. N., Poltawa, Kusnetzkaja, 33 (Russie).

Rohweder, J., Gymnasiallehrer. Husum (Schleswig-Holstein).

Rothenbühler, H., Dr. Bern, Wildhainweg, 12.

Rotrou, A., Pharmacien. La Ferté-Bernard (Sarthe).

Rosa, Prof. Dr. Modena, p. a. Museo Zoologico, Torino.

Rousselet, Ch. London W. Pembridge Crescent 2, Bayswater.

Roux, J., Dr. Basel, Naturhistorisches Museum.

Rubeli, 0., Prof. Dr. Bern, Alpéneckstrasse.

Riitimeyer, L., Dr. Basel.

Rytz, W., Stud. phil. Bern, Marienstrasse.

Saboussow, A., Dr, Kasan, Universität.

S aint-Hila ire, C., Prof. Jurjew (Russland). Universität.

Salensky, W., Prof. Saint-Pétershourg.

Sanchez, Jesus, Dr-med. Mexiko, Ecole Préparatoire.

Sarasin, Ch., Prof. Dr. Genève.

Sarasin, F., Dr. Basel, Spitalstrasse 22.

Sarasin, P., Dr. Basel, Spitalstrasse 22.

* Saussure (de), H. Genève, Tertasse.

Sauvage, E. Dr. Boulogne-sur-Mer (France).

Sch a erer, M., Sanitätsgeschäft. Bern, Marktgasse 63

Scharfi, R.-F., Dr. Dublin, Museum.

Schaudinn, Fr., Dr, Regierungsrat. Berlin-Halensee, Ringbahnstr. 128.

* Schaufuss, C., Meissen, Tierschutzverein.

Schauinsland, H.-H., Prof. Dr. Bremen, Städt. Huseum fïr Naturkunde.

Schenk, Al., Prof. Lausanne.

Scherren, H. London, 9 Cavendish Road, Haarringay.

Schewiakoff, W., Dr. Saint-Petersburg, Wassili Ostrow ö, linie 20.

Schlachter. I., Dr. Basel.

Schlaginhaufen. O..Assistent am Anthropol.' Institut. Zuirich, Dufourstrasse, 47.

Schlumberger, Ch., Ingénieur én chef de la marine. Paris VIIIe, rue Christophe-Colomb, 16.

Schmidt, P., Assistent der Zoologie, Hochschule für Frauen. St-Petersburg, Wassili Ostrow $8,53 / 1$ k.

Schmidt, W., München, Zool. Institut, Alte Akademie. Neuhauserstrasse!

Schneider, A., Dr. Mïnchen, Dachauerstrasse, 1 อ̆

* Schneider, G., Conservator. Basel, Grenzacherstrasse, 67.

Schwner, H. Roma, Via Venti Settembre, 4.

Schulz, A., Assistent der Sammlung des Zoolog. Instituts. Strassburg. 
Schürch, 0., Dr. Langnau.

Schweyer, A., Assistant à l'Institut zootomique. St-l'étersbourg, Université impériale.

Scott, B.-W., Prof. Princeton, New-Jersey (U. S. A.).

Scourfield, D.-J., Dr. Leytonstone (Essex), Queens Road, 63.

Selys-Longch amps (de), M., Dr. Ciney (Belgique).

*Sergent, Ed. $D^{r}$. Paris, Institut Pasteur, Rue Dutot.

Sharp, Benj., Prof. Dr. Philadelphia, Academy of natural sciences.

* Siebeck, 0., Dr. Bern, Bubenbergplatz, 7 .

Siedleki, M., Prof. Dr. Krakau, Collegium Physicum.

Simroth, H., Prof. Dr Leipzig-Gautzsch, Kregelstrasse, 12.

Smalian, K., Dr, Hannover, Göthestrasse, 3 כ̃.

Soukatscholi,, B., Dr. St-Pétersbourg, Institut Zootomique de l'Université.

Spemann, H., Dr. Würzburg, Zoologisches Institut.

Spengel, J.-W., Prof. Dr. Giessen, Zoologisches Institut.

Spiess, C., J)r. Basel, Langegasse, 19.

Spillmann, J. Zürich, Zoologisches Institut.

Standfuss, M., Prof. Dr. Zürich, Kreuzplatz, 5.

Steche, Dr-med. Dresden.

Steck, L., Stud. phil. Bern, Jägerweg, 9.

Steck, Th., Dr. Bern, Tillierstrasse, 8.

Stehlin, H.-G., Dr. Basel, Naturhistorisches Museum.

Stejneger, L., Dr. Washington, D. C. National Museun.

Stelling, $\mathbf{D}^{\mathrm{r}}$, Chemiker. Kopenhagen,

Stieda, S., Prof.-Ir. Königsberg, Anatomisches Institut, Oberlaak 8/9.

Stiles, Ch.-IV., Chef of the Division of Zoology. Washington D. C.

Stingelin, Th., Dr. Olten.

Strasser, II., Prof., Dr. Bern, Finkenhubelweg, 20.

Strasser, Ch., Stud. med. Bern, Finkenhubelweg, 20.

* Strauch, C., Dr. Privat-docent. Berlin, N. W. C., Luisenplatz, 9.

*Surbeck, G., Dr. München, Nymphenburgerstrasse, 176.

Stromer, E., Dr. Mïnchen, Schönfeldstrasse, 26.

Studer, Th., Prot. Dr. Bern, Niesenweg, 2.

T'eichmann, E., 1)r. Frankfurt a. M., Tannenstrasse, 7 II.

Thienemann, A., Stud. zool.. Heidelberg, Gaisbergstr. 78.

Tornier, G., Prof. Dr. Berlin, Zoologisches Museum.

T'scharner (von), L., Dr, Oherst. Bern, Mïnsterplatz.

*Trapel, L.-J. Paris.

*Vaillant, L., Prof. Dr. Paris Ve, rue Geoffroy-St-Ililaire, 36.

Valentin, A., Prof. Dr. Bern., Laupenstrasse, 7.

Vaney, C., Ilaitre de conférences de Zoologie, Faculté des Sciences. Lyon. 
Vejdovsky, F., Prof. Dr. Prag., Myslikgasse, 12. Vischer, A., Stud. med. Bern, Sonnenberg, 14. Vles, P. Paris, XVIe, Villa Mozart, 3.

*Vögler, R., Dr. Frankfurt a. M., Gärtnerweg, 20. Volz, W., Dr.Assistent. Bern, Zoologisches Institut. Vosmaer. G.-C.-J., Prof., Dr, Leiden. Universität.

Wagner, 0., Dr. Bern, Pavillonweg, 5.

* W a gner (de), V. Dr. Moskau, Institut St-Catherine. W ard, H.-B., Prof. Lincoln, University of Nebraska.

W a smann, E., Luxembourg, Bellevue.

* Watten Wyl (von), J., Oberst. Bern, Elfenau.

Wijhe (van), J.-W., Prof. Dr. Groningen, Westersingel, 33.

Williams, D., Miss. Boston (Mass). Biolog. Dep., Normal School.

Winter. P.-H. Frankfurt a. M., Finkenhotstrasse, 27.

Wolterstorf, W., Dr. Magdehurg, Domplatz, 5.

Yung, E., Prof. Dr. Genève, Boulevard Helvétique, 6.

Zavret, J., Prof. Dr. Trebitsch (Mähren).

Zeerleder, F., Forstmeister. Bern, Junkerngasse, „̈l.

Zimmermann. K.-W., Prof. $\mathbf{D}^{\mathrm{r}}$. Bern, Seilerstrasse, $7^{\mathrm{a}}$.

* Zograf (von), G., Assistent. Moskau, Rusa Landgut Mytniki.

*Zograf (von), N., Prof. Dr. Moskau, Rusa Landgut Mytniki.

Zschokke, Fr., Prof. Dr. Basel, Schiitzengraben, 33.

Zuelzer. M.-Frl.. Dr. Berlin. 


\title{
LISTE DES MEMBRES DU CONGRĖS
}

\author{
PAR ORDRE GEOGRAPHIQUE ${ }^{1}$
}

\section{Allemagne.}

Baer (ron), W. B., Tuibingen. Bayern (von), Prinzessin Therese, München.

Berlepsch (von), H., Kassel. Blasius, IV., Braunschweig.

Böhme, R., Posen.

Bourgeois, J., Ste-Marie-aux-Mines.

Brockmeier, H., Gladbach.

Buttel-Reepen (von), H., Oldenburg. Chun, C., Leipzig.

Colmar, Musée d'Histoire Nalurelle. Deegener, P., Berlin.

Driesch, H., Heidelberg.

Driesch, Frau, Heidelberg.

Eckstein, K., Eberswalde.

Ehlers, E., Güttingen.

Escherich, K., Strassburg i. E.

Fischer, R., Veste-Koburg.

Fischer, Frau, Weste-Koburg:

Gruber, A., Freiburg i. B.

Gruber, Frau, Freiburg i. B.

Hrecker, V., Stuttgart.

Hamm, H., Osnabrïck.

Haustein, Berlin, Grosslichterfelde.

Heck, L., Berlin.

Heck, Frau, Berlin.
Hennings, C., Charlottenburg.

Herlst, K., Heidelberg:

Heymons, R., Hannover-Münden.

Heymons, Frau, Hannover-Münden.

Hilzheimer, MI., Strasshurg i. E.

Huene (von), F., Tübingen.

Huene (von), Frau, Tiibingen.

Jacohi, A., Tharandt.

Kallenberger, W., Cannstadt.

Kleinschmidt, 0., Volkmaritz (Halle).

Kolbe. H. J.. Berlin.

Kollibay, P.. Neisse.

Kollibay. II. Fran. Veisse.

Kölliker (von), R. A. Würzhurg.

Lehrs, Ph., Dresden.

Lenz, H., Lübeck.

Liesegang, Diisseldorf.

Linden (von), M., Frl., Bonn.

Lucanus (von), F., Berlin.

Lühe, M., Königsberg i. P.

Maas, 0., München.

Maas, Frau, Miinchen.

Hiehrenthal (von), Fr. C., Berlin.

Malsen (von), H., Lindau.

Malsen (von), Frl., Lindau.

Matschie, P., Berlin.

Meissner, M.. Berlin.

Henneking, F., Oldenburg.

${ }^{1}$ Les noms des dames inscrites pour suivre les travaux dı Congrès figurent dans cette liste. 
Mertens, A., Magdeburg.

Mertens, Frau, Magdeburg.

Merton, H., Heidelberg.

Meyer, A., Essen a. Ruhr.

Möbius, K., Berlin.

Neresheimer, E., München.

Neresheimer, Frau, München.

Neresheimer, Frl., München.

Obst. P., Berlin.

Preske, E., Berlin.

Plate, L., Berlin.

Plate. II. Frau. Berlin.

Reitzenstein (von), Freiburg i. Br.

Rengel, C., Berlin.

Richter, Frau, Lindau.

Roeder, A., Frau, Frankfurt a. II.

Rörig, Frau, Frankfurt a. M.

Rohweder, J., Husum.

Schaudin, Fr., Berlin-Halensee.

Schaufuss, C., Meissen.

Schauinsland, H. H., Bremen.

Schmidt, W., Miinchen.

Schneider, A., München.

Schulz, A., Strassburg i. E.

Simroth, II., Leipzig (Gautsch).

Smalian, K.. Hannover.

Spemann, H., Würzburg.

Spemann, Frau, Würzburg.

Spengel, J. W., Giessen.

Steche. Dresden.

Stieda, S., Königsberg i. P.

Strauch. C... Berlin.

Surbeck, G.. Miinchen.

Stromer, E., Mïnchen.

Teichmann, E.. Frankfurt a. M.

Thienemann, A., Heidelberg.

Tornier. G., Berlin.

Tornier, Frau, Berlin.

Vögrler, K., Frankfurt a. M.

Wertheimer-Rolialoritsch, R., Frau,

Frantifurt a. $\mathbf{I}$.

Winter, F. II., Frankfurt a. M.

Winter. Frau. Frankfurt a. M.
Wolterstorff, W., Magdeburg.

Zuelzer, G., Frl., Berlin.

Zuelzer, M., Frl., Berlin.

Zürn, MI., Frl., Würzburg.

Argentine (République).

Buenos-Ayres, Museo Nazional.

\section{Autriche.}

Cori, C. J., Triest.

Cori, Frau, Triest.

Godlewski, E., Krakau.

Graeffe E.. Triest.

Graff (von), L., Graz.

Kupelwiese, H., Wien.

Marrassowich, Skoardin-Skardona.

Palacky, J., Prag.

Rehah, A., Frl., Prag.

Reifferscheidt, Frl., Meran.

Siedlecki, M., Krakau.

Vejdowski, F., Prag.

Zarret, J., Trebitsch (Mähren).

\section{Belgique.}

Lameere, A., Bruxelles.

Pelseneer, J., Gand.

Pelseneer, P., Nime, Gand.

Pelseneer, A., Mlle, Gand.

Selys-Longchamps (de), M. Ciney.

Brésil.

Goeldi, E. A. Parà.

\section{Chili.}

Porter, C.-E., Valparaiso.

Rivera, M. J., Santiago.

\section{Colonie du Cap.}

Lilchrisı, J.-D.-F., Capetown.

\section{Danemark.}

Hoeck, P. P. C., Copenhague.

Hoeck, IIme, Copenhague.

Stelling, Copenhague. 


\section{Egypte.}

Looss, A., Le Caire.

Looss, Frau, Le Caire.

\section{Espagne.}

Bolivar, J., Madrid.

Bolivar, J. P., Mme, Madrid.

Navàs, L., S. J., Zaragoza.

\section{Etats Unis d'Amérique.}

Bigelow, R. P., Boston.

Bowditch, H. P., Boston.

Clark, Hubert L., Olint, Mich.

Dean, Bashford, New-York.

Harrison, R. G.. Baltimore.

Hay, 0. P., New York.

Hay, Mrs., New York.

Merriam, J. C., Berkeley, California.

Herriam, J. B., Mrs., Berkeley, California.

Miller, Gerrit, Washington D. C.

Miller, Mrs, Washington D. C.

Minot, Ch. S., Boston.

Osborn, II. F., New York.

Osborn, Mrs., New-York.

Scott, B. IV., New Jerser.

Sharp, B., Philadelphia.

Stejneger, I. Washington D. C.

Stejneger, Mrs., Washington D. C.

Stiles, Ch. W., Washington D. C.

Ward, H. B., Lincoln (Nebraska).

Ward, R. H., Mrs., Troy, New-York.

Williams, D., Miss, Boston.

\section{France.}

Allis, E., Menton.

Anthony, R., Paris.

Baer, H.-G., Paris.

Baer, IIme, Paris.

Beauclair, La Ferté-Bernard.

Bignon, F. MHle, Paris.
Blanchard, R., Paris.

Blanchard, $\mathbf{M}^{\text {ine }}$, Paris.

Bonaparte, Prince Roland, Paris:

Bouvier, E.-L., Paris.

Bouvier, $\mathrm{II}^{\mathrm{Hle}}$, Paris.

Brolemann, H. W., Cannes.

Caullery, M., Paris.

Chevreux, E., Bône (Algérie).

Coupey, IIme, La Ferté-Bernard.

Darboux, J.-G., Marseille.

Dautzenberg, Ph., Paris.

Davidoff, M., Villefranche s. M.

Debreuil, Ch., Paris.

Delage, Y., Sceaux.

Fauvel, P., Angers.

Franeois, Ph., Paris.

Gadeau de Kierville, A., Rouen.

Gaudry, A., Paris.

Giard, A., Paris.

Gravier, Ch., Paris.

Guerne (de), J., Paris.

Guiart, J., Paris.

Hérouard, E., Paris.

Hérubel, A. M., Paris.

Ilodgson, $\mathbf{H}^{\mathrm{Hle}}$, Paris.

Janet, A., Paris.

Janet, Ch., Beauvais.

Janet, H., Paris.

Janet, $\mathbf{I}^{\mathrm{me}}$, Beauvais.

Kempen (van), Ch., St.-Omer.

Koehler, R., Lyon.

Linais, Moïse, La Ferté-Bemard.

Loisel, G., l'aris.

Malaquin, A., Lille.

Mare, IIme $^{\text {A., }}$, Rouen.

Ménégaux, Aug., Paris.

Mesuil, F., Paris.

Nibelle, M., Rouen.

Olivier, E., Moulins (Allier).

Oye (van), S., Lille.

Paumier, J., La Ferté-Bernard.

Pellegrin, J., Paris.

Pérez, Ch., Bordeaux. 
Perrier, E.. Paris.

Piéron, H., Paris.

Piéron, IIme, Paris.

Pizon, A., Paris.

Pizon, Mine, Paris.

Pruvot, G., Paris.

Racovitza, E.-G., Banyuls-s.-Mer.

Railliet, A., Alfort.

Raspail, H., Gouvieux.

Rotrou. A., Paris.

Sauvage, E., Boulogne-sur-\$ler.

Schlumberger, Ch., Paris.

Sergent, Ed., Paris.

Trappel L.-J., Paris.

Vaillant, L., Paris.

Vaney, C., Lyon.

Vaney, Mme, Lyon.

Vles, F., Paris.

Vles, IIme $^{\text {me }}$ Paris.

\section{Grande Bretagne et Irlande.}

Anderson, R. J., Galway.

Bles, J. E., Glasgow.

Bles, B., Mrs., Glasgow:

Bund, J. W., London.

Bund, Mrs., London.

Forles, H. O., Liverpool.

Hetscher, W. A. B., Bognor.

Kirkard, J. W., Oxford.

Millet, F. W. Eniscoe-Briaham-Devon.

Nervton, A., Cambridge.

Rousselet, Ch. T., London.

Rousselet. Mrs.. London.

Scharff, R. F., Dublin.

Scharff, Mrs., Dublin.

Scherren, H., London.

Scourlield, J., Leytonstone.

\section{Hollande.}

Andrex, Mlle, Zuidhorn.

Beaufort (de), L. F., Leusden I). Amersfoort.
Bolsius, H., Oudenbosch.

Büıttikofer, J., Rotterdam.

Büttikofer, IIme, Rotterdam.

Jentink, F. A., Leiden.

Jentink, Mlle, Leiden.

Kampen (van), P. N., Amsterdam.

Kerbert, C., Amsterdam.

Kerbert, $\mathbf{M}^{\text {me }}$, Amsterdam.

Landenberg, $\mathbf{M}^{\mathrm{lle}}$, Utrecht.

Lens, MIle, Utrecht.

Nierstrasz, H. Fr., Utrecht.

Oudemans, J. Th., Amsterdam.

Piepers, M. C., La Haye.

Popta, C. M. L., MIle, Leiden.

Vosmaer, G. C. J., Leiden.

Wijhe (van), J. W., Groningen.

\section{Hongrie.}

Agram, Musée national hongrois.

Entz, G., Budapest.

Entz, G., Budapest.

Horvath, G., Budapest.

Méhely (von), L., Budapest.

Nopesa, F. Szacsal par Hatszeg.

\section{Italie.}

Brian, A., Genova.

Brunelli, G., Roma.

Carruccio, A., Roma.

Emery, C., Bologna.

Gighi, A., Bologna.

Gighi, Signora, Bologna.

Grassi, G.-B., Roma.

Issel, R., Modena.

Magretti, P., Milano.

Jonti, A., Pavia.

Monti, Rina, Signorina, Pavia.

Monticelli, F. S., Napoli.

Pierantoni, U., Napoli.

Rosa. D., Modena.

Schoner, H., Roma. 
Japon.

[iujii, K., Tokio.

Jjima, J., Tokio.

\section{Luxembourg.}

Wasmann, E. C. J., Luxembourg.

\section{Mexique.}

Sanchez, J., Mexico.

\section{Monaco.}

Monaco (de), Prince Albert I. Richard, J., Monaco.

\section{Roumanie.}

Cosmovici, L. C. Prof., Jassy. Cosmovici, $\mathbf{I}^{\mathrm{me}}$, Jassy.

\section{Russie.}

Arnold, J., St. Pétersljourg.

Arnold, M'me, St. Pétersbourg.

Bolkowska, G., Mlle, Polen.

Borodine, N., St. Pétersbourg.

Golowin, E., Kasan.

Issakowitsch, A., Odessa.

Issakowitsch, S., Odessa.

Iwanow; P. P., St. Pétersbourg.

Joerus, J., Lorissa, Finlande.

Kennel (von), J., Dorpat.

Kennel (von), Mme, Dorpat.

Kennel (von), Mlle, Dorpat.

Korotneff, Al., Kiew.

Korotneff, Mme, Kiew.

Metalnikoff, Sergino, St. Pétersbourg:

Heyer, E., Kasan.

Petersen, W.., Reval.

Rodzianko, W. N., Poltawa.

Saboussow, N, Kasan.

Saint-Hilaire, C., Dorpat.

Saint-Hilaire, IIme, Dorpat.

Salensky, W., St. Pétersbourg.

Schewiakoff, IV., St. Pétersbourg.

Schmidt, P., St. Pétershourg.
Schweyer, A., St. Pétersbourg.

Schweyer, $\mathbf{M}^{\mathrm{me}}$, St. Pétersbourg.

Soukatschofi, B., St. Pétersbourg.

Soukatschofi, B., Mme, St. Pétersbourg.

Wagner (de), V., Moscou.

Wagner (de), Mme, Hoscou.

Zograf (von), G., Moscou.

Zograf (von), N., Moscou.

\section{Suède.}

Lönnberg, E., Stockholm.

\section{Suisse.}

André, E., Genève.

Auer, G., Frl., Bern.

Auer, L., Frl., Bern.

Beck, G., Bern.

Bedot, M., Genève.

Béraneck, E., Neuchâtel.

Bieler, S., Lausanne.

Blanc, H., Lausanne.

Blanc, $\mathbf{M}^{\mathrm{me}}$, Lausanne.

Bloch, J., Solothurn.

Bloch, L., Solothurn.

Bluntschli, H., Zürich.

Borre (de), Preudhomme A., Genève.

Bourquin, J., Genève.

Bugnion, E., Lausanne.

Bühler, M., Bern.

Burckhardt. R.. Basel.

Büren-von Salis (von), E., Bern.

Büren-von Salis (von), Frau, Bern.

Carl, J., Genève.

Corning, H. K., Basel.

Daiber, M., Frl., Zürich.

Dasen, H., Bern.

Davinet, E. Bern.

Delessert, Eug., Lutry.

Deucher, P., Bern.

Dick, R., Bern.

Diesbach - von Tavel (von), Frau, Bern. 
Dragnewitsch, Bern.

Dragnewitsch, Frau, Bern.

Duerst, U., Zürich.

Eternod, A., Genève.

Eternod, Mme, Genève.

Fatio, V., Genève.

Fatio, I $^{\mathrm{me}}$, Genève.

Fernandez, M., Zürich.

Field, H. H., Zuirich.

Field, Frau, Zürich.

Fischer, Frau, Bern.

Flückiger, E., Bern.

Flïckiger, Frl., Bern.

Flückiger, E., Dürrenroth.

Forel, Aug., Chigny (près Morges).

Forel, F. A., Morges.

Fuhrmann, 0., Neuchâtel.

Gadzikiewicz, W., Zürich.

Gisi, J., Frl., Basel.

Godet, P., Neuchatel.

Goll, H., Lausanne.

Gough, L. H., Basel.

Graf, J.-H., Bern.

Graf, Frau, Bern.

Gross, 0., Basel.

Guillebeau, Frau, Bern.

Gurwitsch, A., Bern.

Heilborn, E., Bern.

Helbing, II., Basel.

Hescheler, K., Zürich.

Hess, E., Bern.

Hess, Frau, Bern.

Hirschfeld, Frau, Bern.

Imhof, 0. E., Windisch.

Isenschmid, M., Bern.

Isenschmid, Frau, Bern.

Isenschmid, Frau, Bern.

Janicki (von), C. Basel.

Kathariner, L., Frilıourg.

Keller, C., Zürich.

Kempe, H., A., Bern.

Kollmann, J., Basel.

Kronecker, I., Bern.
Kronecker, Frau, Bern.

Kronecker, Frl., Bern.

Lang, A., Zürich.

Lang, Frau, Zuirich.

Lang, Th., Bern.

Lang-Zschokke, Frau, Bern.

Langhans, Th., Bern.

La Roche, R., Bern.

Laskowsky, S., Genève.

Lessert (de), R., Genève.

Levien, M., Winterthur.

Linder, Ch., Lausanne.

Lotmar, F., Bern.

Marval (de), L., St-Blaise.

Mayer-Eymar, C., Zuirich.

Merian, P., Basel.

Morton, W., Lausanne.

Hottaz, Ch., Genève.

Miiller, P., Bern.

Müller, Frau, Bern.

Müller, T., Frl., Bern.

Müller, M., Frl., Bern.

Müller (von), D., Frl., Bern.

Müiller (von), F., Frl, Bern.

Murisier, P., Lausanne.

Musy, M., Fribourg.

Noyer, E., Bern.

Noyer, Frau, Bern.

Noyer, Frl., Bern.

Odier H., Bern.

Oxner, M., Zürich.

Penard, E., Genève.

Pictet, A., Genève.

Popofi, N., Lausanne.

Revilliod, P., Genève.

Ritter z. Grünstein (von), Bern.

Rothenbühler, H., Bern.

Roux, J., Basel.

Rubeli, 0., Bern.

Rubeli, Frau, Bern.

Rütimever, L., Basel.

Rytz, W., Bern.

Sarasin. Ch., Genève. 
Sarasin, F., Basel.

Sarasin, P., Basel.

Saussure (de), H., Genève.

Schaerer, M., Bern.

Schenk, Al., Lausanne.

Schlachter, L., Basel.

Schlaginhaufen, 0., Zürich.

Schneider, G., Basel.

Schürch, 0., Langnau.

Siebeck, 0., Bern.

Spiess, C., Basel.

Spillmann, J., Zürich.

Standfuss, M., Zürich.

Steck, L., Bern.

Steck, Th., Bern.

Stehlin, H. G., Basel.

Stingelin, Th., Olten.

Strasser, H., Bern.

Strasser, Frau, Bern.
Strasser, Ch., Bern.

Studer, Th., Bern.

Studer, Frau, Bern.

Tscharner (von), L., Bern.

Valentin, A., Bern.

Valentin, Frau, Bern.

Vischer, A., Bern.

Volz, W., Bern.

Wagner 0., Bern.

Wattenwyl (von), J., Bern.

Yung, E., Genève.

Zeerleder, F., Bern.

Zimmermann, K. W., Bern.

Zimmermann, Frau, Bern.

Zschokke, F., Basel.

Zschokke, Frau, Basel.

\section{Uruguay.}

Arechavaleta, J., IIontevideo. 



\section{PREMIÈRE ASSEMBLÉE GÉNÉRALE}

\section{Lundi 15 août 1904}

à $9 \mathrm{~h} .1 / 2$

dans la salle du Conseil National au Palais du Parlement,

sous la présidence de

\section{M. le Professeur Th. Studer,}

Président du Congrès.

Vice-Présidents: MIM. le Prof. C. Emery (Bologna), le prof. K. Fum (Tolio), le Prof. H.-F. Osionn (New-York) et le Plof. .J.-W. Spencied, (Giessen).

M. (iobat, Conseiller d'Etat du Canton de Berne, déclare ouverte la $6^{\prime \prime}$ session du Congrès international de Zoologie et prononce le discours suivant :

\section{Messieur's,}

Ce Congrès devait être ouvert par un représentant du Conseil fédéral. Comme il s'est trouvé empêché au dernicr moment de remplir cette agréable mission, on vient de me prier de le suppléer. Vous voudrez done bien vous contenter du salut d'un membre du gouvernement bernois. Je vous souhaite à tous cordialement la bienvenue au nom de l'autorité supérieure du Canton de Berne. La Suisse est fière d'être le rendez-vous de tant de savants, de tant d'hommes distingués qui viennent dans notre pays discuter les questions de toute nature, intéressant l'humanité, chercher' en commun les solutions des problèmes scientifiques, politiques et sociaux; fière surtout de former, pour ainsi dire, un lien qui est l'expression de la solidarité des nations. Aujourd'hui cette solidarité ne peut plus être contestée. C'est en vain que les Etats s'isoleraient. Leur's intérêts même leur imposent l'obligation d'unir leurs efforts en vue du progrès dans tous les domaines.

Vous allez travailler en commun sur le vaste champ des sciences naturelles, discuter les origines et les transformations des êtres animés.

Puissent vos délibérations être couronnées de succès et puissiez-vous, après avoir passé quelques jours au milieu de nous, emporter de Berne et de notre pays tout entier un agréable souvenir. 


\section{II. le Prof. STuder, Président du Congrès :}

\section{Hochansehnliche Versammlung!}

Indem ich das Pröisidium über den VI. Intermationalen Zoologenkongre'ss ühernchme, sei es mir vergönnt, Ihnen ein herzliches Willkommen datzubringr'n in Namen unserer hohen Bundeshehörden und im Namen unseres ganzen Landes und Volkes. Ich begrüsse hier besonder's die Vertreter der auswärtigen Staaten, die uns durch ihre Delegationen geehrt haben. die zahlreichen Vertreter der Akademien und gelehrten (iesellschaften: ich hegrüsse hier alle, die von Fern und Nah gekommen sind zu gemeinsamer Arbeit auf dem Felde der Naturforschung. Nicht ohne bangen haben wir den ehrenden Auftrag übernommen, den VI. Intermationalen Zoologenkongress in der Schweiz abzuhalten. Ihr zahlreiches Firscheinen gilot uns das Vertranen und die Zuversicht, dass es uns gelingen möge, denselben zu aller Befriedigung zu Ende zu führen.

Sie kommen hier in ein kieines, aber für den Zoologen in mannigfachen Beziehungen interessantes Land. In wenigen Stunden kïmnen Sie von der tiefsten stelle der Schweiz, 25) Meter über dem Meer, aus Weingeländen und Kastanienlaanen, der Pflanzen- und Tierwelt der mediterranen Regron, hinaufgelangen in eisgepanzerte Höhen, wo die letzten Zeugen der früher'n allgemeinen Vorgletscherung Mittelenropas Sie umgeben. wo PHtanz'n- und Tierwelt der arktisehen Zone Sie in den Polargürtel versetzen. Und wie spiegelt sich in unserer Tierwelt die jüngste geologisehe Geschichte unseres liontinentes wieder. Wohl sehen wir, dass die ganze Fauna den Typus der palararktischen Region trägt, aber wenn wir die Typen am Nord- und Südfusse der Alpen vergleichen, so sehen wir, dass zwar die meisten Grenera miteinander übereinstimmen, aber dic Arten, wenn auch durch kleine Interschiede, von einander getrennt sind: essind Parallelformen. Beispiele liefer'n die kleineren Sängetiere, nördlich der Alpen, der allgemein verbreitete Maulwurf, Talpa europse, südlich die blinde Tulpu ceece, nördlich die grosse Schermaus. MLicrotus terrestris, südlich Mirrotıs Musigmani, und anch wo die Arten als übereinstimmend betrathtet werden können, finden wir Differenzen zwischen Nord und Südformen, die Beide als Varietiiten dentlich sich unterscheiden lassen. A ehnliehe Beispiele liefern die Amphibien, die Fische, besonders dir Cyprinoiden. Hier das gemeine Rothange, secudinius erythrophthenmus, dort serdimius hesperidum, hier Telestes Agassizi, dort Telestes multicellus, hier Burbus vulgaris, dort Barbus plebejus.

Einheitliche Arten verheriteten sich wohl einst über das ganze Gebiet, bis die Alpenbarriere sich so hoch tülmute, dass eine Mischung der Arten zwischen Nord und süd unmöglich wurde und die getrennten Formen sich in versehiedener Weise anpassten und entwickelten. Als mit dem 
Auftroten der Glacialzeit mächtige Lrbenvelnichtende Eismassen von den Höhen nach den T'älern sich senkten, da wurde das organische Leben bis über die Grenzen des Landes weg verdrängnt nach Yorden und nach Süden, bis es Verhältnisse fand, wo eine Anzahl zäher und lrbenskräftiger Formen noch ein kümmerliches Dasein fristete. Ton Vorden und Vordosten mischten sich damit die Tiere der Polarzone, die in den Tundrengebieten, welche sich vor den gewaltigen Gletscherfronten ausdehnten, ein genuines Lehensgebiet fanden; und als dann nach mannigfachen Schwankungen dic Eismassen sich wieder zurückzogen, als der blossgelegte steinübersïtr Boden erst einer 'Tundrenvegrtation, damn aber dem Walde liaum zur Entfaltung gah, da folgte auch die Tierwelt den neuerschlossenen (relieten. Erst zogen die Polartiere den vor den Gletscherfronten sich bildenden Tund'ongebieten, dic immer weiter nach den Höhen sich zuräckzogen, nach. Bis zum Fusse der Alpen wanderte das Mammuth, das wollharige Nashor'n, das Rentier. der Schnechase, das Schnechuhn, aber für die grossen Pflanzenfresser wurde bald das Nahrungsgehiet zu kloin. und sie erlagen dem Hunger und der Inzucht, und nur die kleinen anspruchslosen Arten, Schncehasen, Sichneemäuse, fristen noch auf den örden Höhen ihr Dasein.

In die Gewässer zogen die nordischen Wanderfische, die Coregnnusarten, die Rothforellen, den kalten Gletschorwasern entgegen, vom Inere bis zum Fusse dre Alpen, wo sie durch sinken der (rewässer, abgeschlossen vom Meere, zu ständigen Bewohnern unserer alpinen Seen geworden sind. So erlauhen uns diese Erscheinungen. Tiere zu unterscheiden, rie vor der (ilacialzeit schon das Land bewohnten, und nordische Formen, die rist dor Glacialzeit ihre Gregenwart vordanken und den Alpengürtel nicht überschreiten. Mit dem Aufspriessen des Waldes, dem Wïrmerwerden des Klimas trat aber wieder von allen Seiten die Waldfauna der gemiissigten Zone in das Land, von ()sten, von Westen. von Norden her sehen wir die Arten eindringen, um an Fusse der Ehenen Halt zu machen: aher auch hier suchen sie allmählig ihre Herrschatt zu erstreiten. An den Nordabhängen der Voralpen schen Sie die Alpenfauna und -flora nach oben dräingen, zungenförunig oft in das alpine horeale Gebiet eindringend, hier Fuss fassend, dort wieder zurückgedrängt, in Kleinem wiederholend, was in der Ebene in früher Zoit sich auf grösserem Felde abgespielt hat.

Voch ist die Besiedlung nicht so vollstiendig, dass sich von Osten und Westen einwandernde A rten gleichmässig gemischt hahen; namentlich bei langsam sich verbreitenden wirbellosen Tieren sehen wir, dass sic noch auf dem Wege des Eindringens sich betinden : so bei Mollusken. bei Myriapoden, vielen Inseliten, wir könmen verschiedene Zusammensetzungen der Fauna der Ost- und Westschweiz und der Nord- und Mittelschweiz unterscheiden. Unter den Mollusken z. B. ist Tachea sylutica 
vou Westen her bis zu der Aal gelangt. Camp!lixa ichthyoma vom Osten bis auf die Malserhaide und 'Tarasp.

Andere interessante Phenomene bieten die Veränderungen, welche dir: Arten hei ihrem Aufsteigen zu den höheren Zonen erleiden. Häufig ist wohl unter Einfluss der stälkeren Insolation das Auftreten von Meliznismus. Der Rothfuchs der Ehene erhält in der Höhe immer mehr dunkle Haare, der Bauch wird schwarz, schwarz-graue Fïrbung dominirt beim Eichhörnchen. Die gelberaue Foldmaus, die rothbraune Erotomys glareolus, erhalten dunkle Haarmischungen; noch auffallendre zeigt sich die Tendenz hei Reptilien; melanistische Formen der Bergeidechse, der Viper, treten häufig, an vielen Orten constant auf.

I asselbe Phanomen zeigen Mollusken, so Nacktschnecken, Insekten.

In andern Fällen wirkt Iöhenlage auf dis Körpergrösse, sie bewirlit Kleinerbleiben der Art, so bei vielen Schnecken, Tacher memoralis, sylvatica.

Hochverehte Versammlung, ich will Ihnen hiev keine Abhandlung über unsere thiergeographischen Verhältnisse vorlegen, nur zeigen möchte ich, wie dieses kleine Gebiet, das Ihnen nicht, wie die grossen Centren, ausgedehnte Anstalten und Museen bieten kann, in seiner Natur nahe bei cinander den Schlüssel zur Lösung zahlıeichel Probleme birgt und so für den Forscher stets ein Anziehungspunkt bleiben wird.

Und deswegen zögerten wir auch nicht, Sie in dieses Gebiet einzuladen, und Ihr zahlreiches Erscheinen zeigt, dass Sie die Erwartung hegen, auch hier Anregung und Belehrung zu finden.

Fs wurle und wird von vielen Seiten der Nutzen wissenschaftlicher Kongresse bestritten; froilich sind sie nicht dazu da, wissenschaftliche Fragen durch Stimmenmehrheit zu entscheiden, aber gemeinsame Besprechung, persönliches Verstehen der Ansichten Anderer, hilft Differenzen überbrücken, die in der Einsamkeit des Studirzimmer's gegenseitig zu grimmiger Fehde auswachsen.

Als die Société zoologique de France im Jahre 1889 den ersten internationalen zoologischen Kongress in Paris in's Leben rief, da definirte ihr unvergesslicher Prisident, Prof. Alphonse Micse-Enwaros, den Zweck des Kongresses mit Worten, die ich hicr wörtlich wiederhole:

"Les rémnions sont pleines de charmes, elles provocquent des rapprocherments, elles créent des relations durables, elles fécondent les efforts individucls, elles apprenuent aux membres de la grande famille scientifique à s' connaître et à s'estimer; et en établissant entre eux les liens de confraternité, clles feront disparaître peu à peu les malentendus qui divisent Jes nations. Ici nous n'avons qu'un drapeau. celui de la seience, qu une rivalité, l'émulation du travail et comme l'a si bieu dit un de nos philosophes. nous comprenons que la nation la plus pussante dans lavenir 
sera celle qui aura domné à sa jeume génération la culture intellectuelle la plus fol'te $)$.

Doch wir sind noch weiter gegangen, auf Anregung derselhen ersten longressleitung ist nach jahrelangen Berathungen eine Verständigung über Gesetze der Nomenklatur, gemeinsame Anwendung von Maass und Gewicht zu Stande gekommen. Wir werden an diesem Kiongress Beschlüsse über gemeinsame Massregeln zum Schutze seltener Thierformen fassen, weitere Torschläge zur gemeinsamen Lösung liegen vor. Viclleicht ist auch einmal eino Verständigung möglich über ein zu befolgendes System der Tierwelt, hegründet auf dem Boden der gegenwärtigen Forschung.

Nöge auch der (i. zoologische liongress cine weitere Etappe in dem Fortschritt unserer Wissenschaft bedeuten.

Hiermit erkläre ich den 6. zoologischen Kongress für elöffnet.

M. le Prof. E. Perraer. Président du Comité permanent des Congrès internationaux de Zoologie:

Mes chers Collègues,

Vous mavez fait l'honneur, au Congrès de Berlin, de m'appeler à la présidence du Comité permanent d'organisation des Congrès zoologiques internationaux, en remplacement du regretté Alphonse Mncw-Enwards. Ma participation en cette qualité à notre ourre scientifique (st trop récente pour que je n'aie pas conservé le droit de constater son sucrès et de m'en applaudir. Paris, Moscou, Leyde, Cambridge, Brrlin sont des étapess qui ont laissé à tous les zoologistes d'ineffacables souvenirs auxquels ront s'ajouter ceux que nous emporterons de cotte Suisse si hospitalière et si grandinse. Vous me considérerez sans doute comme votre interpretu fidèle si, an nom du Comité permanent comme aux noms des délégués étrangers qui ont bien voulu me charger de ce soin, j'exprime au gouvernement de la République helvétique, aux représentants du canton et de la municipalité de Berne toute notre reconnaissance pour l'acueil chaleureux qu'ils ont bien voulu nous préparer; si j’adresse toutes mes félicitations aux membres si dévoués du Comité local d'organisation du Congrese, et si jajoute que la Zoologie doit une reconnaissance toute particuliere à mon vieil et éminent ami, M. le professeur S'Trder, présirlent de ce Comité, pour le zèle et le talent avec lequel il a conduit ses travaux de manière à assurer la pleine réussite de son ouvre.

Peut-être me sauriez-vous gré de bornel ce discours à ces témoignages de reconnaissance aux autorités si éclairées de la République belvétique 
et dr profonde estime pour un savant dont l'œuvre si considérable est marrquée par une conscience, une érudition, une finesse d'observation qui ont depuis longtemps entraîné l'admiration de tous les zoologistes, comme sa modestie et son caractère lui ont valu toutes les sympathies. Je vous demande votre indulgence si, après avoir rempli cette tâche tout particulièrement agréable, je me permets d'entrer dans quelques considérations générales sur une partie de l'œuvre que nos Congrès peuvent réaliser.

Notre science brille actuellement par la somme prodigieuse de faits qu'elle a recueillis, par l'habileté qu'elle a déployée pour les découvrir. Elle ne se borne plus, comme encore au XV'II ${ }^{m e}$ siecle, ì observer l'extérieur des animaux et à noter leurs caractères, comme au XVIII ${ }^{\text {we }}$ et a la première moitié du XIX ${ }^{\text {me }}$ siècles à fouiller leur intérieur avec le scalpel. Armée du microscope devenu si pénétrant, disposant de tout un arsenal de produits chimiques subtils et délicats, elle a pu non seulement délimitel les éléments anatomiques, mais saisir en quelque sorte sur le vif les variations de la structure de ces éléments et les phénomènes chimiques ou physiologiques qui s'accomplissent à leur intérieur. A partir de l'u'uf elle a vu se former, se perfectionner, se multiplier, se différencier ces éléments, et l'embryogénie qui observait jadis les transformations des animaux comme un météorologiste étudie les transformations des nuages est devenue une science précise qui confine à l'histologie et ì l'anatomie comparée. A ce point de vue de la récolte des faits, on peut dire que le $\mathrm{XIX}^{\mathrm{me}}$ siècle a accompli une couvre biologique merveilleuse.

Mais pourquoi réeolte-t-on des faits? Dans toutes les branches de la science, à mesure que les faits se multiplient, on les groupe en longues séries dans lesquelles ils sont liés par des relations de cause à effet, et l'on cherche à établir une for'mule simple qui exprime ces relations. C'est ce que l'on appelle constituer une théonif an sens propre de ce mot. Ainsi les mouvements des astres sont reliés par la formule simple de l'attraction universelle établie par Newton; les phénomènes lumineux par les formules du mouvement ondulatoire établies par Fressel et il en est de même des phénomènes électriques, des phénomènes calorifiques, etc. Astronomes et physiciens ont réussi à exprimer mathématiquement les relations simples qui se dégagent du groupement des faits. it constituer de vastes théories mathématiques sur lesquelles ils sont tous d'accord, et dont l'importance est telle qu'elles finissent, dans l'enseignement de leur science, par prendre un rôle prédominant, et qu'elles ont fait naittre l'illusion qu'elles suffisent à constituer ces sciences.

Eilles les ont sans aucun doute affermies et ont assuré leur progrès : clles ont conduit d'ailleurs, relativement à la constitution du monde inorganique, à des conceptions d'une amplitude et d'une profondeur qui inspirent l'admiration et le respect. 
Tout autre est, dans nos sciences biologiques, la place occupéc par les Théories, et la signification même du mot s'y trouve altéréc. Nos théories sont généralement courtes, c'est-à-rlire relatives à un ensemble de faits fort l'estreint. Les relations qui leur servent de base ne sont pas déduites d'un groupement méthodique des faits; nous les créons, pour ainsi dire, a priori, et nous cherchons ensuite à y adapter les faits. Ce sont, en réalité, des hypothieses, et nous en sommes effectivement encore à confondre ces deux mots hypothèses et théorie, à attribuer aux conceptions qu'elles représentent la même fragilité, à envelopper ces conceptions dan.: la même méfiance, à les prendre et à les délaisser à notre gré, souvent dans un seul et même travail, voire à notre insu.

Chacun se croit d'ailleurs le droit de grouper les faits suivant la conception qui lui convient, de telle sorte que sur les plus petites questions comme sur les plus hautes, le désaccord éclate entre nous et que l'accord même qui s'est fait sur certaines lois générales demcure stérile parce que le développement naturel de ces lois se heurte ì chaque instant ì nos idtérs préconçues, et que nous sommes courluits à préférer celles-ci qui sont notre ouvre personmello aux lois gémérales qui existent en dohors de nous.

Les exemples de ees désaccords pourraient être multipliés à l'intini. Chez les Eponges, l'un appelle exoderme, ce que l'autro appelle entoderme ot réciproquement; le mode de constitution de ces animaux, leurs lapports zoologiques domnent lieu aux opinions les plus variées. Les uns commencent l'histoire des Polypes par les Hydres, les autres par les Méduses ou par les Coraux; les faits s'enchaînent comme ils peuvent avec ces points de défrart arhitraires. On fait descendre les Méduses des Hydres par les procéslés les plus variés, on bien au contraire on simplific les Coraux et les Méduses pour en faire des Hydres et l'on ne sait plus si les Siphonophores sont des colonies d'Hydres, des colonies de Méluses ou des organismes autonomes. Comment espérer, dans ce désordé, déméler les causes physiologiques de la production de ces organismes?

La division du colps en segments est un fait d'une telle généralité que Crver en avait fait le caractère de son embranchement des Articulés qui comprenait les Arthropodes et les Vers annelés, et qu'elle se retrouve chez les Veltébrés. Mais deja nous ne mous entendons plus sul sa signification. Nous avons inventé toutes sortes de métaméridations. Nous parlons de métaméridation générale, partielle, extérieure, intérieure. apparente, réelle, fausse ou vraie, simple ou double: nous disputons pour savoir si ces diverses métaméridations dépendent d'une seule cause sur laquelle chacun conserve ses illées ou d'autant de causes que de sortes, et nous sommes incertains s'il faut rattacher ou non aux animaux segmentés des animaux tels que les Echinodermes ou les Mollusques. Les lapplorts découverts par liowatevsix entre les Tuniciers at les Tertébrés. Ioin de 
nous apporter une lumière, nous ont jeté dans le plus grand désarroi. Lowalevsir avait cru trouver un pont entre les Vertébrés et les Invertéhrés; le pont se trouve coupé. On se demande si les Tuniciers, au lieu d'utre les ancetres des Vertébrés, ne seraient pas leurs descendants, et les ascidiologurs de leur coté étahlissent des généalogies de ces animaux qui ne tirment aucun compte de cette question fondamentale: les uns tiounent pour principe que les formes pélagiques sont les formes initiales; dautres que les formes simples et bourgeonmantes sont les formes ancestrales: d'autres encore que ce sont les formes nageuses comme les Appendiculaires, et chacun accumule les arguments en faveur de sa maniere de roir. L'origine des Vertébrés a donné lien à de non moins mémorables désacrords. On les a fait descendre autrefois des Mollusques; on leur a donné de nos jour's pour ancêtres tour à tour les. Tuniciers, les Arachnides, les Crustacés, les Némertes, les Vers annelés, les Balanoglosses, les Echinodermes et pour cousins, les Sayitta, les Plioronis, les Cephulodiscus, les Halilophus, etc. La question s'est d'autre part posée de savoir quels sont des Vers plats ou des Vel's ammelés, ceux que l'on doit considérer comme les ancêtres des autres, et l'on s'est demandé si cesdeux groupes de Vers n'avaient pas respectivement donné naissance à deux groupes de Mollusques que nous aurions eu le tort de confondre.

A voir ainsi les généalogies réversibles et incertaines, certains se détournent de ces problemes d'oripine qui sout cependant capitaux; ils perident confiance dans la doctrine transformiste, et la science se trouve menacée de revenir en arrière.

Les rapports entre les diverses branches de la Biologie ne sont pas concus avec plus de sureté. Sans donte, nous admettons presque tous, ou ilu moins je le pense, que l'embryogénie a pour base une répétition de la phylogénie. Il semblerait des lors qu'une comnaissance approfondie de l'oldrer dans lequel ont évolué les formes organiques doit être le fondement de l'embryogénie qui aurait ensuite pour mission de rechercher comment et pour quelle cause, dans chaque cas particulier, le thème fondamental de la répétition par l'embryon des formes de ses ancêtres a pu etre modifié. Mlais lo probleme est compris tout juste à l'inverse par la plupart des embryogénistes. Ils se considerent volontiers comme les généalogistes par excellence du Riegne animal et vont mème jusqu'a prétendre fonder l'Anatomie comparée ou tout au moins son enseignement sur l'Embryogénie. 'Tout se trouve ainsi renversé. D'ailleurs, les anatomistes à leur tour, pour peu qu'ils s'y trouvent sollicités, oublient volontiers la loi fondamentale de l'embryogénie et ne tiemment qu'un compte médiocre des domnées rmbryogéniques dans leur's cooldinations généalogiques.

Il est certain cependant que les faits se sont déroulés en Biologie comme ailleuss d'une facon déterminée, que les effets dans le monde vi- 
vant ont, comme partout, suivis les causes et ne les ont pas précédées; que li, comme dans le mondo inorganique, les phénomènes sont r'égis juar un déterminisme rigoureux, qu'il n'y a pas en biologie plus (qu'ailleur's, d'eftects sans causes naturelles, et que la logique et Ir raisonnement ont la même valeur dans nos sciences que dans les autres. Notre commaissance des faits est, d'autre part, aujou'l'hui si étendue, que nous pouvons sans témérité, penser que nous avons entre les mains tout ce qui est nécessaire pour établir leur ordre de sucession et leurs rapports, légager de ce travail de coordination les lois fondamentales et créer ainsi une théorie biologique qui aurait pour nous la mème valeur que la théorie de l'attraction universelle pour les astronomes, la théorie mécanique de la chaleur, celle des ondulations lumineuses et autres pour les physiciens, celle des substitutions et des radicaux organiques pour les chimistes?

Quand on fait le sacrifice de ses idées préconçues, quand on reprend les faits un à un dans leur ordre de complication croissante, quand on applique avec une rigouremse logique les principes géneraux qui se sont imposés par leur évidence, malgré le chaos dans lequel nous vivons, on s'aperçoit bien vite que l'écheveau embrouillé jusqu'ici comme à piaisir, so démêle sensiblement et qu'il ne faudrait peut-être pas un très grand effort pour le démêler tout à fait. La chose en vaut la peine puisqu'il s'agit de savoir, en somme, si le monde vivant peut être expliqué tout entier par l'intervention exclusive de causes agissant autour de nous, ou s'il faut réserver dans sa formation une part plus grande qu'ailleurs à des causes surnaturelles. Ce n'est pas seulement la Biologie, c'est la Philosophie tout entière qui est en jeu.

()r, la considération des faits ot des lois que nous possédons déja éclaire singulierement la question: la forme lamifiée du corps semble e'n rapport étroit avec la fixation au sol et la libre locomotion. Le parasitisme ou mème simplement unr immobilité relative fait disparaitre la segmentattion du corps, et cotte considération permet de rattacher les Nématodes' amx Arthopodes, les Vor's plats aux Ver's ammelés par les Trématodes. Les larves d'Echinodermes font do leur coté gauche, le ventro de l'adulte ot de leur côté droit son dos; elles sont assymétriques intérieurement, et il en résulte clairement que les Echinodermes deviennent des Vers pleuronectes; toutes les larves de Mollusque nagent le dos en bas et cela suftit pour faire comprendre le développement de la bosse dorsale contre laquelle ces animaux ont eu à lutter comme l'a si bien montré notre collegue Laxr. Les larvest'. I mphioxus sont assymetriques comme les toles: les ancêtres des Vertébrés étaient donc pleuronectes eux aussi et c'est le point de départ de leure lenversement. dinsi sintroduit dans la science la notion de l'importance des changements d'attitude at des léaletions volontaires de l'animal contre les conditions biologiques mauvaises où il se trouve. Toutes les difficultes que semble rencontrer. all premier abord, 
l'explication des grands types olganiques par des causes physiologiques simples, telles que des changements d'attitude ou la recher'che par l'animal d'un maximum de bien être, disparaissent d'ailleur's et l'on a présente à l'exprit une loi fondamentale de l'hérédité qui permet aussi de grouper méthodiquement les phénomènes embryogéniques, la loi de lincrélération embryogénique ou tachygénèse. Et cela suffit pour que la creation le types organiques sorte du domaine du miracle pour entrel' dans celui des phénomènes scientifiques démontrables.

No serait-ce pas pour nos Congrès une helle œuvre que de s'attacher a cette haute besogne: Ne pourlions-nous pas quand nous sommes tous rémis (ssayer de fixer les principes de notre science de telle façon que devenus clairs pour tout le monde, ils fassent disparaître le singulier état chatique que nous avons accepté jusqu'ici? Ne pourrions-nous pas étahlir sur ces principes une langue qui éliminerait les termes arbitraires, les mots prématurés ne correspondant qu'à des conceptions vagues. incomplètes, hypothétiques, termes qui égarent les jeumes biologistes et ruelquefois aussi les vieux, faussent leur's idées et font ressembler notre science à une façon de tour de Babel? Ne pourrions-nous essayer de provoquer une mise génélale à l'étude de ces questions fondamentales qui aboutirait à une sorte de revision, de nuise au point des principes suivant lesquels nous devons travailler et raisonner. Cette revision sonmise à un prochain Congrès conduirait à la constitution d'une sorte de corpus hiologique, ì la formation en zoologie d'une sorte d'opinion puhlique raisomnée que les rêveur's par trop aventureux n'oseraient plus affironter sans avoir, comme on dit, toumé sept fois leur langue dans leur houche, ce qui serait tout protit pour les progrès rapides de la science et nous éviterait beaucoup) d'imutiles complications ou de travaux d'une portée éphémère.

Je ne sais, mes chers collegues, si en vous parlant ainsi, je nesuis pas moimême un réveur, déreloppant une utopie et si vous ne pensez pas que ma proposition tencl à établir dans notre science un dogmatisme qui enchầnerait la liberté des esprits géniaux et irait ainsi contre son but, le progres. Jo me garderai done d'insister davantage, d'autant plus que l'Association franchise pour lavancement des sciences rous propose de nommer une Commission de cooldination des travaux zoologiques à laquelle ces études pouront itre lenvoyées. Jaurai atteint mon but si de la compalaison de nos methodes de laisomnement et d'enchainement des faits arec (onlles qui ont donne aux autres sciences une si puissante envolér, vous arrive\% il cette conchusion qu il y a tout de meme quelque réfolme il effecetuer dans nos habitudes d'esprit. Vous apporterez le fruit de vos réflexions au prochain Congres pour lequel colui-ci aura contribué à préparel une œuvi'e féconde. 
M. Benot, secrétaire, donne lecture de la liste des délégués des (rouvernements, Académies et Sociétés savantes.

II. le président, au nom du Comité d'organisation, propose à l'assemblée de nommer comme présidents, vice-présidents et secrétaires des assemblées générales et des séances de seetions les memlıros du Congrès dont les noms suivent:

\section{Assemblées génÉRALES}

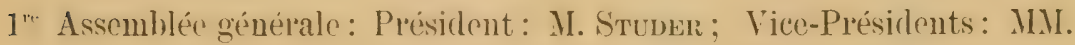
Emery, Fuji, Spengel et Usborn.

$2^{\circ}$ " " Président: M. Мготот; Vice-présidents : MM. von Graff, Peiseneer, Stejneger et Lönberg.

$3^{e}$ " " Président: M. Grassi ; Vice-Présidents: MM. Buanchard, Chun et van Wijile.

$4^{\mathrm{e}}$ 》) 》) Président: M. E. Perrier ; Vice-Présidents : MM. Miller, Horvath, Lang et Stiles.

\section{SÉANCES DES SECTTONS}

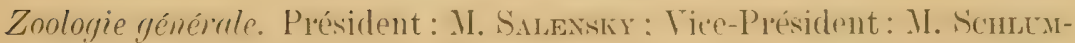
Berger; Secrétaile: M. Gurwitsch.

Vertébrés (Systématique). Président: M. Jenrrnk; Vice-Président: M. SharfF; Secrétaile: M. André.

Vertébrés (Anatomie). Président: M. Montrcelis; Vice-Président: M. B. Dean ; Secrétaire: M. Penard.

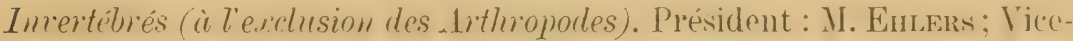
Présidents: MM. Köhler et de Guerne; Secrétaire: M. Funrmanx. Arthropodes. Président: M. Hexmons ; Vice-Président: M. Janet; Secrétaire: М. 'Тн. Stтеск.

Zoologie appliquée. Président: M. Hoek; Vice-Président: M. Plate; Secrétaile: M. Duerst.

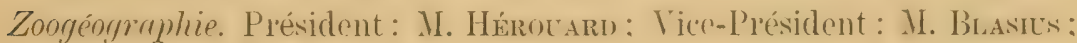
Secrétaire: M. Roux.

L'assemblée adopte cette proposition.

M. Ie président annonce que le bureau a reçu deux propositions relatives au choix du lieu de réunion de la prochainer session du Congrés en 1907, soit:

$1^{\circ}$ une demande des Zonlogistes américains proposant la réunion du Congrès à Boston.

$2^{\circ}$ une demande de S. A. S. le Prince de Movaco proposant la réunion du Congrès à Monaco. 
L'assmblée des membres du Comité permanent et des délégués de gouvernements propose à l'assemblée de choisir Boston.

Cette proposition est adoptée par l'assemblée.

M. Ie Présidrnt exprime, au nom do l'assemblée, ses remerciements à s. A. S. l" Prince de Monaco et espère qu'un des prochains Congrès de Zoologie pourra se réunir à Monaco.

M. Mrxot au nom des Zoologistes américains r'emercie l'assemblée. Il amnonce que le Congrès international de physiologie aura lieu en mème temps à Iioston et il espèce que l'on pourra faciliter aux Zoologistes européens le voyage en Amérique.

Sur la proposition de M. le président, M. BEDOT est nommé secrétaile général du Congrès.

I. le président annonce yue le comité a recu une ploposition de l'association francaise pom lavancement des sciences demandant que le Congrès nommo une commission chargée d'examiner yuels sujets d'études pourraient etre utilement choisis d'une faron coordonnér par les diverses sections et d'indiguer les meilleurs moyens à employer pour aśsurer l'unité de méthode dans les investigations.

Cette proposition est transmise an Comité pelmancut qui est. charcé de l'examiner et de présenter à ce sujet un rapport dans une des prochaines assemblées générales.

M. le Président donne la parole à M. R. Branchard.

\section{ZOOLOGIE E'T MÉDEGINE}

Par le Prof., R. BI_ANCHARD

(')aris).

La question qui va nous occuper n'est pas nouvelle. Je pourrais citel un bon nombre de discours académignes on de dissertations inangurales qui discutent les lapports de la zoologie avec la mérlecine: suivant les préoceupations philosophiques ou les doctrines médicales de l'éporue. ces essais littéraireśs envisagent la question à des points de vue différents, mais la plupart d'entre eux planent dams les hauteurs nebuleuses de la métaphysigue ot tous se ressemblent par l'absence complete d'une base véritablement scientifique.

Au cours rlu XIX min siecle sont nées diverses scionces, telles que lanatomie comparés, la physiologie, l'anthropologie, la mejecine expérimentale ret la palrasitologie : chacune d'elles a éclairé d'un jour noureau lo 
problème de la nature et de l'origine de l'Homme, ot spécialement celui de ses relations avec los Vertéhrés supérieurs. Ce serait une recherche assurément très intéressante, mais dépassant singulièrement les limites d'me simple confélence, que de dégager les notions scientifiques qui dérivent de ces récentes études. Je n'ai pas l'intention d'aborder devant vous une discussion aussi technique, pour laquelle je ne disposerais pas d'assez de temps; mon role sela plus modeste et je veux me borner à vous faire toucher du doigt, par quelques exemples, de quels progrés décisifs les doctrines médicales sont redevables à la zoologie, quelles découvortes ('apitales ont résulté d'une connaissance plus exacte des parasites animaux, quelle lumière inattendue a été projetée par ces notions nouvelles sur l'origine des maladies les plus meurtrieres, quelles hrureuses indications pratiques en déconlent et, à cette époque de vastes entreprises coloniales, a quel point l'acclimatement et le succes de notre racn dans les pays chauds sont liés aux progrès de la zoologie médicale.

Le sang, la lymphe et le tissu conjonctif renfrment deséléments anatomiques depuis longtemps ronnus sous le nom de lencocytes ou globules blancs. C'est une expression banale que de les comparer aux Amibes, auxquelles ils ressemblent, en effet, par leur mode de locomotion et par la facon dont ils englobent les colpuscules solides. (On ('ll connaît plusieurs variétés, dlont la distinction n'était, naguire encore, qu'une curiosité d'histologiste. Or', il se trouve que ces éléments, que leur structure ret leur physiologie rapprochent des animaux les plus inferemeurs, jouent dans l'organisme un rôle capital.

L'é(quilibre physiologique, qui constitue la santé, n'st assuré que par l’incessante surveillance qu'ils exercent : partout disséminés, ils veillent, en tous les points du corps et s'opposent aux perturbations diverses qui peuvent à chaque instant se manifister dans nos organes; en particuliel, ils ont pour mission d'ar'êter au passage les colps étrangers, les Microbes et, d'une faron générale, les parasites qui ('nvahisirnt notre économie par les voies les plus diverses. Suivant que ces derniers sont plus ou moins gros, les leucocytes varient leur moyen d'attaque : ils interviennent isolément ou, au contraire, mettent en commun leurs efforts pour arrêtel' dans sa marche envahissante l'élément parasitaire. Si l'agent infectieux n'est pas représenté par un etro figuré, mais consiste en des sulstances chimiques, doués de propriétés toxiques, ils interviennent d'une autre manière et, s'adaptant ì ces conditions nouvelles, élaborent, eux aussi, et déversent dans les humeurs de l'organisme, des substances capables de neutialiser les premières.

La théorie de la phagocytose, que l'on doit aux sagaces observations de Menshnikov, n'est-elle pas de ce nombre? Chacun sait en quoi elle consiste; chacun, du moins, connait les Amibes, qui vivent dans les fanx stagnantes. C'es animaleules représentent le dernier degré de l'aninalité: 
leur sarcode ou substance plastique émet des prolongements qui lui permettent d'englober les corpuscules solides qui se trourent à son contact; suivant lmur nature, ces dernier's sont digérés et assimilés par l'Amibe ou, au contraire, rejetés au bout d'un certain temps. Rien n'est mieux connu que en phénomène: Dujardrn et d'autres l'ont très bien étudié; ils y vovaient la manifestation la plus simple de l'acte de la nutrition. C'est hien cela, en effet, mais c'est aussi un acte d'une exceptionnelle impoltance puisqußil a été le point de départ de la découverte de la phagocytose, doctrine qui touche aux prohlèmes les plus obseurs de la physiolegie.

Ainsi. un simple fait d'observation zonlogique, bien interprété par un esprit d’une lare pénétration, est venu ruiner de fond en comble les conceptions hésitantes et nuageuses, dérivées de l'humorisme, par lesquelles la médecine essayait d'expliquer le grand fait de la l'ésistance de l'organisme aux infections. La phagocytose a donné la clef du problème. Elle permet aussi, ou va bientôt permettre de comprendre d'une facon tout aussi nette les lois de la vaccination et de l'immunité. au sujet desquelles la mérlecine ne pouvait même pas émettre une hypothèse acceptahle.

Telles sont les conséquences de la théorie phagocytaire. On chercherait vainement, dans une autre branche des sciences biologiques, l'exemple d'une révolution doctrinale aussi profonde, hasée sur un fait d'aussi minime apparence.

Toilat trois ans, notre savant collegue, le professeur b. Grassi, a exposé au Congress ses armirables découvertes sur lo rôle des Moustiques dans la propagation du paludisme: j'aurai garle de revenir sur ce sujet, qu'il a traité avec tant d'autorité, mais il n'est pas inutile de nous arrêter un instant sur ces Insectes qui sont bien plus dangereux quon ne le pourrait croire d'apres sa hrillante conférence. En effet, s’ils propagent le paludisme it la surface presque entière du glohe, ils sont, dans des contrées moins vastes, mais encope trop étendues, les agents de dissémination de diverses maladies qui sont au premier lang des téaux de l'humanite. Dans toute la zone intertropicale, ils inoculent les Filaires du sang : ces Nématorles vivent daus le tissu conjonctif ou l'appareil cireulatoire; leur's cmbryons sont entraînés par le torent sanguin: ils sont en relation avec divers itats pathologiques, tels que l'hématurie des pays chauds et peut-être aussi l'éléphantiasis des Arabes.

Dans une zone plus restreinte, les Moustiques inoculent la fievre jaune. dont le domaine. limité jadis à l'Amérique tropicale, s'étend maintenant a la côte occirlentale d'Afriquru, atteint parfois l'Europe et est peut-citre à la veille de gagner jusqu'a l'Extrème-Or'ient, quand le canal de Panama sera allevé. Les Moustiques ne sont pas, comme on pourlait le eroime. do simples tramsmottrurs inertes des parasites, eomus ou non, qui sont ici en cause; coux-ci, bien au contraire. subisisent daus leur organismo des métamorphoses plus ou moins compliquées. 
L'un des problemes les plus urgents de l'hygiene des pays chauds est done, depuis que ces faits sont comnus, l'extude des Moustiques qui se rencontrent dans les différentes parties du globe. La connaissance exacte de la faune d'un pays, à ce point de vue spécial, est, comme on le voit, đlu plus haut intérét pour la santé publiqur, puisque, suivant la présence ou l'absence des espèces reconnues pathogènes, le pays qui est l'ohjet d'une telle investigation peut être déclaré dangereux ou salubre.

A vrai dire, on ne peut exiger que tout médecin soit capable de déterminer avec tonte la rigueur scientifique les différentes espèces de Moustiques qui peuvent s'offirir à lui, d'autant plus qu'il faut savoir, suivant les circonstances, les recomnaitre à l'état d'o'uf, de larve ou de nymphe, tout aussi bien qu'ì l'état adulte. De telles constatations ne peuvent itre que l'cuvre de naturalistes spécialisés dans ce sens et voici que, par un singulier phénomène. l'entomologiste de cabinet, auquel on aura recours pour la determination des Insectes ailés, recueillis dans les habitations, ou des larves et des nymphes, péchées an filet fin dans les Haques d'eau, devient non seulement l'auxiliaire ohligé, mais mème le conseiller et le guide autorisé de l'hygiéniste et du médecin. Ia question se complique encore, car il est utile de rechercher expérimentalement, chez diverses espéces de Moustiques, le développement éventuel d'organismes parasitaires rencontrés dans le sang de l'Homme ou des animaux. Cela entraine aux rechelches histologiques les plus délicates ct aux expélimentations les plus difticiles. Les récentes découvertes relatives à la filariose et à la fièvre jaune l'ont bien montré.

On connait environ quatre cents espèces de Moustiques : c'est dire l'ampleur imprévue des études qui se poursuivent en ce moment et quel roole prépondérant l'entomologie a conquis dans nos études. Je domnerais une idée très incomplète de son importance, si je m'en tenais à ce qui vient d'ètre dit. D'autres Diptères attirent également l'attention des parasitologues, parce qu'ils transmettent certaines maladies trés meurtrières. Chacun connaît ces épidémies de cause mystérieuse dont sont frappés les animaux domestiques européens que l'on tente d'introduire dans certaines régions de l'Afrique tropicale. Lrvingstone a reconnu qu'elles sont occasionnées par la piqûle de la Mouche 'l'sét.sé (Glossima morsitans), mais on est resté longtemps sans comprendre le mécanisme intime de l'infection. Le probleme est actuellement résolu. La Trétré inocule au bétail un Protozoaire qu'elle a puisé dans le sang d'un animal malade : le parasite inoculé de la sorte se multiplie tres activement dans le sang de son nouvel hôte et celui-ci re tarde pas à présenter les symptômes caractéristique du nagana.

L'animalcule en question est um simple Flagellé, commu sous le nom de Trypanosoma Brucei. Il nage dans le plasma, s'y reproduit par division longitudimale et le sang se charge ainsi de parasites chaque joul 
plus nombreux. Il est dûment établi par l'expérience que ceux-ci sont effectivement la cause de la maladie, qui est presque toujours mortelle. Les Trypanosomes sont done de ledoutables parasites et leur histoire doit singulièrement intéresser le médecin, s'il est prouvé que l'espèce humaine puisse etre attaquée, elle aussi, par des organismes semblables.

()r, la maladie du sommeil, qui sévit dans l'Afrique tropicale avec une redoutable intensité, au point de dévaster des territoires très étendus, comme elle l'a fait ces années dernières au Congo et dans l'Ouganda. n'est pas autre chose qu'une trypanosomose : le parasite spécifique est ici le Trypanosoma gambiense, que transmettent la Glossina palpalis et. vraisemblablement aussi, d'autres espèces du même genre. On connaît chez divers animaux d'autres trypanosomoses, dont les agents de transmission ne sont pas des Glossines, mais des Muscides d'autres types ou divers Tabanides. Bien plus, on sait qu'il existe en Algérie une trypanosomose humaine qui, vu l'absence des Glossines en cette région, rentre également dans cette dernière catégorie. Il s'ensuit que le concours du diptérologiste dans les questions d'épidémiologie est encore plus important que nous ne l'avions supposé.

Au surplus, il ne s'agit pas seulement de préciser la nature des Insectes pathogènes, d'élucider leur's mœurs et leurs métamorphoses, de trouver les moyens les plus aptes à les détruire ou à les écarter, de suivre dans ses moindres détails le cycle évolutif que le parasite peut subir à l'intérieur de leur's organes : tout cela n'est qu'une face de la question et j'ose dire que ce n'est pas la plus importante. En effet, il est indispensable d'expérimenter sur le parasite lui-même, afin d'arrêter. si faire se peut, sa marche envahissante et de déterminel les conditions capables d'atténuer son action pathogène ou de rendre l'organisme de son hôte indifférent à ses attaques. Un Trypanosome pullule dans le sang du Rat, sans que colui-ci en soit incommodé d'une façon appréciable : une telle endurance est sans doute le résultat d'une accoutumance progressive et héréditaire; cela nous donne à penser que l'Homme et les animaux qui sont actuellement sans défense à l'égard des Trypanosomes sont capables d'acquérir, eux aussi, l'immunité. La recherche des conditions suivant lesquelles celle-ci peut s'étahlir est assurément l'un des plus importants prohlimes de l'heure actuelle. Cela nous ramene à la question toujours présente de la phagocytose et de la physiologie pathologique des globules blanes.

Hier inconnus en parasitologie humaine, les Trypanosomes ont done acquis une place importante dans ce domain particulier de la médecine. Méme en supposant résolus les problèmes qui les concernent. ils sont loin de nous avoil livié toute leur histoire et nous en sommes à nous demander maintenant si ces ettres dangereux sont vraiment des Flagellés. 
comme on l'avait cru jusqu’à présent. Le zoologiste a ses classifications bien tranchées, dans lesquelles les classes sont comme des compartiments voisins, mais sans communication les uns avec les autres. ()n s'entendait pour rattacher les Trypanosomes aux Flagellés et l'Hématozoaire du paludisme ou, d'une facon plus large, les Hémosporidies aux Sporozoaires. Les arguments étaient bons, sur lesquels leposait cette répartition.

Or, Schaudiñ nous a récemment appris que ces deux types, en apparence si distincts, pouvaient successivement passer de l'un ì l'autre, soit (lans le sang d'un même ()iseau, soit du Vertéhré au Moustique. Vous nattendez pas de moi la description des métamorphoses vraiment compliquées que subissent les animalcules en question. J'en aurai indiqué toute la valeur en disant que la découverte de Sohauninn, que d'autres observateur's ont déjà controlée pour des types parasitaires différents de ceux qu'il avait envisagés, bien loin de résoudle la question des migrations et des métamorphoses des Hématozoaires, nous montre, je ne dirai pats l'erreur de nos notions actuelles, mais leur tres grande insuffisance. Aussi bien pour les Hémosporidies que pour les Trypanosomes, les phases évolutives admises par tous les observateurs ne sont qu'un simple état passager, se reliant à d'autres formes encore incomnues qu'il va falloir maintenant déceler dans toute l'ur sucession. C'est ainsi que la science progresse, que les questions changent sans cesse de face, que les faits considérés comme les plus définitifs ne sont qu'une simple étape sur la route infinie du progres; c'est ainsi, pour rappeler un mot familier a Claude Bernard, que la science du jour est l'erreur du lendemain.

Il va sans dire que ce n'est pas seulement l'histoire des Hématozoaires des Oiseaux qui se trouve ainsi remise en question, mais que l'incertitude plane également sur les Hématozoaires du paludisme et sur d'autres parasites dont l'existence est certaine, bien que nous n'ayons pas encore su les dérouvirir. De ce nombre est celui de la fiève jaune : on comnaît sa transmission par les Moustiques (Stegomyia calopus), on sait que ceux-ci ne sont infectieux qu'à partir du douzieme jour après qu'ils ont piqué un individu atteint de fièvre jaune, ce qui revient à dire que le parasite subit dans leur organisme des transformations plus on moins analogues à celles dont l'Hématozoaire du paludisme nous donne un si remarquable exemple. Malgré ces indications précises, toute recherche de l'agent infectieux est demeurée vaine, sans doute parce qu'il est de trop prtite taille pour etre accessible à nos moyens d'investigation. Il n'est point le seul dont on en puisse dire autant et, selon toute apparence, la syphilis, la fiève bilieuse hématurique, la rage, pour ne citer que colles-là, appartiennent à cette catégorie d'affections parasitaires dont le germe demeure incomnu. Aussi bien, les recherches de ScrutDrNo nous ont appris que certaines formes do Trypanosomes et de Spi- 
rochètes, dérivées des Hématozoaires des Oiseaux et produites dans le tube digestif (lu Moustique (Culex pipiens), sont assez petites pour traverser les filtres de porcelaine et ne deviemnent apparentes, malgré les plus forts grosisissements, que lorsqu'elles se rassemblent en nombre considérable. On découvrira saus doute des combinaisons optiques permettant de roir et d'étudier ces êtres d'une extraordinaire petitesse : leur investigation ouvre la voie à des recherches particulièrement délicates et intéressantes.

Les faits nouvellement acquis ou les questions récemment soulevées dans le domaine de l'helminthologie ne sont pas non plus sans importance. Voilà vingt-cinq ans à peine, la zoologie médicale se restreignait à une description, voire à une énumération sommaire des quatre ou cinq Helminthes les plus répandus en Europe, c'est-à-dire les deux Ténjas inerme ct armé, l'Ascaride, l'Oxyure et le Trichocéphale. Pour être complet, on citait aussi la Filaire de Médine, à titre de curiosité exotique, pour paraître amateur de raretés, on mentionnait encore le Strongle géant. Quant aux Trématodes, on s'en tenait à la grande et à la petite Douve du foie et on faisait une allusion discrète, et pour cause, it la Bilharzie. Cela prenait dans l'enseignement de nos Facultés de médecine trois ou quatre lecons. J'en sais quelque chose, puisque c'est à ce régime que j’ai été éduqué.

Et notez que les Facultés et Ecoles de médecine françaises sont, dans le monde entier, à peu près les seules à posséder une chaire magistrale d'histoire naturelle. Il est vrai que le professeur devait enseigner en même temps la zoologie et la botanique dans leurs applications à la médecine, comme s'il se pouvait trouver', dans l'état actuel du progrès scientifique, des hommes capables d'enseigner avec antorité deux branches de l'histoire naturelle depuis longtemps si profondément différenciées. Dans la pratique, cette difficulté était tournée, puisque le professeur enseignait telle branche de la science qui lui était plus familière, laissant à l'agrégé Ic soin d'enseigner l'autre. C'est ainsi que mon savant prédécesseur, M. le Professeur BArlan, qui a occupé si longtemps la chaire d'histoire naturelle médicale de la Faculté de Paris et dont les travaux de botanique jouissent de la plus grande réputation, se réservait l'enseignement de la botanique; l'agrégé devait donc enseigner la zoologie.

Jusqu'en 1853, date à laquelle j'ai eu l'honneur de commencer mon enseignement à la Faculté de Paris, le cours de zoologie médicale n'était en réalité qu'un cours ćlémentaire de Faculté des sciences. Il n’y avait a cela que demi-mal, puisqu'il fallait dégrossir des jeunes gens frais émoulus du collège, dont les connaissances en histoire naturelle étaient tout à fait insuffisantes; mais il eût été nécessaire de compléter ces éléments de zoologie générale par une étude aussi létaillée que possible des parasites d'origine animale. 
Convaincu du rôle chaque jour plus important que les parasites do cette nature jouent en pathologie humaine, rôle évidemment méconnu dans une foule de circonstances; instruit par la découverte de nouveaux parasites, en Extrême-Orient, par exemple; persuadé que les expéditions coloniales, qui retrouvaient alors un regain de vogue en Europe, ne tardelaient pas à nous faire commâtre, dans ce même ordre didées, beaucoup de faits nouveaux, je résolus de rompre avec ces errements et dr consacrer mon enseignement presque entier à l'étude des maladies parasitaires. Les résultats ne se firent pas attendre: d'abord un peu déconcertés par la noureauté de cet enseignement, les étudiants ne tardèrent pas à en saisir toute l'importance. Il ne m'appartient pas de dire si le succes fut ou non à la hauteur de l'effort, mais je crois avoir le droit de déclarer qu'une telle innovation, qui équivalait à la création d'un enseignement nouveau. répondait aux besoins de l'époque; j'en vois la preuve dans ce fait, que toutes les Facultés et Ecoles de France suivirent mon excmple et s'en trouverent fort bien. Il en fut de même pour quelques pays, particulièrement pour la Roumanie où furent "réées des chaires d'histoire naturelle médicale.

Ce que j'avais pu l’éaliser dés $188: 3$ comme agrégé, j’ai pu le compléter depuis 1897 comme professeur titulaire. J'ai eu la bonne fortune de monter dans ma chaire au moment où le programme des études médicales venait d'être modifié d'une facon très heureuse. L'histoire naturelle médicale, puisque tel est encore le titre officiel do mon enseignement, figurait désolmais au programme de la troisième année d'études, ce qui permettait de serrer de plus près les importantes questions ressortissant à la parasitologie et d'entrel dans des détails de clinique, de physiologie et d'anatomie pathologique, auxquels jadis les étudiants de première année n'eussent pas compris grand'chose. Il en est résulté une spécialisation beaucoup plus grande de l'enseignement, aimsi qu'une orientation toute nouvelle des travaux pratiques et du laboratoire. La création des dichives de P'urasitologie, dont le huitième volume est maintenant achevé, est encore un témoignage de la profonde réforme que j’ai pu accomplir.

Il va sans dile que. dans un tel enseignement, c'est l'histoile naturelle qui domine et qu'il ne saurait ètre donné avec la compétence requise par un homme dont l'éducation serait surtout médicale. En effet, l'helminthologie n'en est plus à l'âge l'or' que je décrivais tout à l'heure. Quel immense chemin parcouru en vingt-cinq ans! Combien d'espèces parasitaires ajoutées à la liste alor's si restreinte! L'étude complète de ces animaux nécessite des connaissances très techniques de zoologie; il ne suffit pas de déterminer leur structure, de suivre leurs migrations et leurs métamorphoses, de les reconnaître dans leur's diverses transformations, de préciser les lésions dont ils sont la cause; il faut encore connaîtrer 
assez bien les parasites des animaux les plus divers pour discerner les liens de parenté qui peuvent exister entre ces Helminthes de l'Homme et ceux de différentes espèces animales.

Darane a décrit, d'après des échantillons très incomplets, un petit Ténia provenant des Comores, auquel il a donné le nom de Traia madayascuriensis; ConBold a fait connaître sous celui de Distoma Ringeri un Trématode qui vit au Japon et en Chine dans le poumon de l'Homme et cause des hémoptysies fréquentes. Qui donc, sans posséder les notions que je viens d'indiquer et qui ne peuvent s'acquérir que par une longue pratique de la zoologie, aurait pu se douter que le premier de ces parasites appartient à un type qui ne se trouve chez les Mammifères et chez l'Homme qu'à titre tout à fait exceptionnel, mais qui appartient normalement aux Gallinacés? Qui donc, de même, aurait pu reconnaître dans le second un Helminthe déjà signalé par Kerbent chez le Tigre? De tels rapprochements ne constituent point de simples curiosités, comme des espreits superficiels pourraient le croire : ils sont de la plus haute importance, puisqu'ils peuvent mettre sur la voie de l'origine des maladies parasitaires de l'Homme, les seules en somme intéresisantes pour le médecin. Il me serait facile de citer d'autres exemples démontrant d'une façon toute aussi nette cette proposition.

A un point de vue plus strictement médical, les Helminthes sont en train de reprendre en médecine un rôle qui leur était anciennement attribué sans conteste, mais dont les progrès de la bactériologie les avaient dépossédés. La découverte du rôle pathogene des Microbes a été l'origine de progrès surprenants dans l'étiologie, la prophylaxie et le traitement des maladies infecticuses. Par une exagération très compréhensible, on a voulu tout rapporter aux Microbes et ce fut un soulagement singulier pour la médecine que de trourer enfin en eux l'explication de phénomènes pathologiques qui, depuis des siècles, refusaient obstinément de livrer leur secret. Loin de moi l'intention de contester le rôle capital que jouent les infiniment petits dans la production des maladies, mais je suis nettement d'avis que souvent ils ne sont nuisibles que parce qu'ils sont précédés dans leur (ruvre néfaste par divers Helminthes. qui leur ouvrent la voie et leur permettent d'exercer leur action malfaisante.

Gurart a reconnu que l'Ascaris conocephlalus produit dans la muqueuse intestinale du Dauphin des érosions asse\% profondes, glâce aux trois puissants nodules dont sa houche est ar'mée; l'Ascaris lombricoüdes agit de même chez l'Homme, toute proportion gardée. Et, en effet, les cliniciens ont maintes fois noté, mais sans attacher à ce fait l'importance qu'il mérite, l'existence d'Ascarides plus ou moins nombreux chez des individus atteints d'affections intestinales et spécialement de fievvre typhoüde. Rivinerer et Wa(iLER, en 1760 . ont olservé à Göttingen une vio- 
lente épidémie de fièvle typhoüde ou de morbus mucosus, comme ils disaient, au cours de laquelle ils découvrirent le Trichocéphale; ce parasite se trouvait en abondance dans l'intestin des individus dont ils purent faire l'autopsie. On n'ignore pas qu'à une époque tout à fait récente le professeur Metsinikov a reconnu que ce même Helminthe était la cause fréquente, mais non exclusive de l'appendicite.

Est-ce à dire que les Helminthes soient infectieux? En aucune faron: leur rôle pathogene est indubitable, mais il n'est, en quelque sorte, qur préparatoire. L'Ascaride, nous l'avons vu, érode et ulcère la muqueuse intestinale; les dégâts éprouvés par celle-ci sont encore plus graves. quand elle est attaquée par le Trichocéphale, l'Uncinaire et d'autres: Helminthes qui, armés ou non de crochets, la transpercent et s'enfoncent à son intérieur jusqu'au contact des capillaires sanguins. Il se produit de la sorte une série de pertuis minuscules, par où les Microhes pathogènes, qui se rencontrent si fréquemment à l'état de saprophytes dans l'intestin d'individus en bonne santé, peuvent envahir l'organisme et y causel' l'infection. On pourrait donc proclamer cet aphorisme : pas d'infection intestinale sans Helminthes pour frayer la voie aux Microbes infectieux. Voilà qui rendrait aux Helminthes un regain d'actualité, s'ii n'était démontré, d'autre part, grâce aux récentes acquisitions dans le domaine de la médecine coloniale, que les parasites animaux sont beaucoup plus redoutables qu'on ne le croit généralement; ils jouent, en effet, dans la pathologie des pays chauds, un róle alsolument prépondérant.

J'en reviens ainsi à une question qui m'est particulièrement chère. Je suis un partisan convaincu de l'expansion coloniale et je crois fermement que celle-ci ne peut avoir de guide plus sûr que la médecine. Or, les maladies des pays chauds sont en grande majorité de cause parasitaire. et les parasites dont elles relèvent sont pour la plupart de nature animale. Comme la science fait de grands progres dans ce domaine particuliel' et que, d'une année à l'autre, il surgit des questions véritablement imprévues, il m'a semblé nécessaire de créer à Paris, à côté de la Faculté de Médecine, un enseignement complémentaire, d'allure rapide, grâco auquel les médecins coloniaux revenus dans la métropole pussent se mettre au courant de ces questions nouvelles. I) cette préoccupation est né l'Institut de Médecine coloniale, que j'ai été assez heureux pour fonder, grâce à l'appui de l'LTniversité de Paris. Les personnes qui en suivent les cours sont pour la plupart des médecins ayant vécu sous les tropiques et désireux de se perfectionnel dans les nouvelles méthodes d'investigation. Ils retournent là-bas mieux armés pour la recherchr scientifique, connaissant les desiderata de l'heure présente, capables de poursuivre des recherches toujours délicates, l'esprit en éveil et animés du plus vif désir de faire ouvre utile. Il y a lieu d'espérer que leurs 
efforts ne seront pas vains, mais qu'ils pourront élucider quelques-unes des nombreuses questions qui sont encore obscures.

En effet, en élargissant ainsi notre cadre et en étendant nos études à la pathologic exotique, on peut dire qu'un champ immense s'ouvre devant la Zoologie médicale. Si je parlais devant des médecins, je pourrais mpntionnel toute une série de maladies dont l'étiologie est plongée dans la plus profonde obscurité et qui cependant, pour diverses raisons, doivent etre envisagées a mriori comme relevant de la parasitologie animale. La " tick fever" ) du centre de l'Afrique est apparemment de ce nombre. Elle n"est pas sans analogie avec certaines affections parasitaires du bétail, qui sont transmises par la piqûre des Ixodes; il est donc urgent de rechercher si elle ne résulterait pas également de l'inoculation de petits parasites tels que les Babesia.

()n a récemment attribué à ces derniers une forme parasitaire qui se trouve soit dans la peau, dans les cas d'ulcères des pays chauds, soit dans la pulpe splénique, dans les cas de kala-azar et de splénomégalie apyrétique. Les organismes qu'on a confondus avec des Babésies sont, en réalité, bien différents de celles-ci. Imaginez un Trypanosome, qui aurait perdu son tlagelle et sa membrane ondulante et dont le corps se serait condensé en une petite masse ovoïde ayant encore son blépharoplaste: telle est la structure très simple des Leishmania.

Ces parasites ont donc des affinités manifestes avec les Flagellés, bien plus qu'avec les parasites endoglobulaires. Or, quand on les cultive en milieu artificiel, on obtient des petits Trypanosomes. Ces derniers se présentent donc à nous de nouveau comme des organismes paradoxaux qui dérivent, dans certains cas, de formes parasitaires bien différentes d'aspect.

Ces quelques exemples suffisent à montrer l'intérêt des questions que souleve la parasitologie des pays chauds. D'autres problèmes non moins importants seront étudiés demain, et parmi eux figure au premier rang la question de la toxicité des animaux parasites.

(On est familiarisé avec l'idée que les Microbes éliminent des toxines: Roux et Ýrsin ont établi l'existence et le rôle de ces substances dans la diphtérie; depuis cette démonstration magistrale, personne ne doute plus que, dans les maladies infectieuses, certains symptomes ne soient causés par des substances nocives éliminées par les Microbes. Une telle notion doit-elle êtr’e généralisée? Les Helminthes et les autres parasites animaux produisent-ils des substances analogues? Dans quolle mesure agissent-clles et certains phénomènes morbides peuvent-ils leur être attribués? Oui. sans doute, les parasites de nature animale se comportent de la meme fitçon qu' les Microbes et il est vraiment surprenant que l'on ne l'aie pas reconnu plus tôt.

J'en trouve un exemple très démonstratif dans la fièvre paludéenne, l'accès fébrile n'étant que le r'ésultat d'une intoxication de l'organisme. 
En effet. l'Hématozoaire, qui se Inge, grandit et se multiplie it l'intérieur' du globule rouge, obéit à la riggle commune, c'est-ì-dire qu'il assimile des substances étrangères à son organisme, en même temps qu'il désassimile et rejette autour de lui des déchrts solubles. Ceux-ci s'accumulent à l'intérieur du glohule et ne sont déversés dans lo sang qu'au moment où le globule se rompt. Ilssont d'abor'd trop dilués pour être artifs, mais leur quantité augmente à mesure que le nombre des parasites s'élève lui-mème et bientôt ils déterminent une première réaction fébrile. Il est de notion courante que les accès deviennent de plus en plus violents, quand la malarlie n'est pas traitée par la quinine : c'est dire que les toxiness sont déversées dans le plasma sanguin en quantité de plus en plus grande. Cet exemple est, je crois, assez caractéristique; il a clu moins le mérite d'ctre empl'unté ì une maladie dont tout le monde connât la marche et, d'autre part, de donner de la fièvre la seule explication rationnelle.

Cela étant connu, on ne sera pas surpris que les Trypanosomes produisent également des substances toxiques, auxquelles on doit attribuer quelques-uns dess symptômes de la maladie du sommeil. ()n sait déji que le Bothriocéphale cause parfois l'anémire pernicicuse progresisive, non pas parce qu'il cause une hémor'rhagie intristinale, mais par' suite de l'ahsor'p)tion de substances qu'il exereté et qui se trourent déver'sées daus l'intestin; on entrevoit que d'autres Helminthes puissent itre doués de la mime faculté, à un degré plus ou moins accentué. Vivili donc que souver tout un nouveau chapitre de la chimie physiologigue et l'on peut dire que, dès maintenant, il se montre hérissé des pires difficultés.

En vous entretenant de ces questions, je n'ai pas la prétention de vous avoir montré toutes les faces par lesquelles la Zoologie cutte en contact avec la Médecine. Liunion de ces deux sciencess devient chaque jour plus étroite. "Le tempses est proche, me disait récemment sir Patrick Masson, ou chaque Ecole de Médecine devra posséder une chaire de zonlogie: en France, vous avez tranché la question avant les autres pays. ")

Il est trese exact que les Facultés et Exoles francaises sont pourvues d'un enseignement méthodiçue et complet de la parasitologie animale, mais il ne faut pas oublier que, par suite de l'insuffisance des crédits qui lui sont alloués, cet enscignement n'a guère, le plus souvent. qu'un c'aractere thérique. Or', nous avons mis en évidence quelles questions capitales il est urgent de résoudre et dans quuelles voins la science doit matintemant s'engager. Les recherehes dont on attend la solution ne peuvent être conduites à un bon résultat que si l'on dispose de moyens diaction puissants, je veux dire de erédits suffisamment élevés. L'argent n'est pas senlement le nerf de la guerre, il est bien plus encore celui do lat srience. Le succès sourit à ceux qui, sortant des spéculations théoriques ot alsitraites, luttent corps à corps avere les problèmes ot leme arrachent leur secret. 
Les Ecoles de médecine tropicale de Londres et de Liverpool ont fait dans ces dernières années une remarquable besogne dans le domaine de la parasitologie des pays chauds, non pas tant à cause de la valeur, d'ailleurs incontestable, des hommes éminents qui ont pris la direction de ce mouvement nouveau, qu'ì cause des subsides considérables que la générosité publique a mis à leur disposition. D'autres pays ont attaqué la yuestion sous une autre forme. L'Allemagne, par exemple, a créé près de l'Office impérial de la santé publique (Kaiserliches Gesundheitsant) une section de parasitologie animale, à la tête de laquelle le $\mathrm{D}^{\mathrm{r}}$ Schaudinn vient d'être placé avec le titre de conseiller d'Etat; c'est un heureux complément d'une Institution qui a rendu déjà les plus éminents services et c'est pour elle le point de départ de nouveaux progrès. Les Etats-Unis, de leur côté, devenus puissance coloniale par la conquête de Porto-Rico et des Philippines, ont créé à Washington, comme dépendance du Service de l'Hôpital maritime, une IDivision de zoologie médicale dont le chef éminent est le $\mathrm{D}^{\mathrm{r}} \mathrm{Ch}$. Wardell Stimes: le passé répond de l'avenir et, sous son impulsion féronde, la nouvelle Division ne va pas tarder à devenir l'un des foyers de recherche scientifique les plus actifs et les plus productif's. Les deux savants dont je viens de prononcer le nom sont assis dans cet amphithéâtre: il m'est particulièrement agréable de leur rendre publiquement hommage, de les féliciter de la haute situation scientifique à laquelle ils ont été récemment appelés et de leur souhaiter bon augure pour les recherches dont ils vont être les instigateurs.

Ie tels exemples mériteraient d'être suivis par tous les pays possédant des colonies intertropicales; il ne suffit pas, en effet, de constater le progrès du voisin, il faut aussi savoir consacrer à la recherche scientifique les sommes qui lui sont nécessaires. Espérons que les pays qui se sont montrés jusqu'à présent réfractaires comprendront bientôt qu'il y va de leur honneur et de leur bon renom scientifique d'instituer des établissements et laboratoires du même genre ou du moins de doter avec une plus grande libéralité ceux qui existent déjà et auxquels ne manque point la volonté de bien faire.

Quoiqu'il en advienne, il est clair que la Zoologie médicale n'en est encore qu'à ses débuts; d'importantes questions se présentent en foule, qui réclament une solution prochaine et la pénétration des nations civilisées dans les régions encore inexplorées ou insuffisamment connues fera surgir un grand nombre d'autres problemes dont la Parasitologie donnera la solution. Après l'éclatante période que vient de parcourir la Bactériologie, nous saluons avec confiance l'aurore des temps où la Zoologie médicale atteindra son apogée.

M. le Président donne la parole à M. A. Lang. 


\title{
ALEXANDER MORITZI, EIN SGHWEIZERISCHER VORLAEUFER DARWINS
}

\author{
Von Prof. Annot. LANG
}

(Zürich).

\section{Hochansehnliche Versammlung! \\ Verehrte und liebe Kollegen!}

Die Geschichte der Biolngie weist eine grosse Anzahl von Männern, von Naturforschern wie von Dichtern und Philosophen auf, die insofern als Volläufer Darwss gelten kömnen, als sie den Gerlanken der Umbildungsfähigkeit der Organismen vertraten, bald klar, bewusst, mutig und wohl auch temperamentvoll, bald unbestimmt und verhüllt, oder schüchtern und reserviert. Von dem grossen edlen Lamanck, in dessen Geiste sich das grossartige Bild der erdgeschichtlichen Entwicklung der Organismenwelt in scharfen Lmrissen zeichnete, dessen Name heute in aller Munde ist, wo mit dem neuerwachten Vitalismus der Lamarck'sche formbildende Faktor, die direkte Anpassung, wieder lehhaft in I)iskussion steht, bis zu jenen ängstlichen Forschern, die den Arten rine nur beschränkte Teränderlichlieit zuschricben und jenen uralten Philosophen, dic da erkannten, dass alles im Fluss begriffen ist, dass auch die organische Natur dem Werden und Vergehen unterliegt und dass nur aus der nimmerruhenden Betätigung der Kräfte und dem eifrigen Wettstreite Höheres, Vollkommener'es hervorgeht.

Unter diesen Torläufern I Arwss nimmt, wie ich glaube, der Mann eine hervorragende Stelle ein, von dessen Ansichten ich Ihnen heute sprechen will. Was die Originalität und Selbstïndigkrit und die klare Erkenntnis der 'Tragweite der' Probleme anbetrifft, so steht er' nicht sehr' weit hinter Lamarck, während er wohl von keinem der übrigen übertroffen wird. Mit Lamarok teilte der schweizerische Naturforscher Alexander Moritzr von Cliur das Schicksal, dass seine Ideen zu seinen Lebzeiten fast unbeachtet blichen. Das Verdienst, ihn wioder entdeckt zu haben, gebührt dem Botaniker Potoví́, der 1881 und sodann 1899 Auszüge aus seiner diesbezüglichen Schrift verötfentlicht hat. 'Trotzdem ist Moritzr auch von den IBotanikern noch wenig gewiurdigt worden: den Zoologen scheint er noch vollkommen unbekannt zu sein. Ich selbst verdanke Her'n Kollegen I) Bretrcher den Hinweis auf seine Wiederentdeckung durch Potonié. Monitzi's Schrift, die ich mil ungesälumt 
verschaffite, erweckte mein lebhaftes Interesse und ich begann auch sofort biographische Nachfor'schungen, in denen ich besonders durch meinen frïheren Schüler, Her'n Prof. Dr J. Bцосн in Solothurn und durch Herrı D P. Lorkxz in Chur auf das Liebenswürdigste unterstützt wurde, die aber trotzdem noch nicht zu einem befriedigenden Abschluss gekommen sind.

An dieser Stelle mögen einige kurze Daten aus dem Leben Morirzi's genügen. Eine ausfïhrlichere Bingraphie werde ich anderswo veröffentlichen.

Alexander Monrтzi wurde am 24. Februar 1806 in Chur, dem Bürgerort seiner Familie, geboren. E' besuchte die Kantonsschule seiner Vaterstadt und soll nachhel an den Universitäten Basel, München und Leipzig studiert haben. Sicher ist indess nur, dass er das Sommersemester 18: in München zubrachte und sich hier unter ller Leitung Zrcosrrsis, an dessen Exkursionen er teil nahm, fast ausschliesslich botanischen Studien wirlmete. Ton 1828/29 bis 1839/40 lebte er, vorwiegend mit systematischfloristischen Arbeiten heschäftigt, abwechselnd in Chur und Genf. Schon 1832 gab er unter dem Titel "Die Pflanzen der Schweiz n eine SchweizerFlora heraus, der 1839 eine Abhandlung über die Pflanzen Graubündens folgte. In Genf wurle el von Aug.-Pyr. DE Candolde beschäftigt uni unterstïtzt und verfasste auf dessen Anregung hin ein Wörterbuch der Tulgärnamen der Pflanzen in 60 Sprachen und Dialchten, wozu ihn seine Sprachkenntnisse besonders befühigten. Diese Riesenarbeit fand keinen Verleger. Das Manuskipt befindet sich noch heute in den Händen von C. de Candolle. 1839/40 wurle Moritzi als Nachfolger Hu'ir's, zum Professor der Naturgeschichte an der Kantonsschule in Solothurn ernannt.

Auf diese Solothurner-Zeit fällt die Publikation seiner descendenztheoretischen Arbeit und neben andern botanischen und geologischen $\mathrm{Ar}$ beiten die Neuausgabe der "Floru der Schuveiz ». Monrtar scheint sich hier zu viel zugetraut zu haben und es an der nötigen Sorgfalt und Gründlichkeit haben fehlen lassen. Ein kleiner Angriff auf die Zürcher Schule der Botaniker rief einer schonungslos niedel'schmetternden Kritik von ()swald Heer und K. W. Nïnel. Im Jahre 1st6. nach Ablauf del sechsjährigen Amtsperiode, wurde Moniтz nicht wiedergewählt und musste zu Gunsten seines Schülers, Franz Vinzenz Laxo, zurücktreten. der in seiner ohrwüroligen Greisengestalt noch lebhaft in dex Er'inuerung der" meisten lebenden sehweizerischen Naturforscher fortleht. Woritzi zog sich nach Chur zurück, wo er, zum Mitglierl des Bürger- und Erziehungsates ernannt, sich intensiv mit offentlichen und WohlfahrtsAngelenenheiten befasste, in der dortigen naturforschenden (iesellschaft eine grosse Rolle spielte und als deren Präsident schon am 13. Mai 1850 starb. Im Jahre 1 sīg setzten ihm seine Mithürger in den Anlagen am Rosenhügel einen Denkstein mit der Inschrift : 
"Zur Erinnerung an

\author{
Prof. ALEXANDER MORITZI \\ 1806-1850 \\ den verdienten Botaniker \\ und \\ Begründer dieser Anlage \\ 1879. "
}

Im Septemberheft 1800 der" "Archives des Sciences naturelles " widmete ihm Alphonse de Caxborate einen kurzen Nachruf voll Wohlwollen und Anerkennung. Alles woist darauf hin und auch DE CANDoLl_e äussert sich in diesem Simne, dass Moritzr, der eine gewandte, of etwas spitze Ferler führte, ein sehr selbstandiger, unahhängiger, etwas agressiver, im übrigen aber durchaus ehrenhafter ('harakter, dass er von warmer Liehe zur Wissenschaft besecelt und in uneigennützigor. Woise bestreht war, dem öffientlichen Wohl zu dienen.

Seine ketzerischen Ansichten über die Schöpfung dre Organismenwelt hat Moritzi in seiner kleinen. 10! Seiten unfassenden, 1842 in Solothur'n erschienenen Schrift "Réflexions sur l'espèce en histoire naturelle » nicilergelegt. Tie Schritt ist so durch und ilurch originell, dass man den Eindruck belommt, Monrtzr sei ginz von selbst auf seine Ansichten gekommen. Torläufer nemnt el nirgends mit Namen. Es ist allerdings auf-

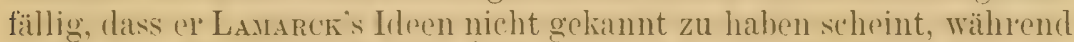
el Crvier zitiert. Ich darf abel' die Bemerkung nicht ühergehen, die sich bei ne Canvor.te findet, obschon ich ihre Richtiglieit fast bezweifoln möchte, dass Mon'tzr seine Ansichten über die Veränderlichkeit der Art aus der Schule HeGETschry

Wiederholte, bissige Ausfälle geoen die Kataklysmen- und die Eiszeittheorie sind zweifellos gegen Louis Arassiz gerichtet, obschon dessen Name in diesem direkten Zusammenlange niræends genannt wird. Offenbar war das etwas sehr geräuschvolle Auftreten les fast gleichaltrigen Neuenburger Professors, dir damals fast auf dem Gipfel seines ruropäischen Ruhmes stand, nicht mach dem Geschmacke Donitzi's, der nach del Aussage von Augustin-Pyrams de Cannolde war " un homme inhabile a se faile valoir". Was man von Arassiz nun nicht gelarle behaupten kann.

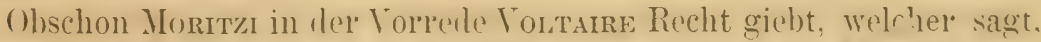
dass in den Erfahrungswissenschaften nichts weniger angehracht sei, als eine poetische Rerleweise und die Telschwendung von Reileblumen. so wild el sich selbst doch später in seinem Eifer über das marktschrejepische Auftreten gewissel Naturforscher" untreu. "Lal vélité, "sagt "1', 
" craint ces moyens violents que les hommes impatiens de gloire emploient pour l'ar'acher de son sanctuaire. C'est une tendre fleur qui ne s'ouvi'e qu'au souffle de l'amour' qu'on lui porte. v

In Ifr Vorrele entschulligt sich Monrtzi in boshafter Weise dafür, dass el nicht deutsch geschrieben habe:

"Indépendemment de cet avantage qu'un livre français est lu par les Allemands tandis qu'un livre allemand ne l'est point par les Français. j’ai une certaine répugnance à lancer', comme on dit, une nouvelle idée (eine neue Ansicht) dans la république des savans allemands. Ces nouvelles idées sont tombées en déconsidération, du moins chez les véritables naturalistes, parce qu'en réalité elles n'ont servi jusqu'ici qu’à embrouiller ce qui était clair et à compliquer par un luxe de nouveaux termes ce qui auparavant paraissait simple. »

Der erste Teil von Moritzi's Schrift enthält eine einlässliche Kritilides Artbegriffes und Erörterung seiner Definitionew. Wenn man als spezifisch identisch alle diejenigen Individuen betrachtet, die untereinander fruchtbar sind oder fruchtbare Nachkommen hervorbringen, so sei dies Criterium nicht allgemein gültig. Er zitiert mit Recht die fortptlanzungsfühigen Kanarienbastarde, die Bastarde zwischen Pferd und Esel, dir hybriden Cirsiumformen. Ausserdem nützen jene Definitionen nichts, wenn es sich darum handle, die Art von der Varietït oder Rasse oder von individuellen Variationen zu unterscheiden. Im Uebrigen anrrkennt Morrez, dass dieses Criterium theoretisch (ideell) noch das am meisten befriedigende sei, besonder's wenn es sich handle, die Art nach olen, gegenüher der Gattung, abzugrenzen. Praktisch lasse es uns aber in den meisten Fällen noch für lange Zeit und in vielen für immer im Stich.

Sorlann nimmt Morixzi jene andere altbekannte Artdefinition unter die Lupe, nach welcher zu einer Art alle Individuen gehören, die aus rinem und demselben Stamme hervorgegangen sind, wobei gemeint ist, lass sie die Merkmale der stammeltern unverändert beibehalten haben.

Diese Definition entspricht im Wesentlichen der Ray'schen Auffassung, welcher Lixwé einen dogmatischen Charakter verliehen hat in dem beliannten Satze: "Species tot numeramus, quot diversie formae in principio sunt creatæ."

Jene Definition, meint Ioriтzr, ist ausgezeichnet für die Zukunft, für dic Vergangenheit aber hilft sie uns nicht viel, denn sie setzt eine Kenntnis der Abstanmung voraus, dio wir im allgemeinen durchaus nicht besitzen. Was wir über die Vergangenheit unserer ()rganismen wissen, beschränkt sich auf einige wonige Fälle, die wohl kaum weitgehende Analogieschlüsse erlauben, beschränlit sich im allgeme'inen auf die Zier- und Kulturpflanz'n und Haustiere, die in historischer Zeit aus Amerilia oder dem Orient ringeführt worden sind. Wenn man sehr skeptisch sein 
wollte, so könnte man sogar noch in Zwwoifel zielien, ob alle heute lebenden Repräsentanten der betreffenden Arten von einem und demselben Individum abstammen. Wenn es nun einerseits sicher ist, dass manche Tiere sich durch lange Zeitrïume hindurch unverändert erhalten haben, so ist anderseits, sagt Montтz, die wichtige Tatsacine wohlbekannt, dass gewisse Organismen, besonders Zierptlanzen, durch die Kunst des Gärtner's wichtige Veränderungen erlitten haben. I)jo Definition leidet aber nach Moritzi noch an dem Hauptfehler, dass sie nicht nur für die Art, sondern auch für die Rassen und Varietaten gültig ist. Gewisse Tierrassen und Ptlanzenvarietaten sind durch Jahrhunderte hindurch lionstant geblieben. Ins Araberpferd ist heute, was es zu SAlomons Zeiten wal und wenn in einem Jahrtausend noch Nachkommen dieser Rasse in den Wüsten Afrikas und Asiens leben werden, so werden es dieselben anmutigen, gescheidten und kräftigen Tiere sein. Es ist bemerkenswert, wie zäh sich gewisse leichte Iodifikationen vom Irerkmalen in der Nachlommenschaft erhalten. Das ist sogar bei Difformitäten der Fall, deren Ursache oft eine zufällige Störung ist. So ist bekannt, dass die SechsFingrigkeit sich mehrere Generationen hindurch erhalten hat. Wer weiss nicht aus Erfahrung, wie frappant sich gewisse Familienzüge von Generation zu Generation wiederholen!

Mit Recht bemerkt Norraz fermer, dass unsere direliten experimentollen [ntelsuchungen über die Konstanz der Artmerkmale wenig zahlreich und wenig heweiskläftig sind. Es genügt durchaus nicht, sagt er, eine Pflanze ein- oder zweimal in einem Garten anzusien. Man muss das eine Reihe von Jahreu hindurch wiederholen, im Norden und im Süden, auf trockenem und sumptigen Borlen, im Waldesschatten und auf von Bäumen enthlössten Felsen, in kieselhaltiger, in kalkreicher und in lehmiger Erde.

Am Ende dieser kritischen Erörterung der Artdefinitionen frägt sich Moritzr, ob nicht dio ()rganisation der Lebewesen selbst ein allgemeines Kilassifikationsprinzip linfore, welches für sich allein eraube, die Arten zu unterscheiden. Auch hier golangt er, in W'ürdigung der ausserordentlich verschiedenartigen Organisation der verschiedenen Tiergruppen, des Umstandes, dass es in den verschiedenen Gruppen ()rgane gibt, die zwar demselben Zwecke dienen, aber einander nicht entsprechen, sich nicht miteinander vergleichen lassen, und der' Tatsache, dass gewisse Gruppen Organe besitzen, die bei andern fehlen und dass die ()roane im Tierreich überhaupt ganz allmählich auftreten und sich homplizieren, zu einem durchaus negativen Resultat.

Zum Schlusse gibt Morimzi noch folgendes Aneliö̈tchen zum besten: Ein Philosophieprofessol hat ihm gesagt und die Sentenz hat ihn im Munde eines Philosophen nicht verwundert: I)ie Art existirt, ganz. gewiss, aber man hat vielleicht ihre richtige Definition noch nicht gefun- 
den. Dazu brmerkt Monrazi höhnisch, dass eine Idee im Kopfe eines Menschen existire, die in den Kopf eines andern nicht hineingehe, sei ja begreiftich, dass abor in einem Kopfe eine Idee, - denn die Art sei eine Idee - existire die ihm selbst unbekannt sei, dies sei eine Absurdität.

Trie liommt man denn dazu, an die S'pezies zu glauben? Diese Frage discutiıt Monitzi in einem lesenswerthen kleinen Kapitel, aus dem ich folgendes hervorhebe. Der Mensch lernt zuerst unterscheiden, bevor er dazu kommt zusammenzufassen. Er ist aus praktischen Gründen gezwungen zu klassifiziren und wählt zu diesem Zwecke die Merkmale, die ¿mm meisten in die Augen springen. Diejenigen Systeme, und mögen sie noch so künstlich sein, passen ihm am besten, die für das Unterscheiden und Bestimmen der Gegenstände am bequemsten sind. Selbst den Naturforschern ist das Bedürfniss nach natürlichen Kilassifikationen erst kürzlich gokommen, weil ihnen die Idee der Verwandtschaft fremd war. Der junge Naturfor'scher, der zu sammeln beginnt, bringt seine Zeit mit Unterscheiden zu. Un schneller und leichter zum Ziele zu gelangen, benützt er Bücher, in denen dir Charaktere notwendig schärfer markirt sind, als in der Natur selbst und in welchen, ohne Rüclisicht auf ihren Werth, die anffälligsten Unterscheidungsmerkmale angegeben sind. Die weniger auffälligen werden äbergangen oder höchstens zur Unterscheidung von Varietiten benutzt. Unsere Beschreibungen sind ausserdem häutig das Ergebniss des fehlerhaften Verfahrens, dass man nur diejenigen Merkmale berücksichtigt, in welchen die meisten Individuen übereinstimmen, während man die abweichenden Nüancen de• Minorität, welche oft Uehergangsformen enthält, ausser Acht lässt.

So kann man junge Botaniker mit dem Buch in der Hand botanisiren, diejenigen Exemplare, die mit der Beschreibung überoinstimmen, auswählen und diejenigen wegwerfen sehen, welche die vom Buche vorgeschriebenen Merkmale nicht besitzen. Dass auf diese Weise die Species zu einer scharf abgegrenzten wird, ist selbst verständlich. Alles das trïgt dazu bei, die Idee der natürlichen Gruppen, die der Erfahrung entspringt, im Geiste der Menschen zu der staren Idee der scharf begrenzten Species zu gestalten.

Wrom os num aber mit der theoretischen (idecllen) Formulirung des Artbegriffes sehr misslich steht, so sicht es mit der praktischen Anwendung vollends schlimm aus. In Wirklichkeit kümmern sich die Entdecker nener Arten sehr selten um die theoretischen Definitionen. In drastischer Weise schildert Morterz das Verfahren hei der Aufstellung nener Species und die wunderbare Zunahme ihrer Zahl seit der Zeit, da resebrüuchlich ist, den Namen des Ertinders hinter den Speciesnamen zu setzen. Es werden neue Arten beschriehen auf (rrund eines einzigen, erhärmlichen, unvollständigen Herbarimmsemplar's, dem vielleicht der Stengel odor die Wurzeln, die Blüthen oder Friühte fehlen. Vergess- 
lichkeit, Zerstreutheit, Verwechslung von Etiqueten u. s. w., spielen eine grosse Rolle und sind die ergiebige ()uelle von Irrthümern. Ein und dieselbe neue Pflanze, die zufällig zu gleicher Zeit in drei verschiedenen Länder'u entleckt wird, wird von dem einen Forschel als Varietät einel Art, von dem ander'n als Varietät einer' ander'n verwandten Art beschrieben, während der dritte Fntdecker eine neue Art daraus macht. Ohne Cintersuchung der Exemplare selbst lässt sich nach den mangelhaften Beschreibungen die Identität der drei formen schwerlich feststellen. Und dann der endlose Streit über den Grad der Wichtigkeit der Untersuchungsmerkmale! Niemand kommt auf die Idee, dass die Unfruchtbarkeit dieses Streites einen tieferen, in den Dingen selbst liegenden (irrund hat, der in der Nichtexistenz der Art beruhen könnte.

Wenn nun schon derartige Erörterungen und Ueberlegungen nicht geeignet sind, die Idee der Art - gemeint ist hier die scharf abgrgrenzte, zeitlich unveränderliche Art - zu stützen, so giebt es verschiedene Wege, die nach Morıtz dazu führen, die Existenz der Art direkt zu bezweifeln.

Einer dieser' Wege ist der ganz specielle der direkten Beobachtung und positiven Feststellung. Er besteht darin, irgend eine Gruppe von ()rganismen aufmerksam und ohne Voreingenommenheit zu studieren. Man muss zu diesem Zwecke einheimische Tiere und Pflanzen wählen, um sie bequem in allen ihren Entwicklungsphasen und in ihrer natiulichen Ungebung, an verschiedenen Lokalitäten und in verschiedenen Bodenverhältnissen studiepen zu können. Man muss ferner Gruppen wählen, die aus nahe verwandten Arten bestehen. Gehören dazu fremolländische Spezies, so müssen auch diese in die Untersuchung einbezogen werden.

In dieser ITeise hat Monitzi selbst cine Reihe von Gruppen untelsucht. Er nemnt Festuca, Primula, Erigeron, fermer die Rosen, Hieracien, Ranunculaceen und teilt oinige Resultate seiner diesbezüglichen Beobachtungen mit. Besouder's die lückenlosen Reihen der ineinander fliessenden Hieracien sind es gewesen, die in ihm die ersten Zweifel über die Existenz der Art erweckten.

Diese Ausführungen sind durchaus zutreffend und es citiert Moritzi Pflanzengruppen, hei denen auch die seitherige Forschung dic Unmöglichkeit der scharfen Abgrenzung der Arten festgestellt hat.

Es giebt aber auch Ueberlegungen allgemeinerer Art, welche zum Zweifel führen. Die vergleichende Anatomie lehrt uns, dass die verschiedenen Organe von den einfachsten Organismen an successive bis zu den komplizierteren ununterbrochene Reihen von Umbildungsstadien darbieten. Die einfachste und für denjenigen, der nach natürlichen U' sachen sucht, auch natürlichste Erkiärung dieser Continuität der Gestaltung sei doch wohi die, dass sie das Resultat einer Continuitat der auf dic Organe einwirkenden Einflüsse sei, die ihre Fol'm verändern. 
Auch die Tatsachen der Geologie führen zum Zweifel. Indem sie uns die erdgeschichtliche Aufeinanderfolge der Organismen oftenbaren, tragen sie mächtig zur Stütze des Gedankiens der stufenweisen Entwicklung der (Jrganismemvelt bei. Die höchsten und vollkommensten Tierformen, die Sïugetiere und Vögel, treten auf der Erdobertläche erst zuletzt auf. Moritzi wendet sich scharf gegen diejenigen Geologen, welche die Continuität der Schöpfung leugnen und prinzipiell die totale Verschiedenheit der ()rganismenwelt der verschiedenen Epochen behaupten, inshesondere gegen diejenigen, welche annehmen, dass eine allgemeine Vergletscherung und Vereisung das ganze Leben auf der Erdohertläche zerstört habe, und welche zu gleicher Zeit, um zu zeigen. dass bei dieser Gelegenheit wirklich alle Lebewesen zu Grunde gingen, alle heute lebenden Organismen als spezifisch verschieden von denjenigen erklären, welche vor der Eiszeit lebten.

Ernste Zweifel über die Constanz der Art müssen auch die Beobachtungen an Culturracen erwecken. Wie kommt es, class die Haustiere und Culturpflanzen die grösste Zahl von Varietäten zeigen, die sich oft durch Merkmale unterscheiden, welchen man sonst spezifischen oder gar generischen Wert beimisst, und denen auch die Constanz nicht fehlt ? Offenbar sind es doch die viel mannigfaltigeren Veränderungen in den Fxistenzbedingungen, denen sie ausgesetzt worden sind, welche diese grössere Variabilität hervorgerufen haben.

Yon allen Seiten bedrängen Zweifel den Geist Morrtzi's. Woher kommen die weitverbreiteten Aehnlichkeiten im Bau der uns umgebenden ()rganismen. Ist es ein Zufall, dass 50)000 Insekten nach demselben Bauplan gebildet wurden? Oder hat der Schöpfer daran Gefallen gefunden, 49,999mal mit nur kleinen Veränderungen sein eigenes. Modell zu kopieren, durch ebensoviele direkte, spontane Schöpfungsakte? Oder ist es nicht vielmehr die Natur selbst, welche durch allmähliche ummerkliche Veränderungen der Existenzbedingungen Veränderungen an den Organismen hervorgerufen hat? Wir sind wohl alle übereinstimmend überzeugt, dass es sich nicht um einen Zufall handeln kann, meint Moriтz: : Aber wenn es sich um direkte Schöpfungsakte handelt, so wäre es doch, nach menschlichem Urteil, des Schöpfers würdigel gewesen, Formen von grösserer Mannigfaltigkeit zu el'zeugen, alssiebeständig nach demselben Plan zu bilden. Inser Staunen und unsere Bewunderung würden in viel höherem Masse erweckt beim Anblick von kl'ystallförmigen Tieren, die ohne Füsse laufen und ohne Mund fressen würden, oder bei der Betrachtung von Bäumen, die so wüchsen, dass sie zum Wohnen bequem eingerichtete Häuser bildeten.

Auffallend ist auch, dass die letzte Analyse der organischen Substanzen lauter Stofte ergieht, die schon in der anorganischen Natur vorkommen. Der Schöpfer hat sich also ohne Zweifel bei der Hervorbringung der zu 
organisierenden lebenden Substanz der schon preexistierenden anorganischen Stoffe bedient. Wenn er aber die Organismen aus anorganischen Substanzen gebildet hat, warum sollte er dem nicht auch ()rganismen aus schon bestehenden Organismen gebildet haben?

Noch viele andere Fragen, man möchte fast sagen indiskrete Fragen. über das Vorgehen des Schöpfers hei der Schöpfung der Organismen stellt sich der grüubelnde, zweifelnde. vor kieiner Consequenz zurückschreckende Geist unseres naturae curiosus. Wir übergehen sie und gelangen zu dem Schlussresultat der Betrachtungen Moritzi's.

Wenn es konstante, scharf umgrenzte Arten giebt, so sind sie als solche ersehaffen worden. Wenn sie er'schaften worden sind, so ist es nicht ander's denkbar, als dass eine jede durch einen besonderen Schöpfungsakt helvorgebracht worden ist. Die Geologie zeigt uns anderseits, dass in dem Masse als die Organismen der älteren Formationen verschwinden, in den darüberliegenden neue organische Formen an ihre Strlle treten. Die Schöpfungsakte müssen sich also widerholt haben. ()b man num sechs oder zwölf oder irgend eine andere Zahl von Schöpfungsaliten annimmt, ist vom theologischen Standpunkt aus einerlei; die Annahme verstösst so wie so gegen das kirchliche Ingma. Anstatt sechs odel zwölf kann man ebenso gut hundert oder tausend Schöpfungsakte annehmen, und am Ende ist es ebenso vernünftig, eine ununterbrochene Schöpfung anzunehmen, welche von einem Geschöpfe zum nächstfolgenden übergehend. die Erde nach und nach mit ihrer Organismenwelt bevölkert hat.

Aber zu diesem Resultate, der Annahme einer stufenweisen Entwicklung der Organisationswelt, der Annahme einer Abstammung der heutigen Organismen von früheren, der Annahme, dass die Natur aus einer fast homogenen Masse, wie sie den Körper der niedersten Tiere bildet, durch allmähliche Spezialisierung und lokale Umbildung die zusammengesetzten ()rganismen habe hervorgehen lassen, sodass die verschiedenen Organe in den Organismenreihen nur successive auftraten, zu dieser Annahme einer liontimirlichen Schö̈fung führten ja auch, sagt Moritzi, alle andern Ueberlegungen allgemeiner und spezieller Natur, botanisele und zoologische Betrachtungen, die berechtigten Zweifel an der Existenz der Art, die Uebergangsreihen, die Variabilitat u. s. w. Was uns aber die Geologie nicht lehren kann, das lehren uns hotanische und zoologische Betrachtungen: die Art und Weise nämlich. wie dic allmähliche Schöpfung erfolgt ist. Sie zeigen uns in. den äusseren fhysischen Einfliissen die bewirkenden Uisachen, die sich als vermittelude Agentien zuischen Schöpfer und Geschöpf einschalten. So wird in den Augen Moritzi's div Entstehung der Organismenwelt buchstäblich zu ciner natiorlichen Schöpfungsyeschichte.

Moritzi sucht anch gewissen Schwierigkeiten seiner Theorie. die ihm nicht entgangen sind, zu begegnen. Zu diesen schwierigkeiten gehören in 
erster Linie die grossen Lücken in den paläontologischen Reihen. Wenn, so sagt Moritzi, die Organismen so entstanden sind, wie ich annehme, so konnte es keine Unterbrechung in ihrer Schöpfung geben. Ebensowenig konnten sich anfänglich andere Verschiedenheiten ausbilden als individuelle Nüancen. Indem sich diese Nüancen immer weiter vom primitiven Typus entfornten, kamen schliesslich differente systematische Einheiten von sehr verschiedenem Werte zu Stande. Wenn in den ältesten sedimentïren Formationen schon Vertreter der vier grossen Tierkreise vorkommen, so ist sehr wohi anzmehmen, dass während der Epoche der Bildung der Uebergangsformationen, welche sehr lange gedauert haben muss, eine schr lange Entwicklungsstrecke zurïckgelegt worden ist, was um so wahrscheinlicher wird, wenn die Anmahme richtig ist, dass die Temperatur in jener Epoche eine höhere und die Reproduktionskraft des Organismen eine energischere war. Dem entsprechend mussten auch die Veränderungen der Organismen noch viel bedeutender sein, als leutzutage selbst in den Tropenländern. Mit Recht schreilst Moritzr die Lïckenhaftigkeit der geologischen Urkunden auch dem Unstande zu, dass viele Organismen nicht versteinerungsfïhig waren. Vergessen wir nicht, sagt er, dass eine Masse von Tieren und Pflanzen dem Geologen notwendig entgehen, weil diese Organismen zu ihren Lebzeiten nur aus weichen und sich rasch zersetzenden Substanzen bestanden. Diese Lebewesen konnten keine Spur ihrer Existenz hinterlassen. Wenn wir nun annehmen, dass zur Zeit der Ablagerung der den versteinerungsführenden Sedimentgesteinen vorausgehenden Formationen nur Organismen mit weichen Teilen lebten, so können sich aus jener Zeit auch keine fossilen Reste erhalten haben.

Morimzi macht ferner darauf aufmerksam, dass die Geologen jälılich Zwischenformationen entdecken, die auch paläontologisch zwischen den darüber und darunter liegenden Schichten vermitteln.

Auch jenen andern Einwurf sieht Moritzi voraus, dass ja heutzutage noch neben den höhern Organismen solche existicren, welche auf den niedersten Stufen tierischer und pflanzlicher Formbildung stehen. Auch diesen Einwurf sucht ex zu entkräften, freilich in ungenügender Weise, die unser Interesse nur wenig zu wecken vermag.

I)er Bedeutung seiner evolutionistischen Auffassung für dic Beurteilung des Systems, für die Aufstellung einer natürlichen Klassifikation, war sich Moritzi wohl bewusst. Er erkannte, wie übrigens schon viele vor ihm und besonders Lanarck, dass die lineare Anordnung eine unnatürliche ist. Wenn die Organismen so entstanden, wie er, Moritzi, annimmt, so können sie nicht eine einzige Reihe hilden, sondel'n sie müssen 'ntsprechend der Verschiedenartigkeit der Existenzbedingungen, in die sie gerieten, eine komplizierte und verästelte Reihe darstellen. Ein Ver- 
gleich, den el vorbringt, wird sie gewiss lebhaft interessieren. Er sagt: Welches System man auch immer adoptieren möge, immer wird die Form in der man es in den Bücher'n zul Darstellung bringt, eine lineare sein, weil man mit $\Lambda$ beginnen und mit 7 anfhören muss. Dieso Anolednung ist aber so wenig natülich und gicht uns so weuig ein getreues Bild von den natürlichen Zusammenhängen, als die abgeschnittenen und in einel Reihe in den Boden gesteckten Zweige eines Baumes uns ein Bild von seiner Ver'zweigung zu geben vel'mögen.

In beredter Weise äussert sich Moritzr über die durch seine Ansichten bedingte Auffussung der. Harmonie und der Zuechimissiglieit in de' ( ) ryanismenuelt. Dic Harmonic, sagt er, welche in der Natur her'scht, wirel allgemein betıachtet als der Austhuss eines tiefen Schöpfungsgedanlens, ler das ganze (xetriele der Organismenwelt zum Voraus und bis in die kleinsten Einzelheiten eingrichtet, der von Anfang an alle kommenden Bedürfnisse vorgeschen und der durch allespezicllen Einzelerscheinungen hindurch es auf den einen Endzweck alogesehen hat, den. Wensehen. Dabei Könne nur das die Aufơabe des Naturforschers sein, diesen Sihöpfungsgedanken in allen Einzelerscheinungen nachzuweisen.

Wir aber, so ruft Moritzi aus, weit entfernt, die Harmonie in der Natur leuguen zu wollen, erkennen sie als rine Notwendigkeit. I)er Luft, dem Wasser, dem Kilima, der Bodenbeschaffenheit, der Nahrung sind die Tiere und PHanzen gerade deshalb angepasst. Weil eben diese Faktoren aus den Tieren und Pflanzen das gemacht haben, was sie sind. Diese konnten nicht Gewohnheiten annehmen, dir den Ursachen, wolche Gewohnheiten hervorrufen, entgegengesetzt sind. Wrnn die Existenzlvedingungen, denen (in Organismus angepasst ist, aufhöpen zu wirken, so muss der Organismus zu Grumle gehen. Wenn sie aher ummerkich und stufenweise sich verändern. so veränder`t sich auch die Organisation, den neuen Bedürfnissen entsplechend.

Wie Sie sehen, sind es die heute sogenannten Lamarck'schen Fattoren, sind es die direkten Anpassungen, denen .Ioriтzi die zweckmässigen L'mwandlungen (ler Organismen zuschreibt. I)abei ist es interessant festzustellen, dass Moriтz in dem festen Glauben leht, damit eine causale Evliburung im (regensatz zu der teloologischen der maistabilirten Harmonie gefunden zu hahen. Tirse heiden Systeme, sagt MoniTzr. schliessen sich gegenseitio aus. Wemm das Finnlitiitssystem in de1 Natul waltet, so kann as lieine cunsale Terknïpfung der Erscheinungen gehen. Herrscht aber Causalitit, so fallen von selbst alle jene Fragren übex den Zweck oder die Absicht dahin, die der schöpfer bei der Bildung diesos oder jenes Organismus haben mochte.

Wenn dereinst unsere Ansichten, wemn dereinst, ruft Morr'sr aus, die Causalität anerkannt sein wird, so wirh man die Frage nicht mehr hören, zu welchem Zwecke sich die Berge erhoben haben. Man wird sich nicht,

VIe CONGR. INT. ZOOL., 1904. 
mehr den Kopf zerbrechen, um zu erfahren, zu welchem Zwecke die dem Menschen schädlichen Thiche crichatfen worden sind. Man wird in solchen Fragen eine allzumenschliche Art der Beurtheilung der Dinge exblicken und man wird es hochmüthig und anmassend finden, dem göttlichen Geist ausichliesslich auf die Existenz des Menschengeschlechtes gerichtete Gedanken zuzuschreiben.

Schon hieraus geht mit aller Klarheit hervor, dass MonrTz die anthropocentrische Wranschaung verwirft. Dass er sogar den ticrischen Ursprung des Menschen annimmt, geht überdies aus einer andern stelle heror", wo er davon spricht, dass die höchsten Thiere erodgeschichtlich zuletzt auftreten. Der Mensch selbst existire im fossilen Zustande nicht. Das. Meisteruerti der. Schüpfuny lomnte erst zu allerletzt auftreten, nicht etur deshulb, weil, wie die Theologen behaupten, alles zu seinem Empfun!f wohl ros.bereitet und beguem cingerichtet sein musste, sondern weil itm nothuendigerweise die' Formen, unf deren Busis er sich entrichelt hat, haben vorausgehen miissen.

Ich bin am Schlusse!

Lassen Sir mich hotfen, hochgeehrte Fachgenossen, dass meinc Ausführungen Sie davon üherzengt haben. dass wie ich eingehends sagte, unserem schweizerischen Naturforscher Alexander Monitzi in der 'Tat eine hervor'agende Stelle in der Geschichte des Entwicklungsgedankens gebührt. 


\title{
DEUXIÈME ASSEMBLÉE GÉNĖRALE
}

Mardi 16 août 1904

ì 9 heures

\author{
dans l'Aula de l'Université. \\ sous la présidence de
}

M. le Professeur Ch. - S. Minot.

Vice-Présidents : MM. le Prof. L. von Grafk (Graz), le Prof. E. Lönxierg (Stockholm), le Prof. P. Pelseneer (Gand) et le $\mathrm{D}^{r}$ L. STEJNeger (Washington).

M. le Prof. Strner, Président du Congres, annonce à l'assemblée que M. H. von lierlepsen étant malade no pourra pas faire la couférence annoncée au ploglamme, mais qu il sera remplacé par M. (). Klemscrumet, qui traitera le même sujet.

M. le Président donne la parole à M. le Prof. Salenskr.

\section{UEBER DIE HAUPTRESULTATE DER ERFORSGHUNG DES IM JAHRE 1901 AM UFER DER BERESOWKA ENTDEGKTEN MÆNNLIGHEN MAMMUTHCADAVERS}

VoII

\author{
Prof. W. SALENSLI
}

(St-Petersburg).

Von seiten dep wissenschaftlichen lommission des VI. Internationalen Zoologenkongresses ist mil del ehrenvolle Wunsch ausgesprochen worden, in einer der algemeinen Sitzungen einen Vortrag über das von mir selbst ausgewählte Thema zu halten. Bei del Auswahl des Themas habe ich mich an die Frgebuisse dex Tntersuchungen des im Jahre 1901 ausgegrabenen Mammuthcadavers gehalten und zwar deswegen, woil es mil schien. dass die Untersuchung eines fossilen, in Folge besomler's güustigen Umständen mit Fleisch und Blut erhaltenen Tieres nicht nur spezielles, sondern ein allgemeines Interesse erregen soll. Inie von del Pe- 
tershurger Akátemie der Wrissenschaften vorgenommene Untersuchung der Mammuthleiche ist schon so weit vorgeschritten, dass ein Teil derselben (Osteologie, Odontographic, Myologie. die Untersuchung des Eises) liepeits im vorigen Jahre veroffentlicht wurde'. Um die Wicderholung der hereits publizierten wissenschaftlichen Resultate zu vermeiden, will ich hier den eben erwähnten Teil der Untersuchungen nicht berïhren. I) Ac Afgabe meines Tortrages besteht erstens in der Aufkläl'ung d(rlo biologischen Existenzbedingungen des Mammuths und zwortens

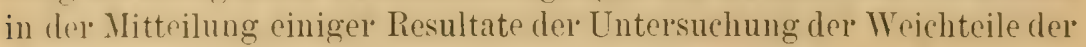
Mammuthleiche, soweit dieselbe bis jetzt volgeschritten ist.

Jas Erscheinen der gut konservierten Mammuthleichen auf der Erodoberfläche stollt bekanntlich in hohen Norden Sibiriens keine hesonders seltene Erscheinung dar. In den von MiddendorfF ${ }^{2}$ und von K. F. von $\mathrm{BAER}^{3}$ musterhaft zusammengestellten Grschichten der Entreckung('n der Mammuthcadaver sind bis zum Jahre 1866 achtzehn Fälle von dem Erscheinen der Mammutheadaver in verschiedenen Gegenden des Norksibiriens angeführt. Wenn man noch die später angemeldeten zwei Mammutheadaver, von denen die Akademie zwei Füsse und ein Stück Fell von Baron v. Maýnes bekam und einen Mammuthcadavel der Ljachoftinsel, von dem wir einen Fuss von Dr. Buxge besitzen, beizählt, so wird die Zahl der im vorigen Jahrhundert aufgefundenen Mammuthleichen bis auf 21 steigen. 'Trotz vieler Bemühungen von seiten der Petershurger' Akademie der. Wisisenschaften, die angemeldeten Mammuthleichen auszugraben und dieselben fül die Wissenschaft zu bewahren, blieben die von der Akademie zu diesem Zwecke ahgesandten Expeditionen meistens wenig erfolgreich. Die Ursache davon liegt in der grossen Entfernung: Nordsibiriens, in den ausserordentlichen Schwierigkeiten, mit denen die Reise nach diesen wilden Gegenden verknüpft ist, und in mehreren anderen zum Teil athmospherischen, die fül die Erhaltung der Leiche ungünstig erscheinen. Die Erötfinung der sibirischen Eisenhahn hat diese Verhältnisse bedentend gebessert, und der gute Erfolg der im Jahre 1901 an das Ufer der Beresowka abgesandten Expedition muss wenigstens teilweise dieser Verbesselung zugeschrieben werden. Die Geschichte der Entdeckung und der Ausglabung del Mammuthleicho an der Beresowlia ist folgende:

1 Wissensch. Resultate d. von d. Akad. der Wiss. in Petersburg ans Ufer der Beresowka für die Ausgrab. d. Mammuthleiche im J. 1901 abgesandten Expedition. Bd. I (russisch).

${ }^{2}$ Middendorfy. Reisen in d. äuss. Norden und Osten Sibiriens, etc. Bd. IV, T. I, Petersburg.

${ }^{3}$ K. E. v. B无. Neue Ariffind. eines vollst. MIammuths mit der Haut u. d. Weichteilen, etc. (Mélanges biologiques de l'Ac. Imp. de St-Pétersb., B. V, 1866.) 
Im April 1901 hat die Akademie der Wissenschaften in Petershurg von dem Gouverneur von Jakutsk Nachricht bekommen, dass am Ufel" der Beresowka, eines Nebenflusses der Kolyma, eine gut erhaltene Mammuthleiche aufgefunden wurde. Die Akademie hat beschlossen, möglichst schnell eine Fxpedition für die Ausgrabung der Mammuthleiche auszurüsten. Inden der Erfolg dieser Expedition hauptsächlich von der schnellsten Ankunft derselhen an del Maumuthstelle abhing, so hat man sich hestrebt, die Expedition möglichst hald abzusenden. Anfangs Mai haben die beiden Mitgliedel der Expedition, dir Heren O. Herz und E. W. Protzamayer, deren Eifel und (xeschichlichkeit wir den guten Erfolg der Expedition verdanlien. Petersburg verlassen. Nach viermonatlicher schwerer Reise hat die Expedition den 9. 2.2. Sept mber die Mammuthstelle erreicht.

Ich brauche hier nicht auf die Beschreibung der schweren Reise der Expedition näher einzugehen, da dieselbe bereits von dem Chef der Expertition, (). Hezz, in einem speziell geschriebenen Auf-at\% ${ }^{1}$, auf den ich verweise, niedergelegt ist. Ich will hier nur einige Stellen daraus anführen, welche hauptsäthlich die Lmgehung und die Lage des Mammuthcarlaver's betreffen.

Ueber die (xegend. in weleher der Mammuth hegrahen wurle, berichtet 0 . Henz ${ }^{1}$ wie folgt: "Der Mammuthearlaver liegt auf einem :35 Meter" übel' dem gegenwärtigen Flussspiegel der Beresowka an linken Ut'en derselben, 11/2 Worst langen, nach ()sten gekohrten Ahsturzfehle, das sich

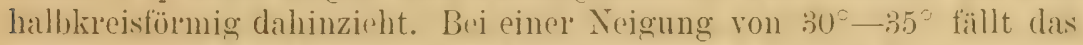
ganz zerrissene und zerklüftete Absturzgebiet von der die Tiaiga trag(nden, oberen Humussehicht 11:) Meter zum Ufer der beresowka ab, während die absolute Hölse derselben 5.5 Meter beträgt; die Entfernung rom Mammutheadaver his zum Flussufer heträgt tie Meter. I)ie obere Humuschicht, mit einer Mooslecke bekleidet, mass ich an reschiedenen Stellen und stellte sich eine Dicke von $30-52 \mathrm{~cm}$. heraus. Darunter liegt cine lehmhaltige Erdmasse, zu ('a. " " Erde und "/, Lehm, die durchschnittlich 2 Meter, stellenweiso aber bis 4 Meter und meln mist und von geschichteten Eisadern von 5 - 1 s ('m. I)icke, mit Steinen, Wruzeln und Holzstüclen remischt, durehzogen ist. Unter dieser alluvialen Erdschicht tritt eine vertikale Eiswand zu Tage, die oberhall) des Mammutlpplatzes :s Meter, an anderen Stellen sogar 7 - i Meter freiliegt. . . p "Auf diesem vermutlichen Eisahhange liegen mächtige, zerrissene Erdmassen und Erdhügel, die durch das allmählige Schmelzen der Eiswand mit hinzulommendem Wasser aus dem oberen Taiga und dem ca.

${ }^{1}$ 0. Herz. Berichte des Leiters der von der Kais. Akad.d. Wiss. zur Ausgrabung eines Mammuthkadavers an die Kolyma-Beresowlia ansgesandten Expedition 1902. (Verl. d. Akad. d. Wiss. zu St. Petersburg.) 
1/. Werst dahinter liegenden Bergrücken von 120) Meter Höhe, hei starken Regengüssen zum Beresowka-L'fer hinabrutschen. Bei einem solehen Erdrutsch orler dem Auseinanderreissen einer grösseren Erdmasse ist auch nach Vermutung der Lamuten (Jakiten) schon vor' 2 Jahren der Mammuthkopf zum Vorschein wekommen, während ein Teil des ührigen Kiörper's er'st Ende August sichtbar' wurde. "

Die unter der Leitung von O. Herz mit grosser Vorsicht vorgenommenen Ausgrabungen haben allmählig die Mammuthleiche blossgelegt. Inie Arboit der ersten Tage hat schon einen sehr wichtigen Fund erbracht: es ist namentlich eine Portion des Futters die in Form einer Platte zwischen den oberen und unteren Zähnen bei dor Freilegung des Schädels entreckt wurde. I) ire wichtige Bedeutung dieses Fundes für die Biologie des. Dammuths werde ich weiter unten genauer besprechen; hier will ich nur hervorheben. dass die Anwesenheit des noch ungekauten Futters zwischen den Zilhnen des oberen und unteren Kiefers auf einen plötzlichen 'I'od des Tieres hinweist. Der 'Tod war in Folge der weiter unten näher zu besprechenden [rsachen so schnell eingetreten, dass das 'Tier' nicht eimmal die Zeit hatte, die in seiner Mundhöhle liegende Nahrung zu verschlucken.

Die weiteren Ausgrabungen, bei denen der Rumpf und die Extremitïten allmïhlig zum Vorschein kamen, haben mehr und mohr die Idce von dem natürlichen Tod des Tieres ausgeschlossen. Der Rumpf der Leiche war aufrecht gestellt; der liopf etwas emporgehoben. I Iic beiden hinteren Extremitäten sind in einer fast horizontalen Lage unter den Bauch geschoben : das Tier erweckte die Ansicht. als ob es auf seinen hinteren Extrenitaten gesessen häte. I) Lage der in den carpalen Gelenken gebogrnen vorderen Extremitäten wejsst darauf hin, dass das Tier kurz vor scinem Tode heftige Anstrengungen geüht hat, um sich zu hefreien. Ein Vorderfuss war etwas höher gelegen, als der andere. Kun\%, die Stellung der Leiche, die Lage der vorderen und der hinteren Extremititen, die gebrochenen Knochen und die ungeheure Ienge des Blutes in der Brust- resp. Bauchhöhle, weisen darauf hin. dass das Tiel in Folge eines gewaltigen Stosses plötzlich verendete.

Beror wir zur Frage üher die T'mstände, welche den plötzlichen Tod unseres Irammuths hervorgerufen haben, ühergehen, wollen wir zuerst die allgemeinen physikalisch-biologischen Verhältuisse unter welehen das Mammuth lebte, etwas näher hetrachten. Die letzten kömnen nur auf Frrund del" mit dem IIammuth aufgefundenen Ueberreste der derzeitigen Flora beurteilt werden. Der berühmte K. E. v. BAER hat schon vor 40 Jahen vollkommen richtig bemerkt, dass " die Bestimmung der Nahl'ungseste, die man im Iarm finden wird, überwiegt an Wichtigkeit sicher alle Untersuchungen üher die spezifische Form des Tieres ». I)iese Salnrungseste wurden aher bis in der letzten Zeit nicht erhalten: des- 
wegen enthehren unsere Kenntuisse über die Naturverhältnisce dor Mammuthzeit eines thatsichlichen firmondes und sind thoilwoise auf Vormuthungen, thrilweise auf die Analogie mit den bofunden longründet. welche man an den Zeitgenosien des Mammuths gomarht hat. So hat

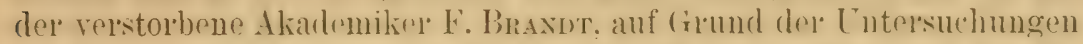
der Futtereste. die zwischen den Zähnen des Zoritgenossen und dos Begleiters des Mammuths, des Rhinoceros ticholluinus. gefunden wurden

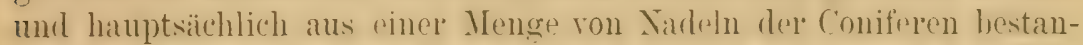

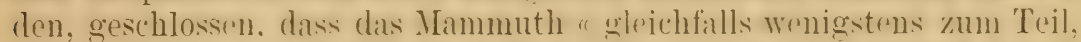
vielleicht selbst gröstrntrils. von Zaptenhämmon sich gemährt halıen

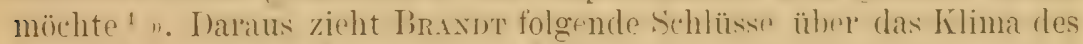
hohen Fordens zur. Mammuthweit ?. Er sagt namentlich: " In num aber" gerade die intacten Leichen in so nördlichen, gegenwärtig so vegetationsarmen, öden Gegenden vorkommen, wo so grosse 'Ticre, wie die

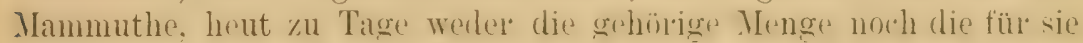
geeignete Nahrung finden könnten, so darf man wohl daran denken, dass das Klima des hohen Nordens von Sibirien früher ein anderes,

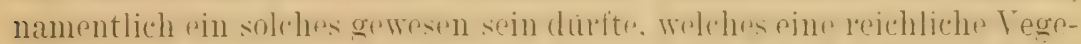
tation, namentlich eine weit grössere Ausdehnung der Wälder nach Norden gestattete. );

Wollen wil" versuchen die Richtigkeit diesel Neinumg von F. BraxdT anf frund des nengefundenen Materials zu prüfen. Fis ist wirklich fin ausielordentlich glürlilicher Zufall gewessn, dass das Mammuth rom

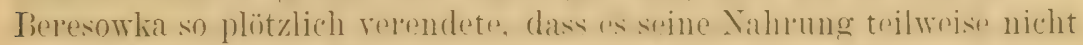

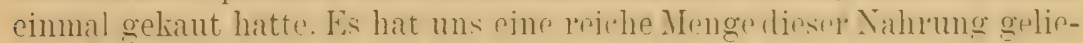
fert. Ausser der schon ohen orwähnten zw ischen den obrem und unteren Zithnen gefundene Platte. Wrehe aus zusammengepuestem Hou hustand. hat man bei der fortgesitzten Ausglahung einen wam\%n Magrn grefunden, welcher ungefähr $12 \mathrm{kgm}$. von unverdautem Futter enthielt.

Die Untersuchung diesel' werthrollen Ausbeute wurde von der Aka-

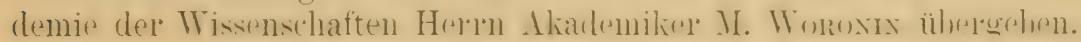
Nach dem unerwarteten und sehr bedauerten Tode desselben, hat die Bearbeitung dieser Futterreste Her' Acarl. J. Bononis übernommen.

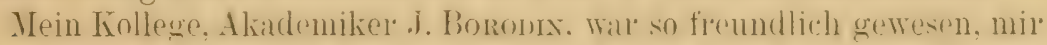
eine Liste der von ihm lestimmten. in Futter aufgofumdenen PHanzen

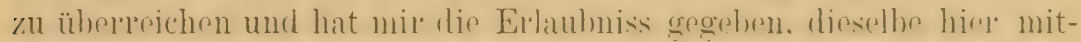
zutheilen, wofür ich ihm hier meinen besten Dank ausspreche.

())wohl die im Futter "nthalteno Flora hemeswes mamnigfaltig ist, hietet sip ein hervorragendes Interese dadureh, daws sir aus den Ptlan-

1 J. F. Brandt. Zur Lebensgeschichte des Mammuths. (Bull, de l'Ac. Impér. de St-Péterslourg, Bd. X, p. 112.)

${ }^{2}$ IDEM, p. 115. 
Z'n bestoht, die noch jetzt an demselben Orte wachsen. Es wurden fast ausichliesslich Gräser gefunden. Die Nadeln der Coniferen sind in ausserordentlich geringer Menge dabei vertreten.

I)i Futterptanzen des Mammuths gehören zu den sechs Ptlanzenfanilien, von denen die Pepräsentanten der Gramineen und der Cyperavearen durch ihre Menge und durch die Mannigfaltigleit ihrer Arten prävaliron. Aus den Framineen gehören die meisten folgenden Arten: 1. Alopecurus alpiuns Sm. (Fuchsschwanz), von denen Stengel, Rispen und pinzelne Aerchen in reichlicher Menge gresammelt wurden; '2. Hordemm jubatrum L. mit sehr vielen Stengeln und einzelnen dehrchen: 3. Aryostis borealis Hartm., Stengel, Rispen, teilweise mit gut erhaltenen Achrehen; 4. Atropis desturs (irideh., nicht besonders gut conservirte teilweise mit Achrchen versehene Stengel; 5. Beclimamia cruceformis Host., wenige Achrchen.

Die Familie der Cyperaceaceen ist nur durch zwei Arten von Carex reprïsentirt : 6. Carer glareosa IVg. von denen viele Hüllpelze und Samen gefunden wurden, und 7. Curex incurva Lightf. durch sehı viele Hüllpelze, Samen und ganze Aehrchen repräsentiert.

l)ie ührigen Familien sind bloss durch einzelne drten vertreten. Von der Familie der Labiaten sind nur 2 schlecht erhaltene Früchte des 8. Thymmes serpillum L. gefunden. In viel grösserer Menge sind im Futter die manchmal gut conservirten Bohnen des 9. Oeytropis compestris De. aus der Familie der Leguminosen vorhanden. Aus der Familie der Papaveraceen wurden einige Samen von 10. Pripacer alpium L. gefunden. Endlich ist die Familir der Ranunculaceen nur durch zwei Frütchte vou 11. Ranunculus acer L. var. borealis, dargestellt.

Alle hier erwïhnten Pflanzemesten gehören den Pflanzen die noch

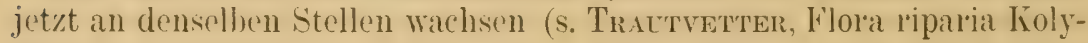
mensis ", Ostenfeld, "Flora arctica, " Cajander, "Lena-Thal ") und stellen sich als charakteristische Repräsentanten der Wiesenflora dar. Die typischen Tundraptlanzen, ausser dem Alopecums alpims Sur. und Papaver alpinum L., welche auch in der Tundra vorkommen, wurden nicht aufgefunden.

Ausser den aufgezihhlten Ptlanzen wurden noch einzelne kleine Stücke von Holz angetroffen, die bis jetzt nicht näher bestimmt sind.

lank den beschriebenen Futterresten haben wir positive Tatsachen (rworlien, welche uns sichere Auskunft über den Charakter der Flora zur Iammuthszeit und zugleich den Grund für die Beurteilung dè damaligen physikalischen Verhältnisse des hohen Tordens Sibiriens liefern.

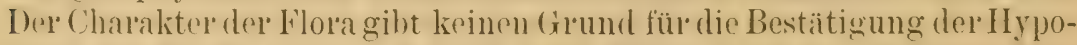
these von Fr. Braxut, nach welcher das Kilima des hohen Nordens sibirens milder als das gargenwärtige gewesen soin dürfte. Dio Identitüt del Wiestnflora, welche im . Mammuthsfutter enteded wurde. mit dereder heu- 
tigen 'Tage, weist vielmehr darauf hin. dass das Maumuth unter ganz anderen Bedingungen als seine gegenwärtigen Verwaulten, der afrikanische und der indische Filophant, lebte. Das Mammuth war ganz entschieden ein hochnordisches 'Tier"; es bewohnte kalte fegenden mud wal dafür durch verseliedeno Einrichtungen, die wir bei don jotzt lobenden Elephanten nicht treffen, angepasst. ()h es sich manchmal von den Nadelhölzern nïhrte, kam ich nicht entschejelen; jeclenfalls hat man jetzt noch keinen Grund für die Annahme, dass die Vadelhölzer irgend rine Rolle bei seiner Jihrung spieiten. I) Möglichlieit eines zeitweiligen viehmehr oder zufailligen Verzehrens der Coniferennadeh will ich aber durchaus nicht in Alnede stellen. Tatsiachlich hahen wil keinen Ground für die Annahme, lass es sich grösstenteils von den Nadelhölzern ernährte. Xach den gehörigen Quantititen der Xahıumg, welche im Man-

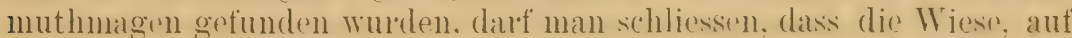
der' es kurz vor' seinem 'Tode weidete, ihm die notwendige Menge des Futters geliefret habe. Dieselbe Wiese ist ihm in Folge eines Unglüchisfalles auch eine Grabstelle geworden. Wio ist es gestorben? Welche gïnstigen Umstände sind es, die soinen Cadiver withrend mehrerer Jahrtau-

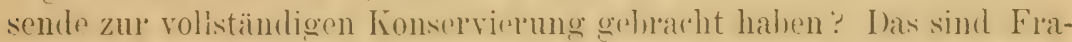
gen, welche sowohl Laien wie auch Gelehrte in holiem Masse interessicren, die aber nur hypothetisch beantwortet werden können.

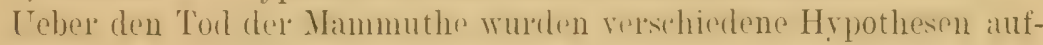
gestellt.

Bekanntlich hat der herühmte schweizerische Naturfor'scher Oswald HeEr die Meinung ausgesplochen, " die in Sibirien mit Haut und Haar his anf unsere Tage robaltenen, im Fis oingeforenen Mammuthes seien vielleicht auf dem Fis verunglülit, in Giletschespalten gofallen und in diesem uralten Eiskeller durch alle Jahrtausende aufbewahrt worden ». F. Brandt bemerkt darüber" " Für Sibirien kann die Theo-

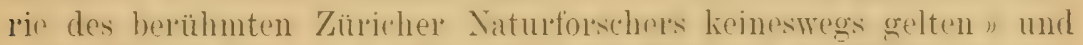
schlägt zur Erklatrung der Irage üher das Verenden der Mammuthe pine andere 'Theorir vor', die sich aut das Vorkommen der aufrechtstehenden Mammuthleichen stütat. Braxnt hat auf diese Eigentünlichlieit besondelen Wert gelegt und dio Ansicht ausgesprochen, "dass die wohl ("lhaltenen Mammuthleichen an ihrem Fundorte solbst (den Flusiuferm)

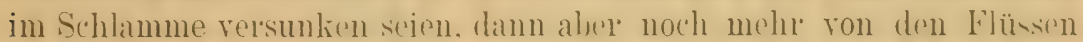
mit Schlamm hedecht wmen. worauf sie sehr hald darmach einfroren, was natürdich nur im Herlst, und in Folge eines bald eingetretenen Frostes geschehen komute. Ein harter darautfolgender Winter tat das ührige, während der kalte Schlamm, womit sie im nïchsten Fröhling und im weiteren Verlaufe der Zeit bedeckt wurden, sie gegen das Aufthauen schützte.... W Was dir liegend gefundenen Leichen anlangt. so werolen sie als solche zu betrachten sein, wolehe entwedere aus ihrem natürlichen 
Fundorte durch Erdstürze (wie namentlich del selbst ron ADAns beobachtere) oder [nterwaschungen losgerissen, oder durch Fluten eine sehr kurze Strecke transportiert wurden. »

Akad. L. Schrenk ${ }^{-1}$ hat diese Theorie einer Kritik unterworfen und dahei die richtige Bemerkung gemacht, dass " die auf solchem Wege, durch Finhettung in Sand-, Thon- oder Schlammschichten erhalten gebliebenen 'Tiere. wenn auch nicht immer aller ihrer Weichteile berauht, doch in der Regel stark mitgenommen worden seien. Wenn sich aber unter ihnen dennoch eimmal eine wohl konservierte Leiche finden sollte, sn müssen wir das nur einem ganz glücklichen Zufall und dor Mitwirkung ganz aussergewöhnlicher, vielleicht nur lokaler Umstände zuschreihen. Solche Fülle dürften daher nicht als liegel, sondern nur als Ausnahme betrachtet werden. ")

Ich schliesse mich der in diesen Zoilen geäusserten Meinung r. Śникжк sehr germe an. Die Stellung des Mammutheadavers von der Beresowka, die unverdaute Nahrung; welche in demselben gefunden wurde. lassen sich nicht mit der Ansicht von F. Branut in Einklang bringen. Wäre das Mammuth in einem Sumpf versunken, so ist dadurch noch kein Grund gogolom, dass sein Tod so plötzlich erfolgte. dass es nicht eimmal die Zeit hatte, die in seinem .Iunde steckende Nahrung zu kauen. Das Versinken grosser Tiere, wie z. I. der Ochsen, Küuhe usw., welches in den sumptigen Gegenden Sibiriens nirht seiten vorkommt, dauert jedenfalls so lange, dass die Tiere noch genug Zeit haben, um ihre Nahrung zu verschluclien. I)ie Stellung der Mammuthleiche weist auf cinen heftigen aber kurzdaucrnden Kampf um das Lehen hin. Die Knochenverletzungen im Humerus und im Becken, in den Lendenwirbeln und im Kreuzbein kïnnen nur darin ilne Erklälung finden, dass das Tier unmittelbar vor seinem Tode an einen festen (regenstand stark gestossen resp. von einer grossen Höhe heruntergefallen wäle. Ias Herunterfallen des 'l'ieres aus einer bedentenden Höhe in irgend eine Frolspalte, ofler in rine Höhle kann auch allein den plötzlichen Tor und die eigentümliche Stellung des Mammuths erklären. Wrir erimner'n gerne an die oben zitierte Stelle von Oswald Ilewr's bildliche Torstellung, welche genau den Terhältnissen, unter denen die Mammuthleiche an der Beresowka gefunden wurde, entspricht. Nur bedarf das Bild von (swald Hewe in der Beziehumg einer Kormektur, dass die vermeintliche Höhle keine Gletscherspalte gewesen sein lomnte, da im Norden Sibiriens keine Gletscher bis jetzt entrleckt wurden. I)ire Höhle liomnte auch nicht im Eis selbst gebildet worden soin, da die Mammuthleiche nicht im Eis, sondern in der gefrorenen Erde

${ }^{1}$ L. v. Schrenk. Bericht über neuerdings im Norden Sibiriens angeblich zum Vorschein gekommene Mammuthe, etc. (Mélanges biol., tirés du Bull. de l'Ac. Imp. de St-Pétersb., T. VII, 1. 745.) 
gefunden wurde. I)ic Eisschichten, wolehe unter dele Begrabungsitelle des Mammuths auftreten, bestehen nach don Angaben des Goologen .I. Toumatscheff, wolcher das Eis untersuchte, aus Schnepeis. Diese Eisschichten liegen unter der Mammuthleiche und waren wahrseheinlich schon vor dem Mammuthtode gebildet.

Deswegen ist es sehr wahrscheinlich, dass die Höhle, in welche das Mammuth gelangte, im Boden arebildet wäre. Dio darunter licugende Fisschicht spielte eine grosie Rolle bei der Erhaltung des Mammuthcadavers während der ganzen Jahrtausende, indem sie die 'Temperatur des Burlens erniedrigte.

Es fragt sich nun: wie konnte auf der Wiese, auf welcher das Mammuth einige Vinuten vol seinem Torle weidete. wine solehe Höhle gehildet werden? O. Herz, der die Gegend, wo dieses Mammuth gefunden wurle. sorgfïltig untersuchte, hat die Meinung ausgesprochen, dass auf der Eisschichte, die el als Gletscher betrachtet, tiefe Einschnitte vorhanden waren, " die durch das aus der Taiga und dem angrenzenden Berglücken liommende 'Than- und Regenwasser mit Eirde, steinen und Baumresten ausgefüllt wurden, worüber sich dann eine Ircke gebildet hat, die sehr reichlichen Pflanzenwuchs gehabt haben muss, wo die Mammuthe und andre Tiere vortreffliche Nahrung fanden. Lesagte Decke hat dann wahsweheinlich noch nicht die richtige Festigheit gehabt, dieschweren Mammuthe ülocrall zu tragon, das Mammuth ist dluehgebrochen und abgestürzt, wie seine Lage und die zerbrochenen starken Knochen, wie das Becken, der l'echte Humerus etc., beweisen. Es hat damn zwar versucht sich empnozuarheiten. Was aus der Ketterstellung der beiden Vorderfüsse zu ersehen ist; aber die Beschädigung ist so gross gewesen, dass es die Kraft dazu nicht mehr hatte und bald verendete. )

Die Bildung dor Höhlen in dere Erede dureh dir Autlösmng des (iestrins ist zu bekannt um an denselben sich aufzuhalten. Die fül Europa bekannten Bedingungen der Höhlenbildungen sind in Sibirien noch durch einen Faktor, nämlich durch den stallen Frost vermehrt, welcher die Bildung der Kilüte resp. der Spalten in Eis oder in gefrorener Erole verusachen kann. Nach den Angahen verschiedenor Tieisenden, die Jordsibiven besuchten, ist es bekannt, dass in Folge starker Fröste, die dort sehr häufig sind, in dem gefrorenen Borlen weite spalten sich bilden, in welchen das Wasser von den anliegenden Wasserbehälter'n 7. B. den Seen, verläuft und dort gefriert.

In demselben Kolymagebiet, wo das Mammuth grefunden wurde, sind solche Fialle von den Reisenden angeguben. I bas von dem Boden der Seen nach den Erdritzen resp. Fidspalten hincinströmende Trasser kamn nicht in allen Fällen verfolgt werlen. Es ist sohr wahrscheinlich, dass es untelirdisch fliesst und unter dem Boden cinige meler oder minder gerimmige 
Höhlen auswäscht, wclche von Aussen durch eine Bodenschicht bedecht und masquirt werden kömnen. In eine dieser Höhlen lï̈nnte las Mannmuth gestül\%t resp. gerutscht sein. I)er gewaltige dureh das Herunterfallen des schworen . Mammuthlörpers verursachte Stoss hat einen weiteren Varhsturz des ungebenden Bodens und d'r Höhlendecke helvorgerufen, welcher das Mammuth sofort bedecken sollte.

Nach der oben anguführten Liste dex im Magen aufgefundenen Ptlanzen kann auch die Jahreszeit zu welcher unser Mammuth verunglürlite ziemlich loicht bestimmt worlen. Alle gefundenen Ptlanzen sind hereits mit Samen versehen; daraus folgt, dass dieselben entweder im spätrston sommer oler im Anfang des Herbstes abgerissen worden. Zu dieso Jahreszeit kommen sehon im hohen Norden, wenigstens in der Nacht starke Fröste vor, bei denen der Boden gefrieren kann; daraus kïnnen wir sehliessen, dass die in Folge des Nachsturzes über den Mammuthkörpor gefallene Frdmasse bereits einen sicheren Sehutz gegen alle Zersetzung des Mammuthkörpers bjeten komnte. Starke stürme, die willyend des Winters eine ungeheure Menge von Schnee und Staub bringen, konnten diese Schutzarbeit vollenden, so dass schon in der ersten Zeit der Mammutheadaver mit einer Erdschichte bedeckt werden komnte, welche die Erhaltung desselben für mehrere Jahrtausende sicherte. Dies sind dir Verhältnisse, welche wir uns vorstellen können, um auf Grund der erworbenen Tatsachen den Tod des Mammuths und die Conservirung seiner Leiche zu erklären.

Gehen wir nun zur Morphologie des Mammuths über.

Der nel gefundene. Mammutheadaver hat unsere lienntnise über die Morphologie dieses intressanten vorwoltichen Tieres in bedentendem

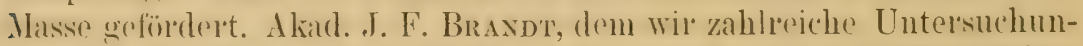
gen ïlıer das Mammuth verelanken. hat vel'sucht, die morphologischen Kennzeichen unseres 'Tieres nälıer zu definieren'. Nach den Angaben dieses Jolschers soll das Mammuth vol\%ugsweise " dureh seine plumpere Gestalt, seine (jedoch nicht sehr viol) hedeutendere (rrösse. seinen längeren Kopt, seine breite Stirn, sehr kleine (0.26.̃ m lang), dicht beharte Ohren, spiralige, grössere Hauer, noch imniger als bei den lehenden Elephanten verbundene Zehen, sowio den dicht mit braunem Wollhaar und zelstrenten, aber leichlichen, am Ialse längeren, wie es scheint eine Art Mähne bildenden, bolstenähnlichen, dunkelschwarzen Haaren bedeckten Kïrper von den lehenden Elephanten sich unterschieden hahen" (p. 111). I)ie angeführte Iniagnose des Elephers primigenius ist duochaus auf die Untrisuchung des im Petelshurgel Museum aufgestellten Adass'sehen

1 J. F. Brandt. Mitteilungen über die Gestalt und Unterscheidungsmerkmale des ITammuths oder Mamont (Elephas primigenius). (Bull. de l'Acad. Imp. de St-Pétersbourg, T. X, p. 93-111.) 
Mammuthslielettes mol der dasellost betindlichen Schädel resp. einzelnon Kuochen des Skelettes begriüdet.

Man hehauptret, dass das Manmuth durch die Grösse somes liörpers die gegenwitrtigen Elephanteuarten (E. indicus und E: (fficums) bedeutend übertraf. I)er lleugefundeno Mammutheadaver war noch zienlich jung gewesen: nach dem Zustande seine Zühlhe zu urteilen. kïmnte man ihm nicht mehr als 25 .Jahpe gehen, vorangesetzt, dass das Altel des. Mammuths nach dem fül die hentigen Filephanten andugelunen sehema des Zahnswstems hestimnt werden kann. Er ist anch kleiner als

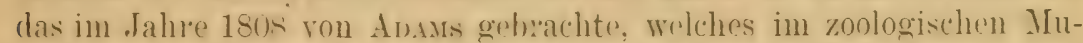
seum der Potershurere Akademie aufgestelt ist. Deswegen kitun freilich das Mammuth von der Beresowka nicht als Mustre für dir Belletrilumg der Crüsse dienen. Nach den Dimensionen der im zoologischen Museum aufhewahrten schädel kann man jerlenfalls schliessen, dass dar Man-

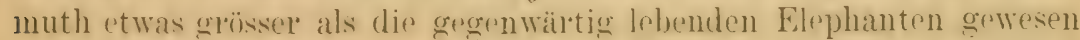

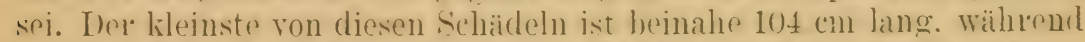
von den dort vorhandenen Elephantenschäuleln nur ein einziger 111 'm. in die Lïnge heträgt, die übrigen ahre nicht mehr als \&1 cm. lang sind.

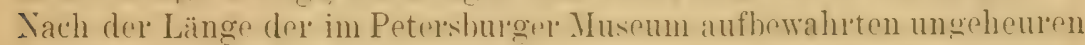
Strsazihne $(4.17$ m.) muss jedenfalls angenommen werden, dass die ohen

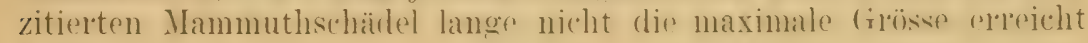
haben.

Es sei hier doch benerkt. dass das Verhälnis zwischen der schädellänge und der Länge des Rumpfes beim Mammuth von dem der Elephanten verschieden ist. I)je Schädellainge des Mammuths übertriftt die Hälfte der Rumptlänge. Wïhrend die Schädellänge der Elephanten nie die Hälfte dre RumpHängr "rereht. I)as Mammuth hesiss also einen rerhältnismaissig gröscren liopt als die Elmphanten. I)iesel L'mstand mus: immer lor der Brurteilumg der Körpergrösse des Mammuths in Betracht gezogen werden.

Ein dichter Haarpelz des Mammuths und eine mächtige bis 9 c'm. ('lreichendo Fettlage seines Unterhautgewebes sind die Ampassungen, welche ihm das Leben im hohen Norden sibiriens ermüglichten. Leider fallen die Haare in der durch den Regen und atmosphereische Einflüsso macerierten Haut der Mammuthleiche sehr leicht aus, so dass es nur in seltenen Fïllen gelingt, eine erhaltene Haarbenteckung zu lionservieren. Thas Mammutl ron der Boresowka ist insoforn wheldicher gewesen, als bri ihm wenigstens an den Füssen die Haare erhalten sind; dureh die Bandagierung der Füsse ist es möglich gewesen, dir behaarten Füsse nach Petersburg zu transportieren. Obwohl die ührigen ron der Haut ausgofallenen Haale unter den Kïrpexstellen, von welchen sie ausgefallen sind, in der Erde lagen. so wurlen diescollen doch sorgtiltig gesinumelt, um an den entsprechenden stellen hefestigt werden zu kïmnen. 
Es ist jetzt kaum zweifelhaft, dass der ganze Mammuthkörper mit Haaren bedeckt war; aber yon diesen Haaren kann man dreierlei Arten unterscheiden, die sich nicht nur durch ihre Länge und Diclie, sondern auch durch ihren bau vou einander unterscheiden. Sie nehmen verschiedene Kïrperstellen ein mud können als Wollhaare, Granenhaare und Bolstenhare bezeichnet werden. Die beiden ersten sind rund in Querschnitt, die dritten stark abgeplattet, sodass sie beinahe handförmig erscheinen.

Die Wollhaare sind die kürzesten Haare des Mammuthliörper's und bilden einen dichten, auf der ganzen Körpertläche ausgebreiteten Haarpelz. Ihre Dicke heträgt ungefïhr $0,1 \mathrm{~mm}$, die Länge $2-2^{1} / 2 \mathrm{~cm}$. Nanchmal sind sie gekräuselt, öfter's geradlinig.

Die Granenhare sind zwischen den Wollharen zerstreut. Ihre Dicke beträgt 0,25 mm. An einigen Körperstellen, wie an den Wangen, an der'schulter, am Oherarm, an der Bauchseite des Körper’s treten sie gruppenweise auf und führen zur Bildung hart- resp. mähnenähnlicher ()rgane. Avasis and Truesius haben hekanntlich dem Mammuth eine Mähne zugeschrieben, olme dieselbe beohachtet zu haben. Leider hat das neugefundene Nammuth kein Material zur Entscheidung del Frage übel die Anwesenheit der Nähne gebracht. Es ist aber sehr wahrscheinlich, dass an den Wangen, unter dem Kinn, an der Schulter, am Oberschenliel und am Unterleib die Steifhare in grosser. Menge vorhanden waren und mähnenartige ()rgane bildeten. Nach der' Stelle, wo die Granenhar'e unter dem Mammutheadaver gefunden wuden, muss man annehmen, dass diese Haare zwei von den Wangen an bis zu den Hinterfüssen sich ziehende Haarfrausen bildeten, die denjenigen vom Jack (Poephagus grumieus) nicht unähnlich wa'en. Es ist sehr interessant, dass die hier angegehene Vertrilung der Granenhare mit den neuerlich von CapiTsx et Brer.u ${ }^{1}$ in der Höhle von Combarelles entdeckten Wandbilder vom Mammuth in sofern ühereinstimmt, als rlort ebenfalls die Haafranzen an den Seiten des Unterleibes abgebildet sind.

Die Borstenhaare; die ihren Namen ihrer Steifheit verdanken, zeichnen sich durch ihre eigenthümliche zusammengepresste bandförmige (iestalt aus. Es scheint, dass diese Hate bloss am Schwanze volhanden waren, wo sie eine stark entwiclelte Haarquaste bildeten. I)ie Borstenhare sind viel dunkler gefärht als die anderen Haararten.

Nach O. Herz soll die Länge derselben 20-35 cm. elreichen; es sei jedoch bemerlit, dass alle Haare vom Mammuth von Beresowka abgubrochen wurden, und dass die angegeh)ene Lünge der'selben wohl wonigstens um $1 / 3$ mehr geschätzt werden soll.

'Capitan et Breunu. Reproduction des dessins palaeolithiques gravés sur les parois de la grotte des Combarelles. (Comptes rendus, 1901, no 24, p. 1038.) 
Was die Farbe der Haare anbetrifft, so darf dieselle im Allememeinen als gelblich-braun bezeichnet werden, obwohl sie von hell--blond bis fast braun variirt. Die Haare des Mammuths von Beresowka sind hell.

Wenden wir uns nun zu den Stosszähnen. Unsere Kenntnisse über die natürliche Stellung dieser Zühne in den Alveolen sind, trotz vielen Untersuchungen üher dir . Morphologie der Stosszähne, nicht vollkommen sicher gestellt. Nach den Angaben vou F. Brande sollen die Stosizithne oder Hauce "bei ihrem Austritte sehr stark divergiren, sich dam zuerst nathAusen, vorn und oben wenden, mit ihrem Endtril orter Spitze abel' nach aussen und hinten etwas gegen die Schulter sich biegen " (Bull. do l'A cad. imp) des se. de St-Pétersbourg, Bal. X. p. 98). Diese Beschroibung stimut mit den auf dem. Mammuth von Bepesowka enteleckten Tatsachen nicht vollkommen überein.

Iie Fxpedition hat hei diesen letzten Mammuth leine Stosizilhne gefunden. Die rechte Alveole des Intermaxillaris war noch vollkommen intact: die linke trug auf ihrer (Oherflïche die sehmittliichen. die offenbal durch das Abhauen entstanden sein mussten. Der rechte Stosszahn war wahrscheinlich schon während des Lebens des Mammuths ausogefallen. Man hat ihn weder gefunden, noch Grund zur Annahme, dass er algehauch gewesen wäre. Glüchlicherweise wurde abor der linke stosizahn für die Wissenschaft gerettet. Er wurde von O. Herz bei einem Lamute (Jakute) zurückgekiuft. Durch die Anpassung der an seinel ()bertlïche vorhandenen Schnittfäthen mit denjenigen del Alveole. konnte derselbe richtig eingesetzt werden. Es ist dadurch erwiesen worden, dass die von F. Br.xot angegehene Beschreihung der Stosszilhne einer Córelitur bedarf, die hauptsächlich die Richtung der Spitzen hetrifft. I) Spoitzen del Stoszähne waren nämlich nicht nach aussen. wie F. BRANDT angiebt, sondern nach innen gerichtet.

Ein anderer sehr wesentlicher Punkt der äusseren Morphologie des Mammuths hetrifft die Form des Schwanzes. Teber die Form und die Grösse dieses Organs beim Mammuth wussten wir hiel in del Jetzten Zeit heinahe gar nichts. Die einzige Angabe darüber rührt von einem Kaufmann Boctwofy, welche die Gelegenheit hatte das Anduschr Mammuth zu beobachten und von ihm eine, offenbal seln grobe und ungenaue Zeichnung zu mathen resp. mathen zu lassen. Nach Jostevorf soll die Länge des Schwanzes o Werschok (ungetiihn 27 em.) hetragen.

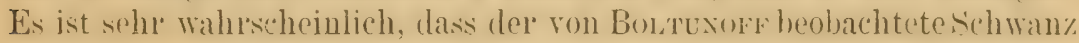
nicht vollständig erhalten wurde und dass der Endteil dessellen abgebrochen wurle, weil die Länge des Schwanzes ron dem nen gefundenen Mammuth mehr als das Doppelte der von Botrexorf angegehenen Lïnge

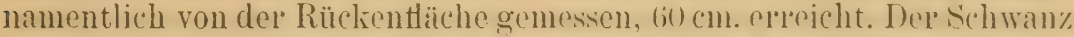
stellt ein lionisches, von vorn nach hinten zu sich stark zugespitztes ()1gan dar, dessen Breite im proximalen 'Teile $36 \mathrm{~cm}$. beträigt. Der stark 
zugespitzte Endteil des Schwanzes wurde von einer ziemlich langen, aus Borstenharen bestehenden Quaste verziert.

Aus dem oben (xesagten ist es exsichtlich, dass die Mammuthleiche von der Berosowka unsere liemntnisse über die äussere Iorphologie dieser Elephantenspecies bedentend gefördert hat, indem wir daraus mehrere äussere Organe kennen gelernt laben, von denen wir bis in der letzten Zeit lieine sichere Vorstellung gehabt hahen. Es bleibt doch eine wesentliche Lück' in unseren Kemntnissen über die äussere Gestalt des Manmuths: sie hezicht sich namentlich auf den Rüssel, von dem wir nur sehr Wenige, anf ungenügende Angaben gegroundete lienntnisse besitzen. In d'r wissenschaftlichen Littratur sind darüber nur einige Bemerkmngen nelst Zeichnungen von Bontunoff und von. Iotscructskr vorhanden, die aber wenig klat und hegründet zu sein scheinen. Bei dem Mammuth von der Belesowka wude der Rüssel nicht aufgefunden, wahrscheinlich ist derselle schon längst vor der Ankunft der Fxperlition an der Ausgrabungsstelle von wilden Tieren gefressen worden. Nach den ohen zitierten Mammuthbildern aus der Höhle von Combarelles soll der Riüssel des Mammuths ansehnlich gewesen sein.

Trotz der angegebenen Lücke in unseren lienntnissen über die äussere Morphologie des Mammuths können wir nun eine mehr tatsïchlich heglündete Inefinition des Mammuths, als die von F. Braxit auf (irund des frühel vorhandenen Materials gegebene, vorschlagen. Ich will hier namentlich folgende Kennzeichen des Mammuths helvorheben.

Das Mammuth war eine hochnordisch lebende Elephantenart, von etwas bedentenderer Grösse und etwas plumperer Gestalt als die gegenwärtigen Elephanten (die Höhe seines Kööpers im Verhältnis zur Länge desselben war kleiner als die der letzten). Soin Kopf war im Verhältuis zur Rumptlänge etwas grösser als bei den gegenwärtigen Elephanten, was mit der starken Fntwicklung der Stosszähne in Zusammenhang strht. I)je Stosszähne zeichnen sich von denen der gegenwärtigen Elephanten nicht nur durch ihre starke Entwicklung, sondern auch durch jhre spiralige Form aus. I)ie Spitzen der Stosszähne waren nach innen gerichtet. I) Haut war mit-Haaren berleckt, die zu beiden Seiten des Bauches eine hesondere Länge erreichten und eine Art von der schulter lis zu den hinteren Extremitäten beirlerseits verlaufender Franzen bildeten. Das ()hr war sehr klein und mit Haaren bedeckt. Del schwanz, war verhältnismässig viel kürzer als derjenige des Elephanten und am Ende mit einer Haarquaste versehen.

Nachdem wir dic äusseren ()rgane des Mammuths hetrachtet haben, gehen wir nun zu den immeren ()rganen über. In Bezug auf diese letzteren muss die Ausbente, welche die Expedition mithrachtr, als sehr leich bezeichnet werden. Dank der Expedition ist jetzt das zoologische Mu- 
seum der Akademie der Wissenschaften in Protersurg mit verschiedenen inneren Teilen des Mammuths: Zunge, Penis, Muskeln, Magen, Blut, Unterhautgewebe mit Fett, Dura mater und Gehirn - letzteres fieilich in sehr zersetztem Zustande - bereichert. Der Erhaltungszustand einige von den angegebenen Organen ist so vollständig gewesen, dass es ๘. B. möglich war, die grossen Nervenstämme der Muskulatur abzupräparieren und die grossen Blutgefässe zu injizieren. Alle Wreichteile des Kürpers wurden in geforenem Zustando nach Petersburg gebracht.

Dic histologische und chemische [ntersuchung der Weichteile der Mammuthleiche ist meinem liollegen, Her'n Akademikel ()Wostrikow, von der Akademie übergeben und werden hoffentlich in kurzer Zeit in extenso publiziert. Hier will ich nur einige von meinen eigenen dieshezüglichen Beobachtungen berichten. Bei dieser Untersuchung handelt es sich naturlich nicht um histologische I)etails, sondern vielmehr um den Nachweis der Veränderung, welche die (iewebe crlitten haben. Ich fange meine kurze Uebersicht mit der Haut an.

Die Haut des Mammuths ist ausserordentlich stark. Sir ist mehr als doppelt so dick wie die Haut des Fiephanten. Nach den Angaben von Fred. Smith ${ }^{1}$ soll die Haut des fetzteren in ihren direkten Teilen am Rumpf nur 1,65 cm (11/16 Zoll) erreichen, während die Dicke des Coriums allein an den Bauchteilen des Mammuths von der Ieresowlka $33 \mathrm{~cm}$. misst. Wemn wir ammehmen, dass die I)icke der Epidermis ein D)rittel ron der der Haut darstellt, so müssen wir annehmen, dass die Haut dos Mammuths wenigstens $2 \frac{1}{2}$ mal dicker gewesen ist als die des Elephantell.

I) fröheren Beohachtel', welche die Mammuthhaut untel'suchten, haben bereits die vollständige Abwesenheit der Fpidermisschicht lionstatiert. Ich kamn diese Angaben vollkommen bestitigen. Die Epidermisschicht ist auf der ganzen Körperobertläche, sowohl an den aus der Erde befreiten, wie an den in der Errde steckenden Körperteilen algelöst; deswegen darf die Ablösung des Epidermis nicht dem Eintluss der atmospharischen feuchten Luft, sonder'n muss viehnehr der Fenchtigkeit del Erde zugeschrieben werden.

Das Corium lesteht aus sehr starken Bindegewebsfisern resp. Bündeln, die schon sehr gut mit unbewaffnetem Auge zu unterscheiden sind. Die Färbung der Schnitte des Coriums gibt wenige neue histologische Details im Vergleich mit den angeführten. Die Faserbündel bilden ein (ieflecht zusammen, welches an der Peripherie dichter, nach innen zu immer lockerer erscheint. Die periphere Schicht ist von den Haarfollikun

1 Vergl. C. Möвius. Die Behaarung des Mammuths und der lebenden Elephanten vergl. untersucht. (Sitzungsber. d. Königl. Preuss. Akad. d. Wiss. zu Berlin, Bd. XXVIII. 1892.) 
durchsetzt, in welchen manchmal die Haarzwiebeln angetroffen werden können: grösstenteils sind aber dieselben leer. Yon den zelligen Elementen konnte ich im Corium nichts entdecken.

Die Haare. Die drei von mir angegebenen Arten der Haare lassen sich wie gesagt, nicht nur durch ihre Stärke, sondern auch durch ihren Bau yon einander unterscheiden. Die stärksten Borstenhaare des Schwanzes sind auch an complicirtesten gebaut.

Möвı's hat schon darauf hingewiesen, dass die Wollhaare vom Mammuth keine Markzellen besitzen. Dies kann ich vollkommen bestätigen. I) iese Haare bestehen aus einer feinen Cuticula und auch aus Rindenzellen, welche durch ihre bekannte langlich ovale, nach den beiden Enden zugespitzte For'm karakterisirt sind. Die von C. M̈̈ви's in diesen Zellen aufgefundenen Kerne konnte ich nicht konstatiren, wie ich überhaupt nicht im Stande war in keinem der Ueberreste von Nammuthgeweben die Kerne hervortreten zu lassen.

Die Granen und die Borstenhaare bestehen aus einer Pinden- und aus (einer Marksubstanz. Die letztere ist in den starken Borstenhaaren viel stäl'ker entwickelt, als in den Granenhaaren.

Merkwürdig genug ist die Anwesenheit der Längskanäle in der Marksubstanz der Borstenhaare, welche von Sniтн' ${ }^{\prime}$ in den Haaren des Elephantenschwanzes beschriehen wurden. Dieselben Kanäle konnte ich ebenfalls in den Schwanzborstenharen des Mammuths beobachten. Besonders deutlich erscheinen sie an den Querschnitten der Haare. Die Zellen dieser Haare färben sich sehr gut mit Hämalaun, wobei in der Marksuhstanz nur die peripherischen Zellteile gefürht erscheinen; die inneren T'eile sind vollkommen hell und farblos. Nan trifft doch manchmal im Inneren dieser Zellen kleine, sich sehr gut färbende Kïrper, welche den Kernen sehr ähnlich sehen. ()b es wirklich lierne sind, konnte ich nicht entscheiden. Zwischen den Markzollen treten in den Querschnitten grosse, scharf begrenzte, runde oder ovale Lücken auf, welche die Querschnitte der Lüngskanäle der Haare darstellen. Sie sind immer durch eine dicke und tinctionsfähige scheide begrenzt. in weleher ich keine deutliche zellige Structur entdecken konnte: da aber die den Kanälen anliegenden Markzellen sich bedeutend abplatten, so bin ich geneigt anzunehmen, dass die Begrenzungsschicht der Kanäle aus zusammengepressten Markzellen entstanden ist. Das Innere der Lüngskanäle ist beim Mammuth leer; bei den Elephanten sollen die Kanäle nach SurTn mit stark lichtbrechenden Zellen erfüllt sein, welche den Zellen der inneren scheide nicht unähnlich sind. Fs ist möglich dass anch beim Mammuth solche Zellen früher vorhanden waren und sich später zersetzt haben.

${ }^{1}$ Fred. Smirr. The histology of the skin of the Elephant. (Journ. of Anat. and Physiol., XXIV, 1892, p. 493-503.) 
Das Unterhautgewebe wurde ebenfalls in ziemlich grosser Menge gefunden. Es war beim Nammuth ausserordentlich stark entwickelt und sehr fettreich. Der paniculus adiposus erreicht an manchen Stellen des Törpers, so z. B. unter der Bauchlaut die ansehnliche Dicke von $9 \mathrm{~cm}$. und stellt mit der Körperbedeckung und der Haarbekleidung einen guten Schutz gegen die strengsten Fröste dar. Er erscheint nun in Form von gelblich-grauer bröckeliger Masse, in wolchel schon bei del Behandlung mit Wasser die Bindrgewebshündel von dem Fett leicht getrennt werden kämnen. Die Bindegewebshündel sind denjenigen der Cutis gleich.

Blut. Das Blut wurde in grosser Menge in der Bauch- und in der Brusthöhle gefunden. Fs stellt eine dunkelbraune, teilweise mit Sand gemischte, harte Masse dar, die lejcht in kleine Stüclie zerfällt. Die grossen Stücke, welche man mitunter findet, sind am besten zur mikroskopischen Untersuchung geeignet, da sie in ihrem Inneren fast lieinen Sand, oder wenigstens nur sehr wenig von demselben enthalten.

Die Stückchen des getrockneten Blutes können auf dem Objoktträger zerrieben werden und erscheinen unter dem Mikroskope in Form von verschieden gestalteten, meist eckigen lö̈rperchen, welche das Aussehen eines getrockneten, lakirt'n Blutes besitzen. Solche Präparate sind nun zu der weiteren Bearleeitung ziemlich gut verwendhar. Die bekannten Eosinreactionen sowie die Behandlung des Blutes mit Essigsäure um dir Blutkisstalle zu belommen, können mit demselben Erfolg am Nammuthblut wie an dem frisch aufgetrockueten Blut der lebenden Elephanten gemacht werden.

Das zerriehene Blut färbt sich in vortrefflicher Weise mit Eosin. Man bekommt unter dem Mikroskop eine ungeheure Menge grosser und kleiner mit Eosin rosa gefübter Blutpartikelchen, welche grösstenteils eine eckige (iestalt besitzen. Zwischen diesen kommen manchmal, und zwal ziemlich selten, kleine, runde schön rosa gefärbte Kürperehen vor, welche den Bluthörperchen ausserordentlich ähnlich sind. Manchmal sind sie gruppenweise angetroffen worden. Iiese Körperchen wurden zuerst von Dr. Frienenthal ( Privat-I)ocent an der L'niversitit in Berlin) entrleckt, als er in Petershurg das Mammuthshlut untersuchte. Später habe ich selbst mehrere Eosinpräparate angefertigt und fast in jeder Probe solche Körrperchen angetroften. Beim Velgleich dieser Präparate mit den in entsprechender Weise behandelten Priparaten des frisch aufgetrockneten Elephantenblutes (welches ich durch die (rüte des Herrn I)r. FrienenTras bekommen habe), tritt die Analogie dieser Körrperchen mit den Blutkörperchen des E. indicus sehr deutlich hervor, nur sind die angeblichen Blutkürperchen des Mammuths etwas kileiner als diejenigen des Elephanten.

Noch deutlicher tritt die Analogie zwisehen dem Mammuth- und dem Elephantenblutr in der Form der Häminlirystalle hervor. I)ie Erhaltung 
der Häminkrystalle aus dem Mammuthblute ist sehr leicht ; nur muss ich bemerken, dass der Zusatz der physiologischen Kochsalzlösung dabei unumgänglich notwondig ist. Die Häminkrystalle sind von verschiedener Grösse und verschieden gestaltet, doch können sie immer zu einer für Elephanten anscheinend charakteristischen Form zurückgeführt werden. Sie haben namentlich die Form eines Tetraeders, von dessen Kanten kleine quergestellte und parallel verlaufende durchsichtige Leistchen sich abspalten. Die Kanten, sowie die Leistchen sind farblos und durchsichtig, während die ührigen Teile des Tetraeder's rot gefürlt erscheinen. Nanchmal setzon sich die Ecken des Tetraeders nach aussen fort; dann nehmen die Krystalle eine eigentümliche lireuzförmige Gestalt an. Nicht selten kommen auch andere Kombinationen der Krystalle vor, welche zu den absonderlichsten Formen führen.

Der Vergleich der Häminkrystalle des Mammuthblutes mit denjenigen des indischen Elephanten, von denen ich einige Präparate anfertigte, weist auf eine vollkommene Uebereinstimmung der Blutkrystalle diser beiden Elephantenarten hin. Es scheint deswegen, dass die eben beschriebeno krystallische Form des Mammuthblutes für alle Elephantenarten charakteristisch sein muss.

Muske] n. Die Muskeln scheinen makroskopisch sehr gut konserviert zu sein und füldas anatomische Präparieren vollkommen tauglich. Ihre innere Struktur hat jedoch wichtige Veränderungen erlitten, welche sich hauptsächlich im Verschwinden der Quersireifung äussern. Die Muskelfasern lassen sich schr leicht in die feinsten Fibrillen zorlegen, sie färhen sich gut mit Hämalaun und mit Methylenblan, doch bleiben sie immer homogen; alle meine Versuche, die Querstreifung hervorzurufen, blieben elfolglos.

Gehirn und Dura mater. Die Gehirnhöhle des Schädels war mit einer pulverartigen Masse gefüllt, die nichts anderes als die zersetzten und ausgetrockneten Gehirnteile darstellen kann. Die mikroskopische Untersuchung lässt jedoch keine Struktur in derselben unterscheiden. Auf der Oherfläche dieser Hirnmasse, gerade unter dem Schädelknochen, befand sich eine derbe Haut, wclche sich als das Ueberbleibsel der Dura mater erweist. Die Jura mater färbt sich mit Methylenblau sehr intensiv blau, hleibt abel' gegen die anderen Farben, z. B. gegen Hämalaun, welcher sonst die Bindegewebefaser'n färbt, indifferent. An den mit Methylenblau gefäloten Stücken von I)ura mater kann jedoch keine innere Struktur unterschieden werden. Diese Haut bleibt homogen.

Interessant sind die Ueberreste der Blutgefässe, die man in der Dura mater an der Farhe leicht erkennt. Ihre gelblich rote Farbe hängt gewiss yom Blut ab, welches die Iblutgefässe erfüllte. I)ie Gefässwände konnten dabei nicht untersehieden werden.

Knochen. In den feinen algebrochenen Knochenplatten treten die 
Tinochenkörperchen mit ihren Vorästelungen sehr deutlich hervor. In den decalcinierten Krnochen zerfällt die Zwischensubstanz in Fasern, an rlen Knochenkörperehen erscheinen kleine Stückchen von braungelber Substanz, die bei de A Afhellung versehwinden. Dic Natur derselben ist mir unbekannt geblieben.

I) Mage nwand. Der in Innern des Mammuthes aufgefundene, mit Hen erfüllte Magen wurde zerrissen; doch hat man den grössten Teil seiner Wände gerettet und konserviert. Die äussere Obertläche der Magenwand ist dunkelbraun, die innere graugelb. Die clunkelbraune Farbe riuhrt wahrscheinlich von der. Wirkung der Gerbsäture her, indem auch andere Kö̈rperteile, wie Kinochen, Haut, teilwoise Muskeln, welche der Maceration unterworfen wurden, elenfalls dunkelgefärbt rischeinen. Diese Farhe schwindet unter der Wirkung des ('hlorwassers. I)ic äussele Obertläche der Magenwand ist glatt; auf der innern erkennt man kleine warzenförmige Erhebungen. Die ganze Magenwand kann sehr leicht mit den Nadeln in fünf Schichten gespalten werden. Zwei von diesen bestehen aus Muskelfasern, dic anderen aus Bindegewebr. Ton den histologischen Elementen sind auch hier Muskel- resp. Bindegewebfasern erhalten, vom Epithel ist keine Spur vorhanden.

Ich werde mich nicht bei der Struktur der Zunge und des Ponis aufhalten. In Bezug auf die erstere will ich mur bemerken, dass an der Wurzel derselben die I)rüsen noch erhalten gehliehen, depen mikroskopische Untersuchung keine zellige Struktur nachweisen lässt. Ueber die Struktu’ des Penis bin ich noch zu keinen sichern Resultaten gekommen.

Fassen wir alles hier über die Erhaltung der Gewebe des Mammuthkörpers Mitgeteilte zusammen, so kommen wir zum Schluss, dass die grösste Erhaltungstähigheit den fasprigen Elementen angehört, während sich die zolligen Körperelemente am leichtesten zerstören. I)as hängt wahrscheinlich von del chenischen Zusammensetzung der Gerebe $\mathrm{ab}$, auf welche ich hier nicht näher" eingehen kann.

Ich schliesse meine Mitteilung mit der Hoffinung, es sei mir gelungen zu beweisen, dass die neu gefundene Nammuthleiche ein sehr wertvolles wissenschaftliches Material gebracht hat, welches in mehreren Beziehungen unsele Kenntnisse ühel die Naturgeschichte dieses ausgestorbenen Tieres befördert.

Einige von den gesammelten Ueberresten wie z. B. die Futterreste haben uns für dic Beurteilung der Biologie dieses Tieres und der physikalischen V'elältuisse unter welchen es lebte, neue That:achen gebracht; andere dienten uns für das richtige Verständnis der Morphologie desselben; aus den dritten hahrn wir die Konntnisse üher den Erhaltungszustand der Gewebe wälrend der mehreren Jahrtausenden Begrabung in gefrorener Erde geschöpft. Die Phylogenio des Mammuths hat auch ein sehr wichtiges Material in den Ueberresten der neu gefundenen 
Mammuthleiche erworben. Bei meinen eigenen osteologischen Untersuchungen ${ }^{\prime}$ bin ich zu dem unerwarteten Schluss gekommen, dass das Mammuth in bedeutender Weise von den gegenwärtigen Elephanten sich unterscheidet, dass es namentlich ein tetradactyles Tier war, während die jetzt lebenden Elephanten einen pentadactylen Fuss besitzen. Durch diese Entdeckung soll die direkte Abstammung der Elephanten vom Mammuth vollkommen in Abrede gestellt werden. Das Mammuth war unzweifelhaft kein Vorfahre der gegenwärtigen Elephanten. Die Vorfahren derselben müssen in irgend welchen anderen Arten der fossilen Elephanten gesucht werden.

M. le Président donne la parole à M. H. F. Ossons.

\title{
TEN YEARS PROGRESS IN THE MAMMALIAN PALÆ.ONTOLOGY OF NORTH AMERICA
}

\author{
By Prof. Hexry fatrfiecd Osbori, LL. D., D. Sc. \\ (New-York.) \\ With 15 Plates.
}

Nembers of the Congress,

I believe that what you as specialists in the many diverse branches of zoology most desire to hear, are the salient results of our recent explorations in America, and their broader bearings on the general principles of zoology.

In 1878, the late Professor O. C. Marsh published his notable address entitled : Introduction and Succession of Vertebrate Life in North America ${ }^{2}$. Fifteen years later I published a somewhat similar review entitled: Rise of the Mammalia in North America ${ }^{3}$. In the ten years which have elapsed exploration has not only been on a larger scale ${ }^{4}$ than ever before,

${ }^{1}$ Osteolog. $u$. odontograph. Untersuch. über Mammuth und Elephanten. (Wiss. Result. der Expedition für die Ausgr. der Mammuthleiche am Ufer von Beresowka. Russisch). - Zur Phylogenie der Elephantiden. (Biol. Centralblatt, 1903.)

2 Proc. Amer. Assoc. Adv. Sci., Nashville, 1877, pp. 211-258.

3 Amer. Jour. Sci. 13, xlvi, 1893, pp. 379-392; 448-466.

${ }^{4}$ Large collections have been secured by the Museums of Princeton University and the University of California, by the Carnegie Nuse um Pittsburgh, the Field Columbian Museum, Chicago, and some few additions have been made to the famous collection brought together by Professor Marsh at Yale University.

The Department of Vertebrate Palæontology in the American Museum of Natural 
but also more thorough as well as guided by the constantly broadening aspects of the science.

The initial plan of the palicozoological survey undertaken by the American Museum was threefold; it was so far as possible to secure not only (1) a complete representation of certain families of mammals, as was done for monographic purposes by Marsu (i. e. I)inocerata, Brontotheriida (2), a complete representation of certain contemporary faunas, as was done chiefly by the late Professor Edward I). Cope (e. g. the Puerco and John Day faunas), but in addition (3) to secure complete phyletic series of various families of mammals in successive geological horizons from their introduction to their extinction (compare Fig. 2). In each of these features of our plan we have been rewarded with a success far beyond our most sanguine expectations. Our large collections studied by friendly cooperation in connection with those of other institutions, and large collections studied independently in other institutions, notably Princeton and the Carnegie Museum, have naturally hrought into a new light some of the important general principles of palæozoology.

\section{PROGRESS IN THE GENERAI PRINGIPLES OF PAL EOZOOLOGY}

Palcogeography. - The first broader bearing is that of past distribution and palaogeography, in which the accuracy of our records ${ }^{1}$ and thoroughness of our search is worling a lerolution. We are finding the remains of animals which have recently arrived from south America, Asia, Europe" and Africa", and it would he impossible to narrow the field of American fossil mammalogy even if we desired to do so. The broad study of the intercontinental evolution and relations of the mammals is absolutely essential to a philosophical understanding. Those who have followed the rapid recent progress of palarontology know that this spirit of uniting palieontology ever more elosely with distribution and

History was founded with the present writer as Curator in 1891. Associated with him at various times were the following zoologists and paleontologists: Messrs. Wortman, Matmhew, Earle, Gidley, and Brown. Fossil mammals brought from the West, secured by exchange, and by purchase, including the entire collection of the late Professor Edward D. Cope, now number 9873. The Cope Reptilian and Amphibian Collection is also in the American Museum.

${ }^{1}$ Matthew, W. D. A Provisional Classification of the Freshwater Tertiary of the West. Bull. Amer. Mus. Nat. Hist. Vol. XII, 1899, pp. 19-77.

2 Osbonn, H. F. Faunal Relations of Europe and America during the Tertiary Period. Ann. N. Y. Acad. Sci. Vol. XIII, 1900, pp. 46-56.

${ }^{3}$ Osbonn, H. F., Theory of Successive Invasions of an African Fauna into Europe. Ann. N. Y. Acad. Sci. Vol. XIII, 1900, pp. 56-58. 
palaengeography is that which constantly animates the older as well as many of the younger workers in this field.

Zoological methods. - Zoology in the sense of studying extinct forms as living organisus is also becoming closer day by day, and we are now enjoying the recognition by mammalogists (WEBER ${ }^{2}$, BEDDARD ${ }^{2}$ ) of the ahsolute necessity of coupling the study of ancestral with that of the recent forms in all questions both of distribution and of classification. In connection with distribution our chief advance has been to determine the exact geographical location and chronological succession of animals, the Jocal conditions of geological deposition in relation to habits and habitat or environment, as well as its bearing upon the study of past climates, or what may be called palæometeorology.

Aduptive radiation, continental. - In connection with the comparison of mammals in their intercontinental as well as in their continental relations, the branching system of LAmareK and the divergence which impresser Darwin is perhaps most clearly expressed by the word " radiation " ". Elsewhere the conception of adaptive radiation has been fully developed in connection with the origin of certain order's 4

It may here be briefly pointed out that Africa ", South America, North America and Eurasia prove to have been the three chief geographical centres of ordinal radiation.

Adaptive radiation. local" - - ()uite as important, although not carried on so grand a scale, is the local adaptive radiation which brings about a diversity of type in the same geographical regions and is the basis of the polyphyletic law of which we shall next speak. It is perhaps best illustrated by the Ungulates. In addition to (1) digital reduction (Kow.LEVsKx) and (2) carpal and tarsal displacement (Cope, Osmors) in relation to the choice of harder and softer ground, there is recognized (3) after the primary conversion of semi-Unguiculate into Ungulate types, a reversed conversion of Ungulate types into clawed types, as seen in Dichobune (Artiodactyla), Chalicotherium (Perissodactyla), and perhaps in an incipient stage in Agrioch(o'ms (Artiodactyla); (4) secondary adoption of aquatic habits, as seen, for example, in the Amynodontida among the Rhi-

1 Die Säugethiere. So, Jena, 1904.

2 Mammalia. The Cambridge Natural History, 80, 1902.

3 Osborn, H. F., Rise of the Mammalia. Proc. Amer. Association. Adv. Sc. Vol. xlii, 1893, p. 215.

${ }^{4}$ Adaptive Radiation of Orders and Families. Ann. N. Y. Acad. Sci. Vol. xiii, 1900, pp. 49-51.

${ }^{5}$ Ann. N. Y. Acad. Sci. XIII, 1900, pp. 56-58.

- Osborn, H. F. The Lav of Allaptive Radiation. Amer. Nat. xxxvi, 1902, pp. 353-363. 
nocerotoidea. Divergence by the above factor's has long been recognized. There ale also to be seen phyletic series combining in various ways either of the following eight conditions of foot, skull and tooth structure, which are not found to be necessarily correlated :

Primitive Condition.

(5) Mesaticephaly

(S) Mesatipody

\section{(11) Brachyodonty}

Secondary Condition.

(6) Elongation (dolichocephaly) of skull

(7) Abbreviation (brachycephaly) " "

(9) Elongation (dolichopody) of limbs

(10) Abbreviation(brachypody) 》 "

(12) Elongation (hypsodonty) of teeth

Law of correlation. - The bearing of these observations on Cuvier's law of correlation is to modify lather than to displace it. It may be restated as follows ${ }^{1}$ : The feet (correlated chiefly with limband body structure) and the teeth (correlated chiefly with skull and neck structure) diverge independently in adaptation respectively to securing (feet) a nd eating (teeth) food under different conditions; each evolvesdirectly for its own mechanical functions or purposes, yet in such a manner that each subserves the other. Thus, for example, there is a frequent correlation between dolichocephaly, dolichopody and hypsodonty, as in certain of the Equidit'; but there are so many exceptions to such correlation, because of the separate adaptive evolution of each organ, that it would be entirely impossible to predict the structure of the tonth from the structure of the claw, or vice versa.

Laue of analorgous evolution. -- One of the most important advances of the past decade, for which the way was largely prepared, in the previous decade, by Scotr's papers on Oipodon, Pö̈brotherimm and Mesohimpus, has been the clear recognition of this law. These phenomena give rise to an enormous number of analogies (homoplasies, parallelisms, convergences) not only of structure but of entire types, of families, and of groups, very confusing to the seeker of real phyletic relationship.

Evolution in part determinate. - As regards the modes and factors of evolution ${ }^{2}$, the continuous stages of evolution which we are securing among the horses, camels, rhinoceroses, and many other families, afford opportunities which have never heen afforded before. We are with adap-

1 Osborn. Amer. Nat. XXXVI, 1902, p. 363.

2 Scomt, W. B. On the Mode of Evolution in the Mammalia and on some of the Factors in the Evolution of the Mammalia. Jour. Morphol. Vol. v, 1891, No. 3, pp. 361-378, 378-402. 
tive characters from their birth or genesis, through their prime, into their decline and death. Through this unique opportunity for observation has been confirmed a view of evolution long shared by most if not all palieontologists, vertehrate and invertebrate, but naturally not understood or

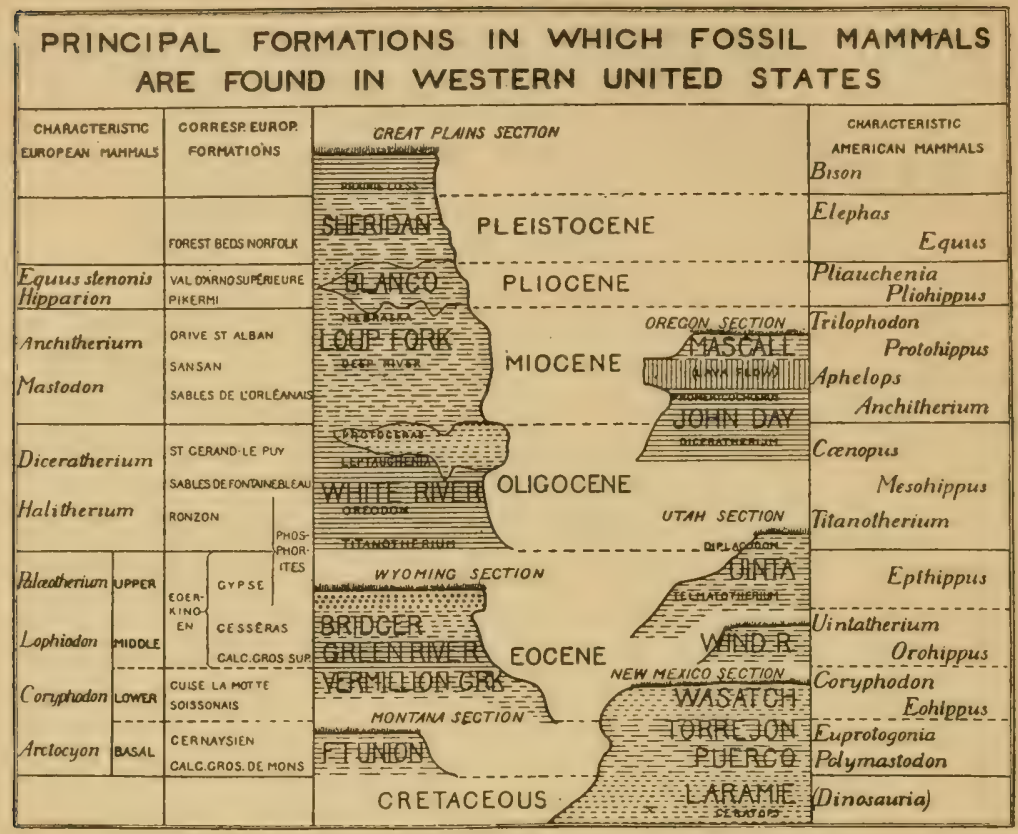

Fig. 1

Most recent geological subdivision of the American Tertiary.

Showing that the successive sections in Montana, New Mexico, Wyoming, Utah, Oregon, and the Great Plains afford a complete history of the Tertiary, homotaxial with that afforded by the corresponding European formations.

shared by other zoologists because of the essentially different nature of evidence. I refer especially to the theory of the definite or determinate $0 l^{\circ}$ igin ${ }^{1}$ and development of certain at least of the new adaptive structures, apparently, but not certainly according to the principle to which

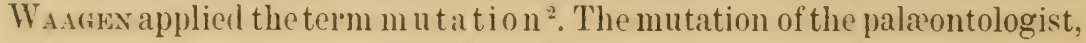

1 Osionn, II. F. The Paliontological Evidence for the Transmission of Acquired Characters. Amer. Naturalist, Vol. xxiii, 1889, p. 562.

${ }^{2}$ Sсотт, W. B. On Variations and Mutations. Amer. Jour. Sci. Vol. xlviii, Nov. 1894, pp. 355-374. 
however, is quite distinct from the phenomena of minute saltations to which de Vries has applied WAarex's term in his valuable experiments?

Potential of similar erolution. - In connection with analogous, but especially with partially determinate evolution, we not only have the similarly moulding influences of similar habits, and the action of the various factor's of evolution ${ }^{2}$ which we cannot stop to discuss, but clear evidence of the existence of a potential of similar evolution, a kind of latent homology which dete minesthat when certain structures are to appear among animals independently derived from a common stock, they will appear at certain definite points and not at random. For example. the genesis of the rudiment of the horn in three independent phyla of Eocene titanotheres is at exactly the same point, namely, at the point of junction of the frontals with the nasals at the side of the face just above the eye.

The polyphyletic low. - Partly as an outgrowth of the synthesis of the above principles and partly as the result of new discoveries and the closer study of types already hinown is the full recognition of the polyphyletic law:". If we 'xamine the phylogenies of Huxury and Cope, and even those of more recent writers (Scotr. Osmors, Worturas) of a decade ago, we find that the attempt is made, for example, to trace the pedigrees of the horses and rhinoceroses in a monophyletic manner. The first known instance of this kind was Huxuex's perdigree of Equns through Hipparion, Anchitherium and Paleotherium, all of which are now known to belong to entirely distinct phyla. Another instance was the comparatively recent effort to trace all rhinoceroses through the oligocene Acerttherium occidentale Leidy as the stem form.

The polyphyletic law is an outgrowth of four different kinds of evidence. First, that the stem forms are very much older than we supposed them to be: we placed them in the Pliocene and Viocene, they have now been traced to the ()ligocene and Eocene. Second, as a consequence of this, certain moder'n genera of mammals have their own ancestry, apart from that of elnsely related genera, as far hack as the Oligocene and perhaps Eocene. The most conspicuous example of this is the tracing back of the Dholes (genus Cyom) among the Canida, to an ()ligocene form, showing that Cyon separated from $C$ aris in the Eocene (Wortasix

1 Elsewhere this profound difference between "palæontological mutations and the mutations of de VRIES is carefully pointed out. See "OsBorN Present Problems of Palæontology ", address before St. Louis Congress of Science and Art, September, 1904, first printed in Popular Science Monthly, December, 1904.

2 Oseorn, H. F. St. Louis Address. Loc. cit. supra.

${ }^{3}$ Osbonn, H. F. The Perissodactyls typically polyphyletic. Science, N. S., Vol. xvi, 1902, p. 715 . 
and MAtruew)'. Thi lod, the polyphyletic law is the result of local adaptive radiation $01^{\circ}$ divergence apparently of habit either by choice ol by necessity. For example, among the horses it separates off the grazing types (Protohipmes), which are naturally proglessive, from the browsing types (H!/pohipmus), which are naturally conservative, both found in the same locality (Fig. 4). It thus splits up animals living in a single region into a number of contemporaneous types or genera which may coexist throughout long periods; it is a segregation, functional rather than arlaptive. Fourth, the polyplivletic law results from the invasion into a region of a generic or specitic phylum which has evolved on another continent: for example, the Eurasiatic Teleoceras came in among the American rhinoceroses in the Middle Miocene (Plate VII).

This polyphyletic law has now been demonstrated (OsBors ${ }^{2}$ ) among the rhinoceroses both of Eurasia and of North America, and is the key to the comprehension of this group; in Fig. 3 printed herewith it is shown that there are not only three families, namely, cursorial (Hyracodontidi(). aquatic (Amynodontida), and terrestrial (Rhinocerotidie), but that the last family splits up into six and possibly seven phyla, many of which are contemporaneous; and the tendency of discovery will be to increase rather than to diminish the number of contemporaneous independent phyla. Similarly the Eocene titanotheres instead of forming a successive monophyletic series, divide into four distinct phyla, to each of which a generic name must be given. Similarly, again, the lower Oligocene titanotheres', as shown in Fig. 7, divide into four phyla, three of which have been traced in successive stages from the bottom to the summit of the (Oligocente, each giving off several collaterals, all living in the same region and found in contiguous beds, but probably having a slightly different local habitat and habits. The law is illustrated again, as shown in Fig. 4, both in the Oligocene and Miocene horses: in the Oligocene, for example, we have five contemporaneous lines of horses (Osiborn:", (index), one of which includes the classic Mesohippus bairdi of LEIDr, which was long considered the single ()ligocene horse, and figured as such in all phylogenies: in the Upper Miocene beside the Pro-

1 The Ancestry of Certain Members of the Canidx, the Viverridx and Procyonidx. Bull. Amer. Mus. Nat. Hist., Vol. xii, 1899, pp. 139-148.

2 Phylogeny of the Rhinoceroses of Europe. Bull. Amer. Mus. Nat. Hist., Vol. xiii, 1900 , pp. 229-267.

${ }^{3}$ New Miocene Rhinoceroses with Revision of Known Species. Bull. Amer. Mus. Nat. Hist., Vol. xx, 1904, pp. 307-326.

* The Four Phyla of Oligocene Titanotheres. Bull. Amer. Mus. Nat. Hist., Vol. xvi, Feb. 1902, pp. 91-109.

${ }_{5}^{5}$ New Oligocene Horses. Bull. Amer. Mus. Nat. Hist., Vol. xx, May, 1904, pp. 167-179. 
tohippus, which still apparently is most nearly ancestral to Eipurs, we find as contemporaries, the low wing, forest-living Hypohipurs (Plate X), and the grazing and highly cursorial Veohipurerion (Plate XI). A comparison of the phylogeny of the Camelide (Fig. 5) published by Wor'T-

Ilodern Fauna, Higher Placental Radiation.

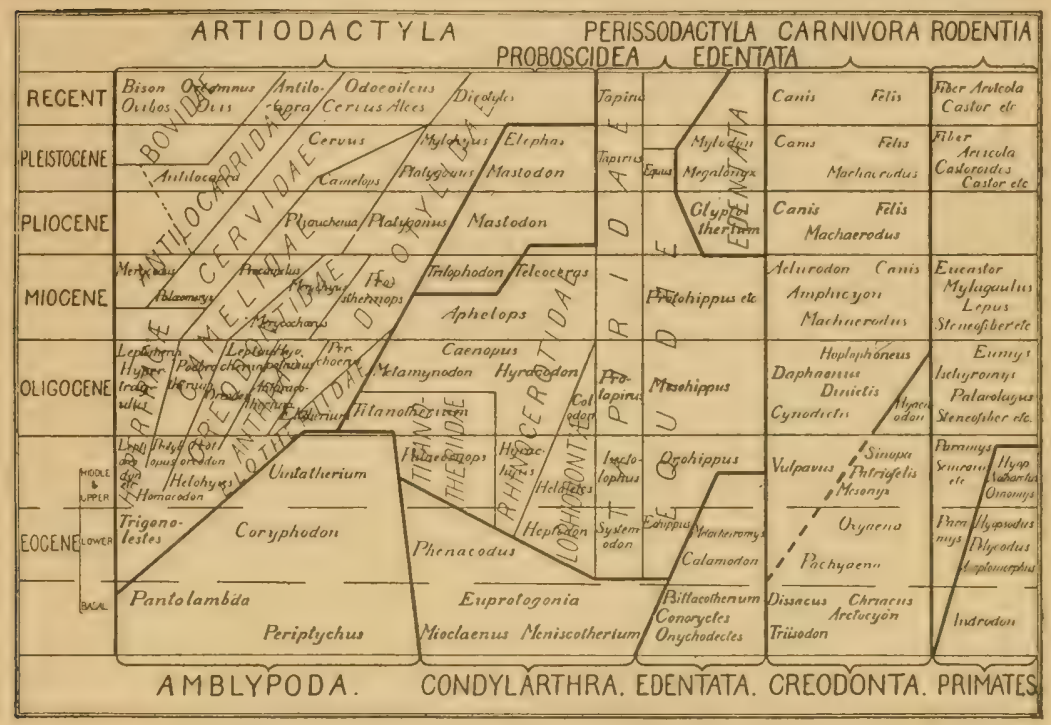

Archaic Fauna, Lower Placental Radiation.

FIG. 2

Extinction of the lower placental radiation of the Cretaceous, and sudden introduction of the higher placental radiation of the Tertiary.

The orders Amblypoda, Condylarthra, Edentata, Creodonta, and earlier Primates disappear in North America. The comparatively modern Rodentia, Carnivora, Perissodactyla, Artiodactyla, and Proboscidea suddenly appear without known ancestors in the Lower Tertiary. No connections have thus far been traced between this older, archaic fauna and the newer fauna.

MAN $^{1}$ in $189 \sin$ the mon op hy le tic basis with that published by MATTHE ${ }^{2}$ in 1904 on the polyphyletic basis, shows the rapid progress which has been made in the demonstration of the polyphyletic law. Similar results are apparent from our preliminary studies of the Proboscidea in Am('-

1 The Extinct Camelidx. Bull. Amer. Mus. Nat. Hist., Vol. x, 1898, pp. 93-142.

3 Notice of two New Oligocene Camels. Bull. Amer. Mus. Nat. Hist., Vol. xx, 1904, pp. 211-215. 
lica. Many able contemporary worker's, especially Ścnlosser and DeréRE'T, are also bringing forth new illustrations of this law in Europe.

\section{PROGRESS OF IMLAOYERY ANO THE VEW PHYLOGENETIC PROBLEMS SUGGESTED THEREBY}

My purpose in this section is to give a brief resume of thr progress during the past ten years, and in our present state of knowledge to point out where exploration and research should principally be rirected.

The general advance has been made in five distinct lines, which appear to mark out also the main lines for future research. First, the biological value of more accurate geological records (Compare Fig. 1), has been recognized; as a result the mammalia have been chronologically segregated into successive life zones similar to those which have long been developed in invertehrate palarontology; these life zones in some cases subdivide not only the periods (Eocene, Miocene, etc.), but also subdivide the stages (Bridger, Uinta), etc. Second, not only have these clearer chronological subdivisions been marle, but the faunas have been separated according to their kinds and the nature of the deposits, into those which inhabited respectively the lowlands and rivers, forests, plains, and uplands. The advance of plysiography has been felt, and by the careful work of Hatcher ${ }^{1}$, Matruew ${ }^{2}$, and Gidser ${ }^{3}$, the theory of Huviatile, flood plain, and arolian drposits has tended to replace the theory of great lakes or lacustrine deposits. Third, there has accordingly been brought about a modification of our views as to the meteorological or climatic phases of the tertiary period, in the direction of extending the idea of the existence of great dry plains with drifting sands favorable to Eolian deposits chietly in the Lower Pleistocene, Pliocene and Miocene; we speak less of a moist, subtropical, and more of a drier climate. Fourth, the zoogeographical relations of the North American faumas to those of other continents have become much more clearly understood $\left(O_{s \text { Born }}{ }^{6}\right)$ in connection with mole exact geological records not

1 Origin of the Oligocene and Miocene Deposits of the Great Plains. Proc. Amer. Philos. Soc., xli, No. 169, Apr. 1902.

${ }^{2}$ Is the White River Tertiary an Eolian Formation. Amer. Nat, xxxiii, May, 1899, pp. 403-408.

3 Fossil Mammals of the Tertiary of Northeastern Colorado. Mem. Amer. Mus. Nat. Hist., Vol. 1, Pt. vii, Nov. 1901.

4 The Freshwater Tertiary of Northwestern Texas. Bull. Amer. Mus. Nat. Hist., Vol. xix, 1903, pp. 617-635.

- Mattiew and Gidley. New or Little Known Mammals from the Miocene of South Dakota: Bull. Amer. Mus. Nat. Ilist.; Vol. xx, pp. 241-268.

${ }^{6}$ Fannal Relations of Europe and America. Science, Vol. xi, April, 1900, pp. $561-514$. 
only by the addition of many new forms from the Eurasiatic radiation hitherto unknown, but also hy observing mole precisely the time of arrival of Eurasiatic migrants in the Lower, Mid-and Upper Miocene and of South American in the Pliocene. F if th, the phylowenetic succession has become much clearer and more direct, although a vast amount remains to be done. The separate branches of the mammalian phyletic tree have been successfully traced back farther and farther toward the beginnings of the Tertiary, with resultant changes in our classification. Perhaps the most signal taxonomic result of this phylogenetic progress is in the clear definition of certain renera, notably among the rhinoceroses (Osmone', Thomas ${ }^{2}$ ), as shown in Fig. 3 ; it has proved to be absolutely necessary for the salie of clantress to recognize a number of genera which many systematists ( F LowER, LxDEKkER ) have considered simply synonyms of the genus Rhimoceros. Sixth, the rhief molpholog ical result is the discrimination of sexual characters, especially among the male and female folms ". which in many ('ases hy Marsi and ('ope had been consideled as distinct species. The recomition (Osmowis ${ }^{i}$ ) that progresive dolichocephaly and brachycephaly profoundly modify all the characters of the skull and the teeth on the principle of correlation, also lepresents a morphological advance.

The independent and more or less cooperative field or museum work of Scott, Osborn, Wortman, Mattheiv, Hatcher, Douglass, Gidley, Peterson, has heen instrumental in forwarding these chief lines of progress.

\section{'The Otder Mesozoid Fauna.}

Unfortunately the efforts of the American Museum to find more of the Protodonta (Dromotherium, Aleroconodon) from tho Upper Trias or Rhatic have proved mavailing. The relation of these animals to the Theriorlont reptiles has been sugrested (Swawr), but the single hone of the jaw rather sustains their relation to the mammalia. The groove on the inner side of the jaw of all Mesozoic and some recent mammals is now recognized as the Meckelian-cartilage groove (Benslex ${ }^{5}$ ).

1 Phylogeny of the Rhinoceroses of Europe. Bull. Amer. Mus. Nat. Hist., Vol. xiii, 1900, pp. 229-267.

${ }^{2}$ Notes on the Type Specimen of Rhinoceros lasiotis Sclater with Remarks on the Generic Position of the Living Species of Rhinoceros. Proc. Zool. Soc., Lond., June 4, 1901, pp. 154-158.

s The Cranial E'volution of Titanotherium. Bull. Amer. Mus. Nat. Hist., Vol. viii, 1896, pp. 157-197.

${ }^{4}$ Dolichocephaly and brachycephaly in the Louer MIammals. Bull. Amer. Mus. Nat. Hist., Vol. xvi, 1902, pp. 77-89.

- On the Identification of Meckelian and Mylohyoid Groores. Univ. of Toronto Studies Biol., Ser. 3, 1902, 1p. 75-81. 
In the Upper Jurassic or Lower Cretaceous mammalia of the Como beds we must also admit that no progress has been made to determine whether these animals represent both Insectivora and Marsupialia and perhaps Monotremata (OsBons), or whether they are all Marsupialia ${ }^{1}$ (most English authors). A re-study (OsBonn ${ }^{2}$ ) of the structure of the upper molars in the Yale Museum collection strengthens the tritubercular theory (Cops, ()sbons) of the origin of the upper molar teeth.

\section{The Upper Cretaceous Fauna.}

Here again the relatively modernized (0seors $\left.{ }^{3}\right)$ animals of the Upper Cretaceous $01^{\circ}$ Laramie, although carefully l'evised, still require elucidation from the rich collection in the Yale Innivel'sity Museum. Marsu's statement that certain of these animals are Marsupials has been fully confirmed by MatтHew, a fact which is striking in the absence of any present evidence of Marsupials in the American basal Eocene.

The present relations of these Laramie animals to those of the Basal Eocene (Puereo, Torrejon) has been somewhat strengthened by the recognition of the ancestors (Meniscoëssus) of Polymastodon, also by the supposed recognition of forms related to the Amblypoda, especially to the Periptychidie (OsBons); but forms certainly ancestral to the Creodonta and other Eocene mammals have not yet been recognized.

\section{The Basal Eocene Fauna.}

In this famna, commonly known as Pterco, great progress has been made.

Two sharply defined faunal stages have been distinguished (Wortans), a lower, Puerco proper, and an upper, Torrejon (MAtruew ${ }^{4}$ ), (Compare Fig. 1, 2). 'The latter is more nearly contemporaneous with the Basal Eocene (Cernaysien) of France. Fortunately, in Montana, a new locality has been discovered for these very archaic mammals in the Fort Union beds (Dovicass ", FArR) which promises to extend our knowledge of this fauna.

1 Ameghino, Fl. Los Diprotodontes del orden de los Plagiaulacideos. An. Mus. Nac., Buenos-Aires, t. ix, 1903, pp. 81-192.

2 Palæontological Evidence for the Original Tritubercular Theory. Amer. Jour. Sci., Vol. xvii, April, 1904, pp. 321-323.

${ }^{3}$ Fossil Mrammals of the Upper Cretaceous. Bull. Amer. Mus. Nat. Hist., Vol. v, 1893, pp. 5311, 330.

* Matthew. A Revision of the Puerco Fauna. Bull. Amer. Mus. Nat., Vol. ix, 1897, pp. 260-261.

${ }^{5}$ A Cretaceous and Lower Tertiary Section in South Central Montana. Proc. Amer. Phil. Soc., Vol. xli, 1902, No. 170, pp. 207-224. 



\section{EXPLANATION OF PLATE I}

Fig. 1. Pantolambdı. - Basal Eocene, Torrejon Beds. (= Cernaysien).

Fig. 2. Coryphorlon. - Lower Eocene, Wasatch Berls. (E Soissunien). 


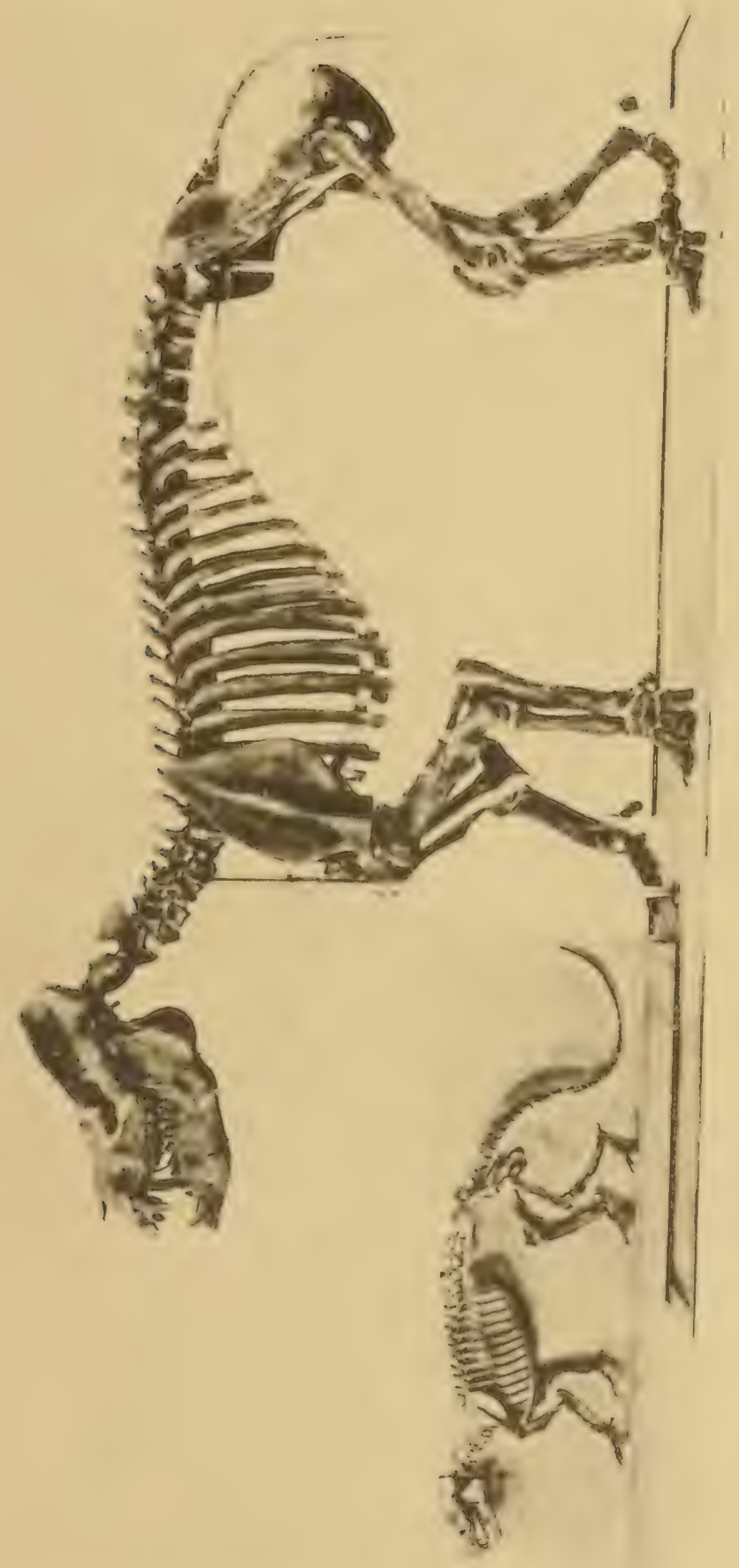

H. F. OSBORN

MAMMALIAN PALEONTOLOGY OF NORTH AMERICA 


EXPIANATION OF PIATE II

Fijg. 1. Pantolambinla. Model to scale.

Fign. 2. Coryphodom. Model to seale. 


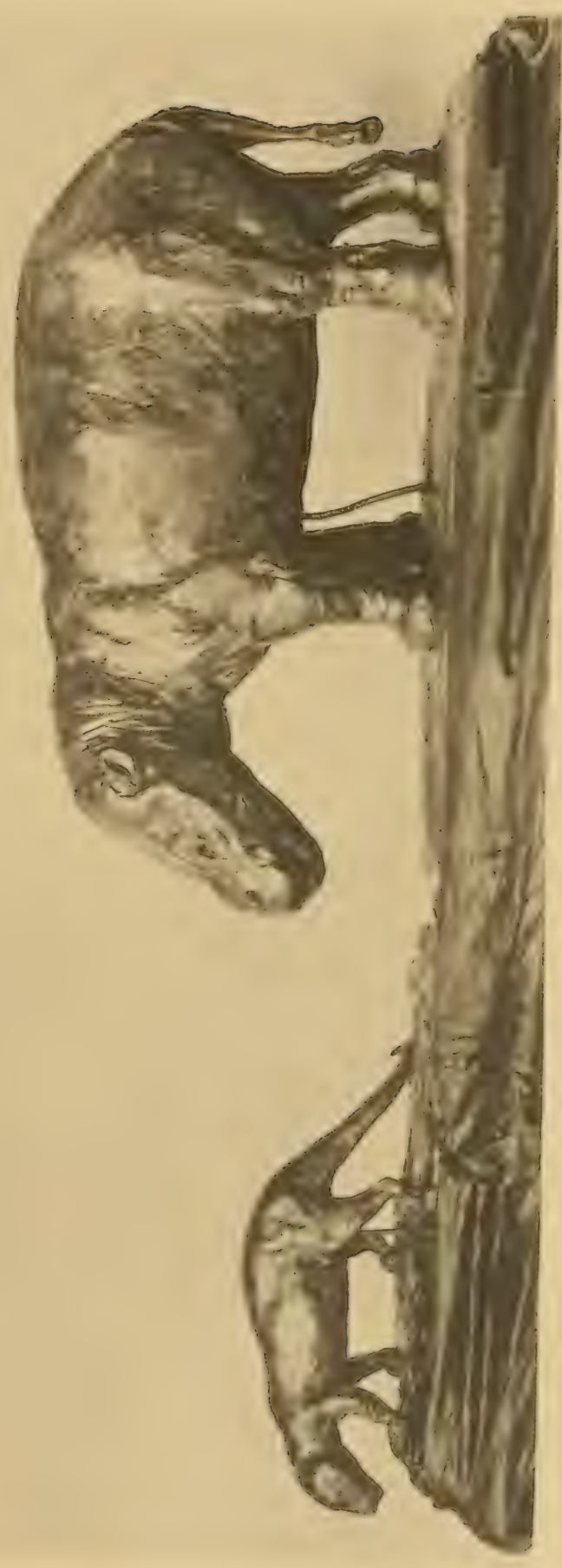






\section{EXPIANATIOS (IF PIAT'E III}

skubls representing four phỵla of Eneme Tilanotheres.

$I=$ horn rudiment arising independently in three phyla.

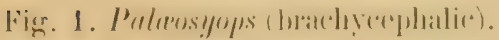

Fin. 2. Telmulotherium, probable ancestor of Titmotherium (dolichocephalic).

Fin. 3. Mremererere, ancestor of Megacerops (mesaticephalie). Middle Eocene (Washakie).

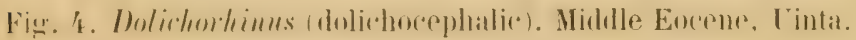



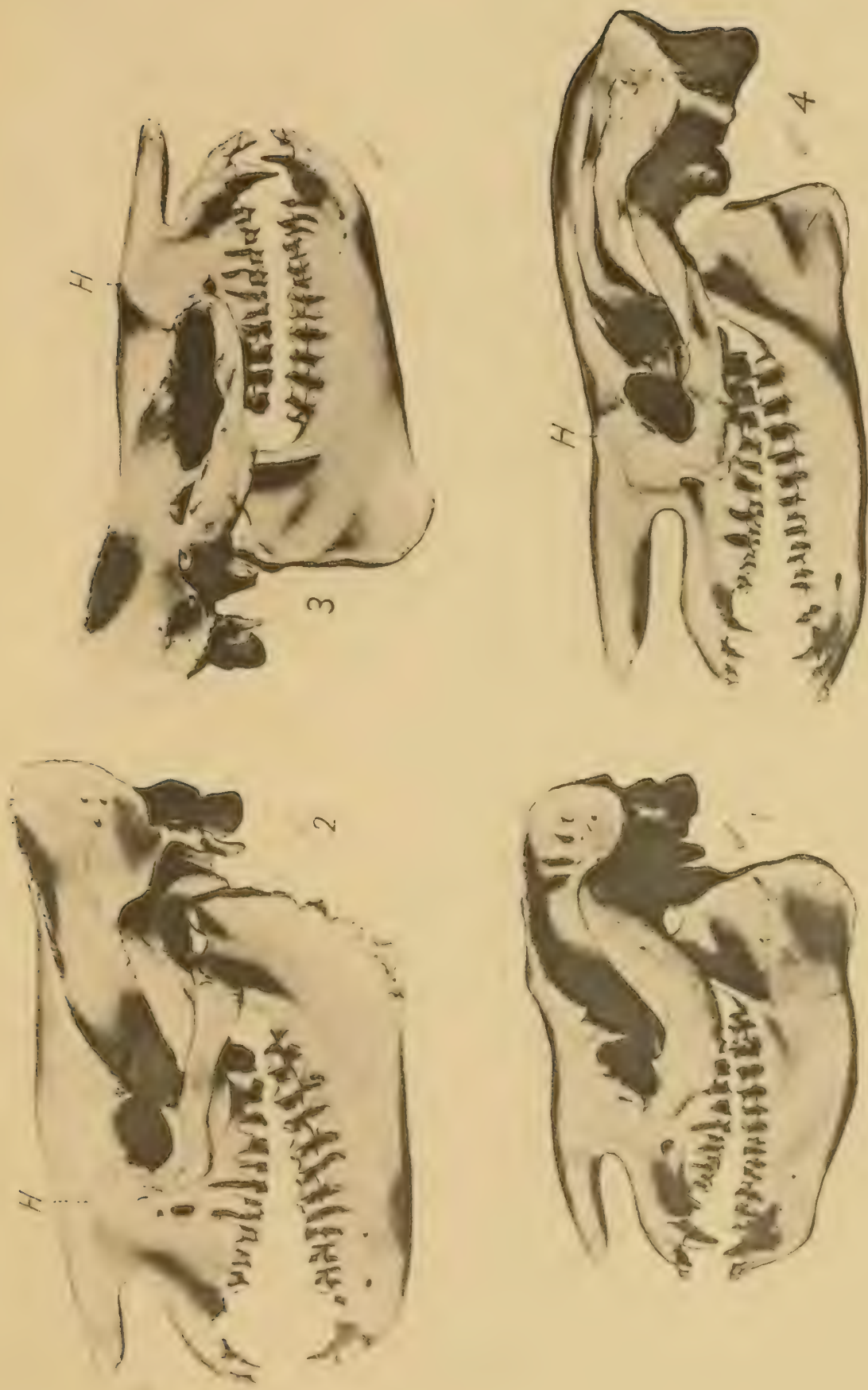

H. F. OSBORN

MAMMIALIAN PALIEONTOLOGY OF NORTH AMERICA 




\section{EXPLANATION OF PIATE IV}

Skulls representing four phyla of Oligocene 'litanotheres.

Fig. 1. Megacerops, probable successor of Manteoceras (brachycephalic).

Fig. 2. T'itanotherium, probable successor of Telmatolherium (dolichocephalic).

Fig. 3. Symborodon (brachycephalic).

Fing. '1. Brontotherium (dolichocephalic to mesaticephalic). 


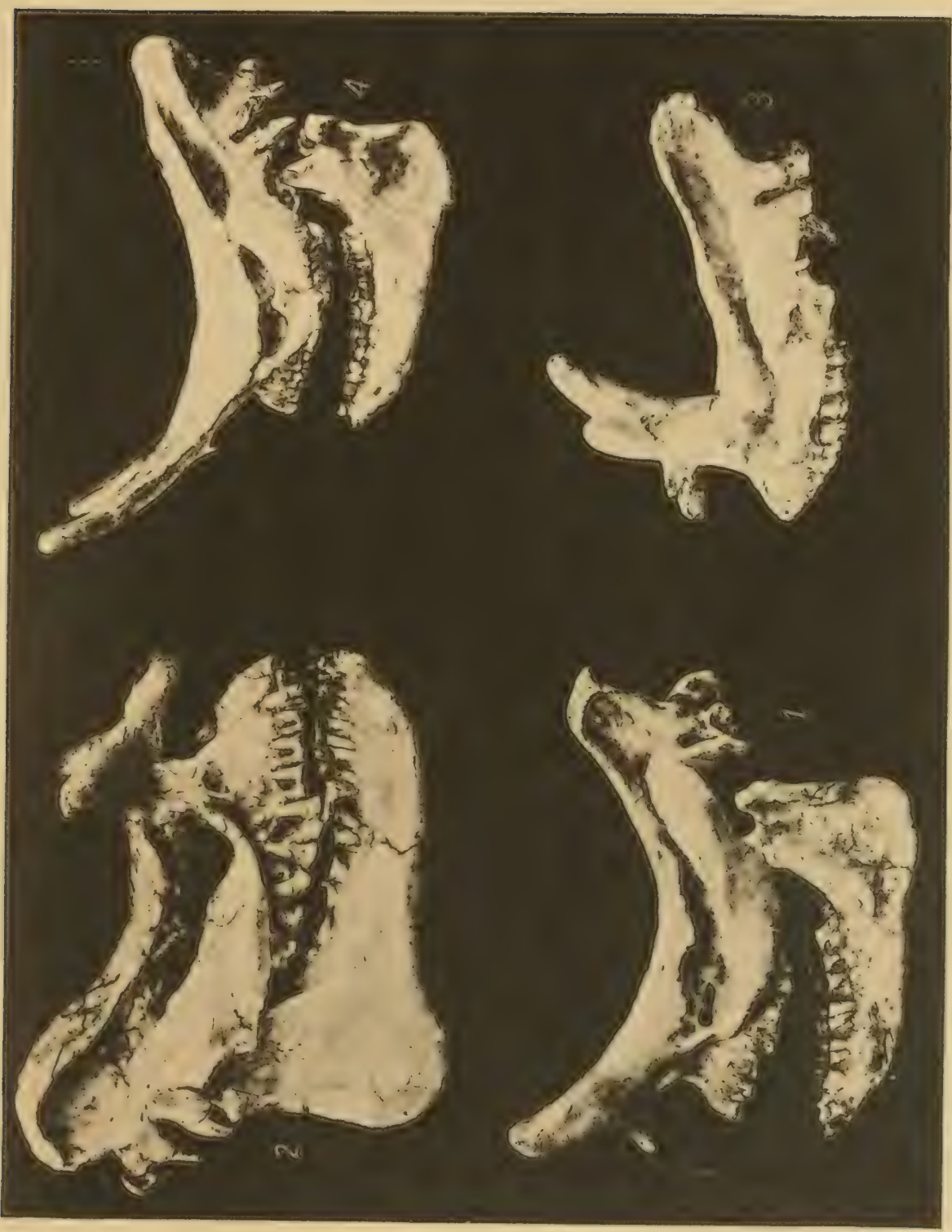

H. F. OSBORN

MAMMALIAN PALEONTOLOGY OF NORTH AMERICA 




\section{EXPLANATION OF PLATE V}

Irodels of Heads of Oligocene Titanotheres four phyla.

Fig. 1. Megacerops (brachycephalic), short horned.

Fig. 2. Titanotherinm (dolichocephalic), short horned.

Fig. 3. Symborodon (mesaticephalic to brachycephalic), long horned.

Fig. 4. Brontollerium (mesaticephalic to dolichocephalic), long horned. 


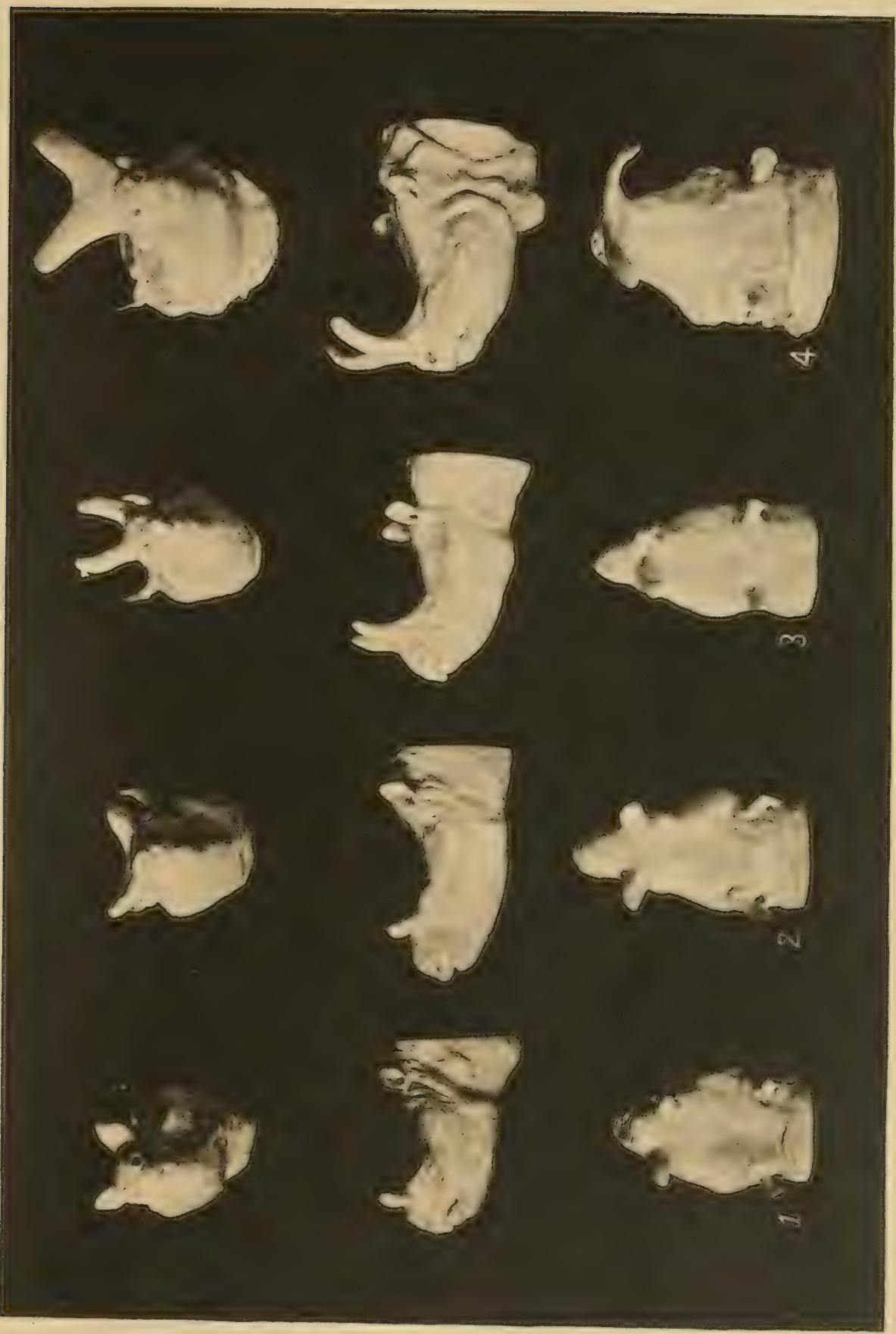

H. F. OSBORN 




\section{EXPLANATION OF PIATE VI}

Merycodus osborni Matthew.

Family Merycodontide Matthew.

Middle Miocene, Pawnee Creek Beds, Colorado.

Resembling the Antilocapride in skull, skeleton and looth structure and in the supraorbilal position of the antlers. Besembling the Gervidir in the branching. deciduous antlers. 


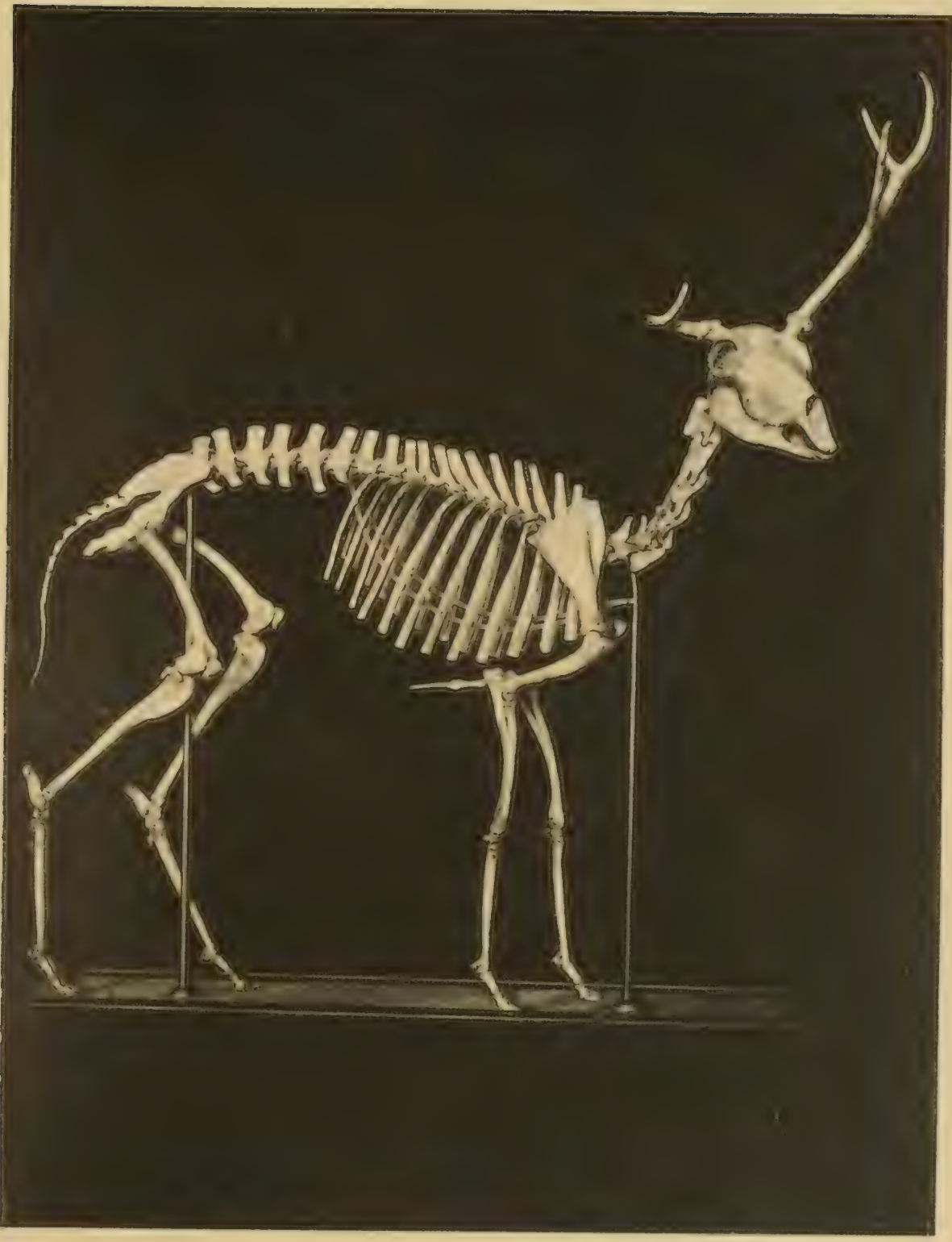

H. F. OSBORN

MAMMALIAN PALEONTOLOGY OF NORTH AMERICA 




\section{EXPIANATION OF PATE VII}

Both types have a very rudimentary frontal horn core at $I I$.

Fig. 1. Toleocerts bicormutus Osb.

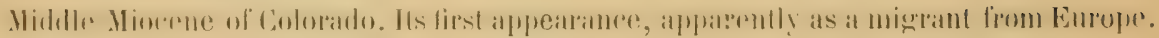
Fig. 2. T'eleocerus aurelianensis.

Lower Miocene of France (Sables de l'Orléanais). 

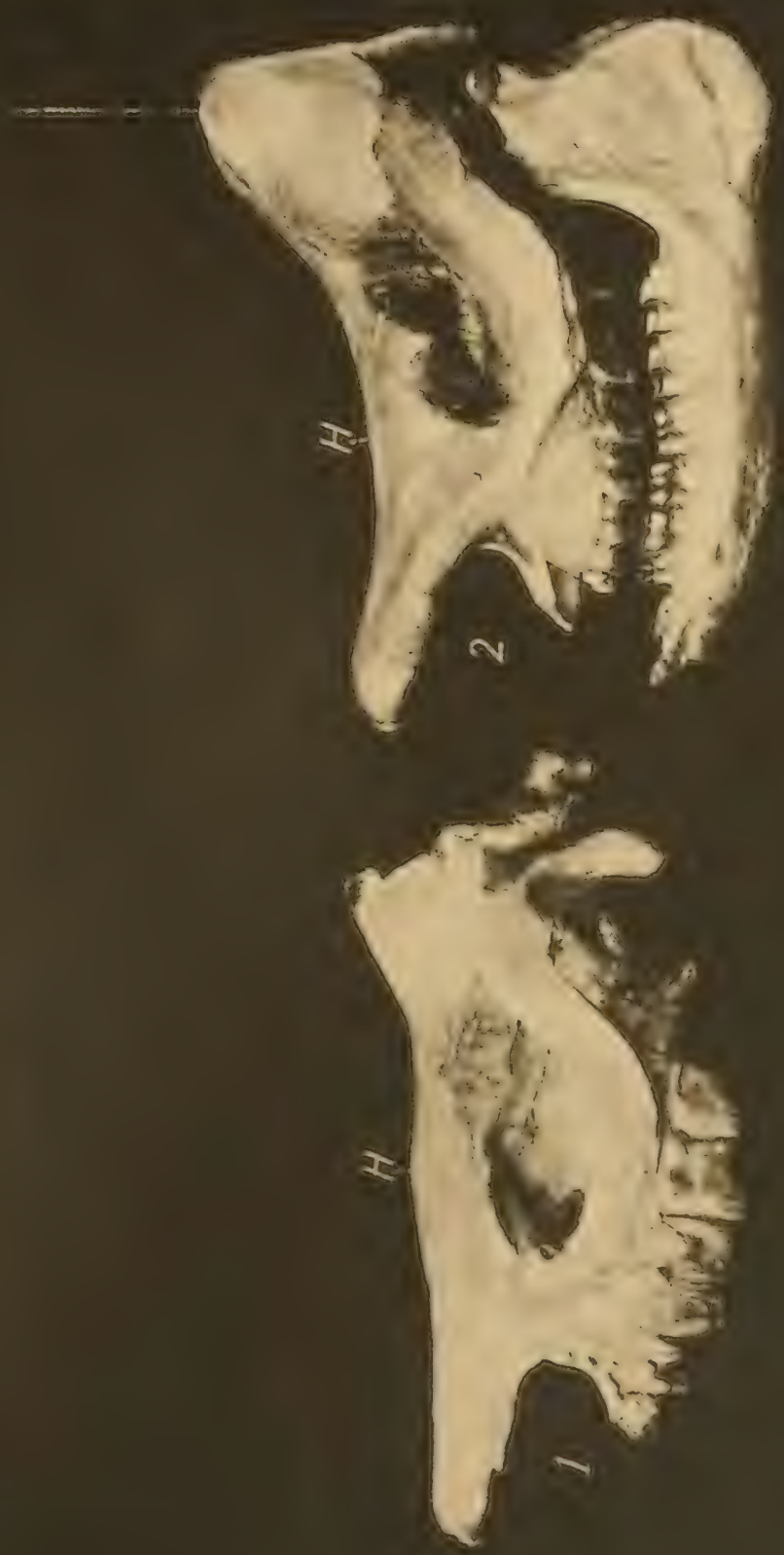

H. F. OSBORN 




\section{EXPLANATION OF PLATE VIII}

Teleoceras fossiger Cope.

Upper Miocene, True Loup Fork.

Short limbed Rhinoceros. with a small terminal horn on the nasals in the males. 


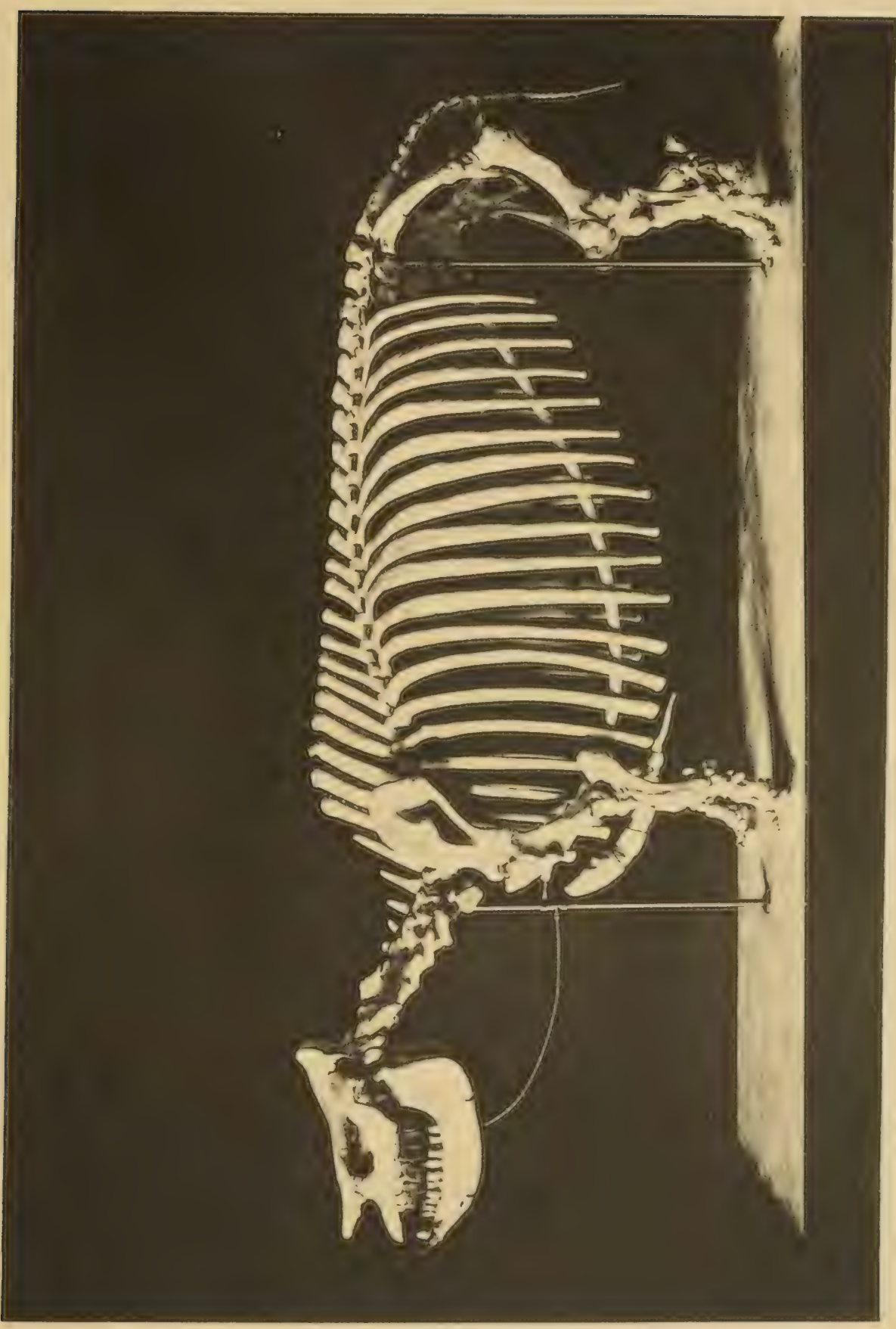

H. F. OSBORN 




\section{EXPLANATION OF PLATE IX}

Models of Protorohipuns to life stale, actual height $121 / 2$ inches (1:32 ('m.). Based on Cope's type skeleton (Plate X). 


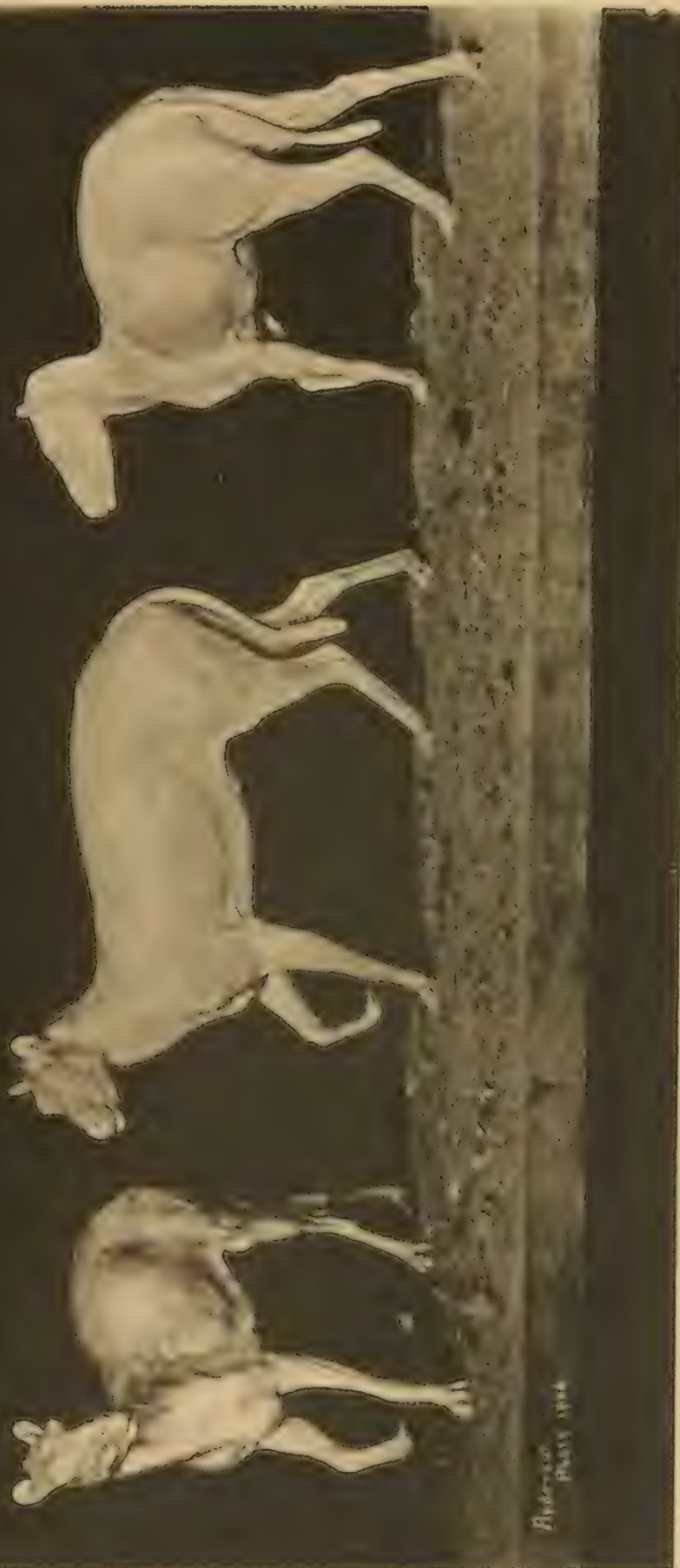






\section{EXPLANATION OF PLATE X}

Protorohippus (small) and II!pohippus (large), the latter a persistent tridactyl. brachyodont, browsing horse from the Upper Miocene. 


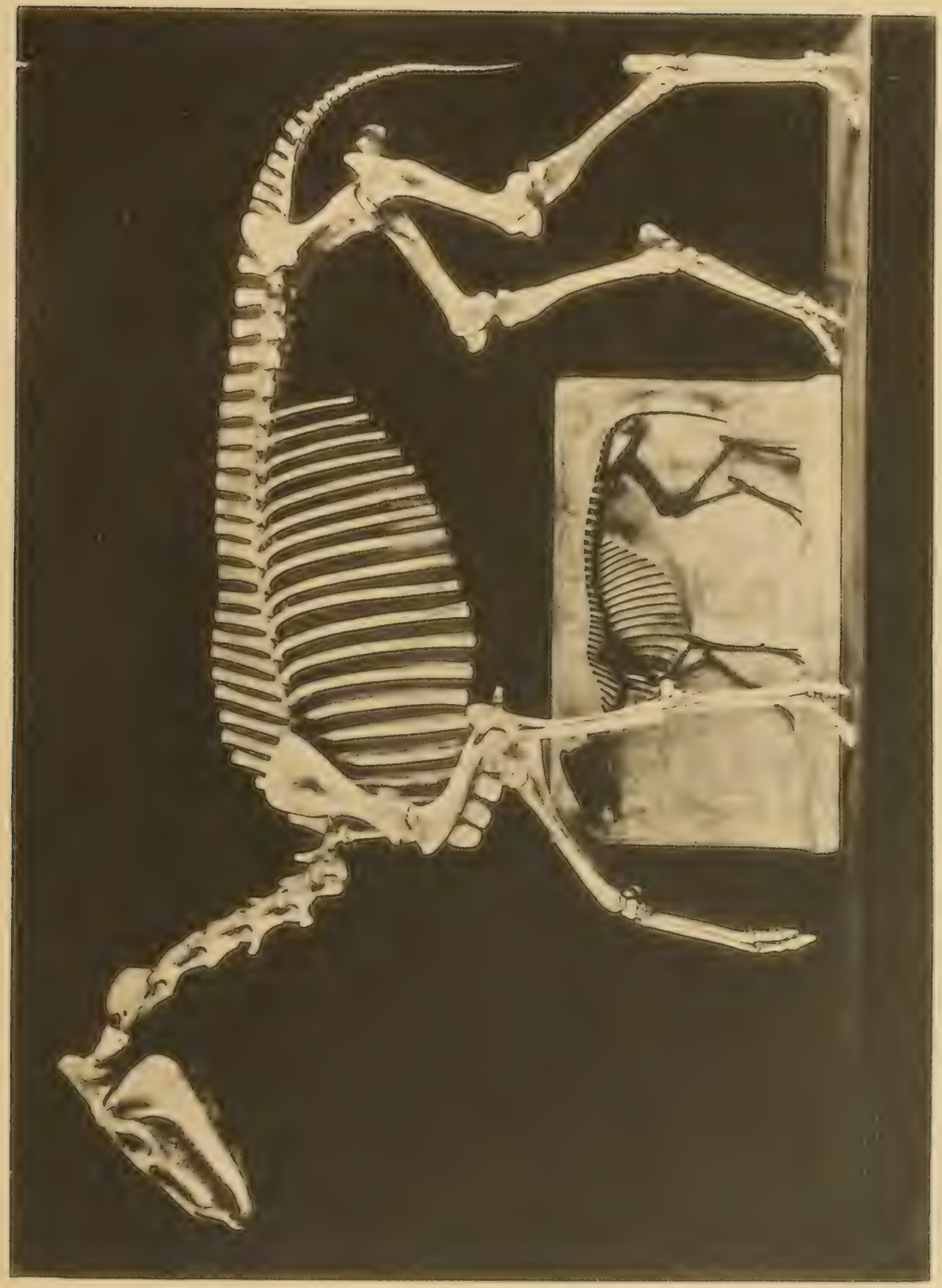

H. F. OSBORN 


EXPIANATION OF PIAATE XI

Neohipparion whimeyi. a cursorial. tridactyl but functionally monodacț̣l, grazing. horse from the Upper Miocene. 


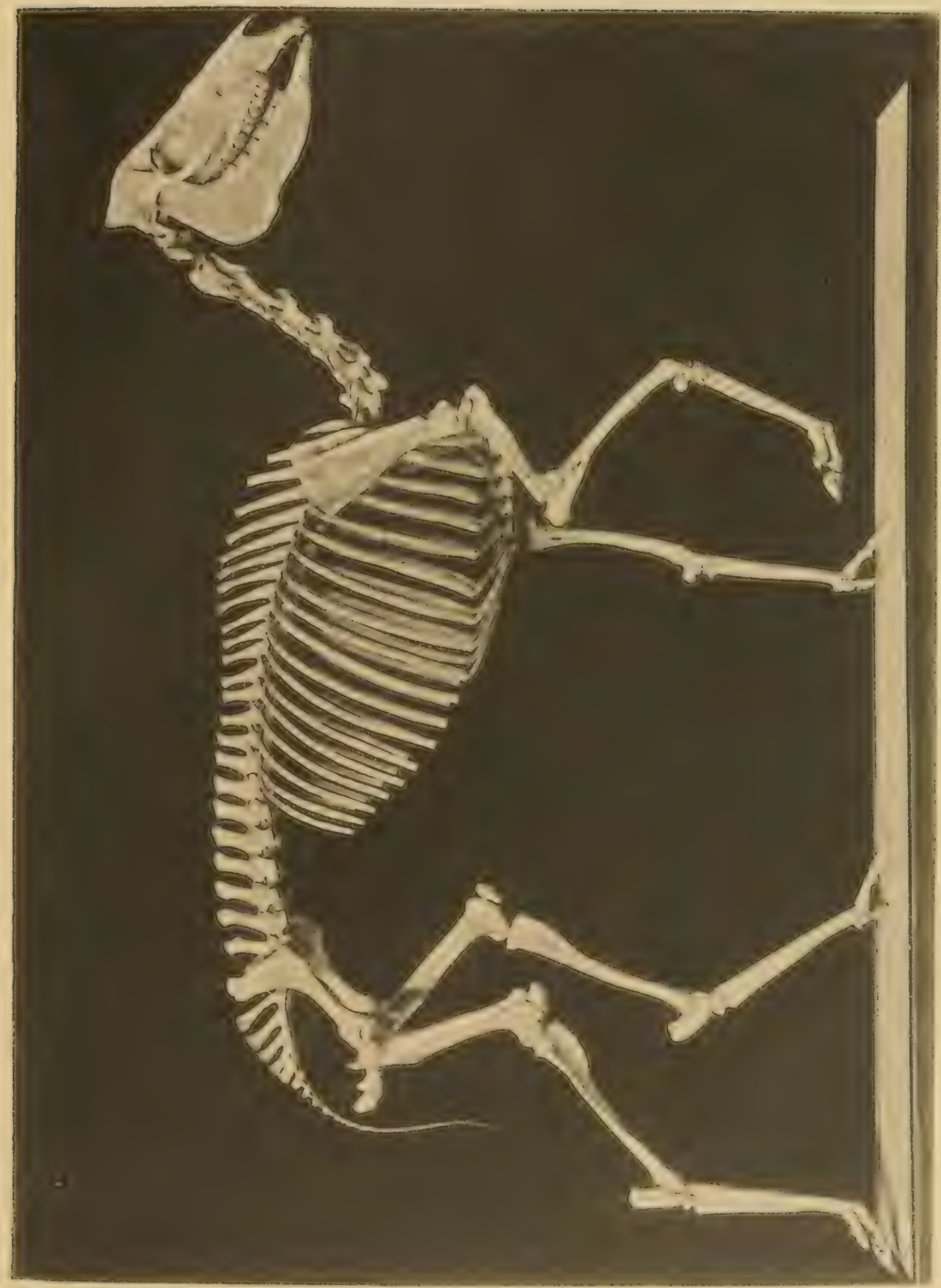

H. F. OSBORN 




\section{EXPLANATION OF PLATE XII}

Fig. 1. Holar of Elephas primigenius Blumenbach.

Fiš. 2. " " " columbi Falconer.

Fig. 3. " " " imperator Leidy. 


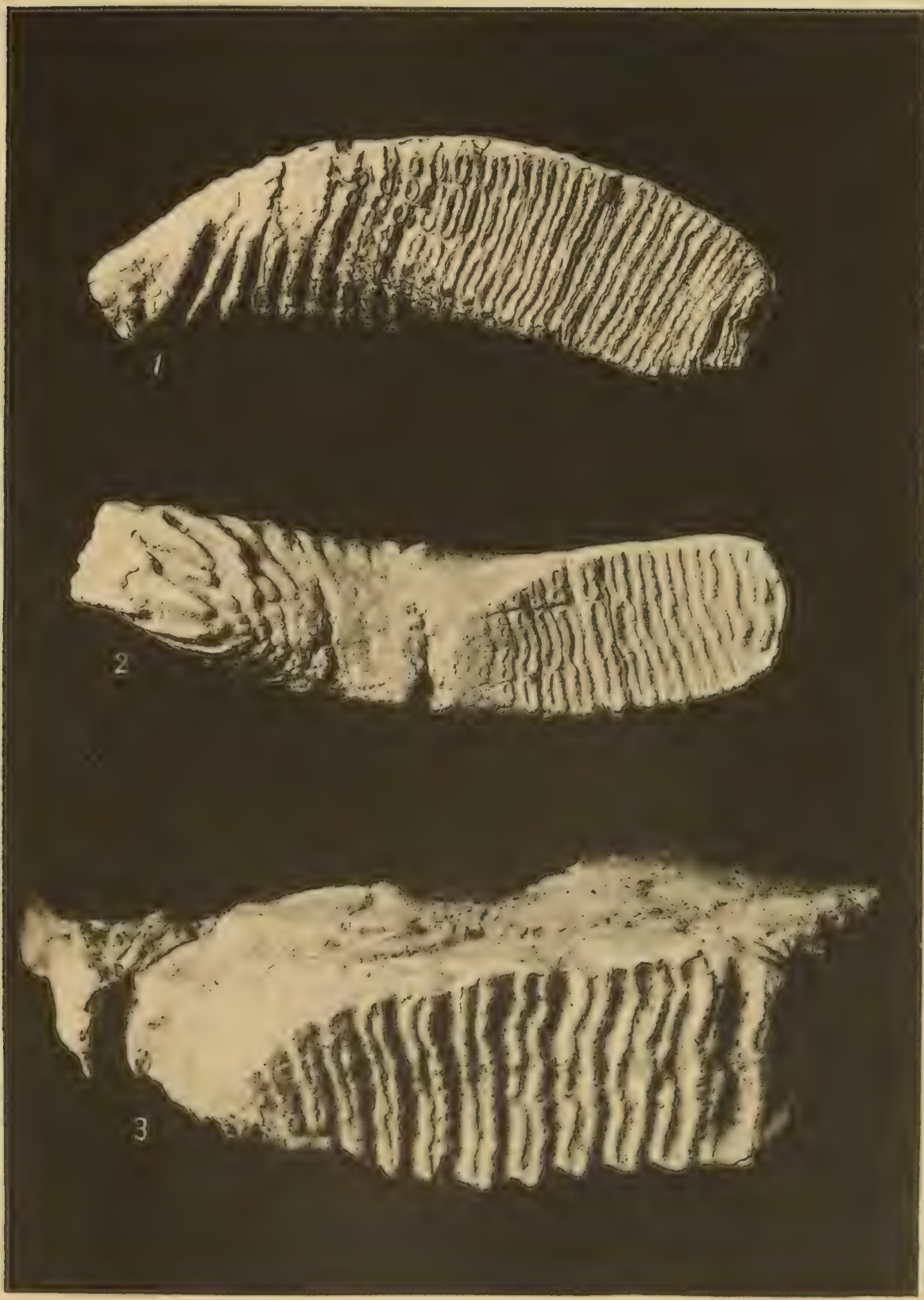

H. F. OSBORN

- mammalian paleontology of north america 




\section{EXPI AANATION OF PLATE XIII}

Tusks and Palate of Elephas imperator Leidr:

The Upper Part of the Skull is restored.

From the Lower Pleistocene of Texas. 


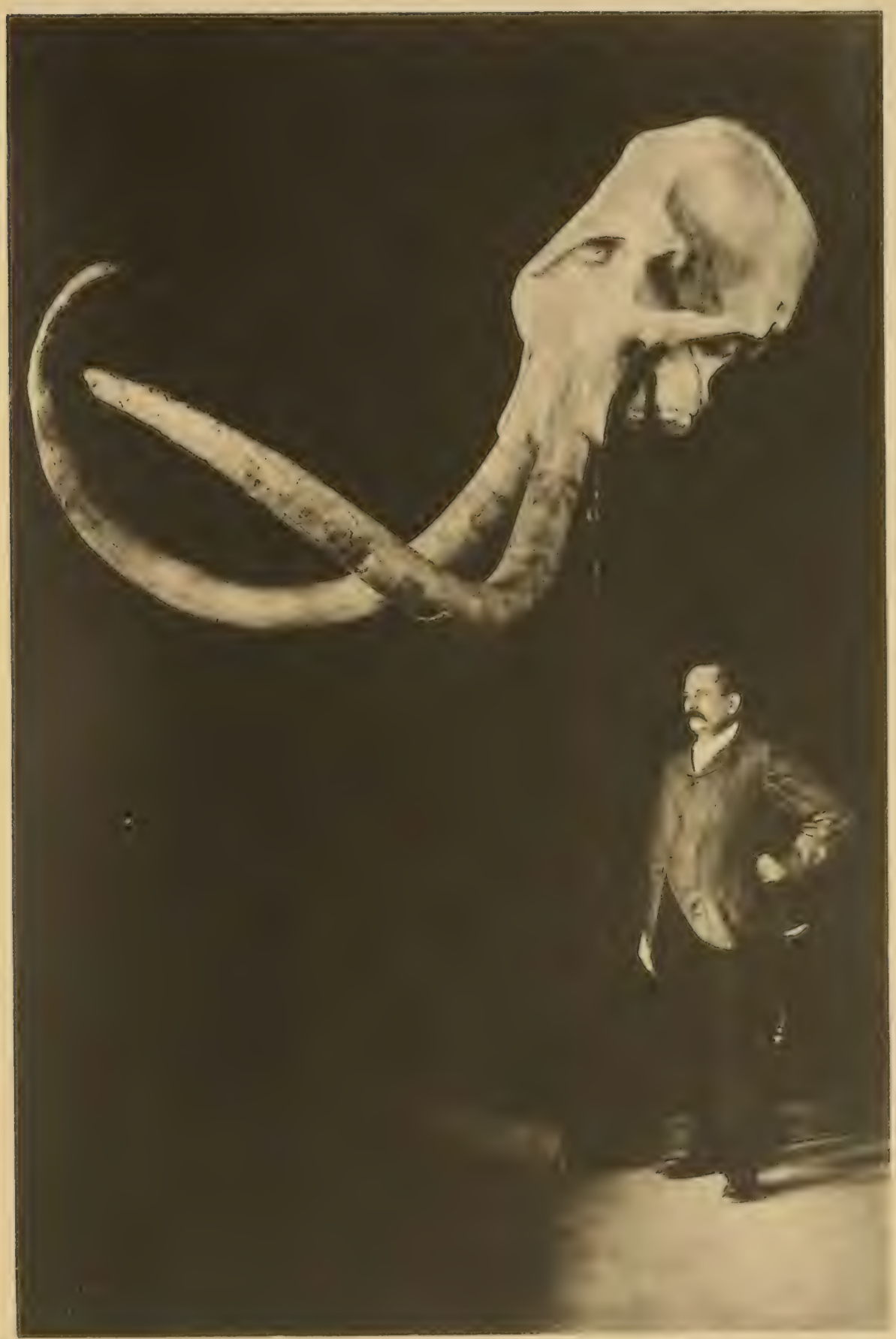

H. F. OSBORN 


EXPIANATION OF PLATE XIV

Hodel of Elephers imperator. 


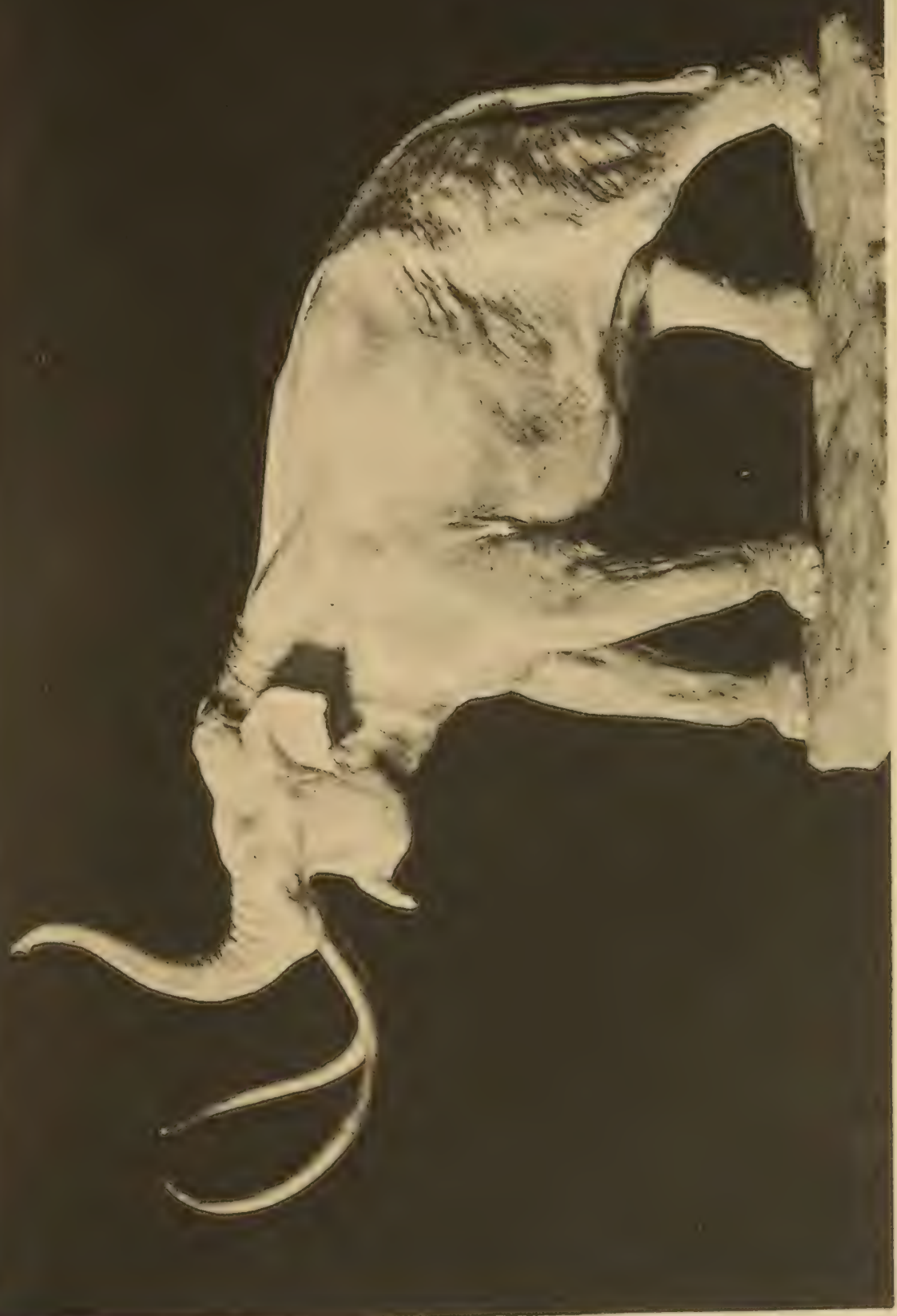

H. F. OSBORN

MAMMALIAN PALEONTOLOGY OF NORTH AMERICA 




\section{EXPIAANATION OF PJATE XV}

The newly discovered Glyptotherinm teramum, heside the skeleton of an armadillo. 


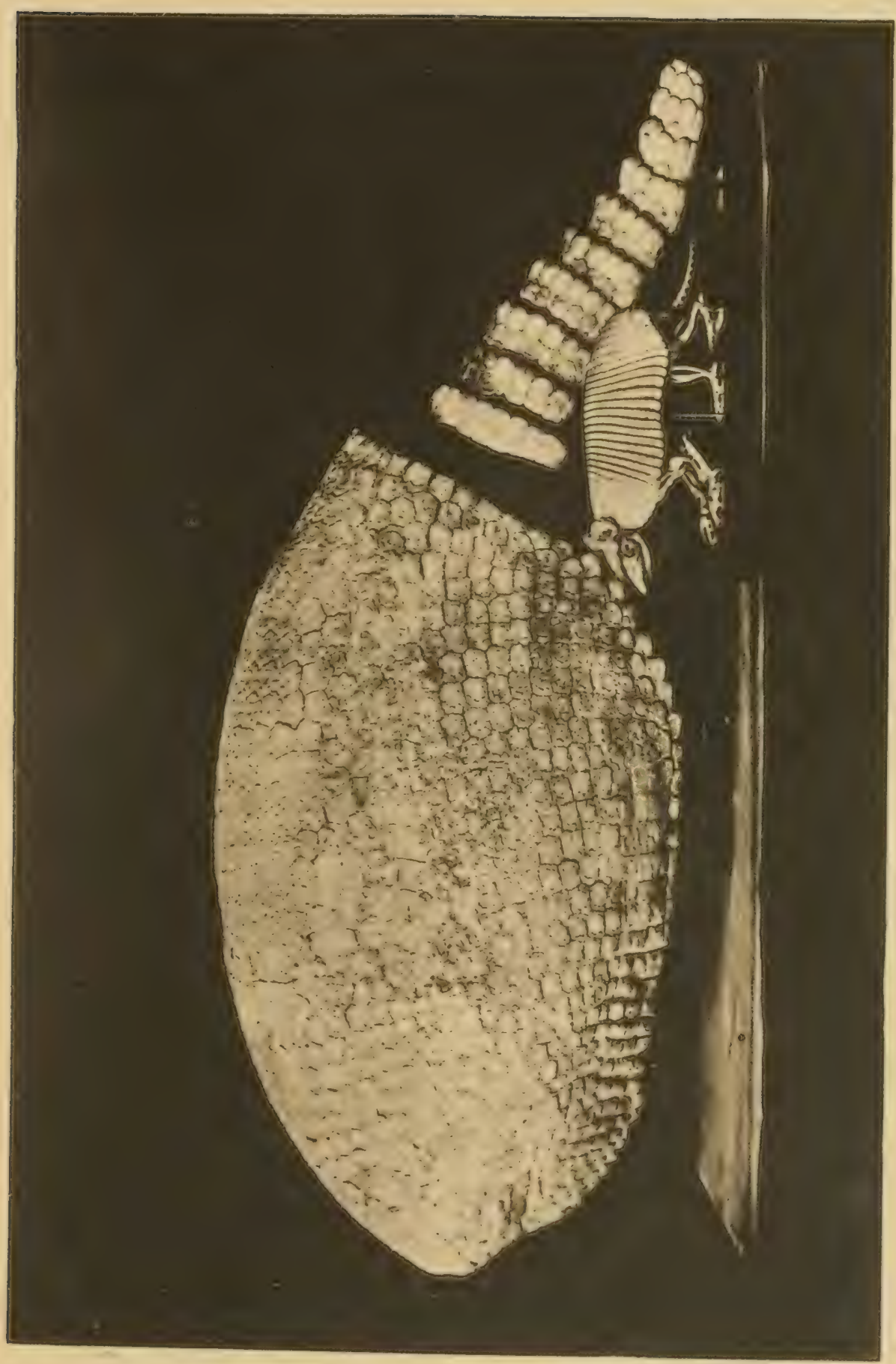

H. F. OSBORN 

The zoogeographical relations of this famma, alroady established by some: parallels with the Cernaysien of France, have been perhaps extended hy discovery of the Notostylops beds in Patagonia (Anecimso ${ }^{1}$ ). Faunal unity with the extremity of South America if confirmed will he of great significance; it appears to be probable hut perhaps not absolutely demonstraterl.

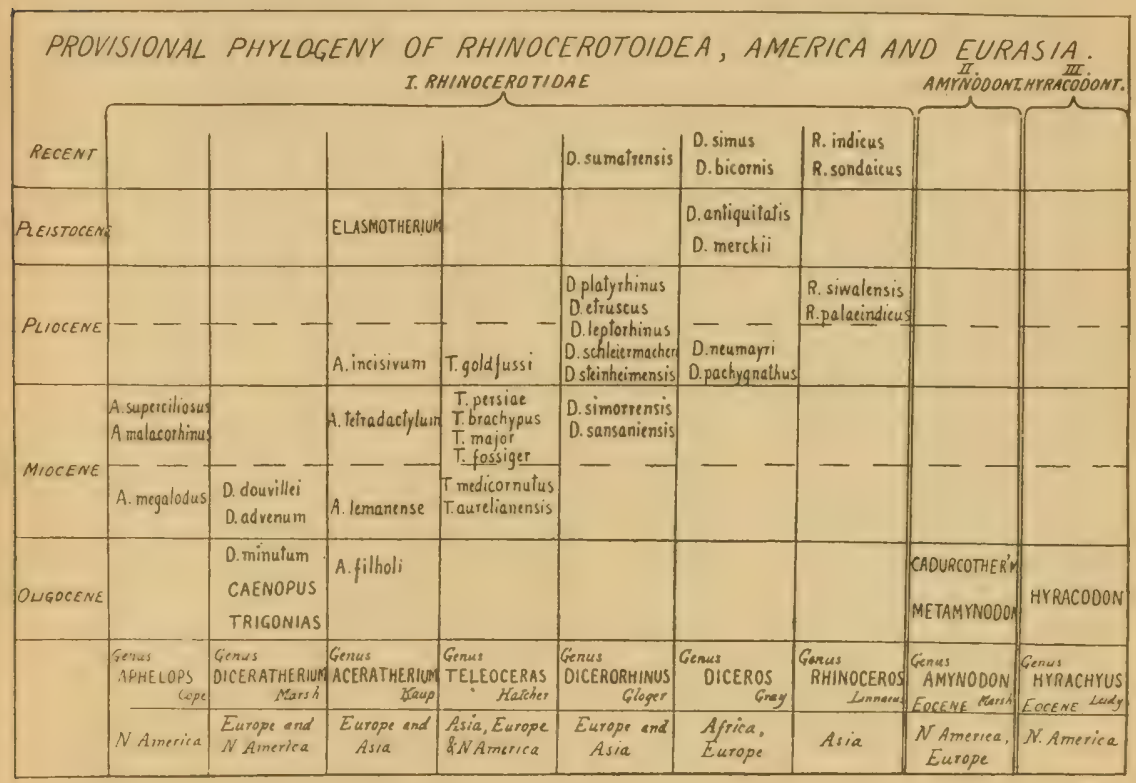

FIG. 3

The Polyphyletic Law Illustrated in the Ithinoceroses.

The Rhinocerotoidea carly divided into the Iyracodontidxe (iii), known only in America, Amynodontidx (ii), known in America and Europe, and the Rhinocerotidx (i). The last family of true rhinoceroses prove to include at least seven distinct phyla corresponding to seven genera which extend back as far as the Middle Miocene if not into the Oligocene.

The most important single phylogenetir result is the strong evidence which has been hrought forward for the ancestral relationship of the Tipniodonta ((ranodonta) of the T'orrejon to the Gravigrade Edentata ( WorTsax $\left.{ }^{2}\right)$, horne out by careful comparison of many parts of the skeletons of Psittencotherinm and allied forms with those of the gravigrade sloths. In-

1 Quadro Sinoptico de las formaciones terciarias y cretaces de la Argentina. An. Mus. Nac. d. Buenos-Aires, t. viii, Julio, 1902.

2 The Ganodonta and their Relationship to the Edentata. Bull. Amer. Mus. Nat. Hist., Vol. ix, 1897, pp. 59-110.

VIo CONGT. INT. ZOOL., 1904. 
direct proof of the early existence of Edentates in North America has come to hand in the discovery of Dasypoda in the Niddle Eocene (Osbors ${ }^{1}$ ).

Another olservation which may prove to have very broad phylogenetic brarings is the evidence of arboreal ancestry in the structure of the feet of the ('reodonta, Condylarthra and Amblypoda (MAtriew); it has not yet been ascertained whether this evidence is of the same nature as that which exists in the feet of the Marsupials (Huxley, Doli.o, Bexsiesy). With this exception attempts to bring these essentially archaic Placentals nea-

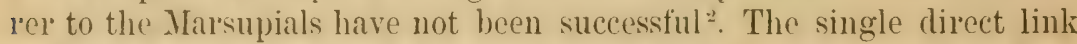
with the higher Placentals which has even been alleged to occur in these heds is the supposed Virervarus of the Torrejon. The opinion has therefore heen expressed (()sison $\left.{ }^{3}\right)$ that these animals should be sharply separated from the higher placentals and placed in the Meseutheria.

Among the minolred problems in this Basal Eocene fauna is also it: source, or ancestry, which has only in part been traced into the Cretaceous fauma. We require fuller evidence as to the relationship with the Notostylops fauma of Patagonia (AMEgniso), also a positive demonstration that the 'Teniodonta are really ancestral to the Eilentata. In other words, the phylogenetic connections of these Basal Eocene Placentals of North America and Europe are circumscribed: the sanguine view of Core that they contain the soures of the modern Placentals which first appear in the Lowel Eocene has not been realized: none of these animals give us the stem forms of the true Carnivoles, Perissodactyls or Artiodactyls of the Lower and Middle Eocene.

\section{Lower, Middie and Upper Eocene Faunas.}

The chicf geological and famal progress has been in the Bridger (Bartonien) and Uinta (Ligurien) slages, corresponding to the Middle and L pper Eocene. which have at last been eleary and sharply divided into two successive faunal stages for the Bridger (MAtruew, Granger), and two sucessive faumal stages for the Uinta (Petrerson, Osibors). The importance of these divisions in the evolution of the Primates, Carnivores and Perissodactyls can hardly be over-estimated.

At the sane time the zoogengraphical relationships of our Lower Eocene (Soissonion) have heen extended by the discovery of a French Creodont (Pularonictis) in Anerica and of an American Creodont (Puchyena) in

1 An Armadillo from the Middle Eocene (Bridger) of North America. Bull. Amer. Mus. Nat. Hist., Vol. xx, 1904, pp. 163-165.

${ }^{2}$ Wortman. Studies of Eocene Mammalia in the Marsh Collection, Part I, Carnivora. Amer. Jour. Sei., Vols. xi-xiv, 1901, 1902.

s A Division of the Eutherian Mammals. Trans. N. Y. Acad. Sci., June 4, 1894, p. 234 . 
France. Still more surplrising and important is the discovery ${ }^{1}$ in the Middle Eocene of I)asypoda (Metecheiromys), armadillos with canine treth and with provision for a stout leathery if not osseous ('arapace. This absolutely establishes the Cretaceous if not Basal Eocene zoogeographical relations of North and South America, and arlds another fact to the elowing evidenee that North and South America were related in the Mid Cretaceous and perhaps Early Tertiary and then separated again until the Pliocene.

Our phylogenetic results have heen most encouraging in some directions and most baffling in others. Still mole striking than ever before is the fact that the Lower and Virlde Eneene fauna of Perissodactyla, Altiodartyla, Carnivora, C'heiroptera. Inukers, and true Rodents, an esssentially moder'n fauna, is without any known direct affiliation with the basal Eocene fauna (Meseutheria) (Compare Fig. 2). Mingled with this essentially modern fiuma are the numerous survivors of the al'chaic fanna, namoly. the Creodonta. Condylarthra. Amblypoda, with which should certainly be reckoned the Edentata (Paratheria. Thomas) and mobably the Insectivol'a.

The phylogenetic sucessions of the families within these archaic orders have been much more clearly traced, namely, the pedigres and adaptive radiation of the Creodonts into specializations of various kinds * Among the Amblypoda the law of long-skulled and short-skiulled phyla has again been found to prevail, in proof that the genera ahout which there was such a heated diseussion, namely, Tinoceros as a relatively short-skulled form and Lorolophodon as a relatively long-skulled form. leally lepresent two valid and distinct phyla.

Among the modernized Placentals, we have added nothing to our linowledge of the supposed Cheiroptrat An important step is the ploposed transfer to the Insectivora of the genus Hyopsodus which has long figured among the Primates (Wortas:"), a relationship which will be settled by material now in our possession. Among the remaining undoubted Primates (Osboris ") there is the series of Anaptomolphiclie which still resmble the Tarsider mole than any of the other lemurs, or true monliess, although their actual relationships are absolutely undeterminol. The second family of Primates, represented by the Notharetidie ( No-

${ }^{1}$ Osborn, H. F. An Armadillo from the Middle Eocene (Bridger) of North America. Bull. Amer. Mus. Nat. Hist., Vol. xx, 1904, pp. 163-165.

2 Matruew, W. D. Additional Observations on the Creodonta. Bull. Amer. Mus. Nat. Hist., Vol. xiv, 1901, pp. 1-38.

${ }^{3}$ Studies of Eocene Mlammalia. Part II, Amer. Jour. Sci., Vol. xv, May 1903, p. 401.

- American Eocene Primates, etc. Bull. Amer. Mus. Nat. Hist., Vol. xvi, 1902, pp. 169-214. 
thurtus and Limmotherium) and other forms, has been placed near the South Amorican Cobidie by Wortase ${ }^{3}$, but this also requires the confirmation ol disproof which will soon be forthcoming; if South American

\begin{tabular}{|c|c|c|c|c|c|c|}
\hline Contermp & $\begin{array}{l}\angle A W \\
\text { sorary Evolut }\end{array}$ & $\begin{array}{l}O F \angle O C A \\
\text { on of } 4-5\end{array}$ & $\begin{array}{l}4 \angle A D A P \\
\text { Phyla of }\end{array}$ & $\begin{array}{l}\text { TIVE RAD } \\
\text { Horses, O }\end{array}$ & $\begin{array}{l}\text { MATION } \\
\text { ligocene to }\end{array}$ & $\begin{array}{l}\text { Pleislocene. } \\
\text { W. Gidley, 1904. }\end{array}$ \\
\hline Pleistorene & $\begin{array}{l}\text { 1. Side phylum } \\
\text { relaled to } 2 \text {. }\end{array}$ & $\begin{array}{l}\text { 2. Hipparrom } \\
\text { phylum }\end{array}$ & $\frac{\substack{\text { Bolyylum } \\
\text { Equus }}}{\text { Equus }}$ & $\begin{array}{c}4 \text { Pliohipous } \\
\text { phylum }\end{array}$ & $\begin{array}{l}5 \text { Hypohipous } \\
\text { phylum" }\end{array}$ & $\begin{array}{l}\text { 6. Side phylum } \\
\text { related to s }\end{array}$ \\
\hline Pliocene & & Hipparion & & & & \\
\hline Miocerre & $\begin{array}{l}--- \\
---\end{array}$ & $\begin{array}{l}\text { Neahipparion } \\
\text { Merychippus } \\
\frac{\text { iosignis }}{\text { Parahippus }} \\
\text { brexideus }\end{array}$ & 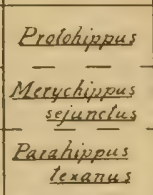 & $\begin{array}{c}\text { Plochupsess } \\
\frac{-}{\text { Merychippus }} \\
-\frac{\text { mirabilis }}{-}- \\
\frac{\text { Parahipous }}{\text { spe }}\end{array}$ & $\begin{array}{l}\text { Hypohippus } \\
\text { daholenses } \\
\text { Bypohippus } \\
- \text { equinus }\end{array}$ & -- \\
\hline \multirow[t]{2}{*}{ Oligocene } & $\begin{array}{l}\text { Miohippus gidlex } \\
\text { M. annes/ons } \\
\text { M. crassicuspis }\end{array}$ & -- & $\begin{array}{l}\text { Mesohippus } \\
\text { validus } \\
\text { M. brachystylus } \\
\text { Mesohippus } \\
\text { bairdi } \\
\text { Mesohippus } \\
\text { montanensi }\end{array}$ & $\begin{array}{c}- \\
\text { Mesohippus } \\
--\end{array}$ & $\begin{array}{l}\text { Mesohippus } \\
\text { obliquidens } \\
-\end{array}$ & $\begin{array}{l}\text { Mesohippos } \\
\text { ineloulophus } \\
\text { Mesohippus } \\
\text { eulophus } \\
\text { Mesohippus } \\
\text { proteulophus }\end{array}$ \\
\hline & \multicolumn{2}{|c|}{$\begin{array}{l}\text { Cusps separated. } \\
\text { Protocone becomes isolated. } \\
\text { Slyles prominent }\end{array}$} & \multicolumn{4}{|c|}{$\begin{array}{l}\text { Ectoloph \& styles flattened, } \\
\text { cusps united into perfect crests } \\
\text { lite those in Tapirus \$ Thinorress }\end{array}$} \\
\hline
\end{tabular}

FIG. 4

The Polyphyletic Law Illustrated in the Evolution of the Equidx of North America.

In the Miocene are at least four contemporary phyla of horses, the extremes being represented by the forest-living horse IIypohippus, and the light-limbed Neohipparion; the central form, Protohippus, apparently leading to Equus.

This true Equus line separated off from other horses as far back as the base of the Oligocene. In this period again five distinct contemporary phyla have already been found.

lelationships are established for these Primates, a very much mooted problem will be solved.

Among the Perissodactyla the Titanotheres (Osbors ${ }^{2}$ ) have split up into four phyla, one of which (Pulwosyops) died out, while the three remaining phyla independently acquired rudimentary holns (Telmatotherimm, Munteoceras. Dotichorhimus) and apparently gave rise to the evo-

1 Wortanan. Op. eit., Amer. Jour. Sci. Vol. XV, 1903, pp. 409-411.

2 The Four Phyla of Oligocene Titanotheres. Bull. Amer. Mus. Nat. Hist., Vol. xvi, 1902, pp. 91-109. 
lution of the four phyla of great Oligocene titanotheres. Among the Artiodactyla the rare Middle Eocene forms still require elucislation, but the Camelide have been traced definitely into the diminutive Ipper Eocene (Uinta) Protylopus (Scot'T ${ }^{\prime}$, Wortana ${ }^{*}$ ). Two distinct phyla of ()reorlontidar have also been traced back in the Lpper Eocene into the genera Protagrichorus and Protoreodon (SorrT). Among the enemies of these animals, the Canidie have been traced into the Upper Focene genera P. rodryphemus and Uintacyon, and Marsu's Middle Eocene Vulprovers has also proved to be a member of the true Canida, although, its relationships are not exactly determined (Wortuas, Matrowe. The supposed ancestry ( Wortus ) of the Felidie in the Eocene in the problematical genus Elurotherium has been disproved (Matтнеw).

Still undiseovered ol unrecognized in the Eocene hoth of Americal and Furasia are the ancestor's of the true Rhinocerotidar which suddenly appear in the Oligocene. 'The Ibasal Focene ancestry of the Rodentia is still satisfied only provisionally by the family Mixodectider, belonging to the somewhat hypothetical Proglires ( $)$ sisore $\left.{ }^{3}\right)$; the treth seem to be approarhing those of the Rodents hut knowledge of the skeleton is necessary to determine whether they may not after all be remotely related to the Lemurs (order Cheiromyoidea) as Cope and Wortaran have suggested. Won'mis is strongly of the opinion that the Eocene Primates (Notharetida', Anaptomorphidar) are not Lemuroidea, and that the former family are elistinctly South American; this also requires confirmation.

Search for the exact relations and points of connection between the Carnivora and Creorlonta, has thus far been entirely without definite success; in other words, the true Carnivora seem to be as separate from the Creodonta as the true Perissodactyla are from the Condylarthra.

As regards the Artiorlactyla, as yet very little is linown of the Midrle and Lower Focene stages, among which it is especially important to test the truth of S'otr's 'broad generalization that the American Artiodact yla should all be resareded as atfiliated to the Tylopola as a stem group from which not only the Camelide evolved hut also the othel distinctively American Artiolactyls, such as the Orendontida, and that "ven the tritguloid forms are of tylopodous affinity and morely parallel or andogous to the true Tragulines of Furavia. There is no doubt that such an adaptive radiation from a Tylopod stem is possible and that there is conside-

1 The Selenodont Artiodactyls of the Uinta Eocene. Trans. Wagner Free Inst. Sci. Phil., vi, 1899 , p. 100.

2 The Extinct Camelidx. Bull. Amer. Mus. Nat. Hist., Vol. x, 1893, pp. 93-142.

$s$ Bull. Amer. Mus. Nat. Hist., XVI, 1902.

4 The Selenodont Artiodactyles of the Uinta Eocene. Trans. Wagner Free Inst. Sci. Phil., vi, 1899, p. 100. 
rable actual evidence for it in the morphology of the skull of these various distinctively American Artiodactyls; but the hypothesis is such a bold one that we must wait for more material.

The chief problem of all, which is also the problem of the European

\begin{tabular}{|c|c|c|}
\hline Provision & $\begin{array}{c}\text { al Phylogeny of } C_{a}^{\prime} \\
\text { Wortman, } 1898\end{array}$ & $\begin{array}{c}\text { melidae, Old \& New World } \\
\text { Matthew, } 1904\end{array}$ \\
\hline Recent & Aucheria & Camelus \\
\hline Pleistocene & Camelus Camelops Auchenia & Camelus Camelops Auchenia \\
\hline Pliocene & Plia & Camelus Pliauchenia Auchenia \\
\hline Miocene & $\begin{array}{l}\text { Procamelus } \\
\text { Miolabis } \\
\text { Paratylopus }\end{array}$ & 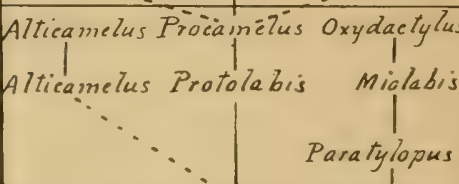 \\
\hline Oligocene & Poëbrotire & $\begin{array}{c}\text { Pseudolabis Protomeryx Paratylopus } \\
\text { Poëbrotherium Paratylopus } \\
\text { Poibrotherium }\end{array}$ \\
\hline Eocene & $\begin{array}{c}\text { Leptotragulus Protylopus } \\
\text { ? Ḧoniaciodon } \\
\text { : Pantolestes }\end{array}$ & 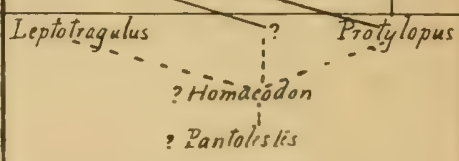 \\
\hline
\end{tabular}

FIG. 5

The Polyphyletic Law and Local Adaptive Radiation Illustratcd in the Phylogeny of the Camels.

On the left is illustrated the older monophyletic view held as recently as 1898 ; on the right, the newer polyphyletic view developed in 190 ' showing three distinct contempovary lines of Camelidx.

palirontologists, is the source and origin of the modern Lower Eocene fauna as a whole, namely, the Carnivora. Perissodactyla, Artiodactyla, Primates, and Rodentia.

\section{American Oligocene Faunas.}

()ur ()ligocene (Lower Oligocene Infra-Tongrien, Niddle Stampien, and ('pper Aquitanien, of Europe) has been the most thoroughly explored of any of the periods, owing to the richness of its fossil fauna. 
The chief geological result is the separation of the fluviatile or channel beds, with ehiefly lowland or hottom fauna, from arolian or hackwater sediments, chiefy with a plains and cursorial fauna. The three subdivisions originally observed by Hornex and Leny are thus divided ats follows :

I. Fluviatile or Channel Beds. II. Eolian or Backwater Sediments. Upper, Protoceras beds......... Leptauchenia beds.

Middle, Metamynodon beds....... Oreodon beds.

Lower, 'Titanotherium beds.

'This separation was chicfly brought ahout hy Matruew's careful analysis of the animals coming from these lespective heds, the former (I) including lowland, forest and rivel-lottom, and aquatic animals, the latter (II) the animals of the plains and uplands. The John Day berls of ()regon apparently contain an overlapping fauna partly equivalent to the Upper Oligocene and partly to the Lower Miocene.

The already well known (C'ope, Finou) and close zoogengraphical rolationships during the ()ligocene of North America and Europe are strengthened by the discovery of European Anthracotheriidar. Mustelide (Bu-

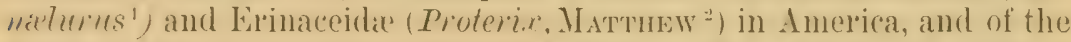
American Titanotheriide in Europe". This leaves as the chief families in Europe still unknown in Anerica the Palatheriida, Anoplotheriidae, Tragulidie.

Our faunal knowledge has heen especially enriched by the diseovery and description of the hitherto unknown microfiuna of the Titanotherium

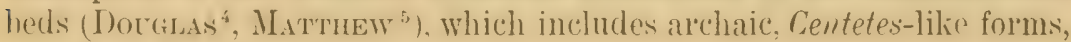
as well as Erinacens-like forms.

The main phylogenetic results are the following. The Crendonta have leen definitely traced to their extinction in the Hranodontida (Table II). fmong the Canide the ancestral line of ('yom (I) noLes) has almost cel'tainly been recognizerl in this period in the genus Temmocyon (Wortas and Matriew ${ }^{\text {) }}$ ( Fig. (i). No trace of Edentata has been found, the forms formery described as such now leing linown to be the peculiar Chalico-

1 Matmiew, W. D. On the Skull of Bunælurus. Bull. Amer. Mus. Nat. Hist, xii, 1902, pp. 137-140.

" A Fossil Hedgehog from the American Oligocene. Bull. Amer. Mus. Nat. Hist., Vol. xix, 1903, pp. 227-229.

s Tovta. Ueber neue Wirbelthierreste aus dem Tertiär CEsterreichs und Rumeliens. Zeitschr. d. Deutsch. geolog. Ges., Jahrg. 1896, pp. 922-924.

4 Foss. Mamm. White River. Trans. Amer. Philos. Soc., n. s., Vol. xx, 1901, p. 1-42.

5 The Fauna of the Titanotherium beds. Bull. Amer. Mus. Nat. Hist., Vol. xix, 1903, pp. 197-226.

${ }^{6}$ Bull. Amer. Mus. Nat. Hist. Vol. XII, 1899, pp). 139-148. 


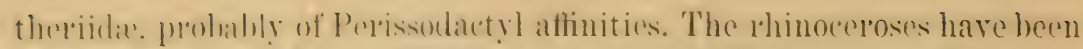
trand batek in the Iower ()ligocene to animals (Trigmemes with several incisol's as well as with canine teeth (Osmons ${ }^{1}$, LuCas $\left.^{2}\right)$.

The law of local aldiptive ladiation with its polyphyletic consequences

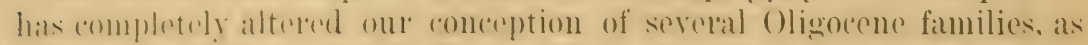

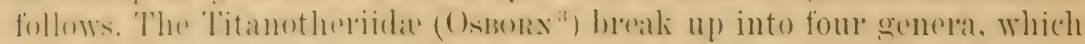
crolve independently from the base to the summit of the () lisgoene. namely.

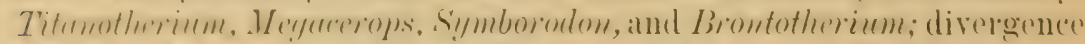
is indicated hy dolichorephaly and hach yeephaly ats well as hy othere eharace-

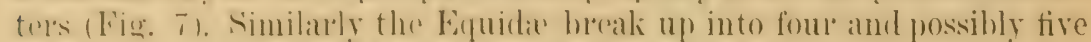

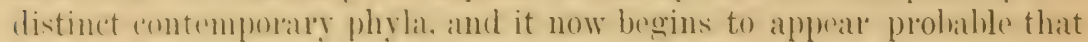
the line giving rise to Eques, separated off from the other horses as cally as the Lowel Oligocene (Osbors, Giduer; Fig. 4). The Oreodontida, represented by two phyla in the Upper Eocene, now present three

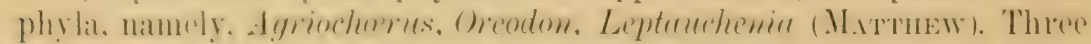
phyla of Camelide are also recognized, namely, those represented by Paratylopus, Poëbrotherium, and Pseudolabis (Matruew Fig. 5). Similarly among the Feldier. the Macharodont division, the only folines represented in Americal at this time. hreaks up into the stont-limbed Ho-

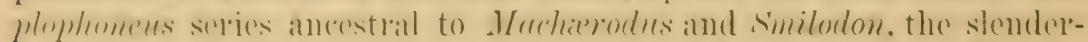
limbed Dinictis ${ }^{4}$, and a third series represented by Nimrams (Fig. 6).

Among the gaps in the Oligocene are the entire absence of Primates,

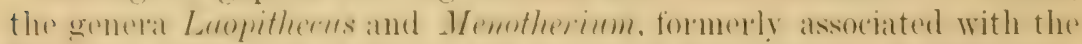

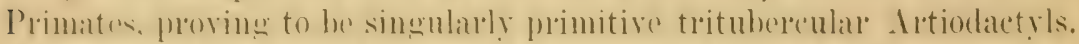

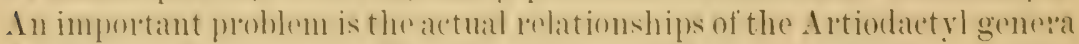

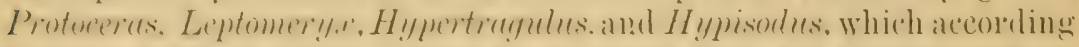

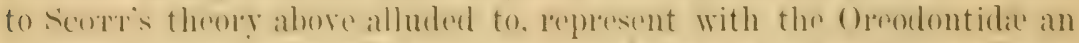

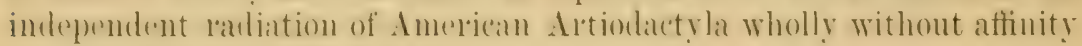
with the European 'Tragulines.

\section{THe Miocene Haund.}

In our Miocene, equivalent to the Langhien (Orléanais), Helvétien (s:ansin. simorre), and Tortonien ((triwe st. Alhan, Bamboli) stages of

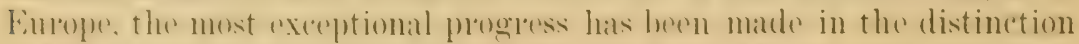

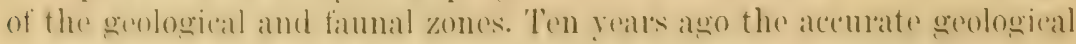
observations of HAYDen were overlooked, and it was believed that formations equivalent to the Middle and Lower Miocene of Europe were

1 The Extinct Rhinoceroses. Nem. Amer. Mus. Nat. Hist., Vol. I, 1898, pp. 75-165.

2 A Nex Rhinoceros, Trigonias Osbomi. Proc. U. S. Nat. Mus. xxiii, No. 1207.

s Bull. Amer. Mus, Nat. Hist. XVI, 1902, pp. 91-109.

+ Matriew. Fiossil Mammals of the Tertiary of Northeastern Colorado. Mem. Amer. MLus. Nat. I1ist., Vol. I. I't. vi. 1901. 


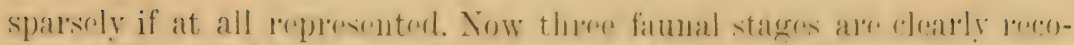

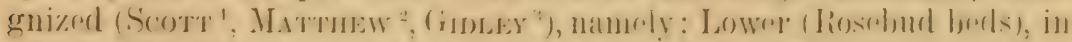

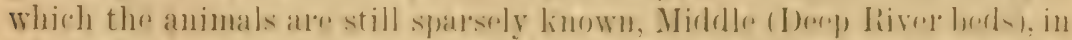

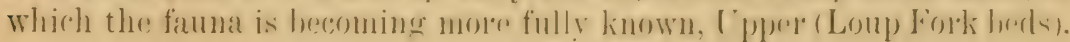

\section{PROVISIONAL PHYLOGENY OF CARNIVORA, OLO \& NEW WORLO.}

WDMatthew. 1904

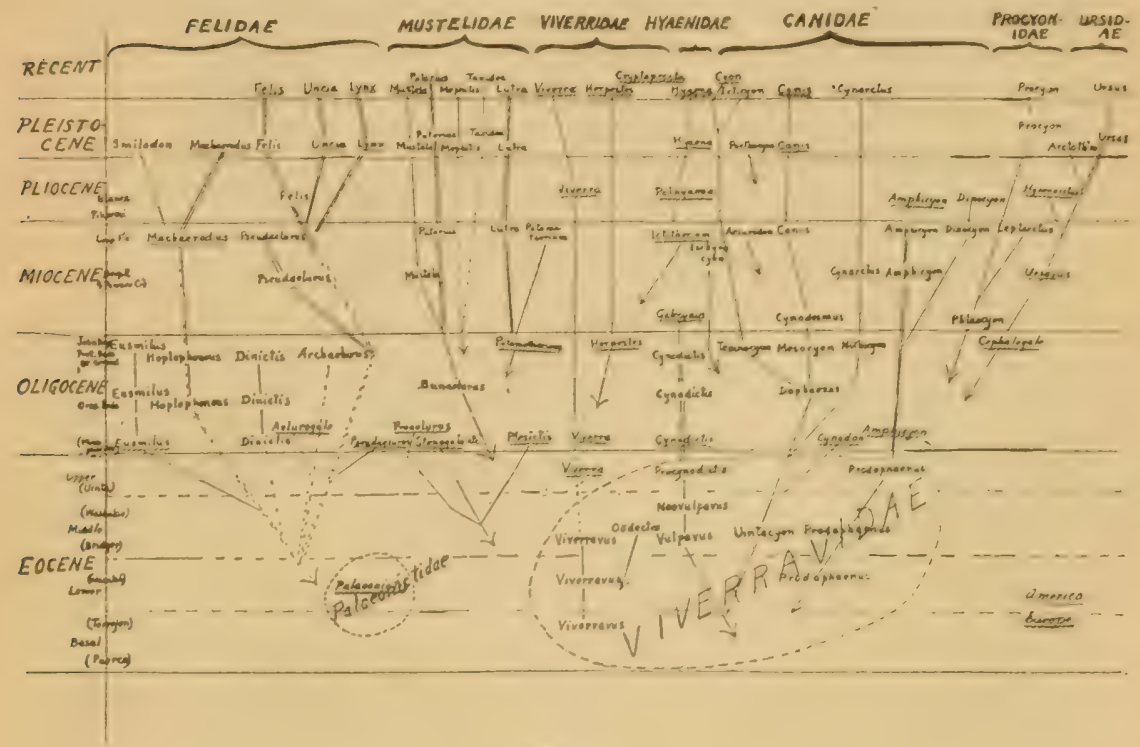

Fis. 6

The hypothetical phylogeny of the Carnivora illustrating especially the great antiquity of some of the modern genera of dogs, such as Cyon, which separated ofl from the other Canidse in the base of the Oligocene if not in the Eocene. This table includes also the European Carnivora and is of a temporary value. I'alseonictis, although a Cireodont may possibly be related to the Felidie.

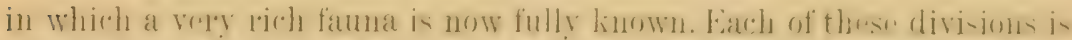

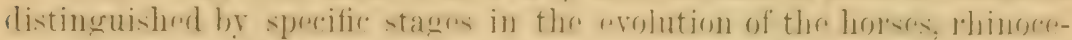
roses, camels, oreodonts, rodents, and carnivoles. These chronological

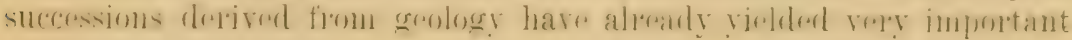
new biological results.

${ }^{1}$ The Mammalia of the Deep River Beds. I'rans. Amer. Philos. Soc., xviii, 1895, pp. $55-185$.

"Foss. Mamm. of the Tertiary, etc. Mem. A. M. N. H. Vol. I, 1901.

3 New or Little Known MLammals from the Miocene. Bull. Amer. Mus. Nat. Ilist.. $\mathrm{xx}, 1904, \mathrm{pp} .241$. 
The zoogeoglaphical relationships with Europe have been strengthened by the discovery fol the first time of Dinocyon (MATTHEW ${ }^{1}$ ), of a new surecies of rhinoceros (Teleoceras bicomutus" () sborn), closely similar to the Teleoceras anvelianensis of the Lower Miocene of France, by the reeognition of new Mustelidie (Lutra), and of the Castoridie (Dipointes). The Proboscidea, now known to be of African origin, are not certainly found in the lower and sparsely known in the middle, but are fully represented in the upper beds. In the middle beds appears Mustodon productus, lather derivable from the Paldomastodon of Africa than from the $M$. angustidens of France.

()u views as to the Miocene climate have also undergone a change, owing to the recognition that most of these deposits are fluviatile and aeolian lather than lacustrine (Matruew, Gind $\mathrm{EY}^{3}$ ), as evidence of a dry climate, mashy plains, and drifting sands, rather than of the moister climatic conditions inferred from the older lake basin theory.

Among the chief phylogenetic results are the addition of at least four kinds of Canids (Fig. (i) and the tracing hack of the Procyonidie to the Lower Niocene I'hlaocyon (MATTHEw ${ }^{\circ}$ ), tending to unite this phylum more closely with the Canidax. The Mustelidae are now represented by Mustele and Lutra. The Viverridie and Urside are still wholly unrepresented in America although evolving contemporaneously in Europe. Among the distinctively American Artiodatyls the Cervidix are now recorded in the Middle Miocene (Palaomeryx), a fact however still requiring confirmation. In this connection should be mentioned the discovery of the full ('haracters of the genus Merycodus (Cosoryx), which with Blastomeryx as the new family Merycodontida has been regarded by MATTuew to be more nearly related to the American Antilocapridie than to the Furopean Cervidar. although its deerlike horms certainly suggest Cervine relationships (Plate VI). The Camelide until recently considered monophyletic have been shown to be in a marked degree polyphyletic $c^{\prime}$, the Lower ()ligocene Paratylopus giving rise to two phyla, one of which in-

${ }^{1}$ A Shull of Dinocyon from the Miocene of T'exas. Bull. Amer. Nus. Nat. Hist., Vol. xvi, 1902, pp. 129-136.

2 New Miocene Rhinoceroses. Bull. Amer. Mus. Nat. Hist., Vol. xx, 1904, pp. 307326.

3 New or Little Known Mammals from the Miocene of South Dakota. Bull. Amer. Mus. Nat. Hist., Vol. xx, 1904, pp. 241-268.

- Foss. MTamm. of the Tertiary, etc. Mem. A. M. N. H. Vol. I, 1901.

'A Complete Skeleton of Merycodus. Bull. Amer. Mus. Nat. Hist., Vol. xx, 1904, pp. 101-129.

' Notice of two New Oligocene Camels. Bull. Amer. Mus. Nat. Hist., Vol. xvi, 1902, pl). 617-635. 
cludes the "giraffo (amel ". Alticamelus (MATruww", which presents a lemarkable analogy in the elongation of its nock and limbs with the giraffes of Africa; similarly Poibrotherium splits into three plyla (Fig. 5. Matthew). Similarly the Oreodont, and Agrichorine pliyla have disappeared without leaving sucessors. The rival cursorial Hylacodontide and aguatic Amynodontidie having died out. the true Rihinocerotidae (Fig. 3) split up into three series, one inchuding the extremoly long-skulled and long-limbed types, possibly related to the true Aceratherium incisivum of Europe, a second including excessively broadskulled types (genera Aphetops and Peruceras (ope), and a third including the short-fonted (brachypodine) types (Teleoceras), almost rertainly ef European origin. 'The 'Tapiridre are still sparsely known. The aherant C'halicotherida terminate in an Lpper Jincene species which nearly equals in sime the Lower Pliocene Ancylotherium of the Pikermi. The most astonishing discovery among the Rodentia is that of a member of the Mylagaulidae with a very large hol'n role on the firont portion of the skull (genus Ceratogaulus Matthew ${ }^{2}$ ).

The principal work still to be done in our. Mincene is the following : to ascertain more fully the character of the Lower Niocene fauna, which is still unkmown: to tix the date of the arriyal of the arliest Proboscidea either early in thr Middle or in the Lower Mioceno: to trace the ancestry of the typical dogs; to ascertain the origin of the Cervidre, which will probably prove to be Asiatic, as well as the origin of the peculiarly American Antilocapridæ.

\section{The Pliocene Fauna.}

Equivalent to Messinien (Piliermi), Plaisancien (Casino), Astien ( Ponsillon), Sicilien (Val d'Arno sup.).

()ur limited dmerican Pliocene fauma still stands in sarl contrast to the rich succession of Pliocene mammals of Europe. The Palo I)uro mammals which Cope included in the Pliocene have proved to he I'pper Viocene. Recent grological and palanntological work ( (iDd.EY ${ }^{3}$ ) shows that the only true Pliocene formation and locality is that of the Blanco beds of Texas, 7i) feet in thickness as against the rich sucessive Pliocene series of Furope. Nor are any sprecies of Erums found here, as Cope supposed, and as might be expected from the presence of Equms (E. stemonis) in the Tpper Plioene of Europe. The chief faunal distinctions are the entire dis-

1 Foss. Mamm. of the Tertiary, etc. Mem. A. M. N. H. Vol. I, pt. VI, 1901.

2 A Horned Rodent. Bull. Amer. Mus. Nat. Hist., Vol. xvi, 1902, pp. 291-310.

$s$ The Freshwater Tertiary of Northwestern Texas. Bull. Amer. Mus. Nat. Hist., Vol. xix, 1903, pp. 617-635. 
apperatance of the Ihinocerotide and the appearance of South American Mammals.

The zongengraphical changes are well known to enter a new relation by the invasion of the South American Edentata, namely, Glyptodon, IErgulon!y, Mylodon. Among these a new Glyptodont. Cilyptotherimm.

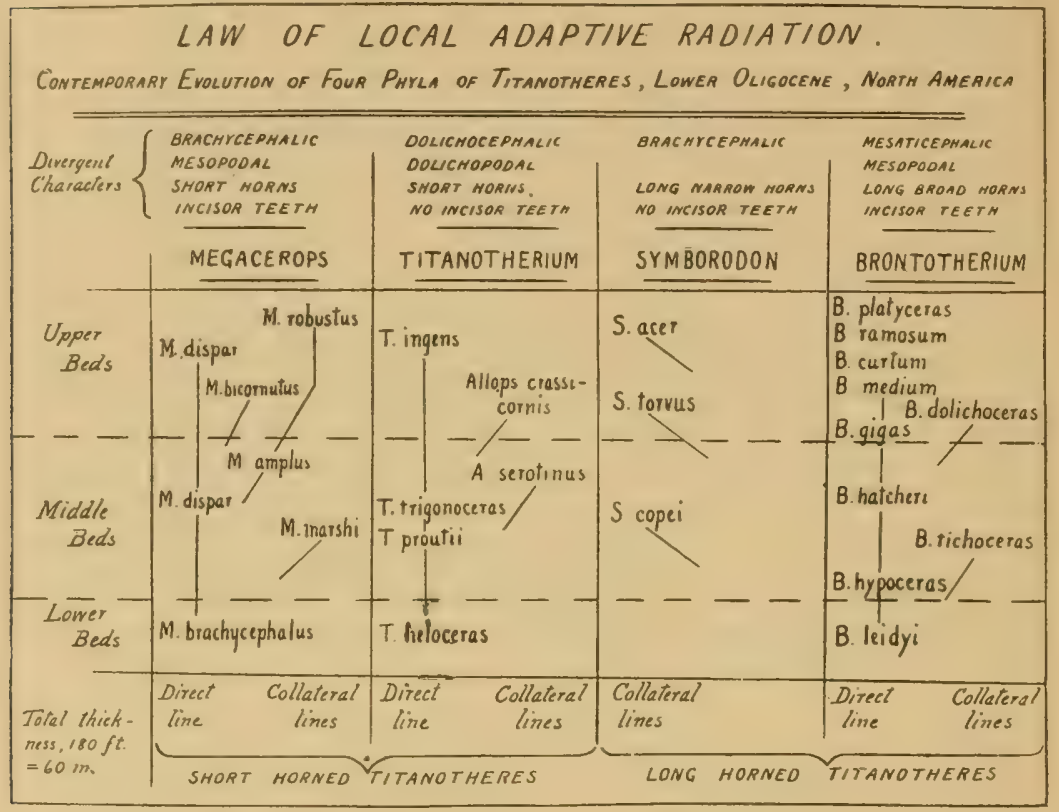

FIG. 7

The law of local adaptive radiation illustrated in the four phyla of Oligocene Titanothercs, one or more of which gase off collateral branches.

Three of these phyla have now been shown to have a separate origin in the Middle Eocene.

teacemm has recently become lnown (Osisons ${ }^{1}$ ) from a nearly complete carapace and partial skeleton, which exhibils primitive aftinities with the Eocene types of Patagonia. Among the Proboscidea the Stegodont stage apperars in the so-called Mustodon mirifiens of LEnD, indicating a late Plioerne age for the Blaneo formation. In the marine Viocene of Japan (Iwasalii and Yoshiwara ${ }^{2}$ ) the lemarkahle discovery has been made of an

1 Glyptotherium texanum. Bull. Amer. Mus. Nat. Hist., xix, 1903, pp. 491-494.

2 Notes on a New Fossil Mammal. Jour. Coll. of Sci. Imp. Univ. Tokjo, Vol. xvi, Art. 5, 1902. 
anomalous skull repuresenting a new fanty (Desmostylide fam. nov.) cither of hypsorlont Sirenia of of Prohoscidea, and Merrand has reeognized as a similar form occurring on the coast of California the genus Desmostylus first noticed by Marsir.

'The phylogenetic series is all too linited, the horses being suarsely re-

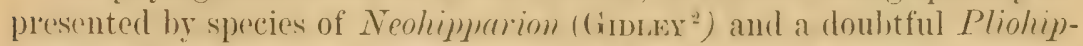
mus, the Camelide hy Plinnchenia, the Dicotylidie hy several spexeses of Platygomus, the Carnivola by an Amphicyon and other doubtful speceses of Canidar. The collateral lines of Camelide, so far as we linow, died out, and the adaptive radiation of the true camels begins.

However, no wencralisations can as vot he made from this seanty fauna; we are confionted with morer gilps in our knowleder and mole unsolved problems than in any other period. Among these, the direct ancestry of the South American cameloids (Aucheria) as well as of the trur camels (Camelus) should be found. We also should find here the stages directly ancestral to the horse (Eqmus), because it now appears certain that Marsu: Pliohipums was an [uper Miocene and not a Pliocene anima], and was, moreover, apparently on a side line not leading directly into Eqquus (Grdery, Fig. 4). Thus not only is the Pliocene plains fauna sparsely known but the Pliocene forest fauna is wholly unkmown.

\section{The Pufistocene Fauna.}

Equivalent to (1) the Preglacial, Forest Beds of Nolfolle (St. Prest, I)urfort. Malhattu. Peyrolles), (22) (ilacial, (Mirl-Ploistoene, Lower Mirl-Pleistocene), (3) Postglacial deposits of Norther'n Europe and Asia.

Here again American palieontology is far hehind that of Europe as to knowledge of thr chenological succession of deposits, and a vast amount of work remains to he done in the discrimination of geological and fatunal stages, in the comparison of Fisternand Western cave - and sand deposits, and in the coordination of the first appearance of man with that of the inammalian succession.

The advent of the true Equus marks the base of our Pleistocene, as shown in the sand deposits of thr Western plains in the so called Eiques beds. The geographical distribution and remarkable adaptive variation of the Pleistocene hol'ses has now hern fully worked out (GIDLEx ${ }^{3}$ ), ploving that there are ten sperese characteristic of different localitios, and ranging in size from E. gigcutens, larger than any molern horse. to the

1 Science, n. S., Vol. xvi, Oct. 31, 1902, p. 714.

${ }^{2}$ A New Three-toed Horse. Bull. Amer. Mus. Nat. Hist., Vol. xix, 1903, pp. $465-476$.

$s$ Tooth Characters and Revision of the Genus Equus. Bull. Amer. Mus. Nat. Hist., Vol. iv, 1901, pp. 91-142. 
diminutive E. montezuma. But nowhere in North America have horses been found contemporaneous with man.

Two chicf advances have been made, first, the distinction of plains and river, from forest fiunas; second, the exploration of two very remarkable cave deposits.

The Western plains fauna of the Equus beds or Lower Pleistocene (MATTuew ${ }^{-1}$ ) contains among the Carnivora, Canis, Dinocyon, Felis; among the Rodentia, Fiber, Arvicola, Cynomys, Thomomis, Castoroides; among the Edentata, Mylodon; among the Perissodactyla, three species of Equns; among the Artiodactyla, two speeies of the Dicotylide; one species of the Camelidix, and two of the Antilocapridie (Capromeryx, a new form, Matrinew), and Antilocamra; anong the Proboscidea, Elephas columbi. A similar plains fauna is that of Silver Lake, Oregon, which includes also two aquatic animals, Custor und Lutro. At Washtuckna Lake, Washington, is found a forest fauna which includes in addition to camels and horses, a badger, Tuxidea, three species of Felis, two of Alces, one of the American deer, Curiacus, and one of the goat ()reamnos (Haploceros).

Our knowledge of the Western cave fauna has been enriched especially by the discoveries of Sinclate ${ }^{2}$ in California. in the Potter Creek Cave, probably of late Pleistocen eage. This includes an extremely rich series chiefly of the mountain and forest type. Of fifty two species. twenty-one are extinct, including a new member of the Ovina in the genus Encercttherium $^{3}$ (Sinclatr). With these animals are associated relics possibly of human origin. In the East, the Port Kennedy Cave, also treated by Cope, has heen exhaustively investigated hy MErcer ${ }^{4}$, and shown to contain fifty species of mammals, including chiefly forest types, among which are the Mastodon americanus, a tapir, and two species of Eques Again no human remains have been found.

As regards phylogeny, the horses are evidently polyphyletic; but we have not as yet worked out the distinction hetween possible representatives of the hor'ses, asses, and zehras. The Proboscidea have been clearly distinguished (Ponur(i. Lucas, ()sвors") into four great types Mastodon

${ }^{1}$ List of the Pleistocene Fauna from Hay Springs. Nebr. Bull. Amer. Mus. Nat. Hist., Vol. xvi, 1902, pp. 317-322.

2 The Exploration of the Potter Creek Cace. Univ. Calif. Publ. Amer. Archæol. \& Ethn., Vol. 2, No. 1, 1904.

${ }^{3}$ Euceratherium. Univ. Calif. Publ. Bull. Dept. Geol., Vol. 3, No. 20, 1904 pp. 411-418.

4 The Bone Cave at Port Kennedy. Jour. Acad. Nat. Sci. Phila., Vol. xi, Pt. 2, 1899.

${ }^{5}$ Evolution of the Proboscidea in North America. Science, N. S. xvii, Feb. 13, 1903 , p. 249. 
americums in the Eastern and Niddle States; Elephus primigenius in the North, practical identical with the north Asiatic Nammoth; Elephas columbi chiefly in the Middle States hut also in the Southern, and Elephas imperator in the South and ranging north to the Middle States: these species represent profoundly different types hoth in skull and tooth structure, (PI. XII-XIV) Elephus columbi is analogous to the Elephas antiauns type of Europe; the Elephas imperator is rather analogous to the E. meridionalis of Europe. It is altogether probable that these species evolved in Eurasia and arrived fully formed in America. Naturally their weoglaphical ranges overlap; but F. imperutor is never found in the extreme North, nor E. primigenius in the extreme South.

In conclusion, the great problem of all is the time of arrival of man amidst the Pleistocene fauna. This event is of such paramount impoltance that wo must prepare for it by definitely determining the chronological stages of lowel mammalian succession. At present man appears to be a late arrival, but personally. I have a strong presentment that human remains will he found in an carlier Plristocene stage than is genelally supposed.

\section{CHIEF CENTRES OF ADAPTIVE RADIATION OF THE ORDERS} OF MAMMALS

\section{I. - Jumassic radiation (Partly Hypothetical).}

Monotremata (Hypothetical, i. e. fossil forms not yet recognized.)

Marsuptalia (Triconodonta).

Placentalia (Insectivora Pinmitiva, = Tmituberculata.)

II. - Minsupial radistion, upper cretaceous and tertiary.

Australia (chief centre), Antarctica and South America. Only one family (DidelPHyDe) certainly known in North America and Eurasia.

III. - First or lower placental radition, upper cretaceous aNd lower tertiair (三 MESEUTHERIA Osborn.)

North America (chief centre), Europe, Afriea (Gieononta), probalı]y extending also to South America.

a. Orders Certuinly Recoynizerl.

Creodonta, surviving to Lower Oligocene.

'lillodontia, Middle Eocene (possibly related to Rodentia).

Tenodonta, probably related to Edentita Gravigimad.

Condrlatma, surviving to Middle Eocene.

Amblтрода, " "Upper Eocene. 
b. Order's not certainly lnown in Basal Eocene but probably belonging to this radiation.

Ixsecrivona, giving rise to modern INsectivora.

LEMUROIDEA.

Rodextis, Not yet certainly known earlier than Middle Eocene.

IV. - Second or Higher placextal radiation (= Cexeutheria Osborn), Middle EOCENE AND T'ERTIARY.

A. Chief centres North America and Eurasia, migrating to Africa and South America.

a. Orders dericed from first placental raliation.

EDextata from Radiation III (North America only).

Insectrona from Radiation I and III.

RODENTIA.

b. Orders characteristic of second placental radiation.

Cheiroptera.

Carivora (Fissipedia and Pinnipedia).

Primates, Anthropoidea, possibly from Radiation III.

Perissodactyla, Lower Eocene.

Artiodactyla, Middle Eocene.

c. Centres of origin unkinown.

Nomartura or Effodiextia (Lower Oligocene of France, Necromanis Filhol).

Tubuldentata (First appearing in Lower Oligocene of France, Palaorycteropus Filhol).

B. Chief centre Africa, migrating in upper Oligocene (SineniA), Lower Miocene (Probosamea), and Pliocene (Hrracomea) to Europe, to Asia (Hrracoidea). Also to North and South America (ProBOSCIDEA).

Sirenta, Middle and Upper Eocene.

Proboscidea, Middle Eocene.

Hriscoides, Upper Eocene.

Ansivolthenicy.

BARYTHERIUM. 
L. Chief centre South America.

a. Autocthonous order's.

Litopterna.

'Toxonoxtia.

TYPOTHERA.

Astripotheria.

Pyrothenia.

b. Autocthonous or derived orders, in part.

Edextata, Suborders: Lonicata (Glyptonontia and Dasypoba), Pilosa (Gravigrada, Tardigrada, Vermilingua).

Thus the degree of zoological kinship of the continents may be expressed as follows :

1. Close kinship of North America, Asia and Europe (= Holarctica), having all pre-Miocene Orders in common, and separated only by the independent radiation of certain families.

2. Separation of Africa as a pre-Miocene centre of at least three order's not found in Holaretica.

3. Strong separation of South America from the Eocene until the Pliocene. Affiliation with Australia.

M. le Président donne la parole à M. C. Chus.

\title{
DIE VERTIKALE VERBREITUNG DES MARINEN PLANKTONS
}

\author{
Von Prof. Carl CHUN \\ (Leipzig).
}

Meine Herren!

(iestatten Sie, dass ich Ihnen über einige Untersuchungen herichtr, die wir während der Fahrt der" "Valdivia » nach Möglichkeit zu förder"n hestreht waren. Sie betreffen die Frage nach der 'Tiefenverhreitung dus marinen Planktons: eine Frage, welche voraussichtlich noch für lüngere Keit eine wichtige Betatigung mariner Fxpeditionen und einzeln' ${ }^{\circ}$ Fors.her, die in der glücklichen Lage sind, über die nötigen Hilfsmittel zu 
verfügen, ahgeben wird. Wir haben denn auch während der Fahr't der "Valdivia " besonderen Wert darauf gelegt, durch zahlreiche Schliesisnetzfïngr jene Erlenntnisse zu vertiefen, welche durch die "Challengel Exprodition a angebahnt wurden, denen Alex. Atissiz seine Aufmerksamlieit zuwendete und die späterhin durch die Untersuchungen von PAutruso, Curx, Fowler, vor allem aber dulch die Plankton-Expedition Hexser's gefürdert wurden. Für alle diese Untersuchungen ist die Verwrudung ron Schliessnetzen die Voraussetzung. Sie durchfischen in rertikaler lichtung eine bestimmte Wassersäule und sind derart eingerichtet, dass sie bei dem Aufwinden geschlossen werden und keine Vurmenoung mit dem in obertlächlichen Schichten enthaltenen Material gestatten. I)a wir bis jetzt nicht in der Lage sind, eine bestimmte Wasselsäulo in horizontaler Richtung zu durchtischen (es hätte dies allerdings den Vorteil für sich, dass wir grosse Strecken durchfischen und ein cntsprechend reiches Quantum an ()rganismen erbeuten könnten), so sind wir oinstweilen noch auf vertikal fischende Schliessnetze angewiesen. I as auf der Fahnt der" "Valdivia " verwendete Schliessnetz, welches sir hier vor sich sehen, ist derart eingerichtet, dass es einerseits Wassersäulen von belicbigel Höhe, und zwar solche, welche zwischen 20 und fio() m.schwanken, durchfischen kamn, und dass anderelseits nach dem Schliessen das Hereingeraten kleinster Organismen, z. B. der Diatomeen, ausgeschlossen ist. Wir haben dieses Netz auf mehr als 100 Schliessuet\%zügen angewendet und sind im Verlaufe der Expedition dazu übergegangen, Serien von Schliessnetzfängen an einer und derselben Stelle auszuführen, welche über die Schichtung der Organismen ein besonders anschauliches Bild lieferten. Ier Inhalt fast jeden Zuges wurde von den Teilnehmern der Expedition sofort nach dem Herauflommen mikiroskopisch geprëuft, bevor man ihn konservierte. Ich glaube wohl versichern zu kïmnen, dass bis jetzt noch niemals ähnlich serupulös verfahren wurle und dass die scharfe Kiritik, welche wir an den einzelnen Zügen ausül)ten, zugleich auch eine Garantie fül das taldellose Funktionieren des Netzes abgab. Wir gewannen denn auch bald eine derartige Uebund in der Beurteiung der einzelnen Fänge, dass wir aus dem Erhaltungszustand des gewomnenen Materiales schon an Bored uns einige allgemeine Vorstellungen über die Tiefenverbleitung des Planktons zu machen vermochten.

Eine wesentliche Ergänzung der gewonnenen Anschaumngen wurde freilich durch die Verwertung unserer grosien offenen Vertikalnetze hedingt. Stufenfänge, die wir mit ihnen an ein und derselben Stelle veranstilteten (dir guantitativen Planktonnetze wurden im Allgemeinen bis 2(x) m.. die gross'n Vertikalnetge in weit hedentendere Tiefen versenkt) bekräftigten in Algemeinen die durch die Schliessnetze gewonnenen Vorstellungen. Ibahei linferten gerade diese offenen Netze eine wahre 


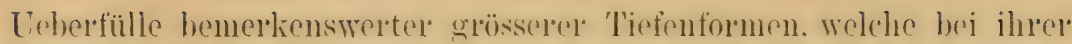
Flürhtigkeit den kleinen und zudem ain heschnïnktes Aleal durchfischenden Schliessnetzen entgehen.

Es wird lange Zeit dauern, bis unsere Funde von den einzelnen Bealloitern eingehend gexichtet und geprüft worden sind. Ich mörhte mich dahes auch darauf beschränken, die allgemeinen Gesichtspunkte in den Fordergrund zu stellen und sie ab und zu dureh die Angaben versehierdener Bearbeiter unseres. Materjales zu illustreren, welehe mir in ausserordentlich zurorkommender Wrise zum Zweclie dieses Vortrages zur Verfügung gestellt wurden.

Es liegt in der Natur del' Sache, dass wir bei Untersuchungen, welche die Vorbeitung von ()ranismen in unbelichteten Tiefen betreffen, zunächst unsere Aufmerlisamkeit auf jene Formen zu richten haben, die unter dem Finfluss des Sommenlichtes zu assimilieren imstande sind und demgemaiss ans annoganischen Bestandteilen ihren Leib auflaten. Wiu writ reicht das assimilierende ptlanzliche Plankton in tiefere Wassepschichten herah, liefert es genügende Uruahrung für dio Vortretel dex Tiefenplanktons, existieren in den kalten, unbelichteten Tiefen charaktel'istische Formen von sch wimmenden ()rganismen und sind diese zalhlerejeh „2enug, um der Bodenfauna als Nahrung zu dienen? I)ies alles sind Fragen. welche riner Kilarumg hedurften und teilweise durch die Fahrt der

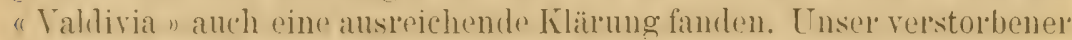
Fremul, Prof. Sommen, lernte halel die Bedentung des Sehliessnetzes schätzen und ging vor allem im antarktischen Meer", wo uns eine fast rimmonatliche Fahrt unter rolativ günstigen Verhälnissen längs der 'Treibeiselenze beschieden war', zu systematischen L'ntersuchungen ühes' dir Tiefonverbueitung (les ptlanzlichen Planktons über. Da zudem in den kalten Wassersehichten die Verhältnisse insofern vereinfacht sincl, als auffällige 'Temperatusplünge zwischen dem ()herflächen- und TiefonWasser nicht vorkommen. so mag an die I)jatomentlora des antarktischede Meeles angeknüpft werden. I)ie "Challenger Fixpedition " hatte bererits dalauf hingewiesen, dass hiog pine nicht minder üppige Wuchel'ung von Diatomern Platz grejet, als in den arktischen Merlen. Wie mir

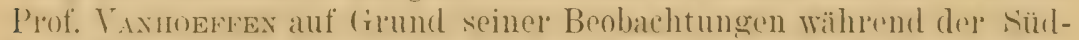
Polar-Expedition mittrilt. so beginnen dir I)iatomeen zu Anfang drs südlichen Frühjahrs, also im November, rejehlicher zu wuchern. Im Laufe des I tezmber bis in den Februar hinein findet dir Hauptentwicklung statt: gegen Endr April heginnen sir abzunehmen und schliesslich zu verschwinden. Während der Fahrt der "Valdivia » in antarktischen Meer (Eurle Vovember bis Ende In'zember) hatte offenbal die Massenentwicklung der I)iatomeren bereits eingesetzt uml gah häufig zu jenre schon von der "Challenger Expedition" erwähnten Verfürbung des 
Oberflichenwassel's Aulass. Es handelt sich hierbei grösstenteils um Gattungen, ja sogar, wir wir späterhin noch betonen werden, um Arten, welche auch in den nordischen kalten Stromgebicten verbreitet sind. Am häufigsten tritt nach den Bestimmungen von Prof. Karsten Chrotoceras criophilum Castr., Rhizosoleniu semispina, inermis und sonstige Rhizosolenia-Arten, weiterhin Thalussiothrix anturctica, synedra sputulata schimp. und Vitzschia serrata auf. Als Nebenbestandteile gesellen sich ihnen 2 neue Arten von Corethrou, Dactyliosolen antarcticum und Frarilaria anturctica, die oft auch mit zu den herrschenden Formen gerechnet werden können. hinzu. ()hne auf sonstige antarktische Diatomeen einzugehen, sei erwähnt, dass die assimilierenden Flagellaten im kalten südlichen Wasser zurücktreten. Immerhin fand sich ein neues Peridinimu, $P$. antarcticum Schimp)., in jedem Fange so regelmässig, dass es pine der besten Leitformen des antarktischen Planktons darstellt.

Was nu! die durch zahlreiche Schliessnetzfänge ermittelte Tiefenverbreitung dieser her'schenden Planktonformen anbelangt, so ist es uns zunächst aufgefallen, dass die oberflächlichsten Schichten - namentlich in der Nähe der Eiskante des antarktischen Kontinentes - den Diatomeen offenhar ungünstige Bedlingungen darbieten. Häufig ist das Plasma kontrahiert und zeigen die Chromatophoren eine Gruppierung, wolche als Wirkung ungünstiger Bedingungæ^n auch künstlich hervorgerufen werden kam. Erst von $40 \mathrm{~m}$. al) stellen sich normale Verhältnisse ein, indem auch gleichzeitig die Masse der Arten zunimmt. Ob nun, wie ich vermute, der Einfluss des Schmelzwassers der Eisherge und des Treibrises sich in dieser Weise geltend macht, müssen weitere Beobachtungen lehren. Die Masse der Diatomeen staut sich in einer relativ flachen Schichte zwischen 40 und $80 \mathrm{~m}$. Tiefe an. Ton hiel an findet eine plötzliche starke Abnahme statt, sodass unterhalb 200 bis höchstens $400 \mathrm{~m}$. 'Tiefe die assimilierende Vegetation äusserst dümn gesät ist. In grösserer Tiefe ausgeführte Schliessnetzzüge förder'n nur noch leele Schalen zu Tage, wohei es sich ergiebt, dass die zarten Skelette von Chretoceras, die ja den Hauptbestandteil des Oberflächenplanktons abgeben, eben so vollständig anfgelöst werden, wie diejenigen von Corethrom?. Bei 600 his 700 m. Tiefe fehlen leere Skelette der genannten Arten schon vollständig.

Es vordient nun besonders hervorgehoben zu werden, dass sich zu diesel I) iatomen-Vegetation, wrehe in gemässigten und tropischen Mreren auch tatsiachlich bis in die oberflichlichsten Schichten hinaufreicht,

\footnotetext{
'Nur die mit relativ mächtigen Zellwänden ausgestatteten Formen der oberen Etage rweisen sich als widerstandsfähig. Prof. Kanstex fand z. B. durch besonders dicke Schalen ausgezeichnete Chætoceras-Arten (z. B. Chætoceras radiculum Castr.) in Grundproben aus 5502 m. (St. 147) unverändert wieder.
} 
rine das intensive Licht moidende "Schattenflora " hinzugesellt. Sie setzt sich hauptsächlich aus zahlreichen Asten der Gattungen Coscinodiscus und Asteromphalus zusammen, zu denen sich in den warmen Mceresgebieten noch die Vertreter der Gattung Planlitomiella und der schon von fruheren Fxpeditionen als Tiefenform nachgewiesenon Algengattung Halosphara hinzugesellen. Im tropischen Indischen ()cean reicht die gernannte Schattenflora von $80 \mathrm{~m}$. bis zu etwa $350 \mathrm{~m}$., im antarktischen Meer setzt sie sich zwal nicht so scharf gegen die oberste Etage ab, herrscht aber doch von etwa 80 bis $200 \mathrm{~m}$. Tiefe derart vor', dass sic dic Leitformen für die genannte zweite Etage ahgiebt. Prof. Kiststw, welcher unsere antarlitischen I)iatomen eingehend prüfte, macht mich daraut aufmerksam, dass die Tertreter dre oberen Etage über das ganze von der "Valdivia " (urehfahrene (xobiet und voraussichtlich auch über dis ganze antarktische Region gleichmässig und rinförmig verhreitet sincl, im fiegensatz zu dem ptlanzlichen Tiofenplankton, welches nahezu bei jede'm Schliesnetzzug ein gewisses Lokialkolorit aufweist. Hier tritt die (rattung Coscinodiscus in nicht weniger als 80 Arten auf, von denen die Iälfte noch unbeschrieben jst. Iazu gesellt sich die Gattung Asteromphalus in 7 Arten (darunter 5 neue) und die Gattung Actinocyclus in 5 Arten (darunter 4 neue). Ich mörhte vermuten, dass die gleichmässige circumpolare Verbreitung der in der oberen Etage vorkommenden I)iatomeen durch die ständigen schweren Weststüme und den gewaltigen Sereang helingt wird, desson Wirkung sich nicht mehr in der unteren Etage gelteurl macht uml dir Aushildung von Lokaltholen hegünstigt.

I)ie Untersuchungon unsers verstorbenen Freundes Scmmpke dürften gerate aus dem Grunde besondren Wert heanspruchen, weil sie uns üher die untere (xpenze pthanzlichen Lehens im Yeere Aufsohluss gegeben hahen. Allererlings sind rinige Peridineen auch noch in tireperen schichten vollständig lebrensliraiftig vertreten: aber os handelt sich hierber um Formen, welche der Chromatophoren enthehren und demgemïss nicht assimilieren. Ia im Allgemeinen unterhalh $600 \mathrm{~m}$. nur noch leere Sehalenpreste von assinnilierenden Organismen gefunden werden, so honzentriert sich das pflanzliche Leben auf eine relativ thache ocem nische Schichte.

Die Schalenreste selbst, welche in grössere 'Tiefen niedersinken, bestrhen denn auch hauptsiichlich aus den etwas widerstandsfähigeren V'r"tretern der sogenannten Schattenflora. Untersucht man daher eine Grumdprobe aus den grosien Tiefen des antarkischen Mreres, so spiegelt sie durchaus nicht das pflanzliche Leben an dere ()berfliche wieder. Es fehlen die in der obersten Etage dominierenden Folmen nahezu vollstïndig, die Rhizosolenien sind oft nur noch mit ihren spitzen Enden elhalten, symedra und Thalassiotlorix: treten häufigel auf, und dir Hauptmasse des Diatomeenschlickes wird aus den Schalenresten der schattenflora, nämlich Coscinodiscus und Asteromphalus, gebildet. 
Auf das pflamzliche Lehen ist das tierische angewiesen. Trotzdem sich das erstere an der. Meresoberfläche auf eine relativ dünne Schichte beschrinlit. so reicht es doch aus, einer eigenartigen tierischen Lebewelt das Inasin \%u frosten. An der Hand unserer [ntersuchungen, welche ja in dies'l' Hinsicht im wesentlichen mit früheren Befunden übereinstimmen, wirl man schwerlich fermerhin noch in Abrede stellen lömmen, dass die gealmmten Wassermassen in vertikalem Sinne his zum Meeresboden von ()pganismen durchsetzt werden. Zwei Schliessnetzzüge, von denen wir den rimen im antarktischen, den anderen im Indischen Meer zwischen 4400 bis s)(n) m. ausführten, enthielten lebende Radiolarien und Crustaceen. Von letzter'on sei aus dem indischen Schliessnetzzug ein grosser blutroter Dekaporle erwähnt, welcher der Gattung Hymenodora angehören dürfte und durch stark rückgebildete Augen auscezeichnet war. Wenn in der genamnten Tiefe dureh einen glücklichen Zufall ein so ansehnlicher blinder Cruster erbentet wurle, so deutet dieser eine Fund darauf hin, dass hirr immerhin ein genügendes Nährmaterial an flottierenden Organismon vorhanden sein muss, um ihm die Existenz zu ermöglichen. I)as Quantum an lebenden planktonischen Formen nimmt zu, je näher der ()herfläche dir Züge ausgeführt werden, wobei indessen nicht ausser Acht gelassen werden darf, dass das tierische Plankton zwischen $200-400 \mathrm{~m}$. sowohl was Arten, als auch Individuenzahl anbelangt, am reichlaligsten vertreten ist. Es scheint also, dass direkt unterhalb der Vegetationsglenze des ptanzlichen Planktons die Ernähıumgsedingungen fül das tierische sich am günstigsten gestalten.

Auf die allmähliche Alnahme an (Yuantum gegen die Ticfe hahen schon flöhlere Erfahlungen hingewiesen und speziell hat Braxd ${ }^{1}$ an der Hand der Frgebuisse der Plankton-Fxpedition die Almut der tieferen Wasserschichten an lebenden Folmen quantitativ zu erläutern versucht. Nach unseren Ergebnissen, die freilich noch einer rechnerischen I)arlegung bediufen. macht as durchaus den Eindruck, als ob unterhalb soo m. eine ziemlich plötzliche Alnahme an schwimmenden tierischen Formen stattfünde. Com diese Verhältnisse wenigstens an cinem Beispiel klarzulegen, so si auf die quantitative Verbreitung der Ostracoden in einer Serie von Schliessnetzzügen hingewiesen, die wir im Indischen Ocean an einer und drrselben Stelle bis 1600 m. Tiefe ausführten. Zum Vergleich mögen damn noch 2 henachlarte Schliessnetzzüge aus grosser Tiefe herangezogen werlen. Wie mir Prof. Mërser, der Bearbeiter unserer ()stracoden. mitteilt. so fanden sich im Inhalt eines Schliessnetzzuges aus $4200 / 4890 \mathrm{~m}$. zwei unbestimmbare Jugendformen von Halocypriden. Ein Zug aus 26(1)2:300) m. ergah sin Exemplar einer neuen Ait von Archironchoecia.

${ }^{2}$ Brandr, K. Ueber die Schliessnetzfünge der Plankton-Expedition. Verh. Ges.d. Natf., Bd. 2, p. 164, 1896 . 
Was nun die Züge an derselben Stelle anbelangt, so "rgab die Untelsuchung folgendes Resultat:

$1600 / 1400 \mathrm{~m} .1$ 요. sp).

$1 \sigma^{x} \cdot 1$ o Conchoecia n. sp.

1 unbest. juv.

$1000 / 800 \mathrm{~m} .2$ unbest. juv.

4 Individuen, 2 Arten

$800 / 600+10^{x} 1$ Individuen

(1) Sars

2 q Conchoecia elegans Sars (= gracitis Cls.)

1 ơ, 1 \& Conchoecia n. sp.

2 ㅇ Conchoecia n. sp.

1 q, 1 juv. Conchoecia n. sp.

1 unbest. juv.

$600 / 400$ m. 2 juv. Conchoecia daphnoides Cls.

11 Individuen, . Arten

1 juv. Conchoecia imbricata Brady (= amata Cls.)

1 q C. curta Lbb.

1 ㅇ. C. spinifera Cls.

$1 \sigma^{T} C$. elegans Sars

1 juv. C. caudata MIIr.

$1 \sigma^{r}$ (! $11 . \mathrm{sp}$.

2 unbest. juv.

400/200 11. 2 ơ Conchoecia magna Cls.

10 Individuen, 7 Al'ten

7 ㅇ, 2 厅 C. curta Lbb.

1 ๆ. $1 \sigma^{x}$ C. spinifera Cls.

$2 \sigma^{x}, 2$ o C. oblonga Cls.

$10^{x}, 2$ o $C$. elegans Sars (= gracilis $\mathrm{Cl}$.)

7 q, 4 O C. rotundata Milr.

$\therefore$ ๆ, 3 o C. procera MIllr.

2 ๆ, $2 \sigma^{\top}$ C. spinirostris Cls.

1 juv. Halocypris inflata Dana (= concha Cls.)

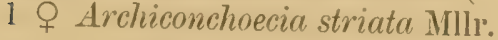

2 ㅇ, 1 or Conchoecia n. sp.

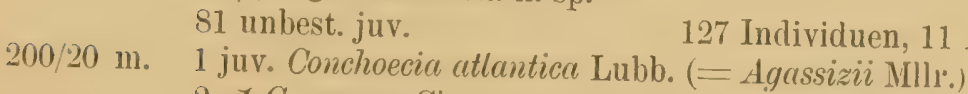

2 O C. magna Cls.

1 ㅇ, $1 \mathrm{O}^{x}$ C. oblonga Cls.

17 ๆ, 4 ơ $C$. rotundata Mllr.

9 Indiv. C. procera Mllı.

$1 \sigma^{x}$ C. spinirostris Cls.

$1 \sigma^{x}$ C. acuminata Cls.

$1 \sigma^{x}$ C. Giesbrechtii (n. n. für C. oblonga Mllr.)

1 ๆ $C$. n. sp.

2 ㅇ Archiconchoecia striata Mllr.

82 unbest. juv.

123 Individuen, 10 Arten 
Es liegt nun in der Natur der Sache, dass die quantitative Abnahme nach grösscren 'Tiefen wesentlich durch die immer spärlicher fliessende Quelle an Nahrung heeinflusst wird. Jenen tierischen Organismen, wolche zwischen 200 und $400 \mathrm{~m}$. sich in besonders reicher Zahl anstauen. stehen ptlanzliche Organismen zur Terfügung, welche nur wenig zersotztes Plasma aufweisen. Unterhalb $600 \mathrm{~m}$. haben wir äusserst selten eine Diatomee nachzuweisen vermocht, die Spuren von Plasma enthielt. Allordings lisst es sich nicht in Abrede stollen, dass viclleicht noch die organische Grundsubstanz der Schalen his in grosse Tiefen orhalten bleiht. Anders wäre es kaum zu erklüren, dass Radiolarien, die wir noch als Bewohner der grossen Tiefen von $3000 \mathrm{~m}$. ab werden kennen lernen, ihren Leib mit Diatomeenschalen vollpfropfen. Was nun die in grösseren Tiefen vou etwa to0 $\mathrm{m}$. ab lebenden Organismen anbelangt, so sind sie im wesentlichen auf ticrische Kost angewiesen. I)ie Radiolarien und die unel'mü̈llich nach Nahrung stöbernden Copepoden und ()stracoden dürften sich vorwingend von den in die Tiefe sinkenden ahgestorbenen Leibern der in höhcren Etagen angestauten tierischen Organismen nähren. Sie fallen ihrerseits wicher den räuberisch lebenden Tiefsceformen zur Beute. Da schliessich alles, was in mittleren Tiefen seine Lehensarbeit verrichtet, zu Grund geht und hinabsinkt, so liefert es auch seinerseits eine Nahrungsquelle für Charakterformen der wrössten Ticfen und für die auf' dem Borlen angesiedelte Grundfauma. Fs liegt auf der Hand, dass von Etappe zu Etappe dir Nahrungsquelle spärlicher fliesst und daclureh dic obenerwähnte Armut an Lehewesen in grösseren Tiefen hedingt. Ob sich über dem Boden eine halbpelagische Fauna anstaut, ist noch nicht sicher erwiesen, wenn anch einige Wahrnehmungen diesel Auffassumg günstig elscheinen ${ }^{1}$.

Fs diüfte vielleicht angezeigt sein, die hier gegehenen Andeutungen durch einige spezielle Beispiele zu illustrieren, welch wir den Radiolarien und Copepoden entlehnen. Was die liarliolarien und zwar speziell die für das Leben in der 'Tiefsec bedeutungsvollen 'Tripyleen anbelangt, so woist Hïkme ${ }^{2}$ hei seiner Besprechung dre vertikalen Verbreitung auf rinige bemerkenswerte Enterschirde zwischen Obertliichen- und 'liefenfolmen hin. Ino in obertlächlichen Schichten bis zu rtwa 400 m. Tiefe lehrndrn Formen setzen sich aus Aulosphäriden. Sagosphäriden, kleinen ('hallengeriden und kleinen Conchariden \%usammen. Es handelt sich hierber in Allgemeinen um Formen von geringer Grösse, die durch einen

\footnotetext{
${ }^{1}$ Ein Schliessnetzfang aus dem südlichen Indischen Ocean aus $3300 / 2700 \mathrm{~m}$. (Station 173. Tiefe 3765 m.) enthielt nach Angabe von Dr. Steuer, Harpacticiden, welche litoralen Formen ähneln.

"V.IÏ̈CGER. Bericht über die Tripyleen-Ausbeute der deutschen Tiefsee-Expedition, in : Verh. D. Zool. Gesellschaft, p. 133, 1904.
} 
zarten Weichkörper ausgezeichnet sind und häufig auch einen reich entwickelten Besatz von Schwehestacheln aufweisen. Ihnen reihen sich derberr Formen an, welche dip Sehichten von 400 bis 1000 pesp. 1500 m. hevölkern. Es handelt sich bei ihnen hauptsächlich um Aulacanthiden (Aulographis, Auloceros, Aulospathis etc.), um Vertreter der reizvollen Gattung Grazelletta, um grosse Tuscaroren und um Crelodendrum furecutissimum. Eine neue Zwisehenform zwischen Culodendriden und Coxlographiden, nämlich Coleshims uputicorms, gibt in deen antarktischen Grbiet rine Leitform für die genanntr mittlere Schichte ab. Die grösiten Tiefen von 1500-5000 m. sind durch einige hochspezialisierte Arten charakterisiort, unter denen speziell Challengeria Naresii und Vertreter der Gattungen Conchopsis, Poreupinia, Porospothis hervorgehoben werden sollen.

Was nun die in mitteren und grosene Tiefen lehenden Radiolarien anbelangt. so zeichnen sie sich dureh relativ ansehnliche (rrösse. durch derbe, delochwohestacheln meist enthehrende Schalen und dureli einen wohlerhaltene'n mit Phaeodellen und Diatomeen vollgepfropften Weichlörperaus.

Offenbar stehen dio L'nterschiede in Haloitus zwischen Ohertlïchenund Tiefenformen mit den Anpassungen an die verschiedenen Existenzhelingungen im Finklang. I)as warme ()hertlächenwasser hesitzt rine geringere innere Trobung uml I)ichte als das kalte Tiefenwasser. Daher kann es nicht überraschen, wenn die Schweherorrichtungen in frestalt

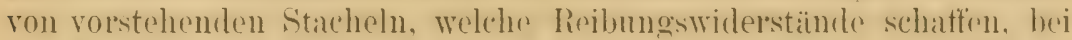
den Ober'flächenformen reichlicher entwickelt sind als bei den Tiefenformen. Was dio geringere frösse der (Obertlächenformen anbelangt. so strht sio wierlerum im Finliang mit den Fixistenzhedingungen. Vergrösser’n wir einen annäher'nd kugeligen Körper, wie ihn z. B. einige Concharden antweisen, um das Inoppelte. so wähst der Inhalt im Cubus. diu ()berflache im (Quadrat. Wis lingt also) auf der Hand, dass bej den kileinen in Warmwasserschichten lehenden Arten die ()her thïhe grünstiged chtwickelt ist als bei den grossen 'Tiefenformen. I)ie (Ohertläche schatfit nun ihrerseits wiedorum Reilumgswiderstände, welche in den wärmeren Wasserschichten mit geringerer innerer Reibung ausgiebiger entwicholt sind, als bei den im kalten Tiefenwasser enthaltenen Formen.

Was nun die Copepoden betrifft, so lässt sich nach dem mir zugegangomen Bericht von s'Tever vorläufig nur schwer eine scharfe Schichtung in vertikale Zonen durchführen. Viele auch in der Nähe der Oberfläche Iebende Arten steigen bis zu der Tiefe von $1850 \mathrm{~m}$. herab. Immerhin scheinen manche Gattungen, so vol Allem spiworelumes und Conere, echte T'iefenformen zu sein, welche fpeilich in den Laltwasser-(robieten woit höher hinaufreichen als in deu warmen Zonen. Iervorgehoben sei nur. dass wir in cinem indischen S(hliessnetzaug zwischen ofo(t) und $4600 \mathrm{~m}$ ein lebendes Weibchen von Euchivella venusta vorfanden. 
Die hishrre gegelnemen Mitteilungen drängen die Frage auf, ob es möglich ist, cine Anzahl von Vertikalzonen zu unterscheiden, welche durch charaliteristische Formen ausgezeichnet sind. Dass jedenfalls eine Zone, nämlich die oberflïchlichste, sich scharf von den tieferen Wasser'schichten ahhoht, geht nicht nur aus allen oceanographischen. sondern auch aus den hiologischen Entersuchungen der Nenzeit hervor. Sie erhält ihpe Hignatur durch rlas Auftreten assimilierender Diatomeen und Peridinorn. Dass diese sich wieder in zwei Etagen gliedern, deren oberste durch die Hauptmasse des assimilierenden Planktons, deren unterste durch die sogenannte Schattenflora charakterisiert ist, wurde bereits ohen hervorgehoben. In den kalten Gebieten dürften wir die Mächtiglieit dieser Schichte zu etwa $200 \mathrm{~m}$ veranschlagen, während in den Warmwassergebioten die monterste Grenze etwas tiefer zu liegen scheint und gelegentlich mit der sogenannten Sprungschichte, d. h. jener Schichte, in welcher eine ziemlich rapide Temperaturalmahme gegen die Tiefe sich geltend macht, zusammenfällt.

Interhalb dieser oberflächlichen, eine relativ dünne Schale darstellenden Schichte staut sich die Hauptmasse des tierischen Planktons zwischen 200 bis $40(0)$ 11. an. Es ist immerhin fraglich, ob wir diese Zone in einen scharfen (regensatz zu den oberthichlichsten Schichten zu setzen herechtigt sind. In ihr ist das Licht hereits so stark geschwächt, dass sie Formen zum Aufenthalt dient, welche die intensive Belichtung der ( )ber'Häche nicht vertragen und nur bei Nacht an die letztere aufsteigen, wihlrend sic andererseits die elste Etappe auf dem Rückzug von der OberHïche für jene pelagischen Organismen abgiebt, welche die hohe sommerliche Oberflächentemperatur nicht vertragen.

Derartige jahreszertliche Wanderungen in vertikalem Simne, wie sie von mir zuerst im Mittehmeer nachgewiesen wurden ${ }^{1}$, erschweren um so mehr eine scharfe Scheidung in vertikale Schichten, als Obertlächenformen in sehr beträichtliche Tiefen hinalozusinken vermögen. ['m nur ein Beispiel anzuführen, so fanden wir in zwei Schliessnetzzügen zwischen 1600) bis 1000 m. untro dem 42. Grad (südlich vom Cap der guten Hoffnung) zahlreiche Exemplare der Solya fusiformis. L'nsere Kenntnisie ïher dir vertikalen Wanderungen der (Obertlächenformen sind freilich, insoweit der freie ()ean in Betracht kommt, noch recht lückenhaft, doch deutet jetzt schon eine Reihe von Erfahrungen, die wir vor allem durch die Anwendung der Vertikalnetze machten, darauf hin, dass sie von zahlrejchen Arten vollzogen werden. Allerdings dürften sie weniger an die Aequatorial-Regionen anknüpfen, in denen das ganze Jahı hindurch eine gleichmässig hohe Temperatur der Obertläche sich geltend macht, als

\footnotetext{
${ }^{1}$ CHUn, C. Die pelagische Tieruelt in grösseren Meerestiefen und ihre Beziehungen zu der Oberflächenfauna. Bibl. Zool. Cassel, Bd. I, 1887.
} 
an dic gemiissigten Zonen mit sinnfälignen jahreszeitlichen T'mperaturdifferenzen. Unter den in grössere Tiofen einwandernden Obertlä('henformen scheint cin 'Teil auch in der Tiefe geschlechtlich tïtig zu sein, während andere Arten 11 u' im Larvenzustand die kalten unbelichteten liegionen bevölkern. Ein besonder's auffïlliges Beispiel hierfür hat in

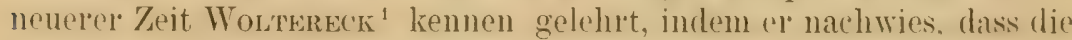
Larven der Velella, also einer Art, die wie kaum eine andere an das Leben auf der Obertläche angepasst ist, in die 'Tiefe niedersinken?2.

Wenn wir davon absehen, dass jenen Tiefenformen, die unter normalen Verhältnissen nicht an die (Herthäche aufsteigen, sich eine supertizicllr Lehewelt heimengt, so dürfen wir an del Hand unselor Erfahrungen unit den Vertikalnetzen die oberste Grenze des Tiefenplanktons auf 700 bis $800 \mathrm{~m}$. ansetzen. Ob es num angezeigt ist, die Fauna, welche in diesen kalten unbelichteten Regionen danernd sich aufhält, nochmals in mehrere Etagen zu gliedern, müssen spätere Erfahrungen lehren. Inie einzelnen Beobachtri" werden, je nach dem sie auf die eine oder auf die andere charakteristische (iruppe von ()romismen vorwiegend Wert legen, vielleicht geneigt sein, zwei oder auch mehr Etagen unterhalb so() m. anzunchmen. Für vine Zweiteilung spricht der Emstand, dass in grösseren Tiefen (von ungefähr $20(0) \mathrm{m}$. ab) Organismen auftreten und bis zum Grunde sich nachweisen lassen, welche in oberen Schichten fehlen.

Weiterhin müssen wir bei einem Versuche, das 'Tiefenplankton nach suiner vertikaleu Verbreitung in Zonen zu gliedern, der Tatsache Rechnung tragen, dass in arktischen und antarktischen (iebieten die hiurver der Tiefenverbreitung erheblich nach der ()herflïche ausbiegt. HaEcker betont dieses Verhalten für die 'Tiefen-Radiolarien, deren obere Verhurejtungsgrenze in den gemässigten und warmen (iebieten bei $400 \mathrm{~m}$., in der antallischen Region dagegen bei $200-150 \mathrm{~m}$. liegt. Um ein writeres Beispiel anzuführen, so sei elwähnt, dass wir nach den Mitteilungen von STEver unter dem 42. südlichen Breitengrade mit dem Srhliessnetz zwischen 1600 und $1000 \mathrm{~m}$. eine Anzahl typischer antarktischer Copepoden (Rhinculanus grandis und Heterorhabdus austrimus) erbeuteten, welche die "Belgiea " viel südlicher, unter dem 70 . Grade, nahe der Oberflüche fischte. Auch wir begegneten den Jugendformen dor gemannten Arten

${ }^{1}$ Woltereck, R. Ueber die Lintwicklung der Velella aus einer in der Tiefe vorkommenden Larve. Zool. Jahrb. Jena, Suppl. VII, 1904.

${ }^{2}$ Für Physalia möchte ich eine ähnliche Entwicklungsweise vermuten. Bis jetzt kennen wir an ihren Genitaltrauben nur die männlichen Gonophoren, nicht aber die weiblichen. Es ist möglich, dass die ganzen Genitaltrauben sich loslösen und dass erst nach dem Niedersinken in die Tiefe die Eier in jenen grossen Medusoiden heranreifen, welche am Ende der Zweige sitzen. An den zahllosen Physalien, die ich im Laufe der Zeit auf ihre Geschlechtsverhältnisse hin prütte, habe ich niemals weibliche Keimzellen nachzuweisen vermocht. 
etwa unter dem 60. Breitengrade zwischen 100 und $200 \mathrm{~m}$. Tiefe. Dasselbe gilt nach den Nitteilungen von Prof. MüLler fül eine Anzahl Ostracoden aus der Gattung Conchoecia.

I)a die T'muperaturunterschiede zwischen Oberflächen- und Tiefenwasser in den kalten Stromgebieten mit ihrer dichothermen Schichtung sich nur um wenige Grade hewegen, so kann es nicht überraschen, dass manche pelagische Formen gegen die zunehmende Belichtung unempfindlich sind und bis zur Oberfläche aufsteigen. Es handelt sich dann um Olganismen, welche in beiden polaren Gebieten auch die oberflächlichen, etwa 150 m. mächtigen Schichten in identischen Formen bevölliern, aber in den gemässigten und tropischen Regionen sich als Tiefenbewohner el'weisen.

Das Vorkommen derartiger pelagischer bipolarer Arten ist unbestreithar, wenn auch erst für wenige, so für Sagittre hanata, der Boweis erluracht wurde, dass sie in gemässigten und tropischen Gebieten als Iestandteile des 'Tiefenplanktons auftreten '. Bei der gemaueren Sichtung unserer Fïnge wird sich dieser Tachweis noch für eine grössere Anzahl ron Formen führen lassen. So sei z. B. erwähnt, dass nach den Angaben von HaEcker eine typische antarktische Rarliolarie, die namentlich in der Nähe des Treibeises auftrat, nämlich Aulospathis pimus, ganz unvermittelt in ainem Schliessnetzzug zwischen 1500 und $1850 \mathrm{~m}$. in der Nähe von Gibraltar gefunden wurde.

Was nun derartigo hipolare Formen anbelangt, so muss für die Diatomeen erst noch der Nachweis geführt werden, ob unter den 17 Arten, welche mach der Mitteilung von Kiskstes sowohl im nordischen, wie im südlichen Planliton verbereitet sind. sich typischo hipolare Formen, d. h. solche, die in den zwischengelegenen Gebieten an der Obertiache fehlen, vorfinden. Unter den Tripyleen sind nach HAsoker mehreve (Camosphara antarctia, Auloscena resticillus, Sagenoscena irmingeriana) als bipolar zu betrachten. Nach meinen Wahrnehmungen liommt unter den Siphonophor'n dir Leitform der arktischen Gewässer, nämlich Diph!yes arctirr, auch im hohen Süılen an der Oberflächr vor. Unter den Copepoden sollen nach (irssizecht ${ }^{2} 6$ Arten bipolar verbreitet sein. Hierbei ist indessen zu herüleksichtigen, dass mit Ausmahme des Psendocalames I'y!gmax's dir aufgeführten Arten auch in mittleren Zonen oberflächlich volkommon und demgemäss als kosmopolitisch zu betrachten sind. Als rin hipolarer (opepode dürfte sich nach STEuen auch Guidius temispims erweisen, da wir ihn in Schljessnet\% unter dem 42 . (rrad südlicher Broite

\footnotetext{
1 Crux, C. Die Beziehungen zwischen dem arktischen und dem antarlitischen Plankton. Stuttgart, 1897.

${ }^{2}$ Giesbrechi, W. Résultats du Voyage du S. Y. Belgica. Copepoden, p. 7, 1902.
} 
zwischen 1600 und $1000 \mathrm{~m}$. erbenteten. Unter den Ostracoden ist Conchnecia elegrons Sar's nach Mǘr.er bipolar, während den nordischen Leitformen, nämlich $C$. obtusatu und $C$. borerılis, sehr nahestehende Arten dic Charakterformen für das antarktische Meer abgeben. Von besonclerem Interessere ist denn auch die Tatsache, dass die charakteristischen arktischen Pteropoden, nämlich Clione limacina Phipps und Limacina helicina Phipps nach den mir gemachten Mitteilungen von Dr. Meiswasueiark in identischer Form die Obertläche der antarlischen .Ieere massenhatt bevölkern.

Wenn wil unsere Darstellungen bisher auf die kleineren Formen heschränliten. wolche fast niemals in den Schliessuetzzügen fehlen, so sei zum Schlusse noch auf jene grossen prachtvollen Vertreter einer pelagischen Tiefenfauna hingewiesen, welche freilich den kileinen Schliessnetzen leicht sich entzichen, aber mit unseren grossen Vertikalnetzen hïutig erbeutet wurden. I) wir diese Formen mit ihren sinntälligen Anpassungen an das Tiefenleben schon hei früherer Gelegenheit schilderten, so mag auf sie nur insofer'n zurüukgekommen werden, als sich über ihre Verbreitung in grössere Tiefen annïhernd sichere Angahen hahen el'mitteln lassen.

Zunächst sei elwähnt, dass unter den Vedusen die auffälligen Vertretru der (iattung Periphylla und Atolla von H.Eoker mit vollem Rechte als echte Tiefenbewohner (bekanntlich wurde diese Auffassung von Fiwks und MaAs bestrotten) bezeichnet wurden. Ein Ex(mplar der Periphylla regina wurde in einem Schliessuctzfang aus 1600 und $1000 \mathrm{~m}$. an der Grenze des antarktischen (rebietes erbeutet und da in demselben Fange auch eine craspedote. Meduse, nämlich Colobonema sericeum, gefund'n wurde, so dürfen wir mit Sicherheit ammehmen, dass auch ein grosser Toil der bereits von Vanuenfex geschilderten Craspedoten zu den echten Tiefenbewohner'n zu zählen ist. Wir vermochten weiterhin dir Existrnz von Tiefsee-Ctenophoren nachzuweisen, die wir niemals oberhalb) $800 \mathrm{~m}$. erhentetrn. Dassolbe gilt für den Pelagonemertes und für die interessante schwimmende Holothurie, nämlich Pelagothuria, die wir in cinem indischen Schliessnetzzug zwischen 1000 mod 800 m. lehend nachwiesen.

Unter den Crustaceen bevölliel'n neben Copepoden und ()stracoden vor allem zahlreiche Amphipoden die tieferen Sichichten. Zwar haben wir die glanzvollsten I'ertreter derselben, nämlich die (iattung Thaumutops, nicht im Schliessnetz nachzuweisen vermocht, wohl aber eine ihr nahestehrnde neue Gattung, welche WoLtereck in einem Schlinssnetzfang aus 1600-1850) m. nachwies und als sphieromimonectes bezeichnete. Ton din meist hochrot gefärbten Vertretern der Gattung Lanceola fanden wir eine noue Art in einem Schliessnetzfang aus grösserer Tiefe, nämlich in 3300 bis $3700 \mathrm{~m}$. Wenn wir weiterhin noch hinzufügen, dass nach den Bestimmungen von WorTereck drei Exemplare cinel' neuen (xattung von 
Cyphocaris aus 1200 bis $1500 \mathrm{~m}$. und eine neue Art von Eutyphis aus 2300 bis 2600 m nachgewiesen wurde, so mögen diese Beispiele immerhin genüøen, um den Antejl der Amphipoden an der Zusammensetzung dru Tirfenfama zu boleuchten. Dass auch die Schizopoden, vorab die (xrnera stylocheiron und Nematoscelis, einen charakteristischen Bestandtoil der Tirefenfauna ausmachen, haben schon meine früheren Untersuchungen gelehrt. I agegen war es bisher unbekannt geblieben, dass die Riesen unter den Schizopoden, nämlich die zahlreichen Arten der Gattung Gruathophernsir, lieine Grundbewohner sind, wie man bisher annahm. sondern gleichfalls einen charakteristischen Bestandteil des Tiefenplanktons alogehen. Zu ihnen gesellen sich als besonders charakteristische und fist in keinem Vertikalzug fehlende Formen die Sergestiden mit ihren Larven, und eine Anzahl meist blutrot gefärbter I)ekapoden aus den Gattungen Acanthephyra, Notostomus und Eryonicus. I)ass wil einen grossen, der (rattung Hymenodor uahestehenden Dekapoden in einem indischen Schliessuetzzug aus der grossen Tiefe von 5000 bis $\$ 400 \mathrm{~m}$. erbenteten, wurde bereits oben hervorgehoben.

Was die grösseren pelagisch lebenden Mollusken anbelangt, so sind die in Schliessnetzen erbeuteten Arten noch nicht genau gesichtet worden und deshalb sei nur helvorgehohen, dass in Schliessnetzfängen unterhalh 1000 m. weder Pteropoden noch Cophalopoden fehlen. So weit die I.ctzteren in Betracht lommen, handelt 's sich freilich nur um einige Larven, da die grossen geschlechtsreifen Tiere als elegante Schwimmer sich den Netzen entzichen. Dasselbe dürfte auch für die Riesenfolmen von Appendikularien, die zudem zu den grössten Seltenheiten gehören, unıl für die überwiegende Mehrzahl der pelagischen 'Tiefenfische gelten. Unter dim letzteren kommen freilich die kleinen Vertreter der Gattung Cyklothore in der 'Tiefe so häutig vor, dass sie niemals in den Vertikalzügen fehlten. sobald die Netze tiefer als 700 bis $800 \mathrm{~m}$. versenkt wurden. Ganz vereinzelt fanden wir sie alleldings auch hisweilen in etwas oberHïchlicheren Zügen. I)ass es sich indessen um echte Tiefenformen handelt, beweisen zwei Schliessnetzzüge, in denen zwischen 800 und $1500 \mathrm{~m}$. Vertretel der Gattung Cylitothone nachgewiesen wurden.

I ass die hier erwähnten pelagischen Tiefenformen durch alle ()eane kosmopolitisch verbreitet sind, kamn insofern nicht überraschen, als die Existenzhedingungen, volab die 'Temperatul' des Tiefenwassels, sich gleichartig gestalten. I)ies gilt allerdings zunächst nur für den von uns mntersuchten atlantischen und indischen Ozean, dürfte aher auch für den Pacifik zutreften. in dem schon frühere Fxpeditionen einen Teil der von uns erheuteten pelagischen Tiefenformen nachwiesen. Die grösseren 'Tiefen der kalten Meeresgebiete, speziell der antarktischen Region, scheine'l allerdings auch eine Anzahl von Formen zu bergen, welche den warmen Gebieten fehlen. während andererseits ein 'Teil der in letzteren 
häufigen Formen gegen höhere Breiten selten wird, oder ganz verschwindet.

Wenn num anch (namentlich insoweit die grösseren Arten in Betracht liommen) (r'st für einen relativ geringen Bruchteil das Auftreten in bestimmten Schichten durch Schliessnetzfänge erwiesen ist, so fehlt es doch nicht an Handhaben, um ein Urteil über ihren Charakter als 'Tiefseeorganismen zu gewinnen. Einiger Wert, freilich nur ein untergenrdneter, kann auf die Fïrbung gelegt werden, welche häufig santschwarze, dunkelviolette oder alle Nüancen des Rot aufweisende T'öne erkennen lässt. Weit wichtiger ist das Verhalten der Sinnesolgane, insbersondere dasjenige der Augen. Manche Vertreter der C'rustaceen, so z. I3. die Eryonicus-Arten und zahlreiche Amphipoden sind völlig blind. oder weisen die Augen in den ver'schiedensten Stadien der Rü̈ckbildung auf. [Tnter den Fischen und Cephalopoden kemen wir allerdings noch keinen blinden pelagischen 'Tiefenbewohner', doch zejgen die Augens. so weit überhaupt die Verhailtnisse bis jetzt bekannt geworden sind, das Pigment in d(ب Dunkelstellung und die Stäbchen ungewöhnlich verlängert. Dass weitrelhin das Vorkommen won sogenannten Teleskopaugen. welches wir in auffïlliger Convergenz bei ('rustaceen, Cephalopoden und Fischen beobachten, wiederum eine Anpassungserscheinung an das Lehen in unbelichteten 'Tirfen darstellt, ist schon fruhherhin so vielfach hetont worden, dass wir an dieser Stelle hierauf nicht erst noch zurtuckzuliommen brauchen. Fntlich kann noch erwïhnt werden, dass in manchen Fällen auch der Mageninhalt einen Rüclischluss auf die Tiefenverbreitung zu lieferm vermag.

Die Zeiten werden nicht fer'n sein, wo wir eine ziemlich ausreichende linntnis der Landfauna, namentlich insoweit es sich um Wirbeltiere nul cinige der mit Vorliehe gesammelten Grupuen von Wirbellosen handolt bexitzen werden. Auch die marine ()bertlächenfiuma, mag sie pelagisch lehen oder an den Strand gebunden sein, wird uns immer vertranter, und wir recinnen bereits mit der 'Tatsache, dass in absehbarer Zeit cigenartige Fol'men, welche neue Organisationstypen darstellen, sich nicht mehr werden auffinden lassen. Wir können sogar die Vermutung aussprechen, dass wir auch über die Charakterformen der auf dem Grunde Jebenden Tiefenfiuna bald auswiehig orientiert sein werden und das Auftauchen von Vertretern unbekannter Klassen nicht mehr erwarten.

Ander's liegen die Verhältnisse für die pelagische Tiefenfauna. Ihre Vertreter sind nur unvollkommen hekannt und verfehlen niemals durch die Anpassungen an die Existenzhedingungen, durch originelle und abenteuerliche (iestalt in hesonderem Maasse das Interesse wachzurufen. Ihre Erforschung wird noch lange Zeit die Zoologen in Athem halten und sie wird auch dann noch durch eigenartige neur formen oder durch 
die bemerkenswerte Lmmodelung einzelnel Organsysteme fesseln, wenn uns die Vertreter sonstiger Faunengebiete längst vertraut geworden sind.

I. le Président donne la parole à M. R. Burcknardt.

Prof. Rud. Burchiardet legt dem Congress das I. Heft der" Zoologischen Annalen " vor an Stelle von Herrn Prof. Max Braus, der zu erscheinen verhindert ist. Man wird angesichts der grossen Zahl unserer Zeitschriften die Gründung einer nenen überflüssig finden. Der Herausgeber liess sich aber von dem Gesichtspunkte leiten, dass die speziellen Aufgaben nomenclatorischer und historischer Art eines besonderen Organs bedürfen. Als solches sollen die "Zoologischen Ammalen » dienen, da die Ausdehnung, welche die Nomenclaturbestrebungen angenommen haleen und die historische Vertiefung, wie sie sich auch in andern empirischesı Wissenschaften anzubahnen beginnt, im Bestreben nach sehiil'ferer Erfassung unserer Allgemeinbegriffe ihr allgemeines Centrum hahen. Die ohnedies weitgehende Spaltung unserer Wissenschaft und ihrer Vertretung in zahlreichen Spezialitäten kann keinen Grund dafür liefern, dass die Geschichtsbetrachtung innerhalb derselben sollte abgelehnt werden. Das lebendige Bewusstsein der Entwicklung unserer Wissenschaft und ihrer Teilgebiete sollte vielmehr recht eigentlich dazu heitragen, dass der Zoologie des beginnenden XX. Jahrhunderts nicht nur Aushreitung über den Erdball, sondern auch die aller wahren Wissenschaft eigene Fühlung mit den Gr'undlagen menschlichen Denkens nachgerühmt werden könne. 


\section{TROISIÈME ASSEMBLÉE GÉNÉRALE \\ Jeudi 18 août 1904}

à 9 heures

dans la salle du Conscil National au Palais du Parlement, sous la présidence de

M. le Professeur G.-B. Grassi

Vice-Présidents : MM. le Prof. R. Blanchard (Paris), le Prof. C. Chun (Leipzig), le Prof. J.-IV. van WiJhe (Groningen).

I. Io Dr Ch.-W. Srules, délégué des Etats-Unis d'Amérique, parlant au nom des Zoologistes américains, propose ì l'assemblée de lésigner I. le professeur Alexander Atissiz comme président de la prochaine session du Congrès qui aura lieu à Boston en 1907. Cette demande est appuyée d'une lettre adressée, an nom des Zoologistes américains, all président du Congrès par M. le prof. C.-S. Мınot.

Cette proposition est adoptée par l'assemblée.

M. le prof. R. Buancirard, secrétaire général du Comité permanent, donne lecture des quatre rapports suivants:

\section{I. - RAPPORT SUR LE PRIX DE S. M. L'EMPEREUR ALEXANDRE III}

Par le Prol:, R. BI.ANCHARD

Secrétaire de la Commission internationale des prix.

Le prix fondé en 1s92 par le Congrès de Ifoscon, en l'homneur de s'. II. l'empereur Alexandre III, est attribué alternativement au C'ongrès international de zoologie et an Congrès international de démographie et d'anthropologie préhistorique. Le Congrès de zoologie n'est appelé à le décerner que tous les six ans. Le prix consiste en une médaille d'or qui est délivrée par la Société impériale des amis des sciences naturelles de Moscou, le Congress de 18y2 ayant attribué à cette société savante, 
avec mission d'en employer les intérêts comme il vient d'être dit, le religuat de la somme importante dont S. M. l'empereur. Alexandre III avait fait don au Congrès.

La Commission internationale des prix comprend les sept membres suivants: M. le professoul E. Perrikr (Paris), président, II. le professeur R. Blanchard (Paris), secrétaire, M. le Dr Jentink (Leyde), M. le profesirilu H. Lumwi (Bomn), M. R.-B. Sharpe (Londres), M. le professello Th. Studer (Berne) et M. le professeur N. Zograf (Moscou).

Le Congrès de Berne devant être appelé à décerner le prix de S. .M l'mpereur Alexandre III, la Commission intermationale, par une note publiée dans le Zoolorjischer Anzeiger, a la date rlu 24 mar's 1902, a fait conuaîtı'e qu'elle mettait au concours la question suivante:

On demande de nouvelles études sur l'anatomie et l'embryologie des Solifuges.

Les travaux présentés, manuscrits ou imprimés, mais, dans ce cas, pubieśr depuis le précédent Congrès, deraient parvenir au Secrétariat avant le $1^{\text {er }}$ mai 1904.

La Commission n'a l'eçu qu'un seul mémoire, ayant pour auteur II. le prof. Ii. Hexross, de l'Académic folestière de Münden (Hanovre), et ayant pour titre: Die fliigelförmigen Oigune (Lateralorgane) der Solifugen und itwe Bedeutuny. Ce travail a été jublié dans les Comptes-rendus de l'Académie des sciences de Berlin, à la date du 11 février 1904, par conséquent dans les délais exigés par le règlement.

M. Hevmons a soumis en outre à la Commission le texte, les dessins el les préparations relatifs à deux autres communications qu'il se proposait re faire, et qu'il a faites effectivement à la section des Arthropodes, ainsi que des documents relatifs a la suite de ses recherehes sur les Galéodes.

Les faits qui découlent de cet ensemble de recherches sont d'une grancle importance. Dans son mémoire imprimé, M. Hersoxs fait conmaîtro des organes aliformes qui sont disposés sur les cotés de l'embryon des colifuges et lui servent d'appareil resporatoire. Il décrit leur structure histologique, leur développement et leur atroplie ultérieure. Ce sont. ì proprement parler, des branchies sanguines, qui démontrent une rolation phylogénétique étroite entro les Galéorles et les Pédipalpes.

Les deux communications orales, dont le texte se trouvera imprimé dans le Compte-remdu du Congress, sont également dordre embryologique. Lime d'elles porte sur la segmentation de l'ouf et la formation des feuillets liastodermiques. Lautre est relative à la morphologie de l'embryon. Elln met en rividence l'identité des faits embrvologiques observés chez les Solifuges ut chy les Arachnides. spécialement chez les Pédipalpes. Au contraire, on constate des diffe'encens considérables arec les Insectes. Les Solifuges ne sont done pas, comme on l'a pu croire, la forme de transition entre les Arachnides et les Insectes. 
La Commission a été unanime à penser que ces importantes recherehes méritaient d'être courommés. Pourtant, lo règlement adopté par lo Congrese de Moscou (1892) exige que les travaux présentés au concours des prix soient écrits en langur francaise et il exprime cette opinion de la facon la plus formelle. Fu rogard au caractipe de plus en plus international des Congres de zoologie et à l'ohligation pratique d'y employer plusicurs langues pour les communications scientifiques et les discussions, la Commission internationale des prix a pensé qu'il était difficile, dans l'état actuel des choses, d'exiger d'une faron absolur que l'article susdit du règlement fut rigoureusement observé. Elle est unanime à penser que des travaux écrits dins l'une ou l'autre des langurs ordinairement admises dans les Congres seientifiques pourraient etre adoptés, ¿̀ la condition ru'ils fussent accompagnés d'un résumé en langue franraise. M. le professem Hernons, anquel ces conditions ont éte communiquées, a déclaré les accepter et être prêt à s'y soumettre.

Dans ces conditions, la Commission internationale des prix a pensé, à l'unanimité, que le prix de S. M. l'emper'eur Alexandre III devait être attribue a M. le professem. Hexuroxs et a l'honneur d'invitel le Congres à émettre un avis favorable.

Les conclusions du présent rapport, mises aux voix, sont adoptées à l'unanimité.

\title{
II. - RAPPORT SUR LE PRIX DE S. M. L'EMPEREUR NICOLAS II
}

\author{
Par le Prof. R. BLANCHARD \\ Secrélaire de la Conmmission internationale is prix.
}

Le prix fondé en 1892 par le Congrès de Moscou, en l'honneur de son A. S. I. le tsarévitch, depuis monté sur le trône sous le nom de S. M. l'empereur Nicolas II, est décerné à chacune de ses sessions par le Congrès international de zoologie. Ce prix consiste en une médaille d'or qui est délivrée par la Société des amis des sciences naturelles de Moscou, le Congrès de 1892 ayant attribué à cette Société, avec mission d'en 'mployer les interets comme il vient d'etre dit, lo reliquat de la somme importante dont S. A. I. le tsarévitch avait fait don au Congrès.

La Commission internationale des prix, par une note publiée dans le

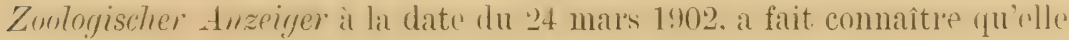
mettait au concours la question suivante:

Nouvelles études su' l'anatomie et l'embryologie des Myzostomides.

Les mémoires présentés, manuscrits ou imprimés, mais, dans ce cas,

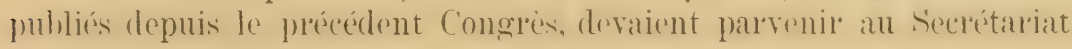
avant le $1^{\text {er }}$ mai 1904. 
Pal' deux lettres 'n date des 16 et 17 mars 1904. les professeurs MrrsuKURI et IJMa, de 'Tokio, ont fait connaître qu'un de leur's élèves, le I) II AnA, était sur le point d'achever la rédaction d'un important mémoir répondant à la question posée, quand la guerre russo-japonaise est venue l'empêcher de terminer en temps utile son travail.

En fait. la Commission internationale n'a recu qu'un seul mémoire, ayant pour auteur le I r Rudolf Ritren von Stumer-Traunfels et ayant pour titre: Beitriige zur Anatomie und Histologie der Myzostomen. I. Myzostomu asterixe Marenz. Ce travail est accompagné de cinq plan('hes in-4"; il a été publié dans la Zeitschrift fiir wissenschaftliche Zoologie en 1903. par conséquent dans les délais exigés par le règlement.

L'auteur a travaillé sous la direction de M. le professeur L. von Graff, ¿ l'T'niversité de Graz. Il donne une description anatomique très détaillée des diftérents organes qui entrent dans la constitution du corps de l'esperce de Myzostomm prise pour type. Cette étude se prete difficil('ment à lanalyse; on doit reconuaître néanmoins qu'elle rst consciencieuse, méthodique et qu'elle apporte une série d'observations intélessantes. On prut regretter qu'elle se borne à la pure description anatomo-histologique et laisse entièrement de côté l'embryologie, qui n'eut pourtant pas manqué de donner des résultats intéressants, au point de vue de la phylogénie toujours controversée des Myzostomides.

Le mémoire est entierement écrit en langue allemande. Sous bénéfice des réserves exprimées dans le précédent rapport et tout en invitant l'auteur à transmettre au Congrès un résumé français de ses observations, la Commission internationale des prix est unanime ì penser que Ir travail du I $)^{r}$ Rudolf Ritter von Stumer-'Trarsfers est digne d'être couronné et elle invite le Congrès à émettre un avis favorable.

Les conclusions du présent rapport, mises aux voix, sont adoptées à l'unanimité.

\title{
III. - RAPPORT SUR UNE PROPOSITION ÉMANANT DE LA SEGTION DE ZOOLOGIE DE L'ASSOCIATION FRANGAISE POUR L'AVANGEMENT DES SGIENGES
}

\author{
Par le Prof. R. BLANCHARD \\ Secrétaire Général du Comité permanent du Congrès.
}

La Section de zoologie de l'Association française pour l'avancement des sciencess, dans la sexsion qui sest récemment tenue à Grenoble, a résolu de soumettre au Congres international de zoologie la proposition suivante: 
"Considérant que les zoologistes ont intérêt à suivre l'exemple des géologues (Congrès international de Vienne, 1903) et à définir les branches de recherches zoologiques pour lesquelles une coopération internationale pourrait s'exercer' avec le plus de fruit;

"Considérant la résolution votée par l'Association inter'nationale des Académies dans sa réunion à Londres, le 25 mai 1904: "que l'initia" tive de toute organisation internationale nouvelle à maintenir par des "subventions de divers Etats demande à être examinée minutieusement " quant à son objet ot à sa valeur, et que les propositions tendant à éta" blir de pareilles organisations soient avant toute action définitive sou" mises à l'Association internationale des Académies »;

"Emet le vœu:

" $1^{\circ}$ Que le Congres international de zoologie, dans sa session de Berne, nomme une Commision chargée d'examinel' quels sujets d'étude pourraient utilement étre choisis d'une façon coordonnée par les diverses sections; cette Commission se prononcerait également sur les meilleurs moyens à employer pour assurer l'unité de méthode dans les investigations.

" $2^{\circ}$ Que les décisions prises par cette Commission et lo programme de recherches qu'elle aurait élaborés soient sommis à l'Association internationale des Académies, ce puissant organisme paraiswant plus que tout autre propre à assurer la réalisation de projets de ce genre.

"I)es aujourd'hui, la section de zoologie désirerait attirer l'attention de la future commission sur les questions suivantes:

" $1^{\circ}$ Etude systématique du plankton de la mer du Norol, l'A tlantique et la Méditerranée, après entente préalable avec le Bureau international fonctionnant déjà à Copenhague;

" $2^{\circ}$ Migrations des Oiseaux;

" $3^{\circ}$ Publication de fiches phototypiques représentant les types originaires des espèces décrites par les anciens auteurs ».

Cette communication, soumise an Congrès dans sa première séance générale, a été l'envoyée au Comité permanent. Colui-ci en a délibéré et, par un vote unanime. a décidé de rejeter les propositions de la Section de zoologie de l'Association française.

Le Comité permanent, tout en reconnaissant la haute valeur scientifique et morale de l'Association internationale des Académies, est d'avis que le Congrès international de zoologie, qui tient actuellement sa sixième session, a suffisamment prouvé sa vitalité pour' n'avoir pas besoin de se mettre sous une tutelle quelconque. Il doit garder son indépendance ot son initiative, qui sont les meilleur's garants de ses succès futurs.

Le Congrès, d'ailleur's, ne saurait rentrer en aucume facon dans la catégorie d'institutions visée par l'Association internationale des Acalé- 
unies, dans sa délibération du 25 mai 1904. Il n'est pas à créer', puisqu’il existe depuis 15 ans; il ne vit pas par des subventions régulièrement inscrites au budget des diver's Etats.

It n'a pas darantage besoin de nommer une commission ayant pou* but d'examiner quels sujets d'étude pourraient être utilement choisis par les diverses sections. Le Comité permanent revendique ce soin comme l'une de ses principales prérogatives; il n'a jamais cessé d'agir dans ce sens, d'accold avec les commissions locales d'organisation, et déjà des pourparler's sont engagés avec les zoologistes américains pour qu'il en soit de même à l'occasion du Congrès de Boston.

L'organisme que la Section de zoologie de l'Association française nous propose de créer existe done depuis le Congrès de Mloscou (1892).

I'autre part, le Comité permanent neéprouve aucunement le besoin de consulter l'Association internationale des Académies au sujet des questions à mettre en discussion. Son initiative particulière, jointe à lentente susdite avec les commissions locales organisées pour chaque Congrès, répond à tous les besoins. Elle donne, en tous cas, des r'ésultats plus favorables que ne saurait le faire un avis émis par un corps scientifique qui comprend assurément de hautes persomnalités, mais où les compétences, c'est-ì-dire les zoologistes, no constituent qu'une faible minorité.

Le Comité permanent ne méconnaît pas, d'ailleurs, que les questions énoncées par l'Association française ne soient particulièrement intéressantes. Il doit faire observer, toutefois, que chacune d'elles est déjà en voie d'étude, grâce à des initiatives diverses. En particulier, la question de la publication de fiches phototypiques représentant les types originaires des espèces décrites par les anciens auteur's n'est plus une question banale, dans le sens juridique du mot.

En ce qui concerue la paléontologie, elle est, grâce à l'initiative de 1I. Ehrent, le savant directeur du Musée de Laval, entrée dans la voie d'exécution, depuis deux ans environ.

En ce qui concerne la zoologie, M. le professeur L. Joubrs, de Paris, a pris une initiative toute semblable: une communication, faite récemment par lui à la Société zoologique de France, en fait foi; et son intention de porter la question sur le terrain international est suffisamment indiquée, d'un côté par les pourparlers qu'il a déjà engagés avec différents Musées, notamment avec celui de Genève, d'un autre côté par' ce fait qu'il figure ¿ l'ordre du jour de cette séance, précisément pour une communication sur ce mème sujet. M. I professeur Jocbix prend part actuellement à ume eroisière de S. A. S. le prince de Monaco; il ne peut donc exposer ses vues sur la question, mais je suis expressément autorisé par lui à déclarer qu'il s'en réserve l'entière propriété.

comme on le voit, la question des fiches bibliographiques n'est plus 
entière ret le Congrés empiéterait, à proprement parler, sur une propriété privée, s'il en abordait la discussion.

Pour ces différents motif's, le Comité prrmanent rst, a l'unanimité, d'avis de rejeter les propositions de la Section de zoologie de l'Association française. Il vous demande d'émettre un avis conforme.

Les conclusions du présent rapport, mises aux voix, sont adoptées à l'unanimité.

\title{
IV. - RAPPORT SUR LES TRAVAUX DU CONGILIUM BIBLIOGRAPHIGUM PENDANT LES ANNÉES 1901-1904
}

\author{
Par le Prof. R. BLANCHARD \\ Sécrétaire Général du Comité permanent.
}

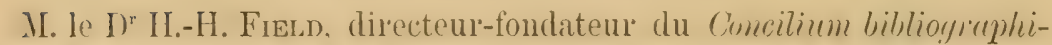
cum, a rendu compte des progrès de son institution à la Commission internationale instituée par le Congrès de Leyde (1895). On trouvera d'autre part le rapport qu'il a présenté.

La Commission est heureuse de constater les éminents selvices que rend le concitum bibliographicum, ainsi que le développenent progressif de cette tres utile institution. File invite le Congrès à se joindre à elle pour exprimer à M. le $\mathrm{D}^{\mathrm{r}}$ Frem ses plus vives félicitations.

\section{ETAT DU CONGILIUM BIBLIOGRAPHIGUM PENDANT LES ANNEES $1901-1904$}

\section{Rapport présenté c̀ la Commission internationale de patronage}

Par le $D^{r}$ H.-H. FIEIdD

Directeur du Concilium bibliographicum.

Le point essentiel qu'il nous semble utile de signaler à la Commission intermationale de patronage, (cest que le veeu émis par M. le professeur Bouvikr (lans le rapport qu'il a présenté au Congrès de Leyde (18950), au nom de la société zoologique de France, a été réalisé. La mortt du très regretté professeur J.-V. Cart's, directeur du Zoologischer Awzeiger, a permis de fusiomer la Bibliographica zoologica avec les publications du Concitium. Cette mesure a eu pour conséquence l'uniformité qu' chacun désirait et dont il est superflu d'indiquer les avantages.

Nous devons, en outre, fare remarquer que plusieurs publications bibliocraphiques sont sur le point de suivere cet exemple et de se grouper autour du Concilium, dont l'importance augmente ainsi d'une facon très appréciable. 
Pour donner une idée du travail accompli par le Concilizm et des services ainsi rendus par lui aux institutions scientifiques, il nous suffira de donner la statistique suivante:

Le nombre total des fiches primaires éditées jusqu'au 31 décembre 1903 est de $12,942,000$.

Le tableau suivant indique le nombre des fiches différentes qui figurent dans une collection complète comprenant un Répertoire par matières et un Répertoire par auteurs. Les années se rapportent à la date de publication des fiches.

A) Répertoire par matières

1. Paléontologie

1896/98 $1899 \quad 1900 \quad 1901 \quad 1902 \quad 1903 \quad$ Total

2. Biologie générale $\begin{array}{lllllll}1460 & 1840 & 2662 & 2035 & 1436 & 2170 & 11603\end{array}$

3. Microscopie, etc.

4. Zoologie $\begin{array}{llll}187 & 151 & 92 & 155\end{array}$

$\begin{array}{llll}266 & 132 & 249 & 263\end{array}$

93200 878

5. Anatomie $\begin{array}{lllllll}18845 & 14271 & 13326 & 16845 & 11059 & 12692 & 87038\end{array}$

6. Physiologie $\begin{array}{lllllll}1940 & 936 & 1875 & 2007 & 1224 & 2009 & 9991\end{array}$ $\begin{array}{lllllll}1380 & 1270 & 433 & - & - & - & 3083\end{array}$

Total $2 4 0 7 8 \quad 1 8 6 0 0 \quad 1 8 6 3 7 2 1 3 0 5 \quad 1 3 9 1 9 \quad 1 7 2 4 0 \longdiv { 1 1 3 7 7 9 }$

B) Répertoire par auteurs

\begin{tabular}{rrrrrrrr} 
& 16165 & 9492 & 10890 & 10119 & 6727 & 8485 & 61878 \\
\hline Total & 40243 & 28092 & 29527 & 31424 & 20646 & 25725 & 175657
\end{tabular}

A la date du 31 décembre 1903 , nous avions publié un total de 1573 fiches divisionnaires, tables des matières. Elles étaient ainsi réparties: Paléontologie 272 - Biologie générale 14 - Microscopie 14 - Zoologie 1071 - Anatomie 98 - Physiologie 114.

La situation financière, à la date du 31 décembre 1903, était la suivante:

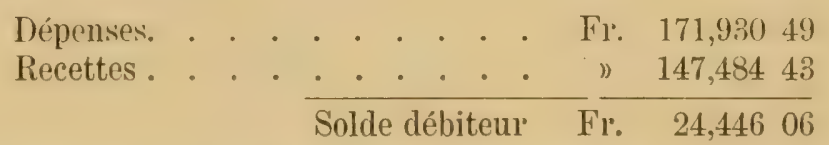

Le personnel du Concilium bibliographicum est actuellement composé de la manière suivante:

Directeur: $D^{r}$ Herbert Haviland FiewD.

Assistants: M" Marie RüHL.

1) $\mathrm{D}^{\mathrm{r}}$ Hermann JoRdan.

Secrétaires: M"le Léonie Locher.

") $\mathrm{M}^{\mathrm{Hl}}$ Lina MÜLLter.

Correcteur: M. Adolph Law WGge.

Deux typographes et trois manœuvres. 
II. Ie prof. R. Blanchard, président de la Commission internationale de nomenclature, transmet ì l'assemblée le va'u suivant que la Commission, dans sa séance en date d'hier, a adopté à l'unanimité.

Vœu proposé par la Commission internationale de nomenclature.

" La Commission internationale de nomenclature,

"Considérant qu'un certain nombre de membres n'ont pris part ni au présent Congrès, ni à celui de Berlin;

"Considérant d'autre part que, par suite de ces absences, le travail de la Commission se trouve entravé:

"Considérant en outre que, par suite du décès du professeur J.-V. Carus et de la démission de MiN. Sciater et Howard Saunderes, la Commission se trouve actuellement réduite à 12 membres au lieu de 15 :

"Emet le vœu:

"Que la Commission, par" voie de tirage au sort, soit autorisée à se diviser en trois sections qui seraient renouvelées à tour de rôle, lors des différents Congrès, les membres sortants étant immédiatement rééligibles $)$.

Ce vœu, mis aux voix, est adopté à l'unanimité.

Eun conséquence, les membres de la Commission se réunissent dans une salle voisine, pour y procéder au tirage au sort énoncé ci-dessus. La répartition des 12 membres restants se fait de la façon suivante:

Premier groupe. - MM. Collett, von Mlehrenthal, Sharp et Sinon.

Deuxième groupe. - Mir. Blanchard, Stiles, Studer et Wright.

Troisième groupe. - MM. Horst, Jentink, Schulze et Stejneger.

Pour remplacer le premier groupe, dont les fonctions viennent de prendre fin, et pour combler les trois lacunes qui existent d'autre part dans son sein, la Commission propose MiI. I) autzentber (*, von Graff, Hoyte, Jordan, Joubin, von Mimirenthat et Osborn. En prévision de l'adoption de cette liste, la Commission a déjà procédé à un vote tendant à désigner les trois personnes qui, à titre de remplacants, devraient ètre attribuées respectivement à chacune des trois sections.

D'apres ce vote, la Commission internationale de nomenclature serait ainsi constituée:

Série sortant en 1907.

$\mathrm{D}^{\mathrm{r}} \mathrm{R}$. Honst (Leyde).

Dr F.-A. Jentink (Leyde).

Prof. David Starr Jordan (Palo Alto).

Prof. F.-E. Schulze (Berlin).

$\mathrm{D}^{r} \mathrm{~L}$. Stejneger (Washington). 
Série sortant en 1910.

Prof. R. Blancharo (Paris), Piésident.

Prof. L. Joubin (Paris).

Dr Ch. Wardell Strues (Washington, D.C.), Secrétaire.

Prof. Th. Studer (Berne).

Prof. R.-R. Wright ('Toronto).

Série sortant en 1913.

I. Ph. Dautzenuerg (Paris).

$D^{r}$ Hoyle (Manchester).

Prof. L. von Graff (Graz).

Prof. F.-C. von Memrenthal (Berlin), Secrétaire.

Prof. Henry-F. Osborn (New-York).

La Commission a l'homneur de proposer au Congres de bien vouloir émettre un vote approuvant cette procédure.

Par un vote unanime, le Congrès approuve la décision ci-dessus.

II. le Président amnonce à l'assemhlée que M. H. von Berdepacir étant malate ne pourra pas prendre part aux travaux du Congress. Il a prié M. O. Kifenschmot de bien vouloir le remplacer.

M. le Président donne la parole à M. O. KLeinscunnot.

\section{B E R I G H T}

über den

\section{BESCHLUSS DES V.INTERNATIONALEN ZOOLOGENCONGRESSES :}

- DER V. INTERNATIONALE ZOOLOGENKONGRESS TRITT für ALLE BESTREBUNGEN ZUR ERHALTUNG DER DURGH DIE KULTURFORTSCHRITTE BEDROHTEN UNSCHÄDLICHEN TIERARTEN BEFÜRWORTEND EIN *

\section{Von 0 . KLELNSCHHIDT}

(Volkmaritz).

Nit 1 Tafel.

Auf Wunseh des lieiherrn von BerLepson, dem sein Gesundheitszustand leider den Besuch des Congresses nicht ermöglichte, habe ich es ührrmonmen, an seiner stelle hier über das angeliundigte Thema einge 
Worte zu leden, um so lieher, weil ein von mir für eine Soktionssitzung angemeldeter Vortrag sich eng mit dem vorliegenden Gegenstand herührt.

Ich bedauere es, dass Freiher' von 'BerLepscir nicht hier an meinel' Stelle steht, und dass es nicht seine Ausfühlungen sind, die ich Ihnen vortragen darf. Er liebt es, überall die Sache in den Vordererund und sirh selbst, scine Person zurüclizustellen. Abrer, wir as Professor Harnack als Vertreter der Universitit Berlin so schön auf dem vorigen Congress ausgesprochen hat haben solche wissenschaftlichen Versanmmlungen unter vielen Anderngerade den Wert, dass man die "Persönlichkeit o kennen und wïrdigen lerent, die hinter der Sache, hinter dem sonst nur geschriebenen und gedruckten Worte steht.

Ich bedauere deshalb, wie gesagt, selur, dass der eifrigste Vertereter des wissenschattlich hegründeten Vogelschutzes hier nicht persön l ich Anregungen geben und, wie es seine Absicht war, bei den Gelehrten - insbesondre dieses Landes - su chen kann.

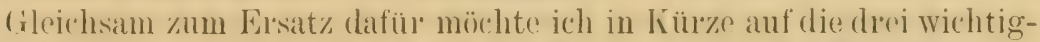
sten Terdienste hinweisen, die sich von Brandessen um die Erbaltung del durch die Kulturfortschritte so gan\% hesonders bedrohten Vogelwelt erworben hat.

Sie bestehen zunitehst in che Konstruktion einer naturgetreuen Nachahmung der künstlichen Spechthöhle, die an Stelle der früheren unpraktischen weil unnatürlichen Nistkisten allen Hühlenbrütern im hentigen Kunstwalde dieselben günstigen Lehenshedingungen bietet wie im Urwalde;

Zweitens in der Trü̈ffentlichung riner Sihlift: "Der gesamte Tongelselutz ", dio nicht in gesetzlichen Masspergeln, sondern in der Sich aff ung von Brutstätton die wichtigste Abhilfe gegen das Vorschwinden del Vogelarten sieht ${ }^{2}$;

Mrittens in der energischen Betonung des Grundsatzes: "Wrg nit aller Sentimentalitat! Die Erhaltung dor Tierwelt ist in unserer Kulturwelt nur möglich, wenn sie auf wissensehaftichel Grundlage, il. h. auf Grund der Ar beit von Fach-Zoologen betrieben wird. »

Es ist sofort klar, dass solche ursprünglich für den Vogelschutz aufgestellten Grundrätze für die Frrhaltung der gesamten höheren Tierwelt Geltung hahen, und dass sie die Zustimmung und Förderung intruat-

\section{Ueber die Wiedereinbürgerung ausgestorbener Alpenvögel.}

2 In 9. Auflage im Verlag von Hermann Gesensus, Halle a. S., erschienen. In dem Buche sind die künstlichen Nisthöhlen ausführlich besprochen. Der Verfasser ist an dem Verkauf des Buches und an der Fabrikation der Nisthöhlen in keiner Weise finanziell beteiligt. I) Schrift wurde von der prensischen Regierung in 5\%00 Lxemplaren als Lehrbuch für Forstbeamte angefordert. 
tionaler Zoologencongresse verdienen. Deshalb hat auch der vorige Congress den Beschluss gefasst, über den ich nun berichten will, indem ich darlege, wie er gemeint war. Ich darf mir das Recht des Interpreten herausmehmen, da ich einerseits jenen Beschluss mit veranlasst, andererseits ihn mit vielen Mitgliedern des damaligen Congresses nachträglich eingehend besprochen habe.

Dabei wurde von mehreren Seiten der Ausdruck "unschädliche» Tierarten beanstandet, und sicherlich sind es meist gerade s ch ä d li e he Tierarten, für die wir trotz ihrer Schädlichkeit ein Wort der Fürsprache ('iulegen müssen, um sie vol dem Aussterben zu schützen. Aber in vielen solchen Fällen wird der wissenschaftliche Zoologe nachweisen können, dass als "schädlich " verschrieene Tiere unschädlich oder sogar nützlich sind, oder dass sich über ihren Nutzen oder Schaden zur Zeit gar kein sicheres Urteil fïllen lässt. Als Beispiele lege ich hier der Yersammlung zwei Präiparate vor, eine grosse Raupe aus dem Magen eines jungen Hausrötlings, eines sonst als Bienenschädling vielfach verfolgten Vogels und Schildlïuse aus dem Kropf und Schnabel eines fütternden Feldsperlingmännchens. (Bekanntlich will sogar Freiherr von Bertersor beide Sperlingsarten mit allen Mitteln verfolgt haben.) Doch im selben Augenblick, wo wir den Nutzen des Vogels durch den Selitionshefund wissenschaftlich hewiesen zu haben meinen, erhebt sich wieder der Zweifel, ob der Vogel durch Verschleppung und Verbreitung seiner Nahrung nicht wiederum elbensoviel schadet wie er durch Verminder"ung der'Beutetiere nützt.

Aher selbst wenn wir leugnen, dass die relativen Begriffe "nützlich " oder" "schädlich » sich wissenschaftlich begründen lassen, so müssen wir doch dem Einzelnen das Recht zugestehen, sich im einzelnen Falle gegen bestimmte Uebergriffe von Tieren auf sein Eigentum zu schützen, auch dem Staate das Recht, sich gegen Schärligung gemeinsamer Kulturgüter zu wehren.

(ob) freilich Yertilgungsprämien immer das richtige Mittel sind ? ()ft genug wird durch Prämien das Gegenteil von dem erreicht, was sie bezwecken sollten, so z. B. wenn daraufhin arme Teufel nun erst recht ihr Lehen wagen, und mit schlechten Waffen geführliche Raubtiere angreifen, um den Lohn zu verdienen. Wo die Hyänen rertilgt werden, wandern Löwen cin, wo man der Jagd zuliebe die Krähen aasrottet, kïnnen Raubrügel ungestört ihr' Wesen treiben, wïhrend sie früher durch erstere im Selhach gehalten wurden. In manchen Fällen sollen Jagdaufseher geradezu das Raubzeug hegen, um aus dem Abschuss der Jungen eine feste jährliche Einnahme an Prämien zu erzielen.

Sicherlich können wir kein Tier schützen, das dem Menschen selbst geffihrlich wird. So bedauerlich vom zoologischen Standpunkt die Ausrottung etwa einer bestimmten Löwentorm erscheinen mag, wir würden den bedenklichen Weg vom Errhahenen zum Lächerlichen beschreiten 
und unsere idealen Bestreloungen zum Gregenstand des Spottes, zum Stoff für Witzblätter machen, wollten wir hier wirklich schädliche Tiere gegen die Kultur in Schutz nelımen.

Es handelt sich ja hier' gar' nieht um den Schutz der 'Tiere, der Individuen, sonder'n auf die Erhaltung von Tiel-A l'ten kommt es uns an, und hier liegt mun das Interesse, das dic Zoologie als Wissenschaft an der ganzen Frage nimmt.

Es ist ein Vortrag hiel gehalton worden über das "Irleal eines naturhistorischen Museums n. Ich habe bedauert, ihn nicht anhören zu liönnen, denn jeder Sammler sucht unahlässig nach nenen Methoden, die liostharsten Stücke seines Materials möglichst gut zu erhalten und zu sichern.

Das idealste Museum wäre ein solches, in dem alle Tiere stets lebend oder frisch getïtet dem for'scher zur Verfügung ständen. Das hietet uns nur die freie Natur draussen. Sie ist unser Idealmusemm; daher liegt ein glosser Roiz darin, wenn es uns vergönnt ist, unsel Untersuchungsmaterial selfst zu sammoln. Meist verbieten es ja Zeit und Arbeitsteilung, aher immerhin werden wir hemüht sein, das lebendige internationale Illealmuseum als Bezugsquelle fïr alle anderen Musern zu crhalten.

Nun hat man freilich gesagt. gerade die zoologischen sammler seien die schlimmsten Feinde der aussterbenden Tiere, weil da jeder noch Stücke der immer seltener werdenden Art haben will und so zu ihrem gänzlichen Ruin beiträgt.

Aber wie oin gowissenhaftel Vogel-Präparator für seine Arbeit zu viol Zeit braucht, als dass a nach Art eines Federhändlers . Iassenmord treiben und Fabrikarbeit tun könnte, so weiss auch ein gewissenhafter sammler, dass ex durch Beschränlung auf bestimmte Gruppen. nicht durch Jagd nach Soltenheiten zum Ziel kommt. Nicht das zoologische Interesse. sondern mangelndes Interesse der Zoologen an Forthestand des 'Tieres ist der (iruml, dass so viele interessunte Tiere noch in letztel" Zeit ausgestorben sind. Ich erinnese nur an den Tarpan, das Wildpterel (les enropäischen Riusslands. Man kömnte von Congress zu Congress eine Liste der schutzbediüfigen Arten anfistellen. Ioch wïrden durch Teröffentlichmng solcher Listen gewissenlose Händlep erst recht auf "seltene Arten ) aufmer'ksam gemacht.

In erster Linie wird man dabei an die grossen afrikanischen Säugetiere zu denken haben, und sir sind vielleicht enttäuscht, wenn ich dat-

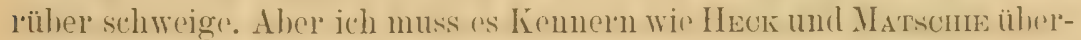
lassen, darüber zu urteilen, denn über die schwierige Frage ob und welche Schutzmasspegeln nötion siud, um das Aussterhen eines Tieres zu hindern, kamn nur ein Spezialist etwas sagen, del das betreftende 'Tier"gebiet eingehender bearheitet und in der Erkenntnis der letrefteuden 'Tiergruppen tiefer' gegraben hat. 
Um zu zeigen, wie fortwährend vor unsern Augen Tiere aussterben, will ich einige lionlirete Fälle aus der jüngsten Geschichte der Alpenfauma anführen.

Vor Ihnen steht hier ein prächtiges Exemplar des Gypatus barbatus (L.) aus dem hiesigen Museum, eines der letzten Stücke der Schweizer Alpen. In Afrika ist der Vogel noch übrraus häufig, aher es rücken von dort keine Vögel nach. weil die Afrikanel zwei kürzel gefiederten Formen' angehïren, die auf den $A l p e n$ sicher frieren müssten. Wenn man Bälge drs ausgestorbenen Schweizers mit ihnen vergleicht, so findet man erst, wie interessant dieser Vogel war durch seine bedeutendere Grösse die etwas abweichende Kopfzeichnung, deren Anlage so merkwürdig an die Zeichnumg eines Gemsenliopfes erimnert, fernel durch das noch inmer nicht genügend erklärte Färbungsproblem. Das Getieder fïrlot sich allmïhlich tief rostrot, aber nur hei starker Abnutzung. Bei rinzelnen Stücken und immer hei (refangen'm hleiht es weiss. Der Vogel wird stets eines der interessantesten Objekte für die zoologische Wissenschaft blejben. I)ie grosse europäische Form scheint, nachdem sie in der Schweiz ausgestollien ist, auch zum mindesten auf der Balkanhalhinsel, wo ich sie 1893 noch am Horst beohachten und das heroliche Flugbild bewundern durfte, rapid alzunehmen, so dass der Vogel in absehbarer Zeit ganz aus Europa ver'schwinden muss.

I) Hirten freilich werden dies als einen erfreulichen Kulturerfolg ansehen. Sie sind schlecht auf den Vogel zu sprechen. Mehr liecht würden dazu die Paleontologen haben, denn dio Hauptnahrung des Bartgeiers besteht weder in frischem Fleisch noch in Aas, sonrlern vichnehr aus trockenen Kinochen. Die Ahnen dieses Togels, der in seiner ganzen Erscheinung einen urweltlichen Eindruck macht, haben gewiss manches wertvolle Shelett ausgestorbener Säugetiele hinweggeräumt, ehe die Erde es schützend bedecken konnte. Zumeist elst damn, wenn die andelen Geier ihre Mahlzeit beendet haben, escheint der Bartgeier, um mit seinem schmaien Schnabel dic Schmen zu zerreissen. Er erheht den losgetremuten Kinochen hoch in die Luft und lässt ihn auf eine Felsenplatte fallen, un damn nachstürzend die Kinochensplitter aufzulesen und zu vel'schlingen. Es ist ganz wunderbat, dass el sich an diesen oft messerscharfen und nadelspitzen Bruchstücken die Schlundwand nicht verletzt. Ich lege der Tersammlung den Magen- mol I)arminhalt des Vogels, sowire einen dem jumgen Bartgeiel von seinen Elterln zugetragenen Kinochen als Bewres des fesagten vor. Wemn der Alpenbartgeier Angrifte auf Tiere und Menschen verübt hat, so sehe ich darin gewissermassen

\footnotetext{
${ }^{1}$ Gypatus barbatus atlantis und ossifraga. Erstere wird in einem von E. FLÜCKIGER in Algerien gesammelten prüchtigen alten $O$ nebst Abbildungen aus der Arbeit v. ErLangers vorgelegt, der diese Form erkannte und beschrieb.
} 
die Verzweiflungstat ansgehungerter. Tiere, eine Parallele \%u dem an anderen Orten brobachteten Angriff eines Bussalis auf ein Pferd, oder eines Uhus auf ein Kind.

Solltr also von Siebenbürgen oder Sardinion her ein Bartgoierpar wioder in der. Schweiz einwandern, so wïre es durchaus nicht geboten, die Vögel zu vernichten. Wenn man ihnen (besonder's im Winter' zu Zeiten, wo alles rom Schnee verschüttet und hedeckt ist) auf einem fiutterplatz Knochen ausstreute, so würden sie eben so harmlos sein, wie es die Bartgeier in Afrika sind, die in Abessinien sich zahlreich, ohne Schaden zu tun, sogal hei den Wohnungen heruntreiben und dort geduldet werden ${ }^{1}$.

Einem ähnlichen Schicksal, wie es den Bartgeiel elreicht hat, geht laugsam der Steinadler der Alpen entegenen. Sorh ist er der Charaktervogel der. Shaweiz, und sein stolzes Finghild vollendet erst den Reiz einel

${ }^{1}$ Baron Carlo ron Ertanger, der die beiden afrikanischen Formen des Bartgeiers auf seinen Reisen beohachtet, die eine davon überhaupt entdeckt hat, schreibt darüber Journ. f. Orn., 1904, p. 202: "Auf jedem Hochgebirge ist er heimisch und daher in dem Gebirgsland Abessinien, die afrikanische Schweiz mit Recht benannt, häufig. In Adis-Abeba sah ich tagtäglich Lämmergeier über den Hütten der Abessinier kreisen, öfters gar nicht hoch über denselben, sodass es sogar Präparator HuGERT cines Tages gelang, einen Gypaetus, während er über unseren abessinischen Hütten, in welchen wir wohnten, kreiste, zu erlegen. In der Nähe derselben lagen mehrere alte Knochen, die Lieblingsspeise der Lämmergeier, und hatte er sich wahrscheinlich diese zur Mahlzeit auserkoren. " Uebereinstimmend berichtet 0 . NeUninn Journ. f. Orn., 1904, p. 368: "Häufig in der Nähe grösserer Städte, so sehr häufig in Adis-Abeba, wo er auf den Wiesen vor der englischen und russischen Gesandtschaft stets anzutreffen ist. Scheint sich ausschliesslich von Aas, besonders von alten Knochen zu ernähren. » Nun sind allerdings die afrikanischen Bartgeier etwas kleiner, aber einerseits ist der Grössenunterschied so gering, dass ich ihn an vielen Skeletten nur mit Mühe feststellen konnte, und andererseits lernten alle gründlichen Beobachter des Vogels ihn auch in Europa und Asien als harmlosen Knochensammler kennen. Die Namen "Lümmergeier • und "Gypxtus " sind unglückliche Bezeichnungen, denn der Vogel gehört wissenschaftlich in die Gruppe der harmlosesten aller Raubvögel, zu den Schmutzgeiern, und hat mit den Adlern gar nichts zu schaffen. Die Uebergriffe einzelner - ja nun unschädlicher - Schweizer Vögel waren sỉcher ganz abnorme Erscheinungen. Am meisten leuchtet mir die Ansicht ein, die Herr Professor STUDER in einem Privatgespräche mir gegenüber äusserte: Seit der Wegebau in den Alpen so grosse Fortschritte gemacht hat, kommt es selten vor, dass Lasttiere abstürzen, was in alten Zeiten, wo die Wege noch sehr mangelhaft waren, sehr häufig geschah. Die letzten Bartgeier fanden kein Aas, bezw. keine Knochen mehr und suchten deshalb aus Hungersnot Tiere in den Abgrund zu stürzen. - Ich füge hinzu: Wären sie wirklich so gefährlich gewesen, wie es nach jenen abnormen Einzelfällen glaubhaft schien, so wären sie nicht aus Nahrungsmangel ausgestorben. In Bosnien hat, soviel ich weiss, die Regierung Schutzmassregeln für den seltenen Vogel angeordnet. 
Alpenlandschaft. Wir in Deutschland haben ihn fast nur noch als Wappentier. Hier haben Sie ihn wirlklich.

$\mathrm{Zu}$ dem zoologischen Interesse kommt hier der allgemeine ästhetische Wert des Tieres als Naturdenkmal. Dem Menschen wird der Adler nicht geführlich. Man liest zwar von Zeit zu Zeit grauenvolle Schilderungen von Kämpfen zwischen Jäger'n und Adlern in der Tagespresse, die dar'um jede Erlegung eines Adlers als eine Heldentat feiert. In Wirklichlieit wird der Steinadler von dem kleinsten Falken an Mut übertroffen. Las Adlerpaar Hieht, sobald es die Nähe des Menschen am Horst gewahr wird und wagt sich nicht herbei, solange man bleibt. Ja, ein alter' Steinadler, den ich im Horst fing, machte keinen Versuch, mich anzugreifen, als ich ihn fasste und auf die Höhe der Felswand emporbrachte.

Wohl vermag der Togel der Jagd und jungem Weidevieh vereinzelt zu schaden, aber schädlich wird eine Tierart doch nur, wenn sie in grösserer Anzahl auftritt. Eine beschränkte Anzahl von SteinadlerBrutpaaren kann manalso unbedenklich an verschiedenen Stellen des Landes schonen. Wenn für den Schaden, den die Jagd an diesen wenigen Plïtzen erleidet, die Staatskasse aufküme', so würde man das Ueherhandnehmen der' Tiere in gewissen Landesteilen sofort merken und jederzeit fähig sein, den Bestand zu r'eguljeren.

Fine der merkw ürdigsten Gestalten der Alpenornis war der Waldrapp oder Kahllkopfibis, der heute nur noch in Afrika und am Euphrat vorkommt. Vor etwa hundert Jahren dürften die letzten Brutpaare dieses Vogels aus Europa verschwunden sein. Ith lege hier zwei Bälge meiner Sammlung aus Naroce und Abessinien samt den alten Abbildungen des Vogels vor. Das eine Stück bringt durch das Fehlen des roten Flügelspiegels den Beweis, dass Geronticus eremitu L. mit Geronticus comatus Hemprr. u. Ehrl). vollstindig identisch war. Der Vogel wurde früher vielfach mit einem Kormoran (Carbo graculus desmaresti) verwechselt und war dadurch lange völlig in Vergessenheit geraten. Merkwürdig, dass anch ein neuaufgefundener Brutplatz in Haroce mir zuerst als Kormoran-Kolonie gemeldet wurde?

1 Dasselbe ist bei uns in Deutschland bezüglich der Saatkrähenkolonien empfehlenswert. Der weitere Umkreis, also das Land, hat Nutzen davon. In der engeren Nähe des Brutplatzes haben die Grundbesitzer Schaden. Es würde im Interesse des ganzen Laudes, mindestens des Kreises liegen, die Wenigen zu entschädigen, welche im Interesse der Gesamtheit den Nachteil tragen.

${ }^{2}$ Ich begegnete nach meinem Vortrag bei anwesenden Ornithologen dem alten Zweifel, ob Gessner nicht s. Zt. mit diesem Vogel betrogen worden sei. Die vielen Spuren einstigen Vorkommens des "Waldrapp " in Europa, die verschiedenen grundverschieden aufgefassten, also selbständigen Abbildungen und endlich die Kenntnis Gessmers von der Entwicklung des Kopfgefieders, dem Mageninhalt, dex Lehensweise und Zugzeit des Vogels schliessen solche Zweifel völlig aus. 



\section{TAFEIERKI,ÄRUNG}

Parus Salicarius rhenamus (Kl.).

Artgenosse des Parus montanus Baldenst., des Parus borealis Selys, und des amerikanischen Parus atricapillus L., nur noch an wenigen isolierten Brutplätzen am Rhein vorkommend, die den hier abgebildeten Landschaftscharakter tragen.

Hinter dem Vogel ist die Oeffnung der nach Art der Spechte angelegten Nisthöhle sichthar.

Die fünf I.inien unter dem Bilde stellen das Maximum der bis jetzt gefundenen Fliigellängre bei den nächstverwandten Formen dar. Das verdickte Endr' dieser Linien bedeutet die teils individuelie, teils durch Alter und Geschlecht bedingte Variationsweite, soweit dieselbe nach den bis jetzt vorliegenden sicheren Messungen ermittelt werden konnte.

Cf. Journal für Ornithologie 1897, p. 112, Taf. III. - Orn. Monatsberichte 1900 , p. 168 (Originalbeschreibung) - Journ. f. Orn. 1903, p. 141, Taf. V. 


\section{Veth. VI. Intern. Zuni Congr.}
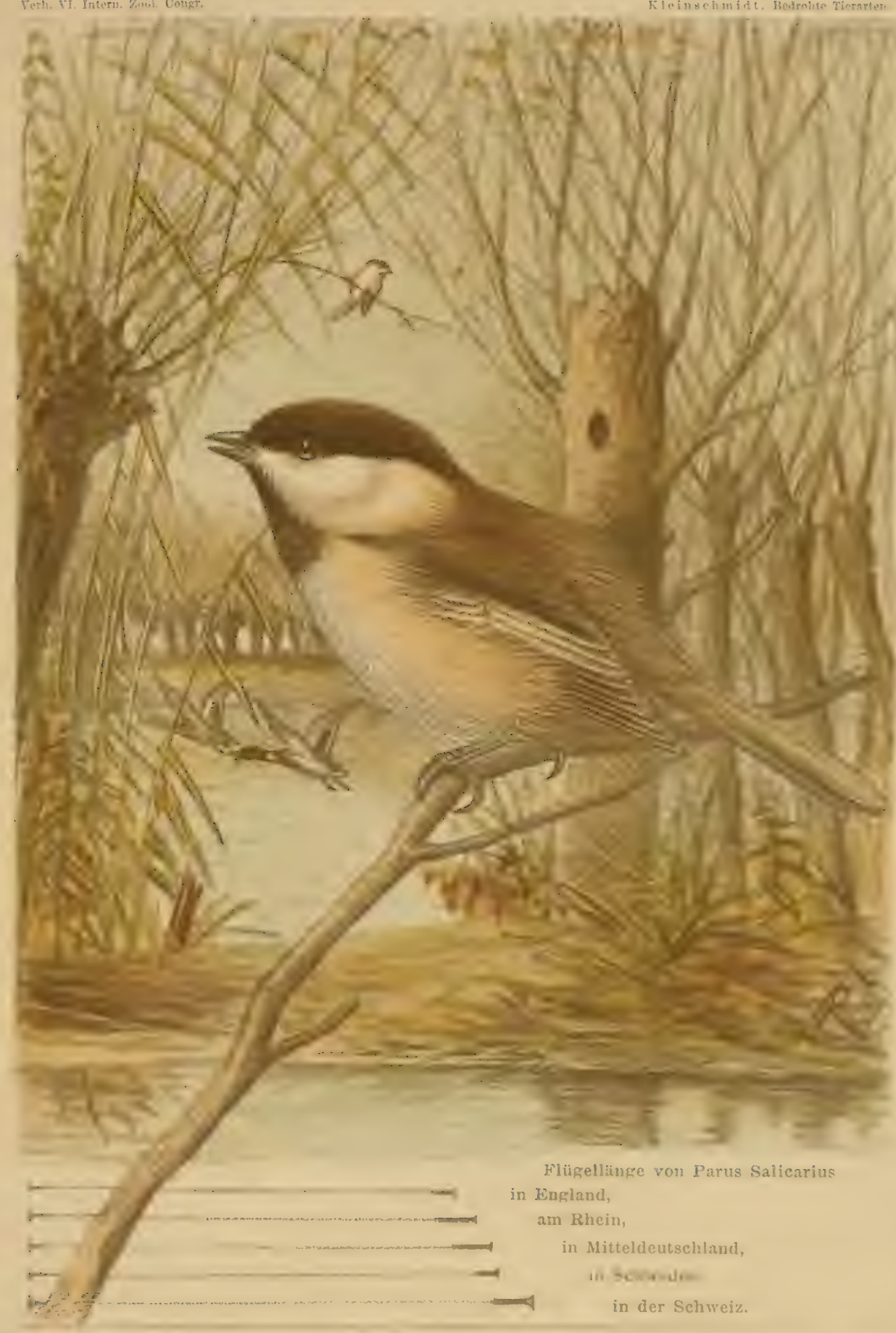

Filugellange von Parus Salicarius

Eugland,

am Rhein,

in Mitteldeutschland,

an sow ande=

in der Schweiz.

Steinzeichn. v. O. Kleinschmidt.

Bunedruck v. Fir. Eugen Fühler. 

Hier ist es nun zu spät, aber bei der Leichtigkeit, mit der sich Ibisse in (refangenschaft zur Foltptlanzung ${ }^{\prime}$ bringen lassen, liönnte man die interessanten Vügel in Volieren züchten, und sogar mit einer freilich seh" geringen Aussicht auf Erfolg einen Wiedereinbürgerungsversuch wagen *.

Zum Schluss muss ich hier noch von einem aktuellen Beispiel reden, wo es sich in den nächsten Jahren entsicheidet, ob ein wissenschaftlich hochinteressantes 'Tiel' sozusagen unter unser'n Augen vor der rastlos fortschreitenden Bodenkultur für immer verschwinden wird oder nicht. Es ist mu ein kleiner Vogel; aher das wissenschaftliche Interesse l'jehtet sich ja nicht nach der Körpergrösse der Tiere. Es ist auch nicht der letzte Rest eimer Art, viehmeln ein Togel, der von England bis nach fapan und Nordamerika in zahlecichen ähnlichen spezifisch identischen Formen verbreitet ist. Neben all diesen Formen kommen in der alten Trelt ihnen tïuschend ïhnliche Formen einer ganz anderen Species vor. Für die morlernsten zoologisehen Probleme ist dies ein Musterberispiel, wie sich wenige in gleicher Vollständigkeit finden. In diese Gruppe hrirht num die Ausrottung gerade des für ihr Verständnis allerwichtigsten Cilierles eine Lürclir. Fs handelt sich um den vor einigen Jahren aufgefundenen in den unzugänglichsten Rheinsüm)fen lebenden Parus sulicarius rhenams oder Parus montanus rhenams, über don ich bereits gestern in der Gelition für Tiergengraphie gesprochen habe und den ich hiel inmitten der tatsächlich ver'wandten und der nur äusserlich ähnlichen formen rorlege. Injse merkwürdige Ineisengruppe hat berejts rine ganze Litteratur hervorgeruf'n und wird die Zoologie, nicht nul' die Spezialisten, noch lebhaft beschäftigen. Wenn aus einem so vollständigen bilıle ein Stück mitten herausegeschnitten wird, so ist das fast norel? ägerlicher als womn das ganze nun dorh pinmal entstelle Bild nicht da wäle. Gewissermassen handelt es sich hier auch um einen Alpenvogel - dem Namen, der Nomenklatur nach, denn der Vogel ist eine dunkel gefärbtr in der Ebene lebende Zweroform der Alpenmeise (s. die Abbil(lnug). Yan lï̈nnte anmehmen, dass der Togel sich von den Alpen aus an den Rheinufern entlang ausgebreitet und gleichzeitig umgehildet hätte, ähnlich wio mit der Flut des Rheines, del ja anch ein Schweizer Kind jst, dio Bergkiristalle himabrollen und damn noch selten als alıgeschliffone "Lhoinlinsel n sich fimlen. Achnlich könnte die Sumptineise zur Alpenforms sich bildend und in die Ehene zurücklehroud dor gewöhnlichen For'm wieder ähnlich gewor

${ }^{1}$ Es sei hier an die interessanten Erfolge des Zoologischen Gartens in Berlin erinnert.

2 Der interessanteste derartige Versuch würde die Uebertragung von Sitta whiteheadi von Corsica in einen isolierten Alpenwald sein. Dem steht nur die Seltenheit des Vogels entgegen. 
charaktere verlor. Abor das (resamtbild zwingt zu ganz anderen Annahmen, und darum ist seine Vollständigkeit für das ganze Verständnis der Alpenfama wie für die künftige zoogeographische Arbeit üborhaupt von ausselordentlichem Wert ${ }^{1}$.

Es würde in diesem Falle genügen, an einzelnen Stellen der Rheinufer dis vollständige und plötzliche Ahholzung von Kopfweidendickichten zu verhindern. I ann wird der Vogel erhalten bleiben und noch zu mancher fördernden Debatte Anlass geben.

Die Cregner haben ja an der Tatsache, üher die sie streiten, das gleiche Interesse. Ind darum ist hior ein (rebiet, in dem wir alle einig sein müssen. Man wird uns in spateren Zeiten I)ank wissen auch fül das Geringe. was wir in der Erhalturg vielleicht nur vereinzelter 'Tierformen vorläufig creschen. Mäge es der hescheidene Anfang zu grösweren Erfolgen sein ${ }^{2}$.

Ich unterbreite dem permanenten Congress-Comité folgende Beschlüsse mit drr. Bitte, sie bis morgen zu prüfen und sodamn eine $A$ bstimmung des Congresses darüber zu bewirken :

Die Beschlüsse, wie ich sie mir vorzuschlagen erlaube, lauten:

1. Ier VI. internationale Zoologencongress spricht den Herren Präsidenten liünftiger intermationaler Zoologencongresse die Bitte aus, die Angelegenheit des Schutzes gefïhrdeter Tierarten vor dem Aussterben weiter im Auge behalten zu wollen.

2. Ier in der Schweiz rersammelte VI. internationale Zoologeneongress stellt an die einzelnen Staaten den Antrag, solche Vertilgungs-Prïmien, die zur (reführoung oder gïnzlichen Vernichtung irgendwelcher Tiere beitragen lömnten, rechtzeitig aufzuheben, wenn diese Tiere einen üherwiegenden wissenschaftlichen oder isthetischen Wert hesitzen und womöglich einzolne sichele Heimstitten fül die Landesfama zu schaffen übreall da, wo die fortschreitrude Kultur dies als geboten erscheinen lässt.

Inor Congress stellt an den hohen Bundespat das Ansuchen, diesen Beschluss den einzelnen Regierungen übermitteln zu wollen.

'Ich freue mich, hier bereits den zahlreichen Congressmitgliedern, die meine $\mathrm{Ab}$ sicht befürwortend begutachtet haben, mitteilen zu können, dass das grossherzogliche Mlinisterium des Innern zu Darmstadt sich inzwisehen bereit erkliart hat, der Angelegenheit seine Förderung angedeihen zu lassen.

${ }^{2}$ Da in Amerika so Grosses auf diesem Gebiet geleistet worden ist, haben wir von dem nächsten Congress sicherlich dankenswerte Anregungen zu erwarten.

M. le Président domme la parole à MI. F. Sarasin. 


\title{
TIERGEOGRAPHISCHES, BIOLOGISGHES UND ANTHRO- POLOGISCHES AUS CELEBES
}

\author{
Von Dr Futz SARASIN
}

(Basel),

Es geschieht eigentlich gegen meinen Wunsch, dass ich hente in einex allgemeinen sitzung einen Tortrag üher ('elehes halte, denn die Ergehnisse unserer letzten Reise sind noch weit davon entfernt, durchgearbeitet zu sein ; aber es ist mir nicht möglich gewesen, dem liebenswürdigen Ibängen unseres verehrten Herrn Kongressprïsirlenten auf die Dauep Widelstand zu loisten, und so muss ich Sie sehon um Entschuldiguug litten, wenn ich auf manches früher schon veröffentlichte zuröckgreife (13).

Die ' $\mathrm{T}$ i er' g o gr a p h i e hat in den letzten Jahren eine dur'chgreifende Umgestaltung ihrer Aufgahen erfahren. Zur Zeit als Sorter in geistreicher Weise die Fro in seine Regionen einteilte, glaubte man, dass diese Regionen Realitäten seien, und man musste es glauben, denn DARIVIN's Werk wal noch nicht erschienen, und die Regionen deckten sich mit dem gewohnten Begriff der schöpfungseentren. Keine Aufgabe schion likarer und dankbarer zu sein, als nach den Grenzen dieser Centren zu suchen und die Regionen weiter in Unterregionen einzuteilen. Da nun aber nach der Descendenzlehre die gesamte Welt der Organismen sich bildlich als ein vicherzwejgtel Riesenbaum darstollt und nicht, wie man früher dachte, als nebeneinander gepflanzte Einzelbäumchen, deren Grenzen wohl bestimmbar wären, so erscheint die Fragestellung wesentlich verïndert. Die sog. Regionen sind num nicht mehr Primare-Divisionen des Erolballs, sondern blos Teile der Firdoberfläche, die während mehr oder minder langer Zeit mehr oder minder vollständio isoliert geblioben sind, wohei sich n'ue Familien, (xattungen und Arten ausgebililet haben orber alte vor dem Verdrïngen bewahrt gebliehen sind. Iabei ist es von jeher der Willkür jedes Einzelnen über'lassen gewesen, für welche Zahl eigener Formen er eine Region oder Subregion creieren mochte, woraus allein schon das Künstliche solchep Einteilungen sich des deutlichsten ergiebt.

Die Aufgabe der 'Tier- und Pflanzengeographic kann also nicht melne sein, nach don Grenzlinien von Plantomen, we es die Regrionen sind, zu suchen, sondern sie ist hente wesentlich rine historische Wissensehatt geworden. Ihr Ziel ist. die Besiedelumgsesehehichte del Kontinente und

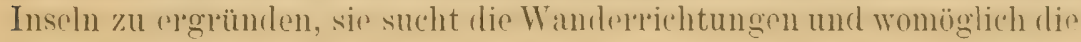


Wanderursachen der Lebewesen in reroangenen Erolperioden zu ermittoln, schlïgt Brö̈cken üher Strecken, wo jetzt Neere liegen und wird dadurch zu einer Hilfswissenschaft der Geologie, einer Art sulmariner Grologie, wemn sie wollen. I)ass sie selber nur denkbar ist in Terbindung nit geologischen studien oler wenigstens Kenntnissen, ist selbstverstindlich. Wïhrend früher die Tiergeographie gewissermassen aus der Vogelperspective die Erde in Regionen teilte, so ergielst sich jotzt, dass jele Insel, und sei sie noch so klein, ja unter Lmständen. wie dies bei Colohes der Fall ist, jeder Teil einer Insel eine eigene verwickelte Besiedelungsgeschichte haben ham, die zu ermitteln eben die Aufuahe unserer Wissenschaft ist.

An lieinem Punlete dor Errle hat der Streit um eine Regionengrenze so heftig getoht als auf dor Insel C'elebes. Hie australische, hie orientalische liegion! tremnte als Feldgeschrei die Lager. wähend In'itte ein ['eberoangsgehiet zwischen den beiden liegionen aufstellten. Als wir vor ca. 10 Jahren unsere Arbeiten in Celebes begannen, sind wir von dem Gedanken ausgegangen, del heutige indo-australische drehipel sei entstanden durch Zersplitterung eines vortertiären Kontinentes, der cinst Asien mit Australien verbunden habe, und es handle sich nun darum, zu verfolgen, in wolcher Weise diese Auflösung vor sich gegangen sei und wo die älesten Bruchlinien dirse Landmasse durehsetzt hätten.

Allein die Geologie der Insel Celebes zeigte bald, dass diese Voraussotzung un ichtig sei. Mächtig entwickelte, frühtertiäle Kalke brwiesen, dass in diesel Periode die Insel unter Wassel gelegen hatte; ihe Erhehumg und Auffialtung hegann, wie es scheint, erst im Miocän. Ablagerungen aus dieser Zeit tragen ihren Einschlüssen nach den Charaliter von Strandbildungen. Erst in's Miocän verlegen wir die cl'ste Besiedelung des neu gehildeten Landes und zwar von der asiatischen Seite here I)ieser 'rsten Invasionsschicht möchten wir die verschiedenen altertünlichen oder heutzutage im Arehipel ohne nähere Verwandte dastehenden For'men in der 'Tierwelt der Insel zuschreiben, unter den Säuger'n volnehmlich den Babirussa, vielleicht den Pavian, den seltsamen Paradoxurus Musschenbroli und einige Muridx, unter den Vögeln Gattungen wiu streptocittu, Citture, scissirostrum, Mulire. Aramidopsis u. a., unter den Reptilien Testudo Forstenii, unter den Mollusken Mirateste u. S. w.

I)ie in Niocän begonnene Hehung von Celebes und mit ihm des ganzen umgebenden Arehipels setzte sich im Laufe des Pliociun fort und führte zu einer Periode ansgedehnter Landrerbindungen mit umliegenden (iehieten. Im num die Herkunft der Fauna von Coldes zu elmitteln, haben wir für jestr species ron Land- und süsswassersehneclen fes waren deren 2:sic). Weiter für die 10s Reptilien und Amphibien der Insel und für eine Anzahl Vögel und Sïugetione Verboreitungskarten angefertigt. Bald 
stelle sich heraus, diss diese Karten für zahlleciche Species überein-

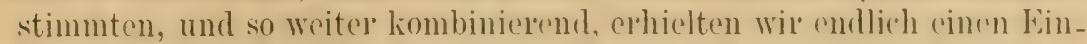
blick sowohl in die Verteilung der Arten auf dex viclgestaltigen Insel selbst, als in die. Beziehungen zu den Nachbarländern.

So rergab sich \%. B., dass Süul-Celebes mit Java chenso viele MolluskenArten gemein hat als mit Nord-Celebes, dass Nord-Colohes in domselluen Vorhältniss zu den Philippinen steht, wie Süd-Celohes zu dava. und daraus schlossen wir. dass Landverbindungen müssen bestanden haben, welehe Java mit Süd-Celebes, die Philippinen, speziell Mindanao, mit NorelColebes vereinigten. Aussel Java und den Philippinen (2loahen sich aber noch zwej woitere Brzugsquellen, nämlich die kileinen sundainseln im Süden und die Molukken im Osten.

Wir erhielten somit das folgende geographische Bild: I) jo nördliche Inalhinsel setzte sich nordwärts fort üher die heutige singrigruppe nach den Philippinen: dio östliche Halhinsel schloss die Sulainsoln oin, gabelte sich dann in zwei Arme, von denen dre nördliche ühere das hentign ()hi

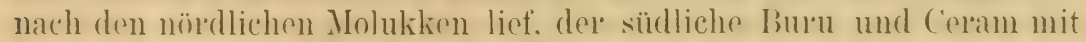
Celebes vernüpfte. I)ir Molukken ihrerseits hingen mit dom zu dieser Zeit märhtig angeschwollenen Neu-(ruinea und somit indiredit mit Australien zusammen. Von del südlichen Halbinsel von celebes endlich gingen zwei Lamdhrüthen aus, von denen die eine nach Java. die andere nach dem likeinen Sumlagebiet, speziell nach Flores führte. Auf allen diesen vier aus der Analyse der Fauna als notwendige Forderungen

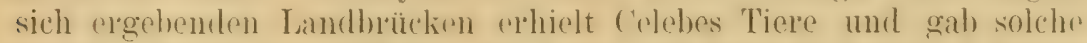
weiter : so lïsst sich vorfolgen, wie javanische 'liere nach Celobes kamen und sich weiter über die Molukkenhlücke nach ()sten verholeiteten, orler

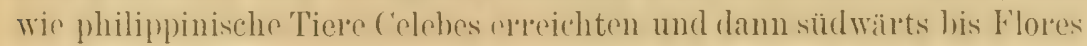
wanderten oder molukkische Elemente über ('olebes nach den Philippinen gelangten. Dahei ist es für unsere Betrachtung gloichoiltig, ob diese Landbüucken alle gleichzeitig bestanden oder ob. was heute noch nicht entscheidbar ist, eine gewisse Abwechslung stattfand.

Ganz andel's als zu Java, den Philippinen, Molukken und kleinen Sumbla-Inseln ist das Verhältniss von Calehes zu borneo. Mit allen den genannten vier (ichieten nämlich hat ('clebes rine ganze Anzalıl von Arten gremeinsam, olme dass sie cine weitere Verbecitung im Arehipel besissen. Solche ausschliesslich gemeinsame und daher deutlich eine alte Verbindung anzeigende Arten hesitzen aber Borneo und ('alebes keine, obschon sie nur durch die schmale und in ihrem südlichen 'Teile wenig tiefe Makassarstrasse von cinander getremnt sind. Mlle Species vielmehr, wolche Bornoo und Colehes zugleich zukommen, finden sich auch auf Java oder auf den Philippinen. I) ie Alt ihrer Wanterung wird hiedureh ohne weiteres klat. Es sind asiatische oder sundaische Tienarten, welche entwerler die Landhrïcle von Java nach süd-Colebes he- 
nützten odel' von Nol'd-Borneo aus, sei es über die Palawan-, sei es über die Sulubräch nach den Philippinen gelangten und von dort aus Celebes erreichten. Dic ursprüngliche Heimat anderer Borneo und Celebes gemeinsamer Arten kann auf den Philippinen gesucht werden, von wo aus sic leicht Nord-Borneo und Tord-Celebes invadieren kounten. Eine direlite ['oherlurückung der Makassarstrasse gah es indessen nicht.

I)ie Einsicht, dass Borneo und Celebes lieine Tierart ausschliesslich beherbergen, erhielten wir zuerst aus dem Studium der Land- und Süsswasser-1lollusken. Auch unsere neuen Sammlungen, die wir auf der langen Reise durch das westliche Central-Celebes, also gerade auf der Borneo zugekphrten Seite der Insel anlegten, haben hierin keine Veränderung gobracht. Es war aber damals, als wir dieses Ergehniss zuerst ausiprachen, auch die Molluskenfauna des ()sthorns von Borneo, welches sich C'elebes so hegehrlich entgegenstreckt, dass man eine alte Verbindung vermuten kömnte, noch unbekannt. Diese Lücke ist nun auch ausgefüllt worden. und es schrieb neuerdings hieriuber yos Martexs (9): Es bleibt also dabei, dass die Celebes nächste Gegend ()st-Bol'neo's in den Landund Süsswassermollusken ganz ('ntichieden auf der Seite von Borneo und im Gegensatz zu Celebes steht.

Dasselbe Ergebniss hatten uns die Reptilien und Amphibien geliefert, und auch unsere neue, von Dr. Roux (11) bearbeitete Sammlung hat nichts daran modifiziert. Füı unsere Annahme der Besiedlung der Insel mittelst Landbrüclien ist es wichtig, dass einige javanische líriechtiere, die bisher nur rom Norden von Celehes bekannt gewesen waren, sich num auch im Süden gefunden haben, da ihre angenommene Wanderung von Java her ihr Vorkommen im Süden der Insel als notwendig voraussetzte.

Nicht anders steht es mit der Verbreitung der Säugetiere und Vögel, und es ist namentlich bei der scheinbar so grosen Beweglichlieit der letzteren Tiergruppe wunderbar genug, dass Bor'neo und Celebes, deren Fï̈sten über 6 Breitengrade hin einander fast parallel laufen, keine einzige Vogelart auschliesslich gemeinsam beherbergen. Auch hieran ändern unsere neuen Sammlungen nichts. Seit dem Abschluss unserer tiergeographischen Arbeit sind noch eine Anzahl weiterer faunistischer Publikationen über unsel Material erschienen. So berichtete Brendix (1), dass von den 239 heute bekannten Rhynchoten-Arten von Celebes keine rinzige ausschliesslich Bolneo und Celebes eigen sei, wogegen zahlreiche mit Java oder den Philippinen oder den Molukken gemeinsame Arten beständeu. Nicht anders äusserte sich Euers (4) über die Ameisen. Ich kann, sagt er, den Satz, dass keine Tierart aussehliesslich Borneo und Celebes zukomme, für die Ameisen bestätigen. Die Süsswassel- und Land-Crustaceen, eine in Celebos reich entwickelte 'Tiergl'uppe, geben nach Schexket. (15) und Rocxis (12) Arbeiten dasselbe Terbreitungsbild, keine ein- 
zige mit Borneo ausschliesslich gemeinsame form gegenüber heispielWeise oiner ganzen Anzahl aussehliesslich mit den kleinen Sunda-Inseln überoinstimmendon Arten. Beiläutig gesagt, ist es möglich, dass dio reiche C'ustacernentwicklung auf Celehes eine Folge der Armut seiner Fischfauna ist.

Nur bei den ()ligochæten glaubte Michaelsen (10) eine näher'e Bezirhung zwischen Borneo und drum Norden von Celehes zu sehen. I ass diese aber lieine direkte war, sondern dass die Wanderung über das heute noch schlecht hekannte Mindanao geführt hat, beweist das Vorkonmen rines Vertroters der einzigen gemeinsamen Altengruppe von Pheretinu Stelleri (Michl.) auf dem Inselchen (iposs-Sangi zwischen Nord-('elehes und Mindanao.

Wir rhalten somit das durchaus gesicherte Resultat, dass linine 'Tierwamderumg von Borneo direkt hinüber nach Colebes stattgefunden hat, sondern dass die Besiedlung von Celebes mit asiatischen Tierformen einerseits von Java aus nach Süd-Celebes und anderer'seits von den Philippine'n aus uach Nord-Celebes vor sich gegangen ist. Die Makassar'strasse ist somit eine tiergeographische scheidelinie, aber durehaus niclst etwa im altrn simne eine Iiegionengrenze, sondern lediglich ain Meeresarm, der in jüngerer geologischer Verwangenheit niemals in festes, verbindendes Land sich verwandelt hat.

Wir haben nun des Weiteren versucht, nachzuweisen, wie etwa in Procenten dir Anteile der vier Landbrücken an Aufbau der celebensischen Tierwelt sich verhalten mörhten und fanden das folgende: Nach Alyzug der weitverheiteten Arten und der endemischen unsicherer Terwandtsehaft. Welcherasammen $26^{\circ}{ }^{\circ}$ der Fama bilden, stellt sich der Anteil

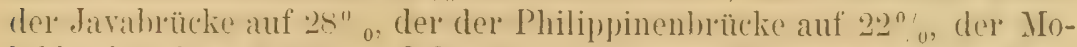

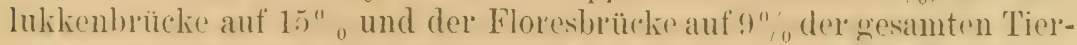
welt. I)iese Zahlen wulden gewommen aus dex Alualyse der Mollusken, Krochtiere und Tögel. I) Javabritcke ist somit die wichtigste der Besiedlungsitrassen, ein Ergebniss, zu dem auch Bredons, vom studium der Wanzen ausgehend, gelangt ist; sie dürfte auch die älteste sein, denn auf ihr vollzog sich vermutlich schon die miocäne Wanderung nach Celebes hin, womit aber nicht gesagt sein soll, dass sie nicht, was sogar wahrscheinlich, zeitweilig Unterhrechungen erlitten haben liönnte.

In zweiter Linic folgt die Philippinenbrücke: beide zusammen lieforten reichlich die Hälfte der celebensischen Fauma. Dagegen tritt die .Io-

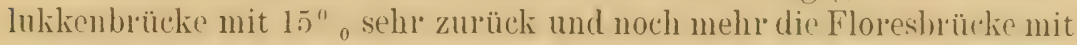
nur $4 \%$. Wem man es nicht allzugnnau nimmt, kann man sagen. dass die Anteile der vier Brücken sich verhalten wie $4: 3: 2: 1$. Die Fauna von Coleber ergiht sich somit als eine verhätnismïssig moderne Vischfauna aus vier benachbarten (iehneten, nämlich aus Java, den Philippinen, den Molukken und den kleinen Sunda-Inseln, wobei der javanisch- 
philippinische Charakter überwingt, vergesellschaftet mit Resten einer älteren Invasionsschicht.

I ie Tierwelt von Celelses zeigt weiter die Eigentümlichkeit, dass einzolne (iruppen reich, andere dagegen schwach vertreten sind. An Artenzahlı reich entwickelt sind beispielsweise die Mollusken, gewisse Insektengruppen, die Landplanarien u. s. w., etwas schwächer die Amphibien, Reptilien und Yägel, noch schwächer die Säugetiere, ganz schwach die Süsswasserfische. Die Erklärung für diese Erscheinung ist unschwer zu finden, sie liegt in der angenommenen Besiedelungsweise der Insel mittelst unserer Landbrücken.

Es ist erstlich klar, dass diese Wandergelegenheiten jeweilen nur von einem gewissen Bruchteil einer Fauna benützt wurden oder benützt werden konnten, und nun zeigt sich, dass nur diejenigen Tis'gr'uppen in Celebes gut vertreten sind, welche in allen vier Quellgebieten eine mehr orler minder reichliche Entwirklung aufweisen. Dies gilt unter anderem für die Mollusken. Für die Süugetiere aber versagten z. B. dir Flores- und die Jolukiken-Brücke fast ganz, weil diese Gebiete selbst an sïuger'n äusserst arm sind. Blos Pluclenger ist eine charakteristische Gattung der Molukken-Brücke. Somit bliehen nur die Philippinen, welehe sellst an Säugern nicht eben reich sind, und Java als Bezugsquellen ührig, und die Folge ist die ziemlich schwache Vertretung dieser Gruppe auf Celohes. Noch mehr gilt dies für die Süsswasserfische. Bei diesen beginnt die Abnahme an Artenzah! bereits deutlich in Java, und die anderen (iebiete kommen als Lieferanten gar nicht in Betracht, daher die ungemeine Armut von Celebes an Süsswasserfischen. Die Vertretung jeder Tiergruppe auf ('elebes -- so kann der Satz etwa lauten - ist proportional der Vertretung derselben in den vier Quellgebieten.

Fs frägt sich nun, wie sich die Resultate der oceanographischen Untersuchungen, wie sie in so ausgezeichneter Weise die niederliandische Siboga-Expedition unter Prof. Nax W EBer s ( 16 u. 17) Leitung im Arehipel vorgenommen hat, zu unseren angenommenen vier pliocänen Landbrücken stellen. Da fand sich erstlich, dass Süd-Celebes mit Ost-Java durch ein wenig tiefes Plateau verbunden ist. welehes die Paternosterund P'ostillon-Inselgruppe, Kangeang und Madura trägt. Dieses Plateau wird durchschnitten von 'Tiefen. welche $600 \mathrm{~m}$. kaum ïherschreiten und fällt nach Osten steil in die bis 5000 m. tiefe Flores-See al) ; (es ist der unverkennbare Rest der alten .Javabrüicke.

Ines weiteren wurde durch die Siboga nachgewiesen, dass die Inseln zwischen Nord-Celehes und Xindanao auf einem Rïtcken liegen, der die kalten tiefen Wasser des pacifischen Oecans ron dem $5000 \mathrm{~m}$. tiefen Becken der wäl'meren Celebes-See abhält. Die tiefste Stelle dieses Rückens wurde südlich von Mindanao zu $1638 \mathrm{~m}$. bestimmt; durch seinen Nachweiserhailt auch unsere Philippinenbrücke ihre oceanographisehe Stütze. 
Sehwierger liegen die Verhältnisse bei den beiden andern tiergeographiseh geforderten Landverbindungen, vor'uehmlich der Molukkenbrücke. Wohl fand sich zwischen den Sula-Inseln und der Molukkeninsel ()bi eine Barre, welche in einer 'Tiefo von nicht mohr als 15 - $1600 \mathrm{~m}$. dic Ceram-See von der Molukken-See trount; ebenso sind Ceram und Buru durch eine Barre vereinigt, aber zwischen Buru und den SulaInseln wurde bei einer Lothung die cnorme Tiefe von über $4000 \mathrm{~m}$. gefunden. Ob nun freilich damit bewiesen ist, dass nicht doch ein untieferer, schmaler Verhindungsiücken existieren könnte, diese Frage scheint hei den so sehı abrupt sich ändernden leoliefverhältnissen im Archipel wohl kaum endgiltig eredigt. Aber selbst wenn diese Tiefen durchgehen sollten, müsston wil bei de Forderıng einer Ladudverbindung zwischen Colohes und Buxu bleihen. Ein Tier wie der Babirussa kann nur über Land und nur von Celebes her Buru erreicht haben, und nicht ander's steht es mit einer ganzen Reihe anderer Lebervesen; hat doch noch unlängst Hantert (6) nachgewiesen, dass die Vogelwelt der Berge von Burul ausseroritentlich starle. Westliche Verwandtschaft hesitze. Wir finden dort z. B. einen Papagei aus der durch lange Schwanzfedern charaktrisirten (xattung Prioniturus, die nur Celobes und den Philippinen eigenthümlich ist, eine ächte Brückenform.

Es ist eine naheliegende, aber sicherlich unrichtige Betrachtungsweise, bej der Rekonstruktion alter Landmassen sich ausschliesslich von den hentigen Tiofonverhältnissen der Mere leiten zu lassen. Fin seichtes Meer kann natürlich sehr wohl und wird auch in der Regel ein junges spin: (si kimn aber auch ein altes, durch Auffüllung seicht gewordenes sein. was namentlich im Arehipel bei der enormen Erolzufuhr der Flüsse leicht denl:har ist: es kamn endlich auch ein in Hebung begrifferes sein.

Anderersoits giebt es tiefe Meerestheile, die ohne Zweifel jung sind. So fand die siboga eine bis $3000 \mathrm{~m}$. tiefe Deplession zwischen dou Aluund Kei-Inselgruppen, dreen Fama einen noch jungen Zusammenhang verlangt. So fischte diesellon Expedition in der Ceram-See zwischen 1300 mul 160u Meter Tiefe. 30 Seemeilen von den Küsten entfernt, mit Mangan üherzogene Fragmente litnaler Korallen, die zu ihrem Gedeilıen ein untief $M$ ser vorausset\%en. Ton den spättertiären Veränderungen des Mittelmeerbeckens wollen wir hier nicht rerlen.

Lokale Senkungen gehen auch heute noch im Arehipel rasch vor sich. So hiaben wil auf der letzten lieise in der Mingkoka-Bai auf der südöstlichen Halbinsel zu unserem Erstaunen bemerkt. dass dort Kokospalmen und ächte Waldbäume bei Fluth im Meerwassel und nul bei niedrigster Elube noch auf trockenem Grumde standen. Die meisten waren auch bereits ahgestorben, und ein chinesischer liaufmann versichelte uns, er habe im Laufe weniger Jahre soine Vorrathshäuser wanz beträcht- 
lich landeinwärts rüicken müssen, um sie vor dem immer weiter vor'dringenden Meerwasser zu schützen.

(ranz dasselhe berichtet Carthaus (3) ron einer Stelle an der Westkïste von C'entral-Celebes. Dort stehen die toten Stämme des Urwaldes sogar wïhrend der Eblue schon fusshoch im Meerwasser. Es sind dies Fälle ausserordentlich rascher, während der Lebensdauer von Ptlanzenindividuen sich abspielenter, lokaler Senkung. denen ebensolche von Hebung angereiht werden könnten. Würde es uns gegeben sein, dic Jahrtausende in Winuten zu verwandeln, und damn den ganzen Archipel zu überblicken, so würden wil das Bild einer wogenden Masse erhalten; wir würden Inseln langsam verschwinden und an andern Stellen Festlïnder auftauchen sehen, nicht willküllich zwar, sondern grossen tektonischen Leitlinien folgend.

Eine ganz besondere Aufmerlsamkeit verdient die Süsswassertierwelt von C'elebes. In Herzen der Insel liegt der grosse und über $300 \mathrm{~m}$. tiefe Posio-See, den wir im Jahre 18.5 besuchten. Disser ist mamentlich an Mollusken ungeheuer reich, so zwar, dass die toten Sehalen den Strand stellenweise wie an Meeresküsten bedecken. Diese Molluskenfauna zeigt einen decidiert altertümlichen Charakter. Ihre Melani'n gehören mit Ausuahme von zwei weitverbreiteten Arten derjenigen Gruppe an, die wir nach Bau von Deckel und (relsiss als Alt- oder Palaeomelanien bezeichnet haben; eine eigeue Gattung mit schwieligem Mundrand musste als Tylomelania abgetrennt werden. Weiter waren die Limnaiden durch zwei merkwürdige, kiementragende Gattungen, Wiratesta und Protancylus vertreten. Eine Fauna ähnlichen Charakters beherbergen die beiden grossen Seen Matanna und Towuti im Nor'den der südöstlichen Halbinsel, welche tektonisch in der Fortsetzung derselben Mulde liegen, die auch den Posso-See trägt.

Das auffallende ist nun, dass die Molluskeu-Wrelt dieser drei obengenannten Seen ausserordentlich abweicht von der der übrigen in Celebes verteilten Süsswasserbecken, des Tondano-, Limbotto- und Tempe-Sees. In diesen dominieren die Neomelanien, und die oben namhaft gemachten drei Gattungen fehlen ganz. Es ist dies aus zwei Gründen bemerkenswert. Fimmal zeigt es, wie schwierig die Verbreitung der SüsswasserMollusken-Fauna vor sich geht und zweitens gestattet es, einen Schluss auf das relative Alter der genannten Seen zu zichen. Die Bildung und Besichlung der drei grossen centralen Becken möchten wir dem Charakter ihrer'Tierwelt nach in's Miocän oder in den Beginn des Pliocäns setzen, während die anderen Seen pleistocäne Bildungen sind.

Wir haben num auf unserer letzten Reise 1902 einen weiteren See zum e'sten Nale untersuchen können, den Lindu-See im westlichen CentralCelebes. cin Beclien von etwa 8 Kílometer Länge und circa $70 \mathrm{~m}$. Tiefe, in grossartiger Beloglandschaft etwa $1000 \mathrm{~m}$. hoch gelıgen. Da er vom 
Pos:o-Sre in Luftlinie nur etwa 90 Kilometer entferent ist, elwarteten wile eine Tebereinstimmung im Charakter der Fauna zu finden. Allein diese. Vermutung traf nicht zu. Unter den Mollusken her'schten hier die Senmelanien vor, und die beiden einzigen Palaeomelanien waren livine eigenen Arten wie in Posso-See, sondern solehe weiter Verbreitung. Ier ührige Bestand setzte sich aus Ampullaria, Vivinara, Limmea, Planorbis, Bithynia und Corbicula zusammen. Die drei eigenen Gattungen des Posso-Sees fehlten. Der Lindu-Sec gehört somit nicht in die Katrogorie der drei Sern des centralcelehensischen Seengrabens mit altertümlicher Fauna, sondern muss jüngerer Entstehung sein.

Einer der Charakterzüge der Süsswasser-Fauna von Celebes ist das Fehlen der Unioniden, und es ist dies um so merkwürdiger, als einerseits im Westen Java. Sumatra und Borneo und andererseits im ()sten Australien und das südliche Neu-Guinea Lnioniden beherberen. Aus den hydrographischen Verhältnissen von Celobes ist dieser Mangel nicht zu rlibären, denn mit tiefen seen und grossen Flüssen ist die Insel überpeich gesegnet. Wir müssen viehmehr auch für die Verbreitung dieser Gruppe nach geologischen Trsachen suchen. Beiläntig gesagt, ist der Inionidrmmangel auf Colebes oin schlagender Buweis für die Ohmmacht der kiunstichen Verbreitungstaktoren. da es den seit Jahrtansenden und Jahrtausenden aus Unioniden reichen fehieten hergetlogenen Myriaden von Wassergetlügel nicht gelungen ist, die Gewisser von Celebes mit Unioniden zu bevölkern.

Wir hahen uns fröher godacht, die Verbereitung der Unioniden nach Australien sei auf einem vortertiären, asiatisch-australischen Continent vol sich gegangen; dieser Continent sei dann zerfallen, Australien isolierend, und bei unserer sckundïlen Brïclienperiode im Pliocian hätten die Unioniden aus irgend einem Grunde, wahrscheinlich wegen ihrer Emptindlichleit gegen Brackwasser, dio neuerdings gegehene Verhreitungsmöglichkeit nach Celebes und den Mlolukken nicht benützen kïnnen.

Allein je weiter die geologische Erforschung des indo-australischen Archipels fortschreitet, um so schwieriger wirel es, fül diesen alten asiatisch-australischen Kontinent einen Platz zu finden. Fin Jurafestland ist durch die Arbeiten von Martix. Wromaxi, Böna u. A. zu1 Unmöglichkeit geworden. In die eretacische Zeit hinübergerettet, wird ihm auch hier durch immer mehr sich häufende Nachweise mariner Kreideablagelungen der Boden Schritt für Schritt strittig gemacht. Nun hat zwar im Beginn der Tertiärzeit cine grössere Festlandentwicklung stattgehaht; ihr gehören die abbruchfühigen Kohlenlager von Sumatra, Java und Borneo an, die auch in C'elebes untrehalb der mächtigen Declie von Numuulitonkalken nachweishar sind. Aber nichts beweist, dass dicses Festland sich ostwärts über die Molukken nach Teu-Guinea und Australien fortgesetzt habe. 
Anderer'seits mehren sich die Stimmen derjenigen Forscher, welche Australien mit Südamerika durch einen alten, erst in der Kreideperiode sich autlösenden, süıllichen, pacifischen Kontinent in Verbindung setzen. So glaubte unlängst ( $\therefore$ BurckHard'T (2) auf geologischem Wege den Nachweis fühıen zu können, dass die Ostküste dieses Kontinentes ungeführ mit rlel heutigen pacifischen Krüste von Chile zusammengefallen sei.

Aus dieser Quelle könnte Australien unter anderem seine Beuteltiere "rhalten haben, und es ist geviss bemerkenswert, dass nach ZscноккE (1S) und v. JAsicki's (7) Befunden dieselbe Cestodengattung Linstowia australische und südamerikanische Bentler heimsucht. Speciell für die Unioniden, von denen wir hier ausgegangen sind, suchte v. Jusring ( $)$ nachzuweisen, dass die australischen Vertreter zu chilenischen in nahen Beziehungen stehen.

Wenn all dies richtig ist, so brauchen wir allerdings nicht mehr nach cinem alten asiatisch-australischen Kontinent zu suchen, und es würde unsere pliocäne Brïckenperiode genügt haben, um Australien und Neu-(xuinea ihre asiatischen, westlichen Formen zuzuführen. Auf dem Wege üher Celebes mud die Molukkenbrücke hätten dann Neu-Guinea und Australien beispielsweise ihre placentalen Säugetiere erhalten, und es ist auch kein Zweifel, dass mehrere australische und neuguinensische Muridengattungen zu Celebes und den Philippinen enge Beziehungen aufweisen. Auf diesem Wege erhielt Australien auch den Mensehen und den Dingo.

Es führt uns dies zur letzten hier zu besprechenden Frage, dem Verhältniss des Menschen zu unseren Landbrücken. Wic bereits mehrfach gesagt, rerlegen wir diese Landverhindungen in's Pliocän; ilre Autlösung mag im Pleistocïn vor sich gegangen sein. Es ist somit kein Grund vorhanden, weshalb der Mensch sie nicht benützt haben sollte.

Wir glauben in der Tat, dass Celebes seine Urbevölkerung auf diesem Landwege von Asien her erhalten habe. Es gelang uns nämlich, in der Bevölkerung der Insel zwei Schichten zu unterscheiden, eine ältere und niedrigere und eine höhere. Die erstere wird dargestellt durch eine Reihe kleinwüchsiger, ziemlich dunkel gefürbter, wellighaariger und breitnasiger Stämme, die sowohl somatisch, als ergologisch eine bedeutsame Achnlichkeit mit den Weldas von Ceylon und auderen indischen Urstämmen aufweisen. Hicher gehören die Toála von Süd-Celcbes, ferner die Tomuna und Tokeja in der südöstlichen Halbinsel. Reste dieser kleinen Urbevölkerung haben wir auch in Central-Celebes mehrfach angetroffen, dort zwar nicht in ihren eigentlichen, abgelegenen Wohnsitzen, sonder'n stets nur als Sklaven der höheren Völker. I)iese kleinen Stämme halten wir für die Nachkommen der auf dem Landwege nach Celobes gelangten Menschen, Reste aus jener alten Wanderperiode, die auch nach Austıalien den Menschen brachte. The höheren celebensischen 
Stämme dagegen möchten wir als spätere, überseeische Zuzüge betrachten, welche sich dann in sehr ungleichem Maasse mit der Urbevölkelung vermischten und dieser einen Teil ihres IBlutes sowohl, als ihres Kulturbesitzes übermittelten. (xanz analog wie die Sprache der Weddas in Ceylon. ist auch die der celebensischen ['rstämme durch die der höheren Völker verdrängt worden.

['eber die frühere Kútur der L'rbevölkerung haben uns Ausgrabumgen in den Höhlen der Toala im Gebiete von Lamontjong, östlich von Makassar, Aufichluss gegehen. Diese Kalksteinhöhlen. welche zum kleinen Teil jetzt noch ron Toala's bewohnt sind, zeigten auf ihrem Boden eine his 80 cm. hohe Lage grauer Asche, und als wir diese durchsuchten, fanden wir darin zahlreiche Artefacte. Dir wesentlichsten waren Messer aus Silex, aber anch ans andorem Gestein, wie Andesit, ja selbst Kalkstein, weiter Schiber und Pteilspitzen, diese letzteren oft durch Finkrroungen am Rande gesïgt, fermer Spitzen aus Kinochen, polin'te Babirussahanel und durchlohrte schneckenschalen als Schnuck. Topfscherben fanden sich in den unteren Lagen keine, sonder'n traten erst gegen oben zu auf; Steinbeile fehlten durchaus.

Ton grossem Interesie wa die Mrenge zerschlagener und angekohltel Knochen und Zïhne, welchr mit den Artrfacten vermengt dir dsche erfüllten; sie zeigten, dass wir es mit einem reinen Jägervolk zu thun haben, ohne den Besitz zur Jahrung dienender Haustiere. Ein einziger Zahn beweist die Anwesenheit des Hundes. Ine heutigen Toala digegen haben, und zwar jedonfalls durch Berührung mit den Bugis, den Acher. zu bebauen gelernt und treiben Jagd nur als Nebenbeschäftigung.

Das Knochenlager in den Höhlen war aber auch nach anderer Richtung von Interesse, nämlich nach der zoologischen und tiergeographischen hin. I)ie Ibicke der Aschen- uni Kunochenschicht liess auf ein langes Bewohntsein dur Hählen sehliessen, und so durften wir hoffen, ein ziemlich altes Lager vor uns zu haben. Die Untersuchung ergab zwal keine für Celebes neue Tierat't, aber doch eine ander'e Verteilung als heute. Tamentlich ist die in vielen Ex'mplaren knnstatierte Anwesenheit des Babirussa wichtig. I)ieser fehlt heute in Sürl-Celebes, so viel man weiss, und bewohnt blos den Osten, das Centrum und den Norden. Da aber der Bahirusia vou der asiatischen Seite her Celebes besiedelt hahen muss, so war uns sein Fehlen in Süd-Celebes immer rätselhaft gewesen, und die Ausfüllung dirser Verbreitungslücke lian somit höchst willkommen. Unter den anderen Nährtieren ist in erster Linie die Anoa zu nennen, deren Ueberreste sich selr zahlreich vorfanden; sie ist heute zwar aus dem Gebiet vou Lamontjong verschwunden, ist aber auf den höheren Waldgebirgen der südlichen Halbinsel noch häutig. Writre. sammelten wir leichliche zerschlagene Skelettstüchr vom Wildschwein, Sus celebensis, vom Affen, Macacus maurus, von den beiden Beuteltier- 
arten, Phalanger msims mul celebensis, von einer Paradoxurusart, mehreren Flederhunden und Waldratten, worunter sich auch die im Süden noch nicht konstatierte grosise Lenomys Meyeri vorfand. Massenhaft zeigten sich ferner angekohlte Reste von Python reticulatus, ferner zerschlagene Sichalen von Muscheln, ('yrena und Batissa, und einer grossen, noch hente im Gebiet lebenden Landschnecke, die wir Namina toalam benannt liaben.

Dagegen fehlte im Höhlenknochenlager der Hirsch, hente ein ungemein häufiger Bewolner des Gebietes. Zwei Schneidezähne von ganz recentem Aussehen und ganz oben autliegend gefunden, bilden seine einzige Spur: kein einziges Artefact erwies sich als aus Hirsch horn hergestellt. Der Hirsch gehört also zweifellos nicht rer ursprünglichen Fauna der Insel an, sondern muss rin später Import des Menschen sein; sein Fehlen als Jagdtier in den Höhlen lässt keine andere Erklärung zu.

Wir haben daraufhin bei Beamten und Missionaren Erkundigungen eingezogen nach allfilligen Traditionen über die Geschichte des Hirschs. In der Minahassa, wo er heute ein häufiges Wild ist, wurde er nachweislich erst in den drejssiger oder anfangs der vicrziger Jahre des letzten Jahrhunderts durch Europäer eingeführt. Sonst sind nicht die Europä‘r', sonder'n die jagdlustigen Bugis die Verbreiter des Hirsches gewesen. I) Toradja's von Central-Celebes erimnern sich teilweise noch an sein elstes Frscheinen: andere lassen dieses Freigniss zu Grossvaters Zeit geschehen sein; in manchen (xehieten fehlt el hente noch. Am frühsten muss er in den huginesischen Rejchen von Süd-Celebes importiert wolden sein, demn Valentyn herichtet im Beginn des 1s. Jalnhunderts, dass Amlıoina seine für Jagdzwecke erforderlichen Hirsche von Java und von Makassar locziohe. Der Hirsch ist somit endgiltig aus der Liste der celohensischen Tiere zu streichen, womit auch die gelegentlich vorgesehlagene Ahtrennung eines Cerrus celebensis von moluccensis als nichtig dahinfällt.

Doch es ist hohe Zeit, zum Schlusse zu eilen. Auf die Periode der Landrerbindungen folgte in Pleistocïn eine solche, wo das Land etwas ticfer untrigetaucht war als heute, wie die zahlreichen subfossilen, üher die Strandhügel ausgest reuten Meerschnecken beweisen, eine Zeit, in der z. B. dir südliche Halbinsel durch einen Meeresarm in der Gegend von 'Tempe quer durchsehnitten war. Vermutungsweise lïnnen wir in dieser' Epoche dje Iesiodelung der Insel mit höheren Völkerschaften begimnen lassen. In Nord-Celebes und auf Singi besteht (nach Gradruand 5) eine Sise, nach welcher die Inseln nördlich von der Minahassa in alter Zeit mit der Sangigruppe und noch weiter nördlich mit einem grossen Land vorbunden gewesen seien; dieses Festland sei vom Meere verschlungen worden, wonach sich die Bewohnersüdwärts nach Celebes gewandt hättron. Sollte vielloicht in diesere uralten Leberlieferung eine Erimnerung durchklingen an jene verschwundene Verbindungsblücke zwisehen Nord-ce- 
lehrs und den Philippinen, welche die 'Tiergeographie zu fordern sich genötigt sah ?

\section{CITIERTE LITTERATUR}

1. Bredin, G. Die Hemipteren ron Cielehes. Abhandungen der Naturforsch. Ges. zu Halle, 24, 1901 .

2. Burckinatr, C. Traces géoloqiques d’un Ancien Continent Pacifique. Revista del Museo de La Plata. 10, 1900.

3. Cintriaus, E. Beobuchtungen auf Celebes und Sumatra, Sammlungen des yeologischen Reichsmuseums in Leinlen, I. Beiträge zur Geologie OstAsiens und Australiens, 6, 1900 .

4. Evenr, C. Formiciden ron Celebes. Zoolog. Jahrb., Alıteil. für Systematik, 14... 14, 1901 .

i. Grafaftaxi, N. De Mimahassa. Eerste deel., Haarlem, 1898.

i. Hantert, E. The birds of Bum. Novit, Zoolog. 7, 1900.

7. Janicki, C. у. Zur Kemtnis einiger Sängetiercestorlen. Zoolog. Anzeig., 27, 1904 .

8. Jhering, H. v. Die georpraphische Verbreitun! der Flussmuscheln. Ausland, 1890.

1. Mantens, E. V. Land-und Süsswasser-Conchylien ron Ost-Bormeo. SitzungsBer. d. Ges. naturforsch. Freunde, Berlin, 1903.

11. Michaelsen, W. Terricolen von verschiedenen Gebieten der Erde. Mitteilungen aus dem Naturhist. Museum Hamburg, 16, 1899.

11. Roux, J. Reptilien und Amphibien aus Celebes. Verhandlungen der Naturforsch. Ges. in Basel, 15, 1904.

12. - Décupodes d'enu donce de Celebes. Revue suisse de Zoologie, 12, 1904.

13. Sarasin, P. u. F. Materialien zur Naturgeschichte der Insel Celebes. Bd. I; Die Sïswasser-Ulolluskien von Celebes. Bd. II, 1898; Die Lamimollusken von Getebes, Bd. III, 1899 ; Ueber die Geolog. Geschichte der Insel Celebes auf Grund der Tierverdreitung, Bd. IV, 1901; Entwuif einer geographisch-yeologischen Beschreibung der Insel Celebes, 1901.

1'1. - Ueber die Torila con Sül-Celebes. Globus, 83, 1903.

1.). Schenkel, E. Beitray zur Kem handlungen der Naturforsch. Ges. in Basel, 13, 1902.

16. Tromarax, G. F. Hydrographic results of the Sibogra-Expedition. SibogaExpeditie, Monographie, III, Leiden, 1903.

17. Weber, M. Introduction et description de l'Expédition. Siboga-Expeditie, Monogr. I, Leiden, 1902.

18. Zscrokke, F. Die Darmcestoden der amerikamischen Benteltiere. Centralblatt f. Bakteriologie, Parasitenkunde, etc., 36, 1904. 
2I. le Président donne la parole à M. C. EMreny.

\title{
ÉTHOLOGIE, PHYLOGÉNIE ET GLASSIFIGATION
}

\author{
Par le Prof. C. EMERY
}

(Bologne).

\begin{abstract}
Wissen möchten wir ob das 20. Jahrhundert nicht, wenn man die Kunst das Leben im Leben $\mathrm{zu}$ beobachten wieder gelernt hat, über die Selbstzufriedenheit des 19. !ächeln wird, mit der es glaubt, aus dem Leichnam das Leben in seiner ganzen Fülle erkennen zu können.
\end{abstract}

K. E. VON B瓜.

Mesdames et Messieurs,

Lorsque, sur les instances de mon ami le professeur Aug. Foreu, j’ai accepté de faire une lecture dans une séance générale de ce Congrès, je me proposais de retracer l'influence exercée par la reprise dés études éthologiques sur les mogrès de la phylogénie et de la classification des animaux. Il m'eût fallu pour cela faire des recherches bibliographiques étendues, dont je vous aurais apporté ici les résultats.

Un ensemble de circonstances est venu mettre à néant mes bonnes intentious. Un mois seulement me séparait encore de l'ouverture du Congrès et mon travail était à peine commencé. Aussi ai-je dî renoncer à mon plan primitif, et ce n'est que pour ne pas manquer à ma parole que je me présente aujourd'hui à cette assemblée. A la place d'un discours longuement préparé, je ne puis vous offrir qu'une causerie pauvre en aperens originaux et, pour ainsi dire, l'expression d'un état d'îme du moment présent, de sentiments personnels, se lapportant au sujet dont le titre figure au programme sous mon nom. Veuillez, je vous prie, m'accorder votre indulgente bienveillance.

Les sciences de la nature montrent de nos jours une tendance toujours plus marquée ver's la forme der sciences exactes. La physique et la chimie sont depuis longtemps sur cette voie; leurs lois générales, tout en ste transformant dans le cours des années, sont susceptibles d'être résumées, dans leur état du moment, en formules qui se prêtent au caleul et aux applications mathématiques. Parmi les seiences de la vie, c'est surtout la physiologie gui s'efforece d'emboîter le pas sur celui de sa sex'ur la chimie. Une nouvelle branche, science encore au berceau, est issue de son sein : la biologie générale, dont le but ultime et idéal est de déterminer les propriétés des particules élémentaires des corps vivants, pour ar- 
liver ì définir et à expliquer la nature de la vie, la raison d'être intime des phénomènes de l'organisation et des fonctions des etres vivants. Elle intel'oge les structures subtiles des cellules et celles de leurs noyaux, mystérieux louages du mécanisme vital, régulateurs des échanges matériels du plasma, déterminateur's des propriétés des cellules, transmetteurs secrets de l'hérédité. Elle serute les fonctions physico-chimiques de ces éléments, pour jeter les fondements d'une physiologie cellulaire.

Aiusi nos regards plongent dans l'abîne de l'ultra-visible, pour y chel'cher la solution des questions que l'étude des phénomènes visihles a fait naître dans notre esprit.

Mais le vif intérêt qu'excitent en nous ces questions, ces hypothèses, ces mysteres de la vie ne doit pas nous faire oublier la source même à laquelle nous en avons puisé les éléments; l'observation directe de la nature vivante, l'étude des fol'mes, des activités des êtres vivants, de leur's conditions d'existence, de leur's rapports réciproques. Chacun de ces itres, quelque compliqué qu'il soit, constitue un tout coorilonné, doué d'une activité proplè et dépendant en memes temps du milieu gui l'environne, ainsi que des autres êtres avec lesquels il se trouve en contact.

C'est là ce qui donne à l'étude éthologique ' des animaux un intérêt tout particulier. La variété infinie des formes et des structures que nous remarquons aussi bien sul le col'ps inanimé que sur l'animal vivant n'a évidemment sa raison d'être que dans la variété non moins grande des fonctions physiologiques et de leurs combinaisons; c'est la somme de leurs activités qui détermine l'harmonie entre les êtres vivants et le miliou qui les entoure, en d'autres termes, ladaptation au milieu ambiant, sans larguelle aucun animal (ni aucun végétal) ne serait capable d'existel.

C'sst dans l'éthologie que nous trouvons l'expression la plus complète de l'espece animale, la manifestation la plus évidente de différences norphologiques, qui ne se montrent parfois qu'ì un examen minutieux et que les recherches les plus exactes n'arrivent même pas toujour's à découvrir. Bien souvent, nous en sommes réduits à supposer des différences morphologiques absolument hypothétiques, pour ne pas laisser sans fondrment matériel les diftérences que révèle l'observation des morurs des animanx; ou ('ncore des différences chimiques non moins hypothétiques, pour nous représenter la raison d'être des différences évidentes qu'offirent des galles produites sur la même espèce de Chêne par deux Insertes que nous ne sommes pas capables de distinguer' l'un de l'autre, ou bien encore pour expliquer la spécificité des parasites qui vivent sur différentes espèces de plantes ou d'animaux.

${ }^{1}$ Pour la signification et l'extension du nom d'éthologie voir WV.-M. WheELer, Natural... history, acology or ethology; in : Science, n. S., v. 15, p. 971-976, 1902. 
Quelle que soit la nature morphologique ou chimique des faits fondamontaux dont dépendent en théorie ou en hypothèse les phénomènes éthologiques, tant que ces faits ne sont pas réellement obser'vés ou observalıles avec les moyens dont la science dispose, nous sommes contraints de nous borner à leconnaîtıe les phénomènes qui tombent sous l'obselvation directe et de chercher à les classer, en attendant que, dans un avonir plus ou moins éloigné, la comaissance plus complète de la molphologie et de la physiologie en ait dévoilé le mécanisme intime.

Un cas très semblable a donné lieu récemment à un débat extrêmement intéressant, dont a fait partie la conférence tenue au Congrès zoologique de Berlin par Auguste Fones ' '. Est-jl légitime de donner le nom de psychologie comparée ou psychologie des animaux à une branche d'études scientifiques:? ou, comme le soutient un groupe de jeunes physiologistes et neurologistes, ce que l'on a appelé ainsi par' le passé n'est-il autre chose qu une physiologie de certains centres nerveux? Il est évident pour moi que les deux partis ont raison, chacun à son point de vue. Pour quiconque "l'admet pas la nature spirituelle c'ost-à-dile transcendentale de l’âme, les manifestations psychologiques, soit instinctives, soit intellectuelles ne sont autre chose que des faits physiologiques, du lessolt de centres dits psychomoteurs. Toutefois, à l'état actuel de la.science, et sans doute pour bien longtemps encore, nous ne sommes ni ne serons en mesure de rapporter les phénomènes qui paraissent d'ordre psychologique à des fait fonctionnels élémentaires des centres nerveux. Je pense done que la thèse de Forer qui soutient la légitimité de la psychologie animale est parfaitement citablie, mais en même temps je suis d'avis que cette thèse est conciliable avec celle de Beser, Bethe. Uexkuelu qui prétend réduire la soi-disant psychologie des animaux à un chapitre de nempophysiologir. L'un et les autres ont pour point de départ une théorie mécaniste de la

${ }^{1}$ Forel, Aug. Die psychischen Fähigkeiten der Ameisen und einiger andern Insekten, etc. Vortrag gehalten auf dem 5. internat. Zoolog.-Kongress in Berlin. München, 1901.

Foner, Aug. Die Berechtigung der vergl. Psychologie und ihre Objekte, in : Journal f. Psychol. u. Neurol., Bd. 1, 1902.

Bethe, A. Dürfen wir den Ameisen und Bienen psychische Qualitäten zuschreiben, in: Arch. ges. Physiol., Bd. 70, 1898.

Beer, Bethe, v. Uexkuelt. Vorschläge zu einer objektivierenden Nomentilatur der Physiologie des Nervensystems, in: Biol. Centralbl., Bd. 19, p. 517-521, 1899.

Wasmann, E. Die psychischen Fähigkeiten der Ameisen, in: Zoologica, Heft 26, 1899.

Wasmann, E. Nervenphysiologie und Tierpsychologie, in : Biol. Centralbl., Bd. 21, 1901.

Buttel-Reepen, H. v. Sind die Bienen Reflexmaschinen? in : Biol. Centralbl, Bd. 20,1900 . 
vie et de tous ses phénomènes, ceux de l'instinct et de l'intelligence non rxclus; seulement Forf, considere l'état actuel do nos connaissances et son avenir prochain; Bethe et ses confrères se rapportent à un état futur plus ou moins lointain, auquel ils prétendent conduire la science. Par contre, je pense qu une conciliation n'est pas possible entre les opinions de ces auteurs et le transcendentalisme de Wasians.

Nous nous trouvons aujourd'hui dans la nécessité d'étudier séparément la psichologic des animaux et les phénomenes plus simples, directement réductibles aux fonctions élémentaires du système nerveux, réservant à l'avenir la fusion des deux domaines actuellement distincts.

Il en est de même du vaste domaine do l'éthologie, se laattachant d'unc part à la psychologie, en ce qui concerne l'étude des instincts et de ses produits, de l'autre ì la physiologie mécanique et chimique, ainsi qu'a la biologie générale. Le domaine de l'éthologie comprend l'ensemble des phénomines dont l'analỵse phỵsiologique n'est pas faite, ou n'est mêmo pas faisable dans le moment actuel; ce que Karl Sexper appelait la "physiologie des organismes' ", par opposition à la physiologie des organes. Elle constitue avec la zoologie descriptive et systématique la branche historique par excellence de la zoologie. l'histoile naturelle des animaux.

I ans un avenir certainement encore lointain, éthologie et physiologir ne feront plus qu'un; les faits de la vie des animaux, leurs conditions d'existence, lcurs instincts d'une part, leur' évolution morphologique dr l'autro pourront alors être réduits à des sommes de fait physiologi(pues élémentaires; des rapports de dépendance mutuelle entre los faits d'observation se montreront toujours plus nombreux, permettant peut-etre un jour d'établir de réritables formules, capables d'expurimel', uon seulement chaque forme réellement existante, mais en outre toutes les formes possibles, comme les formules chimiques, établies sur les corps conmus et bien étudiés, nous mettent en mesure de prévoir des séries entières de composés encore inconnus et d'établir à l'avance leurs propriétés principales ${ }^{1}$.

La zoologie cessera alors d'appartenir à la catégoric des sciences historiques, pour devenir une science exacte. Mais cette pensée qui hante quelques-uns desplus harlis champions de l'école biomécanique moderne

${ }^{1}$ Des tentatives ont été faites d'exprimer par un symbole mathématique l'ensemble des formes vivantes existantes et possibles; notamment: Schiapparelit, G.-V. Studio comparativo tra le forme organiche naturali e le forme geometriche pure. Milano, 1898.

Voir aussi : Enery, C. Osservazioni critiche, in : Riv. Sc. biolog. Como, v. 1, fasc. 4, 1899; et Volterra, V. Sui tentativi di applicazione delle matematiche alle scienze biologiche e sociali, in : Giorn. d. economisti, Bologna, novemb. 1901. 
semble n'ètle actuellement qu un décevant mirage, une illusion magnifique, un rêve prophétique peut-être.

Les lois génélales de la vie, desquelles dépendent les propriétés particulières de chaque forme, lois immuables, conséquence des propriétés memes de la matière, lois communes à notre terre et aux autres corps célestes sur lesquels nous aimons à nous représenter que la vie s'est développée comme chez nous, existent certainement; du moins un besoin logique de notre esprit nous en fait admettre la nécessité, par analogie avec les lois physico-chimiques qui gouvernent uniformément toute la partie à nous pereeptible de l'univers. Mais pour le moment, nous sommes forcés rle le reconnaître, la zoologie n'est pas encore sortie de la période historique. Elle a i rassembler les faits morphologiques et physiologiques, à recomnaître les unités systématiques (especes, sous-especes, etc.) dans toutes lour's manifestations de structure et d'activité vitale, à les coordonner, à en rechercher l'origine, c'est-à-dire la généalogie et les migrations. Ce n'est donc pas la vie animale en général qui fait actuellement l'objet de notre science, mais l'histoire de la vie animale sur la trre. Le résultat synthétiqur auquel elle aspire tout d'abord est la phylogénie.

L'historien de la société humaine commence par rassembler les documents qui établissent les faits et leur ordre de succession, puis il cherche leurs connexions de cause ot d'effet, pour en déduire, autant que possible, des conséquences d'ordle scientitique. Ine la simple chronique naît ainsi la science historique, étudiant les lois de la vie et de l'évolution des sociétés humaines.

Ni la zoologie descriptive pure, ni l'éthologie simplement narrative ne constituent vérital)lement la zoologie scientifique; elles sont la chronicque et non pas la seiene historique du règne animal. Cr n'est pas que je dédlaigne le simple chroniqueur', l'obsel'vateur' méticuleux, le foulleur' patient des archives indéchiffrables de la nature. Honneur à lui lor'squ'il signe Ie Geer, Rowser, lí́aryer ou Heber ! homneur à ceux qui ont suivi la trace de ces maitres! Te saurait itre bon observateur qui n'est capable de penser. Le passant distrait peut trouver sur ses pas une pépite, mais la mine d'or ne sera découverte que par le chercheur intelligent. A la lumière de lévolutionisme ot des théoriess qui s'y rattachent, l'étude éthologique des animaux ac'quiert un intérêt nouveau et l'entomologie, trop négligée, dans un moment où l'attention des zoologistes se portait toute ('ntice sur les formes infimes de la faune marine et sur leur développement, alors prescue inconmu, reprend peu ì peu l'importance qui lui revient. Le nombre immense des Insectes, constituant à eux seuls plus que la moitié du régne animal, leur ubiquité, la variété itomnante de leul's morurs et de leurs métamorphoses, les socictés admirablement organiséces des Abeilles, des (ruepes, des Fourmis, des Termites, avec leurs in- 
nombrables commensaux et parasites offrent un champ d'études sans limites, un champ fécond en résultats souvent imprévus, toujours intéressants.

Sous les mains et par la plume de Paul Marcual, Charles Janet, Geolges et Elisabeth Pecknam, Woldemar WAgrer et d'autres encole, l'ouvre des brillants observateurs de la vieille école revit et se complète; l'histoire des Insectes commence à se développer sur la souche de la chronnique, par la critique sévère des sources, la comparaison des faits, la généralisation prudente et éclairée: Auguste Fores, Lrbisock, Mare Cook, W. M. Wheeler renouvellent la comnaissance des sociétés des Fourmis; v. Butrer-Reepen se fait l'historien attitré du loyaume des Abcilles: Wasuann nous révèle le monde caléidoscopique des mylmécophiles et termitophiles, leurs mours et leur's adaptations merveilleuses.

Tandis que je salue avec joie cette renaissance évolutioniste de l'éthologie entomologique, ma pensét se porte vers un vieillard, digne continuateur de l'école des Ríacaur et des Léon Drforr. L'îge qui anéantit tant d'énergies n'a pas diminué en lui la vigueur d'esprít, ni l'enthousiasme pour la " petite bête », dont nul mieux que lui n'est habile à surprendre les secrets. A Henri FABre, doyen des éthologistes, adressons un hommage de respect et d'admiration.

A plus d'un égard, les Lépidoptères ont acquis un intéleet tout particulier': l'aile du P'apillon, avec ses écailles colorées, composant des dessins si riches et si variés, est un admirable réactif de l'influence du milieu sur l'organisme. Les belies études de Wessaraxs sul le dimorphisme de saison ont ouvert la voie; Merrifiedo, STandfess, Fiscier ont suivi, étudiant l'influence de la température et de l'humidité, éléments constitutifis du climat; leurs études nous ont révélé l'origine et l'importance des aberrations et éclairé d'une lumière inattendue des questions de phylogénèse. Enfin voici les expériences dernières venues de A. PICTET sur l'influence de la qualité de la nourriture sur les couleurs et les formes des papillons et sur leurs effets héréslitaires, qui vienuent ouvril des horizons nouveaux à l'étude de la variation et de l'adaptation.

Les ailes des Papillons me conduisent à la cuestion si embrouillée du minétisme, à propos de laquelle tant d'encre a été versée, depuis que Bates et Fritz Muesler avaient cru l'avoir définitivement résolue. A quoi servent ces taches, ces marbrures délicates'? pourquoi ces ressemblances, évidentes à nos yeux, mais dont la signification éthologique est si fort contestée? Prepers ${ }^{1}$ a-t-il l'aison de combattle avec tant d'acharnement l'interprétation classique? ou bien celle-ci a-t-elle un fond de vérité? Et les colorations et ormements sexuels des Oiseaux et des Insecetes? les chants, les stridulations, les phospholescences? Autant de pro-

1 Piepers, M.-C. Mimicry, Selektion, Darwinismus. Leiden, 1903. 
blèmes dont la solution paraissait aisée au temps de l'enthousiasme pour le darwinisme naissant, et qui réclament aujourd'hui de nouvelles recherches et une revision critique approfondie.

L'influence de la reprise des études éthologiques se fait sentir vivement dans les travaux récents de phylogénie paléontologique: les modifications successives qui ont transformé les espèees animales dans le cours des âges ne sont pas un pur effet du hasard, une somme de variations fortuites: elles se sont formées sous l'étroite dépendance de modifications correspondantes de la manière de vivre, c'est-à-dire de la nourriture, du milieu ambiant liquide, aérie'n, arboréal ou souterrain, du climat, de la flore, etc. Ainsi le développement des Insectes, à partir du carbonifère, a eu lieu parallèlement à celui de la végétation; nous le royons s'accentuer avec l'apparition des angiospermes et atteindre enfin son épanouissement complet par la formation de la symbiose merveilleuse des plantes et des Insectes, se manifestant dans la fleur et sa fécondation croisée, opérée par ses visiteur's ailés ${ }^{1}$.

Les conditions d'existence des ancêtres ont laissé des traces dans la deseendance; nous nous en apercevons tous les jour's davantage. Déjà l'école américaine et surtout Cope avec ses coreligionnaires néolamarckiens se sont servis fréquemment d'observations ot d'hypothèses éthologiques; mais ils visaient, au delà de la phylogénie, la théorie générale de la transformation des organismes.

L'auteur qui, à ma connaissance, a le mieux compris la valeur de l'éthologie pour la phylogénie paléontologique est Dolso". Le travail par lequel il démontre l'origine arboréale des Marsupiaux, ainsi que son récent mémoire sur l'Eochelone brabantica peuvent être cités comme modèles du genre et méritent de trouver des imitateur's. Les rapports que nous observons actuellement entre les structures et les fonctions, entre les conditions anatomiques et le morle d'existence, le milieu, la nourriture habituelle, ont pu servir à Curier à retracer les parties demeurées incomnues des animaux fossiles et leur manière de vivre. Le principe de l'évolution nous conduit à rechercher les vestiges de la transformation éthologique, en rapport avec la transformation morphologique des espèces. L'une doit servir de contrôle à l'autre, car elles ont eu lieu en même temps et sont unies l'une à l'autre par des liens nécessaires de dépendance mutuelle.

' Handuinsch, A. Ueber die Insekten der Vorvelt und ihre Beziehungen zu den Pflanzen, in: Ver. Ges. Wien, p. 114-119, 1904.

${ }^{2}$ Dollo, L. Les ancêtres des Marsupiaux étaient-ils arboricoles? in : Trav. stat. zool. Wimereux, v. 7, p. 188-203, 1899.

Doluo, L. Eochelone brabantica, tortue nouvelle du Bruxellien de la Belgique et l'évolution des Chéloniens marins, in : Bull. Acad. Belgique, 1903. 
Actuellement. les grands problemes do la phylogénie ne sont guères ale. cessibles ì la méthode éthologigue. La conjectuse y tient une large place et l'imagination peut s'y donner libre cour's: aussi les thèses les plus étranges ont-elles été sontenues et les transformations d'organes les plus invaaisamblables exposées sériensement, par exemple par cenx qui prétendent faire dériver les Vertébrés d'Arthropodes plus ou moins limulödes. De pareilles aherrations sont le produit de v'ues unilaterales et d'une méthode mol'phologique imparfaite. dominée par un' auto-suggestion intense et continue. Quelques purs morphologistes ont pu oublier que les organes dont ils étudiaient la forme inerte sur leurs séries microtomiques ont été vivants et fonctionnels, à toutes les périodes de leur évolution phỵlogénique, et qu'ils ont du servir à quelque chose, dans des ormanismes également virants. Il faut combattre ces exagérations de la mesthode morphologique et chorcher it nous rendre compte autant que possib)le du mécanisme éthologique et physiologique de la phylogénèse, auquel se rattache néressairement celui de la transformation morphologique. Le principe du changement de fonetion des organes (Princip des Functionswechsels), fol'mulé par' A. DohrN ${ }^{1}$ dès 1875 , est l'expression d'une juste considération de limportance de la physiologie et de l'éthologie dans les questions de pliylogénie.

La distribution géographique des animaux présente des problemes nombreux, dont la résolution demande une connaissance de l'éthologie bien plus approfondie que celle que nous avons aujourd'hui. Saus doute les changements de la configuration géograplique du globe, ainsi que de la répartition de la terre ferme et do la mer. l'uxhaussement et l'alarissement des montagneséclairent bien des questions, donnent la clef de nombreux problèmes; les brillantes études de Paul et Fritz SARAsin ${ }^{2}$ sur la distribution et la provenance des mollusques de Célébes, pour ne citer qu'un serul exemple, en sont la preure. Elles nous pendent compte de la lignée des formes qui, venues de différentes terres, se sont avancés vers le centre de Célèbes, ainsi que de leur itinéraire, le long des bras rle cette île au contour étrangement découpé.

Mais il ne suffit pas que les barrieres des montagnes s'abaissent, que les bras de mer se dessèchent, pour qu'un groupe faunistique puisse se répandre sur de nouveaux territoires; il faut qu'il y trouve des conditions d'existence favorables, que le sol, le climat, la végétation conviennent à son régime et à ses habitudes: il faut qu'il soit capable de vaincre la concurrence des formes rivales. Les conditions d'existence des esperees,

' Dohri, A. Der Ursprung der Wirbelthiere und das Princip des Functionswechsels. Leipzig, 1875.

2 Sarasin, P. et F. Ueber die geologische Geschichte der Insel Celebes auf Grund der Thierverbreitung. Wiesbaden, 1901. 
leurs rapports réciproques, leurs moyens de transport ì distance, l'inHuence du milieu sur leur structure, quel sujet magnifique d'études! Karl Sexrer ${ }^{1}$ en a tenté autrefois l'esquisse avec une rare compétence dans un livre atlmirahle, mais qui n’a malheureusement pas trouvé de continuateur. Les temps n'étaient pas mûrs: la microtomie (qui venait de se perfectionner, précisément dans le laboratoire de SEmper par l'invention des coupes à sec en paraffine) offirait un champ trop fécond en découvertes faciles !

Cerains faits de la distribution discontinue des especes ont quelque chose de mystérieux : ainsi Bonaparte a décrit autrefois une petite Vipère provenant du massif du Gran Sasso d'Italia, la Tipera ursinii. Extrêmenent rare dans son habitat tỹpique, cette espèce est répandue dans une partie de la vallée du Danube; un Carabe très remarquable $(C$. voriolatus Costa), proplure en Italie au même massif, n'est qu'une variété du C. cavernosus Friv. de la Bosnie et de la Serbie. Je ne sais si d'autres espèces ont une distribution analogue, dont les faits géologiques et géographiques connus ne me semblent pas pouvoir fournir une explication suffisante.

La distribution géographique des animaux est en grande partie l'effet do leur différente capacité de migration active et passive, dont les éléments sont loin d'être suffisamment connus. L'ubiquité de la microfaune d'eau douce est, comme on sait, en rapport avec le transport passif de ses germes par les Insectes et les ()iscaux, et reux-ci sont capables d'accomplir des traversées lointaines, emportant sur leurs pattes le limon des étangs, riche en oufs et en kystes microscopiques. Les détails de cette distribution domment lien à des problèmes variés, dont soccupent les spécialistes do la faune des lacs et des rivières. - Permettez-moi de choisir quelques exemples parmi les Fourmis, dont la chorologie m'est plus familière.

Pourquoi telle espèce, sans avoir été transportée par l'homme ni volontairement ni par hasarel, est-elle largement répandue ou mème cosmopolite, tandis que ses proches parents ont un habitat restreint?

Il y a des Fourmis qui sont capables de se répandre dans les îles à travel's l'océan, tandis que leurs congénères en sont incapables. Leptogemys falcigera Rog., Tollemhovia levithora.r Emery, Pheidole megacephala F., divers Momomorium et Cardiocondyla, Premolepis bourbonien For. en sont des exemples. La plupart sont de petites espèces dont on peut supposer que les femelles ailées se laissent porter par les vents; mais pouryuoi alor's ces especes seulement et non pas unc foule d'autres?' Leptogemys falcigera a probablement une femelle aptère (encore inconnue) comme sés congénères; il faut donc lui supposer d'autres moyens de

' Semper, K. Die natürlichen Existenzbedingungen der Thiere. Leipzig, 1880. 
transport maritime, bois flottés ou autres, car je ne pense pas que la diffusion de la faume entomologique insulaire suftise pour légitimer l'hypothese de vastes continents disparus ou de ces ponts do terre ferme que quelques chorologistes élèvent et submergent au gré de leur imagination. Mais tant que nous ne saurons pas comment vit et ou habite chaque espéce, toute discussion sur ses moyens de diffusion et sur les raisons de ses limites géographiques sera vaine. Et nous nous demanderons en vain pourquoi Crematogaster tricolor Gerst. et Tetramorimm blochmanmi For. ont pu passer' de l'Afrique continentale à Madagascar et aucun de leurs nombreux congénères, ni d'autres Fourmis largement répandues en Afrique et en Inde, comme par exemple Oecophylla smaragdina F.

Ise genre Odontomachus compte une quarantaine d'espèces et sous-espèces, la plupart strictement localisées, ou du moins ne dépassant pas les bornes d'une région zon-géographique. Seule la forme typique du genre, la Formica hæmatoda de LinNé, se trouve dans presque tout lè monde tropical, avec quelques sous-especes locales en Amérique (certaines formes australiennes telles que $O$. coriarius Mayr et $O$. cephalotes F. Smith se rattachent aussi de très près à $($. hæmatoda). Si l'on veut domer une explication purement géographique de ces faits, il faut sup)posel que 0 . Thematoda est la forme primitive, archäque. qui a envahi le monde par drsponts interentimentaux aujourd'hui disparus, émettant de sa souche des branches divergentes localisées. Nais rien ne prouve la primitivité de l'espèce limnéennu': ume raison d'orchre éthologique me palaît bien plus vrajemblable, mais dans l'état actuel de nos connaissances, nous ne saurions faire que de vaines hypothèses, car nous savons fort peu de chose de la manière de vivre d'O. hrmatoda et absolument rien de la plupart de ses congénères.

Sans allor aussi loin, la faune mypruécologique de l'Europe présente des problemes non moins intéressants. La paléontologie montre que le genle Formicr est d'origine boréale, probablenent américaine '; $F$. Hori Mayr abonde dans l'ambre de la Baltique, tandis qu'aucune espècer du genre n'a été vue dans l'ambre de Sicile, où domine une faune de type indo-australien ”. Ces Fourmis sont done pour l'Eurnpe méridionale dos immigrants relativenent modernes et leur distribution présento des détails fort curieux. Ainsi les especes acervicoles ont pour limite sud. en Italie, la plaine du Pô, où aucum olstacle apparent ne s'oppose à leur diffusion ultérieure; elles n'ont pas non plus suivi la montague, car je les ai cherchées en vain dans l'Apennin; $F$. sanguinea Latr., au con-

1 Emery, C. Beiträge zur Kenntnis der nordamerikanischen Ameisenfauna. Allgemeiner T'eil, in : Zool. Jahrb. Syst., Bd. 8, p. 339-358, 1895.

${ }^{2}$ Emery, C. Le formiche dell' Ambra Sicitiana, ecc., in: Mem. Accad. Bologna, (5) v. 1, 1891. 
traile, s'est répandue le long de l'Apennin jusqu'en Sicile. Le littoraal adriatique sahlonneux de la Romagne est littéralement infesté par la Formica cinerea Mayr', tandis que les îles non moins sablonneuses de la lagume vénitienne nont à ma connaissance que $F$. fusca L. D'autres Fourmis très communes en Italie paraissent manquer aux îles de la lagune, p. ex. Pheidole pullidula Nyl., du moins à Lido, où je les ai cherchées. Comme ces îles sont d'émersion récente, il est naturel que leur faune offie de grandes lacunes; une étude détaillée de cette faune serait du plus grand intérêt. Quant aux Fourmis dont je viens de parler, il faudrait, pour expliquer les particularités de leur diffusion, connaitre exactement pour chaque espèce comment se comportent les femelles fécondées, germes ailés de l'espèce, de quelle manière se fondent les nouvelles sociétés et bien d'autres choses encore que nous ignorons complètement. D'après mes observations, les femelles de Pheidole pallidula volent peu; le plus souvent, elles se dépouill'nt de leurs ailes aussitôt après l'accouplement et deviennent ainsi incapables d'être transportées par le vent à de grandes distances. Formicu fuscu vole au contraire fort loin. Je n'ai pas eu occasion d'observer le vol nuptial de $F$. cinerea.

Je me borne à ces exemples, pour ne pas abuser de votre patience.

Si les conditions éthologiques de tout genre entrent en jeu pour déterminer la possibilité de la diffusion au delà de certains obstacles naturels, ce sont elles encore qui favorisent ou arrêtent le progrès de la diffusion et assignent des limites au territorre de chaque forme animale ou végétale. Nous constatons l'existence de ces limites, nous voyons les formes différentes se substituer les unes aux autres dans les territoires contigus, le plus souvent sans en apercevoir les véritables raisons, parce que nous ignorons plosque toujoul's dans quelles conditions chaque espèce peut vive et soutenir victorieusement la concurrence de sos livalns. Ce sont ces conditions qui déterminent le facies faunistique local. Celui-ci trouve son expression daus l'ensemble de la population zoologique d'un territoire, ensemble dans lequel il faut prendre pour unités les individus et non pas les espèces: cet ensemble comprend donc des espèces dominantes, leprésentées chacune par de nombreux exemplaires, espèces qui sont les vainqueurs du moment dans la lutte pour l'existence. Les l'echerches sur la composition numéricue ou statistique des faunes locales et leur's modifications selon les années et les saisons, dans la forme proposée par Adalbert SerTz ${ }^{1}$ et Friederich DAHr ${ }^{2}$ donneront l'expression graphique du facies faunistique, base de faits pour poser des problemes.

${ }^{1}$ Sertz, A. Allgemeine Biologie der Schmetterlinge, in : Zool. Jahrb. Syst., Bd. 5, p. 281-334, 1890 .

2 Darl, Fr. Das Leben der Ameisen im Bismark-Archipel, in : Mitt. Zool, Mus. Berlin, Bd. 2, 1901. 
La solution de ceux-ci ue pourra itre domnée que lol'squ'on aura recomnu les conditions qui favorisent la propagation de telles ou telles espèces au détriment de leur's concurrentes.

Notre époque a une tendance mal'quée vers les méthodes statistiques, tendance qui n'est pas sans inconvénients, contre lesquels il est bon l'ètre en garde: rien n'est plus aisé que d'accumuler des chiffies, de les aligner en colonnes et d'en tirer des sommes, des moyennes, voire même des diagrammes plus ou moins intelligibles. Ce n'est pas que je condamne cette méthode qui, dans des mains intelligentes, conduit à des résultats de haute valeur' mais elle se prête trop facilement (et chacun deva le reconnaître) à couvril d'écriture beaucoup de papier et ì charger la littérature scientifique d'un lest eneombrant. Les effrayants grimoires du dilettantisme biométrique sont là pour le prouver.

C'est précisément dans la concurrence pour la diffusion géographique que se combat la véritable lutte pour l'existence entre les formes spécifiques et subspécifiques. Je laisse de coté la promière formation de l'espèce et les théories qui s'y rattahent; elle est du domaine de la variation, de ses morles et de ses causes que je n'ai pas à traiter ici. Mais une fois constitué, tout nouveau groupe spécifique se trouve nécessairement localisé et en concurrence avec ses prédécesseur's, déjà en possession du terrain et des moyens de subsistance qu'il produit. La lutte ne tarde pas ì s'engager et l'extension du territoire est le signe de la victoire.

Sauf les cas de migration à distance, conduisant à la formation de colonies, le territoire des formes jeunes doit être continu; la discontinuité du territoire de l'espèce fait supposer des défaites suloies dans la lutte entre les formes vieilles ou nouvelles, sous l'influence des changements du milieu climatique ou biologique. La chorologie statistique, en nous l'évélant l'état actuel de la lutte entre les éléments des faunes locales, pourra nous mettre sur la trace des éléments éthologiques déterminateur's de la phylogénie, dans ce sens, qu'ils donnent lieu à la sélection naturelle.

Mais les faits éthologiques eux-mêmes ont leur phylogénie dont l'étude est extremement intéressante; elle se rettete encore sur cette forme appliquée de la phylogénie, qui a pour formule la classification. Des groupes systématiques fondés sur des faits morphologiques sont souvent encore caractérisés par les faits éthologiques saillants, consacrés même par la momenclature : Oligochètes terricoles, Lamellicornes coprophages, V'espides sociaux, Mammifères carnassiers en sont des exemples. Dans la phylogénie de ces groupes, les caractères morphologiques ont dî se déveJopper en connexion nécessaire ou accidentelle avec les caractères étholocriques; l'étude de l'origine des uns pourra nous aider à retracer celle des autres et les deux genres de lecherches se viendront en aide mutuellement. Comme nous avons une morphologie comparée, nous devrions 
avoir une éthologie comparée. J'ai dit "nous devrions" car', si l'on fait exception pour les Oiseaux, les Mammifères et quelques groupes privilégiés des Insectes, l'on peut dire que l'éthologie en est encore à la période primitive de la chronique, à la pénible recherche des faits, à des généralisations timides et trop souvent contredites par des observations ultérieures.

Nul ne soupçonnait l'existence de la vie conjugale chez les Coléoptères, lorsque Fabre' ${ }^{1}$ nous fit connaitre successivement l'idylle du Géotrupe, puis celles du Sisyphe et du Coprobie des Pampas, représentants de différents groupes de la souche des Coprophages. Nous étions accoutumés à regarder les mâles des Hyménopteres comme des êtres incapables de toute autre fonction que celle de la reproduction, et voila les observations de $G$. et E. Peckham ${ }^{2}$ nous apprenant que chez Trypoxylon le mâle garde le nid en l'absence de sa femelle. Et combien de choses étranges et imprévoyables dans la symbiose des myrmécophiles et termitophiles avec leur's hôtes, Fourmis et T'ermites !

Les origines et le développement des faits éthologiques sont au nombre des problemes les plus difficiles. Le fait morphologique est palpable et persiste aprè la mort; il est susceptible d'être conservé en préparation, observé à nouveau et comparé à d'autres objets du même ordre. L'observation de l'animal vivant est au contraire fugitive, lor'squelle ne laisse pas pour trace l'œuvre de l'ouvrier, le nid, le dégât, la victime. Imposibible d'appliquer la " loi hiogénétique "si commode aux morphologistes, malgré les erreur's sans nombre auxquelles elle a conduit ! Où et comment, par exemple, retracer les origines de la vie sociale des Insectes? Nous en sommes léduits à la méthode comparative. Pour les Fourmis et les. Termites, dont nous ne comnaissons pas de proches parents solitaires, il est naturel que nous ne puissions faire que des suppositions plus ou moins fondées. Mais pour les Abeilles et les Guêpes nous ne sommes guère plus avancés, quoique les formes solitaires soient nombreuses. VernerF ${ }^{3}$ avait bien signalé chez les Halictus une'ondition qui paraît être un prélude de vie sociale, et les nouvelles observations de FABRE ${ }^{4}$ sont venues à l'appui. Mais, pour des raisons morphologiques, les Halictus ne peuvent être regardés comme proches des ascendants directs des Abeilles sociales. Puis ils ne font pas de cire et la cire est un matériel commun aux constructions de tous les apides sociaux; leurs ancêtres communs so-

${ }^{1}$ Fabre, J.-H. Soucenirs entomologiques, $5^{\ominus}$ et $6^{e}$ série. Paris.

2 Pecknam, G. and E. On the instincts and habits of the Solitary Wasps. Madison, Wis., 1898.

s Verhoeff, C. Beiträge zur Biologie der Hymenopteren, in: Zool. Jahrb. Syst., Bd. 6, 1892 .

${ }^{4}$ FABRE, J.-H. Souvenirs entomologiques, 8 e série, 1908. 
litaires ont dû v'aisemblablement travailler la cire. L'on a reconnu des traces de sécrétion cireuse sur le corps de certaines Anthophores; tout réermment H. v. Jnering: a signalé un nid d'Abeille du Brésil dont les cellules d'argile sont crépies intérieurement avec de la cire. C'est, me semble-t-il, un pas important sur cette voie difficile. Nous avancons lentrment, mais nous avancous! Que chacun apporte sa brique au chantier, et l'édifice que les beaux travaux de v. Butres-Reepen ${ }^{1}$ et de v. Jhering ${ }^{2}$ ont fondé progressera plus vite.

C'est surtout dans les pays tropicaux, où la vie pullule en formes innombrables; qu'il faudra chereher la solution des problemes que notre faune européemne, appauvie par les frimats et la culture séculaire, nous permet à peine de formuler. Que les naturalistes y travaillent, non pas seulement en chatsseurs avides de rapportel de riches dépouilles, mais en ohservateurs patients et intelligents; les résultats ne se feront pas longtemps attendre.

Ce n'est pas par des généralisations hâtives que l'on arrivera à fonder' des conclusions éthologiques applicables à la phylogénio et a la classification. A mon avis, les grandes lignes du système resteront du domaine à peu près exclusif de la morphologie: celle-ci est en mesure de retrourel plus facilement les traces d'un passé lointain, l'heritagr des antiques génélations, insereit daus la structure générale des organismes on dans des détails 'n apparence insignifiants, qui ont échappé à l'intluence modificatrice de l'adaptation. Les propriétés étholngiques, par contre, se rapportent principal'ment it des conditions d'existence actuelles ou récentes, dont elles sont l'expression la plus intense. Toutefois le passé éthologigue a laissé souvent des traces profondes dams la structure et les coutumes des animanx. Ainsi l'Anguille conserve dans sa repoduction et sa métamorphose les modes de ses palents, labitants des abîmes de la mer" : la Salamandre ter'restre confie à l'eau, domicile de ses ancêtres, sa progéniture branchiée; l'Abeille domestique, suspendue en exaim, attache quelquefois encore un rayon de cire à la branche d'un arbre, comme font certaines espèces de l'Inde (Apis dorsata F., A. florea F.), qui ont conservé dess mours plus primitives. Restes et rudiments éthologiques non moins intéressants que les rudiments morphologiques. Mais les rudiments morphologiques rux-mimes ont une portée éthologique: Les plaques imaginales des ailes dans la nymphe d'un Insecte aptère, les moignons d'ailes de l'Oiscau coureur montrent que les ancêtres de ces animanx ont volé; et la fonction du vol a modifié non seulement lo mem-

'Bottel-Reepen, H. v. Die stammesgeschichtliche Entstehung des Bienenstaates; etc. Leipzig, 1903.

2 Jhering, H. v, Biologie der stachellusen Honigbienen Brasiliens, in : Zool. Jahrb. Syst., Bd. 19, p. 179-287, Taf. 10-22, 1903. 
bre devenu aile, mais le systeme musculaire et le squelette tout entier, y laissant des traces indélébiles, alor's que la locomotion aérienne est depuis Iongtemps abolie; la structure des extrémités des Marsupiaux, quel que soit leur mode actuel de locomotion, révèle les mœurs arboréales de leur's précur'seur's; les fentes viscérales embryounaires des Vertéhrés pulmonés montrent la respiration branchiale et la vie aquatique de leur's progéniteur's paléozoïques.

La morphologie et l'éthologie se completent donc mutuellement, récoltent sur le même champ d'étude des fruits diffélents, signalant l'une ¿l l'autre les questions à poser, les problèmes à résoudre, ainsi que les l'ésultats obtenus, dont découlent à leur tour de nouvelles questions et de nouveaux problèmes.

Pour me servir d'une image exprimée autrefois par notre collègue Armold Lan(r ${ }^{1}$, les serlures qui ferment les portes de la phylogénie sont extrêmement compliquées et ne sauraient être ouvertes au moyen d'une seule clef. Ce n'est que par le concours de toutes les méthodes, employées avec discernoment et sans exclusion a priori, que nous pouvous espérel le succès. L'éthologie est une de ces méthodes, une des clefs's du labyrinthe; elle contribuera sans doute à nous ouviril plus d'unc salle, où personne n'a encore pénétré et dans laquelle sont renfermés des trésor's inestimables.

Ye nous lassons pas d'étudier les êtres vivants; c'est d'eux surtout que nous applendrons à connaître l'histoire de la vie.

'Lang, A. Mittel und Wege phylogenetischer Erkenntniss. Jena, 1887. 


\title{
QUATRIÈME ASSEMBLÉE GÉNÉRALE
}

\section{Vendredi 19 août 1904}

à midi

au Kursaal d'Interlaken

sous la présidence de

II. le Professeur E. Perrier.

Vice-Présidents : MM. G. S. Mrumer (Washington), Dr G. Horvatir (Budapest), Prof. A. Lang (Zurich), Dr C. W. Striess (Washington).

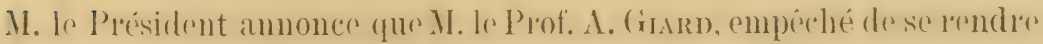

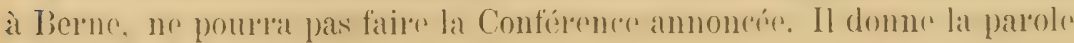
it NI. P. P. ('. Hokk.

\section{ZIELE UND WEGE DER INTERNATIONALEN MEERESFORSGHUNG}

\author{
Von D) P.-P.-C. HOEK \\ (Kopenhageni).
}

Das Meer ist gross und mit kleinen Mitteln ist ihm nicht beizukommen. HEINCKE.

\section{Hochverehrte Versammlung!}

Ich möchte fast um Eintschuldigung hitten, dass ich hicr zwisehen den schönen Sern, am Fusse der herrlichen Alpen, den grauen nordischen Meren und deren mühseliger Firforschung lhre Gedanken zuwende. Ich würde mich gewiss nicht bereit erklärt haben, hier über dieses Thema einen Vortrag zu halten, wäre ich nicht der Ausicht, dass die jetyt international organisierte. Mereresforschung sich auch für dier zoologische Wissensehaft als nutzhringend herausstellen wird, unt hätte ich nicht zugloich die Ueberzengung, dass garade anch die Sichwejz und dir schweizor (ichehrten sich in hohem Grade für die Heerestorschumg in- 
teressielten. Die Schweiz hat uns Agassiz, Vater und Soln, Craparide und Fou geschenkt; hier fand Carl VorT, um von so vielen anderen auch noch lehenden, tüchtigen marinen Zoologen nicht zu leden, ein neues Taterlaud. Ist die Schweiz noch dazu das Land, das sich um die Erforschung der Bimnenseen besonder's verdient gemacht hat, eine Forschung, von der man erwartet, dass ihre Arbeiten liünftig mit den internationalen Meeresuntersuchungen in Einklang stehen werden.

I'nd dass die Idee einer in te r'n a t ion a len Organisation dieser Untersuchungen hier lehhafte Sympathie wach gerufen hat, dafür bülgt uns der Umstand, dass wir uns hier im Lande der internationalen Organisation

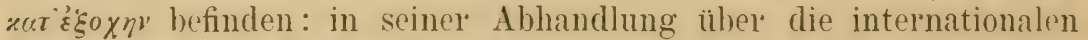
Bureaus sagt der Belgier I)Escames: "La part faite à la Suisse dans l'établissement des bureaux annexés aux unions universelles est hors de pair. Si Berne n'aspire pas à devenir la capitale du monde, elle peut légitimement revendiquer le titre de chef-lieu des offices internationaux. Il en faut rendre hommage au Gouvernement fédélal qui a toujours manifesté l'empressement le plus louable à offrir l"hospitalité du sol helvétique aux nouvelles institutions comme aux conférences qui ont précédé leur création. " "Der Empfang, der unserem Kongress in diesen 'Tagen hier zu Teil wird, ist ein neuer Beweis für die Richtigkeit von Descamps' Behauptung.

I)er Gedauke, die Erforschung der nord-europäischen Meere auf internationalem Wege, nach gemeinschaftlich festgestelltem Programm und nach dem Prinzip der Arbeitsteilung zu organisieren, ist ein so rationeller, dass man sich nicht darüber wundern kann, dass er fast zu gleicher Zeit in verschiedenen und verschiedenen Nationen angehöronden Häupter'n entstanden ist. Das V'erdienst, zu del Austïhrung dieses Credankens die Initiative ergriffen zuhaben, kommt aber unbestritten dem schwedischen Chemiker und Hydrographen Professol Otto Peteressox in Stockhohn zu. Er beschältigte sich schon seit vielen Jahren mit einer umfangreichen Fiforschung der physikalischen Wasserverhältnisse des Skageraks und Kattegats, jenes charakteristischen Grenzgebietes zwischen der salzarmen Ostsee und der salzreichen Nordser, und hatte sich zu dem Ende schon mit Erfolg mit einigen hierbei in erster Linie in Betracht liommenden Uferstaaten in Verbindung gesetzt: in den Jahren 1893-94 wurden von den Schweden Untersuchungen angestellt, an welchen sich auch dänisehe, dentsche, nolwegisthe und schottische Forscher beteiligten, und die somit als die ersten internationalen Intersuchungen auf diesem (iebiete betrachtret werden könnem. I)ureh dir äusseust wichtigen liesultate dieser hydrographischen Forschungen angeregt und in

${ }^{1}$ Descamps, E. Les offices internationaux et leur avenir. Bruxelles, p. 16, 1894. 
der festen Ueberzeugung, dass nur internationale Arbeit nach festen Prinzipien im Stande sein würde, diese Untersuchungen weiter zu führen und zu vertiefen, wandten die schwedischen Forscher G. EkMan und O. Petrerssox sich an ihre Regierung mit der Bitte, eine internationale Konferenz zu Besplechung ihrer Pläne zusammenzurufen. Es wal ihnen ein leichtes, fül ihıe weitblickenden Ansichten die Sympathie ihres Königs zu gewinnen: im Winter 1898-99 richtete die schwedische Regierung an die hier am meisten in Betracht kommenden Staaten NordEuropa's die Einladung, zur Besprechung des Planes, Vertreter auf eine in Stockholn abzuhaltende lionferenz zu beschicken. I)iese Konferenz, an welcher sich acht Staaten (in alphabetischer Reihe: Dänemark, Ieutschland, England, Finnland, Holland, Norwegen, Russland und Schweden) beteiligten, fand im Juni 1899 in Stockholm statt. Nicht bloss sprach sie sich dahin aus, dass es vom rein wissenschaftlichen, wie vom volkswirtschaftlichen Standpunkte im Interesse der Fischerei-Industrie) im höchsten Grade erwünscht sei, dass die Arbeit auf internationalem Wege in Angriff genommen werde; sondern die Konferenz benutzte sofort die Gelegenheit, die Art und Weise, wie zur Ausführung geschritten werden solle, eingehend zu studieren und für diese Ausführung ein vorläufiges Programm aufzustellen.

Nachdem in einer zweiten Konferenz (Kristiania, Mai 1901) eine nähere Prüfung und Besprechung der Pläne, sowie des Programms für die Ausführung, stattgefunden hatte, entschieden sich die oben geuannten Staaten, zu welchen sich später auch Belgien gesellte, an den in den Konferenzen geplanten Unter'suchungen teilzunchmen, und wurken in sämmtlichen Ländern, sowohl fül die Ausführung der eigenen Untersuchungen als für die Einrichtung eines centralen Bureaus und für die Stiftung eines internationalen Lahoratoriums die Mittel bewilligt. Im Juli 1902 fand daraut in Kopenhagen die lionstituierende Versammlung des Central-Ausschusses für die internationale Merresforschung statt. und schon wenige Wochen später lionnte mitgeteilt werden, dass die Untersuchumgen in den meisten Staaten in Angriff genommen waren. Im ()ktober des nämlichen Jahres fingen das in Dänemarks Hauptstadt sesshafte centrale Bureau, und ungefäh zu derselben Zeit das in Kristianja gestiftete internationale Laboratorium ihre Wirksamkeit an.

Möge dieve äusserst skizzenhafte Darstellung der Entwicklungsogeschichte unserel Organisation hier genügen: für eine ausfühlichere Behandlung wird auf die Protokolle der Konferenzen, sowie auf den ersten vom Bureau veröftentlichten Verwaltungs-Bericht verwies'n. Füu Sie, meine Damen und Herren, hat nicht die Frage, wie unsere ()rwanisation zu Stande gekommen, und wie sie num arbeitet, besonderes Interesse. wohl aber diejenige, von welcher Bedeutung die Untersuchungen fül die biologische Wissenschaft sind und werden liömen. Formell sind

VIe CONGR. INT. ZOOL., 1904. 
diese Untersuchungen in zwei Hauptabteilungen getrennt: die hydrographischen Untersuchungen, die man auch das Studium der Physik und der Chemie des Meeres nennen könnte, und die biologischen, die sich mit dem Studium der lebenden Organismen des Meeres, besonders derjenigen heschäftigen, die für die Fischerei-Industric von Wichtiæzkeit sind. Es liegt aber auf der Hand, dass von einer wirklich getrennten Behandlung dieser Wissenschaftszweige der Meeresforschung keine Rede sein kann und rom Anfang an keine Rede sein durfte. Ein prächtiger Beweis dafür liefert gleich der Umstand, dass man won hydrographischer Soite dir sofortige Inangriffnahme des Studiums über Vorkommen und Terhreitung der schwebenden Lebewesen (des sog. Planktons) in den Torderorund gedrängt hat, in der festen Ueberzeugung, dass die kleinen, hier in Betracht kommenden Tiere und PHanzen so viele Leitorganismen für die Unterscheidung ron Wasserschichten und Wasserströmungen abgeben. Ich komme später noch auf das Studium des Planktons zurück; ich habe dessen hiel nur elwähnt, um zu zeigen, dass die $\mathrm{Hy}$ drographie für bestimmte Fragen gewissermassen der biologischen Forschung bedarf, wie sich umgekehrt die Förderung der biologischen Kenntnisse des Meeres ohne genauere Kenntnis der hydrographischen Terhältnisse gar nicht donken läst. Die bekannten Fälle, in welchen es gelungen ist, eine Aenderung in der Zusammensetzung, namentlich in dem Gasgehalt des Meereswassers auf die Lehewesen (d. h. auf das massenhafte Vorknmmen von bestimmten Lehewesen) zurückzuführen, sind schon heute nicht mehr so gering an Zahl : Heringe und Copepoden, die den Siluerstoff des Seewasser's ausnützen. Sauerstoff-Termehrung durch die assimiliepende Wirksamkeit von Diatomeen u.s. W. Noch viel weniger schwierig ist es aber', eine Reihe von Beispielen zu geben, welche die grosse Bedeutung der physikalischen und chemischen Bedingungen für das Torkommen und das Gedeihen der Lebewesen im Meere dartun: Laichreife, die hei einer hestimmten 'Temperatur eintrifft, Rassen-I)iffolenzen, die mit dem Salzgehalt zusammenhängen, Wanderungen bestimmter Fische, des Horings in erster Linie, die von den Meeresströmungen hecinflusst worden 1. s. w.: rin richtiges Verständnis des Lohens und der Fortptlanzung irgend cines Organismus ist ohne genaue liemutnis der physikalischen und chemischen Bedingungen scines LebeMediums absolut undenkl)ar.

Also auch alogesehen von del hohen Bedentumg der Pflege der hydrographischen Wissenschaft um ihrer selbst willen, ist eine möglichst genaue Kentuis der physikalischen Bedingungen und der. Wassorbewro-

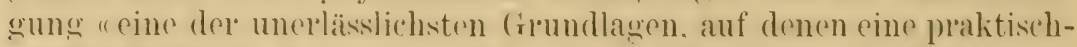
wissenschaftliche Erlienntnis des organischen Lebens der Meore. insbesondere der Ost- und Nordsee, aufgebaut werden muss \%. Und wie für die biologischen Lntersuchungen gilt mzweifellatt anch für die 
Erforsehung der hydrographischen Verhältnisse, dass nur " internationale Arbeit nach festen Prinzipien in stande sein wirol, jene grosse Fülle von korreliten Finzelbeolachtungen in unseren nordischen Meeren zu sammeln, die zul Gewinnung sicherer Schlüsse notwendig sind ". '

Also werden für die Erforschung dieser Verhältnisse seit Anfang unserer Intersuchungen, seit zwei Jahren ungeführ, vierteljährlich von allen beteiligten Staaten (Fngland und Schottland als gesondert zu betrachten) an festegetzten Tagen, mit speziell dafür eingerichteten Forschungsdampforn, eine Reihe von gemeinschaftlich festgestellten Stationen in den Meeren Nord-Europas besucht und dort eine Reihe von Beobachtungen angestellt. Ile liesultate dieser Beobachtungen werden nach der Rückliehr des I)ampfers von dem Hydrographen des betreffenden Landes ausgearbeitet und in tabellarischer For'm der ('entral-()roanisation, der hydrographischen Abtrilung des in Kopenhagen bufindlichen Bureaus, mitgeteilt und von dicsem vierteljährlich veröffentlicht. Mit Anfang des zweiten Jahles sind dieser Publikation, dem sogen. Bulletin. Tafeln beigefügt, auf welehen die Verhältnisse, was Temperatur und Salzgehalt anbetrifft, in sogen. hydrographischen Schnitten, graphisch dargestellt sind. Es ist ein äusserst wertvolles Material, das in dieser Voröffentlichung in übersichtlicher Form zusammengrtragen wird. Um nur ein paar Beispiele zu gehen, so wird s' gewiss mit Erfolg angewandt werden zur näheren Begründung der von Peatersoson und Ekmax anfurestellten Theorie, dass die physilialischen Verhältuisse unserer Meere von dem Gegensatz zwischen dem stark salzigen, ozeanischen Wasser und den süksen Abwässertungen der Festlande, sowie von der besonderen Figuration der Festlande, des Meereshodons und der Verbindungsstrassen zwischen der Nord- und Ostsee und dem Ozean bcherrscht werden. Demnächst wird das nämliche Material äusserst nützlich serin für dic Prüfung der Hypothese, dass die Wanderungen der Fische in erster Linie durch die Strömungen bestimmt werden. Auch wird es sich als wertvoll herausstellen für die nähere Erforschung der von den nämlichen schwedischen Forschern stammenden Vermutung; dass die Meeresströmungen, die durch den Gegensatz des ozeanischen (iolfstromwassors, des kialten, ans dom Eismeer kommenden Polarstromes und der süssen Abwässerungen der Festlande entstehen, sine äusserst wichtige, ja sogal wesentlich bestimmende Rolle fül das Klima Nord- und Mitteleuropas spielen.

${ }^{1}$ Ueber internationale Untersuchungen der nordeuropäischen Meere im Interesse der Seefischerei. Mitteil. d. Deutschen Seefischerei-Vereins XX. 5, S. 117, 1901. 
Also auch für die biologische Erfor'schung der nordischen Neere, zu deren Besprechung ich jetzt schreiten möchte, werden, wie ich oben schon betonte, die Eracbnisse der hydrographischen Untersuchung von sehr grosser Bedeutung sein. Es ist nicht leicht gewesen, auch fül diese biologische For'schung ein gemeinschaftliches Programm aufzustellen. Es jst ein ungemein weites Gebiet, nicht bloss den Dimensionen nach, sonder'n auch nach der Verschiedenheit der Eischeinungen, das man als 7ur Biologie der nordischen Meere gehörend zu betrachten hat; es liann nicht wundern, dass, als eine Anzahl von Gelehrten zusammenkamen, um über die Feststellung cines Programms für die biologische Erfor'schung dieser Meere zu beraten, sehr verschiedene Ausichten darüber laut wurden, was als das wichtigste zuerst in Bearbeitung genommen werden sollte. Und als die Angelegenheit aus dem Stadium der vorbereitenden Konferenzen in das der Ausfühlung übergehen sollte, stellte es sich bald als notwendig heraus, dass, sollte wirklich in alschbarer Zeit etwas wertvolles geleistet werden, die internationalen Untersuchungen sich wenigstens anfänglich auf die Bearbeitung einzelnel Hauptprobleme beschränken müssten. Da nun noch seitens einiger der Staaten, die sich bereit erkiärt hatten, an der, intermationalen Arbeit teilzunehmen, die Bedingung gestellt wurde, dass die Untersuchungen sich in erster Linie mit der Lösung von bestimmten praktischen, für die Nordseestaaten wichtigen Aufgaben beschäftigen sollten, handelte es sich nur noch ditl'um, diese genauel zu bestimmen.

Es drängten sich besonders zwei Hauptfragen in den Vordergrund, welche beide sowohl von rein wissenschaftlichem Standpunkte aus als von ökonomischer Seite als ausserordentlich wichtig betrachtet werden dürften. Und während die einzelnen Forscher die besseren, ihnen jetzt zur Verfügung stehenden Mittel nebenbei zu zahlreichen anderen zur Meeresbiologie gehörenden Nachforschungen benutzen, wird von ihnen Material zur Lösung dieser Hauptprobleme gesammelt und mehr oder weniger ausgearbeitet einem vom Central-Ausschuss ernannten Geschäftsführer, zum Teil auch direkt dem Bureau, zu weiterer Bearbeitung zugeschickt.

Die Probleme, welche hier als Hauptprobleme angedeutet werden, sind a) das Problem der Wanderung der Fische und b) das Problem der sog. Ueberfischung. Während beide besonders das Studium von NordseeFischen und Noldsee-Verhältnissen umfassen, wurde ihnen später die Untersuchung der ()stsee-Fische, der Ostsee-Fischerei-Verhältnisse u.s.w. als ein drittes Problem hinzugefügt. Es möge mir erlaubt sein, Ihnen die Bedentung der beiden Nordsee-Probleme in kurzen Zügen zu erläuter'n.

(Geschäftsführer fül die sub) a gemeinten Untersuchungen ist Herr 1)r. Johan I.ont, Beronen. Die für die Nordseefischelei wichtigsten 
Fische, deren Wanderungen Gegenstand dieser Untersuchungen bilden, sind der Dolsch und dep Hering. Man findet diese Fische nicht das ganze .Tahr hindureh auf den nümlichen Fischgründen in der Nordsee, sondern an bestimmten Stellen nur in riner gewissen Jahneszeit. Seit Jahrhunderten, kann man wohl sagen, ist dies den Fischern bekannt, und der Fischereibetrich hat sich natürlich diesen Wanderungen der Fische ganz angepasst: will man Ende Juni an der Wrestreite del Nordsee Heringe fangen, so muss man diese auf der Höhe der Shetlands Insoln suchen, im September findet man sie aber auf der Höhe von Neweastle, im November in dem südlichen Teile der Nordsee.

An der Ostseite der Nordsee bis in den Skagerak hinein hat man, wenn auch nicht die nämlichen, so doch gleichartige [)ifferenzen : an der Kü̈ste kichwedens kann der Hering vom September bis Febluar vorkommen; an der Küste Norwegens, besonders zwischen Stavangel* und dem Korsfjord, wird der sog. Frühjahrshering (Vaarsild) im Februar und März gefangen, während der sog. Sommerhering und der Fetthering (Fedsild) ausschliesslich in den Sommer- und Herbstmonaten gefangen welelen. Letztgenannte Fischereien finden in der Regel an den Küusten der nördlichon Provinzen Norwegens, ausnahnsweise auch um den Eingang des Kristianiafjords herum statt.

Nicht anders verhailt es sich im Norden mit dem Inorsch und vielleicht auch mit einigen anderen dorsehartigen Fischen (Kö̈hler, Ling u. s. w.) An der norwegischen Kiüste, auf den sog. Kü̈stenbänlen, findet der Fang von sog. "Shreimn ) (laichreifon Iorschen) in den vier ersten .Ionaten des Jahres und besonder's im März und April statt: der Ilaupt-Dorschfang Vorwegens ist also eine richtige Winter-Fischerej. Fs ist dies abel nicht die cinzige Inorschfischerei Norwegens: an der Küste von Finmarken hat man im April und Mai einen zweiten Dor'schfang, der als "Lodde "-Fischerei bezeichnet wird, weil man beobachtet hat, dass die Iorsche sich der Kï̈ste nähernn, um der sog. Lodde (Mallotres rillosus) nachzustellen. Eine richtige Sommerfischerei auf Dorsch findet damm auch hei Island statt. Doch sind nach Hsone die Mengen, die dort gefangen werden. nicht gross genug und die Gehicte, auf welchen sio dort und in Finmarken vorkommen, zu hegrenzt, als diss man annehmen dürfte, dass simmtliche grosse Dorsche sich im Sommer dort auflalten sollten. Es muss hier also noch von einem rätselhaften Verschwinden der grossen Fischschwärme gesprochen werden.

Yit den Dorschen auf der Ioggerbank und auf don sog. Fischerbänken hat es nun insofern cine andere Bewandtnis, als es schwirrig sein würde zu sagen, was dort die beste Zeit für den Dorschfang wäre. Sowohl Iorsich als Schellfisch fischt man dort, sowie an anderen Strollen in dere Nordsee, das ganze Jahr hindurch : seit Jahrhunderten, möchtr ich sagen, ist auch dies eine bekannte Sache, und seit Jahrhunderten werelen die 
auf diesen Bänken mit Angeln gefangenen Fische in den wärmeren XIonaten des Jahres als gesalzene Fische, in den Wintermonaten als frische Fische auf den Markt gebracht.

(1) diese Dorsche von denjenigen Norwegens versehieden sind, ob sie auch wandern, ob ihre Wanderungen sich über ein weniger ausgedehntes Gebiet erstrecken u. s. w., das sind Fragen, deren Beantwortung noch auf sich warten lässt. Wenn auch einzelne Tatsachen bekannt und zum Teil bis in die Einzelheiten studiert sind, irrt man sich doch, wenn man glauben würde, dass diese Fülle von Erscheinungen auch nur so weit erklärt wäre, wie es für den praktischen Bedarf wünschlich ist. Die Wanderungen geschehen zum Teil der Fortpflanzung, zum T'eil der Frnährung wegen - so weit war man schon vor vielen Jahren! In den letzten Wochen, die der Geschlechtsreife vorangehen, nehmen die meisten Fische entweder gar keine oder nur spärliche Nahrung: Fische, die in diesen Wochen wander'n, tun dies nicht der Nahrung wegen, sondern un für die Fintwicklung ihrer' Eier und Brut geeignete Stellen aufzusuchen. Hingegen gilt für die geschlechtlich unentwickelten Fische, die auf der Wanderung sind, ganz iu Allgemeinen, dass sie sich solchen Stellen, wo reichliche Nahrung vorhanden ist, zu näher'n suchen. Die Heringsscharen sind aber oft aus laichreifen, aus ausgelaichten und aus geschlechtlich unentwickelten (sng. Natjes) zusammengesetzt; sie wander'n an der Westseite der Nordsee gegen Sürlen, ohne dass man sagen könnte, dass sie sich dort bestimmten Laichplätzen näher'n, noch dass sie der Nahrung wegen dort hinziehen; die Theorie, die die Wanderungen ausschliesslich entwerler als Fortptlanzungs-Wanderungen oder als Nahrungs-Wanderungen erklären will, scheint also wohl kaum auszureichen.

Die Studien Herncke's, die die Existenz verschiedener Rassen unter den in den nordischen Neeren vorkommenden Heringen nachgewiesen haben, haben viel dazu beigetragen, die alte Ansicht von den sehr weiten Wanderungen der Nutzfische zu widerlegen. Wir nehmen jetzt die Existenz lokaler Fischstämme an, die die einzelnen physikalisch und biologisch verschiedenen (iebiete der nordischen Meere als indigene Rassen bewohnen. Aber auch die Wanderungen dieser Rassen enthalten noch viel Rätselhaftes und unterliegen periodischen Schwankungen, die wir nicht verstchen, die sich aber wirtschaftlich schr unangenehm fühlbar machen kömnen. Dass die physikalischen Verhältnisse des Seewasser's, Temperatur, Salzgehalt. Strömungen u. s. w. dabei eine grosse Rolle spielen, wird jetzt allgemein angenommen: wir sind aber noch weit davon entfernt sagen zu können, dass wir die Ahhängigkeit der Fischwanderungen von diesen Verhältnissen in den einzelnen Fällen auch wirklich verstehen. Der äusserst merkwürdige und von den schwedischen Forschern sorgfältig studierte Fall des Bohuslän-Herings, der allerdings schon sehr oft als Beispiel des engen Zusammenhangs zwischen den Wanderungen des 
Herings und den Meeresströmungen gediont hat, darf hier in Erinnerung gebracht werden.

Der älteren, fast sagenhaften Geschichte der Heringsfischerei Bohuslän's zu geschweigen, scheint es wohl begründet zu sein, dass nach einem langjährigen Ausbleiben der Horinge ungeführ 1746-49 wiederum eine besonders reiche Periorle der Fischerei angefangen hat; diese hatte wohl in den Jahren 1770-so ihren Höhepunkt erreicht, dauerte aber bis in die ersten Jahre des 19. Jahrhunderts tort. I)ie Heringsscharen fingen dann wiederum an, die Bohuslän-Lï̈ste zu meiden, mnl verschwanden allmählich ganz von dort; die Periode ihres Wiedererscheinens begann erst wieder 1s77: in gewaltigen Zügen drang der Hel'ing in diesem Jahre, wie in den folgenden, vom September bis in den Mäl'z wieder in die Scheren der Bohuslän-Küste ein. Man glaubte, dass von neuem eine längere Prriorle von reichen Heringsjahren für Bohusiän angefangen habe - als im Ilerbst und Winter 1sy6-97 der Hering wieder ausblieh mol grosse Schwierigheiten für die Fischer davon die Folge waren. Es sind nun bei dieser Golegenheit die in jenen Monaten herrschenden Zustände des Sliageraks in unfassender Wreise untersucht worden, und es hat sich gereigt, lass sich in Folge anhaltender und starker Winde aus ästlicher Richtung der sog. baltische Strom. der dort dire ()herflïchensehicht hildet, weit nach Westen ausgehleritet und das sng. Binkwasser grösstenteils verdrängt hatte, und dass das Wasser aus der Tiefedas sehr salzige Ozeanwasser - in Folgo dieses Cmstandes in die Höho gezogen war. ()b man auch bei fröheren Gelegenheiten die hydrographischen Verhältnisse so genan studiert hat, dass sich die Umstände mit denjenigen, die jetzt beolachtet wurden, vergleichen licssen. muss ich unentschieden lassen. Für den Fall von 1896-97 stellte man aber diese Erklärung auf: der Helingsfang ist fehlgeschlagen. weil in lolge des ()stwindes das Bankwassel soh" nahe an die (Obertläche gekommen und nur wenig Meter tief war und das salzige Ozeanwasser von 34 und 35 Salz pro Mille ein zu hohes Niveau erreicht hatte.

Eine neulich von dem norwegischen Hydrographen Nordiard aufgestellte 'Theorie kommt der Hauptsache nach zu ähnlichen Resultaten. Norugaro guht von der Annahme aus, dass die Wanderfische im allgemeinen mit dem strom schwimmen : dio Heringe nit dem Obertlächenstrom, die Dorsche mit dem Strom in der Tiefe. Ist also an der Küste Norwegens der (Obereflächeustrom mit anhiltendem Ostwind landabwärts gerichtet, damn bleiben die Huringe aus. Bei Ostwind ist aher der Kompensations-Strom in del 'Tiufo gerarle nach der Küuste ger'ichtet. und dieser Strom also führt die Dorsche mit, sich. Richtung und Kraft der Winde sind also nach ihm dio Hauptlaktoren, die das liesultat der Fischerej bedingen. Mit Ilülfe seiner Theorio lässt sich gut rrklären, dass oft ein gewisser (regensatz in dem Vorkommen des Frühjahl'sherings an der nor- 
wegischen Küuste und des Bohuslänherings beobachtet wird: hat das Vorkommen des cinen ein Maximum, dann hat oft das des anderen ein Minimum und ungekehrt.

Ich halte es fül sehr möglich, dass diese Theorien einen wichtigen Jín ron Wahrheit enthalten, dass man aber doch von ihnen nicht die auschliessliche Erklärung der hier in Betracht kommenden Erscheinungen erwarten darf. [Tnter den Biologen und wohl auch unter den Hydrographen wird es manche geben, denen es wie mil geht, denen is sehr schwel wild anzunehmen, dass es nur von den physikalischen Umständen der letzten Wochen abhängen soll, ol sich der Hering an einer hestimmten Stelle, z. B. an der Küüte von Bohuslän, massenhaft zeigen oder ganz ausbleiben wird, um sich dann sogar an eine andere Stelle zu begeben. Da soll doch erst festgestellt werden - um bei unserem Falle zu hleiben - dass wirklich die Bohuslän- und die norwegischen Frühjahrsheringe die nämlichen (oder verschiedene Altersstufen der nämlichen) Fische seien! Nach dem, was wir jetzt davon wissen, ist dies kaum wahlscheinlich. Auch ist es nicht leicht einzusehen, angenommen die hydrographischen Verhältnisse veranlassen, dass in einem bestimmten Jahre dic Heringsfischerei an einel bestimmten Stelle fehlschlägt, wie sie auch das Weghleiben der Heringe vou einer hestimmten (iegend während riner langjührigen Periode (z. I) vom Anfang des vergangenen Jahrhunderts bis ungefahr 1sit) erklären kïmnen: die Annahme, dass wïhrend einer'so langen Periode immer die nämlichen oder ungefähr die nïmlichen hydrographischen Bedingungen in der Wanderzeit der Heringe geher'scht haben, wird wohl von keinem verteidigt werden.

In allen Fällen liegt hier für die internationale Forschung ein äusserst interessantes und aus praktischen. ökonomischen Rücksichten wichtiges Problem vor! I)ie Bohuslän-Heringsfischer benutzen kleine Boote und betrachten nur solehe Jahre als Heringsjalıe, in welchen die Heringr bis in die Scheren hinein ziehen und sich dort in Iassen fangen lassen. In den Jahren, in welchen der Heringstang an der Bohuslän-Küste fehlschlug, handelte es sich also, aller Wahrscheinlichkeit nach, nicht um ein vollständiges A usbleiben der Heringe, sondern um einen nicht lohnendron Fane infolge des Umstandes, dass die Heringe zum grössten Teil ausserhalb der Scheren blieben. Wolche waren nun die nächsten Stellen, an welchen die Heringe sich in reicheren Scharen ansammelten? Es kömnen sich solhstrerständlich nur dann sehr reiche Scharen der Küiste näher'n, wenn in einer früheren Laichperiode recht viele von der nämlichen liasse oder Varietiit (oder wie man es nemnen will) gehoren und scitdem herangewachsen sind. Desshalb bildon die reicheren Jahre eine (ipuppes, die wohl von Gruppen von ärmeren Jahren unterbrochen werden können, nicht aber von Jahren, in welchen die Heringe dieser Rasse? ganz fohlen. Wie auf ihre Wanderungen üben die physilialischen Bo- 
dingungen auch auf die Entwicklung, auf das Heranwachsen der Heringe cinen überaus mächtigen Einfluss aus. Hencke's Untorsuchungen haben auch schon einiges Licht über die Bohuslän-Heringe (ihre Laichplätze u. s. w.) verbreitet: an diese anküupfend, ohne daboi die Bedeutung der hydrographischen Bedingungen aus dem Auge zu verlieren, wird es wahrscheinlich golingen, der Lösung des Problems näher zu kommen.

Ich möchte hieran anschliessend auf einen schon im Anfang von mir berïhrten Punkt zurückgreifen, nämlich auf dio Bedentung der kleinen Lebewesen des Planktons als Leitorganismen für die Bestimmung von Wasserschichten. Ein äusser'st interessanter Streit ist auch über diesen Gegenstind entbrannt! Fs wird natürlich die Bedeutung der Meeresströmungen für dic Terbreitung der Plankton-Organismen ron keinem bezwoifelt. Wählend abor nach einigen Golehrten die Plankton-()rganismen regulmässig mit dem Wasser einer hestimmten Herkunft kommen und gehen und sie somit viele riesel Organismen als für bestimmte Wasserschichten charakteristisch betrachten, nohmen andere Forschere an, dass dic Strömungen gewissen Plankton-Arten (und wie es sich allmählich herausgestellt hat, gar nicht wenig(n) behülflich gewesen sind, eine sehr weite Verbreitung zu erhalten. Nach ihnen haben aber auch die Plankton-Lebewesen (Diatomeen, Peridineen, Copepoden u. S. w.) eine hestimmte geographische V(rrhreitung: einge eine beschränktere, andere eine weitere, während auch einige fast über die ganze Welt rerbreitet in dem Plankton vorkommen. Zeigen sie sich, wie mit oimmal, in bestimmten Wassorschichten in auffallend grosser Anzahl, dann wird von letztgemamnten Forschern nicht angenommen. dass sie alle mit riesem Wasser mitgekommen sind, wohl aber, dass sie in diesem Wasser für ihre Entwicklung und ihr Gedeihen dir Bedingungen günstig gefunden und sich in Folge dessen dort schr sehnell und seln kräftig entwickolt und vermehrt habon. Letztere Annahme schliesst natürlich nicht aus, dass sich hier und da Plankton-Formen von dem Wasser getragen in Gegenten verioren, wo sir nicht zu Hause sind und also gewissermassen als zufällige Gräste betrachtet werden müssen. und natürlich auch nicht. dass es bestimmte Wanderformen gielot: die grosse Mehroahl del Formen soll aher cine lokale Verbreitung mit einer schwankenden Häufiglesit hahen, je nach den Umstäuden (Jahreszoit, Zusammensetzung des Wasser's u. s. w.).

Man sieht nun leicht ein. dass die Frage, wie man sich die Verhreitung der Plankton-Oreanismeñzu denken hat, auch für die Lösung des Problems der Fischwanderungen von besonderer Bedrutunes ist. Ist die Ansicht richtig, dass bestimmte Planliton-Formen massenhaft mit den Wasserschichten wanderen, so gewinnt auch die Ansicht, dass gewisse Wanderfische ron diesen Wasserschichten getragen und verbereitet wereden, eine grosise Wahrsehrinlichleit. I) Wanderficehe sind aber anch 
in ihrem Wander'n voneinander sehr verschieden, und was für den eine'n gilt, hraucht für den andren kein Gesetz zu sein: wie so oft, wird sich bei fortgesetzter L'ntersuchung herausstellen, dass auch dies Problem viel verwickelter ist, als man Anfangs angenommen und für eino baldige Lösung für erwünscht halten möchte. Alte Probleme spalten sich in neue, grössere allgemeinere in kleinere und speziellere, und die Wissenschaft schreitet fort, indem sie kileinere Irrtümer an die Stelle grösserer' setzt ?

I as Probleu der Ueberfischung ist von rein praktischem Standpunkt aus vielleicht noch wichtigel als das der Fischwanderungen. HuxLer konnte seiner Zeit noch der Meinung sein, dass die Produlitivität des Meeres eine so grosse sei, dass im Vergleich damit der von Menschen auf dessen Reichtum ansgeübte Einfluss kaum in Betracht komme. I)ie Erfahrung der letzten 20 Jahre hat aber für die Nordsee und besonder's für gewisse Fischarten gezeigt, dass die menschliche Tätigleit durchaus so harmlos nicht ist. Besonders hat die Meinung. dass del Fang von hlein'ren (sog. untermässig(n) Plattfischen auf die Dauer für die Erhaltung des Fischbestandes eine (iefahr soi, allmählich viele Anhänger wewonnen. Als im Jahre 1890 eine von der" National Sea Fisheries Protection Association " nach London berufene internationale Konferenz zusammentrat, um über die Schonung und die Entwicklung der Nordseefischereien zu beraten, wurde wohl schon der Wunsch nach einem internationalen Vertrag, dex dem Landen und dem V'ekauf von untermïssigen Plattfischen entgegentreten sollte, ausgesprochen, wurde aher zu wleicher Zeit betont, dass die Massregel nicht genommen werden solle, so lange man nicht über eine genaue Kenntnis, statistisch sowohl als biologisch, des durch den Fang von untermässigen Fischen angerichteten Schadens verfüge. In verschiedenen Staaten hat man sodann über diese Frage Untersuchungen angestellt und auch versucht, der Lösung der allgemeineren Frage, ob wirklich Ueberfischung stattfinde, nïher zu troten. Ueber vieles haben die von einzelnen Ländern unternommenen Forschungen schon Licht verbreitet: je weiter man kam, je mehr stellte sich aher die Notwendigkeit heraus, die verschiedenen hier in Betracht kommenden Fragen nach dem Prinzip der internationalen Arbeitsteilung von nenem zu studieren. Will man für die rationello Bewirtschaftung des Meeres rine wissenschaftliche Grundlage finden, so müssen die I'ntersuchungen sich zu gleicher Zeit über ein möglichst grosses Gehiet erstrecken; dann müssen auch gemeinschaftlich aufyestellte Arbeitsplïne und ühereinstimmende L'ntersuchungsmethoden den einzelnen Forschern der verschiedenen an der Sache interessierten Länder die Zusammenstellung der Resultate ermöglichen und erleichtern.

Ess würde kamm von Höflichkeit für mein gegenwïrtiges Auditorium 
\%eugen, falls jch eine ausführliche Iarstollung der Art und Weise greben würde, in der man sich voryenommen hat, das Problem der Uherfischung zu erfol'schen. Ich möchte mir nur erlauben, über ain parr diesen Gegenstand berührende Punkte etwas zu sagen.

Es ist möglich, dass die Grösse der Produktion des Meeres an belebter Substanz sich theoretisch berechnen lässt - wie dies schon vol einigen Jahren von Hensen versucht wurde. Bei einer Berechnung dor Cirösse del Produktion eines hestimmten Meeres an tür menschliche Nahrung sich eignenden Fischen wit'd schon die. Ungleichnässigkeit der Verteilmng, die lokale und zeitliche Anhäufung von Exemplaren, wie dirse z. B. mit den Wanderfischen vorkommt, die allerörosten Schwierigkeiten in den Weg legen. Nach meiner Meinung wird man also nicht leicht, vielleicht nie, dazu kommen, sagen zu kömnen: das Mreer (oder auch nur: dir Sordsee) produziert pro Tag oder pro Jahr so viel, und da ihm mehr als dies Quantum entnommen wird, findet Ueberfischung statt!

Um die Ueherfischung zu konstatieren, muss also ein anderel Wrgegewählt werden. I)ie Ueberfischung muss, wenn sie stattfindet, nach kürzerer oder längerer Zeit Rülekgang der Fangergebnisse zur Folge haben. Es liegt also auf der Haud, dass man sich von dem in den einzelnen Ländern gesammelten statistischen Materiale für die Beantwortung der Frage, ob [eherfischung wirklich stattfinde, vic] Nutzen versprochen hat. Jedoch würde man sich irren, wenn man glaubte, dass das jetzt schon vorliegende Material von statistischen Angahen sich ohne weiteres für eine endgültige Beurteilung dieser Sache anwenden liesse. Wel sich mit statistischen Untersuchungen beschäftigt hat, woiss, dass, soll die Statistik für die Beantwortung bestimmter Fragen einen entscheidenden Wert hahen, so muss sie auch speziell mit Rücksicht auf diese Fragen gesammelt sein. Nun ist die Fischerei-Statistik fast ausnahmslos im Interesse des Handels gesammelt - nicht im Interesse der Lösung del hier in Betracht kommenden Fragen. So giebt sie z. B. nur selten Auskunft über den Gesammtfang irgend eines Landes, wohl über die Gesammtmasse von Fischen, anch von bestimmten Fischen, die in einem Fischereihafen gelandet sind; sie schweigt meistens über die Fangplätze, sowie über die Zahl der Schiffe', mit wolchen die Fische gefangen und ans Land gebracht sind. Es ist natürlich brsonders die Frage, wo die Fische gefangen sind, von der allergrössten Bedeutung; die statistischen Angaben sind selhstverständlich nur dann unter sich veruleichlar, wenn sie sich auf das nämliche Fischereigobiet bezichon. Hat sich aber die Fischerei, wïhrend die Statistik gesammelt wurde, übcr ein viel grösseres (rebiet verbreitet, so ist der Vergleichswert verloren gegangen. Nur in dem Falle, dass der (resamutfang ungeachtet dieser Ausbreitung nur wenig urösser geworden, lionstant gehlicben oder sogar kleiner geworden ist, ist es klar, dass die Erträge dos ursprünglich befischten engeren Gubiotes 
ahgenommon haben. In der Regel sind die Verhältnisse aber nicht so einfach und setzt die zur Verfügung stehende Statistik uns nur sehr mangelhaft in Stand, derartige Konklusionen zu ziehen, zumal da man in den meisten Länder'n er'st neulich angefangen hat, sie zu sammeln und sie in anderen Länder'n nur insofern existielt, als sie sich auf die exporticlten Fische bezieht. Bedenkt man, wie schwankend die Ergebnisse vieler Fischereien so wie so sind, so sieht man schon deshall ein, dass nur eine sich über längere Perioden erstrockende Statistik wirklich brauchbar sein könne. Dass aber doch eine sorgfältige Bearbeitung der in den einzolnen Ländern bisher veröfentlichten Fischerei-Statistik in Angriff genommen wurde, geschah teils, weil sie in einzelnen Beziehungen, z. 13. was hestimmte Fischarten betrifft, wertvolle Auskunft zu geben verspricht, teils, weil man in allen Fällen von dieser Bearbeitung erwarten darf, dass sie zu einer eingehenden Prüfung und sorofaltigen Beantwortung der Frage fülıren wird, wir soll die Fischerei-Statistik gesammolt und aufgestellt werden, um eine richtige Beurteilung der Abnahme oder Nicht-Abnahme des Fischbestandes zu ermöglichen?

Im engsten Zusammenhang mit der Terwendung der Fischerei-Statistik im allgemeinen strht die Frage, ob es möglich soin wird, auf statistischem Wege den schon oben von mir berührten, den Fang und Verkauf von untermässigen Plattfischen betreffenden Punkt hinlänglich kilar zu legen. An welchen Stellen und in welchen Jahreszeiten werden in der Nordsee die meisten kleinen Plattfische gefangen, welche Grösse haben diese Fische, in welchen Quantitäten werden sie gelandet? Das sind ganz einfache Fragen, deren genaue Beantwortung aber uncriasslich ist, soll es je gelingen, gegen die Vernichtung der kleinen Fische auf entscheidende Weise einzuschreiten.

Der hier zunächst in Betracht kommende Fisch ist einer, der das ganze Jahr hindurch in der Nordsee mit den sog. Trawls gefischt wird und doch gewissermassen als ein Wanderfisch betrachtet werden muss : es ist die Scholle. Die Eier dieses Fisches treiben, schwimmen im Wasser und werden in der Laichzeit über ein sehr ausgedehntes Geloct verbreitet vorgefunder. In unticferem Wasser, ganz in der Nähe der Küste halten sich die gan\% likinen Exomplare auf, und mit der Zunahme ihner Grösse verbreiten sie sich über tieferes Wassel weiter von der Kũste ab. An den tiefsten Stellen, wo Schollen noch ziemlich häufig sind (d. h. bis auf $60 \mathrm{~m}$. Tiefe), findet man auch die grössten Exemplare. Von diesem Fisch wurden und werelen immer noch gewaltige Quantitäten gefangen und ans Land gebracht: "I ist wohl der wichtigste Plattfisch der NordseeSchleppnetztischerei. Mit der Zunahme dieser Fischerei ist aber nicht allein eine Verminderung der Iurchschnittsgrösse dieser Fische Hand in Hand gegangen: man ist wohl darüber rinig, dass sich allmïhlich auch rine Verminderung der Gesammtquantität konstatieren lässt. 
Neben mittelgrossen und grossen Fxemplaren werden nun in gewissen Jahreszeiten auch seh" bedeutende Quantitäten von kleinen Exemplaren dieser Fischart gefangen und gelandet. Das ist auch früher geschehen; weil damals abel die Trawlfischerei noch keine so grosse Ausdehnung hatte, weil also für den damaligen Betrieb hinlänglich grosse Fische da waren, kümmerte man sich nicht um den Fang del kleinen Fische. Jetzt ist die Sachlage eine andere geworden: es werden wohl noch grosse Quantitäten kleiner Fische gefangen und gelandet: man möchte aber gern mehr mittelgrosse und grosse Fische auf den Fischgründen vorfinden und fragt sich nun, ob dies nicht durch Schonung der kleineren Fxemplare zu errerichen sei. Aus Untersuchungen der letzten Jahre über diesen Punkt scheint hervorzugehen, dass die Schollen der sog. zweiten Grössenstufe in bestimmten Gebieten der Noldsec grosse Anhäufungren bilden und sich dort aufhalten, bis sic eine Grösse von wenig über $20 \mathrm{~cm}$. erreicht haben. Nachher sollen sie sich mehr zerstreuen und dic Dichte ihres Vorkommens nie mehr eino so grosse werden, als bevor sie die genannte Grösse erreichten. Die internationalen Untersuchungen nehmen sich nun in erster Linie vor; diesen Punkt näher zu elforschen. Eine speziell zu diesem Zweck gesammelte Statistik soll üher das Vorkommen, die Häufigkeit, die Grösse der jungen Schollen in den hier am meisten in Betracht kommenden Teilen der Nordsee Auskunft geben: dazu werden in den einzelnen Ländern in den verschiedenen Monaten des Jahres an den Hauptanfuhrylätzen zahlreiche Messungen von jungen Plattfischen ausgeführt, von welchen bekannt ist, wo sie gefangen wurden. Dies Material wird dem centralen Bureau zu weiterer Bearbeitung übergeben. Es sollen aber auch die Wanderungen, die Wachstumsgeschwindigkeit, die Grösse, bei welcher sie zuerst laichreif werden, die Fruchtbarkeit u. s. w. dieser Fische so genau wie möglich untersucht werelen. Zu dem Ende sollen sowohl das ganze Jahr hindureh die an hestimmten Stationen in der Nordsee gefangenen Schollen auf das genaueste untersucht und miteinander verglichen, als auch das Narkieren von Schollen in grösserem Massstabe angewandt werolen.

Dies Markieren und Wiederaussetzen von markierten Schollen (um mit den Fischzüchtern zu l'eden) soll nun auch dazu angewandt werden, um zu hontrollieren, ob mit den Uebersiedeln von kleinen Schollen von solchen Stellen, wo man sie in ungemein grosisen Mengen antrifft, nach tieferen und von Schollen nur spärlich besuchten Teilen der Nordsee praktische Resultate erzielt werden können. Es ist dies ein von dem dïnischen Biologen D1. C. (*. Joh. Perersex vorgeschlagenel Versuch. Peatersex stütyt sich dibei auf eine von ihm in dem Limfjord (im Norden Jütlands) gemachte Frfahırung und hält es für wahrscheinlich, dass dort, wo die kleinen S.hollen so äusserst zahleeich sind, ihro Wachstumsgeschwindigkeit eine geringere ist, als sie sein würde, wemn man die jun- 
gen Fische nach anderen in Folge der Fischerei oder anderer Ursachen wogen nur schwach mit Schollen besetzten Toilen umpflanzte. Er hält es somit für möglich, dass was sich mit gutem Erfolg in dem Limfjord hat machen lassen, auch mit Vorteil in der Nordsee anzuwenden wäre. Alle diese Untersuchungen sind jetzt in vollem Gang und versprechen in absehbarer Zeit über viele Punkte aus der Naturgeschichte dieser für den menschlichen Haushalt so ausserordentlich wichtigen Fische Licht zu verhreiten. Yeben den Schollen sollen zu gleicher Zeit besonders die Seezungen und von den runden Fischen die Schellfische berücksichtigt werden. Letztgenannter Fisch, der sich, bevor dir Dampfschleppnetzfischerei sich entwickelt hatte, vom Herbst bis Frühjahr regelmässig und in so grossen Scharen, den südlichen Küsten der Nordsee näherte, dass um die Zeit an der deutschen Küuste, z. B. von der Insel Norderney aus, und an dep holländischen Küste von Helder aus, Angelfischerei auf diesen Fisch als Küstenfischerei, sogar mit offenen Booten, betrieben werden konnte, wird jetzt wohl noch in grossen Mengen von den Trawls gefangen und von den Trawlfahrzeugen gelandet. unter den feilgebotenen hat aber die Zahl der kleinen (3. Sorte) und derjenigen der 4. Sorte allmählich ein sehr bedenkliches Ueberowicht über die der mittelgrossen und grossen bekommen - und die einst als Kïustenfischerei betriebene Schellfischangelfischerei hat ganz eingestellt werden müssen.

Ich will mich hier in diesen Gegenstand nicht weiter vertiefen. Ich mö̈hte nur noch hinzufügen, dass Hand in Hand mit den oben skizzierten Untersuchungen auch solche angestellt werden sollen, die über die Möglichkeit Licht verbreiten sollen, die Schleppnetze und die Fischgeräte im allgemeinen so zu modifizieren, dass ihre Schädlichkeit gemindert wird. Cielingt es, Netze zu lionstruieren, mit welchen ein lohnender Fang gemacht wird, und die doch die kleinen Plattfische sowohl wie die kleinen Rundtische durch diæ Maschen entwischen lassen, so wäre damit der Fischerei-Industrie ein ausserordentlich wichtiger Dienst erwiesen! Sieht dies augenblicklich noch aus wie ein Suchen nach dem Stein der Weisen, die grossen Mittel über welche die internationale Meeresforschung verfügt, berechtigen aber gewiss dazu, sehr bedeutende Leistungern zu erwarten, und dass diese in erster Linie der FischereiIndustrie zu Gute kommen sollen, ist in vollkommener Uebereinstimmung mit den Gedanken, welche dem Zustandekommen unserer Organisation zu Grunde gelegt wurden.

Sie, meine hochverehrten Zuhörer, muss ich nun auch deshalb um Entschuldigung bitten, weil ich Ihre Gedanken in dem Masse von den idealen Bahnen der. reinen Wissenschaft ah auf cin so praktisches (rehiot dee Naturfor'schung hingelenkt habe. Es ist ganz richtig, dass die Wissenschaft, die allein um ihrer selbst willen gepflegt wird, eine höhere libldung vorauswetzt - das strelon aluer, anf wiscensehaftlichem 
Wrege Problence zu erforschen, die für das Wohl der Menschen von hervolragender Bedentung sind, hat doch gewiss auch scine Berechtigung. Nan verliere dabei nicht aus dem Auge, dass, sei es auch das IIauptziel der internationalen Neeresforschung, die Fischereibetriebe zu forrdern und füı internationale Fischereiverträge eine möglichst zurribasige Glundlage zu heschaffen, die Thtersuchungen sich deshalb, weder was Breite noch was Tiefe anbetrifft, zu beschrälien brauchen. Dem einzelnen Fol'scher ist von Anfang an vollkommene Freiheit gewihrleistet. seine ['ntersuchungen so zu unternehmen und seine Aufgabeso auszualberiten, wie es ihm für die Lösmng dieser Aufgabe erwünscht vorkommt. Die für die aemeinschaftlichen Untersuchungen aufgestellten Programme sollen leiten, nicht zwingen oder beschränken. Hieran anknüpfend möchte ich zum Schluss noch einmal auf die grossen Mittol aufmerksam machen, über welche die Meeresforschung dank der internationalen Organisation jetzt verfügt. Ihnen brauche ich nicht zu explizicren. wie ausgezeichnet sich diese Mittel zu gleicher Zeit für die Bearheitung verschiedener in hiologischer Hinsicht wichtiger Fragen anwenden lassen, auch wenn diese Fragen mit den in erster Linie in Behandlung genommenen Problenen nur indirekt in Zusammenluang stehen sollten. Und dass dies jetzt schon geschieht, dafül brauche ich bloss Braxup's Untersuchungen übor den Stoffrechsol im Merere, über dio Bedentung der Stickstoff́verbindungen für die Produlition im Meere als Beispiel anzuführen. welche sich den schon vor dem Zustandekommen unserer ()rganisation ausgeführten Untersuchumgen Hevsex's üher dir Produlition des Xecpes an helebtel Substanz anschliessen. Als Beispiele solcher Untersuchungen nenne ich weiter Gran's Studien über Meeresbakterien, Enrenbaum und Strodtuasx's Untelsuchungen über die

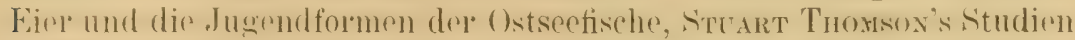
über das periodische Wachstum der Schuppen der Gadidoe, um aus so vielen nenerdings erschienenen grösseren und kleineren Arbeiten nur einiges heranzuzichen.

Meine hochverehrten Damen und Her'en, die die Geduld gehabt haben, mir bis hiel zu folgen, ich möchte meine Skizze hiermit abschliessen. Hoffentlich ist es mir, wenn auch nur einigermassen, gelungen, Ihnen davon die Ueberzengung zu geben, dass, wenn die internationale Moresforshung im Norden Furopas in Foigr der Kopperation von neun Staaten über verhältnismässig grosse Mittel verfügt, die Bedeutung der Ziele, die von ihr angestrebt werden, zu diesen Nitteln in cinem nicht zu ungünstigen Verhältnis steht.

M. Je Président donne la parole à M. le Secrétaire Général du Comité pel'manent. 


\title{
RAPPORT SUR LE VEU DE M. 0. KLEINSGHMIDT, PRÉSENTÉ A LA TROISIÈME ASSEMBLÉE GÉNÉRALE DU CONGRÈS
}

\author{
Par le Prof. R. BLANCHARD \\ Secrétaire général du Comité permanent.
}

Le comité permanent, après avoir examiné le vou de M. O. KuerNschumo, propose à l'Assemblée générale de l'adopter et de le transmettre au Conseil fédéral de la Confédération Suisse.

Après une discussion à laquelle premnent part MM. ZerLeder, Kúcenschind, Strules et Hoек, l'assemblée générale adopte cette proposition.

MI. le Président de l'Assemblée donne la parole à .I. le Président du Congrès:

M. le Prof. Studer, Président du Congrès:

Hochansehnliche Versammlung!

Indem ich hier den VI. Internationalen Zoologenkongress schliesse, möchte ich noch in Ihrem Namen den Dank aussprechen an die hohen Behörden, welche das Gelingen desselben ermöglicht haben. Vor allem dem hohen Bundesrate, der Regierung des Kantons Bern, dem Gemeinderate und dem Bürgerrate der Stadt Bern, den Vorständen der medizinischen, der tierärztlichen und der naturfor'schenden Geselischaften der Schweiz, den Behörden und Vereinen von Interlaken, welche uns heute in so liebenswürdiger Weise empfangen haben. Höge auch der VI. Internationale Zoologenkongress fruchtbringend auf die Weiterentwicklung unserer Wissenschaft gewirkt haben.

Hiermit erkläre ich den Kongress für geschlossen. 


\title{
SEAANCES DES SECTIONS
}

TENTES A L'TNERSTÉ

\section{Ire SECTION}

\section{Z00LOGIE GÉNÉRALE}

Séances du lundi 15, mardi 16 , mercredi 17 et jeudi 18 aoùt $190 \%$

Président: MI. le Prof. W. Saldensky

Vice-Plésident: M. C. Schlumberger

Secrétaile: M. ie Dr A. Gurwrtsch

Stegomyia Fasciata, der das Gelbfieber übertragende Mosquito und der gegenwärtige Stand der Kenntnisse über die Ursache dieser Krankheit.

\author{
Von Prof. E. A. GOELDI (Pari).
}

1. Unter den zahlreichen blutsaugenden Culiciden ouler Mosquitos del' Anazonas-Region haben mothodische Anfsammlungen. die sich hrereits über mehrere Jahre erstrecken, uns als solche kennen gelernt, die rorzugsweise dem Menschen lästig fallen und somit in sanitiurer Beziehung in Betracht gezogen zu werden verdienen, die Arten: 1. Anopheles argyrotarsis und die vielfach gleichzeitig und an den gleichen Oertlichkeiten vorkommende, so nahe verwandte Varietät albitarsis. 2. Panoplites titillans und amazonicus. 3. Truiorhynchus fasciolatus. 4. Janthinosoma Lutzii. 5. Culex confirmatus. 6. Stegomyia fasciata. 7. Culex fatigans.

2. Die כ̆ zuerst aufgezählten Al'ten, neben einigen anderen, die mehr rine seliundiure Rolle spielen, verfolgen den Menseluen, die einen mehr 
des 'Tages, die andern mehr des Nachts, zumal im Ereien: im Walde, auf dem Folde, im Gestrïp) und im Sumpfe, während dio beiden letzteren. Stegom!yu fascinta und Culex futigans so recht eigentliche Hausplage-

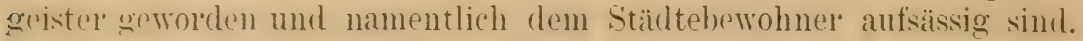
Ihr Lehen und ihr gan\%er Entwicklungsgang spielt sich in und um die menschlichen Behausungen ab. Dabei ist Stegomyia fasciata vorzugswoise Tagtier, mit ausgesprochen photophilen Neigurgen, während Culex fatigans in dorselhen Treise ein naichtliches Insekt genannt werden muss. Dor Tätiglepit beider substituiert mol reänzt sich gegenwärtig in der Stadt Pará zu einem 'lages-Cyklus.

3. Neben den Arten, die durch ihre Blutdürstigkeit und Zudringlichkeit, durch ihre Zahl und den beim Stiche verursachten physischen Sohmorz beschworlich fallen und sich zu cince besonderen Rangordnung einteilen lassen, tritt eine unverkenubare sanitäre Wichtigkeit heute schon klar zu Tage bei den 3 Arten: 1. Anopheles argyrotarsis in Beziehung zur Malaria. 2. Stegomyia fasciata in Beziehung zum gelben Fieber: 3. Culex futigans in Beziehung zum Filariose.

4. Anopheles kommt wenigel für die Stadt Pará, als fül die sumpfigen Niederungen im Innern des Staates, für das Land in Betracht. Da nnseren persönlichen Beobachtungen zufolge Anopheles in der Stadt und in deren Peripherie selten sich einstellt (circa s-12 Exemplare imnerhalh) 2-3 Jahren), glaube ich mit Bestimmtheit vorauszusehen, dass von den zahlreichen Fälen von Fieberkrankheiten, die von der einheimischen Merlizin unter dem Allgemein-Begreift " impaludismo," zusammengewürfelt werden, das Allermeiste an der Hand des mikroskopischen Befundes und methodischer Blutuntersuchung als ausiseloalb des Brgriffes der" "Malatria "stehend erkannt werelen wird. Was an eigentlicher" "Malaria " dol't angetroffen wird, ist woll in der Regel im Innern des Landes erworben und von aussen eingeschleppt.

5. Da das Problem der Nahrung in ilrem Einflusse auf die Dauer des Lebens und auf die Fruchtbarkeit der \{Weibchen, sowie dasjenige der Copula in ihrer Rückwirkung auf die Blutliebhaberei dor Weibchen bisher' bei keiner Culiciden-Art der neuen noch der alten Welt gr'ündlich untersucht worden war, ist während des Jahres 1903 in Pará eine lange Versuchselhe zumal mit. Stegomyur fuscirtu und Culer futiguns angestellt worden, in der an circa.220 erwachsenen Individuen der einen Art und annähernd 260 Individuen der anderen Art diese Verhältnisse auf experimentellem Wrege und nach einem methodischen (irundplane und Frageschema definitiv festgestellt wurden.

6. Unter den Resultaten besagter Unter'suchung, die den Gegenstand einer ausführlichen, portugiesischen, vor einigen Monaten veröffentlichten Abhandlung bildet. möchte ich natehfolgende hesonelerel Beachtung empfehlen: 
a) Honig und Zuckersaft bilden fül ${ }^{\circ}$ das weibliche Individu um de

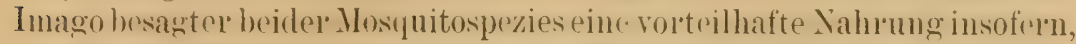
als sie die Lebensdaner verlängert; die Beurtrilumg fält hingengen umgekohrt aus vom Standpunkt der A r terhal tung, demn dieser Frmährungsmodus übt in ersichtlicher Weise einen verzögernden Einfluss aus auf den Reprodulitions - Vorgang, bezichungswoise die Eicrahlage. I)urch ausschliessliche Hongnahrung gelang es die biorablage his über 100'Tage zulückzuhalten, um sie am 102ten 'lage zur sofortigen Auslösung gelangen zu lassen durch Einschaltung von Blutnahrung.

b) Das Blutsaugen ist für die Weibchen der namhaft gemachten Mosquito-Arten ein notwendiges, menthehrliches l'ostulat zur Ablage ent-

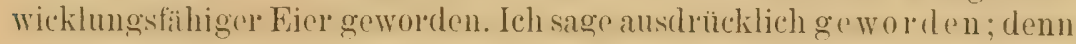
verschiedene Gründe veranlassen mich, anzunehmen, dass hier eine verhältnismässig neue Anpassungs-Erscheinung vorliegt, deren Vorteil, rincrseits vielleicht in einer beschleunigten Auspeifung der Fierstockseier, anderer'seits abel hauptsächlich in einem quantitativen Zuwachs der Eier gefunden werden dürfte.

c) Schon bei einer einmaligen Blutration macht sich die förderliche Wirkung auf die Eier-Ablage bemerklich. Hoch hat eine solehe bloss eine particlle, fraktionierte Fi-Auslösung zur Folgæ. Eigentliche Ersehöpfung des Ei-Vorrates erfolgt bloss nach mindestens 2-3 maliger Blutration in der Schnelligkeit der Verdauung parallel laufenden Intervallen.

d) Bemerkenswert ist nun, dass auch unbefruchtete weibliche Imagi-

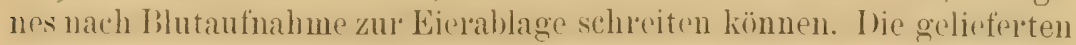
Eier erwiesen sich jedoch in allen Fällen als nicht entwicklungsfähig; ich bezeichne sie als pseudo-parthenogenetische.

e) Durch experimentelles Verfahren ist aber mindestens bei den beiden vorgenamnten Culieiden-Spezies ein Abhängigkeits-Terhältnis zwischen normaler Eier-Ablage unel Blutsaugen bei einem höheren Wirbeltier festgestellt, - Abhängigheits-Verhältnis, das als eine Uehergangsphase zum definitiven Parasitismus wird anerkannt werden müssen. Es wird Sache spezieller Untersuchung sein, nachzusehen, ob und bis zu welchem Grade sich dieses Abhängigkeits-Verhältnis anch bei anderem dem Menselien und den höheren Wirbeltioren durch libutsaugen beschwerlich fallenden Mosquito-Arten nachweisen lässt.

f) Bei Stegomyia fasciata kamn es keinem Zweifel unterliegen, dass auch dir Mämnchen dem Menschen beschwerlich fallen durch Sichlürfen von Schweisströpfchen an den enthlässten Stellen der Kö̈rperoherfläche. Zum wirklichen Durchstechen der Epidermis ist aber ihr Rüssel zu schwach gebaut. Die in dieser Ioyichung besser ausgerüsteten Weiluchen halien sich mun nicht mit dem schweiss- und Sormm-saugen brenügt und sind zum habituellen Anstechen und Anzapfen übergengingen in direkter Absicht auf das Blut der Wirthstiere. Es wird auf solche Weise 
hei dieser Art auf"s Schönste der von der Hamaphilie durchlaufene historische Wro und Fntwicklungsgang dargetan. Es ist auch vom sanitäl'ischen Standpunlite aus hemerkenswert, dass lüngs der Küuste Buasiliens die Zudringlichlieit der Stegomyia, in den Imagines beider Geschlechter (r'sichtlich proportional sich verhält der Temperaturhöhe respektive der Intensität der Schweissabsonderung.

7. Entgegen dor bisher vielfach vertretenen Anmahme, die die Heimat dor Steyom!ju fusciutu in die Antillen-Region verlegt und ihr frühestes Auftreten mit dor Entderkungsfahrt des ('olumbus in Velbindung setzt, scheue ich mich nicht, aus verschiedenen Gründen, zumal aber aus solchen, die sich mir in erster Linie aus einer kritischen Prüfung über die heutige gengraphische Verbreitung des gexammten Genus Stegomyia ergeben, die Meinung aufzustellen, dass Vaterland, Herkunft und I)ispersions-Zentrum dieser gefährlichen Mosquito-Art in Afrika zu suchen sein werde. Ihre Verbreitung über die neotropische Küste atlantischerseits erkläre ich mir (gerade so wie bei manchen anderen Ueborträgern, die hente in Brasilien zum anscheinend einheimischen-Krankheits-Inventar gerechnet zu worlen pttegen) durch den sklavenhandel in früherer Zeit.

S. Was den augenblicklichen Stand der Frage nach der Ursache des Gell b e Fi eber's anbetrifft, so hat die bereits universell acceptierte, auf Versuche basierte Annalıme der nordamerikanischen Aerzte auf Cuba, lierd. Caroll und Agramonte - nämlich. dass diese Krankheit dem Stiche der Mosquito-Art Stegomyin fascintu ihre Fintstehung verdanlie. num in allerneuester Zeit auch in Brasilien und in ganz Südamerika derartig allgemeinen Ankiang gefunden, dass in der medizinischen Presse dieser Länder andere Deutungen und Zweifel sich nur noch vereinzelt hervorwagen.

Ias (xofühl dor sicherheit in dieser Schlussfolgerung hat in Brasilien seine hauptsächliche bestarkung "rfahren dureh die Wrederholungsversuche des cubanischen Experimentes del künstlichen Tebertragung durch eine ärytiche Lommission im Staat São Paulo einerseits und die vom Institut Pasteur in Paris behufs Studiums des (relben Finder's nach

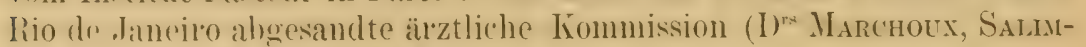
BENI und SuMON) andererseits.

9. In ihrem im November 1903 in den "Annales de l'Institut Pasteur" »

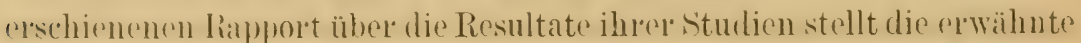
französische liommission in weiteren noch folgende Ergohnisse, Schlüsse und Konjekturen auf:

a) Un Gelbes Fieber hervorzurufen, muss der Mosquito vorerst sich infiziert hahen, indem er Blut eines (relbfioberkanken wänend der :3 risten Tige des Krankheitsverlaufes einsog (pag. 729), Conclusion 17) und dak Selum des Kranken am dritten Tage ist virulent (pag. 7.24, Conclusion 1). 
b) I)ie infizielte Stegomyio ist bloss geführlich nach einem Intervall von mindestens 12 Tagen seit Aufnahme von virulentem Blute (Conclusion 18) und ilne Geführlichkeit nimmt in dem Maassitabe des Zeitunterschiedes zu, welcher zwischen Selbst-Infelition mol abermaligem Stiche liegt (Conclusion 20).

c) Der Stich von zwei inficirten Stegomyias kam V'ranlasisung eines schweren Gelbticher-Falles werden (Conclus. 19). Aher der Stich von inficirten Mosquitos veranlasst nicht nothwendigerweise das gelloe Fichore (" ne donne pas fatalement la fièvl'e jaune ») (Conclus. 21).

d) Die populäre Annahme, dass die Gelbfieberansteckung Nachts erfolge, wird in clee Weise erkliirt, dass das Stegomyju-Weibchen nach rinmaligel IHutaufnahme seine bisherige 'Tages-Lebensweje mmkehre, zu einem nichtlichen Inselite werde (pag. (i9:5). Eine nothwendige complementä1e Schlussfolgerumg dazu ergäbe, dass die inficirendon stegomyiuWeibchen also einzig und allein unter den nächtlicher Weile stechenden Individuen gesucht und gefunden werden müssten.

e) Auch die Copula werde zur Nachtzeit und in der Dunkelheit vollzogen, sei selten und schwer zu beobachten (pag. 681) und überhaupt vollzögen sich unter dem schutze des nächtlichen I)unkels die wichtionsten Lebensfunktionen dieser Art (pag. 686) (" c'est surtout à la faveur de l'obscurité et pendant la nuit que s'accomplissent les fonctions les plus importantes de l'existence de l'espèce n).

f) Obwoh! zugestanden wird, dass die Mission insofer'n negativ verlaufen, als abermals weder im Mosquito selber, noch im Blut das Agens des Gelbfiebers nachgewiesen werden konnte ( nous n’avons réussi jusqu'à présent de mettle en évidence l'agent de la fièvl'e jaune ») (pag. 730, Conclus. 30), so geht doch aus dem ganzen Gange der" Untersuchung, aus der Fragestellung und der Argumentation mit unverkennbarer Deutlichkeit hervor, dass die Existenz und Wirksamkeit eines

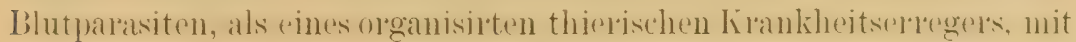
zeitlich bestimmtem Entwicklungsgang und gegebener IncubationsDauer, gebunden an die Uebertragung durch gewisse speziell inficirte Stegomyia-Weibchen-Individuen, die in der Luft schwebende Voraussetzung, Annahme uud Ausgangspunkt bildete - wir können also kurz sagen, ein gคnau kongruentes Analogie-Verhältniss vermuthet wurde zwischen Stegomyia und Gelbfieber, wie es zwischen Anopheles und Malaria duroh das Bindeglied des Plusmodinm maluriar und zwischen ('uler. fatigans und Filariose durch das Intermedium von Filaria sangrinis hominis thatsichlich besteht.

10. Gewissenhafte Originalstudien nun über die Einzelheiten der Lebensweise von Stegomyia fasciata einer'seits, die persönliche Gelegenheit der genauen Beobachtung und Begleitung vom ersten Anfang bis zum leider letalen Ausgange eines typischen Gelbfieberfalles anderscits. 
im Weiteren die Vertiefung in die neueste ${ }^{1}$ und ältere medizinische Literatur üher' (ielbfieber' und schliesslich die kritische Ueberlegung und Sichtung des von mir während 20 Jahren in einem mehr oder weniger als klassisch angesehenen Gellofieber-Nedium selbst Erlebten und Erfahrenen und aus vertrauenswürdiger Quelle (ishörten, hat mich nun zu der felsenfesten Treberzeugung geführt, dass gerade in eimzelnen von denjenigen biologischen Fragen bezüglich der Lebensweise von s'tegomyju, die als springende Punkte für die herwärtige Diskussion in Betracht fallen, die Wahrheit ander's liegt, als es nach den Darstellungen der französischen Mission den Anschein hat. (ierade so, wie meine eigenen hiologischen Resultate bezüglich des Golbfieber-IIosquitos divergiren, so werde ich anch hinsichtlich der Artiologie des Gelbfieber's selbst zu (iner verschiedenen Auffassung gedrängt, die in kurzen Zügen zu charakterisiren mein Bestreben ist:

11. Für das Erste halte auch ich an der Erkenntniss fest, dass in Stegomyin firsciatu der Teherträger und Verbreiter des gelben Fiebers zu suchen ist;

12. Entgregen aber der neuen Meinungsströmung, dass das Agens des gelhen Fiobers in einem organischen Blutparasiten etwa nach Analogie des Verhältnisses zwischen Anopheles und Malaria (Plasmodium mularix) zu erblicken sei, fühle ich mich zu der Annahme gedrängt, dass rine genau deckende Analogie nicht vorliegt, dass das Agens in Form und Gestalt (ines organischen Giftes, eines Toxin's, vorauszusetzen ist, welches in erster Instanz normaler Weise in den Speicheldrüsen von Stegromyin fusciute seinen Sitz und Ausgingspunkt besitzt und durch den Stich dem Menschen eingeimpft wird ${ }^{2}$.

${ }^{1}$ Hiebei kann ich nicht umhin zu betonen, dass mir der umfangreiche, treftliche "Report of the Yellow-Fever-Expedition to Parí 1900 ", herausgegeben von a School of Tropical Medicine " in Liverpool (1902) und verfasst von den englischen Aerzten Durham und Mrers (wovon der letztere als Opfer seines Berufseifers in Pará erlag) durch ihre nüchterne, sachliche Darstelluug von ganz besonderem Werte gewesen ist.

2 Um meine Meinung und Ansicht genauer zu definieren gegenüber den augenblicklich in Mode stehenden Tendenzen "in medizinischen Kreisen, möchte ich hier ein für allemal betonen und festnageln, dass ich weniger gegen die Annahme eines Blutparasiten uberhaupt mich ablehnend verhalte, als gegen diejenige eines verhältnismässig so grossen und relativ hoch entwickelten Blutparasiten wiebei Malaria und Filariose.

Die Vorsicht erheischt, immerhin noch für die Möglichkeit Raum offen zu lassen, dass eventuell schliesslich doch noch eine von jenen winzigen Bakterien-Formen die Hand im Spiele haben könnte, die, zwar als Einzelindividuen für unsere bisherigen optischen und sonstigen Erkenntnismittel nicht direkt fassbare, indessen als kompakte Mengen doch sinnlich wahrnehmbare Form und Gestalt anzunehmen vermögen.

Eine solche Möglichkeit spricht ja auch aus so manchen Stellen des verdienstvollen 
13. Jeder cinzelne Stich wirkt also überhaupt giftig und als normaler Trbertriger dient jedes einzehe weihliche Individum von stergm!nia in Momente des Stiches. Jeder stich ist cine Partial-Infektion: die TotalInfektion wird durch die summierende Wirkung aller Einzelstiche beziehungsweise aller Partial-Infektionen hervorgebracht.

14. Das Speicheldrüsengift von Stegomyia wird durch das Blut von der Peripherje nach innen verfrachtet und die Leber, sei es im Kampfe gegen dasselbe (was ich für das Wahrscheinlichere halte), sei es als Bundergenosse desselben (indem sie möglicherweise dasselhe ihrerseits in nrue, giftige Toxine umsetzen liönte) wird zu einer almor'men Tätigkeit angeregt, die in einem hochgradigen Ictorus immerlich wie äusserlich ihren Ausdruck findet. (Nach übereinstimmendem Urteil ist beim Sektionsbefund die intensiv gelbe, fettig degenerierende Leber ebenso rogelmässig dir vorderstr. die Autmerksamkrit tesselnde Firscheinung, als das mehr oder weniger ausgedehnte Gelbwerden der Körperhaut zu den ständigen nosologischen Merlinalen auf einer gewissen Krankheitsphase zu zählen pflegt - so sehr, dass es ja mit Fug und Recht zur Namengebung der Krankheit selbst herangezogen wurde.

15. Schält man bei den Beschreibungen von Vergiftungen, die anderwoitig durch den Biss giftiger Tiere, also auch durch deren Speicheldrüsenseliret hervorgerufen worden sind, die lokalen Erscheinungen (die der Grösse der Wunde und der Quantität des injizierten Giftes proportional sich verhaltend, natürlich manchmal überriegen), los, so gewinnt das von den Allgemein-Erscheinungen gelieferte und zurïck-

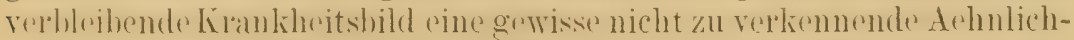
liejt mit dem (iellotieber. (Verol. Simmetomatologie-rles sehlangenhisses (Ophidismus) bei Screube ( Krankheiten der warmen Länder », 1903 (pag. 450-461) pag. 453. Speziell scheint der Icterus als eine gewohnte Begleiterscheinung aufzutreten, was mir beim Durchlesen der einschlä-

Rapportes der englischen Aerzte-Kommission (Dr. Durhan und Dr. Myers) bezüglich ihrer Studien in Pará. Es ist dort die Rede von einer gewissen Bakterien-Art, die teils in den verschiedenen Organen von Gelbfieberkranken, teils auch in der Stegomyja-MIücke selbst angetroffen wurde und es scheint, als ob die Regelmässigkeit ihres Vorkommens nicht verfehlte, auf die erwähnten Autoren Eindruck zu machen.

Wenn sich durch Einräumung einer solchen Möglichkeit einerseits der streng theoretische Standpunkt einigermassen verschiebt, so vermag ich andererseits vom praktischen Gesichtspunkte aus eine sehr wesentliche Veründerung der Sachlage nicht einzusehen. Alle die ins Gebiet der Prophylaxis und Defensive einschlagenden Fragen z. B. würden jedenfalls duich den Entscheid, ob er nun so oder so ausfalle, keineswegs berührt. Auch das Spezifische an meiner Auffassung über die Art und Weise der Uebertragung und Ausbreitung des Gelben Fiebers erleidet nicht die geringste Modifikation, ob nun ein organisches Toxin oder cine unendlich kleine Bakterienform sich als letzte Ursache herausstellt. 
gigen medizinischen Literatul (vide z. B. Schevis, loc. cit., 3. Autl. 1903, jag. 461 sag.) einen besonder's nachhaltigen Eindruck hinterlassen hat. Nan verogleiche doch was dort gesagt wird bezüglich der Symptomatologie hei Biss von Skorpionen (pag. 463-464), von spinnen, z. B. Latrodectes nactans (pag. 465) und Zecken (Ixodiden) (pag. 465-466).

16. Aber auch einzelne andere Vergiftungen mit organischen und anorganischen Stoffen ergeben cin Krankheitsbild, indem der Ieterus und die hemolytische Zerstörung imponierend im Vordergrund stehen; ich brauche $z$. B. bloss an die Phosphor-Vergiftungen zu erinnern.

17. Der Ausbruch des Gelbfiebers bei einem Neu-Ankömmling - der. wenn Horid aussehend, vollh)ütig und stark schwitzend, für die Stegomyja brsondere Anziehungskaft besitzt - scheint in der Regel in del Weise von sich zu gohen, dass nach voraufgegangener Total-Infektion durch Stegomyia, die sich eventuell üher Wochen und Ionate erstrecken kiann. die Auslösung durch eine gastrische Störung (Darmkatarrh, Erkältumg, etc.) (rofolgt. I)ie Situation ist zutreffend derjenigen einer geladonen und gespanten Schusswatfe zu verolejchen, wo die Entladung durch die keine mechanische Leistung am Drückel ausgelëst wi!rl; elfolgt diese nicht, so wirel auch die Entladung ausbleiben: Die Waffe kann, geladen und mit gespanntem Hahn, einrosten - es ist die natürliche Immunisation,

18. Die gastrische Störung wird in letzter Instanz die Funktionen del Leber affizier'en und so dürfte voraussichtlich das von der StegomyiaIntoxication gelirerte und aufoestapeltr Toxin zur Virulenz angeregt und in seiner verheerenden Wirkung unterstützt werden.

19. Es wird also zwischen Intoxication und Begleiterscheinung zu unterscheiden sein. Ine crstere wird am Tage zu stande liommen. wärend letztere in der Regel Nachts aufgelesen wird. So lösst sich denn auch das Rätsel der sogenamnten "I) iarios do Petropolis" (es hrancht bloss noch die an und für sich schon wahrscheinliche Ammahmo hinzuzutreten, dass dere

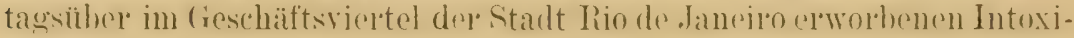
cation durch die von den klimatisehen Veränderungen begünstigte Isildung oines Antitoxins regelmaissig erfolgreich entgegengewirkt wirl) - das so viel Kopfzerbrechen verursacht hat und dem zulieb, "pour le besoin de la cause n, die französische Mission in Rio de Janeiro den Tatsachen Gewalt antat, indem sie das Stegomyia- Weibchen zu einem närhtlichen Tiede strupelte. Was also nachts geholt wird, ist wohl nicht

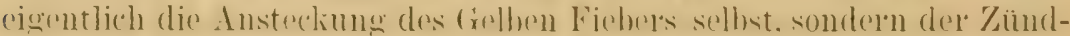
stoff zur Explosion und der Anlass zur Auslösung der Katastrophe.

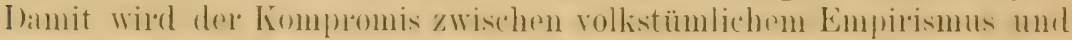
tatsichlichem Sachverhalte auf's einfachste in ein verständliches Licht geruickt.

Das mil vorschwebende gegenseitige Verhältnis zwischen Intoxication 
und Antitoxin möchte ich am chesten mit einer Conto-Corlent-Rechnumg vergleichen, wo im Soll und im Haben tägliche Posten zur Buchung gelangen, die sich nahezu das Gleichgewicht halten: nach Quantität und Zeitdaure summiere Wirkungen nach boiden Seiten hin. Ein lileines tägliehes Plus zu Gumston des Antitoxins rückt für den im GellotichereMedium kontinuierlich Verweilenden die Annäherung der Immunisiorung herbei das ungekehrte Vorhältnis wird z. B. rurch rine gastri-

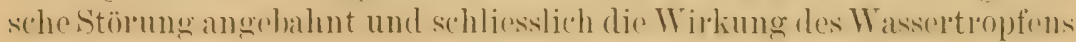
wiederholt, was das volle Glas zum Ueberlaufen bringt. Dass das bei einer jeweiligen Bilanz den dusschlag gebendo I) fizit auf der einen und

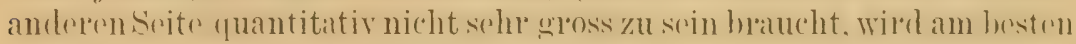
dureh die Tatsache beleuchtet, dass bei den "Diarios de Petropolis » nic eigentlich eine tatsaichliche Immunisierung erfolgt.

20. Mit vorgehendem soll nun keineswegs die Möglichkeit einer Infektion von einem Gesunden durch einen Gelhfieberkranken duxch

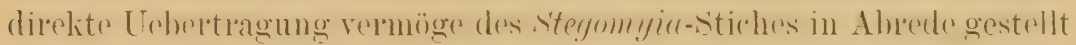
werden. In Gegenteil : meine eigene Ansicht von der Natur und Entstehungsweise des Gelben Fieber's bedarf dieser Möglichkeit und ich vermute sogar, dass solchem Toxin eine erhöhte Virulenz und potenzierte Wirkung zukommt. Ich bringe mit diesem direkten Uebertragungs-

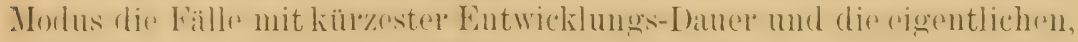
cyklisch um sich grepifouden Epidemien in Zusammenhang, wïhrend irh auf dem langsamen Wege der fraktionierten Intoxication durch die sich summierenden Wirkungen der Finzelstiche die spmungweise Entstehung völlig neuer Krankheitsherde erkläre, vorausgesetzt natürlich, 1. dass überhaupt Stegomyia an der betreffenden Lokalität vorkommt und 2. dass dort die zu ihrer ungehinderten Vermehrung erforderlichen klimatischen Existenzbedingungen gegeben sind.

21. Beim Krankheitsverlauf des Gelben Fieber's wird 1. ein inflammatorisches-congestives Stadium und daranf, 2. ein Remissions-Stadium unter'schieden, welches gegenüber von dem ersteren durch den 'Temperatur-Fall sich abgrenzt. Nun ist es eine bekannte Eigentümlichkeit dieser Krankheit, dass das Eintreten der erwähnten Remission keineswegs sich an eine bestimmte Zeitregel hält und sie kamn ebensowohl nach 2-3 'Tagen, als nach dem doppelten Zeitraum sich einstellen. In dieser laumenhaften Unregelmässigkeit dürfte ein weiteres gewichtiges Argument zu Gunsten der Amnahme eines 'Toxins gegeben sein, denn läge cin Blutparasit vor nach Analogie der Malaria, so stände zu erwarten, dass seine Existenz in ciner zeitlich 1uehr oder weniger scharf ausgeprägten Periodicität, in einer an Regel und Gesetz gebundenen IncubationsDauer ihren äusseltich ersichtlichen und fühlbaren Ausdruck fände.

22. Es sollte möglich sein, durch sorgfïltiges, geduldiges Herauspräparieren "en masse » von frischen Stegomyia-Thorax, wo im vorderen, 
unteren Quadranten die Speicholdrüsen lokalisiert sind, Speicheldrüsensokret in reaktionfïhigem Zustande in solchen Quantitaten anfzusammeln. die direkte physiologische und therapentische Versuche zu unternehmen Mlaubten uml uns so den Intoxications-Vorgang in etwas vergrössertem Maassitahe verdeutlichten. Solche Versuche wären zunächst in zweicrlei Richtung von besonderem Interesse: 1. in Bezug auf die Frage nach dem Verhalton des Blutes überha!tpt bei dem Zusammentreffen mit dem Stegomyia-Spricheldrüsensekret: 2. hinsichtlich des Trohaltens der Leber bei direkter Injektion mit demselben Sekret.

2:3. Eine del hauptsächlichsten medizinischen Fachzeitschriften Bratsiliens, das wöchentlich in Rio-de-Janeiro erscheinende "Brazil-Medico », bringt in einer nouesten, mir kurz vol der Alneise zugekommenen Nummer einen Artikel mit der Nachricht, dass inzwischen erfolgreiche Heilversuche an Gelbfieberkianken vermittelst Injektion von "Anti-Crotalin-Serum » und "Anti-Bothropin-Serum » angestellt worden sind, also durch Finimptung eines durch Schlangengift geronmenen und attenuirten und gegen schlangen-Bisi- und fift gerehteten Heilserums. Auch die hervorragendsten Homöopathen Brasiliens weisen mit Stolz auf die günstigen liesultate hin, diesie seit Jahren und zu 7eiten intensiver Epidomien gegen das Gelbfieber elzielten durch ihr" "Crotalus ) genanntes und ans Kilappreschlangen-Drïsenseliret gewonnenes Spezitikum. Man mag hierüber denken, wie man will, man wird sich der Erinnerung an den Wahlspruch "Similia similibus ) nicht erwehren können und wemn im Allöopathen- wie im Homöopathen-Lager auf erfolgreiche Anwendung soldher aus Schlangengift hergestellter Arzmeistoffe einstweilen als liesultat lesliglich ampirischer Frliemutniss hingewiosen wird, so wird es erlaubt sein, zu konstatiren, dass auch die 'l'heorie heute bereits ihre Erklärung hiefür zu bieten hat und dass meine Auffassung von dem Gelbficber als einer Intoxication durch das Speicheldrüsenseliret der Stegomyia von dieser empirischen Seite her eine bedeulungsvolle Bundesgenossenschaft erfähıt.

24. Wenn meine völlig unabhängig gewonnene Theorie, wie ich fest überzeugt bin, seitens del medizinischen Wissenschaft in nicht allzu

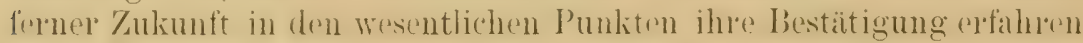
wird, so sehe ich auch wie ein freundliches Gestirn über der leidenden Menschheit die begründete Hoffnung leuchten, dass über kurz oder lang der Kahn auf der Suche nach wirksamer Prophylaxie und Defensive nach wissenschaftlich unanfechtharen Grundsätzen aus dem Bereiche der schaukelnden Wogen auf dem breiten Meere des Zufalles den richtigen Weg finde und einfahre in den sicheren Haten positiven Wissens.

25. In prophylaktischer Hinsicht bleibt nach wie vor als Hauptgrundsatz zu Recht bestehen: "Das letzte Gelbfieber-Vorkommen wird mit

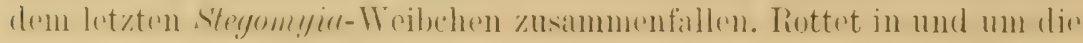


Städte diesen Ueberträger aus, so wird die Krankheit von selbst aussterben. ") Das Gelbe Fieber wird bloss in den Städten nicht erlöschen, wo man und so. lange man nicht will! - Vollste Anerkennung verdient dis auf dieser Erkenntuiss fussende, radikale Vorgehen, welches seitens des ('hefs des hrasilianisehen Sanitits- Wesens, Inr. ()swaldo Croz, neuerlich bezüglich der Stadt Rio-de-Jancimo mit glänzendem Erfolgr angewendet wird, nach dem Muster und Vorbilde der von dem verdienten englischen Arzte Ronald Ross inscenirten " Mosquito-Brigaden ».

\title{
Die Mutationstheorie im Lichte zoologischer Tatsachen.
}

\author{
Von Prof. L. PLATE (Berlin).
}

Da der Tortrag in erweiterter Form und mit den nötigen litterarischen Hinweisen im "Archiv für Rassen- und Gesellschaftsbiologie » Bd. II elscheinen soll, so gebe ich hier nur ein kurzes Résumé meiner * Erörterungen.

\section{Lassen sich Variationen und Mutationen begrifflich streng unter-} scheiden?

Es kommen hier folgende Gesichtspunkte in Betracht:

1. Die Variabilität soll nach DE Vries von der Eınährung abhängen (dieses Wort im weitesten Simne genommen), welche die individuelle Kraft hald gïnstig, hald ungünstig beeinflusst. Sir wird also in letztre. Linie erzeugt von den wechselnden Faktoren der Aussenwelt. Die Mutabilität hingegen soll unbekannten Ursprungs sein. Es ist klar, dass sich hier'mit kein Gegensatz begründen lässt. Auch die Mutationen müssen

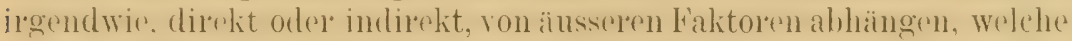
auf das Keimplasma plötzlich verändernd einwirken.

2. Ebenso wenig stichhaltig ist der Untersehied, wonach die Variatione'll nur quantitative Aenderungen natch Plus und Vinus, die Jutationen

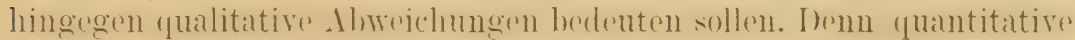
Veränderungen können leicht einen solchen Grad annehmen, dass sie als

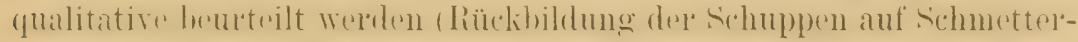

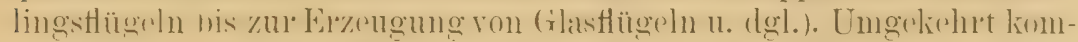
men manche typische Mutationen zustande nur durch Verlust odel Zu-

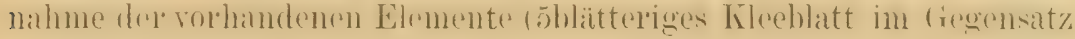
zum 3blätterigen; Auren-Mutationen der Blätter beruhen auf der Zunahme des Xanthophylls gegenüber dem Chlorophyll).

3. Variationen sollen mit Uebergängen auftreten und dem QueTELET'-

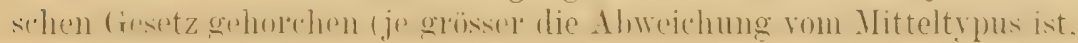


desto soltener wird sie heobachtet); bei den Mutationen fehlen die Uebergänge, sie treten plötzlich und stossweise auf. Ich gebe zu, dass in dieser These viol Wahrheit anthalten ist und dass in vielen Fällen Abweichunge'n von geringer Erhlichkeit "flukturen" um einen Mitteltypus, während umgokehrt stark erbliche Abänderungen "spontan », unvermittelt auftreten. Ein wirklich brauchbares Kriterium erhalten wir aber hierdurch nicht, weil

a. alle sog. moristischen Variationen (übre- lesp. unterzählige Organe: Wrirbel, Rippen, Zähne, Augentlecke et(.) ihrer Yatur nach einen discontinuirlichen Stow odor Schritt bedeuten: derantige Veränderungen sind sehr oft gar nicht oder $11 \mathrm{r}^{\circ}$ in geringem Masse erblich, stehen also dann in der Mitte zwischen typischen Variationen und Mutationen.

b. Dasselbe gilt für viele andere Variationen: zwischen einer links gewundemen Helix und einer normalen gibt es heinen Uebergang, trotzdem ist abre 's'stere duchaus nicht erblich. Fhenso treten viele Farbenvariationen plötzlich, olne Uebergänge auf und zeigen dabei keine oder nur sehr geringe Erblichkeit.

c. Typische Mutationen weisen zuweilen zahlreiche Uebergänge auf, sind also kineswegs immer völlig diskontinuierlich. I) gefüllen Bhımen zeigen oft alle (x)ade der Füllung: Trifolinm pratense quinurefolinm zeigt inmer norh vine dreischeibige Blätter. Plantugo lanceolata ramosa weist noch zahlreiche unverzweigte Achren auf.

4. Nach manchen Autoren bedeuten die Variationen kleine Ahweichungen. Sehritte, während die Nutationengrösser Abändrungen. Sprüunge. darstellen. Sie sehen daher in der Mutationstheorie eine Stütze für eine "sprungartige Evolution,", wie sie von Köurrer in seiner Theorie der " heterogenen Zeugung " vertreten worden ist. Diese Auffassung ist unhaltbar und auch von DE VRies bekämpft worden, denn viele seiner Mutationen stellen nur sehr geringe morphologische Verindermengen dar. Er unterscheidet sogar manche Halhrasien und Vittelasisen als. Mutationen, die sich morphologisch überhaupt nicht. sondorn nur phrsiologisch an ihrer verschicdenen Erblichkeit von einander trennen lassen (tricotyle, syncotyle Mutationen).

5. Variationen sollen bei grossem Material immer nach den verschiedensten Richtungen hin nachweisbar sein, während die Mutationen (三 single variations, Darwiv) selten, meist sogal sehr selten sind. Es liegt auf der Hand, dass auch dieser Gegensatz, wenngleich oft volhanden, nicht scharf ist, zumal einzclne typische nicht erbliche oder schwach erbliche Tariationen (links gewundene oder scalariforme Helix, manche Farbenalureationen der schmetterlinger) zu den grösiten Soltenheiten gehören.

6. Variationen zeigen eine geringe Neigung, als correlative Gruppenabiunderungen anfzutreten. Währemel die Mutationen meist a Habitus- 
änderungen 》, gesetzmässige "Umprägungen 》 der verschiedensten Teile darstellen. DE VRIEs elwähnt aber' auch einzelne Mutationen, bei denen nur ein Charakter sich verindert hat, z. B. Datura stramonizm Frucht mit Dornen, Datura stramonium inermis Frucht glatt.

7. Selection von Variationen soll nach de VIrIES, Correns und JoHANnsen nur zu labilen, erblich nicht gefestigten Zuchtrassen führen, die nach dem Aufhören der Zuehtwahl wieder in die Stammform zurüclischlagen; hingegen sollen llutationen durch Selection rasch von allen fremden Beimischungen sich reinigen lassen und dann schon nach wenigen Generationen ein festes Erblichkeitsverhältnis zeigen, das nach DE VRIES freilich in dem Prozentsatz der Erben erhebliche Unterschiede aufweist. Darnach unterscheidet er reine Rassen mit ca. 100\% Erben, Mittel-

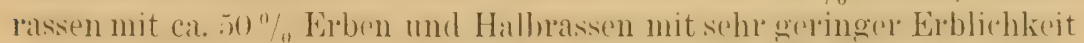
$(1 / 2$ bis $3 \%$. Ob dieser angebliche Gegensatz inder Wirkung der Zuchtwahl wirklich vorhanden ist oder nur auf Täuschung beruht, ist eine der dunkelsten und schwierigsten Fragen der Abstammungslehre. Ianche

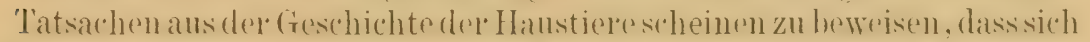
durch andauernde Ausleso kloiner Abänderungen schliesslich konstante

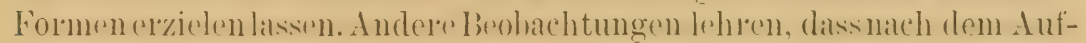
hören der Selection die Rassenmerkmale ungefähr ebenso schnell verschwinden, wie sie durch die Züchtung entstanden sind, woraus zu fol-

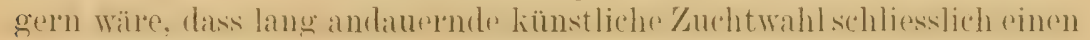
solchen Grad von Festigkeit herbeiführen muss, dass er in praxi einer erblichen Constanz gleichkommt. So wichtig die ganze Frage für die praktische Tiel'- und Pflanzenzüchtung ist, so wenig Bedeutung hat sie fül' die Evolutionstheorie, denn die Natur hört - solange die jeweiligen Fol'men des Kampfes ums Dasein sich nicht ändern - nie auf mit der einmal begonnenen Züchtung, also kann auch kein Rückschlag eintreten.

8. Die Anhänger der Mutationstheorie betonen besonder's, dass Variationen häufig gar nicht oder nu' in sehr geringem Masse erblich sind, während die typischen Mutationen (nota bene bei Selbstbefruchtung) $100 \%$ Erben licfern. Hieraus kömnte man ein physiologisches Criterium ableiten, wenn nicht DE VIres selbst auch solche Abänderungen zu den Mutationen rechnete, die wie die Mittelrassen ca. $50 \%$ oder gar wie die Halbrassen nur $1 \%$ oder noch weniger Erben aufweisen.

Aus den angegebenen Erwägungen folgt, dass alle Uebergänge existieren zwischen nicht oder wenig arblichen Variationen und vollstandig erblichen Mutationen; es kann demnach kein prinzipieller Gegensatz zwischen Variationen und Mutationen vorhanden sein, denn eine

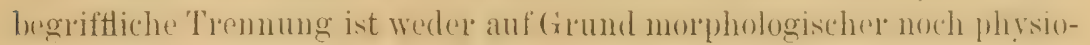
logischer Charaktere durchzuführen.

Sollen wir num deshalb den Begriff der Mutation völlig fallen lassen? 
Ich glaube, dies wäle nieht richtig. denn für die Abstammungslehre ist es ron grö̈ster Wichtigheit, den Grad der Erbblichlieit festzustellen. De Fries hat sich rin sehr grosses Verdienst epworhen durch den Nachweis, dass $A$ binderungen plötzlich auftreten kïmnen, die von vorn hreein im höchsten Grade erblich sind. Solche Formen verdienen auch eine besondere Brzeichumng und mögen daher auch in Zukunft als "Mutationen " gelten, während das andere Extrem die wenig erblichen Variationen darstellen. Beide Gregensiitze sind in der Satur dureh alle Ueberoünge verhunden und lassen sich daher sprachlich nur unterscheiden, wenn man übrerinkommt, den Inenriff jedes Wortes aus Zweckmässigkeitsgründen in irgend einer. Weise zu begrenzen. Ich schlage folgende konventionelle Nomenclatur vor:

Alle individuellen Abweichungen, welcher Art und welchen Erblichkeitsgrades sie auch sein mögen, werden zusammengefasst mit der alten Darwin'schen Bezeichnung "Variationen ». Dadurch wird zugleich angedeutet, dass scharfe principielle Gegensätze sich unter den Variationen nicht aufstellen lassen. Die Variationen zerfallen in drei Gruppen:

a) Die Somationen sind überhaupt nicht erblich, weil sie r'ein somatische Abänderungen darstellen und in ihrer Wirkung nicht bis zum Keimplasma vorgedrungen sind.

b) Die Fluctuationen sind Abanderungen mit einer Erbziffer von

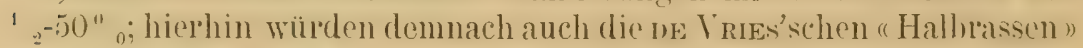
zu rechnen sein. die üh)rigens besser als "Schwachrassen " hezeichnet werilen, da das Wort Halluasse zu leicht nach seiner Bildung mit " Mittelrasse » verwechselt werden kann.

c) Die Mutationen haben eine Erbziffer', von 50-100\%.

Die Mittelrassen von DE VRIEs mit einer um 50\% schwankenden Erblichkeit würden den Uebergang von den Fluctuationen zu den Mutationen bilden ${ }^{1}$.

Bei dieser Auffassung handelt es sich demnach um physiologische Differenzen des Lirimplasmas, die sich in ciner wechselnden Erblichloit äussern. Es ist abre sehr wahrscheinlich, dass diesen liategorien meist auch eine Tendenz zu morphologivehen (irgensitzen inmewohnt, derart, dassdic Fluctuationen in der Regel Lohergïnge und geringe korrelative Verkettung zeigen, während dir Nutationen, namentlich diejenigen mit höchster Enhlichkeit, ohne Uebergïnge, aher als Gruppenabänderungen auftreten. Wir sind gewohnt, Erblichkeit und Variabilitit als Gegen-

\footnotetext{
'In dem mündlichen Vortrag habe ich die Abgrenzung etwas anders formuliert, nämlich: Variationen 0-25\%, Mittelrassen 25-75\%; Mutationen $75-100 \%$; es scheint mir aber praktischer zu sein, für die bis jetzt wenig bekannten Mittelrassen keine besondere Kategorie zu machen, hingegen die nicht erblichen Abweichungen durch einen Fachausdruck hervorzuheben.
} 
sätze aufzufassen. Bei del reinen Rasse mit 100\% Erhlichkeit, schlagen alle Kinder nach den Eitern, so dass eine variable Nachkommenschatt ausgeschlossen ist, während umgekehrt in (ler Mittelrasse nur $50 \%$ der Kinder den elterlichen Charakter erben, während die übrigen hiervon mehr oder weniger abweichen, also tas Bild einer" "Variation mit Ueber-

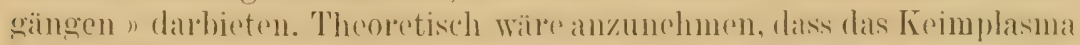
bes don Fluctuationen cine geringlügige, bei den Nutationen cine plitzliche intensive Veränderung erlitten hat, während es bei den Somationen intact geblieben ist.

Es fragt sich nun, ob DE VRIEs Recht hat mit seiner Ansicht, dass die

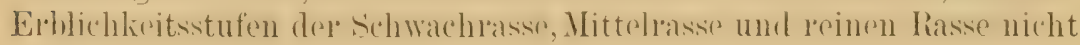
in einander übergehen können.

11. Tatsachen, welche für die Möglichteit einer Steigerung der Erblichkeit eines Char alters im Laufe von Generationen, fïr die Entwiclilung einer. Fluctuation au einer Mutation sprechen.

1. Der Umstand, dass bei derselben Art oder bei nahen Verwandten Schwachıassen, Nittelıassen und reine Rassen auftıeten können, macht es wahrscheinlich, dass eine Steigerung der Erblichkeit unter gewissen Umständen möglich sein muss, zumal de Vries an vielen Beispielen den Finthusi dor äuseren laktoren auf die Vererbungspotenz nachgewiosen hat. Für sehr viele Anomalien gilt der Satz, dass günstige Lebensverhältnisse sie steigern, ungünstige sie schwächen.

2. Bei manchen Atavismen, z. B. wenn gestreifte Blüten, bunte Blätter, Linaria vulgaris peloria, und Plantago lanceolata ramosa zum 'Typus dè Art vollstindig zuräclischlugen, sank dir Fobhlichkeit von der Stufe der Mittelrasse auf die der Schwachrasse. Wenn eine solche Regression möglich ist, darf auch der ungekehrte Prozess angenommen werden.

3. De Vries erwälnnt selbst zwei Fälle letzterer Art, nämlich den Uebergang der Schwachrasse Linaria vulgaris hemipeloria zur Mittelrasse Linaria vulgaris peloria und de Schwachrasse Chrysanthemum segetum grandiflom zur Nitteliasse Chrysanthemum segetum plenum.

4. Die vergleichende Anatomie lehrt an vielen Beispielen, dass die durch Uebergänge miteinander verbundenen und daher als "Fluctuationen " anzusehenden individuellen I)ifferenzen densellen Charaliter haben. wie die zur Unterscheidung von Arten verwendeten Merkmale. Aus dieser morphologischen Firscheinung likst sich nur der bine Schluss ziehen, den ich in meiner" "Anatomie und Phylogenie der Chitonen ) schon ange-

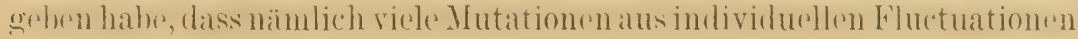
hervorgehen, was eine allmähliche Steigerung der Erblichkeit voraussetzt. Bei jenen 'T'ieren schwankt sehr oft die Zahl der Kiemen auf den briden suiten desselben Individumes oder bei vershiedenen Individuen derselben Art. Auf Grund solcher Schwankungen müssen sich in der 
Phylogenir die merobranchialen (mit wenigen Kiemen) Arten zu den holohnanchialen entwicholt haben. Dasselbe gilt für die im Laufe der phyletischen Entwicklung zu beobachtende Zunahme in der Läuge des Hauptkinals der Niere und des Renopericardialganges, für das allmählichr Anwachsen der Kö̈rperoröse und für die Vermeln’ung der Zahl der. Atrioventricular-Ostien um 2 resp. 3. Paare. Alle diese phyletischen Verت̈nderungen stimmen überein mit den individuellen Fluctuationen, und rine solche Identitit in so vielen heterogenen Punkten kann nicht Zufall sein, sondern muss darauf beruhen, dass neue Arten aus Fluctuationen hervorgehen.

5. Derselbe Schluss folgt aus Beobachtungen, die wiederholt an Arten mit einem grösseren Verbreitungsgebiet gemacht worden sind: bestimme Teränderungen treten an einer Lokalität mit zahlreichen Irobergängen auf, haben also den Charakter von Variationen, während sie an andern Stellen scharf gesondert als Lokalvarietäten resp. Subspezies, als arhlich konstante Rassen auftrete'u. Solche Beobachtungen liegen z. I). vor: von Friese, und v. Wagner über Hummeln, von Forel über Ameisen, von Lang über Helix hortensis, von Davenport über Pecten opercularis.

6. Die Temperaturexperimente von Standfuss und Fischer haben gezeigt, dass sich künstliche "Aberrationen ) elzielen lassen, die wegen ihrer geringen Erblichkeit und ihrer Thbergange als Fluctuationen zu benteilen sind. (ranz ähnliche, ja zum Teil fast idrntische Terïnderungen lingen in erhlich gefestigter Form bei manchen saisondimorphen faltern vor, sind also bei diesen zu Mutationen geworden.

Um zunächst eine Arbeitshypothese zu haben, so kann man annehmen, dass im Keimplasma der Eizellen einer Art jede erbliche Eigenschaft durch eine Anzahl homodynamer Jeterminanten hestimmt wird. Aendern diese aus irgend einem Grunde alle in derselben Weise ab, so haben wir eine Mutation vor uns, dic aber danu im höchsten Grade erblich sein wird (wenigstens wenn durch Reinzucht fremde störende Elomente ferngehalten werlen). Viränderon sich hingegen nur einige dor homodynamen Determinanten, während andere muerändert bleiben oder spalten sich die homodynamen Determinanten in mehrere Gruppen, so wird die Erblichkeit herabgesetzt sein: es resultiert eine Fluctuation, welche die Tendenz haben wird, Uebergänge zu bilden. Es ist a prior’i wahrscheinlich, dass eine Mutation zustande kommt, wenn ein ungewöhnlich intensiver Reiz auf das Keimplasma ausgeübt wird, denn el muss so stark sein, dass alle oder fast alle homodynamen Determinanten in demsellen simne umgestaltet werden. so würde orstens die seltrinheit del Mutationen sich erkiälen und zweitens ihre Neigung zu col'relativer Abänderung, denn ein derartiger Reiz wird wahrscheinlich auch die Interminanten anderer ('hatalitere herintlussen, soweit sie überhaupt für jenen Reiz empfänglich sind. 


\section{Die descendenztheorelische Bedeutung der Flucturtionen und der Mutationen.}

Füı die Abstammungslehre kommen die Somationen selbstverständlich nicht in lietracht. Es fragt sich, ob dic Evolution überwiegend mit Nutationen arbeitet, wie De VRIEs behauptet, oler vormohmlich mit Fluctuationen orlor ob beide Liategorien in dieser. IInsicht als gleichwertig grelten können. Fül den Biologen ist es ebenso wichtig, die relative Constanz der dren innerhall) gevisser Zeiträume wie die Möglichkeit rines Formenwechsels im Strome der Erolgeschichte zu exklären. Nach meinel Auffassung machen uns die Mutationen das erste Prohlem verständlich: sohald der höchste Grad der Erblichkeit erreicht ist, erscheint der betreftemln Charaktel mit Regelmäsigkeit hei den Nachkommen wieder, und ist er erst durch 'Tausende von Generationen hindurch befestigt worden, so scheint er überhaupt nicht mehr aufgehoben werden zu können, denn man hat z. B. noch nie gesehen, dass ein Wirbeltier scine Wirbelsäule oder irgend einen andern fundamentalen Charakter des ganzen Vertebratentypus eingebüsst hätte. Das Auftreten neuer Arten und die allmähliche Aenderung der Formen aber vermag die Mutationstheorie nicht ver'ständlich zu machen, denn es ist keine Errklärung, wenn man hehauptet. die neue Fol'm soi plötzlich aufgetretrn umb eine Mutation habe dir andere ahgelöst. Die Fluctuationen hingegen, leren Erblichkeit noch nicht völlig fixiert ist und die in der Regel in Uebergängen auftreten, vermögen sehr wohl eine allmähliche Umgestaltung, eine "Entwicklung» uns begreiflich zu machen. Sie sind noch labil und daher progressiv oder regressiv wandelbar und erst in dem Masse, als sie stabil unl exhlich constant werden, hört ihre phyletische Bedentung auf. Unhersehen wir das Gebiet der Abstammungslehre, so zeigen sich in der 'Tat viele Erscheinungen, die durch Mutationen nicht erlilärt werden können, sodass dic Fluctuationen für die Deszendenztheorie gar nicht zu entbehren sind.

1. Die Mutationen sind nach DE VRIEs richtungs- und

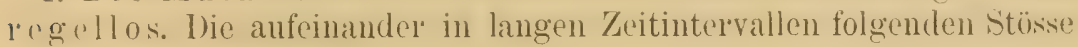
divergieren nach allen Richtungen.

Dadurch werden sie ungeeignet, die 'Tatsachen der" "bestimmt gerichteten phyletischen Entwicklung » zu erklären. Eine solche "Orthevolution ») kann regressiv sein, wodurch Organe zur Rudimentation und schliesslich zum Schwund gebracht werden, oder progressiv, wenn Organe aus kleinen Anfängen zu immer bedeutenderere Grösise mol Verrollkommunng heranwachsen ohne die einmal eingeschlagene Entwicklungshahn zu verlassen, z. B. wenn ein laüssel zuerst so klein ist wie brim 'Tapir, allmählich aber' so gross wird wie heim Elrfanten. Fs ist nicht einzusehen, wie die in ihrem Auftreten keiner Gesetzmässigkeit unterworfenen Mutationen plötzlich im stande sein sollten, S.Jn'itt anf Schritt

VIe CONGR. INT. ZOOL., 1904. 
in drrsellen Richtung anszuführen. Die labilen Tariationen hingegen lömnen whr wohl eine bestimmte Entwicklungslinie einhalten, sei es indem Selelition als richtendes Prinzip wirkt, sei es indem ler Gebranch oder ein beliebiger anderer günstiger Dauer'reiz die Organe und die ihnen entsprechenden Determinanten in den Keimzellen stärkt.

2. Die Mutationen sind nach den derzeitigen Erfahrungen bei ihrem ersten Auftreten äusserst selt e n, d. h. sie zeigen sich nur bei sehr wenigen Individuen. De Vries fand bei seinen Oenotheren im Iaufe von 10 Jahren unter 50,000

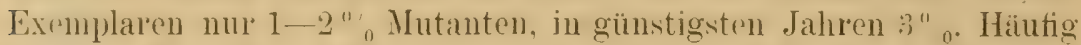
ist der" "Mutationscoefficient " viel geringer, z. B. bei Oenothera rubrinervis $0,1 \%$, bei $O$. gigas $0,01 \%$. Diese Seltenheit tut ihrer Bedeutung für die giirtnerische Praxis keinen Abbruch. da man hei PHanzen durch liünstliche Sollstbefruchtung und bei ginossen liulturen leicht die gewünschte Zahl von Individuen erhalten kann. In der freien Natur aber liegen die Verhältnisse anders. Zunächst sorgt die "katastrophale Fimination " dafür, dass ron allen abgesetzten lieimzellen eines Elternpares weitaus die Mehrzahl durch die Ungunst der äusvren Faktoren zu Grunde geht. mögen sie gut oder schlecht organisicrt sein. Die Wahrscheinlichkeit ist also äusserst gering, dass die so seltemen Iutanten überleben. Trifft aber dieser günstige Zulall einmal ein, so hat ein mutiortes Individum nicht Gelegenheit, mit einem andern in demsellen Sinne mutierten sich zur Foltptlanzung zu vereinigen; dazu sind die Mutanten zu selten. Es wird also cine Kreuzung mit der Stammform erfolgen, wobei die neue Mutante in der ersten oder zweiten Generation zwar wieder erscheinen kann, aber nur bei sog. Mutationsliveuzungen und hei Mexnes.'schen IBastardierungen. Inese gelten aber nach DE Vries nicht für die Fälle von progressiver Mutation, d. h. wenn eine wirklich neue Eigenschaft bei dem Mutanten aufgetreten ist, sondern diese folgen den Regehn der misexuellen Kireuzmus, mach denen keine Spaltung erfolgt, sondern dir neur Anlage nur zur Hälfte auf den Bastard ühergeht. I a der Bastard, wenigstens hei Tiepen, als liegel keine Möglichlieit zur Sellstbefruchtung orler licinzucht hat, so ist el wieder zur lirenzung gezwungen, worlureh die neue Eigensebuft abermals auf die Hälfte reduziert wird, und sofort, bis sie schliesslich durch den velwischenden Einfluss der Kreuzung völig entfornt ist. Dieser letztere kamn für die progresiven Mutanten nicht geleugnet werden, was um so) berdeutungsvollex ist, als diese für die Descendenzlehore in crster Linie in Betracht kommen. Sie allein fügen neur Charaktere zu den vorhandenen hinzu, lewirlen also einen Fortsehritt, withend bei retrogressirel und degressiver Mutation nur eine aktive Anlage latent resp. eine latente wieder aktiviert wird; dadurch können, nach DE VRIES, nahverwandte Formen geschaffen werden, also "Varictäten » in der üblichen Auffas- 
sung, aber nicht wirklich neue Arten. Solange also die Mendel'schen Spaltungsurestze nicht auch für progressive Mutanten nathgewieson sind. halte jch an desu durch viele Beobachtungen gestützten Satze fest. dasis in der freion Natur eine Singularvariation als liegel nicht aufkommen kann, sondern dur'ch den verwischenden Einfluss der Kreuzung wieder heseitigt wird. Da die bis jetzt belsamnten Mutationen (mit Ausnahme der Writeschen Washington Tomate) ausgesprochene Singularvariationen sind, so kann ihnen schon aus diesem (xrunde in der freien Natur nur vine untergeordnete Bedentung für die Evolution zuliommen.

3. Aus Mutationen lassen sich keine Anpassungen ableiten. Wer als Gegner des Vitalismus überzengt ist, dass die Organismen nicht die Fähigkeit zu "direkter Anpassung ) besitzen, sondern dass sie unter bestimmten äusseren Bedingungen nur in einer bestimmten Weise leagielen können, die bald nützlich, bald indifferent. bald schädlich sein wird, für den gibt es nur eine Möglichkeit der Elpkiärung lomplizierter Anpassumgen : die Selektion. Eune ganz einfache Anpassung mag dadureh zustande kommen. dass zutällig die Reaktion auf den äusseren Reiz nützlich ausfïltt. Die meisten Anpassungen ahel sind zusammengesetzter Natur und langsam erworben worden durch Auslese günstiger Variationen. Dies setzt voraus, dass zu jeder Zeit zahlreiche Varianten, die nach den verschiedensten Richtungen von einander differierten, vorhanden waren, eine Bedingung, die von den Mutationen nicht erfüllt wird, denn wie DE VRres selbst zugibt, tritt eine Art nur äusserst selten in eine Mutationsperiode ein, und die dann erzeugten Mutanten sind zwar richtungslos, aber sie bedeuten doch nur Stösse nach einigen wenigen Richtungen. Es fehlt ihnen die Allseitiglirit, und L'niversalität der Abänderungsmöglichlieit, durch wolchr umgekehrt die in zahllosen Cebergïngen auftrentenden Fluctuationen das gesignete Material für die natürliche Zuchtwahl alıgehen. Tatsüichlich bezielen sich die lis jetzt bekannten. Mutationen ganz überwiegend auf indifferente, manchmal auch schädliche, höchst selten auf nützliche

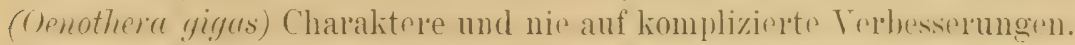
Da nun jodro" Organismus ein "Lomplex von Anpassungen " ist und als solcher erklärt werden muss, so versagt hier die Mutationstheorie.

Aus dem Gesagten ergiht sich als allgemeine Schluss : die Mutationen sind nicht das Material gewesen, welches eine Entwicklung, eine Evolution, ron ursprünglich einfachen zu immer limplizierteren Lebewesen elmöglichte, sie haben also nicht die eigentlich führende Rolle in del Jescrudenz der (Hoganismen gespielt. Dagegen haben sie sehr wohl dazu beitragen können, auf einer bestimmten Stufe der Komplikation den Rerichtum an nahverwandten Formen zu vererös:sern. Wenn ()rganismen durch Migration, Verschleppung oder Domestikation plötzlich in gan\% neue Existenzverhältnisse kommen, so kann das Keimplasma so erschüt- 
tert werden, dass cinzelne Determinanten sich erheblich verandern; das liesultat ist dam eine neur Lokalrasse, Subspecies orler eine neue Zuchtrasse. Hierfür splicht, dass auch die Oenothera lamarchiane, die Stammform der 1)E T'Rres'schen Mutationen, eine von Amerika nach Europa velptlanzte und in Holland verwilderte PHanze war und dass fast alle bis jetzt lokannten Jutationen an Pflanzen beobachtet werden, die seit langer Zeit im Garten orler als Gemüse gepflegt worden und daher durch don Irnschen vielfach wechselnden Lebrnshedingungen ausgesetzt gewesen sind.

\section{Diskussion.}

O. ḰLensiscunow. Sichel müssen zoologisehe Begriffe aus den konkreten Einzeltatsachen gewonnen werden. Da scheint mir zweierlei für "Mutationen ") charakteristisch zu sein: 1. Dass gewissermassen mit einem Schlage etwas Fertiges entsteht. eine Varictit sofort auf ihrer denkibar höchsten Stufe und 2. dass diese Erscheinung da auftritt, wo Ptlanzen oder Tiere in ein andres Land, in ein andres Klima gebracht oder doch unter ejgenartigen geographischen Bedingmuen erzeugt werden. Auf ornithologischem (iebiet vordienen hior besonders :) Fälle Frwähnung. die sogenannten: Corvus varius (leucophræus) auf den Faröern, Sylvia heinekeni auf Madeira und Palma und Athene chiaradize in Italien. Als hoher Grad zufälligen Varierens kïmnen diese Fülle nicht erkiärt werdon, da diese sonderbaren Färbungen regelmässige Erscheinungen sind, die sich wiederholen. Sie finden sich, soviel man bis jetzt weiss, einzeln in demselben Nest mit normalen Geschwistern. Dabei stimmen sie unter sich so sehr überein, dass sie irrtümlich als Spezies beschrieben werden komnten. Ehel lïnnte man sie als hohen (rrad geographischen Varierens auffassen. denn genauere Lntel'suchung hat eroehen, dass auch die Normalstücke der betreffenden Länder ein wenig und zwar in derselben Richtung vou Kontinentaltypus abweichen. Vielleicht verdanlit die Ersheinung iln Aufterten Stïrungen des Gleicherwichts zwischen produktiven und auschleichenden Faktoren. woher es'stere allein wirken. Die Frage. ol man das w i ed erhol te Autteoten dicser geographischen "Mutationen » nicht auf Abstammung von einem zufällig abnormen Individuum zurückführen kann, ist zu verneinen; denn ich erhielt Anklänge

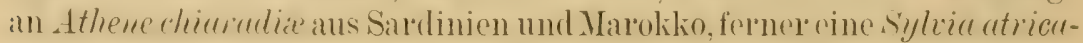
pilla mit schwarzem Nackenstreif von Korsika. Auf Kor'sika variieren aher die normalen s.hwaryplittchen geographisch ähnlich wie auf Maleira, und das Gleiche gilt von den normalen Steinkżuzen von Italien und Malookko. 


\title{
Ueber die Bedeutung der Generationsorgane für die Entstehung der Arten.
}

\author{
Von W. PETERSEN (Revel).
}

Fs mag als Vermessenheit erscheinen, dass ich es unternehmo, hier vor einel so angeschenen Versammlung ein Thema zu behaudeln, an dem sich seit mehr als rinrm Jahrhundert der Scharfinm unserer grössten Biologen geül)t lat. ohne zu einem allseitig befiecligenden Resultat gelangt zu sein. Doch ist die Frage der Artumwandlung und die damit im Zusammenhang stehende Inefinition des Artbegriffes in letzter Zeit wirder in den Vordergrumb des Interesses getreten. Wrem man eine zeitlang in der I ARww'sehen Naturzüchtung den Schlüssel gefunden zu hahen meinte, der uns das Verständniss eröfnete für die ganze Formenfülle der ()rganismenwelt, so muss es doch als soln charakteristisch hezeichnet werden, dass zu ciner Zeit, wo sich die Inswrs'schen Ideen im Siegeslauf' die Welt roberten, dir WArsen sche Miglationstheorie wenigstens voliuberohenel Beachtung finden komnte, mul dass heute cinerseits das Axiom von der Allmacht der Naturzüchtung anfeistellt wil'd, andererseits in schroffem (iegensatz dazu die alten Lawark'schen Anschanungen sich in ein modernes fewand kileiden, während wierlerum die Lehe von den Mutationen bisher scheinbar unhotretene Pfade wandelt.

Alle diese Erscheinungen deuten darauf hin, dass man noch weit daron entfernt ist, in der Frage der Artumwandlung zu einem völlig leefriedigenden Abschluss gelangt zu sein, und lassen dis Bestreben gerechtfertigt elscheinem, dass man immer wienler neue Tatsachen aufsucht, mit deren Hilfe man die Zulänglichlerit oder L'nzulanglichlede de mit einander concurrierenten Theorien und Hypothesen pröft. Sucht man in eincl derelben das IJeil, so bleibt bui dem Exempel stets ein Lest, der uns in die Brüche führt.

Von diesem Gesichtspunkte aus sci es mir nun gestattet, Ihre Autmerksamkeit auf einige Gruppen von Tatsachen zu lenken, die, wir mir scheint, hisher zu wonig borücksichtigung gefunden hahen; ich würde weit mehr Bedenken traginn, Ihnen das Resultat meiner Folschungen vorzutragen. Wenn mire nicht ein Rüstzeng von morphologischen Befunden zur Verfügumg stünde, die an sich der Beachtung wert sind, nnd die vielleicht von ander'n mit mehr (ilück und freschick in oin System gebracht werden.

Mrine Untersuchungen beschäftigen sich seit dem Jahre 1891 mit den 
Generationsorwanen del Insekten, und zwar sind es hauptsächlich die Lepidopteren gerwesen, dir mein specielles Interesse erregten. Ich begann mit der Intersuchung dere innern ()rgane und habe sodann in einer soele'n im I)ruck befindlichen Ahhandlung, als Fortsetzung einer früher'n Arbrit, ' ('יn Kopulationsapparat in den Bereich meiner Lntersuchung gezogen. Es erscheint mir notwendig, hier gleich zu betonen, dass ich ohne jerlo vorgefasste Meinting an die rein morphologische Lntersuchung gegangen bin, und dass elst zum Schluss dir Notwendigkeit, die Fülle der nenen Tatsachen unter einen allgemeinen Gesichtspunkt zu hringen, mich zu den Ideen geführt hat, die ich Ihnen hier in Kürze vorzulegen die Ehre habe. Vor allem konnte ich nachweisen, dass die bis dahin geltende Ansicht von der Monotonie des Banes der innern Generationsorwane riur durehaus irrige war, und dass hei den Blattwespen, den Trichopteren, inshesourlere aber bei den Lepidopteren in Bezugauf die Bildung der im Innern des Ablomens liegenden Trile des Sexualapparates eine schier uncschöptliche Folmemmannigfaltigkeit her'scht, dir zudem gecignet scheint, mo sichelel als andere Organsysteme einen Einblick in die phylogenetischen Beziehungen dex Formen zu gewälnen. Die bedeutenden morphologischen Verschiedenheiten der Keimbrüsen mit ihren Ausfühlıngsgängen und den Anhangsgobilden mussten um so auffallender erscheinen, als es sich hier um Organe und Organteile handelte, dir mehr ak andere Teile des Körper's dem direkten Einfluss äusserer Agentien 'ntzogen sind, und bei deinen füglich von irgend einer Anpassung an neue Lebensberlingungen nicht die Rede sein kann. Um ein Berspiel anzuführen, verweise ich auf die merkwürelige Thatsache, dass immerhalb der Gattung Argymis bei zweifellos nahe stehenden Arten das Pigment der Hodenkapsel bei einigen Arten lebhaft rosenrot, bei andern grün ist, während wiederum bei andern Arten derselben Gattung rlie Hodrnkapsel schwach gellolich tingirt orler fast fallos ist. Ein Eintluss besondrel Froührungsverhältnisse der Raupe ist hiel ausgeschlossen und wenn man mir auch etwa einwenden wollte, dass der rote und grüne Farbstoff dioselbe chemische Zusammensetzung haben, so bleibt die physikalische Terschiedenheit gleichwohl bestehen, und wir hïten noch keine Antwort auf die Frage, warum denn bei einigen der nahestrhenden Arten das Pigment äberhaupt nicht auftritt. Ein ähnliches Brispiel liceten am woiblichen Apparat die Ovarialröhren, die, bei den Schmetterlingen bekanntlich meist 4 auf jeder Seite, sich in sehr verschierdene Wrise zum Oviductus communis vereinigen. Auch hier kamn bei der $A$ bwoichung von dem Grundtypus an irgend rine Anpassung nicht gerlacht werden, und wir werden zu der Annahme gezwun-

${ }^{1}$ Beiträge zur Morphologie der Lepidopteren. K. Akademie der Wissensch. St. Petersburg, 1900. 
gen, dass diese im Laufe der phylogenetischen Entwicklung aufgetretenen erblichen Abweichungen weder durch Gebrauch resp. Nichtgebratch noch auch dureh Naturzüchtung liel'vorgerufon und für Fantile, Gattung oder Art charakteristisch geworden sein können. Es handelt sich eben hier um Merkmale, die wir in dic Kategoric der indifferente n C har aktere setzen müssen.

Anders schien die Sache bei den Formverschiedenheiten der Copulationsorgane zu liegen. Hier weist lie Lilulung des Lortphanzungsapparates bei nahe verwandten Arten in der Tat bisweilen so starke Differenzen auf, dass eine Bastardierung ausgeschlossen erscheint, und die Ansicht von Srabold und Dufour, dass durch derartige Verschiedenheiten

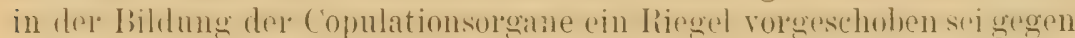
die Vermischung nahe verwandter Arten, hat sich bis heute allgemeiner Zustimmung erfreut. Die Anhänger der Naturzüchtung konnten cinen solchru Bumdesgenossen ruhis annehmon und tiuden es sehr begrestlich, dass die Natur sich auch dieses Mittels bedicne, um einmal crworbene nützliche Charaktere nicht verloren gelıen zu lassen, sondern auf diesem Wege mit dem grössten Erfolge rein weiterzuzüchten. Aus meinen Untrisuchungen haben sich nun schwerwegende Bedrnlien gergen die SieBoLD-Durour'sche Interpretation ergeben. Die Annahme, dass eine, die Bastardierung ausschliessende Verschiedenheit in der Bildung der Copulationsorgane den Zweck habe, die A r t rein zu erhalten, setzt voraus, dass jeder Art bei dem Verlust der' Artmerkmale durch Bastardierung Nachteile irgend welcher Art erwachsen würden, und dass der

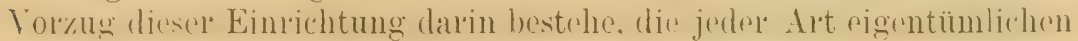
nützlichen Charaktere nach Möglichkeit zu erhalten.

Gelänge es nun, bei Formen mit stark differenziertem Copulationsap-

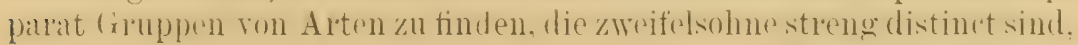
sich aber n $\mathrm{l}^{\circ}$ durch sogenannte indifferente Charaktere unter einander" unterscheiden, so wäre die Siebordo-Durour'sche Ansicht nicht zutreffend, und dio Infferenzierung der (opulationsorgane kïnnte nich t durch Vaturzüchtung ins Lehen gereufen sein, um jene indifferentern ditt tremenden Merkmalezu erhalten und einen Riogol gexen dio Termischung mahestehender Artenzu hilden. Wennes num atuch im einzelnen Falle schwel sein mag zu entscheiden, welehe Merkmale den indifferenten zuzurechnon scien. so haben wir doch bei den Inseliten und sperziell gerade bei den

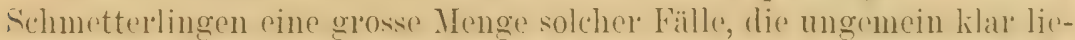
gen, und wo hei nahestehenden Arten die einzigen Art trennenden Jerkmale sicher keinen Nützlichkeitswert für die betreftenden Arten haben können. Ich habe in meiner neuen Arbeit eine Anzahl solcher Fälle aus-

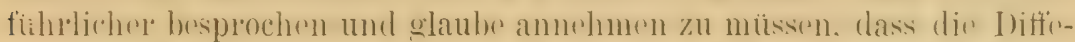

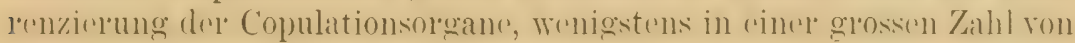
Fällen, nicht durch Naturzüchtung hervorgerufen ist, und dass bei der 
Arthildung anch andere Faktoren als die Natural solection in hervorragendem Masse ihren Anteil haben.

Zur Kilarlegung der hier aufgeworfenen Fragen habe ich folgenden Weg eingeschlagen :

Vor allem suchte ich eine Antwort auf die Frage, ob bei den Lepidopteren alle Arten durch die Beschaffenheit ihree (ienerationsorgane so wohl charakterisiert sint, dass sie sich nach diesen Organen allein unterscheiden lassen. Den Begriff" der" "Art 》 fixierte ich dabei provisorisch im weitesten Simne so, dass ich darunter Gruppen von Individuen verstand, die sich nicht nur morphologisch durch konstante Unterscheidungsmerkmale von allen andern Gruppen tremnen lassen, sondern auch physiologisch getennt sind, d. h. Lei denen eine gesehlechtliche Vermischung normalerweise nicht vorkommt.

I ie Feststellung der physiologischen Trennung lee einzelnen nahe verwandten Arten-Gruppen, bietet in vielen Fällen erhebliche Schwierigkeiten; doch hat speziell bei den Schmetterlingen die grosse Zahl der Liebhaher durch Zudht und Beobachtung der Wissenschaft wesentliche Dienste geleistet und zur Lösung mancher schwierigen Frage beigetragen. Allerdings hat dagegen ein Heer von Unberufenen durch "Artenmacherei " aus materiellen oder Eitelkeitsrückischten vorübergehend manchem Irrtum Vorschub geleistet, doch sind in den hohen Werten soleher Pseudo-Arten genügende, natürliche Sicherheitsventile gegehen, und das Hauptliontingent der sogenannten fraglichen Alten stellen infolge schwieriger liontrolle im Augenblick wohl die Tropen, so dass wir

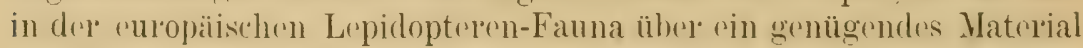
von Formen verfügen, bei denen obige Definition der Art nach der morphologischen und physiologischen Seite zutrifft.

Meine Untersuchungen an zirka 1000 Arten von Selımetterlingen haben nun ergeben, dass in der 'Tat jede Art (mit Ausnahme eines Teiles der sogenamuten geographischen Formen durch ihre (xenerationsorgane so wohl charakterisiert ist, dass man sie mit Sicherheit nach diesem Apparat erkennen und meist mit Leichtigkeit selbst von den nächstverwandten Arten unterscheiden kann. Ich suchte nach Möglichlieit Kiomplexe nahe verwandter Arten aus den heterogensten Gruppen zu berücksichtigen von den Micropterygiden, Nepticuliden, Hepialiden und Tineiden hinauf bis zu den Aretiiden und Noctuen und Pyraliden, überall zongte sich dasselloe Verhalten: bei verhältnismässig geringer oder auf (in Minimum beschrinkter individueller Tariabilitit scharf zu fixierende artliche I Iifferenzierung dere (ienerationsorgane, die bei nahe verwandten Arten bisweilen so weit gehen konnte, dass eine Bastardierung dieser Formen als eine mechanische Unmöglichkeit erscheinen musste.

I) ie männlichen Copulationsorgane der Schmetterlinge sind schon fröhore Gegenstanl der L'ntersuchung gewesen und auch praktiseh zur 
Unterscheidung schwer zu trennender Arten benutzt worden, da man ans einex verhältnissmässig kleinen Zahl von wirklichen Beobachtung('n sich begreiflicherweise zu einer Verallgemeinerung der Beobachtungslesultate himpoissen lixs. Wenn num auch durch meino Untelsuchungen las Beohachtungsmaterial um ein betrïchtliches angewachsen ist, so kömnte immerhin die. Vreallgemeinerung bedenklich erscheinen, und in der Tat hat in jüngster Zeit der Hauptarbeiter auf diesem 'ngern felicet (ler anatomischen ['ntersuchung('n, I)r. K. Jordas, mit voller Fintschiedenheit seine frühere Ansicht, dass die münnlichen Copulationsorgane stets artlich differenzirt seien, als irrthümlich bezeichnet und seine frühere Artenhildung dureh mechanical selection in eine solehe durch geographical isolation verbessert. Her' JoRDAN hat umfangreiche Untersuchungen an tropischen Papilioniden, Apaturiden, Agaristhiden angestellt, vor allem aber in seinel kürzlich erschienenen grossen Monographic der sphinginen nicht allein den Copulationsapparat der Mänmchen untersucht, sondern auch bei den Weibchen auf die Formverschiedenheit an äussern Copulationsorgan aufmerksam gemacht. Trotz dieser gewichtigen und, wie wir sehen, auf zahlreiche Untersuchungen sich stützendrn Einsplache muss ich oloch moine obige Behauptung hezüglich der artlichen Differenzierung der (xemerationsolgane aufercht orhalten und zwar aus folgenden Gründen:

Erstens habe ich mich bei meinen Untersuchungen nicht auf die Copulationsorganc allein bechrinlit. sondern wo diese nicht auspoichten auch die andern Teile des ganzen männlichen Sexualapparats, wie Keimdrüsen, ausführende Gänge, accessorische Drüsen und Duftapparate hinzugezogen, und in dem einen oder' ander'n dieser 'Teile, oft in allen, weitgehende artliche Differenzierungen constatieren können.

Zweitens ergab, was bis dahin nouh nicht Gegenstand einer vergleichenden Untersuchung gewesen war, eine Prüfung der im Innern des Abrlomens liegenden 'Teile der weiblichen Generationsorgane, wie z: B. der Bursa copulatrix und der Receptaculum seminis, eine unerschöpfliche Fiulle von Formverschiedenheiten, worlurch auch bei den Weibchen nahe verwandter Arten sichere Unterscheidungsmittel gefunden warell.

D rittens erwies sich die Zahl der Fälle, wo die Differenzierung der äusseren Copulationsor ga ne beim ơ oder die Bildung des Os tium bursæ beim 우 zur Unterscheidung nahe verwand ter Formen nicht auszureichen schienen, als verschwindend likein im Vergleich zu den Fällen, wo eine präcise artliche Trennung nach diesen Organen möglich war.

Viertens war nicht allein die Quantität sondel'n auch die Qualität del' von mir mit positivem Erfolg untrisuchten Fälle von Berleutung. Meine Stichproben "rstreckten sich auf fast alle grösiceren Gruppen der Schnert-

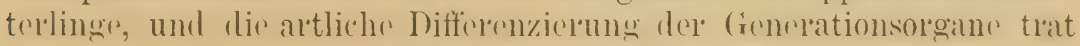


losonders deutlich in frattungen zu Tage, deren Arten dlureh Färbung, Zeichnung und andere äussere Merkmale sich oft nur schwer unterschuiden lassen, wio z. B. Tephrochystia (Épithecia), Scoparin, Nepticula ete.

Fünftens liessen sich gerade bei solchen Arten, wo die Copulationsorgane minime oder gar keine Differenzen aufweisen, starke morphologische E'nterschiede im Bau der Duftorgane feststellen, wir z. B. bei Argymis niobe und adippe oder bei den für Gerüche bekanntlich besonders empfindlichen Apaturiden, wie Apatura iris und ilia. Dass hise mit dieser morphologischen Differenzierung der Duftapparate auch (inc I) ifterenzierung der Function eingetreten ist. liesse sich schon a pr ior i "rwart'n, wird aher dadureh zur Gewisheit erhoben, dass Bastardiel'ungen bei diesen Artenparen nicht vorkommen oder höchstens zu den allergrössten Seltenheiten gehören.

Auf dirse Momente gestützt, glaube ich meine Behauptung aufrecht rylualtun zu dürfen, dass jede Schmetterlingsart durch ihren Fortptlanzungsapparat so gut charakterisirt ist, dass sie sich nach demselben erlemenen und von allen anderen, auch den näthst verwandten Formen mit Sirherheit unterschriden lässt. Eino Ausmahme machen vielleicht einige formen. dir als sogemannte geographische Arten durch locale Isolierung entstanden sind. Im übrigen ist mir eine Schwierigkeit der morphologischen 'Tremung sehr mahe stehender Formen nur dort nicht gelungen, wo die Artherechtigung derselhen üherhaupt noch nicht völlig sicher gestullt ist. Es regal, sich ferner, flass auch die Familien- und Gatturgscharaktere in den Generationsorganen einen mehr oder weniger prägnanten Ausludi fiuden. Somit dürften diese morphologischen Eigentümlirhlieiten wohl geeignet sein fül dir Zukunft in der Systematik und besonders in zweifelhaften Fällen der Artberechtigung ein Factor von Writtragender Iiedeutung zu werden. Wenigstens wird eine artliche Vorschiedenheit zweier Formen in fraglichen Fällen, wo die Prüfung der Inftapparate auf Schwirigkeiten stöst, durch verschiedrnen Bau der Generationsorgane mit Sicherheit zu entecheiden sein, die Variabilitaitsamplitule ist bei den meisten Teilen des Apparates eine selu geringe; in einigen Gruppen, wie z. B. den tropischen Papilioniden scheint sie nach JorDax's Untersuchungen bedeutender zu sein, doch dürfte hier. eine norhmalige Prüfung in Bezug auf allo Teilo des Apparates und besonder's der inneren weiblichon Organe von grossem Interesse sein, da mach meinen Erffihrungen noh)en variahlen auch sohr constante Aluweichungen in dem einen oder ander'n 'Teil vorkommen. Im allgemeinen scheint es, als oh auch in Bowg auf den Sexualapparat gewisse Arten sich in einel Periode der Constanz oder der Variabilität befinden können, wie wir dis in Bezug aut alle andern Merkmale bei allen ()roanismen beobachten kömmen. Nur ist es hier wichtig, zu betonen, dass die Varianten in Fär- 
hung, Zeichnumg ote. oincrseits und den Sexualorganen andererseits im allgemeinen von oinander unahbängige lieihen bilden (einige Ansmahmen cor'relativer Abänderungen werden wir später' kennen lernen).

Im weiteren Verlaufe der Untersuchungen kam es mir nun darauf an festzustellen, ob der von vielen Forschern supponierte Fall häufig auftrecte, dass die Ibiflerenziorung der C'opulationsorgane bei nahe verwandten Arten eine so weitgehende sei, dass eine Bastardierung mechanisch unmöglich würde. Fine genaur Prüfung der entsprechenden Orointerile zeigte, dass diesel Fall wohl vorkomme - klassische Beispicle dafür lieferten Larentia autumnalis Str. (impluviata Brh.) und sordidata F. oder Augiades comma L. und sylvanus Esp., sowic einige TephroclystiaArten; doch steht diesen vereinzelten Fällen ein ganzes Heer von solchen gegenüber, wo streng distincte Arten von ihren nächsten Verwandten durch minime aber sehr konstante Differenzen im Bau der Copulationsorgane morphologisch mit Leichtigheit gotrennt werden können.

Durch diese Tatsachen muss die Annahme, es sei der ver'schiedene Bau der Copulationsorgane als Riegel zur Reinerhaltung der Art durch natürliche Zuchtwahl ins Leben gerufen. im höchston Grade problematisch erscheinen, und wir müssen nach einem andern Urgrunde der Erscheinung suchen. Die praktische Erfahrung zeigt uns, dass die Schmetterlinge und wohl anch die ührigen ()rdnungen der Insektren in ihren I) uft-

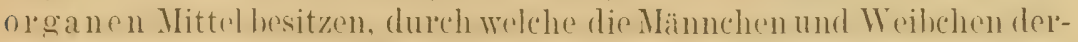
selben Art sich aufzusuchen und zu erkennen im Stande sind, wobei die

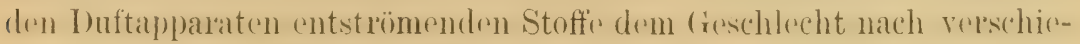
den sein müssen. Wird das Männchen dureh die vom Weibchen ausströmenden Düfte angelockt, so wirken wiederum die von den oft sehr auftal-

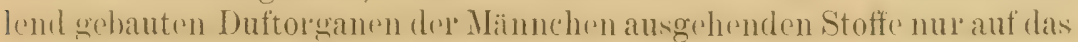
Wribchen derselben Art, und zwar als auslösender Iiejz beim Greschlechtsalit. Ein Männchen wiled von den I)nftstoffen eines andern Männchens deresollon Art nicht angelockt; somit besitzen die Inuftstoffe der Wribchen noch etwas speziell weibliches, das nur für die Männchen derselben Art berechnet ist. Hinzufügen will ich hier gleich, dass diese Art de Yerständigune oder der Auslösung des Geschlechtspeizes nicht die ainzige zu sein hraucht. Ich habe liürzlich heim Sehmetterling ein Organ gefunden, das ich nur als Ohr deuten kann und das ich mir später Ihnen zu demonstricren erlauben werde. Jedenfalls haben wir es hier bei dieser Verständigmgsmethode mit "inem seh" homplizierten Mechanismus zu tun, dep mit einere stamenswerten Sicherheit funktioniert, und wenn wir damit lie geringen Differenzierungen am Copulationsapparat, dru g(ringen For'mver'schiedenheiten der' Valvæ, des Uncus oder' anderer' 'Teile vergleichen, so könntrn wir derartigu Einrichtungen zur physiologischen Tremumg der Arten nur für plump, im hörhsten Grade mangellaft und 
überflüssig crklären. I)ie Möglichkeit müscen wir offen lassen, dass bei Artengruppen, wie wir sie oben kennen lernten, die Differenzierung bei don Copulationsorganen stärlier ist, als bei den Duftapparaten derselhen, und somit durch die grosse Verschiedenheit des Banes trennende Schranken gegen die Artvermischung gesetzt sind, aber im ganzen bleilst dies, wie vorhin ausgeführt, immer nur ein seltener Fall oder - eben nur eine Möglichkeit.

Und doch bilden jene oft sehr geringen Abweichungen im Bau der Valvæ, des Uncus, Penis und anderer Teile des männlichen Copulationsapparates oder jene wunderbaren Formverschiedenheiten der Bursa copulatrix heim Weibchen ein unfehlbares Nittel. die cinzelnen Arten zu unterscheirlen. Ich habe, um die Stichhaltiglesit und Zuverlässigheit dieser morphologischen Artmerkmale eingehend zu prüfen, nach Möglichkeit ganze Gattungen durchgearbeitet und bin \%. B. hei der (xattung Argynnis (rstaunt gewesen, mit welcher Prägnanz die unbedentendsten spreifischen Alweichungen gerule beim Copulationsorgane ihren Ausdruck finden. Dabei liess sich zugleich zeigen, dass in dieser an Varietäten und Aberationen so reichen Gattung nur die wirklich verschiedenen Arten sich nach ihrem Copulationsapparat unterscheiden lassen, während die Aberration und Varietait gleiche Bildung wis die Stammart aufweisen. Es ist eine sehr auffallende und beachtenswerte Tatsache, dass ich bei den zahlreichen Varietäten der Argynnis pales = Gruppe von den verschiedensten Lokalitäten Europas und Asiens nicht die geringsten Ahweichungen von der sogenamnten Stammart im Bau der männlichen Copulationsor'ganc habe fimlen können, während anderersoits niobe und elisa, von denen letztere als "geographische Art " der erstern

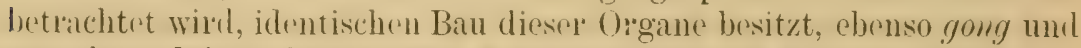
eva orler aglaja und alexamdra. Achnliches habe ich auch in anderon Gattungen bestätigt gefunden und glaube daraus mit Sicherheit folger'n zu müssen, lass geographische Isolic'ung ganz unabhängig von einer molphologischen Embildung der Copulationsorgane zur Bildung von Lokalformen führen kann. In dieser Beziohung kann ich mich den Schlussfolgerungen Jordax's nicht anschliessen, der in der geographischen lsolierung die alleinige (Quelle neuer Artbikhng sieht. Die Isolierung

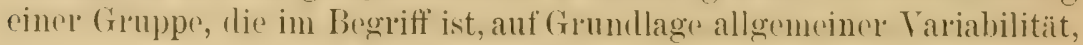
sich zu einer besondern A rt herauszubilden, schien mir eine unerlässliche Forderung in den Fïllen zu sein, wo die betreftende Individuengruppe, die sich als neue Art absondert, als unterscheidende Charaktere der Stammform gegenüber nur indifterente morphologische Verkmale aufzuweisen hat. In einem solchen Fall erweist sich eben die Natural selection ak machtos, denn sio kann nur mit neuen specifischen Charakteren allwitrn, die der nenen (xpuppe von irgend einem Nutzen sein müssen. I) iene Teherelegung hat mich zur Formulirung der" "physiologischen Iso- 
lierung "geführt, welche in ihrem Effect der geographischen Isolicrung gleicht. Dass verschiedene Arten normaler Weise sich geschlechtlich nicht vermischen, hat früher schon öfter dazu geführtt, die sogenannte geschlochtliche Entfromolum als Ausgangspunlit bei dor Bildung neuer Arten eine wichtige Rolle spielen zu lassen. Als rein theoretische Hilfshypothese loistete die "geschlechtliche Fintfremdung "gute D)ienste unel half, wo dio Naturzüchtung nicht gut ausreichen wollte, über manche schwierigkit hinweg. Im frumde genommen aber handelte es sich $11 \mathrm{~m}$ nichts anderes, als um einen Ausd ruck, der die Tatsache wiedergab, dass bei divergenten Formengruppen die geschlechtliche Affinität grössere, goringer od 'l ganz fehlend ist, oder mechanische Hindernisse für den Copulations- resp). Befruchtungsakt vorlagen. Andere nahmen obufalls die Hilfe der geschlechtlichen Entfremdung in Anspruch, stellten sie aber vollstïndig als cino Eirrungenschaft der natürlichen Zuchtwahl hin, da sie cin unfehlbares Mittel bot, neu auftretende, nü tzlich e Charaktere nicht wieder verloren gehen zu lassen, sondern rein weiter zu züchten; hier wurde sie dann in der 'Tat zu einem Mittel der Reinerhaitung der Art oder zu einem Riegel gegen die Vermischung der Arten. Für dic materielle Grundlage der merkwürdigen Escheinumg sind aher nur sehr dürftige Daten gesammelt worden, und wie wir vorhin gesehen hahen, hat man sicherlich die Differenziesung der Copulationsorgane als Grumblage für dir geschlochtliche Entfremdung weit übesschätzt.

Wir müssen num nach dem vorliegenden Tatsachenmaterial die Quelle und Berleutung der geschlechtlichen Fntfremdung festzustellen suchen.

Tatsache ist, dass bei den Schmetterlingen, und wahrscheinlich wohl bei allen Insckten, jede Art einen dermassen differenzierten specitischen Duft besitzt, dass Bastardierungen als durchaus anormale Ausnahmeerscheinungen aufzufassen sind.

Die specifische Verschiedenheit des Duftes geht in den bisher näher untersuchten Fällen, wahrscheinlich aher wohl in allen Faillen, Hand in Hand mit einer morphologischen Verschiedenheit der Duftorgane, wodurch der Schluss nahe gelegt wi’d, dass die Funktion dieser Gebilde in dirohter Abhängigleit von ihrem Bau steht : morphologische Varianten derselben fülıren zu Varianten der producierten Duftstoffe. Damit wäl le abel diegeschlechtliche Entfremdung auf eine morpholog ische Grundlage gebracht. Eine weitere, im höchsten Grade bemerkenswere T'atsache ist nun die, dass die dureh differente Duftstofte physiologisch getremnten Arten nicht nur durch morphologische Figentümlichlieiten der Intapparate, sondern auch dureh den Bau der übrigen Teile des sexualapparates. moistens gerale dere ('npulationsolgane, morphologisch gotoennt werden kïnnen. Wenigstens habe ich das an fiast $10(x)$

${ }^{1}$ Uf. Biolog. Centralblatt, Band XXIII, Nr. 13 u. Band XXIV, Nr. 13 u. 14. 
Schmettellingsarten aus den verschiedensten Gruppen des Systems bestiitigt gefunden und ausserdem liegt, wenigstens füı die Teile des männliche'n Copulationsoraturs, noch eine grosse Zahl von andern Beobachtungen vor, die dasselbe Resultat ergeben haben. Bei Arten dagegen, die sich durch eine gan\% aussergewöhnliche Varbubilitä in Bezug auf Färhung und Zeichnung auszeichnen, bei denen aber selbst zwischen den am meisten ahweichenden Varianten, untereinander und der Stammform gegenïher, lisine geschlechtliche Entfremdung eingetreten ist, wo also die Inftorgane noch in früherer Weise funktionielen, lässt sich oft nicht die geringste Alweichung vom normalen Bau der Generationsorgane auftinden. Eine sorgfältige Prüfung an langen Reihen von Individuen solchel vardahlel Arten gestattet mir. diese Beohachtung als gesichert anzuselien.

Wir haben also hier cinen offenbaren Zusammenhang zwischen ilen morphologischen Eigentümlichkeiten des Copulationsapparates, der Duftorgane und der von letztern producierten Duftstoffe. Durch den Geschlechtsapparat ist jede Art von allen andern scharf geschioden, und die Formverschiedenheiten dieses Apparates bei nahe verwandten Arten bezichen sich der Regel nach gar nicht nur' auf den einen oder andern' T'eil. sondern treten meist gleichzeitiz in mehreren Stücken deutlich zu Tage. I) ies alere zwingt uns eine Correlation zwischen all diesen Troilen anzunchm('n. I lese gle i ch z i i ig e Abänderung in den einzelnen Teilen des ganzen Sexualapparates tritt mit einer solchen Klarheit und Gesetzmässiglieit auf. dlass wir sie elon einfach als Tatsache hinnehmen müssen, wenn wip auch häufig, wenigstens zur Zeit, noch keinen Einblick in den innern Zusimmenhang dieser Fischeinungen gewinnen können. In vielen Fïllen freilich sehen wir klar, wie die ühermäswige Ausbildung einzelner Teile auf' Kosten anderer benachbarter geht. die damn in der Entwicklung haleutend zurücklileiben; ja ich labe sogar beobachten kömmen. dass die correlative Aländerung der Duftorgane andere Teile des Körrpers, wie die Brine, ja sogar das Göider direct in Mitlejelenschaft ziehe'n kann, und es sind auch die sog. Duftflecken, als zu den Elementen der Zcichnung gehörig, nach Vorhandensein oder Fehlen, nach Ausdehnung und Fiallung von den Systematikern vielfach zur Tnterscheidung der Arten in Anspruch genommen worden. Forner habe ich in der Bildung des von mir als ()hr gedeuteten Apparates specifische Differenzen constatiren können.

Auf die lomplizirte Frage, wie der Fortpflanzungsapparat in Cormelation mit den ührigen Teilen des Organismus steht, kamn ich hice natürlich nicht specieller aingehen und muss mich mit dem Hinweis begnügen, dass dieser noch wenig untersuchto Zusammonhang dureh die Kastration und b viden sogenannten halbirten Zwittern deutlich zu Tage tritt.

Ueber die dominierende Stellung des Sexualapparates am Organismus 
dem Soma gegenüber körnen wir uns nicht wundern, wenn wir die Stufenleiter der Lebewesen von den niedersten zu den höchsten Formen durchgehen, und wenn wir uns von dem landläutigen Vorurteil immer mehr frei gemacht haben werden, als sei der rein somatische Teil des Oranismus die Hanptsache und das Wresentliche, während 's nü dex unserer directen Beobachtung an leichtesten zugängliche 'Teil ist. Verimblerungen im C'entrum, Strïrungen des Gleichgewichts irgend woleher Art im Centralorgan, bilden den Ausgangspunkt für Abänderungen nach der Peripherie hin. Zu dieser Annahme werden wir immer mehr gedlängt, und so allein können wir eine plausible Erkklärung für die

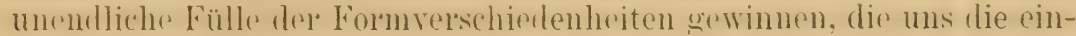

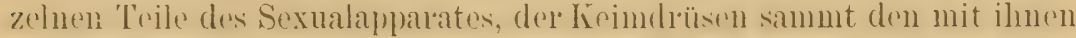
im directesten Zusammenhang stehenden Hilfsapparaten, darbicten.

Nun könnte man freilich meinen, dass umgekehrt Veränderungen an der Peripherie, am Soma, das Ursplrüngliche seien, und dass die Naturzüchtung, hildich gesprochen, sich dos Sixualapparates lediente, um durch physiologische Trennung der Formengruppen neuc Charactere artlich zu fixieren.

Die Untersuchung dieser Möglichkeit hat einen wesentlichen Teil meiner Arbeit gebildet, und ich glaube als Resultat meiner Untersuchungen don Satz verteidigen zu lï̈nnen. dass bei den Sehmotterlingen wenigstens für gamz grose (iruppen von Arten dieser Jodus der Arthil-

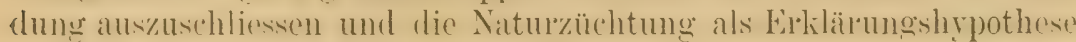
in den Ruhestand zu versetzen sei.

Ich wählte hier grössere Gruppen von Arten aus, bei denen die einzigen arttrennenden Charactere zweifellos ind ifferente waren, wie z. B.

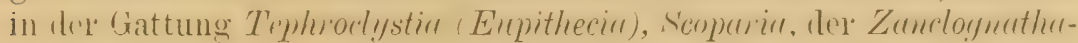
Gruppe und andere. Es zeigte sich nun die erstaunliche Tatsache, dass jone änssern, inlifforenten Unterscheidungsmerkmale für die einzelnen Arten geradezu verschwindend zu nennen waren im Verhältniss zur specitischen I)ifterenzierung des (reschlechtsilpparates und nicht nur in den Teilen des Apparates, wo noch die Spur eines Verdachtes vorliegen kounte, als handle es sich um coadaptive functionelle Abänderungen, und nicht nur in einem einzelnen 'T'eile des Apparates, sonder'n oft auf der ganzen Linie von den Keimdrüsen bis zu den Duftapparaten. Besonder's waren es die im Innern des Abdomens liegenden Teile des weiblichen Apparates, die eine äherraschende Fülle von Formverschiedenheiten boten. I) praktischen Lepidoptrrologen gegenüber werde ich mich vielleicht am deutlichsten ausdrücken, wenn ich sage, dass es nach den innern morphologischen Merkmalen der Bursa copulatrix, des Ductus seminalis und des fereeptaculum seminis nicht schwieriger ist. zwoi nahe velwandte Eupithecien odel' Scoparien artlich zu trennen, als etwa zwei Arten der Gattung Vanessa nach ihren äusseren Merkmalen. 
Hier giebt es keinen andern Ausweg für die Erkkläıung, als die Allmacht der Taturzüchtung fallen zu lassen, da es sich nur um minime arttremnende Merkmale in Färbung und Zeichnung handelt, zu deren Erhaltung und arthicher Fixierung der ganze Sexualapparat umgestaltet sein müsste, während die äussern Merkmale doch durchaus indifferenter Natur sind.

I) a reicht ferner auch die Erliärung durch geographische Isolierung nicht aus, da die betreffenden Arten auf beschränkten Fluggehieten noben cinander leben, - hiel versagt auch das Lamark'sche El'klärungsprincip; donn es handelt sich um ()roane, die der Roger nach nur eimmal im Leben in Function treten.

Fis hleibt somit num noch die Anmahme übrig, dass bei diesen Gruppen von Arten die artliche Divergenz ihren Anfang genommen hat mit einer morphologischen Differenzierung des Geschlechtsapparates, die zu einel physiologischen Isolierung der Individuengruppe geführt hat, während durch diese physiologische Isolierung wiederum auch indifferente Charactere an der Peripherie des Soma durch Reinzüchtung zu Artcharacteren werden konnten.

Wie weit dieses Princip der physiologischen Isolierung auf morplologischer Grundlage zur Entlastung der Naturzüchtung heitragen kann. wird dio Zukunft lehnen; einstweilen handelt ws sich un einen Versuch, eine Summe nener Tatsachen unter einen einheitlichen Gesichtspunkt zu bringen mit dem trostreichen Ausblick, den uns das Wort Baco's von Verulan gewährt: Veritas potius ex evrore emergit, quam ex confusione.

\section{Demonstration des Bakterienkernes.}

Von Prof. F. VEJDOVSKY (Prag).

In dem Garschina-See im Rluatikon lebt in grosser Menge eine Gammans-Art (G. Zschokkei Vejd.), welche von vielen Parasiten infiziert ist. Yon diesen ist gerviss das Interessanteste eine Bakterium-Art, die zu Tausenden in dere Hiemolymphe des Krolses ihr Dasoin fristet. Reforent

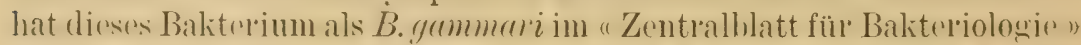

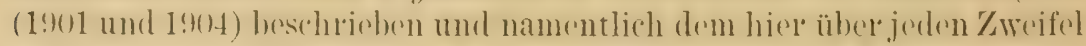

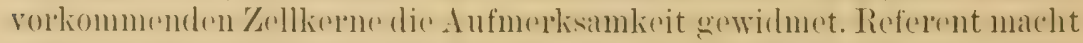

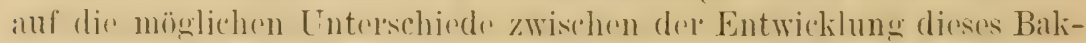

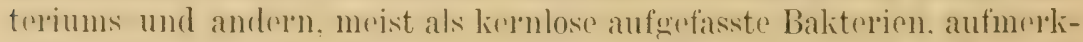
sam. Indessen sind in dirser Ridhtung neue Beohachtungern an ()rt und Stelle anzustellen und in verschiedenen Jahreszeiten zu wiederholen. 


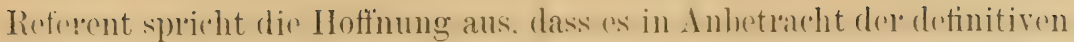
Stollung des Buliterimm yammori im Systeme den cinheiuischen Zoologen gelingen wird, diese Fragen bald und befriedigend zu beantworten.

Referent ladet die Fachgenossen ein, das ausgestellte Präparat zu besichtigen.

Siehe: F. Vejoovsкy. Ueber den Kern der Bukterien und seine Teilung. In: Centralbl. f. Bakteriologic, II. Abt., XI. Bd., No 16/18, 1904.

\title{
Diskussion.
}

Prof. Schaudiny schliesst sich dem Antrag des Referenten an.

\section{Die Veränderung der tierischen Zelle während der Verjüngung und der Veraltung.}

Von Prof. CII. S. MINOT (Boston).

L'auteur n'a pas remis le manuscrit de sa communication.

\section{Die Wanderung der Ancylostomum- und Strongyloides- Larven von der Haut nach dem Darm.}

\author{
Von Dr. A. LOOSS (Cairo).
}

Das Folgende ist eine kurze Uebersicht über die Versuche, die ich mit (ten Larven von A Acylostomme- und strongyloidesarten angestellt hab), um ihre Wandremug von der Hant nach dem Inrme in den Einzelheiten kennen zu lernen. Die ersten Beobachtungen, die mich zu dem Schlusse führten, dass das Ankylostoma des Menschen noch auf einem anderen Wege als dem durch den Mund in seinen Wirt gelangen müsse, liegen bereits eine Reihe von Jahren zurück. Ich hatte versucht, die reifen Larven auf 'liere zu übertragen, um ihre Metamorphose zum geschlechtsprifen Tiere zu studienen. Hjese L'ntersuchumgen wurden in Alexandrien angestellt zur Zeit als die Cholera dort herrschte, und sie wurden in demselben Laboratorium vorgenommen, in dem auch die Leichenteile bohufs bakteriologischer Foststellung dey cholera montersucht wuden. Es ist unshore einzusehen, dass es unter diesen Verhätnissen in meinem ureigenstru Interesse lan, die Beziehungen zwisehen den Händen und dem Munde auf das genaueste zu überwachen; ausserdem wusste ich, 
dass das Anliylostoma ein goührlicher Gast des menschlichen Körper's ist, und dass es durch den Mund erworben wird. Trotz aller bewusst und systematisch angewandten Vorsichtsmassingeln fand ich mich oinige Monate später ausserordentlich stark mit dem Parasiten behaftet; aus dem, was ich gesagt habe, wird es aber verständlich werden, dass ich die Mörlichlieit, diese Infrktion kïnn" durch don Mund erfolgt sein, positiv in . Wrede stellte, obwohl und trotzdem ich zunächst lieinerlei Vermutung darüber hatte, wie sie in Wirklichkeit elfolgt sein konnte. Kurze Zeit später (im Sommer 1897) fand ich durch Zufall, dass die Ankylostomalarven, wenn sie auf die Haut des Menschen gelangen, sich in dieselbe einhohren: vin am lebenden Menschen vorgenommenes Experiment bei dem die Larven auf (in zu amputierendes Bein eine Stunde vor der Operation aufgetragen und später in Schnitten durch die Haut wiedergefunden wurden) zeigte, dass sie dabei hauptsächlich die Haarfollikel als Eintrittspforte benutzen, in diesen bis zur Haarzwiebel vordringen und von da aus in das Corium übertreten. Für mich persönlich war es von vornherein klar, dass es sich in diesem Eindringen der Larven in die Haut nicht um ein zufälliges und bedeutungsloses Phänomen handeln konnte, sondern dass es einen tieferen Sinn haben musste. Es wäre ferner nicht recht einzusehen gewesen, warum die Larven, nachdem sie sichi in die Haut eingebohrt hatten, nun gerade in dieser verbleiben und nicht die Fähigkeit besitzen sollten, von der Haut aus auch noch weiter im Tïrper vorzudringen; glückte es ihnen dabei, auf irgend rine Wrise in den Darm zu gelangen, dann war eine Erklïrung fǜ meine eigene Infektion gegehen, dem die Gelegenheit zum Eindringen in die Haut der Hände hatten die Larven während meiner früheren Experimente reichlich gehaht. Ich habe nach wioderholter, eingehender Prüfung der Terhältnisse dio Ibhauptung ausgesprochen. dass diesel zweite Infelitionsmodus tatsichlich existiere. Neine Angaben sind einem allgemeinen Zweifel begegnet und von Seiten italienischer Autoren sogar positiv als morchtig bezeichnet worden auf Grund von Experimonten, die sintlich rin nexatives liesultat erogen haben sollen. Ich hahe daraufhin nochmals einen Versuch am Menschen gemacht unter bodingungen, dio eino zufaillige anderweite Infektion so gut wie vällig ausschlossen. Fs handelte sich um einen Krankenwïrter des Kasp-el-Aini-Hospitales, der bereits i Jahre im Inenste war, währeud dieser Zeit trils im Hospitale, teils in del Stadt Cairo geleht hatte, und bei der Untersuchung sich als frei von Ankylostoma alwies. 71 lage nach der Auftragung einer reichlichen Anzahl reifer Lalrven auf seinen linken Vorderarm konnten in seinen Stühlen zum ersten Male Ankylostomaeier nachgewiesen werden, und zwar durchschnittlich etwa zwei in einer Kotmasse von ca. $4 \mathrm{cmm}$. In der niichsten Zoit stieg die Anzahl dor Fier merklich und nach etwa anderthalb Monaten fanden sich im Mittel zwischen 25 und 35 in einer Kot- 
masse von del oben angegebenen Grösse. Diese Zahl ist bis heute, d. i. während einer Zeit von $1 \frac{1}{2}$ Jahren, im Grossen und Ganzen konstant geblieben. Leider bemerke ich, dass ich meine speziellen Notizen über die vorgenommenen Zählumgen dor Eier entweder in Cairo golassen oder auf der Reise verloren habe; ich muss mich deshalb hier auf die eben gemachten allgemeinen Angahen beschränlen; dis letzte, Mitte Juli dieses Jahres angestellte Zïhlung ergab bei 3 Präparaten aus demselben Stuhle 39, 29 und 33, im Mittel also 34 Eier. Diese Konstanz in der Zahl der in den Stühlen meiner Versuchsperson erscheinenden Eier weist meines Erachtens positiv darauf hin, dass nach del Infektion durch die Haut kein Zuwachs in der Zahl der vorhandenen Parasiten. d. h. keine neue Infektion erfolgt ist. Der Mann war vor dem Experimente frei von Ankylostoma, und produziert nach dem Experimente eine durch 1\%2 Jahre hindurch sich nahezu gleichbleibende Anzah! von Wurmeiern; es ist mir vorgeworfen worden, dass ich bei diesem Experimente in irgend einer Weise getäuscht worden sei; das, was ich hier über das Resultat gesagt, spricht nicht dafür. Uebrigens soll dieser Ver'such bis zum Ende, d. i. bis zum allmählichen Er|loschen der Infektion auf natürlichem Wege for'tgesetzt werden.

Bei dem eben besprochenen Experimente, ebenso wie bei meiner eigenen Infelition, waren nur der Antang und das Ende eines natürlichen Vorganges objektiv nachweisbar gewesen; was dazwischen lag, entzog sich einstweilen unserer Kenntnis; um es festzustellen, war das Experiment am Tiere unerlässlich. Nachdem es mir gelungen war, das menschliche Ankylostoma - wenn auch immer nur in wenigen Exemplaren auf dem Wege durch den Mund in ganz jungen Hunden bis zur Geschlechtsreife zu erziehen, lag die Wahrscheinlichkeit vor, es in denselben Versuchstieren auch auf dem Wege durch die Haut bis in den Darm zu bringen; spätere Versuche haben ergeben, dass dies in der Tat möglich ist. F'ür den Anfang erschien es mir jedoch wünschenswerter, unter möglichst natürlichen Bedingungen zu arbeiten. und ich wählte zu den Versuchen deshalb das Ancylostomum caninum, welches im Hunde seinen normalen Wirt hat. Bei den beiden ersten Versuchen, welche mit dieser Species angestellt, und in denen eine reichliche Anzahl von Lalven auf die Haut des Rumpfes aufgetragen wurden, erlagen die Versuchstiere bereits an 10. Tage einer akuten Ankylostomiasis, 1. i. genau so schnell, als ob sie dur'ch den Mund infizicrt worden wäl'en. Die Larven mussten hier also innerhalb von etwa 24 Stunden nach dem Darme gelangt sein; dirses Resultat war auftallend. Wähpend beim Mensehen zwischen der Infelition und dem Erstheinen der crsten Eicr in den Stühlen ca. 70 Tage verflossen waren, würde derselbe Prozess bei den Hunden, wenn sie nicht vorzeitig ellegen wären, etwa 30-35 Tage, d. i. also nur die Hälfer der Zeit, in Anspruch genommen habeu. Ich war anfïnglich 
der Unheryoung, dass diese Verschiedenheit ihre Lrsache habe in der verschiedenen Entfernung der Infektionsstelle vom Darme. Beim Menschen waren dic Larven auf den Unterarm dicht am Handgelenke appliziert worden, sie hatten also erst die ganze Länge des Armes zu durchwandern, che sie in die Nähe des Verdaumngstractus gelangen konnten; bei den Hundrn dagegen brauchten sio nur die Bauch-oder Brustwand zu durchsetzen, um sich in umittelharer Nähe des Darmes zu befinden. Etliche weitere Versuche schienen diese Annahme zu bestätigen. So fanden sich bei einem jungen, etwa 3 Monate alten Hunde, der von der Bauchwand aus infiziert und 4 Stunden nach der Infektion getötet worden war, zahlreiche Larven noch im Corium, sehr zahlreiche im subcutanen (xewehe und einige bereits in den obertlächlichen Schichten der Bauchmuskulatur unter der Infektionsstelle. Bei einem anderen, in derselben Weise infizierten, aber bereits ca. 8 Monate alten Hunde, der 6 Stunden nach der Infektion getötet worden war, fanden sich die Larven dagegen sämtlich noch im Corjum der Haut oder in den Harfollikeln und ihren drüsigen Anhängen. Auf diesen letzteren Versuch worde ich noch zurüickzukummen haben.

Auf dies'm Stadium wurden meine Untersuchungen durch eine mehrmonatliche Abwesenheit von Cairo unterbrochen, während deren mir überdies das lebende Infektionsmaterial von Ancylostomum conimum ausging; erst Anfang dieses Jahres (1904) war ich im Stande, sie teils mit den ehengenannten Species, teils mit dem menschlichen Ankilostoma wieder aufzumehmen. Hierbei stellte sich nun immer unzweifelhafter heraus, dass norine bisherige Annahme über den von den Larven eingeschlagenen Wrig zum I)arme nicht richtig sein konnte. Wohl gelang es nach entsprechender Zeit immer, die Larven tiefer in der Muskulatur der Körperwand, zum 'Teil von der Infektionsstelle weit entfernt, ja sogar im Inn'rn des hörpers, anscheinend im Peritonem, aufzutinden; stets aber stand die Zahl dieser Larven in keinem Verhältnis zu der Zahl derjenigen, die auf die Haut appliziert worden waren. In keinem Falle komnten sie dagegen frei in der Leibeshöhle. im Mesenterium oder endlich in der. Wand des I)armes nacherewiesen werden, obwohl von diesem ganze stüclir in sichnitte zerlegt und genau durchgesehen wurden. Der von den Larven in Wirklichkeit genommene Weg musste ein anderer sein als der, auf den die er'sten Versuchsergebnisse scheinbar hingedeutet hatten. Um ihm auf die Spur zu kommen, wurde zunächst das Verhalten der Larven imnerhalb der Haut nochmals einer genaurn Prüfung unterzogen. Hierbei fanden sich bei einem zirka 3 Monace alten Hunde, der mit den Larven von Ancylostomm duodenale infiziert und 20-22 Stunden nach der Infektion getötet worden war, einige Larven vollkommen drutlich im Innern von Ha ut venen. Wonn dieses Verhalten ein normales war, dann liess sich annehmen, dass die Larven mit dem 
IBlutstrome nach dem rechten Herzen und ron dort in die Lungergefiuhrt wïrden. In dieser konnte es ihnen ferner nicht schwer sein, aus den feineren Verästelungen der Lungenarterie in die Luftwege überzutreten, und von diesen aus lag dam durch Trachea, Kohllopt und Oesophagus ein freier Weg nach dem Darme vor ihnen.

Schon die ersten Versuche, die Larven auf diesem Weg zu finden, orgabon ein durohas positives liesultat und liesenn lieinen Zweifol dancïber, dass hier der normale Weg vorlag, auf dem sie nach dem Darm ge-

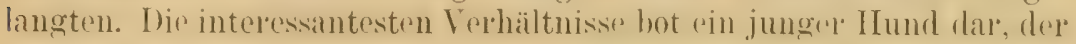
zweimal mit Ancylostomum canimum infiziert und 77 Stunden nach der ersten und 5 Stunden nach der zweiten Infektion getötet worden war. Hier fanden sich auf Schnitten durch die Lunge zahlreiche Larven in den Alveolen und den feineren und gröberen Bronchien; in der frisch abgeschabten Schleimhaut des untersten, etwa $3 \mathrm{~cm}$. langen Teiles der Trachea, der auf Deckglïser ausgestrichen worden war, konnte ich später noch 399 Ankylostomalarven zählen und dabei waren von den wirklich vorhanden gewesenen sicher eine ganze Anzalil verloren gegangen. Ebenso zahlreiche Larven fanden sich auf Schnitten durch den oberen

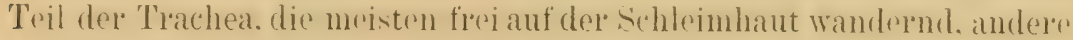
in dieselbe eingedrungen, und noch andere endlich im Innern der

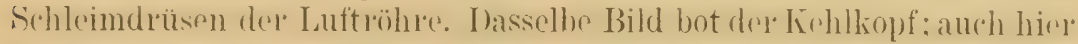
frei wandernde Larven neben anderen, die in das Epithel und zum Teil tief in die I)rüsen ringerdrungen waren. Schliesslich lionnten Lan ven auch im ()esophagus nachgewirsen werden. sowohl in dem frisch abgeschabten Schleime, als anch später ant Schnitten durch die übrig gebliebenen Teile des Desophagus. Im (regensatz zum ()esophagus erwies sich die Mundhöhle vollkommen frei von Larven, obwohl ihl Epithel sowie das del Zunge in nahezu ganzer Ausdelınung abgeschaht und genau untersucht wurde. Die in den Bronchien und in der Trachea vorhandenen Larven waren fast ohne Ausmahme deutlich gewachsen, und die meisten zeigten bereits die verschiedensten Stadien in der Anlage del provisorischen Mundkapsel, dagegen hatte lieine schon dio Häutung durchgemacht, dureh welehe diese Mundliatper frei wird. Im Inarmo endlich fanden sich cine Menge junger Ankylostomen. die grössten etwa „2 mm. lang und von diesen aus alle möglichen Uebergänge zu den Lar'ven, wie sie in der'Trachea vorhanden waren. Bei diesem Hunde fand sich somit ein vollstïndiger, ununterbrochener Strom von Larven von der Lunge aus bis zum Darme und w zejgte sich gleichzeitig, dass der Wachstum der jungen Parasiten schon in der Lunge seinen Anfang nimmt.

Etwas schwierigel wal der Nachweis der Larven auf ihrem Wege von dre Haut nach der Lunge. der mit dem Blutstrome offenhar ziemlieh sehnell zurïckgelegt wird. loch glückte es mil mohrmals, sire in Schnit-

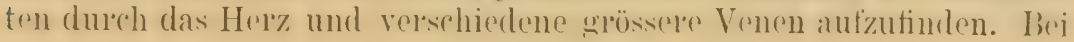


den Versuchen, die Larven in den Blutgefässen zu treffen, ergab sich noch ein anderes wichtiges Faktum. Bei einem ganz jungen, etwa $3 \mathrm{~W}_{0-}$ chen alten Hunde, der mit Ancylostomum duodenale infiziert und etwa 2 Stunden danach getötot worden war, fanden sich auf Flächenschnitten durch die Haut ganze Schwärme von Larven an der Basis des Coriums dieht üher der Hautmuskellage und anscheinend frei im Gewebe. Auf (juerschnittrn dur'h benachbarte Hautstuicke jedoch zeigte sich, dasis einzelne der Larven in Blutcapillaren und andere unzweifelhaft in Lymphgefäsen eingeschlossen lagen. Demnach schien es, als ob auch die letzteren von den Würmern als Weg benutzt würden. Wenn dies del' Fill war: damn muste die Mrhrzahl der Larven die Lymphdrüsen, besonders also die Achsel- und Leistendrüsen, passieren, und in diesen mussten sie aller Voraussicht nach auch leicht nachzuweisen sein. Eine Untersu'humg der Lymphdrüsen zu geeigneter Zeit ergah denn auch ein durchaus positives Resultat. I'h will hicl zunächst heiläufig erwähnen, dasse die Loiston- und Achseddrüsen otwa 3 Stunden nach der Applikation zahlreicher Lav'ren auf die Haut intensiv anzuschwellen heginnen und otwa 4 stunden später das ¿-8 fache ihres ursprünglichen Volumens (rroichrn liönn'm: nach 24 Stunden ist diese Schwellung meist mehr oder mimder vollkommen wieder geschwunden. Entersucht man num die frischen Lymphdıüs'n im Zupf- oder' Quetschpräparat zu einer Zeit, wo die ersten Larven in ihnen ankommen, dann sieht man diese in ihren charakteristischen Bewegungen zwischen den Lymphzellen umherwandern; nur etwas langsamer als sonst sind diese Bewegungen, gleich als ob es den 'Tieren schwerer würde, zwischen den Zellen vol'wärts zu kommen. Untersucht man einige Stunden später, dann finden sich unter den jetzt zahloweher gewordenen frei heweglichen Larven immer einige, anf deren Kö̈perobertläche sich Lymphzellen in grösserer oder geringered Zahl so festgesetzt haben, dass den Laryen das Vorwärtskommen äusserst erschwert oder schliessich gänzlich ummöglich gemacht ist: sie machen noch langsam schläugelnde Bewegungen. ohne sich von der Stelle zu hewegen. Toch später endlich findet man diese Larven von einem dicken Besatz von Lymphzellen umhüllt und völlig hewexungslos, ihre inneren ()rgane tröbe und liörnig, wit anderen Worten dem Absterben nahe orler gämzlich algestorben. Demnach fungieren die Lrmphdrüsen hier, wio auch bei anderen Infoktionen, als Sicherungsol'gane für den Körper, indem sie schäulliche Keime festhalten und abtïten. Die Anliylostomalarven, die auf diese Woise alogefangen und unsehädlich gemacht werden, sind anscheinend diejenigen, denen es nicht innerhalb relativ kurzer Zeit gelingt, den Ausgang aus den Drüsen zu finden; die anderen setzen ihren Weg in den Lymphgefüsisen fort und gelangen schliesslich duch den I)uctus thoracicus oflex den lechtssejtigen kleineren Lymphstamm in den venösen lireislanf. wo sie mit den von Anfane an in die Venen einge- 
drungenon Latren zusammentreffen. Soweit moine bisherigen Beoharhtuntungen rinen Schluss zulassen, scheinen die Ialden zurest die Lyupherofïsse zu bevol'zugen und el'st später in die Venen einzudringen.

Ein Umstand nun, der die systematischen Untersuchungen über die Wanderung der Laven betriithtlich erschwert, ist die ganz auffallemel verschiedene Geschwindigkeit, mit der der Prozess im einzelnen Falle verläuft. Ich habe schon erwähnt, dass ich die Ur'sache dieser'Ver'schiedenheit zuerst in der verschiedonen Länge des Weges suchte, den die Larven im jeweiligen Falle von der Haut nach dem I atrone zurürkzulegen haben. spätere Erfahrungen hahen jedoch gezeigt, lass dieso Erkiärung unhaltbar ist. Ich verweise hier auf das oben elwähnte Experiment an einem \& Monate alten Hund, bei dem sich die Larven des Ancylostomum caminum (also in ihrem natül'lich en Wirte) 6 Stunden nach der Infektion noch sämtlich in der Haut befanden; dagegen waren sie bei einem erst 3 Wochen alten Hunde 2 Stunden nach der Infektion bereits in die Lymphdrüsen vorgedrungen, hatten also einen nicht nur relativ, sondern auch absolut grösseren Weg zurückgelegt, als in dtem ersteren Falle. Demnach scheint es mehl' das Alter' der' 'Tiere zu sein, welches bestimmend auf die Geschwindigkeit der Wanderung der Larven einwirkt; diese Erklärung hat bis anf weiteres die meiste Wahrscheinlichkeit für sich und passt auf alle von mir angestellten Versuche. Ueberdies kann man sich leicht vorstellen, dass die weicheren Gewebe junger Tiere den bohrbewegungen der Parasiten weniger Widerstand entgegensetzen: es gelingt diesen, leichter und schneller in die Gefässe einzudringen, sie gelangen in grösserer Zahl gleichzeitig in die Lunge und von da in den

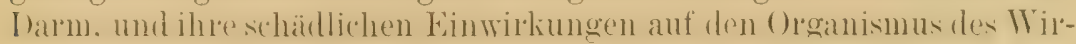
tes summieren sich derart, dass sie tötlich werden können. Bei älteren und alten 'Tieren verlangsamt sich das Eindringen der Larven in die Gefässe etc. immer mehr ; vielen Larven gelingt es, nach an mir selbst ge-

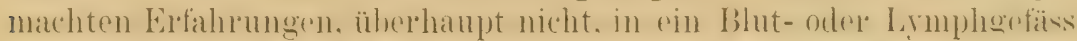

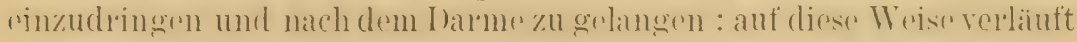

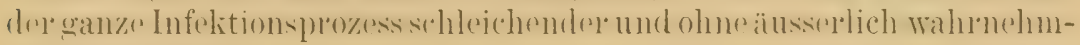
bare Symptome.

Was ich bisher gesagt habe, bezicht sich speziell zunächst auf' die Lalven der Ancylostomumaten, hat aber, soweit meine bisherigen Versuche gezeigt haben, in ganzer Auselehmung atuh Gültigkeit für die Lalven der Arten des Genus Strongyloides. Dass die filariformen Larven von S. stercoralis die Fähigkeit besitzen, aktiv in die Haut von Säugetieren einzudringen, ist von vax Durate an Meerschweinchen elwiesen worden. Mit diesem Nachweise war für mich persönlich bereits die Gewisheit gegehen. dass diese Latrven elentalls vom dor Haut aus nath dem l armo zu wandern vermochten. Ein an mil selhst angestelltes Experiment hat diese Ueherzengung hestätigt. Ich muss hiorzu zunächst hemerlien, 
dass meine frühep Infelition mit Ankylostoma Anfang des Jahres 190:3 spontan erloschen war. Bis gegen Ende des Jahres 1902 war es mir in jodem Falle möglich gewesen, in moinen Stühlen noch vereinzelte Ankylostoma-Fier nachzuweisen ; von Anfang 1903 ab gelang dies nicht mehr: selbst ganze Stühle, die mit Tierkohle zur Kultur angesetzt worden waren, lieferten beim Ausziehen mit Wasser keine Larven mehr. In diesen wirderholten und übereinstimmend negativen Befunden kann wohl der sichere Boweis oblickt werden, dass ich von 1903 ab keine Ankylostomawürmer mehr beherbergte. Anfang dieses Jahres (1904) erhielt ich zur [Tutersuchung aus dem Kasp-e]-Aini-Hospital einen Stuhl, der dir Stromyyloidestarven anscheinend rein, d. i. ohne Beimengung von Ankylostoma-Eiernn enthielt. Diese Stühle wurden zur Vornalıne eines Experimentes kultiviert und dann mit Wasser ausgezogen; es ergab sich dahei. dass aussel den Strongyloideslarven doch einige, wenn auch nur wenige Ankylostomalarven vorhanden waren. Von dieser Kultur applizierte ich Ende März 1904 einige hundert Larven auf meinen linken Unterar'm und breitete die Flüssigkeit mit der Pipette auf eine grössere Fläche aus. Infolge dieses Umstandes und der geringen Zahl der aufgetragenen Larven blieben die früher beobachteten Symptome aus, dagegen zeigte sich in umittelharem Anschluss an das Experiment eine Escheinung, die ich schon während der Dauer meinel ersten Infektion zu wioderholten Malen am eigenen Körper beohachtet hatte und die in der medizinischen Litteratur unter den Namen cresping disease, creeping eruption, Hautmaulwurf mehrfach beschrieben worden ist. Bisher war als Ur'sachn dieser eigentümlichen Hauteruption, soweit ich sehen kann. nur in einem Falle eine kleine, etwa 1 mus. lange Fliegenlarve aufgefunden worden; nach den an mir selbst gemachten Erfahrungen kamn es indessen krinem Zweifel unterliegen, dass dicht unter der Haut wandernde Ancylostommm-und Strong!yloideslarven die gleiche Affektion ebenfalls hervorzubringen im stande sind. Am 64. 'Tage nach dem Experiment fand ich in meinen stühlen zum ersten Male Strongyloideslarven und auch ein Ankylostoma-Ei. Der Befund konnte bei allen späteren Lntersuchungen hestätigt werden, und jetat liegen die Terhältnisse so, diss sich in jedem Kotpartikel von del früher bereits angegebenen (riösce (ca. 4 cmm.) 2--35t,omygloideslarven, dagegen in jedem dritten Präparate erst cin Ankylostoma-Ei findet. Wit diesem Experimente halte ich den Beweis erbracht, dass auch für Strongyloides stercoralis dieselben zwei Infektionsmodi bestehen, wie füı die Ancylostommarten.

Ueber die Details der Wanderung der Strongyloideslarven waren besomdere Intrisuchungen überflüsig. da sich diese Details nebenbei bei einigen Versuchen mit A. canimum ergaben. Einer der jungen, wild ringefingenen Füchse. der mir das Intektionsmaterial mit dem eben genannten Ankylostoma lieferte, war ausser mit diesem auch mit eincr 
Stinngyloidesalt behaftet, deren Larven ich von denjenigen des st. storcoratis bis auf Weiteres nicht $\mathrm{zu}$ unterscheiden vermag. Ich kann deshall) noch nicht sigen, ob ich mit S. stercoralis oder mit ciner anderen Species experimentiext habe: im ganzen dülfte die Speciesfiage hier nu' von untergeordneter Bedeutung sein, da anzunchmen ist, dass alle Strom(f!gloiclesarten sich biologisch gleich verhalten. Pioi den mit dem gemischten Infektionsmateriale volonommenen Experimenten fand ich die St, ongyloirleslarven an allen Stellen unter den Ankylostomalarven wieder, woraus hervorgeht, dass sie mit diesen den gleichen Weg genommen hatten.

Die Resultate meiner bisherigen Versuche lassen sich in folgenden Sätzen zusammurnfassen: Fül dir Arten der Gattumgen Ancylostrmmm und stromgyloides besteht neben dem Wege durch den Ilund ein zweiter Weg durch die Haut, auf dem die reifen Larven in ihren Wirt zurück gelangen können. Ersterer Weg wird passiv, letzterer aktiv, und zwar in der Weise zur ückgelegt, dass die Larven, nachdem sie sich in dir Haut eingehohrt haben, in obertaichlich gelegene Lymphgefüsse oder Venen cindringen und in diesen durch das Her'z nach der Lunge geführt welden. Hier treten sie aus den Blutwegen in die Luftwege über, und wanderu schliesslich durch Trachea, Kichlliopt und Oesophagus nach dem Darme. In den Lymphdrüsen wird anscheinend stets eine Anzahl zupüukgehalten und unschädlich gemacht. I)ic Larven gelangen um so leichter, schmeller und in um so grösserer Zahl an ihren Bestimmungsort, je jüng!l die befallenen Individuen der Wirtstiere sind. Bei alten Wirten bleibt eine grössere oder geringere Zahl von Larven in den Geweben des Körpers zurück, wo sie, wenn sie dicht unter der Oberhaut umherwander'u, die als ('lerping eruption ete. bekannte Hautaffektion horvorlufen können. Sir vermögen sich als wandernde Latrven mugefähr ebenso lange wie die erwachsenen Würmer, i. e. etwa 5 Jahre am Leben zul erhalten.

\title{
Ueber Linsenbildung nach experimenteller Entfernung der primären Linsenbildungszellen.
}

\author{
Von D) II. SPEUANN (Würzburg).
}

J) urch früher mitgeteilte Experinonte hatte sich feststellen lassen. dass dio bildung der Linse des Froschatuges nicht erfolgt, wenn del Augenbreher verhindert wurde die Epidermis zu herühren. Ta direlit 
schärligende Einflüsse ausgeschlossen werden kounten, so ergab sich daraus, dass zur Linsonbildung ein auslösender Reiz des Augenbechers auf die Epidermis nötig ist. Die Experimente, über die ich jetzt zu herichten habe, hetreffen lie durch meine Versuche noch often gelassene, ährigens schon ron andern Autoren behandelte Frage, oh die Linsenlildung nur an (jinel hesonders dazu determinierten Stelle ausgelöst werden kamn, oder' auch an ander'n 'Teilen der Epider'mis.

Die Experimente bestanden darin, dass an Embryonen von Triton tirmintus die Kiuppe dor mimären Augenblase nit den primälen Linsenbildungszellen oder in etwas späterem Stadium der äussere Teil rles Augenbechors mit der eben sichtbar gewordenen ersten Linsenanlage entfernt wurde.

Diese Experimente ergaben:

$1^{\circ}$ In vielen Fällen blieb das mehr oder weniger regenerierte Augenrudiment in der Tiefe, ohne die Epidermis zu berühren; es entstand an dicser keine neue Linsenanlage.

$2^{\circ}$ In anderen zahlveichen Fällen erreichte der regenerierte Augenbecher die Epidermis; es entstand an ihr eine neue Linse, die sich in normaler Weise weiter entwickelte.

$3^{\circ}$ In e in em Fall, wo der Augenbecher die Epidermis nicht erreichen konnte, entwickelte sich am oberen Irisrand eine deutliche kleine Linsenkunospe, wie sie Corcecr nach Verstümmelung des Auges, G. WoLF nach reiner Linsenextraktion gefunden habell.

Aus diesen 'Tatsachen folgt, dass nicht nur die primären Linsenbildungszellen, sondern zum mindesten auch die Zellen in ihrer näheren Lmgehung befähigt sind. auf einen vom Augenbecher ausgehenden Reiz mit Linsenbilalung zu antworten. I)asselbe schloss hekanntlich schon frühel C. Henust mit Wahrscheinlichkeit ans gewissen Misshildungen, 1). Barfurtu ans Experimenten an Hühnerembryonen und nenerdings II. H. Lews aus eigens hiozu angestellten, sehr heweiskraftigen 'Transplantations- und Verlagerungsversuchen an Froschembryonen.

Fernel gestattet der Fall von Linsenregeneration aus dem oberen Irisrand cine otwas eingehendere Analyse der bekannten WoLrF'selen Fntdeckung. Diese ganzo Frage seheint nir durch die num experimentell firtestellte Fihigkeit des Augenbechers, sich aus indifferentem ectodremalem Material cinc Linse aufzubauen. in ein neues Stadium eingetreten zu sein.

Die Belege füı die tatsächlichen Angaben und die nähere Ausfülır'ung der theoretischen Folgerungen werden an anderem Or't nitgeteilt werden. 


\section{Nomenclalure générique.}

Par M. P. DAUTZENBERG (Paris).

L'auteur n'a pas remis le manuscrit de sa communication.

Voil: P. Dautzenberg et G. F. Dollfus. Etudes critiques sur la nomenclature avec examen des genres Pectunculus et Glycimeris. In : Journal de Conchyliologic. Vol. 52, $n^{\circ}$ 2, Paris, 1904.

\section{Der Einfluss des Zentralnervensystems auf die Regeneration bei Tritonen.}

Vou Dr. E. GODLEWSKI (Krakau).

Mit 4 Textfiguren.

Der Redner bespricht zuerst die bisherige Literatur, welche sich auf den Einfluss des Zontralnelonsinstems aud die Regeneration bezieht. I)ie Autoren, welche diesbezügliche Experimente an den embryomalen ()roatnismen angestellt haben, sind übereinstimmend zum Resultat gelangt. dass in einer gewisen frühen Fntwicklungsperiode das Zentralnervensystem lieinered Einfluss auf die Torgänge der ontogenetischen und regenerativen Entwicklung ausübt.

Zu diesem Ergebnis sind auf Grund ihrer Experimente J. Løв, Scra-

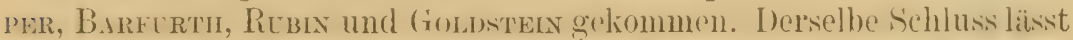
sich auch aus den neuerlich pulbizierten Experimenten von Harssos ziehen.

I) Fe Forcher, welche dionwachsenen Tiere als Untersuchungsmaterial benutzten - und namentlich Rubin und G. WoLff glauben, dass dem Zentralnervensystem die Rolle eines formativen Reizes zuzuschreiben ist.

Der Redner schildert weiter eigene Experimente.

In der ersten Versuchserie wurde den 12 Tritonen der Schwanz in der Jitte seiner Länge mit zwei hehrïgschnitten so ahgetremt, dass das gebliebene Schwanzstück gahelförmig endete. I) urch diese Operation wurde in dem telminalen Schwanztrile der Achsenteil mit dem Rütchemmak ausgeschaltet. Am terminalen schwanzende ging so lange die Regenerattion nicht vor sich, his zwischen den boiden terminalen sichwanzstürlien 
in Proliferationsgewebe das Rückenmark sich differenzierte. Diesem Expreriment, aus welchem man schliessen kömnte, dass die Terzögerung der Regeneration auf Abwesenheit des Nervensystems zurückizuführen ist. könnte jedoch rin Einwand gemacht werden : Man könnte namentlich don Erlfoled des Versuches mit dem von Morrids beschriebenen Resultat d'r Regeneration bei der Fischtlosse analogisieren und dieselben Falitoren, mit welchen Morgan seine Resultate erklärte, auch hier anerkennen.

Aus anderen Versuchen geht jedoch hervor, dass es sich hier um den Eintluss des Nervensystems handelt :

Den 25 'Tritonen wurde der Schwanz in einer Entfernung von cr. $1 \mathrm{~cm}$. von der Afteröffumng amputiert und mit einer glühheissen Nadel von der Sichnittläche kopfwärts das ganze Stück des Rückemmarks, welches den Schwanz innervierte, zerstört. Den Kontrolltieren wurde nach Amputation des Schwanzes das Rückenmark nicht zerstört. Die Kontrolltiere haben die Schwänze regeneriert, bei den 'Tieren mit dem zel'störten Rürckenmark hat sich nur die Wunde bedeckt. (Die Tiere wurden bei dem Vortrage demonstriert.) Bei jenen Tieren, bei welchen nach 6 bis s Wochen die Regeneration begann, honnte auf den histologischen Priparaten (welche im Demonstrationssaal aufgestellt wurden) nachgewiesen werlen, dass das zerstörte Rückenmark schon hergestellt wurde.

I) a bei dies(' Experimentenserie nu das Rückenmark zerstört wurde, die Spinalganglien aber nicht verletzt wuden' - so glaubt der Verfasser zu den Schlüssen berechtigt zu sein :

1. Bei den erwachsenen Tieren ist das Vorhandensein des Rückenmarks zum normalen Verlauf der Regeneration notwendig.

2. Die Spinalganglien selbst sind nicht im Stande, die Rolle des Rückenmarks zu vertreten.

Um den Finfluss der Liontinuitätsunterhrechung des Rüclemmarls auf den Verlauf der Regeneration zu untersuchen, hat sich der Reduer der Methode bedient. Welche von Barfurth (01) zur Untelsuchung der Abhängigkeit der Regenerationserscheinungen angegeben wurde. Den 25 Tritonen wurde die Schwanzspitze amputiert. Einige Millimeter vor dieser Schnittfläche wurde ein Loch mit dem scharfen Scalpel so ausgeschnitten, dass dir dorsalen Wirbelbogen mit dem Rückenmark und den Spinalganglien auf die Länge von $3-4 \mathrm{~mm}$. entfernt wurden und dadureh die Kontinuität des Rückenmarks unterlorochen wurde² (Fig. 1).

Am terminalen Ende, wo die Schwanzspitze ahgeschnitten wurde, ist die liegeneration in allen Fällen ganz normal vor sich gegangen. Die Kontinuitätstrenumg des Nervensystems hat also lieinen Eintluss auf die Ro-

\footnotetext{
1 Das beweisen ebenfalls die histologischen Präparate.

2 Diese Methode hat sich von der von BARfurTu angegebenen dadurch unterschieden, dass die Wirbelkörper mit den Gefässen nicht entfernt wurden.
} 


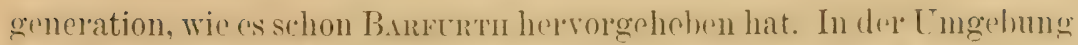
der. Wunde, welche zur Tremumg des Rückemmarlis angelegt wurde, begannen gleichzeitig die Reparationserscheinumgen. In einer Anzahl der Fälle hat sich die Wunde mit dem Proliferationsgewehe ausgefüllt und nachher die Kontinuität des Rückenmarks hergestellt.

In anderen Fällen fällt der obere Gewebsabschnitt (Fig. $1 \alpha$ ) ab, so

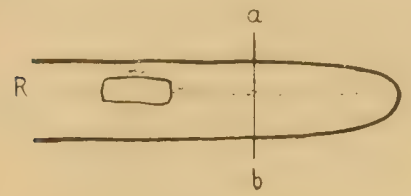

Fig. 1.

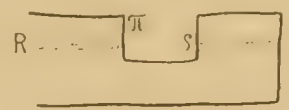

Fig. 2

dass der Schwanz das Bild vorstellt, welches rig. 22 veranschaulicht. An den Wundtächen $\pi$ und $\varrho$ kann jetzt del Regenerationsvorgan nen, welcher zur Entstehung neuer Schwanzanlagen aus dop Schnittfläche $\pi$ (Fig. 3) resp. $\pi$ und $\varrho$ führt (Fig.4). Auf diese Weise können zwoi- (Fig. 3) und dloischwänzige (Fig. 4) Tiele entstehen. Entwicholt

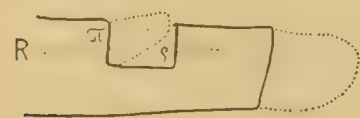

Fig. 3,

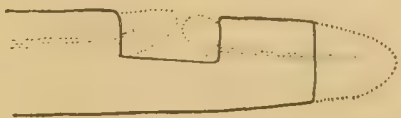

Fig. 4.

sich die Sehwanzanlage auch von der Schnittfläche $Q$ - so wächst sie anfangs kopfwärts, und erst nach ca. 2 Wochen velüudert sich rlureh Wachstumslegulation die Richtung des Wachstums dieser Schwanzanlage!.

Es ist bemerkenswert, dass in diesen Fällen, in welchen von der SchnittHïche $\pi$ und $Q$ neue Sullwanzanlagen entstanden sind, dieselben nicht von dem (puershnitt des ganzen Schwanzes, sondern rom Querschnitt pines Tojles des Schwanzes rntstanden sind. I)je kleineje El'mentenzahl hildet hier den Ausgangspunkt der. Schwanzanlage, welcher doch die Potenz der Ausbildung der Totalität des Organs innewohnt.

Liei Erörterung der Frage, ob das Zentralnervensystem auch in der Prriodr der Auswestaltung einzelner ()rgane pinen Einfluss ausübt - weist der Redner auf die 'Tatsache hin, dass das Zentralnervensystem sich im Proliferationsowehe zuerst diffremziert, dass also dio Mönlichlirit nicht

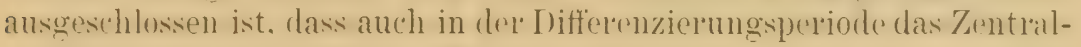
nervensystem eine formative Wirkung haben kann.

1 Das zwei- und dreischwänzige Tier werden demonstriert. 
Auf Grund der auforeürrten Experimente glaubt sich der Redner zu folgenden Schlüssen berechtigt:

Im Einklange mit den Resultaten von RuBin und G. WoLfF muss der Einfluss des Zentralnervensystems als unumwirnglich notwendige Bedingung zum normalen Terlauf der Regeneration betrachtetwerden.

l)ir Spinalganglien vermögen dir formative Wirkung der Rückenmarkszentren nicht zu vertreten.

Die Kontinuitätrennung des Zentralnervensystems resp. des Rückenmarks hat keinen Einfluss aufden nolmalen Verlauf der Regenerationsvorgänge (BARFuTH).

Das Vorhandenseindes Zentralner vensystem s bedingt die Aktivierung der prospektiven Potenzen dieser Elemente, welche durch den operativen Eingriff zur Realisiclung ihrer legenerativen Tätigkeit angeregt worden sind.

Der formative Einfluss des Zentralnervensystems a die Ausgestaltung der Organe aus dem indifferenten Proliferationsgewebe scheint wahreheinlich zu sein.

\section{Entwicklungsmechanische Studien an Schwämmen.}

Von Prof. 0. MAAS (München).

Durch Entzichung des kohlensauren Kalks vor der Metamor'phose werden die jungen Schwämme skelettlos, auch wenn andere Ca-salze, wie Gips. in notmaler Monge vorhanden sind. Die oreanische Grundlage des Skeletts kann gebildet werden, wird aber unlegelmässig, ebenso das ganze Sehwämmehen: der Gastralraum fällt ohne den Halt der Nadeln

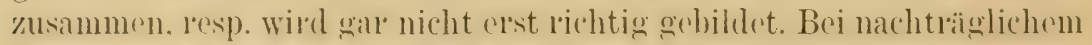
Zusatz von kohlensaurem Falk elscheinen Skelettstücke von anolmaler Form, jodoch aus renelrechtem Kallispat. Bei normaler Motamorphose und nachtriglieher Entzichung des kohlensauren Kallis findet cin Einschmelzen schon gebildeter Nadeln statt.

Die Experimente sprechen für die formative Reizwirkung, die die Nadeln auf andere Schwammteile zur Weiterentwicklung ausüben; sie zeigen ferner, dass bei der Nadelbildung zwei Prozesse auscinanderzubalton sind, oin oreanischer und eine loine Kidistallbildung. Sodann sind 


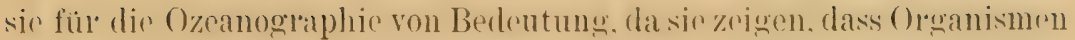
fül ihre Skelette aus kohlensaurom Kalk nicht durch chemische Um-

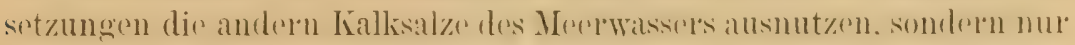
den schon vorhandenen kohlensauren Kalk, wenn er auch nur in Spuren zur Verfügung steht, verwenden können.

Siche: MaAs, O. Ueber den Autbau des Kalkstieletts der Spongien in normalem und in $\mathrm{CaCO}_{3}$ freiem Seewnsser. In : Verhandl. d. Dentsch. Zool. Gesellschaft, 1904.

\section{Sur le polyphylétisme.}

Par le Prof. J. PALACKI (Prague).

L'auteur commence sa communication en réclamant la liberté absolue pour la science, sans respect des autorités, même scientifiques. Le monophylétisme est un reste des idées anté-cuvieriennes et un obstacle au développement de la paléogéographie. Il ohlige la paléogéographie à admettr'e des choses impossibles telles que la Lémurie, l'Atlantis, etc.

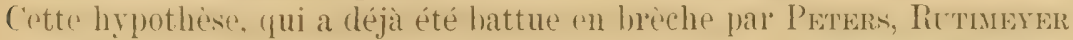
(Bos), WrNGe (Lemur), par l'auteur lui-même et beaucoup d'autres savants, subsiste néanmoins par la force de l'inertie et par le manque de connexion entre la Géographie et la Zoologie. L'auteur cite comme

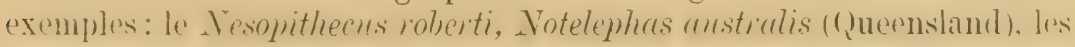
Discoglossides, Hypudeus Wrangeti à Revilla Gigedo etc. Il rappelle aussi ses travaux sur la distribution des Vertébrés. Tant que l'on ne sera pas arrivé à acquérir des notions positives, il vaudra mieux l'avouer que de faire des hypothèses et de chercher à forcer la nature.

Le polyphylétisme doit donc être admis jusqu'à ce que de nouvelles découvertes aient prouvé le contraire.

L'auteur recommande l'étude approfondie des variétés individuelles que l'on trouve dans l'aile de l'épartition de l'espèce, de même que les expériences synthétiques. Il termine par cette parole d'un empereur romain mourant: Laboremus. 



\title{
$2^{\text {me }}$ SECTION
}

\section{ERT TÉ B R É S}

\section{Systématique.}

Séances du lundi 15, mardi 16 et mercredi 17 noût 1901.

\author{
Président: II. Ie Dr F.-A. Jentink \\ Vice-Président: M. le Dr R.-F. ScimarfF \\ Secrétaire: M. le Dr ${ }^{r}$ E. ANDRÉ
}

\section{The Mammalian fauna of the Santa-Gruz beds of Patagonia.}

\author{
By W.-B. SCO'T'T (Princeton).
}

As is probably known to many members of the section, Princeton Uniresity maintained exploring expeditions in Paltagentia during the years 1896 to 1899, under the leadership of the lamented Mr. Hatciner. Owing to the indomitable energy and skill of Mr. Hatcher and his associates, these expeditions were very successful in making extensive and valuable collections in nearly all departments of natural science, but the most important is undoubtedly the remarkable series of fossil mammals gathered from the Santa Cruz beds.

The liberality of J. Pierpont Morgan, Esq. of New-York, has made it possible to publish in an adequate manner the reports of the expeditions, and I take much pleasure in presenting to the members of the Congress, for their inspection, such parts of these reports as have already appeared.

The admirable work of the brother's Anfeghro long since attracted the interest ard attention of palaeontologists to the vertebrate fossils of Patagonia, but much work remains to be done before we can satisfactorily interpret this wonderful assemblage of animal types,

In what follows I shall assume that the Santa-Cruz beds are of Mio- 
cens age, as has, in my opinion been clearly demonstrated by the work of Dr. A. E. Orturans upon the invertebrates of the underlying Patagonian formation. (See Vol. IV the Reports.)

'The Santa-Cruz formation itself is non-marine and largely of terrestrial origin, and where the fossil mammals are found, the matrix almost invariably consits of volcanic ash and tuff. This explains the surprisingly large number of complete and nearly complete skeletons which are found; perhaps many of the animals were overwhelmed alive by the shower's of ash and thus buried in an uninjured state.

On looking over a series of representative Santa-Cruz mammals, we are immediately struck by the strangeness of the assemblage; not a single genus of these mammals occurs in any port of the northern hemisphere and, what is more surprising, the difference from the norther'n faunas is not only one of families and genera, but of orders.

'Thus the beds have yielded no Carnivora, Artiodactyla, Perissodactyla or Proboscidea, and no Rodentia except the Hystricomorpha. The place of the Carnivora was taken by the carnivorous marsupials, of which there is a great variety, more or less resembling the Australian Thylacymus. Numbers of dispropodont marsupials, mostly of extremely small size, accompany the carnivorous types, and of these Cromolestes is an interesting survival to modern times.

One of the largest, most varied and most characteristic elements of the Santa-Cruz fauna is the subclass Edentata, which, so far as at present known, is represented only by three of its suborders, the Dasypoda, Glyptodontia and Gravigrada. Though no trace of the true sloths or of the anteaters has yet been found, it can hardly be doubted that both groups had already become differentiated, but they are to be sought for in some other region of South America.

The armadillos are, for the most part, not ancestral to existing genera, but represent peculiar types, some of which extended into the Pleistocene, while others died out in the Niocene. A peculiarity of the SantaCruz armadillos is the absence of a pectoral buckler, the carapace consisting chietly of movable bands of plates, with a small posterior, $\mathrm{Or}^{\circ}$ pelvic buekler, while in one genus (ficle Axeguro) the entire carapace is made up of movable bands.

'The glyptodonts are relatively less advanced than the armadillos, and are strikingly smallel than their gigantic successors of the Pampean beds. The Santa-Cluz genera are not only smaller', but distinctly more primitive than the latter, as is shown in all parts of the exo- and endoskeleton. The carapace always retains a greater or less number of partially movable bands and the tail sheath is composed throughout of rings, nevel fused into a club-like mass. Several of the genera retain vestiges of incisors and the grinding teeth are mostly of a pattern sim- 
pler than that which prevails among the Pampean genera. The skeleton, though typically glyptolont, yet shows many interesting points of approximation to the armadillos, which indicates that these two oreders are more nearly allied than either is to any other edentate group.

The (rravigrada are extraordinarily numerous and varied, so variable indeed that it is not yet possible to make any satisfactory taxonomic arrangement of them. Nore important is the fact that the three fanilies which occur in the Pleistocene are already recognizable and that the ancestors of nearly all the Pleistocene genera of hoth Nolth and South America may be identified. As in the other groups of edentates, the Santa-Cruz Gravigrada are of relatively small size, some of them extremely small, and in structure they are far more primitive than their Pampean successor's. Time will permit the mention of only a few of the more significant differences between the ground sothes of the two epochs. 1. The dental formula is invariably $5 / 4$ and in each jaw the first tooth is always more or less distinctly caniniform. 2. The trunk is very long and (onsists of numerous vertebrex, as many as 25) (lorso-lumbars; the articulations of the posterior thoracies and of the lumbars is less complicated than in the Pampean ground-sloths or the armadillos and anteaters of the present day. 3. The lumerus always has an epicondylar foramen and the femur has a large third trochanter. 4. All the parts of the manus and pes are free and uncoössified; the feet are always pentadactyl and the digits all have the full number of phalanges, including the claw. From the form of the astragalus and calcaneum it is evident that the fort was plantigrade and that rotation, so as to bring the fibular horder. to the ground, could have been present only in an incipient degree.

A careful stuly of the Santa-Cruz Gravigrarla leveals important resemhlames, not only to later members of the same group. but also to the true sloths and the anteater's, and we have the strongest evidence that all the American edentates are descended from a common ancestry, to which the so-called edentates of the old world have no apparent relationships.

As is well known, continental South America contains no Insectivora at the present day, and it is therefore somewhat surprising to find replesentatives of this ordel in the Santa-Cruz beds. As yet only a single genus (Necrolestes) has been obtained and this is a somewhat problematic type, concerning which I am not yet prepared to express any very definite opinion. Among recent insectivorous genera, Chrysochloris is undoubtedly the one which most resembles Necrolestes and, if this lesemblance is really due to relationship, as it seems to be, it gives interesting suggestions as to the former land connections between South America and Africa.

No Cheiroptera have yet been found, but that is, of course, no sufficient reason to doubt their presence in South America at that period. 
The Rodentia are of particular interest; they consist exclusively of Hystricomoryha: all the myomolphs, sciuromorphs and lagomorphs which now inhabit South America, came in the great migration from the North at the close of the Miocene. Of the six recent families of South American hystricomorphs, all but the Dinomyide and Dasymroctidee are represented in the Santa-Cruz fauna and only one subfamily is now extinct. Several of the genera are plainly ancestral to living types, while others helong to extinct lines: it is interesting to observe that those genera which were most flourishing, most abundant and most varied in Santa-Cruz times, are, for the most part, not those whose descendants persist to the recent epoch.

Taken as whole, the Santa-Cruz rodent fauna is surprisingly modern, but in a number of anatomical details these genera are more primitive and serve to connect existing types with the ancient generalized forms of the Oligocene and Eocene. Some of these more generalized forms persist, with little modification, into the Miocene and throw much light upon the mutual relationships of the hystricomorphous families.

Perhaps more interest attaches to the extraordinary series of SantaCiuz ungulates than to any other group. These remarkable animals all belong to different orders from those which are found in the northern hemisphere and inclucte neither Artiodactyla. Perissorlactyla, Proboscirlea, nor Amblyporlic. Roтu ${ }^{1}$ has lately published a very important paper, in which he shows that three of these ungulate groups agree in a very exceptional development of the auditory region, especially of the periotic, and that they should therefore be regarded as subdivisions of the same or del'. 'This conclusion is no doubt valid, though I must differ from Rotu's opinions regarding the other two groups of Santa-Cruz ungulates, believing them all to be more nearly related to one another than he is disposed to admit.

'The following table represents my present ideas upon the taxonomy of these animals :

\section{Toxodontia.}

\section{Notoungulata.}

1. 'Toxodonta.

2. Typotheria.

3. Homalardotheria.

II. Astrapotheria.

III. Litopterna.

The Toxodonta are extraordinarily abundant in the Santa-Cruz beds, where they are represented by larger and smaller species of the genus

1 Los Ungulados Sudamericanos. La Plata, 1904. 
Nesorton; the number of these species has heen much exagererated, for', as Amegrino has shown, the changes in the appearance of the animal, due to the developmental stages of the teeth, are most unusual. AmeGuno has also pointed out the very remarkable fact that $N^{\top} e s o d o n$ has a complete and functional series of prelacteal incisors and canines. Incredible as this statement may appear, the evidence for it is very strong.

Though perhaps not the actual and direct ancestor of the Pampean Toxodon, Nesodon yet shows us very nearly what that ancestor must have heen. The skull is disproportionately heary, the trunk massive, the limbs short and thick and the feet euriously small. The feet are tridactyl and, at first sight, very like those of the Perissodactyla, but the tarsus is of a radically different character and belongs to the type which all of the Santa-Cruz hoofed animals exemplify, without known exception. It should be added, however, that the feet of Astrapotherium have not yet been found.

Even more abundant individually than the preceeding group and far more varied generically and specifically, is the suborder Typotheria. In the Santa-Cruz beds they are represented by a surprising number of small animals, with a great variety in dental and skeletal structure. The typotheres have a certain resemblance to the Hyracoidea, but this I belive to he 'ntivery analogical, and I quite agree with liotu in the opinion that their closest relationships are with the toxorlonts.

The Homaladotheria are comparatively rare and their structure is much less completely known than it the case of the two preceeding suborders, but sufficient has been learned to make clear their systematic position. 'T'he skull, dentition, carpus and tarsus, are fundamentally similar to those of the Toxodonta, yet the feet have undergone a curious morlification, the position of the phalanges with reference to the metaporlials being quite unusual anong the hoofer animals and the unguals having assumed the for'm of claws. A somewhat similar change has occurred in the Ancylopoda and, among artiodactyls, in the Agriochoeridx, but the resemblance is merely another of those cases of convergence, which the Santa-Cruz fauna has in such a striking degree.

The Astrapotheria still form an unsolved problem, for they are known only from the skull, no other part of the skeleton having been certainly identitied. This is quite curious in view of the fact that skulls of Astrapotherim are not lare in Patagonia and in view of the large number of nearly complete skeletons of other animals which the collectors have obtained.

Astrapotherium does not have the exceptional structure of the auditory region of the skull which characterizes the thres precerling groups: alone among the Santa-cruz ungulates, it has large tusks in both jaws, 
which are canines and not incisors. The grinding teeth have a striking similarity to those of the rhinoceroses, more particularly of Metamynodon and Cudurcotherium, of the North American and European Oligocene respertively. so long as the feet remain unknown it will be impossible definitely to determine the taxonomic position of the Astrapotheria, but the more ancient members of this order, from formations older than the Santa-Cruz, show in their dentition surh an approximation to the characteristic molar-patterns of the other and specifically South Americau ungulate groups, as to indicate, in my opinion, a nearer relationship to the Toxodontia than to the Perissodactyla. It is a highly signifigant fact that in these more ancient genera the molar-patern is less rhinocerotic than it hecame in the Santa-Crou epoch, a fact which, of itself, is suggestive of convergent development.

Most remarkable of all the hoofed animals are the Litopterna. In the Sinta-Cruz beds we find the ancestor of the Pampean IIacranchemin, and also a family which, as Ameghivo long ago pointed out, has paralleled the horses in the most wonderful way. Certain of these genera are tridactyl, with the lateral digits reduced to dew-claws and the weight supported antirely upon the median or third digit, giving to the font a most striking and deceptive resemblance to that of Protohipmes and Hipurarion. Another genus even surpasses Equus in the completeness of its monotactylism, the lateral metaporlials being reduced to minute proximal rudiments, far smaller than the splintbones of the hor'se. Here again the carpus and tarsus show that these amimals are not related to the perissodictyls, but to be much more nearly allied to the Toxodontia. They afford one of the most remarkable and instructive examples of parallel or convergent development known among mammals, but, at the same time, they demonstrate that in so complicated a structure as the mammalian skeleton convergence or parallelism can never result in complete identity, but merely in a greater or less number of striking similarities.

The problem of relationships hetween these south American ungulates and those of the northern hemisphere camnot yet be definitely solved, but it is my present opinion, derived from an examination of the preSantacruzian types of hoofed animals, that all the South American groups are more nearly related to one another than to any northern group, and that both the northern and southern types go back to a common ancestry, which is nearly represented by the Condylarthra. (1) sons's recent discosery of a true armadillo in the Bridger Eocene of North America proves the possibility of a communication, however roundahout and indirect, hetween the two Americas in late Mesozoie or early Tertiary times, so that the existence of Condylarthra in the southern continent is what we should expect. 
Remains of the Primates are very l'ale in the Niocene of Patagonia, but they are futirely rharactristir and unerpuivorally demonstrate the presence of monkeys of South American type in the Santa-Cruz beds.

In the Miocene period Patagonia appears rather to have been an outpost than the principal area of mammalian cvolution in South America; hence it is that many groups which we might confidently expect to find there are not represented in the Santa-Cruz beds. Nevertheless the study of this fauna is of the utmost importance for the understanding of the taxonomic and geographical relations of the modern Mammalia.

\section{A new group of marine Reptiles from the Triassic of California.}

By J.-C. MERRLAII (Berkeley).

In the collections of marine 'riassic vertebrates at the University of California there are a number of specimens representing a heretofore unknown form of swimming Reptile. This form has been made the type of a new genus and species having the following character's.

Thalattosaurus Alexandre, n. gen. n. sp.

Cranium elongated, with slender snout. External nares separated and not far in front of the orbits. Dentigerous portion of the premaxil-

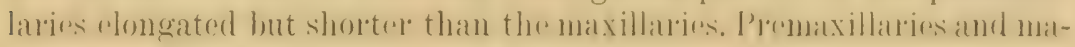
xillaries sculptured on the external surface.

Vomer's with two rows of flat, button-like teeth. Pterygoids with four or more rows of curved, conical teeth. Palatines not known to be dentigerous. Treeth of the premaxillares and of the antreror end of the dentary slemelere conical. Posteriol part of elentary and posibly of maxillares with button-like, flat ol only slightly tuberculate teeth.

Vertebra amphicolous, neural spines slender: Dorsal ribs single-

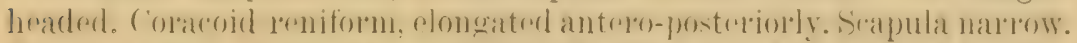
Ilumerus short, much expandord distally. Raulius and uha about half thre length of the humerus; radius with median constriction. Pelvic arch lobust, inferior elements not plate-like.

In its fundamental outlines, the skelctal structure in Thalattosaurus

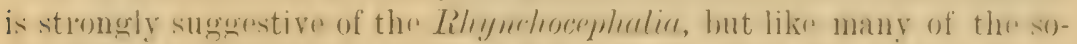

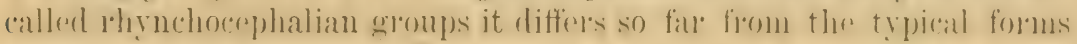

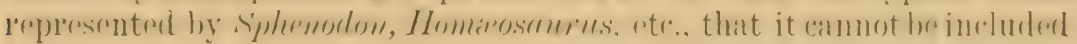
in the same ordinal division. It is likewise so different from all of the other described reptilian families and orders that it must be given an 
independent position. The family name Thalattosaurid.e and the ordinal name linalatrosauria are therefore used to express its position in the scheme of classification.

For the present the Thalattosania may be placed in the superorder Dinptoscurice of OsBors, though it is doubtful whether it will be retained in that division, or even whether the Diaptosanvia can hold together when the various forms included in it become better known.

Inside the Diaptosanrin the closest affinities of the Thalattosanria are with the Protoroscuria and Rlyynchocephatia. From both groups they differ more widely than these two differ from each other. In many respects, particularly in limbstructure, the Thaluttosenvia represent the most highly specialized aquatic forms in the Diaptosauria.

Outside of the Diaptosauria there are noticeable resemblances to the Purusuchin and to the Lacertilians. The common characters are. howerer, almost without exception, primitive or rhynchocephalian characters which we find persisting in the Parasuchia and Squamata.

\section{Sur un Ours nain des Alpes grisonnes (Ursus formicarius)}

Par le $\mathrm{D}^{\mathrm{r}} \mathrm{S}$. BIELER (Lausanne).

Avec 2 figures dans le texte.

J'ai reçu, il y a quelques années, un crâne d'Our's adulte de petite dimension, à peine de la grosseur du crâne d'un Chien saint-bernard. Le crâne est caractérivé en outre par l'alsence presque complète de crête occipitale et par la rectitude des os du nez qui forment une ligne presque droite avec le frontal

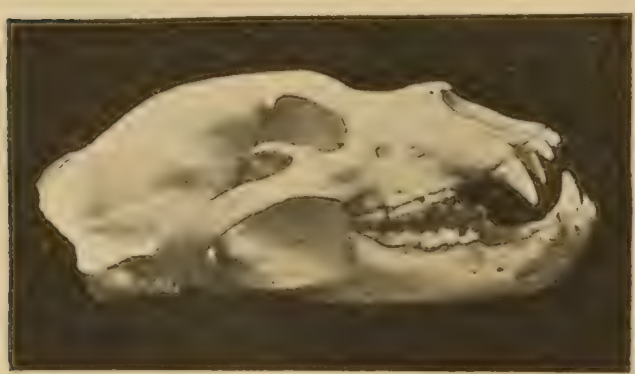

Ursus arctos (1/0) de Lithuanic. tandis que chez l'Ours brun il y a une concavité prononcée à la naissance du nez et que la crête occipitale est très prononcée, comme les figures ci-dessous le montrent très bien.

L'animal dont il s'agit avait été tıé dans les Grisons il y a 5 ou 6 ans.

L'échantillon était resté pour moi à l'état d'énigme, jusqu'à une visite de M. le prof. Galli-Valerio à notre Musée agricole. Il me dit avoir vu dans 
les collections du Gymnase de Sondrio (Valteline) des Ours de petite taille auxquels les montagnards domnent le nom de fimiguren, mungen is de foumis. Ce crâne de petite dimension appartiendrait donc, probablement, à un Ursus formicarius dont le nom se trouve dans tous les livies, mais sans aucune description spéciale sur les caractères de la taille et des mœurs de l'animal.

J'ai cherché des renseignements dans le mémoire d'Eversmann (Moscou 1840). Dans cet ouvrage, les caractères différentiels de taille, de forme, de crâne et de poils sont nettement décrits et correspondent à ce que l'on peut voil soit sur' le crâne du Musée agricole de Lausanne, soit sur les exemplaires empaillés du Gymnase de Sondrio.

D'après Eversmann, les paysans et les chasseurs de la Russic orientale et de la Sibérie connaissent deux espèces d'Our's, déjà décrites

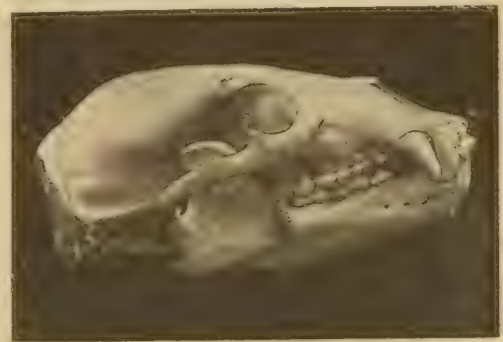

Ursus formicarius $(1 / 6)$ des Grisons. par PaLLAS; un grand Our's nornmé Sterveniki (Aasfiesser, U. arctos), et un petit Ours Muraveinitii (U. formicarius, Ameisenbär). Mais ces désignations populaires n'ont pas une valeur réelle, les animaux des deux espèces mangent les uns et les autres de la chair et des Fourmis. Lorsque les Ours quittent leurs tanières, au printemps, ils se jettent sur les fourmilières et dévorent à la fois les Fourmis, les pupes et les débris d'aiguilles de Pins qui y sont mélangés et qu'on retrouve dans les excréments.

La différence des deux Ours est dans la forme de la tête dont les crânes donnent une idée. Comme taille, l'Aasfresser, Our's brum, est presque le double de l'U.formicarius; dans la four'rure, l'Our's biun jeune porte un collier de poils blancs, qui l'a fait appeler $U$. collaris, et que le petit Ours n'a pas. En outre, le poil de ce dernier est moins brillant et sa fourr'ure a moins de valeur. En dernier lieu, l'U. formicarius est plus plantigrade et a une démar'che plus fel'me que le grand Ours.

Quoi qu'il en soit, on devrait inscrire dans la faune des Alpes des Grisons et de la Valteline la présence de cet Ours puisqu'il y a diver'ses preuves de sa présence.

M. le baron de Nopsca, qui assistait à la séance, dit que cet Ursus formicarius se trouverait aussi dans les montagnes de Transylvanie. 


\title{
Vom Biber an der Elbe.
}

\author{
Von Dr. A. MERTEXS (Magdeburg).
}

Als Zoologen ist es Thnen wohl hekannt, dass unser grösster europäischer Nager. der Biber (Custor fiber), jetzt fast üherall in seinem, sich ursprüuglich von dor Pyrenäenhabinsel his tief nach Sibirien hinein erstreckenden Verbreitungsgebiete auscerottet ist. Nur noch an einigen, weit von eimander entfernten Stellen, die besouder's geeignet waren, ihm Zuflucht vor din Tachstellungen des Ienschon zu gewähren, hat er sich his heute erhalten: er wird noch angetroffen an der unteren Rhone in der Nïhe von Lyon ${ }^{1}$ und im Mündungsgebiete ${ }^{2}$, im westrussischen Sumpfwalde in den Bezirken Polesje, Pinsk, Minsk, Mohilew, Tolhynien, Kiew ${ }^{3}$, in Norwewen ${ }^{4}$ und an der Elbe zwischen Wittenberg und Magdeburg.

I) a ich als Bürger der letztgenannten Stadt in unmittelbarer Nähe der Biber wohne - ein Bau befand sich bis vor drei Jahren sogar im vielbesuchten Starltualke - habe ich häufig Gelegenheit gehabt, das Tier zu lrebachten, und ich glaube lieiuen kehlgriff getan zu haben, wenn ich mir erlaube, Ihnen hier etwas von dem zu berichten, was ich gesehen halur, sellost auf die Gefahr hin, dass ich Ihnen schon Bekamntes bringe.

Die Kürze der mir zur Vertügung stehenden Zeit macht es mir natürlich unmöglich, rinen vollständigen Ueberblick über das Leben des merkwïrdigen 'Tines zu gehen; ich muss mich daher auf einige Einzolheiten beschränken und möchte zunächst über die Wohnung des Bibers und seine Bauten sprechen.

Der Biber legt seinen unterirdischen Bau in den steilen Ufern der Elbe und ihrer Zuthüsse, der. Mulde, Siale, Nuthe und Ehle, lieber aber noeh als an dem von der Schiffahrt helebten Hauptstrome an den stillen, waldumrauschten Seeen und Altwässern - chemaligen, jetzt von der Ellor abgenchnittenen Strombahnen - an. I)er Hauptteil ist ein geriulmiger, an Boden mit weichem Gras, alser auch mit Holzspähnen und dereteichen bedeckter Kessel, der, wemn möglich, so hoch liegt, dass dass Hochwasser ihn nicht erreicht. Er dient dem Tiere den 'Tag über als Aufenthaltsort und wird meist erst mit Einbruch der I) immerung

1 Mündliche Mitteilung yon Prof. Matschie, Berlin.

2 Annales de la Société Entomologique de France, p. 147. Octobre, 1884.

Ilarion. Physionomie zoologique du Département des Bouches-du-Rhône, p. 7. Marseille, 1891.

3 C. Grevé. Zoologischer Garten, H. 3 u. 4. 1903.

' Brenis '́lierleben, II, p. 463. 1890. 
verlassen. Nur wo Stör'ungen gar nicht vorkommen, bringt der' Biber zuweilen auch am Tage rinige Stunden im Freien zu, um sich zu sommen; er sehlät dabei auch wohl ein und kamn dann leicht üherrascht werden. So wurde an einrm schönen Vittage in dere Kerruzhorst bri Magdeburg ein sehlafender Biber in der Nähe seines baues angretroffen, der orst im

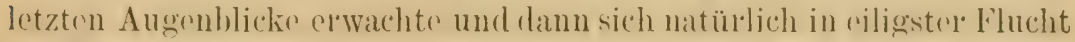

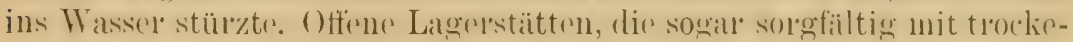
nem Gras gepolstert waren, habe ich mehrfach gefunden.

Vom Kessel führen eine oder mehrere meterlange Röhren schräg zum

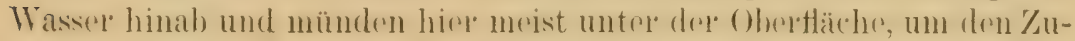
gang zum Bau zu verbergen. Wenn das Wasser an dieser Stelle frei ist, gelingt dies ja auch; wo jedoch Rohr und Schilf das Ufer umsäumen, sind die Halme an den Zugangsstellen abgebrochen und niedergedrüekt, sodlass man daraus crliennen liann, wo das Tiere einzufahreen pthegt, auch wenn das Eingangsloch nicht sichtbar ist.

Wo die Gegend ganz ruhig ist, sieht das 'T'ier von dieser Vorsicht auch wohl ab und legt free und offen mündende Röhren an. So berichtet FransRICH $^{1}$ in seinem Werke über die Biber an der mittleren Elbe von einem solchen Bau auf dem rechten Muldeufer bei Dessau und von anderen im Grosskühnaure Forste, ich selbst sah eine offene Riohne mehrere sehritte vom Wasser in der Kreuzhorst bei Nagdeburg und zwar ganz frisch befahren, wie noch feuchter sechlamm auf den kur\% vorher umgeknickten

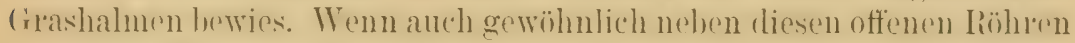
noch unter Wasser mündende für den Fall der Not vorhanden sein mögen, die sich nur der Beobachtung entzogen haben und erst bei besonderen Veranlassungen entdeckt werden ${ }^{2}$, hatte der erwähnte Bau in der Liveluzhorst wohl keine, dernn am gamzen [fere entlang wueherte ein wahres Dickicht von Wasserpflanzen, dur'ch das der Biber nicht hätte zum Eingang hindurchkommen kömmen. olue dentlich sichtbare spuren zu hinterlassen.

Sinkt in helehten Gegenden dep Wassierpiegel, sodass die Zugangsuiffnung zum Bau sichthar wird, so verlassen die Biber wohl ihre Wohnung,

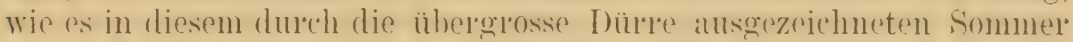
in der Kreuzhorst der Fall war, oder aber sie suchen den Zugang zu verdecken. Zu drm Zwecke schleppen sir algerchnittene Aeste mond Zwoige herbei, packen sie in geschickter Weise in einander und bauen so einen vom Ufer weit in das Wasser hinausragenden Schirm, unter dem sie unbemerkt in das Wasser gelangen können, um er'st weit vom Bau wieder aufzutauchen.

Nach dem Lande haben die IBiberwohnumgen in der Rergen krinen Aus-

1 Friedrich. Die Biber an der mittleren Elbe. Dessau, S. 21. 1894.

¿ Friedrich. A. a. 0., S. 21. 
gang. Der Kescel liegt meist dicht unter der Oberfläche, und da kann es rorkommen. dass die Decke eimmal einbricht. Dann kann der Biber an dieser Stelle wohl heraus. Gewöhnlich aber sucht er diese Oeffinung möglichst schnell wieder zu verdecken, indem er abgeschnittene Aeste und Zweige darüber schichtet, die schliesslich grosse Haufen bilden liönnen. An ein'm Bau aber fand ich im vorigen Winter einen Ausgang, den die Biber, als Eis die unter der Wasseroberfläche mündrnde Röhre unbenutzbar machte, regelmässig befuhren, um zu den benachbarten Stämmen zu gelangen und sie abzuschälen.

solche absichtlich odel unabsichtlich entstandenen ()effinungen dienen wesentlich zur Durehlüftung des Iaues; wo sie nicht rol'handen sind, muss dir Luft jedenfalls durch die dünne Bodendecke hindur'h eindringen.

I lese Höhlenwohnungen sind jedoch nicht die einzigen Bauwerke, die die Biber auftïhren. W'ie hemerlit, werden sie nur an einem hohen Ufer angelegt; wo nun das Ufer flach ist, ist eine solche Bauart unmöglich. Da baut der Biber Burgen, wie sie aus Berichten über den amerikanischen Biber bekannt sind. An der Elhe sind hisher nur einige aufgefunden worden, die beiden schönsten am Grosskühnauer-See bei Dessau. Am Nordufer dieses Sees, eines Altwassers der Elbe, liegt nach dem jetzigen Strome hinüber eine weite, bruchige Wiesenfläche. Das Seeufer ist ein schwankender, sumpfiger Boden, der sich kaum über den Wasserspiegel erheht und von einem dichten Rohr- und Schilfdichicht bedectit ist. Dieses Dickicht wird von einigen B iber ka näl en durchzogen, die dadurch cntstanden sind, dass dir Biber immer denselben Weg zu den mit Nahrung liefernden Bäumen bestandenen Höhen genommen und dadurch den weichen, schwankenden Boden allmählich rertieft haben, his mit Wasser grefüllte Rimnen entstanden sind. Am Rande dieses Dikkichts liegen zwei Burgen. Sio haben die Fol'm eines Bachofens und sind aus Zweigalschnitten, Schilf, Rohr und schlamm aufgebaut; dabei sind sie so fest, dass man sie besteigen kann, ohne durchzubrechen. In die östliche burg führen drei Kanäle. I)je Entstehung dieses Bauwerkes wird in der' Weise zu denken sein, dass die Tiere, um geschützt zu sein, an einer zusagenden Stelle auf obener kide aus Reisig (in Dach gehaut haben, unter das die Kanäle hinuntereichten. Dieses Dach ist damn immer weiter ausgebaut und verdichtet, sodass ein geschlossener Hohlraum entstand, der neben dem Wasser noch Platz zum Lager für die Tiere bot, andrerseits diesen die Möglichlieit liess, bei Störung sofort in einem der Kanäle zu entwischen.

Auch wenn dic Biber, durch ungünstige Verhältnisse gezwungen, längere Zeit ausserhalb ihrer Höhlen zubringen müssen, legen sie sich zum Schutze auf dem Lande wohl solche Reisighaufen an, unter denen sie sich an 'Tage verstecken.

Weit grossartiger aber als die hisher hesprochenen Bawwerke sind die 
Dammbaten der Biber, von denen jeh einige Abbildungen vorzulegen im stande bin. Man kionnte, wenn man ihre Anlage und ilure Festigkeit betrachtet, beinahe zu der Annahme kommen, dass man es dabei mit mensehlichen Erzeugnissen zu tun habe. Diese Dïmme werden dam angelegt, wenn in schmäleren Wasserarmen, an denen IBiberröhren liegen, der Wasserspiegel so sinkt, dass die Ausgänge sichthar werden und zugleich die Tiere in ihrem Filement sich nicht mehr schwimmend und tauchend frei bewegen können. An den verschiedensten Stellen des Velbreitungsgobictes, von Wittenherg his nach Magdehurg, hat man sie unter den angegebenen Bedingungen getroffen, und die Tiere waren so eifr'ig bei dieser Arbeit, dass sie die am 'Tage zerstörten Werke in der Nacht immer wieder auffühten. Dic Dämme werden in der Weise gebaut, dass starke Holzahschnitte an Iter und im Grunde festgeklemmt werden; dünnere Zweige werden dazwischen geflochten; Schilf, Gras und dergl. verstopft die Lücken, und endlich wird durch darauf gebrachten Schlamm völlige Dichtung erzielt, sodass dass Wassel aufgestaut wirel. I)amit der. Damm nicht duch den Wasserelluck zerstört wircl, ist er zweckmässig so eingerichtet, dass er unten breiter als oben ist und die allmählich ansteigende Seite der Druckrichtung entgenstellt.

Wenn diese Biherdïmme an Ausdehnung auch denen in Amerika nicht gleichkommen, dürfen sie doch in der Art ihrer Anlage jedenfalls diesen an die Seite gestellt werden.

So lange das 'Tier sich darauf beschränkt, nur im Ufer zu bauen, mag der anliegende besitzer es noch mit ansehen. Wo aber der strom durch die weite Aue Hiesst, wählt das Tier auch gern die zum Schutze der Niedelungen aufgeführten Deiche zur Anlage seiner Wohnung. I es damu allerdings sehr gefahrlich werden; denu wenn schon das HochWasser durch Mäuse- und Maulwurfiöcher sich leicht hindurchfrisst und dadurch den Damm zel'stört, wie viel mehr wird dass der Fall sein, wo Rïhren von so gewaltigem I)urchmesser, dass rin Biber hindurch liann, die aufgrworfene Erdmasse durchziehen? Da ist damn der Alschuss des Tieres wegen feführdung der ganzen Umgebung gehoten, vorausgesetzt, dass es sich auf andere Weise nicht vertreiben lässt.

Zu diesem Schaden, der jedoch nur hier und da verursacht wird, kommt aber ein anderer, recht bedeutender. Wer im letzten Jahre Gelegenheit latte, dieschöne Auwaldung der Kreuzhorst bej Magdeburg zu besuchen, der konnte am Ufer der Alten Elbe Stellen finden, die aussahen, als ob die Holzhauer hier tätig gewesen wären.

An mehreren Plätzen lagen 12 bis 15 Eichen von 20 selbst $30 \mathrm{~cm}$ Durchmesser fein säuberlich dicht über dor Erdo abgeschnitten in Reihen mehen einander. Die kegelfürnigen Stümple. der kegelförmige schnitt am Stammende, die massenhaft dahoi liegenden langen spähne liessen gar keinen Zweifel aufkommen, dass die Ibiber die Täter gewesen. Ual)- 
rigens waren die Bäume nicht, wie man vielfach wohl lesen kann, nach dem Wasser hin gefallen, sondern lagen sïmtlich ilem Ufer parallel; und vom Wasser her führten mehrere durch den Kü̈rper und den nachschleppenden Schwanz (die Kielle) deutlich ausgeprägte Pfade. Die Zweige waren meist abgeschnitten und zum Wasser geschafft, wo sie dann entweder sofort geschält orler weiter zum Bau befördert waren. An einer solchrn Eiche war sichtbar, dass der Biber auch zu klettern vermag. Der Baum war durch starke Aeste im Fall aufgehalten, sodass der Stamm ganz schräg lag. Auf ihm war das Tier emporgestiegen und dann auf die stälkeren Zweige übergegangen, sie von ohen und an den Seiten schïlend, während die unerreichbaren Unterseiten ihre Rinde behalten latten; erst die dünnen Enden waren dam glatt abgesehnitten, unten aufgenommen und weggeschleppt.

Im Kuhlenhagen, einem anderen Reviere der Kreuzhorst, sind selbst Eichen von über $1 / 2 \mathrm{~m}$. Durchmesser gefährdet; einige solche sind rom Biber gefällt, andere so weit angeschnitten, dass sie auch hald stürzen werden oder doch bald abgehaunn werden müssen, bevor sie absterben. Bei dem südlicher grlegenen I)orfe Ranies ist sogar eine Pappel von 72 cm. Durchmesser umgestürzt.

Solche Schädigungen sind in den staatlichen und stiftischen Waldungen, wo es auf einen Baum melu oder weniger nicht anliommt, schliesslich zu ertragen, nicht dagegen in kleinen Privatforsten, und wenn 1 un gar die 'Tiere in die (Gärten kommen und die Obstbäume fällen oder, wie in einem Fallo eine ganze Obstanlage von etwa 150 Stämmen ahschneiden, dann hann man es wohl verstehen, dass die Besitzer bestrebt sind, sich diese Feinde vom Leibe zu halten.

So wird mancher Biber erlegt. Andere gehen den Fischern in die Netze, velwickoln sich darin und müssen dann ertrinken, wieder andere geraten in die für die Fischottern gelegten Eisen. Das Hochwasser und namentlich das von diesem mitgefühlte Eis bringt viclen Tieren den Torl. Und bedenkt man, dass der listbare Pelz auch Liehhaber findet und daher mancher Biber sein Kileid lassen muss', so versteht man. dass die Zahl der 'Tiere an der' Elbe nicht mehr' allzu gr'oss geblieben ist.

Es mögen auf der ganzen Strecke von Wittenherer bis Magdelourg noch etwa 150-200 Stück volhanden sein; cine ganz genaue Angabe ist bei del verstediten Lehensweise, den vielfachen Ahgängen und der Wanderlust namentlich der Männchen nicht möglich.

Um die völlige Ausrottung zu verhindern, besteht in Preussen seit lange eine Cabinettsordre, wonach in den staatlichen umd stiftischen Forsten das Erlegen der Biber gïnzlich verboten ist. In den Privathesitzungen dagegen war er bis jetzt völlig vogelfrei; jeder, der ihn traf,

1 Die Verwendung des Geils hat fast völlig aufgehört. 
komnte ihn ungestraft töten, und wemn dies nicht mehr ausgenutzt ist, so heruht das wohl auscehliesslich auf Unkenntnis der Sachlage, da man glaubte, der Selıutz im Staatsgebiete gelte allgemein. Seit diesem Jahne ist der Bilner dureh das neue Jagdschutzgesetz als jagdbares Tier anerkannt, d. h. seine Erlegung ist nur dem Jagdherechtigten erlaubt, jedem anderen bei hoher Strafe verboten; und ausserdem ist ihm eine 10 momatlich Schonzeit bewilligt, die noch dureh hesondere Verfügung auf das ganze Jahr ausgedehnt werden kann ${ }^{1}$.

In Anhalt geniesst der Biber eine 4 monatliche Schonzeit, in den herzoglichen Privatforsten völligen Schutz.

So ist denn zu hoffen, dass nummehr der Biber in unseren Elbwaldungen als ein Rest ans alter Zeit noch längele Zeit erhalten bleiht, musomehr als das Interesse für Heimatschutz, der sich auch auf bemerkenswerte Tiere und Pflanzen erstrecht. immer reser wird und die weitesten Lipejse der Bevölkerung ergreift, also auch dem Bilıer zu gute kommen wirl.

\section{Triton Blasii und die Mendel'schen Regeln.}

Von Dr. W. WOLTERSTORFE (Magdelourg).

Triton Blasii de lisle 2, dieser interessante und seltene Molch Zentralfrankreichs, wird bekanntlich seit langem mit mehr oder weniger Ibstimmtheit als Bastardform zwischen $T$. marmoratus und cristatus betrachtet. Auch der Entdecker selbst, DE L'IsLE, hat $1872^{3}, 10$ Jahre nach der ersten Veröffentlichung, in einem Aufsatz über die Hyluridation bei Anuren und Urodelen die Vermutung ausgesprochen, dass Triton Blusii vielleicht ein Kreuzungsprodukt dieser Molche sei. Leider geht er in diesere Arbeit nicht nähes auf seine dieshoüglichen Bastardierungsversuche ein, sei es, dass sie misslangen, sei es, dass sie überhaupt unterblieben sind.

Von anderer Seite, insbesondere von Bedriaga, wurde dagegen die Bastardnatur des T. Blasii bis in die neueste Zeit angezweifelt. Mir selbst gelang es er'st im vorigen Jahre, nach vielen misslungenen Versuchen, in Verlindung nit rinigen Freunden dureh dir gedmgene Lerelzung von Triton marmoratus und $T$. cristatus, insbesondere durch die

\footnotetext{
${ }^{1}$ Diese Verfügung ist jetzt erlassen, sodass der Biber überall das ganze Jahr hindurch Schonzeit hat.

${ }^{2}$ Notice zoologique sur un nouveau Batracien. Ann. Sc. nat.(4) t. 17, p. 364, pl. 12.1862.

${ }^{3}$ Ann. Sc. nat. (5) t. XVII. 1872.
} 
Kreuzung von $T$. marmoratus $\sigma^{x}$ mit einigen $T$. cristatus carnifex 우 von Teapel, den positiven Nachweis zu erl)ringen, dass Triton Blasii tatsïchlich eine hybride Form darstellt ${ }^{1}$. Ausführlicher habe ich hier'über in den "Zoologischen Jahrbücher'n " und im "Zool. Anzeiger" ) berichtet. Es (rubrigt mir daher heute nur noch zu benerken, dass mir inzwischen auch die Kreuzung zwischen T. mamoratus ơ und T. cristatus subsp. typicu o aus Zentralfrankiecich, den eigentlichen Stammformen, gelungen ist, sodass jetzt jeder Einwand gegen die Richtigleit dieser Auffassung hinfällig wird. Auch die Kreuzung zwischen $T$. cristatus or und T. marmoratus $q$ ist mir jetzt gelungen.

Ich erlaube mir, Ihnen hier einen jungen, einjảhrigen Bastard vorzulegen. Vater ist ein T. marmoratus von Porto mit schwarzen Seitenbinden und grüner, gezackter Rückenmittenbinde, Mutter ein T. cristatus carnifex mit orangegelbem, geflecktem Bauch.

Angeregt durch meinen verehrten Freund Boulenger habe ich bei dieser Gelegenheit auch auf die etwaigen Beziehungen zu den Menoei' schen Regeln mein Augenmerk gerichtet.

Wenn sich unsere Beobachtungen auch naturgemäss elst auf dic elste Generation der Hybriden erstrecken, so ergeben sich doch schon jetzt manche interessante Resultate.

Die Variabilität der Bastarde ist weit grösser als jene der Stamm formen.

Im allgemeinen herescht auf der ()berseite der Bastarde die Färbung des $T$. marmoratus, auf der Unterseite jene des $T$. cristatus vor. Im einzelnen ergeben sich aber manche Abweichungen.

Den vorherrschenden dominierenden Einfluss des T. marmoratus heohachten wir mit Sicherheit an einem 'Teil der Bastarde (auch an dem vorliegenden), welche einon $T$. marmoratus $\sigma^{x}$ von Porto zum Vater haben. Die grüne Rückenmitte, die dunkehn Seitenbinden sind auf der Oberseite deutlich kenntlich.

Die I) ̈̈ mp f ung des grellen Grüns bei vielen Bastarden ist dagegen auf den abschwächenden Einfluss des $T$. cristatus zurückzuführen.

I)ie. Terteluallinie. ein schmaler Streifen auf Rücken und Schwanz, ist bald mehr gelb, wie bei Triton cristatus carnifex, bald mehr orangerötlich, wie bei $T$. marmoratus.

Das Colorit der Lnterseite junger kleiner Bastarde stimmt völlig mit T. cristutus überein. I)er Bauch jst orangegelb his orangerot, die Zeichnung besteht in rundlichen dunklen Flecken.

\footnotetext{
1 Sie entspricht ganz dem Rackelhuhn (Tetrao hybridus) der Ornithologen, der Kreuzung zwischen Auer- und Birkhuhn (Tetrao urogallus und T. tetrix). Es erscheint durchaus gerechtfertigt, den Namen "Triton Blasii" als Sammelbegriff für alle Hybriden zwischen $T$. marmoratus und cristatus beizubehalten.
} 
Bei ninem 'Teil der Bastarde, auch dem vorliegenden, ist diese Färhung noch jetzt, nach cinem Jahre, unveräindert. Bei anderen Tieren schicbt sich später von der Brust- und den bauchseiten hor ein hräunlicher Farhenton vor, wolcher das Orange zurülkzudrängen bestimmt ist. Es rntsteht so die aigentümliche Mischungszeichnung, welche die Bauch fïrlung der meisten treilebenden T. Blasii kennzeichnet.

Diese dominierenden Merkmale bleiben in der Kreuzung zwischen $T$. marmoratus und $T$. cristatus im allgemeinen dieselben, ganz gleich, ob Vater oder Mutter Triton marmoratus oder cristatus ist.

Die gleiche Erfahrung lässt sich ühngens an der Mehrzahl der importierten T. Blasii machen. Fast stets überwiegt bei ihnen auf der Oberseite das Colorit des T. marmoratus, auf der Unterseite jene von $T$. cristatus. Abweichende Individuen gehören entweder (ler "2. Generation der Hybriden ") an oder sind Mischlinge zwischen einer der beiden Stammformen mit $T$. Blasii. Solche Individuen stehen im Colorit auf der Oberseite dem $T$. cristatus, oder im Colorit auf der Unterseite dem T. marmoratus nahe. Im ersteren Fall tragen sie vielleicht $3 / 4$ cristatus-, im letzteren Fall ${ }^{3} / 4$ marmoratus-Blut, um den alten, landläufigen Ausdruck zu gebrauchen. Gewöhnlich entspricht dann auch die Gestalt inehr der einen oder anderen der Stammformen.

Da solche Tiere noch nicht gezüchtet, sondern nur in Freien gefangen wurden, enthalte ich mich hier weiterer Ausführungen, welche doch nur Vermutungen wiedergeben würden.

Dagegen lässt sich jetzt schon sagen, dass manche der angeführten Beobachtungen an $\mathrm{Zuch}$ tmaterial mit den Resultaten der Menderschen Untersuchungen gut übereinstimmen. Es würde zu weit führen,

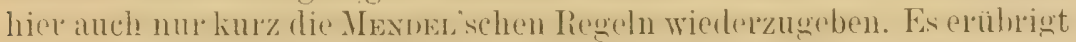
sich um so mehr, ais gerade im letzten Jahre von mehreren Zoologen, wie HaEcker, LANG, ausführliche Arbeiten über diesen Gegenstand erschienen sind, nachdem die Botaniker bereits seit einigen Jahren Mexines lange vernachlässigte Untersuchungen einer gerechten Würdigung unterzogen haben.

I. Es kamn keinem Zweifel unterliegen, dass Mendel's " Prävalenzgesetz », wie es Correns bezeichnet, für $T$. Blasii in Bezug auf die Färbung im Ganzen zutrifft. Im Einzelnen sind aber die Verhältnisse zu verwickelt, um schon jetzt klar sehen zu können, denn T. Blasii ist ja lien Monohyluil, sondern ein Polyhybrid, da sich die Elternformen in vieler Hinsicht unter'scheiden.

II. Nach Mesder ist es für das Zeugungsprodukt durchaus gleichgültiw, ob das dominierende Nerkmal der Samen- oder dex Pollemptlanze entstammt. Dies trifft, wie erwälnt, allem Ansehein nach boi $T$. Blasit.

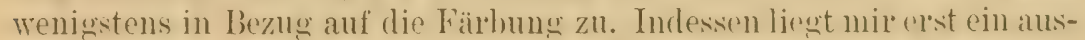
gebildetes, älteres Stück vor, welches $T$. marmoratus zul Mutter, $T$. 
cristatus carnifex zum Vatel hat. Auch hier ist der Rücken wie bei $T$. marmoratus grünlich, der Bauch wie bei $T$. cristatus orangegelb. Bei allen anderen ausgebildeten Bastarden ist T. marmoratus Vater, T. cristatus Nutter. Ich möchte daher mit meinem endgültigen Urteil noch zurückhalten, bis meine disjährigen Bastarde völlig ausgefürbt sind. Jedenfalls überwiegt in der Färbung nach meinen bisherigen Erfahrungen der mütterliche Einfluss n $\mathrm{ich}$ t. Correxs und 'Tschermak sind allerdings, im Gegensatz zu Mexded, der Meinung, dass bei PHanzenhybriden der mütterliche Einfluss üherwiegt.

Fül die übrigen Mexues'schen Regeln fehlt es in meinem Material nicht an Anhaltspunkten, doch sind die Untersuchungen noch nicht zum Abschluss gelangt, da die Züchtung der zweiten Generation der Hybriden noch aussteht. Doch dürften die vorstehenden Ausführungen genügen, um darzutun, dass die "Triton Blasii" oder Bastarde zwischen T. marmoratus und T. cristatus für Arbeiten in diesem Sinne ein ausgezeichnetes Versuchsobjekt darstellen.

Aber nicht nur die Kreuzung von Tierarten, sondern auch jene verschiedener Varietäten oder Rassen würde von Wert sein. Sie wüıde vielleirht für die scheinlar sinnverwirrende Tariation des Farbenkleides mancher Molcharten - ich nenne nur Triton vulgaris - die Aufkläı'ung licfern. Auch hierfür liegen mir bereits manche Anhaltspunkte vor.

\section{Zwergformen der paläarktischen Urodelen.}

Von Dr. W. WOLTERSTORFF (Magdelurg).

Man findet in unseren gemässigten Breiten öfter Lokalitäten, Tümpel und Teiche, in welchen die Molche, seien es nun sinzelne Exemplare oder die Gesamtheit der Individuen, eine besonders stattliche Grösse erreichen. Der Eintluss günstiger Lebensbedingungen ist hiel unabweisbar. Wahrscheinlich wirken hier verschiedene Umstände, als reichliche Nahrung, Tiefe und Tüühle des Wassers, Mangel an natürlichen Foinden, als Raubfischen, zusammen. Dagegen dürfte man meines Erachtens in I) utschland, Frankreich, Oesterreich - mit Ausnahme der südlichsten Gebiete - vergeblich nach $\mathrm{Z}$ we r g for m en suchen. Es fehlt zwar nicht an einzelnen zurückgebliebenen, schwächlichen Individuen, aber diese gehen meist bald im Kampf um das Dasein zu Grunde.

Ander's liegen die Vurhältnisse im Süden. Hier bilden sich, nach meinen 
mehrjährigen Fifahrungen und den Berichten meiner. Korrespondenten zu schliessen, tatsïchlich hin und wieder konstante Zworgformen aus.

Triton palmatus, der interessante westeuropäische Fadenmolch ${ }^{1}$, ist noch in den Pyrenäen sehr häutig und ereecht hior betriichtliche I)imensionen. Aus Nordspanien ist er wiederholt, so von Coruña, bekannt geworden. Im nördlichen Portugal ist er so selten geworden, dass BeDruacia im Gregensatz zu portugiesischen Autoren sein Vorkommen direlit in Abrede stellt. Indessen erhielt ich aus der Ungeloung von Porto seit Jahren unter zahlreichen grossen und starken Triton Boscri regelmässig einige spärliche Fxemplare von Triton pulmutus, welche sich in voller Brunsttracht hefanden, aber durch ihre geringe Grösse auffallend von ihren Stammesgenossen ahweichen. Bei Harhurg nahe Hamburg, de'm nordöstlichsten Fundort in Europa, messen brünstige Mämuchen 70 bis

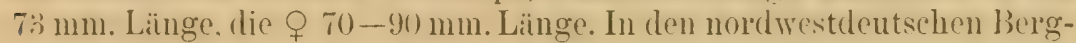
landen sowie in den Pyrenäen erreichen die $\sigma^{x}$ eine Länge von $70-80$, die o eine Länge von 70-92 mm. und mehr, während bei Porto die $\sigma^{x}$ nur ca. $55 \mathrm{~mm}$, die $Q$ nur $60-70 \mathrm{~mm}$. messen. Auch die Färbung ist etwas ahweichend. Bei Porto-Tieren herrscht ein lichter Bronzeton auf eler ()herseite vor. Ich henenne diese ausgesprochene biologische Zwergform bis auf weiteres nach dem Entdeckel' als forma Sequecirai, da es mir noch nicht gelang, diese Rasse in der verworlenen, für mich sehwer zugänglichen portugiesischen Fachlitteratur' wieder' zu finden. Triton yulmotus ist hier, an der Südwestgrenze seines ansgedehnten Verbreitungsbezirkes, wohl unter dem Einfluss der zunehmenden 'Temperatur' zu ein'r' Lummerform geworden, während Triton marmoratus und $T$. Boscui hier frendig gedeihen und häufig sind!

Nach Sequerra's Mitteilungen weist T. palmatus noch an der Nordgr'nze P'ortugals in höheren, kühleren (rebirgsgegenden bedentendere Dimensionen und dunklere Färbung auf. Ich selbst lionstatierte an mehreren der gefangen gehaltenen Tiere von Porto nach Jahr und Tag eine erhebliche Grössenzunahme. So wuchs ein $\sigma^{x}$ von ca. $55 \mathrm{~mm}$. aut ca. 65 mm. an (beide Male in Brunfttracht, mit Schwanzfaden, gemesien). Andere Individuen blieben lifein. schritten aber nichtselestoweniger im zwerten und drittrn Jahre der Gefangensehaft wieder zur Fortptlanzung. Es ist mir wahrscheinlich, dass eine Kolonie dieser Zwergfol'm, in einen Teich etwa der Schweiz oder am Har'zrande verf)tlazt. nach cinigen Generationen zu nolmalen Dimensionen heranwachsen würde.

Auch bei Triton marmoratus, einem del schönsten und grössten Molehe Europas, welcher ganz auf Frankleich und die Prrenäunhalhinsel heschränkt ist, findet sich an der äussersten Gronze srines Vorberitungs-

${ }^{2}$ Vergleiche meinen Aufsatz: T. palmatus bei Harburg. Zool. Anz. 1904. 
bezirkes, in Südspanien, um Cadix und Algeciras, anscheinend eine Zwergform. Es war mir längst aufgefallen, dass die spärlichen Individuen, welehe ich ah und zu von meinen dortigen korrespondenten und Sammlern empting, an Grösse wesentlich hinter ihren Artgenossen in Frankireich und noch in der Nordhälfte Portugals zurückblieben. Ich hielt dir cr'sten Exemplare anfünglich für junge Individuen, musste aber die Beobachtung machen, dass die Wännchen schon bei der geringen Grösse von 95-105 mm. in Brunst traten. So besitze ich seit 4 Jahren ein $\sigma^{x}$ von Cadix, welches 3 Jahre nach einander in Brunst trat, in einem Fall sogar ein $T$. Blasii o erfolgreich befruchtete, ohne im Lauf der Jahre zu wachsen. Es misst jetzt $102 \mathrm{~mm}$. Länge, gegenüber $125-135$ mm. bei normalen geschlechtsreifen $\sigma^{x}$. Fin anderes, frisch importicrtes $\sigma^{\star}$, welches mir im April 1904 zuging, weist jetzt bei $88 \mathrm{~mm}$. Länge bereits alle charakteristischen Merkmale eines $\sigma^{x}$ ausser Brunst auf, insbesondere ist die Rückenfirste, der künftige Kamm, durch schwarze und orangengelbliche Bänderung dentlich gekennzeichnet. Die Oberseite ist saftig gr'ün, mit graubraunen Flecken, welche auf den Flanken zu zackigen Längshinden vertliessen. Zeichnung und Colorit der Oherseite sind daher hei diesem Stück trpiseh. Der Bauch ist auf ursprünglich licht graubriunlichem Grunde stark weisslich geperlt und dunkel gefleckt. Bei einem anderen, im Juni erhaltenen Tiere, einem anscheinend erwachsenen $q$, hetriagt die Lïnge $105 \mathrm{~mm}$., wïhrend alte $Q_{\text {in Frankreich }}$ $130-160 \mathrm{~mm}$. Länge erreichen. Die Oberseite weist auf saftig grünem Grunde joderseits eine Reihe rundlicher schwärzlicher Jarmorttecken auf, welche z. T. mit einander verfliessen. Der Bauch ist auch hier ganz licht, unbestimmt, bräunlich, mit einigen verlowchenen dunkelbraunen Flecken. Beide Exemplare weichen daher höchstens durch hellere Banchfärbung vom 'Typus ab. Andere Stücke waren wieder verschieden gefärbt, doch dürfte es zweckmässig sein, bei der Veränderlichkeit des Colorits bei T. mamoratus und im Hinblick auf die geringe Anzahl ron Individuen, welche ich bisher von Sudspanien erhielt, auf die Färbung noch keine Rücksicht zu nehmen. Lediglich auf Grund der geringen Grösse bezeichne ich die Spielart als T. marmoratus forma pygmæa.

Es muss betont werden, dass der Marmormoleh von der Südspitze Spaniens überhaupt noch nicht sicher nachgewiesen war, während er in Portugal mindestens his Cintra und Lissabon heruntergeht. Die Lebenshedingungen, die klimatischen und hydrographischen Verhältnisse (vielleicht Hitze und Wassermangel ), scheinen seinem Fortkommen ungünstig zu sein. Hiedurch lässt sich sowohl seine Seltenheit - mein Freund in Cadix fing im Lauf der Jahre nur 3 Exemplare, während er von Pleurodeles Wraltlii hunderte Stücke beobachtete - als die geringe Grö̈se erkilären. Weitre Nachforschungen in dem herpetologisch so ungenügend rerforschten Südspanien wärden wohl Kílarheit schatfen. Es ist nicht un- 
möglich, dass $T$. marmoratus schon in den Gebirgslandschaften der Provinzen Cadix und Malaga, sowie an der Sierra Nevada - falls er hier existiert! - betrïchtliche Dimensionen erreicht.

In den südlichsten Teilen Italiens haust ein kileiner Molch, Triton itaficus, welchen Peracea erst vor wenigen Jahren auf einer For'sehungserise entrleckte und im Boll. Mus. Zool. Torino und Soc. Zool. London beschrieb.

Triton italicus ist der kleinste europäische Molch, immerhin erreicht er aber an der Nordgrenze seiner Verbreitung, im Gebiete des Monte Arellino. in Weiluhen his so mm. Länge. I)je Yormallä̈nge der or dürfte 54-65 mm., jene der $\$ 55-74 \mathrm{~mm}$. betragen. Dagegen fand PERACoA bei Leree, nahr dem Meeresstrande, hrünftige Fixemplare von geradezu winzigen Dimensionen, da die $\sigma^{x} \mathrm{im}$ Durchschnitt nur $46^{2} \mathrm{~mm}$., die $ᄋ 50$ mm. Länge erreichten. Wie Peracca vermutet, lässt sich die geringe Grösse an diesem Platze darauf zurückführen, dass die Sümpfe in der heissen Jahneszeit völlig austrocknen, daher dic Larven ihre Entwiclilung und Verwandlung sehr beschleunigen müssen.

Von Interesse ist die 'Tatsache, dass ein $q$ normaler Grösse, welche mil Peracca S. Z. verehrte, binnen 3 Jahren auf $80 \mathrm{~mm}$. Länge heranwuchs, eine Folge der giunstigeren Ernihhungs- und Aufenthaltshedingungen. Sollte die Form von Lecce konstant sein, d. h. an jenem Orte nicht grösser werden, so berechtigen die Grössendifferenzen und biolologischen Unterschiede zur Abgrenzung als eigene For'm.

Eine ganz eigenartige Zwergform, welche bereits Anspruch auf den Rang einer Unterart hat, stellt endlich die for'ma excubitor ${ }^{\prime}$ Wolt. des T. vittatus dar. Auch diese Form kann ich nur mit Vorbehalt in die Tisserschatt einführen, glaube aber. dass die vorliegenden. für mein und

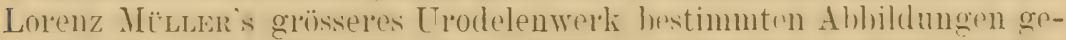

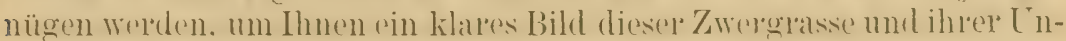
torichiede von der hauliasisch-pontischen Rasse zu gehen. Ich erhiolt die

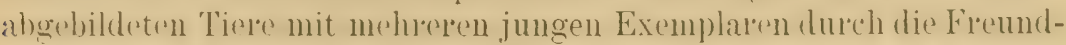
lichkeit der Herrn Hofrat Sterndachner und Dr. Siebenrock aus dem Wiener Hofmusemm leihweise zur Lntersuchung. Inas or unterscheidet sich ausser durch die geringe Grösse - die Länge beträgt in Wirklichkeit nur $85 \mathrm{~mm}$., da die Abbildung etwas vergrössert ist - durch den niederen, schwach gekerbten Rückenkamm, die kürzeren Finger und Zohen, die anscheinend schwäthere entwickelte merkwärdige Tarsalfalte von der grosien Rasse des Kankasus und des Olymp bei brussia bei welcher das ơ 125-144 mm. Länge erreicht. Leider gelang es mir noch

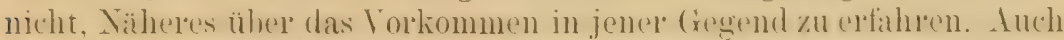

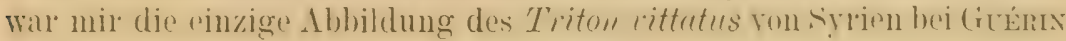

$1=$ vorgeschoben, auf Aussenposten befindlich. 
Méxevilue. Jenn. Règne Animal, p. 17, pl. 28 , fig. 2, bisher nicht zugänglich. Im Norden Syriens, so bei 'Tripolis, erreicht Triton vittatus bedeutendere Ininensionen und besitzt einen höheren gezackten Kamm. Jextenfalls macht das ahgehildete or den Findruck eines völlig erwachsenen Tieres, nach der stallk geschwollenen Kloake zu schliessen. Fundorte der forma ferculitor sind Nashre el Kihebir und Trabulor, Antilibanon.

Alle bishner angreführten Iolche gehörten der Gattung Triton im enger’en Simn án. Aber auch in der Untergattung Pleurodeles, deren typische Tertreter der" allbckannte Triton (Pleurodeles) Waltlii ist, findet sich eine auffallende Zwergform. Sie wurde bereits 1881 von F. Lataste beschrieben und als sellständige Art, Triton Hagemmielleri, bezeichnet. Ihr Torkommen beschränkt sich, soweit der Typus in Frage kommt, auf Bône. Individuen anderer Fundorte, wie von Biskra - mir liegt ein Exemplar des Sinclinberg-Museums in Frankfurt vor - und von Bougie weisen Lehergänge zu Pleurodeles Poireti auf. Wir hahen hier ganz das gleiche Verhältnis wie bei den anderen Zwergformen: $P$. Hagenmiilleri stellt mit $100 \mathrm{~mm}$. Länge die Zwergrasse von $P$. Poireti, welche 140-160 mm. misst, dar. Hierzu treten aber andere Unterschiede. Von $P$. Poireti liegen mir zur Zeit mehrere schöne und lebende Tiere, alte und junge, von Philippeville und 'Tunis vor, welche sich von $P$. Walttii schon durch die geringe Aushildung der Rippenfortsaitze und dementsprechend das Fohlen der orangerötlichen Rippenflecken deutlich unterscheiden ', von der verschiedenen Grösse. der' abweichenden Färbung der Augen und anderen, weniger auffallenden Merkmalen abgesehen.

Pleurodeles Hagemmïlleri unterscheidet sich von P. Poireti abermals durch geringere Grösse, ferner aber durch gestreckteren Kopf, halbelliptischen Lmriss der Kiefer, durch längere, zartere Finger und Zehen. Leider konnte ich diese Form noch nicht lebend untersuchen. Indessen lagen mir zahlreiche typische Exemplare von Bône aus dem Senckenhergianum und dem Baseler Yuseum vor. Ein direkter Vergleich mit jungen, halhjialnrigen $P$. Poireti, welche ganz das gleiche Volumen und Gewicht besitzen, bewies die völlige Verschiedenheit. Die kleinen $P$. Poireti sind durch hreiten Kopf, kur\%e, gedrungene Finger und Zehen, sowie den kurzen Schwanz - letzteres nur Jugendmerkmal - sofort zu unterscherden. Da diese ['nterschiede aber nicht bedentender sind, als sie bei versehiedenen Unterarten derselben Art - vergleiche T. vittcttus! - auch vorkommen, so möchte ich die Zwergform von Bône nach dem äusseren Befund nur als Unterart des P. Poireti betrachten².

\footnotetext{
' Nur bei einzelnen Stïcken beobachtete ich Spuren ron Rippentlecken.

"Auch die Anordnung der Gaumenzähne weicht ab. Diese ist aber auch bei anderen Molchen, so Salamandra maculosa, variabel.
} 
Ist diese Annahme aber richtig, so bleibt noch die schwierige Frage zu lösen, wie diese Zwergform innerhalb des Wohngebietes des P. Poireli zu Stande kam. Bône liegt keineswegs kimatisch oder hydrogratphisch ungünstiger als Tunis und Philippeville. I)ic Molche von Tunis sind allerdings in der Fürbung etwas alwwichend von der Algier-Form, stimmen aber gerade in der Grösse völlig überein. So erwachsen dem Forscher stets neue Räthsel, die, wenn überhaupt, nur durch die rïhrige ['nterstützung jener Zoologen, welne Algier lowohnen oder bereisen, gelöst werden können. Nicht nur das Sammeln, sondern auch das Beobachten, die Aufzucht der Larven und jungen 'Tiere sind hier zu berücksichtigen. Leider ist aus Bône seit vielen Jahren kein einziger

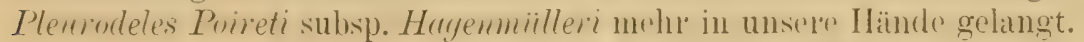

Sehen wir von diesem unaufgeklärten Fall ab, so dürften an der Ausbildung der "Z/Worgformen » der paläarktischen Region im Süulen wohl verschiedene Unstiinde die Sehuld tragen, hald hohe Jahrostemperatur, bald Wassermangel, Futtermangel orler I) generation infolge Isolierung in den Grenzgebieten. Oefter wird alles zusammenwirken.

Ireine Beolatehtungen an gezüchteten Tieren hewejosen, dass es tatsiichlich möglich ist, junge Molche mit odel ohne Alsicht im Warhstum hetrïchtlich zu hemmen. Indessen lassen sich diese Beobachtungen nicht ohne weiteres auf das Freileben übertragen, da diese "Zwerge » zwar oft jahrelang hin regetieren, aber nicht zur (reschlechtsleife gelangen. Jedenfalls spielen inmere, zehrende Krankheiten. Störmugen des Organismus, vielleicht auch Parasiten, hier eine grosse Rolle. Die Untersuchungen über diese Frage sind noch nicht zum Abschluss gelangt. Indss gezüuhtete Aquarientische. inshesondere Exoten, in der Gefangenschaft leicht degenerieren und klein bleiben, dabei aber fortpthanzungsfähig werden, ist ja eine bekannte Tatsache.

Man darf aber nun nicht ohne weiteres annehmen, dass a Ile Molche nach Süden an Grösse abnehmen. In Gegenteil erreicht z. B. Salamandra maculosa, der Feuersalamander, in Kleinasien und Syrien oft die beträchtliche Grösse von 30-32 cm., selbst an Orten, wie Berg Karmel bei Haiffa, wo die Höhe über dem Meer nur ca. $170 \mathrm{~m}$. beträgt ${ }^{`}$. Auch in Algier wird el in der var. algira bis $22 \mathrm{~cm}$. lang, bleibt also kaum hinter dem Durchschnittsmass in Zentraleuropa zurück.

\footnotetext{
${ }^{1}$ Ein riesiges Stück von Haiffi verdanke ich der Freundlichkeit des Herrn Lehrer Fr. LANGE.
} 


\section{Les Clupéidées de la Mer Caspienne.}

Par MI. I3ORODINE (St-Pétersbourg').

Arec 1 planche, 1 tableau et 10 figures dans le texte.

Les Clupéidées de la Mer Caspienne ont une grande importance au point de vue de l'industrie poissonnière en Russie; on les pêche par centaines de millions. La pêche principale a lieu à l'embouchure du Tolga, où les Clupéidées remontaient autrefois en bandes innombrables.

Cependant, on y a constaté très nettement, pendant la dernière douzaine d'annés, une diminution graduelle de la quantité de ces Poissons (voir le diagramme). Fin même temps, on pourrait signaler un accroissement considérable de la péche des CIupéidérs dans la Mer Caspienne même, et assez loin du Volga.

Il était important de savoir s'il y a quelque relation entre ces deux faits, si les Clupéidées pêchées dans la mer sont de lạ même espèce que celles du Volga, si les Clupéidés caspiennes peuvent frayer dans la mer. Si tel est le cas, on peut se demander où a lieu la ponte des différentes espèces, où r'estent leurs alevins, enfin - question principale - quelle est l'apparence des a’ufs ot des alevins des Clupéidées caspiennes et quel est le moyen de les distinguer de ceux des espèces différentes ${ }^{1}$.

Il sera facile de comprendre l'importance considérable des études indiquées, si l'on remarque que l'on connaît déjà, dans la Mer' Caspienne, 4 espèces de Clupea, auxquelles, pour le moment, on peut ajouter encore une nouvelle espèce et 3 variétés faciles à distinguer et que, au point de vue biologique, elles sont toutes différentes.

Cés considerations expliquent lintérêt tout ì fait spécial des études sur l'histoire naturelle des Clupéidées de la Mer Caspienne, ainsi que mes recherches personnelles daus cette direction. Je vais exposer', dans cette communication, quelques r'ésultats de ces recherches.

L'illustre ichtyologue l'usse Kessider a décrit 3 espèces du gomle Clupere habitant le hassin de la Mer Caspienne: Clupea enspia Eichw., Clupera pontica Eichw. et Clupea delicutula Tordm. En parlant de la Clupea poutice (qui, d'apres lui, habite principalement la Mer Noire (Pontus), il urentionne pour la Mer Caspienne une forme spéciale (forma cospru), caractérisée par un museau plus olstus et par un nombre moindre d'épines hranchiales (25 à 28 au linu de 35 à 55 qu'il indique pour la Clupea poutica de la Mer Noire. Kesslen trouvait que cette forme est celle qui ressemble le plus à la Finte (Clupea finta)².

1 Il faut dire que personne ne les a vus jusqu'à cette année-ci.

2 IÍEssler. Poissons des Mers Caspienne et Noire (russe), pp. 8t, 116. 1874. 


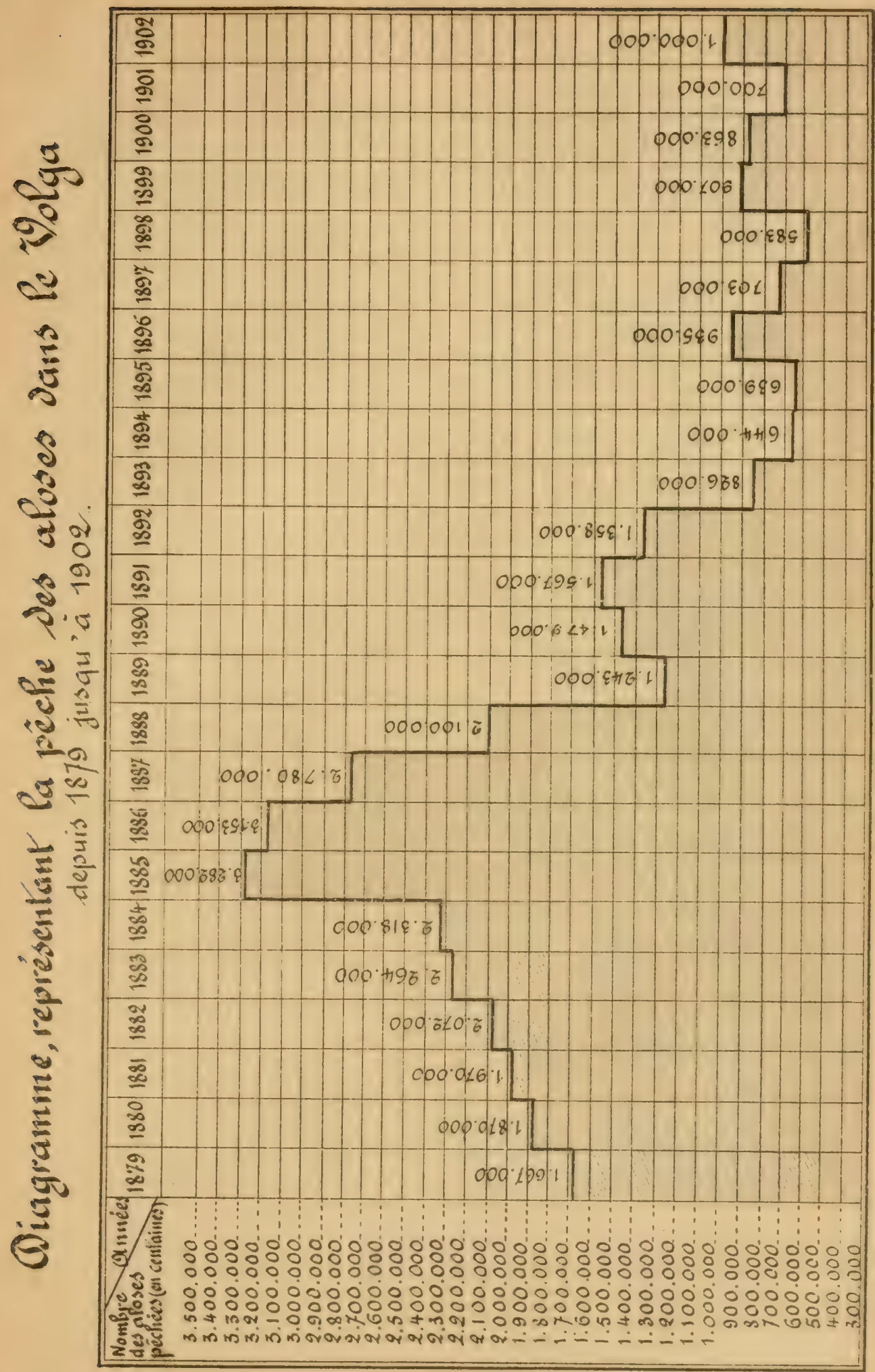



Un autre ichtyologue l'usse, le Dr Grnn, dans un travail très peu connu des zoologistes intitulé "Hareng d'Astraklan ", et publié dans le journal officiel "Economie r'urale et sylviculture " en 1886, a donné la description do deux nouvelles espeees de Clupéidées caspiennes: Cluper Késsleri Gr. et Clupea Suposchnikowii. Cet auteur est d'avis que Kessler a pris 3 formes (C. Kessleri, C. Saposchmikowii et $C$. pontica) pour la même espèce.

Comme Kessian le $\mathrm{D}^{\mathrm{r}}$ Grman a fondé la distinction de ces espèces sur le nombre desépines branchiales, or qui est en offet caractéristique pour les Aloses. nou semlement au point de vue purement morploologigue, mais anssi au point de vue biologique, parce que, de la construction des ares branchiaux, dépend, entre autres, la nourriture du Poisson, c'est-à-dire le genre d'animaux qu'il peut captur'er' et manger. Ainsi, par exemple, Clupea caspia, chez laquelle on trouve plus d'une centaine d'épines branchiales, qui sont fines ot délicates. sesert de cet appareil comme d'un filtre pour retenir les animaux minuscules de l'eau (des petits Copépodes par exemple). Au contraire, ces petits animaux ne peuvent pas devenir la proie de la Clupe Kessleri, qui n'a que 60 à 80 épines branchiales, c'està-dire deux fois moins.

On constate la même chose pour la Chupea Saposchnikowii Gr., qui n'a que 30 à 40 épines branchiales. Les deux dernières espèces ayant parfois la même taille que la Clupea caspia se nourrissent déjà de Poissons.

Le Dr Grims dit que chez la Clupea Kessleri le nombre des épines branchiales est de 60 à 80 et qu'il y a en outre une série d'autres traits

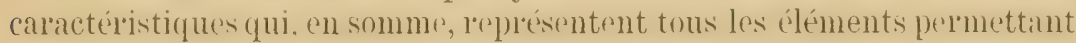
de distinguer une vraie nova species pour le bassin de la Mer Caspienne.

Une autre espèce, Clupea Saposchnitiowii, d'après la description du $\mathrm{D}^{\mathrm{r}}$ Grum, est caractérisée par le nombre desépines branchiales (30) a 4.3), par des dents beaucoup plus développées que chez la Chupea Kesslev $i$ et par une forme du corps différente. Tous les exemplaires de cette espèce du $D^{r}$ Grinm étaient de petite taille (au maximum $248 \mathrm{~mm}$.), mais cet auteur a trouvé parmi eux des exemplaires sans doute adultes. Cette espèce ne se trouvait qu'à l'embouchure même du Volga; elle ne r'emonte pas la r'ivière comme le font deux autres espèces, Clupea Kessleri Gr. et Clupea caspia Eichw.

En 1898, M. Braschstkow a l'marqué que, dans la Mel Caspiemne mime. se trouve une espèco d'Alose qui ressemble heaucoup a la Cluper suposchnikowii Gr., mais d'une taille beaucoup plus grande et avec un nombre moindre d'épines branchiales (24 à 28).

En 1900, le Dr Lönnberg, de Suède, a visité la Mer Caspienne pour des études ichtyologiques. En parlant des (lupéiclées caspiennes. il exprime l'opinion que l'Alose que M. Braschnikow a trouvée dans la mer même

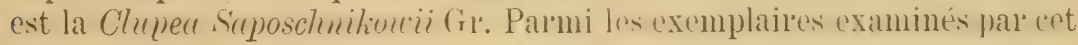


auteur, se trouvaient deux formes: une avec de gros yeux, ayant le colps plus large et l'autre avec des yeux plus petits et le corps plus allongé.

Les auteurs cités ne donnent pas de solution à la question de la relation de cette forme, qui est sans doute marine, avec la Clupea pontica de la Mer Toire. Cependant, si l'on considere l'historre géologique de ces deux bassins voisins, autrefois unis, on ne peut pas supposer qu'il n'y ait pas un lien de parenté entre la forme pontique et la forme caspienne. (I) pourrait dire " priori que Kessien avait raison d'étahlir une forme (aipiemne de la Chupeu pontica, puisque c'est dans la Mer Noire qu'il faut chercher les ancêtres des formes caspiennes.

Il n'est pas douteux que la forme marine établie par M. Braschnikow soit la même que celle que Kessten décrivit comme une forme caspienne de la Clupea pontica.

J'ai eu l'occasion, pendant les deux dernières années, de faire une assez grande collection de Clupéidées de toutes les cotes de la Mer Caspienne et de les examiner de plus près, et je suis arrivé à la conclusion que la forme caspienne de la Clupea pontica est devenue - comme on pouvait le deviner d'avance - après un laps de temps assez long, une espèce distincte, représentée par plus de :3 formes ou variétés locales. La description de cette nouvelle espèce et de ses formes sera donnée plus loin.

Fe domne à l'espece le nom de C'lupen cospio-pontica, puisque c'est la seule forme commune à la Mel Caspienne et à la Mer Noire, où le $\mathrm{D}^{\mathrm{r}}$ Grimm a constaté ausi :) formes paralleles aux tormes caspiennes, savoir C'huper Eichwaldi Gr., Clripea tanaica Gr. et Clupea maeotica Gr., dont la dernière est uno forme marine possédant des caractères semblables à ceux de la forme marine des Clupéidées caspiennes.

Done, cette forme coimmune aux deux mers voisines, autrefois unies, s'appelle Clupea caspio-jontica. A mon avis, il faut placer cette espèce plus près du Hareng, Poisson marin - tandis que deux autres espèces Cluper Lessleri Gr. et Clupea crispia sont de vraies Aloses. Poissons habitant plutôt l'eau douce.

\section{CLUPEA CASPIO-PONTIGA mihi.}

(Fig. 2) ${ }^{3}$.

Syn.: C. pontica Eichwald (ex parte). Bull. des natur. de Moscou, 1838, XI, p. 133. - Funn cuspio-canc, p. 204, tab. XXXII, fig. 2. - Nondus, Faune pomlique (ex parte), p. 5̃20, pl. 20̈, fig. 2. - Güsther, Calaloque of fishes, etc., VII, 1. 4 4 -4.19 (ex parte).

C. pontica Eichw. forma cuspia Kessl. - Kessuen, Poissons des mers Noire et Cirspienne, p. 116.

2 Afin de faciliter la comparaison nous donnons les photographies des trois espèces principales des Clupéidées caspiennes. Les Poissons étaient à peu près de même taille, $150 \mathrm{~mm}$. (fig. 1 à 3 ). 
C. Saposchniliowii Gr., Grimm, Hareng d'Astrakhan. - Braschnowow, Sur la systematique des Clupées caspiemnes. Mess. des pècheries, 1898, p 231. LüxxBEIG, Contribution to the ichtyolog!! of the Caspinu seu. K. Svensea Vet. Akard. Handlingar, Bd. 26, Afd. IV. No 8.

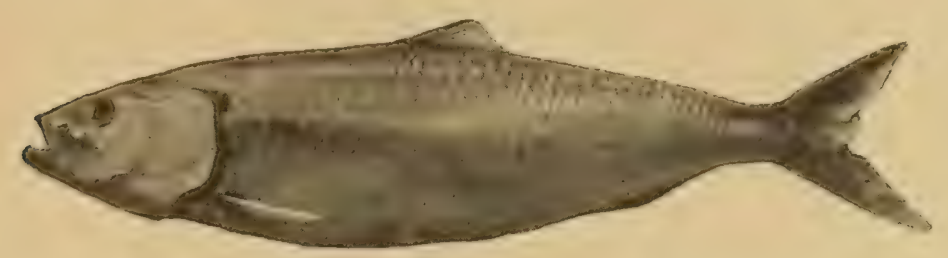

Fig. 1. Chuper crespia Eichw. P

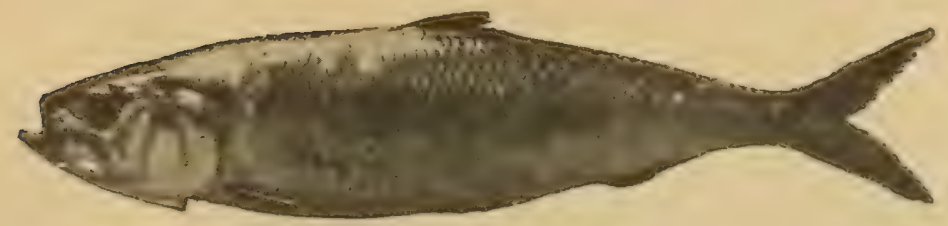

Fing. 2. Clupea caspro-pontica m. C

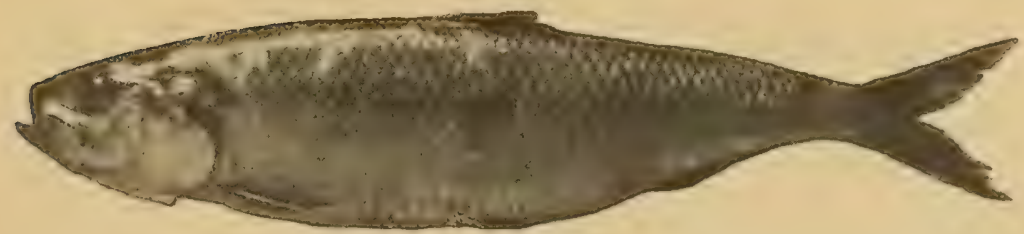

Fig. 3. Chupea Kessleri Gr. $\mathrm{O}$

Pect. I/13-16. Dor's. III/13-15. Ventr. I/S. An. IV/17-18. Caud. V/17/V.

Car a c tè r'es. Il y a, sur le premier arce branchial, 20 à 40 épines plates, osveuses et d'une forme recourhér. Les dents sont fort dévelopjuées ot girlnissent les deux mâchoires, le vomer et la langue. Les yeux sont gros et placés au $1 \frac{1}{3}$ de la distance entre l'extrémité de la houche et le lonpl postérieur de l'opercule, plus près de la partie antérieure de la tête. La coloration du corps 'st blanche. argentée, d'un retlet vert métallique sur le dos. La tête et les nageoires sont pâles, privés de pigment. La taille atteint $448 \mathrm{~mm}$. 
Description. Le corps est assez bien proportionné, plutôt allongé, car la moyenne de sa plus grande largeur est le $22 \%$ de sa longueur. La tête est allongée (sa longueur est un peu plus grande que la plus grande hauteur du corps et fait $1 / 4$ de la longueur totale du corps) et basse (sa hauteur est le $65 \%$ de la longueur, ce qui rend la tête du Hareng pointue). L'avant-œil est deux fois moins grand que l'arrière-œil qui fait à peru pres le $12^{\prime \prime}$ o de la longueur totale. Les yeux sont éloignés vers le bord anterieur de la tête: ils sont assez grands $(4 \vdots 5 \%$ de la longueur totale du ('01'p) (et domnent au Poisson un aspect rapace. L'iris est de couleur blanche-argentée, tirant un peu ver's le jaune ${ }^{1}$. La mâchoire inférieure est saillante. L'opercule est arrondi et strié; les rayons sont faiblement marqués. Il y a presque toujour's, derrière l'opercule, sur le corps, une tache noire, qui ne se trouve jamais sur l'opercule même. On rencontre, par exception, des exemplaires avec une rangée de taches sur le corps (fig. 4).

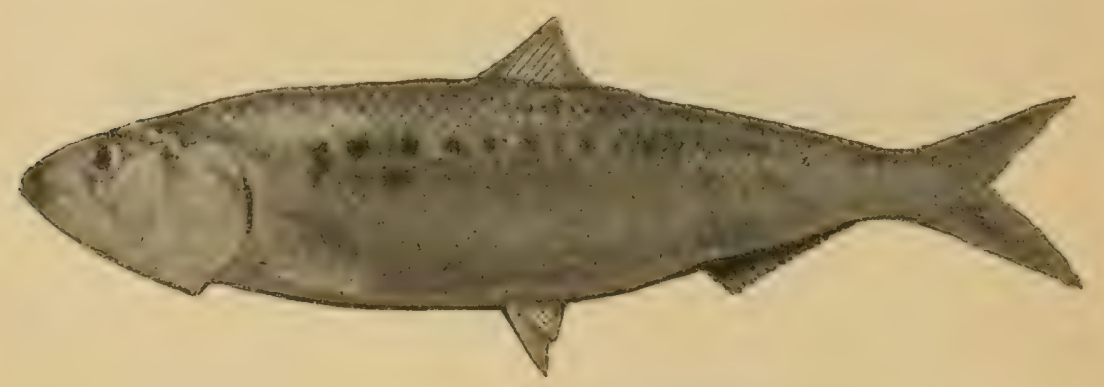

Fig. 4. Chupea caspio-pontica, var Braschnikowi, m.

Leś dents sont développées et garnissent la mâchoire supérieure et inférieure, le vomer et les os palatins. Les épines branchiales sont assez espacées; il y en a de 20 à 35 . Les épines sont courtes, unies et un peu recourbées aux extrémités. La mâchoire supérieure a une échancrure visilıle au milieu: la mâthoire inféricure a l'air d'avoir été coupée par devant, ce qui donne au museau un aspect un peu obtus. L'extrémité postérieure mohile dépasse la verticale du hored postérieur de l'œil. Ecailles caduques. Le Poisson vivant est d'une couleur vert foncé (la couleur de l'eau de la Mer Caspienne) sur le dos et d'un blanc vif argenté sur les flanes et le ventre. La tête est toujour's d'une couleur blanchâtre claire, mais jamais foncée nu noire, comme cest le cas pour les C. cospru Eichw. et $C$. Kessleri Gr. L.es nageoires, près de leurs bases, sont un peu jaunâtres: en qénéral elles sont pâles. ce qui frappe l'xil. La tige de la queue

${ }^{1}$ C'est cette coloration de l'iris qui distingue la $C$. caspia de la $C$. Kessleri, dont l'iris est rougeittre. Chez ces dernières, en outre, la pupille est plus grande. 
est relativement plus mince (sa hauteul n'atteint pas plus de $7,5 \%$ et très rarement $8 \%$ ) que chez les autres especes des Clupéidées caspiennes.

L'exemplaire le plus grand qu'on ait vu avait $448 \mathrm{~mm}$., les plus petits exemplailes adultes avaient de 230 à $240 \mathrm{~mm}$.

On trouve cette espèce, durant toutes les saisons, le long des côtes orientales et méridionales de la Mer Caspienne; la partie du Nord de la mer (près du For't Alexandrovsk) n'est visitée par cette espèce qu'au printemps.

L'examen des exemplaires de cette espèce, provenant de Petrovsk, du Fort Alexandrovsk, de la baie de Krasnovodsk ot du golfe d'Astrabad, a prouvé que le nombre des épines branchiales de ce Poisson diminue graduellement du Nord ver's le Sud. Le Hareng d'Astrakhan n'en a que 20 et quelquefois même 18. Le Hareng du Fort Alexandrorsk et de Pétrovsk a 28 épines et une variété de ce Poisson, découverte par le $\mathrm{D}^{\mathrm{r}}$ Grim, en a 32 et même 43 . On peut donc admettre qu'il existe des formes locales de la même espèce dans lés différentes parties do la Mer Caspiennes, fait qui coïncide entièrement avec les résultats de l'étude fondamentale du $\mathrm{D}^{\mathrm{r}}$ Heincke ${ }^{1}$ sur les Harengs des mer's du Nord. Ces formes ou variétés sont caractérisées pal les traits molphologiques et hiologiques suivants:

1. Forme du Nord. La C. Saposchnikowii Gr. se distingue par sa petite taille (max. $248 \mathrm{~mm}$.), par un corps relativement plus large, une grande tète, des yeux d'un plus grand diamètre et placés plus cn arrière et prin(cipalement par un plus grand nombre d'épines branchiales (la moyenne du nombre $=35$ ). On trouve ce Hareng près de l'embouchure du Volga. On en trouve aussi sur les côtes ouest de la Mer Caspienne.

2. La forme de la zone médiale de la Mer Caspienne ou de Manguischlak, var. Biaschniliowii, est caractérisée par une quantité moyenue d'épines = 27, par un corps plus allongé, par une plus grande taille (jusqu à $390 \mathrm{~mm}$. d'après Braschnikow et jusqu'à $448 \mathrm{~mm}$. d'après mes données) et par une tête plus longue et plus pointue. Ce Hareng a servi de modèle à Liessier pour établir la forma caspia de la Clupea puntica Eichw. (roir cidessus). Cette forme se trouve tout le long de la côte orientale de la Mer Caspienne depuis les îles Dolguy jusqu’à la ville de Krasnovodsk et se rencontre en exemplaires séparés sur la cote occidentale depuis Pétrovsk jusqu'à Astara. Il fraye au mois d'avril, en mer, sans entrer dans les rivières.

3. La forme de la zone du Sud, que nous appellerons var. Grimmi, se distingue par le plus petit nombre d'épines, de 18 à 25 (la moyenne

${ }^{1}$ F. Heincke. Naturgeschichte des Herings. 1. Theil. Die Lokalformen und die Wanderungen des Herings in den Europäischen Meeren, Abhandl. Deutsch. Seefischerei-Vereins, 1898, 2. Bd. Ưn rapport détaillé, en langue russe, concernant cet ouvrage a été publié dans le "Messager des pêcheries " 1900, p. 28. 
$=2(0)$ et par une tete relativement plus petite, des yeux moins gros et particulierement par Io maxillaire inférieur plus court. Elle habite durant toutr lannéc le Sud de la Mer Caspienne où elle fraye aux mois de mar's et d'avril, dans les environs de la baje d'Astrabad.

J'ai constaté, au printemps de 1903 , sur place, les trois variétés de la Cluper corpio-pontica indiquées ci-dessus, après avoir mesaré des exemplail's frais, pris durant un court espace de temps dans différentes parties de la Mor Caspiemne. Tous ces exemplaires, conservés dans de la folmaline. ont été mesurés de nouveau systematiquement par M. Socronow et eroupés d'après leur provenance. Les r'ésultats de ces mensurations sont donnés dans les tableaux I à IV ci-joints.

Les chiffies moyens des caracteres les plus importants (la longueur de la tête, la hauteur du corps, la longueur de l'arrière-cil, le diametre de l'œil, la longueur de l'os maxillaire inférieur et le nombre des épines branchiales) réduits pour les différentes localités en ó prouvent clairement l'existence réelle des variétés de la Clupea caspio-ponticu que nous avons citées ci-dessus.

Afin de pouvoir comparer avec cette espèce une espèce analogue du hassin de la Mer Caspienne. nous indiquons sur le tableau V les mensurations de trois exemplaires de la C. mexotica Gr., espèce décrite par lo I) (x́mim pour la Mer d'Azow. Il est hien difficile de la distinguel de la Cluper caspio-pontica et je me permets de la compter pour une variété de l'espèce commune à la Mer Caspienne et à la Mer Noire, et propre à la Mer d'Azow. Par la longueur de la tête. elle se rapproche de la variété Grimmi, propre au Sud, st à tête courtr; d'après la largeur du corps, elle se rapproche de la var. Sroposchritionvii, prople au Nord; d'apries le nombro des épines hranchiales. cllo se rapproche de la variété Braschnitiorii, propre au Manguischlak (voir tableaux $\mathrm{I}$ à V).

Au point de vue biologique. la Cluper caspio-pontica m. se distingue beaucoup des deux autres esipeces de Clupéidées de la Mer Caspiemne. Tout d'ahord, cette espècer, à l'exeeption de la variété du Nord. (var. Saposchmilionii) n'entre pas dans les rivières; elle habite surtout la côte orientale de la Mer Caspienne, à partir des îles Dolguy jusqu'ì la baie d'Astrabad et lo long de la côte persanne jusqu'à Astara. Flle apparaît dans la partie méridionale de la baie d'Astrabad. prese des cotes. au mois de févirier, y reste en grande quantité jusqu'au 20 avril et fraye dul'ant les mois de mal's et avril, comme j'ai eu l'occasion de m'en assurel' persomnellement. près de l'embouchure de la rivière Gurguène, où j'ai trouvé des femelles de ce Poisson avec des œufs tout à fait mûrs, ainsi que des larves $(17 \mathrm{~mm}$.) et des alevins assez développés (20 à $26 \mathrm{~mm}$.). ("est en "xaminant ces dernier's. qu'il a été possible. il l'aide d'une loupe, on comptimt les épines branchiales, de démontlere que ce sont los alevins de C. caspio-pontica (voir fig. 5). 
Je cite ici les résultats des mensurations d'un alevin (un des plus grand) examiné à l'aide d'une loupe: Longueur générale du corps: $22,5 \mathrm{~mm}$., longueur de la tête: $3,5(16 \%)$, hauteur maximum du corps:

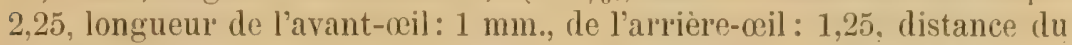
bout du museau jusqu'à la dorsale: 17,25 , même distance jusqu'à la ventrale: 17. Les épines branchiales sont au nombre de 32 à 35 . Le corps a une teinte blanche argentée; les petits sont à demi transparents. On

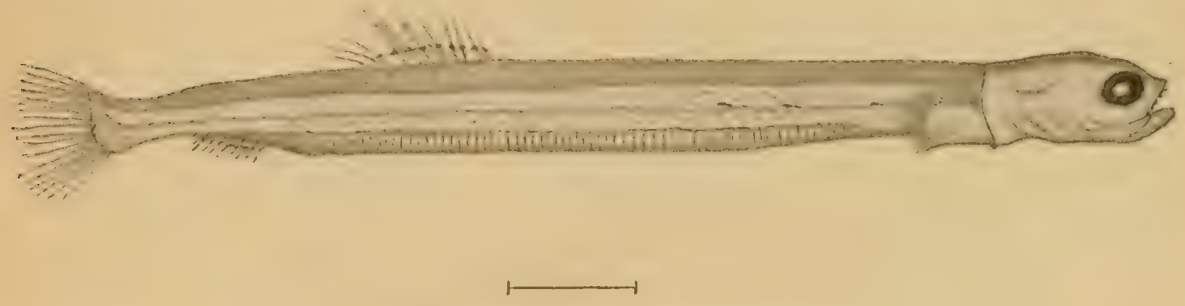

Fig. 5. Larve de la C. caspio-pontica.

peut voir quelques étoiles de pigment sur le ventre et sur le dos. L'iris est d'un éclat argenté et la pupille noire du Poisson vivant attire avant tout l'attention. Lanale commence vis-i-vis de la dorsale; la ventrale est située avant la dorsale. Les plus grands exemplaires mesurent 24 à $25 \mathrm{~mm}$. (jusqu'ì $28 \mathrm{~mm}$.), la longueur de la tète étant de $4,25 \mathrm{~mm}$.; les plus petits de cette collection de 1903 avaient $17 \mathrm{~mm}$. Le corps est tout à fait nu; on voit parfaitement les chevrons musculaires. Dans la partie ventrale, on voit bien la membrane d'une couleur argentée recouvrant l'abdomen à l'intérieur. Dors. I/11-15. Caud. II/18-20-21. Chez les quatre autres exemplaires. j'ai trouvé les nombres suivants dépines branchiales
1) $22+10=32$
2) $17+7=24$
3) $18+8=26$
4) $19+10=29$

L'âge des plus grands alevins, d'après les données sur' la taille des alevins du shad américain, ne doit pas être moins d'un mois, de sorte que Ir conmencement de la ponte de cette Clupéidée doit tomber sur te dernier tiers du mois de mars. Mais, ell même temps, j'ai trouvé ces larves à l'âge de 1 à 2 semaines en forme de fils, sans nageoires formées (17 mm.). Tout cela, et le fait qu'on a pêcché en même temps des exemplaires avec des œufs mûr's, prouve que le temps de la ponte, même avec des conditions analogues de température et an méme emdroit. dure chez

1 Le plus petit exemplaire. 


\section{Tableau I. Clupea caspio-pontica}

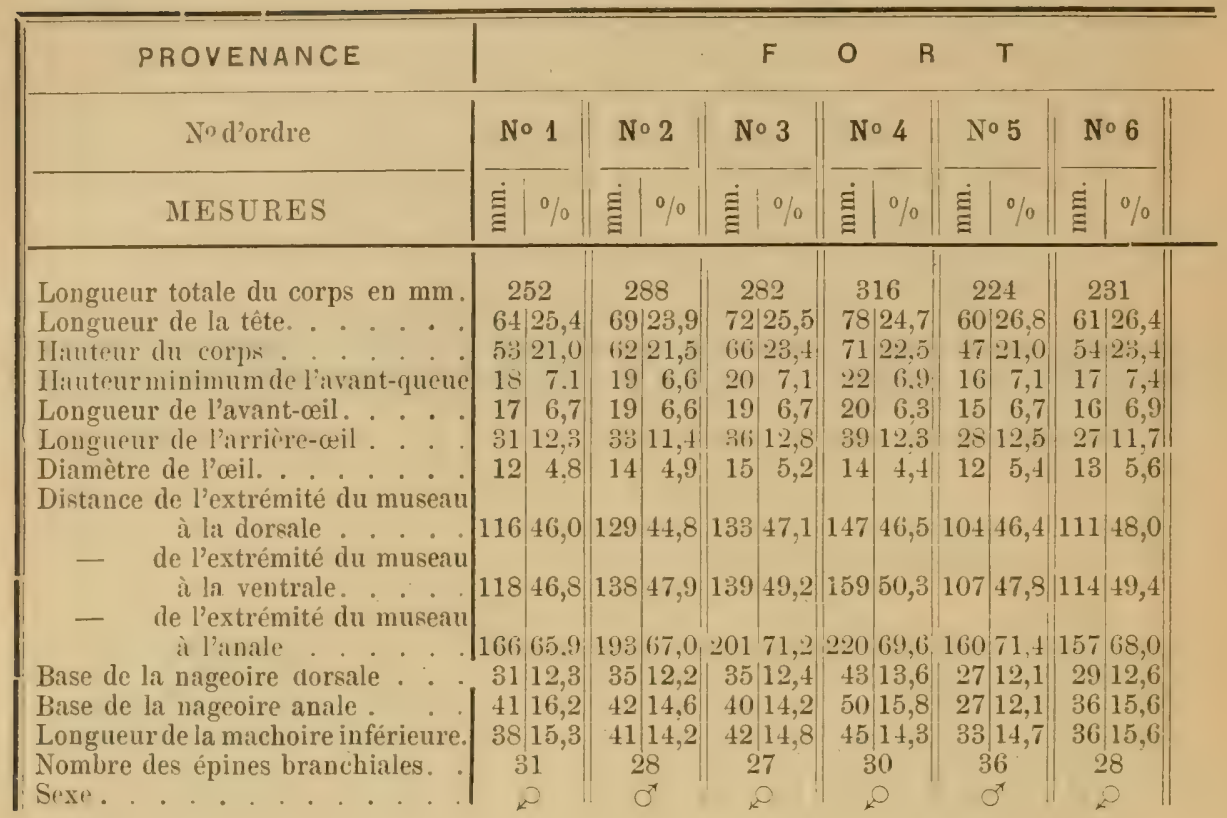

Tableau II. Clupea caspio-pontica var.

\begin{tabular}{|c|c|c|c|c|}
\hline PROVENANCE & \multicolumn{4}{|c|}{ DERBENT } \\
\hline No d'ordre & \multicolumn{2}{|c|}{ No 16} & \multicolumn{2}{|c|}{ No 17} \\
\hline MESURES & $\mathrm{mm}$. & $\%$ & $\mathrm{~mm}$. & $\%$ \\
\hline 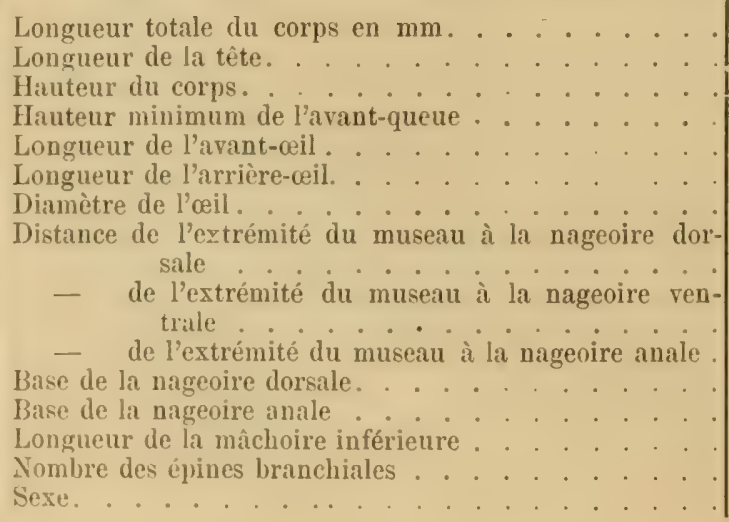 & $\begin{array}{r}14 \\
3 \\
37 \\
12 \\
9 \\
17 \\
11 \\
68 \\
69 \\
69 \\
17 \\
23 \\
23 \\
4 \\
4\end{array}$ & $\begin{array}{r}5 \\
26,2 \\
25,3 \\
8,2 \\
6,2 \\
11,7 \\
7,6 \\
46,9 \\
47,6 \\
66,9 \\
11.7 \\
15,9 \\
15,9\end{array}$ & $\begin{array}{r}1 \\
43 \\
37 \\
12 \\
10 \\
19 \\
12 \\
76 \\
79 \\
110 \\
19 \\
27 \\
27 \\
3\end{array}$ & $\begin{array}{r}5 \\
26,1 \\
22,4 \\
7,3 \\
6,1 \\
11,5 \\
7.3 \\
\\
46,1 \\
\\
47,9 \\
66,7 \\
11.5 \\
16,4 \\
16,4\end{array}$ \\
\hline
\end{tabular}




\section{var. Braschnikowi m.}

\begin{tabular}{|c|c|c|c|c|c|c|c|c|c|c|}
\hline & & A L & $E X A$ & $A N D$ & R O & $V \mathrm{~S} \mathrm{~K}$ & & & & \\
\hline \multicolumn{2}{|c|}{$N \circ 7$} & $N \circ 8$ & $N \circ 9$ & No 10 & No 11 & No 12 & No 13 & No 14 & No 15 & \multirow{2}{*}{$\begin{array}{l}\text { Chitties } \\
\text { moyens }\end{array}$} \\
\hline$\dot{\Xi}$ & $\begin{array}{ll}0 & \\
0 & 0 \\
\end{array}$ & 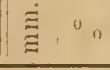 & 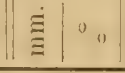 & $\dot{\Xi} \equiv$ & छ் & घ̇ $|\%|$ & $\dot{\Xi} \mid \%$ & 离 $|\%|$ & $\dot{\Xi} \mid \%$ & \\
\hline \multicolumn{2}{|c|}{268} & 218 & 210 & 215 & \multirow{2}{*}{\begin{tabular}{|c|}
190 \\
$53 \mid 27,9$ \\
4021
\end{tabular}} & li 208 & 235 | & \multirow{2}{*}{$\begin{array}{c}266 \\
68 \mid 25,6\end{array}$} & \multirow{2}{*}{$\begin{array}{c}275 \\
70 \mid 25,5\end{array}$} & \multirow[b]{2}{*}{25,7} \\
\hline \multicolumn{2}{|c|}{$65|24,6| \mid$} & $56|25,7|$ & 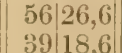 & \begin{tabular}{|l|}
$58 \mid 26,9$ \\
\end{tabular} & & \begin{tabular}{ll|l}
52 & 25,0
\end{tabular} & \begin{tabular}{|l|l|}
60 & 25,5 \\
& 53
\end{tabular} & & & \\
\hline $\begin{array}{l}50 \\
19\end{array}$ & $\begin{array}{r}20,5 \\
7.1\end{array}$ & $\begin{array}{l}47 \mid 21,6 \\
16\end{array}$ & & \begin{tabular}{|r|r|r|}
42 & 19,5 \\
14 & 6,5
\end{tabular} & \begin{tabular}{rr|r|}
40 & 21,1 \\
13 & 68
\end{tabular} & $\begin{array}{rr}52 & 25,0 \\
15 & 7,2\end{array}$ & \begin{tabular}{|r|r|r|r|r|}
53 & 22,6 \\
16 & 6,8
\end{tabular} & 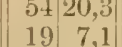 & \begin{tabular}{|l|l|}
20 & 7,3
\end{tabular} & 21,6 \\
\hline 17 & 6,3 & 146,4 & \begin{tabular}{|l|l|}
14 & 6,7
\end{tabular} & \begin{tabular}{|ll} 
& 14 \\
14 & 6,5
\end{tabular} & $\begin{array}{ll}16 & 8.4\end{array}$ & \begin{tabular}{ll|}
14 & 6,7 \\
\end{tabular} & \begin{tabular}{l|l|}
16 & 6,8
\end{tabular} & \begin{tabular}{l|l|}
18 & 6,8
\end{tabular} & $\begin{array}{ll}18 & 6,5\end{array}$ & \multirow{2}{*}{12.2} \\
\hline & $11,6 \mid$ & \begin{tabular}{l|l}
26 & 11,9
\end{tabular} & \begin{tabular}{l|l|}
27 & 10,5
\end{tabular} & 2612,1 & $26 \mid 13,7$ & 2411,5 & \begin{tabular}{l|l|}
30 & 12,8
\end{tabular} & \begin{tabular}{l|l|}
34 & 12,8
\end{tabular} & $36|13,1|$ & \\
\hline 12 & 4,5 & 115,0 & $10 \quad 4,8$ & 125,6 & 115,8 & 115,3 & $\begin{array}{ll}11 & 4,7\end{array}$ & $\begin{array}{ll}12 & 4,5\end{array}$ & $13|4,7|$ & \\
\hline \multicolumn{2}{|c|}{$119+4,4$} & $100 \quad 15,9$ & $9 s+1 ;, 7$ & 9845,6 & (96) 17,4 & $94+5,2$ & $10 \mathrm{sin}+5.9$ & 12145,5 & $125 \quad 15,5$ & $(4,9 ! 9)$ \\
\hline 124 & 46.3 & \begin{tabular}{|l|l|}
101 & 46,3
\end{tabular} & $102+8,6$ & $106+9,3$ & 9550,0 & $102+5,1)$ & 11147,2 & 13149,2 & $131+7.6$ & \\
\hline 182 & $67, !$ & \begin{tabular}{l|l}
15.9 & 72.9
\end{tabular} & $1+160,6$ & $14 \approx 6 \Omega, \Omega$ & $1: 116-.9$ & 14265,3 & $159(6 \overline{7}, 6)$ & 184159.2 & $1 s+6 t^{2}, 9$ & \\
\hline 32 & 11,9 & $23 \mid 10,6$ & || $26|12,4|$ & $|28| 13,0 \mid$ & $23|12,1|$ & $|26| 12,5$ & $|30.12,8|$ & || $35 \mid 13,2$ & $36 \mid 13,1$ & \\
\hline & 16,0 & 3415,6 & $\begin{array}{ll}32 & 15,2\end{array}$ & 3214,9 & \begin{tabular}{l|l}
31 & 16,3
\end{tabular} & \begin{tabular}{l|l|l|}
35 & 16,8
\end{tabular} & 3514,9 & \begin{tabular}{ll|l|}
40 & 15,0
\end{tabular} & $42 \mid 15$, & \\
\hline & 14,0 & 3214,7 & 3416,2 & $\begin{array}{lll}3: 3 & 15,3\end{array}$ & 3116,3 & 3215,4 & 3514,9 & 40 15,0 & 4014,5 & 15,0 \\
\hline & 8 & 29 & 25 & 20 & 23 & 28 & 26 & 27 & 25 & 27,9 \\
\hline & & $0^{\pi}$ & $\sigma^{x}$ & $\sigma^{\top}$ & juv. & juv. & $0^{x}$ & $0^{x}$ & $\sigma^{7}$ & \\
\hline
\end{tabular}

Saposchnikowi m. = Clupea Saposchnikowii Gr.

\begin{tabular}{|c|c|c|c|c|c|c|c|c|c|c|}
\hline \multicolumn{11}{|c|}{ DERBENT } \\
\hline \multicolumn{2}{|c|}{ No 18} & \multicolumn{2}{|c|}{ No 19} & \multicolumn{2}{|c|}{ No 20} & \multicolumn{2}{|c|}{ No 21} & \multicolumn{2}{|c|}{ No 22} & \multirow{2}{*}{$\begin{array}{l}\text { ('hiffirs } \\
\text { moyens }\end{array}$} \\
\hline $\mathrm{mm}$. & $\%$ & $\mathrm{~mm}$. & $\%$ & $\mathrm{~mm}$. & $\%$ & $\mathrm{~mm}$. & $\%$ & $\mathrm{~mm}$. & $\%$ & \\
\hline \multicolumn{2}{|c|}{165} & \multicolumn{2}{|c|}{203} & \multicolumn{2}{|c|}{182} & \multicolumn{2}{|c|}{380} & \multicolumn{2}{|c|}{$3 \pm 7$} & \\
\hline 45 & 27,3 & 48 & 23,6 & 49 & 29,9 & 95 & 25,0 & 86 & 24,8 & 25,2 \\
\hline 36 & 21,8 & 43 & 21,2 & 39 & 21,4 & 93 & 24,5 & 83 & 23,9 & 23,6 \\
\hline 12 & 7,3 & 14 & 6,9 & 13 & 7,1 & 26 & 6,8 & 26 & 7,5 & \\
\hline 10) & ii. 1 & 1:) & 6,1 & 12 & (i.6) & 25 & 6.6 & $2: 3$ & (i.ti & \\
\hline 21 & 12,7 & $2: 3$ & $11, ;$ & 21 & 11.5 & 5) & 13.1 & 42 & 12.1 & $11 . !$ \\
\hline 11 & 6,7 & 10 & 4,9 & 12 & 6,6 & 16 & 4,2 & 15 & 4,3 & 5,5 \\
\hline 32 & 49,7 & 91 & 44,8 & 85 & 46,7 & 167 & 43,9 & 156 & 45,0 & \\
\hline 77 & 46,7 & 100 & 49,3 & 92 & 50,5 & 190 & 50,0 & 167 & 48,7 & \\
\hline 110 & 66,7 & 142 & 69,9 & 126 & 69,2 & 265 & 69,7 & 244 & 70,3 & \\
\hline 20 & 12,1 & 26 & 12,8 & 22 & 12,1 & 53 & 13,9 & 48 & 13,8 & \\
\hline 25 & 15,2 & 32 & 15,8 & 28 & 15,4 & 57 & 15,0 & 55 & 15,8 & 15,2 \\
\hline 27 & 16,4 & 30 & 14,8 & 29 & 15,9 & 56 & 14,7 & 49 & 14,1 & 32,1 \\
\hline \multicolumn{2}{|c|}{29} & \multicolumn{2}{|c|}{29} & \multicolumn{2}{|c|}{29} & \multicolumn{2}{|c|}{38} & \multicolumn{2}{|c|}{$\begin{array}{r}27 \\
\vdots\end{array}$} & \\
\hline
\end{tabular}

VIe CONGR. INT. ZOOL., 1904. 
Tableau III. Clupea caspio-

\begin{tabular}{|c|c|c|c|c|c|c|}
\hline PROVENANCE & & & & & \multicolumn{2}{|c|}{ KRAS N } \\
\hline No d'ordre & \multicolumn{2}{|c|}{ No 23} & \multicolumn{2}{|c|}{ No 24} & \multicolumn{2}{|c|}{$N^{\circ} 25$} \\
\hline MESURES & $\mathrm{mm}$. & $\%$ & $\mathrm{~mm}$. & $\%$ & mm. & $\%$ \\
\hline 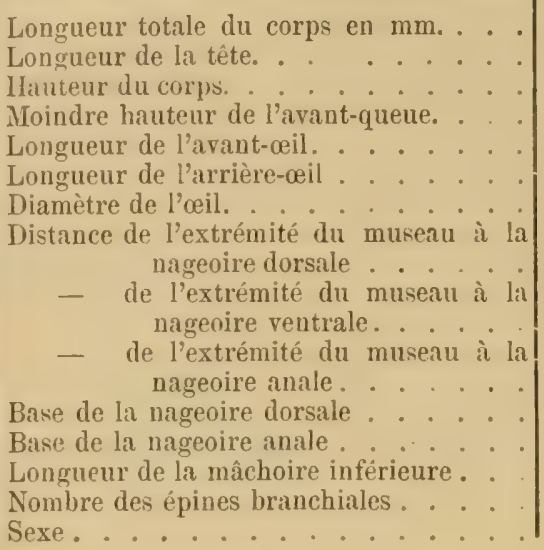 & $\begin{array}{r}17 \\
46 \\
37 \\
12 \\
11 \\
23 \\
10 \\
82 \\
84 \\
117 \\
25 \\
27 \\
28 \\
2 \\
\end{array}$ & $\begin{array}{r}5 \\
26.3 \\
21,1 \\
6,8 \\
6,3 \\
13,1 \\
5,7 \\
46,8 \\
\\
48,0 \\
66,8 \\
14,3 \\
15,4 \\
16,0\end{array}$ & $\begin{array}{r}18 \\
47^{3} \\
35 \\
12 \\
13 \\
24 \\
10 \\
\\
86 \\
\\
90 \\
\\
126 \\
25 \\
28 \\
27 \\
2 \\
\end{array}$ & $\begin{array}{r}1 \\
25,5 \\
19,0 \\
6,5 \\
7,1 \\
13,0 \\
5,4 \\
46,7 \\
48,9 \\
68,5 \\
13,6 \\
15,2 \\
14,7\end{array}$ & $\begin{array}{c}{ }^{1} \\
48 \\
39 \\
13 \\
12 \\
21 \\
11 \\
82 \\
86 \\
\\
120 \\
21 \\
26 \\
28\end{array}$ & $\begin{array}{r}3 \\
27,7 \\
22,5 \\
7,5 \\
7,0 \\
12,1 \\
6,3 \\
\\
47,4 \\
49,7 \\
69,4 \\
12,1 \\
15,0 \\
16,2\end{array}$ \\
\hline
\end{tabular}

Tableau IV. Clupea caspio-pontica var. Grimmi m.

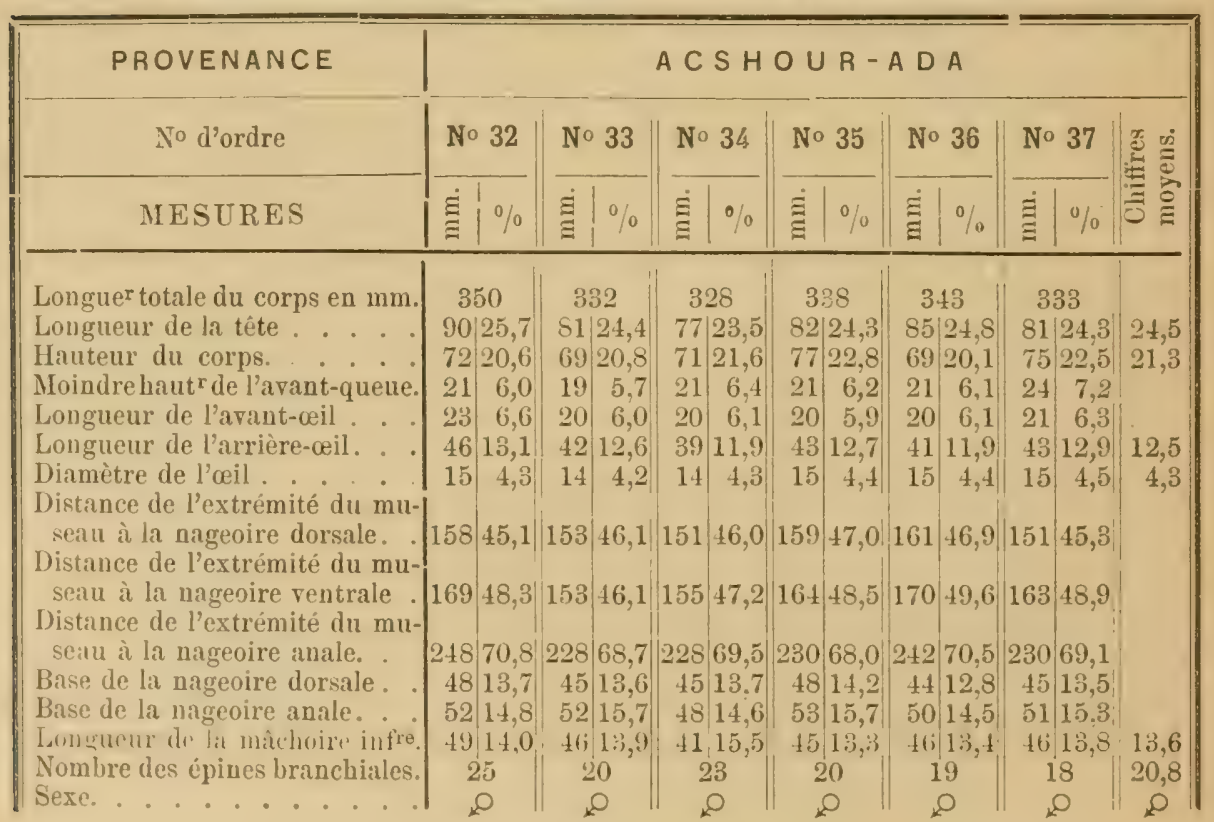


ontica var. Braschnikowi m.

O D S K

\begin{tabular}{|c|c|c|c|c|c|c|c|c|c|c|c|c|}
\hline \multicolumn{2}{|c|}{ N 26} & \multicolumn{2}{|c|}{ No 27} & \multicolumn{2}{|c|}{ No 28} & \multicolumn{2}{|c|}{ No 29} & \multicolumn{2}{|c|}{ N० 30} & \multicolumn{2}{|c|}{ No 31} & \multirow{2}{*}{$\begin{array}{l}\text { Chiffres } \\
\text { moyens }\end{array}$} \\
\hline $\mathrm{mm}$. & $\mathbf{0} / 0$ & $\mathrm{~mm}$. & $\%$ & $\mathrm{~mm}$. & $\%$ & $\mathrm{~mm}$. & $\%$ & $\mathrm{~mm}$. & $\%$ & mm. & $\%$ & \\
\hline \multicolumn{2}{|c|}{202} & \multicolumn{2}{|c|}{199} & \multicolumn{2}{|c|}{222} & \multicolumn{2}{|c|}{260} & \multicolumn{2}{|c|}{271} & \multicolumn{2}{|c|}{370} & \\
\hline 50 & 24,8 & 51 & 25,6 & 54 & 24,3 & 68 & 26,1 & $6 s^{\circ}$ & 25,1 & 92 & 24,9 & 25,5 \\
\hline 43 & 21,3 & 42 & 21,1 & 46 & 20,7 & 58 & 22,3 & 60 & 22,1 & 82 & 22,2 & 21,3 \\
\hline 14 & 6,9 & 14 & 7,0 & 15 & 6,8 & 18 & 6,9 & 19 & 7,0 & 26 & 7,0 & \\
\hline 18 & 6,4 & 13 & 6.5 & 13 & 5,9 & 18 & 6,9 & 18 & 6.6 & 25 & 6,8 & \\
\hline 24 & 11,9 & 24 & 12,1 & 27 & $\begin{array}{l}12.2 \\
\end{array}$ & 32 & 12,3 & 34 & 12.5 & 49 & 13,2 & 12,5 \\
\hline 11 & 5,4 & 10 & 5,0 & 12 & 5.4 & 13 & 5,0 & 12 & 4,4 & 14 & 3,8 & \\
\hline 92 & 45,5 & 93 & 46,7 & 103 & 46,4 & 121 & 46,5 & 127 & 46,9 & 169 & 45,7 & \\
\hline 97 & 48,0 & 99 & 49,7 & 110 & 49,5 & || 129 & 49,6 & 130 & 48,0 & 177 & 47,8 & \\
\hline 138 & 68,3 & 136 & 68.3 & 155 & 69,8 & 185 & 71,1 & 184 & 67,9 & 257 & 69.5 & \\
\hline 24 & 11,9 & 25 & 12,6 & 28 & 12,6 & 36 & 13,8 & 34 & 12,5 & 46 & 12,4 & \\
\hline 29 & 14,4 & 29 & 14.6 & 35 & 158 & 35 & 13,5 & 45 & 16.6 & 56 & 15,1 & 15,4 \\
\hline 32 & 15,8 & 31 & 15,6 & 34 & 15,3 & 40 & 15,4 & 41 & 15,1 & 55 & 14,9 & 26,4 \\
\hline & & & 10,0 & & & & & & & & & \\
\hline
\end{tabular}

Tableau V. Clupea maeotica Gr.

\begin{tabular}{|c|c|c|c|c|c|c|}
\hline PROVENANCE & \multicolumn{6}{|c|}{ MER } \\
\hline No d'ordre. & \multicolumn{2}{|c|}{298} & \multicolumn{2}{|c|}{288} & \multicolumn{2}{|c|}{294} \\
\hline MESURES & $\mathrm{mm}$. & $\%$ & $\mathrm{~mm}$. & $0 \%$ & $\mathrm{~mm}$. & $\%$ \\
\hline Longueur totale du corps en $\mathrm{mm}$. & \multicolumn{2}{|c|}{298} & \multicolumn{2}{|c|}{288} & \multicolumn{2}{|c|}{294} \\
\hline Longueur de la tête. . & 67 & 22,5 & 66 & 22,9 & 66 & 22,4 \\
\hline Hauteur du corps . . . . . & 73 & 24,5 & 76 & 26,4 & 69 & 23,5 \\
\hline Moindre hauteur de l'avant-queue. & 23 & 7,7 & 23 & 8,1 & 22 & 7,5 \\
\hline Longueur de l'avant-œil. . . . . & 15 & 5,0 & 17 & 5,9 & 17 & 5,8 \\
\hline Longueur de l'arrière-œil. & 34 & 11,4 & 33 & 11,4 & 34 & 11,6 \\
\hline Diamètre de l'œil . & 13 & 4,4 & 14 & 4,9 & 12 & 4,1 \\
\hline $\begin{array}{l}\text { Distance de l'extrémité du museau à la } \\
\text { nageoire dorsale. } \\
\text { _ de l'extrémité du museau à la }\end{array}$ & 133 & 44,6 & 128 & 44,4 & 129 & 43,9 \\
\hline $\begin{array}{l}\text { nageoire ventrale . } \\
\text { - de l'ixtrémité du musearu à la }\end{array}$ & 146 & 48,9 & 136 & 17,2 & 142 & 48,3 \\
\hline nageoire anale. . . . . & 212 & 71,1 & 200 & 69,4 & 196 & 66,7 \\
\hline Base de la nageoire dorsale . . . & 36 & 12,1 & 33 & 11,4 & 37 & 12,5 \\
\hline Base de la nageoire anale : . . . & 44 & 14,8 & 43 & 14,9 & 48 & 16,3 \\
\hline $\begin{array}{l}\text { Longueur de la mâchoire inférieure. } \\
\text { Nombre des épines branchiales. . . }\end{array}$ & 40 & 13,4 & 38 & 13,2 & 38 & 12,9 \\
\hline Sexe. & & & & & & \\
\hline
\end{tabular}


la dite espece un mois de plus, ce qui est depuis longtemps connu relativement à la $C$. finta ${ }^{1}$.

Mais, d'un autre côté, lor's de ses recherches sur la faune de la Mer Caspienne. en 1SiO. M. le Dr Grmu a déjà découvert. au commencement du mois de juillet. au milieu de la Mer Caspienne. un petit Poisson nommé par lísslen ('lupermella Grimmi”. (Toir fig. 6). Lejnomlnte d'épines de ce Poisson est, selon Kessler, de près de trente!

Les mensurations d'un des exemplaires de Chupeonella. Grimmi Kessi.

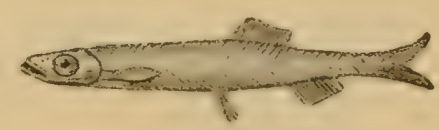

Fig. 6. Clupeonella Grimmi Kessl.

et le dénombrement des épines branchiales m’ont donné les chiffres suivants. Longueur générale du corps : $35 \mathrm{~mm}$., hauteur maximum : $4 \mathrm{~mm}$, longueur de la tête: $6 \mathrm{~mm}$. Les nageoires sont bien développées, mais consistent en rayons séparés, non reliés entre eux (r a y ons proimair es de Poucheт). Les épines branchiales sont courtes et au nombre de 18.

En recherchant ì laquelle des Clupéidées on ponrrait rapporter. comme larve, la Clupeonella Grimmi Kessl., le Dr Grum a émis d'abord la supposition que cela devait être la larve de $C$. caspia (voir: Le Hareng d'Astrakhan, p. 20), mais ensuite il a reconnu que cela devait être plutôt la larve de la $C$. Saposchnitiowii et peut être en partie de la $C$. cas$p_{i a^{3}}$. La forme marine n'était pas encore connue à cette époque, c'est-àdire qu'on ne supposait pas qu'il en existât une grande quantité au large de la Mer Caspienne. Il n'admet pas que cela puisse être la $C$. delicatula, vu les dimensions par trop grandes pour la larve d'un Poisson aussi petit que la $C$. delicatula.

Il me semble que le nombre des épines, le séjour des larves en masse dans les parties du Sud et du centre de la Mer Caspienne", le temps et le lieu de la pounte des différentes espéces des Clupéidées caspiennes - tout parle

${ }^{1}$ Voir le très intéressant mémoire sur ce sujet du Dr HoEk. Neuere Lachs-und Mraifischstudien.

${ }^{2}$ Les exemplaires plus âgés étaient de la longueur de $45 \mathrm{~mm}$., mais il y en avait de $15 \mathrm{~mm}$.

s Pêches de la Mer Caspieme et du Volga (russe), p. 96.

"Les larves des Harengs décrites par Kesscer sous le nom de Clupeonella Grimmi ont été trouvées par MI. Grinm en 1874 dans les endroits suivants : $0^{\circ} 26^{\prime} \mathrm{E} .40^{\circ} 57^{\prime} \mathrm{N}$ (137 à $146 \mathrm{~m}.), 0^{\circ} 26^{\prime} \mathrm{E} .41^{\circ} 6^{\prime} \mathrm{N}(146$ à $164 \mathrm{~m}), 1^{\circ} 9^{\prime} \mathrm{WV} .41^{\circ} 54^{\prime} \mathrm{N}(80,5 \mathrm{~m}$.), par moi en 1903 et par M. Ǩnipowitsh en 1904, près de la baie d'Astrabad (le coin SE de la Mer Caspienne). 
en faveur de la supposition que nous avons affaire. dlans ce cas. à des alevins de la C. caspio-pontica, c'est-à-dire à la Clupée caspienne marine, variétés Braschmiliourii et Grimmi. La larve de C. delicutula devrait être plus petite (voir ci-aplese) et, ce qui est de la plus grande importance, devrait être pigmentée plus fortement. Le nombre de seś épines devrait être plus grand (50). La larve de $C$. cospucu ne pourrait pas parvenir en masses au Sud de la Mer Caspienne où elle est relativement rare et le nombre des épines branchiales devrait etre, dans ce cas-là, beaucoup plus grand. L'alevin de la $C$. Kessleri reste dans l'eau douce; les alevins de la var. Saposchritiowii doivent lui ressembler beaucoup, mais il en existe si peu, que cest douteux qu'on puisse attribuer à cette variété la masse d'alevins trouvés dans le centre et au Sud de la Mer Caspienne.

La région de distribution de la $C$. caspio-pontica est très caractéristique; elle est originaire du Sud de la Mer Caspienne. où elle se trouve pendant toute l'année. On la pêche le long du littoral oriental (par exemple dans la baie de Krrasnovodsk). en été et en hiver. pour servir d'appât, mais on n'observe pas, ici, son apparition en masse, excepté dans les endroits de la baie d'Astrabad où on a signalé le passage de ses troupes. La plus grande masse de ce Poisson apparaît chaque année aux mois de février et de mars dans la partie septentrionale du littoral transcaspien; ses troupes sapprochent de l'île Koulaly. en se dirigeant plus loins ver's les îles Dolguy (les îles Kolpinoy), et entrent aussi dans la baie de Sarytasch. La pêche des Clupéidées commence ici au mois de mar's et continue en avril. On peut supposer avec certitude qu'elle fraie dans la baie de Sarytasch ${ }^{1}$.

Il est à noter que la partie orientale de la Mer Caspienne est, par' excellence. l'endroit où domine cette espèce, tandis que la côte opposée, occidentale. appartient entièrement au domaine de la C. caspice (" pousanok »). La $C$.cospio-pontica se rencontre, ici, en quantité heaucoup moins grande. La nourriture de cette espèce consiste exclusivement en Poissons qui sont, au midi. l'Athérine (Atherina caspra), au nord. diftérentes espèces du gemre Gobius et surtout la Clupea delicatula Nord. La Cluper caspio-pontica est très vorace et se prend facilement à la ligne la plus primitive des aborigènes.

La quatrième espèce des Clupéidées caspiennes est la C. delicatula qu'on distingue aisément de la première ( $C$. Kessleri) et de la troisième espèce (C. cospio-pontica m.) par l'absence complete de dents, ainsi que de la $C$. caspia - dont on peut facilement confondre les alevins avec les exem-

${ }^{1}$ Ce qui a été confirmé récemment par les renseignements recueillis par l'inspecteur de pêche M. Sumpow.

M. ARnold a trouvé dernièrement l'endroit de la ponte de ces troupes près des Iles Kolpinoy. 
plaires adultes de la $C$. delicatula - par la forme de la tête et la position de l'oil ${ }^{1}$. Elle se distingue, en vutre, des trois espèces caspiennes par' le nombre des épines branchiales, qui est de 44 à 53.

Cette espèce dépasse très rarement les dimensions de $120 \mathrm{~mm}$. et on rencontre déji dés Poissons adultes pleins d'oufs parmi des exemplaires ayant unn taille de so i 100 mm. Les ceufs de ce Poisson se reconnaissent très facilement des rufúr des autres C'lupéirlées. et même des antres l'oissons. par'

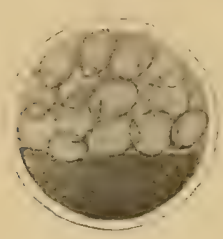

Fig. 7 .

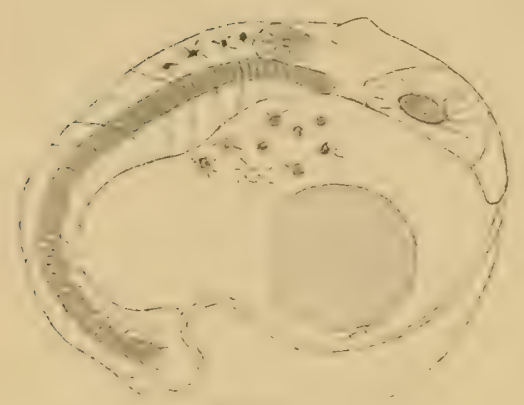

Fig. !

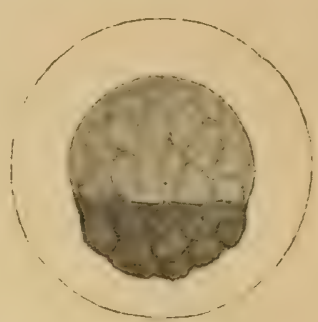

Fig. 8 .

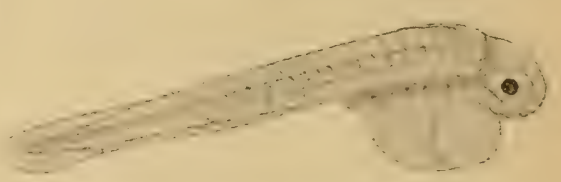

Fig. 10.

Evolution de la Chupea delicatula : Fig. 7. Eufs récemment fécondés dont la membrane n'est pas encore gontlée par l'action de l'eau. Fig. 8. Trois heures après la fécondation. Fig. 9. 32 heures après la fécondation. Fig. 10. Larve de la Clupea delicatula.

lenr trinte rosittere ('e petit Poisson fraye aussi bien dans l'eau donce, que dans l'eau salée. Habitant au large de la mer et r'eprésentant une forme

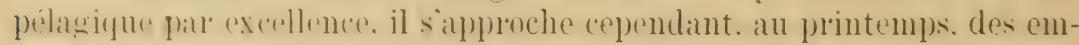
bouchures des rivières où il peut frayer. En 1885, j'ai obtenu des œufs mûrs de ce Poisson dans un bras du Volga, le 24 avril, et je les ai arti-

${ }^{1}$ L'arrièredu muscau à la nageoire dorsale et près de $10 \%$ de la longueur totale. Pour les autres Clupéidées cette longueur est de 12 à $14 \%$. (Lönsberg, Mess. des Pêch. 1901, p. 30.) 
ficiellement féeondés. Les oruf́s féconelés ont été placés, sur une assiette, dans de l'eau de rivière ayant $9^{\circ}, 2 \mathrm{C}$; la température s'est élevée jusqu’à $11^{\circ}, 6$ C. La membrane des œufs mûr's, avant l'immersion dans l'eau, enveloppe fortement le vitellus dans lecpuel on pent distinguel des gouttes graisseuses de couleur lilas (vues à l'œil nu) qui communique à la masse des anfs la teinte rositre. Les oeufs qui, apres la fécondation, sont mis dans l'eau. gonflent et la membrane se détache du contenu presque sur $1 / 3$ du rayon de la sphère (voir fig. 7). Deux heures après la fécondation ${ }^{1}$, on peut voir clairement la segmentation. 'Trois heures après, un assez grand nomlne de segments se sont déja formés (fig. S). En général, l'évolution s'effectue avec une rapidité étonnante. Après 24 heures, on voit distinctement l'embryon avec la corde et les myotomes. Après 32 heures, on peut bien distinguer la tête et la partie caudale. On voit les débuts des yerux ot les étoiles du pigment sur le dos; (olles sont aussi de conleur lilas comme dans les œufs. De la masse générale de la vésicule ombilicale. se détahe un globe de matières graisseuses. diune teinte rositre. et prex d'elles sassemblent de petits corpuseules de pigment lilas en formes d'étoiles (fig. 9). Le 27 avril, c'est-à-dire après 48 heures, l'éclosion des alevins a eu lieu. Les corps de pigment caractéristiques restent dans la vésicule et le globe graissenx rosâtre y occupe $1 / 3$ de tout le sac vitellin. Les alevins de la Clupea delicatula ne sont restés vivants chez moi que 3 à 4 jours. Outre la nageoire embryonnaire, on pouvait voir, le 29 et 30 avril, la formation des nageoires pectorales. A ce moment, les yeux sont devenus noirs (fig. 10).

Outre la Cluper delicatulu Nord, qu'on trouve partout dans la Mero Caspienne en grand nombre, j'ai eu l'occasion, le printemps passé, de trouver une autre espèce, très voisine de l'espèce citée, mais ayant des traits bien distincts, comme on peut le voir dans la description donnéc ci-dessous.

Corps cylindrique très allongé. Sa plus grande hauteur est le $15,3 \%$ de la longucur totale et se trouve près de la nageoire dorsale; elle est un peu plus grande que la hauteur du corps près de la base de la tête et près de la base de l'anale. L'épaisseur du corps n'est que deux fois plus petite que sa hauteur. La longueur de la tête n'est que le $3 \%$ de la longueur totale du corps et est 1 ' $^{\prime}$, fois plus grande que lat hauturur du corps. Locil est tres gros; son diametre ue faisant qu'un quart de la longueur de la tête. La pupille est énorme; elle est seulement $1 \frac{1}{2}$ fois moins grande que le diametre do l'oeil. ('es trois derniers traits la distinguent de la Clupea delicatula qui a la pupille très petite, 2 à 3 fois plus petite que le diamètre de l'œil.

Le maxillaire inférieur est très proéminent et ne porte pas de dents.

1 Température de $9^{\circ}, 2$ à $11^{\circ}, 6 \mathrm{C}$. 
Le nombre desépine: branchiales est. comme chez la Chupea deticatula de 55 à 56.

Dors. 1/13, Pect. 1/13, Ventr.1/7, An. 1/18-20. Les deux dernier's rayons de l'anale sont séparés des autres rayons et sont plus longs. Caud. II-10-10-II. La longueur de la pectorale est presque égale à la hauteul du corps près du bor'd de l'opercule, tandis que chez la Clupea delicrtula cette nageoire est $1 /$ a plus courte. Les épines de la carène dentelée qui. en général, sont moins développées que chez la Chupea deticatulu, sont au nombre de 23 (la Clupea delicatula en a 27). Du reste. cette nouvelle espèce diffè be beacoup de la Clupea delicatula par la coloration beaucoup plus foncée du corps. Le dos est, chez les exemplaires frais. d'un bleu foncé (couleur d'acier'); chez la Clupea delicatula, il est vert clair (couleur de l'eau do mel\%). Les côtes et le ventre sont d'un blanc argenté: les nageoires (sauf la caudale) sont pâles. Les rayons de la caudale sont teintés en noir. L'iris est d'un blanc argenté.

Il faut encore noter une différence entre ces deux espèces, au point de vuc biologique. Tandis que la Clupea delicatula avait des oufs presque mûrs le 11 avril, la nouvelle espèce, à la même époque, avait des œut's trés peu développés. Enfin. nous trouvons la première partout en masse lo long des côtes, tandis que la dernière est relativement très rare près des côtes et habite les parties de la mer les plus profondes et les plus éloignées. Elle a, en général. une taille plus grande que la Clupea delicatula et qui atteint sourent $137^{\mathrm{mm}}$ à $150^{\mathrm{mm}}$. Grâce à la ressemblance frappante de cette espèce avec l'Anchois, jappelle cette nouvelle espèce Clupen engrauliformis $\mathrm{m}$. Personne n'a jamais vu ce Poisson remonter la rivière ou se trouver près de son embouchure.

Nous avons donc. pour la Mer Caspienne, d’apress les recherches récentes, cinc espèces du genre Clupea (C. caspia, Eichw. C. caspia-pontica m., avec trois variétés, C. Kesslerii Gr., C. delicatula Nord., C. engrauliformis m.). 'Trois espèces sont des vraies (lupéidées. Poissons marins, deux espèces ( $C$. caspia et $C$. Kessleri) sont plutôt des Aloses.

Nous connaissons les larves et les alevins de la Chupea caspia-pontica st de la Cluper deticrutula; ceux de la Cluper cospia étaient inconnus jusqu'à présent.

Le printemps passé, j'ai r'éussi à faire à l'embouchure du Volga, le 3 (Ii) mai, la fécondation artificielle des aufs de cette espèce et à obtenir artificiellement des larves. Le développement des oufs ne dure que trois jours, la température étant $20^{\circ} \mathrm{C}$. Les oufs qui nagent deviennent transparents dans l'eau, ce qui facilite beaucoup toutes les observations sur li développenent à l'aide du microscope. Le diamètre des oufs. avant leur misc dans l'eau. est de $1^{\mathrm{mm}}$. Mis dans l'eau. ils gonflent et ont déjà le diamètre de $2^{\mathrm{mm}}$. La larve a $4^{\mathrm{mm}}$ de longueur (voir fig. 8 à 10). Je n'ai pas réussi à les observer vivantes plus d'une semaine. A ce moment- 



\section{EXPLICATION DE LA PLANGHE}

Fig. 1. Oeufs de la Clupea caspia Eichw.

a) 2 heures après la fécondation;

b) 28 heures après la fécondation.

Fig. 2. Larve de la Clupen caspun Eichw. nourellement inose.

Fig. 3. Alevin de la Clupea caspia Eichw. de 7 jours.

Fig. 4. Tête de l'Alevin de la Clupen caspia Eichw. de 7 jours. 

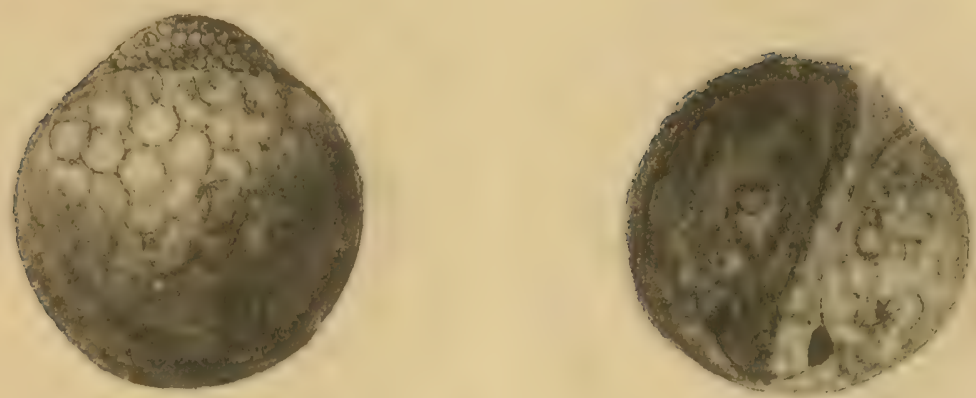

1

(l.

b.
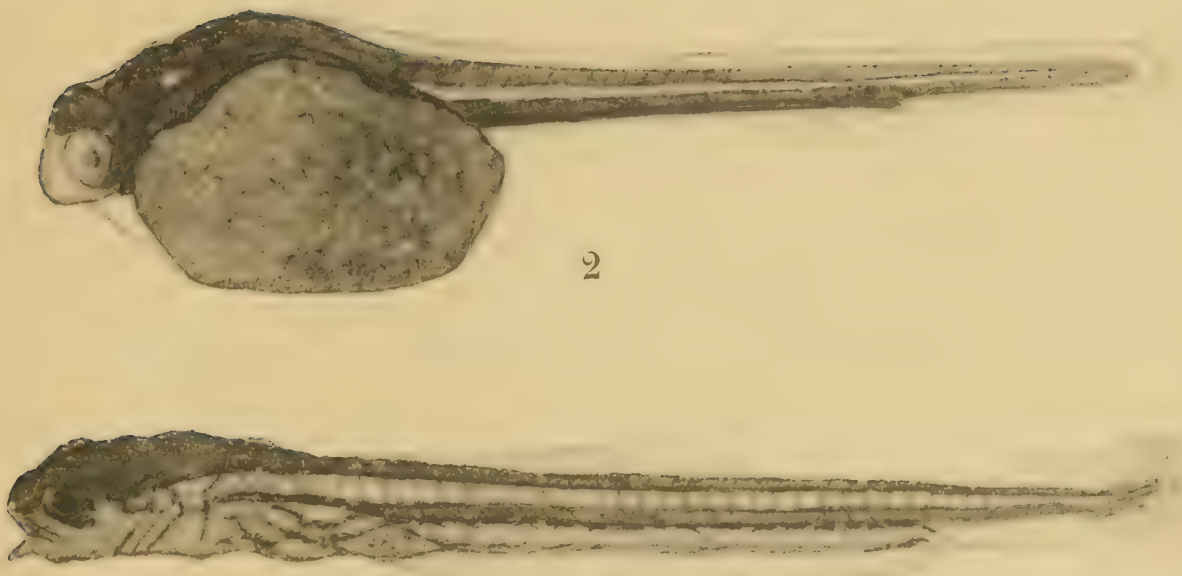

3

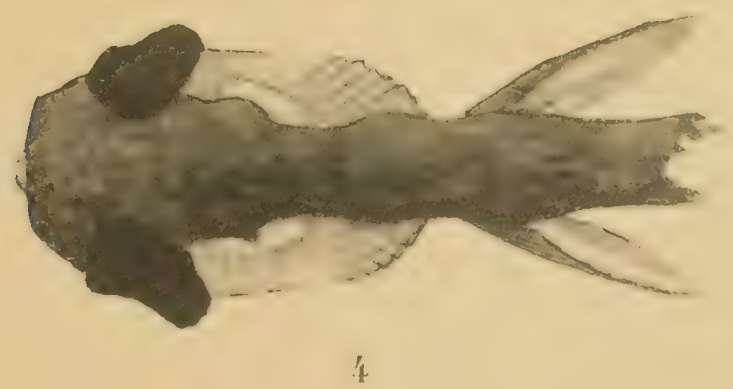



là, on doit les compter toujour's comme des larves, parce qu'elles sont encore privées rle nageoires (sauf les pectorales et les caudales) et sont loin de lessembler au Poisson adulte (voir dessin). On peut les distinguer facilement des larves de la (lupea delicutula, qui sont d'abord d'une taille plus petite et sont pourvues de la pigmentation spéciale (voir ci-clessus). Je ne dirai pas par quels caractères elles diffèrent des plus jeunes larves de la Clupeu caspro-pontica, car je n'ai pas encore eu l'occasion de l'examiner de plus près.

D'un autrecoté. il nous reste à ohtenir et à (xaminer en détails la larve de la Cluper Kessleri, dont la fécondation artiticielle des oufs n'est pas encore faite. La lal've de cette espèce est jusqu'à présent inconnue. Nous ne connaissons que ses alevins, assez développés, ayant la taille de $70^{m n 1}$ et ses yerrlings, c'est-à-dire les Poissons âgés d'une année, ayant la taille de 85 à $100^{\mathrm{mm}}$. On rencontre les premiers dans les eaux douces du Tolga: j'ai trouvé les del'nier's, en abondance, le long des côtes occidentales de la Mer Caspienne. La Clupea cuspia et la Chuper Kessleri se mropagent dans l'eau doure. La promière ne remonte pas très haut dans les rivières et fraye dans les eaux peu profondes, calmes et claires des lacs et des lagunes du delta du Volga. Mon collègue, M. Arsol.d a cu l'occasion. durant le printemps passé. d'Yy olotenir une quantité d'oufs mûs, de les féconder et de faire éclore des milliers: de larves de cette espèce. La dernière remonte le Tolga et ses aftluents à une très grande distance do l'embouchure, ou la ponte doit avoir lieu. Ses alevins restent quelquefois dans les eaux séparées du Volga même, mais dans la règle, ils vont à la mer, oì on les rencontre le printemps suivant en abondance. Ils y restent au moins trois années et ce n'est que la quatrieme année que ces Poissons remontent la rivière pour frayer.

J'ai exposé brièvement presque toutes les données importantes sur l'histoure naturelle et surtout sur la propagation des Clupeidées caspiemnes. On roit qu'il nous manque encore quelques domnées sur la propagation de la Clupea Kessleri Gr. et de la C'lupea engranliformis m. mais on peut espél'er que nous les obtiendrons prochainement.

1 Dans la petite note de M. Roudzky. 


\section{Evolution of the horse. Recent discoveries and studies.}

By Prof. H. F. OSBORi' (New-York).

Exploration for the horse in America has been carried on for the last thirten years by the American IItseum of Satural History, and special explorations during the last three years have heen devoted especially to the history of the hor'se. More or less complete remains of over 700 extinct horss's have been found, including the discovery of complete skeletons of Mesohimpus, Hypohipmus, Neohiprorion and Erums scotti. The history of the American Pliocene horses, and the direct ancestry of the modern horse (Equus caballus) still require more light.

As elaborated in the author's address on another page, the fact of chief importance brought out by these discoveries is that the hor'ses were polyplyyletic, three being four and perhaps five contemporary series in the ()ligocene and again in the Niocene. Unfortunately the series leading directly to Equeus is not completely known; the origin of Eques in North America, therefore, while highly probahle. is not yet absolutely certain.

Comparison of the existing species of horses, asses and zebras also tends to show that. like the dogs. these animals were polyphyletic, and took their origin very far back, probably in the Pliocene.

The authors especially requests in exchange papers, notices and photographs relating to lecent and extinct hol'ses, which should be addressed to the American Museum of Natural History, New-York.

\section{Ueber den phyletischen Verband der Spalax-Arten.}

Von Prof. L. von MÉHELY (Budapest).

L'auteur n'a pas remis le manuscrit de sa communication.

\section{Entstehen und Bedeutung der Hauptfarbkleidmuster der Reptilien.}

Von Prof. G. TORNIER (Berlin).

L'auteur n'a pas remis le manuscrit de sa communication. 


\title{
Zur Biologie der kaspischen Finte, Clupea caspia Eichw.
}

\author{
Von Ir. J. Aln()I,I) (St. Petershurg).
}

Mit 4 'l'extfiguren.

Es gibt im kaspischen Meere fünf Herings-Arten: Cluper Kessleri

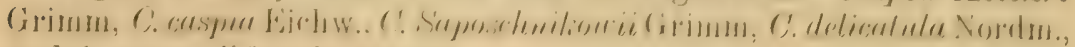
und $C$. engrauliformis Borodin.

Clupea Kessleri, der Kaspische Maifisch, ist die grösste $\Lambda$ rt (bis $52 \mathrm{~cm}$.), die der westeuropäischen Alosa vulguris 'Troschel analog ist. Ijese Art, sowie auch zwei andere: C'. caspia, welche der finte entspricht und $C_{\text {. }}$

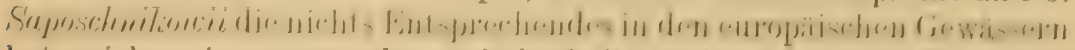
hat, spielen eine grosse ökonomische Rolle in der kaspischen Fischerei, während die übrigen zwei Arten, C. delieabula und C'. engraneliformis, die ganz klein (nicht über $15 \mathrm{~cm}$.) und der (j. sprathus aus de. () stsee ahnlich sind, bis in die letzte Zeit noch keine praktische Anwendung gefunden haben.

Clupea caspia, die kaspische Finte (Fig. 1), die höchstens $98 \mathrm{~cm}$. erreicht, lat cinen breiten, zusammengepressten Leib, einen grossen Kopf mit breiten Kiemendeckeln und besitzt biberemstimmend mit dex Finte, aber durchaus nicht immer, noch eine lioihe von 5 und mehr dunkeln Flecken hinter dem Schulterfleck. Abweichend von der Finte ist bei der. C. caspia die Zahl der. Rrousenzähne (118-136; gegen 38-4:3), was wah"scheinlich mit der Verschiedenheit in der Art der Ernathung dieser beiden lische in Zusammenhang steht.

lis wird allgemein angenommen, dass die finte im Früjahr aus den Meere in die lilüsse hinaufsteigt um hiel ihr Laichgeschäft auszuführen. So steigt sie z. B. in Rhein und in dren abrigen Nordseceflussen Ende Mai ziemlich hoch hinauf; in der ()stsee geht sie abol im Apri] und Maj nur in die Haffe, und nur wenige Exemplare gelargen weiter hinauf in die dasolbst müdenden Flisse. Auch in den Nordserftüssen steigt die linte nicht so weit hinauf wire der Maifisch (Alosa vulgaris) ${ }^{1}$.

Dje kaspische finte scheint auch nicht weit in die Wolga hinaufzusteigen, wenigstens in den letzten Iecennien. Nach J)r. O). (immu's Angaben soll dieser Hering noch withend der achtziger Jahro in den Flussbusen neben der Stalt Sarepta, also circa 500 Kilometer weit von

1 II. Stratzen. Forstliche Produltionslehre. 'T. 2. 'Tübingen, 1903. 


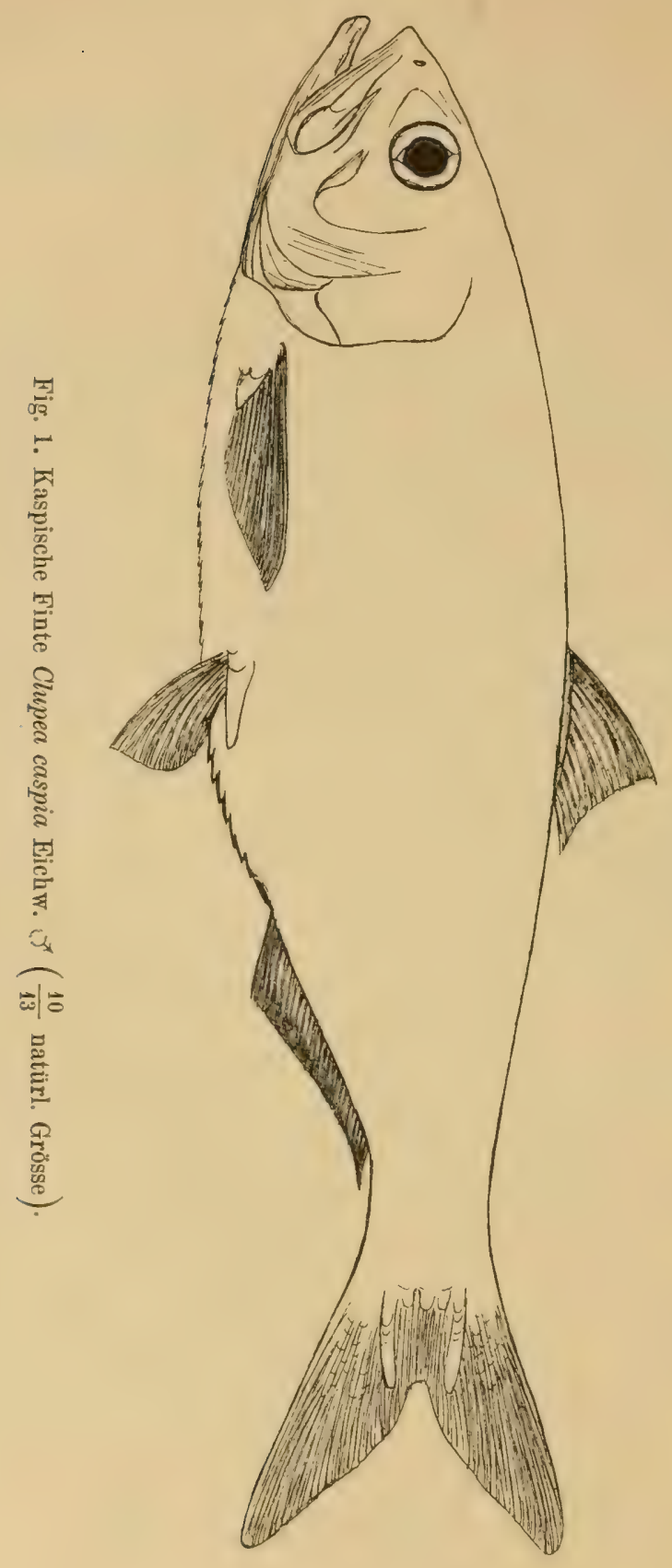


der Wolga-Nündung ${ }^{1}$ gelaicht lıaben. Leider sind aber diese Angaben nicht genügend begrüudet und es bleibt nur ausser Zweifel, dass del (rwähnte Forscher die Gelegenheit gehabt hatte, laichreife Exemplare der C. caspia im Jahre 1885 neben Tscherny Jar, also circa 350 Kilometer von der Mündung der Wolga, zu beobachten.

Obgleich ich die oberste Grenze, bis welcher die Hauptmasse der kaspischen Finte in die Wolga hinaufsteigt, noch nicht genau zu verfolgen im Stande war, bin ich der Meinung, dass dieser Fisch dicht an der WolgaMündung resp. in den zahlreichen Altwässeln, sogenannten "Ilnjony », theils auch in dem kaspischen. Mecre selbst, also im salzigen Wasser laicht. Diese meine Voraussetzung griundet sich theils auf Bexecke's" Angaben, laut denen die verwandte Al't, die westeuropäische Finte, früher in grossen Mengen aus del Ostsee in die Haffe, also nicht weit in die Flüsse hinaufstieg, theils auf meine eigenen Untersuchungen mit der liünstlichen Befruchtung der C. caspru, welche ich diesen Sommer als Mitglied del' kaspischen Expedition und noch fi'üher, iu Jahr 1899, ausgeführt habe.

Da mir die Versuche mit der lïustlichen Befruchtung der haspischen Finteschon im Jahr 1899 gut gelungen sind (die Fischehen, die sich schon in den Eiern energisch bewegten, schlüpften damals nur wegen eines unglücklichen Zufalls nicht aus), bemühte ich mich in diesem Sommel parallele Untersuchungen mit der Befruchtung im Süsswasser und Meerwasser resp. Blackwasser (der Salzgehalt des kaspischen Meeres wird auf circa 10 gegen 30 pro Nille im atlantischen Ocean also auf cirea $1 \%$ taxirt) anzustellen.

Die vereinzelten, laichreifen Exemplare der C. caspia traf ich in der Mündung der Wolga (in den Armen der Wolga, die dem FischereiEtablissement der Gebrüder SAposchrikow angehören), während des ganzen Monats Maj; die Zahl der abgestrichenen Eiel war aber für die erwähnten Zwecke zu beschräinkt. Am 6. Juni habe ich endlich in einem ziemlich dicht mit Potamogeton sp. verwachsenen Altwasser " Ilmjen Charbuta ), dicht an der Mündung der Wolga, eine ganze Menge (450) laichreifer kaspischer Finten getroffen, von denen eine genügende Menge Eier abgestrichen wurde, wobei die eine Hälfte der letzten $\mathrm{jm}$ Süsswasser, die andere aber im reinen Meerwasser mit aller Sorgfalt künstlich befruchtet wurde. Die T'emperatur des Wasser's in den beiden Tellern, wo die Befruchtung ausgeführt wurde, betrug $15^{\circ} \mathrm{C}$. Die ersten zwei Versuche gelangen aber nicht, was ich der ungenügenden Frischheit des Spermas zuschreibe, da zwischen den in den Stellnetzen gefangenen 450 Exemplaren leider nur todte Männchen waren (20 Stück). Erst am Abend desselben Tages gelang die Befruchtung, welche dann

${ }^{1}$ Dr. O. Grim. Diekaspisch-wolgaische Fischerei. S. 94 (russisch),St.Petersburg, 1896.

2 Benecke. Fische, Fischerei und Fischz. in Ost-Preussen. 1886. 
auch später, am 12. Juni (5 Chr Morgens) und 14. Juni (3 Uhr Morgens). mit vollem Erfolge ausgeführt wurde. Die Befruchtung im Meerwasser gelang tadellos, und die Segmentation (Fig. 2) und die ganze Entwick. lung gingen hier eben so gut und schnell wie im Süsswasser vor sich.

Dies stimmt also gänzlich mit den Angaben von Di. NoordiaARd über die Entwickelung der Lachseier in schwacher (bis $1 \%$ ) Salzlösung ${ }^{1}$ überein. Sehr empfindlich erwiesen sich die Eier der C. caspia für die 'T'emperaturschwankungen, so z. B. dauerte bei der Temperatur von $21^{\circ}$ C. die ganze Entwickelung 45-48 Stunden, also 2 Tage, und schon eine Herabsetzung der 'Temperatur um 3 Grad (also auf 18 ) verlängerte diese Periode beinahe auf 3 Tage (circa 72 Stunden). Noch empfindlicher erwiescn sich die Eier für dei Schwankungen des Sanerstoffgehalts im

Wasser, welcher Umstand inzwi-

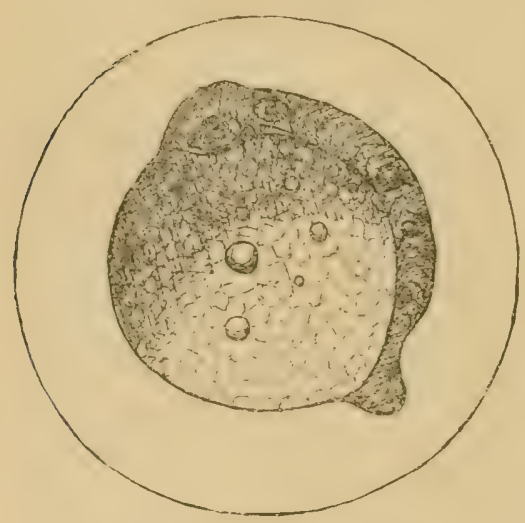

Fig. 3. Ein ca. 36 Stunden alter Embryo von C. caspia. Die Öltropfen entstehen gleich nach dem Absterben.

(Vergrösserung 25.)

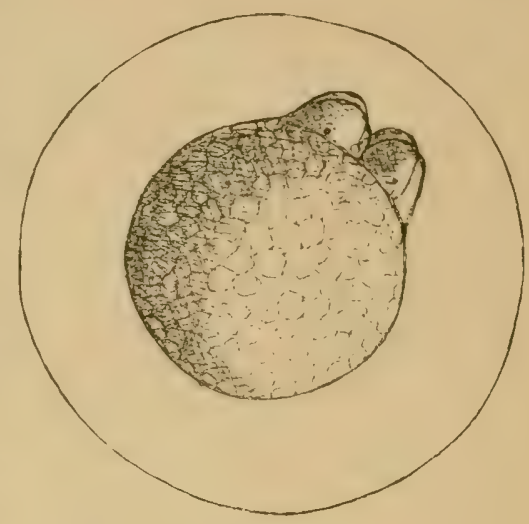

Fig. 2. Beginn der Segmentation. (Vergrörserung 25.) schen viele Misserfolge, die ich früher mit diesem zarten Okjecte hatte, beeinflusste. - Es genügte z. B., dass die Eier anstatt in einer in zwei Schichten - die Eiel der $C$. caspia sind nicht adhrsiv und haben einen Dur'chmesser von 1,7-2 mm. - in dem Brutapparate (als Brutapparate erwiesen sich sehr gut und praktisch die gewöhnlichen Essteller, sowie auch kleine gläserne Untertassen, resp. ganz flache Kristallisationsschalen) lagen, um die Entwickelung stark zu hemmen, wobei die 'Thatsache, dass die Entwickelung immer auf einem und demselben Stadium (Fig. 3) aufhörte, sehr charakteristisch ist.

${ }^{1}$ Dr. O. NoordgaARD. Ueber die Entwickelung der Lachseier im salzenen Wasser. Bergen, 1899. 
Es ist noch zu bemerken, dass der Mangel an Sauer'stoff in kurzer Zeit eine 'Trühung und die Entstehung vieler Ocltropfen in den Eiern hervorl'uft, nach welcher sie alle plötzlich absterben. Am besten ging also die Entwickelung. Wenn die Eier mu in einer schicht auf den Teller gelegt waren, wobei die Wasserschicht, mit der sie bedeckt waren, ganz dünn, also leicht zu durchlüften war. Selbstverständlich habe ich auch von Zeit zu Zeit das Wasser in den 'Tellern künstlich gasiert und durch frisches Wasser ersetzt ${ }^{1}$. Nach diesen Untersuchungen, die also unzweifelhaft gezeigt haben. dass die Befruchtung und die Entwickelung der Eicr von C. caspia im Meerwasser ebenso gut wie in ganz süssem Wasser vor sich geht, ging ich zu einer Reihe von anderen Experimenten über, um den Einfluss des Wasser's verschiedener Beschaffenheit auf die Entwickelmug der Eicr. sowie auch auf die ausgeschlüpte Brut der C.cospia zu untersuchen. Diese Untersuchungen legten inzwischen folgendes klar: 1. dass das stagnierende Wasser aus den Altwässern, die zu wenig Durchfluss haben, wo aber solche Fische wie Schleien, Brachsen, sogar auch Barsche noch gut gedeihen, fü l' die Eier del' C. caspia gar nicht geeignet ist; 2. dass das Wasser aus gut durchfliessenden Altwässeln, sowie auch aus dem Flus Wolga (der Arm "Podstepka ») im Gegenteil ganz passend ist, obgleich es im Frühjahr wegen einer Unmenge vou suspendierten Schlammpartikelchen selır trüb ist.

Was die postembryonale Entwickelung anbelangt, so geht sie im salzigen Wasser sogal bessel vol sich als im süssen, wo die ausgeschlüpfte Brut sehr oft von den Schimmelpilzen angegriffen wird.

Weitere Aufzucht der äusserst zarten, glasdurchsichtigen, kleinen (7 mm.) Heringe (Fig.4) in grossen, stark mit Vallysneria und verschie-

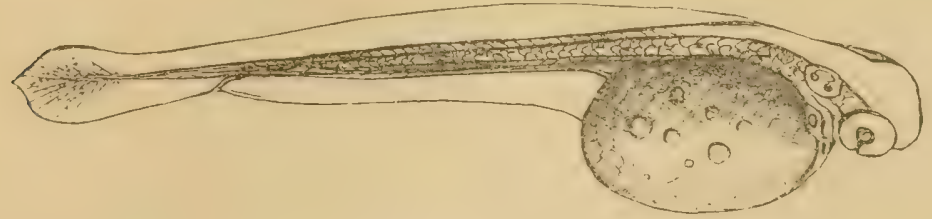

Fig. 4. C. caspia eben ausgeschlüpft. (Vergrösserung 13.)

denen anderen I'tanzen besetzten Aruarion gelang mir leider nicht, mol jedes Mal verschwanden alle kleinen Heringe zwei bis drei Tage nach dem Einsetzen, obgleich keine sichtbaren Fischschädlinge zu bemerken waren.

${ }^{1}$ In einem Falle habe ich auch mit Erfolg mittelst einer ganz dünnen Syphonröhre durchfliessendes Wasser für meine improvisierten Brutapparate geschafft. 
Sehr intelessante Beobachtungen habe ich inzwischen noch über die Zählol)igkeit des Spelmas von C. cospra gemacht. Die Samenfüden sterben in süssen Wasser fast plötzlich ah; im Meerwasser dagegen bewegen sie sich circa "/. Stunden lang sehr energisch (im Strudel) und sterben nachher allmählig ab.

Wir sohen also, dass die Samenfäden der kaspischen Finte sich denen dos Lachses gegenüber diametral entgegengesetzt verhalten, da nach den genannten Untersuchungen von Dr. O. NordfaAkd die Spermatozoen des Salmo salar im salzigen Wasser sehr bald (nach einer ${ }^{1 / 2}$ Minute) ahsterben, wogegen im Süsswasser ihre Bewegungen viel längere Zeit zu beobachten sind.

Alle diese Untersuchungen führten mich zu der Ueberzeugung, dass die kaspische Finteauch im offenen Meerezu laichen im Stande ist und dass also das Aufteigen in die Flüse (Wolga, Emba) für diese Art nicht unbedingt nötig ist. Diese Vol'aussetzung stimmt inzwischen mit den Angaben des Herrn Ir. N. Kímporitson, lant welehen laichreife und ebenso ausgelaichte Exemplare dor C. caspria im kaspischen Jeere schr weit von den Flussmündungen getroffen werden?. Jedenfalls geben diese Ergebnisse meiner Untersuchungen eine wichtige Inective fül weitere Untersuchungen in dieser Richtung.

D) natürlichen Laichplätze der ('. exspiu aufzusuchen, ist desto wichtiger, als dieser Fisch in Rusiland eine sehr grosse ökonomische Rolle in der Tolkseruährung spielt, sein Fang aber wegen der Ueberfischung während der letzten Decennien sehr stark abgenommen hat.

Ich bin überzeugt, dass einheitliche Untersuchungen mit der künstlichen Befruchtung in Wassel von verschiedenem Salzgehalte für die Biologie auch vieler anderer Fische sehr viel leisten könnten, und es ist nur zu bedauern. dass solche Experimente bis jotzt noch in so geringem Maasstabe unternommen worden $\operatorname{sind}^{3}$.

${ }^{1}$ Dr. O. NordgaARd Ueber die Entwickelung der Lachseier im salzenen Wasser Referirt in Wjestnyk Ribopromischlennosty, No 12 (russisch), 1899.

${ }^{2}$ Knıpowrisch, N. Hydrobiol. Untersuch. d. kasp. Meeres. Peterm. Geogr. Mittheil. Hefte $\mathrm{V}$, XII. 1904.

${ }^{3}$ C. H. Eigenmans. The fishes of San Diego. Procedings of t. U. S. Nat. Mus. Vol. XV. IVashington, 1892. 


\section{$3^{\text {me }}$ SECTION}

\section{E R T É B R É S}

Anatomie.

Sénces du lundi 15. mavdi 16, mreredi 17 al jeuli 18 aồ 1901.

Président: M. le Prof. F. S. MLontriceru.

Vice-Président: M. le Dr B. DEAN

Secrétaire: M. le $D^{r}$ E. Penard

\section{Ueber die Eier und Larven von Megalobatrachus maximus Schl.}

Von Dr. C. KERBERT (Amsterdam).

Wie ich bereits im "Zoologischen Anzeiger" ) Bd. XXVII, No. 10, 1904 in einem Aufsatze "Zur Fortpflanzung von Megalobatrachus maximus Schl. (Cryptobranchus japonicus v. d. Hœven) " mitzuteilen Gelegenheit hatte, war' ich im Jahre 1903 in der' glücklichen Lage, im Aquarium der Königlichen Zoologischen Gesellschaft "Natura Artis Magistra ) in Amsterdam zwei Riesen-Salamander zur Fortpflanzung zu bringen. Bis dahin war dieser 'Torgang nicht und nirgendwo gelungen. SASAKI ${ }^{1}$, der,

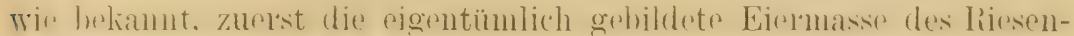
Silamanders in seinem natürlichen Wohngebiete, den kleinen Gohirgsbächen im Isa- und Isegebirge Japans, gesehen und nachher auch beschrieben hat, sagt: "All attemps to make Cryptobranchus breed in captivity have failed hitherto, owing no doubt to the difficulty of obtaining in the city cool water such as the animal is accustomed to in its mountain home. ») Unsere Gesellschaft erhielt die beiden Exemplare im Jahre 1893. Dieselben wurden von mir in einen Behälter ( $2^{\mathrm{m}} 11$ lang, $1^{\text {min }} 92$

1 SASAKI. Some Notes on the Giant Salamander of Japan (Cryptobranchus japonicus v. d. Hoven. Journal of the College of Science, Vol. 1, Part III, 1). 269, Imperial University, Japan. 1887.

VIe Corgn, INT. ZooL., 1904 
breit und 1,63 m. tief) unseres Aquariums untergebracht und zwar mit dem Zwecke, zu versuchen, ob es unter den offenbar für das Leben verschirdener Sep- und Süswassertiereso günstigen Finrichtungen unseres Aquariums vielleicht möglich wäre, die Tiere zur Fortpflanzung zu bringen, vorausgesetzt - was ich zu hoffen wagte, aber im voraus nicht wusste - dass dieselben wirklich als sexuell verschiedene Tiere sich entpuppen würden. Denn sicher ist es, dass der Unterschied zwischen männlichen und weiblichen Individuen des Riesen-Salamanders nur während der Brunstzeit ausgeprägt ist, und zwar durch die von mir später mit voller Bestimmtheit beobachtete Tatsache, dass beim Männchen (August und September) die cloacalen Wülste aussergewöhnlich angeschwollen sind, während dieselben beim Weibchen flach bleiben. Sehon zu Anfang des August 1902 verhielten sich die beiden Tiero anders als gewöhnlich. Während die durchaus trägen, stumpfsimngen Geschöpfe in der Regel Tage und Wochen lang bewegungslos, fast wie tot, auf dem Borlen ihres Behälters lagen, nur äusserst langsam nach den ihnen dargehotenen Fischen schnappten, das Licht schenten und immor die dunkelsten Stellen ihres Behälters aufsuchten, fingen dieselben im August an, sich einnander zu nähern und gegenseitig zu berühren. Manchmal wurden zittrinde und wellenförmige Bewegungen des ganzen Körpers wahrgenommen.

Die Vermutung lag auf der Hand, dass ein Erregungszustand des Nerrensystems als Einleitung zur Zeugung eingetreten war. I as Liebesspiel dauerte nur einige Tage. Während der Nacht des 18. September 1902 fand nun zum ersten Male in unserem Aquarium die Ablage der Eier statt und zwar durch das kleinere $\left(0^{\mathrm{m}} 85\right)$ der beiden im Behälter sich befindenden Individuen.

In Betreff des Geleges kamn ich die Beobachtungen Sasakis ${ }^{1}$ und IsurKAwAs ${ }^{2}$ in del Hauptsache bestätigen. Die photographische Aufnahme dieses Geleges ist deutlich. Das Weibchen hat die schönen, merkwürdig gebildeten Eierschnüre in vielfachen Windungen um eincn im Hintergrunde des Aquariums gelegenen Felsen abgelegt. Sie können ganz dentlich sehen, dass die Eier von Megulobutrachus maximus nicht einzeln abgelegt werden, sondern in Form einer rosenkranz-ähnlichen Schnur -. " in a string that resembles in form a rosary " - wie SASAKI bereits hervorgehoben hat. Von den Kapseln mit den eingeschlossenen Eiern kann ich Ihnen noch zwei Exemplare in Formol conserviert zeigen. Die Grösse der Eikapseln beträgt etwa $20 \mathrm{~mm}$; die Dicke der Kapselwand $2-2^{1} / 2 \mathrm{~mm}$. Die innere Kapselwand ist von ziemlich starker Beschaffen-

Sis.k. L. C., S. 27:;.

- Ismrawa. Ueber den Riesen-Salamander Japans. Mitteilungen der Deutschen Gesellschaft für Natur- und Völkerkunde Ostasiens, Bd. IX, Th. I, S. 92. 1902. 
Leit, stark lichtbrechend und wird nach aussen schichtenweise von andern Hüllen - Isumawa zählt deren 12-15 - umgeben. Die äussere, wohl im untern Teile des Oviductes gebildete Hülle zeigt deutlich eine spiralige Chalaza-förmige I)rehung, scheint also ziemlich zähel Beschaffenheit zu sein.

Die Fier zeigen eine sphäroidale Form mit einem [urchmesser von 7-5 mm. oder 6-4 mm. und eine gelbliche Farbe. Ich schätze die Anzahl der Eikapseln auf ungefihr 500 und mehr, obwohl ich annehme, dass die Zahl del Eikapseln und auch die Grösse dieser Eikapseln und der Eier eines Geleges je nach der Grösse des Tieres verschieden sein können.

Diese äusserst merkwürdige For'm des Geleges steht allerdings unter Berücksichtigung ganz bestimmt hervortretender Verschiedenheiten im Bau der Eihüllen oder der Eikapseln unter den Amphibien nicht ganz vereinzelt da. Bei C'ryptohranchus (Menopoma) Alleghrniensis Har. von Nord-Amerika sind nach Core ${ }^{1}$ die ziemlicin grossen Eier " attached by two strong suspensions at opposite poles ». Har sagt von Amphiume dass "the young, which now constitute the whole contents of the eggs -.- er meint wohl "Kapseln" - are surrounded by a transparent capsule about as thick as a writing-paper and these capsules are comnected by a slender corrd of simular substance. " Eine "rosenkranzförmige " Anord-

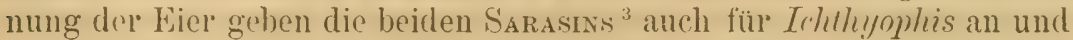
auch C. VogT ${ }^{4}$ hat ein solches Verhältnis bei Alytes obstetricans beschrieben.

Schliesslich finden wir, mach Cope " und Wruer "i hei Desmoguathus fuscus einen ähnlichen Bau des Geleges.

Obwohl nun durch diese Ergebnisse nachgewiesen ist, dass eine "rosenkianz n-förmige Anordnung des Geloges bei Amphibien keine Seltenheit, ja sogar bei sämtlichen Dorotremen Regel ist, muss ich nachdrücklich hetonen, dass sich die Eikayseln vom Riesen-Salamander doch dadurch von den Eikajuseln von verwandten Formen unterscheiden, dass die Eier immer bedeutend kleiner sind, als der innere mit einer Flüssig-

1 E. D. Cope. The Batrachia of North-America. Bulletin of the United States National Museum, No. 34, p. 42, 1889.

${ }^{2}$ O. P. Hay. Observations on Amphizma and its young. American Naturalist, Vol. XXII, p. 318, 1888. Mit einer Abbildung dreier Eikapseln mit Larven.

${ }^{3}$ P. und F. SARASIN. Ergebnisse naturwissenschaftlicher Forschungen auf Ceylon.

S. $10,1887-1893$.

${ }^{4}$ C. Vogt. Untersuchungen über die Entwichlungsgeschichte der Geburtshelferliröte. 1842.

${ }^{5}$ Cole. L. c., p. 196.

${ }^{B}$ Harris H. WiLder. Desmognathus fuscus (Raf.) und Spelerpes bilineatus (Green). American Naturalist, Vol. XXXIII, p. 236, 1899. 
keit gefüllte Kapselraum und also frei in dieser Flüssigkeit flottieren, während die Eier von Ichthyophis, Menopoma, Amphiuma und Desmogruthus fuscus und also auch später deren Embryonen den ganzen Kapselraum einnehmen.

Welche Rolle diese in der Eikapsel sich befindende Flüssigkeit, vielleicht eine ernährende Eiweissschicht, bei der Entwicklung der Embryonen vom Riesen-Salamander spielt, weiss ich noch nicht näher anzugeben. Diese Frage wird aber, wie ich hoffe, an der in diesem Jahre abzulegenden Eiermasse näher untersucht werden.

Die in unserem Aquarium im Jahre 1902 abgelegten Eier waren leider unbefruchtet. Nit dem Gelege vom 19. September 1903 - ungefähr sechs Uhr Nachmittags - erhielt ich aber vollständig günstige Ergebnisse. Wie nun die Befruchtung bei Megalobatrachus maximus stattfindet, kann ich leider noch nicht angeben. Diese Frage ist eine noch vollständig offene. Hoffentlich wird es mir noch gelingen, sie endgültig zu lösen.

Nach Beendigung der Eiablage in 1903 legte sich das Weibchen offenbar in grösster Ermattung in eine Ecke des Behälter's hin und kümmerte sich um das Gelege gar nicht mehı. Das Mïnnchen hingegen hat seitdem die Eiermasse nicht verlassen, ja sogar die Brut fortwährend bewacht.

Die Eiermasse war diesmal in einer sandigen Grube am Boden des Behälters angelegt. Die Bewachung des Geleges durch das Männchen geschieht mit einer solchen Treue, dass ich sogar nach einigen Tagen das Weibchen aus dem Behälter zu entfernen gezwungen war. Denn sobald das Weibchen der Eiermasse zu nahe kam, stürtzte das Männchen in sichtbarer Wut auf die Mutter los und vertrieb sie.. Unter diesen Umständen war die zeitliche Entfernung des Weibchens wohl notwendig.

Der' männliche Riesen-Salamander kriecht zwischen-den verschiedenen Sträingen der Eiermasse hindurch und bleibt dann von der Eiermasse teilweise umhüllt liegen, oder er legt sich einfach neben das Gelege hin. In beiden Fällen aber hält er, hauptsächlich durch eine pendelartige Bewegung des ganzen Körpers von Zeit zu Zeit das ganze Gelege in Bewegung. Durch diese Bewegung entsteht eine für den Atmungsprozess der Eier und Embryonen höchst wichtige Wasserströnung, während die Lage der Eiermasse hiedurch gleichzeitig fortwahtrend wechselt.

Das V'orkommen einer " münnlichen " BrutpHlege bei llegulobatrachns maximus war bisher unbekannt. Ismika wa erwähnt zwar ciner Brutptlege doch meint er, dass das Tier, welches von Anfang August bis zu Anfang Okitober fast in jedem Hansaki-Loch neben einem Eiklumpen gefunden wird, ein " weibliches " Tier sei. Auf welche Ierkmale hin Ismrkawa nun

1 Ishimawa. L. $c .$, p. 94. 
auf die "weibliche ») Sexualität des bei der Eiermasse verweilenden Tieres schliesst, ist aus einem, am 28. November $1900 \mathrm{in}$ der Ieutschen Gesellschaft für Natur- und Völkerkunde Ostasiens gehaltenen und im Jahre 1902 in den Mitteilungen dieser Gesellschaft veröffentlichten Vortrage nicht näher ersichtlich. Auch in der nenen Auflagr dieses Vortrages ${ }^{1}$ - nach dem Erscheinen meine' Mitteilung ${ }^{*}$ mit der Beifügung verschiedener Abbildungen veröftentlicht - wird ron Ismkawa nicht näher angegeben. nach welchen Beobachtungen orler Fiffahrungen "r* auf die " weibliche" Sexualität des 'lieres zu schliessen berechtigt war. Nach meinen Beobachtungen kümmert sich. wie schon hervorgehoben, das Weibchen um das Gelege gar nicht, und ist die "mämmliche " Brutptlege über alle Zweifel festgestellt.

Während der Fntwicklung der Eier vergrösser'n sich die Eikapseln nicht unerheblich. Während nämlich die frisch golegten Eikapseln bei unserem Tiere ungefüh $29 \mathrm{~mm}$. Durchmesser hatten, so ist der Durchmesser in dem Stadium, wo z. B. der Embryo schon eine Länge von 27 mm. ereeicht hat, die drei äussern Kiemenpare und die Anlages dor beiden Extremitaitempare schon unverkenubar entwickelt sind. bereits auf $23-24$ mm. zugenommen. Diese Vergrösserung der Kapsel und ihres flüsvigen, dem Embryo wahrscheinlich als Nährstott' dienenden Inhaltes, mag einer Aufnahme ron umgebendem Wasser durch die Kapselwand zuzuschreihen sein. Mit der Vergrösserung der Eikapseln geht eine allmähliche Abstossung der an der äussern Obertläche gelegenen Schichten del Kapselwand Hand in Hand; diese abgestossenen Schichten sicht man nachher im Wasser in Fetzen schweben.

Am 10. November 1903 waren die ersten, am 26. November fast sämtliche Larven ausgeschlüpft. Die ganze Entwicklung von der Eiablage bis zum Ausschlüpfen aller Lalven dauert also 52-68 Tage oder ungefähl acht bis zehn Wochen bei einel mittleren Temperatur des Wassers von $13^{\circ}$ C. Die ausgeschlüptte Larve hat eine Länge von ungefähr $30 \mathrm{~mm}$. Dic Larven yom 25. Oktober zeigen schon die äussern Kiemen und die Anlage der vordern Extremitäten. Bei den ausgeschlüpften Larven (27. November) ist auch die Anlage der hintern Extremitïten deutlich entwickelt, und die zweier Finger bereits nicht mehr zu ver'kennen. Jie Nundötfnung, noch deutlich ventral, wird jetzt allmählich terminal wie beim ausgewachsenen Tiere.

Auch die jüngsten Larvenstadien des Megalobatrachus maximus waren bisher unbekanut. I)ie kleinsten bisher bekaunten Larvensind beschrieben

${ }^{1}$ Proceedings of the Department of Natural History. Tokio Imperial Nuseum. Vol. I, Part I, 1904.

${ }^{2}$ C. Kerbert. Zur Fortpflanzung von Megalobatrachus maximus Schl. Zool. Anzeiger, Band XXVII, No. 10, 1904. 
von SASAKI ${ }^{1}$, hatten eine Länge von $19-20 \mathrm{~cm}$. und zeigten noch die Kiemen. Bei einem Individuum von $24,5 \mathrm{~cm}$. waren die Kíemen bereits geschwunden. I)as Larvenleben des Megalobatrachus maximus scheint also ziemlich lange zu dauern. Am heutigen Tage sind in unserem Aquarium noch zehn Larven am Leben; dieselben sind 9-10 cm. lang und entwickeln sich bei einer Temperatur von $13-20^{\circ}$ C. ausserordentlich gut. Die übrigen Larven sind ausführlicheren, embryologischen Untersuchungen geopfert.

Schliesslich muss ich mit Rücksicht auf die Priorität nachdrücklich betonen. dass die Abbildungen der Spermatozœn und der jüngsten Larvenstadien des Riesen-Salamander's in den in diesem Jahre von IsHrisw herausgegebenen "Beiträge zur Lienntnis des Riesen-Salamanders ), (Proceedings of the Department of Natural History. Tokyo Imperial Museum, Tol. I. Part I, 1904), na ch dem Frscheinen meiner Mitteilung im Zool. Anzeiger, Bd. XXYII, Yo. 10, 1904, publiziert sind, und dass bereits Dr. P. N. van Kampen in der Sitzung der Ned. Dierk. Ver. 30. Oktober $1903 \mathrm{im}$ Aquarium zu Amsterdam über die eigentümliche Structur der Samenfäden eine Mitteilung gemacht hat.

\title{
Some embryological evidence as to the position of Chimæra.
}

\author{
By Dr BASHFORD DEAN (New-York).
}

During the past half century various investigators have referred to the Chimaeroid fishes as picturing in many regards the ancestral jaw bearing vertebrate. And in this they have based their induction upon an extensive series of anatomical and palrentological facts. On the other hand, these evidences have been controverted vigorously by the upholders of the view that the ancestor of the ancestral vertebrate could not have been widely different from a moder'n shark.

Reviewing these evirlences. one must, I believe, speedily admit that the palicontological evidence in support of the view that the Chimeroid was the more generalized form is sadly defective, for no definite data dating from periods more remote than the Jurassic is at hand. The jaw plates of Ptyctodontids upon which all rely as exemplifying Devonian members of this group certainly rppear.Chimaroid, but as we know nothing clse (e. g., re body structures) regarling this family, it is hardly possible

1 SASAKI. L. c., S. 272. 
to decide whether they were really the ancestral Chimaroids or whether they may not have been equally well aberrant forms of sharks. The socalled tritoral points which are present in these plates resemble closely structures in some of the early Cestraciont sharks.

As to the anatomical evidence, we may note that the peculiar solid (holocephalous) character of the skull was regarded as a secondary condition even in the time of Johannes MüLLER, and that the majority of the much discussed anatomical leatures in this curious group are looked upon at the present time either as primitive, or as neotenial, or even as very highly specialized, according to the standpoint of the individual observer.

The embryological evidence upon this problem, however, still remains to be considered, and to this I purpose to refer briefly in the present paper. In this connection it may he recalled by those who are interested in the problem of the relationships of the Vertebrates, that the middle and late stages of the development of the Australian Chimaroid Callorhynchus, have been lately described by Schaunssuand, and that the younger stages, of the Californian Chimara colliei, have been referred to briefly (1903) by the present writer. At the present time it may be pointed out that in a number of important regards the development of Chimera indicates a remarkahle degree of specialization, and that these conditions can only be interpreted on the ground that the Chimaroid tishes in the course of their evolution have departed developmentally from the simpler features of sharks. In passing, I might refer to the following conditions:

The Chimæroids are highly specialized:

First: In the means fertilizing the eggs: in this regard witness, for example, the erectile frontal and ventro-lateral spines, and the peculiarly modified pterygopods. In the last, as in the genus Horriottu o1 Rhinuchimera, the component elements are fused into a single cartilaginous nailshaped plate.

Second: - In the remarliable egg caspules. These are fal mole highly specialized than in any known Elasmobranch. They are of gigantic size, measuring without their terminal organ for attachment relatively about one quarter the length of the parent fish: they are, moreover, oriented for the young fish at the time of hatching, having a definite region for its snout, trunk, tail and caudal filament, and even for its dorsil and ventral sides. The capsule is constructed, furthermore, so that a system of hreathing pores will weather through the shell at the appropriate time, and that an elaborate operculum will appear at the time of hatching. This capsule altogether affords an interesting case of almost perfect adaptation, one. by the way, whose origin it is difficult to understand on ihe ground of pure natural selection. For how may two morphologically 
independent characters $(i . e$. , in the developing embryo and in the capsule secreted by the oviduct) continue to coincide during selection of fortuitous variations in the different stages in development, so that in the end a species might continue to survive? Nor is it easy to understand its evolution on Lamarckian grounds, since a complete egg capsule would hardly have been moulded around an embryo when the latter was already so perfectly grown that the elaborate capsule could have been but useless.

Third: In the fertilization of the egg. Polyspermy occurs, but judging from stages in the male pronuclei the sperms do not enter the germ simultaneously : and thus fertilization takes place during an appreciable interval of time. Noreover, the supplemental sperm nuclei undergo at once amitotic division, whereas in sharks amitotic division follows only after a series of gradually changing mitotic divisions.

Fourth: In segmentation. Cleavage lines appear only after several divisions of nuclei have taken place. In this regard Chimiarn hehaves somewhat like the highly specialized ray among recent Elasmolranchs.

Fifth: In the precocious growth of the embryo. An embryo which, with its attached blastoderm suggests stage $B$ (BALForr) in the shark, is intrinsically much more perfectly developed e.g. in the details of its head, heart region and tail. In spite of minute size, it resembles more closely the shark stage $D$. The chimuroid embryo tends, accordingly, to assume its ordinal character's at a very early period, and in this regard it is to the shark as the teleost embryo is to the ganoid.

Sixth: In the early arrangement for the nutrition and development of the blastoderm, for it is found that the conditions of the periblast are remarkably complicated. Thus, it is found that even in an early stage of the blastoderm, one corresponding to $B$ in shark, the yolk nuclei are already widely different in different subgerminal regions. Especially clear is the way in which yolk cells are contributed to the blastoderm. Into the periphery of the latter are sometimes budded elements of such large size that they can in no way be confounded with the cells of the blastoderm. These cellular increments, moreover, cannot, I an convinced, be confounded with the so-called primordial germ cells, since I have traced their fate and found that in the majority of cases their descendants contribute to the development of the vascular system. Furthermore, in this precocious development of the blastorlerm, the laws hoth of the germ layers as well as of the behavior of amitotic nuclei appear to have undergone remarkable inversion. In the first regard, for example, there is clear testimony that the vascular system is in part (I refer particulary to the vessels which arise hear the mildle of the blastoderm) formed from ectoderm. In the second regard, in a large number of cases nuclei which have been undergoing amitosis pass into the blastoderm and even in certain cases continue for a time to undergo amitosis in the blastoderm. 
As this condition is seen abundantly in all carly gastrula, and as there are no temporary annexes of the embryo which these amitotic nuclei could form, one is compelled to recall the belief that mitosis is rather useful expression rather than a necessary mechanism of metabolic activity.

Seventh: In provision for the more effective nutrition of the embryo during later stages. Here particularly is to be referred the taking up of nutriment by the embryo via external gills and gut. In the shark the rolk material nourishes the embryo largely if not entirely by umbilical vessels. In Chimara, on the otherband, the mubilical mode of nutrition is less conspicuous since the yolk sac is of miniature size. A large part of the egg, it is found, is not enclosed by the down growing blastoderm. And this larger part of the egg, on the other hand, undergoes a process of fragmentation (which follows repeated division of the yolk nuclei), lesulting in the production of a creamy fluid which bathes the embryo and in which, therefore, the external gills are freely exposel. Interesting accordingly, is the fact that in the gill filaments are found at rarious points large blood knots in which numelous hrmacytes are found undergoing division. It may be mentioned also that for a considerable period the yolk laden ventral wall of the gut huds off directly into the gut lumen many small rolk masses, which, judging from mitoses in the arjacent wall of the gut, are of nutritive value. These conditions, it may he mentioned, are, as far as is at juresent known, unique in the rertebrate line.

\title{
De l'influence de l'alimentation sur la longueur de l'intestin. Expériences sur les larves de Rana esculenta.
}

\author{
Par le prof. E. YUNG (Genève).
}

Je renvoie pour l'historique de la question et la bibliographie au récent travail de Edward BABAK : Je rappelle seulement qu'on admet généralement une influence de la uature de la nourriture sur la longueur de l'intestin des animaux; celui-ci atteindrait son maximum de longueur chez les herbivores et son minimum chez les carnivores. Les omnivores tiendraient à cet egard le milieu entre les deux groupes précédents. De fait, l'observation tout en confirmant d'une manière générale cette r’egles.

${ }^{1}$ Ed. Babak. Ueber den Einfluss' der Nahrung auf die Länge des Darmkanals. Biolog. Centralblatt, Bd. XXIII, p. 477-483 et 519-528, 1903. 
constate qu'elle souffre de singulières anomalies et que dans un même groupe de Mammifères et d'()iseaux s'alimentant de la même facon, ou à jeu près, les chiffres exprimant le rapport entre la longueur de l'intestin ct la longueur du corps varient du simple au double, ou même au triple. ()r, il semble que la question puisse être étudiée expérimentalement et que, par là. on parvienne à déterminer d'une façon précise quels sont les facteur's diver's qui sont susceptibles d'influer sur le développement du tractus intestinal : qualité et quantité de la nourriture, durée de son séjour dans l'intestin, degré de son pouvoir nutritif, etc.

J'ai été conduit aux recherches actuelles par la constatation que chez l's Poissons et les Amphibiens soumis à un jeune prolongé l'intestin se raccourcit progressivement en même temps, d'ailleurs, que sa paroi s'amincit d'une façon très notable.

Ainsi, sur un lot de 10 Rana esculenta adultes $q$, pêchées le 25 octohre à Villeneuve au moment où elles allaient s'envaser pour passer l'hiver, le rapport de la longueur de l'intestin à celle du corps fut trouvé en moyenne de 3.65 et seulement de 3.14 che\% 10 individus ơ de la même provenance.

Dix individus femelles et dix individus mâles de la même taille que les précédents furent isolés et soumis à un jeûne absolu de six mois après lequel les 10 으. et les $7 \sigma^{\top}$ survivants, furent sacrifiés et leur intestin mesuré. Le rapport de la longueur de celui-ci à la longueur du corps fut trouvé en moyenne de 3.02 chez les $q$ et 2.77 chez les $\sigma^{\top}$. La longueur du colps mesuré de l'extrémité du museau à l'extrémité du coccyx étant restéc la même. la différence entre les chiffres indiqués ci-dessus ne peut être attribuée qu'au raccourcissement de l'intestin.

Jai constaté, d'autre part, des variations saisonnières du rapport en question chez les Grenouilles de la mème espèce. Au printemps l’intestin de celles-ci est relativement plus court qu'en automne. Voici, par exemple, les chiffres obtenus en mesurant l'intestin chez 12 femelles de $R$. esculentu dans la première semaine d'avril, et chez 12 fomelles de même taille et de même provenance que les précédentes, dans la dernière semaine d'octobre suivant. Le rapport de l'intestin au cor'ps chez les printanieres s'est trouvé de 3.28 et les automnales de 3.85. J'ai naturellement attribué cette différence au fait que les premières sortaient de leur jeûne hivernal, tandis que les secondes allaient y entrer et avaient derrière elles une période durant laquelle leur intestin n'avait pas chômé. Mais on sait en outre, que l'augmentation de masse des organes reproducteurs chez les femelles ovipares se fait au détriment des autres organes, il y a là un facteur qui peut également avoir joué un roòle dans la réduction de l'intestin au printemps. Je ne possede jas pour le moment de documents rolatifs a l'intluence de la saison sur la longueur de l'intestin des mâles, 
mais je ne doute pas qu'il en soit chez eux de même que chez les femelles.

Quant aux Poissons, je rappelle que nous avons observé, M. le prof. Fumranan et moi ${ }^{4}$ chez le Brochet (Esox lucius) et chez la Lote (Lota rulgaris) après un jêne prolongé durant dix ou donze mois, un raccourcissement de l'intestin pouvant mesurer plusieur's centimètres et atteindre jusqu'à $1 / 6$ de la longueur normale.

Voilà done un ensemble de faits qui démontrent une influence du repos forcé de l'intestin sur ses dimensions. ()uand celui-ci travaille il s'allonge; quand il ne fait rien, il se raccourcit. Or, il pourrait bien se faire que. conformément à l'opinion rappelée plus haut, l'allongement soit en proportion de l'intensité du travail et per'sonne ne contestera que celui-ci ne soit plus considérable lorsqu'il s’agit de digérer des végétaux et moindre lorsqu'il s'agit de substances animales.

Nous nous sommes adresse aux têtards de (irenouilles que l'anciennes expériences nous avaient montré pouvoir être alimentés exclusivement de plantes ou exclusivement de viande, au moins à partir d'un certain âge. Le régime normal de ces larves est mixte, c'est-ì-dire qu'elles introduisent d'ordinaire dans leur intestin du limon du fond des marais auquel se trouvent mêlés de la viande sous forme de Rotateurs, Infusoires et Rhizopodes, ainsi que du légume sous forme d'Algues, de Flagellés, etc. Tos premieres expériences remontent à 1897. Conduites à peu près de la même façon que celles de BABAK, elles ne nous ont pas conduit du premier coup à des résultats aussi satisfaisants. C'est pourquoi nous avions (lifféré leur publication; nous les avons reprises en 1899, puis en 1901 et enfin nous en avons fait une troisième série cette année même à la suite de la lecture du mémoire de notre collègue de Prague. Avant d'indiquer les résultats de 1901 qui sont les plus complets, il me semble nécessaire de résumer ici ceux obtenus par BABAK et de présenter à leur propos quelques remarques relatives aux difficultés d'obtenir dans les expériences de cette nature des résultats strictement comparables.

BABAK ne fait pas allusion à ces difficultés et son mémoire n'est pas explicite sur plusieur's points importants : $1^{\circ}$ On ne sait pas au juste sul les larves de quelle espèce il a travaillé, car il dit bien avoir observé des têtards de Ranu temporaria (et esculenta), mais il ne spécifie pas sur lesquels ses mensurations ont porté. Il importerait cependant de le savoir et d'être certain que ses aquariums ne contenaient pas un mélange des deux espèces de larves, car il résulte des chiffres publiés par Gaup ( $A$ untomie des Frosches, 3. Abth., pg. 65. 1904), que l'intestin de Rana temporaria (firsca) adulte, est moins long que celui de Rana esculenta. Peut-

1 Voir: Compte rendu de la $82 m e$ session de la Société helvétique des Sciences naturelles. Arch. des Sc. phys, et nat. Octobre, 1899. 
être la différence spécifique ici signalée, se manifeste-t-elle dès l'état larvaire, auquel cas on doit en tenir compte. $2^{\circ}$ BABAK est muet sur l'âge des têtards au moment oì ils furent soumis au régime expérimental; où les avaient-ils pris, comment avaient-ils été nourris jusqu'alors, avaientils tous le même âge au début de l'expérience? $3^{\circ}$ Babak n'indique pas si les chiffres qu'il public dans son tableau, p. 523, sont des moyennes prises sur un même nombre d'inclividus dans chaque cas particulier; il se contente de citer une moyenne de 60 mesures dans un seul cas. Il nous sera très difficile dans ces conditions de nous rendre compte des raisons pour lesquelles nos résultats diffèrent des siens sur certains points.

Nous avons constaté, au cours de nos mensulations, des différences individuelles tellement considérables que nous nous sommes posé comme règle d'élaguer les individus qui s'écartaient beaucoup de la moyenne. 1)ans un mème vase où se développent au milieu de conditions identiques, des larves issues d'une même ponte, on voit les unes crôitre avec une excessive rapidité alor's que d'autres demeurent de véritables avortons. Ces individus, géants ou nains, s'écaltant du vulgum pecus, nous n'en avons pas tenu compte. Nous avons aussi supprimé de nos moyennes les individus qui, de taille extérieure normale, offraient un intestin extraordinairement plus long ou plus court que leur's congénères de même taille. Dans chaque catégorie nous avons rencontré des individus évidemment anormaux sous ce rapport qui, introduits dans nos moyennes, les aulaient sîuement faussées. D)ans la règle, les chiffres que l'on trouvera plus loin sont des moyennes tirées de mensurations faites sul 10 individus. Lorsque par défaut de matériel ce nombre de 10 observations n'a pu être atteint, je l'ai indiqué en note.

Babak a trouvé que la longueur de l'intestin mesurée quelques semaines avant la métamorphose du têtard en Grenouille est 7 fois la longueur du corps chez les larves végétariennes et de 4,4 seulement chez les larves carnivores. Le maximum constaté par lui chez ces dernières fut 4,9 et le minimum parmi les végétariennes fut 5,7. (Nous avons constaté des différences individuelles bien plus grandes.)

En comparant le rapport de l'intestin au corpos, à divers stades de développemrent (celui-ci apprécié par le degré de croissance des pattes), BAbak conclut que l'intestin commence par s'accroître très rapidement durant une période qui se termine au moment où les pattes postérieures approchent de leur formation définitive. C'est alor's que l'intestin atteint son maximum de longueur. A partir de lit, il se raccourcit, plus vite naturellement chez les larves végétariennes que chez les carnassières, puisqu'elles ont l'intestin le plus long et que, à la fin des métamorphoses, celui-ci est réduit à la même dimension chez les unes et chez les autres. Jusqu'i cr que la queue du têtard soit completement résorbée et qu'il ait 
été complètement transfor'mé en petite Grenouille, l'intestin des individus nourris aux plantes est un peu plus long que chez ceux nourris à la viande.

Mes expériences de 1901 ont porté exclusivement sur des larves de Rana esculentu nées au laboratoire d'une même ponte d'œufs recueillis le 7 avril. Elles furent durant 15 jour's nourries de l'alhumine de leurs œufs, de limon, de Spirogyres et des cadavres de leurs semblables, alimentation mixte que leur offre normalement la nature. Le '22 avril, 100 larves furent isolées dans un vase $(A)$ dont l'eau était chaque jour renouvelée et où elles ne reçurent dès lor's que des aliments végétaux. Pendant le premier mois ec furent des Spirogyres soigneusement lavées et l'enouvelées afin de combattre la multiplication des Infusoires. (Il va sans dire qu'un certain nombre de ces derniers restaient toujours, en sorte que, pour végétal qu'était le régime, il ne l'était point absolument. Inversément, notons dès maintenant, que dans le vase à viande, il se développait sur celle-ci et contre les parois du vase des Saprolegnia que broutaient les têtards, en sorte que pour animal que fût le régime de ces derniers, il ne l'était pas non plus d'une facon parfaite.) A partir de la fin du mois de mai, ayant eu de la peine à me procurer les algues je les remplaçai par des feuilles de laitue que les têtards mangent fort bien quoiqu'ils ne les rencontrent pas dans les cir'constances ordinaires.

100 autres larves furent placées dans un vase $(B)$ identique au précédent où, toutes choses égales d'ailleurs, elles furent nourries avec de la viande. Au début ce fut pendant qnelques jour's des débris de manteau et de branchies d'Anodontes r'écemment mortes, ensuite, et d'une façon constante jusqu'à la fin, avec de la viande de houcherie (bouf et veaul) coupée en morceaux et renouvelée tous les deux à trois jour's.

Enfin, les larves restantes au nombre de 2 ou 300 dans le vase initial (que nous appellerous vaso ( $\left.C^{y}\right)$ furent soumises à un régime mixte composé d'une couche de limon joris au fond d'un marais et dont, selon leur coutume à l'état de nature, les têtards se r'emplissaient l'intestin, digélant les micro-organismes tant végétaux qu'animaux qui s'y trouvent mêlés à des substances terreuses, puis de plantes aquatiques S'pirogyra, Auncharis, ete., et le viande (cadavres de têtards, viande de Poisson, etc.).

J'ai démontré précédemment' que les larves de Glenouille nourries à la viande s'accroissent plus rapidement que les vécétariennes. Cela est appréciable à l'œil déjà au bout de quelques jours. Le (j mai, les larves du vase $B$ mesuraient en moyenne près de $2 \mathrm{~mm}$. de plus que celles du vase $A$ (la longueur du corps étant toujours prise de l'extrémité du mu-

${ }^{1}$ Arch. des Sc. phys. et nat., t. VII. 1852 et Arch. de Zoologie expérimentale, $2^{\text {tne }}$ série, t. I. 1883. 
s'au à l'anus), leur intestin (mesuré de l'extrémité du museau à l'anus après aroir été déroulé) ('n revanche, était beaucoup plus court que chez. ces dernières. Voici les moyennes:

\section{6 mai.}

Longueur du corps . .

Longueur de l'intestin . .

Rapport
Vase $A$.

$8^{\mathrm{mm}}$
62
7,7

Vase B. Vase $C$.

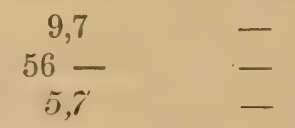

Il est ì remarquer que, dès cette date, c'est-à-dire quinze jour's après lo début de l'expérience la différence de régime se manifeste par une diffélence très notable de la longueur de l'intestin. Les chiffies précédents indiquent une différence moyenne de 6 mm., mais pour apprécier plus justrment celle-ci, il faudrait mesurer des individus de même taille pris dans les deux locaux, car la taille influe sur la longueur de l'intestin. Ainsi, 3individus de même âge, choisis parmi les plus petits, les moyens et les plus grands du vase $A$ et (Lu vasr $B$ fournirent à cet égard les chiffres suivants:

Vase $A$.

$\begin{array}{cccc}\text { Corps } & 5 \frac{1}{2} & \text { intestin } & 41 \\ \text { " } & 7 \frac{1}{2} & \text { " } & 54 \\ \text { » } & 10 & \text { » } & 73\end{array}$

Vase $B$.

$\begin{array}{clcc}\text { corps } & 7 & \text { intestin } & 40 \\ \text { » } & 91 / 2 & » & 52 \\ \text { » } & 12 & \text { " } & 60\end{array}$

On voit que le plus grand du vase $A$ ayant à peu près la même taille que le moyen du vase $B$, portait un intestin plus long de $21 \mathrm{~mm}$. A cet âge et à égalité de taille, les végétariens pouvaient donc avoir déjà $2 \mathrm{~cm}$. environ d'intestin de plus que les carnivores.

Remarquons aussi que rlans les deux vases $A$ et $B$, les frères et soeurs qui s'y développaient sous l'influence d'un même réğime alimentaire, présentaient entre eux des différences de taille allant jusqu'à $5 \mathrm{~mm}$., c'esta-dire (pour ceux du vase $A$ du moins) allant du simple au double et des différences dans la longueur de l'intestin allant à plus de $20 \mathrm{~mm}$. Ces diffélences s'accentuèrent jar la suite, car les plus gros mangeant toujours davantage et les plus petits faisant tout le contraire, leur's divergences ne peuvent qu'aller en croissant. Du reste, j'ai eu dans les deux vases des avortons qui mangeaient très peu, juste de quoi ne pas mourir de faim et qui vécurent plus de trois mois sans grandir. Enfin, je dois ajouter que la mortalité fut all comnencement de l'expérience plus forte dans le vase $B$ que dans le vase $A$. Après la sixième semaine, c'est l'inverse qui se produisit; les larves végétariennes non seulement restèrent en arrière au point de vue de leur croissance, mais leur nombre diminua par le fait de morts fréquentes: les cadarres étaient, bien entendu, soustraits à la 
voracité de leur's congénères. Durant le mois de mai tout alla à soulhait et le 30 , nous obtînmes les moyennes suivantes:

\section{0 mai.}

Longueur du corps . . .

Longueur de l'intestin . . Rapport .
Vase A.

$$
\begin{array}{r}
13,66 \\
116,80 \\
8, \tilde{5} 4
\end{array}
$$

Vase B. 14,20

$78,-$

5,48
Vase $C$.

8,62

A ce moment. aucune des larves n'avait de membres. Cependant l'élauche des pattes postérieures commençait à itre perceptible cher quelquesunes du vase $C$ : Les différences au point de vue qui nous occupe se sont accentuées entre régétariens et carnivores. Ici, nous avons dû̀ éliminer dr nos moyennes deux individus carnivores dont le corps de dimension normale contenait un intestin deux fois et demi seulement plus long que le corps. raccourcissement excessif que j'ai attribué à un arret dlans l'alimentation, car chez ces deux individus le tube digestif était vidle. Le même fait s'est produit chez un individlu végétarien dont l'intestin était relativement beaucoup plus court parce qu'il avait cessé de manger. Nous verrons plus loin l'effet du jeûne expérimental sur l'intestin. Or', dans la nature il arrive que spontanément et saus qu'on sache pourquoi, des larves apparemment saines ne premnent plus de nourriture et vivent quand même durant des semaines, cela est inclépendant de la qualité des aliments, car nous en avons rencontré des exemples dans lo vase C $($, aussi bien que dans les deux autres. Nous avons considéré ces larves comme des malades atin de les exclure dle nos mesures dont elles auraient par trop altéré les moyennes. Et puisque j'on suis à signaler des anomalies, je citerai encore celle offerte par certaines larves dont l'intestin se remplit de gaz à tel point qu'elles flottent et ne réussissent plus à plonger' l'intestin de ces - malheureuses est distendu, déformé, je n'en ai pas tenu compte.

Durant le mois de juin, la mortalité, ainsi que le nombre des lentardataires augmenterent dans tous les vases, mais principalement dans le rase $A$. Chez les bien portants, les pattes postéricures apparurent. mais dans chaque vase à des dates fort espacées, de sorte qu'il devenait difficile de trouver un lot de dix individus à peu près pareils pour être soumis aux mensurations; les uns avaient les pattes un peu plus saillantes que les autres et parmi ces dernier's il en était dont ces organes se trouvaient si courts encore qu'il était impossible de les mesurel exactement. J'envie Babak d'avoil trouvé dans ses aquar'iums un nombre suffisant d'individus ayant les jambes postérieures de $1,2,4$ et $7 \mathrm{~mm}$. pour lui fournir les moyennes (a supposer que ce soient des moyennes) de son tableau. Il est vrai qu'il a pris ses mesures sur des individus conservés. tandis que nous avons opér'é sur des individus frais préalablement chloroformés ou sur rles indivilus récemment trouvés morts dans les vases. 
Je passe donc sur les termes intermédiaires observés sur un nombre insuffisant d'individus morts en cours de route et j'en arrive au tableau suivant datant du 20 juin. Ce jour-là, je pus puiser dans le vase $A$ huit larves et dans chacun des vases $B$ et $C$ dix larves dont les pattes postélieures étaient bien développées sans que les antérieures fussent encore visibles. Je pense que ces larves peuvent être approximativement mises en parallele avec celles du groupe $V$ (pattes postérieures et pied long de $7 \mathrm{~mm}$.) du tableau de BaвaK.

$$
20 \text { juin. }
$$

Longueur du corps . . : .

Longueur de l'intestin. . .

Rapport .
Vase A.

6,9

\section{Vase B. Vase C.}

14,5

$72,-$

$$
4,9
$$

Somme toute, ces chiffres sont supéricur's à ceux trouvés par le physiologiste de Prague et en diffèrent par là, que les larves nourries avec l'aliment mixte ont présenté un intestin relativement plus long que celles alimentées seulement avec les plantes. Pour le reste, quoique la longueur du corps des larves soit demeurée sensiblement la même que celle des larves mesurées le 30 mai, c'est-i-dire que, durant cet intervalle de temps la croissance de leur trone se soit arrêtée ${ }^{1}$. leur intestin a très sensiblement diminué.

J'attribue ce raccourcissement intestinal au fait que pendant la période de genèse des pattes, les têtards mangent peu ou pas du tout et que, par conséquent, ils rentrent dans les conditions de jeùne des animaux à intestin raccourci dont il a été question au début de ce mémoire. Une fois les pattes postérieures sorties, l'appétit ralenti se réveille. C'est là un point intéressant que Babar n'a pas remarqué. Selon lui. à partir du raccourcissement intestinal qui se manifeste lors de la croissance des pattes de derrière, l'intestin ne cesse plus de décroître. Mes mesures démontrent au contraire qu'entre l'époque où les pattes postérieures achèvent de se développer et celle où les pattes antérieures commencent à le faire, il y a un intervalle de temps fort court, il est vrai, pendant lequel les têtards mangent abondamment et allongent de nouveau leur intestin. Je m'en apercus dans les expériences de 1901 que je

${ }^{1}$ A strictement parler les moyennes semblent même indiquer une légère diminution de la longueur du tronc, mais cela peut provenir d'un simple hasard qui m'a fait tomber sur des individus relativement courts; ce ne sont nullement les plus grands de taille qui sont les plus avancés dans leurs métamorphoses. Du reste les moyennes seraient différentes si au lieu de prendre la longueur à l'anus on l'avait prise à l'extrémité de la queue, celle-ci est relativement plus longue chez les tétards munis de leurs jambes de derrière que chez les apodes. 
relate ici, en mesurant l'intestin le 30 juin sur des individus de même taille que la taille moyenne de ceux mesurés le 20 du même mois. Pendant les dix jour's qui séparent ces deux dates, les têtards munis de leur's pattes postérieures, mais n'ayant pas même encore visible à l'œil nu l'ébauche de celles de devant. se montraient fort alertes et mangeaient fréquemment. Or, dans tous les vases, ceux au nombre de trois seulement qui servirent aux mesures, avaient l'intestin plus long que leurs semblables de même taille mesurés dix jour's plus tôt.

$$
\text { 30juin. Vase A. Vase B. Vase C. }
$$

Moyennes de 3 individus dans chaque vase.

$\begin{array}{lcccr}\text { Longueur du corps . . . . } & 13,5 & 14,5 & 14,- \\ \text { Longueur de l'intestin . . . } & 100,- & 76,- & 110,- \\ \text { Rapport . . . . . . . . } & 7,4 & 5,2 & 7,8\end{array}$

Le fait que ces chiff'res mettent en évidence a été confirmé par les têtards que j'ai soumis cette année même à des expériences toutes semblables a celles qui nous occupent en ce moment. Je dois dire cependant que si les têtards de cette année ont, comme ceux de 1901, gardé leur taille au stade do développement intermédiaire à l'apparition des deux paires de membres, ils ont à un moindre degré que ne le marquent les chiffres ci-dessus allongé leur intestin. Il y a du reste, des différences notables à d'autres points de vue entre les têtards de cette année et ceux des années précédentes. Nous en reparlerons ailleur's.

Un autre point sur lequel mes résultats ne coïncident pas avec ceux de B.Bar est relatif à la rapidité avec laquelle l’intestin décroît. Selon lui, le rapport de la longueur de l'intestin à la longueur du corps est, chez les tetards dont les pattes postéricures mesurent $8 \mathrm{~mm}$. et dont les pattes antérieures sont encore repliées sous la peau, à peu près le quart pour les végétariens et la moitié pour les carnassiers de ce qu'il était chez ceux dont les pattes postérieules mesuraient 4 mm. Et lorsque les extrémités antéricuress sont extériorées, ce rappolt aurait encore diminué de plus du tiers. Comme Babak, nous l'avons déjà dit. ne donne pas lés dimensions du corps et de l'intestin, mais seulement leurs rapports, nous ne pouvons marquer "n chiffres absolus l'importance et la vitesse du raccourcissement intestinal, mais elles ont toutes deux dû être très grandes chez les larves sur lesquelles il a operé. Chez les nôtres les choses se sont effectuées plus calmement.

Pour bien en saisir le sens, résumons les points acquis jusqu'au 30 juin. Des larves nées le 7 avril, nourries toutes de la même façon pendant quinze jours, puis alimenteces les unes avec des plantes, les autres arec de la viande et d'autres encor'e avec un régime mixte, ont développé leur' intestin de façon trè diftérente; les régétaricnues et les mixtes l'ont 
développé beaucoup plus, en longueur du moins, que les carnassières. Toutes, d'ailleurs, ont allongé leur intestin jusqu'au 30 mai, puis toutes l'ont raccomrei durant la genèse de leurs pattes postérieures, et toutes encore l'ont de nouveau allongé pendant une courte période se terminant au 30 juin, après que leur's pattes postérieures eurent entièrement poussé et avant que leurs pattes antérieures fussent devenues apparentes.

A partil de ce moment. c'est-à-dire apres que les pattes antérieures en voie de croissance eurent formé derriere les branchies les deux verrucosités annonçant leur apparition prochaine, l'intestin décrît pendant que la taille continua à s'accroître. Le moment précis où commence cette décroissance de l'intestin, laquelle s'accentue dès Iol's jusqu'à la métamorphose dernière, est celui où l'appétit, un moment réveillé après l'apparition des pattes de derrière, s'apaise de nouveau. Les têtards ne cessent point completement de manger, mais la quantité de nourriture qu'ils ingurgitent est beaucoup moindre, ainsi qu'en témoigne l'examen direct du contenu intestinal remarquablement diminué, surtout chez les végétariens dont le tube digestif au lieu d'être distendu comme dans les périodes de fringale est partiellement vide.

Pour apprécier les détails de la marche régressive de l'intestin, il faudrait la mesurer à intervalles de temps plus courts et sur un plus grand nombre d'individus que nous n'avons pu le faire. Il ne nous restait à cette phase de notre expérience qu'un petit nombre de têtards dans chaque vase, ì cause de la mor'talité assez forte, surtout chez les végétariens, et à cause des prises précédentes. Nous dûmes malheureusement réduire nos mesures à cinq individus de chaque catégorie. Les moyennes suivantes sont donc celles de cinq mensurations serlement.

\section{3 juillet. Vase A. Vase B. Vase $C$.}

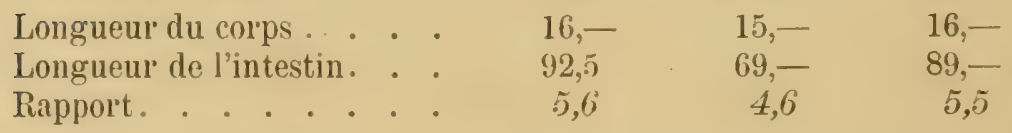

A cette date les pattes antérieures n'étaient point extériorées, mais en voie de croissance chez tous les individus mesurés. Dans chaque hocal il y avait encore des arriérés apodes qui, introduits dans mes moyennes les auraient sûrement moditiées. Dans le vase $C$ 'seul se trouvaient des individus à quatre pattes, mais je n'en ai pas introduit dans mes moyennes. Il est clair qu'on pent me r'eprocher d'avoir choisi mon matériel de mesure, car si j’avais puisé au hasild dans mes vases, les résultats eussent été différents, mais pour le but que je poursuivais, lequel n'était point d'établir une statistique de la croissance des têtards, mais d'observer l'influener 
du régime sur la longueur de l'intestin, il m'a parı indispensable d'écar'ter les individus qui, cessant de manger et de croître, maintenaient à unc: période donnée l'état des choses de la période précédente; ces individus devaient done, comme nous l'avons déjà dit, être considérés comme des anor'maux.

Le 23 juillet, il ne restait plus qu'une vingtaine de têtards dans les bocaux $A$ et $B$ qui, au début de l'expérience, trois mois plus tôt, en avaient recu chacun cent. Les prélèvements pour les mosures ci-dessus et celles dont il sera question plus loin avaieut provoqué ce déficit. Ces vingt survivants croissaient d'ailleurs lentement, surtout daus le vase $A$, beaucoup plus lentement que les têtards dans la nature lesquels avaient, autant que je pus m'en assurer, achevé leur's métamorphoses dés la première quinzaine de juillet. Ce retard s'explique par l'étroitesse de la prison dans laquelle étaient enfermés mes captifs (surface d'aération $\left.346 \mathrm{~cm}^{2}\right)$, par l'absence de soleil dans le laboratoire. l'absence de limon au fond des vases, etc. Ce dernier facteur présent, l'on s'en souvient, dans le vase $C$, suffit à lui seul pour permettre aux têtar's de ce vase de se transformer plus tôt que les autres, les petites grenouilles à queue s'y rencontraient déjà le 23 , et le 30 il y avait une petite grenouille ayant entièrement perdu la queue. Je n'en obtins de telles dans le vase $B$ que le 12 août et parmi les larves végétariennes du vase $A$ aucune n'en arriva à ce point. A la fin d'août, je ne trouvai parmi ces dernières qu'une seule grenouille à peu près achevée, elle mourut le 26 de ce mois sans avoir entièrement résorbé sa queue. A cette date il y avait encore dans le même vase quatre individus apodes et deux munis de leurs seules pattes postérieures.

Néanmoins, je pus me procurer au commencement du mois d'août trois individus à peu près au même point de développement dans chaque vase et possédant les pattes antérieures entièrement développées et une queue non encore en voie de régression. Ils n'ont point été mesurés le même jour: les trois du vase $C$ furent pris le 3 aout, ceux de $B$ le 7 , et ceux de A le 13 août; je les crois, malgré cela, assez comparables pour figurel' sur le même tableau.

\section{Commencement d'aô̂t.}

Longueur du corps . . . Longueur de l'intestin . . Rapport

$\begin{array}{ccc}\text { Vase } A . & \text { Vase } B . & \text { Vase } C \\ 17 & 17 & 17 \\ 48 & 46 & 57 \\ 2,82 & 2,70 & 3,35\end{array}$

On voit que les rapports encore fort différents le $2 \%$ juillet. se ressemblent davantage 15 jour's plus tard. Les individus non seulement ont la même taille, mais aussi à peu près la même longueur d'intestin, et si l'on se reporte au tableau précédent, on conviendra que durant cette der- 
nière période, ce sont les végétariens qui ont eu le plus à faire pour se mettre sur les rangs de leurs frères carnivores. Le moindre raccourcissement de l'intestin des omnivores est probablement dî au limon qu'il contenait encore et qui, nous le verrons bientôt, fait obstacle au retrait de l’intestin sur lui-mème. Mes chiffres, quoique plus élevés que ceux de BaßAк confirment cependant ici ses résultats: réduction générale de l'intestin (quelle que soit la longueur que celui-ci ait atteinte sous l'influence (lu régime) s’accélérant dans les dernières pliases de la métamolphose.

Où nous sommes en désaccord, BabaK et moi, c'est sur la date où commence cette réduction: lui la place pendant la croissance des pattes postérieures et moi seulement après que ces mèmes pattes ont complètement poussé.

Quant aux Grenouilles anoures, je ne puis fournir des moyeunes attendu que si j'en récoltai sept dans le vase $C$, je n'en eus que deux en $B$ et zéro en $A$. Je dirai seulement, à leur propos, que la moyenne des sept omnivores indique le rapport 1,54 et les chiffres obtenus sur les deux carnivores fixent chez l'une ce rapport à 1,54 aussi, et chez l'autr'e à 1,42 , soit en moyenne 1,4s, chiffire peu différent de la moyenne des omnivores. Je tiens done pour acquis le résultat de Babak, relatif à l'identité de la longueur de l'intestin chez les Grenouilles issues de larves soumises à des régimes différents et j'attribue les différences absolues de nos chiffres ou bien à ce que nous n'avons pas opéré sur des larves de même espèce, ou hien à ce que parmi les jeunes Grenouilles de la mème espèce, il est très possible que les différences puissent égaler, celle indiquée par nos chiffres 1,42 contre 1,1 .

On sait en effet, que dans la nature les têtards et les petites Grenouilles qui en naissent ont l'intestin plus long que ceux mis en expérience (BABAK), et que sous des influences encore mal connues, il se produit dans certaines localités des têtards de taille démesurément grandes. 11. Lucoris, directeur de l'établissement de pisciculture de Gremaz (Ain), voit souvent de ceux-ci se développer dans ses hassins ou dans leur voisinage et il les considèe comme des têtards de 2 ou même de 3 ans; peutêtre a-t-il raison, car on connaît en effet de tels têtar'ds dans les sources froides des montagnes où l'hiver est précoce et qui, n'ayant pas le temps de se transformer avant les premiers gels attendent l'été suivant pour le faire; mais comme Gremaz n'est point élevé et que ses eaux ont une température constante de $14-15^{\circ}$, il est possible aussi que ces têtards géants soirnt le résultat d'une suralimentation facile à se procurer dans des eaux comme celles de Gremaz où pullulent les petits Crustacés dont on soigne la multiplieation en vue mène de la pisciculture? Quoiqu'il 'n soit, au mois de juin de cette année, je rapportais de Gremaz une centaine de têtards très actifs dont la taille était au moins double de la 
taille moyenne des têtards de Rana esculenta que j'étais en train d'élever in vitro. Appartenaient-ils à cette espèce? Je ne saurais l'affirmer. En tout cas, il s'agissait de têtards de Grenouille et non de Crapaud, en sorte que, comme nous ne possédons chez nous que deux espèces de Grenouilles $R$. esculenta et $R$. temporaria, il ne peut y avoir d'hésitation qu'entre olles deux. Ces têtards possédaient pour la plupart leus pattes postérieures, quelques-uns étaient aporles et quelques-ms anssi avaient les pattes de devant; comme ils se montraient très voraces, je les nour'ris au laboratoire avec de la viande de boucherie qu'ils mangèrent avidement, je fus curienx de mesurer leur intestin sur des individus aux trois stades de développement qui viennent d'être indiqués. Voici les chiffires moyens obtenus fin juin sur cinq individus de chacune de cesc catégories de grands têtar'ds.

Apodes. Pattes postérieures. Pattes antérieures et queue.

$\begin{array}{lccc}\text { Longueur du corps . } & 26,4 & 37,- & 28,5 \\ \text { Longueur de l'intestin. } & 335,- & 337,5 & 81,- \\ \text { Rapport. . . . . . . } & 12,6 & 9,12 & 2,84\end{array}$

Malgr'é l'énor'me développement de l'intestin de ces têtar'ds nour'ris dans la nature ${ }^{1}$, lequel avant l'apparition des pattes postérieures atteignait jusqu’à $335 \mathrm{~mm}$. on voit cet organe diminuer et réduit au quart quelque temps avant la dernière métamorphose.

Dans ce lot, j'ai obtenu plusieur's petites grenouilles dans la première semaine de juillet; le rapport de la longueur de l'intestin au corps fut trouvé chez elles de 2.58 , chiffire identique à celui obtenu en plenant les memes mesures chez des petites grenouilles recueillies a Gremaz en juin. Il se produit donc dans la nature des Grenouilles issues de larves géantes dont l'intestin présente une longurur mative heancoup plus ('onsidérable que celle observée par BaBAK et moi dans nos expériences. Nous ne pouvons, par conséquent, donner nos chiffres comme typiques, ni prétendre qu'ils représentent un caractere spécifique absolu. Il serait intéressint do mesurele l'intrstin comparativement chez un nombre suffisant de larves de même âge et appartenant à des espèces diverses de grenouilles parfaitement déterminées. Je ne crois pas que cela ait été fait jusqu’à présent.

${ }^{1}$ Babak cite des tétards apodes dont l'intestin de $250 \mathrm{~mm}$. égale 16 fois la longueur du corps. Chez les mêmes, munis de leurs pattes postérieures ce rapport était descendu à 9 puis à 1,7 chez les petites Grenouilles issues d'eux. Il semble donc exister de fortes différences des uns anx autres. 
Toici maintenant. condensés en un seul tableau, les résultats exposés ci-dessus.

Rapports de longueur entre l'intestin et le corps chez les larves $A, B$ et $C$ nées le 7 avril.

Date. Age.

Vase A. Vase B. Vase C. Régime végétal. Régime animal. Rég. mixle.

6 mai

(29 jours) larves apodes . . . 7,70

5,70

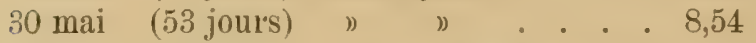

5,48

8,62

20 juin

(73 jours)$$
\text { ave }
$$

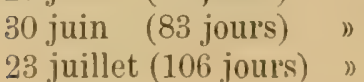

et ébauche des antéres 5,60

) . . 7,40

4,90

5,20

7,40

23 juillet (106 jours)

))

3 au 13 août (117 à 127 jour's) larves avec

Petites Grenouilles

4 pattes 2,82

4,60

5,50

2,70

3,35

1,43

1,56

L'influence du régime est indéniable, les aliments végétaux contribuent à l'allongement de l'intestin davantage que la viande. Le régime mixte conduit à peu près aux mêmes résultats que le régime végétal. En même temps que l'intestin s'allonge, ses parois s'amincissent, ainsi que l'a for't bien vu BarAK; il devient fragile et l'on a beaucoup de peine ¿̀ le dérouler saus le r'ompre. Un autre caractère différentiel sur lequel je n'ai pas pris de mesures, mais qui saute à l'œil à l'ouverture des tétards, est le plus fort diamètre de l'intestin chez les têtards carnivores; il semble, par conséquent, qu'ils gagnent d'un côté ce qu'ils perdent de l'autre. Il y aurait là un joli sujet d'étude pour un naturaliste qui aurait la patience de prendre de nombreuses mesures comparatives. L'intestin, à l'âge tendre où nous avons mis nos larves en expérience, paraît être comme un tube élastique s'allongeant sous la pression d'une plus grande quantité de nouriture (végétariens) et revenant sur lui-même lorsque la quantité de nourriture est moindre (carnivores) ou cesse completement (inanitiés). BaBAK, remontant aux causes de l'inégalité de l'intestin produite par le régime, émet l'hypothèse que ces causes pourraient être à la fois d'ordre mécanique et d'ordre chimique.

Voici quelques faits qui militent en faveur de l'influence mécanique:

\section{Influence de l'inanition sur la longueur de l'intestin.}

Le 30 mai j'isolai dans de l'eau pure $1^{\circ}$ trois têtards pris dans le vase $A$ choisis parmi ceux dont la taille se rapprochait le plus du chiffre moyen 13 mm. 6 indiqué au tableau de ce jour, et dont on pouvait présumer que 
l'intestin mesuraitaussi à peu prois 116 mm., 8 (p)ésomption invérifiable et d'ailleurs discutable, je le reconnais); 20 trois têtards pris dans le vase $B$ et choisis également parmi ceux de taille voisine de $14 \mathrm{~mm}$. 2, moyenne de la taille à laquelie correspondait alor's une longueur de l'intestin égale à $78 \mathrm{~mm}$.

Ces deux lots furent soumis à l'inanition aussi complète que le permettait la nature de l'eau dont je disposais. Malheureusement, l'eau alimentant mes aquariums charriait de nombreux germes de Suprolermic qui bourgeonnaient rapidement contre les parois des vases, et étaient broutés par les têtards. Cés Champignons ne constituent pas pour eux un aliment bién nutritif; je doute même qu'ils soient digérés mais, ainsi que je m'en suis assuré au cours d'expériences relatives à l'inanition, ils s'établissent contre la muqueuse de l'intestin des larves, s'y accroissent et y produsent des excitations diverses dont il faudrait pouvoir tenir compte. En tout cas, ils y tiennent de la place, et c'est à ce titre que je les mentionne ici. Du reste, les têtards dr: Grenouilles supportent admirablement le jeûne, lequel s'il ar'rête leur croissance ne gêne guère et même dans certains cas, favorise leurs métamorphoses. J'en ai gardé pendant plus de trois mois sans rien manger (à l'exception des inévitables Saprolegnias) qui ont achevé la genèse de leurs pattes postérienres ou de leurs pattes antérieures. Ceux dont il s'agit en ce moment étaient apodes au délout du jeûne et ne poussèrent leur's pattes ni les uns ni les autres. L'un d'entre eux étant mort le 21 juillot. soit après gl jours de jeune, je sacrifiais les autres afin de les mesurer tous lessix. Ils avaient sensiblement maigri et pâli, mais leur taille n'avait guère diminué. En revanche, l'intestin que nous supposons avoir'été, au début de l'expérience, de $116 \mathrm{~mm}$. chez les végétariens et de $78 \mathrm{~mm}$. chez les carnivores soit de $38 \mathrm{~mm}$. plus long chez les premiers, fut trouvé à peu près de même longueur après le jêne, c'est-i-dire respectivement de $68 \mathrm{~mm}$. et de 59 mm. Il est assurément probable que l'inanition a joué un rôle clans la production de ce résultat, mais ce rôle n'a pu être que le mêm' dans les deux catégories de têtards; or, comme dans l'une (les végétarieus) le raccourcissement de l'intestin durant ces 51 jours fut de 48 mm. et dans l'autre (les carnivores) de $19 \mathrm{~mm}$. seulement, il faut bien attribuer la diffélence à l'inactivité imposée par le jeûne à tout le tractus digestif.

Voici d'ailleurs ce qui justifie une telle interprétation.

Influence de la réplétion non alimentaire de l'intestin sur su longueur.

Il fallait pour démontrel l'action mécanique du contenu de l'intestiu sur sa longueur, indépendamment de toute action chimique, trouver le moyen de faire ingurgiter aux teitards des substances solides et indi- 
gestes. J'y parvins, en leur offrant tout simplement du papier' à filtrel Bel'zélius: qu’ils dévorent avec avidité, dont ils remplissent entièrement leur tube digestif et qu'ils rendent sous la forme de selles cylindriques parfaitement hanches chez lesquelles l'examen microscopique démontre un assemblage de fibres de papier nullement altérées.

Aucun doute que leur's sues digestif's sont sans action sur la cellulose du papier qui, à en juger par l'abondance des selles, ne fait que traver'ser' l'intestin en se moulant sur' lui et en maintenant sa paroi distendue. Or, ces tetards hourrés de papier peurent vivre pendant des mois, maigrissant lentement, et se pretent par conséquent ì des comparaisons avec ceux qui ne mangent rien. J'ai le so mai institué précisément une expérience comparative entre trois têtards du vase $A$ et trois têtards du vase $B$ se trouvant exactement dans les mêmes conditions que ceux cités plus haut et isolés, comme eux, dans des bocaux contenant de l'eau avec du papier à filtrer à discrétion. Le résultat, quoique ne portant que sur un petit nombre d'individus, fut d'une netteté remarquable. Le 21 juillet ils furent sacrifiés bien que paraissant tous en bonne santé. C'est ce jour-là, on s'en souvient, qur mourut le premier végétarien de l'expérience précédente, lequel par sa mort décida de celle de tous les autres, je tuai ces dernier's dans de l'eau chloroformée et les mesurai de suite. L'intestin des trois végétariens supposé au début de $116 \mathrm{~mm}$., mesurait après 51 jours de pseudo-alimentation au papier, justement $116 \mathrm{~mm}$. chez l'un d'eux, et 99 et 94 chez les deux autres, soit, en moyenne, $103 \mathrm{~mm}$. marquant un raccourcissement de $13 \mathrm{~mm}$. seulement, au lieu de $48 \mathrm{~mm}$. comme ce fut le cas chez ceux soumis au jeûne absolu.

Quant aux trois carnivores, résultat inattendu, mais on ne peut plus démonstratif en faveur de la these que je soutiens ici, leurs intestins ont été trouvés plus longs qu'ils ne devaient l'être au commencement de l'expérience, à en juger d'après leur taille, c'est-à-dire en moyenne de $78 \mathrm{~mm}$. Ils mesuraient en effet 80,86 et $\mathrm{s} s \mathrm{~mm}$. soit en movenue $85 \mathrm{~mm}$., et par conséquent s'étaient allongés au courss de l'expérience. Pressés par la faim, ces têtards nourris jusqu'alors avec de la viande et trouvant dans une masse relativement faible de celle-ci suffisamment de quoi se sustentcr, avaient sans doute ingurgité des quantités de papier beancoup supérieures sous la pression desquelles leur intestin avait cédé en s’allongeant.

\section{Conclusions.}

Je concluerai donc des observations qui viennent d'être décrites:

$1^{\circ}$ Que la nature des aliments est un facteur de la longueur du tube digestif chez les larves de grenouilles qui, à cet égard, offient des variations allant du simple au double et davantage, 
2. Le rógime exclusivement régétal ne modifie guroro les dimensions de l'intestin des larves auxquelles il est imposé. Les chiffi'es exprimant sa longueur s'écartent peu de ceux du même ordre mesurés chez des larves alimentées de limon mố de micco-oronanismes végétaux et animanx qui font leur pâture ordinaire.

$3^{\circ}$ En revanche, les larves nour'ies exclusivement de viande oftrent à l'âge correspondant et, toutes choses égales d'ailleurs, un intestin plus cour't que celles élevées au régime mixte ou au régime végétal. La différence peut aller presque du simple au double.

$4^{\circ}$ Cette différence se manifeste très tôt après l'application du régime sur les jeunes larves, elle s'accentue jusqu'à l'approche de leurs pattes postérieures, puis alle tend a dimimuel lentement mais progressivement pendant la période des metamorphoses. Elle est peru sensible une fois que les pattes antérieures ont achevé leur poussée, et (selon BABaK) elle s'éteint durant la résorption de la queue, de telle sorte que les jeunes Grenouilles issues de larves végétariennes et de larves carnivores redeviendraient toutes semblables à cet égard. Nous n'avons pu dans nos expériences de 1901 dont le détail remplit ce mémoire, obtenir la métamorphose dernière des larves végétariennes, mais le raccourcissement très marqué de leur intestin après la poussée des pattes antérieures ne nous permet pas de douter qu'il aurait continué pendant la phase de phagocytose de l'appendice caudai. Toutefois, il eût été intéressant de constater entre les Grenouilles quelque dissemblance résultant d'un raccourcissement moindre de l'intestin chez les unes que chez les autres. C"est sur ce point que devront porter surtout les recherches futures.

$5^{\circ}$ La cause du raccourcissement intestinal est, pour une part, d'ordre mécanique; celui-ci, en effet, commence avec le ralentissement de l'appétit à l'approche des métamorphoses. Les larves alors mangent peu ou pas, et leur intestin se raccourcit sous l'influence du jeûne; comme ce dernier atteint son maximum d"intensité et de durée pendaut la dernière métamorphose l'intestin est, dans tous les cas, réduit à son minimum de longueur à la fin de celle-ci.

$6^{\circ}$ La preuve que la longueur de l'intestin est en rapport avec son activité mécaniqur et lá quautité deśs aliments qüil renferme, est fournie par le fait que les têtar'ds très gros, ingurgitant une grande masse de substances régetales on minérales. ont un tube digestif relativement plus long que les têtards de taille moyenne qui mangent des substances moins volumineuses et plus nourricières. D'autre part, les têtards soumis à un jeûne expérimental, raccourcissent leur intestin comme ils le font durant leurs métamorphoses. 
$7^{\circ}$ Dans ce dernier cas, le l'accourcissement est al'rêté par l'introduction dans l'intestin d'une substance indigeste mais tenant de la place telle que du papier à filtrer, ce qui prouve bien que les actions chimiques ne jouent qu'un rôle secondaire ou nul dans la production du phénomène.

\title{
Das Centralnervensystem von Geratodus Forsteri.
}

\author{
Von Prof. R. BURCKHARD'T (Basel).
}

Dieses Thema ist bereits mehrfach Gegenstand der L'ntersuchung gewesen, doch hatte bisher das Material nicht ausgereicht, um weiter in die Struktur des Gehirns und seine Fntwicklung einzudringen. Der Arbeit, über die hier kurz soll Bericht erstattet werden, liegt das von R. SEmon auf seiner australischen Reise gesammelte Material zu Grunde; bearbeitet wurde es von dem Vortragenden und Dr. Rob. Brst; welch letzterer den anatomischen Theil der Aufgabe übernahm, während ersterer speziell die Frage nach der zoologisch-systematisehen Beurtheilung des Gehirns von Ceratodus verfolgte im Vergleich mit ausgewählten Materialien des Fischgehirns überhaupt.

Das Hirn von Ceratodus zeigt neben allgemeinen Eigenschaften folgende Eigenthümlichkeiten : Relativ geringe Verdickung aller Hirnmassen, grosse Ventrikel, ein in der Medianzone beträchtlich verdicktes Kleinhirn, eine besonder's complicierte Struktur der Decke des III. Tentrikels, wic sie bei keiner verwandten Form gefunden wird. Die specielle Beschaffenheit dieses Hirntheils wird durch ein Modell erläutert. In der Fntwicklung schliesst sich das Hirn am ehesten an dasjenige von Acipenser an, behält aber vielfach primitivere Verhältnisse in Vorderhirn und Zirbel bej.

Bei der Vergleichung des erwachsenen Gehirns von C'eratodus mit dem verwandter Fische ist in erster Linie die relative Länge des Hirns überhaupt zu berücksichtigen. Aus dem Beispiel des Polypterasgehirns ergiebt sich, wie übrigens auch auf Grund der Erfahrungen am Selachierhirn bestätigt werden kann, dass die Gestalt des Gehirns, die Massenentfaltung seiner verdickten Partieen, namentich aber die Configuration des olfactorischen Gebietes noch im späteren Leben der Fische erhebliche Veränderungen erfährt. Daher ist bei Vergleichungen stets in Rücksicht zu ziehen, dass Gehirne halbwüchsiger Exemplare in Folge der frühzeitigen Entfaltung der optischen und olfactorischen Centralstationen, oberfiachlich betrachtet, den Eindruck erwecken, als ob sie relativ höher differenziert wären. Ein einfaches Kiriterium, wonach wir 
las Hirn von Ceratodus einer Reihe eingliedern könnten, gibt es nicht. Durch eine Summe primitiver Eigenschaften, wie geringe Wandverdickungen der Lateralzonen, grosie Ausdehnung der rein epithelialen Plexus, algemeine Configuration des Vorderhirns, embryonale Stadien des Medianschnitts und Mittelhirns schliesst es ziemlich direct an das Selachierhirn an, ohne nähere Beziehungen zu dem mehrfach primitiveren und durch einseitige Bildungen specialisierten Hirn von Polypyterus oder zu dem mehr in der Richtung auf das Teleostichnirn deutenden höher specialisierten des Stör's zu zeigen. Beim Vergleich mit Protopterus und Lepidosiren ergeben sich als wichtigste Unterschiede die Gestalt drs Vorderhirns und des Kleinhirus. Bei Protopterus ist die I)ehnung zwischen Vorderhirn und Riechschleimhaut auf cinen andern Punkt verlegt als bei Ceratodus, nämlich in die Fila olfactoria. Die Verlegung der Kaufläche in rostraler Richtung beeintlusst nachweisbar die Architectur des Schädels und damit auch des Gehirns. Ausserdem aber erfährt bei Protopterus das Torderhirn eine bedeutendere Massenentfaltung als bei Ceratodus, und dementsprechend werden cigentliche Plexus hemispharium von der Decke des III. Ventrikels her ausgebildet, während diese in L'ebrigen eine Verkürzung erfährt. Dagegen bleibt bei den Dipneumona das Kleinhirn auf relativ primitiver Stufe. Charactere, dic allgemein den I)ipnoern zukämen oder deron specielle Verwandtschaft mit den Crossopterygiern verrathen wül'den, sind nicht nachzuweisen.

\section{Beiträge zu einer Entwicklungstheorie des Hymen.}

Von Dr. H.-A.-E. KEMPE (Rotterdam).

Es sei mir gestattet, mit wenigen Worten nur, (jine nähere Erläuterung meiner Präparate zu gehen, zu deren Demonstration auf dem jetzigen Kongresse das Organisationskomité so freundlich war, mir Mikroscope zur Verfügung zu stellen.

Es ist eine Thatsache, die von einigen Forschern hervorgehoben, von Andern wiederum unherücksichtigt gelassen worden ist, dass es nicht gelingen kann, mit Hülfe der allgemein angenommenen und bekannten Entwicklung:processe eine hefriedigende Entwicklungstheorie der Placentalier-Vagina aufzustellen. Die bekannten Formeigenschaften und For'manlagen des MüLLer'schen Ganges, die man der theoretischen Entwicklung dieser Vagina zu Grunde zu legen pflegt, genügen nicht zul' Fertigstellung (les genannten Organes. Namentlich reichen sie nicht aus, das Hymen entstehen zu lassen. Das Hymen kamn niemals in die El- 
scheinung treten als eine logische Folge einer Velschmelzung von Oriducten.

So empfand auch ich es, als ich vor zwei Jahren bemüht war', die heliannte Frage nach dem Wesen und der morphologischen Bedeutung des Hymen ihl'r Lösung ein wenig näher zu bringen, wobei es von vornherein deutlich war, dass dieses mir nicht gelingen würde ohne die línntnisse cinel gut verstandenen Entwicklungsgeschichte des fraglichen ()rganes. Dem entsprechend suchte ich die Anfänge ciner Lösung dieses Problemes durch eine Tremehrung der bereits behamnten Formeigenschaften des MëLLER schen Ganges zu erreichen oder durch eine Aenderung in der Auffassung desselben, und ich versuchte die Möglichle it

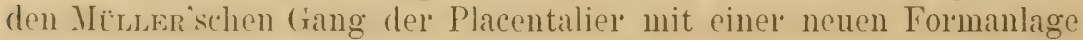
zu versehen.

Man braucht, so meinte ich, die ausgewachsené Placentalier-Vagina nicht aufzufassen als einen aus dem Zusammenfluss zweier Kanäle entstandenen Kanal; man kann sie wohl auffassen als eine zu einem Kanal gewordene Tasche, und clabei wird man dann im Hymen die letzten Reste eines 'Taschenbodens zu erblicken haben. Nur muss man um diese Auffassung aufrecht erhalten zu liömen, weiter gehen. Zu einel geänderten Auffasiung der Form der ausgewachsenen Vagina gehört eine geänderte Auffissung ihrer Formanlagen. So ging ich weiter und stellte folgende Hypothese auf :

Wenigstens in seinem untern distalen Abschnitte wird der MüLler'sche Gang der. Placentalier morphologisch nicht gleichwertig sein mit dem MüLuER'schen Gange der übrigen Amnioten. Man wird das Epithel dieses distalen Abschnittes ehel vergleichen können mit dem Epithele, das enthalten ist in der Oviductausbuchtung, die bei mehreren Didelphen den Vaginalblindsack und bei Einigen sogar die dritte mediale Vagina erzeugt.

Dies war nun durch Thatsachen zu beweisen.

Zu diesem Zwecke nun habe ich mich einige Zeit ausschliesslich dem Studium dex Verschmelzung der MürLfer'schen Gänge bei Placentaliern gewidmet, namentlich habe ich den miliroskopischen Bau rles Productes diesel Verschmelzung bei der "Weissen Ratte" an Schnittselien untersucht. Tach dieser Arbeit aclangte ich zur Ueberzeugung, dass es möglich ist, die morphologische Bederutung des MëLter'schen Ganges der Placentalier in der angerleuteten Weise zu änder'n. Es ist in Einklang zu hringen mit an Placentalier-Fmbryonen beobachteten Thatsachen, dem Mëtuen'schen Gange den neuen Werth, dio gewünschte Folmanlage beizulegen - dic Fähigkeit bei dem Verschmelzungsprocesse einen Vaginalblindsack zu erzeugen. Damit ergibt sich die Möglichlieit zul Aufstollung ciner Futwicklungsthorie, die das Hymen zum Vorschein hrin- 


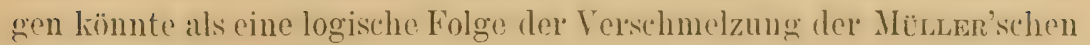
Gänge.

Wie sich diese Entwicklungstheorie am Ende gestaltete, möchte ich jetzt nicht besprechen; eine lichtign Beschreibung ihrer Entwicklungsprocesse würde zuviel Zeit in Anspruch nehmen. Es sei mir erlaubt für diese Beschreibung auf meine Inaugural-I)issertation hinzuweisen, in welcher ich sie so ausführlich als mir möglich war, gegeben habe. Ich möchte Sie hier nur noch einige Augenblicke nit der Betrachtung einiger Stützpunkte dieser 'Theoric beschïftigen, welche sich am besten zu einer Demonstration eignen, nämlich der Besprechung ciniger Schnitte aus den Genitalsträngen von weiblichen Embryonen der "W (Wissen Ratte».

Es hat sich namentlich hei dem Studium der Schnittserien dieser Embryonen herausgestellt, dass bei der Futstehung des bukannten LteroVaginalcamales. an distalen Ende diese's Canales durch die Verschmelzung der medialen Oviductwïnde ein ganz anderes Gebilde erzeust wird, als am proximalen Ende. Während man an diesem proximalen Fnde in dem Durchschnitte dir bekannten Bilder erhält, - die Durchschnittsbilder zweier Oviductwände, die sich dicht zusammenlegen, um allmählich zu einem Septum zu verschmelzen, - so bekommt man am distalen Ende von diesen dicht aneinander schliessenden Oviductwänden nichts zu sehen. Durch rlas Zusammenfliessen dieser Wände wird hier nur eine,

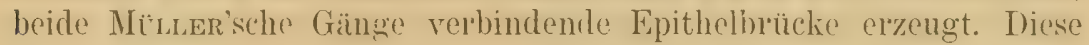
Epithelbrücke meine ich als den epithelialen Boden, oder besser gesagt, als die Bodenanlage des Vaginalblindsackes reconstruieren zu dürfen.

Die zwei ersten meiner zur Demonstration aufgestellten Präparate zeigen Ihnen nun diese Differenz. Sie werden also in den Schnitten durch den proximalen Theil, die $15 \mu$-Dicke haben, ein einheitliches UteroVaginalseptum finden, das proximalwärts in zwei dicht aneinander schliessende Ovirluctwände übergoht. I)iese Oviductwämdr werden damn noch weitel proximalwärts durch ein allmählich breiter werdendes, bindegewebiges Septum von einander getrennt. In den $10 \mu$-dicken Sehnitten des distalen Theiles dagegen werden Sie die Epithelbrürlie finden und wenn Sie weiter distalwäts die Serie verfolgen. wird es sich Ihnen zeigen, dass die Oviductwände nach ihrer Terschmelzung plötzlich auscinander weichen und im ersten Augenblickeschon durch Bindegewebe von einander getrenint sind.

Das folgende dritte Präparat zeigt Ihnen das Gleiche in einem $15 \mu$. dicken Querschnitt. Der Embryo, von welchem dieser Schnitt herrührt, wurde mit Alkohol fixiert und unter dem Einflusse dieses Fixiermittels hat sich das Epithel der MüLLes'schen Gänge stark zusammengezogen. Ehen deswegen zeigt dieser Schnitt das heschriebene Verhältniss hesonders dentlich; denn die Epithelbrücke ist hier nur leicht angesehnitten und gestattet, sich gänzlich davon zu überzengen, dass diese Fpithel- 
brïcke auch in ihrem meist distalen Theile, auf dem Iurchschnitt selbst, keine Kerngruppierungen mehr' zu unterscheiden giebt, die als letzte Spur von zwei aneinander liegenden Oviductwänden gedeutet werden könnten. Der Durchschnitt des die Oviducte trennenden Bindegewebes erscheint schon in der distalen Einstellungsfläche dieses Schnittes.

Die zwei übrigen Prïparate beziehen sich auf einen zweiten bemerkenswerthen Vorgang bei der Entwicklungsgeschichte der Vagina der "Weissen Ratte ".

Es finden namentlich, unabhängig vom bekaunten Verschmelzungsprocesse der MëLuer'schen Gänge. da, wo das Epithel der Genitalgänge sich mit dem Epithele des Sinus urogenitalis verbindet, ausgebreitete Epithelverschmelzungen zwischen den MIÜLLER'schen Güngen und den WoLfF'schen Gängen und der Rückwand des Sinus urogenitalis statt, und ohme auf die Entwicklungsweise dieser Verschmelzungen, die ziemlich ver'wickelt ist, näher einzugehen, will ich hier nur bemerken, dass sie schliesslich zur Bildung einer mächtigen dorsalen Sinuswand führen. Durch eine Faltung der beiden lateralen Wände des Sinus wird diese dorsale Wand zu einer soliden Platte, der Sinus-Rückplatte, umgeformt. Die genannte Faltung schreitet, am oberen Ende des Sinus anfangend, allmählich proximalwärts und führt schliesslich zı einer ausgedehnten longitudinalen Spaltung dieses Canales. Dabei tritt dann die Sinus-Rückplatte aus dem Gebiete des Sinus in das Gebiet der Vagina ein und formt so ihre strangförmige Terlängerung, den distalen Abschnitt del' Vaginal-Endplatte.

Vou den zwei Präparaten zeigt nun das erste die Faltung der lateralen Sinuswände, das zweite die Ablösung der Sinus-Rückplatte vom Sinus urogenitalis.

(Nachher wurden die Präparate gezeigt).

Mit tiefem Bedauern machen wir den Kongressmitgliedern Mitteilung von dem inzwischen (23. Dez. 1904) erfolgten 'Tode des Verfassers dieser' Arbeit. (Red.) 


\section{Ueber die Entwicklung des Kopfskeletts bei Selachiern.}

Von Prof. J. W. Van WIJHE (Groningen).

Hiex werle ich meine hauptsächlichsten Resultate kư zusammenfassen, da die ausführliche Arbeit an anderer Stelle erscheinen soll.

Wenn ich in mancher Hinsicht zu anderen Resultaten gekommen bin als Parker, Sewertzoff u. a., welche lasselbe Thema bearbeitet haben, so liegt dies wohl grossenteils daran, dass man durch die Fürbung mit Methylenblau den Kinorpel von anderen Geweben - auch vom Vorknorpel - scharf unterscheiden kann. Auf Literaturangahen soll aber erst in der ausführlichen Arbeit eingegangen werden.

Das Material verdanke ich der Liebnnswürdigkeit der Vorstände der Stationen auf Helgoland, in Plymouth, Neapel und Helder. Am vollständigsten ist meine Serie von Acanthias-Embryonen.

Neurales Cranium. Der zuerst auftetende Kinorpel des Chondrocraniums, wie des Slieletts überhaupt, ist bei Aconthias (wie bei sicyllium und Pristiurus) das parige Parachordale, welches bald die Lamina basiotica (mesotica) entsendet. Bei Embryonen von 20-30 mm. Länge ist es der einzige Skeletteil. Bei wenig älteren Embryonen bildet das Parachordale zwei weitele Auswüchse, welche die seitliche Schädelwand bilden helfen, nämlich an seinem rostralen Ende die Lamina antotica (das sog. Alisphenoid) und an caudalen Ende den primitiven Occipitalbogen, welcher ein Loch umschliesst. Durch dieses Loch tritt eine ventrale Nelvenwurzel, nach Horruasi diejenige des 10. und letzten hopfsenmentes (Nerv $z^{r}$ nach der Nomenclatul von Fürbringer). [nteldessen ist eine selbständige Kinorpelschale am äusseren Bogengang des Gehörlabyrinthes aufgetreten; auch die Trabecula ist erschienen, und zwischen Trabecula und Parachordale sieht man den kleinen, parigen Polknorpel. Bald verschmelzen an jeder Körperseite Parachordale, Polknorpel und Trabecula mit einander: die Trabecule setzen sich nun rostralwïts in eine unpaare mediane Knorpelplatte fort, die an jeder Körlperseitr zwischen Orbita und Nasensack die Lamina orbito-nasalis abgibt, welche anfangs dor'sal frei endet.

Am späteren Orbitaldach entsteht bei Acanthias (auch bei $R(i j a)$ selb- 
ständig der Supraorhitalknorpel, welcher, den Trochlearis umwachsend, das Foramen für diesen Terven bildet. Der Supraorbitalknorpel verschmilzt hinten und ventral mit dem freien dorsalen Ende der Lamina antutica, vorn mit dem freien Ende der Lamina orbito-nasalis. In dieser Weise wird hei Acanthices-Embryonen von etwa $40 \mathrm{~mm}$. ein geschlossener ()rbitalrahmen gebildet, welcher vor'n durch die Lamina orbito-nasalis, hinten durch die Lamina antotica, dorsal durch die fröhere Cartilago supraorhitalis und ventral dureh die frühere Trabecula und den früheren Polknorpel begrenzt wird. Durch den Orbitalrahmen treten der Opticus, der Oculomotorius, eine Wurzel der Carotis interna und eine Vene. Die Ausstrittöffnungen für diese beiden Crefässe sowie diejenige für den Oculomotorius werden bald vom Knorpel der Lamina antotica umwachsen.

Der 'Trigeninus und der Facialis treten hervor durch die Lücke zwischen der Lamina antotica und der knorpeligen Labyrinthschale, welche sich mit dem Parachordale verbunden hat. Die Lücke ist anfangs dorsal offen, wird hier aber zum Foramen geschlossen, indem die frühere Cart. supraorhitalis caudalwärts wachsend, mit der Labyrinthkapsel in Kontakt tritt und verschmilzt.

Durch die Lacune zwischen der Labyrinthkapsel und dem Occipitalbogen treten bei Acanthias-Embryonen von 35-40 mm. Länge der Vagus und zwei Hypoglossuswurzeln ( $x^{v}$ und $y^{v}$ ) heraus. Diese Lacune schliesst sich zum Foramen, indem der Gipfel des ()ecipitalbogens mit der Labyrinthkapsel verschmilzt. Der Nerv $x^{v}$ ist sehr rudimentär und verschwindet später in der Regel; der Nerv $y^{v}$ dagegen persistirt, und seine Durchtrittsöffuung durch das Cranium wird nachträglich rom linorpel des Occipitalbogens umwachsen.

Ganz anders verhält sich die Occipitallegion bei zwei Embryonen von Heptanchus, resp. 63 und $95 \mathrm{~mm}$. lang. Hier zeigen sich, wie beim ausgebildeten Tiere, vier bis fünf Foramina für ventrale Wurzeln von Oceipitalnerven; drei derselben liegen caudal vom Foramen vagi. Es sind aber deutliche Zeichen vorhanden, welche darauf himweisen, dass die Elemente zweier. Wirbel mit dem Schädel versehmolzen sind, sodass die beiden letzten Occipitalnerven dieses Tieres, welche Fürbrasier mit y und z hezeichnet, eigentlich Spinalnerven (Occipitospinalnerven) sind. Erst der Nerv $x$ muss mit dem oben mit $z$ bezeichneten Nerven, der bei Acanthirs den primitiven Occipitallogen durchbohrt, homologisirt werden.

Tie Angaben von Hoffman, nach welchen der Occipitalteil des primitiven Schädels (d. h. des mit dem primitiven Oceipitalbogen abschliessenden Schädels) bei Acanthias durch Terschmelzung von Wirbeln entstehen sollte, sind schon von Sewertzoff und Brats zurückgewiesen, und ich kann mich ihrem Widel'spruch 11 u ansehliessen. Roswsibur hat aberschon vor langer Zeit eine Assimilation von Wirbelelementen in spätembryonaler Periorle bei Carcharias nachgewiesen und bei meinem mündlichen 
Vortrage glaubte ich, dass dies in späterer cmbryonaler Zeit auch bei Acanthias mit mehreren Wirbeln der Fall sei. Fortgesetzte Untersuchungen haben mir aber gezeigt, dass bei Acanthias nur der erste Wirbel in den schädel aufgenommen ist. Bekanntlich durchlohrt beim ausgebildeten 'Tiel je ein Rumpfnerv mit der' ventralen Wurzel einen Bogen und mit der dorsalen Wurzel das nächstfolgende Intercalare. Nur der erste Rumpfner durchbohrt keinen Bogen, sondern el tritt mit der ventralen Wurzel durch die Lacune zwischen dem Schädel und dem ersten Intercalare, welches seine dorsale Wurzel durchlässt, hervor.

Auch in embryonaler Zeit, sobald die Bogen ausgebildet sind, ist der erste Spinalnerv daran kenntlich, dass er mit der ventralen Wurzel keinen Bogen durchbohrt, sondern durch die ehen erwähnte Lacune austritt. In zugäröige rudimentäre Bogen sant seinem Wirbeleentrum ist mit dem schädel verschmolzen; der Bogen ist in Ausnahmefällen aber frei, wie ich auf einer Körperseite bei einem Fmbryo von $82 \mathrm{~mm}$. fand. In einem andern Falle erstreckte sich das Parachordale hei unem Finhryo von $40 \mathrm{~mm}$. auf jeder Körperseite ausnahmsweise nicht bis in das Gebiet des betreffenden Wirbelcentrums, indem es schon vor dem caudalen Rante des Oceipitalbogens aufhörte. Isei meinen übrigen Embryonen, die den Occipitalbogen zeigen, reicht das Parachordale etwa um die Länge eines Wirbels caudal von diesem Rande.

Nach Braus wirol ein ähnlicher Nerv wie der eben erwähnte bei Spinax-Embryonen mit der ventralen Wurzel nachträglich in den Schädel aufgenommen und mit z bezeichnet. Diese Bezeichnung trifft nicht zu, da der betreffende Ner'v, welcher mit der dor'salen Wurzel das erste Intercalare durchbohrt, dem ersten Spinalnerven bei Acanthias entspricht.

Bei Embryonen von Heptanchus und Torpedo wird am Rumpfe wie bei Acanthias jeder Bogen von der ventralen Wurzel und das caudal folgende Intercalare von der' dor'salen Wurzel eines Spinalnerven durchbohrt. Bei Embryonen der Scyllidær ist die Wirbelsäule aber nach einem ganz anderen Typus gebaut. Hier tritt die ventrale Wurzel durch den Raum zwischen dem Bogen und dem folgenden Intercalare, die dorsale Wurzel durch den Raum zwischen diesem Intercalare und dem Bogen des folgenden Wirbels hervor, wie schon von früheren For'schern bemerkt worden. Es ist nun interessant, dass bei Scyllium- und PristiurusEmbryonen von etwa $30 \mathrm{~mm}$. Länge, die noch keine knorpeligen Wirbelelemente zeigen, ein durchbohrter primitiver Occipitalbogen dem Ende des Parachordale aufsitzt. In der Bildung des primitiven Schädels stimmen die Scyllidæ mit Acunthias überein, obgleich der 'Typus der' Wirbelsäule ein ganz anderer ist.

Auch bei Torpedo-Embryonen habe ich cinen rudimentären, durchbohrten, primitiven Occipitalbogen auf dem caudalen Ende des Parachor- 
dale beobachtet. Mein Torpedo - Material ist aber noch etwas zu unvollstïndig, um die Homologie mit dem Occipitalbogen der Haifische behaupten zu können.

Visceralskelett. Im Kieferbogen treten bei Acanthias das Palatofyuadratum und der Lnterkiefor als sellständige Kinorpelstücke auf, ebrenso im Hyoddbogen das Hyomandibulare, das Hyale und die Hyoid(opula. Der L'nterkiefer besteht anfangs auf jeder hörperseite aus zwei getrennten Knnorpelstücken, die später verschmelzen, wie ich auch bei Vogel-Embryonen fand. Im Kiefor-und Hyoidbogen tritt das Kinorpelskelett fast gleichzeitig auf und hald giebt die starke Entwicklung der linorpel dieser Bogen. während noch lieine knorpeligen Kiemenhogen vorhanden sind, dem Kópfskelett von Haifisch-Embryonen aus dieser Periode ein eigentümliches, auffälliges Gepräge.

Die knorpeligen 'Teile der Kriemenbogen (Hypo-, Cerato-, Epi- und I'harrngohranchiale) (rscheinen beträchtlich spater und werden selbstiindig angelegt. Iie Angaben der Autoren, nach welchen der Kiemenbogen als einheitlicher Kinorpelstah auftrete, der sich nachtraglich gliedere, sind irrig und beruhen auf einer Terwechslung des Knorpels mit Torknorpel. Auch die Copula werden als selbständige unpaare Kinorpelstiucke angelegt.

Bei Scyllium, Pristiurus und Torpedo treten die Teile der Visceralbogen ohenfalls als getrennte hinorpelstiicke auf. Im L'nterkiefer aher habe ich bui diesen formen stets nur ein einziges Kinorpelstück (natürlich algesehen vom Lippenknorpel) an jeder Körrperseite beobachtet.

Schliesslich will ich noch erwähnem, dass in der vorknorpeligen Anlage des Schultergürtels die Scapula und das Coracoid bei Acanthias, ScylTium, Pristiurus und Torpedo als getrennte Knorpelstücke auftreten, wie ich dies auch bei Vogel-Embryonen gesehen habe, während Noonprisos gefunden hat, dass sogar hei Säugetier-Emhryonen der Processus coracoideus als selbständiger Knorpelkern angelegt wird. 


\title{
Demonstration eines Fötus vom westafrikanischen Elefanten, Elephas cyclotis Matschie.
}

\author{
Von Prof. E. LÖNNBERG (Stockholm).
}

Mit 1 Tafel.

Es sind nicht viele Elefanten-Fötus beschrieben worden; so viel ich weiss bisher nu' zwei und zwal ein afrikanischer und ein indischer. Der erste von diesen wurde schon in SwiBa, Thesaurus ${ }^{1}$ abgebildet. El gehört nicht zu der sudanesischen grossohrigen Art, sondern ist wahrscheinlich aus Westafrika nach Europa eingeführt worden. Nach dem 'Tode des holländischen Apothckers SEBA wurde dieser Elefanten-Fötus als eine ganz besonders wertvolle "Kuriosität " von dem schwedischen König Adolf Fredrik angekauft und ist noch jetzt im Naturhistorischen Reichsmuseum in Stockholm aufbewahıt.

Mehr als hundert Jahre später wurde cin Fötus von einem indischen Elefanten von 'Turner abgebildet? ${ }^{2}$. Dieses Exemplar' gehörte dem Anatomischen Museum der. Universität Edinburgh. Die Länge dieses Fötus betrug 21 engl. Zoll. 'Turner gibt in sciner' Beschreibung auch andere Dimensionen an nebst einigen morphologischen Notizen. Der Rüssel dieses Exemplares zeigte schon den medianen fingerförmigen Fortsutz und ist im grossen und ganzen dem eines elwachsenen Elefanten ganz ähnlich.

Der Fötus, den ich jetzt vorzuzeigen die Ehre habe, ist deshalb von grösserem Interesse, weil el vicl klemer als die beiden bis jetzt in der Litteratur bekannt gemachten Elcfanten-Fötus ist. Ich habe es von einem in Kamerun lebenden Frounde, Herm G. Linnesis, erworben und es gehört jetzt dem Naturhistorischen Reichsmuseum in Stockholm. Beim ersten Aublick dieses Fötus ist man erstaunt, dass er schon, wenn el noch so lilein ist, so vollstandig elefantenähnlich aussieht. (Die beigegebene Abbildung zcigt dies genügend, so dass weiter hierüber zu sprechen nicht nötig ist.) Ich möchte aber hervorheben, dass schon in dicsem Stadium diejenigen Merkmale, die den westafrikanischen $E$. cyclotis rom sudane-

\footnotetext{
1 Vol. I. Amstel. 1734.

2 Journ. Anat. Phys, XY. 1881.
} 
sischen E. oxyotis nach Matscue unterscheiden, gut ausgeprägt sind. Ian sicht also bri diesem Fötus, dass die ()hren deutlich abgerundet sind und nicht vorne und unten spitz anslaufende Lappen wie beim sudanesischen hild'n. I) In Interfüsse zeigen vier kleine Hufe beim KamerunElefanten. dagegen scheinen die sudanesischen konstant nur deren drei zu haben. I)er Iïissel dieses Fötus hat die charakteristische (restalt und ist verhailtnismässig so lang wie bei einem erwachsenen, was ja von Interesse ist, da bekamntlich oft, aber irrtumlich, behauptet wird, dass die neugehorenen Elefanten ganz kurzen Rüssel haben. (Übrigens sei auf die bejgegebene Abbildung verwiesen.)

Die Fütalmembranen von Elephes sind auch, soweit die mir zugängliche Litteratur mich belehrt, zweimal beobachtet worden. In beiden Fällen handelt es sich um Elephas indicus. Im Jahre 1858 gab Owen $^{1}$ die crste Beschreibung dieser Teile und wies dann verschiedene Eigentümlichleiten nach. Dabei ist besonder's zu bemerken, dass die Placenta zonal ausgebildet und mit partieller Decidua versehen war, während das Chorion übrigens glatt war und nur an beiden Enden der Fruchtblase je ein kleines villoses Feld aufwies. Weiter fand er, dass der Amuios auf der Innenflache ein granulöses Aussehen hatte und dass eine grosse Zahl von eigentümlichen Körlyerchen auf der inneren Seite der Allantois auftrat. OWex verglich diese in Betreff ihrer Form mit den Cotyledonen der Wiederkäuerplacenta, betonte aber, dass sie nach einwärts ragten und dass sie also gar nichts mit den Cotyledonen zu tun hätten. Da etwa zwei Dezennien später Chaparax ${ }^{2}$ Gelegenheit hatte, eine ähnliche Untersuchung zu machen, bestätigte er Owev's Angaben in allem wesentlichen, fügte aber hinzu, dass er die Zahl der Allantoiskörperchen grösser gefunden haitte als diejenige des Owex'schen Exemphares. Iries ist in Külrze, was wir his jetzt von direliten Beobachtungen an den fë̈talhüllen von Elephanten in dieser Beziehung wissen. Später sind uns Deutungen oder Missdeutungen zugekommen und gar nichts über die Verhailtnisse be den affikanischen Elefanten. Die Kenntnis hierüber ist also recht lückenhaft.

Durch das zu meiner Verfügung stehende Naterial bin ich aber im stande zu bestaitigen, dass die afrikanischen Elefanten in dieser Beziehung sich ähnlich wie die indischen verhalten und dass also die Bemerkung OwEn's in der Hauptsache für beide zutreffend ist.

Die hügelartigen Bildungen auf der inneren Seite der Allantois sind ausserordentlich zahlleich und da sie oft mit einander verschmelzen, ist es schwer, sowohl Zahl wje Grösse mit bestimmten Zahlen auszudrücken. In der äquatorialen Gegend der Fruchtblase unter und in der Nähe der

1 Phil. Transactions, Vol. 147.

2 Journ. Ac. Nat. Sc. Philadelphia, 1879. 



\section{TAFELERKL ÄRUNC;}

Fig. 1. Fötus von Elephas cyclotis Matschie, aus Kamerun.

Fig. 2. Ein Stück von der inneren Seite der Allantois von Elephas cyclotis die eigentïmlichen Körperchen in der Nähe der Gefässe zeigend. Etwa $2 / 3$ nat. Gr. 

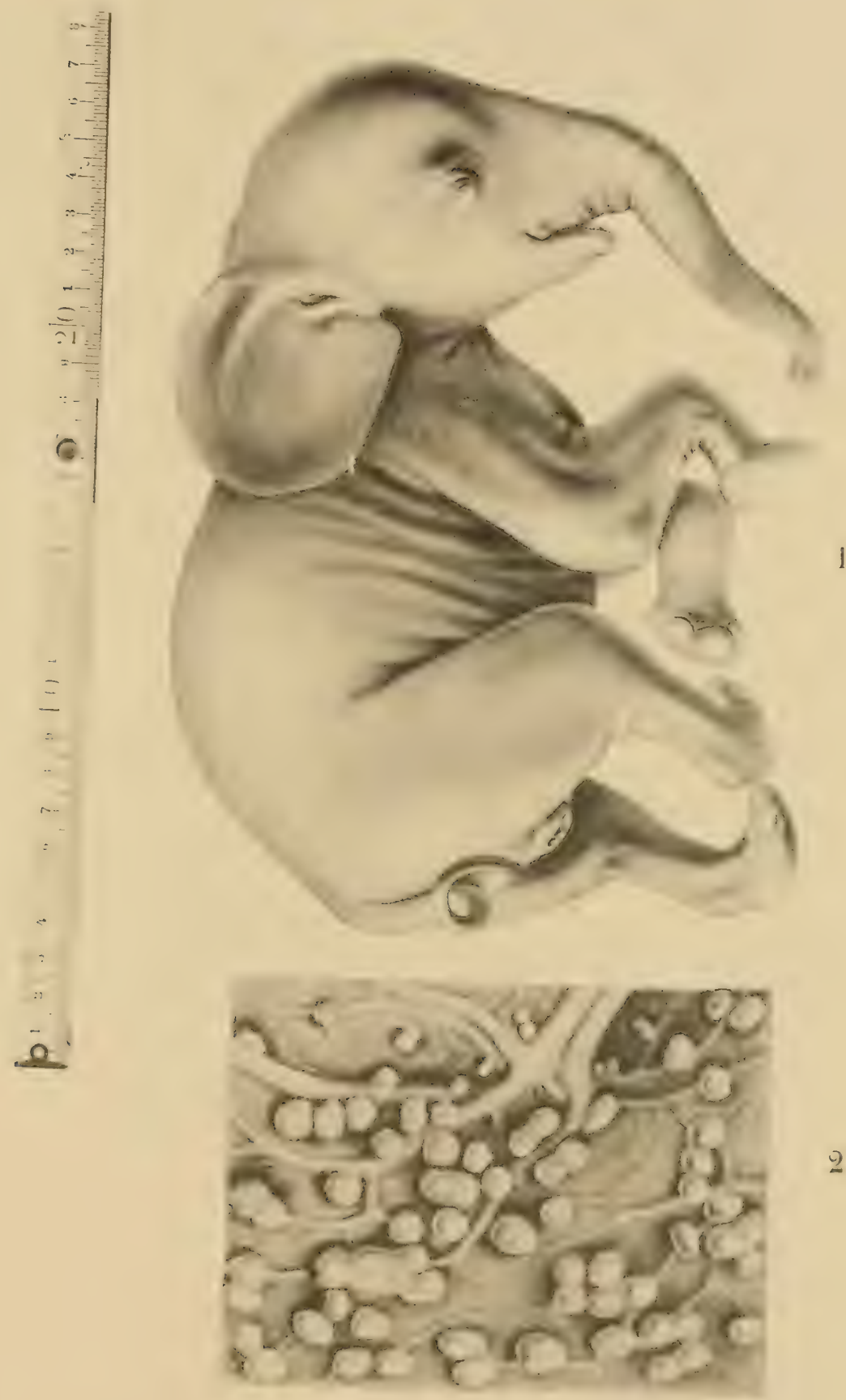

E. LÖNNBERG

ELEPHAS CYCLOTIS 

Placenta sind sie am zahlreichsten; gegen die beiden Pole der Fruchtblase werden sie allmählich spärlicher. Dies steht in Verbindung mit ihrem Auftreten an oder neben den Gefässen. Die beigegebene Figur zeigt eine Partie, wo diese Bildungen ziemlich zahlereich sind. Man sieht daran, dass sie von wechselnder Grösse sind. Die grösseren haben einen Durchmesser von $1-1 \frac{1}{2} \mathrm{~cm}$., die kleinsten von nur $2-3 \mathrm{~mm}$. Ihre Höhe ist viel geringer. Die grössten werden kaum höher als etwa $5-6 \mathrm{~mm}$. Die ()bertlache ist glatt, hisweilen aber durch anfsitzende Vebenhöekepchen etwas maulberaihnlich. Ein (Wuersehnitt durch ein solches Allantoishügelchen nit ['mgebung zeigt ron der inneren Fläthe ausgehend zuerst ein einschichtiges Epithel mit grossen Kernen in beinahe ku. bischen \%ellen. Dann folgt ein Galleituewebe (demjenigen des Tabelstranges ähnlich) mit sternförmigen Zellen, oft etwas in die Länge ausgezogen, in einer glasklaren Zwischen- oder Grundsulstanz. Lapillaren kommen in diesem Gewebe sehr häufig vor. Dieses Gallertgewebe geht nach aussen, d. h. an der Basis des Hügelchens allmählich in ein fibrilläres Bindegewebe über, in welchem die grossen Gefässe der Allantois verlaufen. Diese Bindegewobschichten zeigen bisweilen Lürke'n, dir vielleicht die Grenze zwischen Allantois und Chorion darstellen. Die Chorionschichten, zu denen wohl die äussersten Partien des Querschnittes zu rechnen sind, sind ähnlich, d. h. von fibrillärem Bindegewebe, nur etwas dichter. Sie werden nach aussen von epithelartig angeordneten Zellen begrenzt.

Aus dieser Schilderung geht ja dentlich hervor, wie schon aus der Lage diesere Allantoiskörperehen sich schliessen liess, dass sie gar kejur Cotyledonen oder mit solchen homologe Bildungen sind. Wenn Oscar Scilcutze in seinem übrigens trefflichen liuch ${ }^{1}$ eine solche Dentung sich erliaulst. muss diesellye gleich zurückgewirsen werden, un nicht länger als ein Irrtum in der Handliteratur zu bleiben.

Fragt man aber, was für eine Bedeutung diese Bildungen liaben, so ist dies gar nicht leicht zu entecheiden. Ihre Lage zeigt. dass sie in gerrisser Beziehung zu don (iefïssen stehen. Es seheinen mir zwei I) utungsmöglichkeiten rorzuliexen, ohne dass ich jerloch ein bestimmtes L'rteil darüber aussprechen könnte. Entweder können sie eine gewisse Rolle bei der Absonderung der Allantoisflüssigkeit spielen oder sic können

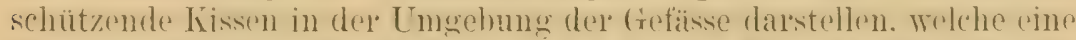
Zusammendrückung der Gefïsse vom schweren Fötus rerhinder'm sollten. Im letzteren Falle sollte also ihre Aufeahe eine Sicherstellung der Zirkulation séin. Vielleicht dienen sie zu beiden Zwecken.

I) Am Amiosgranulationen sind mit diesen verglichen sehe litein, aber immerhin leicht mit dem unbewaffineten Auge sichthar. Ihre (irösse

1 Grundriss d. Entwicklungsgesch d. Menschen u. d. Säuget. Leipzig, 1897. 
ist verschieden, meist weniger als ${ }^{1 / 3} \mathrm{~mm}_{3}$ : aber bisweilen sitzen mehrere zusammen und bilden zusammengesetzte etwa traubenförmige Bildungen, die Millimetrrlänge arreichen lönnen. Diese Amniosgranulationen sind nicht Epithelwucherungen, wie beim Pferde sich solche finden sollen, sondern sir bilden Knötchen, die von einem einschichtigen Epithel wie anderswo auf der Innenseite des Amnios bekleidet sind. Das Innere dieser Kinötchen besteht aus einer kompakten bindegewebigen Masse, in welcher nur verhältnismässig wenige Bindegewelszellen und einige Fibrillen eingeloettet liegen. In Folge der grossen Dichtigkeit fürben sich die Linötchen stärlker mit Eosin als die normalen Amniosschichten. Diese bestchen aus fibrillär'm Bindegewebe und sind äusserlich von einem platteren Epithel als innerlich begrenzt. Die Bedeutung dieser Amniosgranulationen ist mir nicht bekannt.

Die Villositäten an den distalen Enden der. Fruchtblase sind boi Elephus ryclotis, wie es scheint, noch unbedeutender als bei $E$. indicus. Itie übrigens vollständig glänzend glatte Oberfläche des Chorions erleichtert doch das Aufsuchen dieses Feldes. Sogar unter der Lupe ist es sehwer, die Gestalt der Villositäten klar zu bekommen. Auf Schritten sieht man aber, dass sie ganz kleine und schmale zylindrische Fortsïtze sind, die aus der' ('horiontlïche etwas hervorragen. Es ist ganz deutlich, dass diese Bildungen als Rudimente betrachtet werden müssen. Sie sind gar $\mathrm{zu}$ klein und reduziert, um jetzt irgendwelche Bedeutung zu haben, sondern sind nur Erinnerungen ans einer längst vergangenen Zeit, da die Vorfahren der Elefanten eine diffuse Placenta hatten, und eben deshalb sind sie auch von Interesse.

\title{
Homologien der verschiedenen Teile des Vogelschnabels.
}

\author{
Von Prof. E. LöNNBeRG (Stockholm).
}

L'auteur n'a pas remis le manuscrit de sa communication.

Voir: On the homologies of the different pieces of the compond Rhamphootheca of' birds. In : Arkiv för Zoologi, Bd. 1. Stockholm, 1904. 


\section{Experimentelle Ergebnisse über Hydropswasserkopfbil- dung fünf- und mehrbeiniger Individuen.}

Von Prof. G. TORNIER (Berlin).

L'auteur n'a pas remis le manuscrit de sa communication.

\section{Dimostrazione di preparati sullo sviluppo dei denti del Balistes capriscus.}

Del Prof. A. GHIGI (Bologna).

L'autore spiega brevemente alcuni fatti che si possono osservare in una serie di preparati tratti da parechi giovani di questo teleosteo.

La cresta dentaria scorre plofondamente al lato labiale dei denti funzionanti, tanto nella mascella superiore quanto in quella inferiore : i denti si formano ilnuque tutti al lato lingualo della cresta ed i piu distanti dalla medesima sono i più vecchi.

$\mathrm{Per}^{\mathrm{i}}$ sei denti che si trovano al lato interno della mascella superiore, le cose procedono in modo assai diverso : qui non vi è cresta dentaria ed ogni germe ha origine da uno zafto epiteliale che si distacca poi dalla mucosa hoceale quando la nuova formazione dentaria sta per raggiungere lo stadio campaniforme.

Notevole è l'influenza esel'citata dal maggiore o minore spazio sull'accrescimento dei denti di cambio. Due denti vicini non si trovano mai nello stesso stadio, ogni dente calcificato trovandosi in mezzo a due germi campaniformi, i quali si svilupperanno solo quando il primo sarà spuntato. Il cambio dei denti ha luogo, dunque, con sviluppo alterno.

In ciascun individuo si possono osservare fino a tre generazioni di denti, che si trovano rispettivamente nello stadio calcificato, in quello campaniforme ed in quello di bottone.

Sopra altri particolari l'autore si diffonderà quando pubblicherà il lavoro completo, corredato di figure. 


\section{Dimostrazione di preparati sopra una nuova forma di epitrichio nelle penne embrionali di Fulica atra.}

Del Prof. A. GHIGI (Bologna).

Anche su questo argomento l'autore, riservandosi di dare maggiori particolari nel lavoro completo, si limita a richiamare l'attenzione dei presenti sul fatto principale.

Le penne del capo che nel pulcino della folaga circondano la cresta, il becco e gli occhi non hanno nè l'aspetto esterno nè la struttura intima delle penne embrionali degli altri uccelli.

Laspetto esterno papillare è dovuto ad un ispessimento noterolissimo dell'epitrichio il quale offre vari strati concentrici di cellule appiattite, più numerosi allapice che non alla hase della penna. Tale astuccio formato dall'epitrichio non si sfaldta poche ore dopo la nascita del pulcino come avviene per le altre penne, ma persiste molti giorni ancora.

\section{Rekonstruktionsbilder fossiler Wirbeltiere.}

Von Prof.. R. BURCKHARDT (Basel).

Prof. Burckharut legt einigo Relionstruktionen fossiler oder ausgerotteter Wirbeltiere vor. Die nach seinen Skizzen von Herr'n Kunstmaler .Jon auswofühten Blätter in Farbendruck werden 45 meist hisher nicht rekonstruierte oder reproduzierte ausgestorbene Tiere wiedergeben. 


\title{
Beiträge zur Anatomie und Systematik der Læmargiden.
}

\author{
Von Dr. II. HELBING (Basel).
}

Her' Dr. H. Helbing referiert über eine von ihm verfasste Monographie der Lemargiden, die demnächst in den Nova Acta der Leopoldiniseh-Carolinischen Akarlemie 'rscheinen wird. Seiner T'ntersuchung lagen Exemplare von Lrmargus borealis, mehrere Exemplare von $L$. rostratus, sowie vier Stadien der späteren Embryonalentwicklung zu Grunde, ausserilem ein reiches Vergleichsmaterial an iuhrigen Selachiern. Die äussere Körperform der Embryonen von L. rostratus erscheint auf spitren Entwicklumgstadien rigentümlich morlificiert (Bauchkiele, Bauchschuppen, Bauchtlosisen ), sodass man aus Analogie anmehmen muss, dirse Embryonen führen längs der Steilliüsten rin Leben ähnlich rom

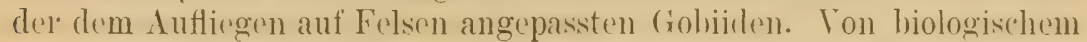
Interesse ist ferner, dass von Lxmargus borealis bisher keine kleineren Exomplane als $1 \mathrm{~m}$. lange gefunden wurden, dass aher die Männehen erst bei ca. $4,5 \mathrm{~m}$. geschlechts'eif' werden. 'In Verbindung mit der' Tatsache, dass der $1.2 \mathrm{~m}$. höchstens ererichende Seymmus seine Jungen erst geliert, wenn sie $30 \mathrm{~cm}$. Länge erreicht haben, ist aus diesem Fehlen kleinerer junger Exemplare von L. borealis der Schluss zu ziehen, die 1,2 m. langen Exemplare seien neugeborene Junge. I)as Skelett von $L$. horeulis und rostratus zeigt ungehener weitgehende Varlation der Elemente sowohl als auch ihrer Verbindung unter sich. Besondere Aufmerlsamkeit wurde dem Stachelrudiment an der I. Dorsalis und dem Subcaudalstrang goschenkt, welcher auch bei Spinaciden nachgewiesen wurde. Die Grösse der Schwanzflosere steht in umgoliehrter Proportion zu der der übrigen Unparflosson; sie rreicht bei $L$. borectis das Maximum, während hier dic drei Cnpartlossen auf das Minimum hrealsinken. L. borealis ist im Gegensatz zu Hasse nicht als primitiv, sonclern als terminale liesenform eimer Reihe aufzufassen, wolehe durch L. rostrutus und Seymmes zu den Spinaciden himabfülı̀t. 


\title{
L'incubation buccale chez le Tilapia galilæa Artédi.
}

\author{
Par le Dr J. PELLEGRIN (Paris).
}

On est habitué à considẻrer les Poissons téléostéens comme des animaux ne prenant aucun soin de leur procéniture. Si le fait est assez exact pour la majorité des espèces qui peuplent les cour's d'eau de nos climats tempér'és, il n'en est pas de même dans certaines régions tropicales. Les conditions différentes d'existence, les conflagrations plus nombreuses, la concurlence vitale plus intense ont amené certains modes de différenciations destinées à assurer autrement que par le nombre des germes produits la perpétuité de l'espèce.

L'un des plus intéressants et en même temps des plus efticaces est saus contredit l'incubation buccale. Les arantages de celle-ci sont nombreux. Les (’’ufs, en effèt, placés daus la cavité bucco-b)ranchiale du mâle ou de la femelle, se trouvent daus des conditions extrêmement favorables de développenent. Ils sont d'abord à l'abri des nombreux ennemis qui ne manquelaient pas de les attaqu'l' s'ils étaient alıandonnés comme c'est la règle hahitucllo au sein des eaux. Fnsuite, ils sont là dans un courant d'eau perpetuellement renouvelée et puisent facilement l'oxygène nécessaire à leur existence. Les soins ne s'arrêtent pas, d'ailleur's, à la naissance; les alevins vont encore, le plus souvent, chercher dans la bouche paternelle ou maternelle, - en cas de danger, - asile et protection.

C'est surtout chez les Siluridés que l'on a étudié l'incubation buccale. Elle est aussi des plus fréquentes chez les Cichlidés, acanthoptélygiens pharyngognathes des eaux douces tropicales africaines et américaines dont je viens de donner une monographie ${ }^{2}$.

("est Louis Arassiz (jui en 1869 signala le premiel' l'incubation buceale chez des Poissons de cettr dernière famille, dans le genre américain Geophagus. Ses observations furent confirmées depuis par L. Hensez.

Pour les Cichlidés de l'Ancien continent, le Pr L. Lor'tex qui a étudié les mœur's curieuses des Poissons de la Syrie, a fourni des détails fort intéressants sur les espèces du genre Chromis ou Tilapia et particulièrement sur le Tilapia Simonis Günther, auquel il avait donné le nom de Gromis puterfumilirs. paree que suivant lui, c'était le mâle qui se char-

1 Mém. Soc. Zool. Fr., T. XVI, p. 41. 1903. 
geait du soin des oufs et des jeunes. J'ai pu constater en disséquant un spécimen de cette espèce ayant la cavité buccale remplie d'cufs, qu'il šagissait d"une femelle; cre fait semble donc intirmer la maniere de voir (lu Pr LoRtet.

I)'ailleurs, des obselvations récentes de Botueswar sur le Tilupin milotica L. et sur des Poissons du lac Tanganyka appartenant aux genres Ectodus et Tropheus, il paraît résulter que c'est la femelle qui se charger des petits. Il en est de même chez un Cichlidé du Congo, le Pelmatochromis luterulis Boulenger, chez lecuel j'ai aussi constaté l'incubation buccale.

Enfin, grâce aux matériaux qui m’ont été adressés par M. Trulier, chef du transit du canal de Suez à Ismailia, j’ai pu faire un certain nombre de remarques sur les soins donnés aux reufs et aux jeunes par le Tilapia gatiliea Artédi, espèce fort commune en Palestine et répandue en Afrique, depuis le Nil jusqu'au Sénégal et au Niger.

Les 4 spécimens étudiés par moi provenaient de la partie la moins sammâtro du lac Mrnzaleh (Egypte). L'autopsie révéla $t$ fomellos. Voici d'ailleur's l'observation détaillée de chacun de ces Poissons.

Spécimen I. - Longueur : $165+45=210$ millimètres.

La cavité branchiale renferme une quantité assez considérable d'œufs volumineux. Ceux-ci sont étroitement pressés les uns contre les autres et oceupent toute la partic antérieure dupharynx ('ntre les ares branchiaux. On peut les évaluer approximativement à 80 environ, mais ils devaient être plus nombreux si l'on en juge par des impressions marquées à la partie supérieure de la cavité buccale et par quelques-uns tombés dans le bocal. Leur total pouvait donc, à peu près, être de 100 à 150 . A l'autopsic on trouve des ovaires assez flasques, réduits, avec des ovules petits, encore peu développés, d'une dimension moyenne de $0^{\mathrm{mm}}, 8$ à $1^{\mathrm{mm}}$.

Spécimen II. - Longueur: $130+40=170$ millimètres.

La bouche et le pharynx sont complètement bourrés d'embryons extrêmement jeunes de $5^{\mathrm{mm}}$ environ, avec une vésicule encol'e énor'me, par lapport à la taille. Les ovair'es sont moyens et les ovules déjà plus avancés que chez le spécimen précédent sont encore de dimensions réduites ( ${ }^{\mathrm{mm}}, 2$ en moyenne).

Spécimen III. - Longueur: $155+40=195$ millimètı's.

Le phal'ynx est rempli de petits alevins étroitement serrés et dans les positions les plus diverses, mais cependant avec la tête le plus souvent tournée du cuté de l'orifice buccal maternol. Ils sont a un stade heancoup plus avancé que ceux du spérimen II, la vésicule a complètrment disparu. Leur longueur est de 8 à 9 millimètres. Leur nombre peut être estimé à 200 si l'on ajoute les exemplaires qui se sont échappés de leur asile et sont tombés dans le bocal ayant servi au transport. Les ovaires sont très développés, les ovules presque mûrs ont une grosseur très voisine de 
celle des œufs et semblent indiquer qu'une nouvelle ponte ne tardera pas à se produire.

Spécimen IV. - Longueur: $170+50=220$ millimètres.

Il est très comparable au précédent. Les alevins, déjà grands $\left(9^{\mathrm{mm}}\right)$, sans vésicule, restent cantonnés dans l'arrière-pharynx, ceux qui se trouvairnt dans la partic antérieure étant tombés. Les ovaires sont également très volumineux, les ovules mûrs $\left(2^{\mathrm{mm}}\right)$, sont aussi gros presque que les oufs's pondus. placés dans la bouche du spécimen I. qui ont à peu plès $2^{\mathrm{mm}}, 4$ de diamètre.

Ces observations permettent de formuler les conclusions suivantes:

$1^{\circ}$ C'est à la femelle, chez le Tilipia galitza Artédi, qu'incombe l'incubation bucco-branchiale.

$2^{\circ}$ Les soins se poursuivent après la naissance au moins jusqu'à complète résorption de la vésicule des alevins.

$3^{\circ}$ Tandis que les œufs se développent dans la cavité branchiale, les ovules dans les ovaires s'accroissent parallèlement de sorte que lorsque les jeunes assez grands s'échappent, une seconde ponte peut s'effectuer is bref délai.

$4^{\circ}$ Dans cette espèce le nombre des œufs et des embryons doit êtr'e évalué de 100 à 200.

\section{On the hatching of anuran tadpoles and the function of Kuppfer's Stirnknospe.}

By E.-J. BLES (Glasgow).

L'auteur n'a pas remis le manuscrit de sa communication. 


\section{Neue Versuche und Beobachtungen über die Entwicklung der peripheren Nerven der Wirbeltiere.}

Von Prot,. R.-G. IIARBISON (Baltimore).

L'auteul' n'a pas remis le manuscrit de sa communication.

Voir: R. G. Harrison. Neue Versuche und Beobachtungen über die Entwickhung der peripheren. Nerven der Wirbeltiere. In: Sitz. Ber. Nieder'hein. Gesellsch. f. Natur- u. Heilkunde zu Bonn, $190 t$.

\section{Die Pneumatisation der Vogelknochen.}

Von Prof. H. STRASSER (Bern).

Der Vortragende bestreitet auf Grund von Untersuchungen, welche von ihm und seiner Schülerin, Frau Budustern, angestellt worden sind, die Annahme von Wildersuth, dass die Ausbreitung des Luftsackes im Knochenmark der Vögel Folge sei einer Verengerung der Arteria nutritiva. Auch ist von einer besondern membrana pneumatica an der OberHäche des Luftraumes nichts zu bemerken und nichts von besondern, eng an diese Obertläche geknüpften Resorptionsprozessen. Der Schwund des Markes stellt sich dar als ein durch die Freilegung des Markes an einer Stelle ermöglichter Schl'umpfungs- und Rückbildungsprozess, wobei unter den geänderten Verhältnissen der mechanischen Inanspruchnahme vor'übergehende besondere Faserungen im Nark auftreten, welche die Bedeutung funktioneller Strukturen haben. Auch bei der Bildung der Pori pneumatici handelt es sich nur um Folgeerscheinumgen der durch das Herantreten des Luftsackes geänderten mechanischen Verhältnisse der Corticallamelle.

Eine ausfühliche Publication wird demuächst in den "Anatomischen Heften » elscheinen. 

$4^{\text {me }}$ SECTION

IN V E R T É B R É S

(à l'exclusion des Arthropodes).

Sémces du lundi 15, mardi 16 , mercredi 17 et jemli 18 noût 1904.

Président: M. le Prof. E. Ehrers.

Vice-Présidents: MI. le Prof. R. Køhler et M. J. doe Guerne.

Secrétaire: M. le Prof. O. Funrmann.

\title{
L'acquisition de la forme arrondie chez les Mollusques acéphales dimyaires fixés en position pleurothétique.
}

\author{
Par le Dr R. ANThony (Paris).
}

En se plaçant au point de vue exclusif de l'attitude, on peut reconnấtre parmi les Mollusques acéphales deux grands groupes:

$1^{\circ}$ Les euthétiques qui peuvent être définis: ceux qui dans les conditions nor'males de leur' existence se trouvent constamment placés de telle façon que leur plan sagittal (bucco-ventro-ano-dorsal) soit perpendiculaire au plan sur lequel ils reposent ou progressent.

$2^{\circ}$ Les pleurothétiques qui peuvent être définis: ceux qui dans les conditions normales de leur existence se trouvent constamment placés de telle façon que leur plan sagittal soit parallèle au plan sur lequel ils reposent.

L'Anodonte, la Pholade sont des exemples des premiers; l'Huître, la Chame des exemples des seconds.

Les lois de la pesanteur faisant que les animaux ne peuvent en général reposer ou progresser que sur un plan sensiblement horizontal ou très légèrement incliné, on peut êtr’e amené à dire que les euthétiques sont ceux dont le plan sagittal est vertical dans les conditions ordinaires de la vie, les pleurothétiques étant ceux dont le plan sagittal est horizontal.

Comme parmi les Nonomyaires, il y a parmi les Dimyaires des formes pleurothétiques; les unes sont libres comme Pandora de la famille des 
Anatinidx, ou Corbula de la famille des Myidx; les autres sont fixées. Ces dernières sont les suivantes:

Dimyidx, formes fossiles semblant se rapprocher des Arcidx.

Myochamida, C'humostreidx, se rattachant ì la famille des Anatimida'.

Chamidx, se rattachant probablement à la famille des Cardiidre. (Je comprends provisoirement dans cette famille les formes enroulées de la fin du Jurassique et du Crétacé inférieur, telles que Diceras, Requienir, Toucasia, etc.).

Etheriidar, se lattachant nettement à la fanille des Unionidie (Mullevia faisant partie de cette famille est Monomyaire).

Rudistæe, c'est-à-dire toutes les formes à arête ligamentaire plus ou moins développée telles Hippurites, Radiolites, Biradiolites, etc.

Chondrodontidx, formes paraissant se rapprocher des Pinna.

La fixation pleurothétique entraîne chez les Acéphales dimyaires des modifications nombreuses parmi lesquelles la tendance à l'acquisition de la forme ar'rondie. En effet, comme toutes les formes animales fixées l'une faron générale, les Dimyaires pleurothétiques tendent à arrondir leur base. Supposons le cas qui est d'ailleur's le plus firéquent où le plan sur lefuel repose l'animal soit sensiblement horizontal : l'arrondissement pent itre expliqué par l'homogénéité des conditions ambiantes. Par le fait qu'il est fixé, l'animal n'a aucune raison de s'allonger ou de se raccourcil' dans un sens plutôt que dans l'autre, comme l'animal qui se déplace; l'action de la pesanteur qui seule peut agir sur lui est précisément dirigée perpern.liculairement au plan horizontal sur lequel il repose et ne peut pas, par conséquent, modifier la forme de son périmètre. Le Dimyaire pleurothétique fixésur un plan sensiblement horizontal, ot tout animal d'une façon plus générale, s'ar'rondit pour les mêmes raisons (qu'une goutte de cipe chaude tombant et s'étalant librement sur un plan horizontal.

Comment se fait l'arrondissement chez les Dimyaires fixés en position pleurothétique?

J'ai essayé de résoudre cette question pour les genres Chama et Etheriar en ćtudiant lo développement post embryonnaire de la coquille de ces animaux. Il peut se faire suivant deux processus, l'un l'emroulement qui s'applique au genre Chama, l'autre auquel je donne le nom de pseudoplicature et qui s'applique au genre Aitheria.

$1^{\circ}$ A r rondissement par enroulement. Si l'on considère la coquille

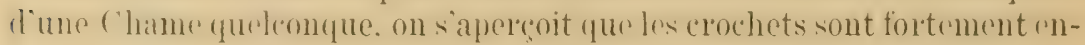
roulés en avant et que le ligament présente un aspect tout spécial : simple 
dans sa partie postérieure la seule fonctionnelle, il se divise en avant en deux branches qui suivent les hords antérieurs des crochets et décrivent une hélice comme cux. En suivant le développement de la Chame, on s'aperçoit que sa prodissoconque a la forme d'un petit isomyaire euthétique. I ans ses stades suivants de développenent, la jeune Chame prend ensuite une forme allongée dorsoventralement et ce n'est que lor'subelle a atteint 1 millimetre de longueur antéro-postérieure, à peu près, qu elle se fixe par son bord antérieur dans beaucoup d'espèces. A partir de ce moment, elle s'ar'ondit. Les couches calcaires successives que secrètent les bords du manteau débutent dans la région postérieure du ligament, de plus en plus loin de celui-ci, dans le sens ventral ; il en résulte que le ligament qui, comme l'on sait, se développe d'avant en arrière sur le bord dorsal de la coquille, suit nécessairement, dans son développement, une ligne courbe. En conséquence, à mesure que l'animal augmente de taille, les valves tendent, lor'squ'elles s'ouvi'ent, à s'écarter de plus en plus l'une de l'autre en avant, exécutant un mouvement de rotation autour d'un axe transversal. Il en résulte que les deux moitiés de toute la région antérieure qui est la plus ancienne du ligament, se séparent de plus en plus. En outre, comme chaque nouvelle couche calcaire tend à remplir constamment l'espace laissé libre par l'entrebaillement des valves, il s'en suit que l'ayport de calcaire s'étend de plus en plus en avant, à mesure que le ligament se déplace ver's l'ar'rière. Il est facile de se r'endre compte qu'un enroulement est le résultat final de tout ceci et que le contour sagittal arrive à être complètement arrondi.

Le même enroulement pent être constaté chez les Diceras, Requienia, Toucasia, etc.

$2^{\circ}$ A r rondissement par pseudoplicature. Sur une coquille d'Ethérie, le ligamrnt apparait sous la forme d'un petit triangle isociele à surface cylindrique, dont la pointe est dirigée en arrière et dont les grands côtés s'insèrent sur l'une et l'autre valves, au fond de sillons très profonds, et dont la présence se traduit, sur la face intérieure de la valve, par une sol'te d'éperon ligamentaire.

J'ai eu la chance de pouvoir étudier la formation de ce sillon et de cet éperon ligamentaires sur diver's jeunes individus d'Etheria senegatica Bgt. et d'Etheria Caillaudi Féruss. Dans l'une et l'autre de ces espèces, la prodissoconque est encore semblable à un prtit isomyaire cuthétique allongé d'avant en arrière. A ce stade prodissoconque, fait suite un autre stade dans lequel l'animal, tout petit encore, affecte la forme d'un petit Unionidé. A ce moment, le ligament est encore très peu développé et les couches calcaires secrétées par la partie postérieure du manteau, se prolongent de plus en plus loin dorsalement, tout an contraire de ce qui se passe chez les Chames. Il en résulte que le bord dor'sal de la coquille devient sinueux, formant comme une boucle qui se resserre de plus en 
plus, et, c'est sur cette ligne sinueuse qu'est obligé de s'étendre le ligament dont le développement est beaucoup plus lent et plus tardif que celui de la région postérieure de la coquille. Il l'ésulte de tout ceci une apparence de plicature qui produit le sillon et l'éperon ligamentaire des Itheries, lesquels sont d'ailleurs indubitablement les analogues de l'arete ligamentaire des Rudistes (Hipmurites, Rudiolites, Biradiolites, (ritc...).

Donc. ('herz less Dimyaires fixés en position plenrothétique, l'arrondissement peut se faire suivant deux processuls absolument indépendants et tout différents, l'enroulement pour les Chamidar, les Chamostreide', la pseudoplicature pour les Etheriidæ et les Rudistes.

\title{
Ueber den Bau des Prototrochs der Echiuruslarven.
}

\author{
Von Prof. W. SALENSKY (St. Petersluurg).
}

Vor mehr als 20 Jahren hat Prof. N. KLeineniberg ${ }^{1}$ ein larvales Nervensystem bei den Larven mancher Anneliden entdeckt. I)ie darauffolgenden Unter'suchungen haben nachgewiesen, dass von allen T'eilen dieses komplizierten Nervensystems der Annelidenlarven die wichtigste Rolle in morphologischer: wahrscheinlich anch in physiologischer Beziehung, dem lingnerven zugeschriehen werden muss. Dersello, an der Basis des vorderen Wimperrings gelagert, wurde bei allen in der letzten Zoit in dieser Beziehung untersuchten Annelidenlarven nachgewiesen Trotz vieler Mühe ist es Prof. Kleineniberg nicht gelungen, den Zusammenhang dieses Nervenrings mit den Wimperzellen zu entdecken. Die daranffolgenden Forscher waren nicht glücklicher gewesen. Die Frage der Innervierung der Wimperzellen durch den Ringnerv bleibt bis jetzt offen.

Während ich mich in der letzten Zeit mit der Anatomie und Metamorphose der Trochophoralarven einiger Ammeliden und Gephyreen beschitf-

${ }^{1}$ N. Kuninexberg. Sull'origine del sistema nervoso centrale degli Annelidi. Atti R. Acad. dei Lincei, Vol. X, p. 420) 1882.

2 N. Kuntnesberg. Die Entstehung des Annelides aus der Lar'e von Lopadorhynchus. Zeitsch. f. wiss. Z., Bd. 44, p. 13. 1886. 
tigte, habe ich meine Aufmerksamkerit auf den Bau des Prototrochs ge-

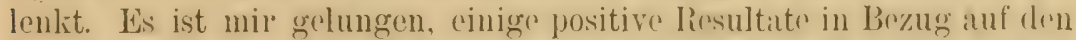
Bau des Prototrochs der Echiuruslatre zu brekommen, dio ich hier kurz mitteilen will.

Die Larven von Echiurus besitzen vier Wimperringe, die als Proto-, Meso-, Meta- und Telotroch bezeichnet werden können. Der Prototroch zeichnet sich von allen ührigen Trochen durch die grösste lionnplikation soines Baues ans und stellt den wichtigsten Iiowegungsapparat der Larve dar. Er tritt in For'm eines vor dem Munde gelegenen Ringwulstesauf, weleher auf seinem mitteren vorragenden T'eile rinige Reihen

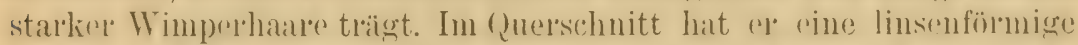

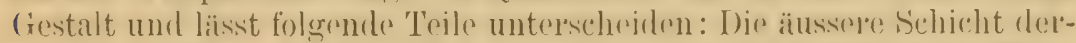

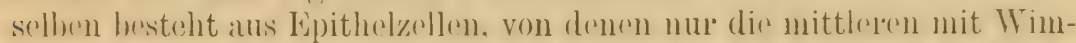
peren besctzt sind, willerend die vorderen und anch die hinteren dere Wim-

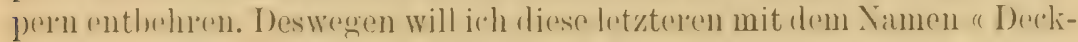
zellen » belegen. Die innere basale Schicht des Prototrochs, welche dem starlien Iingmusiol (Prototrorhmuskil) anlingt, stellt einen Nerveming dar, welcher teilweise aus Nervenfaser'n, teilweise aus Nervenzellen zusammengesetyt ist. Wir wollon num jeden dirser Brstandtrile des P'rototrochs näher betrachten.

Die Deckzellen stellen cylindrische Epithelzellen dar, dessen Pro-

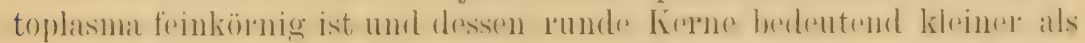
diejenigen der. Wimperzellen sind. Sir bilden zusanmen zwei nach vorme

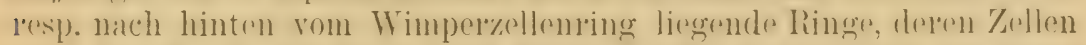

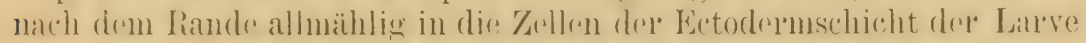

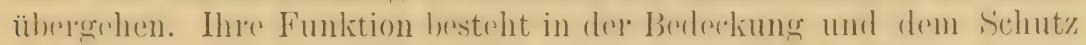
der wesentlichen Elemente des Wimperapparates, der Wimperzellen; deswegen stellen sie nur ein Nebenorgan des Wimperapparates dar.

Die Wimperzellen sind eigentlich in derselben Reihe mit den Deckzellen gepflanzt und stammen wahrscheinlich mit den letzteren

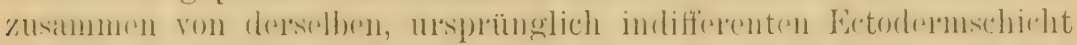
al). Sie unterscheiden sich von den letzteren nicht nur durch ihre viel grösseren Dimensionen, sonder'n auch durch ihre innere Struktur sehr bedeutend. Betrachtet man diese Zellen auf den Querschnitten durch

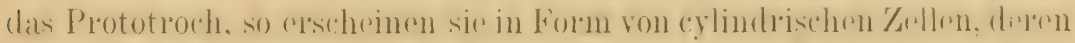

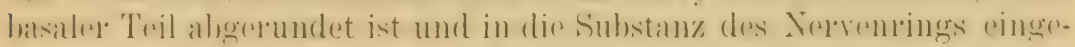

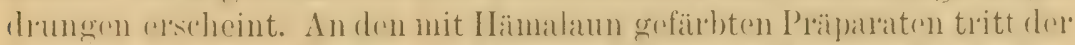

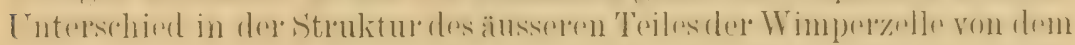
basalen selı' deutlich hervor. Der' basale Teil der Zelle besteht aus einem

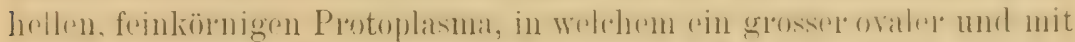
charakteristischen Kernkörperehen versehener Ker'n liegt. Der äussere

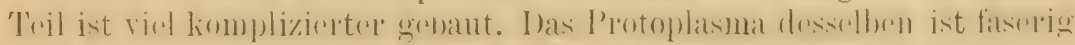

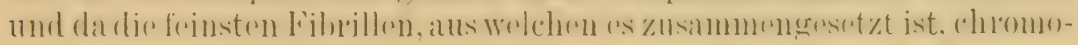


phil sind, so crscheint dasselbe viel intensivel gefärbt als dee innere. Die Fibrillen verlaufen von aussen basalwärts bis ungefähr zur Mitte der Zelle und hören an der Grenze des äusseren und des basalen Teiles auf. Der äussere Rand der' Wimperzelle ist von einer hellen Zone, welche man Basalsaum zu nennen pflegt, begrenzt; die von mehreren Forschern in dirsem Saum rerschiedener Wimperzellen beschriebenen Basalfüssehen und die beiden Reihen Basaikörperehen sind auch hier vorhanden und bei starker Vergrösserung dentlich erkembar. Ihre Zahl ist aber viel geringer als diejenige der Fibrillen.

Die Längsschnitte des Prototrochs (Querschnitte der Larve) lassen weitere Struliturdetails der Wimperzellen entdecken. Anstatt der feinen Fibrillen, die wir an Querschnitten beobachten, treten hier in den äusseren Teilen der' Wimperzellen starke, sich intensiv färbende Streifen hervor, die gerade in derselben Richtung wie die früher erwähnten Fibrillen von aussen an basalwärts sich zichen, sich allmählig verdünnen und teilweise an der Grenze des basalen Teiles der Wimperzelle aufhören, teilweise sich weiter basalwär'ts hineindrängen und beinahe bis an die basalen Rïnder der Zellen verfolgt werden können. Nan könnte auf Grund der Beobachtung der Längsschnitte diese Streifen für Bü̈ndel von Filnrillen halten. Dir Vergleichung der Lüngsschnittbilder mit den Bildern, wolche man an den tangentialen Schnitten bekommt, weist aher darauf hin, dass die angeblichen Streifen eigentlich Platten darstellen, welche die Wimperzellen in der Längsrichtung durehsetzen. Untersucht man eine Serie tangentialer Schnitte des Prototrochs, in denen die Wimperzellen in Quer'schnitten erscheinen, so trifft man in den äussersten Schnitten der Serie immer eine Reihe palissadenartiger Stäbchen, welche nichts anderes als Querschnitte der in den Lüngsschnitten anftretenden Streifen darstellen.

Durch die Kombinierung verschiedener Bilder, die man aus den in verschiedener Richtung geführten Schnitten erhält, bekommt man folgende Vorstelluug vom Bau der Wimperzellen. Jede Wimperzelle besteht aus zwei Teilen: einem basalen, welcher ein feinkörniges Plasma enthilt, und einem äusseren, welcher ausser dem Plasma noch eine Reihe längsgestellter, unter einander parallel liegender Platten enthält, die ihrerseits aus feinsten Fibrillen zusammengesetzt sind. Diese Platten liegen innerlich dem Basalsaum an und stehen durch ihre Fibrillen mit den Basalfüsschen des Basalsaumes in innigster Verbindung.

Einen ähnlichen Batu der Wimper\%ellen hat neuredings Wouteneck hei den Polygordinslarven beobachtet; nur sind zwischen dem Bau und der Lage der Wimperzallen der Polygordius- und der Echiuruslinven bedeutende L'nterschicde hervorzuhehen. Firstens sind die Timperzellen des Prototrochs der Polygordiuslarven zweireihig, während diejenigen der Echiurusharen einreibig angeordnet sind. Zweitens enthalten die 
Wimperzell'n der Polygordinslarven nur je oine Wimperplatte, während die Wimperzellen der Echurustaren mit violen Wimperplatten vel'sehen sind.

Gehen wir nun zum Ringnelv über. Derselbe lässt sich hauptsächlich an (Quer-resp). Tangentialschnitten der Lal've studiren. Ier Ringnerv der Echiumslarven zeichnet sich von dem der' Annelidenlarven besonder's dadureh aus, dass er von den anderen Elementen des Prototrochs nicht so schar'f abgegrenzt ist, wie es z. B. bei den Larven der Lopadorhynchus, wo el zuerst von KLEINENBera entdeckt wurde, der Fall sein muss. Er bildet den Basaitheil des Prototrochs aus und ist am stiuksten in der hinteren Abtheilung des letzteren, über dem Ringmuskel entwickelt; weiter nach vorne plattet er sich belentend ah. Seinem Ia un nach ist er der sog. Scheitelplatte und den Seitennerven volliommen ähnlich, indem el aus einem Geflecht feinster Filsillen und aus den Nervenzellen zusammengesetzt ist. Die Fibrillarsubstanz ist nur im hintern Theile stark entwickelt; in dem vorderen Theile des Prototrochs ist der Ringnerv nur durch Nervenzellen repräsentirt.

Es sind zwei Arten der Nervenzellen im Ringnerv zu unterscheiden. Eine davon ist ganz der fibrillären Substanz einverleibt, so dass von diesen Zellen nur Kerne übrig bleiben, während ihr Protoplasma in feinste Fibrillen zerfallen ist. An den Stellen aber, wo die fibrilläre Substanz, wie im vorderen Theil des Ringuerven stark reducirt ist, bleibt auch ihr Protoplasma erhalten und läuft in feinste Nervenäste aus. Nach der Analogie mit den Zellen der Scheitelplatte und der Seitenneven, welche die filıilläre Substanz produciren, kann man diese Zellen für die Erzeugerimmen der fibrillären Substanz des Ringnervens halten und sie fibrillogene Nerveuzellen nennen. Die Nervenzellen zweiter Art sind in den tieferen Schichten der fibrillären Substanz eingebettet. während diejenigen der ersten Ar't an der Grenze des Nerven und der Wimperzeilen gelagert sind. Sie unterscheiden sich dabei hedeutend dureh ihren Bau von den fibrillogenen Tervenzellen. indem sie die charakteristiche Gestalt der Nervenzellen beibehalten, aus einem sich stark färbenden Protoplasma und zahlreichen Nervenästen bestehen. Ihre Kerme sind rund, während diejenigen der fibrillogenen Vervenzellen immer oval erscheinen.

I) Fibrilläsulstanz, sowie die beiden Arten der Vervenzollen treten in die näthste Bezirhung zu den Wimperzellen, indem sie der Innervation delselben dienen; aber das Verhalten heider Vervenzellenarten zu den Wimperzellen ist verschieden.

Die distale Fläche des Ringnerven stellt keine elone Fläche dar. Sie kommt in Berührung mit der basalen Fläche der Wimperzellen, und da die Wimperzellen basalwälts alogerundet erscheinen, so treften sie nü mit ihren Seitentlächen zusammen : zwischen ihren Basaltlächen bleiben 
kleine Lücken zurück, in welche die fibrillüı Substanz des Ringnerven hineindringt. I)ic Letztere schickt namentlich feine Aestchen nach der Peripherie fort, welche zwischen den einzelnen Wimperzellen verlaufen und auf den Schnitten ziemlich weit (bis $3 / 4$ der. Höhe dieser Zellen) verfolgt werden liönnen. Es entsteht dadurch eine Art Wabenwerkes, in desen Vertiefungen die Wimperzellen eingepflanzt erscheinen. Besonders dentlich treten diese Bauverhältnisse an denjenigen Schnitten auf, in welchen die Wimperzellen zufälligerweise ausgefallen sind. Ich hetrachte diese Fortsetzungen des Ringnerven als Nervenäste, die zur Innervierung der Wimperzellen dienen.

In vorderen Teil des Prototrochs, wo die fibrilläre Substanz des Ringnerven aufhört und die fibrillogenen Nervenzellen allein bleiben, umfassen dieselben die abgerundeten basalen Teile der Wimperzellen und senden Fortsüitze al), welche den Seitentlächen der Wimperzellen anliegen.

Die zweite Art der Nervenzellen verhält sich gegen die Wimperzellen anders. Wir aus der Vergleichung verschiedener tangentialer Schnitte zu schliessen ist, hilden diese Nervenzellen, indem sic durch ihre Acste mit einander in Berührung treten, eine Art Netz aus, welches aber so locker ist, dass es nicht auf einem und demselben Präparat dargestellt werlen liamn. Einzelne Fortsätze dieser Zellen gehen nach der Peripherie aufwärts gegen die Wimperzellen und teilweise ins Innere derselben hincin, teilwoise liegen sie der Peripherie derselhen an. In beiden Fällen scheint es, dass die Aeste der in Rede stehenden Zelien nicht die Wimperzellem solbst unfassen, sondern mit den Wimperzellen in innigster Berührung stehen.

Aus dem hier Mitgeteilten geht herver, dass der Ringnerv zweierlei Nervenarten zu den Wimperzellen abgicbt, von denen die einen die Wimperzellen umfassen, die andern in diesclben hineindringen. Da diese heiderlei Innervierungsarten von verschiedenen Nervenzellenarten ausgehen, so seheint es mir sehr plausibel zu sein, dass sie auch funktionell verschioden sind und dass die beiderlei Nervenzellen funktionell den sensiblen und motorischen Zellen entsprechen. 


\title{
Le mode de nutrition des embryons chez Purpura lapillus.
}

\author{
Par le Prof. P. PELSENEER (Gand).
}

Pendant le développement, on l’emarque assez l'arement, entl'e deux organismes voisins, des différences profondes dans les caractères morphologiques fondamentaux. Le plus souvent, les différences ne portent que sur des caractères adaptatifs de la vie embryonnaire. C'est une différence de ce dernier ordre qui s'observe entre deux Gastropodes rachiglosses bien connus: Nassa reticulata et Purpura lapillus.

$1^{\circ}$ Chez Nassa reticulata, chaque coque de la ponte renferme plusieurs centaines d'urufs, et de rhaque coune ausisi, sort ultérieurement, un nombre égal de larves nageuses (véligères).

On s'explique aisément que ces larves nageuses, exposées, encor'e faibles, aux intempéries et aux ennemis, doivent être nombreuses, pour contrebalancer' ainsi l'effet des chances de destruction; tandis que des embryons qui sortent de l'œuf, plus âgés et presque semblables à leur's parents, et qui ainsi ne sont pas aussi exposés à ces mêmes danger's, peuvent au contraire être en très petit nombre.

$2^{\circ}$ Ce dernier cas se trouve réalisé chez Propura lapillus (et quelques autres formes de Rachiglosses: Buccinum, Fasciolaria, Fulgur, etc.), c'est-à-dire que, de chaque coque de la ponte, il ne sortt qu'une douzaine

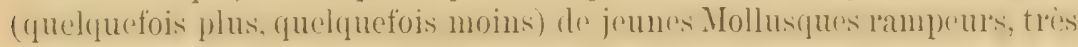
pareils à leurs parents.

Et cependant, à l'origine, chacune de ces coques contient, aussi, des

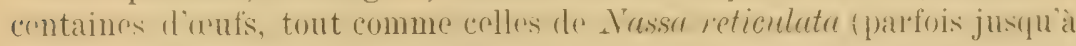
600: chez Purpura, notamment).

On en a naturellement conclu que les oufs non développés ont contribué à assurel le développement des autres. Mais Koren et Davielssen avaient avancé que plusieur's cufs servent à former un embryon, tandis que Carrenter, de son côté, était d'avis que chaque embryon provient bien d'un seul ouf. Ces observations remontent à un demi-siècle, et, personne, depuis, n'a décrit ni observé la façorr dont ces œufs non dévefoppés sont utilisés choz Puppra lruillus (Selexka n’ayant pas tratité ce point spécialement).

L'expérience prouve (qu l'interplétation de lionex ot I)ANersisen citait

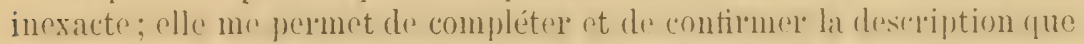
Carpenter a donnée du rôle ultérieur de ces œuf́s. 
$3^{\circ}$ L'examen d'un grand nombre de coques de ponte montre que, dans chacune d'elles, la presque totalité des œufs se segmentent d'une façon anormale, en donnant des (blastomères ) de différentes tailles, qui se trouvent juxtaposés de la façon la plus irrégulière, et qui, tous, sont également chargés de vitellus.

Au contraire, les œufs qui se segmentent régulièrement ou do façon normale, forment l'exception. Ils éliminent des globules polaires, alor's que, constamment, les autres en sont dépourvus (il en est de même chez Fasciolaria, voir: Mac Murrich, p. 406).

Pour le reste, on ne voit pas de différence originelle essentielle entre les deux sortes d'éléments. Carpexter tenait les premiers (à segmentation irrégulière) pour de simples sphères vitellines ou yolk segments (p. 22, 23). Nais l'examen du contenu frais de nombreuses coques, à l'aide de colorants divers, n'a pas fait voir de constitution différente.

On aurait pu supposer qu'il y a un rapport entre le sort de cerlains oufs et l'existence de deux sortes de spermatozoïles (enpyrènes et oligopyrènes) chez Purpura. Nais ces deux sortes de spermies se rencontrent aussi chez bien d'autres Gastropodes, sans qu'on y observe des œufs féconds et des œufs stériles.

$4^{\circ} \mathrm{La}$ segmentation des ovules anormaux s'arrête de bonne heure. Ces œufs irréguliers finissent alor's, comme l'avait vu CARPENTER, par s'accoler les uns aux autres et par constituer ainsi une masse informe de vitellus à surface papilleuse.

Alor's, les jeunes embryons provenant d'œufs normalement segmentés, sont encore de petite taille; il en existe de 6 à 40, nombres extrêmes olsservés a l'intérieur d'une même coque (une douzaine ou une quinzaine en moyenne). A cet état, ils s'appliquent sur la masse vitelline formée par les autres ceufs, tout comme les embryons de Céphalopodes sont attachés sur leur propre vitellus individuel.

Ils viennent, en effet, se fixer, par la bouche, sur une saillie du vitellus et tournent autour d'elle, comme s'ils s'y vissaient, dans le sens du mourement des aiguilles d'une montre (l'embryon étant considéré par' sa face orale). De cette manière, le vitellus s'enfonce peu à peu dans le stomodæum, en s'y moulant pour ainsi dire, et vient s'unir au vitellus propre ou intérieur (souvent plus pâlle) de l'embryon. Parfois, il a été distingué un rapide mouvement péristaltique de l'ossophage, aidant à cette pénétration du vitellus.

A ce moment, les embryons sont donc en quelque sorte parasites sur cette masse vitelline commune, qui ne leur appartient pas en propre, bien qu'elle provienne du même ovaire qu'eux: aussi méritent-ils, jusqu'à un certain point, le nom de "adelphotrophiques".

La masse vitelline collective est alor's absorbée peu à peu et plus ou moins vite, suivant le nombre d'embryons. Ceux-ci deviennent tour' à 
tour indépendants, et les deux derniers restant sont momentanément unis pal une sorte de court cordon vitellin, qui se rompt finalement.

$5^{\circ}$ Entre-temps, et suivant la rapidité de l'absorption du vitellus, les embryons ont pu se développer plus ou moins. Mais le plus généralement, leur évolution n'a pas progressé beaucoup et leur or'ganisation n'est guere modifiér. Habituellement, ils ne posserlent que le stomodieum. Ie velum et l'ébauche de la coquille et des deux reins larvaires, tous organes qu'ils présentaient déjà avant de s'attacher au vitellus nutritif collectif. Leur principale modification consiste en une augmentation considérable de volume (inégale toutefois dans les différ'entes coques, où le nombre des embryons diffère).

Quant aux emblyons qui, accidentellement, ne sont pas fixés à la masse vitelline de leur coque, ils ne grossissent pas, épuisent leur vitellus propre et n'arrivent jamais au terme de leur évolution complète.

$6^{\circ}$ Si l'on compar'e ce mode de développement à celui qui se rencontre chez diver's autres Gastropodes streptoneures, on trouve la série des stades suivants :

a) Nassa reticulata, avec des œuf's pondus sous le niveau de la marée basse et dont sortent de nombreux petits veligers.

b) Purpura lapillus, avec des œuf́s littoraux (c'est-à-dire intercotidaux), dont sortent, souvent pendant la mer basse, un petit nombre de jeunes de grande taille, avec la forme de l'adulte, - stade marquant une preniere condensation emhryogénique. Littorinn littoren et L. obtusata offirent à très peu pres le même deoré de condensation embryogénique. puisque do leur's pontes, également intercotidales, sortent des jemnes peu nombreux ayant aussi la conformation des parents.

c) Littorina rudis, à habitat supra-littoral, dont la ponte est retenue dans l'oviducte où elle éclôt, montrant ainsi le delniel' tel'me de la condensation embryogénique, l'ovoviviparité.

\section{BIBLIOGRAPHIE}

Konen og. Danielssen. Bidrag lil Pectinibranchiernes Udviklingshistorie. Bergen, 18 \% 1 .

Carpenter W.-B. On the Development of the Embryo of Purpura lupillus. Trans. microscop. soc., vol. III. 185̈3.

MaC Munaren. A contribution to the Embryolog!y of the Prosobranchs Gasteropods. Studies from Biol. Labor. John's Hopkins Univ., vol. III. 1887. 


\title{
Ueber den Ursprung der Cephalopoden.
}

\author{
Von Prof. H. SIMKOTH (Leipzig).
}

Mit 1 Tafel.

Leber die Herkunft der Cephalopoden existieren verschiedene Theorien, olne dass man sich über die Fragen hätte einigen können, welche über die allgemeine Morphologie hinausgehen. Die Klasse steht innerhalb der Mollusken nach wie vor isoliert, trotz der Arbeiten von Grobiben, PetSENeER, Lacaze-Duthiers u. A.

Bei einer manchfach veränderten und gefestigten Auffassung, die sich mir in den letzten Jahren, namentlich auf Grund der Pendulationstheorie, üher die Entstehung der Weichtiere aufgedrängt hat, glaube ich auch für die Tintenfische cine bestimmtere Ableitung geben zu kïnnen, namentlich deshalb, weil neuere und neneste Arbeiten über die Entwicklung der Prosolranchien, welche meinen Vorgängern noch nicht zu Gehotr standen, eine positive Grundlage fül' die Spekulation geschaffen hahen.

Yor zwei dahren versuchte ich bereits, die tetrabranchiaten Nautiliden auf Landschnecken zurückzuführen, wenn auch nur in allgemeinen Andentungen. ${ }^{2}$ Heute halte ich mit der Behauptung nicht mehr zurück, dass auch die Diblanchiaten von Schnecken abstammen, und glaube die Behauptung viel mehr ins einzelne begründen zu können. wohei freilich einzelne Veränderungen in der Deutung der Fastropoden unerlaisslich sind. Ich hoffe indess, dass alle Einzelheiten, die ich vorzulringen habe. sich duich Tatsachen werden stützen lassen bis zu einem hohen Wah r scheinlichkeits grade.

Die Gastropoden zunächst fasse ich, wie ich wiederholt ausgesprochen, als Frzengnisse des Landes auf, so dass die ältesten Formen Lungenschnecken waren. Dje Schale ist ein Produkt des Landlebens, ein Rückenschutz genen Trocknis. Ine Vorfahren waren nicht, wie es bisher meist aufgefasst wurde, nur Turbellarien, sondern Turbellarien zusammen mit Gastrotrichen als eine Klasse genommen, eine Vereinigrung, für welche ich Ptingsten in Tübingen die Begrüurlung zu geben versuchte ${ }^{2}$, beidro direkt aus Infusorien entstanden unter dom Finfluss des Landlebens, die Turbellarien aus Holotrichen, die Gastrotrichen aus

1 Simrotr. Das natürliche System der Erde. Verhdlg. d. d. Zoolog. Ges. 1902.

2 Id. Ueber den Ursprung der Echinodermen. Ibid. 1904. 
Iypotrichen. Bei den (iastrotrichen hahen wir in den Borstenluändeln die erste Anlage eines Epipodiums (Fig. 1).

Beiläufig kömnte dieser Teil der Ableitung als nelensichelılich bei Seite gelassen werden, wenn er auch im Einzolnen wieder Verwendung finden mag (s. 11.).

Wohl aber muss, um dem 'Thema näher zu liommen, behauptet werden, das: die (iastropoden sich anfünglich durchweg be gat te te n, und dass die freie Befrochtung der ejaculierten Zengungsstoffe im Seewasse't, wie wir sie bei grossen Weichtiergruppen und so bei den in vieler Hinsicht altertümlichen Scutibranchien kennen, eine secundïre Erwerbung ist, die anf der hallsessilen Lehensweise in der Brandung beruht: der Hinweis mag genügen, datss selhst hochstehende Vorderkiemer, wie Coralliophila unter den Rhachiglossen der Copula verlustig gehen können, sobald sie sesshaft werden. Ferner hat mich die Durcharbeitung des gesammenten die Zeugung hetreffenden Materials für den Bronn zu dere Ueberzengung gehracht, dass die Prosohranchien ursprünglich proterandische Hermaphroditen waren, nach Art der Pulmonaten (für die Begründung verweise ich auf die nächsten Brons-Lieferungen) ${ }^{1}$. Für die nahen Beziehungen selbst der' altertümlichsten Vorderkiemer zum Lande können noch die Struktur und Funktion der Mantelhöhle herangezogen werden. Pleurotomarien haben z. T. ein ächtes Lungengefäisnetz und die Kiemen nur als Secundirrerwerbungen an Rande (Bovvier); Patellen atmen, wie wir von Plymouth aus neuerdings erfahren haben, während der Ehhe direkt Luft, was meiner Deutung ihrer Jantelhöhle als Lunge entspricht (s. Brovs), Trochusarten gehen unter Umständen aus den Wasser und nehmen Luft in den Kiemenraum (JEFFreYs), eine ostasiatische Navicella, die ich als Landform von einem Sammler erhielt, zeigt an der Decke einen mässig entwickelten Lungengefässbaum (Fig. 6 A) u. drgl. m.

Und damit wende ich mich zum eigentlichen 'Thema.

Ich lege eine Trochuslarve zu Grund nach RoBers's ${ }^{2}$ Beschreibung. Die bereits eingerollte Schale hat eine vollkommen exogastrische Stellung, also genau wie bei Argonauta. Das Velum ist noch vorhanden, unmittelhar dahinter tritt als Qnerwulst der Fuss hervor. zwischen beiden würde der Mund liegen (Fig. 2). Mit dem Schrumpfen des Velums treten mummehr nach der. In'ehung der Schale die schon vorher angelegten Tentakel hervor, und daran schliessen sich die Epipodialtaster, pechts und links je vier, allmählich von vorn nach hinten hervorwachsend (Fig. 3). Zunächst ist auch jetzt nur der dem anfänglichen Querwulst

1 Bronn. Klassen und Ordnungen des Tierreichs. Sinroth. Mollusca. Lief. 66-70.

${ }^{2}$ A. Robert. Recherches sur le développement des Troques. Arch. de zool, expér. et gén. (3) 10. 1902. 
entsprechende Vorderfuss da, die ganze hintere Sohlenflitche folgt erst nach. Fig. 3 zeigt aber, was RoBent abbildet, ohne es im Text zu erwähnen, oine merliwürdige Haltung des Vorderfusses; seine Ränder haben sich zusammengekrümmt, so dass eine Rinne entsteht, ganz wie der Nontilustrichter, aus dem man ja allgemein durch Verwachsung der Ränder den Tr ic h te r der Dibranchiaten morphologisch ableitet. Man kann sich ohne weiteres vorstellen. dass aus einem solchen Propodium der Trichter der Cephalopoden hervorgeht ${ }^{\text {. }}$

Eigenartig sind sowohl die $\mathrm{F}$ üh ler, als die Ep i podialtaster, wobei ich zuerst auf die gleiche Ausbildung dieser Organe bei so vielen Rhipidoglossen hinweisen möchte. Sie gehören zusammen der Epipodiallinie an, auf welche Thisue mit Recht so grossen Wert gelegt hat. Bald als Paletten auf der Stirn zwischen den Tentakeln ausgebildet, bald als kleinere Fransen an derselben Stelle, haben sie alle den dichten Besatz von Sinnesknospen geneinsam, der sie oft als gezackt und gefranst erscheinen lïsst. Threr ersten Aulage nach erscheinen sie bei Trochus nach Ronert unter der Form von Hirschgeweihen, wohei die hervorragenden, noch veleinzelten Sinnesknospen deren Enden bilden.

Hier setzt nun ein doppelter Gedankengang ein, der von Grobies, wenn or den Vergleich zwischen Cephalopoden und Scaphopoden zog, und der' yon Pessexerer. wenn er die 'Tentakelschilder der letzteren mit den Captakeln von den Paletten der Rhipidoglossen ableitete.

Jedes Captacalum ist aus einer Sinnesknospe hervorgegangen. Ihre Basis hat sich \%u einem langen muskulösen Sticl ausgezogen. Ihr Ende ist saugnapfartig 'ntwickelt mit Ḱlebdrüsen zum Fassen der im Schlamm verborenen Foraminiferen und anderen Protozoen. Ihre Enpfindlichkeit ist durch Einlage eines Ganglions gesteigert.

Ganz ähnlich darf man olıne weiteres die Sa ug n ä p fe des Cephalopodenal'ues in ihrer. Manchfaltigheit auffassen, bald gestielt, bald sitzend, bald noch wie bei Cirotenthis mit einer centralen Hervorragung versehen, hald mit Chitinhacken wie bei Onychotenthis, oder durch einen Chitinring verstärit, gehn sie auf die hervorragen den Sinnesknospen der Epipodialtaster zurück. Selbst Chitinring und -haken macht keine Schwierigkeit, wemu man bedenlit, wie leicht hewegliche Cilien und Gewebe in stare T'Tastbol'sten übergehen kömmen, oder wie der Wimperschopf der Nomertinenlarve nach Büracr zu einem steifen Dor'n verschmelzen kann.

So fasse ich denn die A rm e der Octopodiden als umgewandelte Epipodialtaster altertümlicher Gastropoden auf. Sie ziehen sich

${ }^{1}$ Eine eigenartige Verwendung des Vorderfusses, gesondert vom Hinterfusse, schildert Ropert bei jungen Tieren, die sich noch innerhalb der Laichgallerte bewegen. Möglich, dass auf diesem Moment die Sonderung des Propodiums und damit sein Gebrauch als Schwimmorgan oder Trichter beruht. 
näher an und um den Kopf heran in der alten Epipodiallinie, weil die Hintersohle der Schnecke nicht zur Ausbildung kommt, sondern der protopodiale Trichter der einzige Fusstril bleiht, von vereinzelter liümmerlicher Sohlemanlage algesehen (Venus'sches Organ). Bei dieser Ableitung ist es jeclenfalls kein Zufall, dass die Zahl der A rme dee ()etopordiden mit der Zahl der' Epipodialtaster' von Trochus genau übereinstimmt.

Die Decapoden sind einen ganz ähnlichen, aber doch eigenen Weg

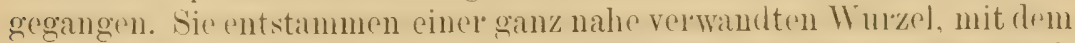

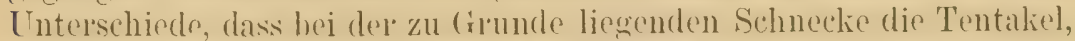
die eigentlichen Fühler. in ihser Aushildung von den Epipodialtastern bereits etwas ver'schieden waren, wie wir es beim Gros der Rhipidoglossen gewohnt sind, und dass sie erhalten blieben. Die decapoden Dibranchiaten haben also vier Parle Epipodialtaster und ein Par ächte Fühler. Ja man kann für die Retractilität dieser Fühler in besondere Taschen loicht Parallelen finden, zumejst bei altretümlichen Stylommatophoren, ()ncidien, Janchliden. aher auch hej Prosolnanehien, die mehr Beziehumgen zum Lande haben, Assiminea nämlich. Alle diese Schnecken vermögen ihne 'Tentakel nicht unzukrämpeln, wie die ächten Landlungenschnecken, wohl abel in Taschen zurückzuziehen.

Die vorgetragene Ableitung bringt zunächst wohl jenen Streit definitiv zur Tiuhe üher die Belentung der Schlundringteile, welche dir A rme innervieren. Sie gehören weder zu ächten Cerebral-, noch zu ächten Pedalganglien, es sind Teile eines epipodialen Centrums, das sich mit den Epiporlialtastern um den Mund herum zusammengezogen hat. I)ie Lösung scheint nach allen Seiten befriedigend und vermittelnd.

Die Ableitung leistet abel' mehr. RoBert el'zählt uns, dass die Trochus-

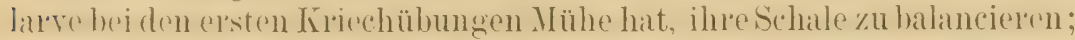
sie schwankt unsicher hin und her. Das muss um so stärker werden, je weniger sich der H'uss in die Länge streckt. Kein Wunder daher', dass diese Schale in solchem Falle durch Anlagerung zweier Arme oder Epipodialtaster gestützt und im Gleichgervicht crhalten wird. Wir erhaltsn unmittelbar die beiden verbreiteten A l'me der weiblichen Aigommen. I annit erschejut diese isolierte Octopodenform lieineswegis als eine Art Relict ron den Ammoniten, sonder'n als eigenartige Neubildung, entsprechend ihrem späteren Auftreten im Pliocaen. Wenn Strmanans auf eine relative Compliziertheit der zarten Schale hingewiesen hat, so braucht man nul zu beachten, dass RoBer' schon bei Trochuslarven versehiedene Stufen der Skulptur und Kalkemlagerung unterseheidet.

'Hierher gehört, als Parallele die Beobachtung Bather's, wonach junge Sepien die eben dem Ei entschlüpft sind, sich mit einem Paare ihrer Arme an der Glaswand des Aquariums anzusaugen vermögen. Die beiden Arme werden dabei ganz ähnlich verbreitert wie die der weiblichen Argonauta, die sich an die Schale anlegen. Dass es ein anderes Armpaar ist, tut dabei nichts zur Sache. 
Aber weiter. Argonanta ist berihnt durch die maximale Steigerung der Hectocotylie, bei welcher der hectocotylisierte Arm sich loslöst und zum selbständig fungiesenden Penis wird. Dabei bleibt das Männchen, das der Schale. die bloss dem ITeibchen als Niststiatte dient, verlustig geht, zwerghaft.

Das bringt mich auf die Frage nach der Fortptlanzung und den Fortpflanzungswerkzrugen. Da lässt sich ohne weiteles behaupten, dass der Penis ursplüuglich aus einer Sinnesknospe hervorgegangen ist. Schon daks seine Tätigkeit mit der höchsten Sinneserregung verbunden ist, dentet in dieser lichtung. Bei den Vorderkiemern aber lässt sich's unmittelbar beobachten. Ich habe längst, gegen die üblichen Anschauungen, cinen kileinen Fortsatz an rechten Tentakel mancher Trochiclen als Penispest gedeutet. Jetzt bildet RonerT bei del Larve eine freie Sinnesknospe ab ummittelbar hinter dem rechten Fühler, welche nachher mit diesem verschmilzt (Fig. 4). Was haben wir hier aber anderes vor uns als den Penis von Paludina? Wir brauchen uns nur des ursprünglichen Verlaufs der (ienitalwege zu erinnern. Bei den Stylommatophoren liegt die Genitalöffinung rechts vorn meben und unter dem Tentakel. Sie liegt aber am Ende einer Rinne, welche vom Mantel schräg nach vorn ( Fig. $5 \mathrm{gr}^{\circ}$ ) herabläuft. Aher noch mehr, die gleiche Rinne läuft symmetrisch dazu anf der linken Seite. Ich lasse es dahingestellt, ob das ursprüunglichste eine Simenrinne ist bei weiter zurücliegendem Genitalporus oder ob sie bloss die Linie bedeutet, unter' welcher' der Genitalschlauch unter der Haut nach vorn zog. Die Tatsachen scheinen mir noch nicht reif zur Entschoidung dieser schwierigen Frage. I) gewöhnlichen Auffissung, nach welcher die Simmentinne vieler Proso- und Opisthobranchien del anfängliche Leitungswog sei, der sich nachträglich zum distalen Vas deferens geschlossen habe, musste ich in vielen Fällen widersprechen; es lässt sich meines Erachtens leicht zeigen, dass häufig genug del Samenleiter nachträglich sich zum Kanal öffnet, aus mechanischen Gründen bei bxyuemerer Leitung, namentlich im Meere, aber auch vereinzelt auf dem Lande, hei (opelte nïmlich: dass aber alle Samenrinnen so entstanden sind, wage ich kaum zu behaupten; ebenso macht die doppelte innere und äussere Lexitung der Oncidien Schwieriglieiten. Genug, diese Frage ist noch nicht genügend geklärt. Wohl aber kann man darauf hinweisen, dass eine solche Rinne bei den Vorderkiemer'n oft genug vorkommt und zwar, was das wichtigste, in beiden Geschlechtern. Meist wird nur das Sperma in ihr nach vorn geleitet, bisweilen aber auch die Eier, so z. B. bei Strombus zur Ablage nach aussen, bei ovoviviparen Melanien in eine distal gelegene Bruttasche, meiner Auffissung nach ein Receptaculum seminis, das nach dem Sperma die Eier anfnimmt. Aber, was noch wichtiger, dieselbe Rinne findet sich bei Strombus auch auf der linken Seite, wo die cienitalim rolliommen fehlen, also wie beiden Stylommatophoren 
(liig. 5). I)as beweist, dass ursplüuglich die (ronitalwege nach beiden Sriten verliefen, ähnlich wie bei den Chitoniden etwa oder wie, un woiter auszuholen, hei den Sagitten. Das regelechte Vorkommen solcher beiderscitigen Furehen bei den Stylommatophoren gibt wierler einen dentlichen Fingerzeig, dass wir von Landformen als ältesten auszugehen haben. Bei den Cephalopoden ist dieses Verhältnis bekanntlich labil geworden, bald ist der Samenleiter nur auf einer Seite vorhanden, bald noch auf beiden.

Ich komme zum Pen is. Er ist bei den Vorderkiemern nach Lage und Ausbildung so verschieden, dass im Allgemeinen hier in den meisten Fällen an sekundäre Neucrwerbungen zu denken ist. Dir mesprüngliche Latge dürfte die der Stylommatophoren sein, oine Simmesknosper nelonen dem rechten Fühler. Aber das Prinzip, das bei diesen her'scht, das der

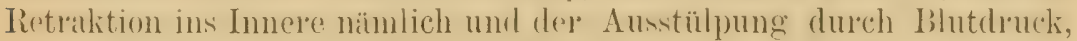
erfordert viel zu viel Kräfteaufwand und greift viel zu tief in die allgemeine Organisation der Tiere ein, als dass es ohne Nötigung im Wasser aufrecht erhalten wäre. Für gewöhnlich.ist bei den Stylommatophoren der Muskeltomus der. Haut darauf eingestent, Koptef und 'Tentaliol auszustülpen; soll der Penis herausgepresst werden, so muss erst der' 'Tonus

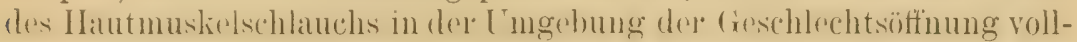
kommen umgestimmt werden, um den gewöhnlich hier her'schenden Widerstand zu beseitigen; dazu bedarf es des oft langen Vorspiels mit

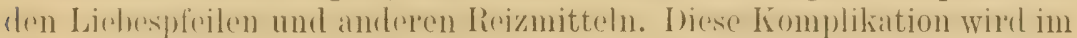
Wasser el'spart. Anfangs aber wird dieselbe Anlage benutzt, eben die el-

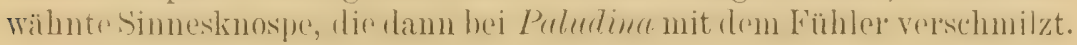
Solche Sinnesknospen finden sich aber mehr, hinter jedem Ep ipodialtaster kommt eine (Fig. 4), anfangs sind sie diesen an Form und Unfang ähnlich genug (Fig. 3). Bedenkt man, dass in der Epipodiallinic auch vor' den 'Tentakeln auf der' Stirn sich Epipodialtaster finden kömmen, die erwähnten Paletten, daun haben wir hier auch anfängliche Simnesknospen zu vermuten; sie sind indess am Vorderende, am Kopfe, am meisten vereinzelt, so gut wie ja die linke Knospe hinter dem 'T'entakel schon bei Trochus und Paludina verloren gegangen ist, wo die rechte bleibt als rudimentiirer orler fungierender Penis. Eine Knospe v $0 r^{\prime}$ dem 'T'entakel aber finden wir' allein noch unter den Rhipidoglossen bei den Neritiden, und zwa im speziellen beiden Navicellen vom Ostpol-

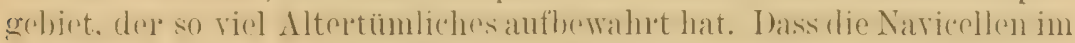
Süsswasser leben, deutet auf die alten Bezichungen zum Lande hin; ja die Form, die mir vorlag, war von meinem Sammler als Landschnecke ringeheinst, vermutleh in den Nachharschaft dines Wasserlaufs (Fig. 6). Nun bei dieser Gattung liegt der Penis vor dem rechten 'Tentakel nach der' Stirn zu, wie seit langem bekannt ist. Seine Form ist eigenartig, ein langgritreckter Heischiger Kï̈rper mit einer tiefen Rinue an der Unter- 
seite (Fig. (i), ansserelem abel mit einer einseitigen blattartigen Erweiterung, die in der Ruhe sich an den Hauptlörper anlegt und zusammenLiummt ( $\mathrm{C}$ und I)): solche blattartigen Erweiterungen liomen aber beim Hectocotylus in ähnlicher Aushildung vor. Wie dieser Penis das Sperma aus der Genitalöffinung, die von ihm getrennt ist, schöpft und bei der Copula ins Weibchen überträgt, wissen wir bei der Navicella so wenig als bei den C'ephalopoden : bei beiden ist der gleiche Hergang zu vermuten.

Die Trennung zwischen den Sinnesknospen oder dem. Penis und der Genitalöffnung bez. dem distalen Ende der Genitalrinne macht es leicht verstïndlich, wie bei don verschiedenen Cephalopodengattungen immer ein anderer Arm hectocotylisiert werelen kann. Erinnern wir uns der Ableitung der Arme aus Epipodialtastern, die mit einer Verschiebung und Konzentration der ganzen Epipodiallinie am Kopf verbunden ist. Da kommt es einfach darauf an, welcher Taster, bez. welche zu ilım gehörige Simnesknospe gerade in die Terlängerung der Gonitalrinne fält. Diese Sinnesknospe, sei es reahts, sei es links, wird zum Penis, der dann mit dem zugehörigen Taster verschmilzt, gerade wie bei Paludina und Trochus; die Verschmelzung liefert den hectocotylisirten Arm.

Die Bezichungen aber zwischen dem Hectocotglus von Argonantu und dem eigentümlichen Penis von Navicella scheinen noch engere zu sein. Einmal ist keineswegs ausgemacht, dass nicht dieser Penis bei seiner komplizierten Form bereits aus der Verschmelzung von einer Sinnesknospe und einem Stirntaster, einer Palette, hervorgegangen ist. Man könnte sich leicht vorstellen, dass das Organ sich, nachdem es die hohe Vascularisierung, Innervation und Muskulatur der Cephalopoden errejicht hätte, als Hectocotylus loslöste mond die Begattung selhständig ausführte, un dimn durch die gerade für dic Schneckenfühler so charalitel'istische Regeneration wieder erzeugt zu werden. Doch das ist eine reine spekulation. Die positife Aehnlichleit grundet sich auf die Tatsache, dass bei Argonauta so gut wie bei Navicella die Männchen zwel'gll aft bleiben. Bei der mir vorliegenden Serie von der Navicella, ca. 60 Stück, die eine kontinuierliche Reihe bilden von 12-27 mm. Schálenlänge, sind alle unter $17,5 \mathrm{~mm}$. männlich, alle darüber weiblich. Zwergmännchen sind ja jetzt von einer Anzahl von Vorderkiemern bekannt geworden, Littorina, Lacuna, Crepidula u. s. w. Wichtiger ist die Feststellung, dass bei anderen vollkommener Hermaphroditismus nachgewiesen ist (parasitische Schnecken, (Jncidiopsis etc.), und dass bei einer dritten Kategorie Zwitterhaftigkeit herrscht, so zwar dass die Gonade anfangs männlich, später weiblich fungicrt. I)ieser proterandrische Hermaphroditismus, der naturemäss schwer, nämlich nur während der kurzen Lobergangszeit, nachzuweisen ist, hat sich namentlich bei altertümlichrn Eormen, hei Seutibanchien, gefunden. Er dentet daranf hin, dass die Schnecken ursprünglich allgemein Zwitter waren: die Zwero- 



\section{TAFELERKLÄRUNG}

Fig. 1. Dasydytes, nach M. Vorgr. B Borstenbündel. Ph Pharynx.

Fig. 2-4. Trochuslarven verschiedenen Alters, nach Robert. 2 und 3 von links, 2 vor, 3 nach der Umdrehung der Schale. 4 von unten. col Anheftungsstelle (Spindelmuskel), m Mantel. os Sinnesknospen. prp Propodium. t Epipodialtaster. v Velum.

Fig. :j. Haut yon Testacella, in eine Ebene auseinandergelegt. gr Genitalrinne. Die Ziffern bedeuten ihre seitlichen Verzweigungen, die zur Bewässerung dienen.

Figr. 6. Eine philippinische Navicella.

A. Die Schnecke ohne Schale, von oben. mr Mantelrand. s Schnauze. sm Schalenmuskel. Jer Penis ist schraffiert.

B. Vorderteil derselben, nach Wegnahme des Mantels.

C. Der Penis, auseinandergefaltet.

D. Der zusammengefaltete Penis im Querschnitt. 
2

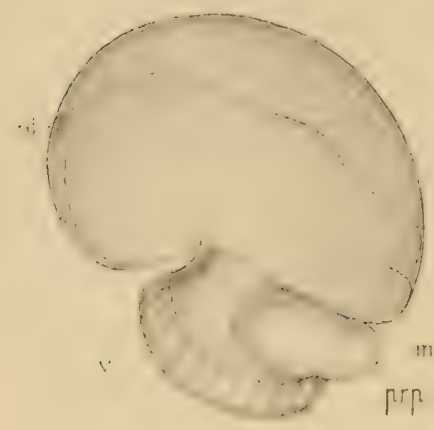

4
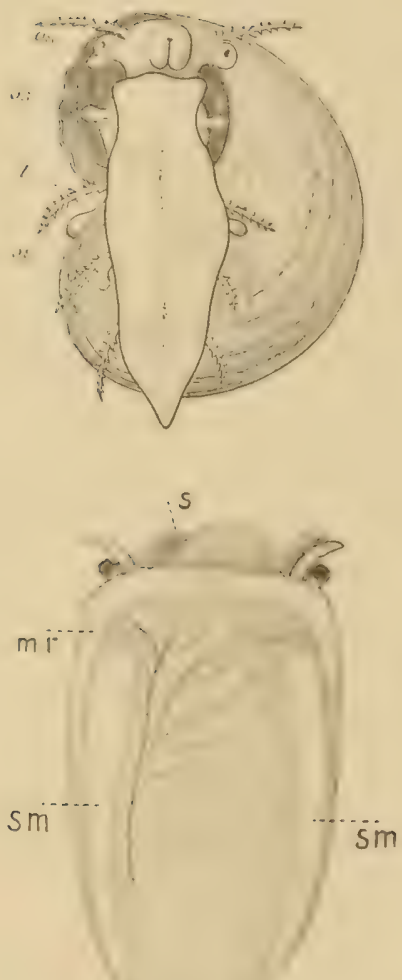

A.

sim
1
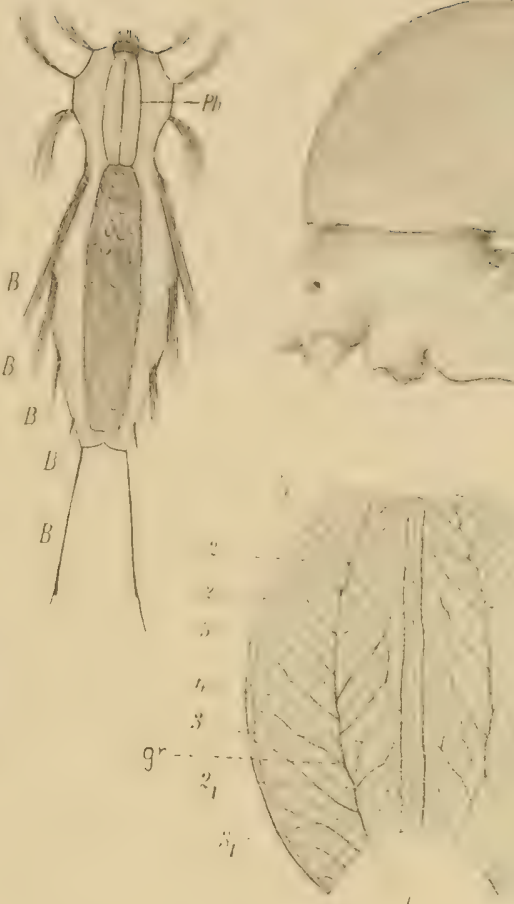

5
3

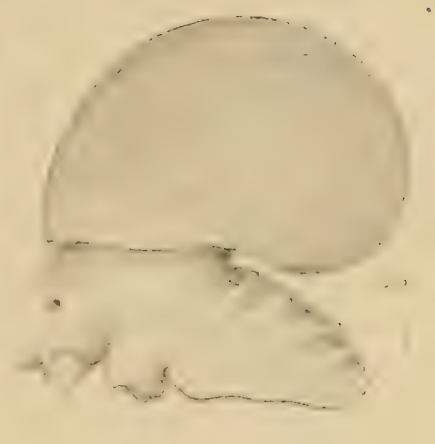

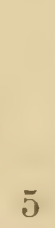

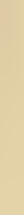

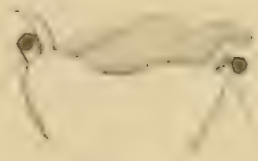

B.

6

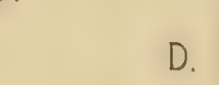

C.

H. SIMRO'TH 

männchen sind dann Formen, die auf der anfänglichen mämnlichen Stufe stehen geblieben sind. Die Deatung gewinnt an Festigkeit durch die Tatsache, dass das umgekehrte Verhiltnis - kleinere Weibehen und grösscre Männchen - bisher bei Schnecken noch nie beobachtet wurde, so verbreitet es sonst im 'Tierreich auch ist. Dieser ganze (redankengang weist mit Sicherheit darauf hin, dass wir nicht in den diøcischen Vorderkiemern, sonder'n in Zwitterschnecken die L'tormen zu suchen hahen. Ina kommt aber noch ein Jloment dazu, welches ausser denen, die ich bisher immer für die Herleitung der" Weichtiere vom Lande geltend gemacht hahe, mit grosser Bestimmtheit an dic stylommatophoren anknüpt: die Spermat ophoren nämlich. Während alle Mollusken bei der Begattung freies Sperma übertrag(n, wird der. Sume nur bei zwei (iruppen in schützende Hüllen eingeschloss'n, hei Cephaloporlen und Stylom matophoren, (ilund genug, auch hier'in einen verwandtschaftlichen Zusammenhang zu erblicken. Dass aber darin eine Anpassung an das Landleben liegt, kann nach allgemeinen Firwigungen kaum einem Zweifel unterliegen.

Wenn somit Cephaloporlen. altertümliche Prosobranchien und Stylommatophoren auf eine gemeinsame Wurzel hinauslaufen, so möchte ich darauf hinweisen, dass auch in der hentigen Form bei den letzteren, bei den Stylommatophoren, wahrscheinlich ein stärkerer Rest der Epipodiallinie vorliegt, als gemeinhin angenommen wird, die drei Paare Fünlne nämlich, d. h. die Ommatophoren, die kleinen Tentakel und die Lipprenfülıler: ja sie zeigen eine ähnliche Zusammenschielsung nach dem Kopf zu wie bei den 'Tintenfischen. Wie aber im Wasser alle Anhänge sich freier zu entfalten vermögen, so ist eine eigentliche Epipodialfalte, als ein Hautsam, dem Fühler und Sinneshnospen eingefügt sind, bei den Pulnonaten zurükgebildet, erhält sich dagegen hei vielen altmptümlichen Vorderliemern und ehenso bei vielen Cephalopoden, als jene Membran, welche die basalen Teile der' Arme verbindet.

Von den Schalen wurde bis jetzt nur der von Argonauta gedacht. Ian kann aher viel weiter gehen. Sprinlu, repiotenthis, die Belemniten, kurz die Decapoden, führt man auf gekammer te, vo n e in em Sipho d urchsetzte Schalen zurück, gleichgiltig ob in exo- oder endogastrischer Stellung, welche lediglich auf die Konservierung der Schale ror oder nach der Drehung hinauslaufen würde. Ich habe bereits vor zwei Jahren die Entstehung der Kammerumg platusibel zu machen gexucht. Ausser bei Cophalopoden liommt es nu bei Schnedion vor, dass sie sich bei zumehmendem Leibesumfang aus dem Anfangsteil der Schale herausziehen und ihn durch eine Querwand abschliessen. Freilich bei den Schnecken ist die Scheidewand nicht durchbohrt, und der Anfangsteil, welchel' weitel' keinen olganischen Zusammenhang mit dem Tiele meh" hat, wird algeworfen : man kamm betone'n, dass solvhe Bildumer herejts hei den Clansilien, die ich für die altertümlichsten halte. zur Beobachtung

VIo CONGR. INT. ZOOL., 1904. 
kommt. Bei drn Tintenfischen dagegen ist die Scheidewand erhalten, weil sie durchlohrt und von rinrm Blutstrang durchsetzt ist, welcher das 'Tier mit dem Anfangsteil in Verbindung erhält. Ier Enterschied zwischon heiden (iruppen beruht aber lediglich darauf, dass die Schale bei den Schnecken asymmetrisch aufgewunden wird, bei den Tintenfischen dagegen in einer Ebene bleibt. Bei den Schnecken wird jener Strang. der nichts anderes ist als der von Haut bedeckte Spindelmuskel, dureh rle mit der Asymmetrie verbundene Kinehelung aus dem Anfangsteil helausgezogen, seine Wurzel gleitet allmählich aus der Spindel herah: und wemn das 'Tier sich gleichfalls aus der Schale hreauszieht, so trifft die neugebildete Scheidewand auf vollkommen verlassene Schalenteile. Ander's bei den 'Tintentischen. Hier bleibt der Retraktor bis in den Schalenanfang orhalten, daher die Scheidewände jedesmal durch ihn durchbrochen worden. Für diesor Spokulation aher, welche auch auf clieser Suite Gastropoden unl Cephalopoden zusammenschwejsen will, lässt sich leicht der embryologische Nachweis führen wieder bei den Scutibranchien. Während bei dem Gros der Gastropoden die Mantelfäche mit der Schalenfläche in engem Kontakt bleibt, entsteht bei Patella und Trochus (Fig. 2) noch in exogastrischer Stellung nach RoBER'T und Patrtex hier ein Hohlraum, der indess durch den Schalenmuskel durchsetzt wird (col): denn der Embryo hat noch nicht den definitiven Schalenmuskel, sondern den winfarhen Spindelmuskel der Pulmonaten. Jene' definitive Bofestigung entsteht 'r'st nachträglich am Mantel l' a nde untel dem Finfluss dor halbsessilon Lebenswejse. Bei rliesem Embryo braucht man nur vom Mantel eine Scheidewand bilden zu lassen, und dar Anfang der

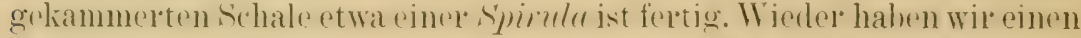
Zusammenhang zwischen Cephalopoden und ein'm alteretumlichen Gastroporlen. der noch nahe Bezichungen zur terestrischen Lehensweise zoigt.

Ganz anders etwa bei Octopus! Hier kommt wohl eine doppel te Schalen bildung in Betracht, beide Male gleich vergänglich. Apperü̈r hat gezoigt, dasis rine ganz zarte Conchinlamelle vorkommt. die dann abfällt. Hierfür kenne ich keine andere Parallele, als die Enbryonalschale von Vagimula nach der Darstellung der SARAsin's, ebenso ein zartes, flach gewölbtes Plättchen, das verloren geht. Beide Bildungen würden auf die astr. Molluskenschale. rine zarte Erhärtung als Trocken-

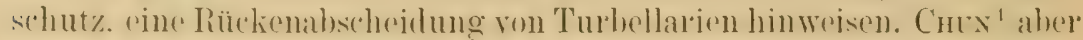
hat von anderen Octopodenlarven erst Pfingsten gezeigt, dass die Haut mit lorstenbündehn hedeckt ist. die anf dem Riücken in (Yuinemnstellung ver'teilt sind. Wenn hier eine Hypothese überhaupt am Platze ist, so kann sie wieder $n u r^{\circ}$ in der vor'hin postulierten vereinigten Klasse der'Turbellarien mnd Gastrotrichen liegen. Manche Gastrotrichen hahen eine Rüelen-

' Crivs. Das Borstenkleid der Cephalopoden. Verhdlgn. d. d.zool. Ges., P. 243. 1904. 


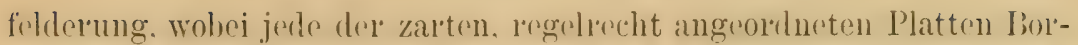
sten trägt. Allerdings ist der Luterschiced in den Borsten noch gross genue.

Da ich einmal die Patellen erwähnt habe, möchte ich darauf aufmerksam machen, dass gerade bei dieser Form eine cuticulare Verdickung ain Boden der Mundhöhle vor der Radula nachgewiesen ist, eine Verdickung, die sich als Gegenstück zum Kiefer leicht weiter entwickeln und den Unterkiefer der Cep hal o poden liefern konnte. Und, um noch rine beziehung zwischen diesen und altertümlichon Srutiluanchien zu erwähnen, so weise ich darauf hin, dass Roвer' bei gewissen Trochusarten eine endständige, seitliche, drüsige Anschwellung des Eileiter's

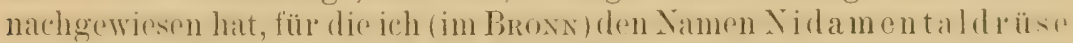
vorgesehlagen habe. wregen der mutmassichen Homologio mit drum gleichnamigen Organ der Cephalopoden. Den 'T' i n t e n b e u tel stellt man in neuerer Zeit wohl allgemein auf eine Stufe mit der Anal-oder Rectaldeüser vicher P'rnsobranchion. und es ist gewiss kein Zufall, dass sich diese Organe ger'ade in den beiden Gruppen vorfinden.

Als ich vor zwei Jahren den Versuch machte, die T'Trabranchiaten in ähnlicher Idrenverbindung auf (xastropoden zurückzuführen, da. kam ich auf altertümliche Pulmonaten, freilich noch mit diffusem Nervensystem, d. h. mit Marksträngen an Stelle konzentrierter Ganglien. Die zwei Mal vier Reihen von Tentakelcirrhen suchte ich auf den Vorderfuss der' Soleoliferen oder Vaginuliden zu bezichen, so zwar, dass diese QuerIristen. wolche dir hörhste Aushildung der Schnediensohle darzustrolem

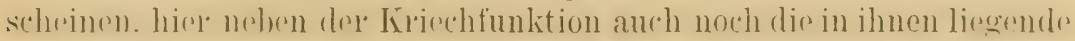
Sangwirkung zur Entfaltung gebracht hätten und dadurch je in eine Reihe von Cirrhen zerfallen wären. Ich ahnte damals noch nicht. dass dir nousen. gleichzoitig erscheinenden Arbeiten über dir Entwicklungs-

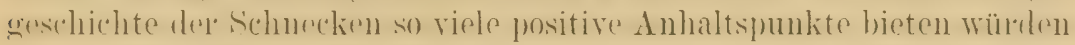
fül die Aufklärung der Phylogenie. Jetzt halte ich die Ableitung der Nautilusar'me in dem angegebenen Sinne noch immer für sehr wahrscheinlich, gestehe aber, dass mir noch keine bestimmten Kriterien vorliegen. oh wir's in ihnen mit umgewandelten vorderen soleolie oder gleichfalls mit Epipodialtastel'n zu tun haben.

Die Ableitung der A m moniten erscheint jetzt wiederum ganz problematisch, nachdem sich Argonanta, für mich wenigstens, als ein besonderer Spross von den Gastropoden dargestellt hat. Dass der' allgemeine Gang der Entwicklung ein ähnlicher war, wie bei den recenten 'Tintenfischen, unterliegt wohl keinem Zweifel. Am ersten darf man wohl noch Aufklärung erhoffen von der Untersuchung der Lobenlinie, d. h. von der Differenzierung des secundären Schalenmuskels. Vielleicht kann man einige Anhaltspunkte erhoften von der Ontogenese des entsprechenden Muskels bei Patellen und Fissurellen, wo dort der kreis- oder hufeisenföronige Muskel bereits in eine Roile von Felelen zerfallen ist. Mir will es 
das nächstliegende dünken, dass die Ammoniten von Bellerophonten sich abgezweigt haben. und das gilit eine gewisse Parallele zu den Dibranchiaten insofer'n, als man die Bellerophonten jetzt zumeist als altertümliche Rhipidoglosen betrachtet. wobei es wiederum unentschieden bleiben mag, ob die Schale von Bellerophon exo- oder endogastrisch war ${ }^{1}$.

Vor zwei Jahren versuchte ich eine von MEYER-Eymar beschriebene problematische Cephalopodenform aus ägyptischem Tertiaer, die Kerunia, auf́ cine Schneckenlarve zurückzuführen. Ich nahm an, dass eine Echinospira, d. h. die pelagische Larve einer Lamellariide, nach ihrem Anlanden ihre Scaphoconcha oder Schwimmschale beibehalten habe und zu viel beträchtlicherer Grösse herangewachsen sei. denn die zarte Schale von Kerunia scheint sich nu’ auf die Schwimmschale der Echinospira bezichen zu lassen. Jetzt möchte ich die letztere Auffassung zwar beibehalten, alser mit der Nodification, dass die Kermuia, so gut wie dlie übrigen von Schneckerlarven abzuleitenden Tintenfische, ein Cephalopod gewesen sein mag, in welchem Falle die Deutung. Meyen-Frman's vollkommen zu Recht bestehen würde.

Noch möchte ich einen Punkt heranziehen, den man oft genug in's Treffen geführt hat, um die Cephalopoden allen übrigen Mollusken scharf gegeuäberzustellen. die Ontogenie nämlich. Die Cephaloporlen sollen meroblast ische alle übrigen Weichticre holoblastische Eier haben. Da genügt es kư darauf hinzuweisen dass die Untersuchungen von Boßretzky. Salensky. Conklin. Hofriann u. A. gerade bei den Volderkiemern, als den ältesten Rüuckwanderer'n des Gastropodenstammes in's Meer. alle Stufen der Versorgung mit Nahrungsdotter aufgerleckt haben, so dass im Falle höchster Steigerung das Ei bereits als meloblastisch bezeichnet werden kitun und auch in dieser Hinsicht Cephalo- und Gastropoden eng aneinander heranrücken. In gleichen Simne kann die hohe Achnlichkeit vieler Laichformen. Fiertrauben und Eikapseln. in beiden Weichtierklassen gedeutet werden.

So weist denn eine lange Reihe von Einzelheiten. zu denen man noch etwa das Spiralcoecum des Magens hinzufügen mag. auf einen engen Zusammenhang zwischen Cephalopoden und Gastropoden hin. engel jedenfalls als zwischen Cephalopoden und anderen Mollusken. wie ich hotfe, in eingehender Begründung. Wir haben bis auf die ältesten Schnecken in ihrer direkten Ableitung vom 'Tubellarien-Gastrotrichenstamme auf dem Lande zurückzugehen.

2 Inzwischen habe ich Gründe vorgebracht dafür, dass die Schale der Bellerophontiden als exogastrisch zu betrachten ist. (Sitzgsber. der naturf. Ges. Leipzig). Danach hätten wir in diesen alten Formen die Klasse der Prorhipidoglossen oder Amphigastropoden zu erblicken. Die Lobenlinie der Ammoniten scheint auf Epipodialanhänge zurückzugehen, deren Muskeln im Integument weiter hinauf griffen und sich an der Schale anhefteten. 
Ueber diese Beziehungen nur noch einige Worte.

Perseneer ist in neucrer Zeit mit besonderer Wärme für die Abstamnumg der Gatstropolen won Anneliden ringetreten. Was demn rine weiterer Controverse hervorgerufen hat. Mir scheint. in den diastrotrichen lingt die gemeinsame Wurzel. und dir Entwicklung ist in mancher Hinsicht parallel gegangen. Die (Bastrotrichen zoigen in ihren loostenbündeln die erste Anlage von Metamerie, die noch nicht weiter ins Innere einschneidet und daher noch als Pseudometamerie zu bezeichnen ist. Dieselbe Rerihr yon Borstenbündeln hildret das erste Epiporlium. I)ir Wimperreihen der Ba u chseit e entsprechen der wimpernden Bauchfurehe viche chatopolen. sie finden sich aber auch in dre Entwicklung

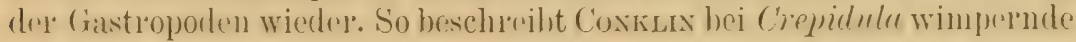
pedale Zellplatten in der Medianlinie des embryonalen Fusses. Sie scheinen später. wohl durch Alwwerfen, verloren zu gehen. und trilen danit velnutlich das Schicksal ähnlicher Zellplatten im Volarfeld. wir dem von Loldia solche provisorische Epithelien durch Inew allgemenin

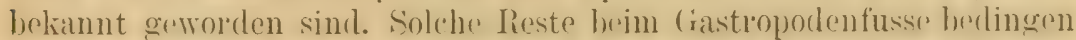
die rerschiedenen Angahen üher dir bilatrade Anlage dieses ()roans, sowie seine Zweiteilung, die wir bei vielen marinen Prosobranchien finden und boj Cyclostoma auf drm Lando maximal gesteigert sthen. Andrerersoits kann dir Gloitsohle namentlich oles Stylommatophoren lud der Eigenart ihre Funktion nu auf die Landp)lanarim zurückgeführt wreden. und zwar mindestens in doppelter Horleitung für dir Aulacopoden und Holopoden. So drängt alles darauf hin, die Weichtiele in breiter Linie, nicht in einfachem Stammbaume, von der vereinigten Tulbellarien-Gastrotrich enklasse abzuleiten, die ich denn wieder unmittelbal auf die Infusorien zurückführen zu müssen meinte.

Zum Schlusse möchte ich versuchen, die Ableitungen in eine Anzahl won Thesen zusammenzufasen. wiewohl solche fireilich oft in ihrer ahgulissenen Schärfe Missdeutungen veranlassen können.

1. Die Gastropoden sind aus der zusammengehörigen TurbellarienGastrotrichen-Klasse a u f $\mathrm{dem}$ La n d e herrorgegangen.

2. Keins der jetzt lebenden Gastropoden entspricht noch röllig einer dor usprüugliehen Formen. doch lassen sich rinzelne msprüngliche Charaktere der von Anfang an bunten Schaal lekonstruieren.

3. Zu den alten Merkmalen gehört ein Epipodium, bezw. eine vorn auf dem Kopf beginnende Fpipoliallinio mit Tentakeln und Sinmeslinospen. Augenträgere. kleine Fühler und Lippentaster der Stylommatophoren sind noch ein Rest davon.

4. Die Genitalöffnung lag rechts vol’n, hinter den Fühlern.

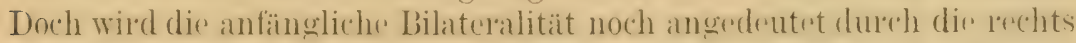
und links vom Mantel zu den vorderen seitlichen Sollenenden herablaufenden (x e n it a l l' in nen. die wohl bei allen Sitylomatophoren und 
bei manchen Prosobranchien noch erhalten sind, bei den ersteren und Strombus auf der linken Seite so gut wie auf der rechten.

๖. Die Tiere waren proterandrische Hermaphroditen; daraus gingen bei eintretender Diœeie vielfach $\mathrm{Z}$ wer g $\mathrm{männchen}$ hervor.

6. Der Same wurde in schützender Hülle, in S p e r mat o p h or e n, übertragen.

7. Aus solchen 'Tieren gingen beim Untertauchen unter den Neeresspiegel teils die jetzigen $\mathrm{Prosobranchien,}$, teils die Cephalopoden hervor.

8. Die Cepha l o pode n entstanden durch Uebergang der Larvenformen zu neuer, teils kriechender, teils schwimmender Lebenswe ise.

9. Das zuerst angelegte Propod i u möhlte sich, wie bei der Trochuslarve, zur Rinne aus, was den Uebergang zum Trichter bildet.

10. Die übrige Sohle kam nur ausnahmsweise und dann in rudimentärer' Anlage zur Entwicklung (VerriLL's ches Organ).

11. Die Arme der Octopoden sind hervorgegangen aus $2 \times 4$ Ep i podialtastern, wie sie Trochus zeigt, indem die der Sohlenentwicklung voratuseilenden Anhänge sich in der Epipodiallinie nach rol'n am Kopfe um den Nund zusammenschieben.

12. Bei den D e c a p o d e $\mathrm{n}$ vollzog sich derselbe Vorgang, doch waren bereits zwei Fühler von den Epipodialtastern differenziert. Diese Fühler waren in Tasehen zurückziehlar. nicht einstülphar, wie noch jetzt bei Oncidien und Janelliden.

13. Der Pen is ist ursprünglich eine $\mathrm{S}$ innesknospe, wie eine solche hinter jedem Fühler und Epipodialtaster angelegt ist.

14. Häufig versehmilzt er mit dem zugehörigen Fühler, unter den Schnerlien bei Pahludiua und Trochens, bei Naricella vermutlich mit vinem vordersten Eipipodialfühler, einer sogen. Palette auf der Stirn. Die Verschmelzung liefert bei den Cephalopoden den $\mathrm{Hect} \mathrm{ocot} \mathrm{y} \mathrm{lus.}$

15. Die Bestimmung, w e l c h e $\mathrm{r} A \mathrm{r}^{\prime} \mathrm{m}$ he $\mathrm{k}$ t o c o t y l is i e r t werden soll. hängt lediglich davon ab), welcher Epipodialtaster bei der Versehiebung der Epipodiallinie gerade auf die Genitalrinne trifft.

16. Eine engere Beziehung besteht zwischen Argonauta und Navicella: Z $\mathrm{W}$ wergmännchen, seitliche Ausladung am Penis und Hectocotylus. Die Wiedererzeugung des ábgelösten Hectocotylus entspricht der Regeneration der Schneckenfühler.

17. Die Spermatophoren haben die Cephalopoden direkt yon Landschnecken ererbt.

18. Die Octopoden zeigen bereits eine ganz verschiedene Ableitung der Se li a le :

a) Die Schale der Argonauta entspricht der La r ve n s cha le von 


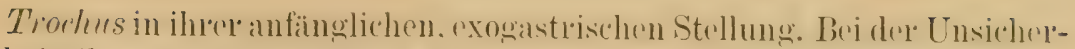

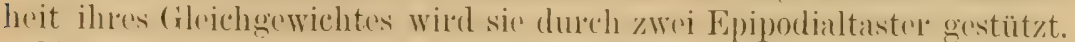

b) Die $\mathrm{za} \mathrm{r}^{\mathrm{t}} \mathrm{e} \mathrm{h}$ ä u $\mathrm{t} \mathrm{g} \mathrm{e} \mathrm{Schale} \mathrm{anderer} \mathrm{Formen} \mathrm{hat} \mathrm{ihr} \mathrm{Pendant}$ in der gleichen Embryonalschale von Vagimula, die ebenso abgeworfen wirel.

c) Die embryonale Rückenbildung mit regelmässig verteilten $\mathrm{B} 0 \mathrm{I}^{\circ}$ stenbüscheln weist wohl auf die Bedeckung mancher G a strot l i c h e n zurüick.

19. Gekammelte Schalen entstehen nach dem Principe der decollierten Gastropodenschalen, wohei die Anfangstrile über den Scheidewïnden erhalten bleiben.

20. Die Erhaltung der oberen Schalenteile und die D u r c h b o h lo u $\mathrm{g}$ der Scheidewände beruht auf der bilateralen Symmetrie der Schale,

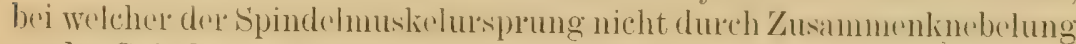
an der Spindel nach abwärts verschoben wurde, wie bei den Schnecken, sonder'n seine anfängliche Lage in del Schalenspitze beibehält.

Der Trochus- und Patellenembryo liefert ein Beispiel für den Hergang.

21. Der O b e $r^{\circ} \mathrm{k}$ i e f e $\mathrm{r}^{\circ}$ der Cephalopoden entspricht dem Oberkiefer der Pulmonaten.

22. Der Un t e r k i e f e r entspricht der cuticularen Verdickung an Boden der Mundhöhle bei den Docoglossen.

23. Das S p i r a l c $\propto \mathrm{c} u \mathrm{~m}$ am Magen ist den Cephalopoden und altertümlichen Gastropoden gemeinsam.

24. Der 'T'inten be ut el der Cephalopoden entspricht der Rectaldrüse der Gastropoden.

25. Die Nidamentald r üse der Cephalopoden entspricht der Nidamentaldrüse mancher Trochus-Arten.

26. Die La i $\mathrm{ch}$ for $\mathrm{m}$ e $\mathrm{n}$ sind beiden Gruppen gemeinsam.

27. Meroblastische Ei er sind nicht auf die Cephalopoden beschränkt, sondern finden sich in allen Uebergängen zu den holoblastischen bei den Gastiopoden.

28. Die Umwandlung von alten Gastropoden zum Mindesten in Octo-

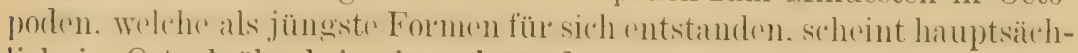
lich in Ostpolnähe bei seinen besonder's weiten Beziehungen zwischen Wasser und Land vol sich gegangen zu sein.

29. Das Auftreten der A m mo niten dagegen erfolgte unter de m Sch wing ung $\mathrm{g}$ i e is, wo Ceratites zucrst in mesozoischen Ablager'ungen gefunden wircl. 
$4^{\text {me }}$ SECTION - INVERTÉBRÉS

\title{
Les Otocystes du Branchiomma vesiculosum Mont.
}

\author{
Par le Prof. Pienne FAUVEL (Angers).
}

Avec 3 figures dans le texte.

Quoique le Branchiomma vesiculosum Mont. soit une espèce très répandue, souvent décrite et étudiéc. ses otocystes ont longtemps échappé aux observatems. Aucun auteur n'en fait mention avant BRunotese (88). à qui nous en devons la première description et les premières figures.

D'après Brunotte, ces organes sont situés de chaque côté du corps, dans le roisinage de la premiere rame thoracique, très rudimentaire. Ils sont for'més d'une petite cavité occupant la base du lobe latéral de la collerette. Ils sont logés dans les téguments, clos de toutes parts, en relation avec le cer'vea u au moyen d'un filet nerveux et ils contiennent de nombreux corpuscules colorés.

L'auteur' n'a pu faire d'observations sur le vivant, vu le peu de transparence des tissus enviromnants, et il ignore si l'otocyste porte des rils vibratiles.

De Saint-Joseph (94) fait remarquer que les yeux et les otocystes ne sont plus apparents chez les adultes et pénètrent plus profondément dans le corps.

Soulier (1909, p. 34) confirme la description de Brunotre qu'il trouve exacte, mais il reconnaît en outre que l'otocyste est pourvu intérieurement de cils vibratiles.

Mes observations, tant sur le vivant que sur les coupes en série, me permettent d'ajouter à ces descriptions quelques rétails nouveaux et quelques rectifications.

La remarque de SAIxT-Josepu est fort juste; chez l'adulte les otocystes sont profondément enfoncés dans les tissus et absolument invisibles à l'extérieur. La pigmentation, jointe au défaut de transparence des tissus. ne permet pas l'étude par transparence, même avec l'aide du compresseur.

Il faut done tourner la difficulté, ce qui peut se faire assez facilement, sachant que l'otoryste est situé à la base du lobe latéral de la collerette, un peu au-dessus du premier mamelon sétigère.

Ce premier sétigèe, dépourvu de rame rentrale, est constitué seulement par un pretit mamelon dorsal portant quelques fines soies capillaires.

Il suffit de faire sauter, arec une aiguille lancéolée, ce petit mamelon seitigere, arec un lambeau de collerette, pris au-dessus, d'étaler ce lam- 
beau sul une lame, dans une goutte d'eau de mer, en ayant soin de tourner "n desus la face interne puis de comprimer légerementave une lamelle, pour pouvoir étudier l'otocyste.

Si la préparation est l'éussie, on aperonit a l'oril nu, au-dessus du bulloe sétigì'e, une petite sphérulo d'un hanc laiteux, opaque. C'est l'otocyste.

Au microscope, par tramsparence, cette sphere paraît foncée, pigmentée, plesque noire, à peu près opaque.

Ce pigment hanc opaque, noir en lumiere transmise, est le même qui for'me les nombreuses taches blanches dont l'épider'me est piqueté. Il rend l'otocyste difficile à distinguer des taches voisines, mais celles-ci sont plus grandes, plus diffiuses et ne contiennent que des cellules claires arrondies, sans traces de grains anguleux, tandis qu'une légère compression permet de distinguer la vésicule de l'otocyste et surtout les nombreux otolithes qu'elle renferme.

Ces otolithes, très nombreux, sont formés de petits grains siliceux, ir'égulier's, anguleux, transparents. Certains sont formés de débris de spicules d'Eponges siliceuses.

Ces otolithes sont animés d'un mouvement très vif, dû à de nombreux cils vibratiles. Ce mouvement est bien différent de la trépidation des otolithes d'Arenicola Grubi et d'A. ecaudata qui est due au mouvement Blownien.

Sur certaines préparations favorables, j’ai même pu apercevoir la projection de l'ouverture du canal cilié faisant communiquel la cavité de l'otocyste avec l'extérieur.

Les coupes transversales, pratiquées sur des individus de différentes tailles, permettent de constater que plus l'individu est jeune plus l'otocyste est gros, r'elativement, et plus il est superficiel.

Sur un jeune Branchiomma, l'otocyste

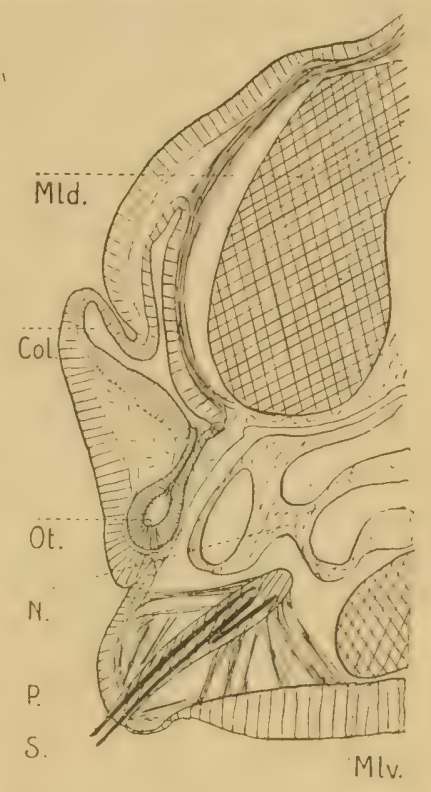

Fig. 1. Branchiomma resiculosum.

Coupe transversale semischématique.

Col. collerette. - Mld. muscle longitudinal dorsal. $\rightarrow$ Mlv. muscle Iongitudinal ventral. N. néphridie. - Ot. otocyste. P. $1^{\mathrm{r}}$ pied. $-\mathrm{S}$. soies. situé juste alt-dessus du mamelon pédieux du premier sétigère est immédiatement au-dessous de l'épithélium de la base de la collerette. C"est une vésicule irrégulierement sphérique. mesurant extérieurement $75 \mu \times 65 \mu$ tandis que le diamètre de sa cavité est de $45 \mu \times 36 \mu$.

Les parois de l'otocyste sont formées d'une seule couche de cellules 
layounantes, à gros noyau à peine ovale, tenant presque toute la largeur de la cellule. Ces cellules sont plus courtes, plus cubiques que chez l'adulte. La cuticule interne est assez épaisse et ciliée. Un canal, égale-

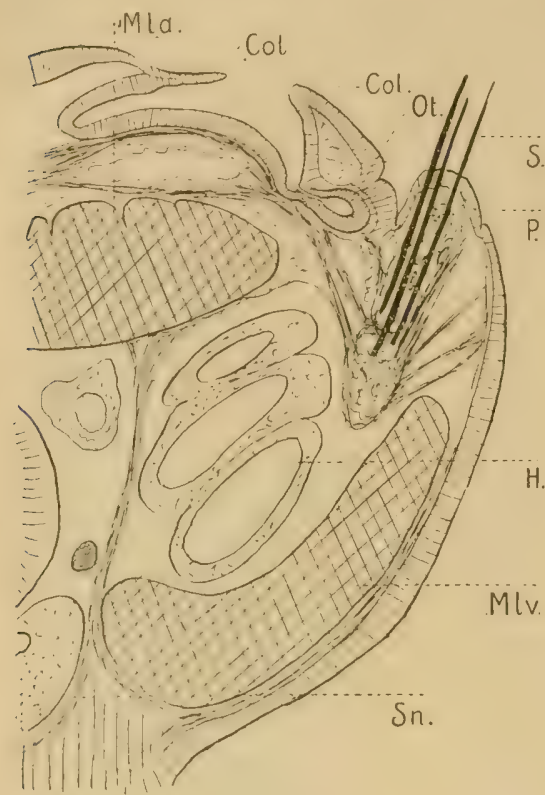

Fig. 2. Branchiomma vesiculosum régénéré.

Coupe transversale semi-schématique.

Col. collerette. - Mld. muscle longitudinal dorsal. - Mlv. muscle longitudinal ventral. - H. néphridie. $-\mathrm{P} .1^{\mathrm{r}}$ pied. S. soies. - Sn. chaîne nerveuse. ment cilié, mais plus court et relativement plus large que chez l'adulte, fait communiquer l'intél'ieur de l'otocyste avec le milieu extérieur. Ce canal cilié débouche sur la face interne de la collerette, dans l'espèce de rigole circulaire que celle-ci fait avec la paroi du col'ps.

Une mince basale sépare l'otocyste des tissus voisins. L'épithélium de la face externe de la collelette est alvéolaire. Il se compose de minces fibro-cellules mélangées de cellules muqueuses à contenu granuleux. Celles - ci sont moins différenciées que chez l'adulte, les deux types de cellules étant moins tranchés. L'épithélium de la face interne de la collerette est formé de longues cellules toutes semblables. On n'y rencontre pas de cellules à mucus. Les cellules de épithélium de la paroi du corps, qui y fait face, sont presque cubiques, à gros noyau arrondi.

L'étroit triangle entre l'otocyste et les deux lames de la collerette est rempli de cellules conjonctives encole peu différenciées. De l'autre coté, une batsale répare l'otocyste de la cavité du mammelon pédieux.

Sur un adulte de taille moyenne, l'aspect est un peu différent.

L'otocyste est bien aussi situé immédiatement au-dossous de l'épithélium externe de la collerette et au-dessus de la cavité du premier pied, mais l'épithélium de la base de la collerette est mieux différencié. Il se compose de longues et minces fibro-cellules ot de grosses cellules à mucus. en massue, se colorant en violet foncé par l'hématoxyline. Liotocyste en est séparé par une basale très nette, qui le sépare également de la cavité pédieuse. Lejpithélium de la face interne de la collerette est formé de cellules toutes semblables, minces, allongées, à plateau cuticulaire 
assez épais. Sauf au voisinage de l'ouverture du canal cilié, ces collules ne portent pas de cils vibratiles. I Leur extrémité basale se divise en fibrilles qui vont se perdre dans le tisinl conjonctif hien développé qui remplit l'expace triangulaire entre l'otocyste et les denx lames épithéliales de la collerette. Liotocyste est sépraré de la lame épithéliale interno par une épaisseur assez grande de tissu conjonctif que traverse le canal cilié.

Les cellules radiales de l'otocyste sont de deux sortes: les unes relativement lareges, ì novau arrondi, les autres, minces. fusiformes, aropuées, à noyau allongé, se terminant par un filament basal sinueux (cellules sensitives).

Le plateau cuticulaire de la vésicule auditive est r'elativement épais et porte des cils vibratiles longs et minces. Le canal cilié est plus long et plus étroit que chez les formes jeunes.

Chez les Polychètes, les otocystes sont d'ordinaire trop profondément situés pour qu'il soit possible d'en faire l'ablation sans produire de graves traumatismes auxquels l'animal ne survit pas.

Chez le Brunchiomma, vu la situation de cet organe au-dessus du premier sétigère, à la base de la collerette, j'ai pu, en employant la technique indiquée plus haut, r'éussir à enlever' les otocystes sans produire de blessures trop graves.

Je dois dire que je n'ai constaté aucun changement dans les habitudes des Branchiomma ainsi opérés. Remis dans son tube après l'opération l'animal semble normal. Il lentre et sor't son panache comme d'habitude. Lor'squ'il est bien étalé, la moindre alerte: ombre projetée, lumière vive, agitation de l'eau, trépidation, le fait rentrel' avec la même vivacité qu'auparavant.

Naturellement, j'avais soin de

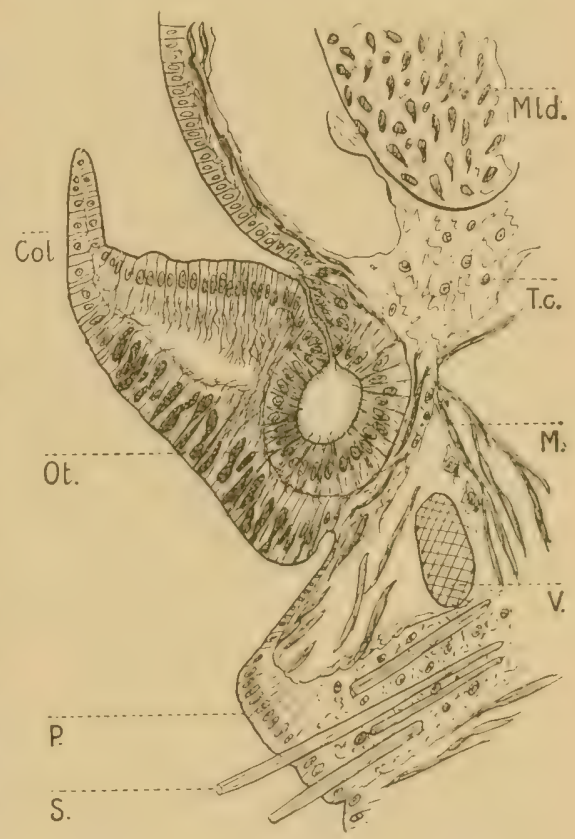

Fig. 3. Branchiomma resiculosum. Otocyste régénéré.

Col. collerette. - M. muscles. - Mld. muscle longitudinal dorsal. - Ot. otocyste. P. pied. - S. soies. - V. vaissenu. m'assurer par l'examen microscopique des fragments enlevés que l'ntocyste citait bien radicalement axtirpé.

Soucier $(91$, p. 47) a l'emarqué que les blessures de peu d'importance guérissent parfois en captivité. mais ra rement et il n'a pu olitenir de ré- 
génération en ayuarium. Beaucoup de mes opérés sont morts dans un délai variant cntre quelques jours et quelques semaines; mais avec un petit spécimen mrsurant 40 mm. sur 3̀ mm. j’ai été plus heureux. Après lui avoir enlevé les deux otocystes j'ai r'éussi à le consel'ver en vie, sans ru'aucune de ses labitudes parût modifiée. Au bout de 5 mois je l'ai fixé pour le sectionner. Les pieds enlevés étaient complètement régénérés ainsi que la collerette dont les bords seuls présentaient encore une fente longitudinale a la place du repli dorsal ordinaire. La taille, par suite de l'alimentation un peu insuffisante, avait légèrement diminué.

Liexamen des sections transversales montre les deux otocystes parfaitement régénérés. L'aspect général est intermédiaire entre celui du jeune et celui de l'adulte. L'otocyste est nettement cilié. il communique avec l'extérieur par un court canal, également cilié, mais il ne renfer'me pas d'otolithes.

Lotocyste est séparé des lames épithéliales de la collerette par une hasale très nette qui l'entoure. L'épiderme de la collerette est bien régénéré. Il a la structure alvéolaire, sur la face externe, avec grosses cellulés à mucus. Les fillro-cellules des deux lames envoient de longs prolongements basilaires dans la cavité qui les sépare.

Les gros muscles longitudinaux du corps montrent une histolyse très accentuée, l'animal mal alimenté s'étant reconstitué au dépens de ses. proples tissus.

Contrairement à l'opinion de Bruxo'te et de Soutuer, je ne considèe pas l'otocyste comme innervé pal' le cerveau, car son nerf n'est qu'un filet du premier nelf pédieux. Celui-ei tire bien son origine d'un des ganglions latéraux de l'œsophage, mais ces ganglions sont en réalité sousasophagiens. Ils représentent la première paire ventrale qui est un pen remontee.

\section{INDEX BIBLIOGRAPHIQUE}

1888. Bruxotte. Recherches anatomiques sur une espèce du qenre Branchiomma. Travaux de la station zoologique de Cette. Nancy.

1902. P. Fauvel. Les Olocystes des Annélides Polychètes. C. R. Acad. Sc., Paris, T. 135, p. 1362-1365.

1894. de Saint-Joseph. Les Annélides Polychètes des Cốtes de Dinard, $3^{e}$ part.

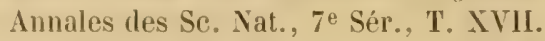

1891. Soulan. Etudes sur quelques points de l'anatomie des Amnélides de la région de Cette. Montpellier.

1903. Soulisen. Revision des Amélides de la rénion de Getle. Travaux de la

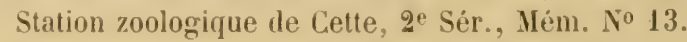




\title{
Beobachtungen an Land- und Süsswasserschnecken.
}

\author{
Von Dr. H. BROCKMEIER (M.-Gladbach).
}

\section{Limnxa ovata und Limmaa peregra:}

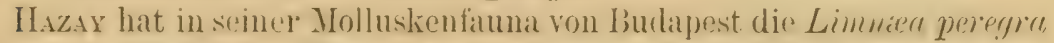

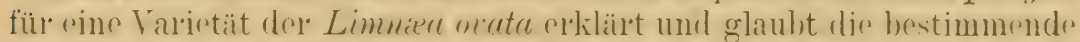
Ursache für die Futstehung dirser. Formen in dem verschiodenen Kallgehalte des Wassers gefunden zu haben. Schon seit längerer Zoit hin auch ich überzeugt, dass $L$. ovata und $L$. peregra zusammengehören, halte aber den Kalligehalt des Wassers hierbei für nehensächlich. In

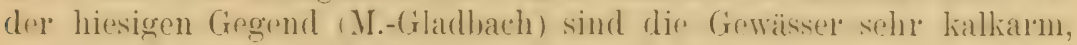
trotzdem kommen beide Formen nicht selten vor.

In den Gewässern, welche das ganze Jahr hindurch, namentlich aber während der warmen Jahresurit, günstige Eirnährungshodingungen hirten, ist mit Sicherheit $L$. ovata zu elwarten; $L$. peregra findet sich höchstens in eingeschwemmten Individuen.

T'ümpel und fliessende Gewässer', welche zeitweise austrocknen oder

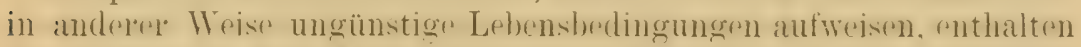
die L. peregror. Eine Ausmahme hiervon habe ich noch nicht feststellen können und in der Literatur habe ich auch noch keine Angabe gefunden. Welche nit dieser Anschaumg nicht in Einklang zu bringen wäre. An anderer Stelle gedenke ich hierauf näher einzugehen.

2. Die Kalkleisten einiger Süsswasserschnecken.

Manche Süsswasserschnecken, z. B. L. peregra, L. elongata und L.

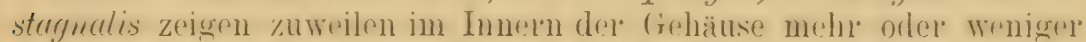
dicke Kalkstreifm. Welche parallel zur Mundlippe verlaufen. Man hat diese Kalkleisten wohl zur bestimmung des Alters der Schnechen vorwertet (Chessis): dazu sind sie aber nur in Ausmahnefallen hatuchhar.

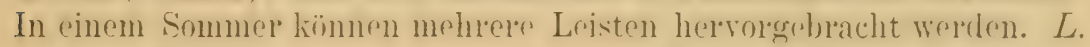
stagnalis zeigt die Leisten seltener, während sie bei $L$. peregra und $L$.

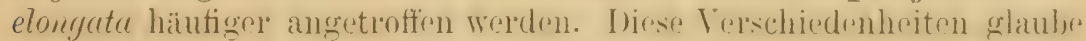
ich in einfacher Weise erklären zu können.

$L$. peregra und $L$. elongata finden sich in der Regel in Gewässern, welche zeitweise austrocknen. Während der 'Trockenzeit sind die Be-

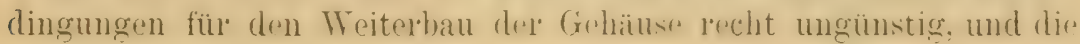


Abscheirlung einer Kalkleiste ist die Folge. Mehrere Trockenperioden in einem Sommel rufen in der Regel auch mehrere Kalkleisten hervor. Tor einigen Jahren sammelte ich in der Umgebung von M.-Gladbach L. elourfutu an rinem Tage, an 3 verschiedenen Stellen. In einem wasserfreien (r aben sassen die Schnecken frei an PHanzenstengeln; alle Tiere hatten sich etwas in das Gehäuse zurückgezogen. In einem zweiten wasierfreien (irahen sass $L$. elongata zwischen feuchten Baumblättern. Die Srhnecken lebten ebenfalls zurückgezogen, füllten aber das Gehüuse ganz aus. An einer dritten Stelle lebte $L$. elongata in einem wasserhaltigen 'Tümpel; hier waren die Bewegungen der Tiere vollständig ungehindert.

Jecle Schneclie aus dem ersten Grahen zeigte einige Millimeter hinter del' rechten Mundlippe eine Kalkleiste. Jedes Tjer aus dem zweiten Graben latte dic Kalkleiste unmittelbar an der Mündung, und die Schneclien des wasserhaltigen Tümpels waren ohne Kalkleisten, übertrafen aber au Grösse alle anderen ; sie hatten eben ungestört weiterbauen können.

L. stannalis zoigt solche Kalkleisten seltener, weil diese weitmündige Schnecke nicht so häufig in austrocknenden Gewissern angetroffen wird.

Ein vollständiges Austrocknen der Tümpel ist aber gar nicht einmal erforderlich, um die in Rede stehende Bildung hervorzurufen. Sinkt der Wasserspiegel, so bleiben in der flachen Randzone schon zahlreiche Schnecken auf dem wasserfreien Schlammgrunde zurück und Kalkleisten werden ausgeschieden. Kehren diese Verhältnisse nach kurzel Zeit wieder. so treten mehrere Kallileisten auf. Derartige Gehäuse habe ich aus einem 'Tümpel bei Plön in Holstein erhalten. In den meisten Fällen wird man aus dem Vorhandensein von Kalkleisten in den Gehä̈sen auf unbeständige Wasserverhältnisse am Wohnorte der Schnekken schliessen können. Die Kalkleisten entstehen in den Gehäusen der Süsswasserschnecken, wenn die 'Tiere während der Wachstumsperiode durch ungünstige Vorhältnisse gezwungen werden, den Weiterhan der Schale einige Zeit zu unterbrechen.

3. Das Alter einiger Landschnecken (Helix memoralis und H. hortensis).

Die Angaben, welche man hierüber finden kann, stimmen mit meinen Zuchtversuchen nicht üborein. Nach O. Goldeuss (Dir Bimnemmollusken Mittoldeutschlands, $19(0)$ erreichen die schnirlielschnecken ein Alter von 2 bis 3 Jahlen.

Unter gewöhnlichen Verhältnissen vollenden die genannten Arten ihre Gehäuse im zweiten Jahre, leben aber dann noch eine Reihe von Jahren. Bastarde von beiden Arten, welche ich züchtete, erreichten ein Alter von 10 Jahren und 1 Monat. Eine erwachsene $H$. hortensis setzte ich in ein 'Terrarium; sie hatte mindestens ein Alter von 2 Jahren und Jcbte noch 4 Jahre. Vom Gehäuse lässt sich das Alter nicht ablesen, wohl 
abcr kamn man unter einمr grösseren Anzahl dieser Schnecken von demsollen Fundorte ältre und jüngere Exemplare mit Leichtiglieit untrrescheiden. Dies wurde an verschiedenen Gehäusen von Gartenschnecken (H. hortensis) gezeigt, welcho in riner orösseren Brennessolgruppe aufgewachsen waren.

4. Bei diesel Gelegenheit wurde darauf hingewiesen, dass Brennnesseln gute Futterpflanzen fül. Schnecken sind, obgleich nach S'xum, (Pthanzen und Sichnecken) diese Pflanzen gegen schnechirnfrass vorzüglich geschützt sein sollen.

\title{
Theoretische Betrachtungen über die ersten Anfänge des ambulacralen Wassergefässsystems der Echinodermen.
}

\author{
Von Prof. E. MEYER (Kasan).
}

Iieferent vergleicht die restem Anlagen des Echinodermen-Hydrocoels mit den zur S'hwellung der Kopftentakel dienenden I iaphragmasäcken der Terebelloiden, welche, ähnlich den ersteren, Aussackungen eines vorderen Coloms gegen einen grösser'en, hinteren Cœelomraum hin vorstellen. Darnach könnte man sich die dipleuren Urahnen der Echinodermen als eine etwa sipunculoidenartige Vermalienform vorstellen, die

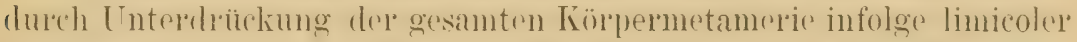
Lebenswejse von terehelloblenibnlichen. halbsedentären Anmeliden ihren Ursprung genommen und sich, nach Umbildung der anfangs offenen I)iaphragmasäckr zu einem abgeschloss'nen, zunäidhst vollhommen symmetrischen Hydrokanal- und Ampullensystem, bei der definitiven Festsetzung mittelst der Kopftentakel der rechten Seite in das pentactreaartige, asymmetrisch-ladiäre Urechinoderm verwandelt hätte. 


\title{
Le sperme de la Hæmenteria costata, du spermatophore à l'oviducte.
}

\author{
Par le Prof. H. BOLSIUS (Oudenbosch).
}

$1^{\circ}$ Dans la nature, on rencontre souvent des particularités très exceptionnelles dans la manière dont se passent des phénomènes très communs. Quoi de plus commun que la rencontre des spermatozoïdes avec les ovules? Et néammoins quelle prodigieuse et étonnante variété dans e' phénomène!

$2^{\circ}$ Nous n'entendons pas parler de la fécondation proprement dite, mais nous nous proposons, comme il convient ici, vu l'auditos d'élite et le temps dont nous disposons, de décrire succinctement comment, chez la Hæmenteria costata, les spermatozoïdes s'avancent ver's les ovules. Nous omettrons même les comparaisons avec les autıes Glosiphonides, où rien de pareil ne se produit.

3. Dans cette description, nous devrons nous éloigner, dans des détails fondamentaux, des vues de notre ami regrette, le Prof. Alex. Kowalewskx, auquel nous devons les principaux matériaux sur lesquels nous avons pu travailler' Lui-même, cependant, de son vivant, était le premier à nous encourager : partant, nous n'hésitons pas.

$4^{\circ}$ Kowalewsky a fort bien vu que, dans cette espèce d'Hirudinée, comme dans bien d'autres, l'échange de sperme entre deux individus se fait par spermatophore. Seulement, - et ceci est une particularité digne de remar'que, - le savant russe constatait, à l'encontre d'autres observateurs, que l'insertion du spermatophore a lieu régulierement, non pas dans l'or'ifice externe femelle, mais dans l'orifice extel'ne male.

$5^{\circ}$ Les nombreux individus que nous avons reçus de Kowalewskr, fixés et conservés d'après nos indications, nous ont prouvé le même fait, comme le démontrent l'exemplaire que nous avons apporté, et les nombreuses préparations microtomiques.

$6^{\circ}$ Mais, dès le moment où il s'agit de faire passer les spermatozoïdes du spermatophore à l'intérieur du corps de lanimal, il y a divergence entre les indications de Kowalewsiry et les données de mes recherches personnelles.

7" Pour Kowalewsi, le bout supérieur du spermatophore doit perfo- 
rer la paroi qui le sépare de la cavité périviscérale. Les préparations, ici présentes, démontrent a l'évidence que lo col du spermatophore, qui ressemble à une bouteille de champagne, est nettement alrété par l'ocelusion de l'orifice mâle externe; et au surplus, - ce que Kowalewsiry a parfaitement remarqué et reproduit dans ses figures, - ce col est surmonté d'une sorte de tampon muqueux ou gélatineux, peu approprié à une perforation d'une paroi puissante.

$8^{\circ}$ Mais cette perforation hypothétique ne saurait même rendre compte de la sortie des spermatozoïdes de leur spermatophore. Celui-ci a une paroi anhyste, absolument dépourvue, par conséquent, de muscles constricteurs, qui chasseraient les spermatozoïdes de leur prison. Ensuite, le spermatophore, au fur' et à mesure qu'il se vide, est absolument ratatiné, ce qui indique un mécanisme aspirateur extérieur.

$9^{\circ}$ Voici ce mécanisme, tel que le révẻlent les relevés de centaines de m’éparations en séries inintrrompues. pratiqués dans les trois directions.

La paroi puissante qui entoure la cavité où le col du spermatophore est emurisonné, est formée par une infinité de cellules musculaires généralement dorso-ventrales. Ces muscles forment des sortes de piliers mobiles. Par leurs mouvements, les piliers les plus internes tendent à agrandir la cavité. Il se formerait un vide relatif autour du col du spermatophore, et, pour y obvier, le tampon se détache. Alor's, la contraction des premiers piliers repousserait le tampon; mais les piliers suivants, se mettant en mouvement, aspirent la matière muqueuse entre les premier's piliers. Cette même manœuvre est répétée de proche en proche par los sé lies consécutives de pilier's de la paroi très épaisse, et il se produit une sorte de déglutition.

Par une série de ces mêmes mourements aspirateur's, les spermatozoïdes sont comme sucés, tirés du spermatophore et transportés dans la cavité périviscérale.

$10^{\circ}$ 'Toute la structure du spermatophore vise l'extraction par aspiration. Le col possède une paroi d'une épaisseur relativement énor'me, on plutôt le col est une pièce chitineuse massive, percée seulement de deux canaux extrêmement étroits, qui sont en communication chacun avec une des deux cavités spermatophoriques. Par contre, ces deux cavités ont des parois très minces. Il s'ensuit que les canaux, dans le col, supportent la pression sans se fermer pendaut l'aspiration. tandis que les parois minees des cavités cèdent à la pression extérieure produite par l'aspiration.

$11^{\circ}$ Une preuve plus biologique ou physiologique, démontrant que la perforation dont parle Kowarewsir n'existe pas, est celle-ci :

L'accouplement entre les Hrementeria costrata ne se borne pas à une seule tois: c'est encore Kómalewsizy qui l'a constaté. Mais à peine un sprematophore est-il vidé, (quil se fait un nouvel atconplenent et échange de spermatophores. Or, nos recher'ches personnelles démontrent, - et les

VIe CoNGR. INT. ZOOL., 1904. 
préparations que nous arons apportées en font foi, - que ce deuxième spermatophore se retrouve arec son extrémité supérieure et son tampon devant une paroi intacte, et dont les cellules musculaires ne présentent ni déchirure, ni régénération.

La paroi a donc fonctionné physiologiquement et sans être endommagée, et se trouve prête à fonctionner encore. C'est d'ailleur's ce à quoi ou devait s'attendre; car il serait bien étonnant qu'un tissu qui doit servir aussi à fermer la cavité périviscérale et à empêcher que son contenu liquide ne se déver'se à l'extérieur, soit normalement déchiré par l'introduction physiologiquement nécessaire du sperme.

$12^{\circ}$ Le tissu de la paroi en question est donc de telle nature et de telle structure que, d'une part, il for'me une cloison étanche contre l'écoulement du liquide intérieur, et que, de l'autre, il permet, ou plutôt il force le sperme à entrer dans cette même cavité. C'est tout simplement la fonction d'une pompe aspirante et foulante.

Il est donc acquis que l'entrée du sperme clans la cavité périviscérale se fait par un fonctionnement normal et physiologique de la paroi qui entoure le vestiluule. Reste à suivre le sperme, sur sa route vers les ovules.

$13^{\circ}$ Les flots de spermatozoïdes, une fois qu'ils ont passé la paroi musculeuse, sont mêlés au liquide qui circule dans la cavité périviscérale.

Les préflatations macroscopiques, telles que les a figurées et décrites notre l'egretté collegue de Saint-Pétersbourg, aussi bien que les sections microtomiques que nous avons pratiquées dans les matériaux gracieusement fournis par le dit prof. Kowalewski, démontrent clairement que le sperme se porte en masse sur les deux portions symétriquement disposées de la matrice. Peu de temps après l'accouplement, dit liowalewsky, on voit des flocons de sperme recouvrir toute la partie inférieure des oviductes. Nous l'avons constaté comme lui. Ce sperme doit traverser la paroi pour atteindre les ovules.

$14^{\circ}$ Comment se fait cette traversée? Ici encore, nos recherches personnelles ne sauraient donner raison aux conclusions de notre savant confrere. C"est encore à la perforation qu'il en appelle. Les spermatozoïdes perforent, dit-il, la paroi de la matrice.

A la première vue, certes, on a l'impression, même en voyant des préparations microtomiques excellentes, que les spermatozoüdes font des trouées dans cette paroi excessivement épaisse. Cependant, en explorant patiemment, et surtout en portant toute son attention sur des points de la paroi où, pour le moment, ces masses de sperme ne sont pas encore enclavées dans l'épaisseur même, on peut observer', grâce à une coloration bien nette, que la surface externe n'est pas continue et lisse, mais qu'il y a nombre de petits enfoncements.

$15^{\circ}$ Èn examinant spécialement ces enfoncements, on s'aperçoit que ce 
sont des formations normales qui nous donneront la clef d'une explication très rationnelle.

Ces invaginations sont des entonnoirs microscopiques, placés sur des canaux plus microscopiques encore, préformés dans l'épaisseur de la paroi.

Le sper'me qui afflue en masse et entoure la partie inféricure de l'oviducte, s'infiltre dans cette paroi d'épaisseur énol'me; c'est un fait absolument certain, et prouvé par d'innombrables préparations. Seulement, le sperme ne perfore pas la paroi, il la traverse sans endommager les tissus.

$16^{\circ}$ Le chemin du sperme est préparé et indiqué par la nature, ce sont les entonnoirs et les canalicules mentionnés ci-dessus.

D’abord, quelques l'ares spermatozoïdes, détachés des masses de sperme, s'insèrent dans ces trouées minuscules. Ainls, par un travail physiologique que nous n'entreprendrons pas d'analyser jei, les canaux commencent à se dilater, et les spermatozoïdes afiluent en plus grand nombre.

Enfin, quelque temps après, la masse pariétale, grâce aux canaux qui so sont dilatés outre mesure, est littéralement bourée de sperme. L'aspect que présente à ce moment la portion inféricure de l'oviducte est. celui d'une éponge, et non pas des plus fines, dont toutes les cavités seraient occupées par des mass's de spermatozoüdes non pas placés à la file, mais amassés en boule. Ce sont ces boules de spermatozoïdes qui tombent enfin dans la cavité de l'oviducte.

$17^{\circ}$ Nous maintenons qu'il n'y a ni perforation, ni même lésion des tissus, malgré cette dilatation outrée des canalicules primitifs. Et ceci pour deux raisons.

La première, d'ol'dre purement physique, est celle-ci. Dans les préparations que nous avons faites, les parois des canalicules, et anssi des cavités nées par la dilatation de ceux-ci, sont toujours formées par un tisisu ne présentant aucune déchirure, ni autre endommagement : les parois sont normales.

La deuxième raison est une raison plutôt physiologique. 'Très peu de temps apres cette énorme dilatation, la paroi a déja replis sa physionomie première, et elle est prête à fonctionner de nouveau vis-à-vis du sperme que va apporter un second spermatophore. Les préparations ne montrent pas la moindre trace de reconstruction des tissus soj-disant perforés: il y a contraction de tissus dilatés, ou plutôt de cellules allongées par un travail pliysiologique, lequel, pour etre microscopique, n'en est pas moins certain.

$18^{\circ}$ Deux détails d'anatomie pour finir.

a) Le sperme, qui a été rejeté par la paroi à mouvements quasi péristaltiques, dont nous avons parlé en premier lien. ne semble pas itre projeté sans une direction spéciale dans la cavité péririscérale. Les sections microseopiques, pratiquées parallèlement à la face ventrale, indiquent plutot que des muscles dorso-ventraux sont disposés en masse vers l'oviducte 
et dirigent le sperme en majeure partie sur ce dernier. Nous constatons qu'eu réalité cet effet est obtenu.

b) En dehor's de la portion terminale, la paroi de l'oviducte est très mince. Or, jamais on ne constate la pénétration du sperme dans cette paroi mince, quoique, lì aussi, il envirome l'oviducte. Nais cette paroi mince ne possede pas d'entonuoirs, ni de canaux : la paroi épaisse seule les possède, et là seulement se prooduit la pénétration ou la traversée.

\title{
Sur un nouveau genre de Syllidien, Alluaudella nov. gen., madagascariensis nov. sp.
}

\author{
Par C. GRAVIER (Paris).
}

Avec 5 figures dans le texte.

En 1901, M. Ch. Altudud a recueilli à la pointe sud de la baie des Galions, it Fort-Tauphin (Madagasear) cing exemplaires d'un type nouveau de Syllidien. Le seul individu entier qui a servi de type à la description suivante mesure $10^{\mathrm{mm}}$ de longueur, $0^{\mathrm{mm}}, 85$ de largeur, parapodes non (onlpris, $1^{\text {mun }}$.30 en y incorporant ces appendices. Le nombre des segments

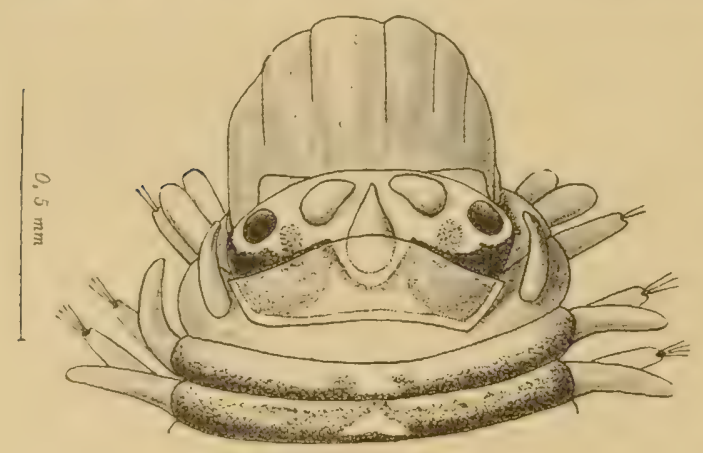

Fig. 1. Partie antérieure de l'animal; face dorsale. La trompe est en partie dévaginée. est de 51 ; les quatre der'niers sont régénérés, transparents et sensiblement plus étroits que ceux qui les précèdent immédiatement.

Le prostomium (fig. 1) presque deux fois aussi large que long est convexe sur son bord antérieur, concave sur son bord postérieur. Il porte une antenne médiane, épaisse et conique et deux antennes latérales insérées bien en avant de la précedente, plus courtes et terminées en pointe mousse. Deux grosses 
taches oculaires, sans lentille apparente, sont situéses de chaque cóté tout près ću bor'd antérieur. Une plage pigmentaire brun très foncé occupant la région postérieul'e du prostomium, encadre les yeux et l'antenne médiane. Un peu en dedans des yeux, on observe de chaque côté une tache ovale d'un beau jaune brun. La seconde paire d'yeux, si elle existe, est indiscernable dans la zone pigmentée de la moitié postérieure du prostomium; celle-ci est en grande partie couverte, de même que la base de l'antenne médiane, par' une membrane qui s'attache en alrière sur le premier segment porteur des deux paires de cirres tentaculaires cylindriques et courts.

Les deux palpes très peu développés, aplatis, soudés dans toute leur étendue, dépassent à peine le bor'd antérieur du prostomium.

La trompe rectiligne et courte (fig. 2) présente trois parties distinctes: $1^{\circ}$ une gaîne pharyngienne dont la paroi semble renforcée, lor'sque l'organe est dévaginé, par' d'épaisses

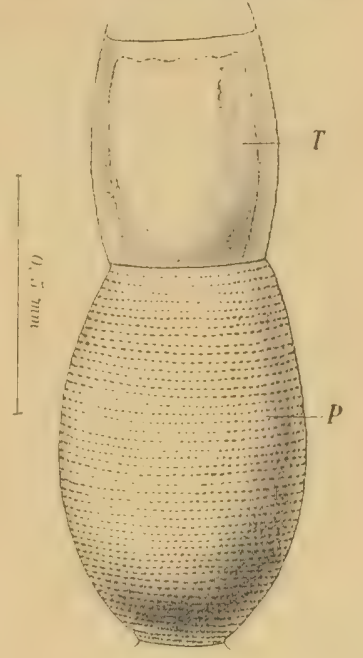

Fig. 2. Trompe.

T. trompe; P. proventricule. colonnes musculaires (fig. 1 ); $2^{\circ}$ une trompe pharyngienne 'I' courte, à paroi d'épaisseur uniforme; au-dessous de la couronne de papilles qui la termine au sommet, je n'ai pu découvrir aucun trépan; $3^{\circ}$ un proventicule $\mathrm{P}$ à musculature puissante, avec ses ran-

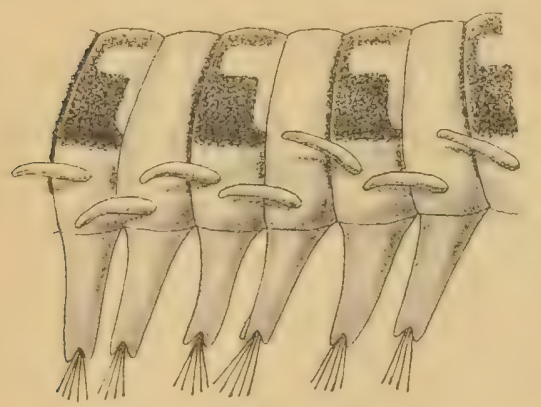

Fig. 3. Parapodes de la région moyenne du corps; les cirres dorsaux sont insérés à deux niveaux différents. gées transversales de ponctuations sombres régulièrement espacées, et qui ne dépasse pas, en arrière, le dixième segment.

La face dorsale est fortement convexe d'un bout à l'autre du corps. La face ventrale, sur toute la longueur de la trompe, c'est-à-dire jusqu'au dixième sétigère, est plane ou même très légèrement convexe; au delà, elle se creuse profondément jusqu’à l'extrémité postérieure. Les parapodtes insérés très bas semblent continuel les bords de la rigole ventrale. Le premior segment sétigère. non pigmenté, poler do chaque coté 111 
cirre dorsal cylindrique et une rame située beaucoup plus bas, au-dessous du niveau du cirre tentaculaire inférieur.

Les sétigères suivants sont armés également d'un parapode uniramé dans lequel le cirre dorsal est moins éloigné du bulbe sétigère. Les $2^{\text {me }}$, $3^{\text {me }}, 4^{\text {me }}, 5^{\text {me }}$ et $6^{\text {we }}$ sétigères portent, sur la face dorsale, une bande pigmentaire très foncée qui s'étend sur la moitié postérieure de chaque segment. En arrière, la pigmentation change de caractère (fig. 3). Certains segments n'ont qu'une simple ligne pigmentaire à leur limite postérieure, les autres ont deux larges bandes très foncées placées symétri-

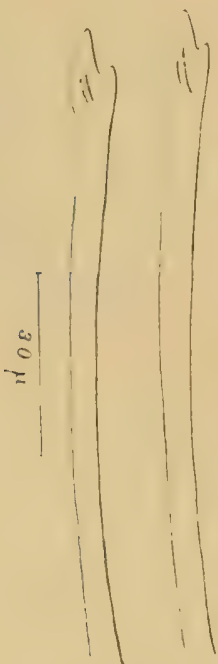

Fig. 5. Soies.

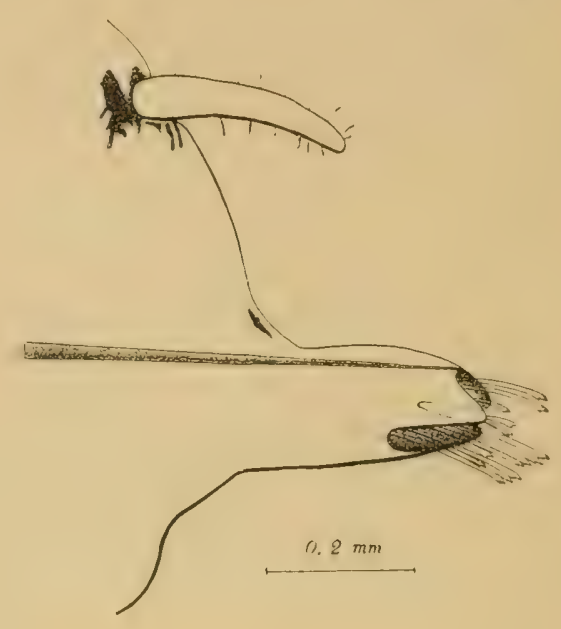

Fig. 4: Parapode.

qu'ment par rapport à une petite plage médiane incolore. Ces deux séries alternent régulièrement et soulignent la segmentation. Sur la face ventrale, la rigole médiane est blanche; de chaque côté, il existe, à la base des parapodes, une bande pigmentaire presque continue.

Les cirres dorsaux (fig. 3 et 4) presque cylindriques, portent des cils raides, comme chez la plupart des Autolytés et des Eusyllidés. Ces appendices offrent ici une particularité curieuse: ils sont insérés sur deux lignes parallèles aux bords latéraux; l'alternance est régulière.

Les bulbessétigères insérés très bas, obliquement, comme pour continuer la paroi de la gouttiere ventrale, sont cylindriques et très saillants; leur extrémité distale est divisée en trois lobes, un antérieur et deux postéricur's, séparés entre eux par de profondes échancrures, qui enve- 
loppent la base du faisceau de soies. Chacun d'eux est soutenu par un seul acicule situé à la portie supéricure. Les soies, toutes semblables les unes aux autres, sont simples, grêles, un peu aryuées, légèrement renflées au sommet qui se termine par deux pointes coniques très inégales (fig. 5). Ces soies simples proviennent vraisemblablement, comme l'a fait Pemarquer le Professeur E. EnLens, de soies composées dans lesquelles la serpe se serait soudée à la hampe.

A l'extrémité postérieure qui est régénérée et de taille encore réduitr, il existe deux cirres anaux ayant la même forme et à peu près les mêmes dimensions que les cirres dorsaux voisins.

La multiplicité des affinités du Syllidien décrit ci-dessus rend assez difficile la fixation de sa position systématique. La longueur médiocre et l'aplatissement des palpes soudés dans toute leur étendue, caractères morphologiques de haute valeur chez les Syllidiens, ne s'observent que dans la tribu des Autolytés de Malaquin'. Mais les caractères de la trompe qui jouent également un rôle important dans la spécification de ces Ammélides l'éloignent des Autolytés et le rapprochent beaucoup des Eusyllidés du même auteur ; ce rapprochement est corroboré par la forme? trapue du corps, si grêle chez les Autolytés, et par la lame recouvrant l'organe nucal, que l'on retrouve chez le genre ()lontosyllis Claparede et à un moindre degré de développement, chez le gemre Éusyllis Malıngren. Cette membrane protectrice existe aussi chez le genre Fauvelia Gravier " qui, par ses affinités avec le genre Platysyllis Grube, rappelle les Syllidés, mais se range plutôt parmi les Eusyllidés.

D'autre part, un certain nombre de traits donnent au Syllidien en question une physionomie tout à fait spéciale.

$1^{\circ}$ Les appendices céphaliques sont ici exceptionnellement courts, de même que les cirres dorsaux; une pareille brièveté ne se constate que chez quelques Fxogonés, comme chez l'Exogone (Pxidophylax) clrviges' Claparède ${ }^{3}$, par exemple.

$2^{\circ}$ Les cirres dorsaux sont insérés à deux niveaux distincts, d'un segment il l'autre; cette disposition, insolite chez les Syllidiens, se montre a ussi, ainsi que l'a indiqué le Professeur E. Enlers, chez certains Amphinomiens. On observe l'alternance, non de position, mais de grandeur, dans les cirres dorsaux de certaines espeess du genro Firchowia Langer-

1 A. Malaquin. Recherches sur les Syllidiens (Morphologie, anatomie, reproduction, développement). $14 \mathrm{pl}$., fig. dans le texte, $481 \mathrm{p}$. Mémoires de la Société des Sciences et Arts de Lille. 1893.

${ }^{2}$ Ch. Gravier. Sur un type nouveau de Syllidien, Fauvelia (nov. gen.) martinensis (nov. sp.). Bull. du Mus. d'hist. natur., p. 371-374, 7 fig. 1900.

${ }^{3}$ Ed. Claparède. Les Annélides Chétopodes du Golfe de Naples. Mém. de la Soc. de Phys, et d'Hist. Nat. de Genève, t. 19, p. 123, pl. XII, fig. 3. 
hans et aussi chez certains Autolytus: A. Tongeferiens de Saint Joseph, A. paradoxus de Saint Joseph ${ }^{1}$.

$33^{\circ}$ Les sojes simples, fort répandues chez les Syllidiens, se mélangent presque toujours aux soies composées qui, très généralement, prédominent largement par le nombre. Cependant, les especes du sous-genre Haplosyllis Langerhans ne possedent aussi que des soies simples a crochet bidenté d'une forme différente de celle qui est représentée ici dans la figure 5.

Xous donnerons au type nouveau de Syllidien dont la description précede le nom d'Alluandella nov. gen. madagascariensis nov. sp., rappelant le nom du voyageur naturaliste qui l'a recueilli et celui du pays d'où il provient.

La diagnose du genre Alluaudella sera la suivante:

Prostomium avec trois antennes très courtes, recouvert dans sa moitié postérieure par unelamefixéesur le premier segment; celui-ci portant, de chaque côté, deux cirres tentaculaires cylindriques courts. Palpes aplatis, très peu développés, soudés dans toute leur longueur. Cirres dorsaux cylindriques et courts. Pas de cirres ventraux. Des soies simples seulement. Trompe rectiligne courte, sans dent, ni trépan.

Au point de vue morphologique, le genre Alluaudella présente plusieurs traits caractéristiques des Autolytés; mais par sa physionomie générale et surtout par la constitution de sa trompe, il se rapproche davantage, comme nous l'a fait remarquer Malaquin, des Eusyllidés, parmi lesquels il prend une place à part.

Cet exemple met une fois de plus en évidence le côté artificiel de nos classifications les mienux citahlies et justifie l'opinion émise par de QuatreFAfES " dans ses généralités sur la famille des Syllidiens: "Toutes les espèces qui la composent ont certains caractères communs et, en particulier, un fucies en général difficile it méconnaittre. Cependant, c'est une de celles qui présentent le plus de variabilité. On y constate surtout une dégradation croissante portant sur les parties les plus caractéristiques, sur les pieds, par exemple, qui ne se montre nulle part ailleur's. Il semble qu'à elle seule, cette famille constitue un petit monde à part où la petitesse du corps est la règle générale et où les caractères jouent dans des linites de variation plus étendues et plus multiples que dans aucune autre famille $)$.

' De Saint-Joserir. Les Annélides Polychètes des Côtes de Dinard. $1^{\mathrm{r}}$ partie,

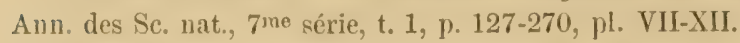

${ }^{2}$ A. de Quatrefages. Histoire naturelle des Annelés marin et d'eau donce. T. 2, $1^{\text {re }}$ partie, p. 7. 1865. 


\title{
Ueber die Bildung des Mesoblastes bei den Echiuruslarven ${ }^{~ .}$
}

\author{
Von Prof. W. SALExSKY (St. Petersburg).
}

Eine der Aufgaben, die ich bei meinen Untesuchungen übre dir Trochophora immer im Vordererund gehalten habe, ist die Entselpeidung der Frage, ob in den 'Trochophoralarven, bei welchen die Bildung des Mesolblastes hereits hegonnen, eventucll auch ziomlich vorgeschrittru ist. die telohlastischen Mesolblastzellen, resp. die primitiven Mosoblasten noch vorhanden sind und ob sie an der Bildung des Mesoblastes teilnehmen? Bekanntlich hat Hatscheck zuerst die hervorragende Rolle der primitiven Mrsoblasten hervolgehoben mol dir letatren hei allen von ihm untersuchten Larven aus verschiedenen Tiertypen nachgewiesen.

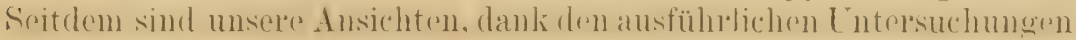
der Embryologie der Anneliden in bedeutender. Weise geåndert, und olwohl der toloblastische [rsprung des Mesolulastes auch in den neneren Untersuchungen eine Bestatigung findet. ist jodenfalls die Taitiolent dele Mrsoblasten und das Vorkommen derselben in die frublere, embrvonale Periode der Entwicklung zurückgeschoben. Es fehlt jedoch nicht an rinzelnen Beobachtungen, dir noch die Hatscmess schen Behauptungen

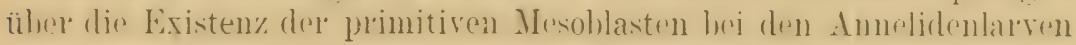
bestätigen (Mexer, Wortereck bei den Polygordiuslarven).

Das erste Objekt, zu dem ich mich wendete, war eine Polygordiuslarve, die ich im vorigen Jahre in Sebastopol in einer ziemlich grossen

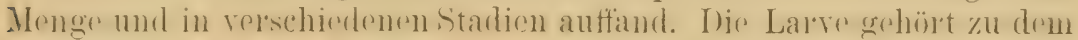

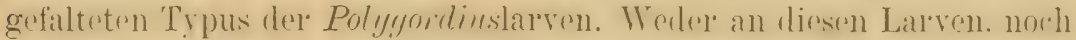
an den Larven der ungefalteten Typen, die mir durch die Güte der russischen zoologischen Station in Villeflanche aus d'm . Iittelmere geljefert wurden, konnte ich selbst bei den allerjüngsten Larven primitive Mesoblasten entdecken. Später wendete ich mich zu den Larven von Echiu'us, von denen ich ein grosses, in der zoologischen Station zu

\footnotetext{
1 Unter dem Namen "Mesohlast " rerstehe ich das "Colothel * und das " secundäre Nesenchym ». Diese Bezeichnung will ich in meiner ausführlichen, im Druck sich befindenden Arbeit anwenden, wo ich auch die Gründe für diesen Ausdruck anführen werde.
} 
Neapel angesammeltes Material besitze. Um die Stadien dieser Larve genau zu bestimmen, habe ich die Larven mit Camera unter derselben Vergrö̈serung (40 Mal) wie die Hatscheck'schen gezeichnet und die Grösse meiner Abbildungen mit der Grösse der Hatscheck'schen Figuren verglichen. Diese Procedur' wurde für mich deswegen notwendig, weil ich nur auf diesem Wege das Entsprechen der von mir beobachteten Stadien mit denen von Hatscheck beschriebenen feststellen kann; bei Hатыснек findet man keine Angaben der Grösse des Objektes, sonder'n nur die Vergrösser'ung der Figuren ist angegeben.

Die Untersuchung der von den jüngsten Larven angefertigten Schnitte hat mich sofort überzeugt, dass, 1. selbst bei den jüngsten Larven keine primitiven Mesoblasten vorhanden sind; 2. dass die Entwicklung des Iesoblastes bei ihnen soweit vorgeschritten ist, dass in demselben bereits die Myotome zu unterscheiden sind und 3. dass die Entwicklung des Mesoblastes hier in einem innigsten Zusammenhange mit dem Entoderm resp. mit dem Nahrungskanal steht.

Der Darmkanal der Echiuruslarven besteht aus 3 Teilen: 1. aus einem gerïumigen und dorsoventral etwas abgepatteten, gebogenen Oesophagus, welches in der Episphare gelagert ist; 2. aus cinem sehr weiten und kompliziert gebauten Nagendarm, welcher in Form eines sphärischen Sackes beinahe die ganze Höhle der Hyposphrere erfüllt und 3. aus einem sehr kurzen Enddarm, welcher am hintern Pol durch die Analöffnung ausmündet. An der Grrenze des Magendarmsackes und des Enddarmrohres liegen grosse einzellige Drüsen, die schon durch die Kïrrperbedeckungen durchschimmern. Ihrer Lage und Form nach sind sie den von Hatscheck abgebildeten primitiven Mesoblasten ("Polzellen des Mesoderms y von Hatscheck) so ähnlich und liegen den Mesoder'mstrei-

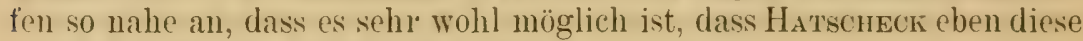
Zellen für Mesoblasten gehalten hat.

Ausser den eben genannten Teilen des Darmkanals sind bei den jungen Larven (aus der von Hatscheck als "Trochophorastadium " bezeichneten Periode) noch zwei Bildungen vorhanden, welche die wichtigste Rolle bei der Entwicklung des Coelomesoblastes spielen. Es sind nämlich zwei symetrisch gestellte Aussackungen der Bauchwand des Mitteldarmes, die im hintern Teile des Mitteldarmes genau an der Grenze des letztern gegen den Enddar'm gelagert sind. Ihre Form ist halbspherisch, ihre Wände hestehen aus einem Epithel. welches im Veroleich mit dem des eigentlichen Mitteldarmes bedeutend kleiner erscheint. Betrachtet man eine Reihe der Lüngsichnitte durch die Larve (Trochophorastadium von H.tśnе(к), so trifft man Folgendes an: Erstens, constatiert man, dass die beiden Aussackungen in den axialen Teilen so zu einander stehen, dass sie sich beinahe berühren. Zweitens, kamn man ersehen, dass die medialen Teile ihrer Wände aus einer einzigen Fpithelschicht hestehen ; 
weiter nach aussen nehmen dieselben an Dicke zu und sind nicht meh" einschichtig, sonder'n aus mehreren Lagen, teilweise cylindrischer, teilweise zugespitzter, einander seh' nahe liegender Zellen zusammengesetzt.

Auf den folgenden seitwärts geführten Schnitten erscheinen die Wïnde der Aussackungen bedeutend stärker verdickt und gehen ununterbrochen in die Mesorlermstreifen, resp. in das Coelomesoblast über. An den darauf folgenden Schnitten hören die Mitteldarmsäcke auf und treten nur die Mesoder'mstreifen auf'.

Die auf den Längsschnitten gewomnenen Resultate, namentlich die Constatirrung eines unmittelbaren Ueberganges der Magendarmsäcke in das Mesoblast, können auch dureh die Beobachtung der Querschnitte bestätigt werden. An diesen letzter'n kann man sich überzeugen, dass die Mesodermstreifen aus einer Fortsetzung der seitlichen und untern Wände der Magensäcke ihren Ursprung nehmen und dass sie als einfache Auswüchse dieser Säcke betrachtet werden müssen. Auf Grund dieser Brobachtungen kömnen die beiden Magensäcke als Colomsäcke bezeichnet werden, welche als den Colomsïcken anderer Tiere, wie Echinodermen, Surgittu, Brachiopoden, Amphiox s, etc. homolog angesehen werden müssen. Die Mesodermstreifen stellen nun nichts anderes als unmittelbare Auswüchse der' Colomsïcke dar.

Jie Höhle jedes Coelomsackes, welche ziemlich gexäunig erscheint, nimmt in den Mesodermstreifen in Folge der starken Verdickung seiner Wand an Umfang ab) und verwandelt sich in eine Spalte, die sich in dem entsprechenden Mesodenstreifen fortsetzt. An einigen Präparaten habe ich sellost gesehen, dass diese Höhle sich stellenweise erweitort: diese Erweiterungen sind die Anlagen der Metamerie. Bei den jüngsten Larven konnte ich bereits fünf solcher Colomeren unterscheiden.

In den etwas späteren, den Fig. 3 von Hatsoneck lorrespondierenden Stadien hört die Terbindung der Mesodermstreifen mit dem Darninanal auf. Die Coolomsäcke gehen allmälig zu Grunde, die Mesodermstreifen liegen nun der Wand des Magendarms an, ohne mit derselhen in Verbindung zu stehen.

Ueber die Art und Weise, in welcher die Metamerie des Mesoblastes sich herausbildet, bin ich noch nicht ganz sicher. Ich kann aber ganz sicher hehaupten, dass die Metamerie schon sehr früh, bereits in dem sog. "Trochophorastadium ) von HATscheok von aussen her deutlich kenntlich ist. In den Längsschnitten kann man sich überzeugen, lass die Motameren bereits in dieser Entwicklungsperiode mit Höhlen versehen sincl. Daher ist es mir sehr wahrscheinlich, dass die C'olomhöhlen der Metameren wirklich aus der Erweiterung der ursprünglichen Höhle der Coelomsäcke ihren Ursprung nehmen.

Ich will mich hiel nur bei den beiden vordersten Metameren authalten, die im Zusammenhange mit den zwoi speziellen ()roanen des Larvenlei- 
bes, namentlich mit den vorderen Borstensäcken und mit den sog. Protonephridien stehen.

Die Borstensäcke sind in Form von soliden Verdickungen des zweiten Myouners angelegt. Diesie Verdickungen entstehen schon frühzoitig an den äuscren Ib̈̈ndern des rechten und des linken zweiten Myomerss und stellen zapfenförmige solide A uswüchse dar, die sich allmählig ins bitoderm vordringen und schliesslich durch dic Obertlache desselben durchbrechen.

Die von Grobrex bei den Echiumslarven entdeckten Nepridien wurden von Hatscheck genau beschrieben. Sic sollen zwei symmetrisch gelegene, verïstelte und durch einen besonderen Ausführumgskanal resp.

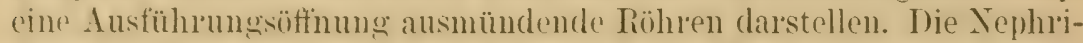
dien der" Echimuslarren, wie diejenigen der 'Trochophora überhaupt. sind den Protonephridien heigezählt. Ihr Zusammrnhang mit den Mesodermstreifen resp. mit der Coelomhöhle ist sehon durch ihre Definition in Abrede gestellt. Ich kann auf Grund meiner Beobachtungen dieser Ansicht nicht beistimmen.

Aus den Abhildumgen von Hatscheck und anderen Forschern, die sich mit der Anatomie der Trochophora beschäftigen, ist ersichtlich. dass das proximale Ende drs sog. Protonephridiums auf den vorderen Rand des Ilesodormstreifons sich stützt. Dieselben Verhältnisse sind bei den lehenden Echiumslarven beobachtet, jedoch nicht vollkommen verwertet worlen. Untersucht man eine Serio der Längsschnitte, so kamn man sich

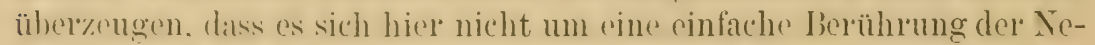
phridien mit den Mesodermstreifen, sondern um eine vollständige Kontimuitat der (xewe handelt. Inir angeblichen Protonephridien exscheinen als Foltsetzungen des Mresolementreifens. Auf den gut geratenen Längsishnitten sieht man folgendes: Del vordere Rand des ersten Mỵotoms und namentlich seines Seitenteiles setzt sich nach vorn in einen Auswuchs fort, der schon bei seinem Anfange in zwei Aeste sich teilt. Finer von diesen richtet sich durch das blistocol nach aussen gegen das Ectoderm, wo er nach aussen ausmündet. Derselbe stellt den Ausführungsgang des Nephridiums dar. Del andere Zweig setzt sich mach vorne hin fort, schliesst sich dem grossen Baüchmuskel an und stellt den eigentlichen Stamm des Nephridiums dar. Ijeser Zweig gibt mohrere Verästelungen ab, welche in der schon von HAtscueck beschriebenen Woise mit don aheseshlosenen Wimperzellen resp. Solenoeyten endigen. Die letztern lassen sich an Schnitten nur bruchstückweise beobachten.

Jie Austrittsstelle des Nephridiums vom Mesoderunstreifen licgt genau üher der Höhle des ersten Myotoms, weleher lotztere bei der Bildung des Nephridiums nicht untätig bloibt. Sire eribt nämlich zwei Acste ab: rinen in den Lusführungszug, den andern in den Hauptstamm des Ne-

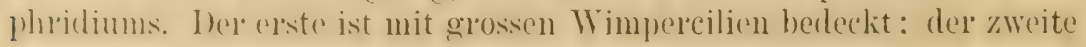




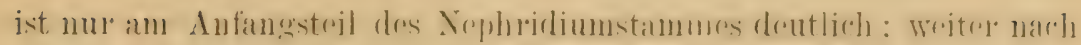
vorne verdünnt er sich so beträichtlich, dass er auf den Schnitten nicht mehr wahrgenommen werden kann.

Aus diesel kurzen Dar'stellung des Baues vom Nephridium geht her-

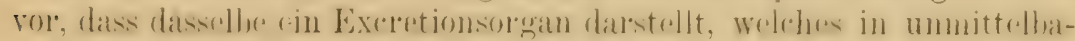
rem Zusammenhange mit dem Colom steht, dass es nach der Art der

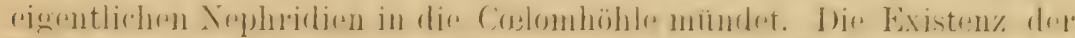

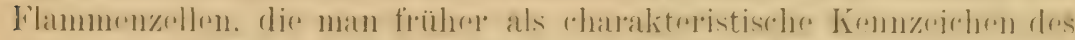
primitiven '/ustandes der Excretionsorgane hielt, hat, nachdem von

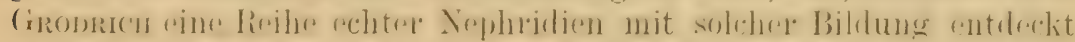

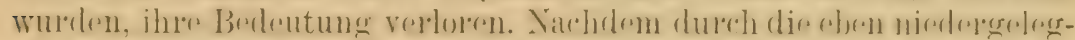

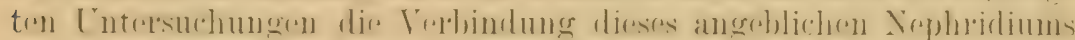
mit der Leibeshöhle nachgewiesen ist, verliert die frühere Ansicht von

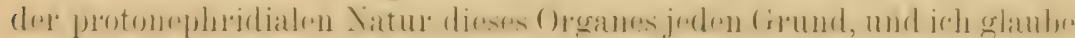
mich berechtigt, dasselhe für ein echtes Nephridium zu halten.

\section{Zur Morphologie der Cardialorgane der Appendicularien.}

Von Prof. W. SALENSKY (St. Petersluurg).

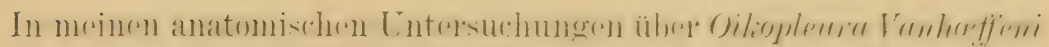
Lohm. habe ich gezeigt, dass das Herz in dem sinne, in welchem es bei den Ascidien auftritt, hier eigentlich nicht vorhanden ist, und dass das Centralorgan des Blutgefuisssystems hier in einer Form zum Vorschein

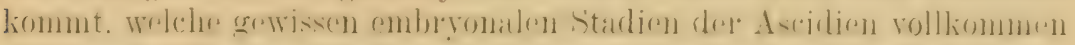
entspricht. Meine weiteren, an anderen Appendicularienarten ange-

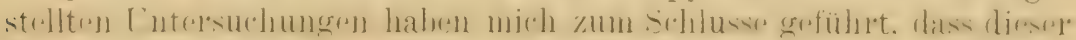
Typus der centralen Circulationsorgane bei verschiedenen Arten gewissen Modificationen unterliegt, welche ich hier hervorheben will.

Die Cardioprocardial-Organe der Oikopleura Vunhoffeni, wie ich diese Vertreter des Herzens genannt habe, stellen zwei grosse, nach hinten

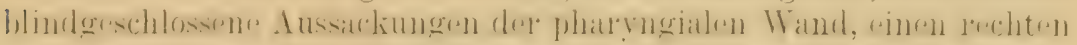
und einen linken dar, die sich aus einer gemeinschaftlichen unparen

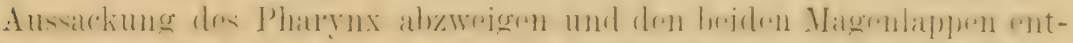

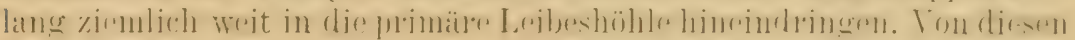
beiden Säcken, die ich nach der Analogie mit den entsprechenden Or-

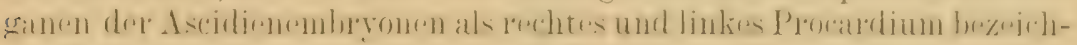
net habe, spielt nur das letzte die Rolle eines Herzens, während das 
erste, resp. rechte Procardium der Wand des Proctodeums von der Bauchseite anliegt und dieselbe teilweise umhüllt. Das linke Procardium ist mit Muskelfaser'n versehen, welche uur auf seiner linken und dorsalen Wand sich entwickeln, während die rechte und ventrale Wand keine Muskelfasern enthält. Deswegen habe ich die linke Wand als Homolog des eigentlichen Herzens, die rechte als Homolog des Pericardiums, betrachtet.

Bei ()itopleura rufescens Fol sind die beiden Procardia, obwohl in einer stark modificierten Form, vorhanden. Dats rechte Procardium ist stark roduziert; sein distaler, auch bei O. T'anhoffeni stark crweitertel' Teil bleibt allein vorhanden; der proximale ist verschwunden. Deswegen hat das rechte Procardium seine Verbindung mit der Pharynxhöhle verloren. Jas linke Procardium ist ebenfalls gegen die Pharynxhöhle abgeschlossen und stellt einen ganz geschlossenen Sack dar, welcher an der Wand des linken Kiemenganges befestigt ist. Sonst ist der Bau desselben demjenigen vom $O$. Vanhoffeni vollkommen ähnlich.

Oitiopleura cophocerea Gghr. und O. albicans Leuck. stimmen im Bau ihrer procardialen Säcke mit $O$. rufescens vollkommen überein.

Oikopleura dioica Fol stellt eine weitere Reduktion der beiden Procardialsäclie dar. Tas rechte Procardium ist bei dieser Art volliommen verschwunden; das linke hat seine Höhle nur in einem kleinen distalen Stück heibehalten; sonst erscheint dieselbe in Form einer aus zwei Zellenschichten bestehenden Platte, dio sich an der Magenwand zwischen den beiden Lappen desselben befestigt.

Wenn wir nun zur Familie der Fritillaridon übergehen, so treften wir noch oine stärliere Reduktion der Procardialsäcke an. Bei den zwei Arten der Gattung Fritilluia, dir ich zu untessuchen Gelegenheit hatte, ist das rechte Procardium volliommen verschwunden. Ilas linke Procardium verwandelt sich in ein Helz, welches hei den besden Arten verschiedene Entwickilungsstufen repräsentiert.

Das Herz von Fritillaria pellucida Vogt, stellt einen weiten quergelagerten Sack dar. Wolcher mittelst eines kur\%en Rohres an der Wand des Pharynx befestigt ist. IDe Verteilung der Muskelfasern, resp. Muskelzellen auf der hintern Wand desselben stimmt mit der des Procardiums der Oikopleuriden vollknmmen überein. I)eswegen fühle ich mich berechtigt, ihn für das Homolog des Procardiums der Oikopleuriden zu halten

Das Her\% von Fritillaria borealis Lohm. ist weiter reduziert und erscheint in Form eines kleinen, zwischen dem Magen und dem Darm gelegenen rackes. I)ie Muskelzellen finden sich hier ebenfalls an der hintel'n Wand angeheftet.

Aus den eben niedergelegten anatomischen 'Tatsachen lïnnen folgende Schlüsse gezogen wel'den :

1" I) centralen ()rane des Circulationsapparates der Appendicularien 
und der Ascidien kömmen aus einer gemeinschaftlichen Urform, nämlich aus procardialen Organen abgeleitet werden.

$2^{\circ}$ Während bei den Ascidien diese Urform nur als transitorisches Entwicklungsstadium el'scheint, bleiht dieselbe bei einigen Appendicularien permanent.

$3^{\circ}$ Dic Cardioprocardial-Organe der ()ikopleuren sind am wenigsten von der' Stammform abgewichen.

$4^{\circ}$ In der Entwicklung der Cardioprocardial-Organe anderer Oitropleura-und Fritillario-Arten ist der regressive Entwicklungstang deutlich ausgeprägt. Dorsellhe äussert sich anfänglich in der lierluktion des rechten. spuiter aber auch des linken Procardiums, welch letztres seine Höhle verliert und in eine solide muskulöse Platte sich verwandelt.

Schliesslich will ich die Velmutung aussprechen, dass der Mangen des

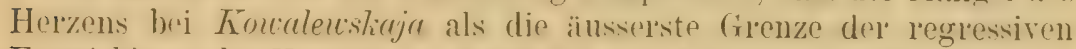
Entwicklung des Horzens in der Roilı dre Appendicularien betrachtet werden kann.

\section{Deux parasites cœlomiques d'Annélides. 1. Pelmatosphæra polycirri. 2. Sphæractinomyxon stolci.}

Par les prof. M. CaUlderi et F. MESNit, (Paris).

Mi. Caullery et Meswir apportent au Congrès des préparations de parasites qu'ils ont décrits récemment: 1. Pelmatosphæra polycirri C. et M. (In : C. R. Sne. Biol., Paris, t. 56, Janvier 1904), parasite d'un Térebellien Polycirrus himutodes Clap. ("est une forme voisine des Onthonectides, formant, dans la carité générale de l'hóte, des spheres nombreuses, dams lesquelles se développent, aux dépens d une masse plurinucléép. une

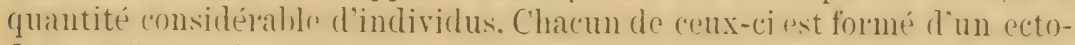
derme cilié et d'une file axiale interne de cellules; mais, à la différence des ()rthometidns. celles-ci ne sont pas dittélenciées en élóments sexurls.

2. Sphæractinomyxon stolci C. et M. (In : C. R. Soc. Biol., Paris, t. 56. Mar's lo().4), premier leprésentant marin du groupe des Actinomyxidies.

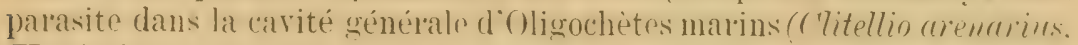
Hemitubifex beredii). Les préparations montrent en particulier' les divers stades du dévelopurement des lipstes de cos formes qui se rattarhont aux Myxosporidies. 


\title{
Le protozoaire du bouton d'Orient.
}

\author{
Par le Prof. F. MESNIL (Paris).
}

Démonstration d'une préparation du Protozoaire du bouton d'Orient. L'auteur attire l'attention sur la structure très particulière du Protozoaire découvert récemment par J.-H. Wright dans le bouton d'Orient et qu'il a nommé Helcosoma tropicum. C"est une petite masse oroüde de $4 \mu$ sul 3 u avec deux masses chromatiques, une grosse et une petite. Ces masses rappellent de très près le noyau et le centrosome d'un Trypanosoma.

Le Protozoaire de Wright est très voisin du Piroplasma donovani Laveran et Mesnil, agent d'une fièrre irréguliere de l'Inde et du Kiala-azar d'Assam.

La préparation exposée est un frottis coloré par la méthode de LAvERAx; il provient d'un bouton de 4 mois contracté à Alep même (voir : Mesnil, M. Nicolde et Remlinger, C. R. Soc. Biologie, Paris, t. 57, 23 juillet 1904).

\section{Evolution des Hématozoaires de l'Athene noctua, d'après F. Schaudinn. Recherches expérimentales.}

Par les Drs Edmond et Etienne SERGENT (Paris).

Les recherches de Schaudinn ${ }^{1}$, parues en janvier derniel (1904), sur le cycle évolutif de certains Hématozoaires d'un Oiseau rapace nocturne, la Cheviche, Athene noctur, ont 'u un retentissement consilerable, mérité par l'importance capitale des faits tout à fait imprévus, signalés par l'éminent protistologiste.

D'après lui, les deux Hématozoaires endoglobulaires de la Chouette commus sous les noms de Halteridium noctua' C'olli et San Folice ot Hitmamoba ziemami Laveran, évoluent chez le Moustique commun (Culex pipiens) ot s'y transforment en verpitables Trypanosomess, le point de départ de cette évolution étant l'ookinète, c'est-à-dir'e le résultat de la féconda-

${ }^{1}$ F. Schaddinn. Generations- und Wirtuechsel bei Trypanosoma und Spirochate. Arb. a. d. kaiseri. Gesundheitsamte, t. XX, p. 387. 1904. 
tion d'un macrogamète par un microgamète. Les Trypanosomes se multipliant chez le Moustique, y déterminent une infection, une véritable trypanosomiase. C'est sous la forme 'Trypanosome que l'un et l'autre des Hématozoaires dont il est question, sont réinoculés à l'Oiscau. Ils y passent par toute une série de formes alternativement trypanosomiques et endoglobulaires; finalement on a les formes sexuées endoglobulaires mâles et femelles, seules connues jusqu'alor's. Ajoutons qu'un des deux Hématozoaires, l'Hrmamoba ziemami, donnerait naissance à des Trypanosomes très fins, de structure identique aux Spirochètes les plus typiques (jusqu'ici classés par tous les auteurs parmi les Bactériacées).

La découverte de Schaudinn a été accueillic avec admiration, mais aussi avec étonnement. Etant donné d'une part son importance capitale au point de vue morphologique, d'autre part les conséquences que l'auteur lui-même en tire au sujet du paludisme, des piroplasmoses, des spirilloses et en même temps de la fièvre jaune, et tout naturellement aussi des trypanosomiases, nous avons eu le désir d'apporter notre contribution personnelle à la question et nous avons pensé que la meilleure manière de l'aborder était de chercher à reproduire les observations mêmes de Schaudinn, d'autant plus que le long séjour que nous faisions cette année en Algérie nous en fournissait les moyens.

Nous nous sommes donc préoccupés, dès notre arrivée en Algérie, de nous procurer des Athene noctua. Grâce à l'appui officiel de M. le gouverneur général d'Algérie, nous avons pu obteniı', par l'intermédiaire des administrateurs des cominumes mixtes, assez rapidement, une quinzaine de Chouettes vivantes; 2 ou 3 seulement étaient infectées par l'Halteridium noctuæ, une seule présentait une infection à la foùs à $H$. noctux et à $H x m-$ amoba ziemami. Nous avons eu aussi quelques Effiraies (Strix flammert) ainsi qu'un Petit-Duc (Scops giu) parasités par' Halteridium noctuae.

Le sang de la Chevêche ì infection mixte renfermait de très nombreux Halteridium noctux, des Hæmamoba ziemanni non l'ares et de très rares Trypanosomes. Une première remarque que nous avons faite est la grande

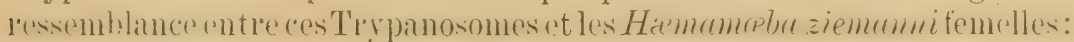
même structure du protoplasme, très cyanophile dans les deux cas; présence chez l' $H$. ziemanni de 2 masses accolées, l'une de 3 à $4 \mu$ de diamètree, colorée en lilas assez pâle, l'autre de $1 \mu$ de diamètr'e, violet foncét. Ces deux masses rappellent tout à fait le noyau et le centrosome (ou blépharoplaste) de la forme Trypanosome.

Jusqu'ici nous n'avons pu suivre l'évolution de l'Hrmamoeba ziemanni chez le Culex pipiens ${ }^{2}$.

1 Il est bien entendu que nous parlons toujours de préparations colorées par.l'éosinate de bleu de méthylène (méthode de Ronanovskx modifiée ou méthode de LAVERAN).

2 Depuis le Congrès de Berne, nous avons eu l'occasion de faire piquer d'autres

VIo CoNGR. INT. ZOOL., 1904. 
Examinons ce que devient le sang de l'Oisean infecté par Halteridium noctuæ quand il est sucé par des Culex pipiens. Pour cela, il faut sacrifice les Cule les jours qui suivent le repas de sang parasité. Si on les sacrifie 36 ou 48 heures après, on constate que le quart environ (exactement 14 sur 50) des Culex mis en expérience renferment dans leur estomac les formes que nous allons décrire. Jamais nous n'avons constaté l'existence de pareilles formes chez les Culex qui n'ont pas sucé de sang parasité, bien que, pour les recherches en question et aussi pour d'autres, nous en ayons disséqué des centaines.

Chez un Culex sacrifié 48 heures après la succion, on voit des ookinètes d'Halteridium noctuce en train de devenir des Trypanosomes et aussi des 'Trypanosomes de $25 \mu$ de long sur' $3 \mu$ de large, lépondant tout à fait à la description de Sorardin des Trypanosomes du type indifférent provenant de l'Halteridium noctux (v. sa fig $1 h$, p. 393); le centrosome est bien visible sur les préparations colorées.

Chez un autre Culex, sacrifié 3 jours après la succion de l'Oiseau infecté et 24 heures après la succion d'un Oiseau neuf ${ }^{2}$, on observe dans le contenu stomacal, al l'état frais, des Trypanosomes bien mobiles. Ils n'ont. pas la même façon de se mouvoil' que les Trypanosomes du sang des Mammifères, les mouvements sont beaucoup plus tortueux; après une secousse, le corpsite farfois rectiligne à travers le champ du microscope. Sur préparations colorées, le contenu stomacal montre surtout, - à côté do Trypanosomes d u tỵ pe ind if fére n t de grande taille ( $36 \mu$ de long sul lesquels le cor'ps mesure la moitié, le flagelle l'autre moitié) - de petits Trypanosomes de 14 à $15 \mu$ de long en moyenne, dont le noyau est composé de '2 partieś et qui répondent absolmuent à ce que décrit ScHatDisx comme Trypanosomes mâles. Enfin, nous avons vu de rares formes femelles, - caractérisées comme telles par la colorabilité plus forte et la présence de granulations (matières de réserve?) à l'extrémité non tlagellée -, en voie de modification nucléaire.

En somme, nous avons retrouvé ce que décrit Schaudinn pour l'Halteridium noctuce, avec cette légère différence que les Trypanosomes que

Chevêches avec nombreux $H$. ziemanni par des Culex; 36 heures après, l'un d'eux montrait, dans les tubes de MaLPIGHI, des formes spirochétiennes très minces et très mobiles de 25 à $30 \mu$ de long sur $1 \mu$ de large (voir fig. 17 de Schaudins). Un autre de ces Culex nous a montré des formes spirochètiennes possédant plusieurs couples de noyaux, manifestement en voie de division (fig. $17, d$. de Sonaudins) et aussi des circulaires, ou en haltères à noyau central, ressemblant tout à fait aux figures de Schaudins (fig. 17,g et $h$ ). Nous avons trouvé trois Culex iufectés sur 17 ayant sucé le sang d'une Chevêche

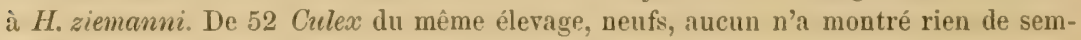
blable.

${ }^{1}$ Cette $2 \theta$ succion a été faite conformément à la recommandation de Schacdixn, pour provoquer la pullulation des formes Trypanosomes. 
nous avons vus, sont plus effilés, moins rentlés que ceux figurés par Schaudins. Etant donné, d'une part que nous avons pu suivre la transformation des ookinètes, d'autre part que les Trypanosomes existent seulement chez les Culex ayant sucé du sang à Halteridium noctux (et où de pareilles formes Trypanosomes sont absentes), il y a peuà douter de la véracité des faits avancés par Schaudins que les Halteridium de la Chouette ont des formes Trypanosomes dans leur cycle évolutif.

Ce point acquis ${ }^{1}$, nous avous provisoirement laissé de côté l'étude morbhologique de ces transformations, si magistralement ot si complitement faite par Sonaudinn et nous nous sommes attachés à léaliser, en partant des Culex infectés, des infections de Chevêches neuves.

Nous nous sommes servis de jeunes Chevêches âgées de 2 à 3 mois et qui étaient depuis un mois enviı'on en notre possession. Nous les conservions sous moustiquaire pour éviter toute infection accidentelle. Leur sang, examiné avec le plus grand soin à plusieurs reprises, n'a jamais montré trace d'Hématozoaire. Nous croyons done pouvoir affirmer que les Chevêchettes, qui ont servi aux expériences que nous allons résumer, étaient réellement n e uves.

Ces jeunes Chevêches ont été infectées par deux modes différ'ents: deux d'entr'e elles l'ont été par inoculation à la seringue de contenu stomacal (renfermant de nombreux Trypanosomes) de: Culex ayant sucé 36 ou 48 heur'es auparavant du sang infecté d'Eftraic; une $3^{\circ}$ Chevêche a été infectée par les piqûres de Culex ayant sucé un mois environ auparavant le sang parasité d'une Etfiraie.

Dans le premier cas, voici comment nous opérions.

Les Culex étaient disséqués sur une lame stérile; leur contenu stomacal, dilué dans de l'eau physiologique citratée stérile, était l'ecouvert d'une lamelle également stérile et examiné au microscope. Si des 'Trypanosomes nombreux étaient présents, le contenu stomacal était recueilli dans un verre de montre stérile, encor'e dilué d'eau citratée, et inoculé à la seringue à une des Chevêchettes neuves.

Une première Chevêche a été inoculée le 16 juillet 1904 avec le contenu stomacal d'un Culex ayant sucé le sang d'une Effiraie le 14 juillet. Les 19 et 24 juillet, l'examen du sang est négatif. Le 27 juillet, on observe des prtites formes endoglobulaires sams pigment de 1 " environ de diametre. Le 30 juillet, les formes ont atteint un diamètre de $3 \mu$ environ, elles

1 Les faits qui précèdent ont étẻ résumés brièvement, en notre nom, par M. MESNIL, à la séance de la Société de Biologie du 23 juillet 1904, à lasuited'une communication de M. Billet sur les rapports possibles entre les Hémogrégarines de la Rana esculenta et la Tryponosoma inopinatum Sergent (voir Comptes Rendus,t. LVII, p. 164). Depuis lors, M. Bullet a signalé (C. R. Acad. Sciences, séance du 10 octobre 1901) de nouveaux faits en faveur de sa manière de voir. 
renferment du pigment et sont parfaitement reconnaissables pour de jeunes Halteridium noctuæ; elles ont continué à croître.

Une deuxième Chevêche a été inoculée le 24 juillet avec les contenus stomacaux de deux Culex disséqués 36 heures après piqûre. Dès le 27 juillet. on olsereve des petites formes endoglobulaires sans pigment de $1 \mu$ environ de diamètre. Le 30 juillet, les parasites ont 3 à $4 \mu$ de diamètre et du pigment; ce sont de jeunes Halteridium noctuæ.

Une autre Chevêche inoculée, dans des conditions identiques à celles de la précédente, avec trois contenus stomacaux, n'a rien contracté.

La scule Chevêche infectée par piqûre de Culex l'a été dans les conditions suivantes. Les Culex en question, au nombre de six, avaient sucé du sang d'Effraie le 19 juin ; depuis, ils avaient été nourris sur Canaris (indemnes d'Hématozoaires) les 24, 27 juin et 14 juillet; ils ont piqué la jeune Chevêche les $16,17,19$ et 22 juillet. Le 24 juillet, nous avons constaté la présence de jeunes Halteridium de 3,75 $\mu$ de diamètre; le 25, quelques formes libres non thagellees sont vues; depuis, les Halteridium ont grandi, devenant de mieux en mieux caractérisés.

Deux autres Chevêchettes, piquées par les mêmes Culex ou par des $C u$ lex " préparés » de la même façon, n’ont rien contracté.

En résumé, trois jeunes Chevêches, à la suite de l'inoculation, - par une aiguille de seringue ou par une trompe de Culex, - d'un matériel où l'analyse microscopique ne décèle que des formes 'Trypanosomes, ont montré dans leur sang, après une incubation de trois à onze jours, une infection à Halteridium. Nous n'avons pas observé de formes Trypanosomes dans le sang de ces jeunes Chevêches infectées expérimentalement '.

'Tous les faits que nous venons d'exposel nous paraissent une confirmation, partielle tout an moins, re ceux avancés par Schaudinn dans son mémorable travail.

Nous nous permettons d'appelel' l'attention d'une façon particulière sur ceux de ces faits l'elatifs à l'infection expérimentale des Athene noctua par Halteridium noctuæ, en passant par les Culex pipiens. Nos expériences nous paraissent rigoureuses et toute considération d'alternance de genélations entere Trypanosomes ot Hématozoaires endoglobulaires mise à part, elles prouvent d'une façon incontestable, nous semble-t-il, qu'une espèce au moins d'Halteridium a pour second hôte un Culicide.

\footnotetext{
1 Nous tenons à bien faire remarquer qu'il n'y a pas, sur ce point particulier, discordance arec les résultits de Schaddiss qui dit que, quelquefois, on trouve des I'rypanosomes dans le sang périphérique la nuit, (nous n’en avons pas trouvé), mais que lia où on les trouve régulièrement la nuit, c'est dans les organes internes. Or, poux diverses raisons, nous n'avons pas sacrifié nos animaux.
} 


\section{Note sur la Dolchinie.}

Par le Prof. A. Kono'TEFF (Kieli).

La Dolchinie ressemble à un cordon gélatineur et transparent, couvert d'ritres salpiformes, fixés a l'aide de pédoncules yui se détachent assez fitcilement de la tige et mènent une existence libre.

J'ai déjà décrit la Dolchinie ${ }^{2}$ et montré qu'elle était composée d'un tube colonial portant des zooides disposés assez rénulierement des deux cotés d'un sillon qui parcourt la tige dans toute la longueur de sa partie supérieure et où se trouvent les plus jeunes zooïdes. Mais à mesure de leur éloignement du sillon, lấge et la grandeur des zooüles augmentent; les plus adultes sont les plus éloignés.

Mon étonnement a été grand en constatant que quelques tronçons d'une Dolchinie que j’ai trouvée deruierement it Japles posiélaient des zooïdes différents de ceux que j'avais observés plécédemment, et d'une taille plus grande, grâce à laquelle ils s'élevaient au-dessus du niveau commun. Un examen attentif a prouvé que, morphologiquement, nous avions affaire à des êtres tout différents des zooïdes ordinaires de la Dolchinie, ayant une ressemblance très marquée avec la for'me latérale du Dotiolum.

La forme en question atteint une longueur de $\mathrm{S}^{\mathrm{mm}}$ et présente l'aspect l'extérieur d'une cuillère, dont les bor'ds correspondent à l'orifice du pharynx; ces bords ne sont pas lobés comme chez le Doliolum denticulatum. La cavité du pharynx communique avec l'extérieur par des fentes branchiales, lesquelles sont très nombreuses chez la Dolchinie, ce qui n'est pas le cas chez le Doliohum. Ce dernier n'a que 12 fentes, tandis que la Dolchinie en possède 40 à 42 ; les premières sont des ouvertures larges, les secondes de vraies fentes étroites et longues. Le troisième trait caractéristique des formes latérales do la Dolchinie consiste dans l'alsencer des prolongements tactiles qui se trouvent ordinairement sur la face dorsale de l'animal. Le rôle des formes latérales de la Dolchinie, ainsi que du Doliolum est plutot respilatoire que nutritif, la nutrition pouvant

1 La Dolchinia mirabilis (noureau Tunicier). Aitth. Z. Stat., Neapel, 10. Bd. 1891. 
être produite par toutes les formes du tronçon de la Dolchinie ou de la queue du Doliolum et non pas seulement par les formes latérales.

Parmi les nombreux tronçons que j'ai obtenus à Naples, il n'y en avait qu' un qui purtait les formes laterales. tandis que les autres ne possédaient que des zooïdes déjà connus, et même ces formes ne se trouvaient qu'à un seul bout de ces tronçons, où elles étaient disposées d'une façon irrégulier. mais toujours plus loin du sillon ventral, parmi les zooüdes jeunes.

Le tube colonial que j'ai décrit dans mon premier mémoire avait un sillon insignifiant, qui servait comme point de clépart aux bourgeons amhulants. ()uant aux ichantillons que j'ai examinés dernièrement à Naples,

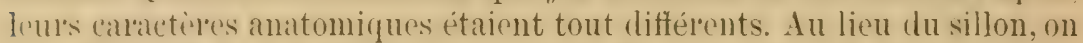
voyait plutit une gonttiere assez profonde qui contenait des bourgeons en profusion, la remplissant et s'entassant les uns sur les autres. Le mouvement de ces bourgeons se produisait à l'aide de cellules spéciales dont l'existrince et le rôle ont rété démontrés par Barzors et moi. Daus une communication préliminaine, j'ai mentionné ces cellules sous le nom de phorocytes (collules-porteul's). Mes dernières recherches mont démontré yue lo caratere des cellules, qui prennent part à la biologir des bourgeons, est bien spécifié. Les phorocytes ne servent qu'à les transporter' mais diutres éments haptocytes) servent à les fixer dans un endroit détrelminé. Mais avant cela, nous sommes déjà en état d'observer le fait suivant. Ir'secellules de lépithélium du tube colonial commencent à se multiplior lappidement ot produisent un amas de petites cellules dim caractère embryonnaire; cet amas présente un sol fertile qui attire les cellules fixatrices. Ces dernières s'entassent et attendent le bourgeon qui est traîné par les phorocytes et dès que celui-ci arrive, ils le fixent. Quand le bourgeon est fixé, on voit les cellules tixatrices se disposer tont autour (en cercles) et la quantité de ces cellules augmente selon la grandeur du bourgeon. Cette manière de se fixer est provisoire, car les bourgeons adultes (zooïles) se détachent à l'aide des mouvements de leur's muscles, ce qui ne selait pas le cas si les bourgeons étaient fixés par toute leur base.

Il n'a pas été difficile de voir que le tronçon de la Dolchinie qui possédait des formes latérales avait aussi la plus large gouttière et la plus Gramer yuantite de bourgeons grimpants. Je me suis déja exprime de la manière suivante sur la provenance de ces bourgeons: " ils doivent certainement leur origine à une source étrangère et le tube ne les porte que pendant la durér de leur dévoloppement. En nous rapportant a l'analogie que présente la Dolchinie avec l'Anchinie et le Doliolum nous devons supposer que la forme agame-mère de notre animal possède un stolon prolifère dont les bourgeons se détachent pour aller se planter le long de la queue. „) Il est évident, d'après cette description, que les tronçons

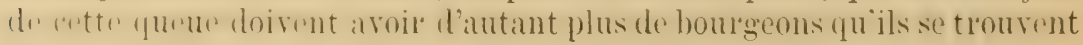
plus r’applochés de la for'me agame-mère. Par conséquent, les tronçons 
que j’ai examinés la première fois étaient très éloignés, et ceux de cette

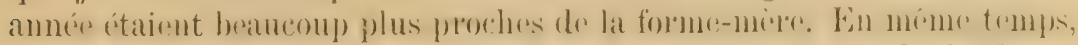
la quantité de ces bourgeons coüncidait avec la présence de la forme

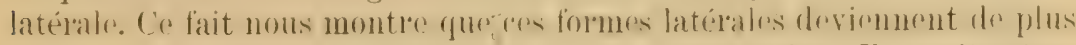
en plus nombreuses en s'approchant de la for'me-mère. Il serait même

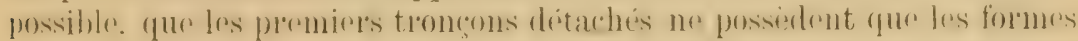
latérales et qu'avec l'éloignement, celles-ci deviennent de plus en plus rares pour disparaitre complètement et être remplacées par de simples

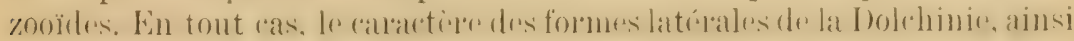
que leur disposition, est déjà un fait assez important pour le désigner comme caractère spécifique d'un nouveau genre.

\title{
Sur la structure intime du tube digestif d'Aulastoma gulo Moq.-Tand.
}

\author{
Par le I)r C..SPIESS (Genève).
}

Avec 1 planche.

Outre l'intérôt qui s'attache à l'étude de la digestion, qui présente une grande importance au point de vue de la biologie générale, le groupe des

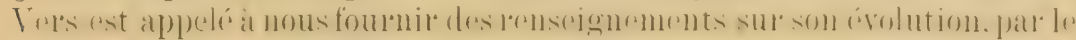
fait que chez eux, dans l'absence en général d'organes annexes du tube digestif, la division clu travail physiologique est encore peu avancée.

Nous savons que primitivement, dans les premières phases de son réveloppement ontogénétique, l'appareil digestif apparaît sous la forme d'un tube, s'étendant en ligne droite de la bouche à l'anus. Ce n'est que secondairement (à partir de la $3^{\text {me }}$ semaine), par un effet d'accroissement en longueur, qu'apparaissent les anses intestinales ainsi que les circon-

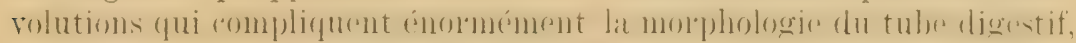
et qui sont une conséquence de la différenciation histologique et fonctionnelle du tractus.

Nos connaissances sur la phylogénie de l'appareil digestif laissent encore beaucoup à désirer, aussi avons-nous entrepris chez les Vers, et particulièrement chez les Hirudinées $(10,13)$ des recherches à la fois mol-

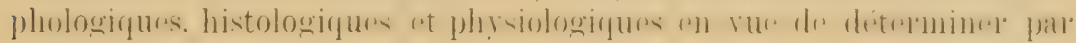


quels moyens ces animaux digèrent, et qui nous conduiront à quelques conclusions génél'ales sur l'évolution de la fonction digestive.

L'état de simplicité, qui demeure pendant toute la vie caractéristique du tube digestif dles Hirudinées. est tout à fait comparable à celui que présente le tube digestif des Vertébrés supérieur's dans les prewières phases de son développement ontogénétique, c'est-à-dire à l'état embryonnaire.

Lianatomie comparée du tube digestif des Hirudinées nous montre en effet que chez ces animaux, l'appareil de la digestion présente les caractères qui distinguent celui d'un embryon de Vertébré, avant la $3^{\text {me }}$ semaine.

Il s'étend en ligne droite de la bouche à l'anus, et ne présente chez ce dernicr. aucune diffírenciation morphologique bien accentuée, (le renHement stomacial ne posiede pas de glandes). A ce stade de son évolution phỵlogénétique et ontogénétique le tube digestif ne présente en outre pas dorganes annoxes, c'est-ì-dive de glandes digestives différencices (hépato-pancréas, pancréas). A côté de cet état de simplicité, qui est la régle générale du tube digestif des Hirudinées, ce dernier peut présentel de grandes modifications sous l’influence du régime alimentaire (12).

Il peut se modifier à la fois dans sa forme, sa structure et ses fonctions, en sorte que nous pouvons avoir des types tout à fait différents (Hivudo, Nephelis).

En passant en revue la structure intime du tube digestif de l'Aulastome, qui occupe une situation intermédiaire entre celui d'Hirudo et de Nephelis, nous verrons que l'intérêt qui s'attache à l'étude de ses différenciations épithéliales résicle non seulement dans leur degré dr complication dî au régime alimentaire, mais encore dans la présence de glandes, qui offrent un intérêt au point de vue de l'évolution phylogénétique des for:nations glandulaires de la muqueuse intestinale.

Les modifications ${ }^{1}$ que présente le tube digestif des Hirudinées (formation de lobes, etc.) doivent êtr'e envisagées comme autant d'adaptations à des conditions biologigues particulieres: elles sont la conséquence d'une differenciation fonctionnelle, particuliere des différentes parties du tractus; elles nous fournissent un certain nombre de faits positifs, qui semblent justifier cette parole de WoLfF: "La fonction est le seul élément qui engendre la forme. ")

Le tube digestif d'Aulastoma ne présente pas de complications mor-

${ }^{1}$ Én soumettant la Sangsue médicinale à une inanition de plus d'une année, c'estì-dire en la plaçant dans des conditions telles que son tube digestif ne fonctionne pas comme réservoir de nourriture, nous avons obtenu une notable réduction des poches stomacales, et l'acheminement vers le type que réalise le tube digestif des Hirudinés voraces, qui ne possèdent pas d'estomac-réservoir. 


\section{EXPLICATION DE IAA PIANCHE}

Fig. 1. Fragment d'une coupe transversale, passant par la muqueuse de la portion cardiaque de l'extomac. Fixation : sublimé acétique. Double coloration à l'éosine et à l'hématoxyline. $\times \mathbf{5 2 0}$.

ep, cellules épithéliales prismatiques; cc, couche conjonctive; $\mathrm{cm}$, cellules migratrices à l'intérieur des cellules épithéliales; cp, cellule de l'endothélium coelomique péritonéal; fml, fibre musculaire à direction longitudinale; fme, fibre à direction circulaire; vs, vaisseau sanguin; $r p$, travées du réseau cytoplasmique des cellules épithéliales, $\mathbf{N}$, leur noyau et $\mathrm{n}$, leur nucléole.

Fig. 2. Une cellule épithéliale glandulaire de la portion pylorique de l'estomac. Coloration au bleu de toluidine, apris fixation au formol-alcool. $\times 2340$.

$\mathrm{pg}$, portion glandulaire; ps, portion supérieure; re, réseau cytoplasmique; gp, granulations protéiques; ps', produit de sécrétion expulsé dans la cavité stomacale par rupture des mailles du réseau cytoplasmique.

Fig. 3. Portion d'une glande intestinale. Fixation : formol-alcool. Coloration hématoxyline au fer. $\times 23 \% 0$.

rc, réseau cỵtoplasmique; s, substance homogène remplissant les mailles du réseau; gp, granulation protéique se colorant en noir par la laque ferrique; fn, granulations nucléaires, formant une masse vivement colorée.

Fig. 4. Tube digestif d'Aulasloma gulo, d'après une préparation injectée avec de l'albumine (grandeur naturelle). A, face dorsale; B, face ventrale.

ch, cavité buccale; $œ$, œsophage, e, estomac, $c$, sa portion cardiaque; $p$, pylore; ap, appendices pyloriques; ci, cacums intestinaux; $i$, intestin; $c$, rectum; a, anus.

Fìn. ö. Une glande pylorique, à l'état de repos. Coloration à l'éosine après lixation au formol et à l'alcool. $\times 23$ '0.

$\mathrm{cg}$, corps glandulaire; $\mathrm{c}$, col; $\mathrm{ce}$, canal excréteur; $\mathrm{N}$, novau et $\mathrm{n}$, son nucléole; s, substance homogène éosinophile; ve, vacuole du cytoplasma renfermant le produit de la sécrétion, sous forme d'une substance homogène oxyphile.

Fìn. 6. Une cellule cylindro-conique de l'épithélium tapissant la muqueuse intestinale. Fixation : Formol-alcool. Coloration : éosine-hématoxyline ferrique. $\times \mathbf{2 3 4 0}$.

$\mathrm{pg}$, portion glandulaire; $\mathrm{ps}$, portion supérieure; $\mathrm{N}$, noyau et $\mathrm{n}$, son nucléole; pc, réseau du cytoplasma, dont les mailles renferment une substance homogène s, éosinophile; bb, bordure en brosse et b, ses batonnets; gb, granulations sidérophiles. 


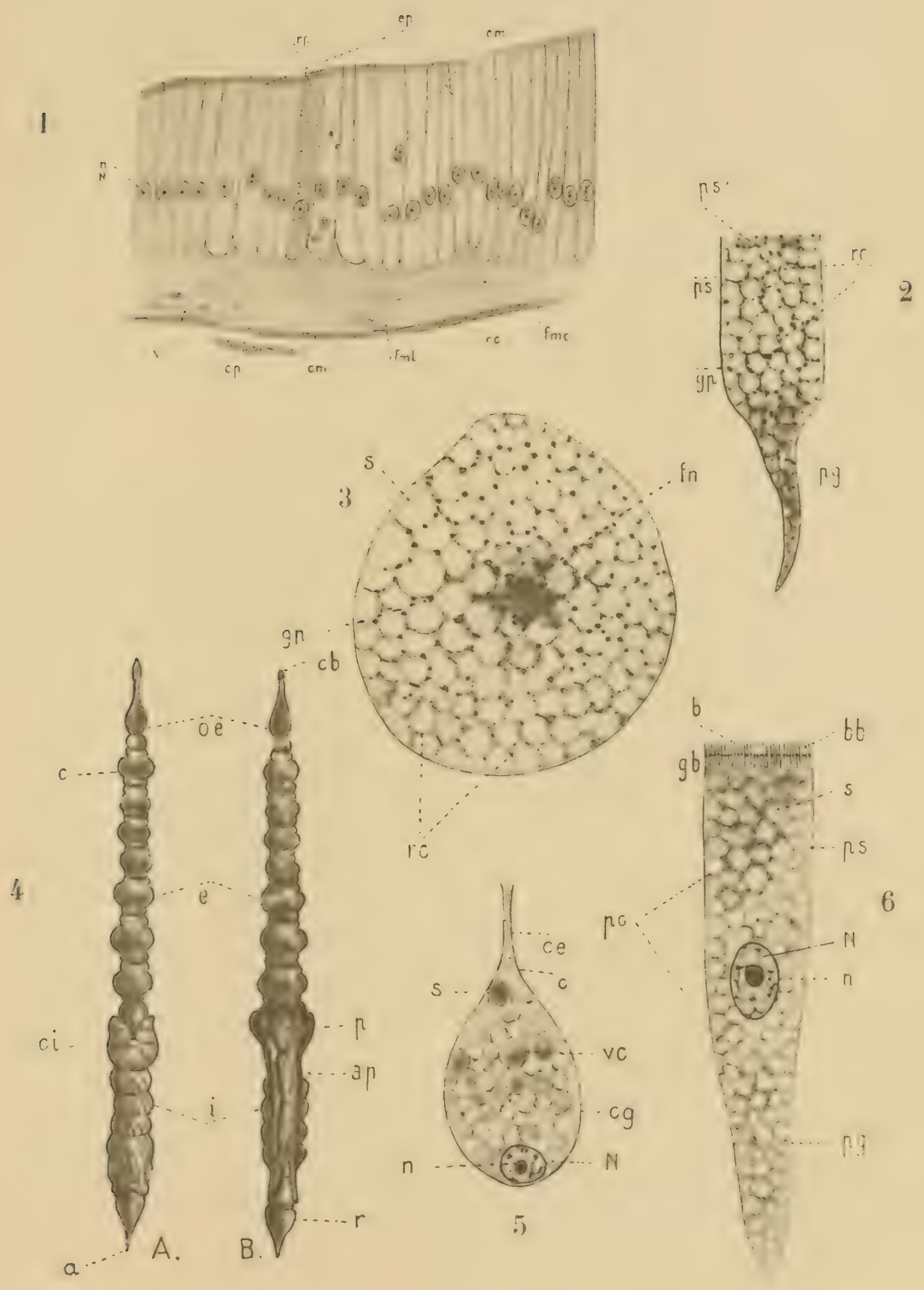

C. SPIESS 

phologiques bien importantes ot crpendant son revêtement épithélial n'est pas uniforme, mais témoigne, au contraire, d'une différenciation assez avancée de ses éléments, qui nous autorise à lui distinguer les parties suivantes:

$1^{\circ}$ Une cavité buccale (fig. 1. cb).

$2^{\circ}$ Un osophage $(\infty)$.

$3^{\circ}$ Un estomac (e), présentant 9 paires de renflements latéraux et deux longs appendices pyloriques.

$4^{\circ}$ Un intestin $(i)$, présentant dans sa portion initiale, une paire d'expansions latérales en forme de cæcum, 5 paires de renflements latéraux et une portion terminale renflée (rectum ou cloaque).

Cavité buccale. -- L'épithélium qui tapisse la cavité buccale fait suite à l'épiderme, dont il procede par invagination; il appartient au type d'ép ithélium pavimen teux simple, formé par une seule couche de cellules plates. Chaque cellule présente un cytoplasma granuleux et une membrane, qui, dans sa partie apicale, s'épaissit pour for'mer un plateau non strié.

Oesophage. - L'épithélium de la cavité buccale passe insensiblement à celui de l'œsopliage, qui appartjent au type d'épi thél ium cylindrique simple. Il est formé par des cellules prismatiques peu élevées, dont l'extrémité libre est terminée par un plateau non strié, qui se colore par le brun de Bismarck. Ajoutons que la muqueuse oesophagienne présente de nombreux plis longitudinaux, sans qu'il soit question de? glandes. L'absence de fibres musculaires radiaires dans la paroi de l'œesophage, ne lui per'met pas d'exécuter les mouvements de succion caractéristiques du pharynx de la Sangsue médicinale. Par ce fait, Aulastoma no pent utilisel le produit de sécétion de ses glandes périoesophagienues. qui, chez la Sangsue, par suite de son régime alimentaire particulier, intervient dans les phénomènes de la digestion; nous verıons que l'épithélium intestinal d'Aulastoma s'est différencié en glandes, dans le but spécial de sécrétel des ferments digestifs.

L'œesophage est la portion dı tractus où les aliments sont engloutis pour arrivel dans l'estomac. On sait que l'Aulastome peut avaler de grosses ploies, dont le passage est facilité par le large lumen oesophagien, ainsi que par les mouvements de ses parois, qui sont dus aux contractions alternatives de ses muscles longitudinaux et circulaires (constricteurs).

Chez la Sangsue médicinale, le produit de sécrétion wes glandes de la région antérieure du corps est utilisé par le fait qu il arrive directement pendant la succion, au contact du sang, dont elle se nourrit exclusivement, et qu'il imprègne, ce qui n'est pas le cas de l'Aulastome, dont l'œesophage est incapable de pomper du sang.

Nous arons lì un exemple des modifications que peut subir l'appareil digestif' sous l'intluence du r'égime alimentaire; le régime carnivore de l'A ula- 
stome a cutraîné les particularités de forme et de structure que nous ve-

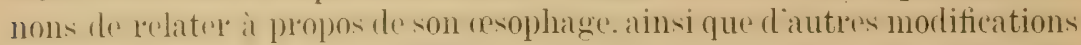
impoltanter lolatives aux différenc'iations épithéliales de son tube digestif.

Estomac. - Le revêtement épithélial de la région moyenne du tube digestif, comprise entre l'osophage et l'intestin, est distinct de celui des autres parties rlu tractus; ce fait nous autorise à admettre qu'Aulastoma possède un véritable estomac, c'est-à-dile une r'égion médiane du tube rligrestif nuttrnent calautérisée au point de rue histologigue. Nous arons montré (10) qu'on ne pouvait faire une telle distinction chez la Sangsue, lont lépithélium intestinal présente dans toutes ses parties, une répartition uniforme de ses éléments.

La muqueuse gastrique forme à elle seule la totalité de la paroi stomacale qui, sur toute son étendue, est formée de deux membranes: $1^{\circ}$ l'épithélium, $2^{\circ}$ une membrane conjonctivo-musculaire.

Nous distinguons dans l'estomac deux portions bien distinctes : $1^{\circ}$ Une portion cardiaque, qui s'étend sur la première moitié de l'estomac, recouverte par un épithélium prismatique, mais absolument dépourvue de glandes; $2^{\circ}$ une portion pylorique, qui s'étend sur la seconde moitié de l'estomac, y compris les appendices pyloriques, pourvue de glandes.

L'épithélium qui tapisse la portion cardiaque de l'estomac (fig. 1, ep), appartient au type d'épithélium cylindrique simple; il est formé par une seule couche de cellules prismatiques très élevées, mesurant $47,6 \mu$ de hauteur sur $3,5 \mu$ de largeur.

Chaque cellule est limitée par une membrane qui fait défaut à son extrémité libre, en sorte que dans cette région, le eytoplasma lui-même limite le bord libre de la cellule. Le noyau petit et globuleux oceupe le centre de la cellule et renferme un gros nucléole, facilement colorable par lhématoxyline.

Le cytoplasma des cellules de l'épithélium cardiaque est l’éduit à l'état d'un réseau (fig. 1,rp) dont les mailles sphériques présentent une grande régularité; elles renferment un produit de sécrétion sous forme d'une substance homogène. présentant les réactions microchimiques du mucus. (1) peut distingued dans les collules épithéliales deux portions distinctes, par la façon différente dont elles se comportent vis-à-vis des réactif́s : $1^{\circ}$ une portion glandulaire qui occupe la partie basale périnucléaire de la cellule et qui r'este incolor e dans l'hématoxyline; $2^{\circ}$ une portion supérieure qui se colore vivement dans les réactifs et qui expulse peu à peu le produit de la sécrétion dans la cavité stomacale. Le produit de sécrétion des cellules de l'épithélium cardiaque, après son expulsion dans la cavité stomacale, se colore en bleu par l'hématoxyline'. Un grand nombré de

2. Plusieurs auteurs considèrent l'hématoxyline comme un colorant spécifique de la mucine, qu’il colore en blen-violet. 
coupes provenant de préparations au sublimé ont éte traitées par lo lurum de Bismar'ck qui colore d'une façon intense le contenu des cellules caliciformes de l'épithélium intestinal des Vertébrés. Ce réactif colore d'une façon générale le contenu des cellules épithéliales avec une intensité très variable. Dans certaines cellules, le brun de Bismarck met en évidence de très fines granulations disséminées irrégulièrement sur les travées formant les mailles du réseau cytoplasmique; ces granulations basophiles ne se rencontrent pas à l'intérieur des mailles, qui renferment une substance parfaitement homogène. Nous les envisageons comme un produit

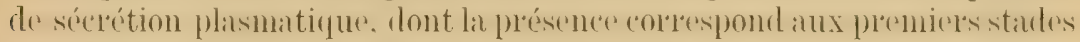
de la phase d'activité des cellules glandulaires.

L'épithélium de la portion cardiaque de l'estomac est for'mé de cellules sécrétantes, du type muqueux, que l'on peut envisager' comme autant de glandes unicellulaires, mais ayant conservé leur caractère épithélial.

La portion pylorique de l'estomac diffère de la portion cardiaque non seulement par son revêtement épithélial, mais encore par la présence de véritables glandes. L'épithélium est formé par des cellules pyramidales (fig. 2), rappelant les cellules caliciformes. Nous pouvons distinguer dans les cellules de l'épithélium pylorique une portion glandulaire ( $p g)$, qui occupe la portion basale effilée de la cellule et se colore vivement dans les réactifs, une portion supérieure qui occupe la partie apicale de la cellule. Le cytoplasma est réduit dans sa totalité, à l'état d'un réseau, dont les mailles sphériques renferment une substance homogène (oxyphile).

Le produit de sécrétion de ces cellules est formé par une substance homogène qui présente un grand nombre des réactions dont nous disposons actuellement pour révéler la présence de cellules zymo-poiétiques ${ }^{1}$.

Il partage avec le produit de sécrétion des glandes pyloriques la propriété de prendre une coloration d'un beau vert émeraude par le bleu de toluidine. On sait que c'est là une métachromasie que présentent les grains zymogènes des cellules à ferment

Les cellules épithéliales de la inuqueuse pylorique se sont diffél’enciées en glandes unicellulaires.

Ces éléments glandulaires de la muqueuse pylorique ont perclu leur caractèı épithélial; nous les envisageons comme la première ébauche des formations glandulaires que présente la muqueuse intestinale au cours de son développement phylogénétique.

Nous ne pouvons qu'appuyer les vues d'EDinger (3) qui considère que les glandes sont ontogénétiquement et phylogénétiquement des formations secondaires.

Les glandes pyloriques d'Aulastoma sont r'eprésentées par de grandes

\footnotetext{
1 Spiess. Recherches anatomiques et histologiques sur l'appareil digestif de l'Aulastome (Aulastoma gulo Moq.-Tand). Rev. Suisse de Zool., avec 2 pl., 1904.
} 


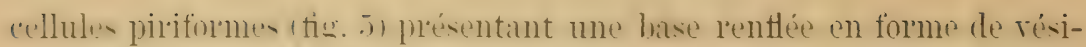
cule. La portion proximale représente le corps de la glande (cg), tandis que la portion distale forme son canal excréteur (ce); entre ces deux parties se troure une portion rétrécie, le col (c).

La membrane de la glande unicellulaire correspond à la membrana propria entourant la glande et son canal excréteur.

Levckart (6) est le seul auteur qui ait fait allusion à l'existence de glandes dans la paroi intestinale d'Aulastoma; il parle de "zahlreiche l'unllich In'ür'n-äclie , mais sans en reconnaître la réritable signification. Il les envisage comme étant des cavités formées par les plis de la paroi intestinale et r'ecouvertes par l'épithélium.

Nos observations nous ont permis de constater que les sacs glandulailes de LEuckarT ne sont autre chose que le corps de glandes unicellulaires intercalées entre les cellules épithéliales.

Le glandes prloriques di Aulastoma sont représentes par des cellules diun tyju spécial. disséminés résulièrement entre les cellules épithéliales. Le cytoplasma présente une structure alvéolaire (fig. $5, \imath c$ ), il sécretr des gouttelftes homogenes ésinophiles et que la laque ferrique noireit. Les traveses du résealu cy toplasmique présentent un grand nombre de granulations protéiques, dont la présence ou l'absence est d'une grande importance four comprendre le mécanisme de la sécrétion de ces cellules.

Les granulations noclales yui avoisinent la région périnucléaire, sont basophites: olles preventent les memes reations chromatiques que le noyau ainsj qur les diverses formations eloaktoplasmiques. Des faits analogues ont été constatés dans un grand nombre de cellules glandulaires ${ }^{1}$.

Les glandes proloiques ainsi que les collules ejpithéliales, sont dépourvues de pretrment parietal: le croplasma limite lui-meme le bord libre des cellules. Nous n'avons pas constaté la présence de cils vibratiles, admis par Bourxe $(1,2)$ dans le canal alimentaire de l'Aulastome.

Pendant leur phase d'activité, le noyau des glandes pyloriques présente d'importantes métamorphoses.

La chromatine diminue, le nombre des nucléoles augmente et le morau finit par or disoulde au sein du crtoplasma. Ces phenomenes de

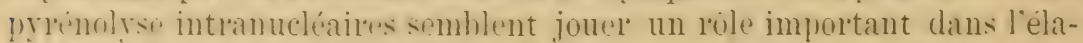
lusrition du produit desécrétion d'un grand nombre de cellules glandulaires.

L' produit l. revetion des glandes prolorques présente les réactions

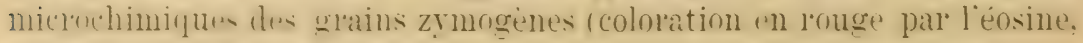
en noir par la laque ferrique).

${ }^{1}$ Lausor. Ann. Sc. Nat., Znol., (8), t. XVIII, 1903. - BrasiL. Arch. de Zool. exp. et gén., te sér., t. II, 1904. - Prexast, etc. Traité d'Histologie, t. I, Paris 1904. 
Intestin. - A partir du sphincter pylorique jusqu'au niveau du rectum. la muquense intestimal me caractériser par la présence de nombrex plis a direction oblique. formant la valvule spiroüde de GraTIOLET (4).

Le plan fondamental de structure de la muqueuse intestinale ne differe pas essentiellement de celui de la muguense prororiqur. Sur toute son étrurlue. la muqueuse intestinale présente des glandes semblables aux glandes pyloriques (fig. 3).

Lépithélium est formé par des cellules cylindrịues on plutiot cylindro-coniques de nature glandulaire dont la base est effiles ot dont l'extrémité distale est linitée par un plateau strié (fig. (j). La striation trans-

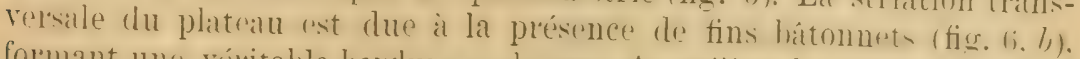
formant mor véritable borelure en brosise. Au milieu de chaqur hátonnet il existe une granulation hasilaire (gl)). que nous arons mise an évidonce par la laque ferriqur. ('es granulations siderophiles paraissent diviser le plateau en deux zones.

Le cytoplasma des cellules épithéliales est constitué par un réscau protoplasmique basplile. dont les alvéoles renferment une substance homogène oxyphile.

Le noyau ovale présente un nucléole de grande dimension.

Glandes intestinales. - Ce sont de grandes cellules en forme

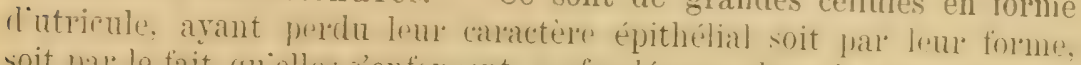
soit par le fait quelles s'enfoncent profondément dans le tisule conjonctif sous-fiphélial, jouvant oceuper toutr lépaisseur de la paroi intesti-

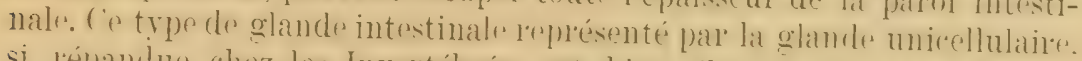
si répanilue cluez les Invertélores ret lien éloigné dlu type glandu-

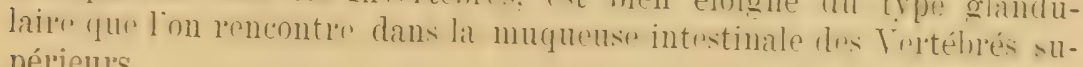
périeul's.

En outre, l'étude des glandes intestinales d'Aulastoma nous montre

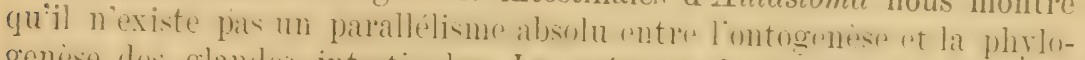
genese des glandes intestinales. La présence de glandes lans la mu-

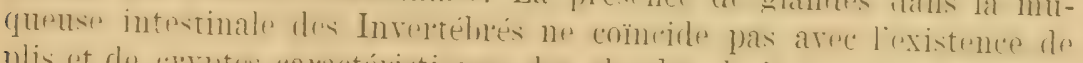
plis ot de cryptes caraletélistiques des glandes de lat muquense intrsti-

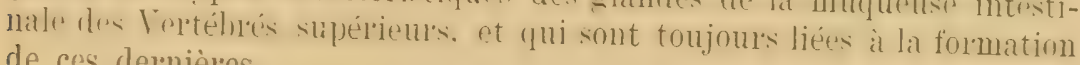
de ceśs derinières.

La formation des culs-de-sac glandulailes des glandes intestinales

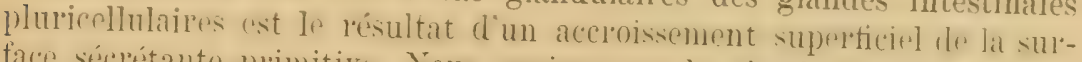
face séprétante primitive. Sous envisageons la glande inte-tinale profrement dite comme le rexultat dime différenciation morphologiqu" de lépithélium intestinal. tanclis que la glande intestinale unicellulaires

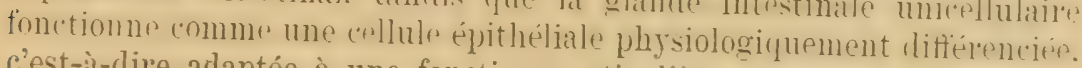
c'est-ì-clire adaptée à une fonction particulière.

Les glandes intestinalus d"Aulustomu peuvent etre envisagers anmm 
une forme de passage entre la cellule épithéliale glandulaire et la glande pluricellularie de la muquense intestinale des Vertébrés supérieul's.

\section{Conclusions.}

l" Histologiquement différencié en une portion cardiaque et pylorique, l'estomac d'Aulastoma présente un stade inter'médiaire entre celui des animaux dont l'épithélium est uniforme, comme chez la sangsue, et celui que possèdent les animaux dont la muqueuse s'est non seulement différenciée en deux régions, mais qui présentent en plus des glandes pluricellulaires.

$2^{\circ}$ La dilatation médiane du tube digestif d'un grand nombre d'Hirudinées u'a pas la même signification physiologique que l'estomac des animaux supérieur's; elle correspond cependant à une différenciation morphologique particulière et fonctionne comme un estomacrésel'voir:

$3^{\circ}$ L'étude de la structure intime du tube digestif de l'Aulastome nous donne un apereu de la phylogenese des glandes de la muqueuse intestinale.

4. Elle ćtablit lem origine épithéliale et nous montre que leur différenciation est progressive, en ce sens que la glande unicellulaire de la muquense intestinale forme le passage entre la cellule épithéliale glandulaire et lia slande pluricellulaire de la muqueuse intestinale des Vertébrés supéricur's.

$5^{\circ}$ Elle ne nous permet pas d'établir une r'olation entre les cellules épithéliales glandulaires et les glandes joroprement dites, au point de vue de la genèse de ces dernières.

$6^{\circ}$ Elle nous montre enfin que l'existence de la glande intestinale unicellulaire n'est pas liée à la formation de cryptes, caractéristiques des glandes phurcellulaires de la muqueuse intestinale des Vertébrés supérieur's.

\section{BIBI.IOGRAPHIE}

1. Bounse, A.-G. Contributions to the Anatomy of the Hirndinea. Proc. of the Roy. Soc. London, vol. XXXV, 1883.

2. - Contributions to the Anatomy of the Hirudined. Quarterly Journ. of microscopical Science, vol. XXIV, 1884.

3. Edingen, L. Ueber die Schleimhaut des Fischdarmes, nebst Bemerkungen sur Phylogenese der Dirisen des Darmrohres. Arch. f. mikrosk. Anat., Bd. XIII, p. 689, 1877. 
4. Gratiolet, P. Recherches sur l'onganisation du systeme vasculaire de la Sangsue et de l'A ulastome vorace. Ann. d. Sc. Nat., t. XIV, 1830.

כ. Johnson, J.-R. A Treatise on the medicinal Leech, including its medical and natural History, with a description of its anatomical structure. London, 1816.

6. Leuchant, R. Die Parasiten des Menschen, 2. Aufl., 1894.

7. Mogun-Tandon, A. Monographie de la famille des Hirudinées, 2me éd. avec atlas, Paris, 1846.

8. Peluetier et Huzarid. Recherches sur le genre Himdo. Journ. pham., t. XI, 182:-

9. Spiess, C. Recherches sur la structure intime du tube digestif de la Sangsue (Hirudo medicinalis). Arch. des Sc. phys. et nat., t. XIV, 1902.

10. - Recherches morphologiques, histologiques et physiologiques sur l'appareil digestif de la Sangsue (Himdo medicinalis, Lin.). Rev. Suisse de Zool. arec 3 pl., t. XI, 1903.

11. - Sur les differenciations épithéliales du tube digestif d'Homopis sunguisuga. C. R. de la Société de Biologie, t. LVI, Paris, $190 \%$.

12. - Modifications subies par l'appareil digestif sous l'influence du régime alimentaire. C. R. de l'Académie des Sciences de Paris, t. CXXXVIII, 1904.

13. - Recherches anatomiques et histologiques sur l'appareil digestif de l'Aulastome (Aulastoma gulo Hoq.-Tand.). Rev. Suisse de Zool., avec 2 pl., 1901 .

\title{
Getrennt-geschlechtliche Cestoden.
}

\author{
Von Dr O. FCHRMANN (Neuchatel).
}

L'auteur n'a pas remis le manuscrit de sa communication.

Voir: Fuhriann, O. Ein getrennt-geschlechtiger Cestode. In: Zoologische Jahrlï̈cher. Abth. für Systematik, Iid. 20, Heft 2. 1) 1:31. pl. 10.
1904. 


\title{
Sul ciclo biologico dell'Ichthyonema globiceps Rudolphi.
}

\author{
Del Prof. Fr.-Sav. HONTICELLI (Napoli).
}

Il rinvenimento di forme larvali e giovanili di Nematodi nelle Sagitte è un fatto noto da molto tempo per le osservazioni di più autori che ne hanno descritte diverse, ora parassite di specie note, ora di specie indeterminate di Sagitte. Il Busch ${ }^{1}$, fra questi, fin dal 1851, ha, con molti particolari, descritto il modo come, il nematode asessuato, da lui rinvenuto nella Spadella cephaloptera Busch ${ }^{2}$, era allogato nell'ospite: e pare egli abbia constatato esser questo nematode abbastanza frequente nelle Sagitte da lui esaminate a giudicare da quello che egli scrive che cioè esso è il nemico di questa specie (der ärgste Feind) di Sagitta; la quale finisce per soccombere per il parassita che alberga, nella maniera che nar'a il Busch. Pertanto, finora, non era riuscito ad alcuno di poter determinare qual fosse la specie alla quale appartenerano queste forme larvali e giovanili di nematodi parassiti delle Sagitte; che, appunto, per esser tali, lasciavan suppore fossero in attessa di passare in un altro ospite per compiere il loro sviluppo e raggiungere la maturità sessuale. Il caso mi ha messo in grado di risolvere la questione; ed ecco in che morlo. Nelle Sagitta bipunctata Quoy. Gaim. del Golfo di Napoli, da aprile a settembre, si osservano ora più, ora meno frequenti, o rade, delle piccole larve e forme giovanili di Nematodi: quest'anno, nel 28 scorso luglio, il Dtt. Lo Bruxco richiamava la mia attenzione su di una di queste larve, ahbastanzal grande. coloratit in grallo brumo. allogata nel mezzo del corpo dell'ospite che l'occunava tutto in larghezza, ravvolta a nodo su se stessa. Esaminando questo Nematode, con non poca sorpresa, riconobbi in riso una forma giovane dell'Ichthyoneme globiceps, scoperta per la prima volta a Napoli dal RudowpH ${ }^{3}$ nell'addome e nei genitali del Uranoscopus scaber (e poi ritrovata pure in altri pesci); della quale specie,

1 W. Buscr. Beobachtungen über Anatomie und Entwicklung einiger Wirbelloser Seethiere. Berlin, 1851.

${ }^{2}$ S. Strodtuann. Die Systematik der Chaetognathen und die geographische Verbreitung der einzelnen Arten im. Nordatlantischen Ocean. Arch. Naturg., Bd. 58, p. 362, 1892. [Sagitta cephaloptera Busch = Spadella cephaloptera Busch (Strodtman)].

${ }^{8}$ C.-A. Rudolphi. Entozoor. Synops, p. 215. (Filaria globiceps). 1819. - WilleMoes-Sunm. Zeit. WWiss. Zool., 21. Bd., p. 190, T'afel 13. 1870-71. 
appunto, molti anni or sono avevo intrapreso un studio, che poi non ho pubblicato. Questo rinvenimento stimolo la mia curiosità per spiegarmi come potessero trovarsi nelle Sagitte la larve di una specie parasita di pesci che vivono nel fondo del mare ed a piccola profonditi. Indagando e ricereando sono riuscito a ricostruire il reiclo biologico dell'Ielithyonema, dell Uranoscopus seguendo la biologia di questo, ad a spirganmi come esso possa infettarsi delle lal've di Ichthyonema, Le uova di ITronoscropus sono galleggianti ${ }^{1}$ e si rinvengono da maggio a settembre: da esse vengon fuori dei piecoli individui pelagici, che cominciano ad apparire nel plankton da luglio od ottobre ${ }^{2}$ : e precisamente, anche in questo anno, nello scorso luglio. a poechi giol’ni di distanza dalla cattural della Sergitta trovata infetta dalla larva di Irhthyonema, ne fu pescato qualehe exemplare nel Golfo di Napoli. Ciò posto, le deduzioni scorrono facili dalla coordinazione dei fatti: coincidono, dunque, la presenza di Nematodi nelle Sagitte a delle giovani larve pelagiche di Uremoscopus. ()uesto fatto lascia concludere circa un rapporto di preslatismo dei giovani Uirenoseromus pelagici sullo śagittu; che. digerite. mettono cosi in libertì il nematode in esse contenuto. perehè possa raggiungere il suo luogo di elezione nell'ospite; e, col erescere di questo, diventare anch'esso sessualmente maturo, cosi da produrre le numerose larve che riempiono il corpo delle femmine di Ichthyoneme. Larve che, partoritr dalla madre, fuorescono all'esterno, con ogni probabiliti. insieme con le nova dell'ospite a misura che queste cominciano a maturare e vengono deposte (da aprile ad agosto) : meso in liberta infestano he Sagitte. Questo si dresume lal fatto che le larve di Nematodi. nelle Sagitte, come ho detto si riscontrano, per l'appunto, a cominciare dall'aprile (a settembre), e dall'osservazione del Busch che questi Nematodi sono i peggior nemici delle Sagitta.

Non posso offirie la prova sperimentale delle mie deduzioni, la quale, in condizioni favorevoli, potrà, forse, tentarsi; come non posso asserire che le Sagitta sieno il solo ospite intermedio di Ichthyonema globiceps liud. Ma dalla cooldinazione dei fatti osselevati e dei dati messi in rilievo, eso si solgano cosi losicamente, che, dalla interpertazione di guesti. il ciclo hiologico di Ichthyoneme globiceps parmi risulti evidentrmente ricostrutto e dimostrato. Delle interescanti vicende del qual ciclo. poiche mi è stato dato di rintraceiarlo, ho cleduto non privo d"interexses farne questa breve communicazione sommaria riassuntiva.

${ }^{1}$ Fed. Raffaele. Le uova galleggiati ecc. Mitth. Z. Stat. Neapel. 8. Bd., p. 27. 1888 .

2 S. Lo Branco. Notizie biologiche ecc. Mitth. Zool. Stat. Neapel, 13. Bd, p. 572.1899 . 


\section{Il gruppo delle Temnocefale.}

Del Prol. Fr.-Sav. Moxtlceld (Napoli).

In possesso di un ricco materiale di Temmocephala, nel quale ho rinvenute interessanti nuove forme, attendo da alcuni anni ad una revisione generale di questo gruppo. Dalla quale ne risulta rinnovata la classificazione, per nuovi dati che ho potuti stabilire come criterio di una diversa ripartizione delle specie; massime per quelle dell'antico genere Temocephala, che viene smembrato in nuovi generi.

Il gruppo acquista, conseguentemente, estensione maggiole nelle forme che lo compongono e più importanza nel fatto che meglio può diseutersi la questione del posto che esso occupa fra i Platelminti.

In seguito ai fatti osservati della anatomia e di quel tanto che, dalla rmbriologia di qualche formal americana puo ricaransi. ho dovuto modificale l'opinione da me sostenuta e difesa che le 'Temnocefale fossero da considerarsi dei Trematodi, giusta il pensiero espresso pel primo dal Semper e poi seguito dall'Haswer e condiviso dagli altri autori. Ciò che, d'altronde, è giustificato dalle conoscenze che di queste forme si avevano; e gia costituiva un passo imnanzi nell'apprezzamento della loro posizione sistematica, per aver riconosciuto in questi vermi - che, pel solo fatto della ventosa posteriore. erano stati considerati dagli autori precedenti, come degli Irudinei Branchiobdellidi - dei Platelminti.

Già nella mia recente proposta di una nuova classificazione dei T'rematodi eterocotilei ho escluso, di fatto, da questi le T'emnocetale, riservandomi di discutere, in altro tempo, della loro posizione sistematica ${ }^{2}$. Ed ol'a, appunto, voglio riassumere, in maniera sommariamente sintetica, le conclusioni alle quali mi hanno condotto le mic ricerche e le comparazioni istituite per studiare l'argomento. Da queste dero dedure che le 'Jemnocefale, come, del resto, da altri già ne era stato espresso il dubbio - pur considerandole sempre dei Trematodi - costituiscono un cruppo del tutto a parte fra i Platelminti, distinto cosi dai 'Trematodi, come dai Rabdoceli e dai l'urbellari in genere; nè è possibile ammettere che essi, come alcuno vor'rebbe, rappresentino un

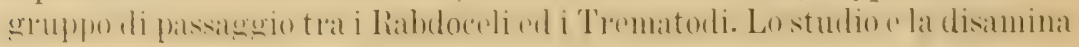

${ }^{1}$ Fr. Siv. Monticelli. Per una mova classificazione dei Trematodi. Monit. 'Z. Ital. (Rend. Convegno limini M. Z. I.), Auno 14, pag. 234. 1903. 


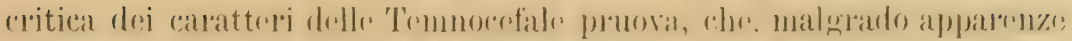

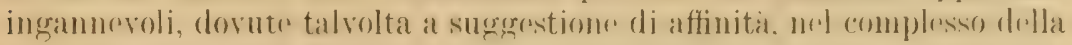
loro organizzazione e sviluppo, esse dimostrano una somma di caratte-

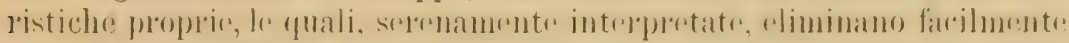

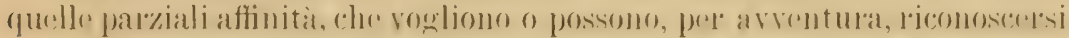
con i T'rematodi e con i Rabdoceli. Ond'è io credo debbano le 'Temnocefale considerarsi come un gruppo distinto dei Platelminti ed equivalente agli altri dei Rabdoceli, T'urbellari, 'T'rematodi, Cestodaria

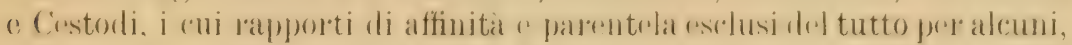
restano per lo meno molto dubbi e discutibili, per gli altri gruppi : e certo non tali da dare agomento ad indagare in qual modo il gruppo delle Temnocefale si sia differenziato nel comun tronco dei Platelminti.

Questa conclusione, di considerare le 'lemnocefale come un gruppo distinto dagli altri fra i Platelminti, vado già da qualche an no svolgendo nelle mie lezioni pubbliche, riservandomi di esporla nel lavoro di revisione delle Temnocefale al quale sono intorno. Frattanto il Bexran, nel trattare i Vermi, nella Zoologia del LANKEsTER ${ }^{1}$, di fatto, in base ad una sommaria discussione e senza un esame diretto delle for'me, crea, per le 'Temnocefale, il nuovo gruppo di Platelminti delle Temnocepholoidea; che, pertanto, come si rileverebbe (lal contesto, pare egli intenda presenti

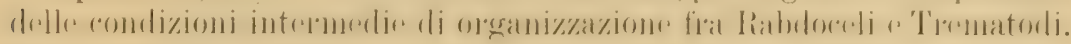
Non entro a discutere se il gruppo delle 'T'mmocefale debra costituire una classe of un ordine dei Platelminti; ciò ì questione di divel'so apprezzamento tassinomico: d'accordo col Benmar nel fatto della conclusione alla quale siamo pervenuti, per diversa via, non sono - nè posso esserlo per le cose innanzi dette, - della stessa opinione sul modo di considerare il gruppo. Questo, a mio modo di vedere, per lo studio diretto dei fatti, son condotto a ritenere non essere intermedio fra nessuno dei gruppi dei Platelminti, ma da tutti indipendente, occupando un posto isolato nel sisterna, che può integrarsi nell'ordine dei Dactyloda; nome col quale io propongo di distinguere le 'Temnocefale, il quale meglio corrisponde a ricordare il carrattere principale del gruppo - cioè l'aspetto digitato della parte anteriore del corpo delle forme che lo compongono, del nome proposto dal Bexnas; che, pel essere già usato in senso più ristretto, è pregiudicato nel caso attuale e può valcre solo pel' una delle famiglie del gruppo; quella che accoglie il recchio genere Temnocephula. 1901.

${ }^{1}$ BL. W. Bexhax. I'lathyelmia. Lankiester's Traitise of Zoology, Part. 4, pag. 43. 


\title{
Une nouvelle application de la chronophotographie: la Biotachygraphie.
}

\author{
Par le Prof. A. PIZON (Paris).
}

Arec 4 figures dans le texte.

Chacun connait le principe de la chronophotorgraphie, cette méthode à la fois si précise et si féconde qui, entre les mains de son inventeur, le savant ct regretté professeur MAREr, nous a ouvert des horizons si inprévus sur la mécanique animale. Grâce à cette méthode, des mouvements rapides tels qu'un coup d'aile d'()iseau ou d'Insecte qui nous paraissaient défier toute analyse et constituer chacun un tout, furent divisés, dissociés en phases distinctes et successives; la mécanique du cœur, du muscle, du vol, de la mal'che, etc., n'eut plus de secrets pour le savant expérimentateur qu'était le professeur Marex.

Ce fut sur ses conseils qu'il y a un an j'essayai d'appliquer la méthode chlonophotographique a l'enregistrement de l'évolution d'animaux qui pourraient s'y prèter. L'opération est généralement délicate à conduire, mais le principe en est très simple. Imaginons un embryon qui soit susceptible d'itre photographié et qui soit braqué devant un ohjectif, avec un systeme automatique qui permette de prendre des images successives a des intervalles égaux et plus ou moins rapprochés, par exemple tous les quarts d'heure ou toutes les clemi-heures suivant la rapidité de l'évolution de l'embryon. Il suffira ensuite de projeter au cinématographe la série des photographies ainsi obtenues, et l'on verra se dérouler sous les yeux les transformations successives de l'embryon.

Il ne s'agit done plus ici d'enregistrer une phase isolée, mais les changements suecessifs qui se produisent au cours de l'évolution pendant un temps donné, rhangements que l'on fait revivre ensuite sous les yeux du spectateur à l'aide du cinématographe.

Pour mes premier's essais dans cette voie, je me suis adressé à des Tuniciors coloniaux, les Botryllidés, qui forment, comme on sait, de belles ritniles multirolores sur les Algues et les rocher's. Mon choix a été déterminé par plusieurs considérations. La première, c'est que ces animaux se laksent assez facilement transplanter sur des lames de verre ou ils con- 
tinuent à vivle sans subir de déplacrnents uotahles, ce qui facilite singulièrement les opérations photographiques.

De plus, les colonies de ces T'unicier's présentent, dans une période relativement courte, par suite de phénomènes d'h istolyse et de bourgeomement, de profondes modifications morphologiques qui en rendent la lecture au cinématographe extrêmement saisissante ${ }^{1}$.

Les ascidiozoides adultes de la colonie ont. en effet, une existence assez

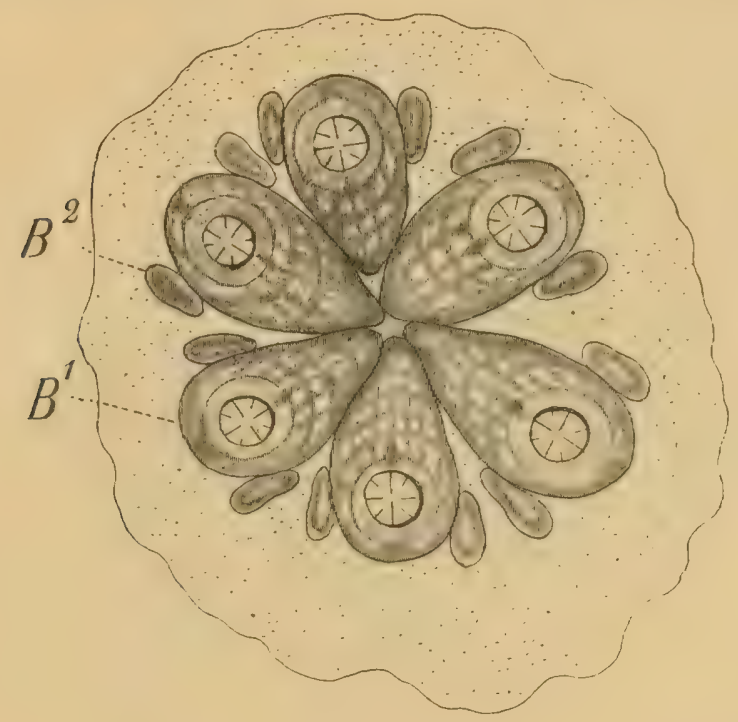

Fig. 1. Colonie de Botrylles comprenant six ascidiozoïdes adultes $B^{1}$ et douze autres $B^{2}$ qui sont en voie de développement sur les sacs branchiaux des premiers.

éphémèle: au bout de cinq ou six jours, tout au moins dans la belle saison, ils entrent en régression et disparaissent progressivement dans l'espace de deux à trois jours; pendant ce temus, d'autres ascidiozoïdes, nés par voie de hourgeonuement sur les Hanes des anciens, grandissent peu à peu en se nourrissant des tissus en histolyse de leurs parents, ot édifient peu à peu sur les carlavres de ceux-ci une nouvelle colonie étoilée qui, au bout de quelques jours, aura le mème sort que la premiere. C'est l'intérêt de ces phénomènes évolutifs et la possibilité de les photogra-

${ }^{1}$ A. Pizon. Etudes biologiques sur les Tuniciers coloniaux fixés. Bull. de la Société des Sciences naturelles de l'Ouest à Nantes, nos du 1er avril 1899 et 30 juin 1900 , avec 18 planches. 
phier qui m'ont particulièrement poussé à m'adresser aux Botrỵllidés pour ces premiers essais.

Je n’ai encore obtenu que deux pellicules cinématographiques, avee la très intelligente collaboration de M. Lucien BubL, chef des travaux à l'Institut Marny, à qui revient l'honneur de toute la partie mécanique et à qui je suis heureux d'adresser ici mes bien vifs remerciements.

La première de ces pellicules a une longueur de $15 \mathrm{~m}$. 50 et compte

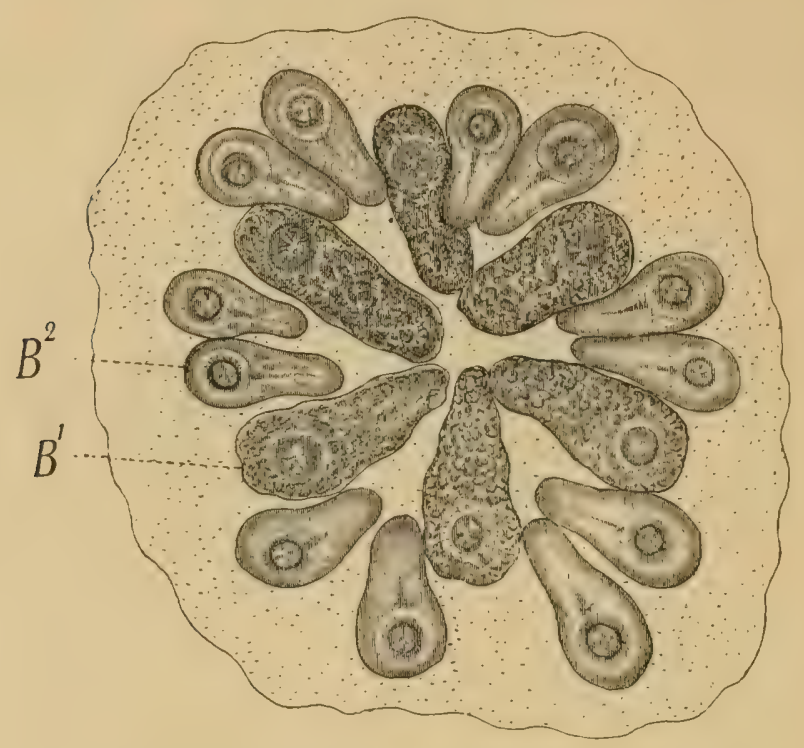

Fig. 2. La même colonie trois jours plus tard; les ascidiozoïdes $\mathrm{B}^{1}$ sont en régression et les jeunes $\mathrm{B}^{2}$ sont plus volumineux.

775 images, prises a raison de trois à l'heure. Elle représente les transformations successives d'une colonip de Botrylles pendant une période de 10 jours 18 heures.

Au point de départ, la colonie comprend six ascidiozoïdes adultes $B^{t}$ (fig. 1 ) avec douze jeunes bourgeons $B^{2}$ qui sont encore de très faibles dimensions.

Trois jours plus tard, les adultes $\mathrm{B}^{1}$ sont en régression (fig. 2), et à la périphérie se voient les douze nouveaux ascidiozoïdes $\mathrm{B}^{2}$ dont le volume a déja consirlérablement augmenté. Entre la phase représentée par la fig. 1 ut la phase représentée par la fig. 2, il a été pris 250 images successives, correspondant à une durée de trois jour's et demi. 
La fig. 3 représente la même colonie ayant encore quinze heures de plus; les ascidiozoüdes $B^{1}$ sont maintenant très réduits et ont presque donblé de volume, en mème temps qu’ils se rapprochent progressivement du centre pour se grouper en étoile. Cinquante nouvelles images ont été prises entre l'état de la fig. 2 et celui de la fig. 3.

Enfiu, dix heures plus tard encore, les premier's ascidiozoïdes $B^{1}$ ont completement disparu (fig. $t$ ) et les nouveaux individus $B^{2}$ se rappro-

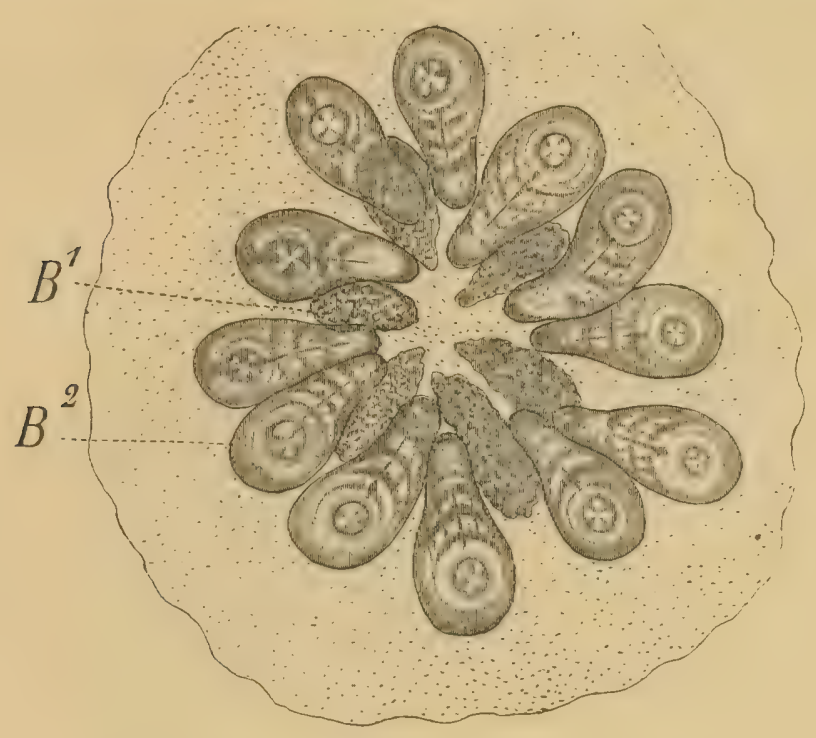

Fig. 3. La même colonie quinze heures plus tard; les ascidiozoïdes $B^{1}$ sont très réduits; les nouveaux $B^{2}$ ont grandi et se rapprochent du centre.

chent de plusen plus du centre pour se grouper suivant une étoile allongée. De la figure 4 jusqu'à l'extrémité de la pellicule, on compte 450 autres images montrant le grossissement progressif des douze ascidiozoüdes $\mathrm{B}^{2}$; sur les dernières, les individus ont atteint leur taille adulte et commencent à bourgeonner à leur tour.

Au cinématographe, cette série d'images représente l'évolution ininterrompue de la colonie pendant la période corrospondante de dix jours et demi. On voit les jeunes bourgeons $B^{2}$ (fig. 1) grandir peu à peu, les adultes $B^{1}$ contracter leur's sacs hranchiaux ot so transformer en petites masses granuleuses (tig. 2) qui diminuent prowressivement de volume (fig. :3) et finissent par disparaitre completement (tig. 4). tandis (que les 
jeunes $B^{2}$ grossissent, se rapprochent du centre et édifient une nouvelle colonie à la place des cadavres de leur's ascendants.

La seconde pellicule, plus courte que la première, ne mesure que cinq metres ot compte 20jo images. Elle présente une petite colonic composée de deux étoiles voisines, l'une de sept individus, l'autie de six. La série des intages commence al moment où ces ascirliozoüles vont entrel en dégénéresence; olle se termine quand leur répression est complete et que

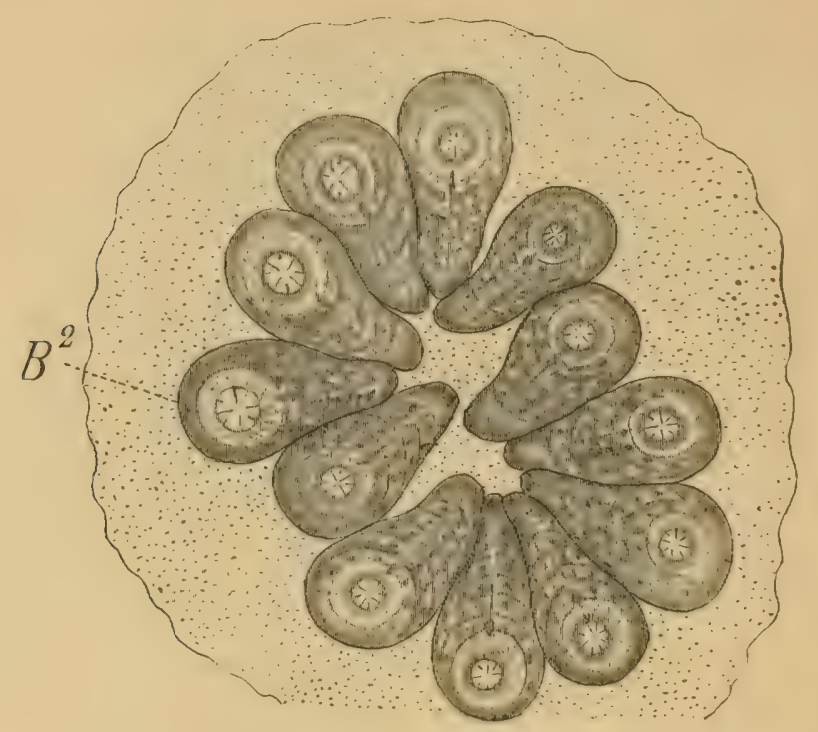

Fig. 4. La même colonie encore dix heures plus tard; les anciens individus $\mathrm{B}^{1}$ ont complètement disparu; les nouveaux $\mathrm{B}^{2}$ se rapprochent pour former une nouvelle étoile.

la première étoile est remplacée par une autre de doure individus, la seconde par une nouvelle étoile de onze individus.

Dans la série précédente, la période de la dégénérescence, qui avait duré un peu plus de deux jour's, n'était représentée que par 150 photographies environ qui, pour passer devant les yeux au cinématographe,

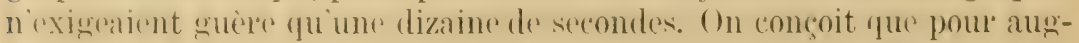
mentere la durée de cette périorle au cinématographe, il suftit de multiplier le nombre des images. Aussi, dans cette seconde série, qui a spécia-

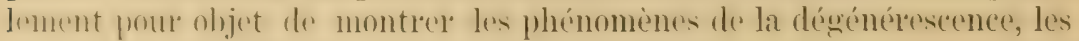
images ont-elle été prises à raison de six à l'heure, au lieu de trois. Dans 
ces conditions, les diverses transformations mettent un temps double poul" passer devant les yeux au cinématographe.

Cette série montre d'une façon vraiment saisissante les contractions des sales hamehiaux des adultes an moment de la mort, leur disparition progressive et surtout les mouvements des jeunes ascidiozoïdes qui, d'abord épars, se rapprochent peu à peu du centre et finissent par se grouper en deux étoiles.

Je terminerai en faisant remarquer' la différence que présente l'inscription de ces phénomènes avec l'inscription du vol des Oiseaux ou des Insectes qu'effectuait le professeur Marer. Celui-ci inscrivait des mouvements qui s'exécutent avec une grande rapidité et il les décomposait ensuite pour en analyser les phases successives.

Ici, au contraire, il s'agit de phénomènes qui mettent une assez longur périnde à siffecture at que le cinematographe condense sous les yeux en l'espace de quelques minutes. Les 750 images de la première pellicule représentent, comme nous l'avons vu, la vie d'une colonie pendant dix jours et demi et il suffit d'une minute environ pour les faire défiler sous les yeux par la cinématographie.

C'est donc exactement le contraire de ce que faisait le professeur Marey quand il photographiait des mourements très accélépés tols que lo vol des Insectes. Aussi me semble-t-il bon de donner une appellation spéciale à cette méthode, nouvelle en zoologie, pa ' laquelle des phénomènes de longue durée sont enregistrés, puis reproduits avec l'accélération du cinémat 0 graphe. Je l'appellerai la biotachygraphie 1.

On entrevoit, d'ores et déjà, toute une série de phénomènes qu'il sera

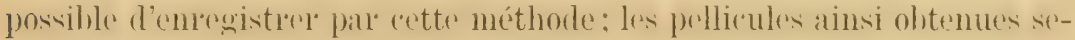
ront non seulement d'un puissant intérêt démonstratif dans les cours scientifiques ou les conférences publiques, mais dans les cas où elles représentront, par exemple, une érolution embryogénique, les inages citant prises à des intervalles réguliers, constitueront en même temps des documents précis sur l'état de l'embryon à chacun des stades de son développement.

La communication de M. le Prof. A. Przon, accompagnée de la projeetion cincimatographique des denx pellicules ci-dessus décrites, a été faite à l'Aula de l'Université.

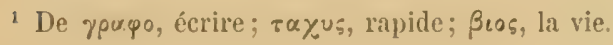




\title{
Nouvelles observations sur le mécanisme de la circulation chez les Tuniciers.
}

\author{
Par le Prof. A. PIZON (Paris).
}

Dans des travaux antérieurs, M. Pizon avait montré que le renversement de la circulation chez les Tuniciers est dû̀ à une différence de pression sanguino sur les deux extrémités opposées du tube cardiaque. En sectionnant le corps des Clavelines au-dessous de la branchie de telle sorte que tout le sang s'écoule au dehor's, on voit néanmoins le cœur. battre à vide et renverser le seus de ses contractions comme auparavant; il faut donc faire intervenir l'hérédité pour l'explication de cette persistance des contractions. Des phénomènes de même ordre, mais plus curieux "ncole, s'observent dans des stolons de Pérophores ne portant plus d'individus, chez lespuels l'auteur a ru la circulation se continuer lentement pendant douze jour's, aree ses renver'sements caractéristiques.

\section{La spermatogénèse du Lombric (L. agricola).}

\author{
Par le Prof. E. BUGNION et N. POPOFF (Lausanne).
}

Avec 5 planches.

Les quatre petits corps, décrits par Hering en 1856 sous le nom de testicules, présentent une partie sexuelle et une partie vasculaire. La partir sexuclle ronferme des cellules germinales, qui, se multipliant par divisions successives, sans se séparer les unes des autres, forment des colonies sper matiques (follicules de von Erranger 1896). La partie vasculaire est caractérisée par la présence d'un glomérule et parfois de sinus sanguins, entourés de lacunes plasmatiques. Semblables a de petits houquets pédiculés, attachés à la paroi des vaisseaux, les cellules de cette rrécion ne forment pas de spermies et semblent dévolues plutit à quelque sécrétion interne.

Les six organes volumineux considérés par Hering comme des vésicu- 
les séminales sont une deuxième forme de testicules (glands testicules) offrant une substance médullaire avec une lacune centrale et une substance corticale traversée par un système de cloisons vascularisées à direction ladiaire. Les alvéoles de la substance corticale sont remplis de colonies spermatiques (follicules) qui forment un parenchyme compact. Cette structure, très caractéristique, ne peut être observée que sur un testicule indemne de parasites (Pl. I).

Les grands testicules antérieur's ot moyens déhouchent, par leur cavité centrale, dans le carrofour séminal antérieur (mediane Samenkapsel de Bergh, 1886), espace médian, quadrilatère, détaché du colome, limité par une membrane propre, compris entre le tube digestif et la paroi ventrale, occupant la partie movenne du dixième segment, renfermant aussi les deux petits testicules et les deux pavillons antériemr.s. Les grands testicules postérieur's s'ouvrent de même dans le car'lefour séminal postérieur qui occupe la partie moyenne du onzième segment et contient aussi les deux petits testicules et les deux pavillons postérieurs.

Les jeunes Lombrics offrent d'ordinaire deux paires supplémentaires de grands testicules (parfois de glandes hermaphrodites) qui plus tard s'atrophient et disparaissent ${ }^{1}$.

Plus précoces que les grands, les petits testicules se développent en même temps et sur la même ligne que les ovaires. Ces ébauches dérivées de l'épithélium du coelome. se voient sur les coupes sériées horizontales dans un plan plus profond que celles des grands testicules (plus pres de la paroi ventrale) des deux côtés du cordon nerveux.

La spermatogénèse du Lombric peut être divisée en trois phases ${ }^{2}$ :

a. Une première phase de prolifération qui, partant de la cellule germinale, conduit à la formation du follicule. Cette prolifération a pour siege les testicules petits et grands; olle donne lieu ì des colonies (follicules) de $2,4,8,16,32,64$ parfois 128 cellules, unies par une masse protoplasmique centrale ou par de courts pédicules englobés dans cette masse.

b. Une phase de dissociation, qui, divisant le follicule en deux moitiés égales et se répétant à plusieur's leprises, donne lieu ì des groupes de $64,32,16,8$, rarement à des groupes de 4,2 , peut-être à des spermatogonies libres (?). Les groupes cellulaires résultant de la dissociation des

${ }^{1}$ Ces glandes supplémentaires ont été décrites par E. Perrier (1872) et plus récemment par M. F. WOODWard (1893).

2 Les deux premières phases correspondent à la période germinative ou préspermatogénèse (Prenant, 1904), la troisième à la période de maturation ou spermatogénèse proprement dite. 
follicules se poconnaissont à ce que leurs déments (piriformes, séparés les uns des autres) sont unis par des pédicules gréles convergeant vers le centre. (PI. III).

c. Une deuxième phase de prolifération (Segmentation), qui pro. cédant de nouveau par progression géométrique et se faisant toujours dans des plans radiaires, donne lieu en définitive à des groupes de 64, 12. - parfois 256 i éléments ${ }^{1}$ disposés symétriquement antour d'une boule protoplasuique centrale (cytophore ou blastophore). Destinés à subir les diverses phases de la spermatogénese, les groupes de ce genre sont désignés sous les noms de morules spermatiques ou spermatosp hères. Lem's éléments (spermatocytes de $1^{\text {er }}$ ordre) subissent deux divisions, après lesquelles ils se transfor'ment en spermatides.

Parfois la phase de dissociation étant raccourcie ou supprimée, le follicule se transforme directement en morule.

Les follicules, ainsi que les mor'ules, qui se préparent à la spermatogénèse se reconnaissent à un état particulier de leurs noyaux désigné sous le nom de "condensation chromatique». Les granules chromatiques précédemment dispersés sur le réseau nucléaire, se réunissent ì cee moment en une masse compacte, coloréo sur les préparations à l'hémalun en violet foncé, constituée elle-même par de petits chromosomes ar'ondis, groupés en amas. Les éléments chromatiques du cytoplasme subissent eux aussi une modification concomitante, car le col'ps cellulaire jusque-là assez oparque, de couleur violacee, devient en même temps parfaitement clair (hyalin) et offre un contour beaucoup mieux maroué. Pcutêtre y a-t-il condensation de certains éléments cytoplasmiques sur le noyau en même temps qu'à la surface de la cellule. On distingue des noyaux condensés à masse chromatique plus grande mesurant $3 \mu, 3$ (gros grain " et des noyaux condensés à masse chromatique plus petite, mesurant $2 \mu$ (petit grain). L'état condensé correspondant vaisemblablement à la prophase de la mitose, la cellule à gros grain représente,

${ }^{1}$ La numération des éléments se fait au moyen de la chambre claire. On dessine exactement les cellules et e'est sur le papier qu'on les marque et qu'on les compte. La photographie peut rendre le même service, à condition que la préparation soit assez aplatie pour que tous les éléments se trouvent dans un plan unique. BloonFIELD, qui a essayé de compter les cellules sous le microscope, n'est pas allé au delà du chiffre 16.

" La masse chromatique entière est désignée sous le nom de grain, parce que les follicules et les morules à l'état de condensation offrent dans chacune de leurs cellules un amas foncé, semblable à un grain compact. Il faut distinguer toutefois entre cette première condensation, dans laquelle le noyau, observé à un grossissement suffisant, se montre constitué par un amas de chromosomes arrondis (difficiles à compter mais encore distincts) et la condensation définitive, propre à la spermatide, dans laquelle les chromosomes sont entièrement fusionnés. 
semble-t-il, le spermatocyte de Ir ordre, au momont où il va se divisel' La cellule à petit grain répondrait au spermatocyte de II" or l'o olsservé, lui aussi, avant sa cinèse. Toutefois comme la spermatide en formation renferme une masse de nucléine plus petite encor'e, plus condensée et déjà plus allongée (ovalaire), il y aurait lieu peut-être de distinguer le gros grain appartenant au spermatocyte I, le grain moyen.(moins compact, encore arrondi), répondant au spermatocyte II et le petit grain plus dense et allongé, qui caractériscrait spécialement la spermatide. ()n trouve en effet dans la plupart des préparations de "petits grains" de grosseur's diverses et de formes plus ou moins allongées.

C'est au cours de ces divisions que se produit la réduction chromatique déjà déerite par CaLrins (1895).

Un fait qu'il importe de constater, est que les "spermatocytes I prêts à se diviser ne sont (contrairement à ce qu'on observe chez les animaux supérieurs) pas plus volumineux que les éléments des phases précédentes. Au contraire, leur taille est plutôt moindre et leur noyau plus potit. Des mesures exactes ont constamment donné pour les noyaux des spermatogonies dissociées $6.4 \mu$ et pour les spermatocytes I (cellules à gros grain $3,3 \mu$. Il y a donc une phase de prolifération, mais non pas d'accroissement de la cellule et du noyau.

Insérées sur le cytophore par un pédicule errêle, les spermatides s'observent d'ordinaire sur des morules à 128 , exceptionnellement sur des molules à 64 ou à 256 (chiffre maximum observé chez le Lombric) ${ }^{1}$.

Chaque spermatide, s'allongeant peu à peu, se transforme en une spermie qui reste quelque temps encore attachée au cytophore, puis finit par se détacher et, Hottant librement à l'intérieur du carrefour, gagne bientôt l'un des plis du pavillon.

La planche II représente les éléments du petit testicule, olservés dans Ir carrefour après la déhiscence de la glande. On remarque surtout des follicules de 16, 32 et 64 , la plupart un peu aplatis en suite de la compression qu'ils ont subie dans les al véoles, quelques-uns en voie de division.

La planche III montre le contenu du grand testicule (laitance étalée sul la lamelle) en voie de dissociation. On voit des groupes pédiculés de $16+16,8+8,4+4$, unis par leurs pédicules. Le Ver ayant été tué dans la saison froide (s (lécembre), la spermatogínèso paraît entièrement interrompue.

La planche IV, enfin. présente le contenu du grand testicule (laitance étálée) en voie de spermatogénèse avec des follicules à 16, 32, 64 et de grandes mor'ules à 256 .

${ }^{2}$ La Sangsue médicinale offre des morules plus volumineuses, avec un grand cytophore (privé de noyau) et des spermatides très déliées, probablement au nombre de 512. 
La spermie offre une forme courte mesurant $40 \mu$ et une forme longue rui en a so i sti. La forme courte procede. croyons-nous, de morules à 128 ou 256, la forme longue de mor'ules à 32 ou 64 . Observés à l'état frais, les zoospermes ne montrent que deux parties distinctes, la tête, allongée en forme de bâtonnet, plus épaisse et réfringente, le flagellum, trois fois 'nviron aussi long que la tête, mais plus grêle, pâle, parfois à peine visible. Le spermatozoaire mûr, conveuablement color'é, apres fixation par le sublimé acétique ou de préférence par le liquide de Fummor, présente quatre segments caractérisés comme suit:

$1^{\circ}$ Le perforateur, long de $4 \mu$, cylindrique, légèrement apointi, de même largeur que la têtr, distinct de celle-ci sur les préparations colorées à l'hémalun-éosine par sa teinte pâle, d'un rose lilas;

¿2o La tète. allongée en forme de bâtonnet cylindrique, d'épaisseur' uniforme, de longueur variable (16 à $30 \mu$, moyenne $20 \mu$ ) colorée en bleu violet dans l'hémalun, en bleu noir dans l'hématoxyline ferrique, en rouge dans le picrocarmin, en vert dans les préparations traitées par le bleu de toluidine et le mélange de van Gieson;

$3^{\circ}$ Le cou, très court $(2 \mu)$, de même largeur que la tête, distinct par sa teinte rose dans les préparations à l'hémalun-éosine;

$4^{0}$ Le Hagellum, beaucoup plus grêle que la tête, mesurant $50 \mu$ dans la forme longue, se colorant à peine cn lilas pâle dans l'hémalun-ciosine.

Le perforateur paraît se former d'un petit col'puscule (c. proximal ou procéphalique, acrosome) contenu dans la base du pédicule, déjà visible sur les jeunes spermatocytes. La tête procède comme toujour's du noyau de la spermatide, progressivement condensé et allongé. Le cou semble bien provenir du corpuscule juxta-nucleare distal (archoplasma), comme l'ont reconnu Calisins et von Erlanger. En traitant pal l'hématoxyline ferrique, on fait apparaître dans le segment intermédiaire 2 à 3 petits grains noirs, qui correspondent sans doute aux controsomes décrits par M ${ }^{\text {Hes }}$ K. Foot et E. C. Strebela (1902).

Le flagellum enfin apparait sous la forme d'un petit prolongement cỵtoplasmique visible à l'extrémité périphérique de la spermatide. Ce prolongement, dans lequel nous n'avons reconnu aucun filament axile (même à un grossissement de 1200), n'acquiert sa longueur définitive quà l'époque ou le noyau de la spermatide s'est lui-mème allongé et étiré.

Le cytophore, qui occupe le centre de la morule, se forme par afflux du cytoplasme des cellules spermatiques dans l'intérieur du groupe. C'est d'ordinaire dans la phase à 64 qu'il commence à se montrer. Les sphères protoplasmiques obserées apres fixation sur la lamelle. paraissent géneralement un peu déformées, à contour indécis. Sur les coupes, au contraire, après fixation dans le liquide de Gruson, le cytophore se montre exactement sphérique (diam. 30 à $36, \mu$ ) et sa surface limitée par un trait parfaitement net. Bien que le cytophore soit privé de noyau (chez le Lombric), 
on peut, semble-t-il, l'assimiler a la cellule de Yerson des Arthropodes, at la cellule basale de Mollusques ou encore au syncytium sertolien qui, chez les Vertéhrés, oceupe les interstices des élements spermatiques et sert, lui aussi, à les porter et à les nour'irir.

Outre les éléments spermatiques, les testicules grands et petits renferment des cellules éosinophiles cor'espondant aux éléments à corpuscules brunâtres décrits par Broomfraco (1880). Leur rôle est probablement nutritif. On voit, en eftet, les éléments de ce genre, détachés des cloisons testiculaires, s'attachel aux follicules en voie de développement ou s'accumuler en nombre dans les alvéoles qui les renferment. On remarque encore que les corpuscules caractéristiques (éosinophiles), suspendus dans le cytoplasme de ces cellules, diminuent peu à peu, à mesure que celles-ci approchent du terme de leurs fonctions.

La déhiscence du petit testicule se fait, à l'époque de la maturité, sur la surface de la partie sexuelle (postérieure) de la glande. Les follicules encole petits, plus ou moins aplatis, tombent dans le carrefour, subissent parfois une dissociation qui ne va guère au delà des chiffies 16 ou 8, puis proliférant de nouveau, se transforment dans le carrefour en morules spermatigues. I'our les grands testicules, la délhiscence se fait des alvéoles dans la lacune centrale et de là dans le carrefour correspondant par le hile creux de la glande. Les phénomènes de dissociation, de multiplication ef de spermatoginese. loaucoup plus actifs et importants que daus les petits testicules, se passent en partie au sein de la glande et en partie dans les carrefours.

Les coupes microscopiques pratiquées au niveau des pavillons séminaux donnent lieu à une obselvation curieuse (Pl. V). Les spermatozoaires qui se trouvent par millier's à l'intérieur sont arrangés avec une régularité si parfaite, que toutes les têtes se trouvent du côté de la paroi, alignées sur un r'ang, perpendiculairement à la surface de l'épithélium cilié, tandis que les queues réunies en faisceaux un peu courbés et inclinés, l'emplissent l'intérieur de la cavité. Cet arrangement des spermies, qui se montre dans les entonnoirs ciliés et non dans les canaux déférents, s'explique vraisemblablement par la propriété des corpuscules allongés, mobiles, connus sous le nom de $r^{\circ}$ hé 0 tax isme. (Voir à ce sujet l'intéressant mémoire du Dr Rorn, 1904).

La spermatogénèse des Invertébıés diffère de celle des animaux supérieurs en ce que la prolifération de la cellule germinale donne lieu à une colon ie spermatique mieux définie et que l'on voit plus distinctement le groupe ou faisceau de spermies qui en résulte.

Cette colonie (spermatogemme de LA Valette Santo-Gierories, spermopolyblaste de Bloomfreld) se distingue par cette particularité qu'elle 
augmente par progression géométrique et que toutes ses cellules, se divisant par cinèses simultanées, se trouvent constamment dans la même phase de développement. Le nombre des cellules atteint pour chaque colonie (avant la transformation en spermatides) un maximum qui varie entre $32,64,128,256$ et 512 chez les types observés.

L' développement ultérieur des éléments spermatiques diffè̀ dans les diverses classes : chez les Annélides, la colonie (follicule de von ErLaxcien) formant un amas mûriforme isolé et bien délimité, subit d'ordinaire une dissociation avant de se transformer. Il est rare (et meme improbablo) que cette dissociation donne lieu ì des spermatogonies libres. (on voit, en l'evanche. des follicules de 64 se diviser en deux groupes égaux $(32+32)$ unis par un pont protoplasmique, des follicules de 32 se diviser en groupes de $16+16$ et ceux-ci en groupes de $8+8$. Les groupes l’ésultant de dissociation se l'econnaissent à ce qu'ils sont formés d'éléments pédiculés, réunis par leurs pédicules. Ces groupes proliférant à nouveau par cineses simultanées ot les divisions se faisant pour chaque cellule dans une direction radiaire, on voit apparaitre les formes caractéristiques connues sous le nom de spermatosphères ou morules spermatiques, dont les éléments rayonnent autour d'un cytophore central.

La morule qui compte ordinairement 12s spermatides (maximum $25(6)$ chez lo Lombric, lorsque sa prolifération est terminée, donne lieu à un nomble correspondant de spermies.

Le hastophole est tantot une houle protoplasmique sans noyau distinct ( Lumbricus, Hirudo d'après nos observations, Branchiobrlella d'apress VorGt, 1885), tantôt une masse semée de noyaux (Clitellio d'après JENswa, 188:3). Il est probable que, dans ce dernier cas, quelques cellules germinales sont, au début déjà, englohées dans le cytophore en formation.

Lopinion généralement admise est que, tout en supportant les éléments spermatiques, le blastophore joue le rôle d'une collule nourricière. les spermatides étant, ensuite de la condensation chromatique. devenus incapables de commandel aux fonctions de nutrition. (Toir à ce sujet les travaux de Prenant 1892, Peter 1898, Benda 1898.)

Dos morules à (ytophore central, semblables à celles des Annélides. ont été ohservéses chez les Turbellariés par JENsen (1883) et chez un Bryozoaire par Konotnefre (1S\$8). Montucendu (1892) décrit chez les Distomes des groupes d'éléments spermatiques qui ressemblent d'une manière frappante aux groupes pédiculés de 4, 8, etc., observés chez lo Lombric. Les formes plus avancées (spermatides) different toutefois de celles du Lombric, en ce qu'il n'y a pas de cytophore central.

Lal spermatosphere de Clitellio figurée par Chaparìne (19)1, Pl. III, Fir. (1) 12) (itablit un passage des Annélides aux Arthropodes, dans ce sems qur les spermies insérées obliquement sur lo eytophore, se dirigent 


\section{EXPICLATION DE LA PLANCHE I}

Coupe transversale d'un Lombric adulte au niveau du grand testicule postérieur. Gross. : 73. On voit le grand testicule (indemne de parasites) attaché à la gaine péri-intestinale par une partie de sa surface, recourbé sur lui-même et prolongé en pointe du còté ventral. La substance médullaire est reconnaissable à sa teinte gris-clair. On distingue encore les cloisons à direction radiaire et les alvéoles remplis de follicules formant la substance corticale. Le hile creux et les lacunes de la substance médullaire ne sont pas visibles sur cette coupe.

A droite se voit l'intestin, au-dessus le vaisseau sus-intestinal, entre l'intestin et le testicule, la glande calcaire de Monres.

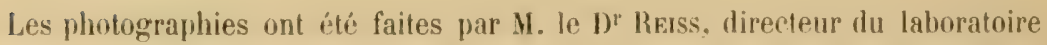
photograjhique de l'Université de Lausanne. 


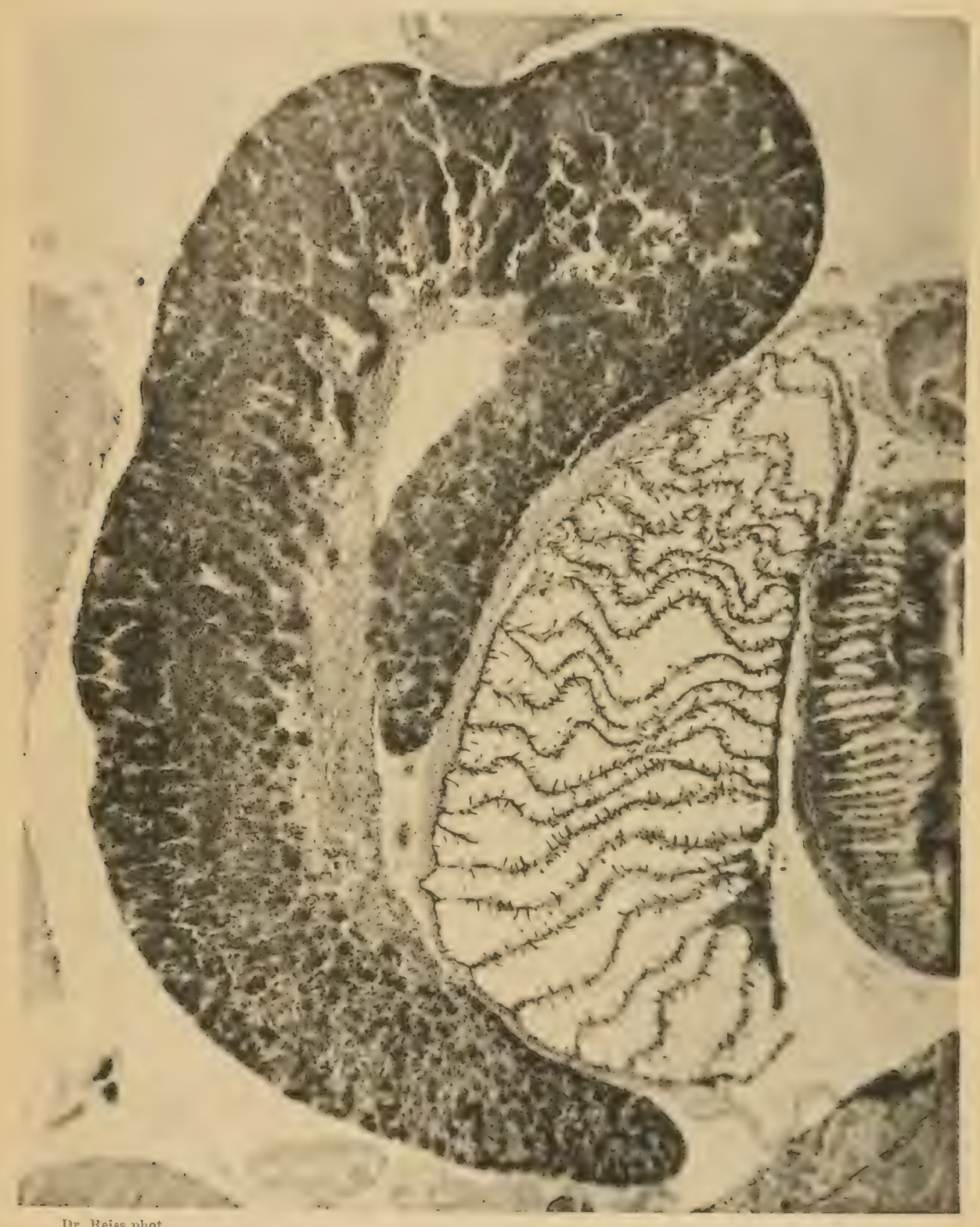

E. BUGNION et N. POPOFF 


EXPLICATION DE LA PIAACHE II

Coupe sagittale du Lombric adulte. Contenu du iarrefour au voisinage du petit testicule postérieur. Gross. : 606 .

La photographie montre:

$1^{0}$ Un grand nombre de petits follicules de 16, la plupart un peu aplatis, avec un seul rang de cellules superficielles, et un espace central allongé occupé par du cytoplasme.

20 De nombreux follicules ou fragments de follicules de 32 et 61, aver leurs noyaux à l'état de condensation. On distingue facilement les groupes à gros grains et les groupes à petits grains.

$3^{\circ}$ Des spermatides et spermatozoaires coupés en sens divers. 


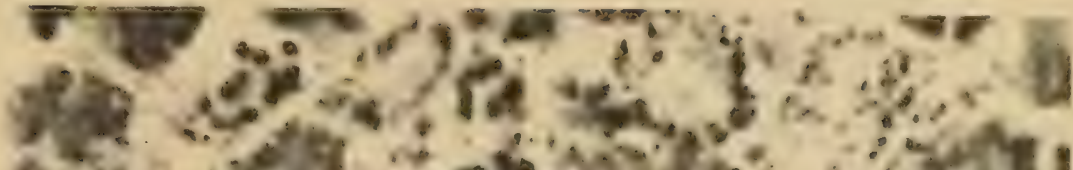

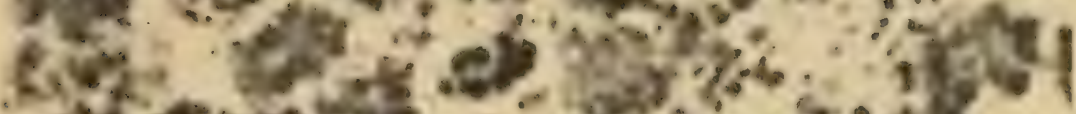

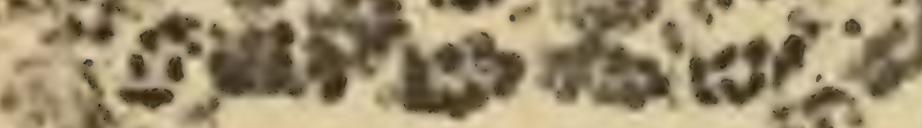

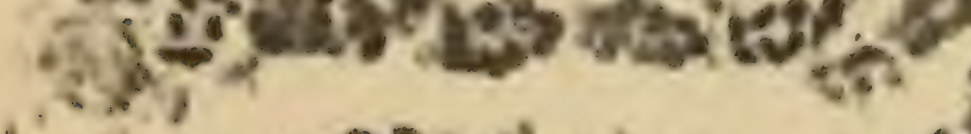

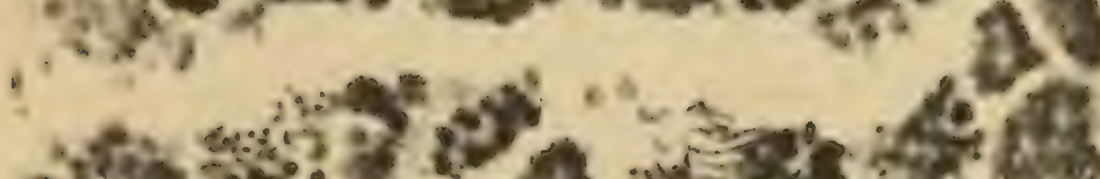

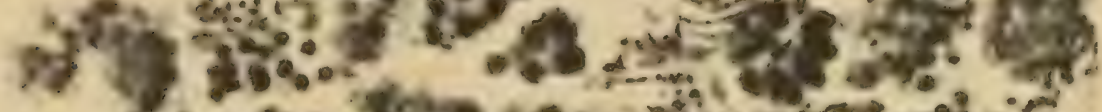
(f)

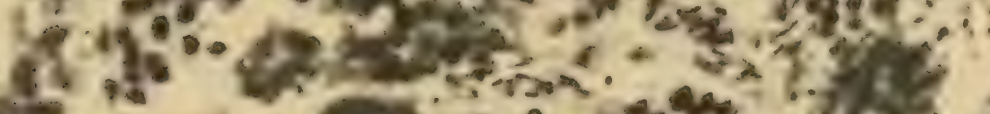

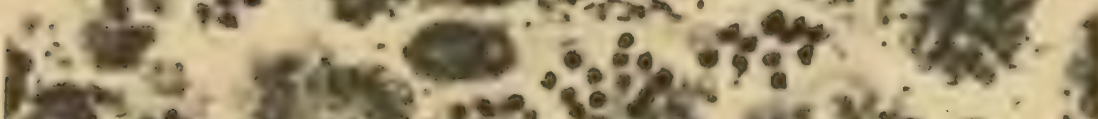

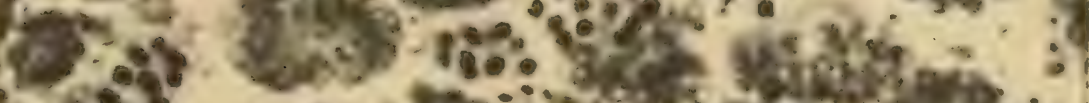

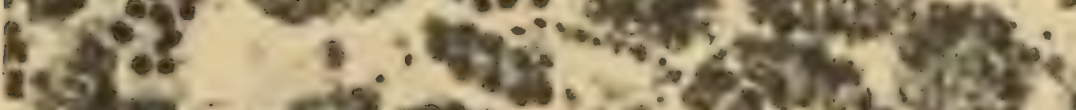

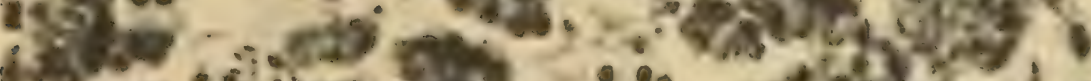

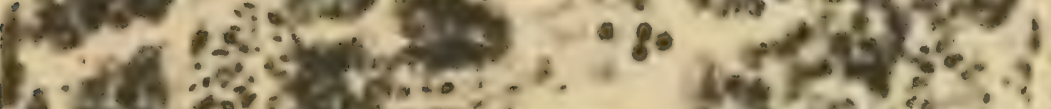

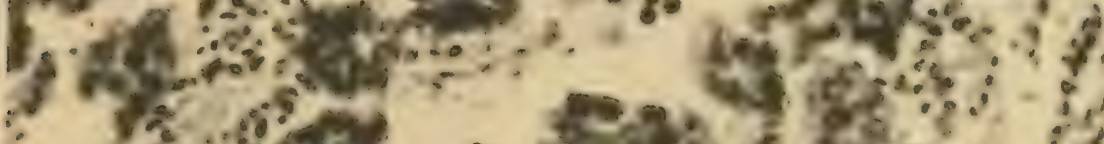

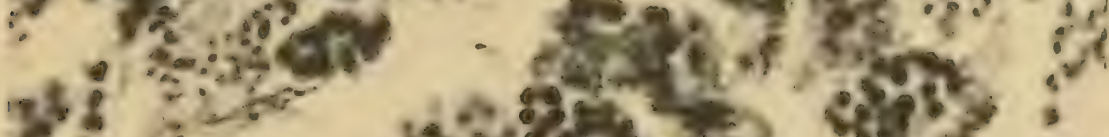
6 6.

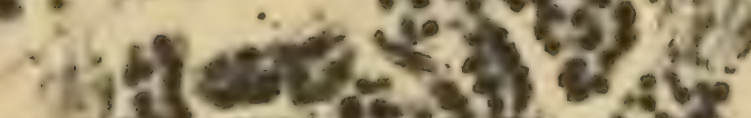

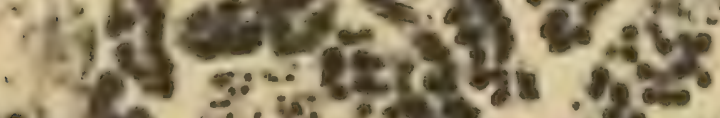

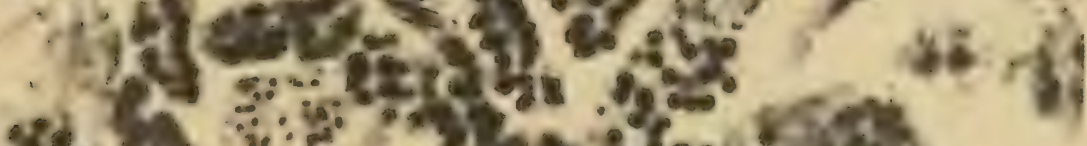

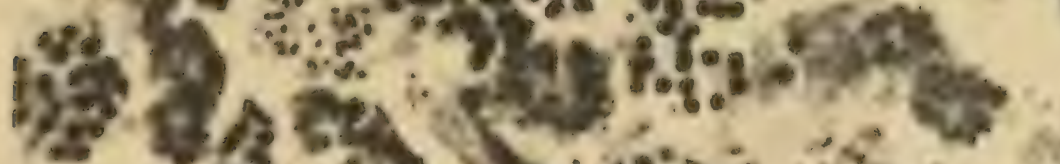

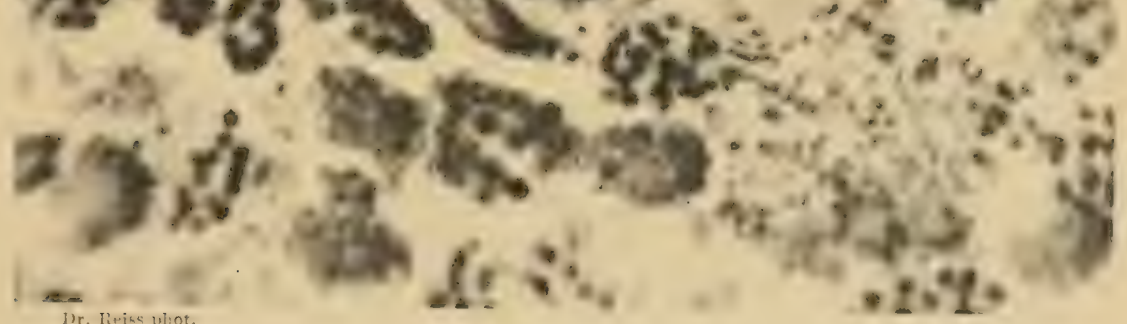






\section{EXPLICATION DE HA PIANCHE III}

Contenu du grand testicule postérieur d'un Lombric adulte, indemne de parasites, tué le 8 décembre. Lailance étalée sur la lamelle. Fixation par le liquide de Gusox renloré, Coloration par l'hematoxyline ferricue et le mélange de van Guson. Gross. : 1200 .

Follicules an roie de dissociation olỉrant des groupes prédiculés de $16+16$, $8+8,4+4$ unis par des ponts protoplasmiques.

Il n'y a. ì cause de la saison avancée. peu de traces de spermatogénèse (quelques cellules seulement à l'itat (ondensí): quelques grroupes offerent cependant des divisions radiaires. indiquant une segmentation yui succede at la dissociation. 


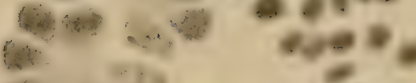

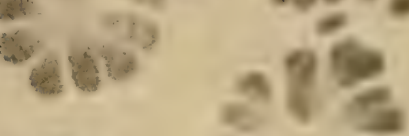

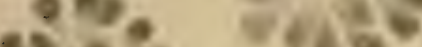





\section{EXPLICATION DE LA PIANCHE IN}

Contenu du grand testicule d'un Lombric adulte étalé sur la lamelle. Liquide de Gilson. Ilémalun-éosine. Gross. : 630 .

La photographie fait voir :

10 Une dizaine de morules de la dernicre phase à 256 éléments; dans l'une d'elles, placée au-dessus du milieu, les cellules s'allongent déjà en spermatides.

20 Quelques follicules à 6ł éléments à l'état de condensation chromatique, plus ou moins nettement divisés en deux moitiés $(32+32)$; les cellules à cytoplasme clair olïrent des contours polygonaux bien accusés.

$3^{\circ}$ Quatre follicules plus petits, foncés et compacts, probablement de 32 éléments divisés en deux moitiés $(16+16)$.

$4^{0}$ Quelques cellules détachées, quelques spermatozoaires. 


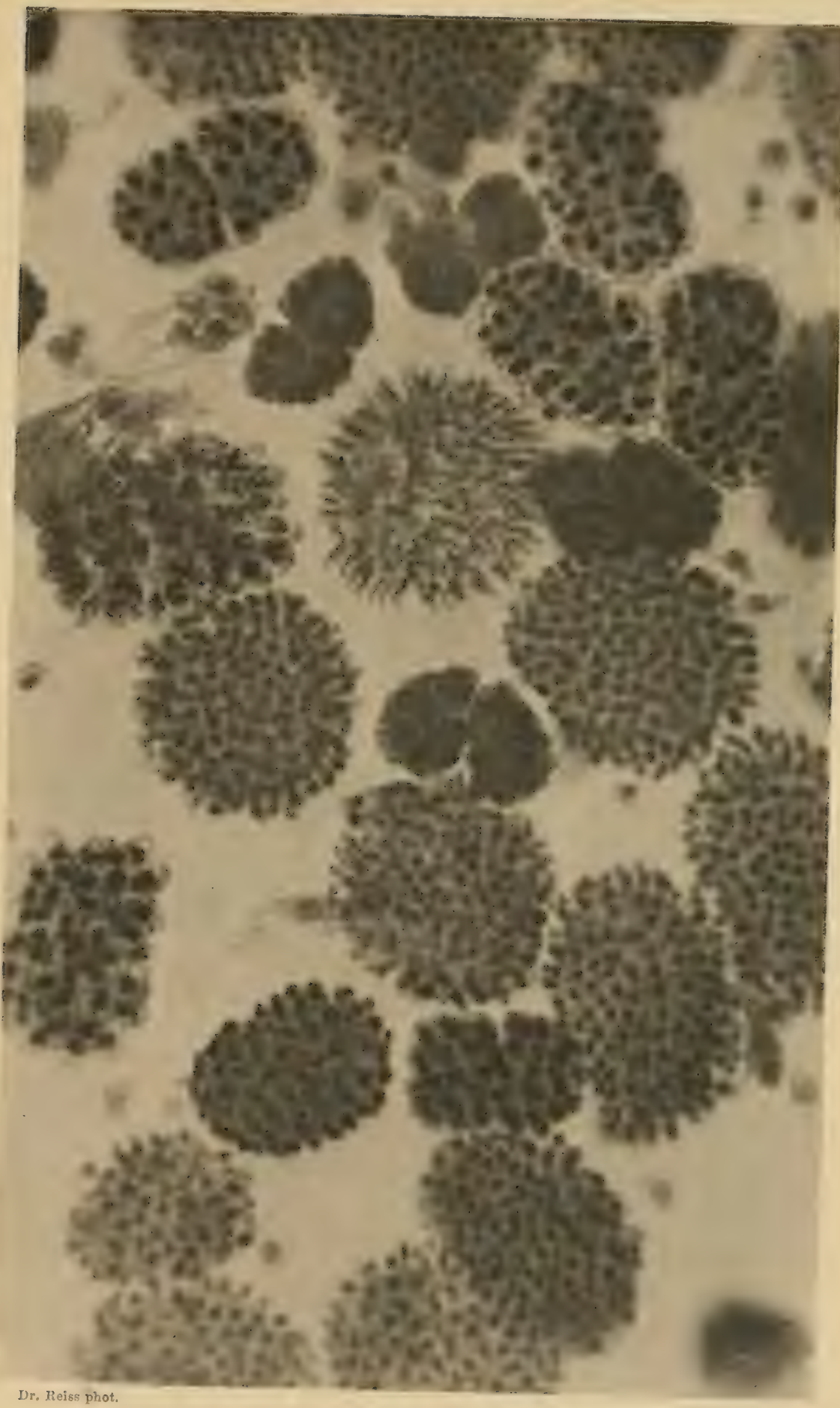

E. BUGNION et N. POPOFF 




\section{EXPLICATION DE LA PLANCHE V}

Coupe sagittule du Lombric adulte. Alignement des spermies dans le pavillon séminal postírieur. Gross.: 130. On voit en haut et à droite le contour de la loge du carrefour qui renferme le pavillon et rę̧oil les éléments spermatiques issus du grand testicule postérieur. 
$6^{\circ}$ Congr. Intern. de Zool.

Bugnion at Popoff. Pl. V.

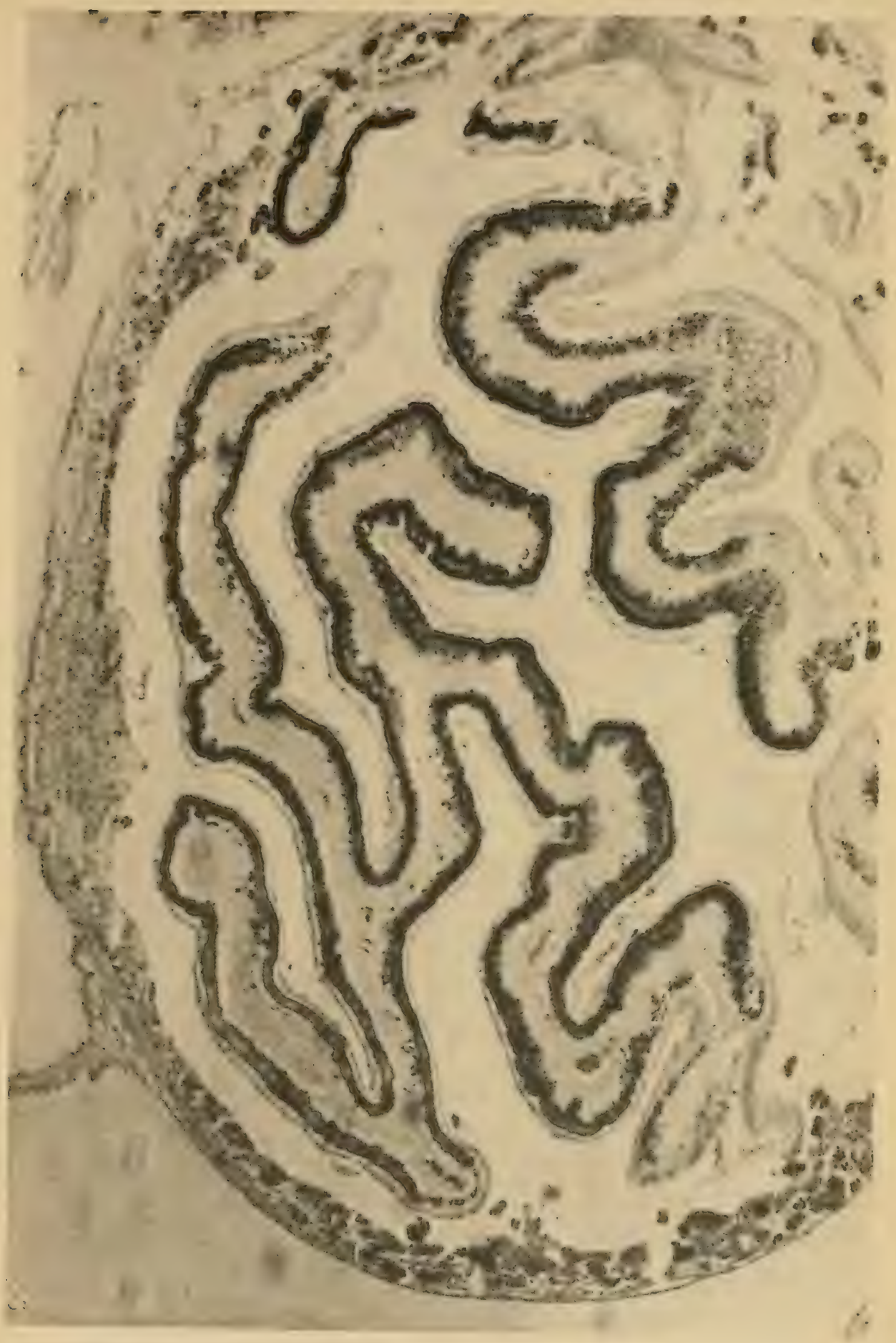

Dr. Reiss phot.

E. BUGNION et N. POPOFF 

toutes du même côté et tendent (au lieu de rayonner) à formel' un faisceau parallèle.

Chez les Arthropodes, la cellule gelminale prolifere d'abold de la meme. façon que celle des Annélides et donne lieu, elle aussi, à une colonie libre plus ou moins sphérique (colonie de métrocytes de Gruson, 1885̃). Mais le développement ultérieur de la colonie diffère : $1^{\circ}$ en ce qu'elle ne subit aucune dissociation avant de se transformer ; $2^{\circ}$ en ce que les éléments spermatiques, au lien de rayonner autour d'une boule centrale, se disposent parallèlement en un faisceau, avec toutes les têtes dirigées daus le même sens et à peu près juxtaposées. Le blastophore est remplacé ici par la cellule de Versox (1889), dont le corps, renfermant le noyau, se voit au bout du faisceau, du côté des têtes, tandis que les prolongements protoplasmiques (amalogues aux prolongenents des collules dre Skrtous) s'insinuent entr’e les éléments à l'intérieur du faisceau. Ce derniel fait ı'essort de l'examen des coupes transverses colorées à l'hématoxyline. On voit par exemple chez la Cétoine dorée, les têtes des sper'mies comme de petits points violets régulièrement espacés, séparés par une substance claire, teintée en lilas. Cette dernière substance est précisément le protoplasma nutritif (dépendance de la cellule de VErsox) dans lequel les spermatozoaires sont englobés. Ayant compté les spermies qui composent le faisceau, nous avons trouvé chez Hyponomenta cognatella (testicule d'une chenille débitée en coupes transverses) le chiffre 256, chez la Cétoine 512. Le chiffre indiqué nous a paru constant pour chacun des faisceaux.

Les auteul's modernes (Toyana, Ziegler, vom Rath, Tichomirofr, de Sinety, cités d'après Hexnegur, 1904, p. 647) admettent généralement qu'il n'y a aucun rapport génétique entre les cellules sexuelles et la cellule de VERson; celle-ci serait une cellule connective émanée des éléments de la paroi du testicule. Il se pourrait toutefois que la cellule de VERson fît une cellule sexuelle primordiale transformée et différenciée de bonne heure. Cette dernière opinion, qui a été soutenue par Versox (1859) et par Gríneng (1902-3) s'accordelait mieux avec ce que l'on sait des animaux supérieurs. Il a été en effet démontré par Regaud (1899) et Bours (1899) que, chez les Mammifères, la cellule nourricière et la lignée spermatique ont une origine commune.

Chez les Mollusques (Escargot) la colonie spermatique diffère de celle des classes précédentes, en ce qu'elle n'est pas libre à l'intérieur' d'un alvéole, mais unie pendant toute la durée de la spermatogénèse à la paroi de sa loge, par l'inter'médiaire de la cellule basale ou pédieuse ${ }^{\text {. }}$

Les éléments, pédiculés comme ceux du Lombric, diffèrent de ces delnier's en ce que les pédicules, au lieu de converger ver's le centre du groupe, sont tous dirigés en dehors du côté de la cellule basale et sus-

1 Décrite par Platner (1885), Bolles Lee (1897), etc.

VIe CoNgr. Int. ZooL, 1904. 
pendus à celle-ci comme les tiges d'un bouquet. Il résulte de cette disposition que les spermies sont arrangées en faisceaux comme chez les Arthropoles. mais arec cette difféerence que les têtes, au lieu d'être exactement juxtaposécs, sont étagées à divers niveaux comme l'étaient déjà les noyaux de la colonie primitive. La cellule basale, très volumineuse, se trouve ici encor'e placée à l'extrémité du faisceau, du côté des têtes ; cllo rolespond sans doute ì la cellule do Versox. Le nombre des éléments du faisceau n'a pas été compté.

Chez les Vertébrés inférieurs (Poissons et Amphibiens) les éléments spermatiques en roie de développement sont disposés de méme en faisceaux volumineux, comparables à ceux des Arthropodes et des Mollusques. Chez le 'Triton, par exemple, la partie germinale du testicule montre, dans chaque loge (spermatocyste), un certain nombre d'amas bien délimités (spermatogemmes) formés, comme les colonies spermatiques dos Inscetes, de petites cellules rondes, toutes de même grosseur et de même aspect (à la même phase de développement) et issues elles aussi de la prolifération d'une cellule germinale unique. La partie mûre de la glande (souvent visible sur la même coupe) oftíe d'autre part, dans chacun des kystes, un nombre correspondant de faisceaux spermatiques parfaitement isolés les uns des autres, avec les têtes juxtaposées et les queues recourbées, toutes dirigées dans le même sens. Chaque faisceau se folmant d'u n e colon ie de jeunes cellules, chacun d'eux possédant aussi sa cellule nourricière (cellule de Bloomfield) placée, comme chez les Arthropodes, à son extrémité céphalique, on voit que l'analogie est à peu près complete.

Quelques essais de numération, pratiqués sur des faisceaux de spermies coupés en travers, ont domné pour le 'T'riton le chiffre constant de 512. Chaque spermatogonie engendrant 4 spermies, on devra, si nos prévisions sont exactes, trouver pour les spermatogemmes le chiffre 128. Ce dernier dénombrement ne pouvant se faire que sur des colonies is()lées, préparées sur la lamelle, nous ne l'avons pas encore tenté.

Les spermies des Vertébrés supérieur's (Oiseaux et Mammifères) ne sont pas rémnies en faisceaux aussi volumineux, aussi compacts et exactement juxtaposés que ceux des Amphibiens. Il est toutefois aisé de constater que lis éléments spelmatiques en voie de développement forment. le long des parois du canalicule, une série de groupes en forme de petits bouquets régulièrement répartis et que dans chacun de ces groupes (spermatoblastes (le row Euner) les têtes sont, comme dans la plupart des classes, tournées en dehor's, du côté de la paroi. On remarque, en outre, que chaque groupe est plus spécialement en rapport avec une cellule nourlicière (cellule de Sertor ou de Renson) que cette cellule envoic des prolongements entre les éléments spermatiques et les englobe dans une masse protoplasmique commune. 
Il est des lors probahle que chaque spermatoblaste prociede de la prolifération d'une cellule germinale unirue, détachée de la cellule de S.rerTor I au début de la formation, et qu'il y a chez les animaux supérieurs aussi bien que chez Jes Invertébrés, des colonies spormatiques indépendantes, se multipliant par progression géométrique avant d'arriver au chiffre définitif typique pour chaque espèce.

Le nombre des spermies constituant chaque groupe paraît être (diapres une évaluation rapide) de 128 pour le Moineau et de 32 pour le Rat. (?)

Le spermatocyte de premier ordre se divisant encore deux fois et donnant lieu à 4 spermatides, il y aurait ainsi 32 spermatocytes I, issus d'une spermatogonio unique, dans le faisecau spermatique du Moineau ret

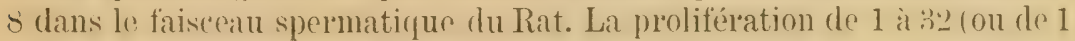
a s) correspondrait aux phases généralement désignées sous le nom de

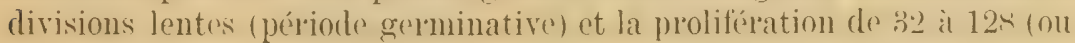
de 8 à 32) aux deux divisions rapides (période de maturation). Si ces déductions sont exactes, le groupement des spermatozoairess m faisceaux typiques, offirant toujours le mème nombre d'éléments, régulièrement disposés à l'intérieur des canalicules, s'expliquerait d'une façon toute naturelle, chez les Vertébrés, aussi bien que chez les Invertébrés, par la prolifiration d'une cellule germinale unicpue, sans qu'il soit nécessaire de recourir à des effets plus ou moins hypothétiques de tropisme ou d'attraction ${ }^{1}$.

On voit que l'étude du Lombric jette quelques clartés nouvelles sur la préspermatogénese, ainsi que sur l'origine des faiscraux spermatiques. non seulement chez les Invertébrés, mais aussi chez les animaux supélients.

\section{OUVRAGES CITES}

Hebrag, E. Zur Anatomie u. Physiologie der Generationsoryane des Regenumems. Zeitschr. f. wiss. Zool., vol. 8. 18 อ̈6.

Claparíde, E. Etudes analomiques sur les Amélides. Mém. soc. de phys. Genève, t. 16, pl. III. 1861 .

Pentien, Ed. Rech. pour servir ì l'hist. des Lombriciens terrestres. Nour. arch. du Muséum. T. 8. 1872.

BLoomfield, J. E. On the development of the spermatozoa. Quart. Journal of micr. Se., vol. 20. 1880.

Jarsex, 0.-S. Rech. sur la spermatogénèse. Arch. de Biologie, vol. \&. 1883.

GiLsox. Lu spermatoginèse des Arthropodes. La Cellule, vol. 1, 2, 4. 1880̈-86-88.

1 Voyez à ce sujet les mémoires de Groben (1899), Loisel (1902). 
Platxer. Ueber die Spermatogenese bei den Pulmonaten. Arch. f. micr. Anat, vol. ㄹํ. 188 วั.

Torgt. Ueber Ei-u. Samenbildung bei Branchiobdella. Arb. Zool., Zoot. Inst. Würzburg, vol. 7.1885 .

Bengr. Unters. über den Bau u. die Entw. der Geschlechtsorgane des Regenwu'ms. Zeitschr. f. wiss. Zool., vol. 44. 1886.

Lionotverf, A. Beitrïge zur Spermatologie. Arch. f. micr. Anat., vol. 31. 1888.

Vensox. Zur Spermatogenesis. Zool. Anz., vol. 12. 1889.

Moxticeili, F.-S. Ricerche sulla Spermatogenesi nei Trematodi. Intern. Monatsschrift f. Anat. u. Phys., vol. 9. 1892.

Prexaxt. Sur la signification de la cellule accessoive du testicule. Journal de l'anat. et de la physs. $28^{\text {me }}$ année. 1892.

Woodwan, M. F. On veriations in the Genitulia of british Earthworms. Proc. \%ool. Soc. 1893.

Vensox. Zur Spermatorgenesis bei der Seidemanpe. Zeitschr: f. wiss. Zool., vol. \%8. 1894 .

Culkuxs, G. X. The Spermutogenesis of Lumbricus. Journal of Morph., vol. 11. 189.5 .

Yox Eirdasen, R. Zur henntnis des feineren Banes des Regenwumhodens u. der Hodenzellen. Areh. f. mier. Anat., vol. 47. 1896.

Bolles LeE, A. Les cinèses spermatogénétiques chez l'Helix pomatia. La Cellule, t. 13.1897.

Bexp., C. Ueber die Spermatonenese der Vertebraten u. höheren Exertebraten. Arch. f. Anat. u. Phỵs., phỵs. Aluth. 1898.

Peten, h. Die Berleutung der Nëhrzelle im Hoden. Arch. f. micr. Anat, vol. ก3. 1898 .

Regaud, C. Sur lu morphologie de lu cellule de Sertoli. Association des anatotomistes, I $^{\text {re }}$ session et Bibl. anat. 1899 , I $^{\text {er }}$ fase.

Botrx, P. A jropos du noyan de li cellule de Sertoli. Bibl. anat. 1899, 5"me fase.

Gronbex, K. Eeber die Anordmung der Samentiörper zu Bündeln. Zool. Anz., vol. 292. 1899.

Foot, K. et Struebeld, E. C. The Spermatozon of Allobophore fotidu. The amer. Journal of Anatomy, vol. 1. 1902.

Lorsel, G. Etudes sur la spermatogénèse du moineau domestique. Journal de l'anatomie et de la phrsiologie, $38^{\text {me }}$ année 1902.

Gnüxiberig. Zool. Anz., 1902 et Zeitsch. f. wiss. Zool. 1903.

Hexwegcy. Les Insectes. 1904.

Prexast, Bocix et Malland. Traité d'histologie. I. 1904.

Roтn, A. Zur hemutmis der Benegung der Spermien. Arch. f. Anat. u. Phỵs., physiol. Abth. 1904. 


\section{SECTION}

\section{A R T H R O P O D ES}

Séances du lundi 15. mavdi $16 \mathrm{ot}$ mercredi 17 aoùt 1904.

Président: M. le Prof. R. Heymons.

Vice-Président: M. le D ${ }^{r}$ Ch. Janet.

Secrétaire: M. le Dr Th. Streok.

\section{Sur les cornicules ou nectaires des Aphidiens.}

Par le Dr G. HORVATH (Budapest).

Les cornicules ou nectaires des Aphidiens sont deux petits tubes chitincux. implantés sur les deux Hanes du cinquieme segment abdominal et

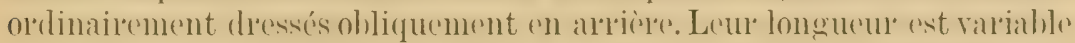
selon les espèces, ainsi que leur forme et leur coloration. Ils sont tantôt cylindriques, tantôt plus ou moins épaissis ver's la base ou vers l'extrémité et atteignent parfois la moitié de la longueur du corps. Ils sont souvent très raccourcis et ne représentent que deux tubercules plus ou moins saillants; che\% eretains groupes ils satrophient ot manquent totalement. Si l'on presse ou exeite un peu brusquement l'Insecte, on voit bientôt apparaître à l'extrémité des cornicules une petite goutte d'un liquide visqueux.

Les fonctions de ces organes particuliers ne sont pas expliquées, jusquüi présent. d'une manière suffisante. (on pense généralement let les traités de zoologie et d'entomologie le répètent souvent) que c'est par' ces cornicules que les Aphidiens font sortir de leur corps la liqueur douce connue sous le nom de la "miellée " et si recherchée par les Fourmis. Cette opinion date déjà du $18^{e}$ siècle; elle remonte aux premier's observateurs des Aphidiens. 
RÉAumur (1737) a été le premier qui s'en soit occupé. Il a pensé que les Aphidiens ont deux sortes différentes dexeréments quils rendent par l'ouverture de l'anus et par celle des cornicules. La liqueur claire et sucrée, issue de l'anus, qui est cherchée et sucée par les Fourmis, serait, d'après lui, analogue à l'urine, tandis que les cornicules laisseraient sortir des excréments analogues aux matières grossières r’ejetées par•l'anus des autles animaux.

Ch. Bonnet (1745) était d'avis que le fluide sucré que, d'ailleurs, il a pris aussi pour de l'urine, sort par les cornicules et que les cornicules pourraient bien être, en partie, des organes de la respiration qui serviraient peut-être à l'éjaculation du fluide.

Lrnnḱ (1758) s'est exprimé, en parlant des Aphidiens, en ces termes: "Pleræque duo cornua postica abdominis gel'unt, quibus excernunt rorem melleum hre Formicarum vaceæ! » (Syst., Nat., ed. X, p. 45̃2).

C'est sans doute à cette phrase et à la grande autorité de Linné qu'on doit attribuer l'extension générale de l'opinion que les Aphidiens font sortir par leur's cornicules une matière sucrée, la miellée. Les obser-

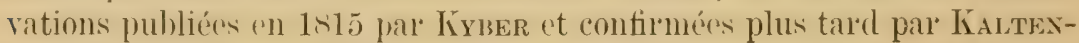
BACH (1843), Fores (1874) et autres, n'en ont rien changé. La théorie de Bonnet s'est conservée, chez la grande majorité des zoologistes, jusqu'à nos joul's.

Cependant, on peut s'assurer bien facilement que le liquide doux et transparent qui répond à la miellée, sol't toujour's et exclusivement par l'anus et jamais par les cornicules. Quand une Fourmi se rapproche d'un Puceron et le frappe avec ses antennes, la gouttelette émise par celui-ci ret lapér aussitot par la Fourmi, apparait toujour's au bout de lablomen. tandis que les colnicules ne sécrètent rien. Mais, si on prend un Aphidien entre les doigts ou si on le touche avec un brin d'herbe ou avec une aiguille émoussere. une petite gouttelette fait immédiatement son apparition à l'extrémité de l'un ou des deux colnnicules. Cette gouttelette n'est jamais limpide et transparente, mais toujour's color'ée, c'est-à-dire verte, jaune, rouge, brune ou noire.

La supposition de BoxNer que les cornicules appartiennent peut-être au systeme l'espiratoire, a été arloptée par Kr Kuer (1815), Morrex (1836) et Kaltenbach (1843). Kyber leur avait attribué, en même temps, le rôle des leviors particuliers dont l'Insecte se servirait pendant léjaculation du fluide transparent issu de l'anus. (Magazin der Entomologie, I, 2 , p. 38.) Morrex a pensé que le cornicule n'est qu'un stigmate prolongé, à sa base avec un lacis considérable de trachées et avec une glande qui sécrète le liquide sucré. Ce liquide est poussé et éjaculé, d'après lui, jar lair de ces traheres pendant la respiration. Anmales des Sciences Natul’elles. 2 cér. VI, p. 91.) Kaltenbach, en acceptant cette explication, s'est imaginé que tout cet arrangement servirait à l'oxydation plus rapide 
des substances nutritives consommées par le Puceron. (Monogr. der Pflanzenläuse, p. XVI.)

Cotte hypothese est eependant tout il fait eronné, puisque les connicules ne sont aucunement en communication directe avec l'appareil l'espilatoire. Writhaczil qui a si bien étudié l'anatomie des Aphidiens (1882), l'avait démontré avec certitude. Il serait, en effet, fort étrange que les cornicules soient des stigmates, car on trouve sur le même segment abdominal qui porte les cornicules, déjà les deux stigmates ordinaires. Ce segment aurait alors quatre stigmates; ce serait vraiment trop bizarre!

Les corpuscules ronds ou elliptiques qu'on voit sous le microscope dans le canal central des cornicules et dans l'intérieur des gouttelettes apparaissant à leur extrémité, ressemblent bien à des bulles d'air qui seraient sorties des trachées; mais, en réalité, ils ne sont que des cellules adipeuses modifiées, Hottant dans le liquide dont les cornicules sont r'emplis.

L'idée de Bonnet que ce liquide serait de l'urine, a été ressuscitée dans les derniers temps par W WT, ACzIL. Cet auteur, qui a pris, en 1882, la sé"rétion issue par les cornicules pour une matièe sucrée (Blattlauszuclier), a écrit, en 1884, que c'était peut-être... de l'urine. Il faut bien avouer qu'on ne trouve pas chez les Aphidiens de tubes de MaLpigh ou d'autres organes semblables; par conséquent, on pourrait supposer queles glandes situées à la base des cornicules et dont le produit se déver'se dans ceux-ci, jouent le rôle des tubes de MaLPighi. Mais le produit de ces glandes ne montre aucune des réactions chimiques de l'urine. En outre, les recherches de Kowalevsky (1892) ont démontré que c'est l'intestin terminal qui remplit, chez les Aphidiens, les fonctions d'organes urinaires.

Il résulte de ce qui précède que les cornicules n'appartiennent ni au canal intestinal, ni à l'appareil respiratoire, ni au système urinaire. Quelle est donc la nature du liquirlo que les Aphidiens font solotir bar les cornicules? Et quelle est la fonction de ces deux appendices abdominaux?

L'analyse chimique faite par le prof. KNore à Iéna et publiée par BüsGen (Der Honigtau, p. 83, 1891), prouve que le liquide visqueux qui apparaît à l'extrémité des cornicules, est une matière cireuse. Les dleux glandes qui la produisent, et qui se trouvent à la base des cornicules, rentrent dans la catégorie des glandes cérogènes.

Pour se rendre compte de la destination de cette matière cireuse, il faut observer une colonie de Pucerons vivants. On peut bien voir, alors, que les membres de la colonie rendent leurs excréments par l'anus sous for'me de petites gouttes claires, surtout quand ils sont visités et frottés par leur's amies, les Fourmis; durant cette opération, leur's cornicules ne fonctionnent nullement et ne montrent aucun changement. Mais, si un Insecte aphidophage ot particulièrement une Coceinelle ou la larve diune Chrysope se rapproche d'un Puceron, celui-ci fait sortir à l'extrémité de 
l'un ou de l'autre cornicule une petite goutte visqueuse, dirige le corni-

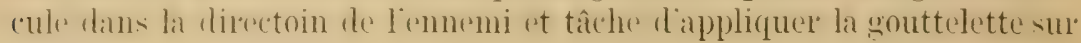
sa tête, ses mandibules ou son cor'selet. Si cette manœuvre réussit, l'ennemi se retire tout de suite et fait tout son possible pour se délivrer de la gouttelette adhérente qui se dessèche immédiatement et qui lui est apparemment très désagréable.

Il est done évident que les cornicules jouent le rôle de moyens de défense. Quoique cette défense ne soit pas absolue, elle assure pourtant au Puceron attaqué une protection, au moins temporaire.

Ces observations confirmées aussi par Büsgen (op. cit., p. 85), permettent de donner la définition suivante des cornicules :

Les cornicules des Aphidiens sont des canaux excléteur's des glandes cérogènes différenciées dans un sens spécial et dont le produit est un moyen de défense contreles Coccinelles et les Chrysopes.

Les Pucerons qui sont dépourvus de cornicules, n'ont pas besoin de cette défense, car ils vivent sous la terre ou dans des galles et n'y sont pas exposés aux attaques des Coccinelles ou des larves de Chrysopes. Les quelques espèeres qui ne posèdent pas des comicules quoiqu olles virent à l'air libre, sont couvertes d'une riche sécrétion cireuse qui les protège non seulement contre les intempéries, mais aussi contre les Insectes carnassiel's. Il n'y a que peu de Pucerons qui n'aient ni col'nicules, ni duvet cotonneux, bien qu'ils vivent à découvert (p. ex. Anœcia cormi, Vacuma dryophila, Phylloxera coccinea, etc.).

\section{Discussion.}

M. JANET ajoute quelques observations a la communication de M. Horvatr. Il faut lui savoir greé de contribuer ainsi à réfuter l'erreur, encore très répandue, que les cornicules secrètent le liquide sucré si recherché par les Fourmis. Il rappelle le travail de BüsGer qui a bien mis en évidence la faron dont les Aphidiens peuvent fare pénétrer dans les tissus végétaux leurs organes buccaux si ténus. 


\title{
Un Caprellidé dans le lac Léman.
}

\author{
Par le prof. H. BLANG (Lausanne).
}

Avec 4 figures dans le texte.

Désireux d'élucidel certaines questions biologiques relatives au plancton du lac Léman, je récolte celui-ci depuis deux ans, d'une façon méthodique. en pratiquant chargue mois, arece des filets à mailles différenter. deux séries de pêches, diurnes et nocturnes, horizontales et verticales. Aujourd'hui, je suis en possession des produits de 176 coups de filet fixés sur le bateau, auxquels s'ajouteront les produits des pêches que je désire faire encore pendant cette année.

Or. c'est en examinant, le 10 mar's 1904, le produit fixé d'une pêche

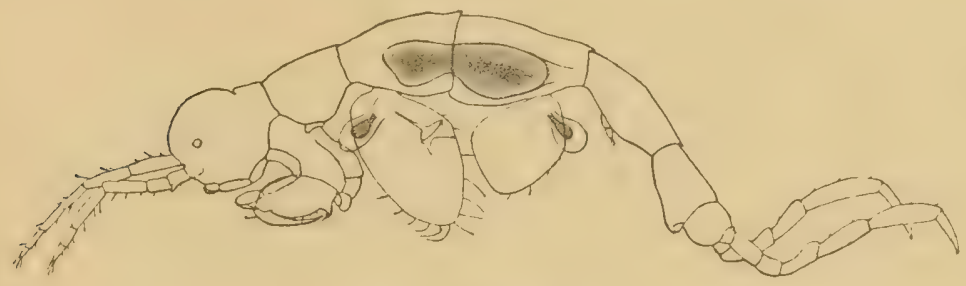

Fig. 1. Podalirius?

Gross. $\times 33$.

verticale faite derant Ouchy le 15 mai 1908 à 40 metres de profonderur. que je découvris, à ma grande surprise, en triant des Bythotrephes perdus dans la masse de Dinobryon et de Bosmina qui composent le plancton à ce moment de l'année, un Caprellidé femelle, avec poche incubatrice développée, prête à recevoir des œuf́s et mesurant $1^{\mathrm{mm}}, 8$ de longueur. (Fig. 1.)

Tous les Lémodipodes connus étant des animaux marins et aucun dintre eux n'ayant été jusqu’ici signalé dans l'eau douce, la découverte diun 
de ces Crustacés dans un filet à plancton n'était pas banale, mais je devais chercher à me procurer l'animal vivant. Connaissant l'habitat des Lémodipoles pour en avoir sourent récolté dans le golfe de Kiel parmi les Zoostères et les colonies d'Hydropolypes', sachant qu'ils marchent plus volontiel's qu'ils ne nagent et qu'ils ne se trouvent qu'accidentellement 'n pleine cau, je commençai par fouillex les touffes de Cerentophyllum, de Myriophyllum, de Potumogeton croissant pres du rivage, à une faible profondeur. Puis, je pronnenai la drague rateau dans les Char qui végètent entre 20 et 30 mètres. Ne réussissant pas à découvrix mon Crustacé dans la flore des eaux littorales, je le cherchai alor's avec la drague à 50, 80 et 150 mètres de profondeur. supposant quil pouvait vive sur le limon comme certains de ses congénères marins et qu'il s'était trouvé pris dans un tilet à plancton, entraîné hos's de son habitat, par quelque conl'ant; mais les 44 draguages que je fis, du 11 mar's au $1^{\text {er }}$ août, restèrent infructueux.

L'exemplaire que je présente est done le seul que je possède en ce moment, c'est pour cela que je n'ai pas voulu le disséquer avant de l'avoir montré au Congrès, conservé in toto. Il a été fixé, sul le bateau de pêche, avec le plancton dans lequel il se trouvait, par le liquide Perenyi. puis traité à l'alcool, coloré à la cochenille alcoolique et monté en préparation microscopique dans la glycérine. La fig. 1 représente l'animal dessiné à la chambre claire: les appendices du coté gauche ont seuls été représentés, mais les pattes thoraciques des $6^{\mathrm{mc}}$ et $7^{\mathrm{me}}$ segments thoraciques. qui manquaient de cer coté, ont dûtere l'emplacées par celles du côté droit.

Sans dissection, j'ai pu déter'miner les caractères essentiels de ma trouvaille et fixer sa place dans le genre Podalirius Kröyer de la famille des Caprellidés. En effet, comme tous les l'eprésentants de ce genl'e, cet Amphipode n'a pas de pattes lienn développées au $5^{\mathrm{mu}}$ seonment; celles-ci sont rudimentaires, faites de

Fig. 2. Patte rudimentaire du $5^{\text {me }}$ segment thoracique.

Gross. $\times 140$. deux articles (fig. 2) et il ne porte des branchies qu'aux $3^{\text {me }}$ et $4^{\text {me }}$ segments thoraciques. Les figures 3 et 4 représentent l'une, le gros mamelon du canal excréteur de la glande antennaire porté par l'article basilaire de l'antemne externe. lautre, Je tuldereule situé sur le bolod interne de la main préhensile de la 2me paire de pattes thoraciques. placé près du bord proximal de son article cilarefi : ces deux différenciations sont conformes à celles que lon olserve che\% les Podalivins. Je n'ai pas pu étudier sans dissection, la strue-

${ }^{2}$ H. Branc. Die Amphipoden der Kieler Bucht. Nova Acta der K. Leop. Carol. Dentschen Akademie der Naturforscher, Bd. XLVII, No 2, S. 87, 90. 1884. 
ture des appendices huceaux, en particulier colle de la mandiluke, qui ne porte pas de palpe chez ces Crustacés. D'après P. Mayer ${ }^{1}$, le genre Podalirius compte trois espèces : $P$. Kröyerii Haller', $P$.typicus Kröyer, $P$. mi-

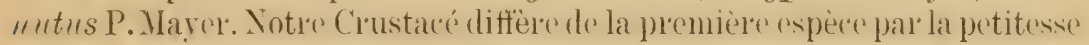
de ses pattes thoraciques, il se différencie de $P$. typicus par la longueur

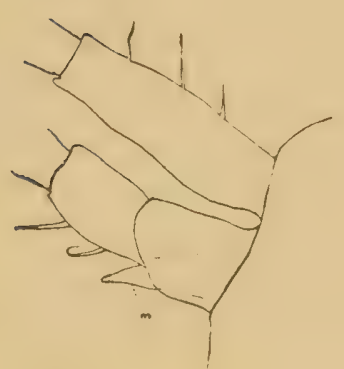

Fig. 3. Articles basilaires des 2 antennes gauches. $\mathrm{m}$, mamelon de la glaude antennaire.

Gross. $\times 140$.

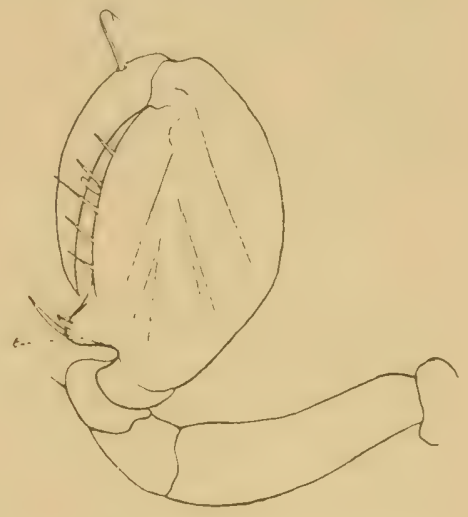

Fig. 4. Patte gauche de la $2^{\mathrm{e}}$ paire. $t$, tubercule.

Gross. $\times 140$.

restreinte du doigt de la main de la deuxième paire de pattes, qui chez cette espèce dépasse de beaucoup le tubercule du bor d externe. (Paul Mayer, fig. 30, p. 75.) Par contre, il lessemble beaucoup à $P$. minutus tout en étant encole plus petit. Nais je laisse cette détermination en suspens jusqu'à ce que je puisse procéder, comme il le convient, à la dissection des divers appendices, quand j'aurai à ma disposition des individus des deux sexes.

De mes draguages infructueux, on pourrait inférer que le Caprellidé dont il est question a été apporté ou semé dans le Léman par un Oiseau migrateur. Palmiperle ou Echassier. et que. par un hasard bien singulier, je l'ai recueilli dans mon filet à plancton alor's qu'il tombait comme cadavie au fond de l'eau, devant Ouchy, le 15 mai 1903 entre trois et

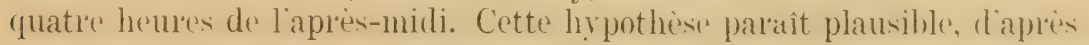
certains faits connus de migrations passives de diver's animaux et de leu distribution géngraphique. mais discutée, elle ne impose pas nécéssairement. Je rappellerai que, pendant vingt-cinq ans, MII. Fores, DU

${ }^{1}$ P. Mayer. Die Caprelliden des Golfes von Neapel. Fauna und Flora des Golfes von Neapel, VI. Monographie. 1882. 
Praessis et moi-même avons vainement cherché l'Asellus aquaticus cité déjà par le doyen Brides comme habitant le Léman ${ }^{2}$ et ce n'est qu'en janvier 1899 que je réussis à décourrir cet Isopode, en secouant vivement des Ceratophyllum où il a l'habitude de se tenir solidement cramponné. Quelques jour's après, M. Fores, auquel j'avais communiqué le fait, l'écoltait plusieur's Asellus devant Morges dans le même habitat.

Contre l'apport du Caprellidé du lac par quelque Oiseau de passage, parle son bon état de conservation. Les muscles du corps, de ses divers appendices, n'auraient pas pu se colorer par la cochenille, révéler leur striation caractéristique, s'ils avaient été desséchés, ne fût-ce même que quelques heures. C'est parce que toute la musculature et les gros œufs, ont bien absorbé le colorant, qu'il m’a été impossible d'étudier le contenu du tube digestif, déterminer la nature des aliments ingérés, ce qui peut-être m'aurait fourni quelques indications sur le milieu habité par l'animal.

Il faut encore remarquer que le passage du printemps, pour lés Oiseaux de rivage et de marais, beaucoup plus court que celui d'automne, est entièrement terminé dans les premier's jour's de mai, que les espèces qui ferment la marche sont quelques petits Echassiers qui ne passent plus qu'isolés sur les bords du lac pour se rendre vers le Nord et qui ne se prêtent guère au transport d'animaux.

Pourquoi est-ce qu'un Caprellidé ne pourtait pas être lacustre, vivre dans le lac Léman, faire partie d'une de ses sociétés animales littorale ou abyssale? La faune de celui-ci renferme déjà plusieurs espèces à facies marin très caractérisé.

Je citerai parmi les Ostracodes : Limmocythæ relicta Vernet, Cytheridea lacustris Vernet, Lencocythere mirabilis Kaufmann. Prostoma lacustre (Emea lacustris) du Plessis est un beau Némertien fréquent dans la faune littorale. Monotus morgiense, Plagiostoma Lemani du Plessis, sont des Turbellariés appartenant à des groupes dont tous les représentants sont marins; la Gromia Brumeri Blanc ne ressemble qu'à l'espèce marine Gromia oviformis Duj. Les recherches des Pavesi, Sars, Wesenuerg-Lund, Sayter et Veltner, Dybowskr, Entz, Graviar ont démontré que l'adaptation importante de la vie marine à la vie lacustre peut très bien se faire sans entraîner avec elle de changements notables dans l'organisation des êtres adaptés.

Le Palamon lacustris du lac de Garde, la Mysis relicta des lacs scandimares ot du Danemark, la Pontopureia affiris, la Pulusiplla quadrispimosa, la Corophium longicome des lacs de l'Allemagne du nord, plusieur's Crustacés du lac Baïkal, quelques Protozoaires du lac Balaton et enfin certains Annélides polychètes de la Guyane française, sont autant de formes lacustres identiques aux formes marines des mers voisines. De

\footnotetext{
1 Le doyen Brider. Essai sur le Léman. Conservateur Suisse, V. 1-93, Lausanne, 1814.
} 
toutes ces espèces adaptées à l'eau douce, quelles sont celles qui peuvent être considérées comme formes reléguées, quelles sont celles qui ont été l'objet d'un transpolt passif de la mel dans l'eau douce? La discussion de ces questions n'a pas sa place ici. Ces faits étant rappelés, il me semble que l'existence d'une espèce de Caprellidé vivant dans le lac Léman me parait bien certaine et j'espère qu'en perfectionnant mes procédés de pêche et de draguage, je pourrai en fournir un jour la preuve. En attendant, je prie ceux de mes collègues qui s'occupent de recherches lacustres de bien vouloir m'arlresser les exemplaires des Caprellidés qu'ils pourraient trourer, afin que je puisse faire un jour une monographie plus complète de ce Crustacé intéressant.

\title{
Discussion.
}

M. Javet ne croit pas que le Crustacé en question ait pu êtı’e appol’té à l'état adulte d'un milieu marin dans le lac Léman, mais il n'y aurait rien d'impossible à ce que cette espèce ait été apportée à l'état d'œuf.

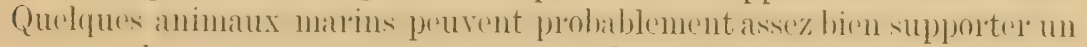
passage de ce genle et peut-être même, dans le cas où il ne peut y avoir adaptation définitive au nouveau mi ieu, donner naissance à un certain nombre de générations.

\section{Ueber die Entwicklungsgeschichte und Morphologie der Solifugen.}

\author{
Von Prof. R. HEYMoxs (Hannover-Müinden).
}

Die Gruppe der Solifugen nimmt in verschiedener Hinsicht eine etwas isolierte Stellung unter den Arthropoden ein. In ihrem Aeusseren den Spinnentieren gleichend, weichen die Solifugen doch in auffallender Weise von dem bekannten Arachnoidentypus ab.

Die Dreiteilung ihres Körpers in einen vorderen, Extremitäten tragenden "Cephalothorax 》, in drei freie mit Gangbeinen versehene "Rumpfsegmente ) und in ein hinteres sackartiges und gegliedertes "Abdomen " ist nicht für Arachnoiden charakteristisch, sondern erinnert lebhaft an den bekannten Bauplan der Insekten. Eine weitere Annïherung an die Organisation der Inseliten kommt noch durch das Vorhalten der Respirationsorgane zu stande, die hei den solifugen aus hamm- 
förming verästelten Röhrentracheen bestehen. In Gegrensatz zu der überwiegenden Mrorzahl anderer Arachnoiden, bei denen die Oeftnungen der Atmungsorgane ausschliesslich auf den hinteren Kïrperabschnitt (Abdomen) beschriult bleiben, treften wir bei den Solifugen aussel drei zum Teil verschmolzenen Abdominalstigmen auch ein sogenanntes thorakales Stigmenpaar im zweiten Beinsegment an.

Es ist hier nicht der Ort, ausführlich auf die bisherige Litteratur cinzugehen, es ist nicht möglich, hier die verschiedenen. Autoren zu zitieren, die sich teils für, teils gegen eine Torwandtschaftsbeziehung der Solifugen mit den Insekten ausgesprochen und ihre Meinungen mit mebr od 'r minder stichhaltigen Gründen motiviert haben. Nur so viel sei gesagrt, dass einstweilen eine definitive Klärung der verschiedenartigen Ansichten nicht erzielt ist. Dis Existenz dreier gesonderter (Thorakal-) Sogmente wird von Manchen als cine ursprïngliche Eigentümlichkeit, von Anderen als das Resultat der Wiederauflosung eines früher einheitlichen Arachnoiden-Cephalothorax angesehen. Die Erlikärung der Stigmen bietet Anlass zu sehr verschiedenartigen Spekulationen, und anch in anderen morphologischen und anatomischen Fragen bestehen zum Teil nicht unerhebliche Meinungsverschiedenheiten.

So viel dürfte feststehen : allein auf veroleichend-anatomischem Wege ist es nicht felungen, der I)eutung der morphologischen Eigentümlichliriten dieser aberranten Tiergruppe ein sicheres Fundament zu geben. Es ist also elforderlich, entwicklungsgeschichtliche 'latsachen zur Beurteilung heranzuzichen.

Nach vielen Schwierigkeiten ist es mir gelungen, in den Steppen Turkistans die wichtigsten Entwicklungsstadien von Graleodes caspins IBirula zu erlangen. Ich teile hier in Kürze einige meiner Untersuchungsergehnise mit, beschränke mich aher auf die Besprechung derjenigen Erscheinungen, welche die Entwickhung der äusseren Körpergestalt hetreffen.

I Die hei der Bildung der velschiedenen Funbryonalschichten, des Elitoderms, Mrsoderms und Fntoderms auf der (b)etläche des liugeligen Eies erscheinemde Embryonalanlage ist anfangs von rundlicher Gestalt: sie wäichst aber lasch in die Länge und gewinnt damit rin streifenförmiges Aussehen.

Embryonalhüllen fehlen, wie bereits Brrula ${ }^{1}$ festgestellt hatte, dem Greleodes-Keimstreif. In der Tat sind auch nicht die geringsten Andeutumaren von Amnion und Serosa. den für die Skorpione unı fül die Inscliten so charakteristischen Irüllen, nachweishar, ein T'mstand, del um so hemerkenswerter ist, als die Achnichlorit in den Entwicklungshedin-

\footnotetext{
${ }^{1}$ A. Brrula. Beiträge zur Kenntnis des anatomischen Baues der Geschlechtsorgane
} bei den Galeodiden. Biolog. Centralblatt, 12. Band. 1892. 
gungen bei den Eicrn der viviparen Skorpione und den Eiern der viviparen Solifugen anch eine Achnlichkeit in der Entwicklungsweise häte el'warten lassen können.

I)ic Sogmentierung der vorderen Kö̈rperregion eilt del' Segmentierung der hinteren Rumpfegion nicht unerheblich voraus. In ciner anderen Arbeit" habe ich dargelect, dass der vordere Körperabschnitt der sogenannte ('ephalothorax der Arachnoiden, im wesentlichen dem Kinpforles Cephalon anderer Arthropoden homolog ist, und ich werde den letztgenannten Namen auch für die Solifugen verwenden.

Das Cephalon setzt sich bei der Embryonalanlage von Galeodes aus 7 segmentalen Zonen zusammen, die in der Reihenfolge von vorn nach hinten entsprechen: 1. den paarigen KopHappen, 2. dem Segment der Cheliceren, 3. dem Segment der Maxillarpalpen (Pedipalpen), 4. bis 7. den Segmenten der vier Gangbeine.

Ueber die Reihenfolge in dem Auftreten der Segmente im cephalen Abschnitte vermag ich nur soviel anzugeben, dass das Segment der Cheliceren in seine Entwicklung im Voroleich zu derjenigen der folgenden Kopfsegmente etwas verzögert ist. Dasselbe ist auch für verschiedene Arachnoiden festgestellt worden.

Die Kopflappen sind an Grösse den übrigen Körpersegmenten nicht unwerentlich üherlegen. Hhe Gestalt ist rine rundlich schoibenförmige, im Inner'n enthalten sie Mesoderm. Der weitere Verlauf der Entwicklung lehrt, dass die hoptlappen von Greorles aus zwei heterogenen ahere mit einander verschmolzenen Abschnitten bestehen, nämlich erstens aus cinem primälen präolalen Ahschnitt (Acron) und zweitens aus oinem prächeliceren Segment (1. Metamer). Diese beiden Abschnitte bleiben dauernd mit einander vereinigt, und nur die Gliederung des Nervensystems und zum Teil diejenige des Mesoderms deuten auf ihre gesonderte Existenz hin. Diese aus dem inneren Bau zu erschliessende Zu-

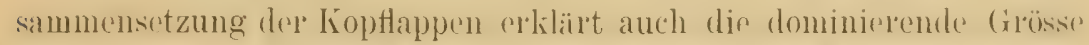
der letzteron in Verole

Auffallend ist die breite mediane Querbrücke, dic sich am Vorderende des Körpers zwischen den Koptlappen vorfindet und wie die übrigen Treile durch eine Anhäufung von Elitorlermzellen und Mrsodermzellen bedingt wird. In der medianen queren Brücke hat man die erste Anlage der Oberlippe vor Augen.

Die Oberlippenanlage von Galeodes ist somit ein unpaares Gebilde, während bei den Skorpionen und manchen anderen Arthropoden (verschiedenen Inseliten) die ()herlippo aus zwei Lüngswülsten horolgoht und somit eine paarige Anlage besitzt.

${ }^{2}$ R. Heymons. Die Entwicklungsgeschichte der Scolopender. Zoologica, Heft 33. 1901. 
I) Go Gliederung des hinter dem Cephalon folgenden Rumpfabschnittes: ("Ahelomen ) der früheren Nomenklatur") vollzieht sich langsam in der Richtung von vol'n nach hinten. Im ganzen bilden sich zehn typische Rumpfsegmente (Ablominalsegmente) aus. Ije Bezeichnumg typisch rechtfortigt sich damit. dass cin jeles dieser Seymente cine Ganglionanlage und ein Cölomsäckchenpaar besitzt. Rechnet man die bereits erwïhnten cephalen Segmente hinzu, so eroibt sich also für den Guleodesembryo die Gesamtzahl von 17 Metameren.

Hintel dem letzten Metamer (dem 10. Rumpfsegment) folgt ein verhältnismässig grosser Endahschnitt, dor' den Rest des sich von vorn nach hinten wliedernden Körpers darstellt und welcher als Telson bezeichnet werden kann. Dieses 'Telson enthält Mesoderm, doch konnte ich in ihm heine Cölomsïchehen und auch lieine Ganglionanlage mehr nachweisen, so dass eine eventuelle weitele segmentale Zusammensetzung des telsonalen Endabschnittes bei Galeodes nicht festzustellen war.

Das Telson ist in der Medianlinie mit einer schmalen Längsspalte versehen, die in die Aftereinsenkung hineinführt. Schon etwas früher ist dir Mundeinstülpung entstanden, dic in For'm einer rundlichen Grube unmittelbar hinter der Oberlippe sich eingesenkt hat.

In den hesprochemen Stadirn sind die Giliednassenanlagen schon vollzählig worhanden. I)jo Cheliceren laswen ein hasales (ilied und ein hereits scheerenförmiges distales Endstück elkennen. Ilie Maxillarpalpen und dip Gangleine sind ehenfalls sämtlich grogliedert. An dem Basalgliede der 2. bis 6. cephalen Extremität habe ich je cinen kleinen höckerförmigen Vorspr'ung bemerkt, der' als Coxalhöckel bezeichnet werden kann. Diese Coxalhörker sind an dem Basalgliede der Maxillarpalpen am dentlichsten auscrebildet. wahrend sie an den folgenden (iangbeinen weniger statk markiert sind. 1)ie Coxalhöcher der Maxillarpalpen werden zu Kitufortsätzen (Kauladen), die bei den erwachsenen Solifugen an dem genannten Extremitätenpaar deutlich zu erkennen sind.

Am vordersten Rumpfsegment (dem 8. Metamer) fehlen eigentliche Extremitatenhöcler. Das betreffonde soment ist aber durch intersogmentale Fuechen und durch eine Ganglionanlage deutlich gekemuedichnet. so diss' an der Existenz dieses nliedmassenlosen Segments durchaus kein Zweifel herrschen kann.

Die folgenden Rumpfsegmente (9--17. Metamer) besitzen sämtlich (ilielluassenanlagen, dic indessen auf dem Stadium knopttörmiger Höckel’ stehen bleiben, ohne eine Gliederung zu gewinnen. Die Gliedmassenhöcker des 9. Metamer's (des späteren Genitalsegments) sind stark ent-

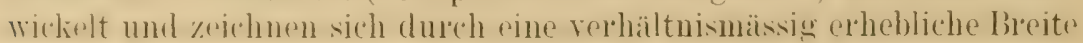
ans. Die Gliedmassenhöcker des 10.-17. Metamer's besitzen eine ziemlich übereinstimmende Gestalt, wobei sie in der Richtung von vorn nach linten eine allmähliche Abnahme ihrer Grösse erkennen lassen. 
Aus der embryonalen Oberlippe (Labrum) geht der vor'dere (obere) Teil des Rostrums hervor. Der hintere (untere) Teil des Rostrums oder die sogenannte Unterlippe entsteht beim Embryo aus zwei Höchern, die medial von den Coxalfortsätzen der Maxillarpalpen sich entwickeln. I)ie morphologische Deutung dieser häufig als Unterlippe bocichneten hinteren Partie des Rostrums hat vielfach Schwierigkeiten gemacht. Nenerdings ist die Meinung geälssert worden. dass die Unterlippe der Solifugen das Sternit (Bauchplatte) des (heliecrensegments darstelle'. Ohne das Für und Wider dieser Hypothese zu erörtern, sei hier nur die Tatsache hervorgehoben, das die fragliche Unterlippe als pariges Gebilde im Maxillarpalpensegment angelegt wird.

Ungefähr gleichzeitig mit den Unterlippenanlagen lassen sich die Stigmen erkennen. Zunäuhst erscheint das cephale Stigmenpar' in For'm

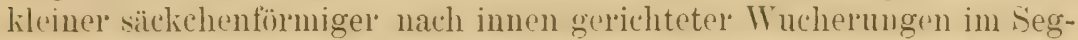
ment der 2. Gangbeine. Es folgen alsdann die Rumpfstignen (Abdoninalstigmen), die als paarige im 3., 4. und 5. Rumpfsegment gelegere Einstülpungen sich bilden. Tie Stigmen werden sowleich in situ angelegt. Für die neuerdings geltend gemachte Annahme einer Verschiebung des cephalen Stigmenpaares, das aus einem nach vorn reschobenen abdominalen Par entstanden sein soll, finden sich leine Anhaltspunkte.

Mit einem am hinteren Törperende hegimnenden Einsinken und Verseluwinden del medianen (ianglionkette sowie mit einer intrnsiven Verliürzung der Lïngsachse des lirimstreif's, der allmählich eine rhombische Gestalt anmmmt. leitet sich die Lmollumg ein. Der Umrollungsprocess bei Galeodes gleicht dem entwprechenden Vorgange bei den Arachnoiden.

Nach der Umrollung wird rasch die Umwachsung des Dotter's vollendet. Der Körper ist ventral eingekr'ümmt, sein cephaler Abschnitt, der zunächst den mit Dotter prall gefüllten kugeligen Rumpfe fest anfgrelagert ist, hebt sich nach der Geburt und dem Platzen der Eischale ab. Schon vorher hatte sich eine Cuticula gebildet. Durch allmähliches Wachstum erreicht der Embryonalkörper alsclann diejenige Fntwicklungsstufe, die schon vor geraumer Zeit durch die Beschreibung von Conseberc" heliannt gewolden ist. Schliesslich platzt die embryonale Cuticula, es erfolgt eine Hüutung, und die junge Solifuge ist nummehr freier Bewegungen fähig.

I)er hier natürlich nu' in oinigen Glundzügen skizzirte Entwichlungsverlaut lässt keine Zweifel mehr obwalten. das's es sich bei den Solifugen um typische Arachnoiden handelt.

${ }^{1}$ C. Börner. Arachnologische Studien. Zoolog. Anz., Bd. XXV, Nr. 673/674. 1902.

2 A. Croneberg. Ueher ein Entwicklungsstadium von Galeodes. Zoolog. Anzeiger, Bd. X. 1887. 
Die Alt und Weise ller Segmentirung, das Aufreten eines auch bei Spinnon beschriehenen präicheliceren Segments, die (hier nicht näher heschriehene) Konfiguration der Segmente und die Gestalt der an ihnen herorsprossenden (iliedmaassen, die Tatsache, dass das erste Rumptserment wliedmaassenlos bleibt, der Lmstand, dass das zweite Rumpfsoment zum Crenitalsegment wird, die Bildung des Rostrums und endlich auch noch viele Eigentümlichleiten der Organogenese beweisen zur Evidenz die vollkommene Arachnoidennatur von Galeodes.

Von irgend einer Annäherung an die Körperbildung der Insecten ist bui der Embryologie dieser Solifuge nichts zu bemerken, dagegen lässt es sich nicht verkennen, dass manche principielle Lebereinstimmungen mit der Embryologie von Limulus existiren.

Die Uebereinstimmungen in der Entwicklung der Xiphosuren und Solifugen kommen z. B. darin zum Ausdruck, dass bei den Embryonen von fialendes auch an den Gangheinen kleine den Coxalfortsitzen ron Limulus offenbar entsprechende Höclier hervortleten, und sie zeigen sich vol allem in der Bildung parriger homologer Lateralorgane, die ich schon früher eingehend beschriehen habe. Rechnet man hierzu die für Arachnoiden und Xiphosuren überhaupt übereinstimmende Art der Kö̈rperbildung und Segmentirung, die bei beiden Gruppen übereinstimmende Anlage der Geschleclitsöftnungen am 2. Rumpfsegment (dem 9. postoralen Metamer). so lïnnen diese Tatsachen zusammenenommen wohl nieht anders als Beweise dines gemeinsamen Oromanisationsplanes angeschen werden.

Bekamntlich sind gegen die Limulus-Hypothese, d. h. gegen die Annahme einer Verwandschaft zwischen den Xiphosuren und Arachnoiden vielfach Bedenken erhoben werden, die anch noch neuerdings durch Ziegrer ${ }^{1}$ wiederum ihren Ausdruck fanden.

Ich kann mich diesen Bedenken nicht anschliessen, sondern betrachte die Gigantostraken nebst Xiphosuren und Arachnoiden als eine zusimmengehörige natürliche Arthropodenabteilung, für die ich in meiner oben citirten Arbeit den Namen Chelicerata in Volschlag gehracht habe.

Wenn man die Cheliceraten als eine gemeinsame und im Grunde cinheitliche Tiergluppe anerkennt. so scherint mir der Streit, ob man num die Cheliceraten von wasserbewohnenden Gigantostraken oder oh man sie von landbewohnenden Arachnoiden abzuleiten habe, von verhïltnissmässig geringer. Wichtigleit zu sein. Irh sellst hin geneigt, in

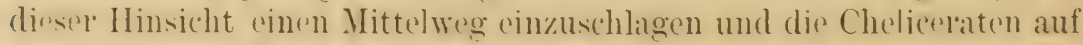
gemeinsame an feuchten Orten lebende Vorfahren (Ufertiere) zurück-

${ }^{1}$ H. E. Ziealer. Das zoologische System im Unterricht. Verhandlungen d. deutschen zoolog. Gesellschaft. 1904. 
zufühıren, bei denen die heutzutage allerelings sehr auffallende Differenzirung der Atmungsorgane noch nicht so stark ausgeprägt war. In analoger. Weise ptlegen noch jetzt bei den reeenten nioderen Inscliten und niederen Myriapoden, die nur an feuchten Orten vorkommen, dic: Atmungsorgane noch nicht so einseitig ausgebildet zu scin, indem sowohl Tracheen als auch gleichzeitig kiemenähnliche in diesen Fällen ausstülpbare Bläschen zur Atmung dienen.

Ueber den Körperbau der Cheliceraten-Stammformen werden wir imlessen nicht über Vermutungen hinauskommen. Börnen ' hat allerdings gemeint, dass wir " dem gemeinsamen Ahnen der heute zu unterscheidenden Gruppen der Xiphosuren, (ijgantostraka und Arachnida bereits das Fehlen von Extremitaten an seinem hinteren aus 6 freien Segmenten bestehenden Kürperabschnitt zuschreiben " dürftrn. Derartige Erkläıungen scheinen verfrüht zu sein. Bei den Embryonen von Guleodes sind sämmtliche Iimmpfsegmente (mit alleiniger Ausnahme des ersten) mit deutlichen später wieder verschwindenden Extremititenanlagen versehen, und in der Ausbildung der vorderen und hinteren Rimpfsesmente findet sich kein Unterschiod. I)ie Polypodie der Gruleodes-embryonen deutet auf polyporle Ahnenformen wenigstens für die Solifugen hin. In der 'Tat ist es auch schwer zu verstehen, warum die Urformen der Cheliceraten nicht chenfalls polypod gewesen sein sollen, wie dies zweifollos bei den Stammformen dep hriden anderen Hauptgruppen von Arthropoden den 'T'eleiocerata (Trilobita, Crustacea) und Atelocerata (Myriapoda, Insecta) der Fall gewesen sein muss.

Jedenfills hat die von Börsen angenommene Einteilung des Cheliceratenkï̈rpers in ein Prosoma, Mesosoma und Iretasoma für die solifugen leine Gültigkeit; denn bei ihnen ist die Rumpfgliederung eine vollkommene homonome, und es liegt keine Veranlassung vol', diese homonome Gliederung des Rumpfes als eine sekundäre anzusehen.

Meiner Ansicht nach haben die Solifugen in diesem wie auch in verschjedenen anderen Merkmalen noch ursprüngliche Charaktere bewahrt. Ob) dies auch für die Abgliederung dreier freier cephaler Segmente (sogenannter" "Thoraxsegmente 》) gilt, lässt sich allein auf Grund der Embryologie nicht entecheiden, doch glauhe ich die Absonderung dieser Segmente als eine sekundäır Figentümlichkeit ansehen zu können, wie ich auch die Aushildung cines cephalen Stigmenpaares für rine sekundäre Er'werbung dieser 'liere halte.

Die Frage mit welcher Arachnoidengruppe die Solifugen am nächsten verwandt sind, kamn hiel nicht erschöpfend hehandelt werden. Ich mache nur darauf aufmerksam, dass sie in der Embryologie Cobereinstimmum-

1 Loc. cit. 
gen mit den Pedipalpen (Telyphonus) zu erkennen geben, die namentlich in der Aehnlichkeit der Keimanlagen sowie in der Ausbildung homologer Lateralorgane beruhen.

Die während des Vortrages erläuterten Zeichnungen verschiedener Embrynalstadien von Galeodes werden in der ausfülnrlichen Arbeit veröffentlicht werden.

\title{
Die phylogenetische Umbildung ostindischer Ameisengäste in Termitengäste.
}

\author{
Von E. WASMANN (Luxemburg).
}

Mit 1 Tafel.

Vielleicht erweckt der Titel dieses Vortrages in Manchem von Ihnen die Vermutung, es handle sich um eine jener vagen phylogenetischen Spekulationen, nach denen eine For'm $b$ aus einer Form a sich entwickelt haben soll, während man vielleicht mit demselben Rechte die Form a von der For'm $b$ ableiten und die ganze Entwicklungsieihe umkelnen liönnte. Ich war niemals ein Freund solcher phylogenetischer Stammhäume, welche mehr der Phantasie als den Tatsachen entspringen, indem sie kühne Möglichkeiten oder blasse Wahrscheinlichkeiten für wirkliche Beweise einer stammesgeschichtlichen Entwicklung hinnehmen. In Gegenteil, obwohl ich die Entwicklungstheorie für wissenschaftlich unentbehrlich halte, so stehe ich doch ihren einzclnen Hypothesen mit kritischor Skepsis gegenüber und halte es für die strenge Pflicht eines jeden wahrheitsuchenden Naturforschers, nicht bloss das p ro sondern auch das contra derselben möglichst allseitig zu untersuchen ${ }^{1}$.

Wenn ich zu Ihnen also trotzdem in dieser Stunde über die phylo-

1 Meine Anschauungen über die Descendenztheorie und ihren heuristischen Wert habe ich in einem soeben erschienenen Buche "Die moderne Biologie und die Entwicklungstheorie ». Freiburg i. B., 1904, näher dargelegt. 
genetische IJmbildung ostindischer Ameisengäste in Tormitengïste sprechen will, so muss ich von vornherein bemerken, dass ich dieses kühn klingende Thema gar nicht gewählt hahen würde, wenn nicht so schwerwiegende tatsächliche Beweise für meine Hypothese existierten. Ich will Ihnen den Sachverhalt möglichst kurz mit Hilfe einger Abbildungen vorführen, damit Sie sich ein Ureteil darüber bilden können, inwieweit meine Erklärung der betreffenden Erscheinungen wirklich beweiskräftig ist. Ferner habe ich in rinem Kä̈stchen eine Peihe der einschlägigen Staphylinirlen-Arten aus meiner Sammlung mitgebracht, die ich Ihnen zur Besichtigung vorlege.

Meine These umfasst folgende Teile:

1. Innerhalh der dorylophilen Kurytlüglergattung Doryloxemen, deren simtliche Anpassungscharaktere ant die Lebensweise bei Wanderameisen hinweisen, hat in Ostindien vor verhältnismässig kurzer geologischer Zeit ein biologischer Wirtwechsel stattgefunden, durch den mehrere termitophile Doryloxenus-Arten entstanden sind.

2. Diese telmitophilen Doryloxemus Ostindiens bilden einen Uebergang zur ostindischen termitophilen Gattung Dismenus, welche bereits zum termitophilen Trutztypus der Alencharinenverwandten gehört, und welcher daher stammesgeschichtlich wahrscheinlich von dem dorylophilen Trutztypus der Gattung Doryloxems abzuleiten ist.

3. Die Arten der Gattung Discoxems bilden ihrerseits wiederum einen ['obergang zu den Arten der Gattung Termiturlisons, welche den vollkommensten 'Trutztypus der ternitophilen Aleocharinen verwandten Ostindiens und Südafrikas repräsentiert.

4. Die Entstehung des termitophilen Trutztypus diesel' Gattungen ist somit stammrsgeschichtlich sehl wahrscheinlich dal'auf zurüchizuführen. dass kleine Ameisengäste des dorylophilen Trutztypus bei Raubzügen von Wanderameisen auf den Ameisen - wie gewöhnlich - reitend in 'T'ermitennester' gelangten, dort zurückblieben und zu Termitengästen wich mmbildeten. I)ie termitophile (rruppe dex Termitodiscini ist somit stammeseschichtlich ron der dorylophilen (iruppe der Pygosterini abzuleiten.

5. Zur Erklärung der stammosgeschichtlichro Fntwicklung der tormitophilen Doryloxenus, Discoxenus und Termitodiscus müssen wir 
vou Dompluxems-Ferwandten zur termitophilen Lebensweise annehmen, und zwal fül Ostindien allein einen zweimaligen, für den afrikanisch-indische'n liontinent einen einmaligen. Iem jüngsten jenel biologischen Uebergänge, der wahrscheinlich crst in der Diluvialzeit erfolgte, entstammen dir heutigen termitophilen Doryloxems-Arten Ostindiens. I)'m mittleren jener Uel)ergänge, der' wahr'scheinlich in das Ende der Tertiälzoit fillt, entstammen die Arten der termitophilen Gattung Discoremus ()stindiens. Dem ältesten jener drei Uebergänge endlich, der wahrscheinlich schon in der Mitte der Tertiäzoit erfolgte, entstammen die Arten der trimitophilen Gattung Termitudiscus Afrikas und (ostindiens.

Ich will nun die einzelnen Punkte näher erklären.

A d. 1. Die Arten der Gattung Doryloxemus (Fig. 1-3), zu Fauvel's Pygrosterimi gehörig. sind kleine Kurztüglel von fast spindelförmigel Grestalt, nit stark gewölbtem Vorlerkörper und kegelförmig zugespitztem Hinterleib. I) liopf ist in den Vorderrand des Prothorax so eingefügt, dass seine Seiten eine gemeinsame Curve mit denjenigen des Prothorax bilden. I)io dicken hornförmigen Fühler 'ntspringen auf der Lntriseite des Kopfes. welche auch die Mundtrile trägt. Die Beine sind sehr kurz, dicht bedornt und haben völlig rulimentäre Tarsen, welche statt der Tarsenglieder lange Stacholn und weisse Hafthaare tragen (Fig. 3).

Schon bevol noch die Lebensweise dieser Tierehen durch Beohachtung feststanl. hatte ich hei Beschreibung des rosten Doryloxemus aus Sürlafrikia, der unter. Steinen bei Dorylus heleolus gefunden wurde, die Ansicht ausgesprochen, dass diese kleinen Käfer mittelst ilner zu Haftorganen ungewandelten Tarsen an ihre Wirthe orler an deren Larven sich festklammern, um so den Zügen derselben folgen zu könmen. Spätere Beobachtungen hahen dies bestätigt. Doryloxems eques wurle von P. Kons am oberen Congo auf Ammma burmeisteri-rubella reitend gefundern. Der Käfer sitzt auf der ()herseite des Hinterleibes der Ameise, an den er sich durch die Gestalt seines Vorderkörpers so anschmiegt, dass man iln, zumal seine Färbung derjenigen des Hinterleibes der Wirte gleicht. nur dann bemerken kann. wenn er seine Hinterleibspitze bewegt. In ähnlichel Wrise reitet nach P. Kons's Beolachtungen Doryloxemes Kühli auf Ammmu Wilcerthi am ohern Congo, ferner nach den Funden jedoch nicht hlos einen einmaligen, sonder'n wahrscheinlich einen deeimaligen. in verschiodenen geologischen Epochen erfolgten Uehergang E. LuJA's Doryloxenus Lujæe, den ich Ihnen in Figur 1 stark vergrösirert vorführe auf derselben Treiberameise an untern Congo, und Doryloxems anmulatus auf Anomma Bumeisteri-molesta am Zambesi .

' Vgl. Zur Kenntnis der Gäste der Treiberameisen und ihrer Wirthe am oberen Congo. Zool. Jahrb., Suppl. VII, p. 611-682 u. Taf. 31-33, S. 650 u. 667. 1904. 
Bitte nun die Figuren 1 und \&े zu betrachten. Die ganze Körpergestalt von Doryloxemus bildet einen vollendeten Trutztypus (Fig. 1) der die kleinen Kïfer unangreifbar macht für die Kirefer ihrer Wirthe und der ihnen zugleich das Reiten auf dem Rürken derselben durch die Haftbildungen dex Tas'sen elmöglicht. Fig. 3 zoigt Vorderfuss und Schienen-

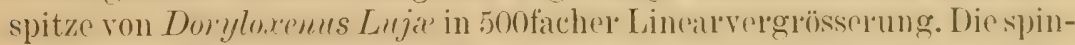
delförmige Körpererestalt von Doryloxemes, sein dureh den seitenrand des Ilalsschildes geschützter Kopf, die hornförmig zugespitzten, auf der Unterseite des Kopfes entsplingenden Fühler, sowie endlich auch die Verkümmerung seiner Tarsen und ihne Umwandlung in Haftorgane sind die systematischen Gattungscharatere von Doryloxems. Da dieselben zugleich Anpassungschalaktere sind, welcheden dorylophilen 'Trutztypus von Doryloxems bilden und ihn zugleich befähigen, seine unsteten Wirthe als Reitel zu begleiten, müssen wir vom entwicklungstheoretischen Standpunkte aus zweifellos sagen: I) ie Gattung Doryloxemus ist durch und durch ein Anpassungsprodukt an die dorylophile Lebensweise.

Ich war daher nicht wenig erstaunt, als mir der erste termitophile Doryloxemus aus Ostindien zugesandt wurde. Ex befand sich zugleich mit anderen Termitengästen in einem Gläschen, das Termes obesus subsp. vallonensis aus Wallon im Ahmodnagardistrikt enthielt. mud das mil von meinem Hoissigen Corpespondenten P. .J. I. Heni s. J. zugesandt worden war. Ich glaubte sicher annehmen zu müssen, der Finder habe sich vergriffen und aus Versehen einen Dorylinengast in das Gläschen mit den Termitengästen gestrekt. In diesem sinne selurjeh ich anch sofort

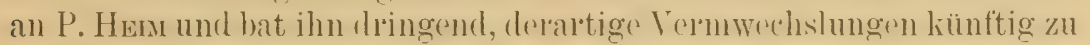
vermeiden. Aber der Irrtum war auf meiner Seite, und dem Entdecker des ersten ostindischen Doryloxemus, den ich später als Doryloxenus

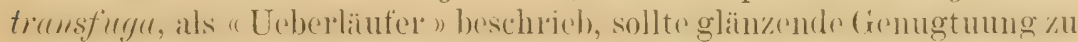
'Teil werden. Bevor noch mein Brief an P. Heis in Indien angelangt sein konnte, erhielt ich von einem andern meiner ostindischen Correspondenten, P. J. Assuutr, eine Sendung von Gästen des Termes obesus i. sp. aus Khandala im Bombay-Distrikt. Da fanden sich Doryloxenus in beträchtlichel Anzabl aus verschiedenen Bauten jener Termite und zwar ausser dem Doryloxenus transfuga noch eine zweite, häutigere Art, die ich als Doryloxenus termitophilus beschrieh. An der Tatsache, dass es in Ostindien termitophile Doryloxems gebe, war somit nicht meh" zu zweifeln. Es konnte sich nur noch darum handeln, dieses biologische Rätsel entwicklungsteoretisch zu lösen.

Vergleichen wir nun die Fig. 1, welche den dorylophilen Doryloxenus Lujæe darstellt, mit Fig. 2, die den termitophilen Doryloxemus transfuga widergibt. Die Körpergestal t, die dem Trutztypus der Iborylinengäste angohört, ist bei heiden dieselbe. Ebenso auch, wie 
ich dureh mikrosliopische Präparate der Beine mich überzeugt habe, ist die Tarsen bild ung bei beiden identisch. Die Fig. 3, welche Vorderfuss und Schienenspitze von Doryloxemes Luje darstellt, könnte ebensogut auch von Dorylorems transfuga oder termitophitus genommen seiu. Iie wesentlichen Anpassungscharaktere, welche die Vertreter der Gattung Dorylorems zu Dorylinengästen des Trutztypus machen, die auf Wanderameisen reiten, sind somit auch bei den termitophilen Arten dorsellon Gattung völlig erhalten geblieben. Enterschiede zwischen den dorylophilen und den termitophilen Doryloxemes zeigen sich nur in folgenden Punkten (vgl. Fig. 1 u. 2):

1. Der Vorderteil des Kopfes ist bei den termitophilen Arten tief eingedrüclit mod herahgedrüclit. als ob er im Begriffe stehe, auf die Tnterseite des Körlpers hinalızuwandern. Bei den dorylophilen Arten dagegen ist er flach oder nur schwach eingedrüuckt.

2. Die Fühler der termitophilen Arten sind zwar ebenfalls spitz hornförmig wie bei den dorylophilen Arten, aber hedeutend länger und dazu weiter vol'ragend.

3. Ibe Skulptur des Kïrpers ist hei den dorylophilen Arten wenigstrus auf dem Hintrleih) stets reibeisenartig rauh punktiert, und mehr odre reniger dicht beborstet. Bei den termitophilen Arten dagegen ist die ganze Oberseite des lörper's spiegelglatt, unpunktiert und unbehart. nur die Seiten und die Spitze des Hinterleibes sind mit langen schwarzen Borsten besetzt.

Das Verständnis dieser Eigentünlichkeiten der termitophilen Dor!yloxemes wird uns später dureh den Vergleich derselben mit der termito-

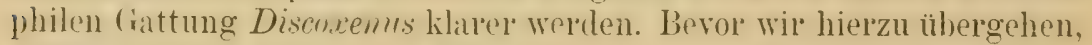
müssen wir jedoch noch die Schlusifolgerung aus den bisher erwähnten Tatsachen ziehen.

Wie sollen wir uns das gesetzmässige Vorkommen von termitophilen Doryloxeme-Arten in Ostindien stammesgeschichtlich erliaren? Inass dieselben einer Anpasiung an die gegenwärtige termitophile Lebensweise ihren Gattungscharaktel verdanken. der sie zu unzweifelhaften. echten Doryloremes macht, das düufte vollkommen ausgeschlossen sein. Jagegen suricht nämlich nicht bloss ihre ganze Körpergestalt, die dem dorylophilen Trutztypus der afrikanischen Doryloremus entspricht. sonder'n vor Allem anch die Verkümmerung der Tarsen und die Verwandlung dersellen in Haftorgane, die nur bei dorylophilen Staphyliniden vorkommt. Dieser Anpassungscharakter kann nur entstanden sein zu drm biologischen Zwecke, seinen Besitzern das Reiten auf dem Rücken von unsteten Wanderameisen zu ermöglichen; bei Gästen del durchaus sesshaten Termiten. deren Kolonien in denselben festen Banten . Jahrzhhnte lang wohnen, ist die Entstehung eines derartigen An- 


\section{TAFELERKLÄRUNG}

Fig. 1. Doryloxenus Luja Wasm., $22: 1$.

Fig. 2. Doryloxenus transfuga Wasm., $22: 1$.

Fig. 3. Vorderfuss und Schienenspitze von Doryloxenus Luja, 5000:1.

Fig. 4. Discoxenus lepisma Wasm., 22: 1.

Fig. 5. Termitodiscus Heimi Wasm., 22 : 1. 

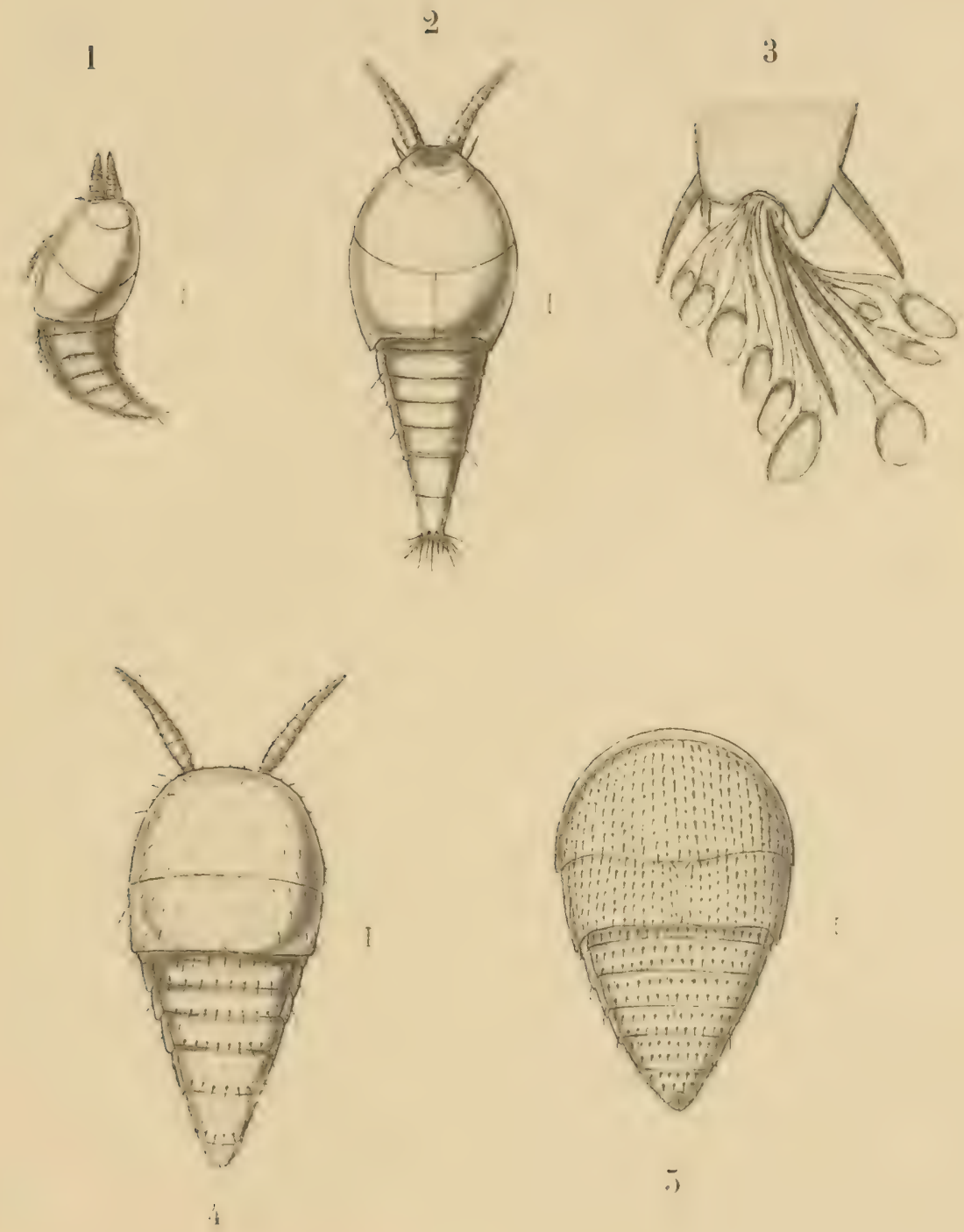

E. WASMANN 

passungscharakters fast ebenso undenkbar wie bei cinem Maulwurf die Entwicklung von Flugorganen.

Die gegenwïrtig in Termitennestern Ostindiens lebenden beiden Doryloxemis-Arten müssen also früher Dorylinengäste gewesen sein, wie ihre afrikanischen Verwandten es heute noch sind. Also hat in der Gattung Doryloxenus in Ostindien ein stammesgeschichtlicher Wirtswechsel stattgefunden, durch welchen ehemalige Dorylinengäste $z$ u 'Termitengästen wurden. Diesen ersten Teil meiner These halte ich nicht bloss für wahrscheinlich, sondern für sicher feststehend. Die Frage kann nur noch sein, wann und durch welche Ursachen jener Wirtswechsel erfolgte.

Da die termitophilen Doryloxenus-Arten Ostindiens von ibren dorylophilen Vorwanden in Afrika nur in relativ unbedeutenden spezifischen Charakteren abweichen, müssen wir annehmen, dass ihre Verwandlung aus Ameisengästen in Tormitengäste vor verhältnismäsigg kurzer geologischer 'zeit erfolgte, vielleicht erst während der Iiluvialepoche. Auch vermögen wir die äusseren Ursachen, die zu jener Umwandlung den Anlass gaben. ziemlich sicher anzugeben. Ina die Itorylinen gefräsige Insphtemrüuber sind, die auf ihren Raubzügen besonders gerne Termitennester üherfallen, un deren weichhäutige Bewolnner als Beute zu verzehren, wofür auch direkte Beobachtungen E. LuJA's vom unteren Congo mir vorliegen ${ }^{1}$; da ferner die Dorylinengäste und namentlich die auf ihren Wirten reitenden kleinen Doryloxemus an diesen Raubzügon der Wanderaneisen teilnehmen, liann es leicht geschehen, dass doryophile Doryloremus-Arten in Termitennester welangten. Wurden sie in den engen Gängen der Termitenbauten zufällig von ihren Reittieren abgestreift, so lionnte es lei(jut vorkommen, dass sie beim Abzug der Räuber in dem Termitenneste zurückbleiben mussten. Dann Waren sie eben, wenn sie nieht unliommen wollten, anf die Gresellschatt der 'Termiten fürder'hin angewiesen.

Aber warum kennt man denn bisher nur aus Ostindien termitophile Doryloxenus ${ }^{2}$, nicht auch aus Afrika, wo die dorylophilen Arten derselben Gattung zahlreich vorkommen?

Dass nicht auch in Afrika noch eine derartige Entrleckung gemacht werden könnte, will ich keineswegs behaupten; denn hier gilt das bekannte Sprichwolt der Franzosen : il ne faut jamais dire "jamais ". Im-

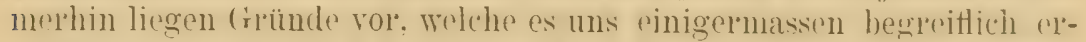
scheinen lassen. Weshalb gerarle aus Ostindien termitophilo Doryloremes bekiant sind. während man von dort noch keine dorylophilen drten

${ }^{1}$ Vgl. Zur Kenntnis der Gäste der Treiberameisen, u. S. w., S. 673.

2 Wahrscheinlich ist auch Doryloxenus (Himocete) phoca Fauv. aus Colombo (Ceylon) termitophil, da er dem Doryloxenus transfuga sehr ähnlich ist. 
derselben (iattung kennt. Gegenwärtig gibt es nämlich daselbst keine oberirdisch wandernden Dorylinen mehr, sondern nur unterirdisch wandernde aus den Gattungen Dorylus s. str. und Aenictus. Zur Zeit dagegen, als ()stindien mit Mittelafrika zu cinem einzigen Kontinente verbunden war, verbreiteten sich wahrscheinlich die heute noch in Afrika zahlreich vorkommenten oberirdisch wandernden Anomma auch über Indien. Als mit dem Ende der Tertiärzeit Ostindien von Afrika durch einen ()zean getrennt wurde, traten die oberirdisch Iebenden Dorylinen in Indien allmählich an biologischer Bedeutung zurück, bis nur unterirdisch lebende Arten übrig blieben. Eine derartige Aenderung in der Lebensweise der Dorylinen Indiens konnte von wichtigen Folgen füı manche ihrer angestammiten Gäste scin. I)a es den Doryloxemus, die auf dem Hinterleib ihrer. Wirte reiten, ohne Zweifel schwerer ist, den hypogäen Dorylimen zu folgen als den epigäen, weil sie in den engen unterirdischen Gängen leichter abgestreift werden, so konnte es von wirklichem Vorteil für sie sein, von der dorylophilen Lebensweise zur termitophilen üborzugehen. Ilje Anpassungscharakteje, welche sie zu Dorylinengästen des 'l'utztypus machen, schadeten ihnen wonigstens für ihren Aufenthalt bei Termiten gar nicht; im Gegenteil, sie nützten ihnen auch hier, indem sie die Gäste weniger angreifhar für die Kiefer der Soldaten und Arbeiter der Termiten machten. Weshalb sich die rigentümliche Tarsenbildung auch bei den termitophilen Doryloxems so lange erhalten hat, ist allerdings schwerer zu sagen. Vielleicht ist sie ihnen von Nutzen, um hei dem Ausschwärmen der geflügelten Termiten auf dem Kïrper derselben sich festzuklammern und so in die neu zu gründenden Ter'mitenkolonien zu gelangen.

A d. 2 und 3. Wir kommen jetzt zum zweiteu und dritten Teil unserer Untelsuchung, wo eigentlich elst die Hypothese beginnt; denn dass die ostindischen Doryloxemus ursprünglich Iborylinengäste waren und erst später 'Termitengäste wurden, ist nicht mehr als hypothetisch, sondern als sicher zu bezeichnen. Vergleichen wir nun die termitophilen Doryloremes ()stindiens (Fig. 2) mit der ostindischen termitophilen Gattung Discoremus (Fig.4). Unter den Gästen von Termes ubesus, wolche P. Assiutu mil sandte, waren neben den termitophilen Doryloxenus auch zwei Arten von sehr ähnlichen Staphyliniden vertreten, die aber bei näherer ITntersuchung als zu einer rigenen neuen (rattung gehörig sich erwiesen, welche ich als Disroxemus beschrieb)'. Die beiden, in ihreer Gritalt kloinen Lepismen ähnlichen Arten sind Discoxems leprisma (Fig. 4) und Assmuthi. Die erstere wurde später von P. Hen auch bei Termes obesus-uallonensis gefunden. Ine Gattung Discorems, die ich

1 Zur Kenntnis der Gäste der Treiberameisen, u. s. w., S. 65ว̆-656. 
Ihnen in Fig. 4 stark vergrössert vorführe, steht morphologisch genau in der Mitte zwischen den termitophilen Doryloxemes und dor termitophilen Gattung Termitodiscus, welche den vollkommensten Trutztypus der indischen und afrikanischen termitophilen Aleocharinenverwandten repriascntiont. Termitodiscus Heimi (Fig. 5) ist in fast allen Nestern von Termes obesus und T. obesus-uvellonensis in Vorderindien häufig und lag in hunderten von Exemplaren vor.

Betrachten wir nun Discoxemus lepisma (Fig. 4) im Vergleich zu Doryloxemus transtiuga (Fig. 2) etwas nïher. I)ie habituolle Aehnlichle it zwischen beiden, die sic auf den ersten Blick fast verwechseln lässt, beruht darauf, dass auch bei Discoxemus die hörpergestalt hinten kegolförmig zugespitzt ist und dic hornförmigen, ja fast spondolförmigen Fühler auf der Unterseite des Kopfes vorragen. Aber Discoxenus ist bedeutend hreiter und flacher gobaut als Doryloxemes und nïhert sich hiorin bereits Termitodiscus (Fig. 5). Ferner ist der Kopf, der bei Doryloxenus von oben sichtbar und bei den termitophilen Arten vorne tief einged'ückt ist, bei Discoxenus bereits vollkommen auf die Unterscite des Halsschilds hinabgerückt, so dass man ihn von oben gar nicht mehr sehen kann; auch hierin nähert sich Discoxenus dem Termitodiscus. Endlich ist die Tarsenhildung von loiscoxemus ganz verschied('n von jener der Doryloxenus: die 'T'arsen sind normal, viergliedrig wie bei Termitodisrus, nicht rudinentär, eingliedrig und zu Haftorganen umgewandelt wie bei Doryloxenus.

Discoremes gehört durch den scheibenförnigen, Hachgewöll,ten Vorderkörper, von dem el seinen Gattungsnamen erlielt, und durch den välig vontral gelegenon Kónf bereit zum Trutztypus der termitophilen Alrocharinenverwanlten Ostindiens. Auch seine Skulptur und Behaaı'ung nähert sich letrtrom Typus, indem dir spiegelglatte Ohneflächr mit nach hinten gerichteten reihenweisen Börstchen besetzt ist, die auch bei den neotropischen Aleocharinenvorwanden des termitophilen 'Trutztypus, bei Trichopsenius, Termitopsenius und Kallopsenius vorhanden sind und in verkürzter und verdickter For'm auch bei T'ermitodiscus sich wiedertinden.

Wenn eine Doryloxenus-Art vom dorylophilen Trutztypus der Pygostenini zum termitophilen der Termitorliscini übergehen wolltr, so musste sie erst zu einer Discoxenus-Form werden, bevor sie die Vollkommenheit dieses letzteren Typus in Termitorlisens erreichen konnte. Vergloichend morphologisch betrachtet bildet somit Discoxenus (Fig. 4) die zweite Stufe des [ehergangs vom dorylophilen 'Trutztypus zum termitophilen. Die erste Stufe jenes Ueberganges lernten wir bereits in den termitophilen Doigloxemes-Arten (Fig. 2) kemnen, doren Kopphildung und Skulptur zu Discoxenus überleitet. Dic dritte und letzte Stufe ist endlich in Termitodisens (Fig. 5) repräsentirt, dessen Vorderlïrpere 
vollkommen scheibenförmig flach von halbkreisformigem Umriss des Halswchildes ist, mit ringsum abgesetzten und etwas aufgebogenen Rändern, die sich desshalb an die Unterlage eng anlegen, während der Hinterleib platt und liurz kegelför'mig erscheint: bei eingezogenem Hinterleih ist sogar der ganze Kör'per scheibenförmig: daher der Gattungsname "Termitenscheibchen ». Die stark glänzende Oberseite ist mit rauhen Punkten (Körnchen) besetzt, aus denen ein kurzes gelbes Bürstehen entspringt. Bei Termitodiscus liegt fermer der Kopf nicht bloss vollkommen auf der Unterseite des Halsschildes, sondern die bei Discorems noch langen, spindelförmigen Fühler sind hier sehr kurz und breit, r'uderför'mig plattgedrückt, ganz ähnlich wie bei den Cephaloplectini (Xenocephalini) der neotropischen Ecitongäste. Diese liurzen, platten Fühler bilden ein ausgesprochenes Element des Trutztypus, indem sie ganz unter dem Halsschilde verborgen werden können, an dessen Unterseite (vor den Vorderhüften) sie sich enge anlegen, so dass sie den Angriffen der Ameisenkiefer oder Ter'mitenkiefer gänzlich entzogen sind.

Vom vergleichend morphologischen Standpunkte aus sind wir daher vollkommen berechtigt, die termitophilen Doryloxemus-Arten, die termitophile Gattung Discoxemus und die termitophile Gattung Termitodiscus als drei a u feinanderfolgende stammesgeschichtliche Stufen der Entwicklung des termitophilen Trutztypus aus dem dorylophilen Trutztypus von Doryloxenus zu betrachten. Sehr' merkwürdig ist hiehei, dass die beiden letzteren Gattungen, wie bereits hervorgehohen wurde, völlig normale, viergliedrige Tarsen haben. Die Imwandlung der Tarsen von Doryloxemes zu Haftorganen ist also hier scheinhat wiederum r'ückgäingig gemacht worden. Warum ich "scheinbar 》 sage, wird sich später (ad. 5) zeigen.

Der zweite und der dritte 'Theil meiner eingangs aufgestellten These dürfte jetzt hinreichend klargelegt sein, zumal wir uns auf eine Erörterung der morphogenetischen Faktoren, die jenem Umwandlungsprozesse zu gr'unde lagen, nicht einlassen können. Ich gehe daher zum vierten Theil der' These über.

Ad. 4. Die Eintstehung des termitophilen Trutztypus der Gattungen Disroremes und Termitodisens ist somit stammesgeschichtlich sehr wahrscheinlich darauf zurück zuführen, dass kleine Ameisengäste des dorỵlophilen Trutztypus bei Raubzügen von Wanderameisen, auf den Ameisen - wie gewöhnilich - reitend, in Termitemnester" gelangten. dort zurüekbleken und zu Termitengästen sich umbildeten. Die termitophile Gruppe der Termitodiscini ist somit stammesueschichtlich von der dorylophilen Gruppe der Pygostenini abzuleiten. 
Vom vergleichend morphologischen Standpunkte ans ist dieser Theil unserer These bereits hinreichend erörtert worden. In biologischer Beziehung haben wir schon olven (ad. 1) den Nachweis erbracht, dass die termitophilen Doryloxemes-Arten Ostindiens dadurch entstanden sein müssen, dass ihre Vorfahren auf rüuberischen Iorylinen reitend in die Termitennester eindrangen, dort zurückgelassen wurden und dann allmählich die Richtung zum termitophilen Trutztypus einschlugen, insbesondere durch die Aenderung ihrer Kopfstellung und ihrer Skulptur. Es scheint mir sehr naheliegend, ja fast unabweisbar zu sein, dass wir auf demselben Wege, durch konsequente Fortsetzung desselben Umbildungsprocesses auch die stammesgeschichtliche Entstehung der Gattungen Discoxemus und Termitodiscus zu erklären haben. Wir können daher bereits zum 5. Theile unserer These übergehen:

Ad. 5. Zur Hirklärung der stammesgeschichtlichen Entwicklung der termitophilen Doryloremus, Discoxemus und Termitodiscus müssen wir jedoch nicht blos einen eimmaligen, sondern wahrscheinlich einen dreimaligen in rerschiedenen geologischen Epochen erfolgten Uehergang ron Doryloxemus-Verwandten zur termitophilen Lebensweise annehmen, und zwar für Ostindien allein einen zweimaligen, für den afrikanisch-indischen liontinent einen rimmaligen. Iem jüngsten jener binlngischen Ueherginge, der wahrscheinlich erst in der Diluvialzeit refolgte, entstammen die heutigen termitophilen Doryloxemus-Arten Ostindiens. Dem mittleren jener Uebergänge, der wahrscheinlich in das Ende der Tertiärzeit fällt, entstammen die Arten der termitophilen Gattung Discoxenus Ostindiens. Dem ältesten jener drei Uebergänge endlich, der wahrscheinlich schon in der Mitte der Tertiärzeit erfolgte, entstammen die Arten der termitophilen Gattung Termitodiscus Afrikas und Ostindiens.

Der oben skizierte stammesgeschichtliche Prozess ist nicht so einfach, wie er auf den ersten Blick zu sein scheint. Zwischen den termitophilen Doryloxemus und der Gattung Discoxemus in Ostindien liegt vom vergleichend morphologischen Standpunkte aus noch eine weite Kluft. Die vermittelnden Uebergangsstadien. die uns das eigentlicher Herabrüeken

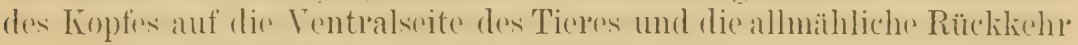
zur normalen Tarsenbildung veranschaulichen, fehlen einstweilen noch. Eine mindestens ebenso weite Kluft besteht ferner auch zwischen den (rattungen Liscruemus und Termitrdisrus; zwischen diesen beiden Entmicklungsstufen des termitophilen Trutztypus liegt nicht blos eine Re'lhe von Aenderungen der Körperform und Skulptur, sondern auch eine gainzliehe Umbildung der Fühlergestalt. Ioürfen wir demnach annehmen. die Gattung Discoxenus stamme unmittelbar ab von unseren heutigen 
termitophilen Dorylems und die Gattung Termtiodiscns ron unseren heutigen Discoxemus?

Nein. das dürfen wir meines Erachtens nicht. Die hentigen termitophilen Doryloxemus, Discoxems und Termitodiscus stellen zwar drei verschiedene stammesgeschichtliche Entwicklungsstufen ein und dessclben Entwicklungsprozesses dar, der vom dorylophilen Trutztypus zum termitophilen führt. Aber wir dürfen uns diesen Entwicklungsprozess nicht so vorstellen, als ob ein ummittelbarer genetischer Zusanmenhang zwischen den heutigen Vertretern jener drei Stufen existiere. Ich glaube vielmehr, dass die termitophile Doryloxenus-Gruppe viel jüngeren geologischen Ursprungs ist als die Gattung Discoxemus und diese wierlerum viel jüngeren geologischen Ur'sprungs als die Gattung Termitodiscus. Daher dürfen wir in den heutigen termitophilen Doryloxemus keineswegs die wirklichen Vorfahren von Discoxemes und in den heutigen Discoxemus keineswegs die wirklichen Vorfahren von Termitudiscus sehen; denn geologisch ältere Formen kïnnen ja nicht von geologisch jüngeren abstammen!

Zur Lösung dieser Schwierigkeiten müssen wir einen dreimaligen, zu drei verschiedenen Zeiten erfolgten Uebergang von Doryloxems-Verwand ten zur termitophilen Lebensweise annehmen. Dafür, dass in relativ recenter Zeit ostindische Doryloxemus aus Dorylinengästen zu Termitengästen geworden sind. halen wil in den Tatsachen so schwerwicgende Anhaltspunlite, dass wir diese Anmahne als tatsïchlich gesichert schon oben (ad. 1) bezeichnen durften. Wenn aber ein solcher biologischer Wirtswechsel e in mal vorgekommen ist, so kann el auch mehrmals vorgekommen scin; denn die äusser'n Bedingungen hiefür waren ja schon so lange volhanden, als es termitematubende Dorylinen und auf Dorylinen reitende kleine Gäste des 'Trutztypus gab.

Die Entstehung der heutigen termitophilen Doryloxemus-Arten Ost-

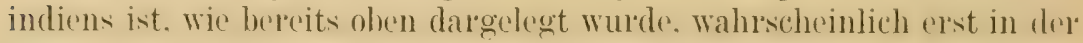
Diluvialzeit "rfolgt, nachdem Ostindien von Afrika dureh den indischen Ocean schon völlig getrennt war. (Vgl. oben ad. 1). Dies ist der letzte und jüngste der drei biologischen Uebergänge ron DoryloxemesVerwandten zur termitophilen Lebensweise. Vorfahren dex jetzigen termitophilen Doryloremus waren ohne Zweifel echte dorylophile Doryloarmus mit rodimentüren, zu Haftorganen umgewandelten Tarsen. da ja die tremitophilen Arten diese sonst völlig unerkiärliche Tarsenbildung noch beibehalten haben.

Wir kommen nun zum vorletzten, mittleren jener drei biologischen Uebergïnge. Da die Gattung Discoxenus durch eine weite Kluft von den trrmitophilen Doryluremes morphologisch getrennt ist; da sie forner bisher nur in Ostindien, nicht aber in Afrika gefunden wurde, so 
müssen wil den stammesgeschichtlichen Ursprung von Discresemes wahrscheinlich in das Ende der Tertiårepoche verlegen, und zwar in

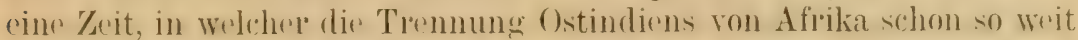
fortgeschritten war. dasis die Aushreitung der (iattung Discreremes auf Indien beschränkt blicb.

Der erste und älteste Uebergang von Dorylinengästen zur termitophilen Lebenswoise, dem die heutige (rattung Termitudisens entetanmut.

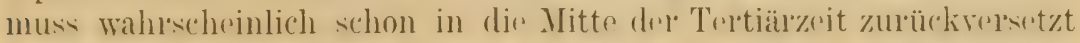
werden und zwar in eine Zeit, in welcher Ostindien und Mittelafrika noch einen gemeinsamen indisch-afrikinischen hontinent bilseten; denn arotrus ist Termitodisars durch rine weitr morpholngische Kluft von Discoxenus getrennt und stellt einen vicl vollkommeren termitophilen 'Trutztypus dar. als letztrer (xattung: zwoitons lommt Tremitodiscus nicht blos in Termes-Nestern Ostindiens vor (Termitodiscus Heimi Wasm. bei Termes obesus Ramb. und dessen Rassen), sondern

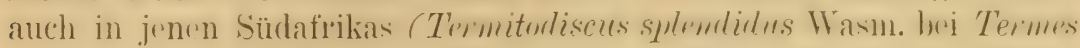
vulgaris Havil. in Natal und Termitodiscus Braunsi n. sp. bei Têrmes tubicole Wasm.) : wir müssen daher annehmen, dass diese Gastgattung sich von ihrem Entstehungsorte aus zugleich mit ihrer Wirtsgattung Termes über das indisch-afrikanische Gebiet ausgebreitet habe.

Was die jungtertiären, bezw. mitteltertiären Stammformen unserer heutigen Discoxemus und Termitodiscus anbelangt, so dürfen wir uns dieselben zwar wohl als mit den Aleocharinen verwandte kleine Dorylinengäise de Trutztypus vorstellen. die ihne. Wirte auf den Raubzügen

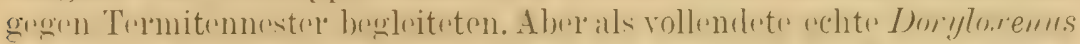

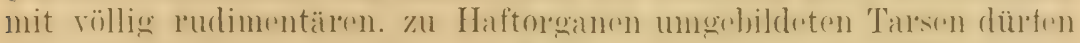
sie wohl noch nicht aufgefasst werden; denn Discoxenus und Termitodiscus haben ja normale, viergliedrige Tar'sen, und eine spätere Rückkehr'

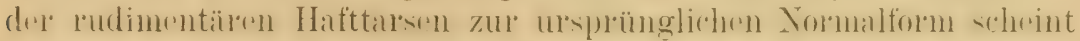
mil phylogenetisch weit weniger wahrscheinlich zu sein, als die Beibe-

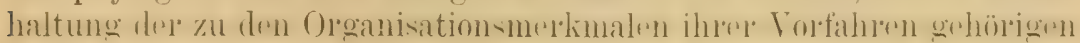
'Tarsenbildung.

Wir können somit das Ergebnis unserer Studie über die phylogene-

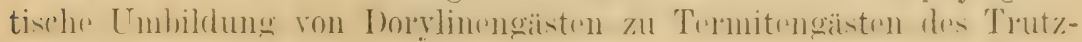
typus in das folgende Schema zusammenfassen: 
Schema der Entwicklung des termitopliten Trutztypus der Termitodiscini.

Termitodiscus (Indisch-Afrikanisch)

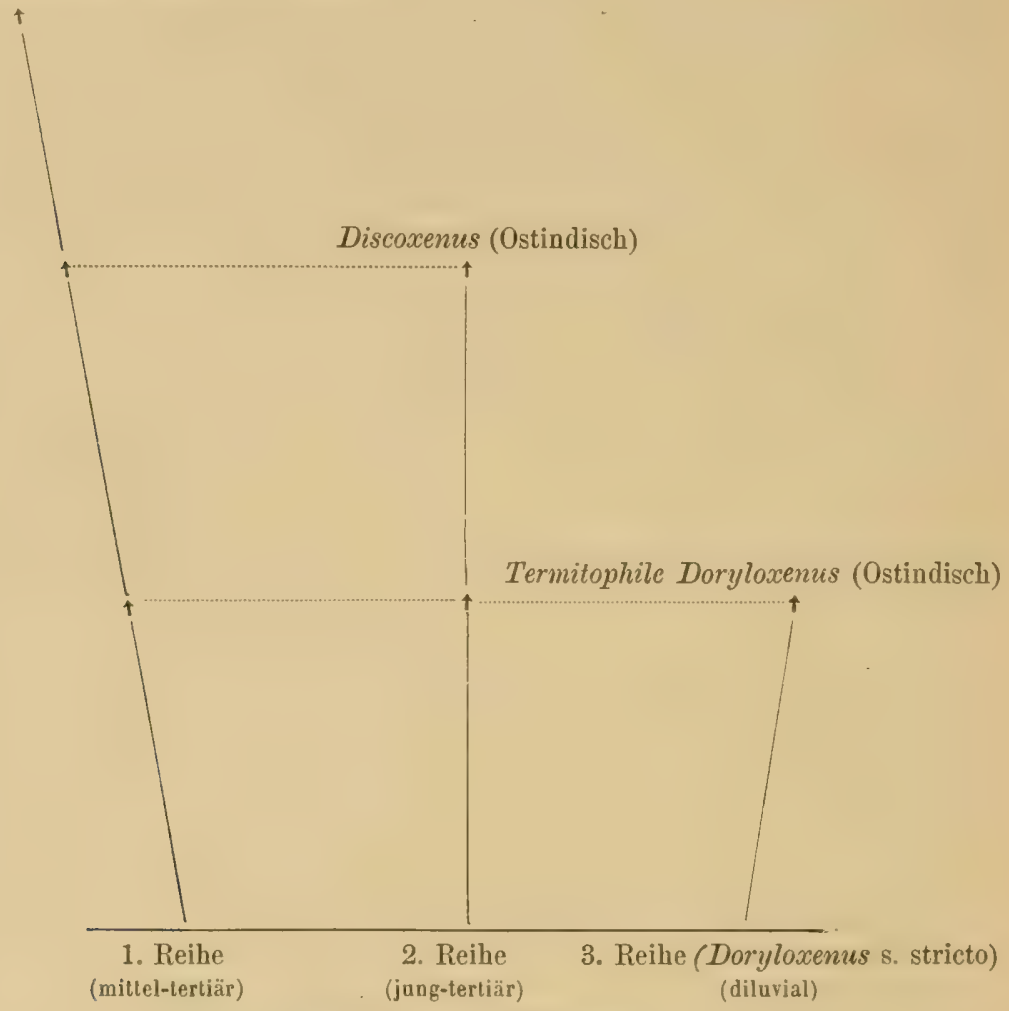

Dorylophile Doryloxemus-Verwandte.

\section{Diskussion.}

Dr. Fores lobt die genaue und vorsichtige logische Verwertung der Tatsachen v. s. des Verfasser's. Er' erinnert daran, dass es auch in Afrika untrrirdisch jagende Doryline'n gibt und meint auf solche hätten sich auch Termitophilen aus Dorylophilen entwickehn kömnen. Ferner frägt er den Vortragenden. ob a sich solche evolutive Spezies-Reihen wie die ron

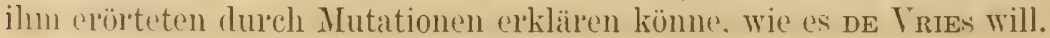


Her" Wasmann erwidert auf die erste Frage, dass es wenigstens wahr-

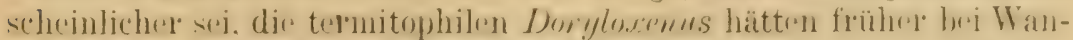

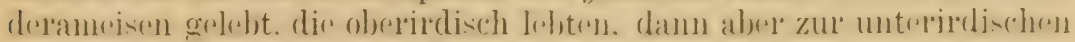
Lebensweise (wie heute in Indien) sich zurückzogen. Auf die zweite Frage bemerkt ere dass nach seiner Anvicht rlie Mutation in Wirklichlieit bestimmt gerichtet sei, obwohl dies schwerer zu konstatieren ist als bei den kleineren Variationen; dann dürfte aber die Nutation in

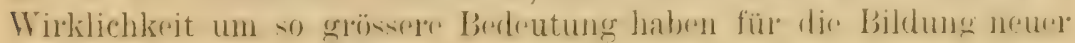
Arten und neuer Instinkte.

Dr. Escherich bemerkt, dass die Frage, ob Termitodiscus etc. ein starlium mit rudinentären Tarsen durchlaufon habe. wohl ülyerflü-irg

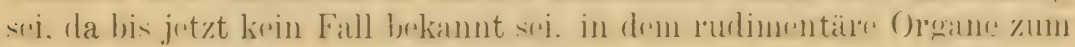
ursprünglichen Zustand zurückgekehrtt seien.

IHere Wasmax erwidert. übertlüsig scheine die Frage zwar nieht alwe

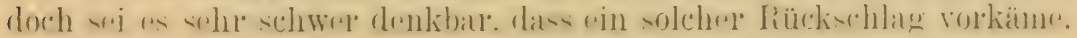
Daher habe Redner ihn auch für ausgeschlossen erachtet.

\section{Einige neue biologische Beobachtungen über Ameisen.}

Von Dr. A. FOREL (Chigny-sur-Morges).

Dir Familie der alle sozial fobenden Formiciden zählt gegenwärtig

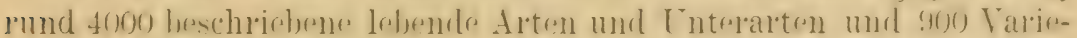

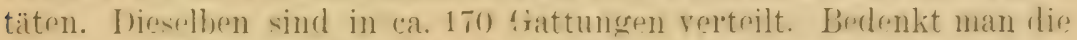

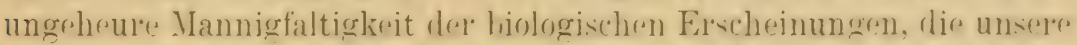
(ii. 150 europäischen Formen allein darbirten, sn kann man sich rinen Begriff davon machen, wie viel Interesantes noch leri genauerem Stuđium der Sitten der or reichen tropischen Fauna zu erwarten ist. Ich will hrute nur einige, im Laufe der letzten Jahre zu Tage aretretrne Erscheinungen erwähnen.

1. In seiner längeren Reise im Staat Amazonas, Brasilien, hat der

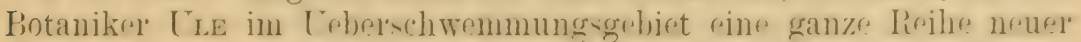

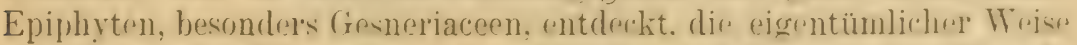

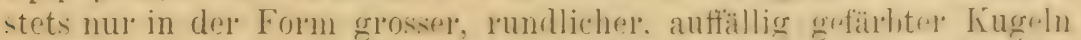

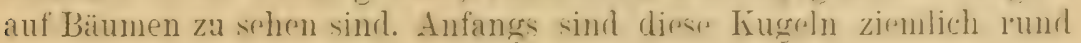


und zeigen nur den Beginn eines PHanzenwuchses. Später wachsen die Epiphyten und bilden förmliche Medusenköpfe, deren Originalphotographie, die ich Her'rn ULE verdanke, ich Ihnen hier vorlege. Nun bestehen genannte Kugeln aus Humus, aus Walderde, in welcher sich die Wurzeln der Epiphyten verfilzen, bevor sie in das Holz des Baumastes eindringen. Ausserdem bildet jede solche Kugel stets ein Ameisennest. Her' ULE will beobachtet haben, dass die Ameisen den Humus selbst auf den Baumast bringen und glaubt, annehmen zu können, dass die Ameisen den Samen der Epiphyten direkt darin pflanzen. Er sah wenigstens die Ameisen Erde und Epiphytensamen tragen und betont, dass jene Ameisen-Epiphyten grössere Samen besitzen, als ihre nächsten Verwandten. Er hat daher diese Kugeln Ameisengärten genannt und ist der Ansicht, dass auf solche Teise sowohl die Ameisen, wie die Epiphyten, vor den Ucherschwemmungen geschützt werden. Aus einem winzigen Fragment eines solchen Nestes, das ich der Güte des Herrn Urw verdanke, steht so viel fest, dass es sich nicht um ein festes Ameisen-Carton, sondern höchsteus um ein ganz lockeres handelt, das den gewöhnlichen Erdnestern sehr nahe kormmt.

Eigentumlich genug ist es, dass die zahlreichen Epiphytenarten, die auf diese Weise vegetieren, nach TLE sonst nirgends vorkommen und gemeinschaftliche Merkuale hahen, die sie von ihren Verwandten unterscheiden.

Herr Ute hat mir nun die hezüglichen Ameisen zur Untersuchung gesandt und es hat sich Folgendes herausgestellt. Die eine Art, die die gröberen, weniger zierlichen Ameisengärten bildet, ist der Camponotus femoratus Fabricius. Diese Anrise, deren ursplünglicher Typus im Museum von Kopenhagen steht, wo ich ihn sellst untersuchte, war bis jetzt 11 u duch einzeln gefundene Individuen in den Sammlungen vertreten; ihre Lebensweise war total unbekaunt. Alle übrigen zierlicheren Ameisengärten gehörten Arten aus der Gattung Azteca und zwar den folgenden :

1. Azteca Traili Emery.

2. Azteca Traili Emery, var. filicis n. val.

3. Azteca Ulei n. sp.

4. Azteca olitrix n. sp.

Etwas zweifelhaft bleilst mir noch die Cremastogaster limata Smith, die Herr Ula in Ameisengärten (zwischen Authurum, einer Fpiphyte) fand. Eigentümlich ist es, dass bereits Bates (Sumtu's Formiciden-Katit$\log 185 \mathrm{~S}$ ) von Cremastogaster limata sagt: "Das Nest befindet sich in durchbohrten, drüsigen Anschwellungen der in der Luft hängenden Wurzeln einer parasitischen Pflanze. " Immerhin hedarf die Frage, ob (: Timatr wirklich selbst Ameisengärten haut, noch der Bestätigung.

Von den genannten Aztecarten sind Ulei und olitrix bisher nur in 
Ameisengärten, Traili dagegen auch noch in den Anschwellungen des Blattstiels von Tococa bullifera gefunden worden. Doch sind auch noch die Varietät Tococx, n. var. der Traili, in den Anschwellungen einer anderen Tococa, und die Subspecies nigricomis (neu), sowie die Varietät Cordix (neu) der Azteca Ulei in den Zweiganschwellungen von Cordir nodosa von Herrn Ure gefunden worden. Fs unterliegt somit keinem Zweifel, dass diejenigen Aztecaformen, die Ameisengärten bauen, auf das Allernächste mit solchen verwandt sind, die in Anschwellungen leben, zum Teil auch selbst auf beide Weisen nisten.

Diese sonderbare neue Form von Symbiose oder Anpassung verdient jedenfalls nähere biologische Untersuchungen. I)ie Gattung Aztece, die ich vor 26. Jahren auf Grund von ca. 3 Arten aufgestellt habe, zählt gegenwïrtig über so rerschiedene For'men, die samt und sonder's im ne'0tropischen Wald als ausschliessliche Baumbewohner leben. Die Einen bilden grosse Cartonnester, die man von Weitem auf den Bammzeigen sieht; Andere leben in ausgehöhlten Bäumen (z. B. velox und irstribitis). und Weitere zeigen alle erdenklichen Anpassungen an physiologische Pflanzenhöhlungen ; wir kommen bei einer ander’n Frage darauf zurïck.

Eigentümlich ist es, dass ron Juening bereits früher dio hängenden Cartonnester beschrieben hat, die in Süd-Brasilien rom Camporotus rufipes, Fab. im Ueberschwemmungsgebiet gebaut werden. Dieser Camponotus ist von femorates nicht entfernt und baut nach von JHERING in nicht überschwemmten Gegenden andere Nester.

2. Schon lange hatten dir Botaniker (Hutu und Andere) die Symbiose gewisser stechender Ameisen mit den tropisch-amerikanischen Pflanzen der (xattune Triplaris beschrieben. Die Triplarisarten besitzen in del' Mitte des Stammes und der Zweige eine sehr schmale Markhöhle. wie Sie hiel sehen (hiele ist die Narkhöhle dureh Austrocknen ('lweiteret). Sie hatten auch erwähnt. wio die Ameisen joden Angriff auf dio Triploris durch wätendes Beissen und Stechen abwehren. Ich konnte mich sellst davon überzengen. als ich 1s96 in Columbien zufällig die Hand auf den Stamm einel Triplaris setzte. Ich liess den jungen, ganz unversehrten und frischen Bamm abhauen und fand dir sehmalen Makhöhlen desselhen rom Stamm bis zum Ende der noch greünen A estehen mit ciner langen Ameise samt ihler Brut und geftügelten Männchen ausgefüllt, die ('inen förmulichen Gïnsemasch in dieser so ausgedelenten und vel'zweigten frin-rylindrischen Wohnung bildeten, in welehor sie knapp) rinandel" krexuzen lonnten. Auch die Puppen lagen in gleicher Weise hintereinander. Erst nach langem Suchen entdeckte ich unten am Stamme ein kleines, dürres Aestehen. das die Ein- und Ausgangstüne dop Amerorn bildete.

Nun stellt sich. I)ank den schönen Sendumgen der Herern Professoren

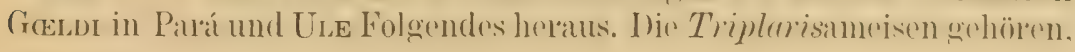


soweit bisher gefunden, samt und sonders zu einer bestimmten Gruppe nahe ver'wandter Pseudomyrmaarten. Es sind dies folgende:

a) Psendomyrma arboris-sanctx, Emery aus Bolivien und Peru.

b) Psendomyrma arboris-sancta, Emery. Subspecies symbiotica, Forel, Columbien (deren Wohnung ich eben beschrieb).

c) Pseudomyrma dendroica, Forel, Rio Purus, Amazonas von Herrn André GæLDi gesammelt.

d) Pseudomyrma dendroica, Forel, var. emarginata, neue Varietät, von Herrn ULE in den Markhöhlen der Triplaris Schomburgkiana gesammelt.

e) Psendomyima Triplaridis, nova species, von Her'm Ule im Amazonas in den Markhöhlen ron Triplaris surinamensis gesammelt. Professor GeELDr hat die Pseudomyjima dendroira in den botanischen Garten in Pará verpflanzt und beobachitet, wie diese Tiere in neue, bis jetzt von Ameisen nicht besetzten Triplarisbäume eindrangen und darin Colonien bildeten. Nach Professor GeLdor zeigen die dortigen Triplaris unter den Blattachseln verdünnte Stellen, die später oft reröden und den Pseudomyrme Eingang verschaffen.

Neu ist hier nur die Feststellung, dass die Triplarisameisen eine bestimmte morphologische Gruppe bilden. Diese Feststellung scheint mir aber wichtig, weil dadurch der Charakter der Symbiose zweifellos dargetan wird. Fs handelt sich nicht, wie man bisher anzunchmen neigte, um die zufällige Benutzung einer P'tlanzenhöhlung durch beliebige Ameisen. sonder'n um eine gesetzmässige Symbiose. Sie können hier die bezüglichen Pseudomyrmaformen sehen.

3. Ich möchte Ihnen fer'ner die cigentümliche biologische Bedeutung der Kopffor'm der. Soldaten und Weibehen gewisser Ameisenarten zeigen. Ich habe schon früher in meinen Ameisen der Schweiz die Rolle gezeigt, die der Soldat der' Untergattung Colobopsis von Camponotus, wenigstens bei unserer "uropäischen Colobrosis truncata Spinola. spielt. Diese Ameise lebt, sei es in Nestern, die sie in hartes Holz meisselt, sei es in Eichengallen. sei es, wie ich neuerdings zeigte, in den hohlen, dürren Arstchen unserer Nussbäume. Ueberall wird die schmale Eingangsgalerie des Nestes durch den scharf und breit gestutzten liopf eines dicht am Eingang stehenden Soldaten verstopft. So schützt der Soldat den Eingang des Nestes vor jedem fremden Eindringling und lässt nur die rigenen Nesthewohner ain. Er spielt die Rolle eines lebenden Stöpsels und zugleich eines Portiers. Während ich neuerdings meine alten Beobachtungen hier in der Schweiz von neuem bestätigte. wurden roriges Jahr von Professor Wheeler in New - York amerikanische ColobopsisArten (nämlich Colobrysis abdita Forel v. etiolata Wheder und Pylartes Wheeler) studiert. Auch bei diesen Arten funktioniert der Soldat genau wie derjenige del trumetu. Da ausserdem die grossen Arbeiter 


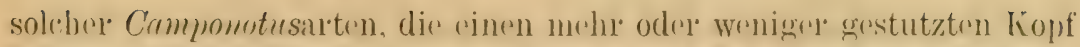
haben, ähnliche (xewohnheiten besitzen und den Fingang des Nestes mit ihrem Kopfe reretridigen, darf man wohl, ohme zu ire'n, ammehnen. dass die genamnte Kopfform sich allmälig an jene Sitte vollständign angerpasst hat. Sie sehen hier bezügliche Abbildungen. sowie das Nest unsereer Colobopsis truncrete Spinola in hohlen Nussbaumästchen. ondlich dis

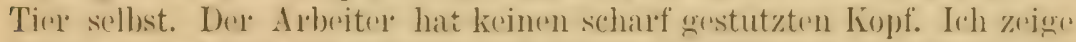

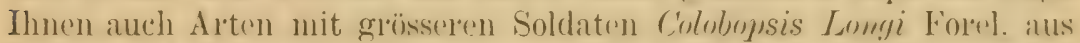
Indien und clevedendiri Emory, aus den Sundainseln. Irei woldehen sie

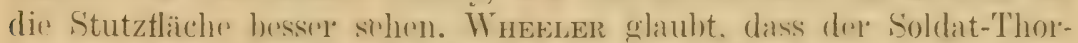

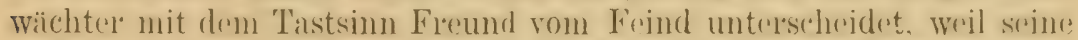
Fühlhörnex hintere der Stutzflache des Lopfes aingelonkt sind. Ich halte

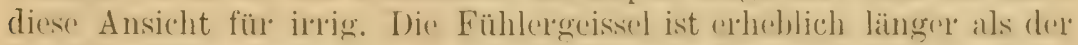

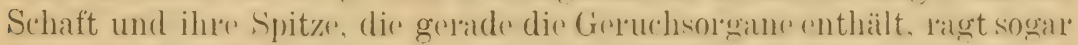

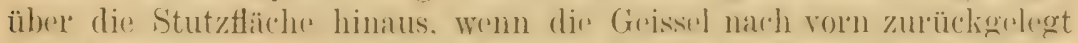
ist. I)ies genügert vollständig unn dem Soldat die Grovelsefualität des

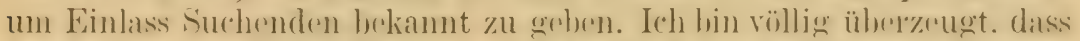
(in Suldat ohne Fühloregeissel soinen I)ienst nicht mohr richtig verschen könnte.

Aber es gibt noch andere Anpassungsformen. Wir sprachen vorhin

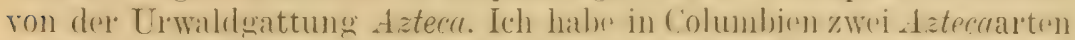

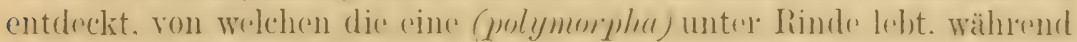

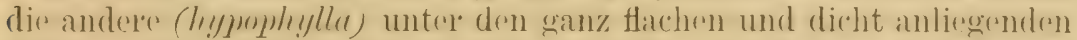
Blätter'n einer Schlingptlange nistet. die sich an den Stamm von Urwaldbäumen anpmesst. Unter jene Blättex schleicht sirh dir Amerise und haut finen harten Carton. dere die Blatträndere an die Ibamminde kittret und

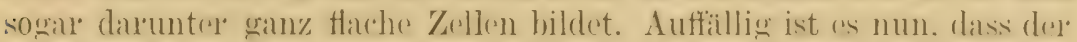

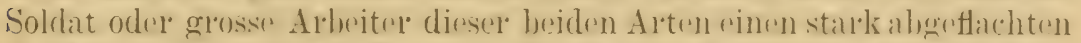

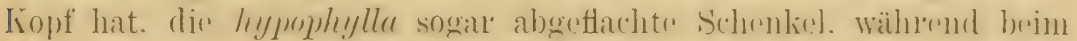

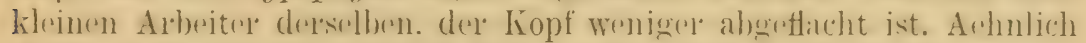
dürftr die Harhlioptige in Columbion v. i. K. Hohreit der P'rinzessin Theres: v. BAYERN entdeckte Azteca Theresia Forel, leben.

Fel'nel finden wir bei der Azteca Emeryi nova species, sowohl beim

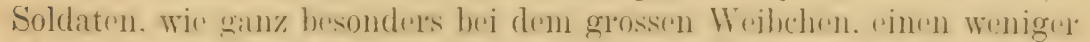

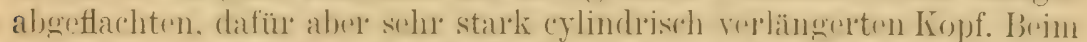
ganz likinen Albeiter ist der Kopf nue weng länglich. Irim grossen

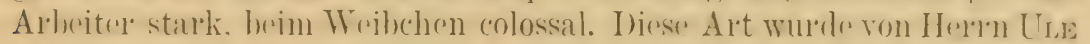
in den cylindrischen Internodien einer Cecropia entdeckt. Professor

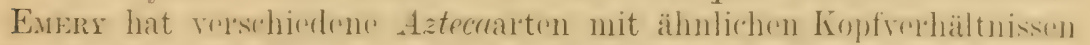

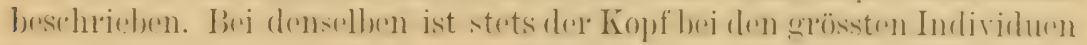
del Colonie, das heisst bei den Weibchen am längsten, dann bei den

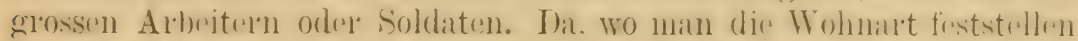
konnte, so bei del angusticeps Emery, war das Nest eine schmale cylin- 
drische Pflanzenhöhle. Die Azteca angusticejs lebt nach Exery's Angabe in der Höhlung des Stieles von Duroia petiolaris. Nun ist es aber characteristisch, dass die Anpassung der Kopfformen an die Wohnungsformen nur bei den grossen Individuen, und zwar bei den grössten am stärksten stattfindet. Die kleinsten haben ja daneben immer Platz genug.

Dass dem anch wirklich so ist, und dass es sich nicht um eine Eigentümlichkeit del Gattung Aztecu handelt, geht daraus hervor, dass bei anderen Aztecaformen der Kopf des Weibchens und des grossen Arbeiters keineswegs länglichel oder abgetlachter ist, als beim kleinen Arbeiter: Sie sehen hier die Aztea trigona Emery, deren Weibchen und grosse Arbeiter umgekehrt einen sehr kurzen und dicken Kopf haben, sowie die Azteca velox Forel, deren Weibchen einen, demjenigen des ziemlich monomorphen Arbeiters entsprechenden Kopf besitzt. Aus den hier vorliegenden Photographien von Prof. Gresdr sehen Sie, wie ein Cartonnest der Gattung Azteca an Bäumen des Tropenwaldes aussieht.

Auch bei derjenigen neotropischen Ameisengattung, die bezüglich Anpassung an die Bäume des Urwaldes Azteca am nächsten steht, nämlich hei der Gattung Pseudomyrma, finden wir ähnliche Verhältnisse. Die Pseudomyrma sind, wie Sie sehen, auffällig längliche Ameisen und wir fanden bereits deren Triplarisgruppe an schmale Pflanzenhöhlungen angepasst. Ich selbst sah in Columbien die allergemeinste Form, Psendomyrma gracilis Fab., in den hohlen Grashalmen der Savanne nisten, gewiss ein schmaler Wohmraum. Die Pseudomyma delicatula entdeclite ich in den schmalen Höhlungen dür'rer Baumästchen in Jamaica; sie ist auch recht schmal. Die allersonderbarste Form ist wohl die Pseudomyrma filiformis Fab. Von den Sitten dieser kolossal schmalen und langen Art kennen wir nur eine Notiz von Bates. Er sagt, dass sie in diurren Acstchen nistet. Die breitere Psendomyman Kïnckeli Emery fand ich dagegen in woiteren, faulen Baumästen, wo sie weitere Wohnungen anlegt. Eine einzige Psendomyrmart nistet meines Wissens in der Erde, wo sie sehr schmale Gänge miniert; das ist die Pseudomyrma elegans Smith. Man könnte mir freilich dieses Beispiel entgegenhalten und mir sagen, dass auch diese schmale Pseudomyrma, ohne durch ihre Wohnung dazu gezwungen zu sein, einen schmalen Körper besitzt. I)a jedoch die Lebonsweise dieser Art eine wahrseheinlich einzig dastehende Ausuahme in der sehr artenreichen Gattung bildet, möchte ich vermuten, dass es sich bei ihr um eine sekundäre Biologie handelt, das heisst, dass diese Art aus Psendomyrmaformen abstammt, die in Pflanzenhöhl'n wohnten, wolurch sich die Sache erklären würde. Ihre bereits vorhandrue schmale Form komnte sich damn auch recht gut an schmale minierte Erdgänge anpassen. 
4. Man kennt schon lange Ameisennester, die aus gewobener Scide bestehen. Verstanden hatte man jedoch ihre Entstehung bis vor Kur'zem nicht. Hier sehen Sie das weisse Seidengewebe der Oecophylla smararydima aus Ost-Indien, die ihre grossen Nester im Laube webt, sowie das zierliche Seiden-Labyrinth des Camponotus senex Smith, Varietas textor Forel aus Costa Rica; endlich das einzellige Nest der Polyrhuchis arachne Emery aus Java, das aus Pflanzenstückchen besteht, welche mit einer Art Spinngewebe zusammengewoben sind. Artken glaubte gesehen zu baben, wie die Ameisen selbst mit ihren Kieferdrüsen das Gewebe spinnen. Er hat sich aber geirrt. Rrbley bewies 1890 im Journal Straits asiatic Society, dass die Oecophylla ihre spinnenden Larven als Webschiffe benutzen, indem sie sie im Maul halten und mit ihrer Hülfe das kunstvolle Gewebe weben. Die Sache ist aber neuerdings zum vollendeten wissenschaftlichen Gemeingut durch die Beobachtumgen des Her'm Professor Grisd in Pará geworden, der die ganze Kunstweberei sehr genau beim Camponotus senex im botanischen Garten zu Pará beobachtet hat. Die Beobachtung wird dadurch erschwert, dass die Ameise von innen nach aussen arbeitet, indem sie ihre Larven als Spinmmaschine benutzt und so das wunderbare Labyrinth ausarbeitet, von welchem Sie hier Fragmente sehen. Im Pariser Museum befinden sich einige prachtvolle Nester von Camponotus senex, die um Baumzweige herum gewohen sind. Diese wunderbare Industrie del Ameisen eröffinet wiedel ein ganz neues biologisehes Kiapitel : die Benutzung der eigenen Brut als Industriemaschine! Nachdem die Sache in Amerika und Ost-Indien für verschiedene Gattungen feststeht, dürfte es liaum zweifelhaft sein, dass alle wesponnenen Nester der Ameisen, vor allem diejenigen der Gattung Polyrhachis, anf diese Weise entstehen. Unsicher hleiht dagegen noch die Sache für die Cartonnester. Sie sehen hier das Cartonnest eines amerikanischen Camponotus unter einem Blatt. Unser europäischer Lasius fuliginosus, der auch Cartonnester baut, besitzt sohr stark entwickelte Kieferdrüsen und die Kieferdrüsen liefer'n ein harziges Seliret. Es grelang mir bis jetzt nicht, seine Carton-Fabrikation direkt zu beobachten, die stets nur im Inulilen geschicht. Entweder littet er die Holzmohlteilchen und Steinchen, aus welchen das Cartonnest hesteht. selbst mit Irüsenkitt, wie die Wespen, oder er benutzt dazu seine Larven. Die Zukuntt wird die Sache entscheiden. Alle diese Brobachtungen erfordern viel Zeit, und diese fehlt mir leider am meisten.

Her'r Andr. Gacdr, Bruder von Prof. Geldr, hat am Rio Purus (Amazonas) Nester von Camponotus senex entdeckt, in deren Mitte das Nest einer kleinen Melipone (amerikanische Biene) sich befindet. Ioje Meliponen tiegen durch rinen besonderen Kanal aus und ein. Hier sehen sie eine bezügliche Originalphotographie von Herrn GELDI. 


\section{Diskussion.}

Prof. Emery bemerkt:

1. dass das Spinnen der Oecophylla-Nester durch Larven auch von den Herren Sarasin in Celebes beobachtet wurde;

2. dass wenn es auch keine Ameisenpflanzen geben sollte, es doch zweifellos Pflanzenameisen gibt.

3. Entwickelung des Kopfes in die Lünge bei grossen Arbeiter'n wird bei andern Ameisen, die in schmalen Gängen leben, beobachtet, so z. B. bei den unterirdischen Dorylus-Arten, im Gegensatz zu den Aromma, die im Freien leben.

Herr Wasuann macht darauf aufmerksam: 1. dass der Begriff " Ameisengarten " ULE's nicht verwechselt werden dürfe mit den von den Ameisen angelegten "Pilzgärten "; 2. dass die starke Entwickelung der. Spinndrüsen der Oerophyllu-Larven bereits morphologisch durch Schnittserien bestätigt worden ist.

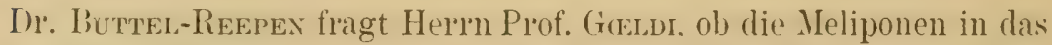
camponotus-Nest einwander'n oder ob camponotus sich später um das Meliponennest ansiedelt? Prof. G(eld vermag hirrauf keine bestimmte Antwort zu erteilen.

I)r. Eschericin macht darauf aufmer'ksam, dass durch einen Schüler Curss bei den Oecophylla-Larven riesige Spinndrüsen festgestellt worden sind, wie in dem Werk "Aus den Tiefen des Weltmeers " angegeben ist. Er bemerkt, dass die Botaniker in neuerer Zeit der Srmbiose von Ameisen und Pthanzen sehr skeptisch gegeuübel stehen: bezüglich des Zusammenlebens von Iridomyrmex und Myrmecodia wird eine Symbiose direkt bestritten. 


\title{
Beobachtungen über die erste Anlage einer neuen Kolonie von Atta cephalotes.
}

\author{
Von Prof. E. GOELDI (Pari).
}

Ein frisch eingefangenes, noch getlügeltes Atta-Weibchen wulde von mir in einen eigens konstruierten und speziell zu diesem Zwocke auselsommenen Zuchtkasten gesetzt. Ierselbe hat die Form rines sehr lang gezogenen Prismas, Vorder-und Rückseite bestehen aus einer einheitlichen, nach oben heweglichen Glasscheibe; die schmalen Seitenwände bestehen aus Holz, indessen sind eine Reihe von grossen, mit Draht vorschlossenen Ventilations-Fenstern angebracht; Boden von Holz; Deckel mobil, mit Drahtgitter zur Tentilation. I)ie I)icke des mismatischen Kastens beträgt nicht über $2^{1 / 2}-3 \mathrm{~cm}$. Der Kasten war gut zu $2 / 3$ mit feinster Gartencrde angefüllt.

Das Atta-Weibchen bohrte sich, nachdem es sich seiner Flügel entledigr, eine Galerie in die Erde, die handhoch unter der Oberfläche zu einer kleinen, zirka $5 \mathrm{~cm}$. in der Länge und etwa $2 \mathrm{~cm}$. in der Höhe betragenden Höhle führte. Was geschah? I)as Atta-Weibchen legtr ungefäh 1: Dutzend wie Milchglas gefürbte, zirka 1 mm. lange, schöne Eier, die zu einem Häufchen zusammengruppiert, von der Mutter mit sichtbarer Sorgfalt fortwïhrend beleckt, geputzt und gehätschelt wurden. Nach zirlia 8- 14 Tagen gewahrte ich jedoch auf eimmal, dass der Eierlilumpen, wie eine Tasse auf einem Unterteller, auf rin napfartiges weisses Gebilde zu stehen kam, das ich mit del (i-s mal vergrössirnden Lupe deutlich als einen Anfang zu einem Pilzgarten zu erkennen vel• mochte. Derselle hatte cin grobliörniges fiefüge, und von jeclem einzelnen Korm aus erstreckten sich, bestens sichthar, wie Igelstacheln, junge Myceltiaden des Nïhrpilzes. I) er Napf wuchs, auch die Eier gediehen ersichtlich und hereits waren nach einem Monat dem Ausschlüpten nahe fast reife Larven daraus geworden, als durch einen Zufall leider (parasitische Psociden schienen dahei die Hauptrolle zu spielen) die ganze junge Kolonie sammt Nutter, Pilzgarten und Nymphen in Zeit von wenigen Stunden einging und abstarb. Ein technischer Fehler in dor Konstruktion des Zuchtliastens, rine Ungeschicklichkeit meinerseits hatte die Möglichkeit dieses Zufalles veranlasst.

Was waren num die groben, weissen Kíörner des Pilzgarten-Napfes in 
seiner ersten, ursprünglichen Anlage? - Positiv konstatiert habe ich es nicht, beweisen kann ich es nicht, und doch vermag ich keine andere Lösung und Erklärung zu finden, als dass es Eier, Eireste und Eipartikel sein müssen, auf die das im Hypopharynx aus dem Elternhause als Mitgift mitgehrachte Mycel gleichsam wie auf ein Gartenbeet ausgesäet wird: es ist das erste Nährsubstrat für die ursprüngliche Anlage der Champignon-Kultur. Vom brasilianischen Strauss behauptet der Volksmund, dass er überzählige Eier lege, die er verfaulen und durch die Gährungsgase krepieren lasse, damit die durch den Fäulnisgeruch angezogenen Fliegen als erste Nahrung für die jungen Strausse der bebrüteten Eiel dienen sollten. Das lässt sich im Innern Brasiliens kein SertãoBewohner ausieden. Hier läge also ein Analogie-Fall vor:

Jedenfalls ist eines im Auge zu behalten, bei der Frage: "Was tritt zuerst auf in der neuen Atta-Kolonie, die Eier oder der Pilzgarten?" - Das positive Ergebnis meiner Experimente, dass nämlich die Eier wirklich zuerst anftreten und der Pilzgarten nachher entsteht, als Unterlage für den Eiklumpen und dass es eine Partie geopferter Eier zu sein scheint, die als erstes Nährsubstrat für die Pilz-Kútur zu dienen hat.

\section{Diskussion.}

Dr. Eschericu wirft die Frago nach der morphologischen Redentung der Infrabuccaltasche auf.

M. JANET rappelle les figures qu'il a domnées dans ses précédents travaux et qui précisent la situation de la poche infrabuccale dans laquelle les Foumis, comme les Guêpes, moulent une houlette agglutinée par des liquides salivaires, qu'elles rejettent et qui contient les matières solides extraites de leur nourriture et les produits du nettoyage de leur corps. Sur une coupe sagittale on voit que cette poche est située entre l'orifice buceal et l'orifice impair de la glande labiale qui correspond à la glande séricigène des larves.

R. Heynons bemerkt, dass die von M. JAnet festgestellte Lage der Infrabucealtasche der Region des Hypopharynx entspricht. Da der $\mathrm{Hy}$ popharynx hei niederen Insektenformen gleichfalls zwischen der Mundöffinung und del Oeftnung des Speichelganges gelegen ist, so wird man die Infrabucealtasche der Hymenopteren morphologisch als ein zum $\mathrm{Hy}$ popharyux gehörendes Gebilde auffassen können, während sie zur Mundhöhle oder dem Oesophagus in keiner Beziehung steht. 


\title{
Sur l'origine des fourmilières.
}

\author{
Par le Prof. C. E.IERY (Bologne).
}

Les observations faites juscu'à ce jour sur la fondation des fourmilières montrent que des fomelles fécondées, après šêtre dépouilléres de lours ailes, se creusent une case fermée, dans laquelle elles pondent des œufs et élèvent quelques larves qui deviennent les premières ouvrières, toujours très petites; elles les élèvent sans prendre de nourriture, c'est-àdire en tirant de leur propre corps l'aliment des larves, soit sous forme d'outs, soit sous forme de sécrétions buccales. L'on a souvent trouvé des nids naissants à dives's stades de leur développement, mais l'observation directe de la fondation d'une fourmilière na jas été faite jusqu ici completement. Lobservation la plus complète que je comnaisse est colle diune temelle de Camponotus ligniprerdre trouvéc par moi dans sa case à Montricher et remise pour observation ultérieure à mon ami le professeur Aug. Forel qui en a publié le résultat. La femelle ar'riva à élevel deux ouvrières, puis elle cessa de s'occuper de sa progéniture et le nid naissant périt.

J'ai à vous rapporter une expérience très bien réussie en nid ar'tificiel; elle se rapporte à Crematogaster scutellaris.

Une femelle privée d'ailes fut trouvée par mes enfants dans un trou d'une peeche jaune, le 11 netohere 1903. Je la placai dans un petit appareil

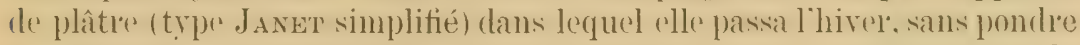
d'œufs, entre les deux vitrages d'une double fenêtre au laboratoire de zoologie à Bologne.

Le 16 avril, je r'emarquai un paquet d'œufs que j'estimai à 12 au moins. Le 2 mai il n'y en avait plus que 10, ce qui me fait supposer que la mère cn avait mangé. Le 11 juin je vois de petites larves; le 21 juin, 2 nymphes, 1 larve, plusieurs ouf's. Il n'est pas admissible que ces oufs soient de ceux du 16 avril. Une partie des œufs a done disparu pour servir de nourriture aux larves et peut-être aussi à la mère.

Le y juillet la première ouvrièrese transforme; le 12. juillot, la deuxième qui est défectueuse et n'ar'rive pas à bien se colorer (elle est morte quelques jours après). Je donne du miel aux Fourmis et elles en mangent. La femelle continue à avoir soin des œufs et des larves, dont le nombre augmente. Je transporte la fourmiliere naisiante dans un petit nird de rerere, système Fielde, où je la nourris avec dui miel et du jaune d'œuf. 
Le 6 août il y arait 6 nymphes, dont l'une se transforme le 12 , une autre le 14, comme me l'écrit mon fils, après mon départ de Bologne. La fourmilière est en voie de croissance : elle est désormais définitivement fondée.

Chose remarquable, mes Fourmis se sont habituées à la lumière et ne recherchent même pas l'obscurité, de sorte que je puis les observer à la loupe, sans nullement les déranger.

Crematogaster scutellaris vole en automne, alors que la température baisse dans nos climats. La longue période d'inertie, qui précède la première ponte, doit avoir là sa raison d'être.

D'autres Fourmis sont plus hâtives: ainsi Pheidole pallidula Nyl. qui role en Italie en juin-juillet pond au lendemain de laccouplement un grand nombre d'œufs. Liometopum microcephahum Panz., accouplée le

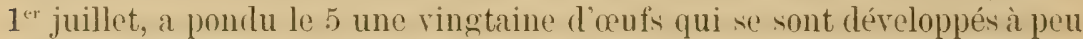
près simultanément. Je me réserve de publier une autre fois les résultats de mes observations encore incomplètes, sur cotte Fourmi et sur d'autres encore, sur lesquelles mes expériences se poursuivent.

D'autres Fourmis pondent leurs œufs petit à petit. Par exemple Camponotus xthiops Latr. et deux espèces de Leptothorax que j'ai en observation.

Une femelle privée d'ailes de Leptothorax recedens Nyl., prise errante à Bologne le 2 juillet dernier, a été mise aussitot sans nourriture dans un appareil FreLde. Le 8 elle avait deux œufs; le 14, cinq œufs; le 21, une grosse larve, une petite et deux œufs; il faut done qu'un œuf au moins ait été mangé; le 31 il y avait une nymphe, une grosse larve, une petite et un œuf; le 5 août, deux nymphes et deux larves de différente taille. La première ourriere s'est transformée le 13 anut; du miel a été fourni alor's aux Fourmis qui en ont mangé.

Ici, un mois et demi ont suffi à la mère pour élever sa première ouvrière. Crematogaster y a mis 10 mois, pendant lesquels elle n'a pris aucune nourriture.

J'ai en élevage une autre femelle de Leptothorax (espèce indéterminée de couleur très foncée), prise au vol et renfermée dans un nid de plâtre. Chose remarquable, prise dans les premiers jours de juillet, elle ne s'est pas dépouilléer de sés ailes, qu elle conserve eneore, sauf une des ailes postérieures qui est tombée après quelques jour's: cela ma a fait douter qu'elle füt féconlér. Quniqu il 'n soit, elle a pondu successivement comme l'autre quelques oufs et élevé des larves dont l'une est devenue nymphe d'ouvrière ${ }^{4}$.

Toutes ces expériences ont été faites sans donner de nourriture aux

'Depuis la présentation de cette note, j’ai pu déterminer l'espèce d'après les ouvrières. C'est L. tuberum leviceps Emery. 
femelles, jusculü léelosion de la première ouvrière. Ceest à mon avis la condition normale pour un grand nomlite de Fourmis; mais je pense que cette reggle n'est pas sans exceptions, peut-être nombreuses. Il ne me paraît guère possible que certaines femelles de Ponérines ot Myrmicines trourent dans leur corps de quoi noureir une ouvrière pas beaucoup moindre qu clles. Certains insuecès do mes expériences d'élevage mo font "roine quil y a des espèces dont les femelles, tout en étant heaucoup plus rrandes que lours ourrieres, ne sont pas disposérs à söisoler dans une collule murée, ni à vivie un mois ou davantage sans mangel.

Et les Foumis parasites et exclavagistes, par exemple Polyergus? Wassaxs a formulé à l'égand de cette espèce me hypothèse qui ne me satisfait pas entièrement. Il suppose la formation diune société dalliance, entre la femelle Poylergus et des ouvrières étrangères de Formica fusca ou rufibarlis. Je hasalderai une autre hypothèse. Les observations de Fores, semblent montrer que la femelle de Polyergus est moins dégénérée dans ses instincts que l'ouvrière: il a vu une femelle aidre une nymphe it se dépouiller de sa peau. D'autre part. Fores, et moi-méme nous avons vu des femelles vierges prende part à des expéditions. Jo supjose donc que la femelle de Polyergus, apprès setete dépouillée de ses ailes est capable de ravir à une faible colonie de Formica une on plusieurs nymphes d'ouvières qui donneront naissance à ses premières auxiliaires.

Ayant capturé cet été une femelle fécondée de Polyergus, je m'étais proposé de tenter une expérience, en lui fournissant loceasion dentrer. en rapport, dans un appareil artificiel, avec une faible société de $F$. fusere. La molt de cette fenele ma a empêché de mettre à exécution ce projet. J" me propose de le reprendre à loceasion et je le lecommande à ceux qui seraient en mesure de tenter l'expérience.

J'ai cité Polyergus comme un cas extrême, mais je suis convaincu que la fondation de fourmilières de la part dautres espèces offere une grande variété de conditions et mérite d'être l'olujet de recherehes expérimentales compalées.

Je présente à lassemblée un nid de Dolichoderns attelaboides F., pronvenant de Silinte-Catherine (Brésil) : il est hâti en carton sur une feuille et renfermait une femelle isolée.

\section{Discussion.}

M. JANET considère que la question de la fondation d'une colonie nouvelle par une femelle isolée doit être considérée comme à peu près tranchée. Tous ceux qui ohservent l'éthologie des Fourmis sarent que lon rencontre souvent une reine isolée qui, après sètre desailée, a creusé une petite cavité, ordinairement sous une pieree plate qui forme le plafond de 
la chamhre, et que cette reine soigne les oeufs qu'elle a pondus et quelle a réunis en un petit paquet. Elle nourrit ses premières larves et les conduit jusquà l'éclosion imagrinale. Les premières ouvrières qui apparaissent ainsi sont souvent plus petites que les ouvièeres normales. Les produits salivaires sont un des éléments de la nourriture que la reine distribue à ses premières larves. C'est l'histolyse des muscles vibrateur's du vol qui fournit les substances alimentaires nécessaires : $1^{\circ}$ à la sécrétion des glandes qui remplissent ainsi une fonction nutritive pour la progéniture; $2^{\circ}$ à la sustentation de la reine ; $3^{\circ}$ au développement énorme que prennent assez rapidement les ovaires.

\section{Biologische und soziologische Momente aus den Insektenstaaten*.}

Von Dr. v. BUTTEL-REEPEN (Oldenburg i. Gr.).

In der Arbeitsteilung erkennen wir ein wichtiges soziologisches Moment. Die höchste Organisation ergiebt sich in der' an weitesten durchgeführten Teilung der Arbeit (Näresr). Sehen wir so in den Kulturländern die Menschheit dieser höchsten Organisation stets mehr und mehr zuschreiten und den National-Oekonomen und Soziologen beschäftigt, den Bedingungen. Gesetzen, den Fntwicklungsprozessen ete. dieses Werdeganges nachzuspülen, lesp. diese zu zergliedern und ihre Bedeutung für den volkswirtschaftlichen und kulturellen Fortschritt zu ermessen. so elwächst dem Zoologen in gesteigertem Maasse das Bedürfnis, die Vorgänge der Arbeitsteilung und Arbeitsvereinigung im Tierereich dal'zulegen, die Bedingungen und Anpassungsursachen zu erforschen, welche diese hochinteressanten Verhältnisse auf dem Tege des langsamen phylogenetischen Werdens bewirkt haben liönnten. sowie anch auf den Wert oder Unwert von Parallel-Erscheinungen andeutend hinzuweisen.

* Der angekündigte Vortrag über « Die Bastardierungsrerhältnisse bei der Honigbiene und das Mender'sche Gesetz » wurde vor Anfang der Kongress-Verhandlungen zurückgezogen. Die nachstehenden, ursprünglich nicht für einen Vortrag bestimmten Mitteilungen, erfolgten auf besonderen Wunsch Prof. Dr. A. Forel's. 
Einer ausgeprïgten, auf das Wunderbarste differenzierten Arbeitsspezialisierung begegnen wil in der Klasse der Insekten bei der Ordnung der Urtlügler (Archipteren) und zwar bei den 'Tex'miten (von denen wir in unserer Betrachtung absehen) und in der Ordnung der Hautflügler (Hymenopteren) bei den Ameisen, Wespen, Hummeln und bei den Bienen.

In den letzten Jahrzehnten ist auf diesem Arbeitsgebiete, wie wir auch aus den gestrigen Verhandlungen ersehen haben, Baustein zu Baustein gefügt worden. so dass unsere Kenntnisse sich erweitert haben, wenn auch noch ganz ausserordentlich viel zu erforschen übrig bleibt. Immerhin komnte ich den bescheidenen Versuch unternehmen, den eingehenderen Fntwicklungsgang darzulegen. del uns von den einsaun lebenden Bienen hinaufführt zu der komplizierten Staatenbildung der sozialen Bienen, zu den Meliponen. Trigonen und zu unserer Honigbiene (Apis mellifica) ${ }^{1}$.

Die charakteristischen Phasen dieser phylogenetischen Entwicklung zeigen uns folgende Zustände:

I. Phase. Zuerst sehen wir das Weibehen einsam ganz für sich lebend. Dis Mämnchen hat sich nach der Hochzeit auf Nimmerwiedersehen entfornt. lesp. ist zu Grunde gegangen. Dic einzellige Wohnung - das Nest - befindet sich weit ab von den Behausungen der Mitgenossen. Die Mutter lebt ihr solitäres nur der angestrengtesten Arbeit gerwidnetes Leben zu Finde, bevor die Jungen el'scheinen, deren jedes sich wieder allein in einem besonderen Nest (Frdloch) befindet (Osmia papareris ete.).

II. Phase. Die Mutter erleichtert sich die Bauarbeit, indem sie mehrcren Fidzellen einen gemeinsamen Ausgang giebt, so wereden mehrere Eier in einem Nest vereinigt. (Anthophora etc.).

III. P hase. In den beiden elsten Phasen erscheinen die Jungen erst in Frühjahr. während die Mutter spätestens im Herbst des vorhergegangenen Jahres zu Grunde gegangen ist. Hiel war also noch nicht einmal die Möglichkeit einer Familienbildung vorhanden. Jetzt verkïrzt sich die Entwicklungsdauer. I)ie Gefahren (Feuchtiglicit. Pilze. Frost etc.) des Winters werden dadurch - wenigstens für eine Generation - beseitigt. Schon Ende Januar kriechen die Jungen aus, um in den Herbsttagen noch eine zweite Generation zu erzeugen, deren Ausschlüpfzeit in den Frühling fällt. (Halictus).

IV. Phase. Infolge noch beschleunigterer Fintwicklung und der Ablage zahlreicher Eiel ereignete es sich, dass die Mutter noch beim Bau der letzten Firdzellen beschäftigt ist. während bereits aus den ersten Zellen die Jungen auskriechen. Hier sehen wir zum ersten Mal den Kontakt

1 Buttel-Reepen. Die stammesgeschichtliche Entstehung des Bienenstuates, sowie Beiträge zur Lebensweise der solitären und sozialen Bienen (Hummeln, Meliponinen, etc.), Leipzig, 1903. 
zmischen "Mutte» ) und "Kind ) herogestellt. Der Anfang der" "Familienbildung ") ist gemacht. (Halictus sexcinctus und quadricinctus).

V. Phase. Unter besonder's günstigen Umständen ereignete es sich. dass die weiblichen Jungen nicht sofort zur Begattung ausflogen, sonder'n bein Anblick der noch unvollendeten, erst ungenügend mit Nahrung gofüllten Zellen sofort den Bau- und Fütterinstinkten gehorchten, also der" "Mutter" ) halfen. Mit dieser ersten Familiengemeinschaft musste naturgemäss - wie bei der menschlichen Familie - sofort Arbeitsteilung cintreten. Ich kann mich hiel nicht auf die nähelen Ausgestaltungen einlasien und velweise auf meine hezüglichen Arbeiten.

In dieser Phase dürfte sich wahrscheinlich auch der Uebergang von der Familien-zur State $\mathrm{n} b i l d u n g$ vollzogen haben. Wir wissen, dass versehiedene einsam lebende Hymenopterenarten die Fähigkeit besitzen, ans parthenogenetisch abgelegten Eiern fortptlanzungsfahige Nachkommen zu elzeugen. War nun diese Fähigkeit bei einer Bienenart, deren häusliche Zustände in der Y. Phase geschildert wurden, vorhanden, so dürften die "Kinder » nicht nur del "Mutter" ) beim Füttern und Bauen geholfen haben, sondern auch beim Eicrlegen. Es ist ein anscheinend bei vielen Insekten gültiges Gesetz, dass nit der Eiablage auch die Brunst für immer erlischt. Diese unbefruchteten Eierlegerinnen blieben demmach unbegattet. Die primitivste Stufe der sog. Staatenbildung war damit ereicht. Diese Stufe dokumentiert sich also durch das Torhandensein einer befruchteten Mutter und mehr oder minder zahlreicher unbegattet bleibender Töchter (Arbeiterinnen) und der Söhne, die schl wahrscheinlich aber keine ständigen Bewohner des Nestes blieben, sondern - wie es auch heute noch vielfach der Fall ist - bald auf Liebesabentener ausgingen und wohl selten oder gar nicht zum Nest zurückkehrten.

Die V I. P hase zeigt uns Zustände, wie wir sie bei den Wespen und Hummeln antreffen. Das im Herbst befiruchtete junge Weibchen überwintert einsam in eineu Versteck, um im Frühling den Neubau zu begimnen, Eier zu legen und die heranwachsenden Jungen mit Nahrung zu versoron. I)ie ausschlüpfenden Tüchter helfen als(ann der Mutter in der eben angegebenen Weise.

Bis hierher hat sich das Muttertier die Fähigkeit bewahrt, ganz allein im Kampfe ums I)ascin bestehen zu kömnen. Trotz der im bevölkerten Neste scharf ausgeprëgten Arbeitsteilung sind die Instinkte des Muttertieres dic alten geblieben. Es vermag ganz allein ohne Beihülfe ein ncues Nest - also eine neue Staatenbildung - zu begründen und den Iatu-, lerteidigungs- und BrutpHegeinstinkten voll nachzukommen.

VII. Ph a se. I)je weitere Stufe dokmmentiert sich durch die einseitige Adaptation der Königin.

Hiel rrgeben sich nun eigentümliche, interessante Kontraste zwi- 
schen den heiden höchst organisiorten Staatenbildungeu, die wir liennen), und zwar zwischen dem Staate der Ameisen und dem der Bienen. Wir sehen einerseits bei den Bienen, die vielleicht am meisten vorgeschrittene Arbeitstrilung und demgemäss cine sehr hohe Leistungsfähigheit. I)as Muttertier - die Königin - hat fast alle Instinkte eingebüsst, sie ist von ihrer alten Höhe herahgesunken ", unfähig sich allein zu cruähren und allein der Winterkälte zu widerstehen, aller Bau- und Broutphlegeinstinkte verlustig, sinkt sie zul Eierlegemaschine herab, vollführt in dieser Eigenschaft aber Erstaunliches. Taneben die ebonso einseitig angepassten Alheiterinnen, die im normalen Zustand dio Eiablage ganz eingebüsst haben und nur noch den Bau-, Verteidigungs- und BrutpHegeinstinkten roll nachgehen. Auch sie kömnen wie die Königin nu• noch im Staatsverbaude lehen. Inas Tolk muss demnach auch im Winter zusammen bleiben. Allein auf sich angewiesen, gehen sie meist in wenigren Stunden schon zu Grunde. Andererseits haben wir bei den Ameisen eine in gewisse. Weise noch weitergehende Spezialisiorung. Tohen der nolmalen Künigin mit Flügelanlagen lommen hei gewissen Arten noch sehr fruchtbare Königimnen ohne Flügelanlagen ror, die Arbeiter scheiden sich bei besonderen Species in grosse, mittlere und kleine, die verschiedene Funktionen zu elfüllen haben. Kur der Polymorphismus ist ein viel grösserer. Ich sehe hier ganz ab von den besonderen Spezialisierungen der schmarotzenden oder Sklaven haltenden Arten ${ }^{3}$.

Wie aber steht es mit der Königin? Ist auch die Ameisenkönigin in der Spezialisierung vorgeschritten, hat sie demnach ihre Selbstïndigkeit eingebuisst oder vermag sie noch einen Stat ganz allein zu begründen, sind die alten Instinlite wach gebliehen, wie hei den Wespen- und Hummelköniginnen?

Wie wir aus den gestrigen Verhandlungen ersehen hahen, beschäftigt diese Frage auch noch unsere hervorragendsten Ameisenforscher und wenn auch hisher verhältnissmässig wenige Beobachtungen vorliegen, so ist doch liein Zweifel mehr, dass diese. Fïhigkeiten bei Ameisenkïniginnen gewisser Arten gefunden werden. Bei der Knappheit der vorliegenden und melnfach nicht ganz durchgeführten Beobachtumgen wei es mir gestattet, meine bescheidenen Resultate hinzuzufügen.

* Somit zeigen sich die Nachteile der Arbeitsteilung für das einzelne Individuum. Die einseitige Anpassung hewirkt hier - wie hei den Menschen - einen Vorteil für das Ganze aber unter Umständen einen Nachteil für den Einzelnen.

3 Ueber Polymorphismus der Ameisen vgl. Aug. Fonel, Ueb. Polymorphismus und Variation bei den Ameisen. Zool. Jahrb. Suppl. VII, 1904, Festschrift f. Wetsurann; ferner Euery, Le Polymorphisme des Fourmis et la Castration alimentaire. C. R. d. Séances du Troisième Congrès int. de zool. 1896 ; derselbe, Zur Kenntniss des Polymorphismus der Ameisen. Zool. Jahrb. Suppl. VII, 1904. Festschrift f. Weismann.

VIe CONGR, INT. ZONL., 1904. 
Wie entsteht ein Ameisenstat? Mustern wir die ausseroldentlich reiche Litteratur über Ameisen, so finden sich, wie erwähnt, nur wenige Jotizen. Es liommen im Wesentlichen die folgenden Autoren in Betracht: Pierre Huber ${ }^{4}$, Forel ${ }^{5}$, Mac Cook ${ }^{6}$, Lubbock ${ }^{7}$, BlochMANN ${ }^{8}$ und neuerdings WheELER ${ }^{9}$ und nochmals Forel ${ }^{10}$, wie auch $J_{\text {ANET }}{ }^{22}$. Diesen Forschern gliedert sich nach den gestrigen Verhandlungen auch EMerY an.

Aus den Beobachtungen der Vorstehenden darf man wohl im grossen Ganzen entnehmen, dass sich bei einigen Arten der höheren Ameisen dir Königin, nach dem Hochzeitsausfluge eine Art Höhlung, einen Kiessel (oft unter einem Steine) selbst bereitet, in diesem Gemache mit der Eiab)lage beginnt, die Fütterung der Larven und Wartung derselben durchführt, bis dann die Jungen sich einen Weg aus den Kessel bahnen und nunmehr die Ptlege der Mutter und der Larven auf sich nehmen.

Wie wenig sicher resp. ausgebreitet unsere Kenntnisse hierüber sind, ergiebt sich auch daraus, dass über die Gründung einer Kolonie bei der sch! weit verhreiteten Formica rufa nichts bekannt ist und WheELer (A weu type of Social Parasitism among Ants. Bull. Am. Mus. Nat. Hist. Vol. XX, p. 347-375. 1904) sogar der Ansicht ist, dass möglicherweise bei dieser Ameise ein eigentümliches parasitüres Stadium statt hat, indem die junge, nestgründende Könnigin zuerst z. B. bei F. firsca unterschlüpft, in deren Nest ihr Volk begründet, un damn allmählich das gastliche Tolk zu verdrängen.

Auf Adoptionsinstinkte von Formicu fusce hat übrigens Wasmaxy (Neues über die zusammengesetzten Nester und gemischten Kolonien der Ameisen. Allg. Zeitschr. f. Entomol. 1901 bis 1902, Bd. 6 u. 7, p. 20 u. 77 des Sonderdruckes) schon frühre aufmerksam gemacht. Er fand zweimal weisellose Kolonien von Formica fusea mit befruchteten Weibchen vou truncicola, und weist auch in verschiedenen anderen Arbeiten auf a'mischt? fusca-holonien lin, desgleichen Fores. ', der schon vor langen Jahren und auch mehrfach neuerdings analoge Terhältnisse schilderte. Neu ist dagegen die Ansicht WneELens, dass wir es in manchen dieser Fälle wahrscheinlich mit normalen Vorgängen zu thun

4 Les Fourmis indigènes. Genève, 1810.

5 Fourmis de la Suisse. Zürich, 1874.

${ }^{6}$ How a Carpenter antqueen founds a formicary. Proc. acad. nat. se. Philad. 1883.

7 Ameisen, Bienen und Wespen. Leipzig, 1883.

8 Zeitschr. wiss. Zool. 41. Bd., p. 719-727. 1884.

9 Biolog. Bull. Vol. II, 1900. Die WheELeR'schen Beobachtungen beziehen sich auf niedere Ameisen, Ponerinen. Wheeler schliesst, dass bei diesen mit der jungen Königin auch stets Arbeiter zur Gründtng einer neuen Kólonie ausziehen. Eine directe Beobachtung liegt jedoch nicht vor.

${ }^{10}$ Ann. Soc. entom. Belg. Bd. 56, p. 180 u. 193.1902. 
haben, dass also Ameisenweibehen gewissel Alten stets in Kinlonien gewisser anderer Arten unterschlüpten müssen, um überhaupt die Gründung einer eigenen Kolonie zu ermöglichen. Von hier aus diüfte auch Licht fallen auf die Entstehung des Instinktes Sklaven zu halten.

Nachstehend seien meine Beobachtungen über Koloniegr'ündung bei Lasius niger mitgetheilt:

Das Nest der Königin. Von mehreren auf dem Hochzeitsfluge abgefangenen Weibchen ron Lasius miger (22. Juli 1903) elwiesen sich zwei als befruchtet, da sic nach mehreren Tagen rie Flügel abwarfon. Sie wurden am 1. Aug. gemeinsam in ein kleines Nest gebracht.

Da ich mir selbst eine Bestätigung bezüglich der Bauinstinkte verschaffen wollte, kam für diesen Versuch nur ein LuBBock'sches künstliches Erdnest in Frage.

A uffälige individuelle Verschiedenheiten ergaben sich sehr bald bei den Weibchen. Während das eine nach kurzer Zeit den Boden an einer Stelle von Erdpartikelchen gesäubert hatte und sofort anfing, einen nach Innen überhängenden Erdwall von ovalem Grundpiss um sich helum aufzulauen, mühte sich die andere liönigin vergeblich ab. das Gleiche zurege zu bringen. Hier und dort trug sie die Fidkïrnehen bei Seite und legte den Glasboden frei, aber ein oldnungsmässiges Gremach kam nicht zu Stande, schliesslich entstand rin kleines sehr unl'egelmässiges Stückchen überhängenden Walles.

Am 8. August zeigte sich das lïnigliche Gemach rollendet. I)ie Kü̈niginnen waren demnach von einander getrennt. Am 15. finden sich bei dem nestlosen Weibchen 10 Eier, bei dem anderen 4. Bei Störungen werden die Eier möglichst ins Dunkle geschleppt, 1. h. unter den überhängenden Erdwall.

Es rrgieht sich also, dass Bau- und Brutptlegeinstinlite vorhanden sind.

Nachdem sich bei der freilebenden Königin die Eier auf 14, bei der anderen auf 10 vermehrt haben, erlebe ich am 21. eine seltsame Ueberlatschung: Uober Jacht ist die freilebente Königin mit Sack und Pack, d. h. mit ihrem Eierpacket in die wohlverschlossene Behausung der anderen eingehrochen und hat sich dort heimisch gomacht. Friedlich hausen die beiden zusammen. Die Eiel finden sich auf einem Haufen vereinigt.

Welche Instinkte mögeu diese Vereinigung herbeigeführt haben?

Dass zwoi Kö̈niginnen und mehr in demselhen Nest gefunden werden. ist eine häufige Erscheinung. Da das Nest normaler Weise von einer Krïnigin hegründet wird, sind die anderen Kö̈niginnen oft wohl 'Tüchter der ersten Königin, die im Nest befruchtet wurden. Doch sind noch andere Vergesellschaftsmöglichlieiten sehr wohl in Betracht zu zichen (vol. JANET $^{22}$ ). Dieser Zustand dürfte aber vielleicht, - es sei dies mit allel Reserve hemerkt, - nur dadurch ak dauerend möglich erscheinen. weil 
in sehr volkreichen Kolonien die Königinnen räumlich von einander getrennt sind und sein können. Bei Arten, die nur kleine Kolonien bilden, finden wir daher auch in den allermeisten Fällen nur èine Königin. Aus einer weiterhin erfolgenden Beobachtung (S. 473) dürfte vielleicht ersichtlich sein, dass - zum mindesten bei einigen Species - zwei Königinnen im selben Raume dicht beieinander nicht der Norm entsprechen.

Man könnte bei dieser Tereinigung der beiden Königinnen ja leicht von einem Unbefriedigtsein der einen Königin reden, da sie nicht in Stande war, die ordnungsmässige Zelle herzurichten. Woher aber konnte sie die ()r(nung. der sie vielleicht instinktmässig zustrebte, hinter dem geschlossenen Erdwall als vorhanden annehmen. sichtbar war ihr der normale Bau des anderen Weibchens nicht.

Manche wer'den hierin wieder einen Beweis für die "Klugheit » der" Ameisen erblicken, aber wie ich schon melurfach an anderer Stelle darl'gte, führt der so maheliegende Weg, speciell bei Beurtheilung der sozialen Insekten die menschliche Auffassung allzusehl walten zu lassen, sehr häufig in falsche Richtung. Das so oft gefundene ausserewewhnlich starke Hineintragen anthropomorpher Ideen in diese Staatenbildungen, wird durch die Erkenntniss unterstützt, dass nicht principielle sondern nur graduelle Unterschiede zwischen Menschen- und Tirrseele vorhanden sind, aber gar zu leicht wird bei Betrachtung der complizierten und anscheinend so hoch organisierten Terhältnisse, die uns diese Kerfvereinigungen licten. vergessen, welch' ausserordentlich weite Kluft den Menschen von diesen trotz wunderbarster Fähigkeiten sehr niedrig olganisierten Insekten trennt. Anstandslos hat die alte Tierpsychologie die höchsten soziologischen Folgerungen diesen Kerfen vindiziert, wie schon der Vame "Königin " für die Mutterameise oder" -bienc besagt und vielfach wird noch gewlaubt, dass es sich damit thatsächlich um eine "Herrscherin 》) handle, die ihre "Unterthanen »-die Arbeiterinnen - nach ihrem Willen leite, königliche Ehren geniesse und was dergleichen mehr ist. In Wahrheit ruhen alle Volksinstincte bei den Arbeiterinnen. Ich habe versucht, - soweit der Bienenstaat in Betracht kommt, - diese Terhältnisse in die richtige Beleuchtung zu rücken und muss den sich hierfür Interessierenden auf diese Arbeiten verweisen, da mich ein näheres Eingehen zu weit führen würde. Nur eines sei hier bemerkt, dass wir der Analogieschlüsse bei Beurteilung der Tiersecle selbstverständlich nicht entraten kömnen ". Bezüglich der psychischen Fïhigkeiten hei den Ameisen etc. seien die Arbeiten von Forel und Wasmann elwähnt ${ }^{12}$.

11 "Sind die Bienen Reflexmaschinen? " Leipzig, 1900, und "Die stammesgeschichtliche Entstehung des Bienenstaates. „ Leipzig, 1903.

${ }^{12}$ E. Wasmann. Die psychischen Fähigkeiten der Ameisen. Zoologica, Heft 26, 
Ueber die Ursachen der Staatenbildung. Wenn ich oben den Kontakt von " Mutter und Kind » erwähnt habe, wie er bei den Apiden erstmalig entstanden ist und von dem Anfang der" "Familienbildung " spreche. so sind auch hier alle anthropomorphen Vorstellungen auszuschalten. Es handelt sich bei diesen Inseliten weder um ein intimeres Terhältnis zwischen Mutter und Sprössling, also um Mutterliebe oder Kindesliebe im menschlichen Sinne, noch um irgend ein Familienzusammengehörigkeitsgefühl.

Wenn nach verschiedene'n Autoren in der. Yutterliebe die Grundlage der Soziologie gefunden wird, so müssen wir meines Erachtens bei den hier zur l'mage stehenden tierischen Vergesellschaftungen dieses anthropomorph psychische Doment ganz ausscheiden. Wir sehen nämlich rine'seits eine ständige Brutptlege bei einigen einsam lebenden Hymennpteren (Cerceris, Bembex, Crabro u. s. w.) d. h. ein andauerndes "liebevolles ) Füttern der Jungen his zur Verpuppung - also ständigen Kontakt zwischen "Mutter" und " Kind " - und anderer'seits bei einer hoch komplizierten sozialen Gemeinschaft bei dem Staate der stachellosen Bienen (Meliponen und Trignnen) keine Brutfütterumg dieser Art, wie mil sie sonst bei allen anderen Insekten-Vergesellschaftungen antreffen (Termiten, Ameisen, Honighiene). Bei den Meliponen und Trigonen wird das Ei auf den Futterbrei gelegt, die Zelle geschlossen und das Junge sich selhst üherlassen. I as ist also genau dasselbe Terfahren. welches alle solitär lebenden Bienen anwenden, die nie zur Fanilien- geschweige denu zur Staatenbildung aufgestiegen sind. Wenn wir demmach dieselbe Art dieser speziellen Brutpflege bei sozialen und solitären Inseliten antreffen, so ist nicht anzunehmen, dass di ese besondere Ausführung der Brutptlege, die hei anthropomorpher Auffassung der Sachlage am deutlichsten eine "Mutterliebe » zu dokumentieren scheint, also das ständ ig e Füttern, das "Aufpäppeln ", zur Familien-resp.zur Staatenbildung hinübergeleitet haben sollte.

Inie Ptlege der Nachkommen und die daraus ahgeleitete Mutter- resp. Elternliebre ist ein reiner Selektionsworgang, ein einfacher Zuchtwahlprozess und Naturzüchtung rermag, wie sich aus Vorstehendem crgibt, auf verschiedenen Wegen dasselbe (Staatenbildung) zu erreichen.

Wie ich an anderer Stelle nähtr auszuführen versuchte und wie ich

1899 ; derselbe, Instinkt und Intelligenz im Thierreich, 2. Aufl. Freiburg i. Breisg. 1899 ; derselbe, Vergl. Studien über das Seelenleben d. Ameisen und der höheren Thiere, 2. Aufl. ebenda 1900. Forel, Aug. Die psychischen Fähigkeiten d. Ameisen u. einiger anderer Insekten. München 1901; derselbe, Expériences et remarques critiques sur les sensations des Insectes, I.-V. Theil. München, Paris 1901; derselbe, Die Berechtigung der vergleichenden Psychologie und ihre Objecte, Journ. f. Psychol. u. Neurol. I. Bd. 1902. 
es vorhin schon kurz berührte, ist in letzter Linie - aber doch als wesentlicher Faktor - sehr wahrscheinlich ein bei vielen Inselkten gültiges Gesetz für die Entst ehung der Statenbildung verantwortlich zu machen, das (iesetz, dass der Begattungstrieb für immer erlischt, wenn ein unbefruchtetes Weibchen mit der Eiablage begonnen hat. Nur durch das Walten eines anscheinend weitverbreiteten Gesetzes erscheint, so will mir dünken, das an verschiedenen Stellen des Insektenstammes eingetretene Aufsteigen zu dem seltsamsten aller Yerbände erklärlicher.

I)er biologische Wert der Staatenbildung liegt nicht in gemeinsamer Abwehr von Feinden oder gemeinsamer' Beseitigung sehädlicher Faktoren, sondern, so glaube ich, lediglich in der Vielbeit der Bruternährerinnen, so dass, wenngleich viele zu Grunde gehen -- und dieses zu Grundegehen geschieht gewöhnlich e in zel n ausserhalb des Nestes dennoch genügend erhalten bleiben, um für die Brut zu sorgen etc. und die Erhaltung der Art zu sichern.

Dass die Staatenbildung entstanden sei aus einer Tergesellschaftung von mehreren befruchteten Weibchen, wie auch angenommen wird ${ }^{13}$, erscheint mir sehr' un wahrscheinlich, da das eigentümliche Bindeglied des Kerfverbandes - nämlich die Entstehung von unbefruchtet bleibenden "Arbeiterinnen " - bei dieser Hypothese vollkommen u n e rklärt bleiht, wie auch das schliessliche Ueberwiegen eines Nuttertieres. Ich halte das Auftreten polygamer Vergesellschaftungen, wie wir sie auch bei tropischen Hummehn und Wexpen beohachten, fül sekundäre Anpassungen.

Kehren wir zı unseren beiden Ameisenköniginnen zurück, so sehen wir eine friedliche Fortentwicklung. Die erste Larve zeigt sich einen IIonat nach der ersten Eiablage und allmählich schlüpfen die anderen 23 aus. Trotz der Tag und Nacht ziemlich gleichmässigen Zimmertemperatur schreiten die Larven nicht zur Verpuppung. Monat anf Monat rergeht bis Anfang Iai dieses Jahres ein Wachstum der Larven zu bemerken ist und am 20. Mai, also ca. 8 Nonate nach dem Ausschlüpfen der ersten Larve, finden sich die ersten liokons. Nach weiteren zwei Monaten, genau am Hochzeitstage der beiden Königinnen — am 22. Juli kriecht die erste winzige Arbeiterin aus.

Dass die Königinnen, wie zu erwarten war, anch die weitere Brutptlege ausüblon, zoigt mir der nächste Tag. Die beiden Weibchen belecken auf das Eifrigste rin fast noch ganz farbloses, allyufrüh aus dem Kiokon befreites Junge. Sie halten es in der Schwebe, die eine leckt den Vorderkörper, die andere das Abdomen und in der Nitte steht ein Liliput, die gestern ausgekrochene Arbeiterin, die kaum so gross ist wie die Hälfte

is R. v. Inering. Zur Frage nach dem Ursprung der Staatenbildung bei den sozialen Hymenopteren. Zool. Anz., 27. Bd., Nr. 4. 1903. 
des Brustkastens der so sehr viel grösseren Mutter, und beleckt nach

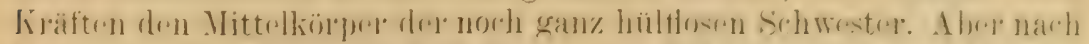

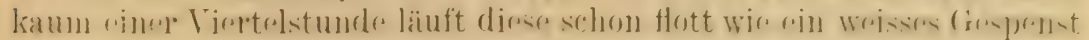
unter den braunen fienossen herum, um elst allmählich im Laufe der nächsten 'Tage das dunkle Pigmentkleid anzulegen.

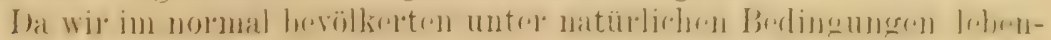
den Ameisenstaat die Königin bei vielen Arten im Wesentlichen sich

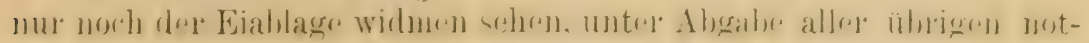
wendigen Verrichtungen an die Arbeiter, so ist es immerhin von Interesse zu erfahren, wann diese Geschäftsabgabe, dieses Nachlassen der übrigen Instinkte, zu geschehen pflegt.

Veränder n der Instinkte. Wenn ich von meinen Beobachtungen auf die Norm schliessen darf, was natürlich mit aller Reserve geschieht, so wird schon alsbald der Batinstinkt nach dem Erscheinen der ersten

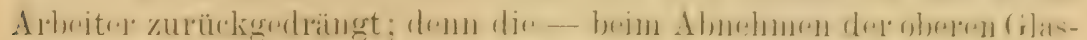

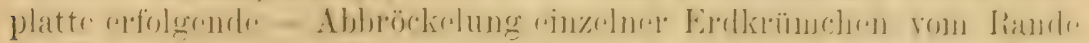

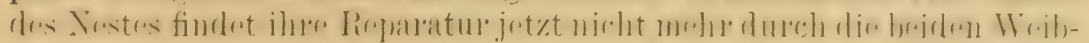
chen, wie fr'üher', sonder'n ausschliesslich dur'ch die Arbeiter'14. Nach dem

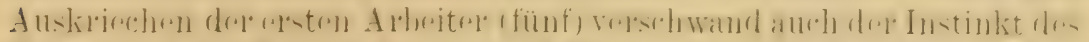

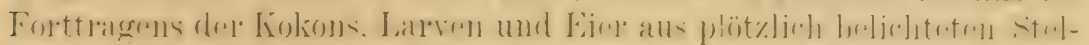
'len in das Dunkle, das wird jetzt ebenfalls nur den Arbeitern überlas$\operatorname{sen}^{15}$. Dagegen füttern die Königinnen sich gegenseitig; von beiden Seiten lief in einem Falle (29. Juli) eine Arbeiterin herzu, "so hängen alle vier an einem 'l'ropfen $1{ }^{16}$. Diese Beobachtung dürte aber keinen

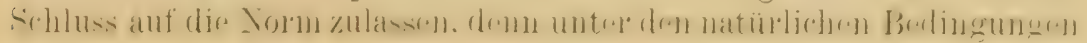

14 Interessant ist die Bauvollziehung. Die in den Mandibeln herbeigeholten Erdpartikelchen werden durch Kopfbewegungen in die Baulücke geschoben und dann durch tretende Bewegungen der Vorderfüsse noch mehr befestigt. Als Mörtel dient der Speichel.

${ }^{15}$ Eine seltsame Ausnahme, dle nach dieser Richtung fällt, verdient Erwähnung. Am 3. August sehe ich eine Arbeiterin ungefähr in der Mitte des Kessel krank auf der Seite liegen, Kopf und Irundwerkzeuge sind in ständiger Bewegung, dis Beine jedoch regungslos. Das eine Weibchen kommt herzu, beleckt die Kranke eifrig und trägt sie dann unter den überhängenden Wall. Dort lässt sie sie liegen. Am übernächsten Tage war die Arbeiterin todt. JAxET ${ }^{22}$ berichtet von Königinnen verschiedener Species, dass sie "en cas de danger s trotz bereits vorhandener Arbeiter Eier und Larven fortgetragen hätten. Auch geht aus einer seither veröffentlichten weiteren Beobachtung JANET's hervor, dass auch vorjährige Iöniginnen, die bereits einmal einen Staat begründet hatten, wenn sie isoliert wurden, aufs Nene die Fähigkeit besassen, alle die notwendigen Instinkte zur Gründung einer Kolonie zu entfalten.

${ }^{16}$ Dieser Tropfen, der gross und breit hervorquoll, sodass der halbe Kopf der Futter heischenden Königin davon benetzt wurde, war offenbar nichts anderes nach Farbe und Geschmack als der gefütterte Invertzucker und kein Drüsensekret. 
ist anzunchmen. dass die Königin eingeschlossen in ihrem selbstgeschaffenen Kerker ohne jede Füt ter ung auszuharren hat, bis die ersten Arbeiter ausgelirochen sind. diese schaften alsdann dir Nahrung herbei. I)je gestrige Selitionsverhandlung berührte ja auch diese Verhältnisse. In der Natur' geht die Entwicklung der Arbeiter schneller von statten, übredies hat Foreu experimentell festgelegt. dass oine Ameisenkönigin in der Tat sehr lange zu hungern vermag ${ }^{17}$. Ein Camponotus ligniperdus- Ireibchen wurde von ihm vom August bis zum nächsten Nai ungefüttert gelassen. In dieser Zeit legte sie eine ganze Anzahl Eier und zog einen Arbeiter und mehrere Puppen heran.

Da mir diese Frage entschieden zu sein scheint, gab ich, um eine gute Entwicklung zu erzielen, den beiden liöniginnen ungefähı alle Monate eimmal einen 'Tropfen Fruchtzucker' (Invertzucker), der stets sofort aufgenommen wurde.

Womit nährt aber (lie normaler" Weise ungefüttert bleibende Kïnngin die Larven, da ihr . lagen nicht immer in Betracht kommen dürfte? Wohl zweifellos mit den Sekreten der Speicheldrüsen, wie wir diese Art der Fütterung auch bei den Termiten und wenigstens teilweise bei der Honigbiene sehen ${ }^{18}$.

Auf diese Weise dürften die beiden Königinnen auch den nötigen Stickstoff den Larven gereicht haben, da in ausschliesslich gegehenen Invertzucker kein Stickstoff' vorhanden ist. Eine Zufuhr von Stickstoff geschieht vielleicht in minimalsten Mengen durch das Anfeuchten der Erde mit Wasser, das dem eingeschlossenen Weibchen auch zur I)urststillung dient und ferner durch das Verzehren der eigenen Kinder (Eier und Larven), wie es mehrfach beobachtet wurde ${ }^{17}$ und wie auch ich es - wenigstens für Larven — bestätigen kann ${ }^{19}$. Normaler Weise muss aher die Quelle des Stickstoffes in den eigenen Körpervorräten der Mutter gefunden werden. So wies JaNet ja auch in der Diskussion der gestrigen Sitzung auf eine neue bisher nicht beachtete Nahrungsquelle der bufruchteten Lönigin und zwar auf die alhuählich atrophierenden verhältnismässig sehr starken Flügelmuskeln hin. Dieses Schwinden der

17 Aug. Forec. Quatre noticcs myrmécologiques. Ann. de la Soc. Ent. de Belgique. 46. Bd., p. 180 u. 294, 1902 ; ferner JANET ${ }^{22}$.

18 Auch in wissenschaftlichen Kreisen trifft man noch auf die Ansicht, dass die Honigbiene Honig und Blütenstaub (Pollen) füttere; sie reicht jedoch den Larven einen fast schneeweissen, geléeartigen Futterbrei, der nach Ansicht von Schimmenz (Z. wiss. Zool., 38. Bd., 1883) ein Sekret der Speicheldrüsen, nach Ansicht von ScröxFELD (Ernähr. d. Honigb., Freib. i. Br., 1897) Chylus aus dem Chymusmagen ist. Ueber "Futtersaft " der Ameisen s. a. WheELER "Ethological observations on an (merican Ant." Journ. f. Psych. u. Neurol.. Bd. II, Heft 1 u. 2. 1903.

${ }^{19}$ Von den 24 überwinterten Larven waren bis Anfang August 11 allmäblich verzehrt worden. 
Flügelmuskoln wirel ührigens auch in eigentümlicher. Weise henutzt, um ältere Kö̈niginnen von jungen zu unterschejolen. Nach WHEEL:e (A wew type of social parisitism among ants. Bull. Am. Mus. Nat. Hist. Vol. 20, 1904, p. 356(i), sinken ältere Kö̈niginnen im Wasser order Alkohol nicht unter. da an Stelle del verschwundenen .Juskeln Luft im Brustkastrn vorhanden ist.

Der monogame Staat. Das friedliche Zusammenleben der Königinnen sollte bald eine Störung erfahren. Am 5. August sah ich beide Weibchen in Kampfe miteinander, dem einen ist bereits das cine Bein zwischen Femur und Tibia algebisson und die Vorstummelte wehrt sich nur schwach. Mit Geschick versucht die Siegerin stets die schwichste stelle. die dünne Vrobindung zwischen Abdomen mor Thorax, zu attaguioren. Ich treme die Raufenden, die das Finerpacket bereits auscinandergewilzt halen. Nit Stamen sche ich aher, dass auch rinige Arbeiter feindlich gegen die Verstümmelte sind und ihr Bisse in die Beine versetzen, ohme allerdings bei ihrer Kleinheit viel auszurichten. Was ist hier vorgegangen? Welche Instinkte habru dirse Auflehnung, diese Frindschaft nach so langen, frodlichen Zusammenloben hewirkt? Man darf vielle ich t folger'n, dass die 'Tendenz zum monogamen Staat auch in der Ameisengesellschaft hegründet liegt, wemngleich die Kïniginnen sich nicht mit der Eifersucht begegnen, wie wil' es z. B. im Bienenstaat sehen; wird doch auch, wie schon erwähnt, jecler Ameisenstaat stets nur durch e in e Königin begründet. Finden wir auch öfter's zwei

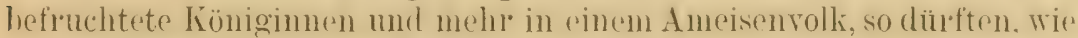
ich bereits anführte, dies Kö̈niginnen öptlich von vinander gotrennt sein und sich nicht in demselben "königlichen (iemache " brfinden. Solange noch krine eigentlich Kolonie begründet war, schlummerten rlie monogamen Instinkte, sowio dio Kolonie sich aber zu entwickoln anfing, golangte rlas phyletische Herkommen ehenfalls zur Entwielslung mel die stälkero Königin (resp). (lie Nesterbauerin) suchte die "Alleinherrseliaft " zu erlangen, nicht um hier nun wirklich "Alleinherrscherin " zu sein, - wie sehon ausgeführt. sinct alle derartigen authroponorphen Uehertragungen nidht entsprechend --, sondern vielleicht weil die phylogenetische Entstrhung eines Ameisenstaates nur e in Woibchen lodingt. Man muss jedoch hier mit Terallgemeinerungen sehr vorsichtig sein. I)ass dir Arbeiterinnen die a ängstlich nsich Wrhende, bereits Terstümmolte nun auch anfallen, ist wahrscheinlich auf rinfachste Reflexe zurüclizuführen (z. B. Reaktion auf Angstgeruch oder "ängstliche » uns nicht vernehmbare Laute).

Da der Kampf bald von Neuem begann und ein weiteres Bein abgetissen wurdr: entfernte ich die Unterlirgende und sotztr sio in rin Nost für sich. Dort fand ich sie am ander'n Tage todt.

Anfallen der eigenen Nestgenossen. Wie leicht die Arbeiter 
dazu zu bringen sind, infolge besonderer Reflexe selbst ihre eigenen Witarbeiterinnen anzufallen und auf das Wütendste zu beissen. zeigt folgende Beobachtung.

In ein künstliches Nest von Myrmica lrvinodis. Nyl. setzte ich eines Tages eine sehr kräftige Musca romitoria (Schmeisstliege), die wie toll umherwirbelte, sowie sie eine Ameise an den Beinen spürte. Dieses stürmische Gobahren brachte eine Aufregung sonder Gleichen in der kleinen Kolonie hervor. Während die Fliege in rasendem Rundtanz die Ameisen, Lar'ven und Eier umherschleuderte, gelang es einer Arbeiterin, sich an den Beinen festzubeissen und num passierte etwas seltsames, in kurzer Zeit hing ein Klumpen von Arbeiterinnen nicht direlit an dem Bein der Fliege sondern an dieser Arbeiterin ineinander festgebissen. In die zweite biss eine dritte, vierte und fünfte u. s. w. Diese Klumpen schleuderte die Fliege mehrfach ah, selhst mehrere derartige Haufen an den verschiedenen Beinen flogen durch starke Drehung fort. In blinder Wut fallen nun erst einzelne Arbeiter einer Instinktsirrung zum Opfer, gegenseitig packen sie sich und verbeissen sich incinander und plötzlich, während die Fliege ermattet ihren Wirbeltug unterbricht, fast verlassen von ihren Verfolgern, sehe ich es wie eine Láhmung über die Kolonie hingehen. siumtliche Arbeitre ohne Ausnahme sind in kleinen Gruppen und Haufen regungslos heieinander und die Lupe zeigt mir, dass sich alle in einander verbissen haben und sich zu erstechen versuchen. Deutlich ist der herausschnellende Stachel bei vielen zu sehen. Ich nehme nun die Fliege heraus und befreie sie von den letzten Anhängenden. Zwei der verbissenen Arbeiter tauche ich unter Wasser aber erst nach gelaumer Zeit lösen sich die Mandibeln. Fünf Minuten lange V'erdunkelung des Nestes bringt einen Teil der drolligen Kampfhähne wieder zur Besinnung aber nach 10 Minuten liegt noch ein Klumpen fest ineinander verbissener Alloeiter da. Trotz dieses langen Gefechtes fand ich hernach weder Verletzte noch Tote.

JANET hat nach seiner mir kurz vor dem Kongress zugegangenen neusten Veröffentlichung ${ }^{2-2}$ oft beolachtet, dass wenn er Tiolnnien von Tetramorium cerspitum und solennysis furyx, welche mehrere Königinnen besassen, in künstliche Nester biachte, die Königinnen nach und nach bis auf eine verschwanden. Auch in einem starken Volke von Lasius flavus wurden die Königinnen bis auf eine "von den Arbeitern ) beseitigt. JANET findet den Grund darin, dass " les colonies qui ne sont pas dans les conditions voulues pour prospérer cherchent et ar'ivent à réduire à une seule le nombre de leurs reines ). Aber von der Lasius-Kolonie heisst es: "la colonie étant restée très prospère 》, und trotzdem sohen wir das bescitign der Königimen. Fs kommen demmach rixlleicht auch noch andere Momente in Frage.

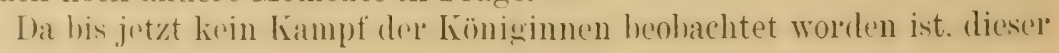


aber wie meine Beobachtung zeigt, dennoch stattfindet, so l'ücken diese ', erhältnisse daduch vielleicht in eine andere Belenchtung wenigstens in Bezum auf Lasius-Arten. I)ir J JxET-Nester hegünstigen abor solche Feindschaften durch den in Wesentlichen ganz froien Wohnraum ohne Erdg:inge bei sehr geringen Separationsmöglichkeiten.

Benehmen sich unbefruchtet gebliebene Königinnen wie Arbeiter? Wenn die vorstehenden Beobachtungen eine weitere Bestätigung dafür geben, dass die Differenzir’ung der Kö̈nigin hei manchen höheren Ameisen nicht soweit vorgeschritten ist, wie die der Bionenkönigin und sich alle Albeiterinstinkte erhalten zoigen, die ein Autsichalleingestelltsein ermöglichen, so müsste der Schluss richtigsein.

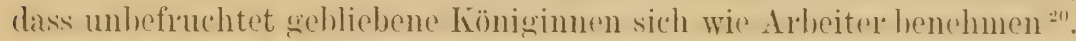
Heber qielot auch an, dass dem so sei " Bei Lasius niger fand ich jedoch anscheinend ein anderes Verhalten, während meine Beolachtungen bei Formica senguinea die Hruer'sche Angahe zu hestätigen scheinen, aher auch in diesem letztelen Falle hlich) eine gewisse Diff(renz bestelen, die sich nicht so schr im eigenen Verhalten, wohl aber in dem besonderen Benohmen der Arlreiter gegen das unbefiuchtete Wreibehen kund wall. So hefreundeten sich zwei likeine $F$. sanmumea-Kolonien. die selon ohne befiuchtete Weiluchen mehrere Monate in liünstlichen Nestern wehalten waren, heim Vreinigen swhe schnoll miteinander. Boi dem einun Volke befand sich ein unhefruchtetes Woibehen und seltsamel'weise wurde mur dieses noch tagelang gezerrt und gebissen, ohne sich je auch nur im Geringsten zu wehren. (iewisse Geruchselfferenzen dürften ständig bestehen bleiben.

Del Lasius-Fall ver'lief wie folgt:

In einer mutter- und männchenlosen Lasius niger Kolonie schlüpten in der ersten Hälfte des Augusts 1903 einige Königinnen aus ihren Kokons, die fast stets von eifrig leckenden, "liebkosenden ") Arbeiterinnen umgehen sind. Sehr häufig reiten Arbeiter auf den breiten Flügraln.

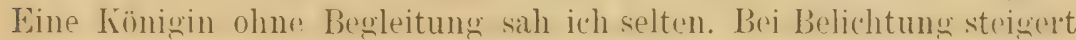
sich die "Sorge 》 und in dem Bestreben, die Weibchen nach dunklen Partien des Nestes hinzuziehen, werden sie oft stark gezerrt. Am 10. Septbr. findet sich ein Weibchen mit halh algehissenen Flügeln und sterbend. Leib und Flügel werden ständig beleckt. Am 4. October gesellt sich zu zwei weiteren inzwischen gestorbenen Weibchen noch ein viertes und am 2.2. ()ct. läuft nur noch eine Kïnigin mit stark zerschlis-

${ }^{20}$ Bei den niederen Ameisen, den Pouerinen, ist auch die befruchtete Königin, wie der tüchtige Ameisenforscher WheELER feststellte, zeitlebens den Instincten nach Arbeiterin, sie geniesst keine besondere Pflege, muss sich selbst ernähren u. s. w. Siehe auch Escherich, Ueber die Biologie d. Ameisen. Zool. Centrallbl. No. 7/8, 1903.

${ }^{21}$ Les Fourmis indigènes, Genève, 1810. 
senen Flügeln herum, die am 1. Novbr. auch stirlot. lis zuletzt gepflegt, grfüttert und heleckt. Die älteste der Königinnen lelite fast drei Monate, aher von Arleiterinstinkten ist nichts zu Tage gekommen. WheELer (Ethological Observations on an american Ant. Journ. f. Psych. u. Neurol. Bd. II, Heft 1 u. 2, 1903) beobachtete dagegen, dass sowohl « virgin and the dealated females of Leptothorax Emersoni Wheeler behaved in all pesperts like the worker's n. Gleiches elgahen WhenLer's Beobachtungen an Myrmica brevinodis Emery sowie hei Atta-Arten (Atta [Trachymyrmex] tardigrada Buckley und $A$. [T.] turifex Wheeler).

Dass die Ameisenlarven selbständig fressen, resp. auch feste Nilhung erhalten. wurde von mir auch an dem Verhleih der Leberreste einer diescr Königinnen konstatiert. Da diese Tatsache zur Zeit der Beohachtung (Juni 1903) im Allgemeinen unbekannt war, sei dieser die Entstehung der verschiedenen Kasten im Staate wieder rätselhafter gestaltende Vorgang hier kurz erwähnt. So gab Escherich in seiner vortreftlichen Uehersicht ${ }^{20}$ noch im vorigen Jahre mit Recht seiner Ueberzeugung dahin Ausdruck, dass "die höheren Ameisenlarven mit Flüssigkeitstropfen gefüttert werden s) und daher keiner so starken Mandilieln bedürfen wie die Ponerinen-Larven, bei denen Wheeler ${ }^{9}$ ein selbststänliges Fressen konstatiert hatte. Finige liurze Tageluchnotizen dürften den Befund am Besten illustrieren. Die Beobachtungen rurden sämtlich an der bereits erwähnten mutterlosen Kolonie von Lasius niger gemacht.

12. Juni 1903. Eine tote Arheiterin findet sich in drei Theile zerst ückelt bei dem Larvenhaufen. Die Larven sind beschäftigt das Innere anzunacen. Das Abdomen zeigt an der Unterseite kreisrunde dem Lmfang der Larven entsprechende Löcher. Möglich ist, dass die Imagines zuer'st angefressen haben.

10. Juli. Hineingelegte Fliegen werden ausgenagt und theilweise den Larven zum sellständigen Ausfessen gegehen. Wenigstens finden sich fast immer Theile auf rlem Larvenhaufen.

30. Juli. Ein zu früh aufgebissener Kokon weist eine anscheinend tote weihliche Puppe auf, die sich nachher auf den Lavenhaufen getragen vorfindet. Zahlreiche Larven fressen daran.

4. Oktoher. Fin Fliegenkopf findet sich auf cinem Larvenhaufen, fost da rangesoyen drei Larven. Nan sieht dentlich das Losreissen, wie eine Arbeiterin eine solche festgesogene Larve packt und ins Dunkle trïgt.

2.). ()ktoloer. Ias Alodomen einer der kiüzlich gestorbenen unbefruchteten Weiluchen lient in der "Kinderstube " und eine Larve saugt daran. Noch deutlicher sah man das Saugen an der Bruch- resp. Bissstelle eines lose liogenden Beines derselben hönigin, das vom regelmaissigen Saugen oder Nagen am Munde der Larve rythmisch berregt wurde.

Hat man die Entstehung der polymorphen Formen zum Theil auch 
auf' die Quantität und Qualität des Futter's resp). der nährenden Sekrete geschoben (EMERY"), so dürfte sich (lic beurteilung wenigstens für die Ameisen nunmehr noch schwieriger gestalten, da von "qualitativer Dosiirung ») wie Fores sich in einer seither erschienenen Arbeit ${ }^{3}$ ausdrückit, nun nicht mehr geprochen werelen kamn. Fores stützt sich auf die erwähnten Angahen von WHEesen üher die Ponerinen und auf eine nicht näher bezeichnete Beobachtung von JANET. Dass sich die JANET' sche Beobachtung auch auf eine Lasius-Form bezog, ersah ich aus der erwälinten mir el'st vor wenigen 'Tagen zugegangenen Arbeit ${ }^{22}$. Hiernach hat JANET bereits 1897 in einer, wie es scheint, nicht allgemein beachteten Note ${ }^{23}$ darauf hingewiesen, "que les Fourmis distribuent à leurs larves non seulement de la nourriture liquide, mais aussi de la nourriture solide ". Er fügt dieser älteren Notiz eine neue hinzu, nach welcher man in Tetramorium und in Tupinome-Kolonien zwischen den Larven kleine Stückchen Fleisch findet, welche die Arbeiter von der Fütterstelle hereinholen und den Larven gehen, "man sieht die letzteren ihren Mund daran heften und begierig saugen ${ }^{\prime}$.

Finde ich so eine erwünschte Bestitigung für meine Beohachtung, so ergiebt diese neueste Arbeit von JanEr auch einige Notizen über die Gründung von Lasius niger-Kolonien. Während eine August-Königin ihm erst im nächsten Frühjahr Arbeiterinnen lieferte, brachte eine andere August-Königin bereits in den ersten Tagen des Octobers die Arbeiter zur Reife. Eine an s. Juli entflügelte Formica fusca lieferte die erste Imago bereits am 22. August!

Selbständigkeit der einzelnen Staatsglieder. Haben wir nunmehr gesehen, dass die lö̈nigin der höheren Ameisen noch eine volle Selbstiundigheit besitzt und dass sogar auch die Larven - wenigstens bei einigen Arten - Zeichen selbständigen Torgehens, wenn auch in sehr beschränkter Weise zur Leusserung bringen, so finden wir diese Fähighoit auch bei den erwachsenen Arbeitern ausgeprägt. Während Bienenköniminmen oder -Arbeiterinnen vom Volke losgetrennt, wie erwähnt, meistens in wenigen Stunden zu Grunde gehen, kamn man eine Ameisenarbeiterin ein halbes Jahr lang isoliert halten ${ }^{24}$.

Stellen wir diese beiden Staatengebilde zur soziologischen Abwägung noch eimmal einander gegenüher, so ergehen sich die nachstehenden Verhältnisse. Auf der einen Seite - bei der Honigbiene eine fest geschlossene Organisation, Unselbstïndigkeit aller Glieder,

${ }^{22}$ Charles JANET. Observations sur les Fourmis. Limoges, 1904.

${ }^{29}$ Charles JaNet. Sur le Lasius mixtus, l'Antennophorus uhlmanni, etc. Limoges. 1897.

${ }_{24}$ Adele Fielde. Notes on an ant. Proc. Acad. Nat. Sciences of Philadelphia, p. 599.1902 . 
Einseitigkeit in Bezug auf Wohnung, Arbeit und Nahrung und demgemaiss eine der einseitigen Arheitsteilung entsprechende körperliche Organisation, die an streng vorgeschrieliene Tätigkeiten gebunden (Nectar, Blüthenstaub, Wachsschwitzen etc.) die höchsten Werte erzielt. (Selhstrerständlich kommen hier nicht die Werte für die Menschheit in Betracht sondern die biologischen Werte für das Bienenvolk sellst.) Stark und ausserordentlich leistungsfähig als Ganzes, schwach, hülttos und verloren bei der Auttösung resp. Ahtrennung der einzelnen Glieder. Im Kampfe ums Dasein in Folge der einseitigen Anpassung im Verhältniss leichteres Zugrundegehen auch des ganzen Staates ${ }^{25}$. Auf der anderen Seite - bei viclen höheren Ameisen - sehen wir ehenfalls eine feste unter Unständen aber modifikationsfähige Organisation (Aufnahme von Gästen, Sklaven u. s. w.), grössere Selbständigkeit aller Grlieder, cine vielseitige, wechselnde Anpassungsfïhigkeit an Wohnort und Nahrung und demgemäss Bestehenlleiben auch unter misslichen V'erhältnissen, grosse Leistungsfähigkeit im Einzelnen wie als Ganzes, hedeutendere Widerstandsfähigkeit im liampfe ums Dasein und demgemäss Verbreitung unter schwierigsten Existenzbedingungen.

Knüpfen wir an den Anfang an, so hiess es, dass in der am weitesten durchgeführten Teilung der Arbeit die höchste Organisation zu sehen sei; aber dieser Ausspr'tuch NAEgeur's lässt sich, so glaube ich, nur in seiner' wirklichen Bedeutung auf diejenigen Organisationen anwenden, deren einzelne Theile untrennbar mit einander verhunden sind ( $z$. B. der Zellenstaat des menschlichen Körpers). Dort wo die einzelnen Teile ein mehr oder weniger selbständiges Dasein zu führen vermögen, bedingt eine zu weit gehende Arheitsteilung eine so einseitige Organisation, dass trotz grösster Leistung im Verbande eine geringere Stellung im Kampfe ums Dasein - sowohl für den Einzelnen als auch für das Ganze - das Resultat ist. Fs scheint mil fraglos, dass wir dem Staatsverbande vieler höherer Ameisen-Arten, der eine grössere Selbständigkeit und vielseitigeres Handeln des Individumus zeigt, in dieser Beziehung den Vorrang überlassen müssen.

Wenngleich in vorstehender Skizze nur beiläufig Bezug genommen ist, auf die complicierten Verhältnisse hei den zusammengesetzten und gemischten Kolonien der Ameisen so möchte ich doch erwähnen, dass Whemar bei Leptothora $x$-Arten Verhältnisse gefunden hat, welche den Schluss zulassen, dass - wie beim Menschen - die Instinkte so gene-

${ }^{25}$ Ich erinnere daran, dass die einzelne Biene nicht einmal mehr die Fähigkeit besitzt, sich bei sinkender Temperatur allein zu erwärmen. Selbst Völker vın 5-10,000 Individuen sind kaum überwinterungsfähig und gehen auf sich allein angewiesen oft zu Grunde. 
ralisiert erscheinen, dass ein und dieselle Species je nach verschiedenen Umständen Herr oder Sklave zu sein vermag. (Journ. f. Psych.u. Neurol. l. c.)

Ich muss es dem Soziologen üherlasisen, anthropomorphe Parallelen, die in obigen Ausführungen nur anklingen, auf ihren Wert oder Unwert zu untersuchen. Die wissenschaftliche Volkswirtschaftslehre hat uns. wie es scheint, hisher nur wenig hefriedigende Resultate reliefert. Die deductiven und inductiven Forschungsmethoden kranken in diesem Zweige der Wissenschatt an ungenauen Pramissen (FHrfasersi). Die Forschungsmethode der Natur wissenschaften schien bisher dieser "Geisteswissenschaft " versagt, obgleich das " 'THünex'sche Gesetz " seit langen Jahren auf diese Wege wies ${ }^{26}$. Hier dürften wir jetzt aber neuen Zielen entgegengehen ${ }^{27}$.

\section{Diskussion.}

Prof. Eyary hat öfters mehrere $q$ Q $q$ zusammen in natürlichen Nestern beobachtet. Bei Ameisen werden zweifellos Eier als Nahrung für die ersten Larven benutzt. Viele Ameisenlarven sind gewiss fähig, feste Nahrung zu verzehren, es sind die langgestreckten, beweglichen, mit endständiøem Kopf versehenen Larven der Ponerinen, vieler Camponotinen etc., wohl aber nicht die tomnenfïrmigen, mit hauchständigem liopf versehenen Larven vieler Myrmicinen, z. I3. Pheidole und Crematogaster.

Prof. Dr. Standfuss. Die von Herrn von Buttel-Reepen mit Recht als üheraus wichtig betonte Eigentümlichkeit der sozialen Hymonopteren 우우, den Paarungstrieb zu verlieren, wenn sie begonnen haben unbefluchtete Fiel ahzulegen, geht keineswegs durch die ganze Insektenwolt durels.

Zumal die $q$ Q , vielel kurz lebenden Lepidopteren-Arten legen unbefruchtet leicht Fier ab und zeigen doch darauf den grössten Paarungstrieb. Auch viele Coleopteren $q$ Q , die bereits gepaart wurden und Eier allegtrn, zeigen nochmals wieder Parangstrieb, werden wieder gepart und logen writere Eier. I)ieser Torgang kann sich hei demsellen Cole-

26 Johann Heinrich von TuÜnen. Der isolierte Staat in Beziehung auf Landwirthschaft und Nationalökonomie. Hamburg, 1826. III. Aufl. Berlin. 1875.

${ }^{27}$ Vgl. Richard Ehrexberg, Das Thünen-A rchiv, Jena, 190כ. Beachtenswert für vorliegende Fragen ist auch H. E. ZiEgler, Die Natuwissenschaft $u$. d. sozialdemolratische Theorie, Stuttgart, 1894, da in diesem Werk auch die Insektenstaaten herangezogen werden. Ich verweise noch auf den Artikel SchaLLMayer's: "Zum Einbruch der Naturuissenschaften in das Gebiet der Geistesuissenschaften ». Arch. £. Rassen- u. Gesellschafts-Biologie, Heft 4, p. 586-597. 1904. 
opteren $q$ sogar mehrfach wiederholen. Zumal Arten der Familien der Curculiomidx, Chrysomelidx, Coccinellidx zeigen diese Erscheinung, wie der Sprechende selbst beobachten konnte.

[)]'. Fiscinerich fragt, ob seit P. Huber genaue Untersuchungen darüher" angestellt worden sind, ob die Eier der Ameisen unter dem Einfluss der Bespeichelung an Volumen zunehmen.

Prof. Forel. Die höchst interessanten Mitteilungen, die wir hörten, mahnen zur Vorsicht bezüglich Verallgemeinerungen. Prof. Exery weist mit Recht auf gewisse Myrmicidenlarven, die so steif sind, dass sie unmöglich selbst essen kömmen. Dies gilt z. B. von Tetramorium, aber nicht von Myrmica. Anderseits finden wir bei Dolichoderinen ebenso steife Larven, z. B. bei Tapinoma, nicht aber bei anderen (xattungen $\mathrm{u}$. s. f. Ehenfalls fand ich bei Tapinoma, Plagiolepis, Formica, sowie in kleinen Íolonien, schr oft Nester mit seh» vielen Hügellosen Weibchen, die friedlich zusammen leliten. Ich fand auch früher Associationen von flügellosen Formica und andern Weibchen. die wahrscheinlich neve Kolonien bilden. Ich glaube, dass die Beobachtungen der Herren v. Butrei und

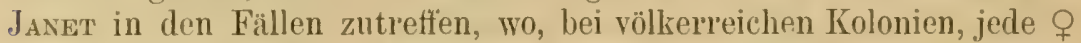
ihren eigenen Hof von Arbeitern hesitzt. Ina mag Eifersucht zwischen den Weibchen allmählig entstehen, wenn sie eng zusammen zu leheli gezwungen werden, so dass in künstlichen Nestern die überzühligen $q$ von ihren Kolleginnen oder den Arbeitern getötet werden.

In der Ernährungsfrage der Kóoloniemütter dürfte auch je nach den Arten die cine oder andere Quelle (Eierfrass, Speichelselret. MundsackInlaalt, Fettkörper etc.) am meisten zutreffen. Her'r v. Butтes warnt mit liecht vor Vermenschlichung der Ameisenseele. Anderseits gibt es gewisse allgemeine biologische Eischeinungen, die zweifellos bei allen Tieren, die ein Gehirn hahen (inkl. Mensch), zutreffen. Wir kïmmen zwar nicht unsere innere psychologisehe Empfindungsqualitait auf Ameisen oder Bienen übertragen. Aber wir können doch feststellen, dass rine Reihe Reaktionen das Vorhandensein psychischer Frscheinungen bei Tieren verraten, die wir per Analogie mit unseren bezüglichen Eigensehaften als derart verwandt betrachten müssen, dass ein gemeinschaftlicher Name zestattet ist, so z. B. Gedächtnis, Angstgefühl, Sympathie und Antipathie, Mlut, Entmutigung, Wille ete.

lindlich möchte ich hezüglich sexueller Sympathie und Sozialgefüh! diran fexthalten, dass die Sozialgefühle phyletische Abliömmlinge del sixmellen Sympathiegrefühle sind, da die sexuellen Sympathiegefühle zwisehen Individuen die phyletiseh ersten und ältesten zu sein pttegen. [moh soll dins lieinesweers sagen, dass jede (iesellschaft sich ausschliess- 


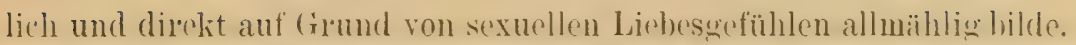
Freundschaftliche Verosellschaftumen zum Zweck gemeinschaftlichere Verteidigung und depoldichen mehrspielen im konkreten Fall sicher oft eine Hauptrolle. Doch liegt phyletisch die Quelle zur Entstehmusmöglichleit solcher Fleundschaftsgefühle in den erhlichen sexuellen Instinkten, die die Individuen der gleichen Art zuerst einander näher bringen.

Dr. von Buttel-Reepen. Aut die Bemerkung des Herrn Dr. Escherich möchte ich bemerken, dass meine Messungen an Ameiseneiern nur die verschiedenen Grössenverhältnisse der Eier kur'z nach der Ablage betreffen, die hin und wieder zu beobachten sind; so ergab sich in einem Falle bei Formica sunguinea die Hälfte der normalen Grösse. Während die normalen Eier : Länge $0,704 \mathrm{~mm}$., Breite $0,384 \mathrm{~mm}$. aufweisen, zeigte das kleine Ei folgende Maasse : Länge 0,496 mm., Breite 0,224 mm. Hin-

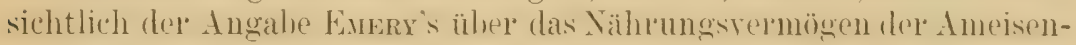
königin habe ich es als selbstverständlich angesehen, dass bei gewissen Ameisenarten der stark ausuchildete Fettiörper dor Königin indirelit als Nährquelle beim Füttern der Larven in Betracht kommt, so lange die Königin eingeschlossen ist in dem "königlichen Gemach ». In Bezug auf die Fores'schen Ausführungen, soweit sie die psychologischen Verhältnisse betreffen, bemerke ich, dass ich ilnen nur zustimmen kann, wie auch aus meinen früheren Publikationen hervorgeht. Wie schon im Vortrage erwähnt, können wir der Analogieschlüsse nicht entbehren, sie werden nach wie vor das Fundament einer vergleichenden Psychologie bilden. Da JANE' ${ }^{22}$ grade bei Tapinoma trotz der Steifheit der Lal'ven ein selbständiges Fressen beobachtete, so scheint diese besondere Kör'per-Eigenschaft der Larven untel' Umständen kein Hinderniss zu bilden. Hinsichtlich del Eifersucht der Königimnen möchte ich darauf hinweisen, dass es sich in dem von mir beobachteten Falle nicht um eine volliperche Kolonie handelte. Die gemenschaftliche Verteidigung spielt meines Erachtens, wie ich früher schon ausführlich zu begründen ver'suchte (vgl. ${ }^{1}$ d. Textes), wohl kaum eine Rolle als Motiv zur' Staatenbildung, da die allermeisten natürlichen Feinde der sozialen Insekten so gut wie ausschliesslich nicht das Nest resp. das Volk angreifen, sonder’n, wie schon erwähnt, das einzelne Individuum ausserhalb des Nestes. Auch finden wir", wie ich desgleichen in der" "stammesgeschichtlichen Entstehung des Bienenstaates » ausführte, eine gemeinschaftliche Vertheidigung bei solitär lebenden Insekten (Anthophora etc.). Andererseits sehen wir dort, wo ein natürlicher Feind das Nest angreift (Ameisenbär) grade einen Nachteil im Kampfe ums Dasein durch die Vielheit der Insassen entstehen, ohne dass die gemeinsame Abwehl irgend welchen Vorteil erzengte. Die Standfuss'sche Angabe ist mir von höchstem Interesse, da ich keine Angabe darüber in der Litteratur 
gefundrn habe. Fs dürften wohl aher die gesammten Coloopteren-Fälle auszuschoiden soin. da es sich nur um unbegattet gebliebene Weibchen handelt.

JANET, Ch.: La reine fondatrice d'un nid semble être incapable de se procurer aucune nourriture autre que celle qui lui est fournie par son orpanisme. Elle possède à ce moment des réserves dans son sang, dans son tissu adipeux et, surtout dans ses muscles vilurateurs du vol, muscles qui sont histolysés pendant le développement des premières larves.

Il y aurait lieu de rechercher si, au moment du vol nuptial, les reines n'emportent pas aussi dans leur jahot une petite provision d'un liquide mutritif riche qui lour aurait été fourni par les ouvrières du nid qu'elles abandonnent.

Au sujet de ses nids artificiels en substance poleuse, M. JaxeT rappelle que ses appareils comportent des chambres d'hahitation garnies de terre fine, mais que cette ter're ne peut être conservée dans les appareils habités par des colonies nomhreuses pares que les ouvrières emportent cette teree et la déposent dans la chambre éclairée et vide. Elles la déposent de préférence sur les matières alimentaires miel, sucre, viande. jaunes d'œuf) mises à leur disposition.

\section{Contribution à l'étude du problème de la reconnaissance chez les Fourmis.}

Par H. PIÉRON (Paris).

Un des problemen les plus étudiés et les moins résolus do la psycholougie des Insectes est certainement, malgre les observations tres justes de XI. Forel, celui de la reconnaissance des Fourmis.

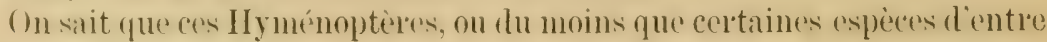
eux, possèdent des instincts belliqueux qui provoquent des luttes violentes, non senlement, ot cela est interessint, ontre Fourmis dispeces différentes. mais encore entre Fourmis de même espèer. Quelle différenere

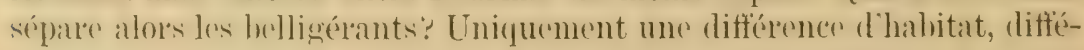
rence qui ne tient pas au milieu, aux conditions physiologiques de la vie, (an on prut constater des batailles anter des lommis tres voisines, vivant d'une vie identique, et ne se différenciant que par le groupement social. Cette diftérenciation sociale, par analogie avec les sociétés humaines, a 


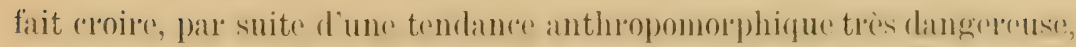
qu'il devait s'agir d'un procédé de reconnaissance par mot de passe. Et

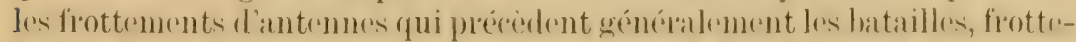
ments quon olserve très génélaloment quand deux foumis se feneon-

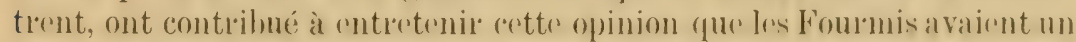

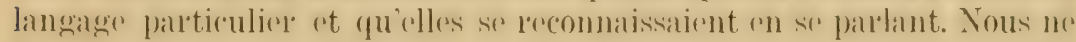
discutelons pas cette opinion, cal l'hypothèse, très complexe, ne repose

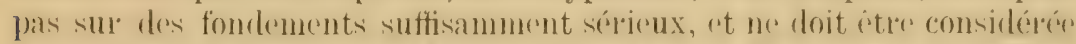
que comme un pis-aller, un dernier lecours aprese démonstration de l'im-

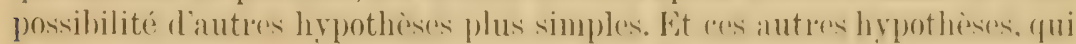
sont nombreuses, et dont aucune n'est entièrement vérifiée, ont cependant, pour certaines d'entre elles, des faits en leur faveur, plus probants que la simple constatation du foottrment des antrumes, ce fortement qui paraît être une simple exploration réciproque destinée à fournir des sensations plutôt qu'à exprimer des idées, car l'exploration antennaire est constamment employée par les fourmis avec les objets les plus divers.

Pour avancer la solution du problème, qui est sans doute complexe, il nous semble qu'il vaut micux, au lieu de chercher une théorie générale

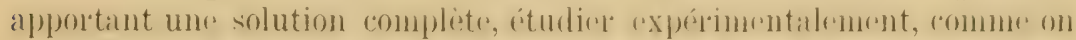
l'a d'ailleur's déjà fait, des faits particulier's et préeis, dont la synthèse,

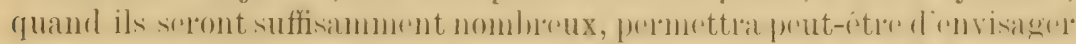

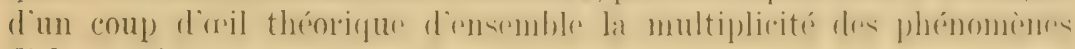
d'olsel'vation.

C'est une contribution particulière de cet ordre que nous voulons apporter.

Les expériences ont visé à vérifier le rôle possible de l’odorat dans la reconnaissance des Four'mis, et les expériences ont été faites proincipalement sur trois espèces, les Aphanogaster Barbara nigra, les Formica

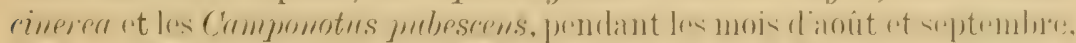

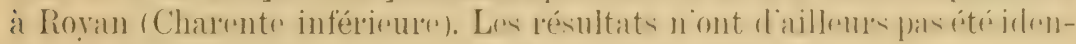
tiques, pour des raisons que nous tâcherons de détel'miner.

\section{$1^{\circ}$ Ajhanogaster barbara nigra.}

Ces Fourmis, d'un noil luisant, dont les yeux sont peu développés, comprennent des individus de toutes tailles, depuis 4 ou 5 jusqu'à 15 ou 16 ;

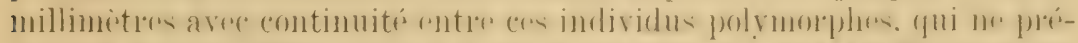

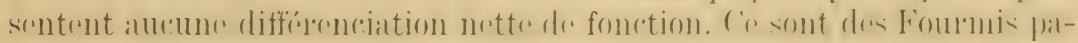
cifiques et qu'on dit moissonneuses parce qu'elles accumulent des graines dans leur fourmilière, creuséc au bol'd des champs, et dont l'orifice est constitué par un cratère qu’elles bâtissent avec les débris des graines qu'elles lecueillent.

De mar'che lente, n'allant pas dans leur's excursions excessivement loin, 
les Aphaxnoygater ont des foumilièles souvent très voisines, de 30 centimètres parfois. () pruand des individus de deux fourmilières, des neutres bien entendu, se rencontrent, elles se battent avec un acharnement tiès car'actéristique, bien qu'elles ne se recherchent pas pour se battre. Il n'est pas rare de rencontrer de véritables champs de carnage dans la zone intermédiaire entre deux fourmilières, dus à ces rencontres fortuites et sanglantes. La lenteur de marche de ces Fourmis qui cherchent très rarement à fuir, la posibilité demployer de gros individus plus facilenent observables, et dont on peut suivie les mouvements des mandibules qui sont énormes chez les sujets de grande taille, enfin leur acharnement à se battre, tout cela faisait des Aphrnogaster des sujets de choix pour le expériences à entreprendre. Ajoutons-y ce détail, qui est très important que, probablement parce qu'elles sont à peu près aveugles, elles ne sont pas troublées par la présence d'un observateur immobile.

Voici quelles furent alor's mes expériences. Je procédai de deux façons inverses, avec un procédé analogue. Le procédé fut le suivant. Avec des Fourmis prises dans une fourmilière je préparai, par écrasement, un bouillon que je filtrai. et posérlant lodeur caractéristique des Fourmis de l'espèce, et peut-être celle des Fourmis de la fourmilière, qui est en tout cas indécelable pour notre odorat. Je n'employai point d'alcool, dont lodeur exerce une action p)rople qui fausse entièrement les léxultats, mais l'eau pure, cela est important. De ces "bouillons" de Four'mis, j'en fis alors avec un certain nombre de fourmilières soigneusement repérées, voisines ou éloignées, en prenant garde de ne prendre les exemplaires qu'à l'intérieur de la fourmilière pour éviter toute erreur, grâce à un procédé de "pêche ", pour ainsi dire: en enfonçant une fine baguette dans l'orifice, on la retire pleine de neutres qui s'y accrochent de toute l'énergie de leurs mandibules.

Les bouillons préparés, les expériences répétées un très grand nombre de fois, dommèrent les résultats suivants que je résume, pour la commodité de l'exposé.

I. Expériences sur le terrain. A. Soit une foumilièle $a$. Je possède un bouillon a fait avec des Fourmis que j'y ai prises. Je prends deux neutres d'une fourmilière $b$, voisine. Je mets l'une des neutres de $b$ (en évitant de la toucher avec les mains, car l'odeur humaine peut avoir dans ces expériences une intluence perturbatrice des plus dangereuses) au milieu des Fourmis de $a$. Elle est immédiatement attaquée, après palpation antennaire, saisie par les mandibules, les pattes et les antennes, et, ou bien elle est entraînée au loin et abandonnée, ou bien clle est tuée par section du métathorax et cela de façon absolument constante. Je prends l'autre neutre de $b$ et je la trempe dans le bouillon 


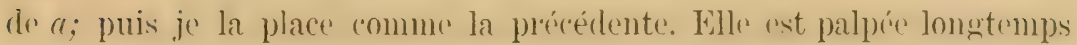
et par un grand nombre de neutres de $a$. Neuf fois sur dix elle s'éloigne sans être attaquée. Et quand elle est attaquée, elle l'est avec moins de persistance que la Fourmi témoin non trempée dans be bouillon a. C"est à dire qu'elle subit une sorte de bousculade d'une Fourmi qui la laisse bientôt, et est seule au milieu d'autres à manifester son hostilité, alor's que dans le cas témoin, la plupart des neutres de a se comportent de manière analogue, quoique non identique is vali dire, et rnfin, l'attarque a lieu après un temps plus long que dans le cas témoin où clle se produit généralement tout de suite.

Mais lorsque la Fourmi $b$ trempée dans le bouillon a l'este longtemps en présence des Fourmis de $\alpha$, elle commence à subir des attaques de plus en plus répeitées et qui finissent par ressembler alsolument a la lutte quion peut constater de suite dans le cas témoin. Cela semble montrer que la Four'mi de même espèce, de fourmilière étrangère, n'est pas reconnue

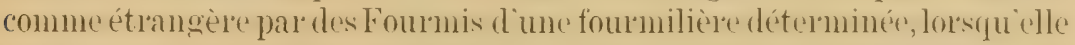
exhale l'odeur, du moins tout paraît l'indiquer, des Fourmis de cette derniere fourmiliere, et n'est attaquee qur lnsque lodeur sest évaporér. En revanche, l'attitude de la Fourmi ainsi trempée, gênée d'abord par ce bain intempestif, est celle d'une égarée qui ne cherche qu'à sor'tir' de l'endroit dangereux ou clle a été placée. Elle n'attaque pas, mais clle ('hereche à s'en aller. Nous verrons que, quand on la mettra en présence d'une seule ennemie, elle attaquera, au contraire.

'Tel a été le résultat d'une centaine d'épreuves sur le terrain, en des endroits diffélents, résultats qui furent les memes en employant comme fourmilières $a$ et $b$, des fourmilières très voisines ou des fourmilières très éloignées.

B. Une expérience inver'se était à faire. Si une Fourmi étrangère est tolérér quand elle émant une odeur identique, une Fourmi de mémur fourmilière ne sera-t-elle pas attaquée quand elle émanera une odeur différente? J'ai pris alor's des Fourmis de $b$ dont je fis un bouillon (et les résultats furent encore les mêmes que $b$ fût voisine ou éloignée de $a$ ), et, prenant deux neutres de $a$, j'en mis une comme témoin au milieu des autres de $a$. Inutile de dire, le fait est trop évident, qu'elle ne fut pas attaquée ".

Quant à l'autre, je la trempai dans le bouillon de $b$ et la mis au milieu des neutres de $a$. O1 elle fut effectivement attaquée de façon à peu près constante, mais, à vrai dire, moins vite qu'une Four'mi de $b$ mise dans $a$.

'A condition de ne pas lui donner avec les doigts une odeur étrangère" : j’ai vu des Lasius fuliginosus, d'odeur forte, attaqués par les neutres de même fourmilière, quand je les avais tenus dans la main. Cela montre les précautions qu'il faut prendre pour ne pas faire intervenir d'odeur étrangère. 
Mais au bout d'un certain temps. les attaques cessent en général. On perut admettre que cette cessation des attaques dans ce cas, comme le redoublement des attrupues dans l'expérience précédente sont des phénomènes dus ì l'évaporation de l'odeur étrangère apportée par le bouillon dans lequel on a trempé la Fourmi. Je dois dire d'ailleur's que j'ai vu parfois une Four'mi être attaquée par une autre de même fourmilière, ce cas formant une exception très rare ${ }^{1}$, tandis que dans ces observations la constance a été remarquable.

II. Expériences en récipients clos. Les séries d'expériences A et B ont été refaites dans des flacons clos où je mettais en présence non plus une Fourmi contre une fourmilière, mais une ou deux Fourmis contre une ou deux autres.

Ces expériences furent beaucoup moins nettes que les précédentes parce que, placées dans des conditions anormales, les Fourmis cherchai'nt surtout à s'enfuir. Néanmoins, dans la moitié des cas à peu près j'eus des résultats du même ordre que précédemment.

A. Une Four'mi $a$ n'attaque pas, ou du moins pas de suite une Fourmi $b$ trempée dans le bouillon de $a$. Au contraire, la Fourmi $b$ attaque en général très vivement la Fourmi $a$ qui se défend à peine; et en effet si $a$ trouve bien que $b$, qui a été trempée dans son bouillon, a bien son odeur, en revanche b reconnaît en a une odeur différente, ce qui explique cette attaque unilatérale.

B. Une Fourmi $a$ trempée dans le bouillon de $b$ est généralement attaquée par une autre Four'mi $a$.

C. Enfin une de mes expériences m'a donné des résultats également très nets: une Fourmi $a$ trempée dans le bouillon de $b$ est mise avec une Fourmi $b$ trempée dans le bouillon de $a$. Or, au lieu d'une attaque réciproque immédiate, je n’ai jamais constaté d'attaque avant au moins une heure. Ces résultats confirment done les précédents, bien que la proportion des succès soit moindre, ce qui est dû aux conditions anormales, artificielles de l'expérience. Il vaut toujour's mieux opérer sur le terrain malgré les difficultés d'observation plus grandes.

\section{$2^{\circ}$ Formica cinerea et Camponotus pubescens.}

Ces deux sortes de Fourmis sont très agiles, et elles sont douées d'une vue excellente. Aussi, d'une part se battent-elles très peu, parce qu'elles

\footnotetext{
${ }^{1}$ Sauf pour les estropiées chez certaines espèces. En estropiant une Formica cinereu ou un Lasius fuliginosus, j’ai vu en général la malheureuse Fourmi attaquée ou du moins éloignée par les autres; nous sommes loiu des "sentiments d'humanité »(?) des Fourmis.
} 
cherchent à peu près toujours leur salut en s'enfuyant ou en se cachant; et, d'autre part l'observation est excessivement délicate, car l'observateur', malgré son immobilité, est vu par les Fourmis qui s'inquiètent et ne se comportent plus normalement.

Est-ce à cause de ces difficultés d'expérimentation et d'une plus grande tolérance, ou bien, ce qui est possible, un mécanisme tout différent intervient-il dans la leconnaissance de ces Fourmis, en tout cas, je n'ai eu que très l’arement des faits du même or’dre que les précédents, en procédant aux mêmes expériences. Dans quatre ou cinq cas sur dix pour les Camponotus, dans deux seulement, environ, pour les Formica, j'ai eu des succès.

Dans la grande majorité des cas, je n'avais. pas d'attaque; mais je n'ai réussi que très rarement à faire attaquer une Four'mi de tourmilière différente, même en Hacon clos, car la fuite la soustrayait toujour's à des attaques possibles mais peu ardentes. Aussi de ces dernières expériences ne m'est-il pas possible de conclure, ni dans un sens ni dans l'autre, sur le rôle de l'odorat dans le mécanisme de reconnaissance des Fourmis.

\section{$3^{\circ}$ Fomica cinerea avec Aphxnogaster barbara nigra.}

Une dernière série d'expériences a consisté à procéder avec des Fourmis d'espèces différentes. Une Formica trempée dans le bouillon d'une fourmilière d'Aphænoguster est placée au milieu des Aphærnogaster de cette fourmilière, et réciploquement une Aphænogaster trempée au milieu de Formica.

Les résultats ont été tı’ès mérliocres sur le tel'ain : en effet. Ia Formica cinerer mise au milieu d'Aphanogaster s'enfuyait toujours et jamais il n'y avait véritablement de rencontre; et une Aphærongaster burbara nigra témoin placée au milieu des Formica n'est pas toujour's attaquée, en sorte que, du fait qu'une Aphranogaster n'est pas attaquée, on ne peut à peu près rien conclure.

En Hacon clos, une Aphrenogaster trempée dans le bouillon de Formica n'est pas attaquée, mais une Aphanogaster témoin ne l'est pas non plus. En revanche, dans l'un ou l'autre cas, la Formica cinerea est attaquée, quand l'Aphanogaster peut s'en approcher. Dans le cas inverse, une Formica trempée n'est, huit fois sur dix environ, pas attaquée par une Aphenogaster alor's que, régulièrement, une Formica témoin est attaquée. Nais, au bout d'un certain temps, la Formica trempée est attaquée à son tour.

Enfin, une Aphrenogaster trempée dans du bouillon de Formica est attaquée par les autı'es, de même fourmilière qu'elle, sur le terrain et en Hacon clos. Rien de net pour une Formica trempée dans le bouillon d'Aphrnogaster. Ces expériences ne nous apprennent donc rien au sujet 
du mode de reconnaissance par les Formica cinerea, mais confirment l'importance de l'odorat dans la reconnaissance par l'Aphænogaster barbara nigra, qui n'attaque pas une Fourmi émanant la même odeur qu' elle. non seul'ment quand il sagit d'une neutre de mème expèce et de foumilieper différentr. mais encore quand il sagit d'une neutre d'espèce très diffémente. moins diffépente à vai dire des Aphernogaster de petite taille plus effilées, que de celles de grande taille, excesirement massives.

Je n'ai pu encore répéter ces expériences, longues et minutieuses, comme préparatifs. sur diutres mèèes jai commencé à expérimenter avec des animaux, des Insectes, ou des Vers, trempés dans des bouillons de Fourmis, mais je n'ai pas encore obtenu de résultats qui puissent être

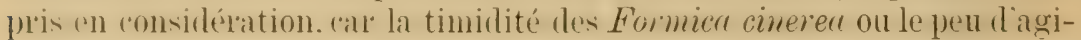
lité des Aphrenogaster nigra ${ }^{1}$ et, d'autre part, la rapidité de fuite des animaux direr's se sentant menacés, empêchent généralement de se produire ure rencontre qui commencerait par une exploration antennaire de la part de la Fourmi et finirait par une attaque ou la manifestation d'une tolérance.

Que pouvons-nous donc conclure de ces expériences très particulières? Je dis très particulières parce que, non seulement elles ne concernent vraiment que deux ou trois espèces de Fourmis, mais aussi parce qu'elles ne concernent que les neutres, car', pour les mères, j'ai r'cncontré, et beaucoup d'observateurs ont rencontré très fréquemment, une grande tolérance, non seulement d'une mère pour' une mère étrangère, mais encore d'une mère pour des neutres étrangères ou des neutres pour une mère étrangère.

Et les conclusions derront se particulariser encore. puisque, en ce qui concerne le rôle de l'odorat dans la reconnaissance, je n'ai rien pu dégager d'assez net dans un sens ni dans l'autre en ce qui concerne le Camponotus pubescens et surtout la Formica cinerea.

Je n'ai donc pu établir quelque chose qu'en ce qui concerne l'A phænogaster barbara nigia. Mais, pour celle-ci, tous les faits s'accordent pour mettre en évidence la part prise par l'odorat dans la reconnaissance. Des Fourmis de fourmilière ou d'espèce étrangère sont tolérées quand elles exhalent la même odeur; des Fourmis de même espèce et de même fourmilière sont attarquérs quand elles exhalent une odeur de fourmilierer ou d'espèce étrangère.

On comprend qu'il y ait une odeur d'espèce étrangère; on comprend moins qu'il $y$ en ait une, car quelle doit être sa subtilité, pour chaque

1 Il y a, dans les fourmilières des Aphrnogaster moissonneuses, un nombre considérable de parasites mjrmicophiles qui rivent dans leurs greniers et ne semblent tolérés que par l'impuissance des Aphanogaster à les saisir. 
fourmilière! Et cette odeur persiste, se maintient longtemps, car en gardant en captivité des Aphrenoguster pendant un mois, avec une nour'riture différente, un habitat différent, malgré tout, elles étaient tolérées quand on les remettait auprès de leur fourmilière d'or'igine.

Mais la subtilité de cette odeur n'est pas une objection sans réplique. Un Chien ne reconnaît-il pas l'odeul' de son maitu'e entre mille, et cette odeur ne persiste-t-elle pas au milieu de bien des changements de r'égime, d'habitude, etc. Et l'on sait que certaines Fourmis, car les différences spécifiques sont ici considérables ${ }^{1}$, ont l'odlorat très développé.

Le siège de l'odorat paraît bien être l'antenne, bien qu'histologiquement je n'aic pu le mettre encore en évidence, mais la physiologie permet

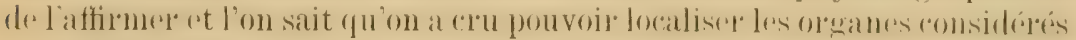
comme olfactif's de l'antenne.

J'ai constaté qu'une Aphxenogaster barbara nigra dont les antennes sont sectionnées est tolérée par les neutres de même fourmilière et attaquée (car les A. n'attaquent en général pas les estropiés) par celles de fourmilières différentes, tandis qu'elle-même attaque indistinctement tout ce qu'elle rencontre, objet inanimé, Fourmi étrangère ou de même fourmilière. Elle ne reconnait plus rien. L'organe de la reconnaissance doit done bien être l'antenne dans ce eas, et l'on comprend le róle de la palpation, qui est une exploration olfactive et non l'échange d'un mot de passe. Et, en passant une Fourmi étrangèe ou un peu de bouillon de Fourmis étrangères sur une feuille de papier où l'on a placé une Aphanogaster, on voit celle-ci, alor's même qu'aucune trace visible parfois ne reste sur le papier, palper de ses antennes et frapper avec force le papicr de ses mandibules comme pour mordre un ennemi imaginaire. Je crois done pouvoir affirmer, jusqu'à preuve du contraire, que c'est essentiellement, sinon uniquement par l'odorat que les neutres d'Aphxnogaster barbara nigra se reconnaissent. Et ce mode de reconnaissance s'il n'est pas universel, doit se rencontrer chez d'autres espèces. Le Lasius fuliginosus qui s'oriente surtout par l'orlorat et exhale une odeur excessivement forte et caractéristique, se leconnait probablement aussi par ce moyen. Et chez une petite Pheidole, dont je n'ai malheureusement pas déterminé l'espèce. ce petit fait curieux montre également l'importance de l'olfaction :

Assistant à un déménagenent de fou'milière, le long d'une allée, je l’épandis quelques gouttes d'un bouillon d'Aphanogaster sur l'entr'ée de la fourmilière abandonnée et sur celle de la nouvelle, dont les neutres, pol-

1 J'ai montré ces différences en étudiant l'orientation chez Aphænogaster barbara nigra qui se guide suxtout par le sens musculaire, Formica cinerea, qui se sert principalement de la vue, et Lasius fuliginosus, chez lequel l'odorat joue le rôle prédominant. (Bulletin de l'Institut psychologique international, no 2, mars-avril 1904, p. 168.) 
tant les larves, prenaient possession. Or, d'un côté comme de l'autre, les survenantes passèrent outre ou s'éloignèrent, et celles qui sortirent ne rentrènt plus pendant Inngtemps, alors quancune trace visible ne subsistait plus.

Au bout d'une heure environ, elles commencèrent à entrel dans la fourmilière qu'elles abandonnaient et qui, si mon hypothèse est juste, exhalait l'odeur caractéristique des Pheidole; mais au bout de plusieur's heures je ne les vis pas encore rentrer dans la nouvelle fourmilière et je crois qu'elles renoncèrent cette fois au déménagement.

Enfin, je puis signaler que, chez plusieur's espèces de Lasizs, j’ai provorguédes fuites à distance, en déposant, sur leur passage, une goutte d'un bouillon de Fourmi étrangère, et la fuite a toujours été précédée d'une tension des antemnes dirigées vers le foyer de l'odeur.

Aussi, tout en ne le considélant pas comme universel, je pense que le procédé olfactif de reconnaissance chez les Fourmis doit avoir une assez grande généralité, comme déjà beaucoup d'observateur's l'admettent, y (ompris lo trex compétent profiscur Fones dont je suis heureux de contirmer, partiellement les vues pénétrantes. Il est souhaitable que des expé-

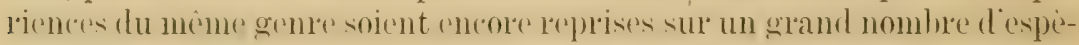
ces différentes, et dans différents pays, y compris les régions exotiques.

\section{Discussion.}

Ch. Janet. Les expériences de M. Piḱron apportent une sérieuse confirmation à l'opinion que les Four'mis d'une même colonie se reconnaissent par l'odorat. L'odeur qui permet la reconnaissance provient-elle du nid ou provient-elle des individus? Le fait, si fréquent, que les Fourmis acceptent les øufs, les larves, les nymphes et même les jeunes individus

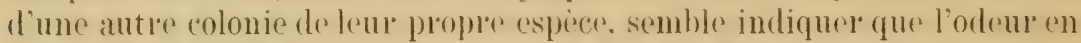
question provient des individus arrivés à l'état d'imago plutot que de la progéniture, ou des matériaux, ou de l'atmosphère du nid. J'ai émis l'opinion que cette odeur était fournie par la paire de glandes qui se trouve à la partie inférieure du stèthe, parce que ces glandes sont les seules qui, chez les Fourmis, produisent un liquide rapidement volatil.

Quant à la raison pour laquelle tous les individus d'une même fourmilière produisent une odeur caractéristique de la colonie, elle peut être rechreché dans l'homogénéité de la nourriture. En effet. tous les indiridus d'une mème colonie mettent pour ainsi dire en commun leur nourliture: puisque les récoltes liquidrs emmagasinées dans le jabot et apporetries par un pretit nombre d'apporovisionneuses sont distribueses des le reton au nid, it un tres grand nombro d’individus qui les conservent a leur

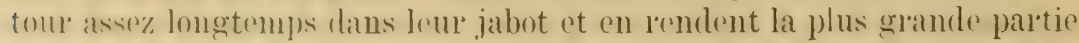
jome les hesoins de la communauté. Il résulte de ces distributions et de 
ces échanges un mélange qui tend à uniformiser la nourriture de tous les individus d'une colonie.

Her' Wasmaxn bemerkt: 1. dass es bei del" psychologischen Beurteilung der Badeexperimente für die Aufnahme fremder Ameisen nicht bloss auf die erste Reaktion, sondern auch auf den weitern Verlauf der Behandlungsweise ankommt. 2. Erwähnt er, dass der individuelle Gel'uch der Ameisen sich bekanntermassen in den elsten lagen des lmagolehens entwichelt. 3. Beschereibt or die Komplikation des Problems der Erkennung von Freund und Foind in den gemischten Kolonien dere Aneisen, speziell an cinem Betrachtungsnest von Polyergus, dem el Sklavenkokons der verschiedensten Ameisenarten gab. 4. Macht el aufmerksam auf die Bedeutung des Gesichtssinnes für die Erkennung von Freund und Feind bei den " internationalen Beziehungen » del Ameisengäste, besonder's bei der Aufnahme von Dinarda in fremden Ameisenkolonien. Auch auf die Beteiligung mannigfacher pschischer Faktoren an jenrm Problem weist W Asmand (gegenüber Berme's Retlextheorie) hin.

\title{
Ueber den Einfluss der Sauerstoffentziehung während des Puppenlebens auf die Gestaltung der Schmetterlinge.
}

\author{
Von Dr. M. vos LIXYDEN .(Bonn).
}

Durch die Experimente zahlreicher For'scher, ich nemne nur STAxDfuss, Merrifield, Fickert, Frings, Staniffuss' Schülel Fischer, wissen wir. dass dir Färbung und Zejehnung der Schmetterlinge in hohem Giad heerinflusshar ist durch Temperaturedze, die den Falter wihrend seiner Puppenentwicklum troffen. Jahreszeitenabarten. Lokalrasien. klimatische Varietïten stellen sich auf Grund der gemachten Experimente als dio Produkte von Tempreaturschwankungen dar, die sich während dor Stammesgeschichte ciner Form in ihrem Terhreitungshezirk fühlhar wemacht haben. So entwickelte sich aus dem im Norden nur in der einen Generation, der Vanessa levana fliegenden "Landkä l'tch ell » in wärmeren Gegenden eine zweite von der ersten verschieden gefärbte und gezeichnete Sommerform, die I'anessu prorsa und so kïmnen wir heute die in Lappland fliegende $V$. poldr is und chenso dio aut Sardinien und Corsica hoinische Vanesse ichmen als nahestehende klimatische Varietaten unserer Vanessa urticx hezeichnen. Denn dieselben Veränderungen, die 


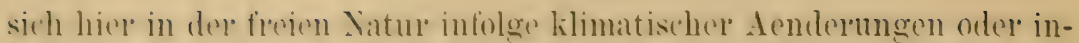
folge von duwandern der sehmetterlinge im Laufe von violleicht lanwen Zeiträmen vollzogen haben. sind im Experiment in wenigen Tagen und Wochen zu erreichen. Verbringen wir, um ein Beispiel zu nennen, dic Puppen von Vanessa urtice in eine konstant erhöhte Temperatur von zirka $35^{\circ} \mathrm{C}$. so entwickeln sich immerhalb liürzerer Zeit, als es bei normal hehandelten l'uppen der Fitl ist. Schmetterlinge, die alle hemnzeichen der südlichen Verwandten des Falters, der Tanessa ichmusa,

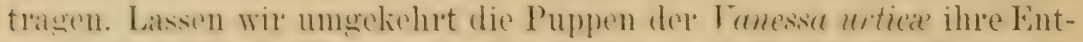
wicklume he tiefer 'Tompreatur durchmahen. so worden wir Falter elhalten, die der nordischen Varietät V.polaris ähnlich sehen, oder mit

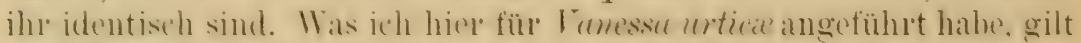

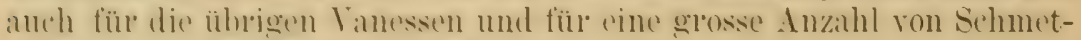
terlingen aus der Gruppe der 'T'agtalter.

Wenn wir nun hei den Vanessen besonders bei T'anessa urtica die

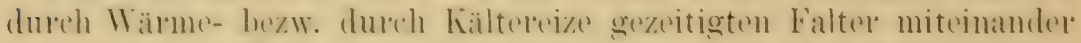
vergleichen, so fillt uns auf, dass dieselben nach zwei verschiedenen Richtungen von del bei uns heimischen Vanessa urtica abweichen. Die her uns unter normalen bedingumgen entstehenden Falter haben rotgello

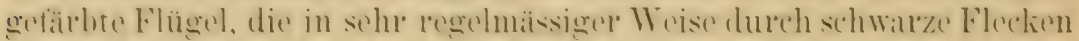

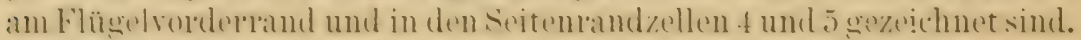

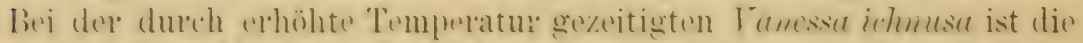

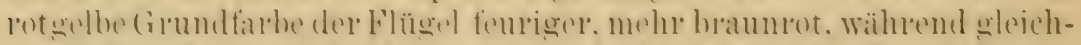

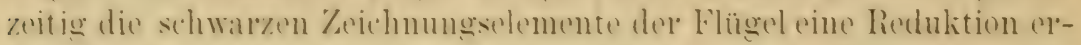
fahren; die rote Beschupung dehnt sich auf liosten der schwarzen aus. Bei der durch Kälte entstandenen $V$. polaris und noth mehr hei der nordamerilianischen Art r. Millerti verdrangen umwekehut schwarze. Shuppen die lote troundfarbe. so diss man sagen kann: Bei Vanessa urtica begünstigt höhere 'Temperatur die Bildung roter, niedere Temperatur die Bildung sehwarzer Pi@mente.

Die Ucberraschung war daher sehr gross, als Experimente mit 'Temperaturen über $40^{\circ}$ und unter $0^{\circ}$, als die lrost- und Hitzeexperimente

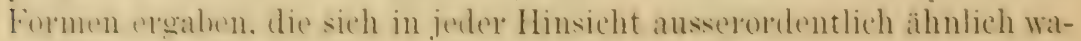
ren. Formen die sich in beiden Fällen ron dem normalen Falter

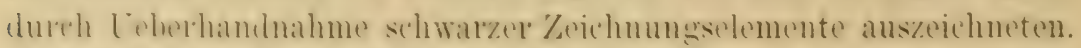
Sowohl bei den Hitze- wie bei den Frostaberrationen waren die schwar-

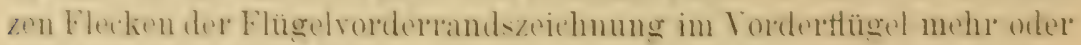
weniger zusammengeflossen, die dunlieln Pigmente hatten also in charakteristischer Weise zugenommen, und im Hinterthügel war sogar bei

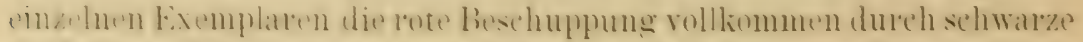
Ferdrängt. Nebeu dieser ausgedehnten Schwirzung del Flügel zeigten die Aberrationen auch noch charakteristische Veränderungen in der 


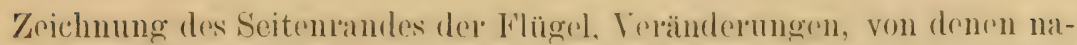

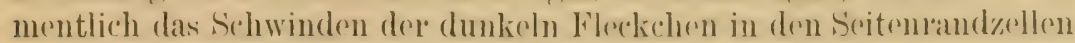
4 und 5 zu elwähnen ist.

Wenn bei den ersten Experimenten mit mässiger Wärme und Kälte von ciner sperifischen Wirkung der 'T'muperatur gesprochem werdem musste, so komnte in der zweiten Serie von Experimenten von rinem sperzifischen Käite- oder Wärmereiz nicht mehr die Rede sein, denn hier hatten verschiodene licize nicht versediedene, sondern greichgestaltete Individuen gezeitigt. Wie war nun die Entstehung dieser neurn unerwarteten formen, dieser allerdings auch in frecier Natme damn und wann

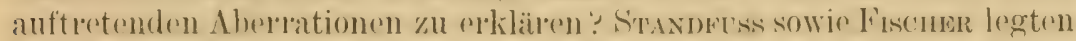

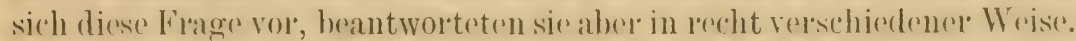
Flscher wal del Mrinung, es müsse sich hoi dom Zustandelommen dieser eigenartigen Aberrationen um Rückschlagsformen handeln,

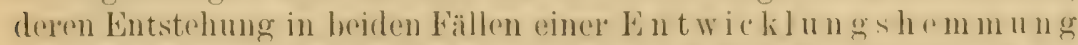
zuzuschreiben sej. Es ist ja hekannt, dass sich die Fiügolzeichnung dor Schmetterlinge ganz allmählich in dor Pupperentwicloclt, dass sio nicht am Schluss dor Puppenrulue plötzlich auftritt, sondern in den letzten Tagen des P’upuenlebens verschiodene Starlion durehläuft, die nicht ohne phylogenetische Bedentume sind. Fiscrea nahm an, dass sowohl dureh

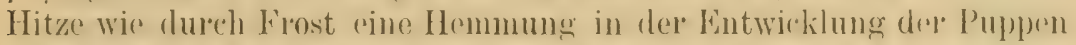
herbeigeführt würde, oine Ilemmmmg, die mubeschadet der woiteren Ent-

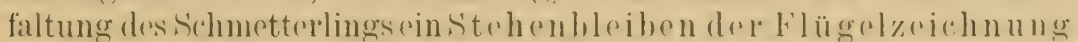
auf einer meh" oder wenigel fröhen Entwicklungsstufe hedinge. I) ie Fintwicklung der Flügelzeichnung sollte je nach der Stälir dres Rerizes

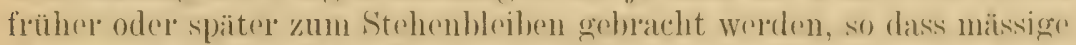

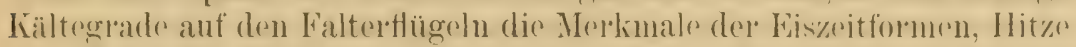
und Frost die zeichnumgseharaktere dor. Miocenfalter tixieren sollten. Standerss verwarf die Hypothese Fiscuen's, da dieselbe weder durch unsere liemutnis über die phylogenetische Entwicklung des Falterlideides. noch durch das Studium der ontogenetisehen Entwicklung der schnetterdingszoichnume gestützt wurde. Phylogenretisch wie ontogenetiseh tretru die dunkeln Zejehnungsmerlimale bei unsern Sinmetterlingern verhältnismaissig spät auf und zwar zuallenletzt auf denjenigen Strellen des Flügels, de sich bri den Aloreationen durch besondere Schwärzung

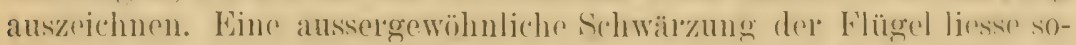
mit viol ehere anf ontogenctisches wie phylogenctisches Alter schliessen, nicht auf ein jugendliches frepräge, wio es doch Eiszeit- und Miocenfor-

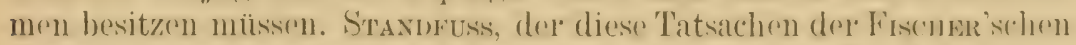

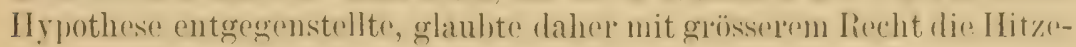
und Frostformen als Abänderungen individueller. Natur, als F ä $r^{\circ}$ bungsanomalien ansprechen zu dürfen. Wir werden aus dem folgenden essehen, dass das physiologische Experpinent dir Auftas- 
sung Staxnfus' hestätigt. Um ülee das Wesen und das Zustandelommen der" Wärme- und Kïlte-, der Hitze- und Frostformen ins Klare zu kommen, stelle ich mir die Frage: wie müssen einerseits Wärme- und liälereize, wie müssen andererseits Hitze und Frost den Puppenorganismus in seinel Lehenstätigkeit beeinflussen? Auf Grund der zahlreichen Ieobachtungen, die über das Verhalten der Insekten gegenüber gesteigerter oder herahgesetzter" Temperatur gemacht worlen sind, ist "rwiesen. dass sie sich in dieser Beziehung den übrigen wechselwalmen Tieren gleich verhalten. Auch die Beobachtungen an Schmetterlingspuppen zeigen, dass bis zu einem bestimmten Grad Temperaturerhöhungen del Umgebung stoffwechselsteigernd, Abkühlung stoffwechsel h erabsetzend wirkt. Mässige Wärme- und Kältereize beeintlussen somit nicht nur die Farbenbildung, sondern auch die gesamte Lehenstitiglieit der Puppe in entgegengesetzter. Teise: indem im ersten Fall eine Beschleunigung, im zweiten Fall eine Verzögerung der Pup)penentwicklung eintritt. Ganz anders ist dagegen die Wirkung von Frost und Hitze, überhaupt von Grenztemperaturen, die von dem Inselit noch oben ertragen werden können. Inreh beide, durch Hitze wie durch Frost, wird der Puppenorganismus in mehr oder weniger emptindlicher Weise geschädigt, wie schon aus dem grossen Prozentsatz von Sterblichkeit, der auf derartige Experimente entfillt, hervorgeht. Die Wirkungswrise der Hitze mud des Frostes ist den Schädigumgen des Hitzschlags und des Frtriepens zu vergleichen; in bejden Fällen wird das lebendige Plasma in einen Zustand velsentzt, in dem sich die ()xydationsrorgänge in den (iewehen nicht mehr vollziehen kömmen, ein Zustand, der von Eiweisszerfall gefolgt wird. Der angewandte T'emperaturgrad sowie die Iraktionsïhiglipit des einzelnen P’uppenorganismus muss daun entscheiden, ob der Falter die Schädigungen über'windet, oder ob er daran zu Grunde geht, ob er trotzdem einer normalen Gestaltung fähig bleibt, oder ob sich in seiner Färbung und Zeidnnung dir Spuren der überstandenen hehïdigungen geltend machen. Ist diese Schlussfolgermen richtig, so muss, sagte ich mir, eine Aberration auch noch auf andere Weise, wie dureh Temperaturwirkung, erziclt werden können; sie muss dann durch jelen Fingrift entstehen. der den Puppenorwanismus in ähnlicher Weise herinflusst oder schädigt wio Hitze und Frost. Mit andern Worten: Ist die zeitweise Herabsetzung der oxydativen Vorgänge in den Geweben des Puppenkörpers für die Bildung der Aberrationenvon wesentlicher Bedeutung, somusses a uch möglich sein, durch zeitweilige Sauerstoffentziehung die abel'ativen Formen zu erziehen, vorausgesetzt natürlich. dass dic sehmetterlingspuppen eine derartige Behandlung üherstehen könmen. Un die Richtigkeit meiner Voraussetzung zu prüfen, verbrachte ich die Puppen von Vanessa urtice und io elstmals in eine 
Atmosphäle von Kohlensäure. Der Versuch war so angeordnet,

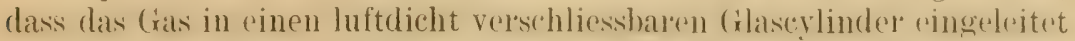
wurde, in dem sich die Puppen hefanden. Die Füllung mit Kohlensäures wurde so lange fortgesetzt, lis das (tas ülorlief, so dass ein Licht, welehes an den unteren Rand des Cylinders gohalten wurde, verlöschte. Das Vorehalten der Puppen in liohlensämreatmosphä̈r war sehr charaliteristisch. Sie bewegten sich meist erst sehr lebhaft, wurlen clann plitzlich still und reagiorten nicht mehr auf änsise lieize, rrlangten indessen, wenn sie nach 12-24 Stunden dem Glas entnommen wurden, ihre Beweglichkeit oft recht bald wieder. Die 24 Stunden in Kohlensäure weilenden Puppen ergaben, wie zu vermuten war, A ber'ratio ne n und zwal' in recht rohehlichem Prozentsatz, es hatte sich hier also tatsächlich unter dem Einfluss der Sauerstoffentzichumg ein ähnlicher Volgang in Pup-

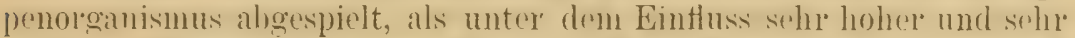
niedriger 'Temperaturen: von Frost und Ilitze. Es zeigte sich alore auch hier, wie bei den Frost- und Hitzexperimenten, dass die jungen sehmetterlingspuppen bei weitem empfindlicher waren wis die altoren und eine viel grössere Anzahl von Aberrationen crgaben. Durch regelmässige Tägungen der in Kónlensäuro gowesenen Pupjen verschiedenen Alters liess sich feststellen, dass der Organismus von jüngeren Puppen durch die abnormen Lebensbedingungen viel stärker affiziert wurde, wie der der älteren Puppen. Während nämlich rlie älteren Puppen gegenüber

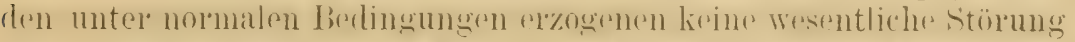
ihnerewichtsahnahme und damit ilıre vitalen Tätgkeit erkemmen liessen, zeigte sich der Verlauf der die Gewichtsabnahme der Puppen bestimmenden Kurve in höchst auffallender Weise beeinflusst. Während normaler Weise die Puppen in den ersten 48 Stunden nach ihrer Verpuppung selu elohblich an frowicht almahmen, war dio (iomichtsilifferenz bei etwa 24 Stunden alten Puppen in Kohlensäure eine sehr gelinge, bei noch jüngeren Tieren war sie gleich Null, und in einem Fall konnte ich bei ganz frischen, noch grün gefärbten Puppen sogal eine Gewichtszunahme konstatieren. Der Aufenthalt in Kohlensäure setzt somit

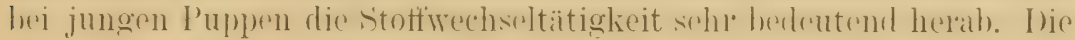
geringe Gewichtsabnahme während des Experimentes pflegt stets von einem sehr starken Abfall gefolgt zu sein, sobald die Puppen wieder normalen Atmungsbedingungen ausgesetgt werden. Es findret also narhträg-

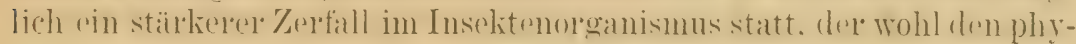
siologischen Folgen der Frost- und Hitzebehandlung an die Seite zu stellen ist. Der Ver'such hatte jedenfalls gezeigt, dass der zeitweilige Aufenthalt der schmetterlingspuppen in Kohlensïuratmophïre dirselhen Resultate hinsichtlich dex Entstehung von thereationen hervolbringt, wie die Behandlung der Puppen mit Frost und Hitze. Da aber die liohlensüure ein giftiges (ias ist. so war dimit die Fragen noch nicht 
entschieden, ob tatsïchlich nur die O-Entziehung die Veränderungen in Färloung und Zeichnung der Schmetterlinge zur Folge hat, ob nicht vielmehr die Aherrationsbildung auf Rechnung der Giftwirkung des Gases zu setzen sej. Die Berechtigung einel derartigen Auffassung schien mir um so grösser, da Fischer durch die Einwirkung der Aethernarkose ebenfalls Abrrationen in holem Prozentsatz erzogen hat. Wir wissen durch dir Untersuchungen Paul Berts, Nexckis und Sieberts, dass die Giftwirkung der Kohlensïureatmung die Reaktionsfähigkeit des lebendigen Plasmas in derselben Woise heeinflusst, wie die Aether- und Chloroformnarkose. In jedem Fall wird das Zellplasma, in dem sich die Oxydationsvorginge vollziehen, in einen vorübergehenden Zustand der (rerimnung versetzt, was eine Abnahme der Reaktionsfähiglicit, eine Herahsetzung der oxydativen Vorginge zur Folge hat, wodurch dann in zweiter' Linie ein mehr' orler' weniger'starker'Zerfall des Körpereiweisses bedingt wird. Um nun zu entscheiden, ob in dem gegebenen Fall die Veränderungen in Fïrbung und Zeichnung der Falter auf die giftige Wirkung des Gases, oder aber auf' die Sauerstoffentziehung zurückzuführen sei. wiederholte ich den Versuch, indem ich die Puppen diesmal in eine Stickstoffatmosphäre verbrachte. Das Ergebnis mit Stickstoff war aber von dem mit Kohlensäure nicht verschieden. Auch hier ergaben sich Aberrationen, die in ansgesprochener Weise die Eigenschaften der Frost- und Hitzeformen zeigten, und ich halte damit den Beweis für erbracht, dass die eigentümliche Gestaltung der A berrationen auf eine Störung in der Pigmentbildung zurückgeführtwerden muss, deren letzte Ursacheineinerzeitweilig herabgesetzten Oxydation und in einem daraus folgenden Eiweisszerfall im Puppenorganismus zu suchen ist. An diesem Eiweisszerfall ist, wie wil aus den Fïrbungsunterschieden der normalen und aberrativen Falter ersehen, der rote Flügelfarbstoff. der ja sellst einen eiweissartigen lïurper darstellt, in hohem Masse heteiligt. und es kommt für den letyten Erfolg nicht darauf an, auf welche Weise der physiologische Prozess eingeleitet wird, der die alerrative Gestaltung der Schmetterlinge zur Folge hat.

\section{Diskussion.}

Herr W. Petersen führt im Anschluss an den Vortrag an, dass er vol Jahren älnnliche Experimente nur mit erhöhter Sauerstoffzufuhr angestellt halse, indem er zur Begünstigung der Oxydationsrorgänge ozonisierte Luft verwandte. I) Experimente gahen keine deutlich messbaren Resultate. Bei der Kontrolle der Gewichtsänderung der Puppe aber wurder auch damals. wie jetzt die (röafin von Lisioex beobachtet hat, die merkwülige Tatsate hemerkt, dass die Puppe in der" allerersten Zerit 
(im sog. kritischen Stadium) an Gewicht etwas zunimmt, damn eine zeitlang sich kaum ver:indert und dass schliesslich in der letzten Zeit, kurz vor dem Ausschlüpfen, eine rapide Gewichtsabnahmo eintritt. Dieses letztere Stadium sei besonders für eine Fortsetzung der aussichtsiceichen Fxperimente zu empfehlen, da ein grosser 'Teil der Pigmentbildungen in diesem letzten Stadium den Abschluss findet.

Prof. Kronecker bemerkt, dass ausser der Temperatur auch die Belichtung die Firrloung beeinflusst, da auf hohen. wohl nicht warmen Alpen die Farben del Pflanzen und auch Inseliten besonders lebhaft sind. Man weiss, dass die Insekten pecht emptindlich gegen CO,-Anhäufung in de Umgebung sind, darum im Wasser schnell sterben. Mosso hat angegeben, dass in luftverdünnten Raum gehrachte Hunde von den Flöh'n verlassen werden.

Fische halten sich lange in gastreiem Wasser, wenn man die ausgeatmete $\mathrm{CO}_{2}$ durch schwache Alkalien bindet. Wenn $\mathrm{N}$, ähnlich wir C() die Puppen beeinflusst, so wäre zu beweisen, dass O-Mangel, aber nicht COOAnhäufung schuld sei. () ist heilvoll, weil er die Alscheidung der C(). begünstigt. Konzentrierte ('(), bringt das Protoplasma zum Gerinnen. wie höhere Temperatur (etwa $41^{\circ}$ bei Kaltblütern).

Auf den Einwand von Her'rn Prof. Kronecker wurde el'vidert, dass eine Anhäufung ron liohlensiure, dass eine liohlensäurevergiftung loei dem Stickstoffversuche dadurch vermieden wurde, dass der Stickistoff jeden Tag gewechselt wurde.

M. A. Puctet, après avoir félicité $M^{1 \text { le }}$ de LINDEn, attire l'attention sur' la similitude de ses résulats et de quelques-uns de ceux du Prof. StrandFuss, relativement à l'influence du chaud et du froid, ce qui montre que ce n'est pas le milieu lui-même qui amène ces variations, mais bien le changement de milieu. Les expériences que M. Prctex va exposel dans sa communication tendent aussi à prouver cette hypothèse. 


\title{
Des variations des Papillons provenant des changements d'alimentation de leurs chenilles et de l'humidité.
}

\author{
Par Arnold PICTET (Genève).
}

\section{INFluence de l'almientation.}

Les principales variations des Lépidoptères qui ont été étudiées,

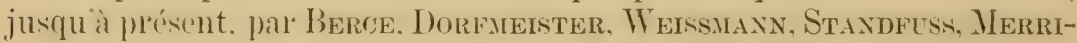
FreLd, ete. proviennent, en grande partie de l'intluence de la température, mais l'éturle de l'intluenee de l'alimentation des chenilles sur la coloration de leurs Papillons n'a pas été faite jusqu'à présent, d'une façon suffisante.

On sait que, en général, chaque espèce de chenille a sa plante nouricière qui lui est propre et qu'elle se refuse absolument à se contenter d'une autre plante, se laissant plutôt mourir de faim, que d'en consommel la plus petite parcelle. Cependant, il existe un certain nombre de "honilles qui sont polyphages ot qui virent égalenent bien des feuilles de plusieur's espèces végétales. Parmi ces chenilles polyphages, il en est quelques-unes, appartenant aux espèces les plus communes, que l'on rencontre maintenant sur des plantes et des arbres qui n'avaient jamais été, auparavant, signalés comme constituant leur nourriture normale.

Ainsi, Lasiocampa quercus, conmu du temps de Linné pour virre sur le Chêne (ainsi que son nom l'indique) et sur les arbustes des haies, se rencontre maintenant couramment sur le Lierre, le Saule, la Bruyère, le Peuplier, le Bouleau, le Groseiller, etc. Il en est de même d'Abraxas grossulariata qui a longtemps été indiqué comme se nourrissant exclusivement de Groseiller (et c'est pour cela qu'il porte le nom de cette plante) et qui se rencontre souvent, maintenant, sur le Chêne, l'Epine noire. l'Aulripine, le Fusain d'Furope. Une yuantite d'autres expexes sont dans le même cas.

Les espèces qui s'étendent facilement et dont les chenilles s'adaptent souvent à de nouvelles plantes nourricières, étant justement celles qui varient le plus ${ }^{1}$, je me suis demandé, il y a cinq ans, si ces fréquents "langenents dams labimentation normale des chenilles pouvaient etre la

\footnotetext{
${ }^{1}$ On connaît du reste quelques variations naturelles provenant du régime alimentaire.
} 
cause de quelques-unes de ces variations. J'ai done entrepris une série d'expériences dont les principaux résultats ont été exposés, soit à la Société de Physique et d'Histoire Naturelle de Geneve, soit à la Société Helvétique des Sciences Naturelles. Ces expériences, au nombre d'une trentaine, ont été faites chacune avec plus de cinquante individus et plusieur's d'entre elles ont été poursuivies, avec la même plante, pendant trois ou quatre générations.

Mais, arant de parler des delnier's lésultats que j'ai obtenus, je tiens à dire quelques mots de mes expériences antérieures.

Des chenilles d'Ocneria dispar (nouriture nol'male: Chêne) élevées avec des feuilles de Noyer, donnent, au bout d'une génération de ce régime, des Papillons dont la couleur du fond, chez les mâles, est devenue jaune au lieu de brune qu'elle est normalement et dont les dessins sont moins marqués; les femelles présentent peu de variations. A la seconde génération de ce régime alimentaire, la couleur du fond devient blanche, chez les mâles; les dessins s'éclaiłcissent encore dans les deux sexes, qui accusent, en outre, une notable diminution de taille. A la troisième génération, mâles et femelles ont diminué de plus de $50 \%$ de leur taille primitive et les dessins de leurs ailes ne sont presque plus reconnaissables; en outre, les mâles sont presque complètement blancs.

Des expériences analogues montrent que les feuilles d'autres arbres, tels que le Nèflier (Mespilus germanica), le Marronnier (Asculus hippocastaneum), le Sorbier (Pyrus aucuparia), etc., ont, à peu de chose près, la même influence que le Noyer.

Des chenilles d'Ocneria dispar élevées avec les feuilles d'Esparcette (Onobrychis sativa), de Pimprenelle (Poterium) et de Dent-de-lion (Taraxacum), donnent après une génération, des Papillons chez lesquels la couleur du fond s'est assombrie et dont les dessins sont devenus plus intenses. Au bout de deux générations, ces caractères augmentent encore. La Dent-de-lion produit, en outre, des Papillons qui sont sensiblement plus grands que les individus normaux.

Une série d'autres expériences montre que la nourriture normale, domnéc de nouveau à celles des chenilles qui ont eu, à la génération précédente, une nomriture étrangère, n'enlève pas les car'actères précédemment obtenus, et que ces caractères persistent encore après deux générations d'élevage nor'mal. Ce n'est qu’à la troisième génération que les caractères aber'ants disparaissent, mais non pas chez tous les sujets, quelques-uns l’estant encore sous l'influence de l'alimentation étrangère.

Des chenilles de cette même espèce qui ont reçu, à la première génération, du Noyer, à la seconde de l'Esparcette et à la troisieme leur nourriture normale, présentent les caractères des trois nourritures consommées, pendant les trois générations consécutives. 
Je ne me suis pas borné à étudier les variations provenant de l'alimentation sur cette seule espèce, mais j'ai refait des expériences analogues sur d'autres.

Chez Biston hirtarius et Himera pennaria, nous voyons que le Noyer et la Pimprenelle ont les mêmes effets que chez $O$. dispar.

Chez Lasiocampa quercus, le Noyer, au bout d'une génération, produit un éclailcissement de la bande fauve. A vec l'Esparcette, les Papillons de cette espèce deviennent très foncés et lex femelles rappellent la var. alpina; avec le Laurier-cerise (Laurocerasus vulgaris) ce sont les mâles qui deviennent plus foncés.

Chez Abraxas grossulariata, Evonymus japonicus donné à la place d'Evonymus enropxus, qui est la nourriture normale, ne produit aux deux premières générations qu'une faible variation, qui devient plus acentuée à la troisième génération ; elle consiste en une forte diminution des taches noires et en un éclaircissement de la bande jaune qui tend même à disparaitre completement.

Des variations analogues s'obtiennent chez Saturnia pavonia avec la Pivoine et le Noyer, et chez Bombyx lanestris ayec le Laurier-cerise.

Porthesia chrysorvhoea, élevé avec des grosses feuilles de Laurier-cerise (au lieu de Chêne et d'arbers fruitiers) ne domne pas de varlations; mais, si on l'élève avec des jeunes pousses de cet arbuste, on obtient, en très grande proportion, l'ab. punctate.

Chez Bumbys: reustria, la coloration brune des femelles solstient, chez les mâles, lor'sque les chenilles de cette espèce ont l'eçu, pendant une seule génération, des jeunes foulles de Laurier-ecrise comme unique aliment.

Chez Psilura monacha, élevé avec du Noyer (au lieu de Chêne et de C0nifères) on ohtient la proportion suivante de sujets aberrants : $250{ }^{0}$, de l'ab. eremita, et $35 \%$ de l'ab. nigra, contre $40 \%$ de la forme typique. Une proportion aussi élevée de ces variations ne s'obtient pas avec l'alimentation normale.

Chez Vanessa urticx, l'élevage des chenilles avec des fleur's d'Orties, au lieu de feuilles, produit l'ab. urticoüdes.

Depuis lors, les nouvelles expériences que j’ai entreprises dans ce domaine mont permis de clasise les variations provenant des changenents d'alimentation des chenilles, en trois catégories distinctes, d'après la nourriture donnée :

l" Varbations provenant de chenilles qui ont été nourvies uniquement de feuilles de Noyer, Nèflier (Mespilus germanica), Liere, Laurier-cerise (Laurocerasus vulyaris), Sorbier (Pyrus aucuparia), Fusain (Evonymus jupuricus, Houlilon (Humulus lumulus), cost-it-dire de feuilles de différents albres. 
2" Variations provenant de'henilles qui nont enonsommé que dex fenilles de Dent-de-lion, Laitue, Esparcette (Onobrychis sativa), Pimprenelle

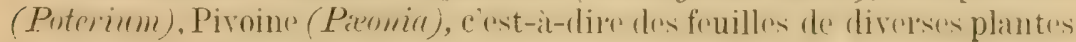
basses.

Il va sans dire que les feuilles utilisées dans ces expériences ne doivent pas être celles de la nourriture nolmale des espèces étudiées.

$3^{\circ}$ Dans la troisième catégorie, je place toutes les exceptions qui, mallumeusement, viennent toujours encombrer les résultats des expériences de ce genre, mais dont il est nécessaire de tenir largement compte, vu l'importance de leur's caractères.

Si nous examinons en détail les résultats que j’ai obtenus et dont les principaux ont été exposés, nous remargureons que les Papilloms dont les chenilles ont été nourries avec les feuilles de la première catégorie présentent tous des variations albinisantes et que ceux de la seconde catéworie au contraire, prexentent des variations melenisontes. Nous arrivons

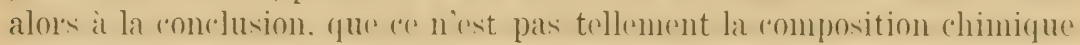
des feuilles consommées qui produit ces deux sortes de variations, mais bien plutrot leur qualité. et que lo régine alimentaire de la première catégorie, difficile à digérer et profitant mal, a une maturaise influener sur le développement de la chenille, ot sur la pigmentation du Papillon. Tandis

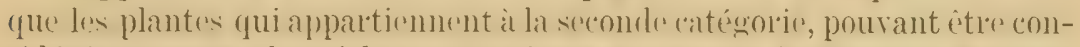
sirlérés rommo plus riches en matiènes nutritives, farcilitent le développrement de la chenille et amenent une cololation pigmentaine plus intense.

Ce que nous conmaissons de la formation de l'albinisme et du mélanisme semble pouroir s'adapter à ce qui vient d'être dit.

Cette hypothèse se trouve en partie confirmée par la suite de mes expériences à plusieur's générations.

Nous avons vu que les spécimens d'Ocneria dispar obtenus après deux générations d'élevage avec du Noyer et une génération intermédiaire de nourriture normale, accusent des variations franchement albinisantes.

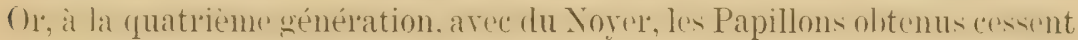
de présenter les variations albinisantes caractéristiques de cette nour'riture et retournent, en partie, à l'espèce typique, ne présentant plus ce manque de cololation des pigments. Cela nous montre que ces chenilles ont fini, apres quatre génélations, par sacentumer à la différence du ré-

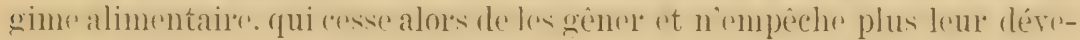
loppement de se faire normalement.

Le mème cas se présente chez Abraxas grossulariata qui, élevé pendant trois générations consécutives avec du Fusain du Japon (Evonymus japonicus) au lieu d'Evonymus europaus et Groseiller, produit une

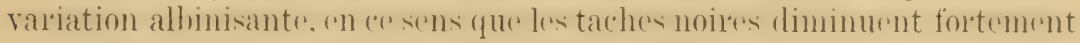
et que la bande jaune tend à disparaître. A la quatrième génération de ce régime, toute variation a cessé et non seulement les spécimens obtenus 
ont repris la forme typique, mais il s'en trouve quelques-uns qui ont pris une coloration plus intense que les Papillons nolmaux.

Cette accoutumance aux changements d'alimentation après quelques générations, montré que les variations provenant du régime alimentaire ne peuvent pas devenir fixes, et ne seront généralement qu'accidentelles. Nais, nous pouvons nous demander jusqu'à quel point elles peuvent se produire dans la nature et les expériences avec $A$. grossulariata nous fournissent une précieuse indication.

Les chenilles de la troisième génération d'expérience ont été divisées en deux lots : un premier lot qui a été encole nourri d'Evomymus japonicus et qui a donné les Papillons dont je viens de parler (ceux qui n'ont plus présenté de variations, par suite de l'accoutumance) et un second lot qui a été nourri de jeunes feuilles de Laurier-cerise. Les Papillons de ce dernier lot présentaient, à peu de chose près, les mêmes variations albinisantes que celles de la troisième génération nourrie avec l'Evonymus. Nous en concluons que c'est le changement de régime alimentaire plutôt que le régime alimentaire lui-même, qui produit ces variations.

Du reste, depuis deux ans, j’ai trouvé des chenilles de cette espèce en liberté sur le Chêne, et les Papillons en provenant, présentaient une variation albinisante analogue, montrant ainsi que le Chêne, comme nouvelle nourriture, produit les mêmes effets que l'Evonymus donné pour la première fois. Le Chêne peut donc être également classé parmi les arbres de la première catégoric.

Expériences de suralimentation. Il est facile de donner aux chenilles une alimentation insuffisante, mais on ne peut pas leur faire absorber plus de nourriture qu'elles n'en veulent. Aussi, pour les expériences de suralimentation,' est-il nécessaire d'employer des feuilles riches en matières nutritives et qui, à dose égale, donnent aux chenilles un développement plus rapide et une taille plus grande que la nourriture normale. Ces feuilles sont celles qui ont été classées dans la seconde catégorie et nous avons vu que les Papillons des chenilles qui les ont consommées, accusent des variations mélanisantes, c'est-à-dire qui présentent une coloration intense, avec acheminement vers la couleur noire. Une seconde conséquence de ces expériences de suralimentation est que, pour les espèces chez lesquelles existe le dimorphisme sexuel larvaire, ces plantes donnent à toutes les chenilles qui les consomment les caractères sexuels secondaires des femelles.

Dans le même or'dre d'idées, on remarque que les feuilles de la première catégorie produisent, chez les mêmes chenilles, les caractères sexuels secondaires des mâles, et que ces caractìres persistent encore après une génération d'élevage avec la nourriture normale. C'est ce que j'ai pu observer prineipalement avec $O$. dispar. 


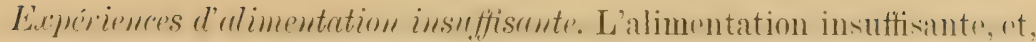
lans cortains cas, la suppression complete dr toute alimentation, à un monment donné, sont deux facteur's de variation. Mais, en général, le premier cas agit seul d'une façon appréeiable.

si lon supprime subitement toute sipecer dalimentation, at des chemilles de Vanessa urticx, à une celtaine époque de leur existence que j’ai essayé de déterminer et qui doit être de quelques jour's postérieure à la dernière mue, ces chenilles se chrysalident quand mème et donnent des Papillons nains, mais ne présentant pas la moindre variation.

Au contraire, si l'on prend des chenilles de cette espèce encore jeunes et qu'on les soumette à un régime spécial, où l'alimentation devienne chaque jour plus insuffisante, elles se chrysalident souvent (autant qu'on peut le reconnaître) avant la (lernière mue et donnent naissance à des Papillons nains également, mais qui présentent en outre une forte variation. Le nanisme obtenu dans ces deux expériences est très accentué, et dans le second cas j'ai eu quelques-uns de ces Papillons dont la taille avait diminué de $50 \%$.

Les variations dont on vient de parler peuvent être classées parmi les variations mélanisantes, en ce sens qu'elles possèdent des dessins estompés bruns et noir's, qui leur doment un aspect particulier.

Il convient, par conséquent, de remarquer que ces variations mélanisantes de Vanessa urticxe constituent une exception à l'hypothèse que j'ai émise plus haut. De nouvelles expériences seront nécessaires pour montrer s'il y a une différence entre ces variations provenant d'une alimentation insuffisante, mais avec la nourriture normale, et les var‘iations albinisantes déjà citées, provenant de chenilles nour'ries abondamment, mais arec des feuilles étrangères, leur profitant mal.

Je dois encore signaler, comme exception, le fait que les chenilles de Psilura monacha élevées avec du Noyer ont donné en assez grande proportion des ab. eremita et nigra qui sont mélanisantes et que la variété mélanisante d'Arctia caja, bien connue des entomologistes, s'obtient, paraît-il, après deux génélations d'élevage avec du Noyer.

Mais, en général, il semble que l'alimentation insuffisante soit une cause de défaut de coloration des pigments, ainsi qu'Aporia cratargi en fournit un exemple. Les chenilles de cette espèce qui ont été, à l'état adulte, soumises à un ou deux jeûnes forcés, donnent des Papillons aux ailes absolument transparentes qui indiquent un albinisme poussé à l'extrême.

Alimentation avec des fleurs. Les chenilles consomment souvent les fleurs de leur's plantes nourricières, sans que cela produise le moindre changement dans la coloration des ailes de leurs Papillons. Il semble cependant que les tleurs, pour des chenilles qui virent de feuilles d'arbres, produisent les mêmes variations albinisantes déjà constatées et 
quelles peuvent être classées dans la première catégorie. Ainsi O. disprar élevé avec des roses devient presque complètement blanc, comme ceux élevés avec du Noyer.

Mais, une exception analogue à celle constatée dans les expériences dialimentation insuffisante se présente ici arec r. urtire, qui. nourrie de Heurs d'( )rtie au lieu do feuilles. produit laal). niticoüdes, qui est plus colorée et plus foncée que l'espèce typique.

Coloration des chenilles. La couleur des chenilles participe souvent de colle des feuilles qu clles ahsorhent. et cest principalement chez les chenilles glabres que cette particularité se remarque, à cause de la transparence de leur peau. Sans entrer dans des détails à ce sujet, je ferai remarquer qu une mème plante peut être digérée de direx'ses manières par des chemilles despexes differentes. mais parfois assez roisines et que. par exemple, la chlorophylle. qui domne aux chenilles leur principale coloration, peut colorer le sang en vert chez les unes et en rouge ou brun chez les autres. Ainsi, il existe une chenille de Noctuelle qui est verte, lor'sque, dans la nature, elle consonme des feuilles d Espateette et de Treftes, et rose ou rouge, lor'squ'elle se nourrit des theur's de ces deux plantes. Par contre, une autre chenille verte se nourrit de feuilles de Chêne. et la larve d'une espèce très voisine, qui consomme la même nourriture, est rougeâtre.

Les observations que j'ai pu faire dans ce domaine, au cours de mes expériences d'alimentation, montrent qu'il faut peu de temps (5 à 10 jour's suivant les cas), pour changer la coloration et souvent l'aspect des chenilles. J'ai montré à la Société de Physique et d'Histoire naturelle de Genève, des chenilles vivantes de Lasiocampa quercus dont la coloration variait passablement suivant l'alimentation ${ }^{1}$. Nourries avec du Lamier-cerise, elles acquièrent, au bout d'une huitaine de jours, sur chaque anneau dol'sal, un losange blanc, tout à fait curieux et anormal. Avec du Lierre, elles ont la ligne dor'sale grisâtre, avec de l'Esparcette, elles deviennent jaunâtres, avec du Saule, brunes, couleur brique.

Les chenilles d'Ocueria dispar deviennent très foncées avec de l'Esparcette et de la Dent-de-lion et ont les tubercules très accentués (caractères sexuels secondaires des femelles). Elles deviennent grises avec de la Pimprenelle, verdâtres avec du Marronnier. Nomries avec du Noyer, elles ont une coloration très claire avec la partie antéricure jaune; elles ont alor's l'aspect des chenilles mâles.

Celles d'Arctia eaja deviennent également verdâtres avec du Marronnicr, et perdent un peu leur's poils lor'squ'elles sont nourries de Pivoine.

Chez Saturnia pavonia, les chenilles deviennent brunes avec de la Pi-

${ }^{1}$ Des aquarelles représentant ces chenilles ont été présentées à l'auditoire. 


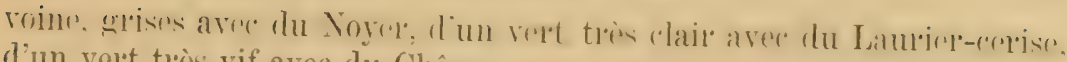
d'un vert très vif avec dlu Cliêne.

Les chenilles de Vanessa urticre ont, dans leur vie normale, deux co-

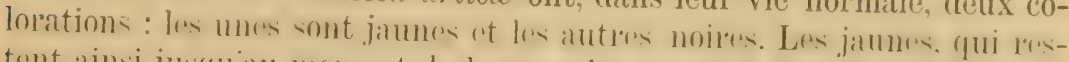

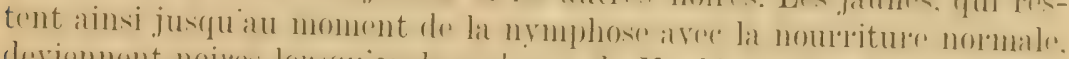

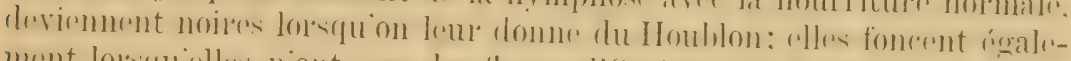

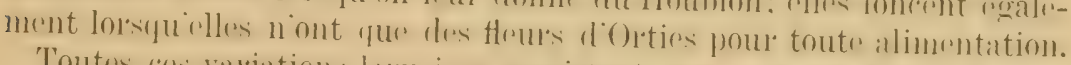

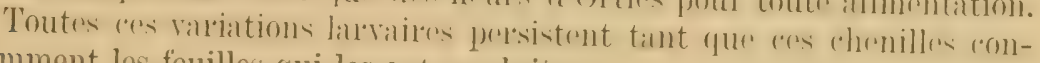
somment les feuilles qui les ont produites.

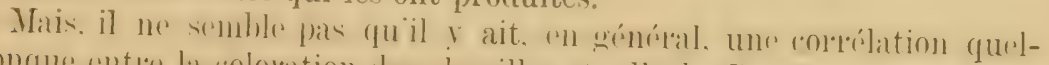

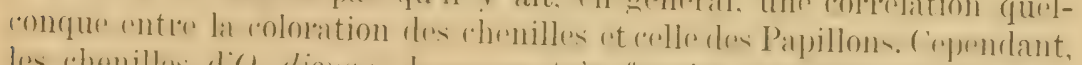

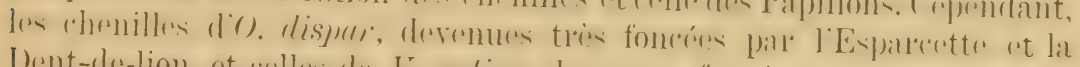

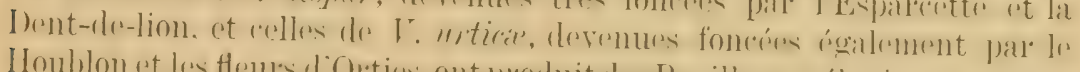

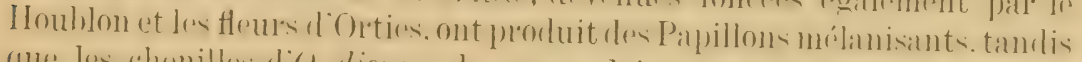

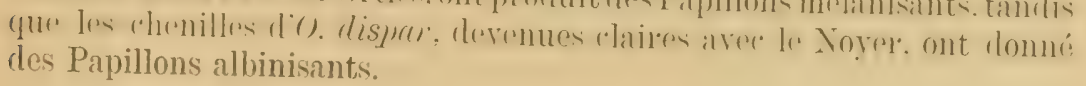

Conclusions. Il résulterait doncede ces cxpériences :

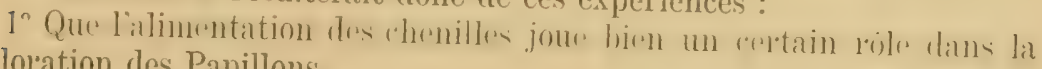
coloration des Papillons.

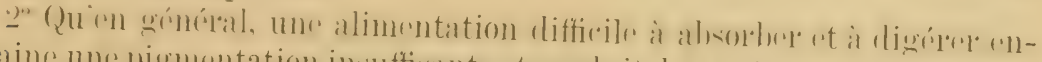

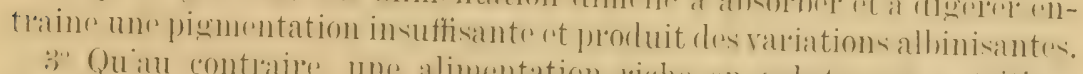

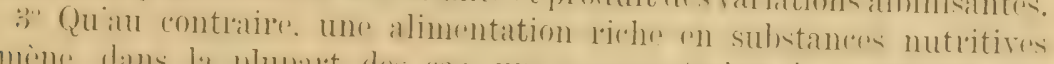
amene, dans la plupart dre ras, mor augmeutation dre roloration dos pigments et produit des variations mélanisantes.

4. Que certaines espèces, telles que V. urticx, V. polychloros, V. io,

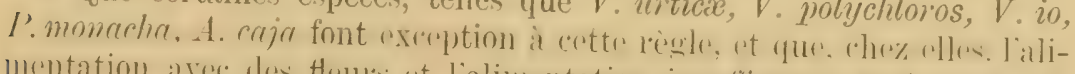

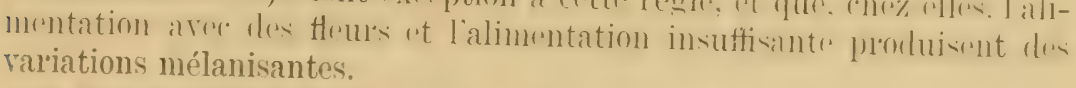

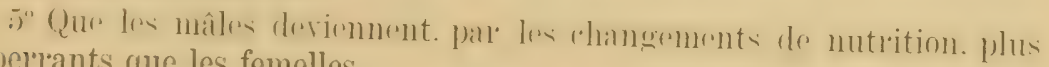
aberrants que les femelles.

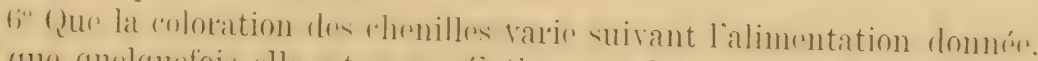

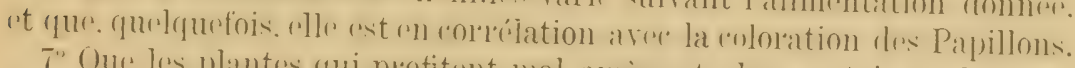

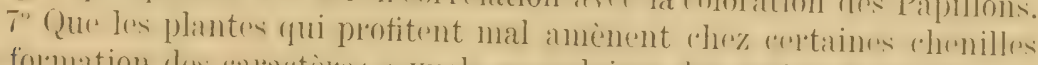

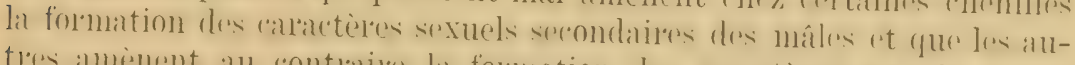

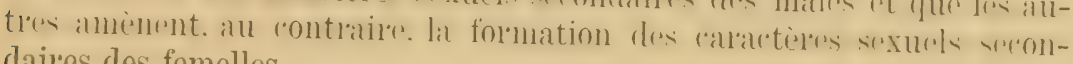
daires des femelles.

\section{INFLUENCE DE L'HUMIDITÉ.}

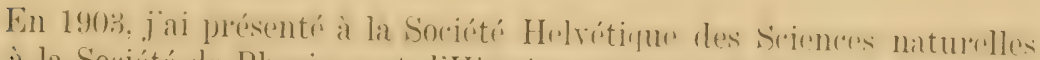

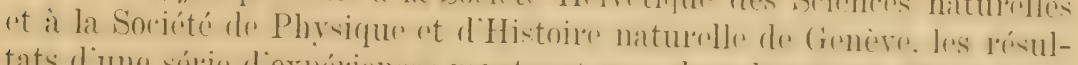

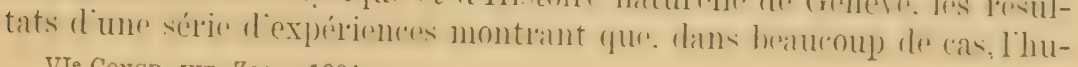

VIE CONGR. INT. ZOOL., 1904. 
midité atrit suffisimmment sur le dérelnppement des Lépidoptèreśs pour amener des différences sensibles de coloration. Les principales expériences ont montré que des chenilles adultes ( $V$. urticæe, V. polychloros, O. dispar) nourries avec des feuilles de leur nourriture normale, constamment humides, représentant ainsi ce qui se passe pendant une période pluvieuse de 6 à 8 jours, produisent des Papillons dont les ailes sont parsemées de dessins noirs normaux. Des chrysalicles placées dans une atmosphere humide. ou reserant la pluie sous forme artificielle pendant une huitaine de jours, donnent naissance à des Papillons beaucoup plus foncés que les normaux. Vanessa urticie, par exemple, éclôt avec des nervures fortement marquées en noir, avec la bordure externe des quatre ailes envahissant presque complètement les taches bleues, qui sont alor's très petites, mais avec une intensité remarquable.

Ces expériences tendent donc à montrer que l'humidité est bien un facteur de mélanisme partiel, ainsi que cela a été constaté déjà chez d'autres espèces animales. Toutes ces expériences ont été faites à la température ambiante.

Cette année, j’ai étudié les effets de l'humidité froide et de l'humidité chaude.

L'humidité chaude (30 à $35^{\circ} \mathrm{C}$.), ne semble pas avoir d'eftets bien appréciables sur les chrysalides et voici pourquoi :

Les chrysalides d'espèces qui n'ont pas coutume de se construire un cocon et qui sont simplement suspendues en l'air, sont recouvertes d'une substance graisseuse qui les protège contre la pluie et il faut un certain temps à l'humidité (environ huit jours) pour arliver à dissoudre cette substance graisseuse. Or, on sait que la chaleur active le développement des chrysalides et celles de Varessa io et $V$. urticæ que j'ai sommises à ces expériences ayant éclôt au bout de huit jours (au lieu de 15 à 18 qu'il en faut normalement), ne purent, pendant cette courte nymphose, recevoil les effets de l'humidité. Cependant, un petit nombre de chrysalides de T. urticx présentaient, en outre de la coloration spéciale produite par la chaleur, les dessins noirs spéciaux produits par l'humidité.

Humidité froide. Le froid, au contraire, ralentit le développenent des chrysalides. Dans une atmosphère humide de 8 à $15^{\circ} \mathrm{C}$, V. unticæ et $V$. io n'éclosent qu'au bout de troois semaines; de 5 à $10^{\circ} \mathrm{C}$., au bout de vingtcinq jours. Dans ces conditions, ces chrysalides subissent les influences de l'humidité pendant une période où le développement se fait plus lentement que dans la température nol'male et les résultats montrent

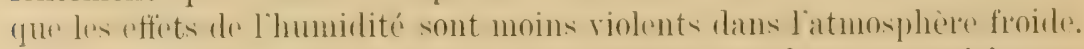
Je ne puis entrer ici dans tous les détails de ces nombreuses expériences, dont les résultats varient suivant la longueur d'exposition au froid et à l'humiclité, mais j’ai l’etrouvé, cette année, dans le $90 \%$ des cas, les mêmes

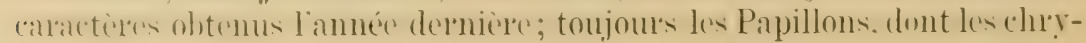




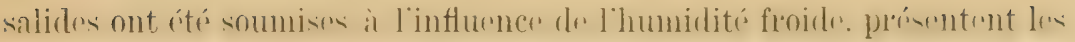
nervures noires et la bordure externe noire envahissant les taches bleues. Mais il faut tenir compte des effets du froid qui tendent à éclaircir la coloration générale; aussi, n’y a-t-il rien d'anor'mal dans le fait que, suivant la température et suivant les expériences faites, les nervures ne sont

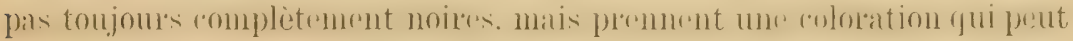
varier du brun-l'ouge au noir. Dans certains cas, une partie des nervures est seule colorée.

Les chrysalides de Vamessa io semblent être moins influencées par l'humidité que celles de $V$. urticæ. Les mêmes expériences ontété faites avec les deux espèces, et alor's que, chez cette dernière, j'obtenais toute une série de variations, c'est à peine si, chez $V$. io, le quart des spécimens obtenus présentaient, aux ailes supérieures, un léger dessin noir aber'rant.

L'accoutumance, dont il a été fait mention dans la première partie de ce travail, semble exister également Jol'squ'il s'agit des effets de l'humidité. Des chenilles d'Ocneria dispar ayant mangé des feuilles constamment humides pendant une assez longue période de leur vie (un mois environ), arec intermittence de sécheresse, donnent, à la première génération, des Papillons dont les femelles possèdent, aux ailes inférieures, un dessin grisâtre. A la seconde génération de cette expérience, la variation en question n'a pas l'eparu.

\section{Discussion.}

Prof. Forel. Ich erwälune hier eine Beobachtung, die ich in meiner Jugend oft gemacht habe. Die Raupe der Saturnia Carpini wird im erwachsenen Zustand grün. Doch gibt es eine Variation mit schwarzen Binden. Nun habe ich ganze Nester dieser Raupen oft in dunkeln

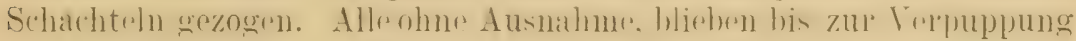
mit bald grösseren, bald kleineren schwarzen Binden, manchmal fast ganz schwarz. Und zwar konnte ich feststellen, dass ihre Geschwister,

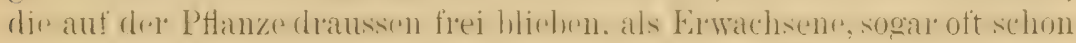
in den vorletzten Häutungen völlig grün wurden. Mir schien hier das Licht, d. h. die Dunkelheit, die Ursache gewesen zu sein. Diese Raupen wurden mit der gleichen Pflanze gefüttert wie die frei bleibenden Raupen, die ich in der Füttel'ung vernachlässigte, und die sich doch velpuppten; sie gaben einfach Zwergschmetterlinge ab. 


\title{
Myrmecologische Mitteilung das Wachsen des Pilzgartens bei Atta cephalotes betreffend.
}

\author{
Von Prof. E. GOELDI (Pará).
}

Es wird die Sachlago in's Auge gefasst, wie sie sich ergiebt beim Einbringen der etwa in Fingernagel-Grösse heimwärts zu transportirenden Blattabschnitzel, welche von der grosien Kaste der Blattschneider-Ameisen am Vortage, in der Vornacht abgeschnitten wurden und meistens rings um den creplünderten und geschändeten Baum hel'um auf Stunden oder einen halben Tag liegen hlehen. Dies Liegenlassen hat offenbal seinen Zweck, das Material soll andürren, heziehungsweise mindestens gehörig welk sein; so dient es besser zur Champignon-Cultur, während das völlig grün Zeug zu diesem Behufe sicherlich wenig thunlich wäre. Ihas Blatt-Parenchym muss abgrätet und in einen Compostdüngerartigen Zustand überofeührt werden, bis es die beabsichtigten Eigenschaften (rhält. I)ass ein solches Bestreloen wirklich vorliegt, (rogieht sich aus Beobachtungen. die zuerst von meinem Collegen, Dr. Jacques Hriser, Chref der hotanischen Abteilung des Museums in Pará, au Laboratoriums-Zuchten von Atta cephalotes angestellt und von mir seither oft und vielfach bestätigt worden sind: sowohl die grossen Blattabschnitzel, als namentlich die kleineren zweiter und dritter Grösse, werden systematisch mit den Kieforn rings am freien Rande herum eingekerht, gekniffen, - Operation, die wohl chen kaum anders sich erklären lässt als durch das Bestreben, das Parenchym rasch abzutöten.

Der Pilzgarten hat das bekannte schwammig-cavernöse Gepräge; es liesse sich von zelligen Hohlräumen reden, wenn man dabei nicht viel Anderes als höchst unregehmässig gestaltete, anscheinend völlig amorphe, kammerartige Gelilde sucht. Der Zuwachs besteht in successiver Apposition n'uer Blattabschnitzel kleinsten Formates auf der freien, oberen Kante des Randwalles der obersten, ohen in Arbeit stehenden Cavernen. Eine grosse Transport-Ameise bringt den eingeheimsten Blattabschnitzel im ()rinal-Format ron Fingernagelerösse herbei; sie neigt sich über den Rand der im Bau hegriffenen Höhlung und hält ihre Last so lange bis dieselbe stückweise von den herbeieilenden Arbeitsameisen heruntergesiigt und aufgebraucht wurde. Dahei hat sio oft cine Viertel-bis eine halle Stunde zu warten und ausser ihrer Last das (iewicht ron 4, 5, 6 mit der Z ' (1'kloincrung betrauten Colleginnen zu tragen, - eine ancliennenswerthe Leistung. sowoh! was die erforderliche Geduld als auch was die in Anwendung kommende Muskelkraft anhetrifft. Jas Aufsetzen der gehörig am

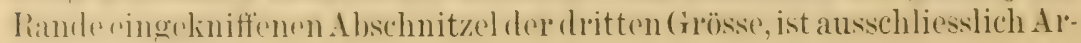


beit dep kleinsten Kasto von Individuen im Atte-Haushalte; es sind dies die eigentlichen Bauleute und Gärtuerinnen im vollsten Simne des Wortes.

Dass sie dieses letztere Prädikat - Gärtnerinnen - thatsächlich veldienen, eregielot sich aus folgender, ahsolut feststehenden Beobachtung. Es war für uns fröhler immor oin Rätlsed geblichen, wie las rasche liewachsen mit Rhozites-1ycel an den neuen Partion des Pilzaratens hei Atta cephalotes zu crklären sei, ob durch spontane Wucherung aus den writer unten liegenden Partien oler durch absichtliche loteiligung der kleinen Atta-Albeitre. Genaues, violtach wioderholtes Zusehen hat nun ergeben, dass zwischen die neuen, noch grünen Partien von den kleinen Arbeitern Bütschel von Mycelfärlen in den Kiofern herbeigeschleppt und rogelmässig hineingesteclit wurlon, ganz nach Art del Beschickung

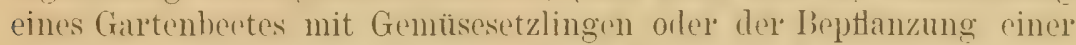
Böschung mit Grasinternodien und Gras-Rhizomen. So erklät sich das rascho Wrisiwerden, das schnecige Aussehen, das die neurn Partien schon nach Stunden zeigen und sie bald nicht mehl unterscheiden lässt in ihrem Habitus von den an den Vortagen angelegten.

\title{
Entomologica.
}

\author{
Von $\mathrm{D}^{\mathrm{r}}$ 0.-E. IMHOF (Windisch).
}

Mit 4 Textfiguren.

Bau der Culicidenflïgel (Fig. 1). Soviel mil bekannt, findet sich in der Literatur nur die Angabe, dass die Culicidenflügel Schuppen tragen, ohne Formbeschreibung.

Wir erkennen am gleichen Flügel zwei Typen von blattartigen Schüppchen in mehreren Grössen.

Der eine 'Typus sind kurzgestielte lanzettförmige oder langgestielt lineale, distal quer abgestutzte Schuppen, der andere vollkommen elegantlanzettfölmige Schuppen.

Alle Schuppen sind sehr fein parallellängsrippig. Die Rippen der Schuppen des ersten Typus enden als kurze vorstehende Spitzchen. Die Rippenzahl ist 2-5.

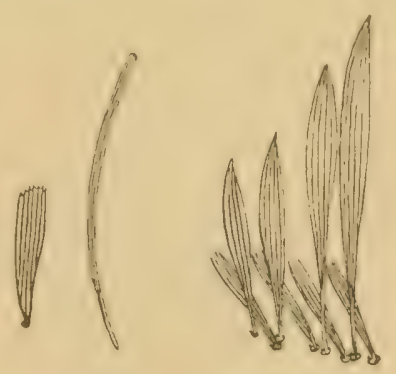

Rippen - Rand-
Schuppen.

Fig. 1.

Die gestutzten Schuppen finden sich besonders am Flügelhinterrand 
und auf don Flügelrippen, die schmal langlinealen nur auf den Flügelrippen, dic vollkommen lanzettförmigen fast ausschliesslich auf dem Flügelvorderrand.

Alle Schuppen sind in einem Ringwulst heweglich eingefügt - ob

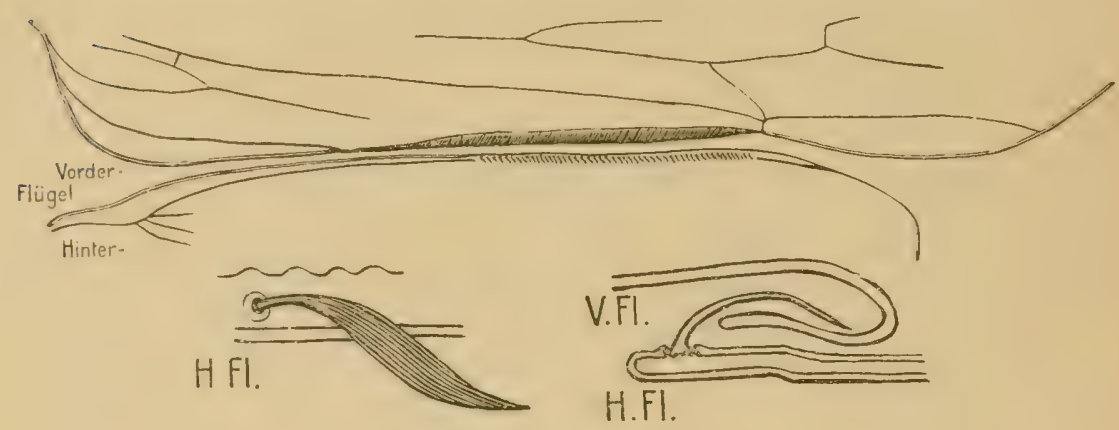

Fig. 2.?

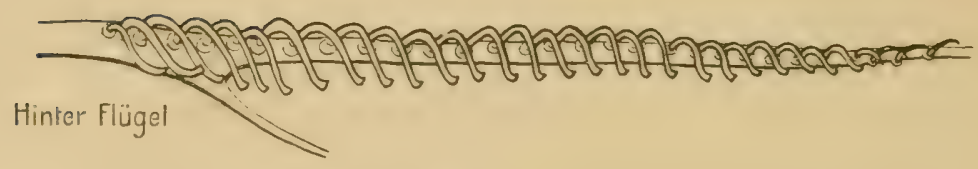

Fig. 3.

drehbar - niederleghbar und aufrichthar. An den Ringwülsten crkennt man einen äusserst feinen Ansatz.

Vorder-nul HinterHiigelierbindung der Inseliten (Fig. 2) und 3). Zwei der lompliziertesten Terbindungsein!

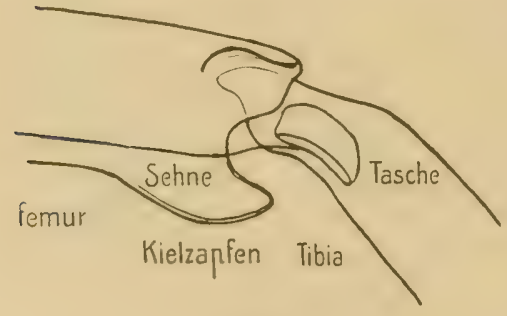

Fig. 4 . Hügels begegnen wir bei dem Genus Leptocerus und bei Vespa crabro L.

Leptocerus hat 51 lanzettförmige doppeltgebogene, bewegliche, gestielte, feinparallelrippige Lamellchen am Vorderrand des Hinterflügels, die durch Vor- und Rückdrehung in den unterwärts umgebogenen Hinter'and des $\mathrm{V}^{\mathrm{O}} \mathrm{r}$ dertlügels eingehängt werden.

Vespa crabro L. hat am Vorderrand des Hintertlügels gegen das

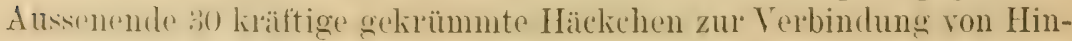
ter- mit Vordertlingel. 
Kniegelenksicherung einer Chironomide (Fig. 4). An der Unterseite des Femurendes findet sich ein kräftiger kielförmiger Fortsatz in Gestalt eines Lamellibranchiatenfusses. Am Anfang der 'Tibia crkennen wir eine formentsprechende Tasche. I) ureh Finsenken des Fortsitzes in die Tasche wird das Kiniegelenk gegen laterale Drehung gesichert. An proximaten Rand der Taschenöftumng beginnt rine sehnenartige Leiste, die sich in das Femur sich fortsetzend erkennen lässt.

\title{
Les œufs pédiculés et la tarière de Rhyssa persuasoria.
}

\author{
Par le prof. E. BUGNION (Lausanne).
}

Avec 1 planche et 2 figures dans le texte.

Les œufs de $R$. persuasoria sont caractérisés par la présence d'un pédicule long et grêle en rapport lui-même avec la longueur et l'étroitesse du canal de ponte renfermé dans le dard.

La description qui va suivre se rapporte à une préparation fixée dans une faible solution d'acide osmique, montée sur le porte-objet dans un mélange d'eau et de glycérine:

Placés dans la partie antérieure de l'abrlomen, les ovaires sont composés chacun de six à sept tubes, lâchement unis par les rameaux trachéens et se réunissant dans leur partie postérieure pour former de chaque côté un oviducte fort long, dans lequel se voient par transparence les pédicules des œufs, brunâtres, un peu sinueux, disposés en un faisceau, faisant à première vue l'effet d'un paquet de soies rigides.

L'œuf mûr de $R$. persuasoria est long de 12 à $13^{\mathrm{mm}}, 5$, dont 3,5 pour' le corps de l'œuf et 9 à 10 pour le pédicule seul. Filiforme, régulièrement cylindrique (épaisseur uniforme : $85 \mu$ ), cet appendice n'offre, à l'opposé de ce qu'on observe chez les Cynipides et les Chalcidiens, aucun rentlement terminal; il se termine simplement par une extrémité arrondie, sans micropyle distinet.

On constate, en outre, que le pédicule est tourné du côté de l'oviducte et doit, par conséquent, pénétrer en premier dans le canal du dard, tandis que chez les Cynipides, c'est le gros bout de l'œuf qui est dirigé en arrière et qui, en conséquence, s'engage tout d'abord dans le canal.

Examiné à un grossissement plus fort (3 à 400 fois), l'œuf mûr offre une coque chitineuse assez épaisse $(10 \mu)$, formée de deux couches, et un 
contenu liquide parfaitement homogene. Je n'ai vu, meme en emplorant des colorants, ni vésicule germinative, ni chromosomes; je n'ai pas observé de granules vitellins dans le cytoplasme.

Il y avait dans chaque tuhe ovarique deux de ces œufs mûrs, blanes, à peu près de même taille, adossés l'un contre l'autre comme le montre la figure. L'ovail'e entier étant composé de 6 à 7 tubes et ces tubes se réunissant pour former l'oviducte, il en résulte que chaque ovaire renfermait 12 à 14 ovules mûn's et chaque oviducte 12 à 14 pédicules juxtaposés, soit pour chaque Rhyssa 24 à 28 ovules prêts à être pondus.

Le tube ovarique comprend: $1^{\circ}$ une membrane d'enveloppe (cuticule) arec des ramifications trachéennes; ; $2^{\circ}$ un épithélium pavimenteux: $3^{\circ}$ un épithélium cylindrique entourant les ovules; $4^{\circ}$ dans sa partie initiale, des cellules germinatives, dont les unes se différencient et deviennent des ovules, tandis que le rôle des autres est purement nutritif. La cuticule forme, au bout du tube, un ligament suspenseui très court, qui, avec les tlachées, fixe l'ovaire dans sa position. L'épithélium pavimenteux, peu distinct sur les pièces fraîches, se voit plus nettement sur les préparations colorées a l'hémalun: il paraît alor's comme un revêtement de cellules polygonales, chacune avec un petit noyau ar'rondi, de couleur violette. L'épithélium cylindrique est destiné plus spécialement à nourril le vitellus et à sécréter la coque. ('et épithélium, qu'on peut appoler folliculaire (correspondant à la granulosa du follicule de GraAF), se voit distinctement à la surface des jeunes ovules, tandis que, sur les œufs mûs, il est plus ou moins atrophié. Il est probable, cependant, que l'épithélium folliculaire accompagne l'ovule au cours de sa descente et qu’il ne disparaît que lorsque son rôle est terminé.

La partic initiale du tube ovarique renferme ordinairement trois jennes ovules, chacun d'eux étant séparé de l'ovule suivant par le groupe de cellules nutritives (vitellogènesi) affecté à ce dernier. Je compte donc après un petit germigène formé d'éléments encore indifférents et indistincts: $1^{\circ}$ un premier groupe de cellules nutritives et, en dessous de lui, un ovule en voie de formation placé transversalement et déjà entouré d'un ípithélium cylindrique; $2^{\circ}$ un deuxième groupe de cellules nutritives ot un deuxieme ovule, à peine plus développé, (ncore placé en travers; $3^{\prime \prime}$ un troisième groupe d'éléments nutritifs (une douzaine environ), semblables à ceux des groupes précédents, mais plus rolumineux et mirux dessinés que ces derniers. ("est, en effet, dans cette partie du tube ovarique qu' les collules vitellogènes atteignent leur maximum de développement, tandis que, dans les groupes qui suivent (4 et 5), elles sulissent une atrophie à peu pres complète. Lovule correspondant (3) a pris une position longitudinale. Appliqué contre lo bont supérieur de l'uf loancoup) plus volumineux qui vient ensuite, il attrint une longuenr der ()$^{\mathrm{mm}}$.s. soit la seizieme partie de la dimension de l'osuf mûr. Cet orule. 


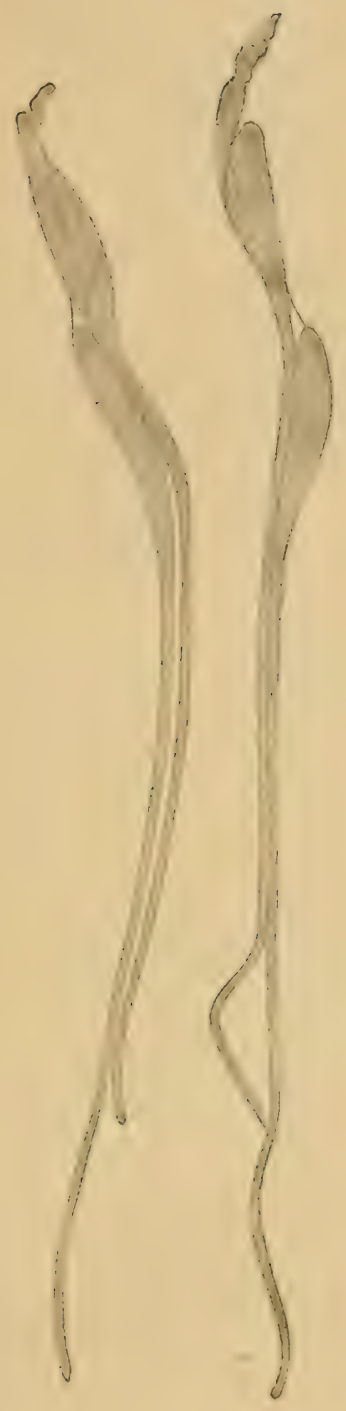

Fig. 1. Deux tubes ovariques montrant chacun denx œu fs mûrs a vec l’ur quédicules. Gross.: \& fris. Acide osmique ${ }^{1}, 2^{0},{ }^{\circ}$ eau et glycérine.

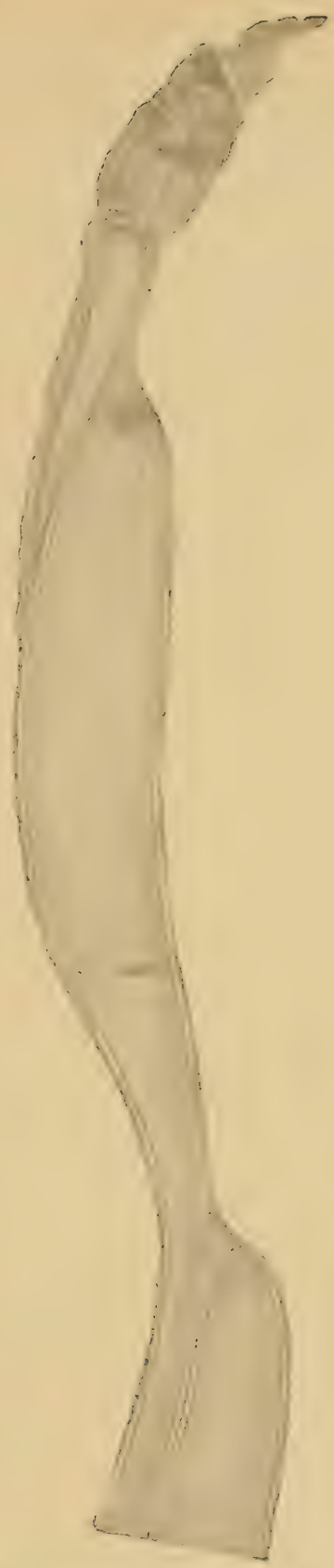

Fig. 2. Bout antérieur de l'un de ces tuber grosi in fois. moutrant l, esernigène, les groupes de cellules nutritives, les ovules en formation et les œufs mûrs. 
dont la coque est encore très mince, est entouré d'un épithélium cylindrique parfaitement distinct. ()n remarque, en outre, que ce troisième ovule est mal délimité au niveau de sa pointe, cette région représentant sans doute la zone d'accroissement du pédicule et n'ayant en conséqueuce pas encore de coque. Comme d'ailleur's dans les œufs mûr's, je n'ai pu découvrir dans ce troisième ovule aucune trace de vésicule ou de noyau. Viennent ensuite les deux oufs mûrs (4 et 5) déjà décrits ci-dessus, avec leur long pédicule et leur coque chitineuse épaisse.

On voit qu'il y a, au point de vue de la croissance, une distance considérable entre l'ovule 3 et l'ovule 4, tandis qu'entre les ovules 4 et 5 , l'écart est au contraire à peu près nul. L'œuf 4 est aussi long et aussi gros que l'œuf 5 . La vie sexuelle de $R$. persuasoria étant probablement limitée à un seul été, on peut conclure de ces faits que, si la ponte avait suivi son cours normal, les ovules 1 à 3 n'auraient pas pu arriver à maturité en temps utile. En revanche, la maturation simultanée des deux dernier's ovules doit être, pour ces Insectes, une disposition favorable. En effet, lorsqu'après maintes recherches, la Rhyssa 우 a découvert un tronc d'arbre approprié à sa ponte, il est clair qu'il est avantageux pour son espèce de pouvoir, au moment propice, disposer de 24 ou 28 œuts.

Le receptaculum est une petite vésicule sphérique (diam. $148 \mu$ ) brune, chitineuse, s'ouvrant par un court canal au point de jonction des oviductes dans le conduit génital. Il était, sur un exemplaire débité en coupes (capturé le 4 juillet 1904), rempli de zoospermes parfaitement consel'vés.

Le canal du receptaculum est en rapport avec des cordons glandulaires, irréculièrement bosselés, qui montrent à l'intérieur un canal collecteur, chitineux. dre calibre uniforme et, hanchés sur celui-ci, une multitude de ramuscules correspondant aux cellules de la paroi. Il s'agit, comme on voit, d'unc glande at canalicules intracellulaires, semblable à la gla nde a p pendicula i e de l'Abeille reine.

Les autres glandes en rapport avec l'appareil génital femelle sont: $1^{\circ}$ les ampoules des oviductes, $2^{\circ}$ la glande multifide, $3^{\circ}$-deux corps ovalaires désignés sous le nom de corps blancs.

La glande accessoire, décrite par Bordas (1897), chez les Ichneumonides, n'a pas été observée.

Les a mpoules ne sont pas situées dans l'axe des oviductes, mais s'élèvent en saillie sur leur bord interne, un peu au-dessus du point de jonction de sesconduits. ()n voit l'oviducte, strié de lignes brunes (pédicules desosuf's). suivie le coté externe de l'ampoule, tandis que l'ampoule elle-meme se distingue par sil couleur blanche, un peu translucide. Les coupes seriexs mont permis de constater gue loviducte forme. au niveau 
de l'amponle. une succession do plis transverses revêtus d'un bo épithélium cylindrique et destinés sans doute à augmenter l'étendue de la surface de sécrétion.

La glande $\mathrm{m}$ u l t if i d e correspond à la glande vénénifique acide des Hyménopteres porte-aiguillons. Flottant librement dans l'ablomen, cest appareil comprend: $1^{\circ}$ une vésicule allongée, continuée par un tube excréteur très fin, inséré à la jonction des oviductes sur le conduit génital (sa longueur avec la vésicule $\left.=8^{\mathrm{mm}}\right), 2^{\circ}$ six à huit tubes glandulaires, grêles, ramifiés, la plupart terminés en fourchette, implantés sur le sourmet de la vésicule, formant énsemble un arbuste délicat de couleur blanchâtre.

Les corps bla n es (tubercules calloso-charnus de L. Dufour) sont des glandes d'un type spécial, caractériscés par la prés 'nce d'un épithélium superficiel, cylindrique, disposé en une couche, et d'une multitude de canalicules simples, convergeant vers une cavité centrale. Longs de $3^{\mathrm{mm}}$ sur $1^{\mathrm{mn}}, 7$, ovalaires, un peu rentlés dans leur partie postérieure, ces $01^{\circ}$ ganes, d'un blanc de lait, ont une cavité centrale et un canal collecteur commun large et court $\left(2^{\mathrm{mm}}, 5\right)$ qui se dégage de leur face profonde et s'unit à celui du côté opposé pour s'ouvrir, vis-à-vis du receptaculum, à l'entrée du canal de ponte. Ces glandes, quoique peut-être homolngues des glandes sébacées des Cynipides, ne fournissent point de granules graisseux et paraissent sécréter plutôt un liquide riche en albumine, coagulé et color'é par les réactifs.

De ces trois espèces de glandes, ce sont les ampoules des oviductes qui paraissent spécialenuent adaptées à fournir le liquide nécessaire à la prorgression de l'œuf.

L'ahsence de micropyle rend l'acte de la fécondation difficile à expliquer. L'ceuf mûr étant entomré d'une coque épaisse, il est peu probable que les spermies puissent passer au traver's.

Faut-il admettre que la fécondation a lieu avant la formation de la coque? que les spermatozoaires remontent à cet effet dans la partie supérieure du tube ovarique au niveau du $3^{\text {me }}$ œuf, alor's que le pédicule est encore en roie d'accroissement? Cette supposition ne s'accorde guère avec ce que l'on sait des autres Insectes. Chez l'Abeille, par exemple, il est étahli que la fécondation so produit. non pas daus le tube ovarique, mais dans l'oviducte, au moment où l'œuf passe devant le canal du receptaculum. Il faudrait, pour répondre à ces questions, pouvoir disséquer un ovaire de Rhyssa, à l'époque de l'imprégnation.

Il ressort d'une observation de Harrington (1887) que l'éclosion de Thalessa hunator espèce américaine voisine de notre Rhyssa, a lieu ordinairement en juin et que les mâles, sortant les premier's de leur cachette, ont la faculté de découvril' (au moyen de leurs antennes, siège 
de l'odorat) le point précis de l'écorce par lequel une femelle va s'échapper. Dans un cas particulier, Harring'ton a constaté qu'un grand nombre de mâles attendaient à cette place déjà deux jour's ì l'avance. La femelle. à peine dégagér de sa galerie, est saisie par le plus habile, avant même qu'elle ait eu le temps de prendre son vol.

Les indications de l'auteur américain peurent probablement s’appliquer' it $R$. persucusoria' . C'est ver's la fin de juin que le rapprochement des sexes se prorluirait d'ordinaire. Toutefois, la Rhysse ayant un receptaculum et pourant en conséquence emmagasiner les spermies, il se peut que la fécondation des oufs n ait lieu çue plus tard, en juillet ou en août (saison de la ponte).

Les hôtes préférés de la Rhysse sont, d'après les olservations de Ratrzeburg (1844), Brischie (1880) et Bignell (cité par Dalla Torre, 1892), les larves de Sirex gigas, spectrum, et juvencus.

Au dire de Provancher (cité par Dalda Torre) la même espèce se reetrouve au Canada et serait parasite de Monohammus scutellator et confusor.

Les métamorphoses de $R$. persuasoria ont été décrites par SNellex vax Voldexhoren (1860); d'autres indications ont été publiées par Eraxé (1877). On ne sait toutefois pas encore exactement si le parasitisme est interne ou externe 2 .

Quant à la duréc du développement, nous pourons, à défaut d'observations précises tirer quelques déductions des faits constatés chez les Sirex. Kíluar (cité d'après Rilex, 1888. p. 179) a observé que la larve de S. gigus termine sa croissance en sept semaines à dater de la ponte, l'Insecte pouvant, d'autre part, après la période nymphale, demeurer plusieur's années sous l'écorce à l'état d'imago. La larve de Rhyssa s'attaquant, paraît-il, à une seule larve de Sirex, ou peut, d'après ces données, conclure que le parasite doit terminer son développement pendant cette même période de sept semaines au maximum (un peu moins, puisque la larve de Sirex a atteint une certaine taille, au moment où la larve de Rlyyssa commence à la sucer). La phase nymphale étant vraisemblabloment de 3 à 4 semaines (?), l'imago éclorait en septembre ou octcbre, passerait l'hiver sous l'écorce et attendrait l'été suivant pour prendre son vol, s'accoupler' et déposer' ses œufs.

La tarière, longue de $4^{1} / 2^{-5}$ centimètres, comprend le fourreau bivalve

${ }^{1}$ En Suisse, on observe la $R$. persuasoria dans les forêts de Conifères dès la fin de juin ou le commencement de juillet (d'après les observations de A. vox Schulthess et L. Hermann.)

${ }^{2}$ RnLex (1888) a observé que Thalessa lunator, se développe comme ectoparasite aux dépens des larves de Tremex (Sirex). 
et à l'intérieur le darod très fin et acéré, spécialement actif dans l’acte de sciel et de forer"

Les valves couvertes de petits poils à l'extérieur sont creusées en gouttière et revêtues sur leur face interne d'une membrane lisse de couleur blanchâtre. Destinées à protéger et ì diriger le dard, elles ne s'rnfoncent pas avec lui dans l'acte de forer, mais s'appuient seulement a la surface du bois. Parfois même les valves s'écartent en divergeant ì droite et ì gauche.

Le dar $d$, qui paraît simple à première vue, semblable à un crin épais de $0^{\mathrm{mm}}, 14$, est en réalité formé de trois pièces, le gor ger et au côté dorsal, les stylets au côté ventral, recourbées à leur base en forme d'ares (ares du gorgeret et des stylets). Les stylets, dentelés ver's le bout en forme de scie, sont articulés avec le gorgeret au moyen de deux raiuures longitudinalus dans lesquelles s'engagent tres exactement deux crètes taillée's en forme de rail, portées par le gorgeret. Frâtee à cette disposition, les stylets peuvent exécuter un mouvement de va-et-vient le long du gorgeret, sans s'écarter de ce dernier. La coaptation des trois pieces est si parfaite que l'on a. mime en s'aidant de deux aiguilles. beaucoup de peine à les sépal'er les unes des autres. Le canal de ponte, très fin et très étroit, est ainsi qu'on le voit sur' la coupe (Pl., fig. 1) compris entre les deux stylets, évidés à cet effet sur leur face interne ${ }^{2}$. Sa largeur, lorsque les stylets sont rapprochés, est exactement de $0^{\mathrm{mm}}, 08$. Un espace libre ménagé en dehor's du canal, dans la partie évidée, r’enferme une trachée (visible sur la coupe) et peut-itre des filets neveux". Cet espace est séparé du canal de ponte pal une membrane plissée (extensible). Le gorgeret l'enferme lui aussi un canal double (diam. 0 ${ }^{\text {man }}, 04$ ) qui se prolonge, paraît-il, jusque dans la cavité somatique et renferme vraisemblablement du sang, des trachées et des filets nerveux. On remarque, en outre, que le gorger'et est divisé par une fissure qui pénètre aux $2 / 3$ environ de son épaisseur et contribue sans doute à faciliter l'écartement des stylets au moment du passage de l'ouf'. Ainsi conformée, la tarière de Rhysso constitue un outil admirablement adapté à sou but, capable de pereer non seulement le bois vermoulu, mais aussi l'écorce et le bois sain en un temps très court (quelques minutes) et de porter les œufs à la profondeur voulue.

1 J'ai sous les yeux une Thalessa leucographa (cadeau du Dr STEck) dont la tarière mesure exactement 88 millimètres.

${ }^{2}$ La coupe dessinée Fig. 1, a été obtenue par M. Popofr, assistant au laboratoire d'embryologie (Lausanne.) Le dard, qui est dur et cassant, avait été, avant l'inclusion dans la parafine ramolli pendant trois semaines dans une solution d'acide azotique à $3 \%$.

${ }^{3}$ Des poils tactiles insérés le long du canal de ponte (Cynipides) et en rapport avec des fibres' nerveuses serviraient d'après ADLER et BEYERLNCK à renseigner l'Insecte sur la marche et la position de l'œuf. 
La tarière est portée par le $8^{\text {e }}$ segment abdominal, dont la forme et la structure sont spécialement affectés à cet usage. (Pl.. fig. 2.) Comprimé d'un côté à l'autre, long de 9 à $10^{\mathrm{mm}}$, ce segment représente une sorte de c a r'èn e qui proémine en dessous de l'abdomen et dont l'extrémité postérieure (pygidium) est seule visible au côté dorsal, en arrière du $7^{\mathrm{e}}$ anneau. En avant, la carène se termine par deux bords arrondis, un droit et un gauche, divergents en forme de $\mathrm{V}$, sur lesquels !es ar'es des stylets et du gorgeret sont exactement appliqués. En arrière, à une distance de $6^{\mathrm{mm}}$ des bords antérieurs, se trouvent les articulations des valves du four'eau. Le dessous de la carène offre une crête formée par la juxtaposition des lames ventrales et, le long de cette crête, une rainure dans laquelle le dard vient se placer. Le dessus, élargi en forme de gouttière, proémine dans l'intérieur de l'abdomen et se voit à traver's les masses musculaires, lor'sque les segments 6 et 7 ont été fendus du côté dor'sal. C'est vers la partie antérieure de cette gouttière que convergent les deux oviductes pour s'ouvrir dans le canal du dard; c'est à ce niveau également que débouchent le receptaculum, la glande multifide et les conduits excréteur's des corps blancs. Chez le vivant, la carène entière peut, grâce à l'élasticité de ses connexions membraneuses, s'enfoncer plus ou moins à l'intéreur de l'abdomen. entre les parties écailleuses des seguents fot 7 .

La dissction au moyen des aiguilles montre que la carène est constituée par deux paires de lames chitineuses: $1^{\circ}$ Deux la mes ventrales (écailles latérales de L. Duthrers, 1849, oblonge Platten de Krxpelin, 1873, Se sternite de Seurat, 1899), situées au côté ventral, formant par leur juxtaposition la rainure médiane mentionnée ci-dessus; $2^{\circ}$ Deux la mes la térales (écailles anales de L. Duthers, quadratische Platten de Krapelin, S tergite de Seurat), situées latéralement, articulées avec

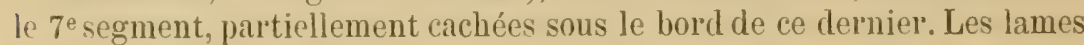
ventrales se terminent par une extrémité tronquée au niveau des articulations des valves. taudis que les launes latérales, prolongées en arrière du $7^{\circ}$ segment, se soudent l'une à l'autre pour former le pygidium. On voit encore: $1^{\circ}$ que l'arc du gorgeret (renforcé par un petit crochet) est appliqué sur le bord antérieur de la lame ventrale, et qu'il paraît attaché à celle-ci ; $2^{\circ}$ que l'arc du stylet glisse le long de l'arc du gorger'et au moyen de sa rainure et peut alternativement avancer et reculer $; 3^{\circ}$ que l'arc du stylet porte dans sa concavité un aileron en forme de demilune (Winkel de KK kPELin, épimère de Seurat) qui s'intercale dans l'espace compris entre les deux lames et s'articule avec l'une et l'autre par ses deux extrémités.

La figure 3 représente les deux moitiés de la carène étalées et sépal'ées l'une de l'autre avec les lames ventrales en dedans et les lames latérales en dehor's; ces dernières ont été séparées du pygidium. L'aileron $a$ se voit entre les 2 lames. La ligne courbe $m$ indique l'insertion de 
la membrane par laquelle la carène s'unit au $7^{\mathrm{e}}$ segment. Les figures 5 et 6 montrent la lame ventrale gauche et la lame latérale correspondante, séparées l'une de l'autre.

Le jeu des diverses pièces a été très bien expliqué par AdLer dans son étude des Cynipides (1877, p. 309), et plus récemment par Seurat (1899). On constate, tout d'abord, que l'articulation de l'aileron avec la lame latérale est plus mobile que l'articulation avec la lame ventrale. Tandis que l'angle interne de l'aileron pivote sur place à la manière d'une charnière, l'angle exter'ne subit des déplacements plus étendus qui se répercutent sur le stylet at produisent le va-et-vient caractéristique de ce dernier. Au surplus, il importe de remarquer que les muscles n'agissent pas directement sur l'aileron, mais sur les deux lames articulées avec lui. C'est done sur les lames (et non sur l'aileron) que sont insérés à l'intérieur de l'abdomen les muscles pro- et rétracteurs. Les lames, juxtaposées au moyen d'une rainure, effectuent l'une par rapport à l'autre de légers glissements qui, se transmettant à l'aileron, le font pivoter sur lui-même. Le stylet entraîné par le déplacement de l'aileron, exécute son va-ctvient le long du gorgeret, avec la précision d'un balancier de machine à vapeur faisant agir une bielle.

Nous avons dit, ci-dessus, que l'ouf de $R$. persuasoria a une épaisseur de $0^{\text {ma }}, 46$ (presque $1 / 2$ millimètre) au niveau de son corps. Le canal de ponte mesuré au micromètre sur la coupe, oftre d'autre part un diamètre

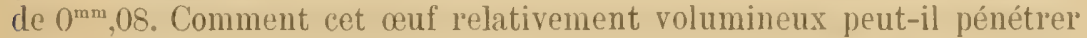
dans un canal qui, d'après ces mesures, est six fois plus petit que lui?

Le passage des ouf́s dans le canal du dard est à promière vue si difficile à expliquer que plusicurs auteur's n'ont pu y croire.

Lacaze-Duthers qui a décrit minutieusement les pièces de la tarière d'Ephialtes manifestator (1849) soutient, contrairement à l'opinion de Burmeister (1832), Westwood (1840) et Hartig (1840), que le canal du dard est trop étroit pour pouvoir servir au passage des oufs et que l'Insecte doit retirer celui-ci avant de pondre. AdLer (1877), se fondant sur ce qu'on sait des Tryphonides, arrive à cette conclusion que. (chez les Cynipides) le pédicule seul s'engage entre les stylets, tandis que le corps de l'ouf glisse le long du dard en restant suspendu en dessous de lui.

Remarquons que l'œuf des 'Tryphonides, qui a un pédicule plein et court, entièrement chitineux, ne saurait être comparé à l'œuf de Rhyssu, dont le pédicule creux est flexible et allongé. Il est clair que si, dans la ponte de Rhyssa, le pédicule glissait seul entre les stylets, le corps de l'nuf resterait en chemin, accroché aux aspérités du bois et serait bientôt déformé et déchiré. Les observateurs qui ont assisté à la ponte des Ichneumonides n'ont jamais rien vu de prareil. On sait d'ailleur's, grâce aux travaux de Beyerinck (1882) et de Krefrer (1897), que l'ouf pédi- 
culé des Cynipides passe tout entier dans le canal du dard et que la présence du pédicule est justement en rapport avec le mécanisme de la ponte. Ces auteurs ont montré que, grâce à l'élasticité de sa coque, le corps de l'œuf se rétrécit au moment où il s'engage dans le canal et refoule son contenu dans le pédicule, puis que, los'sque le corps de l'ouf a paśsé, le pédicule se vide et se rétrécit à son tour. Bien que les conditions ne soient pas tout à fait les mêmes =héch Rhyssa, d'abord parce que le pédicule s'engage le prenier, ensuite parce que la tarière étant envil'on quatre fois plus longue que l'oeuf, celui-ci doit (même s'il a la faculté (le s'allonger) être à un certain moment contenu en entier dans le canal du dard, je pense que le mécanisme de la ponte doit être à peu près le même dans ces deux groupes d'Insectes.

I'admets donc que le pédicule de l'ouf' s'engnge de toute sa longueur dans le canal du clard, en conservant d'abord son calibre primitif, puis que le corps de l'œuf pénétrant à son tour, celui-ci refoule dans le pédicule une partie de son contenu et parvient de cette façon à s'amincir et à s'allonger. Je suppose, en outre, que les stylets, jouant sur les rails clu gorgeret ${ }^{1}$, s'écartent l'un de l'autre au noment du passage de l'œuf, de manière à augmenter un peu la largeur dú canal.

La progression de l'exuf, comprimé ct allongé, s'expliquerait non par l'effort de fibres musculaires disposées autour du canal (la compe n'en montre pas trace), mais par l'effort de la musculature abdominale agissant comme une presse hydraulique sur l'œuf baigné de liquide.

Il faudrait, pour rérifier cessuppositions, surprendre une Rhyssa occupér à pondre, la chloroformer sur place et, la soumettant ensuite à une dissection minutieuse, observer la forme. la dimension et la position de l'rufu déjà engagé dans l'étroit canal. Il faudrait en outre étudier en détail la disposition de l'appareil contractile de l'abdomen, noter le degré de réplétion des glandes au moment de la ponte et suive le jeu des diverses pièces sur un exemplaire vivant. Ces observations, qui seraient fort intéressantes, n’ont pas encor' été faites. Malgré cette lacune, on peut conclure, semble-t-il, que la formation des ceufs pédiculés est en rapport avec la structure de la tarière; la longueur de celle-ci étant déterminée it son tour par le genre de vie de l'Ichneumon et l'obligation où il se trouve de pondre dans la profondeur du bois.

\section{AUTEURS CITÉS}

Burmerster. Handb. der Entomologie. 1832-ãว̆.

Hantig. Ueber die Familie der Gallwespen. Germar's Zeits. f. Entom. 1840-43.

${ }^{1}$ Les rails sont eux aussi, grâce à la fissure du gorgeret (fig. 1), susceptibles de s'écarter un peu l'un de l'autre. 


\section{EXPLICATION DE IAA PLANCHE}

Fig. 1. Rhyssa persuasoria.

Coupe transverse du dard. Gross. 187 fois.

g) Gorgeret.

p) Canal de ponte.

sly) Stylets.

Fig. 2. Abdomen de Thalessu leucographa $q$ vu de profil, avec la carène formée par le $8^{\text {me }}$ segment. Gross. 4 fois.

a) Aileron du stylet (Winkel de Kr.epelix, Epimère de Seunat).

d) Dard.

h) Hanche postérieure.

(i) Lame latérale (quadrilatère).

lv) Lame ventrale (oblongue).

py) Pygidium avec l'orifice anal caché en dessous.

stmi Sternite du segment médiaire (d'après Seurat, 1899, p. 17).

va) Valves du fourreau.

Fign. 3. Rhyssa persuasoria. La carène fendue sur la ligne médiane: les deux moitiés étalées, un peu écartées pour mieux montrer le dard. Face ventrale. Gross. 6 fois.

m) Ligne courbe limitant la partie membraneuse de la lame latérale (cachée sous le bord du 7 me segment).

Les autres lettres comme Fig. 2.

Fig. '. Rhyssa persuasoria. Extrémités d'une des valves du fourreau et des trois pièces du dard séparées au moyen des aiguilles.

g) Gorgeret.

sty) Stylets.

va) Une valve.

Fig. ö. Rhyssa persuasoria. La lame ventrale de la carène, isolée avec l'aileron du stylet articulé sur elle. On a fait basculer l'aileron d'un coup d'aiguille, de manière à faire sortir le stylet de la glissière du gorgeret. Gross. 6 fois.

r) Rainure dans laquelle s'engage le bord rentrant de la lame latérale. Les autres lettres comme Fig. 2 et 4 .

Fìg. 6. Ryssa persuasoria. La lame latérale correspondante détachée avec le pygidium. Gross. 6 fois.

$\left.a^{1}\right)$ Pièce sur laquelle s'articule l'angle externe de l'aileron, lorsqu'il est en place.

bv) Bord ventral de la lame latérale.

jo) Pièce podicale ou sous-anale.

Les autres lettres comme Fig. 2 et 3 . 

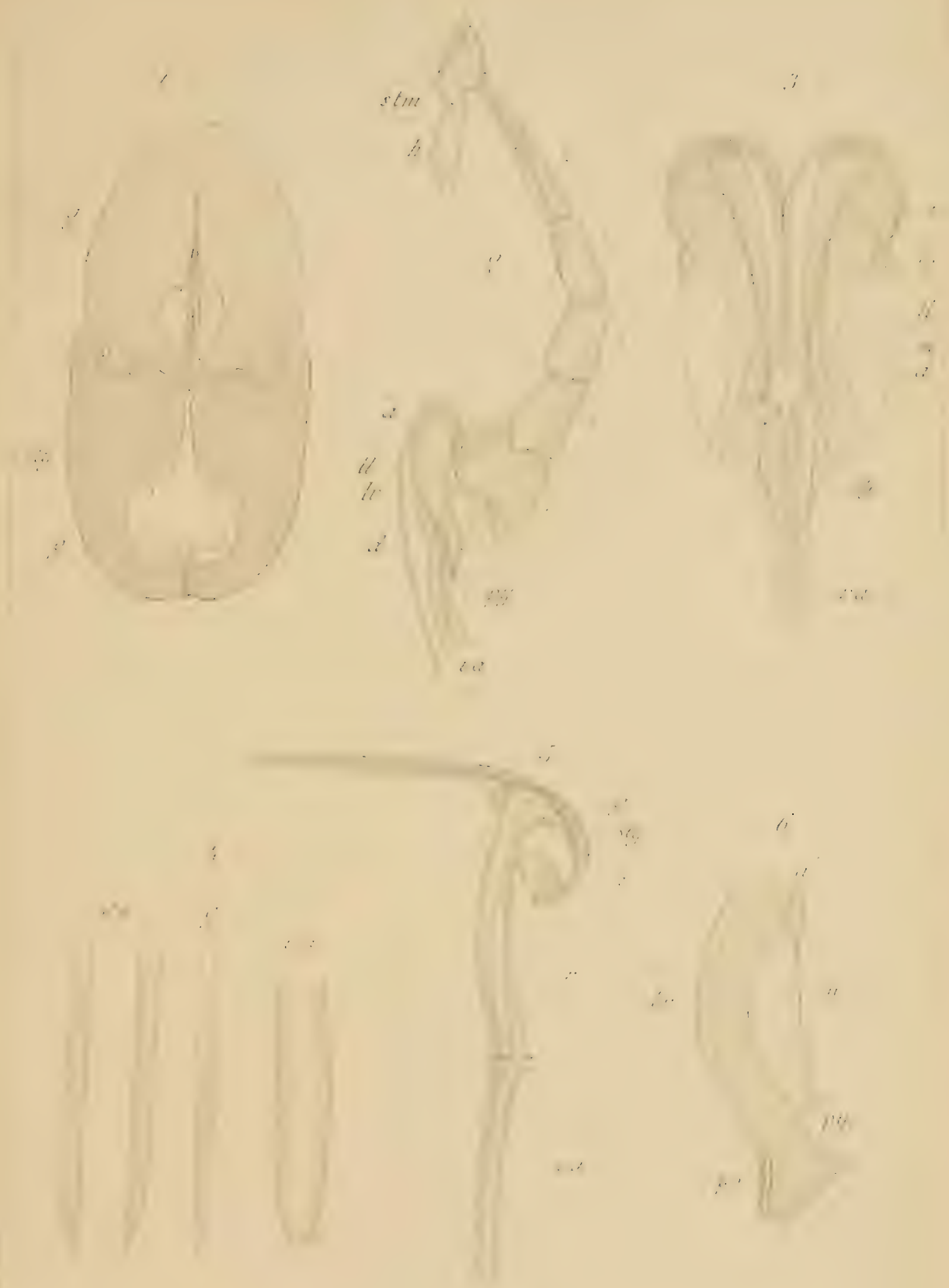

E.Bugrion. Rlys - a 

Westwood. Introd. to the modern ulassif. of Insects. Vol. 2. 1840.

Ratzeburg. Die Ichnemonen der Forstinseliten. 1844-3̋2.

Lacaze-Duthens. Rech. sur l'armure génitale les insectes. An. sc. nat. $3^{\text {e sér. }}$ Vol. 12, 14, 17, 19. 1849-53.

Sneulen van Vollenhoren. Mélamorphoses de Rhyssa persuasoria. Tijdschrift voor Entom., vol. 4, p. 176. 1860.

Kräpelin. Bau, Mech. und Entw. des Stuchels der bienenartigen T'hiere. Zeitsch. f. wiss. Zool., vol. 23. 1873.

Enné. Beob. über die Entw. einiger Inseliten. Mitth. der Schw, ent. Ges., vol, 4, p. 5̈18. 1877.

ADLER. Legeapparat $u$. Eierlegen der Gallwespen. Deutsche entom. Zeits. 21. Jahrg. 1877.

Brischke. Die Ichneumoniden der Prov. W'est- u. Ostpreussen. Sehriften d. naturf. Ges. Dantzig. Neue Folge, vol. 4. 1880.

Berenrack. Beob. über die ersten Entwichelungsphasen einiger Gymipidengallen. Verh. Acad. d. IViss. Amsterdam. 1882.

Harnisgton: The Nuptials of Thalesse. Canadian Entomologist, vol. 19, p. 206. 1887.

RiLey. The Habits of Thalessa and Tremex. Insect Life. 1888-89.

Dalda Torne. Catalogus IIymenoyterorum. 10 vol. Lipsie. 1892-1902.

Bordas. Descr. anat. et hist. des glandes à venin des Hyménoptères. Paris. 1897.

Kiefrer. Les Gynipides. Species des Hym. T. 7. 1897.

Seurat. Contrib. à l'étude des Hymén. entomophages. Ann. Sc. nat. Zool. $8^{\mathrm{e}}$ s., vol. 10. 1899. 

$6^{\text {ine }}$ SECTION

\section{ZOOLOGIE APPLIQUÉE}

Séance du mercredli 17 août 1904.

Plésident: M. le Dr P.-P.-C. Hoek.

Vice-Président: M. le Prof. L. Plate.

Secrétaire : M. le $\mathrm{D}^{\mathrm{r}}$ J.-U. Duerst.

Dr P.-P.-C. Howk (Kopenhagen) eröffnet die Sitzung mit der Ueberreichung der hisherigen Publikationen des a Conseil permanent international pour l'Exploration de la mer" ", zu 'Handen der Universitäts-Bibliothek in Bern.

\section{Das Ideal eines naturhistorischen Museums.}

Von Dr. F. A. JENTINK (Leiden).

Mit 2 Textfiguren.

Jedermann, der die Magazine gekannt hat, worin noch vor 2. Jahren in den europäischen Inetropolen die zoologischen Schätze aufeinander gestapelt waren, bisweilen zu unerreichlaren Höhen, und jetzt die Prachtbauten in London und Paris, in Berlin und Wien besucht, muss dankhar anerkennen, dass die Sachen so viel hühscher ausgestellt, so viel besser zu sehen sind und muss staunen üher die meistenteils ansserordentlich pompösen Eimrichtungen und effectvollen Palïste. Ior Sachverständige lïsst sich aher" nicht blenden durch das imponierende Acussere sonder'n schaut hinter die Coulissen und erlault sich zu fragen: "Sind wirklich diese wunderschönen (rebäude, diese neuen Finrichtungen, worauf so und so vicle Villionen verwendet sind, zweckmissig; kann einerseits der wissenschaftliche Besucher dort ruhig arbeiten und ist andrerseits diss Publikum zufrieden und sind die Schätze gut gehorgen und gregen L'ntergang 
völlig geschützt "? Mit del Beantwoltung dieser Fragen hängt cine andere prinzipielle Frage innig zusammen, nämlich diese: Was ist eigentlich das Ziel eines maturhistorischen Museums? Und vor einigen Jahren war selbstverständlich die Antwort, dass, da Gebäude sowohl wie Inhalt vom Publikim bezahlt wird. also Eigentum des Publikums ist. dasselbe Publikum doch auch billigerweise sein Eigentum besichtigen lïmne: woraus logisch folgt, dass alle Sachen für das Publikum sichthar aufgestellt werden müssen; (las soll belehrend auf das Publikum einwirken, das Publikum wird dadurch gebildet, und Sinn für schöne Formen und Liebe für Ir Mttel Natur sollen in den Museen entstehen und genährt werden u. S. w. Es scheint, dass man die Unhaltbarkeit dieser Logik nicht einselıen wollte, denn warum sind nicht alle anderen vom Publikum hezahlten Gebäude füı Jedermann zugänglich gemacht, z. B. alle Laboratorien und Observatorien, Rathäuser und Gerichtshöfe, Ministexien und Staatsichulen. Solbst fül die liberalsten Republikaner wüude so otwas und ('nlibar sein! Ausserdem weiss jeder Musem ms besucher aus Erfalnung, wie äusserst langweilig diese ('ndlosen Reihen von Schränken sind und wie weniw von diesen ['mmasen aufgestellter Exemplare zu genissen ist. Unul was für ein Publilum ist das Museumbesuchende? Lente die sich langweilen, orler Freunde dir unter angenehmem freplauder Freunden die Morkwürdigheiten der Stalt zeigen; andere besuchen die Museen, weil sie im Sommer angenehm kühl und im Winter angenehm war'm sind, man trifft sich dort und giebt sich rendez-vous, und bald ermüdet, setzt man sich bequem hin u. s. w. und alle diese grossen Mengen gleichguiltiger Museumshesucher genieren noch ausserdem de'n " raram avem ), der' wirklich als Interessierter gekommen ist! Man meint, dass durch den Museumsbesuch beim Publikum Liebe für die Natur angeregt werde - ich kénne hiefür kein einziges Beispiel und meine dagegen, dass ein denkendes Tresen, das eimmal so pine lange Ahnengalerie hesucht hat. sich später des "non his in idem " erinner'n wird. Dazu kommt noch, dass jedes Musem am Ende ein Stadtviertel einnehmen würde. Aher das schlimmste liommt noch: "Alle aufgestellen Tiere. Säuger und Vägel so gut wie Conchylien. gehen. dem Tageslicht ausgesetzt, früh oder spät aber sicher' und unwider'utlich zu Grunde! Allmählich hat man eingesehen, wie unverantwortlich es ist, Exemplare, die niemals wieder $\mathrm{zu}$ ersetzen sind, weil die Arten ausgerottet oder ausgestorben sind, dem sicheren Cntergang auszusetz'n und wie unberechenbar grosse Schätze hei dieser allgemeinen Aufstellumg verloren gehen, und andredeseits hat man angefangen zu bemerken, dass zum richtigen Studium der 'Tiergerogaphio noch ganz andere Serien gefordert werden als bis jetzt, und wo wülde man enden, wenn auch dies alles aufgestellt werden sollte! Und so hat sich allmählich die Ueberzeugung festen Boden erobert, dass in jedem Museum eine Trennung von wertvollen und ersetzbaren 
Fxemplaren dureligeführt werden kamn und muss, cine wissenschaftliche und eine Schausammlung also. Zu dersellen Zeit nun als diese neur Ideen in den Kö̈pfen der Zoologen reiften, sollten überall neue Alusecn gebaut werden. Schade nur, dass die Pläne, die Entwürfe meistentcils ganz ohne Zuratezichung der Direktoren der betreffenden NInseen gemacht sind: der Baumeister linnstruiert einen prachtrollen Rellame-Bau, selhstrerstandlich nach den veralteten Prinzipien und der I)irelitor mag sehen. wie er seine Sammlungen in solch einem "aut of time » Museum unterbringt! Es darf denn auch liein Befremden erregen. diss unter derartigen Umständen entstandene Gehäule nicht dem Zweck ('ntoprechen kömmen. dass man gestehen muss, dass bis jetzt jedenfalls in Europa liein "up to date" Museum existiert. Die ganze Sache muss umgeliehrt werden ; Ziel eines Museums soll jetzt sein : "Wissenschaft Nr. 1, P'uhlilikum Nr. 2!" An ein " up to date " Museum soll man die folgenden Medingungen stellen: Vollkommene Trennung von Dienstgebäude, wissenschaftlicher. Sammlung, Spiritus-Sammlung und SchauSammlung; die Custoden sollen ihre Arbeitszimmer ganz in der Nähe ihrer Sammlungen haben; die Objekte sollen staubrei geborgen sein, das Tageslichtsollgänzlich ausgeschlossen werden; die Schränke sollen feuerfrei konstruiert sein und, last not least, der ganze Bau soll unbrennbar sein! Diese äusserst schwere Aufgabe und das Ziel, "ein Ideal-1Iuseum ", haben vielen pralitischen köpfen zu grübeln gegeben. Es sind unter den Gelehrten, die ilne Gedanken in bestimmterer' For'm darzustellen versucht haben, vornehmlich Mörres in Berlin. MeYer in Dresden, und die Engländer Schater, Pitr-Rivgrs und Frower zu nennen.

Nicht ganz neu, aher doch sehr scharfsinnig war dre Plan. den freneral PrTT-Rivens in the Anthropological Section of the British Association at Bath in 1888, und zwei Jahre später in the Society of Arts demonstrierte. Das Insemm denlit er sich hestehend aus einer Serie in Kireisen konzentrisch angeordneter und überall mit cinander in Verbindung stehender" Galerien. Jeder Kreis soll cine Periods in der "Weltgeschichte vorstellen, im Zentrum anfangend und im grössten Kreise endend, also unsere jetzige Weltperiode vorstellend... Fuower (Presidental address to the Museum Association at the Meeting in London, $3^{\text {ra }}$ July 1893) sagte - und dem kann ich völlig beistimmen - ( such a building would undoubtedly offer difficulties in practical construction: but even if these could be got over, our extremely imperfect linowledge of the past history of animal and plant life would make its arrangement, with all the gaps and irregularities that would become evident, too unsalisfactory that I can scarcely hope to see it adopted in the near future "). Ich sagte, " der Plan von Pitr-Rivers war nicht ganz neu ", denn schon 1869 hat 


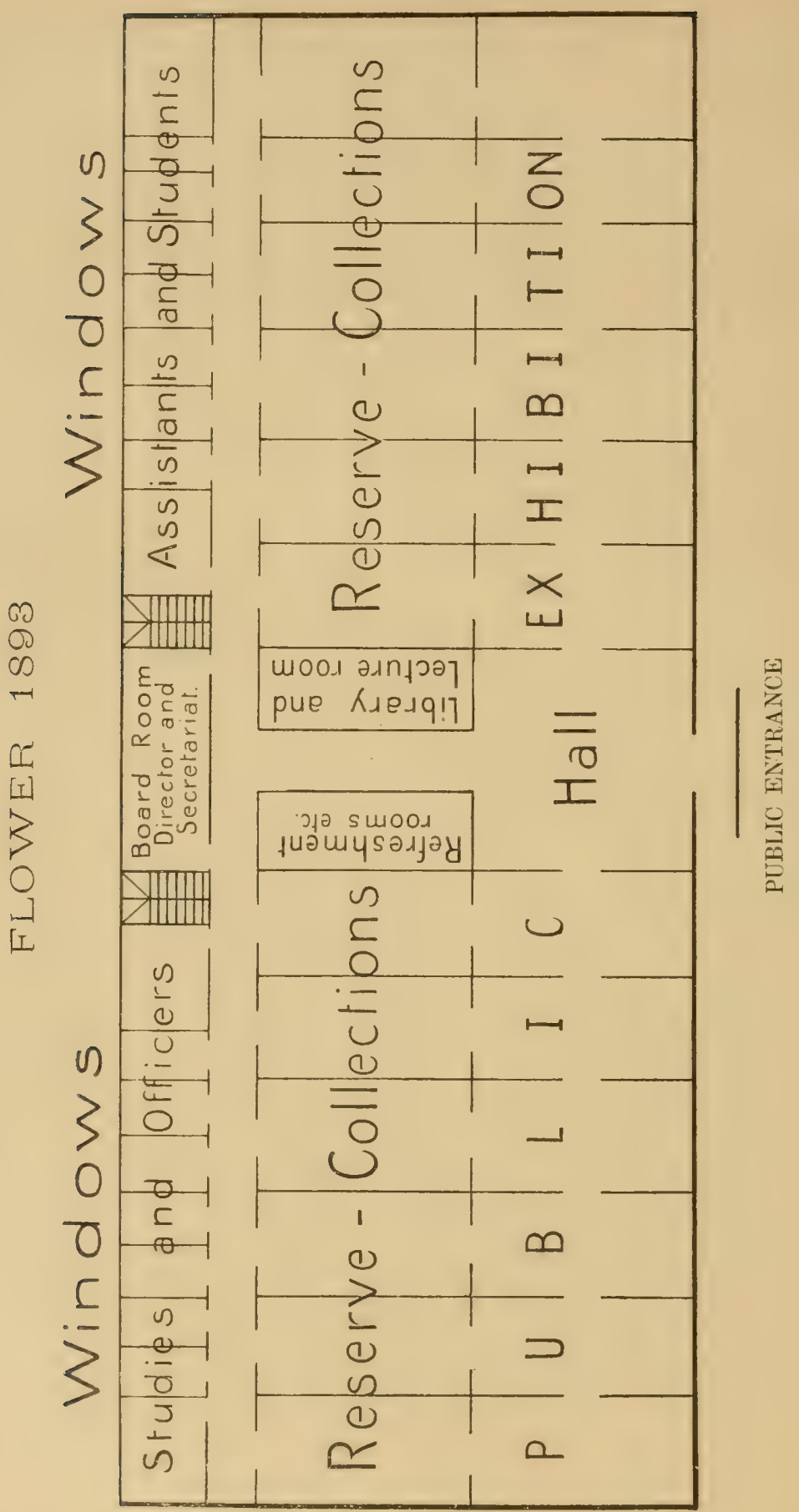

Fig. 1. 
M. Jules Marcou (Le MLuseum d' Histoire naturelle ou Jardin des Plantes. De la Science en France, $3^{m}$ fascicule, Paris, 1869) einen analogen Plan beschrieben und eine schematische liarte dazu gegeben, die nach eine'm Projekt von ihm 1860 oder 1861 für Agassiz hergestellt worden war.

Viel besser und praktischer, wie man aus Fig. 1 sehen kann, war del Plan Frowers 1893, demonstriert in the Museums Association in London. Meine Bedenken gegen diesen Plan sind u. a. folgende: Die Spiritussammlung ist nicht von dem Bau getrennt gedacht, Schansammlung und wissenschaftliche Sammlung sind nicht feuersicher genug geschieden, und der Bau ist einstöclig, also mit Oberbelenchtung - sehr schön und bequem, wenn die Sammlung klein ist, aber, wenn immer angebaut wird, am Ende sehr unbequem. weil die Inistanzen von dem I bienstgebäude zu gross werden und einc (Oberbeleuchtung nur in Ländern angewandt werden kann, wo kein oder wenig Schnee im Winter fällt. Es scheint mir auch sehr gefährlich, "Refreshment rooms " in der Mitte des Gebäudes einzurichten u. s. w. Mündliche liesprechungen mit F'sower und das Studium des F Lower'schen Planes, zugleich muter Beratung mit Jhr. M. V. E. L. De Stuers, J. A. Royer, J. von Lokhorst, W. F. Leemans und G. J. de Jongr haben uns zu dem Entwurf eines Museums geführt. den ich auf Fïg. 2 schematisch darzustellen gesucht habe. Die Vorteile dieses Planes sind folgende: Dienstgebäude, Schausammlung, wissenschaftliche Sammlung und Spiritus-Abteilung sind völlig und f'nersicher von cinander getrennt; der hetreffende Konservator hat sein Arbeitszimmer ganz in der Nähe sciner sammlungen; in Spiritus- und wissenschaftlichen Sammlungen sind die Objekte in mit eisernen Türen geschlossenen Schränkeu geborgen, also ganz im Dunkel, ein niemals zu überschätzender Vorteil - und dazu von grosser ökonomischer Wichtigkeit, weil die sehr kostspieligen Gardinen gänzlich weggelassen werdenn können - ; die osteologische Abteilung hat Raum anch für die allergrössten Skelette u. s. w.. wie cin genaues Studium meines Planes jedem zeigen kann. Ich bemerke nur noch, dass sich überall zwischen den Hauptabteilungen Luft- und Lichträume befinden und also bei Mangel an Raum immer Etagen auf Etagen gebaut werden können, ohne Nachteil, was Luft und Licht anbetrifft.

Nach diesem Plan hat man seit 1900 angefangen, in Leiden ein neues Iuseum zu bauen - das Ideal ist verkörpert! In einigen Monaten können Sipiritus-Sammlungen und seltene Tiere nebst typischen Exemplaren aus allen ()rdnungen untergebracht werden, weil damn das Spiritus-Mragazin und das erste der Magazine der wissenschaftlichen Sammlungen fertig scin werden, mitsammt den Zimmern der betreffenden honservatoren und dem Treppenhaus; das Ganze wird mit warmem Wasser geheizt. Es ist selbstverständlich unmöglich, hier eine nur einigermassen detaillierte Beschreibung der Einrichtung zu geben, ohne den mir 
JENTINK 1900

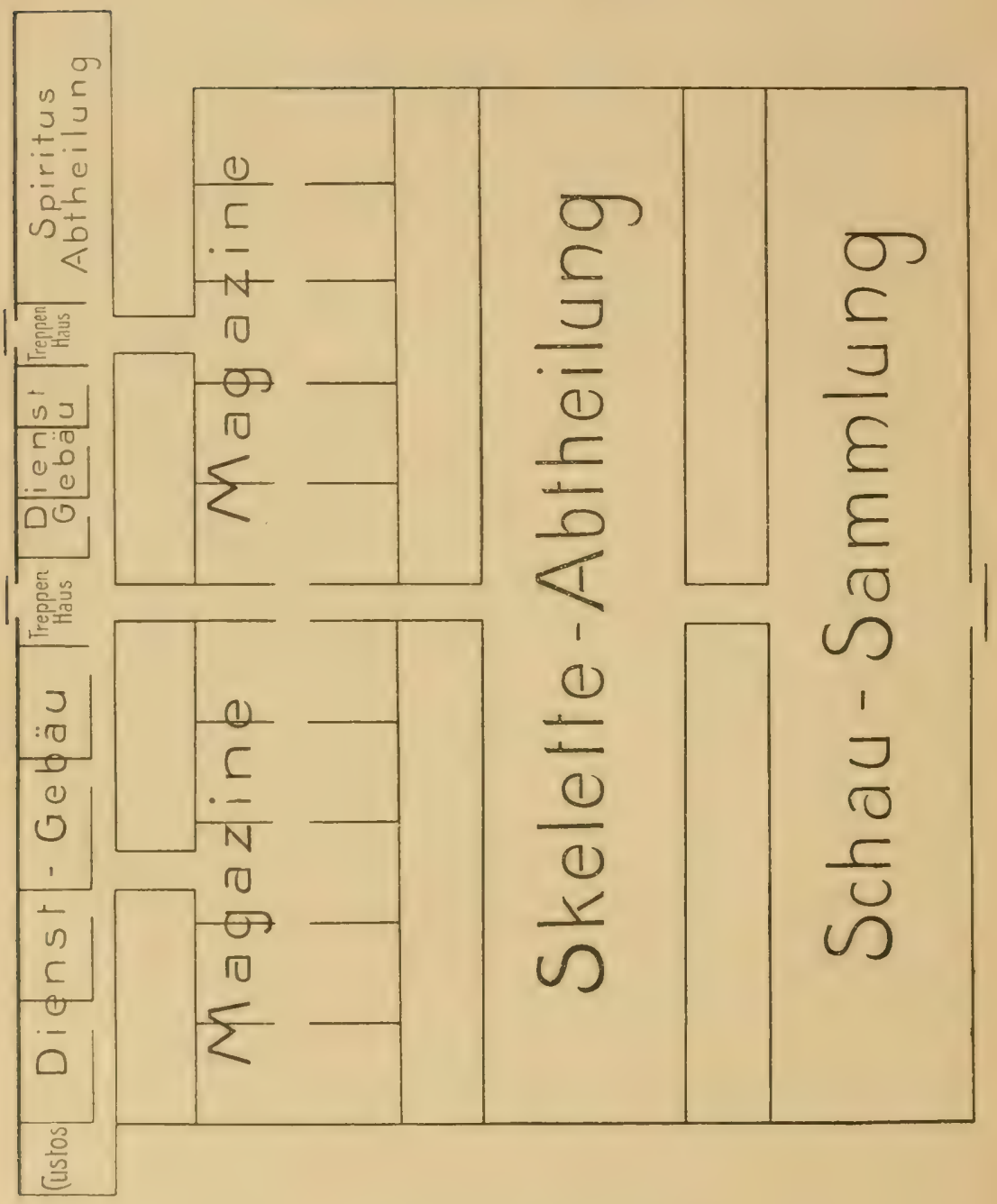

Fig. 2. 
gestatteten Raum bei weitem zu überschreiten. Ich hann nur dankbar'

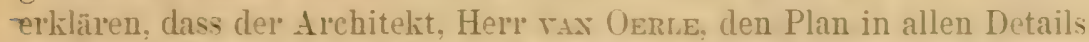
pünktlich ausgearbeitet hat, dass del Bau rollständig den hỏchsten Erwartungen entspricht und dass viele Fachgenossen u. a. aus Moshau.

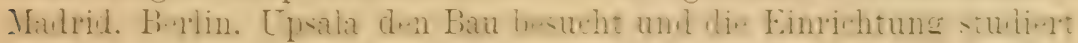
und unisono sich dahin ausgesprochen haben, dass wir hier das Beste.

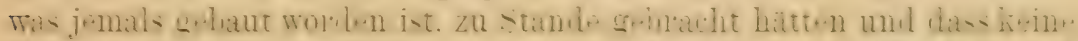

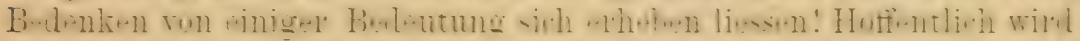

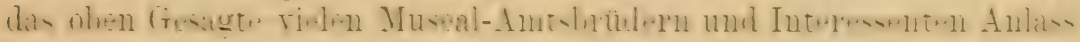
geben, nach Leiden zu kommen, um Seubau und Einrichtung zu stıdielen :

\title{
Demonstration eines Schau-Mikroskopes für öffentliche Museen.
}

\author{
Von Prof. L. PLAte (Berlin).
}

Mit 1 Testfigur.

Herr Prof. L. Prate (Berlin) demonstriert ein Schan-Mikloskop) für Museumszreche, melches nach seinen Angaben ron del Firma Leitz (Wetzlar) hergestelit morden ist. Das Instrument besitzt statt des festeu Objehttisches eine drehbare Trommel mit 12 Fläkhen, auf del ebensoviele Objekttrïgel durch je 2 Klammer'n festgelegt rerden hönnen. Jede Fläche trïgt in der Mitte eine Oettinung, durch welche das rom Spiegel reflektierte Licht auf das Prieparat fallt. Der Spiegel selbst befindet sich im Zentrum der Trommel. Damit das Publikum die Proparate nicht berühren kann, wir'd über" die Trommel eine crlindrische Schutzhülse gestülpt und mit Schrauben betestigt, welche 12 fortlaufend nummerierte und über

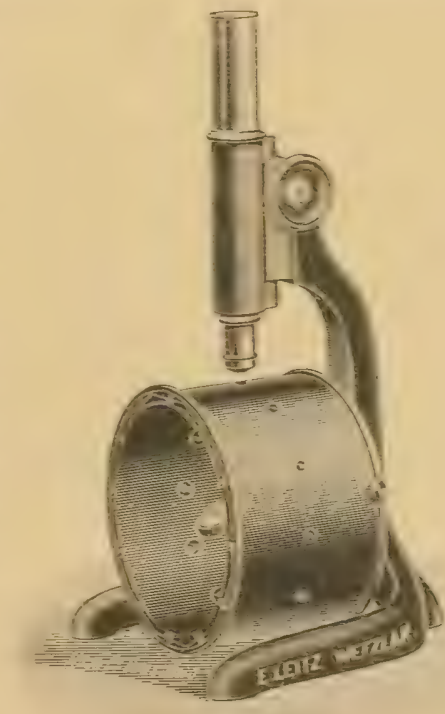
den Priparaten befindliche Oeftnungen besitzt. Die Trommel hat

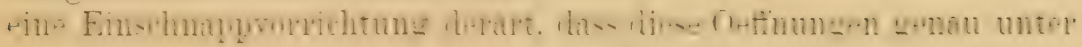




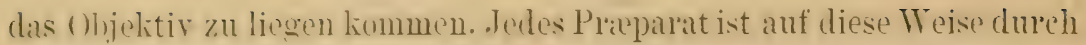
eine Nummer gekennzeichnet und kann dann weiter durch eine Etiquette older pin Bild erläutert werden. Ine genaue Einstellung des Ohjektivs hesoret der Beschauer selbst, jedoch ist der Tubus so eingerichtet dass dis (bhjoktiv der Schutzhülse nicht his zur Berührung genihhert. und dahere auch nicht beschädigt werden hann. ()hjelitis und Oliular sind durch s.hrauben am Tuhus befestigt, so dass sie vor Diebstahl geesichert siml. und desgleichen lassen sich die drei Füssedes stativsan der Tischplattr anschrauhen. Das zur Zeit in (iründung befindliche Museum fül Mexpeskunde hesitzt his jetzt zwei derartige Instrumente mit einel' 1(n)- resu). 1s(1-fichen Vergrässerung. Der Prejs heträgt etwa 50 M. mohe wie ein gewöhnliches Instrument mit del gleichen Veropösserung. Der

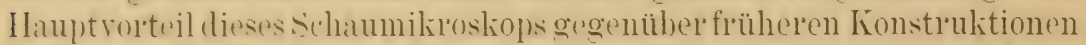
(z. B. dem im Hamhuroer Musem aufgestellten Instrument) besteht in dere geringen Gröste die diejenige eines gewöhnlichen Miliroskops nur

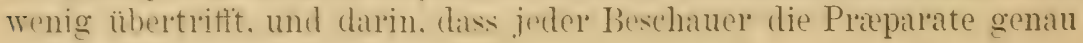
fül sein Auge einstellen kann.

\title{
La pêche sur les fauberts.
}

\author{
Par le Prof. F.-A. FOREL (Morges).
}

L'emploi des dragues pour l'étude de la faune profonde des lacs de-

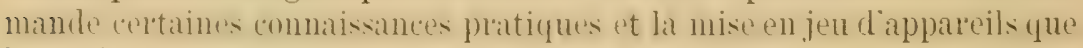
les zoologistes ne possèdent pas toujours. De là, pent-être, la rareté des recherches faites sur la punulation. pourtant tres interessante du fond des lacs, en comparaison de la fréquence des études sur le plancton qui sont heanum plus ficile. La méthode suivante permettra de jeter un premiere

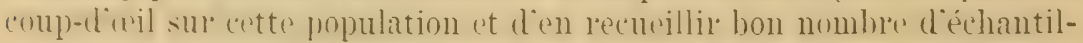
lons sans aucune difficulté technique et sans la mise en œuvre d'instruments compliqués; on peut faire faire toute l'opération par un simple pêcheur sans que le naturaliste ait à intervenir pour la pose et la levée des engins.

Laissez reposer" sur le fond du lac pendant un ou plusieur's jours quelques fauberts (balais de fil de coton) et quelques paquets d'étoupes, attachés à une corde, ancrée sur le sol et signalée par une bouée. Sitôt relevés ces paquets et filaments seront lavés à grande eau et celle-ci sera passée sur un tamis fin. 
C'est par centaines et par milliers que les ol'ganismes mobiles de la faune profonde seront ainsi capturés. Hydrachnides, Crustacés, larves d'Insectes. Annélides, Planariés, etc.; les especes linicoles par contron no se laissent prendre que plus rarement.

\title{
Kleinere Beiträge zur Mikrotechnik.
}

\author{
Von Prof. K. FUJII (Tokvo).
}

I. Amvendung der Glaskapillarröhre zu mikrochemischer Analyse. Bei

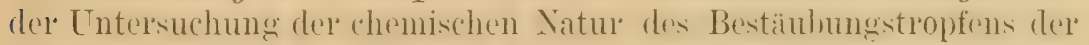

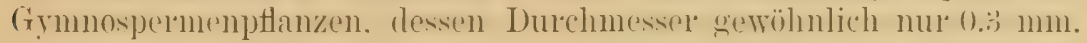
bis $1.0 \mathrm{~mm}$. beträgt, so dass eine sehr geringe Menge del Flüssigkeit zur Verfügung steht. hat der Tortragende Glaskapillarröhren, deren in-

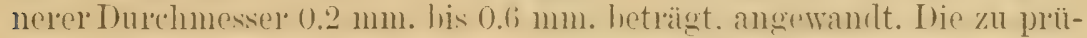
fende Flüssigkeit und das Reagens sollen in die Tapillar ö̈hreautgesogen werden. und falls die Erwälmung rommöten ist, sind die beirlen Enden der Kapillar'öhre in einiger Entfer'nung vom Inhale mittelst (iastlamme zu verschlissen, worauf das (xanze im IVasserbarle "roülmt wircl. Solche Präparate können nun leicht mikroskopisch untersucht werden. Der Vortragende bemerkte, dass überall wo es sich um die Untersuchung: geringer Quantitäten der Flüsigheit handeln mag, man diese Mrethode anwenden kamm. sei es für modizinische, besonders forensische Zweche, sei ex für zonlogiseh-phrsingoische Zweche, z. B. bei dex L'ntersuchung der chemischen Eigenschaft des Drüsensekiets der' Insekten, etc.

II. Eine einfache Einrichtung zum gleichzeitigen Auswaschen einer -Anzahl der firierten Materialien. Diesello hesteht in ein'm rielstrahligen Stromverteiler, der mittelst rines Gummischlauches mit rinem Wasserhahn zu vellinden und äber die (refïsise, die Materialien enthalten, zu hängen ist.

III. Uebertragungszettel. Auf demselben sind die aufeinanderfolsenden zur Anwendung liommenden Alloholprozente gerlucht; "r. wirl auf der Wand des Gefässes aufgeklebt, welches das Material, das vom Wasser his zum Autkiärungsmittel übertragen werden soll, entlialt. Ziffern fül vorleigegangene Alkoholprozente sind rinfach durchzustreichen. 


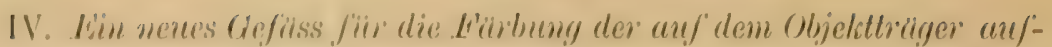

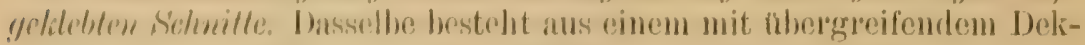
kel verselunen ('ylimler und einem binsatz, welcher von 3 horizontalen

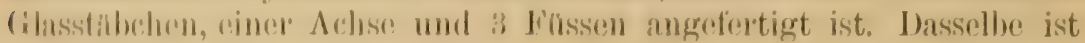

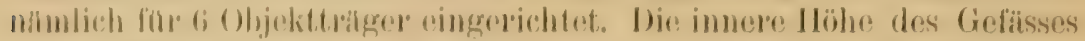

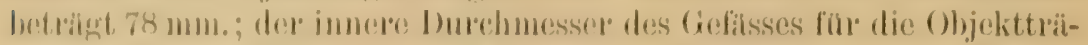

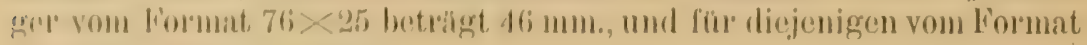
$76 \times$ <is lectoigh or 6i2 mm.

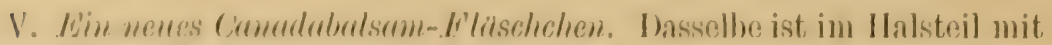
oinem freim Ringe versehen. I)er lotytere strht mittelst zweice horizontaler (ilasstabchen in fester Vorbindung mit den Malsteil des Flasehchens. Dor (ilasstal ist im lange line cingesteckt. Der ling dient dazu, die aberflussigo Mengo des Camadabalsams vom (ilasstab abzustreichen

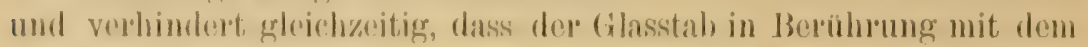
Rambe des cieditsoses liommot.

\section{Demonstration der Einrichtungen des Concilium bibliographicum.}

Vom H) II.-H. Nild) (Zürich).

M. Herhert-llaviland limas expose la bibliographe sur fiches du Comeilinm Bibliographiom ot donne des explications anx membes de lat section sill l'histore de l'ontreprise et smo son fonctionnement. 


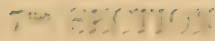

\section{ZOOGEOORAYHIE}

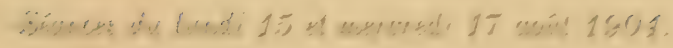

:

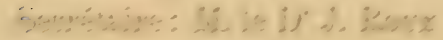

\section{Unser heutiges Wiwer uber die Systematik und die gesyraphiwche Verbusitung der Cladoceren.}

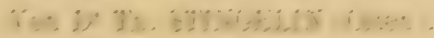

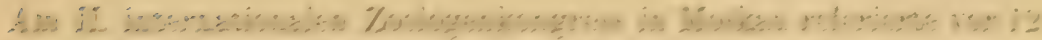

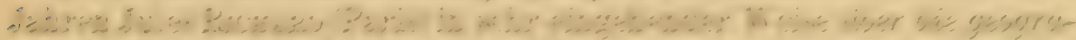

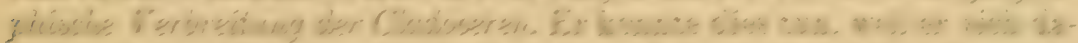

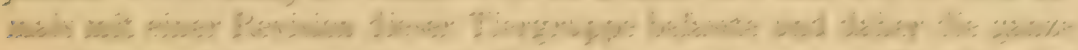

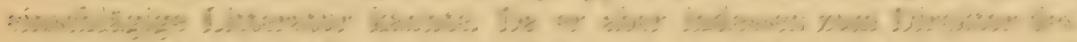

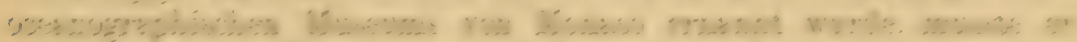

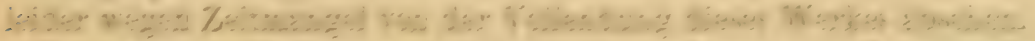

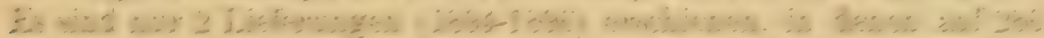

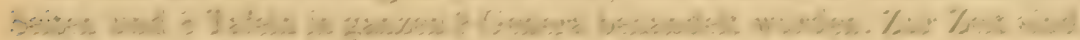

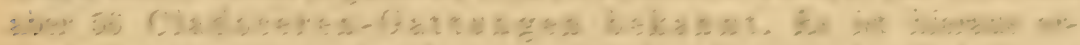

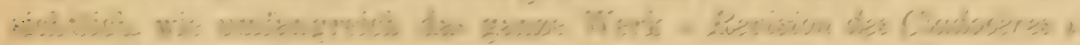

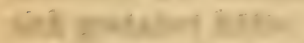

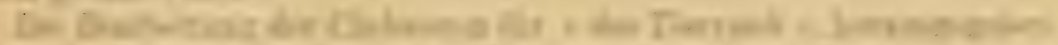

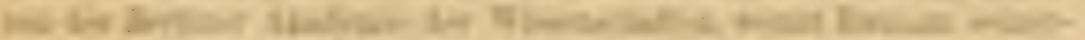

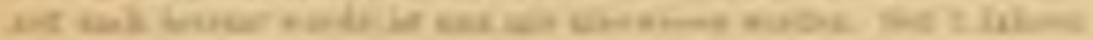

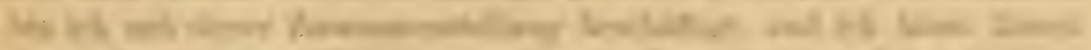

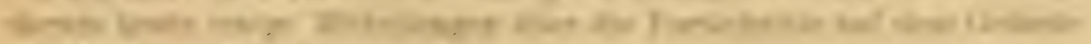

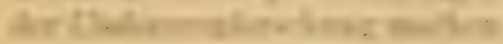

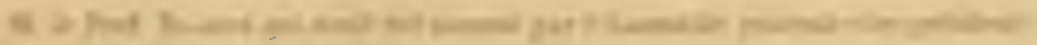

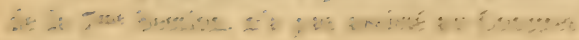


Ton einel ausführlichen, die einzelnen Arten berücksichtigenden

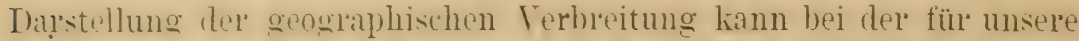
Sultionswortrige so liurz benesienen Zeit natürlich nicht die Rede sein. Ingegen will ich in einem kurzen L'eherblick über die einzelnen Fannlien das Tichtigste, was sich auf die Fortschritte der letzten 12 Jahre bezint. erwähnen. Besonder's herorzuhehen sind die hiolowischen El'ungenschaften der letzten Jahre, sowie ihr Eintluss auf die Srrstematik und mosere heutigen Anschamngen über die geographische Verbreitung der Cladoceren.

Xummeriache Angaben über die Anzahl der his hente heliannt getrordenen Gattungen, Arten und Varietäten - die zwar z. Z. noch nicht auf ahsolute (renaugkeit Anspruch erheben kïmnen - sollen die Darstellung begleiten.

Tregen ihrem hizareen Bau und ihrex Durchsichtigheit gohören die Cladoceren schon in morpholngischer Hinsicht zu den interessantesten ['ntersuchungohjuliten. Iazu hommt. dass sich bei dieser Tielgruppe die Fortptamzungsielhälniso ganz besonders kompliciert gestalten und dass in hiologischer Hinsicht - zumal was die Variahilitait der Individurn nate Altre. Fundort und Jahresent anhetriffit - wohl in

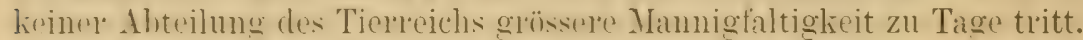
Fein Wunder daher. dass schon eine so grose Zahl ron Forschern sich mit dem Studium dieser Tiere befasste. Sie rerden mit Erstaunen vernehmen, dass mein Zettelkatalog bereits 1200 Schriften aufweist, in welchen Cladoceren Berükdisichtigumg tinulen. Tie Cladocerenforschung ist ron Vorelrupopa ausgegangen. allwo sio noch jetzt in hörhster. Blüte steht. Dann verbreitete sie sich über Mitteleuropa, nachher über die Vereinigten Staaten von Nordamerika und erst in neuerer Zeit wurde auch in tropischen Ländern Heisigg geximmelt. so kennt man nun Cladocepen aus all'n Erelteilen und Zonen. Yach meiner Zusammenstellung beliuft sich die Zahl del his lo eute heschrielenen Formen (Species, Varietates und Forma) auf etwa 600. Dieselben verteilen sich auf 56 Genel'a und $8 \mathrm{Fam}$ ilien. Die Vertreter von 5 Gattungen sind lem malin. I)ie meisten Clidoceren halten sich jedoch ausichliesslich im Süsswasser auf. Sie leben volzugsweise in ruhigen Gewässel'n, von den kleinsten 'Tümpeln bis zu den grössten Seen, in letzteren die vershimlenen fergionen hewohnend. Fs wurlen aber auch zahlreiche Formen beobachtet, die vom Süsswasser in's Meer übergehen. Eine

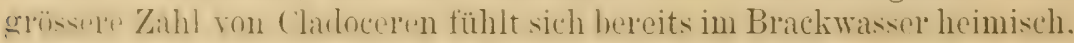
andere scheinen für immer in's Meer ausgewandert zu sein.

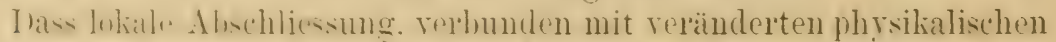
Einflüwn. ('in'n tirfen Fingrift auf die ganze Kïrperorganisation auszun̈hrn in stande sind. ueht hesonders ans den hochinteressanten Arbeiten von Plof. G. O. Sars: Polyphemiden der Kaspisee hervor. Derselbe 


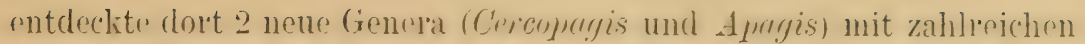
Alten. Welche sich als dirnte Abkömmlinge muserer brannten semfol'm Bythotrephes ausweisen. Aus südrussischen Seen und Flüssen

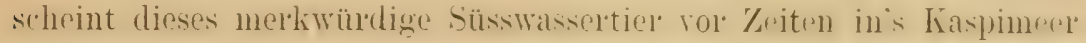
eingervandert zu sein, allwo es sich progressiv zu zahlreichen, äusserst bizarren, neuen Varietäten und Arten umbildete. Es ist das Kaspimeer ein wahres Schöpfungscentrum auch füı Cladocelen geworden!

\section{Ueberbticki ïber die einzelnen Cladoceren-Familien.}

Die 1. Familic “Sidid „ " umfasst zu Zeit 7 Genera mit circa 30 Arten und Varietäten. Das verbreitetste, durchaus cosmopolitische Genus, heisst Diaplınosoma. Demselben gehören allein 15 Formen an. Langezeit kannte man nur 2 europäische Species mit etlichen Varietäten. Neuerdings sind aber' aus Südasien, wo dieses Genus seine grösste Entfaltung zeigt, andere, zum Teil sehr variable Arten, bekannt gerrolden. Von Diaphanosoma excisum Sars und D. sarsi Pichard sind mir

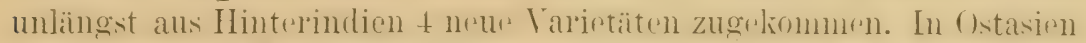
fand man auch Leheroangsformen zwischen eulopuaisch-asiatischen und südasiatischen Arten wie z. B. D. singalense var. intermedia Daday.

Zur 2. Familie "Holopedid » gehörte bis vol kurzen nul eine Species, das merkwürdige Holopedimm giblormm Zauldach. rlas in alpinen Gegenlen z. B. St. (iottharl. sowje im hohen Norlen seine höchste Entfintung aufweist. Zu Anfang dieses Jahres entdeckte ich nun eine zweite,

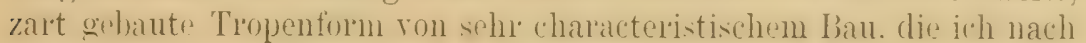
ihrem Fundorte $H$. amazomicum benannte.

In der 3. Familie (DAPHNID $)$ finden wir 7 Genera mit circa 190 Formen.

Die grösste Aufmerksamkeit wurde bisher dem Genus Daphmia, welchem allein etwa 100 F'ormen angehölen, geschenkt. Aus allen Firdteilen - ausgenommen das tropische Süd-Amerika und Süd-Asien, ron wo nur 3 unsichere Angaben vorliegen - wurden fortwährend neue Arten beschrieben. - Erst vor etwa 10 Jahren trat die Daphnienforschung in eine neue Phase ein. Nachdem schon 1891 E. v. DADAY und

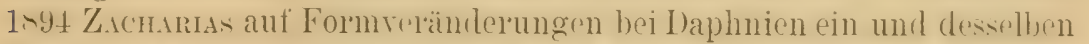

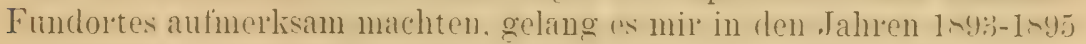
- ohne dass ich damals von DaDaY's und Zacharias' Befunden Kenntnis gebaht häte -- durch unausgesitzte Benbachtung der Formen unes bestimmten Teiches, den durch zahlreiche Generationen vermittelten Tohergang der Duphuia permatr (). F. M. ( Frühlingstorm !) zur Duphuin pulex De Geer (Herbstfor'm !) festzustellen.

Diese Entdecliung rines Saisonpolymolphismus, dex die gemeinsten. uns an längsten bekannten Arten dè "Xobenkamm tragumbn Iaph- 
nien " zu einer Species vereinigte, regte bald zu weiteren Nachforschungen an. Es war besonders G. BurchHardt, welcher anlässlich seiner Planlitonstulien in schweizerseen den verbroitetsten Arten der" "Nehenkamm-losen Daphnien ". der Daphnia longisprina O. F. M. und Daphrnia

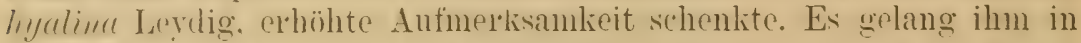
der Folge anch. viele früher als selbstandig betrachtete Arten den beiden ohen grmannten Species als Varictïten oder Localformen unter"zuorelnen. Nicht weniger als 25 neue Localformen hat dieser Zoologe aus verschicdenen Schweizerseen beschrieben. - Den wichtigsten Schritt aber hat nenerdings unser glosser Meister und Begründer des Cladocerensystems, Prof. G. O. Sars (Christiania) getan. Durch Vergleich eines umfangreichen Materials aus verschiedenen Gegenden der gemässigten Zone Asiens ist er zul Ueberzeugung gekommen, dass auch Daphnia longispina und Daphnia hyalina nicht mehr als 2 besondere Arten gelten können. Es war überhaupt nie möglich, wirklich wichtige, konstante Unterscheidungsmerkuale zwischen diesen beiden Arten herauszufinden. Nun hat Sars von vielen Uebergangsformen aus Centralasien (Territorium Akmolinsk und Seen im Altaigebirge!) eine ächt limnetische Zwischenform als Daphnix longispina var. tenutesta beschrieben, welche jegliche Spur einer Grenze zwischen $D$. longispina und $D$. hyalina verwischt. Alle Formen der $D$. hyalina sind infolge dessen in Zukunft als Varietäten oder Formæ der D. longispina unterzuordnen. Daphmia longispina O. Fr. Müller ist somit der Name einer Species,

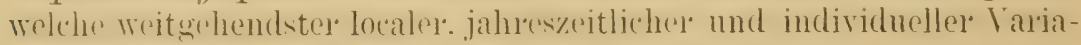
tion unterworfen ist. So viele Fundorte, so vicle Formen!

Durch solche Entdeckungen wird die Zahl der Arten des Genus Daphnia mit der Zeit auf einige wenige Haupttypen reduciert werden können. Die Zahl der Varietäten und Localformen aber wird beinahe in's Unendliche steigen.

In ähnlicher Weise hat die biologische For'schung im Laufe der letzten Jahre in del 4. Familie "Bosmnide », welche heute 2 Genera,

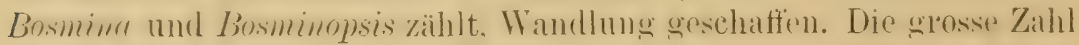

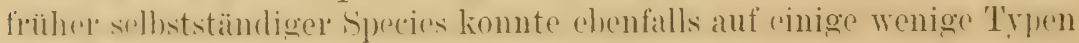
zur'ückgeführt werden. Achnlich wie im Genus Daphnia gelang es mir auch hier zuerst einen F $0 l^{\circ} \mathrm{m}$ en cy c l u s festzustellen, der sich im Laufe eines Jahres zwischen Bosmina longirostris (). F. M ülle r (Winterfol'm!) und Bosmina cornuta Jurine (Sommerfor'm!) abspielte. Bosmina cormuta Jur. ist nur eine Saisonvarietit der Bosmina longirostris !

Wieder ver'suchte G. Burckmardt auf Grund äussel'st sorgfältiger Messungen dic meisten der damals bekannten 56 Bosminen als Varictäten und Forma den beiden Species Bosmina longirostris (O. F. Müller') und Bosmina coregoni Baird unterzuordnen. Aus verschiedenen Schweizerseen beschrieb G. BurcknardT solann noch 15 neue Varietäten del 
Bosmina coregoni. So sind heute vom Genus Bosminc im Ganzen etwas über so Formen beschrieben.

IV. LiLluebong behandelt in seinem grossen Werke Cladocera Suecize 19 Arten und 30 Varietäten nordischer Bosminen. - Aus allem geht hervor, dass dieses Genus in nördlichen und in alpinen Gegenden sich am besten entfaltet. Aus Skandinavien und Finnland sind besonders bizar're Formen bekannt geworden. Dass die Gattung Bosmina aber auch im tropischen Südamerika ihre Vertreter hat, habe ich unlängst nachweisen können mit der Beschreibung von Bosmina hagmami n. sp. aus dem Mündungsgebiet des Amazonas. Nur aus dem tropischen Asien und Australien ist noch keine Bosmina gemeldet worden.

Die 5. Familie "Lrncodarnide » zelfällt bis jetzt in 12 Gattungen mit zusammen 48 Arten und Varietäten. 10 dieser Genera weisen nur je eine oder zwei Arten auf. Vom Genus Ilyocryptus sind 7 Formen beschricben, währeud zum Genus Macrothrix circa 28 Species gehören, die hauptsächlich in Süd-Amerika (14!) und Asien (11!) leben. In Europa kommen bloss 5 Formen vor.

6. Familic "CHydoride 》 (früher Lynceidz). Sie ist die grösste und formenreichste Cladocerenfamilie. Ich zähle 20 Genera mit circa 195 For'men. Starke Tendenz zur Varietätenbildung wird auch hier zu einel' bedeutenden Reduktion der Arten-Zahl führen. In einzelnen Fällen steht sogar die Zugehörigkeit zum Genus noch im Zweifel. Es wird vielleicht auch noch nötig, neue Genusnamen einzuführen, oxler schon vorhandene auszuschalten.

Die wichtigsten Genera sind: Das Genus Alona mit circa 72 For'men, das Genus Alonella-Pleuroxus mit c. 30, und das Genus Chydorus mit c. $32 \mathrm{Hormen}$; alle drei über die ganze Erde verbreitet.

Der als "Kosmopolit » so oft citierte Chydor "us sphrericus, der" "Spatz » unter den Cladoceren, scheint nicht so allgemein verbreitet zu sein, wie früher angenommen wurde. Wohl gibt es in allen Erdteilen zahlieiche Chydorusformen, welche äusserlich dem Chydorus sphxricus auffallend ähnlich sind; bei genauer Untersuchung stellen sich jedoch konstante Entersheidungsmerkmale - hesomders im Bau des Lippenanhangs und des Postabdomens - heraus, was besonder's DADAY's und meine jüngsten Lntersuchungen an Südasiatischen Cladoeeren dartun. Bezüwlich Individuenzalıl und Häufigkeit des Vorkommens scheint in süd-Asien Ch!/dorus barroisi Richard den C. spharicus zu verteten. Diese scharf umschriehene Specins ist anch in den tropischen fiehieten von rüul-Amerika und Afrika, sowie im Kaplande, in Palästina und in Neu-Seeland gefunden worden.

Die 7. Familie "Polypneside ») beherbergt lauter eigentümliche und äusserst bizarre Tierformen. Man kennt num 6 Genera mit zusammen 4.3 Arten und Varietäten. Bis vor kurzem waren bloss 2 Süsswassel- 
genera. Polyphemus und Bythotrephes, sowie 2 marine Gattungen, Podon und Evadne, mit einigen wenigen Arten bekannt.

Wieschon in der Finleitung bemerkt wurde, bereicherto aber unlängst G. O. SARs durch die Entrleckung der höchst eigenartigen Laspiseeformen der (rattungen Cercopagis, Apargis und Evadne, unsere Kenntnisse von den Polyphemiden ganz ausserordentlich.

Zu1 8. und letzten Familie "LEPTODoRID 2 " gehört nur eine Alt, die Leptodora kindtii Focke. Sie ist die grösste Cladocere und wird bis 8 mm. lang. Ferner zeichnet sie sich vor allen anderen Clarloceren dadurch aus, dass ihre Jungen eine Jetamorphose durchmachen. Leptodora ist vorzugsweise ein Bewohner der nördlich gemässigten Zonen von Europa. Asien und Amerika, sie wurde $189 \mathrm{~s}$ aber auch durch E. v. Damar aus Ceylon gemeldet.

Nach diesem kưnn Ueberblick über die einzelnen Familien und die wichtigsten liologischen und systematischen Errungenschaften der letzten 10 Jahre, lasse ich eine gedrängte Zusammenstellung über dic Anzahl ler in den verschiedenen Erelteilen bis herute gefundenen Cladocerenformen folgen. Zum Vorgleich, und damit die bedentenden Fortschritte der letzten 12. Jahre sofort ersichtlich werden. stelle ich meinen Angahen von la)t diejenigen von J. Richaro aus dem Jahre 1892 gecrenüber.

1892

1904

Asien

Nöldlich kalte und gemässigte Zone:

47 Formen (woron 7 unbestimmt)

si spec. 41 var.. total 128 Fol'men (30 Genera).

Tropisches Asien und seine Inseln:

11 spec.

55 spec., 29 var., total 84 For'men (27 Genera).

AMERIKA

Vereinigte Staaten und Grönland:

90 Fol'men

105 spec., 37 var., total 142 For'men (39 Genel'a).

'Tropisches Amelika (Mexiko, Inseln, bis und mit Brasilien): 10 Firmun (5:.5 unbestimmt) (j0 spece, 2 var., total 62 Formen (28 Genela). 
1892

1904

Gemässigtes Südamerika (Argentinien, Chile, Patagonien, Falkland-Inseln):

7 Formen (5 aus Chile zweifelhaft bestimmt)
53 spec., 8 val., total 61 Folmen (17 Genera).

Afrika

40 Formen (zur Hälfte zweifelhaft oder gar nicht bestimmt, 10 aus Aegypten, 15 aus Algerien-Tunis)
60 spec.. 9 var., total 69 Formen (23 Genera)

(die meisten aus Ostafrika).

\section{Australien}

Festland :

30 Formen (1.2 zxweifelhaft bestimmt)
38 spec.. 1 val'.. total 39 Formen (17 Genera).

In seln (Neu-Guinea, Fidji, Neu-Secland, Hawai):

5 Formen (zum Tril zweifollhaft bestimmt)
2 (i spece. 3 var.. total 29 Folmen (16 Genera).

\section{Europa}

J. Richard (1892) zählte die europ. Formen nicht! Letzte Zählung bei HeLLich (1877): 153 spec. (34 Genera)
Zirka 148 spec., 145 var., zahlleiche Formx, total etrva 300 beschriebene Formen. - (44 Genera).

Obige Zusammenstellung zeigt, welch bedeutende Fortschritte die Clarlocerenforschung im Laufe der letzten 12. Jahre in allen Eirdteilen gemacht hat. Nur in wenigen Ländern der Erde sind noch gar keine Nachforschungen angestellt worden.

Aus den drei südeuropäischen Halbinseln liegen immer noch sehr späilliche Angaben vor. Zu einem gewissen Abschlusse ist die Cladocerenforschung einzig in Skandinavien gekommen durch die rorzügliche

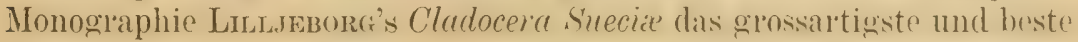
Cladocerenwerk der Neuzeit. Noch nicht nachgeforscht wurde u. a. in Britisch-Nordanerilia, in grösseren Gebieten ron Mittel- und Südamerika, ferner in Zentralafrika und vielen australischen Inselgruppen. 


\section{Ueber die geographische Verbreitung der 56 Genera.}

1. Eine kosmopolitische Verbreitung weisen 14 Genera auf: Diaphanosoma, Simocephulus, Scapholeberis (?), Ceriodaphnia, Moina (?), Ma-

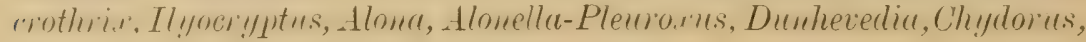
Podon-Evadne (marin).

2. Noch nicht sicher erwiesen ist die kosmopolitische Verbreitung der Genera : Daphnia, Bosmina, Pseudalona, Leydigia.

3. Nur in Europa waren bis jetzt zu finden die Gattungen: Limmosida (Skandinavien und Russland) und Wlassicsia (Ungal’n).

4. Nicht in Europa sind vertreten die 12 Genera: Penitia, Pseudosida, Latonopsis, Daphniopsis, Moinodaphnia, Grimaldina, Guernella, Theringula, Euryulona, Leydigiopsis, Dadaya, Saycia (SARs 1904).

๖. Nur in den Tropen leben : Penilia (marin), Guernella (Afrika, Asien), Grimaldinu (Afrika, Australien, Südamerika), Iheringula (Süd-

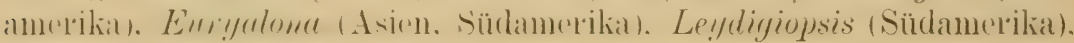
Dadaya (Asien, Südamerika).

'Tropischen Ursprunges sind aber offenbar auch die Gattungen : Latonopsis, Psendosida und Moinodaphria, welche mitunter noch in den Südstaaten der Union gefunden wurden.

6. In den 'T'ropen scheinen nicht vorzukommen 20 Genera, nämlich: Sida, Limmosida, Latona, Daphmiopsis, Ophryoxus, Bunops, Whassicsia, Drepanothrix, deantholeberis, Latonura, Eurycercus, Acroperus, Lep-

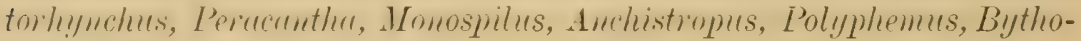
trephes, Cercopagis, Apagis.

7. Am meisten Gattungen haben gemeinsam die nördlichen und gemïssigten Gegenelen ron Asien, Europa und Nordamerika.

8. Aus den tropischen Gegenden von Asien, Afrika und Australien kemnt man zur Zeit kein einziges Genus meht, welches einem dieser drei Erdteile allein zukäme. Nur Südamerika weist noch 2 ihm eigene Genera auf: Iheringula und Leydigiopsis.

Wir gelangen somit zu folgenden Schlüssen :

1. Ueberall auf der Fı̀de sind die Existenzbedingungen fül Cladoceren vorhanden. Nicht nul in den verschiedenen klimatischen Zonen, sondern anch in allen Höbrulagen, vom Iochgobirgsee bis hinab zum tiofgelegenen

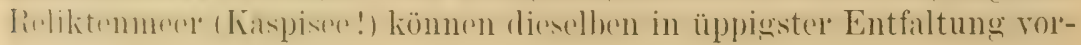
komment. 
2. Die frühere Ansicht, dass die Cladocerenfauna in nördlichen Gegenden der Er'de sich am schönsten entfalte, verliert immer mehr ihre Berechtigung. Auch in den bisher auf Cladoceren leider erst oberflächlich erforschten tropischen Gegenden trat ein ausserordentlicher Reichtum an Arten und Individuen zu Tage. Es felnlt also bloss noch an einer' grüundlichen, systematischen Fr'for'schung dieser Gebiete.

3. Das Klima ist nicht allein massgebend fül das Vorkommen gewisser Genera und Arten. Die tropischen Gebiete von Asien und Südamerika zum Beispiel weisen mituntel ganz verschiedene Arten und Gattungen auf, der Nor'drand von Afrika die gleichen Arten wie das gemässigte Asien und Europa. Die Einteilung der Erde in tiergeographische Regionen lässt sich auch aufdie Cladocerenfauna a $n$ wenden. In der leichten und passiven Ueber-

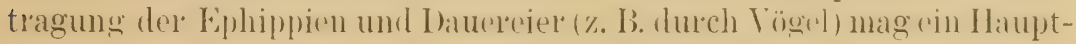
grund gesucht werden für die weite kosmopolitische Verbreitung vieler Arten.

\section{Les Mouettes du Léman.}

Par le Prof. F.-A. FOREL (Vorges).

Le Larus ridibundus, la Monette des eaux douces, passe l'hiver sur' notre lac où elle se compte par milliers d'individus, peut-être 3 à 4 mille. Elle a depuis vingt à vingt-cinq ans appris à mendier le pain que lui jettent à profusion les âmes charitables des villes riveraines. Au premier printemps, vers le 20 ou le 25 mars, elles partent pour aller nicher sur les îlots des lacs du nord, Pologne, Finlande, Pomélanie, Scandinavie. Elles rentrent en octobre sur nos eaux qui ne gèlent jamais.

Une faible partie du contingent des Mouettes rieuses n'émigle pas. Deux ou trois cents sont restées sur le Léman au printemps de 1904. D'après le nombre des jeunes de l'année que nous avons comptés en juillet, une centaine au plus, elles n'auraient pas toutes niché; à 3 œufs par' nid il n'y aurait eu qu'une trentaine de paires ayant mené leur couvée à bien.

Tandis que le grand retour des Mouettes émigrées avec leur's jeunes n'a lieu qu'en octobre, il y a déjà un retour partiel dans les premier's jour's de juillet. Cette année, environ quinze cents Mouettes, toutes adultes, sont rentrées ainsi hâtivement. Comment expliquer 'e retom' prématmé. a vant 
que le froid ou la famine aient chassé ces Oiseaux de leur villégiature estivale dans le Tord : Tronis solutions de ce petit problème zoologique s'offrent en ces termes:

$1^{\circ}$ Ou bien ce seraient des mâles surnuméraires qui n’ont pas trouvé à s'apparier et viennent se consoler de leur célibat par un voyage en Suisse.

$2^{\circ}$ Ou bien ce seraient des parents qui ont abandouné leur nid après que leur couvée a été émancipée, les jeunes ayant appris à nager, à voler, à se nourrir par eux-mêmes.

$3^{\circ} \mathrm{Ou}$ bien ce seraient des parents dont les nichées ont été troublées ou détruites par les paysans, trés friands des oufs d'oiseaux; ils ont quitté leurs roctieries dérastés et sont venus cherchel chez nous des eaux plus hospitalières.

Nova zoologica aus der Amazonas-Region. Neue Wirbeltiere.

Von Prof. E. GOELDI (Pará).

1. Unter den neuweltlichen Affen bilden die Hapalidæe (Eichhornäftchen, Singes-Ecureuils. Tamarins) der Artenzahl und Mannigfaltigkeit nach die überwienende Gruppe, das Gros. Sculesen, sorgfältiger Monograph. nahn im Jahre 157526 Arten an: Forbes führt in scinem neuen Haudhuche (Homilbook of 1/onlieys. London, 1s94) bloss 14 Spezies aut. I) Ansichten gehen in solchem Grade auseinander, dass kaum zwei Autoren völlig übereinstimmen.

I ie Artenzahl ist am grössten in der Aequatorial-Zone des AmazonasGebietes und neben einigen Spezies, die, wohl zugïnglichen Teilen des I'nterlaufes des Hauptstromes zugehörig, heute als leidlich genug bekannt gelten dürfen, giebt es eine ganze Reihe von Formen, zumal an den weiter abgelegenen Seitenflüssen und Bimenlandpartien, die noch lecht wenig beliannt sind, stellenwoise arg verkannt wurden oder auch geradezu unbrelnioben, nen, sind. Es ist dies ein zoologisches Tertain, suf dem methodische Erforschmng des Innern noch viele wichtige und intel'essante Bereicherungen zu Tage förder'n wird.

Fine schöptende Monographie der amazonischen Hapalicle, wie wil sir als zulunfts-I xideratum träumen, wird allerdings noch manches Jahy auf sich warten lassen. Es dürfte indessen nicht ohne Interesse srin. hentr drm Zoologisehen Kongresse einen Finblick zu elöffuen, wie 
weit es dem Museum in Pará bei 10-jährigen Bemühungen gelungen ist, Materialien und Bausteine zum Aushau dieser Frage herbeizutühren.

Hiebri möchte ich mich aber heute speziell anf die Resultate heschränlin, die auf zwei von unserem Institute abgesandten Expeditionen el'zielt worden sind, welche sich üher ein ganzes Jahl erstreckten (190)3-1904) und dem oberen Rio Pur'ús galten. ()perations-C'cntrum war” "Bom Lugar»), eine der typischen Kautschukgewinnungs-Niodellassungen, eine Tagereise unterlialb der Mündung des in neurerer Zeit aus den Grenzhändeln mit Bolivien vielgenannten Rio Acre (Aquiry) gelegen, und das Arbeitsfeld unfasste eine Area, die nach ohen eine Tagereise über die Nündung dieses Seitenflusses hinaufging, nach unten bis Canacury, zwei gute Tagereisen weiter flussabwärts reichte und rechts und links von den Flussrändern eine bis zwei Tagoreisen nach dem beiderseitigen Binnenland, von den Ipurina-Indian'rn hewohnt, sich ausdehnte. In dieser (irgend war bisher noch ron keinem Naturforscher ordentlich gesammelt und gearbeitet worden; berührt worden ist sie in den $60 \mathrm{er}$ Jahren von dem englischen Geographen CHaxdess und zu Finde der 80 er Jahre wiederum von dem deutschen Ethnographen und Anthpopologen Enrexreicir.

2. Die Hapalidxe werden nach dem Vorgange von GraY von den meisten Säugetierforschern in die 2 (irnera Hujrele und Midus aufuetrennt. Zum ersteren, Hapale, werden die Formen mit verlängerten unteren Schneidezähnen gerechnot, üher die dir Eckzähne nicht wesentlich durch Länge und I)iclie herauslagen : zum (ienus Midas werden im Gegenteil alle diejenigen Arten gezogen, wo die Fekzähne wenigstens 1/3 länger sind als die anstoxsenden Incisivi. Dio ächten Honjule sind in der Minderzahl und gehören fast alle dem Süden Brasiliens an.

3. Die 5 Arten, die hier aus der Purús-Gegend zur Betrachtung gelangen sollen, roihen sich allo dem (ienus Midrs ein. Mitgeh)achte Bailge, eine liolorierte ()riginaltafel und detaillierte Vorbeitungskaten orientieren mehr als viele Worte und gestatten mir, mich kurz zu fassen. Sie gehören zufälliger Wrise sümtlich derjenigen Unteroruppe an, dir sich durch weisses Maul und weisse Tasengegend auszeichnot, boj stollonwojse mehr oder weniger stark ausgeprägter Tendenz zur Biklung von weissen Schnurrbärten.

4. Wie sie sehen, liegen hier zu Figur 1 unserer Orjginaltafel 5. Bälge (:) $\sigma^{x}, 2$ O ) ciner farbenprälitigen Affenart vor (zwei writere Fxemplare, $1 \sigma^{x}$ und 1 o, sind hier in ausgestopftem, fertigem Zustande zu sehen), die zwar nach den sehr unzulänglichen Jeschreibungen hei (indy. Fon

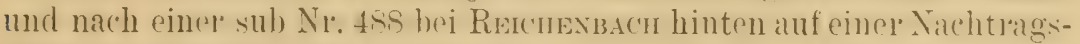
Tafel abgebildeten Figur zu schliessen, dem im Jahre 1843 von Gray aufgestellten . Midus rufienter' sehr mahe zu kommen siheint. immerhin sich aber sofort durch den weissen bis weissgrauen Scheitelfieck auszeichnet, der für $\boldsymbol{M}$.rufiventer allenthalben als rot oder goldgelb ange- 
geben wird. Das von GraY beschriebene Exemplar von M. rufiventer soll von Bates bei Tonantins, nahe am Zusammenfluss des Rio Javary mit dem Maranon, crlegt worden sein. - Sicherheit, ob wirklich $\boldsymbol{M}$. rufiventer vorliegt und die ursprüngliche Original-Artbeschreibung nicht umfassend genug redigiert wurde oder ob man -- was wahrscheinlicher - eine neue, vikarierende Art anzunehmen hat, wird er'st nach Linsicht und genauem Vergleich mit dem Rates'schen Exemplare im British Museum in London zu erlangen sein.

5. Von Midas pileatus Isidor Geoffroy und Deville (Figur 2 unserer Tafel), 1848 von Geoffroy in den "Archives du Museum », T. 5, Taf. 31 abgebildet und unter dem Namen "Tamarin à calotte rousse " beschrieben, (pag. jli9) scheint bisher in einem einzigen Originalexemplare, rom Rio Javary herstammend, im Pariser Museum zu stehen. (Dort habe ich es erst dieser 'Tage, zwal' etiquettenlos und offenbar verkannt, in einem Seitenschrank wieder aufgefunden.) Wie Sie sehen, besitzen wir 2 weitere Exemplare, 1 Paar vom oberen Rio Purús. Die prächtige Art besitzt ihr vorzüglichstes Erlienmungsmerlimal in der zimmtfarbenen Scheitel-

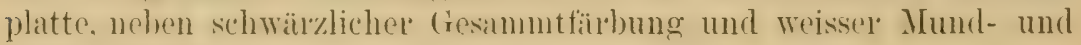
Nasen-Gegend.

6. Midas fuscicollis (Fig. 3 unserer 'Tafel) ist zwar' schon längere Zeit brschrieben, indem sie 1823 von dem hayrischen Forscher v. Sprx in die I'iscuschaft eingeführt wurde nach vom Rio Javary mitgehrachten Exemplaren, aber es hat sich aus unserem viel reicheren Material (8 Exemplare, $6 \sigma^{\Upsilon}, 2$ ) ) vom Rio Purús ergeben, dass das Alterskleid des ausgewachsenen Tirres bisher niemals ordentlich fostgestellt und abgebildet worden war. Indem ich mir erlaube, zu einem Vergleiche der beiden Fi-

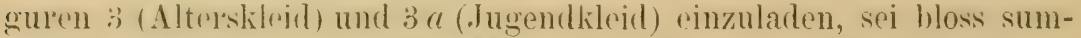
marisch angedentet, dass das Alterskleid durch die tief schwarzhraune Färbung des Vorderkörpers ausgezeichnet ist.

7. Midas mystax Spix (Fig. 4 unserer Tafel) ist zwar ebenfalls schon aus der gleichen I'eriode und aus del'selben Quelle her wissenschaftich lekannt gegehen. Sprx hatte seine Exrmplare vom Solimoes mitgebracht; ein $q$ ist auf 'Taf. 22 seines Werkes abgebildet. Unser' wesentlich dunkleres männliches Exemplar stammt vom Rio Jurua.

8. Eime den Arten Midas labiatus Geoffiroy (1812), M. Illigeri (1845) und $M$. Weddellii (1849) offenbar nahestehende, aber vermutlich neue Art liegt Ihnen hier in Fig. 5 unserer 'Tafel und 5 Bälgen $\left(3 \sigma^{x}, 2\right.$ 우) vor, wovon 2 vom lío Acre und 3 vom oberen Rio Purús stammen. Sie zrichnet sich dureh rötlich grau-lnaume Gesammtfärbung der ()herseitr. licht lostfinbene Unterseite, dunkle Füsise mol dunklen liopf ans, an dem

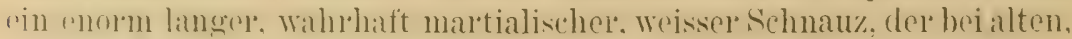
ausgewachisenen, männlichen Exemplaren bis hinter die Ohren reicht, das auffïlligste Merkmal darstellt. 
9. Unter dem Namen Putorius paraensis habe ich 1897 in Spenger's "Zoolog. Jahrbüthern n zum erstru Male cine brasilianische Wieselform

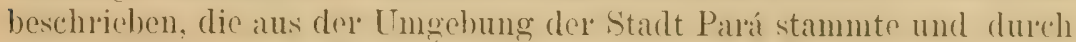
einen dunkelbramen Längsstreif in ockerfarbenem Grunde am Bauche sich von allen mir zugänglichen Beschroibungen und Abbiklungen auszeichnet. Seither sind 2 weitere Exemplare aus derselben Gegend hinzugekommen, die ich hier im Originale vorzulegen das Vergnügen habr und äber die sich in dem neurelich veröffentlichten Katalog der Par:iensel Säugetier-Sammlung die hauptsächlichsten wissenschaftlichen Angaben niedergelegt finden. Es ist ein seltenes 'Tier, das bisher auf drei Exemplare brschränkt gehliehen ist. - Iemerlenswert erscheint, dass in Putorius parapusis transandinischerseits die sürlichste Form rorliegt: die Wieselgattung ist zwar in Nord-und Zentralimerika starli vertreten; bisher kannte man indessen aus dem dem atlantische'n Ozean zugeliehl'ten 'Teile Südamerikas bloss Marder', aber' keine Wiesel.

10. Dinomys Branickii Peters ist der Name eines grossen südamerikanischen Nagetieres, das an Seltenheit füglich mit der neuerdings aus dem tropische'n Afrika bekannt gewordenen Giraften-Art, dem Okap)i, sich messen kann. Denn es war bisher überhaupt nur ein einziges Exemplar zur Kenntnis gelangt: das 1873 von Prof. Perers in Berlin beschriebene und aus den peruanischen Anden stammende, männliche Originalexemplar, von $83 \mathrm{~cm}$. Totallänge, welches von dem polnischen Sammler Constantin IEssiz erlegt worden war und dem zoologischen Iluseum in Warschau angehört. Peter's hat das wohlerhaltene Fell und das bis auf einige Wirbel vollständige skelett zur Terfügung gelabt und an der Hand dieses Materiales eine Ahhandlung geliefert, in der von dem, was zu sehen war, so ziemlich alles verweltet wurde und der auch eine kolorierte Habitus-Tafel beigegehon ist, der man im Grossen und Ganzen alle Anerkennung zollen muss!

11. Dem Museum in Pará ist jüngst das ausserordentliche Glück widerfahren, auf einmal zwei lebende Exemplare dieses überaus seltenen Nagers zu erhalten - Mutter und Soln. Die genau festgestellte Herkunft ist jefloch diesmal rine ganz andere. als diejonige jenes ersten OriginalExemplares: unsere Individuen stammen vom Rio-Muaco, einem der oberen Quellflüsse des Rio Pauhiny, der einerseits wieder ein linksufriger Tributarthuss des Rio Purus ist. Die Heimat liegt also in diesem

${ }^{1}$ Peters. Ueber Dinomys, eine merkwiödige neue Gattung von Nagetieren aus Peru, in: Festschrift der Gesellschaft Naturforschender Freunde zu Berlin, 1873, mit 4 Tafeln, und

Peters. Ueber Dinomys, über eine merlwürdige neue Gattung der stachelschweinartigen Nagetiere aus dem Hochgebirge von Peru, in: Monatsberichten der Kionnigl. Preuss. Akademie der Wissenschaften zu Berlin, Juli-August 1873, pag. 551. 
Falle auf dem teils Brasilien, teils Peru, teils Bolivien zugehörigen Quellund (Oberlaufgelbiet der Flüsse Juruá, Pur'us und Acre, und die Findstelle muss orographisch als ein Teil des dem üstlichen Andenabhang vorgelagert'n Hochplateau's bezeichnet werden - eines Gebietes, das auch in paläontologischer Beziehung, zumal in Hinblick auf die Fülle der dort aufgestapelten Ueherreste mächtiger Sïuger (Mastodon und T'ur:odon) und Saurier (Crocodilier und ('helonier) für die Wissenschaft der nächsten Zukunft ein Eldorado von hochwichtigen Uelerraschungen zu werden verspricht.

12. Von den heiden Dinomys-Exemplaren ist das ältere, die Mutter $85 \mathrm{~cm}$. lang - leider an den Folgen einer schwierigen Geburt eingegegangen; die beiden Fœtus, zusammen ein Paar', liegen hier vor, nehst dem ausgestopften Weibchen. Das vorhin cr'wähnte, grössere, mehr als halbwüchsige Junge lebt hingegen hente noch im zoologischen Garten des Dluseums in Pará, augenscheinlich munter bei seinem Lieblingsfutter, das in Speise-Kürbissen besteht.

13. Ueber den änsseren Habitus des Thieres, seine Haltung, seine Bewegungen, sein Brnehmen orientiren eine grosse Venge von Augenhlicksphotographien, die wir im Laufe der letzten Monate aufgenommen haben in allen möglichen Stellungen.

14. Dinomys Branickii ist ein Paca-ähnliches Thier im allgemeinen Aussehen, in Grösse, in den weissen zu Lüngsteihen angeordneten Flekken der hinteren Körperhälfte. Doch unterscheirlet es sich sofort wieder durch den cylindrischen Schwanz, der' stets mit ciner leichten Biegung: nach oben, gerade ausgestreckt getragen wird, dann durch sehr verschiedenen, eigenartigen und zu dem stachelkleide der EchinomyideLoncheride hinüberführenden, etwas steifen Haarpelz und den wuchtigeren, mehr sub-pyramidalen Kopf, der am meisten an den von Hydrocherms apibura erinnert. Immerhin sind die in seiner Heimat gebräuchlichen Trivial-Namen "paca-rana ") (d. h. Pseudo-paca) und "paca de ralon" (paca mit Schwanz) als sehr zutreffend zu bezeichnen.

15. I je kleinen, schön nussbraunen Augen lassen bei Tage bloss einen 'ngen. verticalen Pupillenschlitz sehen, ähneln daher denjenigen des grossen Faulthiers (Cholepus didretylus). obwohl die Pupille hier tagsübor einen centralen schwarzen, r'unden Fleck darstellt und führen zum Analogie-Sichluss, dass Dinom!gs der Hauptsache nach eine nächtliche Lebensweise führt.

16. Die Füsse sind vorn und hinten 4-zehig, plantigrad. Die starken, langen Klauen weisen auf ein zum Graben geschicktes Thier.

17. Was der Physiognomie des Thieres ein besonderes Gepräge verJeriht, ist dre phänont nal starke Schnurbart, bestehend aus einem Bündel weisser Vibrissen die nach hinten noch erheblich über die Ohren hinausreichen. 
15. Hinsichtlich seines Charaktel's und T'emperamentes fällt bei Dimomys das Gemächliche, Langsame aller seinel Bewegungen auf hoi cinel ïusserst gutartigen (remüths-Disposition. Es trachtet weder zu Hichen, noch zu beissen. Das hochgradige Phlegma dieses Thieres ist im Kampfe um's Dasein offenbal eine bedenkliche Nitgift und die Vermutung, dass Dinomys sich auf dem Aussterbe-Etat betindet, ist aus mehrfachen fründen berechtigt.

19. Schon Perers hat vor 30 Jahren betont, dass Dinomys sich mit keiner der bisher bekannten (iruppen der stachelschweinartigen Nager, denen es sich zunächst anschliesst, vereinigen lasse und dass es als Vertreter einer besonderen, neuen Familie - Dinomyidx - aufzufassen sei.

20. Osteolowisch bieten sich verwandtschattliche Züge mit (len I) usyproctinx ("Cutias »), Caviinx ("Préas »), Chinchillinx, Echinomyinx (Stachelratten), speziell Camomys. Aber dasjenige Tier. dem es craneologisch am näuhsten kommt, ist entschieden Hydrochorns capibara, der Riese unter den heutigen Nagern.

21. Zwei Merkmale sind an dex Patwrs'schen Beschreihung des Schädels auf Rechnung individueller Eigentümlichkeit zu nehmen und aus dem Verbande der Spezies-Charaktere auszuscheiden: 1. der länglich elliptische Schaltknochen (1)s intercalare) zwischen dem einfachen Parietale und den beiden Frontalia (vide Tafel II, Fig. 1). 2. Die nach vorn und innen offene Schlinge der hintersten der \& Schmelzröhren des vierten oberen Molarzahnes und die mittlere Scheidewand an dex mittleren Schmelzröhre des entsprechenden unteren Molaren.

22. Die Anatomie der Weichteile ist bisher noch nicht untersucht worden, ist aber als Gegenstand spezieller Studien für die nïchste Zukunft in Aussicht genommen.

23. Gestatten Sie mir, dass ich Sie ins Gebiet der Ornithologio hinüber'führe und Ihnen einige bemerkenswerte Nova aus denselben Landstrichen des tropischen Süd-Amerika vorführe.

Die Familie der Gulbulide besteht aus Eisvogel-ïhnlichen, nit langen, geraden Pfriemenschnabeln ausgerüstreten, vielfach mit ächt tropischem Prunkgefieder ausgezeichneten Vägeln, die gegenwärtig in 6 Genera und 21 Alten, rein neotropisch, sich über ein Gohiet erstrecken, das einerseits mit Grelbula melenogenia im südlichen Mexico serine Nordgr'nze, andererseits mit. Jacemaralcyon tridactyla in Paraguay seine Südgrenze errevicht und blos auf den Antillen und im Hochgebirge von Peru zu fehlen scheint. I)ie Artendichtigkeit ist am grössten in (ler amazonischen sul)region (mit 14 Arten).

24. Eine sehr eigentümliche Stellung innerhalb dieser Fanilie nimmt die bisher monotyp geblicbene (xattung Galbuleyrynchus ein, mit dev einzigen Art ( + . leucotis, 1845 von IEs . Iurs in der "Revue zoologique ") 
aufgestellt. Sie hetrifft rine Togelart, die sich durch ihre intensiv rostfarlene (iesammtfürbung und im männlichen Geschlecht durch einen leuchtend weissen Ohrfleck anszeichnet; ihre Heimat ist Oler-Amazonien und das östliche Ecuador [Pebas -, Ucayale -, Iquitos, - Envira]. Sie muss dort ohen ziemlich häufig sein, denn im Britischen Museum stehen allein 15 Exemplare laut dem 1891 von Freund $D^{r} \mathrm{Ph}$. L. Sclater abgefassten, respektiven Band des grossen Vogelkataloges.

25. Vom oberen Rio Purus und Acre brachten nun unsere MuseumsExpeditionen zahlreiche Exemplare einer zweiten Art mit, die sehr' nahe verwandt ist, aber sich durch das konstante Fehlen der "plaga postocularis alha ) bei sämmtlichen Individuen mänulichen Geschlechtes sofort als nel ergiebt. Ausserdem sind Schuabel und Füsse nicht weiss, wie es laut S'Later's. Ionographie (18'2, Taf. X VII) und Catalog (1891) von $G$. leucotis heisst, sonder'n ebonfalls rot. Ich sehlage vor, diese unbeschriebene Art unter dem Namen Galbaleyrynchus purusianus in die Wissenschaft einzuführen.

26. I) ne nerdings der Gruppe dor Cotingidar (Schmuckvögel) eingereihte und in die Nähe der Typromiderestellte Familie der Pipridie ist ebenso ausschliesslich neotropisch. Sie reicht nordwärts mit Pipra mentalis nach dem südlichen Mexico, südwärts mit Chiroxiphia condete bis hinunter nach Paraguay. I) ie eigentlichen Pipriden (nach Aussehluss (ley ihnen vielfach beigerechneten Ptilochloride) unfassen heute circa 19 Genera und in die 70 Arten. Die wichtigste Rolle fällt dem Genus Pifru (sensu stricto) zu mit 18 Arten und einer mehr auf das AmazonenGebirt konzentrierten geographischen Ausbreitung. Es sind allerliebste likeine Creaturen; bei der' Mehrzahl ist dis männliche (ieschlecht schwar" mit in irgend eine leuchtende Prunkfarbe (rot, orange, weiss, blau) gekleidete Kopf- und Scheitelplatte, während das Weibchen sich in einem eintönigen, ziemlich unscheinharen Grün präsentiert. Neben diesem schwar\% $\left(O^{\top}\right)$-grünen ( $($ ) Pipra-Arten kannte man aber bisher auch :S Spezies, hei denen auch das Männchen eine grünliche (xesammtfürbung hei rerschiedenfarbiger Kopfplatte anfweist. (Pimro nattereri Sclater 1864, P. virescens Pelzeln 1874 und Pipra opalizans Pelzeln 1874). Ihr Habitus ergiebt sich aus der Pipriden-Tafel meines "Vogelalbums", während oine hirre ebenfalls vorliegende Kartenskizze ihre geographische Aushereiturg soweit spezialisiert, als es an der Hand der gegenwiurtigen Kenntnis über'laupt möglich ist.

27. Unsere Purus-Expeditionen haben nun wiederum eine weitere, bis-

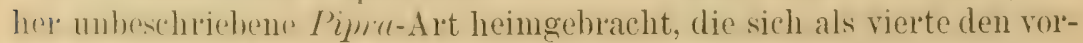
hin wwilhnten :3 grounen anreiht und sich auf den ersten Blick durch ihren

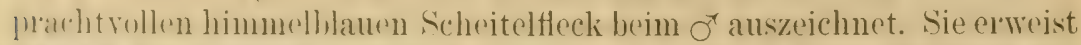

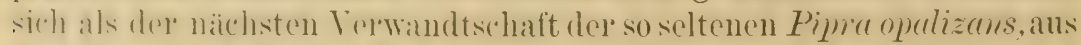
d.m Inne'n des Stalates Pará, angehörig. Es liegen 3 Exemplare vor. 2 
ơ und 1 Q. Ich schlage vor, sie als Pipra czelesti-pileata in die Wissenschaft einzutühren.

28. Ich habe Ihnen neue Säugetiere und neue Vögcl aus dem Amazonen-Gebirt vorgeführt. Erlauben Sir mir, dass ich Ihnen schliesslich noch eine höchst merkwürdige, neue Fischform vorlege - einen winzigen, blinden /swergwels ans dem Wasser ciner Bimenlandzisterne tief im Inner'n der der Mündung des Amazonenstromes vorgelagerten Riciseninsel Marajó.

Er besitzt einen zylindrischen Leib, am Kopfe 6 Barteln (2 obere und 4 untere) und eine Schnauze mit vor'springendem Unterkiefer, eine der breiten Inorsalflosice opponiorte unseheinhare Alolominalfiosse, als auffälligstes Merkmal jedoch einen fast lie ganze hintere Kö̈rperhälfte einnehmenden, medianen Flossensaum, welcher nach unten und hinten die Anal- und Caudalflosse vollständig in sich aufgehen lässt und nach volne über sich bis in die Nachbarschaft der Dorsalflosse er'streckt.

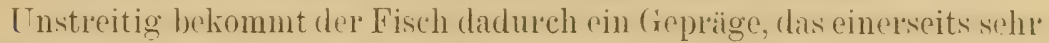
alter'ümlich, andererseits auch wieder embryonal aussieht. Und doch sind seine ührigen . Merkmale dir eines fertigen. ausgewachsenen Fisches. Die Augen sind als kleine, unter der Haut versteckte Punkte erkenntlich - ein Verhalten, das an die zum Parasitismus tendierenden Cetopsiden erinnert. Ueberhaupt lassen sich eine Reihe von Berülırungspunkten auffinden, die teils zu den Trichomycterinen, teils zu den Cetopsiden hinüberweisen. Dabei bleiben aber doch auf der anderen Seite wieder gewichtige Charaktere zur (ronügr, die auf eine isolierte Stellumg zwischen diesen 2 henannten siluriden-(xattungen schliessen lassen. Es liegen von diesem äusserst seltenen, blinden Zwergwelse bisher blos 2 einzige Exemplare vor, ein grösseres und ein kleineres. Ich führe denselben unter dem Namen Phreatobius cisternarum in die W'issenschaft ein und hoffe, dass dis vorliegende Material zun Ausgangspunlite cincr genaueren, monographischen Bearbeitung werden möchte. 


\section{La Ligne de Weber, limite zoologique de l'Asie et de l'Australie.}

Par le Prof. P. PELSENEER (Gand).

T) 's trois grands royaumes zoologiques: Arctogée, Néogée et Notogée, les deux premier's sont nettement séparés l'un de l'autre, de même que les deuxième et troisième.

P'rudant longtemps la limite naturelle de l'Aretogée et de la Notogée a été considérée aussi comme bien définie. En effet, dans la plupart des travaux où cette question a été envisagée et daus la génélalité des ouvrages, mime tres récents, servant à l'enscignement de la géographie zoologique, la limite séparative de l'Aretogée et de la Notogée (c'est-ìdire la séparation zoologique de l'Asie et de l'Australie) est faite par' la ligne dite " de Wallace ", ligne qui passe à l'est de Bali et de Bornéo et à l'ouest de Lombok et de Célèbes.

Cependant, malgré l'adhésion si générale des zoologistes à cette " ligne », quelque opposition s'est déjà manifestée, à diverses reprises, sur ce sujet, par l'opinion que cette limite - au point de vue zoologique et anthropologicque - devait etre transportée plus rers l'Est, soit partiellement (dans la région Nord, en englobant Célébes, ou dans la région Sud, en englolant Lombok, Sumbava, Florès, Timor, etc.,) soit en totalité.

Queldues auteur's plus récents sont même d'avis de déplacer la limite beaucoup plus loin encore à l'Est, de facon à séparer de la Notogée, notamment les Moluques, la Nouvelle-Guinée, etc. (Henter, pour les Mollusques on général; Strupsos, jour les Unionidie; Pascoe, pour les Coléoptèl'es, etc.).

D’autre part, une opinion intermédiaire considérait tout ou partie (C'élihes) de la zono litigieuse, comme une région de transition entre la province orientale de l'Aretogée et la province australienne de la Notogér (Forsy'ti-Major, Heilprin, Kǘnentual, Lydekker, von Martens, etc.).

Mais une limite destinée à remplacer la ligne de WALLACE n'a pas encol'e été définie ni l’eprésentée d'une façon précise.

Cependant, des considérations fauniques, basées sur des travilux reratifs it des wroupes tres divers, permettent de le faire aujourd'hui. 
Ainsi, par exemple, les Oiseaux, sur lesquels WaLface s'était surtout basé, ont été réétudiés pour Célèbes, pal Meyer et Wrastaswontr. qui ont montré que la faune ornithologique de cette île est plutôt orientale.

Pour les Poissons et les Crustacés, Max Weber a constaté que les îles de la Sonde, à l'Est de la ligne de WALraCE, ont une faune essentiellement orientale, dont naturellement le caractère oricntal typique va en s'affaiblissant à mesure qu'on avance vers l'Est.

Pour les Mollusques, Fischer et Cooks ont déjì fait des constatations analogues, concluant à l'invalidité de la (ligne de WALLACE». On peut trouver une confirmation de cette manière de voir, pour les Mollusques de Célèbes, dans les publications plus récentes de P. et F. SARAsin. Et l'examen comparatif de la faune malacologique ter'restre et fluviale de toute la région litigieuse m'a démontré fimalement que la limite F. de la province orientale (et de l'A 'octogée conséquemment) doit passer à l'Est de Célèbes et de 'Timor, à l'Ouest des Moluques et de Key-Islands (qui appartiennent à la province australienne et à la Notogée).

Sans doute il y a une légère pénétration réciproque des deux provinces l'une dans l'autre, au delà de la limite séparative (par exemple de la province australienne dans Timor', et de la province orientale dans les Moluques); mais il en est ainsi partout, et nulle part une limite zoologique n'est une ligne sans seconde dimension. Si l'on devait pour ce motif établir des régions de transition, cela compliquerait la question en nécessitant chaque fois deux lignes-limites au lieu d'une seule. Il est certain que la prédominance d'une faune or'ientale d'un côté, et d'une faume australieme de l'autre, suffit pour démontrel le bien fondé de la frontière proposée.

Au reste, des considérations d'or'lle géoph hsique viennent lui donner un appui particulierement solide.

On sait que l'union ancienne (et d'ailleurs temporaire) de l'Asie et de l'Australie a été rompue à la fin du Crétacé.

Dans le creusement qui s'est opér'é entre les deux terres, la ligne sur laquelle existent les plus grandes profondeurs sous-marines indique avec le plus de vraisemblance la direction suivant laquelle la séparation a commencé et suivant laquelle, conséquemment, les deux faumes ont étí le plus complètement et le plus longuement séparées.

C'est sur ce principe (et sur' des différences supposées des faumes mammalogique et olnithologique) que WaLcace s'est surtout basé poul' ('onfirmer le passage de sa ligne de démareation entre Bali et Lombok: on croyait alors, en effet, à une grande profondeur du détroit de Lombol.

Jais jusqu'ici, dans ce domaine, les connaissances étaient demeurées insuftisantes, et diver's géophysiciens ne considéraient pas la ligne de Wallace comme bien fondée à ce point de vue (Krüniser, Supan). 


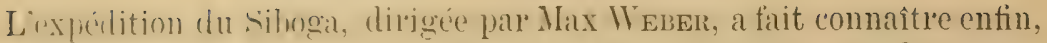
avec beaucoup plus de certitude, les grands traits de la configuration sous-marine de l'Archipel Malais.

La carte publiée dans les résultats hydrographiques de cette expédition (en 1903), est toute différente des plus anciennes, par exemple de colle de Berguaus. Elle montre notamment que la grande profondeur dur détroit de Lombok est erronée; et elle permet de déterminer le thal-

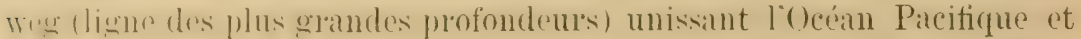
l'Océan Indien, c'est-à-dire la limite géophysique de l'Asie et de l'Australir: il wis constitué par les profoudeur's partout supérieures a $1500 \mathrm{~m}$., et se trouve reporté sur la carte exhibée à l'appui de la présente communiation (la plus grande carte marine de l'Archipel malais: Admiralty, Char't of Eastern Archipelago, 941 B, 942 A, 942 B).

On voit ainsi la concordance de la limite géographique et de la limite faunique; car ce thalweg passe en effet entre les îles à caractère faunique oricutal prédominant, ot colles a caractere famique australien prédominant.

Pour désigner cette ligne ainsi déterminée par deux éléments qui se confirment mutuellement, je propose le nom de "Ligne de WEBER », en l'homneur du zoologiste qui a si bien étudié les faunes terrestres et fluviales de la plupart des îles de la Sonde et qui dirigea l'exploration la plus complète qui ait été faite jusqu'ici, des mers de cet Archipel.

\section{Discussion.}

M. le $\mathrm{D}^{\mathrm{r}}$ J. Roux fait remarquer que le nom de "ligne de Weber " domné par M. Pelseneer à cette nouvelle limite zoologique entre l'Asie et l'Australie n'est pas très heureux, étant donné que Weber n'est pas partisan de ces lignes-limites zoologiques.

Du reste, dans l'état actuel de nos connaissances, ces limites n'offirent plus lointereit quolles pouvaient présenter avant lapparition de la théorio de Dariwis. Les recherches récentes, notamment celles de MI. Sarasin,

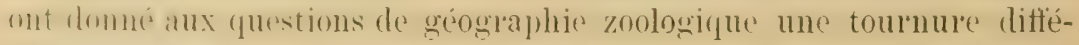
rente et une orientation toute nouvelle. 


\section{Principales lignes de passage des Oiseaux à travers la Suisse et les Alpes.}

Par le $D^{r}$ V. FATIO (Genève).

Quelques auteurs, depuis tantôt quarante ans, ont publié de temps à autre des notes relatives aux migrations de certaines espèces dans le pays. ou aux passages dans telle on telle localité '. Toutefois. ces données, si intéressantes fussent-elles, étaient ou trop circonscrites, ou, par contre, trop générales pour permettre d'indiquer avec un peu de précision les principales routes suivies par les Oiseaux it traver's la Suisse ou par-dessus les Alpes. Le sujet est vaste; je tâcherai de le résumer autant que possible.

Des 357 ou $35 \check{8}$ espèces d'Oiseaux qui se sont rencontrées en Suisse, 36 sont franchement sédentailes, en ce sens qu'olles ne fournisisint gnere de contingent à lémigration, 39 sont à la fois sédentaires ret de pastagere, 107 sont hôtes d'été ou purement nicheuses, 37 sont hôtes d'hiver', 70 sont

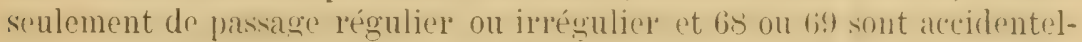
les ou tout à fait exceptionnelles. Si l'on retranche du total 36 espèces

1 Citons plus particulièrement: De LA HaRPE. Renseignements sur la migration des Hirondelles, Bull. Soc. vaud. Sc. nat., VIII, 1865. - SAlis (vov). Beobachtungen über das Wandern der Vögel, Jahresb. der Naturf. Gesellsch. Graubündens, XVI, 1871. - Gulluadme Ch., Migration des Hirondelles, Rameau de Sapin, IX et X, 1875 et 1876. - Мєеsсн C. Wohin und Warum ziehen unsere Vögel; Neujahrsblatt, Zürich, 1877. - Fatio V. Les Hirondelles (passages el séjour); Diana, III, nos 9 et 10, 1885-1886. - Schweizerische Blätter (Auct?), Die Vogelzugstrassen der Schueiz (Innthal), 1889. - Zschokke Fr. Wandertrieb und Wanderungen der Vögel; Vortrag in der ornith. Gesell. zu Basel, 1892. - Fatio et Studer. Catalogue des Oiseaux de la Suisse (avec cartes de distribution), fasc. I-III, Genève et Berne, 1889-1901. - Schenk J., Die Beobachtungen des Vogelzuges in der Schweiz; Orn. Beobachter, Berne, 1903. - Fatio V. Faune des Vertébrés de la Suisse, Vol. II, part. 1 et 2, 1897 et 1904. - Fischer-Sig wart, Biologie der Ornis; Eröffnungsrede der Schw. Naturf. Gesell. in Zofingen, 1901. - Bung G. (von), Ueber den Zug der Vögel durch die Schweiz; Ornith. Beobachter, III, Jahrg. Heft 1, 1904; article en roie de publication, paraissant traiter surtout de la plaine suisse et, plus particulièrement des environs de Soleure.

VI• CONGR. INT. ZOOL., 1904. 
completement sédentaires, on se trouve en face de 321 ou 322 qui toutes se déplacent plus ou moins.

Les Oiseaux, ver's la fin de l'hiver et au printemps, nous viennent, pour' la plupart, du sud, des contrées méridionales de l'Europe ou du nord de l'Afrique, où ils ont passé la mauvaise saison. Les uns vont nichel à diffél’ents nireaux, sur notre sol ou dans les pays voisins du Rhin et du Haut Danube; les autres passent pour aller se reproduire plus loin du côté du nork, en Scandinavie, quelques-uns même jusque dans les régions polaires. C'est de ces contrées plus septentrionales que nous arrivent, en sens inver'se, nol'd-nord-est à sud-sud-ouest à peu près, entre la mi-été et le commencement de l'hiver, soit les nombreux Oiseaux qui ont traversé la Suisse au printemps, soit diverses espèces qui, chassées par les frimas

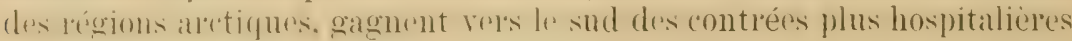
et prennent en partie leurs quartier's d'hiver chez nous, sur nos lacs principalement.

Quelques espèces seulement nous viennent de l'est, comme la Mésange

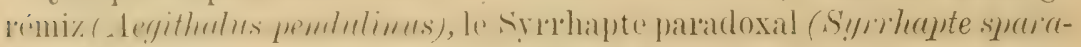

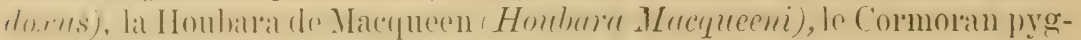
méc (Phatacrocorax pygmauns), le Pélican blane (Pelecanus onocrotalus) et d'autres. Ce sont des Oiseaux, rares daus le pays, qui ne font chez nous que des apparitions irrégulières, et cela, le plus souvent, en dehor's des époques de migration habituelles. Nous n'avons pas, en réalité, de ligne de communication avec l'est, et les espèces orientales qui font parfois des incursions en Suisse peuvent y être considérées, pour la plupart, comme Oiscaux étranger's crrants ou égarés.

De l'ouest, enfin, nous ne r'ecevons rien, si ce n'est, de temps à autro, un sujet dévoyé d'espèce marine ou même pélagique, habitant de l'Atlantique jeté sur le continent par quelque tempête, qui s'est égaré jusque dans nos parages où il arrive généralement harassé et plus ou moins malmené. C'est ainsi que l'on a r'encontré quelquefois en Suisse le Pétr'el glacial (Fulmarus glaciatis), les Puffins cendrés et Manks (Puffinus cinereus et $P$. amglonum), le Thalassidrome tempête (Procellaria pelagica), le Guillemot à capuchon (Uria troile), le Lumme à miroir (Cepphus grille), le Pingouin macroptère (Alca torda) et le Nacareux moine (Fratercula (a) cticu).

Ne pourant traiter ici, en détail, ni des agissements de nos différentes especes en voyage, ni des circonstances et des perceptions qui plus ou moins servent à guider celles-ei dans lem's migrations, je me bolnerai à répartir daus trois grands groupes tous les Oiseaux qui, en diverses conditions, traversent notre pays; trois groupes, un peu arbitraires peutétre, mais capables néammoins d'expliquer jusqu'à un certain point l'abondance ou la rareté compar'ée des royageurs sur telle ou telle route. de jour ou de nuit. 
Je distinguerai done: $1^{\circ}$ de forts voiliers qui, voyageant haut, volontiers en bandes et fournissant de longues citapes, comme les Grues, les ('igognes ou les Oies, peuvent traverser la Suisse en quelques heures, en passant par dessus les Alpes, parfois mème auldessus de soou it 3500 metres d'altitude, ainsi que je l'ai pu voir sur le Théodule, à 3322 mètres s. $/$ m. $-2^{\circ}$ De moyens voiliers volant moins haut, en troupes plus ou moins lâches ou compactes, et faisant des haltes fréquentes, comme les Colneilles, les Etourneaux, les Hirondelles, les Pigeons, diver's Lichassier's, des Laridés, etc., qui suivent tour à tour les chaînes de montagnes favorablement orientées ou les cours d'eau, coupant ou enfilant les vallées selon l'élévation de celles-ci et passant souvent des cols entre 1700 et 2500 mètres s./m. - $3^{\circ}$ Des migrateurs plus petits ou de moindre envergure, qui voyagent plus près du sol, de proche en proche, en plus ou moins nombreuse compagnie, en famille, par paires ou isolément, s'arrêtent à tout propos, en suivant les vallées, des lisières de forêts ou les moindres ruisseaux, comme beaucoup de Passereaux (Bec-fins, Fringilles, Bruants, Pipits, et autres), des Rallidés, etc., et sont susceptibles d'atteindre ainsi, en passage, au même niveari que les précédents.

Signalons, en passant, que, au moins pour nos hôtes d'été dans les deux derniers groupes, les représentants de certaines espèces qui ont niché dans des régions élevées préfèrent souvent redescendre en automne la vallée dans laquelle ils se sont engagés au printemps, pour gagner plus bas une route plus large et plus sûre, plutôt que de se hasar'der sur les glaces et les neiges éternelles. C'est ce qui se voit plus ou moins chez les Hirondelles, les Pouillots, les Bruants et d'autı'es, même chez la Bécasse. Il s'agit là de véritables émigrants qu'il ne faut pas confondre avec les sujets erratiques de quelques espèces sédentaires qui changent de niveau à l'approche de la mạuvaise saison.

La direction générale des vents dominants peut guider plus ou moins les Oiseaux migrateur's, aussi bien s'ils volent contre le vent, comme de forts voiliers, que s'ils marchent vent arrière, ainsi que beaucoup de petits Passereaux, ou s'ils prennent momentanément le biais du courant, quand la pression devient trop forte. Cependant, un vent trop violent ou en tempête coupera généralement le passage, ou égarera bon nombre de voyageurs.

Il semble que des lueurs, crépusculaires ou autres, puissent exercel aussi une attraction sur les Oiseaux très nombréux qui voyagent de préférence de nuit ou soir et matin, avant le level' du soleil. L'éclairage brillant d'une ville ou l'éclat d'un phare suffisent, entre autres, à tromper' et dérouter jusqu'à un certain point les migrateurs passant dans le voisinage. Qu'il me suffise de citel comme exemple les pluies de Cailles qui, assez souvent, sont tombées sur la ville de Genève, placée précisément au point de concentration des passages de la plaine suisse. La lueur du gaz 
attire, en effet, et fait abattre, parfois en très grande quantité, dans les lues et promenades de cette ville, les Cailles voyageuses que les promeneur's matinenx peuvent voir encole courir dans les squares ou le long des trottoirs.

La vue, si développée chez les Oiseaux, doit contribuer encore, doublée de la mémoire, à diriger mu grand nombre de migrateur's dans nos trois groupes, qu'ils voient de haut et de loin les contrées déjà traver'sées ou qu'ils recommaissent, plus près du sol, les moindres particularités du terrain déjì paı'couru. Le fait que, pour diverses espèces rares ou accidentelles, ce sont d'ordinaire des jeunes seulement que nous rencontrons égarés dans le pays, semble bien corroborer l'importance du souvenir dans la question du choix ou du maintien de la bonne direction. Selon la saison, lus conditions locales et les cirenntances atmosphériques, les diffél'ents Oiseaux ne conservent, du reste, pas toujour's la ligne de passage la plus directe et sont contraints souvent à d'assez grands détours.

Le voyage vers le nord et les lieux de nichée, au printemps, est toujour's plus court, plus pressé et souvent plus direct que celui du retour au sud, cu automne, entrepris en vue de la nourriture nécessaire et tou-

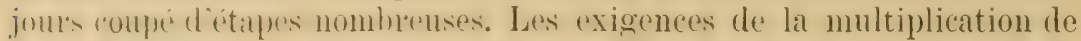

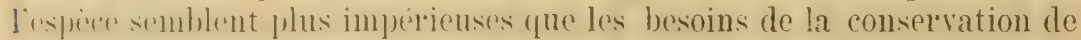
l'individu. L'amour', avec le souvenir du berceau, de la famille ou de la patrie, est un grand mobile. Le mal du pays, le spleen, peut devenir, en effet, chez quelques animaux, l'Oiseau migrateur entre autres, au printemps, une importante force directrice, quasi-instinctive; résultant de perceptions délicates reçues par des organes particuliers. Pourquoi n'y aurait-il pas, par exemple, un certain rapport entre la grande extension de l'air dans les os évidés et les sacs aéxiens, à la fois baromètres, hygro-

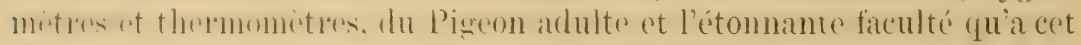
Oiseau de retourner à son pigeonnier, même à de grandes distances, par' des chemins qu'il n'a jamais parcourus.

Ajoutons que, pour bien des Oiseaux qui vont nicher assez avant dans le nord et font, au printemps, une mue presque complète, comme beaucoup d'Anatidés, des Colymbidés et autres, il importe fort de ne pas se laissce surprendıe en voyage par la chute de leur's rémiges qui, pour quelques semaines, les priverait de leurs moyens de locomotion.

En abordant maintenant la question des principales lignes de passage it traver's la Suisse et les Alpes, but de ces pages, je in'occuperai done surtout des migrations d'automne (commençant déjà dès la mijuillet pour certains (Oiseaux), puisque, comme nous venons de le voir, le's royages vel's le sud sont généralement moins rapides et par le fait plus faciles à étudier que ceux qui sopèrent au printemps, en sens invelse, ver's le nord et les lieux de nichée. 
La plus la r'ge voie qui s'offle aux Oiseaux arrivant, du côté du nord, sur nos frontieres septrintrionales, et de brameoup) la plus frérpuentér, est, sans contredit, celle qui passe par la plaine suisse, entre Alpes et Jura et le long de ce dernier, conduisant du nord-est au sud-ouest et au midi, sans passol' par les Alpes; cela, à traver's les régions inférieures des bassins de l'Aar', de l'Emme, de la Sarine, de la Broye et du Léman, dans un pays riche en bois, cultures divel'ses, cour's d'cau, lacs et marais, en majeure partie entre 252 et 850 mètres d'altitude. Il y a là comme une sorte d'entonnoir qui rassemble non seulement la majeure partie de ce qui nous vient des pays du Rhin, mais encore quantité de nos hôtes d'été des régions limitrophes qui, cux aussi, profitent de la grande route pour gagner leurs quartier's d'hiver. C'est au rétrécissement dudit entonnoir ver's le sud-ouest, près de Genève, que les environs de cette ville doivent de compter au passage presque tous les visiteurs régulier's de la Suisse. Dans la même direction, une ligne voisine passe aussi, à l'ouest de Bâle, de l'autre côté du Jur’a, joignant le Doubs et la France.

Une seconde route, qui conduit une moindre proportion des émigrants du Rhin par le centre de notre pays, diverge de la précérlente avec le cours de la Reuss et quelques vallées avoisinantes, se dirigeant, du nord au sud, directement vers le Tessin et l'Italie, par le lac des Quatre-Cantons, le Gothard, la Léventine et la Riviera. Quoique plus directe que la première, elle compte moins de voyageurs, probablement par le fait de l'étroitesse et de la pauvreté relative de son parcours supérieur aboutissant à un col élevé, 2114 mètres sur mer.

Une trois i è me voi e, bien plus fréquentée que la précédente, reçoit, à l'est $\mathrm{du}$ pays, diver's contingents d'émigrants arrivant: les uns par le coude transversal que fait le Rhin sur notre frontiere septentrionale, les autres du lac de Constance ou du bassin de la Thour et. en suivant

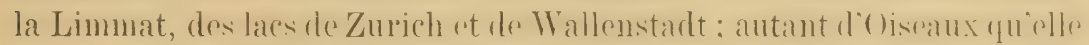
dirige, par le Rheinthal, directement vers le sud, à travers divers cols élevés conduisant soit en Italie, comme le Splügen à 2117 mètres, soit dans le 'T'essin, comme le Bernhardin à 2063 mètres ou le Lückmanier à 1987 mètres s./m. Cette ligne voit peut-être un peu moins de voyageurs que celle de la plaine suisse qui évite les Alpes; mais, plus courte, elle semble nous amener certaines espèces un peu plus tôt au printemps que celle-ci.

Enfin, une quatrième route, à l'extrême est du pays, passe par' l'Engadine, menant, du nord-est au sud-ouest et au sud, les Oiseaux qui ont suivi le cour's supérieur de l'Inn et passent les uns par le col de la Bernina, à 2334 mètres s./1m., pour tomber dans la Valteline, les autres jar la Maloja, à 1811 mètres, pour rejoindre la route précérlente en Italie. Cette dernière voie, quoiqu'une des principales, parâtt bien moins fré- 
quentée que celle du Rheinthal. Peut-être cela tient-il à la forte inclinaison ver's l'est de la vallée en dehor's de nos frontières; car', bien que tributaire du Danube, l'Inn ne nous amène pas d'autres espèces que celles qui nous vicnnent des pays du Rhin. Cependant, si bien des Passereaux font défaut à la Haute-Engadine, on y voit par contre, grâce à

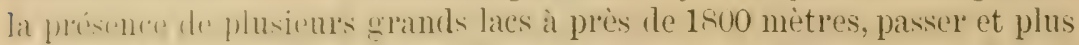

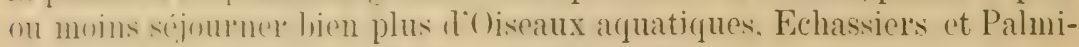
pèdes, que dans la plupart de nos vallées alpines.

La vallée du Rhône, en majeure partie orientée de l'est-nord-est à l'ouest-sud-ouest et l'une des plus grandes du pays, n'a pas à vrai dire de ligne de passage propre. C'est comme un grand collecteur lecevant de diver's côtés les voyageur's qui, depuis le Rheinthal à l'est, juriulau Léman ì l'ouest, ont manqué les loutes latérales, passages déjà en partie cités, pouvant les mener directement au sud. Bien des Oiseaux qui, en suivant le cours supérieur du Rhin, puis le bras principal de la limss, ont traverse sucessivement loberaly à 2052 metres et la Furka à 2436 mètres s./m., ar'rivent, en effet, dans le Valais, entre les hautes barrieres do névés ot de glaciers des Alpes septentrionales et méridionales; prulant que diutres, mgagés, plus au nord, daus les régions supérieures des bassins de l'Aar, de la Kander, de la Simme et de la Sarine, sont igalement amenés dans la vallée du Rhome par des cols felévés. comme le

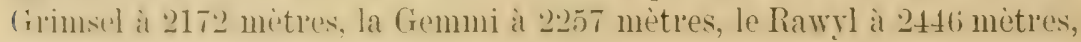
et le Sanetsch à $230 t$ mètres s./m. où l'on a trouvé divers Oiseaux morts sur la neige, une Foulque (Fulica atra) et une Canepetière (Otistetrax) par exemple. Un nombre relativement très restreint de ces voyageurs

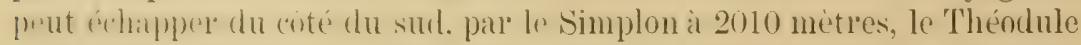
à 3322 mètres ou le Grand St-Bernard à 2492 mètres s./m., quand il n'y a pas de neige flitiche sur les hauteurs. La plupart, guirlés par le Rhône, sont emmenés, après ce long détour, vers l'extrémité orientale du lac Léman et à Genève, par la rive savoyarde. Ceux-là même qui, en passant la Forclaz, à 1523 mètres s./m., ont gagné la vallée de Chamonix reviennent aussi en partic du côté de Genève, en suivant le cours de l'Arve. 


\title{
Ueber die Entwicklung der Fisch-Fauna von Mittel-Borneo.
}

\author{
Von Dr. C. POPT'A (Leiden).
}

Dank den kühnen Reisen von Prof. Dr. A. W. Nieunenuurs und Di: J. B̈̈тTKofer besitzt das zoologische Musenm in Leiden eine reiche, gut konservierte Sammlung von Fïschen aus Nittel-Bol'neo. I) ie Sammlung, welhe genamnte Herren in den Jahren 189:-1897 am Ober-liapuas und Ober-Mahakam für das Leidener Juseum zu Stande gelneacht haben, ist von Professor L. Varluant ${ }^{1}$ vorzüglich bearbeitet worden. Die Fischsammlung dagegen, welche Nibuwexur is in dru Jahren 1s98- 190() aus dem Bongan, einem (quellthuss des ()ber-Kapuas, aus dem Howong und Bo, Seitenflüssen des Ober-Mahakam, und ans dem Ober-Kajan mitgebracht hat, sind mir zur Bearbeitung übergeben worden.

Von den mannigfaltigen interessanten Beobachtungen, welche ich bei dre Cntersuchung der erwähnten Sammlung habe machen lï̈nnen, sei mir hier gestattet, die folgende mitzuteilen.

Reine Süsswassel- Fischarten von verschiedenen Inseln oder aus velschiedenen Gegenden, welche sich also über Ströme, die gar keine Verbindung mit einander besitzen, verbreitet haben, müssen ältere Arten sein als solche, welche kleine Gebiete, z. B. ein Flusssystem oder auch noch damit in Terbindung stehende (rewässer brwohnen. Das gilt natürlich nur so limnge, als keine Menschenhände in dio Zustande cingeroriffen haben. Denn wenn zwei Inseln in ihren Flüssen mehrere gleiche Süsswasser-Fischarten miteinander gemein haben, kann man sich vorstellen, dass in früheren Perioden zwischen ihmen eine Verbindung bestanden hat. Aus diesen älteren, weit verbreiteten Arten können sich später auf jeder Insel oder in jedem Strom durch irgendwelche Einflüsse ihrer Umgebung neue Arten entwickelt, differenziert haben. Betrachten wil von diesem Standpunkte aus dir Fische Mittol-Bol'neos, so ist es nicht ohne Interesse zu untersuchen, in welchem Verhältnis die Oberläufe des Kapuas, Mahakam und Kajan ihne msprünglichen Fische lohalten hathen und in welchem Verhältnis dagegen Differenzied'ung derselben stat1gefunden hat. Zu diesem Zweck betrachten wir von den in den genann-

${ }^{1}$ Notes from the Leyden MIuseum, Vol. XXIV. 
t’u ()herläufen vorkommenden Fïschen die folgenden siehen echten Süss-

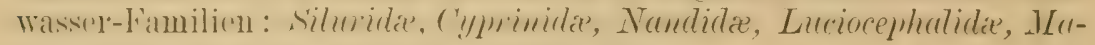
stacembetida, Ophiocephatidae, Labyrinthici.

Wie bekannt, sind die Arten dieser Familien über dass Süsswasser mehrerer Inseln oder mehrerer Gegenden des Festlandes verbreitet. In Mittel-Borneo sind von ihnen bis jetzt vierzig Genera mit zusammen

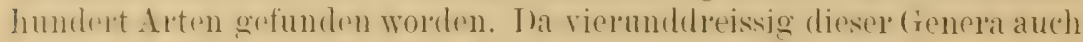
auf anderen Inseln beobachtet worden sind, müssen sie aus einer Zeit herstammen, wo Borneo noch nicht seine jetzige Gestalt besass. Sechs Genera sind daher Borneo eigen, also $15 \%$, von den hundert Arten gehölen neununddreissig, also $39 \%$, speziell Borneo an.

Dies sind natürlich keine absoluten Zahlen, denn wir kennen noch nicht sïmtliche Fische Mittel-Lorneos. Sie conen num den jetzigen. lülikenlaften Stand der Kenntnis von dreien der Uberläufe Mittel-Borneos an, doch liefern sie den interessanten Beweis, dass Borneo ausser der älteren Fischlovölkerung des Süsswassers, wolche auch auf anderen Inseln oder in verschiedenen Festlandgegenden verbreitet ist, eine ziemlich

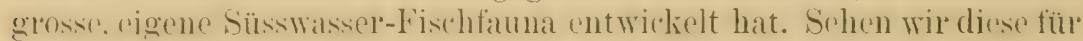
Mittel-Bol'neo noch einen Augenblick an. Die neuen Genera und Arten gehören alle zu den Siluriden und Cypriniden. Von deu fünf anderen Süsswasser-Familien sind für Mittel-Borneo noeh keine neuen Arten be-

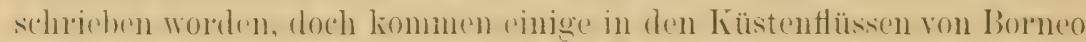
vor, wie der Ophiocephalus rhodotienia Blkr. im Sambas, an der Westküste. Von den sechs Genera sind zwei Silwiden, vier Cypriniden. Gustrom!zam ist sowohl im Mingalongfluss (Nolol-Borneo) als im (OberKapuas mol ()her-Mahakam gefunden worelen; die fünt anderen hat man bis jetzt nur in e in em Fluss entdeckt. Gastromyzon, der in m ehrer en Flüssen der Insel vorkommt, kann also älter sein als die fünf anderen;

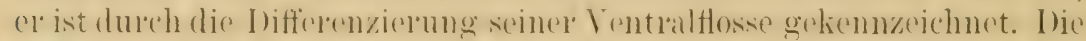

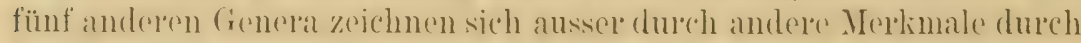
kleinere oder grössere Verschiedenheiten am Kopfe aus. Bei Gyrinocheilus ist der Mund typisch ausqehildet. seine Atmungs- und Verdaunges-

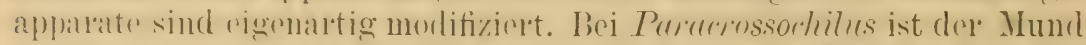
ohenfalls charaliteristisch. Pulhomaloptere ist an dor Modifizierung der

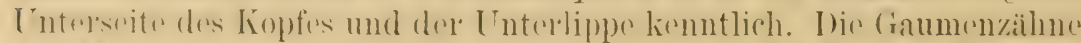

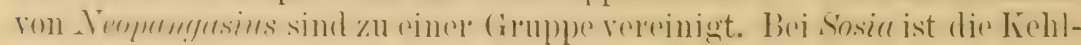
anheftung hinter einer Falte der Oberhaut versteckt.

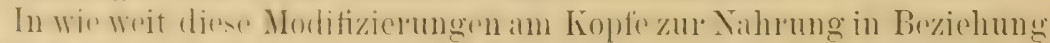

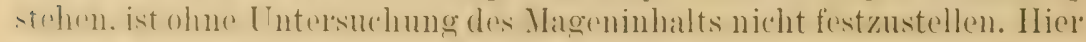
will ich nur mitteilen, dass ich den Mageninlalt derjenigen Arten der letzten Fischsammlung aus Borneo, von denen mehr als drei Exemplare

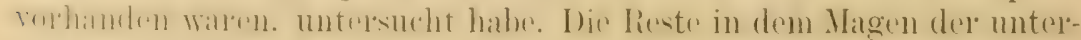
suchten Siluriden-Arten waren erkemubar, und zwar waren sie bei jeder 
Art verschieden; bei keinen zwei Arten fand ich gleichsortige Reste. Der Magen der bis jetzt untersuchten Cypriniden-Arten war entweder leer, oder sein Inhalt war nahezu unerkennbar.

Von den neunundlreissig neuen Arten Mittel-Borneos gehören achtzehn zu den Siluriden, einundzwanzig zu den Cypriniden. Nun fragt es sich, in wie weit die drei Oberläufe einc eigene Entwicklung besitzen. Dreissig Arten sind bisher nur in ei in e mluss gefunden worden, von diesen gehören.dem Ober-Kapuas sieben, dem Ober-Mahakan zwanzig, dem Ober-Kajan di'ei an. Der' Ober-Kapuas hat drei Arten mit Nord- und Nordwest-Borneo, zwei mit Süd-Borneo, drei mit dem OberMahakam gemeinschaftlich. Dieser besitzt, ausser den genannten drei Arten mit dem Ober-Kapuas, noch je eine Art mit Nor'd-Borneo, eine mit Süd-Borneo und eine mit dem Ober-Kajan gemeinsam. Der Ober-Kajan hat, wie gesagt, eine Al't mit dem Ober-Mlahakam gemein.

In meiner demnächst in den "Notes from the Leyden Muscum " erschemenden Arbeit über die letzte von Nieurvenhurs aus Bolneo mitge-

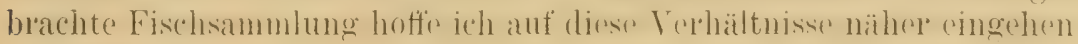
zu können.

\title{
Jeber die Verbreitung der Fische im nördlichen Stillen Ozean und die damit zusammenhängenden zoogeographischen Probleme.
}

\author{
Von P. SCHMidd (St. Petersilsurg)
}

Mit 2 Textfiguren.

Die Ichthyofauna des nördlichen Stillen Ozeans war bis zur letzten Zeit sehr wenig erforscht. Wenn wir yon den alten Arbeiten von Stelder, Tilesius und Pallas absehen, die für die Kemntnis der Ichthyofauna wohl grundlegend, doch aber gänzlich unzureichend waren, ein Bild von der Zusammenstrellung und Vorblestung der Ichthyofiuma zu gehen, so hahen wir nur eine Reihe kleinerer Arbeiten von Kner, Steindachner, Gurcheмот u. a., die nur eine Anzahl von Beschreibungen neuer Arten enthalten, zu verzeichnen. Erst die in den 80-er Jahren vorigen Jahr'-

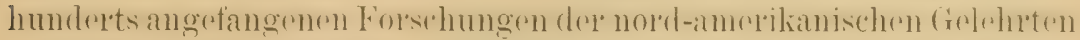
warfen einen Lichtstrahl auf die Zusammensetzung der Ichthyofauna des Berings-Nleeres und del' Gewässer der westlichen Küste NordAmerikas. Besonders ergiebig waren die Resultate der mehrjährigen 
Forsehungen des berühnten amerikanischen Expeditionsschiftes "Albatross», der vom Jallve 1888 an und bis zum heutigen Tage in pacifischen Gewässern eine reiche wissenschaftliche Ernte sammelt. Die gesammten Resultate der amerikanischen Fol'scher aut ichthyologisehem Gebiete sind vol kurzem in 2 grösseren Arbeiten von Jordan - A. GILBERT und von Jordan - A. Evermann zusammengefasst worden und dies. Werke bilden bis jetzt ras Wichtigste und Unfangreichste was auf diesen Gebiete geleistet wurde.

Wir gesagt, el'streckten sich die For'shungen der englischen Ichthyologen mur auf die amerikanischen Gewässer und das Berings-Meer. der westliche Teil des nördlichen Stillen Ozeans dagegen. nämlich das Ochotslisiche und das Japanische Meere, die als Randmeere sich an den Ozean anschliessen, blieben gämzlich unerforscht. Dic Ichthyofauna von Japan war seit der bahnbrechenden Arbeit von Schlegel viel untersucht worden, - sie unter'scheidet sich aber von der Fauna der nördlicheren Gewässer ganz bedeutend und hat mit ihr beinahe gar nichts Gemeinsames.

In der Inoffuung ciniges zur linntnis der Fauna dieser ( zutragen, unternahm ich auf Teranlassung und mit Unterstützung unsener Kais. Russischen Grographischen Gesellschaft in den Jahren 1900 bis 1901 eine Reise nach dem Weiten Osten und arbeitete während beinahe 2 .Jahren an den Ufern des Japanischen und des ()chotskischen Meres. Leider verfügte ich mur üher sehr geringe Geldmittel und hatte auch kein eigenes Fahrzeug, - auf diese Weise konnte ich während

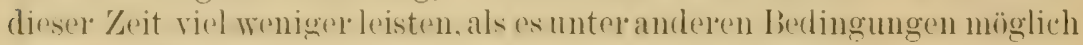
gewesen wïre. Dessenungeachtet gelang es mir ziemlich reiche Sammlungen nach Hause zu bringen, die jetzt teilweise an Spezialisten verteilt, trilweise sehon beatheitet sind. I) Bearlestung der idhthyologischen Sammlung übernahm ich selbt und habe im Laufe der letzten 2 Jahre ausser den meinigen auch alle in unseren Museen befiudlichen aus dem Nord-Japanjichen und drum Ochotskischen Meree stammenden Fische bearbeitet. Es ergaben sich im Ganzen 144 Arten Fische, darunter 23 neue Arten und 5 neue Genera. Ausserdem habe ich auch, wie mir scheint, ziemlich interessante Resultate in Bezug auf die Zusammensetzung und den Charakter der Fauna des Nord-Pacifischen Ozeans erhalten und über diese Resultate möchte ich der hochverehrten Versammlung heute berichten, zumal ich einen Versuch gemacht habe, meine Befunde auch mit einigen Fragen von allgemeinem Interesse in Zusammenhang zu bringen.

Vor allen Dingen ist es notwendig eine, wenn auch ganz allgemeine Vurtellung ühor dir phỵikalisehen Bedingungen der zu briplechenden Gewïsser zu geben.

Es genügt, einen Blick auf die Karte zu werfen, um sich zu überzengen, 
dass sich der nördliche Stille Ozean in ganz anderen Bedingungen hefindet als der nördliche Atlantische. Der letztere steht in einer breiten Terbindung mit dem Eismeere, wogegen sich der Stille ()zean mit ihm nur durch die schmale und nur jo Meter ticfe Beringsstrasse verbundru ist. Ausserdem richtet sich, dank den topographischen und bathymetrischen Bedingungen, die grossartige warme Strömung des Atlantischon Ozeans, der Golfstrom, direkt nach Norden und bringt Wärme und Leben an die nördlichen Gestade von West-Europa. Fine gleichartige Strömung des Stillen Ozeans - der Kuro-Shiwo - wird durch eine ununterbrochene Inselkette, die sich von den Philippinen his nach del Halbinsel Alaska hinzieht, von den asiatischen liontinente abgelenkt und richtet sich im Süden von Alaska nach Kalifornien hin. In das Japanische Meer dringt nur ein schwacher Zweig dieses Stromes hinein, der sich der West-Küuste von Japan und Sachalin entlang hinzicht; das ()hotskische Meer wirl gar nicht von den warmen Strome heeintlusst, das Beringsmeer auch nur verhältnismässig wenig in seinem südlicheren Teile. Diese Umstände nun veranlassen in dem Japanischen, Ochotskischen und in dem Berings-Meere die Anwesenheit von Bedingungen die von denjenigen an den europäischen Ufern im Grunde verschieden sind, einigermassen aber au die Bedingungen an den Ost-Ufern von Nor(l-Amerikit erinnern. Alle drei.Meere sind verhältnismäsigsehr kalt, besonders das Ochotskische, das während 5-6 Monate im Jahre mit Eis bedeckt ist und schon in der Tiefe von 100-200 Meter eine Wasser-'Temperatur von nahe an $0^{\circ}$ oder sogar unter $0^{\circ}$ aufweist. Das Beringsmeer ist auch kalt in seinem nördlichen Teile. Das Japanische Meer zerfällt in 2 Teile: das Nord-Japanische Meer wird in Winter teilweise mit Treibeis bedeckt und friert an den Ufern zu, das Süd-Japanische ist dank der schon erwähnten warmen Strömung, die den WestUfer'n von Japan und Sachalin entlang zieht, bedeutend wärmer', die Grenze zwischen den 'Teilen muss aber aus faunistischen Gründen nicht dem Parallelkreise entlang, sondern schräg gezogen werden - etwa von der Brounhton-Bai zur Südspitze von Sachalin. In den tieferen Scinichten ist auch das Japanische Meer sehr kalt, besonder's das Nord-Japanische - hier finden wir schon überall an 100 Meter Tiefe $+2-+1^{\circ} \mathrm{C}$. und an 200-400 Meter sogar $+1-0^{\circ}$ C. Im Gegenteil werden dic oberen Schichten und die geringeren T'efen im Japanischen Meere, wio anch in dem südlichen Teile des ()ehotskischen. im Sommer stark durchwäl'ment, da diese Meere doch unter verhältnismässig niederen Breiten liegen und einer bedeutenden Insolation unterliegen.

Wir sehen, dass das Nord-Japanische, das Ochotskische und das Berings-Neer, obgleich sie sich in Einzelheiten von einander unterscheiden, doch darin mit einander übereinstimmen, dass sie alle drei kalte Meere vorstellen; sie unterscheiden sich in dieser Beziehung 
ganz bedeutend von den Nachbargewässern - nämlich von dem SüdJapanischen Merere, von den Gewitserm der Ost-Küuste Japatns und von den Gewäissern der West-Küste von Nord-Amerika im Süclen von der AlaskaHallsincel, da alle diese Mrerestheile nie zufrieren und auch vom Packeis gar nicht bedeckt werden, ausserdem haben sie auch viel höhere 'T'emperituren in den tieferen frhichten und eine berleutend gleichmässigere Oherflüchentemperatur während des ganzen Jahres. Wenn man unsere drei östlichen Meere mit den verschiedenen Theilen des Atlantischen Oceans zusammenstellen wollte, so könnte man sie nur mit den Gewässern nördlich von Cap Cod, bei New-Founland, bei Labrador, bei Grönland. hei spitzhergen und Vowaja-Semlja, teilweise auch hei der Murman-Küste (Lappland) vergleichen.

Wenden wir uns nun zur Fischfauna und versuchen wir zu ermitteln, inwioforn sio mit den physikialischen Bodingungen üboreinstimmt.

Wenn wil die Resultate sowohl meiner Forschungen, wie auch diejenigun frührere Forscher und inshesondere der anerikanischen Ichthyologen in Form einer Verbreitungs-Tabelle zusammenfassen, so ergibt sich, dass in dem Nord-Japanischen, dem Ochotslischen und dem Beringsinere 250 drten Fische (Tiefsecticche ausgeschlossen) im ganzen bekamnt sind. Wenn wir nun die Verbreitung dieser 250 Arten in den betreffenden drei Meeren genauer untersuchen, so überzeugen wir uns vor allen Dingen davon, dass alle drei Meere eine ungemein ähnliche Ichthyofama bexitzen; die allen diesen . Iereren gemeinsamen Arteu hilden 38" "der gesammten Ichthyofauna des Vord-Japanischen Veeres. eloenfalls $38 \%$ - des Ochotskischen Meeres und $27 \%$ - des Berings-Meeres. Wenn man aber je beide benachbarten Meere vergleicht, so bemerkt man cine noch gröscre Uoberinstimmung der Fannen, so hat das TordJapanische Meer mit dem Ochotskischen $65 \%$ gemeinsamer Arten, das Ochotskische mit dem Berings-Meer - 56\% gemeinsamer Arten.

Andererseits strllt aber die Ichthyofauna dor betreftenden 8 Meere ein sehr eigenartiges Ganzes vor und ist sehr wenig mit den Faunen des Süd-Japanischen Mereses. del ()st-Küuste Japaus und der West-Küste von Nord-America verwandt. So hat das Nord-Japanische Neer mur $17 \%$ Arten mit dem Süd-Japanischen gemeinsam, das Ochotskische Meer sogar nur $2 \%$ und das Berings-Neer gar keine; mit der Fauna der Gewässer der West-Küste von Nord-America hat das Nold-Japanische Neer $19 \%$ Arten gemeinsam, das Ochotskische $22 \%$ und das BeringsMere :39)" "alue meistentheils dringen diese gemeinsamen Arten nicht südlicher als bis nach dem Golfe von Alasca vor', und nur ein kleiner

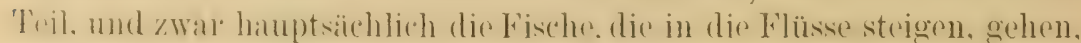
noch südlicher - bis nach Californien hin.

Schr interessante Verhältnisse ergeben sich nun auch bei ciner näheren Untersuchung der Zusammensetzung der Faunen von 3 unserel 
östlichen Neere. An dex Zusammensetzung dep Fauma des Tord-Japanischen, des Ochotskischen und des Berings-1Deeres nehmen hamptsächlich die Fanilien theil, die für die kalten. nordischen Gewässele characteristisch sind. I) He Hauptrolle spielen: Cottidex, Agomidle, Golmonider, Blenniidx, Pleuronectidx, - und diese 5 Familien bilden in jedem von den 3 Meeren ungeführ $2 / 3$ der gesammten Ichthyofauna. Bekanntlich sind die 3 ersten Familien hauptraichlich fül die kilten (rewïssere chatrakteristisch und von Pleuronectiden und Blenniiden sind auch nur die arctischen Formen in den uns interessierenden Meeren vertreten.

J)ic sehr ähnliche Zusimmensetzung der Ichthyofauma des Nolol-Japanischen, des () thotskischen und des licrings-Mecher weist wiedel darauf hin, dass diese Faunen in einer sehr nahen Beziehung zu einander stehen. Die Nachbargewässer sind aus vollkommen anderen nicht nur Arten, sondern auch familien zusammengesetzt. So finden wir schon im Sürl-Japanischen Meere eine hohe Fntwickelung von Serraniden, Sipariden, Labriden, Sciaeniden und anderen südlichen Gruppen; dasselbe finden wir auch an der. West-Küste Amerika's, wo südlicher voin AlaskaGolf eine gainzlich verschiedene, wenn anch nicht so scharf abgegrenzte Fauna auftritt.

Ich kann hier nicht in die Einzelheiten eingehen, glaube aber', dass es mir in meinem demnächst zu "l'scheinenden buche gelungen ist, durch eine solgfältige Analyse der Faunen zwei Thatsachen festzustellen:

$1^{\circ}$ Dass die Ichthyofaunen des Nord-Japanischen, des Ochotskischen und des Berings-Neeres untereinander sehr ähnlich sind und ron den benachbarten Gewässer’n stark abweichen, und

$2^{\circ}$ Dass sie von einem vollkommen nordischen Charakter sind, wogegen die benachbarten Gewässer einen viel südlicheren zeigen.

Diese letzte Thatsache stimmt aber vollkommen mit dem, was wir früher üher die physikalische Natur der betreffenden frevïsser gesagt. haben, überein, da wir gesehen haben, dass die Gewässer selbst nach ihren kimatischen Terhältnissen einen nordischen Charakter tragen und in dieser Bezichung von den benachbarten, die unter dem Eintlusse des warmen Kuro-Shiwo stehen, abweichen.

Auf dieselbe Weise wie nach der physikalischen Natur können auch nach der faunistischen Zusammensetzung die von mir untersuchteu Gewässer nur mit dem Gebiete nördlich von der Golfstromgrenze im Atlantischen Ocean verglichen worlen, also mit den Gewiissern nördlich von ('ap) Corl, hei Labrador', (rrönland, Spitzhelgen, Nowaja Zemlja und theilweise bei der Murman-Küste.

Dieser Vergleich zwingt uns aber auch, die Frage aufzuwerfen, welchem (iohicte dem die uns interessierenden (iowässer zugerechuet werden müssen und wir üherhaupt der Norel-Pacitische und der Nord-Atlantische()ean in zongeographischer Beziehung eingetheilt werden müssen? 
Versuche, auf zoogeographischel Grundlage die Faunen der beiden Oceane einzuteilen, sind schon mehrmals gemacht worden, - so von Dand, Agassiz, Woodward, Günther, Palacky, u. a. Die natüllichste Einteilung wurde aber von OrTsans ${ }^{1}$ gegeben, der zur Grundlage sei-

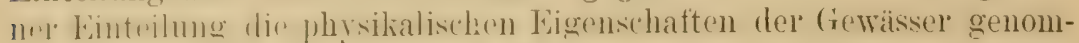
men hatte, die von den anderen Autoren weniger in Betracht gezogen worden waren.

Plof. Ortanan unterscheidet vol allen Dingen 2 Hauptgebiete des Weltmeeres: das trop is che Gebiet zeichnet sich durch verhältnismässig hohe und dabei im Laufe des ganzen Jahres beinahe k 0 n s t a $n$ te Wassertemperaturen, das a $\mathrm{l}^{\circ} \mathrm{k}$ t i s c h e dagegen durech niedrige Temperaturen aus. Dabei wird das arktische Gebiet wiederum in zwei Zonen

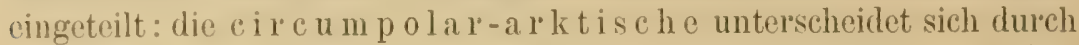
nicdrige und während des ganzen Jahres beinahe konstante und dem Getrierpunkt nahe 'Temperatul', die als b o r eale von Ortunn bezeichnete Zone aber auch durch verhältnismässig niedrige aber dabei wäh-

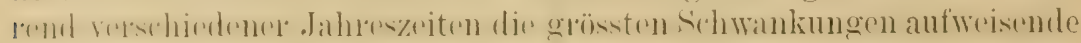
Temperaturverhältnisse. Als Grenze zwischen der borealen und der circumpolar-arkischen Zone nimmt OrTanas die Grenze der Verbreitung des 'Treibeises im Sommer an.

Diese auf der physikalischen Grundlage durchgeführte Einteilung stimmt nach OrTarans mit der Verbreitung der Clustaceen vollkommen ïberein.

Obgleich ich den grossen Verdienst Prof. Ortanans's die erste auf

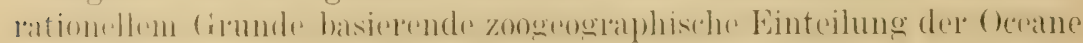
geschaffen zu haben, vollkommen anerkenne, kann ich mich doch nicht in Allem seinen Ansichten anschlicssen. und das besondel's in Betreft des arktischen und des horealen Gebietes.

Die Unzulïnglichkeit der von Ortuax gegebenen Abgrenzung dieser Gebiete ist besonder's angenfällig, wenn wir die Verhältnisse im NordPacifischen Ozean ins Auge fassen: hier wird die Grenze zwischen dem cilcumpolar-alkitischen und dem bolealen Gebiete von Ortwañ del Sommer-Fisgrenze entlang nördlich von der Beringstrasse gezogen, sodass das sïmtliche im Süden yon der Beringstrasse gelogene Gebiet somohl das Berings-Meer, das Nord-Japanische und das Ochotskische Meer. wie auch die Gewässer der Westküste von Nordamerika zu einem gemeinsamen Gebiete, das von Ortumaxs als pacifisch-bol'e al es bezeichnet wird, zusammengezogen werden. Nun habe ich aber schon gesagt, dass der östliche und der westliche Teil des Pacifischen ()zeans, nach meinen Ergebnissen, in der '/usammensetzung der Fauna gainzlich versehieden sind und dabei die östliche Hälfte, das heisst, das

1. (Onтмaxw, Grundzüge der marinen Thiergeographie. Jena, 1896. 

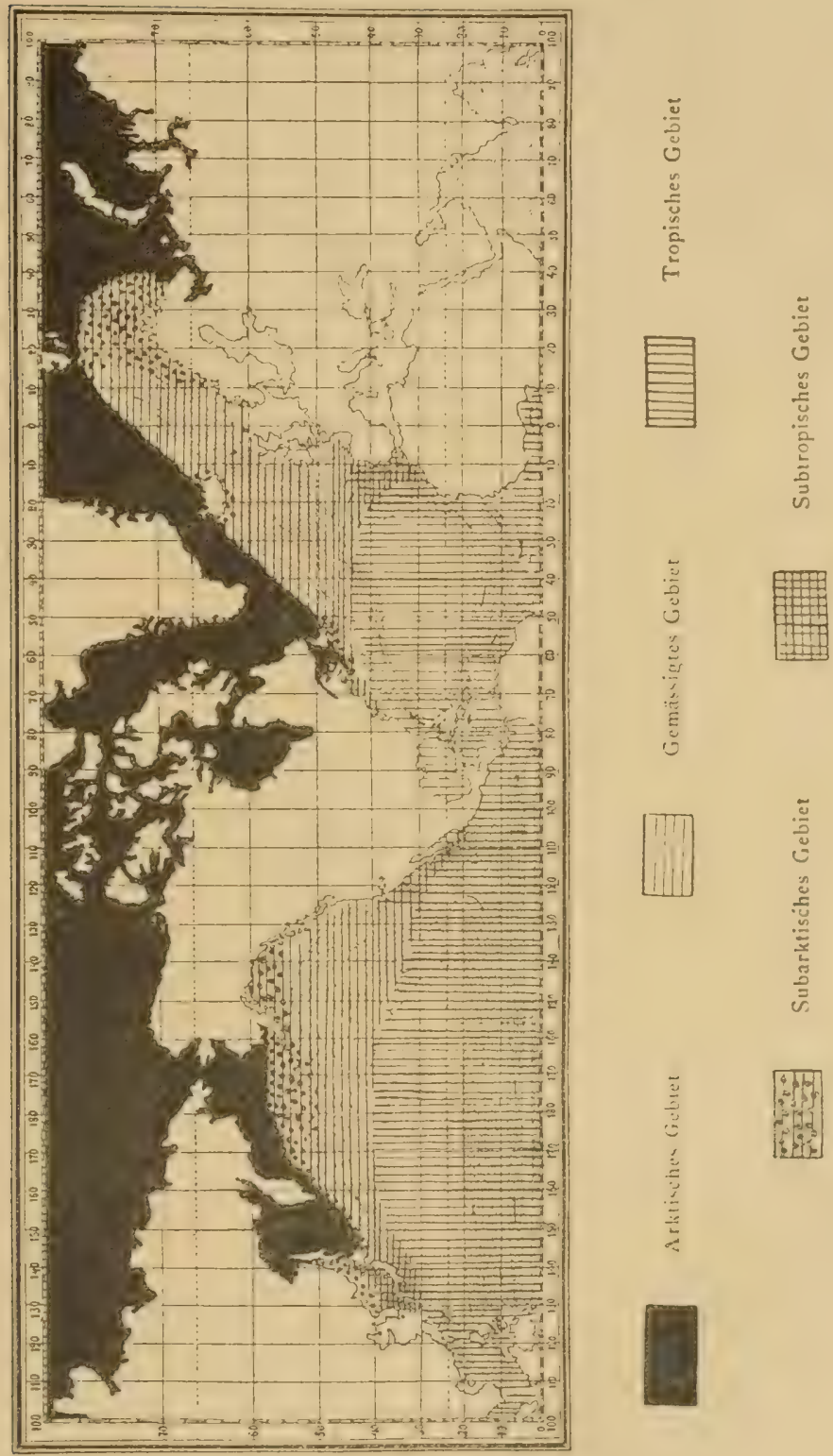

Fị. 1 


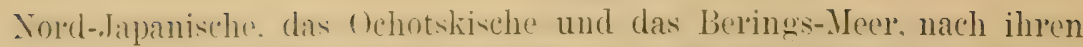
fannistisehen Verhältnissen vollkommen mit den Gewässern ron Labrata-

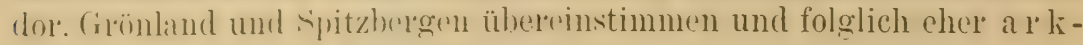
t i s c h genannt werden könnten.

Es scheint mir, dass die von Ortanan gewählte Grundlage zur Begrenzung del arktischen Fauna unzureichend ist und dass nämlich nicht die Eisgrenze i m Som m er, sondern die Eisglenze im W i n $\mathrm{t} e \mathrm{r}^{\circ}$ als Grenzlinie zwischen dem arktischen und dem borealen Gebiete angesehen werden muss. Für das arktische Gebiet halte ich von dem physikalischen Standpunte aus dasjenige. das jührlich wïhrend einer lïngeren Zeitperiode von dem Treibeise bedeckt ist, an den Ufer'n zufricrt und in dem Gobiete der. Kiontinentalstufe eine den Gefrierpunlit malhe oder sogar unter dem (iefrierpunkt liegende Bodentemperatur aufweist.

Wenn wir auf diese Weise das arktische Gebiet auffassen und begienzen. so sehen wir, dass es in den beiden Ozeanen viel übereinstimmender verteilt erscheint, dass es nämlich auch im Stillen Ozean im Westen weit nach Süden l'eicht. (Fig. 1.)

Ich lin zur Unher\%eugung gekommen, dass man überhaupt viel nattürlichel' die beiden Ozeane im Nol'den vom Aequator' in 3 grosse und gleichwertige Gebiete einteilen kann: 1 . a $\mathrm{r} \mathrm{k}$ is $\mathrm{ch}$ es $\mathrm{Geb}$ i et, dass so aufzufasssen ist. wie ich eben auseinandergesetzt habe; 2 . tropis ches Gebiet, das ich, vollständig mit Ormans übereintsimmend, als ein Gebiet der hohen und konstanten Temperaturen auftasse und 3. rin dazwischenliegrendes Uebergangsgebiet, das man wohl treffender Weise zusammen mit den älteren Autoren als gemässigtes Geb i e t bezeichnen könnte.

Nun kamn man abee auf del faunistischen Grumblage in dem gemässigten Gebiote an don europäischen und westamerikanischen Kü̈sten noch weitere Untrialsteilungen unterseheiden. nämlich im Norden eine Zone die ich als sub-arktische bezeichnen möchte, da sie sich durch (in Jaherwiengen von arktischen Formen und vollstandeges Fehlen der Repräsentanten der tropisehen Gruppen auszeichnet. Im Süden dagegen findet man eine sub-tropische Zone, die sich umgekehrt durch Ueberwiegen der tropischen und Fehlen der arktischen Formen auszeichnet.

Die Grenzen dieser Gebiete, wie ich sie auf Grund der Verbreitung (der Fische auftasse, sind aus der Karte ersichtlich (Fig. 1).

I jese Einthrilune scheint mir sowohl mit den physikalisehen. wie mit d'n faunistischen Ionlingungen ühereinzustimmen und natülicher die Mecresgebiete abzuglenzen, als die von Ortuans gegebene. Wenn wil

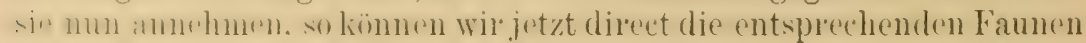
des Nor'd-Atlantischen und des Nord-Pacifischen Oceans untereinander 
verglejchen und zwar um die interessanteste Frage zu lösen: in welchen Beziehungen die atlantische Fauna zu der pacifischen steht. Ich habe einen Versuch gemacht, die Zusammensetzung der arktischen und suh-arktischen Ichthrofauna der heiden Derane zu vergleichen und bin zu folgenden Schlüssen gelangt:

$1^{\circ}$ Die atlantische Fauna steht sehr nahe zu der pacifischen im Bereich desselben Gebietes; einige, aber verhältnismässig wenige Arten sind identisch und das sind hauptsächlich hoch-arktische Arten, dio aus dem Eis-Neere in die beiden Oceane eindringen; andere Arten, aber auch verhältnissmässig wenige, werden in beiden Oceanen durch zwei wenig verschiedene Varietäten dargestellt; dagegen gibt es eine Menge von Arten im Atlantischen Ocean, die den pacifischen sehr nahe stehen und sie hier so zu sagen repräsentieren. Ich erlaube mir sogar zu behaupten, dass es im Atlantischen Ocean im Bereiche des arktischen und subarktischen (iebietes nur wenige Arten gibt, die mit den pacifisehen $\mathrm{n}$ ich $\mathrm{t}$ in Verwandtschaft stehen!

$2^{\circ}$ I) pacifische Ichthyofanna ist hroleutend reicher als die atlantische in demselben Gebiete - sie enthält nicht nur bedeutend mehr Arten heinahe in jedem frenus, sonder'n auch viel mele Genera und sogar einige zusammenhängende Gruppen und Fanilipn, die jm Atlantischen ()cean gar nicht vertreten sind, wie z. B. die Hexagrammiden.

Mangel an Zeit ellaubt mir nicht, weitel in Einzelheiten einzugehen, und ich werde nur ein Beispiel anführen, das diese beiden von mir aufgestellten Thesen erläutert. Die so eigenartige und nach ihrer Verbreitung typisch arktische Familie der $\mathrm{A}$ go $\mathrm{n}$ id en hat in den atlantischen Gewässern nur 4 Repräsentanten - 2 Arten von Agomus und 2 von Aspidophoroides; alle 4 sind durch nahe Formen (Agonus - durch die Arten von Podothecus) in dem Stillen Ocean vertreten, ausserdem finden wir aber hier auch als arktische und subarktische Formen, 13 Genera mit 2.2 Arten von A gonislen, darunter eine sehr charaliteristische (ripuppe (Agonomalus, Percis, Hypsagonus), die in dem Atlantischen Ocean gar nicht representiert ist.

Wenn man im Grossen und Ganzen die beiden Faunen vergleicht, so macht es den Eindruck, als ob sich die beiden so zueinander verhielten, wie lie Fatunen der Nold-See und der ()st-Sre, oder des Mittelländischen Meeres und des Schwarzen Meeres - in allen drei Fällen sehen wil einerseits eine l'eicher'e, andererseits eine ärmel'e abel' mit del' ersten in einer nahen Verwandtschaft stehende Fauna.

Wie hönnen wil nun diese eigentümlichen Beziehungen zwischen den beiden Famen erkiären! Jedenfalls liegt die Frlklïung nicht in den gegenwätigen Bedingungen, sondern in der Vergangenleit, und nur die geologische (reschichte der heiden ()ceane kann uns dar üher ins Kilare setzen - leider bleibt noch in dieser Beziehmo sehn vicl zn lösen übrig!

VIe CONGR. INT. ZOOL., 1904. 
Es scheint mir aber dennoch, dass wir uns schon heute über die Volgänge die rlen merkwürdigen Zusammenhang und dic noch eigenartigere Verschiedenheit der atlantischen und der pacifischen Fauna hervorgerufen hahen. eine Vorstellung machen kïmnen. Es war bis jetzt noch kein Versuch gemacht worden, diese aufzuklären.

Ich stell" mir die geologische Entwicklung der beiden Famen folgendel'massen vol. Tor dem Begimne der Tertiärzeit und vielleicht noch im Lianf' del' ersten Hälfte derselben bestand, wie ron mehreren Geologen und vor allen ron Neratarer angenommen wircl, eine offene Communication zwischen den beiden Oceanen, da die Panama-Brücke noch nicht existiente. Ijei der Gleichmässighe it des Klimas musste damals in den beiden ()eanen auch eine vollständig oder boinahe identische und wanz gewiss tropische Fauna verbreitet gewesen sein. Nun begann aber am Anfange

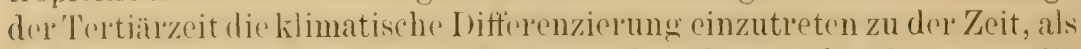
dis Communication der heiden Becken noch nicht unterbrochen war: die gemäsigtrn, suli-arktisehen und arkischen Formen besamen sich aus denselben oder sehr nahen tropischen Formen unter dem Einfluss derselben Bodingungen in den beiden ()eanen zu entwickeln und diesem Tmstande muss man, glanbe ich, die nahe Verwandtschaft der beiden Faunen zuschreiben.

Wenn die äusseren Bedingungen an den beiden Oceanen auch weiter diexulhen gehliehen wären, so würden sich wahrscheinlich anch die faunistischen Ijedingungen ungefähr gloich gestaltet haben und die beiden Faunen würden jetzt kaum bedeutende Unterschiede erkennen lassen. Nun war es aber n icht der Fall - es trat die Eiszeit an und - ein Umstand, auf den noch Niemand, wie mir scheint, bis jetzt aufmerksam gemacht hat - während dieser Zeit waren die Schicksale del beiden Oceane sehr verschieden.

Fs hrfand sich hekanntlich wïhrend dieser Periode die nördliche Hälfte Furopa's unter einer dicken Eisdecke, die ähnlich derjenigen von Grönland oder dere Südpolallïnder war' an der atlantischen Küste reichte die

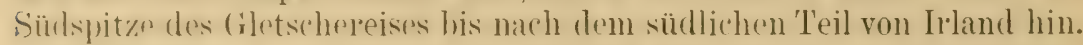

Auch der nord-amerikanische Kontinent befand sich unter einer mächtigen Eiskappe die sich bis nach New-York erstreckte. Wenn wir uns mun solche Verhältnisse vorstellen, so gelangen wir unumgänglich zu dem Sihlusire dass wibrend der Kulminationsperiode der Eiszeit die Grenze des 'I'reibeises im Atlantischen Ozean bedeutend nach Süden verschoben war und jedenfalls nicht nördlicher als an der die beiden äussersten Sitzen de.e die Kontinentalgletscher verbindenden Linie lag. wahrseheinlich aber noch weiter im Süden sich hinzog. Es wal folglich das ganze Gebiet, welches nördlich von der auf der Karte (Fig. 2) punktierten Linie liegt, ungefülı in denselben Bedindungen gewesen, wie jetzt die Gew:isser von Spitzbergen, Grönland und Nowạa Semlja. 


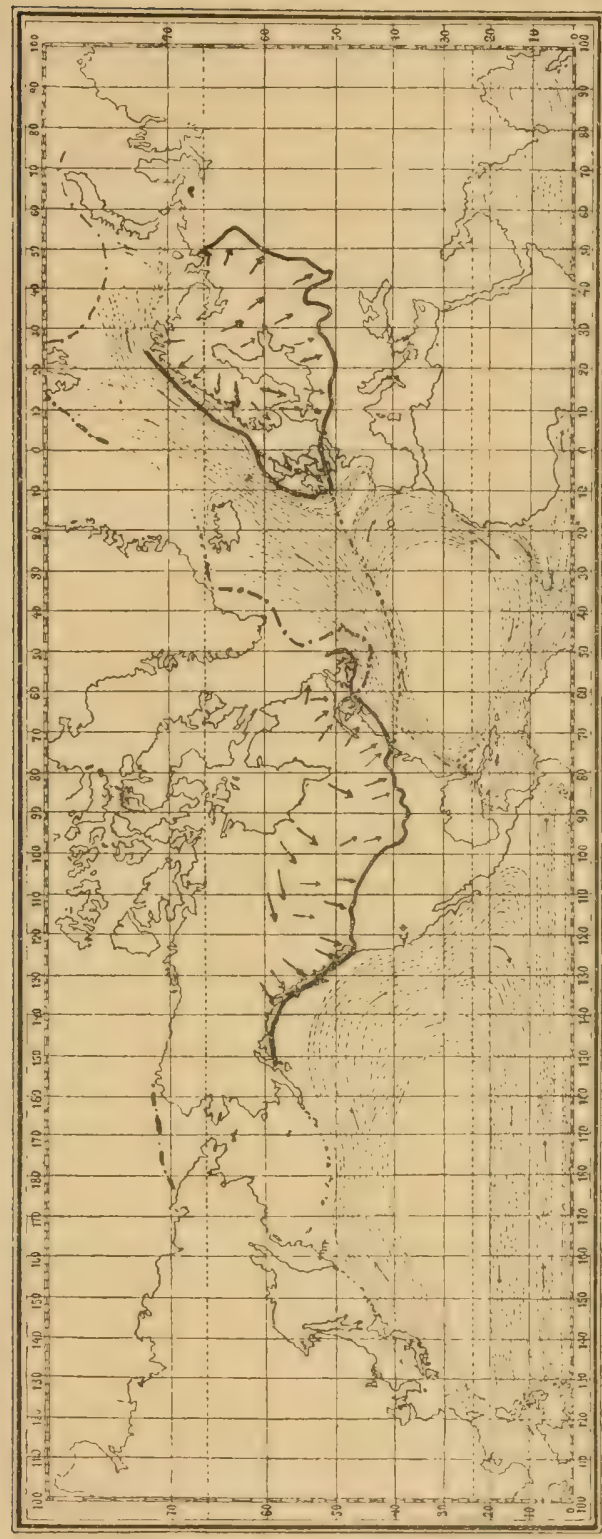

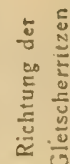

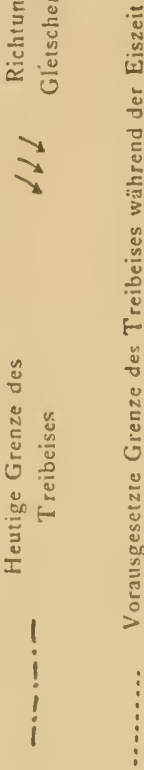

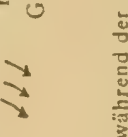

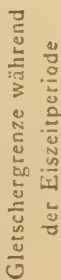

I

Fig. : 
Wie war es nun mit dem Stillen Ozean? Die Erfahrungen der amerilianischen (imologen zoigen uns, dass an seinen Ufern die Bedingungen selhst wiihrend dor hö̈hsten Entwicklung der Eiszeitperiode ganz andere wallen. Es wurlen nämlich gir keine Spuren von einer allgemeinen Ver-

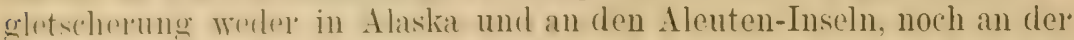

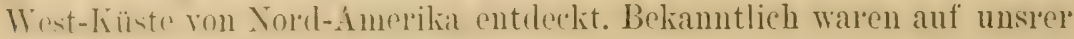
Seite des Ozeans auch gar keine grosse Gletscher vorhanden. Die noch

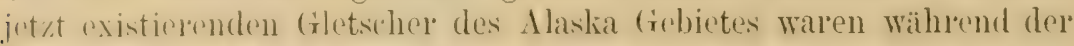

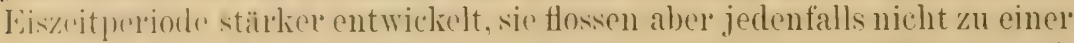
Eiskappe zusammen 1. Ausserdem existierte die Bering-Strasse noch nirht und das Eis ans den Polarg(b)ieten lomnte in die südlicheren Gebicte nicht eindringen.

Auf diese Weise war während der Eiszeit der Stille Ocean keinen so) ungünstigen Bodingungen unterworfen, wie der Atlantische, wenn auch eine allgemeine Erniedrigung der 'Temperatur auch in ihm zur (roltung lann; seine kimatischen Verhïltnisse unterschieden sich ahel verhältnismässig wenig und eine solche Veleisung wie an dem Atlantischen wurde an ihm nicht beobachtet.

Sellostrerstimllich musste dieser Untersehied auch auf die faunistisehen Vorhailtnisso nidht ohme Einfluss bleiben. und wahrscheinlich liegt oben

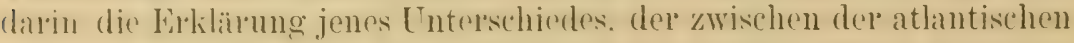
und der pacifischen Fauna existiert. Im Atlantischen Ocean waren gewiss währond der Eiszeit sehr viele sul-anktische und gemässigte Formen zu firmule greangen. die im Stillen Orean, wo die Bedingungen wenigex ungünstig waren, sich erhalten haben. Dass für die gemässigte und sub-arktische Formen die Bedingungen im Atlantischen Ocean tatsïlhlich seho ungünstig gewesen sein müssen, ist schon darans ersichtlich, dass dort die Eiscrenze schr nahe an die Tropenzone rorgeschohen

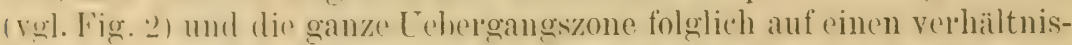
miissigr schmilen streifen zusanmengedrängt war. Eine solche Verschiehume fand vermutich im Stillen ()zean nur in unberlentendem Massstabe statt.

fowiss kommen diese Betrachtungen nicht über eine Hypothese heraus, a scheint mil jedoch, dass diese Jypothese sowohl in den famistischen, wie in den geologischen Tatsachen ihre Stütze findet und uns hilft, die Verschiedenheiten der Faunen zu verstehen.

${ }^{1}$ Cf. Wrignt. Ice age in N. America, IV. ed., pp. 143-149. 1900. 


\title{
Ueber Erfolge und Ziele zoogeographischer Forschungen, insbesondere über die Zeichnung der Vogelfedern und Schmetterlingsflügel.
}

\author{
Von 0. KLEINSCHMIDT (Volkmaritz).
}

Mit 2 Tafeln.

Durch ein Versehen (jedenfalls ungenaue Angabe meiner'seits) war das Thema meines Vortrags im Programm unvollstandig mitgeteilt und für die erste Sektion vorgemerkt. Es war und ist meine Absicht, über die Tragweite vergleichend zoogeographischer Studien ein parr einleitende fredanken mitzuteilen, gewissermassen zur liechtfertigung des: Vorschlages, den ich auf dem vorigen Congress im Anschluss an einen Vortrag von JАСові, Forderungen der Tiergeographie, machte. Mehr noch, als es meine Worte vermögen, hat ja die Bereitwilligkeit, mit der die Leitung dieses Congresses diese Sektion einrichtete und die stattliche Zahl von Vortrïgen das wachsende Interesse an zoogeographischen Fragen hewiesen. Ich gehe aber noch weiter und bin der Ansicht, dass die Zoogeographie nicht eine Aloteilung der Zonlogie ist, sondern dass sie durch I arbietung nener (iesichtspunkte und Erweiterung des Gesichtslipeises fast das gesante zoologische II issen beeinflussen wird.

(I)er' Yortragende bittet die Anwesenden, zucrst sine vorliutige Besichtigung des ausgelegten I) emonstrationsmaterials vorzunehmen und fithrt, nachdem dies geschehen ist, fort.)

Die Hauptarheit des Srrtematikers besteht nicht darin. leieht erkennbaren Formen neue Namen zu geben und vorhandene Namen zu registrieren, sondern zu crkennen, was trotz äusserlicher Verschiedenheit zusammengehört und was trotz ïusserlicher A chnlichkeit versehieden ist.

So liegen hier ein P $^{T}$ von Saxicola finschi und ein abnormes ơ von Saxicole lugens aus Palïstina ersterem verblüffend ähnlich. Aufmerksam gemacht durch die Zugehörigkeit heider Yügel zu ganz verschieden gefärbten Wribchen, findet man die versteckten liennzeichen, die boide trennen.

In ganz ähnlicher Weise, wie Nännchen und Weihchen, die sich st:in- 
dig paaren, zusammengehören, mögen sie auch in Färbung und selbst teilweise in der Gestalt ver'schieden sein, so gehören Formen zu einander, die sich in verschiedenen geographischen Gebieten vollständig biologisch vertreten und an der' Berührungsgrenze in regelmässigem Blutaustausch ${ }^{1}$ stehen.

Aufmerksim gemacht dureh die Zugehörigkeit zweier ganz ähnlicher Formen eines und desselben Landes zu deutlich verschiedenen geographischen Vertreter'n, findet man oft erst die versteckten Unterschiede, zwischen zwei Tierformen, die die Wissenschaft vorher nicht einmal als Rassen anerki'nnen wollte und die sich nun als Glieder ganz verschiedener Artoruppen entpuppen. Die Beschränkung auf bestimmte faunistische Sondergeliete ist zuweilen gerade das Gegenteil von zoogengraphischer Methode. denn durch die Arbeitsteilung nach Faunengebieten ist es gekommen, dass man meinte, die Art sei etwas Geographisches, während nur ihre Formen (die Subspecies) geographisch bedingt sind. Die wirklich geographisch vergleichende Methode führt daher zur Entdecking zahlreicher - sagen wir eimmal "Mimikryäulle ", durch die sich die Systematiker oft Jahrzehnte lang haben täuschen lassen.

Zunächst lege ich hier als bexonder's deutliches Beispiel einer Reihe oder richtiger eines Rings von geographischen Vertretern die wichtigsten Formen der Blaukehlchen vor, die nach Grösse, Fïrbung des Kehlschildes und Sternflecks, Flügelgestalt u. s. w. deutlich verschieden sind und doch durch einheitlichen Typus sich leicht als Ausprägungen desselben Tierlebens zu erkennen geben.

Ganz disssellhe zeigen hier die in Grösse und Färbung verschiedenen Formen von Alcedo ispidda. Dass die kleine tieflhlaue Ceylonform (Alcedo

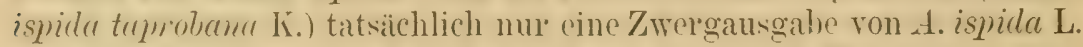
ist, wird al)gesehen von den dazwischen wohnenden IIittelstufen sehr hübsch durch 'in hier mit vorliegendes deutsches Stück hewiesen, das auf der linken Körrperhälfte ispida, auf der rechten taprobana gleicht. Noch zwei weitere Stücke meiner Sammlung zeigen dieselbe Erscheinung in schwächerem Grade.

Wie weit der' (irössemuntersehied geographischer Formen desselben 'Tieres gehen kitmn, beweist der winzige liolkrabe von Somaliland, Corvus corax edithix.

In all solchen Fällen ist das Erkennen der eng verwandten Formen sehr leicht. Nehmen wir nun aber die bis vor kurzem unter dem Namen Purus pulustris L. zusamme'ngeworfenen Meisen oder die gleich interessante (iruppe der Haubenlerehen vor. so stehen wir anfangs vor einem

\footnotetext{
${ }^{1}$ Von Bastardierung darf man meines Erachtens bei dieser Blutauffrischung nicht reden, denn die zahlreichen Mischlinge sind nicht nur fruchtbar, sie scheinen auch zuweilen besonders gross und kräftig zu sein.
} 
Labyrinth von Formen und täuschenden Achnlichkeiten. während gengraphisch geordnet diese Gruppen ganz klar und einfach werden.

Wählt man zum Beispiel von Galerida cristata und thelkle etwa die beiden Vertreter aus Spanien und dem mittleren Tunis und zwar schwachschnäblige Stücke von thelile und starkschnäblige von cristate aus, so wird ein Laie nur mit Mühe davon zu ülor'zeugen sein, dass von diesen vier Lerchen nicht die so verblüffend ïhnlichen, sondern die verschieden gefärbten artlich zusammengehören.

Die Arbeiten Carlo von ErLangers ${ }^{1}$ (dessen Verbleitungskarte und Formenübersicht aus J. f. Orn., 1898 und 1899 werolen vorgolegt) finden eine wertvolle Ergänzung durch die Sammelreisen des hier anwesenden Herrn Fü̈chrtikn nach Algerien. Derselbe hat auf meinen Rat eine grosse Anzahl ron Haubenlerchen mit Er(l- (bezw. Sand-) Prohen von den Fundstellen gesammelt. ()ft, aber nicht immer, stimmt, wie sie an den mitgebrachten Stücken sehen, die Bodenfarbe mit dem Grundton des (refieders ganz wundel'har übelein. Fül diese ja bekannte Erscheinung wird sich wahrscheinlich eine neue Erklärımg ergeben, wenn überall in dieser Weise gesammelt wird.

(Es werden noch zihlpeiche andere Beispiele rorgezeigt, die sich durch die Anschaumw rasch übersehen lassen. wïhrend nit Worten und Namen sich schwer ein anschauliches Bild davon geben lässt. Ich deute deshall, dieselben hier nur in Tabellenform an, wie ich sie auf dem Kongress in geographisch geordneten Balgserien zeigte:)

\section{Dïmnschäblige Sumpfineisen.}

$\begin{array}{cccccccc}\text { Amerika } & \text { England } & \text { Rhein } & \text { Alpen } & \begin{array}{c}\text { Mittel- } \\ \text { Dentschl. }\end{array} & \text { Osteuropa Japan } & - \\ \text { zahlreiche } & \text { klein u. } & \text { ähnlich } & \text { sehr } & \text { mittel- } & \text { gross und kleiner } & - \\ \text { Formen } & \text { dunkel } & \text { England } & \text { gross } & \text { gross } & \text { hell dunkler. }\end{array}$

\section{Dickschnüblige Sumpfmeisen.}

$\begin{array}{cccccccc}\text { Amerika England } & \text { Rhein } & \text { Alpen } & \text { Mittel- } \\ \text { Dentschl. } & \text { Osteuropa Japan } & \text { China } \\ \text { fehlend klein und ähnlich } & \text { gross } & \text { mittel- gross und } & \text { kleiner klein und } \\ \text { dunkel England } & & \text { gross } & \text { hell } & \text { dunkler dunkel } \\ \text { fast wie in } \\ \text { England. }\end{array}$

${ }_{1}$ Der hoffnungsvolle junge Gelehrte und Afrikaforscher wurde uns inzwischen durch einen tragischen Unglücksfall im Beginn seiner wissenschaftlichen Laufbahn entrissen. Sein Tod bedeutet für die Ornithologie und ganz besonders für die 'Zoogeographie einen unersetzlichen Verlust. 


\section{Hansrotschwainze.}

\section{Europa}

Unterseite grauweisslich, selten Anklänge an die östliche Form ${ }^{1}$

\section{Kaukasus}

Unterseite grau, mit gelbrotem Mittelfleck oder Anflug.

\section{Asien}

Unterseite ganz gelbrot. Paradoxa-Jugendkleid. einem europäischen Baumrotschwanz verblüftend ähnlich.

\section{Baumrotschuimze.}

\section{Europa}

Unterseite matt gelbrot

\section{Westasien}

Unterseite intensiver rot.

$\mathrm{Zu}$ diesen Formen werden die Jugendkleider gefügt und zwar die sogenannten Paradoxa-Kleider. Gruppiert man diese Vögel, statt sie geographisch zu ordnen, nach dem Gradr ihrer Achnlichlieit, so entsteht cin ganz falsches Bild, während durch die geographische Anordnung die wirkliche Vorwandtschaft aufgedeckt und durch Auffindung der wirklichen Artcharaktere bestätigt wurdc.

\section{Habichtsadler.}

\section{Europa}

braun und weiss, matter gezeichnet

\section{Tropisches Afrika}

tiefbraunschwarz und weiss, krüftiger gezeichnet.

\section{Zicergatlen:}

\section{Europa}

braun und weiss, matter gezeichnet, oder ganz braun

\section{Tropisches Afrika}

tiefbraunschwarz und weiss, kräftiger gezeichnet, oder sehr dicht gefleckt.

Dic beiden 'Tropenformen wurden bis vor' Kurzem für eine und die-

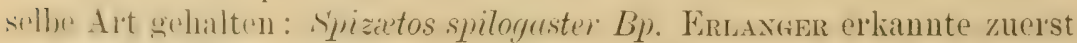
ihn artliche Vorschiedenheit auf (round der Tatsache, dass sie nicht geographische Vertreter sind, da sie nebeneinander brüten, ohne sich zu vermischen. (Vergl. J. f. Orn., p. 186, 1904.) Dass aber Hieraztos spilo-

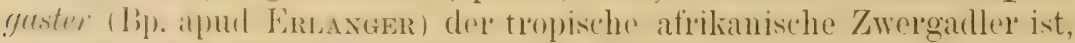
scheint noh nimmand gemerkt zu haben. Wiederum bestätigt dies ein

${ }^{1}$ Line Parallele zu dieser Zwischenform (Ruticilla ochwura $\mathrm{Gm}$.) bilden die von Graf ros BerLepsci auf dem vorigen Kongress vorgezeigten Mittelglieder ron Diglossa brumeicentris und carbonaria. 


\section{TAFEL I.}

\section{FIGURENERKI_ÄRUNG}

Fig. 1 links Falco pereqrinus leucogenys (Brm.) ㅇ juv. rechts Falco peregrimus brookei (Sharpe) $Q$ juv.

Fig. 2 links Astur gentilis astur (Pall.) o ad. rechts Astur gentilis arrigonii $\mathrm{Kl}$. $q$ ad.

Fig. 3 links Accipiter nisus subsp. $q$ ad. rechts Accipiter nisus wolterstorffi (Kl.) 우 ad.

Fig. 4 links Cuculus canorus telephonus (Heine) juv. rechts Cuculus canorus subsp. juv.

Figur 3 links mit der darüber und der rechts daneben stehenden Figur verglichen zeigt den relativen Begriff der Art, ist nicht Mittelglied zwischen beiden, sondern wirkliche Art im Vergleich zu der einen, neben der andern nur geographische Form.

Die unter rinander stehenden Figuren verglichen stellen das geographische Gleichgewicht der Variationsschwankung dar.

Die n the n einander stehenden Figuren die geographisch bedingte Versehiedenheit in der Stärke der Pigmentierung, besonders der Querbänderung. 

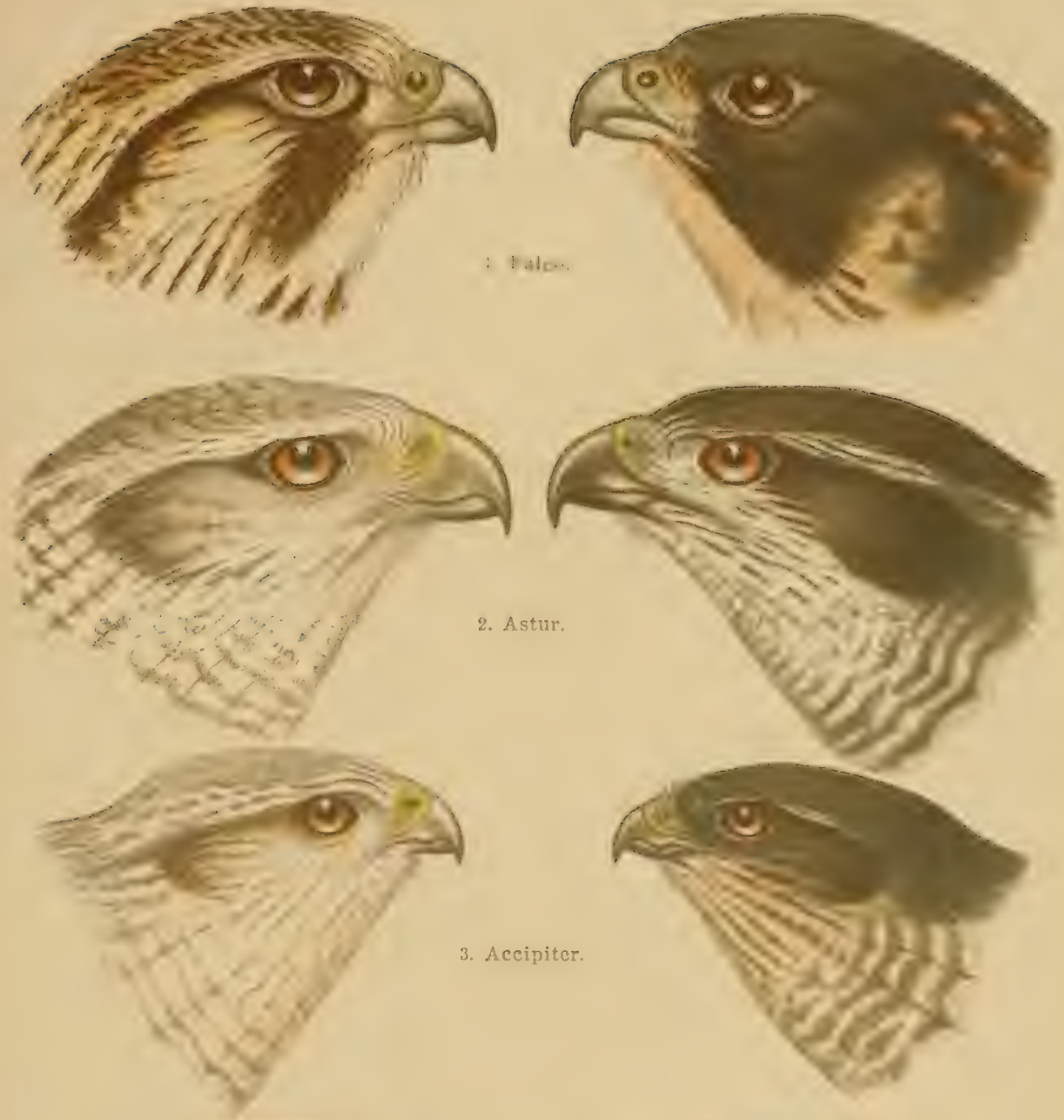

3. Accipiter

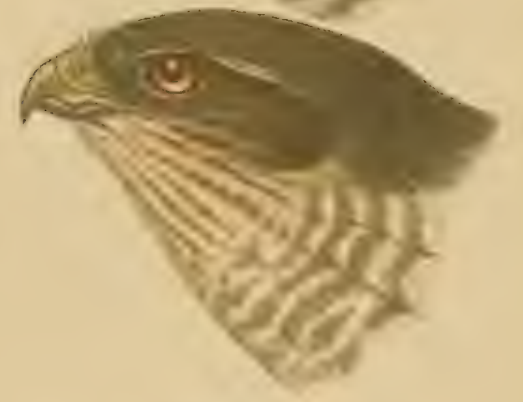

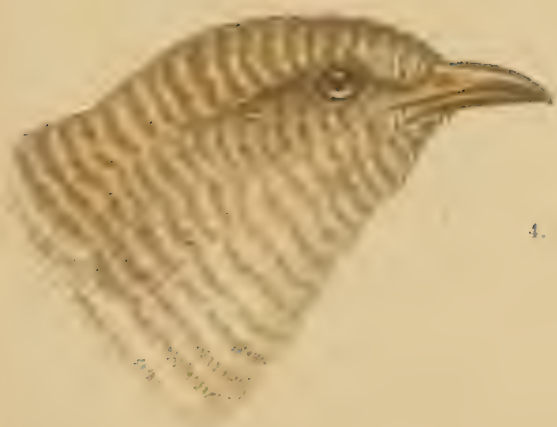

Sibirien.

(Heller.)

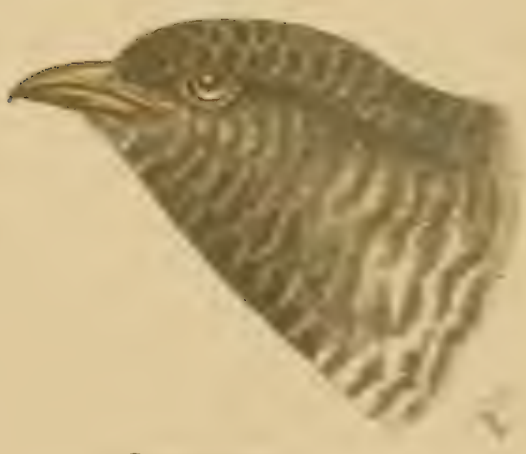

Sardinien.

(Dunkler.)

(5)

Steinzeichn. v. O. Kleinschmidt. 



\section{TAFEL II.}

\section{FIGURENERKLÄRUNG}

1. Schwanzfeder einer sardinischen Saxicola renanthe vor der Mauser. Die pigmentierte Spitze bleibt unverletzt und als Steuerruder brauchbar.

2. Dieselbe Feder rekonstruiert.

3. Haubenfeder einer Galerida cristaia, abgenutzt.

1. " " "

:i. Innere Sekundärschwinge cines Albinos | von Alauda arven-

6. " " normalen Exemplares| sis (Dezember).

7. Brustfeder eines briitenden $q$ juv, von Accipiter nisus 13. Juni. Die dauerhafteren dunkeln Binden halten die Fahnenstrahlen in Zusammenhang.

8. Fahnenstrahlen der in Figur 7 abgebildeten Feder verg rössert, links cin Fahnenstrahl rekonstruiert vor der Abnutzung der hellen Teile.

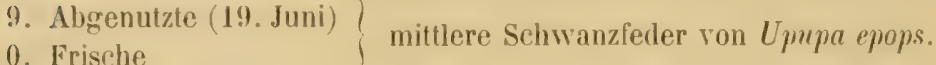

11. Hodell der schützenden Pigmentierung von Schmetterlingsflügeln.

12. Unterer Teil von Fig. 13 vergrössert. Annahme eines $R$ is ses wahrscheinlicher als die eines Bisses.

13. Vorderflïgel / von Pieris brassicre mit Verletzungen vor den am meisten

14. Hinterflïgel gefährdeten und daher durch Pioment geschiitzten Stellen. 


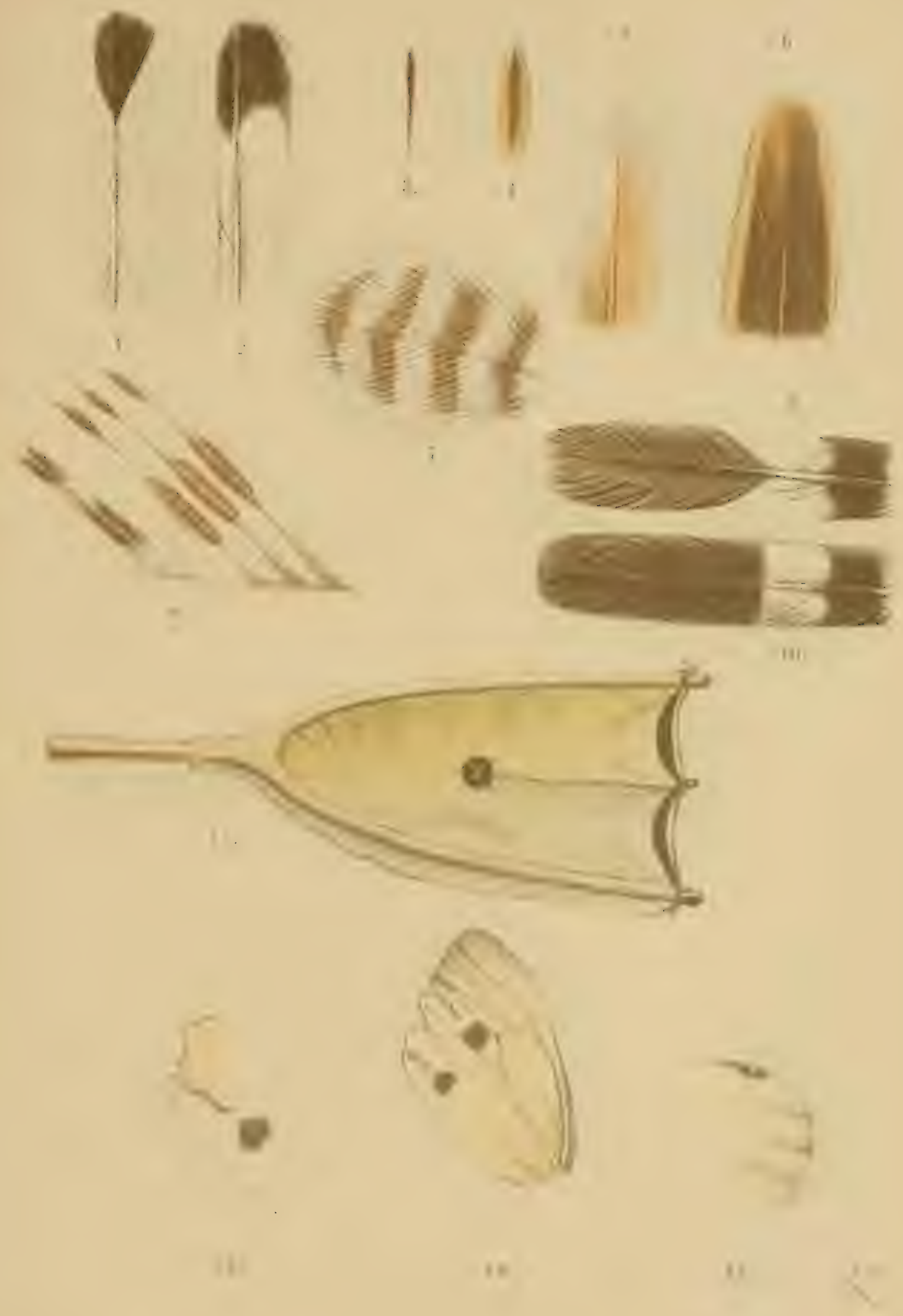



verstecktes Altkrnmzeichen. Eimmal elkannt ist diese geographisehr Mimikry so deutlich, dass an geographiseh geoldnetem Material ein Kind das Richtige sieht.

Die geographiseh orduender und verwleichende Methorle wird norh auserendentlich viel" Irlümer der Systematik aufzuklälen haben. Es. war moine Absicht, hier zu sagen, dass es bei Sohmetterlingen älunliche Fälle geben kömnte, nämlich Minikry, die man kamm feststellen kilnn, während hestätigendr Kennzeichen der Artdifferenz heim Vogel (in der Stimme und vielen andren Lebensïuscerungen) sich viol lojichter feststellen lassen. Nun hat mil Her' W. Petersen im Anschluss an seinen hochinteressanten in Selition I gehaltenen Vortrag mitgeteilt, dass' ("r in der Tat äuserlich kaum unterscheidbare Schmetterlinge durch anatomische Lntersuchung der (renerationsorgane als total verschiodene Alten erkennen konnte.

Es erfüllt sich hier das Wolt, das Bernard auf dem letzten Congress aussprach: "Auf diesem Wege werden wir zum ersten Male erkennen lernen, was eine Art in Wirklichkeit bedeutet (p. 893 del $^{\circ}$ Verhandlungen).

Das zweite. was die gengraphisch veroleichende systematik aufeckt, ist eine ganz wunderbare Iegehässigkeit der geographischen Varation, Fassen wir die hier zusammengestellten Arten ins Auge:

(Vergleiche Tafel I.)

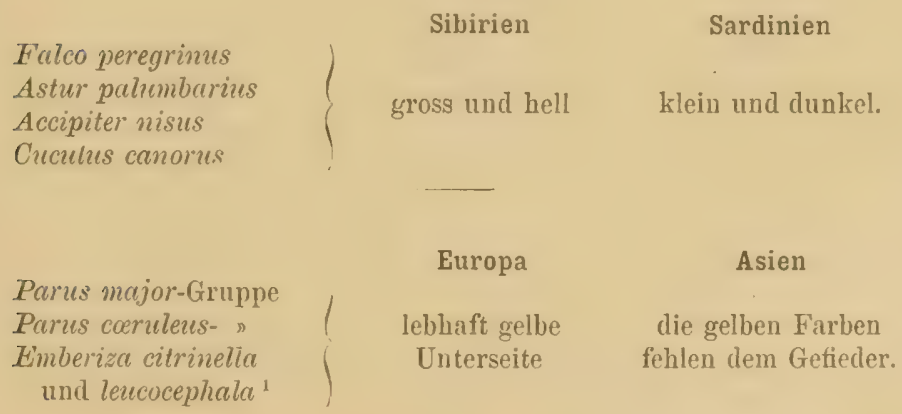

Am interesiantestem ist in zoogeographischer Hinsicht unser simplep Ilausperling, von dem ich hier vine lange Reihs von gengraphischen Formen vorlege. Presser hisymminensis scheint dir wilde Stammform in

\footnotetext{
${ }^{1}$ Es wird eine Serie von Bälgen aus der Sammlung des Herrn JoHansen in Tomsk vorgelegt, welche die artliche Einheit beider Ammern durch eine vollständige Stufenreihe beweisen.
} 
Nordafrika und andern Ländern zu sein. Dieser Palmenbewohner hatte vermutlich rershohlene geographische Vertreter. Einer derselben, del Ilausiperling. breitet sich mit den Ansiedlungen dep Xenschen immer mehr ans. Tohol den Entwicklungsgang kamn man nur Hypothesen und zwar die velschiedensten Ansichten aufstellen. Auftallend nur, dass alte Lulturentron wie Indien, Aegyten. Italien ilne eigenen Formen haben. Sicher aber ist, dass in Algerien, wo der Passer domesticus mit Passer hispmiolensis zusimmentrifft, beide zu cinem oft undefinierbaren Gemisch zusammenschmelzen und zwar so, dass trotz starlier Zufuhl von domestirus-Islut der einheimische hispaniolensis die Oberhand behält, Wiilnend domesticus laum roin zu finden ist, sondern in allen Individuen mehr oder minder hispeniolensis-Charaktere anfweist. Mit andern Worten Pussen domesticus muss wieder Presser hispuniolensis werden. Nahvorwande Formen lï̈ne'n sich im gleichen Gebiet nicht auf die Dauel' nebencinander erhalten.

I) geographischen Faktoren heschräinlen dic For'men und machen sie lionstant. Fe wäle sonst nicht möglich, dass so viele ganz relschiedene Arten in denselleen Länder'n genau his zum gleichen Förloungsgrade va-

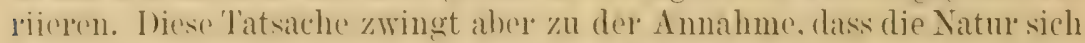
mindestens nicht überall in einer ständigen Fortentwickl ung befindet, sondern in einem Gleichgewichte zwischen strigernelen und ausgleichenden Falitoren. Wo dies Gleichgewicht wie

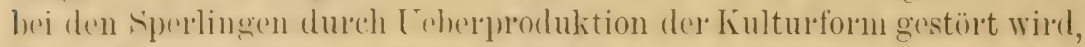
ist die Natu' dennoch bemüht, es wieder zu gewinnen.

Jic Beolatehtung zoogeographischer Tatsachen ist in manchen Fällen

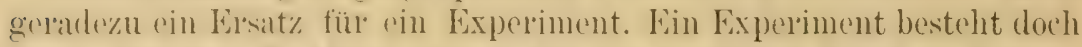
dau in, dass man die Natul zwingt, auf eine bestimme Frage zu antwol-

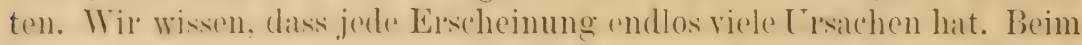
Experiment lassen wil in rinem Fall rine neue Ursache hinzulemmen, oder wir sehalton eine einzige aus. Wïhrend sonst die beiden Fialle (ror mol nath dem Experiment) genau die gleichen Bedingungen haben müssen.

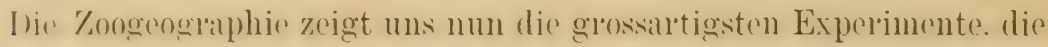
die Natur gemacht hat und die wir nu' abzulesen brauchen. Sie lehrt uns, was wirklich dieselbe Art ist. Nun wissen wir, in dieser Tiergruppe sind dir gerelenen bedingungen gleieh. Anders sind nur die geogratphischen Faktoren. Wir sehen \%. B., dass in Ländern mit grösserer Re-

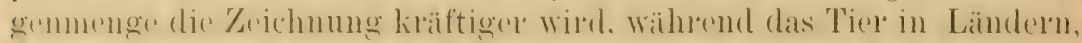

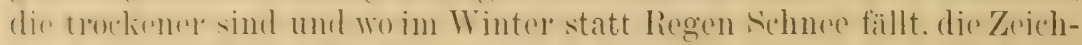

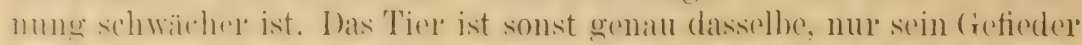

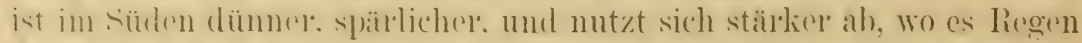

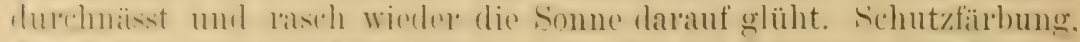

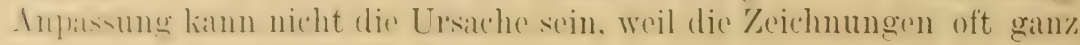




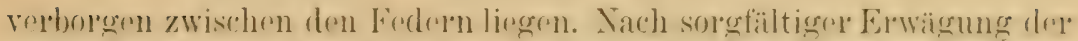

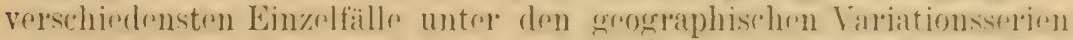

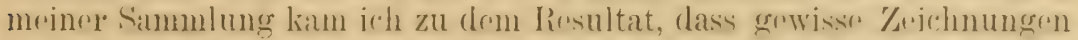
namentlich bianderungenn eine Festigung des Kusammonhangs für dir

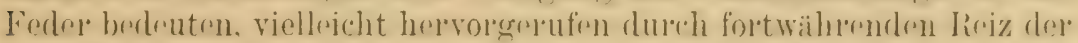

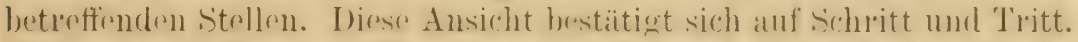

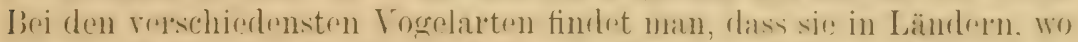
sie an spourlichsten gereirhnet sind, ann schönstent bleibrn, während sie sich in Ländern mit stalker Fleckung stark abmutzen. V'or der Mauser

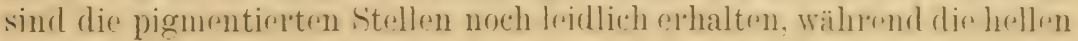
Gefiederteile stark beschädigt sind.

Ob das Pigment der Grund der Festigkeit oder eine Begleiterschei-

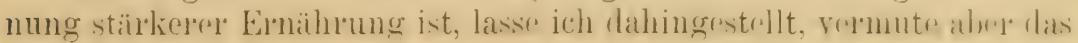
Erstere.

Wüıde sich die ganze Feder gleichmässig abnutzen, so würden die

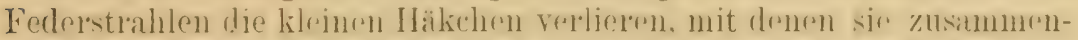

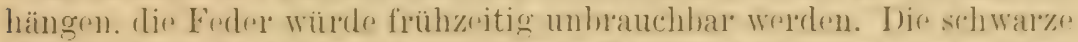

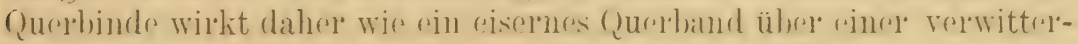
ten Lattenthüre.

Unter dem Mikroskop zeigt sich die raschere Abnutzung der hellen

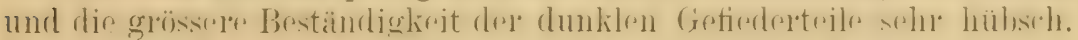
In zahllosen Fällen erkennt man sie schon mit blossem Auge.

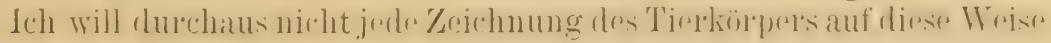
erklären, aber sicher' werden viele Farbenverteilungen dadurch verständlich. Hier nur einige Beispiele.

Die jungen Eulen und viele andere junge Vögel mit zartem Gefieder

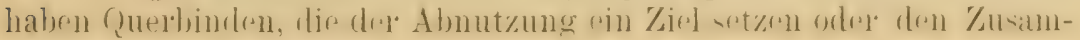
menhang der Federstiahlen erhalten.

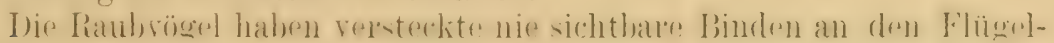
federn, die sie besonder's oft gebrauchen.

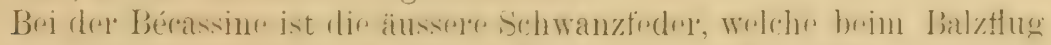
ganz einzeln (nach meiner sichern Beobachtung) frei hervorsteht, im

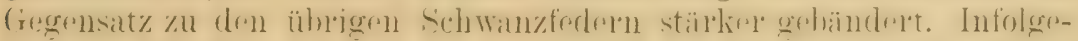
dessen verliert diese Feder, wenn sie vibrierend den meckernden Balzton hervorbringt, nicht den Zusammenhang ihrer Fahnen.

Die Weibchen der Rebhühner und Haubenlerchen, die auf dem Nest

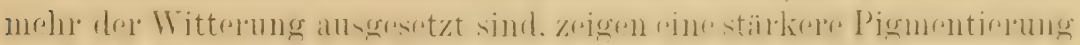
der Rückenfedernn als die Männchen.

Vergleicht man die einzelnen T'eile z. B. bei einer Haubenlerche, so

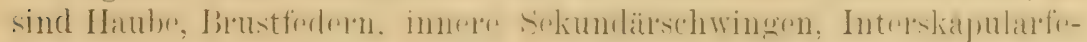
der'n und mittlere Schwanzfeder'n am meisten pigmentiel't, genau die Körperstellen, an denen das Gefieder. wenn der Vogel auf dem Boden sitzt, vom Winde gezaust und vom Regen durchnässt wird. 


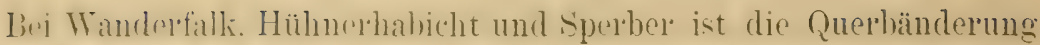

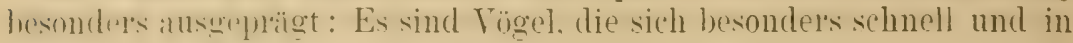
stürmischem Fluge bewegen.

Fehlen die Zeichungen, wie bei diesem Feldlelchenalbino, so nutzen sich die Feder'n überaus schnell ab, während in gleicher* Jahreszeit el'-

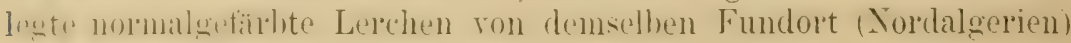
noch unverletztes Geficder haben.

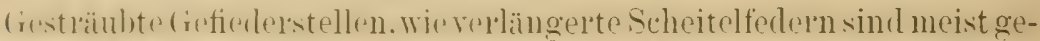
zuilhnet : Man lïmntr diese Beispiele noch ins Lneluessliche vermehren.

Auch bei Säugetieren schcinen die Teile des Felles, welche mehr auszuhalten habre, durch stiuldere Pigmentierung der Beschädigung zu trot\%en. besonders an Hautkanten (Iiückenstreif bei Ziegen und Eseln).

liei s.hmettelingen wilken offonbar vielo Quesbinden ganz ähnlich

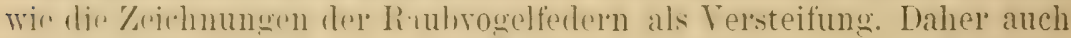

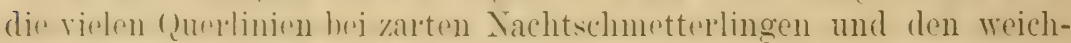

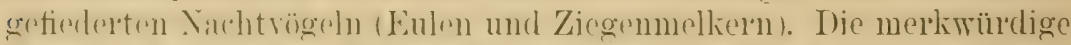

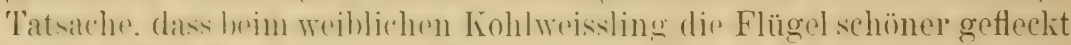
sinel als brim Männchon. hat in dren Tiejbungs- und Almutzungsrerhältnissen ihre einfache Lrsache. Wirdermm zeigen die geographischen Formen der sehmetterlinge sehr dentlich. wie die Pigmentierung von der

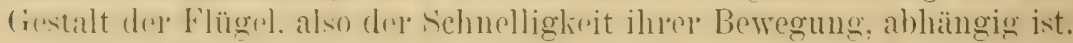

Professor Poulton versuchte auf dem V. Internationalen Zoologencon"ross den Vachwris zu führen. dasi Flecken und pigmentierte Schwalbenschwämze hri sohmettorlingen die Feinde tänschen solden und oft bej

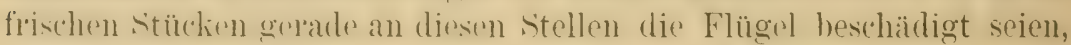
vermutlich durch Schnabelbisse von verfolgenden Vögeln ${ }^{1}$.

Es ist aber gerade umgelieht". Ino Flügel sind nicht rerletzt. Weil sie

' Ueber die Frage, bis zu welchem Grade überhaupt die Schmetterlinge als imago noch unter Verfolgungen seitens der Vögel zu leiden haben, ist viel gestritten worden. Vergleiche u. a. Societas entomologica, Jahrg. XV, p. $76,129,187$, XVI, p. 10, 33, 65, 81, 114 etc. Dass Vögel Scbmetterlinge fressen, darüber bedarf es keiner Diskussion. Man denke nur an Caprimulgiden. Tagschmetterlinge, besonders Weisslinge, werden zuweilen verfolgt z. B. von Muscicapa grisola. Doch lässt der Vogel $99 \%$ Weisslinge, die an ihm vorüberfliegen, unbeachtet und $99 \%$ der Vögel kümmern sich nicht darum. Jinen Vogel sah ich sogar sich erschrocken ducken, als ihm von hinten her ein Weissling dicht am Kopf vorbeiflog. Aber "r verfolgte ihn nicht.

- Beim Aufschlagen einiger Nummern der Societas entomologica fällt mein Blick zufallig auf eine Notiz von Karl Frixgs über ein monströses đon Saturnia pyri (XTT, p. 69). Der linke Vorderflügel zeigt einen bogenförmigen bis an die Doppelwellenlinien reichenden Ausschnitt. Auf Ober- und Unterseite hat dieser Flügel unter dem normalen Augenfleck einen zweiten etwas kleineren. Das scheint ein schöner Beweis für meine Ansicht zu sein, dass nicht der Augenfleck Anlass zur Verletzung, sondern die Verletzung Ursache der Bildung des Augenflecks ist, den hier wohl ein 
an diesen Stellen anffallende Zeichnungen haben. sonder'n sie haben an dies'n Stellen kräftign Pigmentierung, weil die betreftenden Toile besonders viol auszuhalten haben und oft Verletzungen releiden. P'igmentiert sind daher meist die 'T'eile, die einer V e r'ste if u $\mathrm{ng}$ bedürfen, zunächst die Adern, sodimn que über die Adern laufende Querlinien. Die Gestalt dieser Linien ist nicht zufällig, sondern hat etwas, was das Auge (mindestens das eines modrenen Küustlers) entzülclit. Sic hat also einen Simn, einen biologischen Wert. 'In der Tat verlaufrn diese seheinbar zufälligen Zickzacklinien so, dass sie das betreflende Feld an seiner schwächsten Stelle stützen. Punkte sitzen meist am Ende einer Falte oder an der Spitze einer Ader.

['m diese Befestigungen des Schmetterlingstlügels deutlich zu machen, wird ein Modell voroczeigt. bestehend in einem gegahelten Zwreig (der zwrei Flügeladern entspricht) und mit dümnem Seidenpapier über\%ogen. (Vergl. Abbildung auf' Tafel II.) Dieser Eächer würde bei rascher Bewegung leicht einreisien. wenn nicht die Gabelspitzen durch eine eingeklehte Schnul verbunden wïren. Ioieser Schnur entsprechen die bei vielen Schmetterlingen häntigen Streifen und mehrfachen dunklen Hall)mondreihen am Flügellande, die in der Form sehr oft an gewisse Zeichnungstiguren auf Vogelfoder'n elimmern (I)ie verschiedene Form solcher Halbmondreilen hei vorgozeigten geographischrn Formen von P'upilio machaon wird besprochen). Zwischen der Gabel des Modells ist vom Ramle nach der Mitte gehend ein Stähchen eingeklebt, welehes die zwischen den Ader'n verlaufende Falte darstellen soll. Bewegt man num den Fácher rasch hin und her, so wird sich am Ende des Stäbchens das Seidenpapier zerknittern und schliesslich reissen. Die Stelle ist auf dem Modell durch einen l'unden schwar'zen Fleck bezeichnet.

Reiz während der Entwicklung der Puppe hervorrief. Auch ich habe früher die Augenflecke für Schreckmittel gehalten, weil ich z. B. sah, wie meine junge schottische Schäferhündin, die sonst Weisslinge und Hummeln eifrig verfolgte und fing, ein Tagpfauenauge wütend verbellte, ohne es anzurühren. Die Hündin war aber aussergewönlich ängstlich und wagte z. B. einen Kürbis nicht anzurühren; weil er sie an einen Igel erinnerte, der ihr viel zu schaffen machte und sich sogar in ihre Hütte begab. Ich will nicht in Abrede stellen, dass lebhafte Augenflecken noch zugleich mehrere andere biologische Werte haben kömmen, aber wie die überzähligen Augenbildungen beweisen, ist ein Augenfleck zunächst ein ringförmig erweiterter Punktfleck. Diese Flecken stehen meist zwischen zwei Adern. Als ich nach dem Kongress eine Fahrt über den Brienzer See unternahm, sah ich zwischen den Wellen, die der Dampfer auf dem Spiegel des Sees zog, ring- oder augenförmige Spiegelungen des Sonnenlichts so deutlich, wie mir dies früher noch nie aufgefallen war. Die Oberfläche des Wassers muss doch eine entsprechende Gestalt annehmen, um diese regelmässigen Lichtringe reflektieren zu können. Vielleicht sind daher auch bestimmte Schwingungen der Flügelfläche an der Bildung der Augen als Reize beteiligt. 
Tatsächlich sitzen nun bei Pieris-Arten und viclen anderen Schmetterlingen schwarze Punkte genau an diesen Stellen, und diese Stellen sind es, die besonders leicht einreissen, wie eine Anzahl verletzter Schmetterlinge deutlich zeigt. Ein solcher Kohlweissling ist auf Tafel II abgebildet. Die Verletzungen sind zu schmal, als dass sie von einem Scholnahelbiss herühren kïnnten. Die zarten Schmetterlingsflügel reissen ja leicht, wenn sie irgendwo anschlagen, z. B. an Halmen oder scharfen Grïsern. In der Zeit, wo das Tier eben ausgekrochen ist und die Flügel noch wejeh sind und sodann in der Zeit, wo die oft rorragenden und schützenden Schuppen abgewischt sind, ereiguen sich solche Beschädigungen naturgemäss lejchter. Der lileck verhütet hicr rin weiteres Einreissen, das die Flugfähigkeit aufheben würde. Ist eimmal ein Flügel verletzt. so peitschen die Riss-Stellen den andern Flügè oder reiben ihn leim ruhigen Sitzen. so dassauf der andern Seite ein genau entsprechender, meist etwas kleinerer Riss entsteht.

Besonders interesiunt ist unter diesom resichtspunlit die Zeichnung auf der Unterseite von Kallima. Es erlklärt sich da, warum die Zeichnung blattähnlich ist und vor allem auch. warum ein Tril der Zeichnung n ic le t mit dem Bilde crines Blattes übereinstimmt. I)ie Seitemrippen dere Blattzoichnung finclen sich nur da. wo die Flügelarlerung del Quephinden bedarf. Die Mittelrippe biegt von der Querlinie ab und mündet in die auscuzogenen Spitzen dor Torder-und Hinterflügel. Das liann man auch bri andern Schmetterliugen heobachten, die ähnlich geschweifte Flügelfor'men besitzen und bei denen die betreffende Linie sicher keine Blattrippe darstrollen soll. Fs ist dius din Zeichnungsgesotz. das ganz elonso für die Vogelfeder gilt. Am schönsten sieht man es an einer Reihe von gehänderten Sihwumgfedern. Je spitzel da das Federende ist. desto meh" biegen dir Querbinden ab und worden gleichsam in die Spitze ausgezogen. Jan pthicke zwei Blätter von demselhen Baum. I as stumpfer gestaltete Blatt wird an Ende weniger gehogene Seitrmippen haben, während an einem spitzeren Blatt die letzten Seitennerven sich mehr nach der Spitze umbiegen und mit in diese auslanfen. Die sehwarzen Fleck(hen und Ringreihen des Krollimettügels, die man für Nachahmungen von Pilzen hält, sitzen am Ende von Flügelfalten. Die durchsichtigen Strilen, din Lörhere oder "Thautropten vorspiegeln sollten, erkiären sich

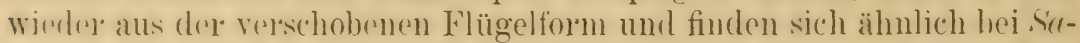
turnia pernyi mit geschweifter. Flügelspitze verbunden. Ich habe Kindron, die ja doch in ihrem Denke'n unhefangener sind als Erwachsene,

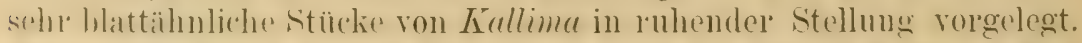
Nur rinmal gelang mir dio Täuschung dureh dio direlite Frage: "Was fül ein Blatt ist das? ) Auf die Frage : "Was ist das?" ) erhielt ich sonst die Antwor't "Ich weiss nicht » oder " Ein Schmetterling" ». Ich selbst hentreite die Blattahnlichkeit gar nicht. hestreite auch nicht. dass diese 
dem Sichmetterling nützt und dass er sich in seiner 'Tarnkappe sicher fühlt, aber wie dieser Schmetterling zu dieser Zeichnumg kommt, das kann man erst durch das auf geographisch vergleichendem Wege gefundene Gesetz der versteifenden Zeichnungen besreifen. Wan vergleiche noch Thelilu betule? mit Kullime und man wird den Einfluss der Flügelform auf den Linienverlauf deutlich sohen. Ein Gesetz, das sich im Vogelgefieder, Schmetterlingsflügel und in den Blattnerven übereinstimmend bestätigt, wild wohl greignet sein, das Rätsel dre Tierzeichnungen in noch vielen Fällen aufzuliären, ohne dass deshalb jode liontrastfärbung auf diesem Wege erklärt zu werden braucht.

Meine Ansicht, dass die Stellen, welche stärke $\mathrm{l}^{\circ} \mathrm{l}^{\circ} \mathrm{Ab}$ butzung unterworfen sind, stärker pigmentiert werden, illustriert

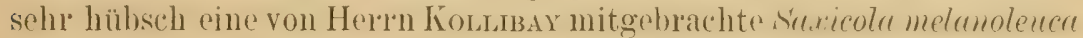
(strpazina) aus Dalmatien. Diese hat pinen abnormen schwarzen Fleck genau an der Stelle auf dem Hinterliopf, welche bei nolmalen Tägeln im Sommer bis anf die Federwurzeln abgerieben ist. Es scheint, dass dieser Schmätzer sich sein weisses, daher ungeschütztes Gefieder', auf dem Scheitel abschleift, wenn er sich unter Steine verkriecht. Dieser Vogel stellt eine sogenannte Nutation dar. Im Wintergetieder haben auch die normalen Vügel, wenigstens dir del östlichen Form, die reinere Farben, und deshalb anscheinend auch stärliele Almutzung zeigt, cinen schützenden gramen Anflug an den Spitzen der Schoitelfedern. Die regelmätssig hei jedem Stück sich wiederholende geographische Variation beweist es: dass wir es auch bei einem solchen abnormen Einzelfall nicht mit einer zufälligen oder bedeutungslosen Erscheinung zu tun haben.

Die genaueste Erforschung auch der kleinsten geographischen $\mathrm{Ab}$ weichungen wird also eine dankhare Aufgabrem. In dou vorwelegten und unzähligen anderen Serien (bezüglich Ringen) varalleler gengraphischer Variationen wiederholt sich das interessante Problem "Iensch und Mrnschenaffe') ', (lessen Klärung sich mit der Kilarstellung zahlecicher Analogion rinst ron selbst ergeben dürfte. Angesichts der lö̈ufigen Uehereinstimmung der zoogeographischen firenzen und sprachgerenzen wird man annehmen dürfen, dass unsere Resultate auch für die geschichtlichen Wissenschaften nicht gleichgültig sein werden.

Wie die voredegten Formen konediniert siud und nicht subordiniert. so wird sich auch hies zuletzt ergeben dis Gleichlerechtigung aller Nationen und Rassen.

1 Ich habe deshalb für solche Parallelerscheinungen den Namen A ffenformen rorgeschlagen. 


\section{Diskussion.}

Her' Wasmann splicht dem Vortrage seine volle Anerkennung aus. I) ie in deuscelhen ausgefuhrten Grundsätze über die Bedeutung der Tiergerographie für die systematische Algrenzung der Arten u. s. w. hat nicht hoss auf ornithologischem Gehiete Geltung, sondern viclfach auch auf ('ntomologischem. I)ie Gattungen Curalues (GAx(thBALER und Bors) und G'umponotus (Forer und Enery) bieten vortrefthiche Bulego dlafür. dass nur durch die Tiergeographie Licht in die Klassifikation der zu jenen (rattungen gehörigen Formenreihen gebracht werden kann. Auch die Ausführungen des Redners über die biologische Bedeutung der Bänderzrichumng auf dem Vogelgefieder dürften fül das tiefere Verständnis der Fïlloungs- und Zeichungsgesstze vieler Inseliten neue interessante Gesichtspunkte bieten.

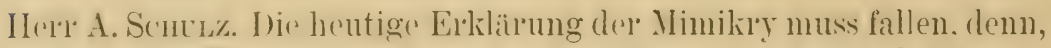
wio ich in den Sitzungsberichten der Kigl. Bayer. Akademie d. Wissensehatten, IBd. XXXIII, S. s11, 1945, und früher schon an anderen (orten. Z. B. Berliner entomolog. Zeitschrift, 48. Bd., S. 261, 1903, auseinandergesetzt habe, kommen ganz ähnliche Eischeinungen auch bei exotischen Stechwespen ( $\mathrm{A}$ eu leaten), speziell bei südamerikanischen Faltenwespen, Diplopteru, vor. Bei ihnen kann, da auch die "Nachahmer " nit einem (xiftstachel hewaffnet sind, ron cinem durch Zuchtwahl esworbenen "Schutze » überhaupt keine Rede mehr sein. Einige willkürlich herausgegriftene Beispiele, denen genug andere angefügt werden könnten, mögen dies elläutern.

Polistes anulis F., eine im Gehiete des Amazonenstromes und in Guiana lo'mische gesellige Faltenwespe war schon frühel als "Nachahmerin " der in denselben Ländern lebenden, ebenfalls den Vespiden zugehörigen Art Polybia flavicans (F.) bekannt, ohne dass einzusehen wäre, was hiel die "Mimikry » bezwecken soll. Vor einigen Jahren habe ich nun aber auch noch (aus Oher-Amazonien [Ecuador]) eine dritte, den h.rilen vorenamnten täuschend ähnliche, zwar auch den Faltenwespen, aber einer anderen Unterfamilie, den einsam lebenden Eumeniden angehörende Spezies erhalten, die von mir inzwischen als Eumenes

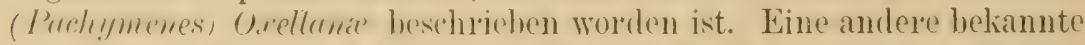
neotropischer Polylia-Art, liliacen (F.) hat zu I)oppelgängerne Polistes lilinciosus Sauss. und weiterhin die Fumenide Montezumin liliacen (irib., die beide mit ihr zusammen in den gleichen Gebieten zu hause sind. Fast sicht es nun so aus, als ob unter den tropisch-amerikanischen lonmun der (a)tungen Polybia, Polistes, Puchymenes bezw. Montezumia, etwa in der Art, wie wir es bei gewissen, ebendort fliegenden 
Tagschmetterlings-Gattungen. z. B. den It homiden: Vrueorgenes, Ithomia, Hypoleria, gewohnt sind, gauz gesetzmässige, vielleicht physiologisch erliärbare Affenformen-Zyklen bestehen. So erkaunte ich erst kürzlich noch zufällig in meiner eigenen Sammlung und gleich danach im Strassburger Museum einen neuen Polistes aus Surinam, deceptor m.. heraus, der mit Polybir angulatu (F.), von der nämlichen Gegend, eine so verblüffende Achnlichkeit hat, dass er meiner und wohl auch schon anderer Hymenopterologen Aufmerksamkeit entgehen konnte. Die mit den beiden letztgenamnten Wespen genau korpespondierende Eumenide wird wohl auch noch auforefunden werden, da Montezumirt-Formen von etwas entfernterer Aehnlichkeit aus Südamerika schon beschrieben sind.

Uebrigens hat Dr. OHaus unlängst - in ider Berliner entomol. Zeitschr., S. 230, 1903 - etwas Aehnliches bei neotropischen Coleopteren (Familie der Ruteliden) bekannt gemacht, wo auch je 4 am gleichen Orte vorkommende Arten der heiden Gattungen Macraspis und Pseudomacraspis einander täuschend ähnlich sehen. (Onats glaubt diese Erscheinung damit erklälen zu kiomnen, dass die korrespondierenden Arten beider angeführten Genera möglicherweise sowohl als Larven wie auch als Käfer dieselbe Futterpflanze haben, dass mithin die gleiche Körperform und Färbung ein Resultat der gleichen Lebensweise wäre, die woh! Einfluss luäte auf die allgemeine Körperform und Fürbung, die charakteristischen Gattungsmer'kmale jedoch unberührt liesse.

Verwickelter wird der. Mimetismus bei südamerikanischen I)iplopteren noch dadurch, dass nicht nur Angehörige verschiedener Gattungen einander nachäffen, sondern dass es solche Spottformen auch innerhalb eines und desselben Genus gibt. So ähneln Polybia liliacea (F.) und P. pseudomimetica M. in (xösse, Farbung und Zeichnung einander zum Verwechseln und sind nur durch scharfes Zusehen zu unterscheiden. In gleicher Treise wird Montezumia litincen Grih. von M. liliaciosa (rril). liopient.

Zur Erklärung für die hier vorgebrachten beiden Arten von Nachahmung reicht die landläutige. von BATEs und WALLACE. vornehmlich auf Grund von Schmetterlingsstudien aufgestellte, wesentlich tel eo logische Mimikry-Theorie nicht aus. Noch viel weniger tut sie es abel fül eine dritte Form von Mimetismus untel Hymenopteren, dio ich zum Schluss kurz streifen will. Eine von mir neubeschriehene Trigonalid e 1 -Gattung und-Alt, Orthogonalys boliviana, sieht gewissen, in ihrer Heimat rorkommenden Schlupfwespen der Familien der Ich n e u moniden und C:ryptiden dermassen ähnlich, dass es auch fül ein geübtes Auge vom Zufall abhängen wird, sie darunter aufzutinden. Nun machen die Trigonaliden ihre Entwicklung als Sclimarotzer in den Nestern geselliger. Faltenwespen durch. denen manche von ihnen in Tracht und Fïrbung annähernd ähneln (echte "Mimikry s). Welchen 
Nutzen hat es dagegen für Orthogounlys botiviana, die offenbar ebenfalls ein Vespiden-Parasit ist, in dem Kleide einer $\mathrm{Cr}^{r} \mathrm{y}$ p tide, also in Feindes-oder Trutzogstalt zu erscheinen? Müsste sie nicht gerade durch die natürliche Zuchtwahl veranlasst worden sein, das schützende Vespiden-Kleid anzunehmen, wie es viele ihrer Verwandten getan haben?

Man sieht, wohin selbst noch so liehgewonnene Theorien führen, wenn einmal das ihnen zu grunde lirgende, jedermann geläufige Tatsachenmaterial beiseite gelassen und neue Daten aus abgelegeneren Wissensgobieten zur Tachprüfung herangezogen werlen. Der Fehler der Bates, Wallace, IARWr bestand im rorliegenden Falle ehen darin. dass sie bei ihren Spekulationen fast nur natürlich wehrlose, indifferente Insektenformen in Betracht zogen, dagegen die mit einem Giftstachel bewaffineten Hymenopteren ausser acht liessen. olowohl unter diesen schon vorher durch Saussre Fïlle von frappanter Aehnlichkeit zwischen Angehörigen verschiedener Gattungen veröffentlicht worden waren.

Auch selbst unter unseren bedeutendsten Lepidopterologen macht sich in den letzten Jahren eine gewisse Skepsis gegenüber der hisherigen Erkiärung der Mimikry-Erseheinungen hemerkbar. Es wird immer mehr zwischen " echter" ) und "falscher" Mimikry unterschieden und auf den Einfluss hingewiesen, den die Insektenhändlex durch Zurschaustellung und Vertrieb von mimetischen Beispielen haben, aus denen die Allmacht und Gïte der Natur zu ersehen sein soll.

Prof. Echstern bestätigt die Abnutzung del hellen Federteile beim (ioldregenpteifer ( vol. Forstliche Zoologic. Berlin, 1^97. p. 212, Fig. 201); als Ursache des Meckerns der Becassine bezeichnet er den schiffsschrauhenähnlich gewundenen unteren Teil der Fahno der äussersten Schwanzfoder, welehe heim Inurehschneiden der Luft in Schwingungen versetzt wird; el glaubt, dass ähnliche Färbungsunterschiede, wie Vorredner durch Hühnerhabicht, Wanderfalke, Kuchuck u. a. illustriert, auch ohne Zuhilfenahme der geographischen Varietiiten "rliärt werden liönnen, da sie sich mit den normal gefärbten Frormen zusammen finden.

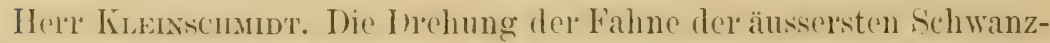
forler der Breassine (Aufloiegung der Innenfahne, welche sich auch bei anderen Vögeln findet) macht es umsomehr begreiflich, dass die Fahnenstrahlen rines besondreren Zusammenhangs bedüfen, um sich in dem auscerordentlichen scharfen Luftzum nicht zu zerteilen. I)iesen Zusammenhang sicher'n die Binden.

Vom Wanderfallien und Hühmerhabicht findet man allerdings auch in I) entschland sehe helle und sehr dunkle Stücke. Trennt man sorgfältig in dens Sammlungen Wintergäste oder Zugrögel und Brutrögel. so wird das bild schon gan\% anders. Soclann muss man die drei zoogeographi- 
schen Gebiete "Ostpreussen - Mitteldeutschland - Rheingegend" tremnen, die ostprenssischen Vögel ähneln den sibirischen, die vom Rhein den dunklen Formen des westlichsten Europas, wie dies sehr dentlich die hier mit vorgelegten Zwergspechte und Meisen erkennen lassen.

Was nach dieser Sonder'ung übrig hleibt, ist die normale Variationsweite, der einzelnen geographischen Formen. Diese mag teilweise in dem Sinn zufällig sein wie z. B. Inucke desselben Bildes oder Abzüge von derselben photographischen Platte, hell oder dunkel ausfallen. Zum Teil beruht diese Variation - sagen wir einmal mitteldeutseher" Wanderfalken und Hühnerhabichte - aber auch wieder auf geographischen Einflüssen, indem bald dunkle südliche Yägel einwanderm, bald helle Zuzügler aus dem Nordosten zurückbleiben, um zu brüten.

Auch in Sibirien variiert der Hühnerhabicht zwischen der hier vorgelegten und einer noch weit helleren ganz weisslichen Form. Man muss, um richtig zu urteilen, die ganze Variationsbreite der Sibirier mit der ganzen Variationsbreite mitteldeutscher Brutvögel vergleichen. Ich konnte natürlich nicht die umfangreichen Serien meiner Sammlung mit hierherbringen und beschränkte mich auf die Auswahl dere charakteristischen Typen. Wie die vorgelegten Skeletteile zeigen, lassen sich die sardinischen Hahichte (auch Wanderfalken und Sperher') an ihrer liürzeren Gestalt leicht erkennen.

Wenn ich meine Intersuchungen auf die gengraphiseht- mod nicht auf die individuelle Variation stütze, so geschieht dies, weil hier der Einwand, es handle sich um zufällige, daher bedeutungslose Erscheinungen. unmöglich ist.

Was die Bemerkung des Her'rn Prof. JACoBr, Tharandt, betrifft, der' den Ausdruck "Mimikry " beanstandet, so stimme ich ihm bei und erlaube mir hinzuzufügen: "Quod erat demonstrandum!" Ich habe den Ausdruck « Mimikry » gewissermassen ironisch gebraucht - "Pseudomimikry " hätte ich also vielleicht sagen sollen - weil gerade die wunderharsten Aehnlichkeiten im Tierreich nicht auf Nachahmung und nicht auf Verwandtschaft zurückzuführen sind. 


\title{
Zur Natur- und Entstehungsgeschichte der Südalpen.
}

\author{
Von Prof. H. SIMROTH (Leipzig).
}

\section{A. Einteitende Bemerkungen.}

Was ich über die Naturgeschichte der Südalpen Positives und Theoretisches vorzublingen habe, beruht lediglich auf Autopsie; es stellt eine Auswahl der Tatsachen dar, die ich bei mehrmonatlichen Herbstreisen in den Jahren 190): und 1903 auftand ${ }^{1}$, sowie die Schlüsse, die sich mir im Zusammenhange mit der kurz vorher hervorgetretenen Pendulationstheorie aufiriangten. Damit ist notwendigerweise ein Nachteil verbunden, insofer'n als die Tatsachen nur als Stichproben erscheinen, welche der gründlichen Prüfung im Gesamtgebiete entbehren, daher die Gefahr 'ntsteht, dass dem Einzelnen eine zu grosse Tragweite beigelegt werde. Andererseits ist der Vorteil einer möglichst intensiven Prüfung umschränkter Gebiete nicht zu unterschätzen, und es ist ja der allgemeine Gang der Wissenschaft, neue Gesetze am Einzelnen zu entdecken und vom I)tail aus kritisch auf das Ganze zu ühertragen, da meist nur so ein wirklicher Fortschritt erreicht wird.

Der" ur'sprüngliche Plan war der, womöglich die gesetzmïssige Umwandlung unserel' grössten Laudschnecke, des Limax maximus, zu ergründen. Ilieses Tler, bekanntlich nördlich der Aipen in Fleckung und IBänderung zwischen Woiss durch Grau und Ocker bis Schwarz ausserordentlich wechselnd, erreicht in den südlichen, besonder's südwestlichen Alpenländern eine'n wunderlaren Reichtum, indem die Zeichnung nicht nur' noch mehr' authlüht, sondern gelbe und lebhaft rote 'Töne den ganz'n Kö̈rper überzichen und schliesslich gelber und roter Farhstofí dem Schleim beigemischt wird. Pant, Lessona. Pollonera u. a. haben uns herrliche Abbildungen von der schier unerschöptlichen Manchfaltigkeit gegeben. I) ie Frage nach der Auflösmug in einzelne Arten mag dabei ganz merörtert bleiben, da sie ausführliche anatomische Untersuchung an rinem reichen Seriemmaterial erfordern würde. Bemerkt mag noch werden, dass Limax muximus das grösste weiter verbreitete wirbellose Land-

${ }^{1}$ Für Reiseunterstützungen schulde ich der königlich preussischen Akademie der Wissenschaften zu Berlin und der Gesellschaft der Wissenschaften zu Göttingen elirerbietigen Dank. 
tiel' mindestens del palaeatktischen Region, vielleicht der ganzen Frole darstellt, daher es bei seiner Varialilität wohl das allergenguetsto ()bjekt für meine Zwecke abgiebt.

Ich logann meine Forschungen an der niedrigsten Wasserscheide, d. h. am Brenner, und folgte dann der alten Strasse zum Gardasee, immer von Zeit zu Zeit Halt machend und eine einzelne Lokalität in Bezug auf Wald- und Baumgrenze, Exposition ote. möglichst ringehend nach Längs- und Quertäleı'n explorierend. An Gardasee bildrut Salo eine'n

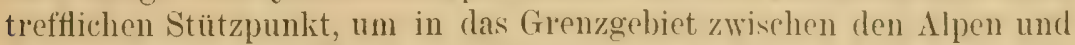
dem lombardischen Hügelland Einblick zu gewimnen. Ton da wandte ich mich nach der Adamellogruppe, beziehungsweise dem 'Tal zwischen ihr und der Brenta, weiter ging ich in die Bergamasher-Alpen, von denen ich das Seriotal meiner' Arbeit zu Grunde legte, weiter wurde am ('omersee das auch den Paläontologen wohlbeliannte Tal von Fsino näher mutersucht, dann gieng's über den Langensee und den Gotthardt zurück.

Das nächste Jahr brachte mich zunächst wieder in das Seriotal zul' Nachprüfung. welche die Resultate von 1902 volliommen hestatigte. Fis wurde diesem Punlite besonderes Augenmerk zugewendet, weil er dem Schwingungskieise, dem $10^{\circ}$ o. Br., del durch die Beringsstrasse geht, zunächst liegt und mir die roten Schnecken zuelst geliefert hatte als deren nördlichsten Torstoss. Damn gieng es nach Piemont, wo ich im Val d'Aosta Station machte und auch den südlichen Quertäler'n, dem Steinbockgebiet des Val Savaranche und Val de Rléme Pesuche abstattete. Die Umgegend von 'lurin und Genua folgte. Bei Genua wurden dir Schluchten des Apennin trotz der Trocknis mit Erfolg abgesucht, ebenso führte manche Schleimspur in der ligurischen Kette auf die crwünschten Tiere. I)ie Seealpen wurden in der oberen Hälfte des Rojatales durchstöbert. Am Mont Cenis wurde nochmals dio höhere Wasserscheide nach der Rhone zu vorgenommen und schliesslich weiter unten in Savoyen ein Einblick in den veränderten Stand der Fauna geronnen. Das, was ich im Einzelnen gefunden, erhielt durch die mündlichen Aussagen der Herren vom Turiner Museum, in erstel Linie Poldoxkra, Bestätigung und Erweiterung, so dass es mir hoffentlich trotz dex sporarlischen Arbeit gelungen ist, eine hinreichende Tehersicht üher die allgemeinen Verhältnisse zu bekommen.

Um ein Wort über die Vegotation voraususchicken, so befinden wir uns in den italienischen Südalpen in einer überaus glücklichen Lage. Während in anderen fíulturländern, wie bei uns in Deutschland, ein Waldbestand zunächst gar keinen Schluss gestattet auf die anfïngliche Pflanzendecke, da del Mensch nach Willkür und wirtschaftlichen Rücksichten die Physiognomie der Gegend absichtlich ändert, auf Laubholz Nadelholz, auf Nadelholy Laubholz folgen lässt, eine Willkï̈, dio in modernen Siedelungsländern, den V'ereinigten Staten z. B., noch viel weiter 
Platz greilt. ist in Italien trotz der weit älteren Kululur die Vegetation ein reines. ursprüngliches Naturprodukt, in das der Mensch nur selundïr modrelnd eingreift und seit alter Zeit eingegriffen hat. Hier gilt seit dem. Altertum das römische Recht: der Wald zwar ist Privatbesitz, aber der Borlen gehört der Gemeinde, die das Weiderecht darauf ausübt. So wird rine Aufforstung, ein Bestandwechsel ummöglich. Und das morlerne italienische For'stgesetz wirkt im gleichen Sinne, es erlaubt nur die Bäme einzeln in betriahtlichem Abstand von einander zu schlagen. Hier sind Kahlschlay, Ahtrieh, Schonung, Saatkamp, die uns Deutschen so vertraut sind, unbekamnte Begriffe. Die Ptlanzen sïen sich selber an. Ja es scheint, dass Eindringlinge nur schwel Fuss fassen: die römischen Landgüter am Comeree darunter das des Pusnus, haben kaum veründernd auf die Vogetation eingewirkt, vielleicht von der Feige abgesehen, die Orangen sind Fremdlinge geblichen; erst dic amerikanischen Gewächse von ähnlichem Boden, Agene und Opuntia, namentlich dazu die Rolsiviu, erwerben hie und da Bürgerrecht. wie es der Nussbaum in: unbekannter früherer Zeit getan hat. Freilich schont der Italiener das Holz nicht, das er allein. frisch oder zu Kohle gehrannt. zur Fenerung benutzt. Aber dass er so wenig Holz in seinen Bauten zu verwenden gewohnt ist. heweist doch wohl, dass der geringere Holzwuchs weniger Folge der Wirtschaft ist, als des Klimas. Man kamn die Stetigkeit der Verhältnisse seit dem Altertum in der Natur so gut wie in der Kultur verfolgen. worauf ich hier mich nicht weiter einzulassen hrauche'. Die italienischn' Sennhü̈tte, die Malga, ist eine Steinhütte, die deutsche drüben über dem Gehirgskimm ein Blockhaus. Aechten L'rwald treffen wir an der oberen Waldgrenze am Brenner, z. B. in dem kleinen Vennatal, wo die Arven entworlev vom Sturm gebrochen alskahle Gerippe umherliegen, oder in etwis dichterem Bestande alte Stumpen ron mehreren Ietern Höhe moderu lassen, dass man knietief in den Muln einsinkt. wie es uns sonst meist aus fernen Weltteilen geschildert wird.

Ich glaubte diese bemerkmgen voranschicken zu sollen, als Stütze für die Schlüsse, die etwa aus der 'T'ier- und Pflanzenverbreitung zu ziehen sind. Wenn irgendwo in Mitteleuropa, so hat der Naturforscher ein Recht, die Verhältnisse in den Südalpen als stetig zu betrachten und seinen Folger'ungen ohne Weiteres zu Grunde zu legen.

1 Die zahlreichen Einzelheiten, die ich sowohl für die ungemeine Stetigkeit der Kultur, als für die nach fast jeder Richtung hin viel grössere Verwendung der Steine statt des Holzes in Italien nach eigenen Beobachtungen anzuführen hätte, mögen gelegentlich an anderer Stelle vorgebracht werden. Der Uebergang zwischen germanischen und italienischen Sitten lässt sich gerade in den Südalpen trefflich verfolgen. Betreff's jeder Kultur möchte man behaupten, dass ihre konservative Stahilität zu ihrem Alter in direktem Verhältnis steht. 
Sie machen sich in einer Hinsicht für das Studium der Nacktschnekken, besonders der glossen Limm, anffälig benerklich. Einem (resetze zufolge, das ich früher aufstellte, ist die allgemeine Fi'nährung rle' T'iero von grümen Ptlanzen elst ein selinudiurer Zustand. Dep ursprüngliche knüpft an chlorophyllfere an, Bakterien, Pilze, Moler, woraus nach del eimen Seite Carnivorie, mach der anderen Herlivoris hervorgeganeren ist. Dom entsprechend finden wir hei uns fast die ganz' (besellschatt alteltümlichster Lanltiele untel' der Rinde alter' Bammstumpen, namentlich

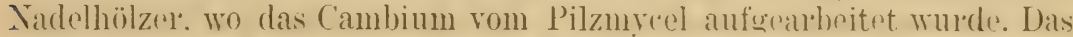
ist nördlich der Alpen die typische Stelle an dor man don Limax ma,rimus bei trockenem Wetter erbeutet. Sie fehlt in den oberitalienischen Wäldern. Die Ausnutzung des Waldes geht hier so weit, dass der Besitzer sich fient, wenn der Waldhoden kostenlos gereinigt wird, der Arme aber jedes Rindenstück sorgfältig wegnimmt und an dre Somno trodknet. um es als Zunder beim Feueranzünden zu verwenden, - gewiss in unserer Zeit fortseschrittener Bulnulutungsmothorlon ein eigenartiges Culturrelilit. Aber es hat. neben dem Kilima. zur Folge. dass auch dio grosie Schneckr gezwungen ist, unter. Strinen Schutz zu suchen, wie die ührigen Tiere von ähnlicher Lebensweise.

Noch möchte ich von allgemeineren Dingı'n eine Folgerıng der Pendulationstheorie vorausnehmen. Da die kurze Erdachse zwischen Nord-

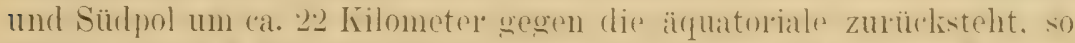

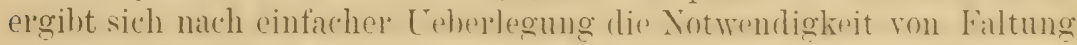
und Gehirgsbildung bei der mach dem Nord-orler südpol zugerichteten Schwingumgsphase. Denlit man sich z. B. den Halbkreis Ecualno-ItalienSumatra oder spreieller Ecuador-Bergamo-Sumatra so verschohen, dass die Schwingpole Ecuador und Sumatra fest bleiben, Italien, bezw. Ber-

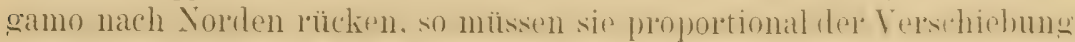
immer weiter über den Meeresspiegel sich erheben. Das mag eine Zeit

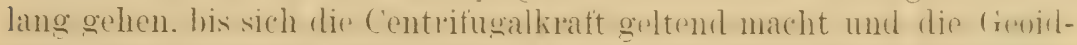

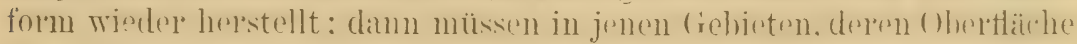
für die Fol'm zu gross ist, die Faltungen eintreten. Wir werden nachlier

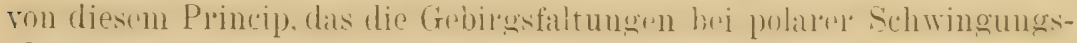
phase eintreten lässt, die Anwendung zu machen haben.

Und damit komme ich auf den Limax maximus, den ich in seinem writesten Unfange nehme, wir bereits angedentet, olne Rüchicht auf die etwaige Gliederung in Arten.

\section{B. Limax maximus nördlich der Alpen.}

Wir haben bei uns bekanntlich zu unterscheiden zwischen der freilebenden Form, die als $L$. cinereoniger bezeichnet zu iverden pflegt, und

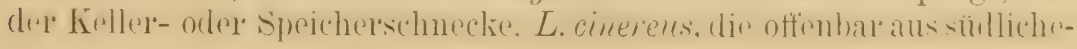


rem Kilima stanment und durch Anschluss an die wirtschaftlichen Terhältnisse des Denschen verschleppt ist. L. cinereoniger setzt in der Jugend mit einer' meist rötlichen For'm ein, welche auf dem Rücken jederseits vine dunlilere Stammbinde trïgt, im Anschluss an den Blutsinus; dazu kommt. mehr oder weniger deutlich, eine ähnliche Binde auf dem Mantel. Die weitere Yerfärloung bringt sehr verschiedene Muster zu Stande: bald entsteht ein einfarbig schwarzes Tier (dlie Sohle lasse ich hoi Seite), bald bleibt auf granem Grunde einfach die dunkle Riückenstammbinde. I)iese aber kann durch stärkere Pigmentconcentration jederseits von einem helien Streifen gesïumt werden, so dass nun noch eine innere mediane und eine nach unten verschwommene äussere Binde auftreten. I)ie beiden inneren Binden rechts und links werden durch die hellere Kiellinie getrennt. Innerhalb aller Binden kamn abermals Pigmentconcentration cintreten; dann löst sich die Binde in cine geringere oder grössere Anzahl von schwarzen Flecken auf. Der Grund wechselt dabei beinahe von Weiss bis Schwarz unter Beimischung ockeriger und rötlicher 'Töne. In Allgemrinen zeigt sich, dass in feuchten kühlen Gerbirgslagen die dunkeln, bezw. schwarze'n Formen vorwiegen, auf wälmerem, trockenerem Haideboden dagegen die helleren, stärker gefleckten. Der Mantel bleibt bei den freilebenden Tieren stets einfarbig ohne Pigmentroncentration. (Von einer unbedeutenden Aufhellung an seinem Rand mag abgesehen werden.)

Umgekehrt wird der $L$. cinereus scharf an den dunklen, schwarzen Flecken des Mantels unterschieden. Auch der Rücken ist gefleckt, meist weit reicher als beim $L$. cinereoniger. Niemals ist er dunkel einfarbig, die mittleren und helleren Töne überwiegen. Relative Trockniss und Wärme haben offenbar auf dieses Tier länger eingewirkt, so dass das Princip, das sich unter den gleichen Bedingungen bei der germanischen Freilandform auf dem Rücken geltend macht, auch auf den Iantel übertragen hat. Soviel wir wissen, entsteht die Syeicherform bei uns nie durch Eindringen freilebender Tiere in die Keller, sondern ist durch Vererbung gefestigt.

\section{Limax maximus in den Südalpen 1 .}

Wie bei uns, ist die Form der südlichen Alpenabhänge eine reine Waldschnecke. Inei Arten von Nackitschnecken fand ich am Brenner über die obere Waldgrenze hinausgehend, Limux arborum s. marginatus, Agriolimax agrestis und Arion subfuscus, us sind dieselben drei Arten, welche

${ }^{1}$ Die bunten Tafeln, durch die der Vortrag illustriert wurde und denen Skizzen nach dem Leben zи Grunde lagen, habe ich hier lieber nicht reproduzieren wollen, da sie besser in eine ausführliche Abhandlung passen dürften. 
auch den hohen Norden bewohnen, mir liegen alle drei als einzige Nacktschnecken von Island vor, aus dem Senckenheres'schen Museun. Limu: arborum steigt mit den Flechten an Felsen empor, die Ackirschnecke autfälligerweise, wie der Arion normaliter, an Hutpilzen, und zwar in der. kleineren weisslichen Form. die für die Iforgegenden des weissen Meeras charakteristisch ist. Es spricht sich also gleich in diesem Vorkommniss ein allgemeines Gesetz aus. L. moximus hält sich an den Nadelwald und zwar an die Pilze, welche ihm eigentümlich sind und vielfach von ihm aus in den Laubwald ausstrahlen. Die eigentliche Hoinat sind zweifellos die Nadelwïlder, Beweis genug für das Alter. (irosse, grohzerfressene Hutpilze deuten fast immer seine (regenwart an. Bedenlit man, dass die Symbiose zwischen dem Pilzmycel und den Baumwurzeln, die Mycorrhiza, zuerst und am allgemeinsten an den Coniferen erwiesen wurde, dann tritt die Beziehung un so klarer hervor. So wenigg genau w ir leider üher die Pilzarten, welche die Mrcorrhiza bilden, unterrichtet sind. so lässt doch gerade der Zusammenhang mit dem Limar die nähere Beziehung leicht erkennen, und die Botanik lïnnte hier von der Zoologie profitieren. Ich jrüfte im Camonicatal groxse Mengen von Agaricus capsareus, dem grossen, delikaten fliegenpilzälınlichen Schwamm mit dem gelben Fleische, der zusammen mit Boletus edulis im weitauscehreiteten Buschwald der Edelkastanien gesammelt war; es zeigte sich keine Spur von Schneckenfrass: gleichwohl fand ich die Schnecke in demselben Tal im Lärchenwald, von wo sic auch in die Kastanienbestände dicht daruntel" eingedrungen war, wenu diesel gewöhnliche Ausdruck erlaubt wäre (s. u.).

Am Brenner war Limax maximus einfach als $L$. cinereoniger dunkelgrau mit schwarzer Stammbinde, weiter nach Süden (Bozen, Adamellogruppe) trat er ähnlich auf, oft mit den Flecken und Bändel’n. Es jst meist nicht schwer, nacholem man die (iesetze der Exposition erkannt hat, das Tier aufzutinden; man hält sich an die untere Grenze des Nadelwaldes. Er wird stets üppiger an den regenreicheren südlichen und östlichen Abhängen, vom 'Tal aus gerechnet, d. h. in westlicher und nördlicher Exposition, wo er auch weiter herunterreicht. Mir ist es geglückt, am elsten Tage in einer Gegend heim ersten A ustlug dir Schnechr zu erbeuten, ohne dasis ich während der ganzen folgenden Woche noch eine zweite Fundstelle in der Umgegend aufgetrieben hätte.

Etwas weiter südlich, etwa bei Klaussen nach Vilnoes zu, treten zu den dunklen Formen hellere, weisslich mit ganz schwacher Rückenstammbinde, dabei ockerig übergossen. Fs mag bemerlit werden, dass niemals eine Form streng an rine Localität gebunden war, sondern dass je eine Serie den allmählichen Uehergang zeigte. Ire heliere form entspricht etwa dem L. montanus Leydig:

Auf dem Mendelgebirge hat sich aus dieser Form eine merkwürdige 
Mimikry heransgehildet. Die Reisehandbücher gehen bereits an, dass an der Hascllumg hei Boz'n die Schildviper vorkomme. Nach meiner Hüchtigen Erfahrung dürfte sie an der Mendel ihr Hauptquartier haben. Hier stiess ich auf ein zortretenes Tiel ohne Kopf, dass ich nach del Rückenzeichnung für eine Coronella lrevis hielt, bis ich bald eines besseren belehrt wurde. In diesem Gebiet bei ca $800 \mathrm{~m}$. erbeutete ich unter' Steinen in Wald mehr L.muxims als an irgend einer anderen Stelle. Darumter aber waren Exemplare, die bei hellgrauem oder weisslichem Rücken, drsosn Runzeln in den Furchen durch dunkleres Pigment abgegrenzt wurlen, einzelne kileine dunklere Flecke hatten. wie eine Coronella, deren Schuppen ausserdem durch die Runzeln vorgetäuscht wurden ". Der Mantel aber hatte eine wunderliche Zeichnung angenommen, die völlig cinem Schlangenkopf glich; es waren aus grauem Pigment hellere Flecke ausgespart, hinten grössere, nach vorn zu kleinere, wie bei einer Schlange. Die Augentriger mögen dazu die gespaltene Zunge vortäuschen. - Neben diesem kommen andere Schnecken mit ganz derselben Zeichmung vor, aber noch mit eincle tief schwarzen Rüchenstammbinde, die auf der oberen, medianen Seite von einem weisslichen Streifen gesïunt wird. Auch für diese Zachnung fand sich sogleich die Erklärung. In greller Jittags:onne kr iecht mir eine junge Srhildviper üher den Wog. offenbar hall)verhungert; denn die Haut bildete jederseits eine scharf alogesitzte helle Falte. unter welcher oin schwar\%" Schlagsehatten entstand, genau wie Stammbinde und Streifen der Schnecke?. Es scheint also, dass junge hungrige Vipern auch bei Tage ihrer Nahrung nachwehen. Wir Mimikiy dep Vacktichnecke nach den fiftschlangen sowohl mit glatter als mit faltiger Seitenhaut aber dürfte sich nach jeder

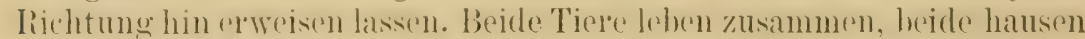

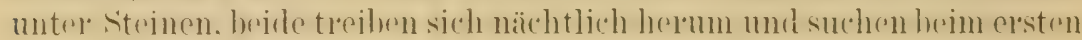

1 Selbst von gewöhnlichen Formen des $L$. cinereoniger, wenn er auf dem Waldboden dahingleitet, ist eine gewisse Schlangenähnlichkeit schon mehr als einem Beobachter aufgefallen. Einen besonderen Fall beschrieb ich in den "Nacktschnecken des russischen Reiches ". Doch handelt sich's dabei mehr um den allgemeinen Habitus der Zeichnung. Schärfer ist die Achnlichkeit bei dem Philomycus viperinus, den ich von Japan melden konnte. Hier wird die Aehnlichkeit mit der Kreuzotter im Zickzackband so stark, dass ich auf das frühere Vorkommen der Vipera berus in Japan schliessen möchte. Aehnlich liegt die Sache bei dem Paralimax salamandroides, den ich aus dem zentralen Kaukasus abbilden konnte. Auch bei ihm springt der Nutzen, den die Mimikry nach einem giftigen Tier gewährt, in die Augen. Nirgends aber wird der Beweis so scharf als in dem vorliegenden Beispiel von der Mendel.

Die Untersuchung der Giftzähne an der erlegten Schlange gab mir volle Sicherheit. Leider habe ich das Tier, da mir der Zusammenhang erst in der nächsten Viertelstunde danach klar zum Bewusstsein kam, nicht mitgenommen; doch wird man unschwer ähnliche Exemplare wieder auftreiben können. 
Strahl der Morgensonne ihre Verstecke auf. Die Schnecke aber hat besondere Verfolger in Vögeln, wahrscheinlich alpinen Corviden. Dafüur fand ich einen interessanten Beweis in einer zerhackten und ihres Inhalts beraubten Arionta arbustorum am Brenner See, von der nur der gequollene Spermovidulit oder Uterus übrig sebliehen war, ein fall von Hexenbutter oder Stermschuppenregen, der auf dem gequollenen Fileiter nicht eines Frosches, sondern einer Schnecke beruhte.

Ich will noch hinzufügen, dass in den hereuzungen der Linien, wolche auf dem Mantel die Lopfischilder der Schlange dastellten. einige schwarze Spritzflecken eingestrout waren, also ein kemmedchen nicht fül $L$. cinereoniger, sondern für $L$. cinereus; und nun lässt sich der Uebergang zwischen diesen beiden Formen nach Süden hin immer schärfer verfolgen, in dentlicher Abhïngigkeit von Exposition und Höhenlage. Als Beispiel führe ich zwei Tiere aus dem Camonicatal an, die sich zwischen gewöhnlichen cinerenniger fanden, in s(x) m. in I axpehenwald ein helleres Stück mit dunkleren Binden, je drei auf dem Rücken; in diesen war das Pjgment vielflach zu schwarzen Flecken concentriert; rin Par solcher Flecke finden sich aber auch auf dem Mantol, und zwall zwei über dem rechten Lungenflügel. einer übex dem linken, also recht deutlich unter meteorischen Einflüssen, wo ron innen und aussen die Luft herantritt. Xul $150 \mathrm{~m}$. tiofer im Kastanionbain vine ganz ähnliche Schnecke, aber mit weit zahlreicheren schwarzen Flecken auf Rücken und Mantel, ein typischer cinereus, während das erstere Stück noch als Uebergangsglied zwischen beiden Formen gelten mag.

Die ächte rote For'm mit rotem Schleim tritt zuerst im mittleren Seriotal auf, bei Ponte della Selva und Groppino. Weiter oben hat das Tal die verschiedenen erwähnten Formen von $L$. cinereoniger und cinereus, einen grell roten Ariou subfiscus u. a. Sie fehlen vollkommen weiter unten im Gebiete des roten L. maximus, wie abgeschnitten. Das rote Tier muterliegt wiederedentlich den Einflussen der Exposition. In Kroferenwald und an den einzehnen Fichten de. 'Talsohle und des ()stahhanges ( Westexposition in scharfer Ahhïngigheit von Wolkenschichten und Vegetation, worauf ich nicht weiter eingehe) lebt die Form, die den dunklen L. cinereoniger (ntspricht, cinfarhig lebhaft hraun mit grell rotre Kiellinie, oder aber die vorschiedenen Rückenhinden hram herausgenolen, so dass helle Streifen bleiben, die wieder auf einzelnen Runzeln das grello Rot aufweisen. Fin stiuck des trockneren Wratahhanges, an dem die Cultur mit den Dörfer'n weit höher ansteigt, und der auch weit weniger durch Regengüsse zerfressen und erodiert ist, war ähnlich gestreift, doch so, dass sich in den Binden durch Pigmentconcentration schwarze Flecken heraushoben. Dahei sind die Schnecken ungemein kräftig; die Seitensohle ist dunkel. Die Rückenrunzeln sind ausserordentlich bewrolich und comtralitil, das Pigment sitzt nicht in den Ful- 
chen, sonder'n in den gekielten Kämmen : die Runzeln können sich scharf zuspitzen und in Längslinien mit einander verschmelzen, so dass der Riücken ansieht, wie in längsgeptlügtes Ackerfeld, wobei die Kämme dunkelhraun aus hellerem Grunde hervortreten '; die Kiellinir legt sich dahei in dichte Schlängelungen. Auf Reiz entleert die Schnecke zunächst. blassen Schleim, ehenso wie beim Kíliechen ein blasses Schleimband zurüickbleibt. In Alkohol hüllt sie sich zuerst in dicken weissen Schleim, erst zuletzt, wenn die Reizwirkung am tiefsten eindringt, entleert sie auch den roten, so dass man aussen weisse, unten rote Hüllen abziehen kann. ('enau so verhält sie sich im Leben. Nur in der stärksten Erregung, d. h. wïhrend dex Copula und deren Vorspiel, wird auch das rote Exeret abgeschieden. Ich traf derartige Schleimbänder von hoher Beständigkeit von je zwei Tieren wiederholt an. Die Steigerung der gesammten Lebensenergie zeigt sich noch in anderer Weise beim Vorspiel. Vorgestern (s. o.) wins ich darauf hin, dass die Bedeutung des Torspiels der Stylommatophore'n auf die Aenderung des Muskeltonus in der ganzen Haut hinausläuft. Er ist für gewöhnlich darauf eingestellt, dureh Blutdruck die retrahierten Teile des Vorderkörpers, Kopf und Fühler, auszustülpen. Fürr die Copula aber wird es nötig, ihn so unzustimmen, dass der Widerstand in der' L'mgehung des Genitalpor'us beseitigt ist, so dass die gewaltsame Gesammintcontraction während dos Begattungsaktes den grossen Penis hervortreibt. Dazu alle die Reizmittel, der Liebespfeil, das Belecken u. dergl. Nirgends aber erreicht der Penis den Umfang, wie bei unserem Limar und rielleicht heim kaukasischen Paralimax. Bei diesem aber wies ich bereits auf eine Erscheinung hin, die ich bei unserem roten L. maximus wiederholt fand. Die Tiere belecken sich gegenseitig so heftig. dass an dem rechten vorderen Rande des Mantels, der gefühllosesten Stelle des ganzen Körrper's, die bei Angriffen sich am festesten dem Boden andrückt, ein oft beträchtlicher Substanzverlust entsteht, gerade üher dem Genitalpor'us, hei contrahierter Körrperstellung. Nebenhei mag bemerkt werden, dass damit eine Aenderung des Fühlerspieles auch ausserhalb der Copula erzielt wird. Der rechte Augenträger liommt hei der contrahierten Schnecke viel öfter und schneller hervor als der linke, ganz einfach mechanisch, weil dem Blutdruck bei dem Fehlen des rechten Mantelrandes auf dieser Seite weniger Widerstand entgegengesetzt wird.

I) bese roten Schnecken nun mit einfarbigem Mantel, welche somit dem L. cinereoniger 'ntsprechen, haben eine cigenartige Verbreitung. Im Tal von Esino traf ich ein einzelnes halbwüehsiges Stück, anscheinend ein gewöhnlichne L. cinereminger; doch schied er in Alkohol gelben Schleim ah. Sonst bildet für die ächte'n grossen roten Tiere der Fundort in den

\footnotetext{
'Achnlich wie bei kräftigen kontrahierten Stücken von Arion empiricorum.
} 
Bergamasker Alpen ren nördlichsten Punkt. Von hier grohen sie nach Jitteilung dèes Herru Poldonera bis Verona nach ()ston und bis zum unteren Val Sesia nach Westen, wo der Fluss aus dem (xebirge heraustritt. Sie sind eben Gehirgsschnecken. Die Lombardei hat rote Formen, aber weniger grell; überhaupt wird der Osten Oberitaliens äımer, aus dem einfachen Grunde, weil wil uns hier an der Südostgrenzo der grossen Schnecke zu befinden scheinen. Sie hat sich von hier aus nach Westen entwickelt und macht in dieser Richtung eine immer stäliere Entwicklung durch. Wirklich reicht sie aber nicht nux his zum unteren Val Sesia, sondern sie tritt in gleicher', kräftiger Ausbildung auch noch bei Genua auf, wo ich sie selbst erbeutete, nicht an der Riviera selbst, sondern ctwas weiter oben in den Gebirgsschluchten. So beschreibt das Gobiet einen nach oben convexen Bogen, dessen höchster Punkt unter dem Schwingungskreis in den Bergamasker Alpen liegt.

Die Entwicklungsreihe geht aber noch weiter. In Südpiemont, auf den Nordabhängen der ligurischen und Seealpen, wo sich diese nach der piemontesischen Ebene hinziehen und über und über mit Edelkastanienhainen bedeclit sind, da ereicht die Schnecke die höchste Farbenpracht in Rot und Gello (Limux Perosinii Less. (t Poll.), und, was wichtiger, die Binden tragen sehr grelle schwarze Flecken und ebenso der Mantel. Mit anderen Worten, hier liegt das Optimum der klimatischen Ausfürbung. Vom Seriotal bis hierher durchläuft die Schnecke dieselben Stufen des $L$. rinereoniger zum $L$. cinereus, wie an den Abhängen der Sürlalpen vom Brenner bis etwa nach Bergamo, eine scharfe Parallele, nur dass die letztgenannte Reihe, die an das deutsche Material anknüpft. hloss blassen Schleim hat, die südliche Reihe aber die gelben und roten Farbzellen des Integumentes nach aussen öffnet und ein buntes Secret orler Excret dem blassen Schleim beimischt.

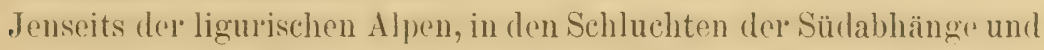
des Apennin, ist das Optimum üherschritten. Wir erhalten eine Schneclie, bei der sich das dunkle Pigment auf Mantel und Rücken in einer grossen Menge schwarzer Punkte concentriert ohne Rücksicht auf Bindenstellung, so dass auf mässig ockerigem Grunde eine dichte unregelmässige Fleckung sich ahhebt (L. millepunctutus). Die Jungen haben noch die ussprüngliche Zeichnung, eine dunkelgraue Rückenstammbinde, dazu eine rotbraune innere Binde, die auf den Mantel übrergeht und hiel hellere Felder ohme Regel ausspart. Während in den Südalpen eine ganz ungemeine Mannigfaltigkeit her'scht von den Formen des $L$. cinereoniger und cinerens mit und ohne rotes und gelbes Excret, bleiben die südlichen 'Tiere nach Uehrowhreitung des Optimum weit gleichartiger", so dass die Funde von Neapel etwa oder von Madeira (L.pardalis) der Schnecke der nördlichen Apenninen sehr ähnlich sind.

Es ist also sicherlich das Klima des bezeichneten Gebietes in den Süd- 
alpen die I'rsache der grossartigen Umfärbung. wohei autfälligerweise der Bogen sich mit seiner Convexität gerarle unter den Schwingungskreis legt.

\section{Folyen der Pendulution fiir die Vertneitung der Tier-und PHrnzen- welt in den Siidalpen.}

Eine aufmerksame Betrachtung zeigte, dass das Vordringen des $L$. marimus nach Süden heineswegs anf alitivel Wanderung beruht, sondern streng an die nordischen Nadelhölzer mit ihren Pilzen gebunden ist (s. 0.). Es (rgab sich aber weiter, dass diese Nadelhölzer, die nach Sürlen zu immer spärlicher werden, ebenfalls keineswegs in Aushreitung hewriffon sind, dor sich violmehr alle kimatischen Verhältnisse hindernd entgegenstellen. In cregenteil. so weit meine Erfahrungen reichen. sind alle die vereinzelten Coniferen bis zum Südfusse der Alpen Reste einel urspriunglichen PHanzendecke. über welche sich oine südlichere Flora bis hoch auf die Alpen hinant hinwegeseshoben hat. Als typisches Beispiel mögen die Befunde gelten, die ich in dem engen Tale von Esino antraf. Unten am Comer See dringen, so weit es auf zusammenhängenden Bestand ankomment. Kastanien ein. dio dam in der oberen Talmulde ansgezeichnete Bestände bieten. Die Hänge werden überall von den Rotbuchen beherrscht, die in der Form von starkem Stangenholz alle Talwände einnehmen, so weit das Auge und das Fernglas reichen. Die herhstliche Verfürhung gah oinen so gleichmässig mtvioletten Ton. dass jede Alweichung in Bestande, vor allen jedes grüune Nadelholz. hervortreten musste. Nebenbei hilden der aufstrigende Ranch von den Kö̈hlerhütten und dex Maultielschlitten aus Buchenstangen Culturelemente auf gleicher Iasis. Nadelhol\% bemerkt man hloss um die Kirehe in Esino und im Wirtshansgarten, namentlich Fichten. zapfentragend. von niedrigem, hreitem IVuchs. Sie liömuton als angeptlanzt wenigstens gerlacht werelen, wrnn nicht andele Vorkommnisse lystimmteren Aufschluss gäben. I'nten bei etwa $350 \mathrm{~m}$. steht unter den Kastanien mit Nuss und Hasel ein T'usus, vielleicht zwanzigjährg, nicht niederedrückt hall strauchartig wie bei uns, sondern von dem schlanken Wuchs einer Tanne, unsere gewöhnliche Form verhält sich dazu fast wie das Knieholz zur normalen Föhle; in der Höhe der Kirche, zwischen 800 und 900 m., vereinzelt im Walde. berzw. im Hain kleine Kiefern und Fichten. und als ich ohne Wog durch die Buchen bis zu einer Spitze von ca. $1500 \mathrm{~m}$. aufsteige, komme ich hej 1100 m. an cine minimale Lärehe. dann folgt rine Schicht Alpenrosen in of wa 1:50) 11. Höhe; alles das, genau und typisch gerordnet und werchichtet. im Buchenhusehwald, wer darüber nach ohen hinwegzieht, v'rmischt mit Cotoneaster, Mespilus, Cytisus, Helleborus und den Teu-

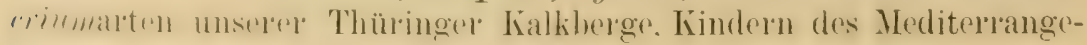


hietes. Wohl weiss ich, dass hie und da die Alpenrose auch auf der Nordseite tiefer hinabsteigt, und bei Esino selbst traf ich sie noch in $855 \mathrm{~m}$. an kalten Bach im Erlengebüsch; aber als Unterholy im Laubwald wirel sie nur von der Südseite angegeben, nach Beispielen der r'egelrechten Schichtenfolge habe ich mich in der Literatur vergehens umgesehen.

Alpenrose und Nadelhölzer sind aber alte. lionservative Pflanzen, und wenn man nicht auf jede Erkläıung verzichten will, bleibt einem nur ührig, eine nördliche V'egetation passiv nach Südon versotzt sein zu lassen auf ein tieferes Niveau, als ihren kimatischen Anforderungen entspricht, in völligem Einklange nit der Penclulationstheorie. Wenn das Auge einmal dieses Verhaitnis hetrachten gelernt hat, dam entelects es auf Schritt und Tritt, in der Nähe und Ferne Beweise, auf die ich mich hiel nicht weiter einlassen kann. Jeder kann die Prüfung vornehmen. Wohl aber* ist es nicht ohne Interesse, noch weitere Algumente herbeizuholen. Da ist zunächst aus denselben Pflanzengruppen die Azalea pontica, die ausser dem Kaukasus in Südwesteuropa auftritt, wo ich sie selbst in der Serra de Monchique blühen sah. Als vermittelndes Glied hat sie WETTs'tern fossil in den Alpen nachgewiesen. Sie berwohnte einst die ganze Gebirgskette ques. durch Emopa. geret aber bej der polaren Pondulation wilnend der Fiszeit unter drum schwingungskejs zu weit nach Norden und wurde in grosser Breit? ausgelöscht, so dass nur dir beidru südlichsten Endzipfel erhalten sind. Von den Coniferen ist es in erster Linie die Conce, die ein ähulich diskontinuierliches Areal bewohnt, durch das der Schwingungsklejs mitten hindurch geht, Libanon und Taurus auf der einen, der Atlas auf der andern Seite. Ich überlasse es den Botaniliern und Palaenntologen zu pröfen, ob nicht die nahe verwandte Lürche chen unter dem schwingungslipejs bei polarer P'endulationsphase aus ihr hervolgegangen sei ${ }^{\prime}$. Ich besitze eine lange Liste von Pflanzen, Bäumen und Kräutern, und von 'Tieren, die entwedel ein bogenförmiges Gebiet bewohnen mit der höchsten nördlichen Ausladung unter dem Schwingungsireis, oder deren Areal diskontinuierlich ist, jerlesmal ästlich und westlich von diesem Meridian. Marshalu, hat eine Reihe von Inschten zusammengestollt, die sich in solcher Lage befinden. mehrere Jahre vor der Geburt der Pendulationstheorie. Auch nicht in einem einzigen Falle liegen die diskontinuierlichen (rebiete so, dass der eine Teil rom Schwingungskereis geschnitten wärde, sondern stets symmetrisch oder annähernd symmetrisch rechts und links davon. Und wenn ich vor

${ }^{1}$ Lärchen der tiefsten Lagen, wie etwa auf der Promenade von Locarno neben den Palmen, wo sie bei ihrem störenden Standort mitten im Kiesweg sicherlich nicht künstlich angepflanzt worden sind, haben ganz den breit ausladenden Habitus der Ceder und auffällig lange Nadeln, so dass man erst bei genauem Augenschein die Art erkennt. 
zwei Jahren den Nachweis zu führen suchte, dass die Ost- und Westpolgebiete um Sumatra und Ecuador Sammelstellen für altertümliche Tropenbewohner darstellen, so hält das Gesetz auch vollkommen Stand, wenn wir es jetzt unter dem Schwingungskreis in den Alpen auf seine Giltigkeit untersuchen.

Für den Limax maximus aber mag noch auf ein Moment hingewiesen werden. Sein Wohngebiet erstreckt sich nirgends in meridionaler Richtung auch nur annähernd so weit als unter dem Schwingungskreis, wo es etwa vom Nordcap bis Neapel reicht. Hier mag er anfänglich entstanden sein, vielleicht in dem Gebiete, das he ute die Alpen einnehmen, oder unser Deutschland, und zwar in den Nadelwäldern mit ihren Pilzen. Nach der Eiszeit ist el passiv nach Süden verschoben und hat in dem Bereich der Südalpen die auffallenden Veränderungen durchgemacht, zunächst vom $L$. cinereoniger zum cinereus, damn nochnals eine ïhnliche Ueherwangsserie mit der Steigerung der Excrete zu buntem Schleim. Wenn wir bei uns im Freien die Schnecke durchweg an Pilzen antreffen, so wissen wir andererseits, dass sie sich in Gefangenschaft auch mit anderen zarten und nahrungsreichen Pflanzenteilen unterhalten lässt. Vorlïufig muss ich's dahingestellt sein lassen, ob beim Herabdrücken der Form nach Süden ein ähnlicher erzwungener Nahrungswechsel statt hatte. Dass die höchste Steigerung der Türbung und Zeichnung sich in den grossen Kastanienhainen des südlichen Piemont vollzog, deutet wohl darauf hin, dass hier andere Nahrung, mindestens andere Pizarten genossen werden. Doch vermag ich leider nicht nach Autopsie zu beurteilen, inwieweit unsere Nadelhölzer vereinzelt noch in jenes Gebiet vordringen. Bei der Bahnfahrt sieht man nur üppige Kastanien.

\section{E. Zur Entstehungsgeschichte der piemontesischen Alpen.}

Nachdem ich in den Bergamasker-Alpen die roten Limax maximus aufgefunden und ihre prïzise Grenze gegenüber den germanischen Formen festgelegt hatte. wandte ich mich im vorigen Sommer nach Südwesten, in der Hoffinmo, nun eine fortlaufende Entwicklungskette aufdecken zu kömnen. IDje Hoffnung wurde gründlich getäuscht. Ich machte zunächst halt in Aostatale in Ville neuve am Eingang zu den Tälern, die in ihren höchsten Teilen noch den Steinbock beherbergen, Val Savaranche und Val de Rheme. Ich fand auch die Schnecke gleich in geeigneter Nordwestexposition an der unteren Grenze des Nadelwaldes, aber nur in der gemeinen germanischen Form. Tachher war alles Suchen talauf- und ahwälts his zu den Steinböcken hinauf und bis zum Ausgang des Tales in der lihene. wo die Serra von Irrea vorspringt, gïnzlich umsonst. Nirgends eine Spur der roten Tiere, zum Verzweifeln. 
Da wurde mir denn in Turin durch Herrn Polsonera die Richtigkeit meiner Beobachtung hestitigt. I)ie Schnecke fehlt im ganzen westlichen Piemont, (t. 1. an den östlichen Abhängen des gewaltigen Amphitheaters's, das man bei glücklicher Beleuchtung so wundervoll vom Monte Capucino oder von der Superga bei Turin erblickt, vom Monte Rosa bis mindestens zum Monte Viso. Aber mehr, hier fehlen auch andere Südformen, zunächst von den Weichtieren die Landileckelschuecken Cyclostoma, Acme, Pomatios, mit wenigen Ausnahmen, welche die allgemeine Regel nicht umstossen. Die Landleckelschnecken sind aber 'Tiere, die nur' ganz spärlich bis Deutschland und Dïnemark hereinleichen, in den 'I'ropen aber", zumal am Ost- und Westpol, sich ausserordentlich reich entfalten; sie reichen unter dem Schwingungskreis sowohl in Europa als im Amurlande am weitesten nach Norden; speziell die drei bei uns vorliommenden Familien der Aciculiden (Acme), Pomatiatiden und Cyclostomatiden haben ein bogenförmiges Wohngebiet, dessen höchste Convexitüt unter dem Schwingungskireis nach Norden sieht. Von den Arionen finden sich nur vereinzelt kleine Formen, dazu driunculus insolartig mehr am Fuss des Gebirges mit dem ebenso vereiuzelten Alpenveilchen. Drüben in Savoyen aber im Rhonetal taucht der Arion empiricomm mit anderen Arten in Masse auf.

Da führte drnn eine nähere Betrachtung der Tier- und Ptlanzenwelt zusammen mit der (xebirgsbildung bald zu eine bestimmten Auffassung. Sie lautet:

Das ganze piemontesische Halbrund vom Monte Rosa bis zum MonteVisomitdem Mont Blancals Culminationspunktist die jüngste Erhebung innerhalb der Alpen. Sie hat erst während der letzten polaren Pendulationsphase in der Eiszeit ihre jetzige Höhe er reicht. Während ihre Gipfel, wie die übrigen Alpenhöhen über der Schneegrenze, noch inder Glacialzeit stecken, befinden sich ih r $^{\circ}$ östlichen piemontesischen Abhängenoch in der Steppenper iode, die ja nach allgemein verbreiteter Anschaunng auf die Eiszeit folgte.

Es velsteht sich von selbst, dass bei der Untersuchung im Einzelnen sich mancherlei Abweichungen ergeben müssen, da bei der südlichen Lage nach dem Abschmelzen der grössten Gletschermassen ciner Menge Mediterranformen der Einzug in die Täler gestattet ist. Im Grossen und Ganzen aber scheint mir der Satz leicht beweisbar.

Versuchen wir, von dem gewaltigsten Massiv, dem des Mont Blanc, im Aostatal auszugehn und die hier gewonnenen Schlüsse auf das Gesammtgebiet zu übertragen!

Bei Villa nova verläuft das Tal ungefähr von West nach Ost. Die Nordseite bildet einen fortlaufenden Steilabhang mit wenigen, schwach 
vertieften Schluchten. Entsprechend der Südexposition beginnt der Wald erst in etwas beträchtlicherer Höhe, die Kultur dringt mit Dörfern und Weilern weit aufwärts. Ein Aufstieg bis zur letzten Ortschaft in $1900 \mathrm{~m}$. Höhe gewährt einen prachtvollen Ueberblick über den Abhang der Gegenseite mit den grajischen Alpen.

Dieser Südabhang verhält sich ganz anders. In ihm sind die tiefen, gewaltigen Täler eingeschnitten, die gegen Villeneuve zusammenstrahlen, Val Cogne, Tal Savaranche, Val de Phême und Val Grisanche, aus deren Hintergrunde übcrall grosse Gletscher herüber grüssen. Die Pässe zwischen ihnen liexen in etwa $3000 \mathrm{~m}$. Höhe. Villa nova liegt in $6-700 \mathrm{~m}$., der Wald beginnt in zirka $900-950 \mathrm{~m}$., und in diesem Niveau dringen anch die Strassen in die Täler ein, die mithin erst steil, bezw. in Zickzacklinien, am Abhang hinaufsteigen müssen, denn das untere Ende der Täler ist noch mehrere $100 \mathrm{~m}$. tief, als enge, unzugängliche Steilschlucht in den Berg eingeschnitten.

Mir erscheint diese Configuration als eine einfache Folge der Exposition; der Süılabhang mit nördlicher Exposition ist der niederschlagsreiche, daher hier das Wasser die Täler und Schluchten ausgewaschen hat, wïrend die trocknere Nordscite des Aostatals viel gleichmässiger geblichen ist. I)ie Schrottheit der Schluchten ist wohl nur im Simne junger Bildungen zu deuten; bei lïngerer Dauer würden sie sich durch Abwitterung der Wände verbreitern. Dass das Tal einst ein gewaltiges G]etscher- und Morinenmaterial beherbergte, beweist die Serra von Ivrea, die sich als der grösste Moränenwall Europas von $600 \mathrm{~m}$. Höhe und $20 \mathrm{~km}$. Länge, wie ein riesiger Bahndamm in die piemontesisch-lombardische Ehene hinausschiebt. Auf der Südseite scheint bei Villeneuve der Moränenschutt durch die stälieren Wassermassen jetzt entfernt zu sein. Nicht so auf der trockneren Nordscite. Hier stehu am Abhange grosse Erdpyramiden, die aus solchem Gletschermaterial bestehen. Ihre Erhaltung scheint aber eine Folge nicht nur der geringeren Niederschläge, sondern auch der schützenden Decke auf ihrer Spitze. Diese Decke ist wieder nur eine Folge der Exposition. Sie besteht, so weit ich's beurteilen kann, aus dem an der Oberfläche durch den Kalkgehalt des Bodens fest cementierten Gletscherschutt.

Zunächst einige Beweise fül diese Auffassung! Ein Bahnviadukt gestattete einem Wildhach zwischen seinen in Mor:inenschutt eingelassenen Pfeilern den Durchitritt. Bei meiner Anwesenheit gegen den Herbst hin nur unbedeutend, muss er doch im Frühjahr grosse Wassermassen brino'n, denn er hatte das Material weguespült und die Basis des Pfeilers auf ziemlich $2 \mathrm{~m}$. Höhe freig(legt. Man sah aber noch die ursprüngliche (b) Bodengrenze auf's deutlichste. Denn wïhrend das untere Pfeilercucle völlig blank wewaschen war, haftete oben noch ein fest angebackner Streifen des alten Bodens am Gemäuer. 
Und so sieht man's bei den Mor:inen der Südalpen häutig genug. Beispielsweise ist der Boden des Seriotals bei Ponte della Selva von ainer Noräne ausgelüllt, in welche der Fluss wiederum sin tiefes secundäres Bett eingeschnitten hat. Gegen die Moräne hin hat es Steilwände von eirca 20 Meter Höhe u. un. Diese IVände bestehen aus lockerem Geschiebelehm, der von einer fest cementierten Decke des gleichen Materials überlagert wird, die an vielen Stellen frei in die Luft hinausragt, bis auch sie schliesslich nachstürzt. Auf der Deche steht der Kiefernwald. An der Nordseite des Aostatals aber sah ich, etwas weiter anfwärts als dic Erdpyraniden, viele völlig ausgehöhlte und unterwaschene Moränenleste, wenn auch von kleinerem Umfange.

Wie schnell die Kalkabscheidung vor sich geht, das bewies mir ein eigenartiges Vorkommniss unter einer Grotte, einem Wegübrogang bei Menaggio an Comersee. Hiel waren frische S'hneckenhäuser, die lreren Puppenhäute von Tagfaltel'u u. derol., mit weissem lialksintel übelzogen, ja das Netz einer Röhrenspinne trug den dicken ITelierzug, wic bei uns gelegentlich die Spinnengewele voll Reif sitzen: eine überaus flotte drt der Petriticierung, wie sie wohl den italienischen Travertinen eigen ist.

Die Erklärung des Vorganges dürfte leicht sein. I)ie Wälme des südlichen Sommers macht sich geltend. Wie bej uns der loppeltiohlensaure Kalk des harten Wassers beim Erwärme'n die eine Hälfte der Kohlensäure verlicrt und somit dere einfachkohlensaure Kalk als Kesselstein sich niederschlägt, so dürfte der gleiche Vorgang im italienischen Sommer langsamer zwar als beim Kochen, immerhin weit energischer als bei uns im Freien sich vollziehen und alle die geschilderten Frscheinungen hervor'ufen. Die Chemie hat wohl die Temperaturgrenz'n für diese' Unterschiede noch nicht festgestellt. Doch erscheint mir's unberlenlilich, die Erdpyramiden von Villa nova auf diesen Prozess zurückzuführen.

Scheint somit die Configuration des Aostatals, in Abhäugigheit von der Exposition, durchaus jungen Datums zu sein, so entspricht dieser Auffassung volkommen die Waldbedeckung. Bei (n) his 1000) Meter liegt. wie elwähnt, die untere Waldgrenze auf der Südseite. Hipr setzen zwei Wachholderarten ein und unmittelhar darüber Pims montenc in den verschiedenen, von Sunörser erst neuerdings abgebildeten Wachstumsformen. Darüber folgen die übrigen Coniferen, mit Ausnahme des Kínieholzes, das bereits weiter östlich seine Westgrenze hat. Lärche und Arve bilden somit die ohere Waldgrenze. Nun verhält sich die Kinfer sehr eigenartig insofern, als sie sich zwal um scharfe Höhengrenzen nieht liümmert, trotzdem aber in rlie ewähnten seitentäler, von denen ich Val Savaranche und Val de Rihême genauer durchwanderte, durehaus nicht eindringt, wiewohl deren Talsohle, auf welcher die Strasse goht, gerate 
in der Kiefernzone liegt, so dass man die ersten Schritte in diesen Tälern zwar durch Kieferwald macht, nachher aber, weiter talaufwärts, keine Föhre mehr antriftt. Die Föhre springt indess nicht über die erwähnten Schluchten, mit denen die Seitentäler gegen das Aostatal ausklingen, hinweg, sondern steigt, so weit sie Fuss fassen kann, in gerader Linie, be\% in derselben Vertikalehene, in sie hinab. Wir erhalten also bloss im Haupttal eine Kífernzone, die sich in hestimmter Höhe am Abhang hinzieht und inzwischen den Weg in möglichst gerader Linie durch die Schluchten nimmt, ohne in die Seitentäler einzudringen.

I) Deutung kann wohl nur die sein, dass die Kiefer noch nicht Zeit gehabt hat, den Höhenlinien, den Isohypsen, in klimatischer Anpassung zu folgen. Sie nimmt noch genau die Stelle ein, die sie unmittelbar nach der Gletscherzeit sich eroberte, als noch das ganze Tal mit Moränenschutt angrefült war. Wo ein Bach in ihr Gebict einschneidet und ihr gewissermassen den Boden unter den Füssen entzieht, da folgt sie ihm in lotrechtor Fichtung nach unten und wahrt ihren ursprünglichen Standort auch in der Schlucht. Mit anderen Worten, in Bezug auf die Nadelhölzel verhält sich das ganze Talsystem wie ein einziges Tal. Die Quertäler kommen noch gar nicht zur Geltung, sie sind zu jung dazu. Umgekehrt scheint es, dass im Ilaupttale die Liefernzone sich talwärts immer weiter hinabsenkt. wieder entsprechend seiner Auswaschung und Ausfurchung.

Nit den Nadelhölzern stimmt aber der übrige Baumwuchs. Ein eigentlicher Lauhwald kommt gar nicht zu Stande. I)ie Buche, am Lago Maggiore noch gemein, fehlt vollkommen und gehört in ganz Piemont zu den Seltenheiten. Dic vorwiegenden wildwachsenden Laubhölzer sind, so weit sie als bestandhildend in Frage kommen, Salicaceen, verschiedene Weiden und von Populus namentlich die Aspe und Zitterpappel. Ias sind aber typische Steppenpflanzen, die hei uns weniger im Walde, als am Anger gedleihen und sonst in Steppe und Wüste vordringen, so weit ihre Wurzeln genügendes Grundwasser finden. Nan erinnere sich \%. B, der Pappeln, die Sven Hedorn als die letzten Bäume in der innerasiatischen Wüstr antraf. Ias ganze Aostatal entlang stehen am Fluss, oft in grossen und breiten Gruppen, hohe Wriden- und Pappelbäume; und an den Hängen sieht man überall vereinzelt die hellen birkenähnlichen Stämme der Aspe und das zweifarbige Laub der Zitterpappeln, dessen weissfilzige Unterseite, ein Schutz für die Spaltöffnungen, doch das ächte Zeichen eines Xerophyten ist. Selbst in den Kunlturen tritt die Kastanie ganz zurück, während überall der Nussbaum einen breiten Schatten auf die Wiese wirft. Es ist, als wäre auch in der Verbreitung dieses Bammes, des freiwilligen Einwanderers im Gefolge des Menschen (s. o.). das Land um eine Stufe weiter nach Norden gerückt, als ihm nach del gar)graphischen Lage von Rechtswegen zukommt. Die Kräuter wird 
man weniger in Rechnung ziohen, da sie leichter ihl (iob) atuszudehnen wissen. Immerhin übergeugt man sich leicht, dass der Nordabhing mit seiner Südexposition roicher an mediterranen Xerophyten ist, an Compositen, aetherischen Labiaten $u$. a.; man mag sie auf das Mediter'angebiet oder auf steppen hezichen, was wohl häufig genug zusammenfïllt. Fine achte Strppenptlanze ist stipu (wohl s. piumuter), das Federgras, das nicht nur auf der Ruine bei Villa nova wächst, sondern von dem auch oben im Val Savaranche ein Strauss im Gasthaus zu sehen war, an de'u umliegenden Hängen gesimmolt. Uir strppruptlanzen gehen hoch hinat im oberen Engadin und am höchsten im oberen Rhonetal.

Freilich, wer die üppigen Culturen, die Massen ansteigender Reben und die noch im Hochsommer und Herbst frisch grouncnelen Matten hertrachtet, wird den Eindruck der Steppe nicht hahen. wio drem auch die hier vertretene Auffassung noch neu zu sein scheint. Und doch, nähere I'berlegumg bestatigt sie selu bald. I) ganze Fruchtharkeit beruht auf künstlicher Bewïsserung, und zwar anf rinem so grosidetig angulegten und bis in's Kleinste fein ausgearbeiteten System, wie es sich nur

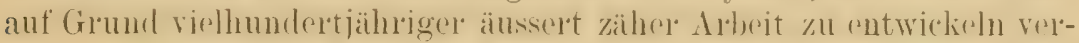
mochte. Iteberall am Steilhang bis zur. Waldgrenze himauf ist del ("ulturbodren mühsam in Terrasisen abgestuft, oft gemug rin tischgrosives Feldstück durch eine Mauel gestützt, wie sie die anspl'uchsrollere moderne Zeit niemals mit hinreichendem Nutzen herzustellen vermöchte. Und über das Ganze breitet sich eine grossartige Wasserleitung aus, welche viele Stunden weit den Gletscherbach heranholt und ihn nach

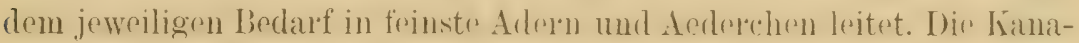
lisierung der Lombardei mit ilurem Reisbau ist nur die letzte, consequente und am meisten in die Augen fallende Fortsetzung dieser Gebirgsarbeit. Wo oben in den Bergen der Wasserfall rauscht, da beruht er halb auf Natur, halb auf Kunst; überall sehen wir geeignete Steine

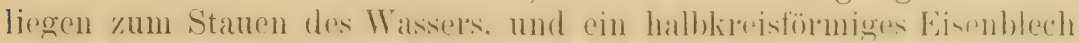

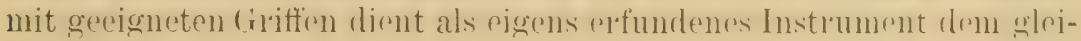
chen Zweck.

Auch in dieser Hinsicht haben wir dieselbe Stetiglieit, dasselbe Verwachsen von Mensch und Boden. das in Italien den Vaturstudien dinen besonders soliden Halt gibt. Man braucht sich nur vorzustellen, dass eine gräunliche politische L’mwälzung l'iemont beträfre, wie sie etwa in Spanien mit dre V'ernichtung der manrisehen Kultur gergeben war. und das gewaltige Piemontesische Amphithater wäldo wiecler zur Strppes herabsinken; oben ewiger Schnee und filetsher, ust den hochalpinen, blühenden Alpenmatten, darunter der Vardelwald. darunter abre melne odel weniger öde Ahhänge, in Tälern und sichluchten an den Wassereläufen von Weiden und Pappeln unterbrochen. 
Es ist kein Zufall, dass der Steinbock trotz mancher Hege nirgends mehr in den Alpen gedeihen will, als eben in den königlich italienischen Jagdrevieren; sie bilden den letzten natiilichen ZuHluchtsort des stattlichen Tieres, wo denn die Pflege leicht wird. Nirgends scheint das Mur'meltier so häufig als in den gleichen Gebieten, von wo der Savoyardenknabe mit der Narmotte als bekannte Figur in das Land hinauszieht. Ton der Gemse gilt wohl das Gleiche. Auch die Säuger bestätigen, was die Nacktschnecken zuerst lehrten. Der Skorpion, im Eisacktal bei Kilansen im Nadelwald bei 1200 Deter äussert gemein, bei Campiglio, in der Nähe des Schwingungskleises his 1500 Neter hoch emporgehoben, dringt nur spärlich in die piemontesischen Täler ein. Ich rechne mit alten Tierformen. Die beweglichen Insekten verschleiern das Gesetz vielleicht ein wenig, wiewohl auch sie sich im Ganzen fügen. Immerhin scheinen sie, vielen Kräutern ähnlich, der jeweiligen Besonnung schneller zu folgen.

Unu noch einen Punkt der Biologie zu berühren, man hat den Cretinismus der Alpentäler oft auf langdauernde Inzucht zurückführen wollen. Nirgends wohl tritt die erschreckende Erscheinung so intensiv auf, wie im Aostatal, abermals ein Boweis für die Stetigkeit der Verhaältnisse und das Alter der Bevölkerung.

Zum Schluss einige allgemeine tektonische und geologische Erwägungen! Die Verwertung und Abtragung der höheren Erhebungen ist auf unserer Erde so gross, dass nach Anschauung der Geographen das höchste ('rebirge ceteris paribus als das jüngste bezeichnet werden muss. Danach allein schon würde sich das piemontesische Amphitheater als das Glied kennzeichnen, das im Alpensystem zuletzt emporgeschoben, emporgehoben, emporgestaut ist. Der Nont Blane mit seinen $4800 \mathrm{~m}$. bildet die Kirehturmspitze Europas, der IIonte Rosa. die Gruppen zwrisehen beiden und auf dem südlichen Flügel in Anlehnung an den Riesen ragen im I)urchschnitt höher empor als die Berge der Schweiz. Das entzückende Bild des Halbrundes von Turin aus schliesst sich so ganz einer solchen Auffassung an in seiner harmonischen Gliederung.

Selbstrerständlich kann aber die Höhe allein den Schluss auf das geologische Alter nicht begründen. Da kommt denn sofort die Form der Berge hinzu. Der entsprechend grossartige Blick auf die Kette des Berner Ohorlandes, etwa vom Gurten aus, zeigt ein ganz anderes Bild, charakterisiert durch die einzeln sich heraushebenden liolosse. Jungfirau. Eiger, In̈nch, die verschiedenen Hörner ete. Jeder von ihnen hat seine eigene Geschichte in den Annalen der Bergsteiger. Der Nont Blanc trägt den einfachen Xamen von seiner Schneemenge, eine Jungfrau war er den Aplpler'u nicht, demn trotz der gewaltigen Höhe erforder't seine Besteigung keine hesondepe fieschicklichkeit in! Kettern und keine Kiraftleistung, dir üher die cinfarhe physische Anstrengung, mit der sich jeder höhere 
Anstieg naturgemäss verbindet, wesentlich hinausgienge. Mit anderen Worten, die Kette des Berner (Oberlandes verdankt ihre wilde Romantik einer stälkeren Erosion, und zu dieser gehörte längere Zeit. Ias piemontesische Amphitheater erscheint auch unter diesem Gesichtsjunkt als die jüngste Bildung.

So viel ich beurteilen kamn, sind auch von streng geologischor Seite Ausichten geäussert, die sich mit meiner Auffassung im Ganzen decken. Ein Blick auf die geologische Karte der Westalpen lehrt aber ganz dasselbe; man nehme etwa das gute Uebersichtsbild in der neuesten Auflage von Meyer's Konversationslexikon, um etwas allgemein hekanntes und zugängliches heranzuziehen. Hier gehen die Farben dre Schichten im piemontesischen Bogen mit weit grösserem Gleichmass hindurch, während das Berner Oberland eine viel stärkere Unterbrechung und Zerrissemheit zeigt.

Denkt man sich Piemont in solcher Weise vielleicht gegen den Schluss der Glacialzeit noch in nördlicherer Lage, so ist wohl zuzugeben, dass nach der Exposition die Gletscher auf der feuchteren Westseite stärkm sein mochten. Gleichwohl musite die (Hesammtwirkung auf der konkaven Ostseite, die ja jetzt noch genug ewigen Schnee trägt, ganz ausserordentlich sich verstïlken, da alle Gletscher nach einem Mittelpunlite zusammenstrahlen. Hier musste die Abkühlung auch bei der aequatorialen Pendulation in südlichere Breiten an längsten vorhalten und das Bild ergeben, das ich entwerfen zu müss's glaubte. Allmählich hat die sürlichere Wärme Schnee und Fis auch hier so weit zur tuckgedrängt, dass kaum noch ein Unter'schied gegen die ül)rigen Alpen hervortritt und der frühere Zustand nur aus anderen Falitoren des Bodens und seiner Bewohner erschlossen werden kann.

Skizzenhaft nur kionnten meine Ausführungen sein. Fins, hoffe ich, wird man ihnen zugestehen müssen, objektive Plüfung del Tatsach'sn ohne gewaltsames Schematisier'n nach vorgefaster Meinumg. Ich gieng in die Südalpen mit der Absicht und Aufgaber, die reiche Umlïrbung unseres grössten Evertebraten an Ortt und Stelle zu studieren, um gleichzeitig die nervösen Folgen der mit der Pendulationsther'ie verhundenen heftigen Veränderung meinet cesammen Naturanschaumg loszuwelden. Aher selbst bei der möglichst intensiven. fast monngraphisch'n Beschäftigung mit der einzelnen Formengluppe brath die zurülekgedrängte Theorie wiederum mit plementarer Crewalt dureh und elschloss mir das Ver'ständnis für das durchwanderte Gebint, soweit es mir meine naturwissenschaftiche Ausbildung zu durchdringen vermochte. Wor lïmnte vermessen das Ganze umfassen?: Und doch sind nur vom streben zum Ganzen wirkliehe Fortschritte unseres Erkennens zu rrhoffen. IDic Falitoren, welche den Limax marimus umfärhen. im Einzohnen aufzuhellen, wie die Insekten mit ihrer durehgebildeten morphologischen und 
biolngischen Gliederung den Wechsel der Süd- und Nordformen neuerdings viofich exalit zu analysieren erlaubten, ist mir nicht gelungen, es lässt sich vorläufig hloss die veränderte Stellung zur Sonne im allgemeinc'n verantwortlich machen. I)iese aber zeigt sich im Einfluss auf den gesamnten anorganischen und organischen Habitus des Gebietes, wie ich denke, in hinreichender Klarheit.

\title{
La faune ichtyologique du lac Tchad et du Chari.
}

\author{
Par le Dr J. PELLEGRIN (Paris).
}

La faune ichtyologique du lac Tchad et du Chari était inconnue jusqu'ici. Grâce aux richrs materiaux rapportés par la mission Auguste CueVAlier ot Inecorses cette lacune peut être aujourd'bui, en partic, comblée. Sans doute de nouveaux élémonts derront éure ajoutés à la liste d'espèces domnéc jei pour arriver à une connaissance vraiment exacte des Poissons habitant cettr intéressante légion de l'Afrique, mais les collections recueillics sont suffisantes, semhle-t-il, pour permettre de formuler déji quelques conclusions générales.

Les Poissons récoltés par la mission Auguste Chevalier et Decorse proviennent du lac Tchad même, de Kousíi dans le bas cours du Chari, à son confluent avec le Logone et de Fort-Archambault, localité située bien plus en amont, à l'endroit où le Hleuve reçoit la rivière Boungoul. Onze familles, comprenant 40 esperes, ont rité rencontrées. Parmi cellesci deux sont nouvelles: un Cryprinidé du genre Labeo et un curienx Miormỵridé du genre Hyperopisus. Un petit Siluridé appartenant au genre Synodontis pourrait, sans doute, aussi etre ronsidéré comme une forme encore incomuce, mais il est représenté seulenent par un spécimen un peu jeune.

Voici la liste par familles des espèces recueillies:

Lepidosirenida: Protopterus annectens Owen.

Polypterida: Polypterus bichir Geoffioy, P. Delhezi Boulenger.

Tetrodontidre: Tetrodon fahaka Hasselquist.

siluridar: Éutronius Grenfelli. Boul., schilbe mystus Linné, Clarotes lati"eps liüprel. Chiysichthys macrops Günther. Syynodont is schall Bloch Schneider, S. membranacens Geoffroy, Symodontis sp."? 
Cyprinidae: Labeo coubie Rüppel, L. Selti Valenciennes, L. senergalensis Val., L. chariensis nov. sp.

Characinida: Starcoduces odoë BI., Hydrocyon Forshali Cuvier, Alestes Kotschyi Heckel, A. macrolepidotus Cuv. Tal., Ichtyoborus microlepis Günther, Distichodus altus Boul., Citharinus Geoffroyi Cuv.

Mormyrida: Petrocephalus bane Lacépède, Marcusemins Lhu!ssi Steindachner, Gnathonemus cyprinoïdes L., G. senegalensis Steind., G. trmandua Günther, Hyperopisus bebe Sonnini, H. tenuicauda nov. sp., Mormyrus Jubelini Cuv. Val., Gymmarchus niloticus Cuvier.

Cichlide: Hemichromis fusciatus Peters, H. bimaculatus Gill, Tilapia nilotica L., T. Heudeloti A. Duméril, T. melanopleura A. Dum., T. Zillii Gervais.

Anabantidre: Anabas Weeksi Boul.

Ophiocephalidæ: Ophiocephalus obscurus Günther.

Mastacembelidæ: Mastacembelus Loennbergi Boulengel ${ }^{1}$.

La plupart des especes qui figurent ici ont pour distribution géographique soit le bassin du Nil, soit celui du Sénégal, soit celui du Congo ou encore plusicurs de ceux-ci. La fiume ichtyologique du Sémégal offire de grandes affinités arec cello du Nil, il n'est donc pas étonnant qu'on rencontre dans le Chari et dans le lac Tchad qui sont intermédiaires boaucoup d'espèces communes à ces deux régions. Au sud lo Chari est en leelations plus ou moins étroitos avec l'Oubanghi. impol'tant afthent de la rive droite du Congo, ce qui explique la présence dans ses eaux de certaines formes nouvellement décrites du bassin de ce dernier fleuve.

En somme, le lac Tchad ne semble pas avoir une faume ichtyologique particulière, très spéciale, très diftéronciée comme celle du lac Tanganyika par exemple; les especes qu'il possède ainsi que le Chari, son tributaire, se retrouvent en effert, en général, dans les grands tleuves africains voisins : Nil, Sénégal, Niger et Congo.

${ }^{1}$ Autant qu'on en peut juger sur des photographies, communiquées par MI. le Dr DECORSE, deux espèces peuvent être jointes à cette liste, un Percidé, le Lates niloticus Gmelin, représenté par un énorme spécimen de $1 \mathrm{~m}, 20$ de longueur et un Ostéoglossidé aussi de grande taille, l'Heterotis niloticus Cuvier. Ces deux espèces se retrouvent aussi sur des dessins et des photographies recueillies par la mission Foureau. 


\title{
Holothuries abyssales recueillies par l' "Investigator " dans l'Océan Indien.
}

\author{
Par le Prof. R. KOEHLFR et le $\mathrm{D}^{r}$ C. VANEY (Lyon).
}

II. Alconk a bien voulu nous charger de l'étude des Holothuries recueillies par l'" Investigator" ». Nous avons l'honneur de présenter au Congrès nos observations sur les formes de mer profonde.

La collection qui nous a été remise comprend en tout 75 espèces ou variétés, la plupart nouvelles, car 13 seulement appartiennent à des espèces déjà connues.

Ces espèces se groupent en familles de la façon suivante:

$\begin{array}{lr}\text { Synallactidés } & 29 \\ \text { Deimatidés } & 8 \\ \text { Elpidiidés } & 4 \\ \text { Psychropotidés } & 5 \\ \text { Cucumariidés } & 4 \\ \text { Rhopalodinidés } & 1 \\ \text { Molpadiidés } & 15 \\ \text { Synaptidés } & 7\end{array}$

Une espèce forme le type d'une nouvelle famille à laguelle nous avons donné le nom de Géphyrothuridés, enfin une dernière espèce n'a pu ètre classée avec certitude.

Yous attirerons plus particulièrement l'attention d'abord sur le nombre considérable des espèces nouvelles et ensuite sur l'importance que prennent les 3 groupes des Synallactidés, Molpadiidés et Srnaptidés.

Nous ne pouvons pas entrer ici dans l'étude systématique de ces Holothuries: nous nous bornerons ì présenter quelques remarques sur la faune ahrssale des Holothuries draguées par l'u Investigator" ", considérée au point de vue de la géographie zoologique.

Les espèces déjai connues qui ont èté retrouvées par l'" Investigator" " proviennent. soit de l'Océan indien, soit de l'Océan pacificue, soit enfin 
de l’Océan atlantique. Si nous groupons ces espèces d'après leur provenance nous trouvons que:

Six avaient déjà été r'encontrées dans l'Océan indien, ce sont:

Mesothuria multipes Ludwig

Synallactes Wood-Masoni (Walsh) ${ }^{1}$

Laetmogone violacea Théel

Ypsilothuria bitentaculata (Ludwig)

Trochostoma granulatum Ludwig

Ankyroderma musculus (Risso);

Cinq étaient connues dans le Pacifique:

Mesothuria multipes Ludwig

Benthodytes sanguinolenta Théel

Ypsilothuria bitentaculata (Ludwig)

Trochostoma granulatum Ludwig

Ankyroderma musculus (Risso);

Six avaient été trouvées dans l'Atlantique:

Deima Blakei Théel

Laetmogone violacea Théel

Benthodytes glutinosa R. Perrier

Ypsilothuria bitentaculata (Ludwig) ${ }^{2}$

Trochostoma albicans Théel

Antyroderma musculus (Risso).

Les espèces déjà connues dans l'Océan indien et dans le Pacifique et que l'" Investigator" » a retrouvées dans l'Océan indien ne donnent lieu à aucune lemarque particulière. Quant aux espèces de l'A tlantique et que l' Investigator " a draguées dans l'Océan indien, trois paraissent très cosmopolites: l'Anliyroderma musculus (Risso), à laquelle nous réunissons les A. Danielsseni Théel et A. spinosum Ludwig, est très répandue dans toutes les mes's; c'est une espece très polymorphe; l'I Insilothuria bitentaculata (Ludwig), dont l' $Y$. attenuatı E. ot R. Perrier ne peut pas être distinguée, a été trouvée au Japon, dans le Pacifique, dans l’Al’chipel de la Sonde et dans l'Atlantique: la Laetmogome violacea Théel était déjà

${ }^{1}$ Le Pannychia Woodmasoni Walsh est synonyme du Synallactes reticulatus Sluiter.

${ }^{2}$ Nous faisons rentrer 1'Y. attemuata de R. Perrier dans l'Y. bitentaculata (Ludwig). 
connue dans l'Atlantique et l"Océan Indien. Les autres espèces, Deima Blakiei Théel, Benthodytes glutinosa R. Perrier et Trochostoma albicans Théel n'avaient pas encole été trouvées hor's de l'Atlantique et les dragages de l' " Investigator" "étendent notablement leur répartition géographique.

Il résulte de cette comparaison que les espèces déjà connues et retrouvéres par l'(Investigator ) comprennent à peu près le même nombre d'especes du Pacifique et de l'Océan indien que de l'Océan atlantique. Si, en effet, nous laissons de côté des formes évidemment cosmopolites telles que Lrretmogone violacen Théel, Ypsilothuria bitentaculutu (Ludwig) et Anhyroderma musculus (Risso), nous remarquons que parmi les espèces déjà connues, deux ont été trouvées à la fois dans les Océans indien et pacifique (Mesothuria multipes Ludwig et Trochostoma gramulatum Ludwig), une n'avait encol'e été trouvée que dans le Pacifique (Benthodytes somguinolenta Théel). une seule dans l'Ucéan indien (Synullactes Wood-1Lasoni Walsh), enfin trois n'étaient connues que dans l'A tlantique (Deima Blakei Théel, Benthodytes ylutinosa R. Perrier et Trochostoma albicuns Théel).

D'autre part, si nous comparons les formes profondes d'Holothuries draguées par le: "Siboga " daus l'Archipel de la Sonde, nous constatons, ell laissant de côté les espèces cosmopolites, telles que Luetmogone violacer Théel, Ypsilothuria bitentaculata (Ludwig), Anliyroderma Danielsseni Théel et $A$. spinosum Ludwig (que nous l'éunissons toutes deux à l'A. musculus), que les espèces retrouvées par l'u Investigator ") dans l'Océan indien ne sont quau nombre de trois, ce sont: Symulluctes IVood-_Masoni (Walsh), Mesothura multipes Ludwig et Trochostoma gramulatum Ludwig.

Il résulte de cette comparaison que deux territoires assez voisins d'un mème océan, l'Archipel de la Sonde d'une part, le golfe de Bengale et la Mer d'Oman d'autre part, ont fourni deux faunes très différentes d'Holothuries et n'offrent qu'un très petit nombre de formes communes, si l'on ne considère que les espèces; mais si l'on considere la répartition en genres et en familles, on arrive à une conclusion toute différente et l'on constate au contraire une grande analogie entre la faune des Holothuries de locéan indien et celle de l'Arehipel de la Sonde. Nous avons fait remarouer" plus haut que la collectiou de l'" Investigator" "était tre's riche en Synallactidés, en Molpadiides et en Synaptes; la meme remarque s'applique également aux Holothuries recueillies par le "siboga ". Ians les deux collections nous remarquons que les gentes Mesothuia, Buthyplotes et Pelopatides parmi les Synallactidés, Anhygroderma et Trochostoma parmi les. Molpadiidés, Protanliyia parmi les Synaptidés, sont représentés par plusieur's especes chacun. Aussi, malgré une composition spécifique toute différente, les faunes de ces deux régions ont une ressemblance marquée. 
Une comparaison des Holothuries draguées par l’a Albatross ) dans la région orientale du Pacifique, avec la collection de l' "Investigatol" » et du "Siboga " montre que non seulement les espèces communes sont très peu nombreuses, mais que la répartition en genres est toute différente.

La faune profonde des Holothuries de l'Atlantique, que nous connaissons par plusieurs dragages, anssi bien dans les rónions occidentales que dans les régions orientales de cet océan, diffèrent aussi considérablement de celle de l'Océan indien tant au point de vue de la composition en espèces que de la répartition en genres.

Des particularités analogues ont été constatées pour d'autres groupes et l'un de nous a déjà eu l'occasion de développer des considérations analogues en étudiant les Ophiures abyssales de l'Océan indien. Plus les explorations sous-marines se multiplient et plus on trouve d'exemples de semblables localisations dans les faumes absssales dont le cosmopolitisme est loin d'être aussi absolu qu'on l'avait cru autrefois. 

A VIEXES 

ANNEXE I

\title{
TRAVAUX REMIS AU CONGRĖS ET QUI N'ONT PAS ÉTÉ LUS DANS LES SÉANCES
}

\section{La Pœcilogonie.}

\author{
Par le Prof. A. GIARD (Paris).
}

Une des questions les plus importantes et en même temps un des prohèmes les plus difficiles à résoudre de la zoologie moderne ost de sivoir si, dans l'appréeiation des rapports de parenté entre animanx semblables ¿̀ l'état parfait, mais présentant une cmlnyogénie différente, il convient d'attacher plus d'importance aux dissemblances évolutives qu'à la similitude des adultes.

La question se résoudrait immédiatement par l'affirmative, si le principe de Fritz Mereluer, la loi hiogénétique fondamentale de Serness et de H.wicke était constamment applicable dans toute sa rigueur, c'est-it-dire si les divers stades ontogénétiques d'un animal répétaient exactement la série phylogénetique ou les formes ancestrales successives. En offet, s̈il en était ainsi, l'embryogénie nous indiquerait les véritables rapports de parenté et la ressemblance plus ou moins grande des adultes chez des types it embryons dissemblables devait ètre interprétée comme le résultat d'une convergenere due à l'éthologie similaire de ces formess adultes.

Mais il arrive fréquemment que le développement embryonnaire est simplifié et abrégé chez certains types dit cœnogénétiques et, chez les animaux à embryogénie explicite, les formes larvaires palingénétiques sont soumises pendant une longue période à l'action modificatrice des milieux et souvent aussi leur évolution dépend dans une large mesure de l'éthologie de l'adulte. Des lor's. Ie principe de Fritz Mreulater devient d'une application délicate et. chose singuliere, mais pourtant très réelle. la détermination des rapports de parenté devient d'autant plus ardue que l'on connait mieux les diverses phases évolutives. Il est vrai que si une demi-science vient ainsi compliquer le problème, nous pouvons es- 
pérel qu'une science plus complète - je veux dire la connaissance de la morphodrnamique embryonnaire d'un plus grand nombre d'espèces nous facilitera la solution.

Dès à présent, nous pouvons distinguer deux grandes catégories d'animanx se lessemblant à l'état adulte et présentant des dissemblances plus ou moins grandes aux diverses périodes de l'ontogénie.

$1^{\circ}$ Certaines formes appartenant à un même genre ou à dès genres distincts. parfois même assez éloignés, mais présentant en tous cas des larves bien différentes. ont des états adultes très roisins rquelquefois difficiles it séparer') par suite de convorgences dues aux conditions de milieu. quelle que soit d'ailleurs la cause de ces convergences (homochromice, ressemblance protectrice, mimétisme direct ou indirect, isotypie, etc.).

$2^{\circ}$ Chez d'autres animaux, les diver's individus ou les diverses générations d'une même espèce considérés aux divers points de la distribution géographique, aux diverses saisons de l'année, ou dans des conditions de nutrition différentes, ont des larves qui ne se ressemblent pas, bien que l'adulte leste constamment semblable a lui-meme, ou ne présente que des modifications trés légères. ("est la particularité que j’ai désignée naguère (92), sous le nom de poecilogonie. Les larves sont devenues divergentes cn s'arlaptant à des milieux différents. L'hérédité a maintenu la similitude des adultes.

Le résultat final est le même dans les deux cas : variété daus l'évolution, ressemblance tres grande ou prescue identité à l'état parfait. Mais au point de vue de la consanguinité et par suite de la classification naturelle (généalogique), ces deux catégories de faits sont loin d'avoir la même signification. Les espèces voisines dorigine precilogèn ont entre elles la parenté la plus étroite et doivent être rapprochées les unes des autres, malgré leurs divergences embryonnaires; les espéces qui se resscmblent par convergence peuvent, au contraire, n'avoir que des rapports phylogíniques asser. éloignés et doivent être considélées comme nettement distinctes.

Lorsque je signalai, il y a une quinzaine d'années, les premiers exemples counus de poecilogonie, ces faits paraissaient rares et exceptionnels. Depuis, on les a observés très souvent et dans presque tous les groupes d'animaux. Peut-être, cependant, n'ont-ils pas été encore étudiés avec un soin suffisant. En outre, parmi les zoologistes qui les ont fait connaître, il cn est peu qui en aient saisi toute la portée. La plupart n'ont pas su se dégager d'un cercle vicieux dont on retrouve plus ou moins la trace dans leur's écrits. Ils ont considéré les modifications embryonnaires qu'ils araient constatées comme des caractères taxonomiques suffisants pour l'établissement de nourelles espèces et ne se sont pas préoccupés de rat- 
tacher ces variations à des causes éthologiques ou, en général, aux facteurs primaires de l'évolution.

Il m'a donc paru qu'il serait utile d'attirer à nouveau l'attention sur ces phénomènes si curieux et de faire ressortir la valeur des arguments qu'ils fournissent à la théorie de la descendance modifiée.

Le premier fait qui semble souvent conditionner la poecilogonie est l'apparition de l'endotokie chez des espexes appartenant à dres groupes où la règle est l'exotokie. Colrélativement, et probablement comme conséquence de l'accumulation de réserves plus abondantes dans l'œuf, la viviparité se substitue à l'oviparité et l'ontogénie tend à prondre une allure plus ou moins conogénétique.

Coele n tera ta. - Chez les C'œlentérés, le facteur primaire qui paraît déterminer ces modifications est l'habitat dans des eaux plus froides, soit qu'il s'agisse d'animaux vivant dans les grandes profondeurs, tels que le Caiail noble (de Lacaze-Duthiers) ou le Sympodium coralloides (A. Kowalewsky et Marion); soit que l'on ait affaire à des types à la fois abyssaux et subpolaires: diverses espèces de Nephthya (Koren et DANIELLSEN), Gorgonia capensis (S. J. Hrokson).

Lor'sque, chez une espèce. l'influence modificatrice se fait sentir seulement chez un certain nombre d'individus placés dans des conditions spéciales, nous voyons se réaliser un exemple typique de pœecilogonie. C'est ce qui a lieu chez un Alcyonaire, Clavularia crassa M. Edw., qui habite généralement dans la zolir littoraie de la Mérliterranée tout à fait près du rivage, au milieu des rhizomes de Posidonia. Marion et KowaLEwskx ont fait connaître, en 1883, une variété de cette espèce qui se fixe dans les eaux un peu plus profondes, à la face inférieure des pierres, et que pour cette raison ils ont nommée Clavularia petricola. C. petricola ne differe morphologiqurment de $C$. crasse que par une légère divergence dans la forme des spicules ou sclérites. Mais j’ai pu constater maintes fois, chez les Synascidies et chez les Bryozoaires, combien ces productions peuyent varier dans une même espèce et, sans sortir du groupe des Alcyonaires, Marion rappelle qu'il a eu l'occasion d'observer des variations analogues chez deux formes d'Alcyonimm (A. palmatum des fonds vaseux et $A$. palmatum forme acaule des fonds coralligènes) sans les consacrer par un terme spécifique nouveau (7S).

" Mais, ajoute-t-il, nous avons ici, à propos des Clavulaires, un élément de plus, un phénomène biologique important qui, ailleurs, dans d'autres groupes, aurait incontestablement une valeur assez considérable; nous voulons dire la viviparité régulière des Clavulaires pétricoles opposée à l'ovipar'ité constante des Clavulaires des Posidonies. Cela suffirait pour admettre une espèce physiologique en l'absence de toutes différences morphologiques y (83). 
Dans cet exemple, nous pouvons admettre que la variation déterminée par les facteur's primaires n'est pas encore fixée par l'hérédité. Les deux formes vivent côte à côte; il n'y a pas d'amixie (pas d'amixie géograjhique tout au moins); mais que l'isolation ou la sélection physiologique entrent en jeu, et nous pouvons prévoir la naissance d'une espèce nouvalle, ou. si l'une des formes vient à disparaître, étant moins bien armée dans la lutte, tout se passera comme si une des deux Clacularia se transformait lentement en l'espèce voisine.

Un exemple du méme genre nous est offert dans un groupe roisin des Alcyons, les Actiniaires ou Anémones de mer.

On sait que le docteur Carliren, de Stockholm, a observé récemment la viviparité chez un grand nombre d'Actinies des mer's arctiques.

Parmi ces espèces vivipares, se trouve une forme qu'Appeclas a également rencontrée dans les dragages au large de Bergen et qui ne diffère d'Urticina (Tealia) crassicornis O. F. Mueller que par la faible dimension des pajilles verrucitormes adhésives. Cardiren l'a désignée sous le nom de $U$. crassicornis, forma levis. Chez les nombreux individus de cette forme qu'il a recueillis au Spitzberg, il a trouvé la cavité du corps remplie de jeunes à diver's états de développement. ()r, sur nos livages, $U$. crassicornis type est constamment ovipare.

U. lartis semble donc être une forme poeilogonique spéciale aux mers polaires et séparée uniquement de la souche, au point de vue morphologiçue, par le caractère insignifiant de la dimension des verrues.

Chez les Discoméduses, Schnemer, puis Heckel ont vu que, suivant less quantités de réserves nutritives contenues dans l'ouf, le scyphopolype d'une même espèce, Aurelic aurita, donne naissance, par bourgeonnement successif, à une série nombreuse d'Ephyra ou se transfor'me directement par hy pogénèse (Нжскец) en une seule Ephyra qui, d'abord fixée, devient nageuse au moment de la transformation en Méduse

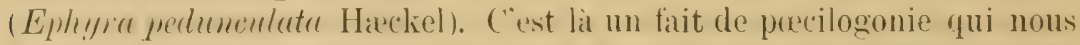
premet de mienx complende de quello farcon s'est établic l'érolution coenogénétique très condensée d'antres Méduses, les Pelagia par exemple’.

$\checkmark$ Bien qu'ils se rattachent étroitement à la pœcilngonie, il convient de distinguer et d'étudier ì part les faits que j'ai groupés (98) sous le nom d'allogonie et dont j'ai cité un exemple très net chez Campanularia calyculata Hincrs.

Les Hydraires allogoniques peuvent être comparés aux Insectes qui, présentant des lurres identiques, diffèrent à l'état adulte (voir ci-dessous p. 632, note). Dans un mémoire tout récent, HaRgITT (04) a fait connaître de nouveaux cas fort intéressants d'allogonie chez les Hydraires Gymnoblastiques.

Podocoryme conchicola Philippi est une forme allogonique (Méduse à 4 tentacules) de Podocoryne carnea Sars (Méduse à 8 tentacules).

De mème Gemmaria implexa Alder, dont la Méduse a deux tentacules (Allman, 
Ech inodermata. - C"est encore par le même processus que les espéces porcilogoniques paraissent prendre naissance che\% les Echinodermes.

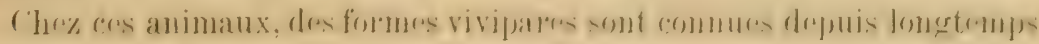
et l'endotokie se présente d'une faron plus compliquée que chez les Cirs-

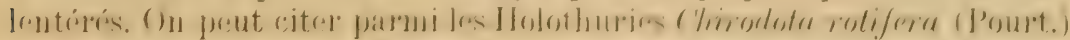
et Phyllophorus urna (Grube) où les jeunes se développent dans la cavité du corps du parent, Psolus eplippifer W. Thomson et quelques espèces de Cucumaria qui ont des chambres incubatrices. Parmi les Astéries, sans parler du cas bien connu d'Asterina giblinsa, les Blakiaster et les Pteraster ont un développement direct. Chro les premiers, les reufs sont incubés dans les espaces en arcades de la surface abactinale; chez les seconds, la surface dorsale forme un vaste marsupiun. Parmi les

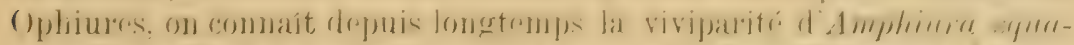

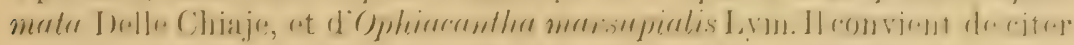
encore Amphizu'a magellanica Sturler: Ophiacantha vivipara Studer et Ophiomyxa vivipara d'apres les recherches de Simer et S'runen sur los Echinodermes des mers antaretiques.

Enfin chez les Oursins, il suffit de rappeler parmi les cas de viviparité signalés par Wyville 'lrouson, les curieux marsupiums ambulacraires d'Hemiaster Philippii (76).

Ln étudiant la distribution géographique de ces diverses especes, on comprendra comment nous avons été conduit à formuler, dés 1878, la règle suivante:

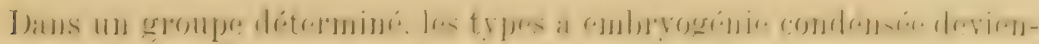
nent de plus en plus nombreux à mesure que l'on s'avance vers les pôles. Les embryons pélagiques sont plus spécialement adaptés aux eáux des mer's chaudes.

Ces propositions sont vraies surtout pour les animaux littoraux et ne peuvent sappliquer sans restriction à ceux qui vivent dans les profondeurs ou qui suivent les courants marius.

Quoiqu'il en soit, nous devons nous attendre d'aprés ce qui précède, à retrouver la precilogonie chez les Echinorlermes. Et en effet, Luwwis: a fait la remarque intéressante qu'Asterina cepher Val., de la mer Rouge et de l'Océan Indien, espéce si voisine d'A. gibbosa de nos mexs qu'on

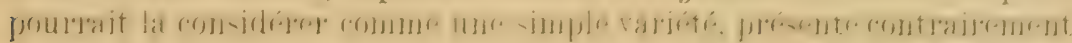
à cette dernière, et conformément à la règle générale, les pol'es génitaux

Hapgrts), présente à Naples une forme allogonique dont la Iéduke possède quatre tentacules (Du P'Lessis).

Les formes Méduses à 2 ou à 4 tentacules sont respectivement progénétiques par rapport aux formes Méduses à 4 ou ì 8 tentacules. 
¿ la partie dorsale. Il a constaté le fait sur plusieurs exemplaires venant des Philippines. Asterinu cephen habitant les mers chandes. albundonne au hasard de la vie pélagique des cuf́s qui doivent domner naissance à des larves mageuses; - t. gibbose dre mers temperées rassemble et protege sous sa face ventrale de gros cuf́s d'où sortent des embryons très peu mobiles et déjà fort avancés dans leur développement.

A. gibbosa n'est done qu'une fol'me poecilogonique de $A$. cepliea.

De même Asterias Muelleri n'est sans doute qu'une variéte poecilogonique septentrionale du vulgaire Asterius glacialis des cotes de France [Sirs (44)].

Nous parlerons plus loin des finits très intéressants de poecilogonie sai-

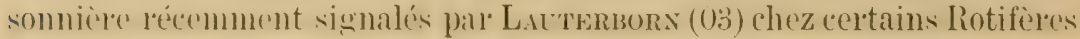
(Anurxa).

LOphiothrix fragilis Müller, espèce si variable et à si large dispersion. est égillunent un type puecilngonique 'n vole d'évolution et qui se divisera probablement en plusieurs espèces distinctes dans un avenir plus oll moins éloigné.

Suivant les conditions éthologrigues, l'évolution de cette ()phiure se fait tantot par des l'luteus normaux (comme dans la Méditerramée), tantot par des Pluteus imparfaits tels que ceux étudiés par Apostonides (à lioscoffi), tantôt même par des embryons très condensés, incapables de mager et qui doment me Ophime prescue sams métamorphoses (it Wimereux, etc.).

Gymnotoca. - Parmi les Annélides, la famille des Polygordiens nous offire un cas de poecilogonie tout à fait comparable à ceux des Colentérés et des Echinodermes.

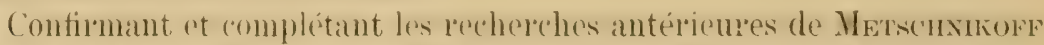

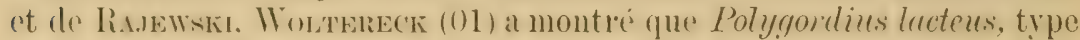
de la mer du Nord, suit un développement très diftérent de celui de sa variété à prine distincte $P$. neupolitumes, de la Méditeranées étuliée is Naples par Hatscien. Ici encore, c'est le type septentrional qui est cœnogénétique. Les discordances ne portent pas uniquement sur les procossus corolutifs mais ausi sur des particularites histologigues profondes (structure des néphridies et du système nerveux). La température ne semble pas d'ailleur's être le seul facteur agissant, cal Keinenberg et Spengel ont recueilli dans le plankton, à Messine et à Naples, des larves ressemblant à celles de la mer du Nord.

L'aspect différent et les dimensions également différentes des oufs chez les Chétopodes néréidiens selon qu'ils sont pondus par la formè Nereis ou par la forme Heteronereis, me porte à supposer qu'il existe

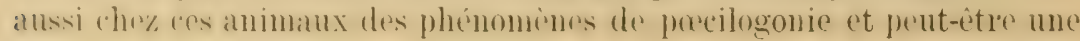


adaptation plus parfaite à la vie pélagique chez les descendants de la forme hétéronéréidienne.

Enfin c'est avec raison, pensons-nous, que CAuldery et Meswis, (98) ont

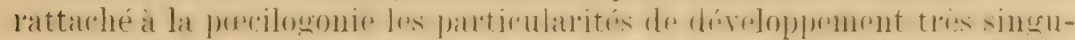

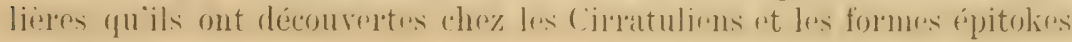
de Dodecacerin.

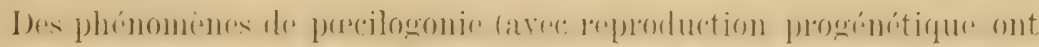

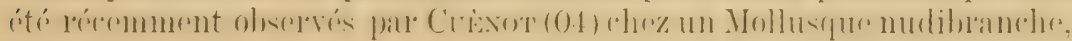
Staurodoris verrucosa. Cuénot fait ressortir l'importance de cette constatation pour l'étude taxonomique des Doridiens.

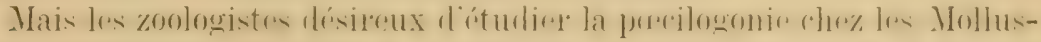
ques devront principalement porter leur attention sur les faits récem-

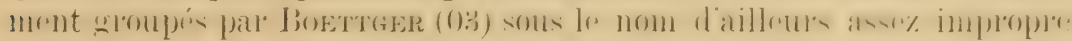
d'hétérostylie. Les données malheureusement trop rares que je possède

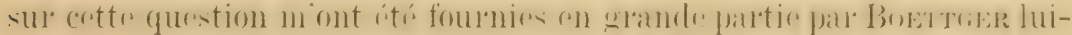
mème et surtout par mes amis H. Fischer, P. Pelaseneer et Ph. DautzenBERG que je tiens à remercier de leur obligeante assistance.

Par hétérostylie, Boertaen désigne la particularité présentée par certains genres de Mollusques gastéropodes (Pleurotomides, Purpura des mer's chaudes, etc.) où l'on rencontre des groupes d'espèces conjuguées ayant la même coquille mais avec des apex différents.

Les meilleur's exemples se trouvent, d'après Boert'Ger, dans le mémoil’e de R. Sturaxy : Expeditionen S'. M. Schiff Pola in das Rote Meer; Zool. Ergebrisse(Denkschr. math. nat. Cl. K. Akad.d. Wiss. Wien, Bd. 74, 1903). Il s'agit de Murex tribulus L. (p. 219) et de Fusus bifrons Strur. (p. 220). Les figures $3 a$ et $4 a$ de la Pl. I, relatives à Fusus bifrous et à sa variété yaucicostata, sont particilièrement instructives ${ }^{1}$. La protoconque, rudimentaire dans le type, est très développée chez la variété.

'Toutefois il n'y a pas cliez les formes adultes identité absolue, car dans la variété paucicostata les varices longitudinales disparaissent plus vite et sont alosentes sur les dernier's toul's, comme cela est expliqué dans le texte (p. 221). Il n'est pas rare d'olser'ver' chez notre Sipho gracilis de pareilles lifférences dans l'embryon avec des coquilles adultes beaucoup plus semblables que dans le cas signalé par Struaxy.

Des espèces d'une hétél'ostylie approximative, c'est-à-dire montrant des apex embryonnaires très diffélents avec des coquilles adultes à peu près semblables, se rencontrent chez les Pyramidellidés où des formes appartenant au genre Parthemina ont le sommet embryonnaire immergé, tandis que d'autres très voisines pour les dernier's tours de l'adulte ont lo sommet hétérostrophe bien visible et très clégagé.

${ }^{1}$ Fusus bifrons a été décrit par Sturaxy en 1900 (K. Akad. d. Wirs. Wien. Sit\%. Math. Naturw. (1., pp. 197-198). 
Des cas assez précis d'hétérostylie peuvent aussi être constatés chez Purpura haemostoma L. (voil fig. dans : Dautzenberg, Contributions à la funue malacologinue des iles Aroos. Résultats Camp. Monaco, 1s.90,

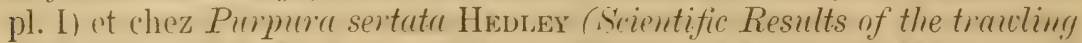
Eneclition of H. M. C. S. "Thetis ". Memoir's of Australian Museum, IV 1903 ; p. 303, fig. 96).

Chez les Pleurotoma et particulièrement chez les Pleurotoma des grandes profondeurs qui présentent ausisi des cas d'hétérostylie, les choses ont moins de netteté en ce sens que la séparation n'est plus aussi marquée rntre la protoconque et les tours suivants. Toutefois on pourra examinel arec profit, au point de vue qui nous occupe, deux espèces signalés par Watson (Challenger"s Reports. Part. XLII): Clathrella inmess (Pl. XIX, fig. 9) et Clathella porcellana (Pl. XXVI, fig. 13).

Entin il existe des Triforis dextres ayant le sommet caréné et treillisé des Triforis. mais dont la partie adulte rappelle à y méprendre la partic similaire des Cerithiopsis qui ont l'apex sans carène, lisse et de forme bien différente. On peut trouver aussi des Bittium et des Cerithiopsis réalisant re phénomene dhétérostyle entre especes de gemes distincts.

Dans ces derniers cas il est évidemment très difficile de décider si l'on doit faire intervenir la peréilogonio ou si la ressemblance constatée n'ext pas due plutot ia la convergenee des adultes sous linfluence de conditions étholociques similaires ou par laction do l'orthogénèse. La mème difticulté se retrouve comme nous le verrons dans d'autres groupes et principalement chez les Insectes (voir ci-dessous p. 630).

Maris le plus souvent la puecilogonie hétérostylique des Mollusques gastéropodes trouve, pensons-nous. une explication satisfaisante dans les conditions d'existence très diverses où peut se rencontrer l'embryon de ces animaux qui tantot se déreloppe aux livages et tantot mène une existenee pélagique.

C'est ainsi que chez Purpura lapillus, espèce de la zone des Fucus, le déroloppement est alwolument direct (avec nue eurieuse adelphophagie

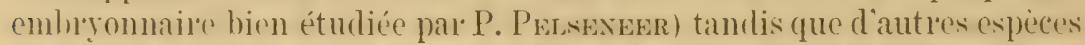
du mène gente ont. comme nous l'avons dit. une protoconque pélacique trè̀s différente des autres tour's et séparée de ceux-ci par un bour'relet saillant. Cos formes larvaires pélagigues à volum très étendu sont même assez tranchées pour avoir été considérées naguère comme des genres distincts (Simsigera d'Orbigny, Cheletropis Forbes). Leur présence ou lem suppresion dans une espece suffit. comme le remareque Sinroth (04) à faire apparaître l'hétérostylie.

Platy el mia. - Le phylum des Ver's plats et en particulier le groupe dos Trimatolus semble etre la terre promise des trpes porilogoniques. Nulle part ailleurs, si ce n'est dans la classe des Batraciens, on ne trouve 
une pareille plasticite des formes embryonnaires ot une si grande farilité d'adaptation des larves aux diver'ses conditions du milieu.

Nous rappelerons tout d'abord les bean mémoires do Zacilems sur Polystomum integerrimum où se trouvent si bien décrites les deux taxies évolutives suivies par co parasite. selon qu il sarete sur les branchies du têtard pour y achever son évolution, ou que. pénétrant dans l'intestin. il arrive finalement à l'état parfait dans la vessie de la Grenouille transformée. Chez Polystomm la puecilogonie est divmontr. cest-iblire que les formes adultes elles-mémes no se ressemblent plus. meme an point de vue anatomique, et, chose intéressante, la forme ectoparasite du têta'd concorde par son organisation avec une autre espèce le $P$. ocellatum Rud. parasite externe de la 'Tortue Emys lutraria Bp.

Mais aujourd'hui que nous savons d'une façon certaine que les germes contenus dans les rédies et les sporocystes ont la valeur de véritables oeufs, nous pouvons dive que les diverses complications du cycle ontogernique de tous les Trématorles appelés digémétiques śexpliquent merveillensement par une poecilogonie due a labondaner plus ou moins giando de nourriture. et accompagnée de progénèse dans une partie des phases évolutives.

Les formes poecilogoniques plus ou moins progénétiques (rédies et sporocystes) so succedent sans interruption jusqu'au moment ou. les l'ésel'ves de l'hôte étant épuisées et la nécessité de la dissémination se faisant alors sentir, on voit se produire en effet une forme disséminatrice active, le cereaire, qui ahoutit rapidement a l'etat adulte normal du Distome.

Ainsi disparaît du cadre de la (rénésiologie un des modes exolutifs les plus embar lassints à expliqu'p parmi les phénomènes réunis arbitrair"rment autrefois sous le nom de générations alternantes.

Chez les Turbellariés, les belles recherches de E. Bresslau (04) ont commencé a jeter quelqur lumiere sur lin probleme tres complexe du di-

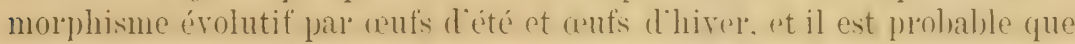
les résultats obtenus daus er groupe pouront etre fitendus à d'autres Métazoaires tels que les Rotifères, les Gastrotriches, etc.

La dissogonie des C'témopholes, gue C'mox nous a révélén dans une série de travaux intéressants, se l'elie en partie aux faits précédents, en même temps qu'elle présente des analogies frappantes avec la néoténie des Axolotls et la progénèse (avec évolution ultérieure) des Salmonides ${ }^{1}$.

1 La néoténie ne doit pas être confondue avec la progénèse: Il y a néoténie quand un animal, en devenant adulte, retient certains caractères infantiles. Un homme adulte qui a gardé ses dents de, lait (nous en connaissons des exemples), est un eas de 
I e matelmia. - Chez les Nématodes, Ercoraxi (75) a signalé depuis longtemps; sous le nom de dimorphobiose des particularités qui semblent bien devoir être rattachées à la pocilogonie.

Mais ce sont surtout les recherches expérimentales de A. Conte (00) qui nous permettent d'apprécier le rôle que les variations introduites par laction des milieux dans l'embryogén ie des Vers ronds ont pu jouer dans lo polymorphisme évolutif remarouable de ces animaux à organisation si uniforme.

Coste opérait sur des espèces à vie libre qu il cultivait sur des milieux artificiels de composition variée.

Sur colle de pâte. Rhabditis monohystera s'ust maintenu pendant six mois constamment vivipare; dans ces conditions, les aufs se développent dans l'utérus, y éclosent, et les embryons sont ensuite expulsés à l'extéricur. Tres exceptiomnellement, dans les débuts de culture sur lames, on constate des pontes d'œuf́s non éclos, mais toujour's à des stades très avancés. Lactivité reproductive et la taille des individus adultes varient proportiomellement à la lichesse nutritive du milieu. Mais (pue celui-ci soit liche ou appaurri, qur le nombre des emblyons soit grand ou petit. il y a toujours viviparité. Sur pommes de terre, les résultats sont analogues, mais la réduction de taille des individus est presque immédiate.

Dans les cultures sur peptone, les résultats sont au contraire bien différents: imméliatement, loviparité devient la règle, et l'on rencontre dans les cultures de nombreux oufs dont beaucoup n'ont pas encore atteint le stade à deux blastomères. Cette oviparité ne peut d'ailleurs ètre attribuée simplement à laccumulation excessive des cruf's dans l'uté-

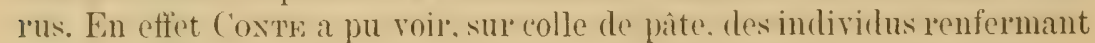
jusqu'à 105 œufs et 20 larves; ils ne pondaient que des embryons éclos, tandis que, sur peptone, des femelles ne renfermant que 6 à $S$ ruf's les pondaient au délut de lent développement. Une femelle ovipare transportée sur colle de pâte r'edevient rapidement vivipare.

Dans ses admirables recherches sur les mues et l'enkystement des Né-

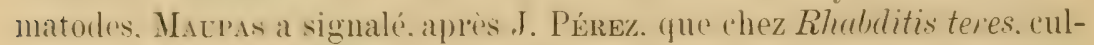
tivé 'm milienx artiticiels, les embryons éclosent dans liuténs maternel,

néoténie partielle. Chez les néoténiques, la croissance continue, mais avec des arrêts de développement plus ou moins nombreux. Il y a progénèse, au contraire, quand dans un développement normal et avec une croissance normale, les organes génitaux éroluent prématurément et permettent à l'animal de se reproduire avant que sa croissance'soit terminée et qu'il ait pris les caractères de l'adulte. L'apparition des signes de la puberté et le fonctionnement des organes génitaux chez les enfants de l'un et l'autre sexe constituent des cas bien connus de progénèse chez l'espèce humaine. Pratiquement, chez les animaux inférieurs surtout, il est parfois assez difficile de distinguer la néoténie de la progénèse. La pédogénèse est la progénèse des larves. 
en perforent les parois et dévorent leur neere. Malpas attribur co parasitisme accidentel à l'inanition ou à la sénilité. Conte a vu les mémes phénomènes se produire chez $R$. monohysterr lorsgue la colle de pâte entre en putréfaction. Sur une autre espece, Diploryaster lomgicanda ('lp.. il a pu également en provoquer l'apparition en faisant. sur lanes. des cultures dans la colle de pâte. Cette espèce, au début, ovipare, devient vivipare quand le milieu s'épuise, ot les larves arrivent enfin is se développer dans le corps de la mère et à s'y enkyster".

Lorsque je m'occupais, on lis7t, du développennent do Molynlu socintis, j'ai montré l'influence que pouvait avoir sur l'évolution ontogénétique d'un animal le genı de vie du progéniteur, et j’ai tenté d'expliques" do cette manière les différences de formes embryonnaires des Molgula à larves urodèles et des Anurella si semblables entre elles à l'état adulte. L'exemple des Nematodes étudiés par Coste confirme alsolument cette interprétation.

Crusta cea. - Un exemple plus curieux et plus démonstratif cncore nous est fourni par la poecilogonie d'un Palémon, lo Pulemonetes iurians Leach, qui vit tantot dans les eaux samuates des estuairrs. tantot dans des lacs depuis longtemps séplurés dr la mer et dont lean est devenue tout à fait douce. Ici, en effet, il s'agit d'animaux appartenant à la même espèce, absolument identiques entre eux à l'état adulte, et présentant un développenent différent suivant qu'ils rivent dans l'uan douce ou dans l'eau salée.

Mon attention a été attirée sur ce fait au cour's de l'étude très minutieuse que j'ai dû entreprendre des divers types de Palémons pour la

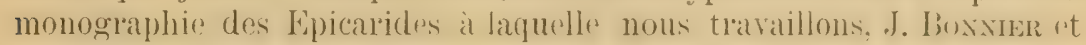
moi, depuis plusieurs années.

Des femelles de Palrmonetes varians provenant de lacs de l'Italie méridionale, comparées avec celles que l'on peut recueillir dans le vieux lit du Wimereux ou dans la Liane, à Boulogne-sur-mer, présentaient avec ces dernières une différence très étonnante au moment de la gestation.

Deux femelles, l'une de Naples, l'autre de Wimereux, prises au hasa'd, mais à peu près de même taille (4 centimètres environ de l'extrémité du rostre à celle du telson), incubaient la première des œufs longs de 3 demi-millimetres, la secondre des rufs mesurant un peu plus d'un demimillimetre seulement; d'où les noms de macrogenitor et de microgenitor donnés respectivement à ces deux types.

Comme conséquence, tandis que la femelle de Wimereux portait 321 cufs, celle de Naples n'en avait que 25, et néanmoins cette dernière paraissait la plus chargée.

Le développement des Pulremonetes de Naples a été tracé de main de 
maître par Paul MAYER. Yous avons étudir presque simultanément, Boas et moi (s.3), l’ontogénie des Pulemonetes de la mer du Nord et du Pas-deCalais, et nous avons reconnu chacun de notre côté que l'évolution de cette forme marine est heaucoup plus explicite et dilatée que celle de la forme d'eau douce dont les orufs sont chargés d'un vitellus nutritif plus abondant (à peu près dans la proportion de 27 à 1).

Il est interessant de noter que chez l'Ecrevisse fluviatile, les aufs sont également trix rolumineux et le déreloppement plus direct et plus condensé que chez les types voisins habitant la mer.

Cette loi pourıait être étendue à beaucoup d'animaux d'eau douce appartenant aux groupes les plus divers (Planaires, Oligochætes, etc.) et comprarés aux types marins correspondants. La cause déterminante de cette condensation embr'yogénique doit être cherchéc très vraisemblablement dans le fait que la concurrence vitale est moins active dans les eaux douces. Par suite, le nombre des embryons peut être réduit sans danger pour l'espeser et dés lors ces embryons per nombreux ont une réserve nutritive suffisante pour atteindre le plus rapidement possible, parfois même avant de quitter l'ouf. la forme définitive. La ségrégation maintient la particularité acquise et la rend constante.

J'avais pensí naguère que, pent-être, la température différente dans les deux habitats pouvait avoir joué un rôle dans létablissement de la pureilogonie chez Palimonetes revious. Mais, depuis, la connaissance de deux cas nouveaux et fort importants d'évolution poecilogonique, signalés également chez des Crustacés décapodes par W.-K. Brooks et F.-H. Herrick, m'a fait l'enoncer à cette idée.

Un Crustacé macroure assez abondant sur la côte atlantique de l'Amérique du Norol, Alpheus heterocheles, présente trois modes de développement suivant les localités où on l'étudie. A liey-ITest (Floride), l'embryon sort rle l'neuf, comme la jeuno Ecrevisse, arec tous les caracteres de l'animal adulte: le développenent est condensé (couogénétique), ainsi que l'avait constaté PAckard. Aux îles Bahama, au contraire, l'A. heterocheles a une larve qui passe par cinq états différents avant de l'essembler à l'adulte: le développenent est dilaté (palingénétique). Fnfin. à Beraufort (Caroline). la même espèce solt de l'(xuf sous une forme assiez semblable aux stades embryonnaires Il re III des larves observées aux îles Bahama.

Le second cas est encore plus curieux. Dans une même localité (NewP'mvidence, aux Bahama), l'Alphens saulcyi commensal de deux sortes d'Eponges, l'une vorte et l'autre brune, presente deux modes d'embryogéne différents suivant les conditions de milieu. Les individus qui vivent diuns l'Fungere verte ont un grand nombre d'aufs très petits dont le díveloppement we dilate; ceux qui vivent dans l'Eponge brune portent un 
petit nombre d'oufs tres gros, d'où sortiront, par développenent condensé, des larves beaucoup plus rapprochées de l'état adiulte.

Le cas de l'Alpheus Saulcyi est donc tout à fait comparable à celui du Palrmonetes varians, mais comme les deux formes de cet Alpheus ont été observées dans une mème localité, on peut ('n déduire que le factem. température n'entre pas en jeu non plus daus la prouluction des variétés macrogenitor et microgenitor de Palamonetes.

Dans ces exemples comme dans celui des Clavularia de Marseille; il serait intéressant de savoir jusqu'à quel point les variétés porecilogoniques sont transmissibles par hérédité et dans quelle mesure olles peuvent etre produites directement par l'action des factems primaires. Henrick paraît croile que chez Alpheus Saulcyi les individus parasites de l'Eponge verte (var. microgenitor) sont nés parfois de la variété de l'Eponge brune (var. macrogenitor) et, qu'arrivées à une certaine taille, les larves ont émigré dans l'Eponge verte et se sont adapties à ce nouvel habitat. Cette opinion ne mo paraît pas absolument démontrée, hien que d'autres exemples semblent venir l'appuyer': celui de Leptodora hyalima notamment et celui de Leptoclinum Lacazei dont nous parlerons plus Inin. Pour Palamonetes, la question pourrait êtle facilement tranchée par l'expérience dans un lahoratoine maritime suffisamment installé. Les arlversaires du transformisme réclament souvent des exemples do moditications accomplies pour ainsi dire sous nos yeux; il serait difficile, ce me semble, d'imaginer une expérience plus convaincante que celle réalisée par la nature sur $P$. varians, expérience qu'on pourrait varier dans diverssens, notanment en repremant pour ce Pritimon le moderoperatoire suivi par Schunkerwisch dans ses observations sur la transtormation des Artémies en Branchipes. T'rès importante aussi serait l'étude comparative de l'embryogénie de Palxmonetes varians, en eau donce, dans les diver's endroits où il a été signalé comme habitant des lacs. Car il est bien évident qu'en deux points très différents de son très large habitat, la ségrégation a dû s'effectuer à des époques différentes, et l'on peut s'attendre à rencontrol des modifications ontogéniques variables ot progressives avec la diminution de la salure de l'eau.

Peut-être aussi les variations dans le développement correspondent-elles seulement à un petit nombre d'états d'équilibre biologique réalisables et, dans ce cas, la poecilogonie apparaîtrait par mutation, c'est-à-dire brusquement et sous des formes identiques en diverses localités.

Pour en revenir aux Alpheidix, Coutuére (99), dans sa belle monographie de cette famillo de Macroures. a braucoup augmenté les domnés que nous possédions relativement à la pœcilogonie des diverses espèces, et surtout à celle des espèces du genre Synalpheus. Mais la question demeure encore très obscurce et ne pourra sans doute être résolue que sur 
place par un emhryologiste comprenant on même temps limportance de la systématique et l'intérêt des observations éthologiques.

Chry les Copépodes, le développement dimorphe bien connu de Leptodora hygrina est manifestement en rapportt avec les époques de ponte. C'est un excellent exemple de pœcilogonie saisonnière ditaxique. Pai les gradations continues. les faits de ce gemre nous conduisent insensihlement vers un cas limite fort important : Ie dimorphisme saisomier de certains Insectes adultes (Lépidoptères, Diptères, etc.).

A rachnida. - En étudiant les hypopes des Acariens de la famille des Tyroglyphides, Trouessart a reconnu que; chez les Trichotarsus, ces hypopes peuvent revêtir deux formes différentes qu'il a désignées sous le nom d'Hypope enkysté et d'Hypope migratile. Les deux formes semblent devoir apparaitre chacune suivant les conditions éthologiques. On les rencontre en hiver, dans les colonies de Trichotarsus osmix et $T$. Ludurigi, installées dans les nids d'osmiu cormuta et de Megachite lomalup. La forme enkystée est la plus nombreuse. Les deux formes sont provoquées par la disette qui régne en hiver dans les nids d'Abeilles où toutes les provisions ont été consommés. L'hypope enkysté est une forme d'hibermation; l'hypope migratile une forme de dissémination. Ici. comme chez les Trématorles, nous nous trouvons en présence d'une precilogonie éthologique dépendant de l'abondance des réserves nutritives.

Insecta. - Chez les Insectes, des phénomènes de pœcilogonie comparables à coux observés chez les Trématodes ct compliqués comme chez ces derniers par la progénèse et la parthénogénèse, ont été signalés depuis longtemps chez les Pucerons (Homopteres), les Cynipides (Hyménoptères), les Cécidomyides et les Chironomides (Diptères). Ces développements poecilogoniques sont détrmmines en partie par les facteurs primaires température et alimentation.

Mais dans un grand nombre d'autres (’on moinsbien étudies jusqu ì pré sent, il est plus difficile de démêler les causes qui ont occasionné le polymorphisme (généralement polychroüsme) des états jennes d'une mème espèce.

C'est un fait bien connu de tous les entomologistes que certains Insectes, très semblables entre eux à l'état adulte. ont des larves fort différentes. De nombrenx exemples de cotte particularité ont été signalés, principalrment chez les Lépidoptères.

Heliothis maritima et Heliothis dipsacea, Acronycta psi et Acronycta cuspis sont des Papillons qu'on a quelque peine à distinguer, mais dont les chenilles sont bien caractérisées. Cucullia lactucæ et C. lucifuga ne pourraient pour ainsi dire être séparés si on ne les avait élevés de che- 
nilles qui sont tres différentes. Ch. Onentrür raconte que les Nymphalides Gymecir dirce et rymocir dircenides sont tellement parcils al l'état parfait que Constant Bar ne put distinguer, à Timnes. les Papillons de ces deux espèces que lui-même avait élevés à l'île Portal, en Guyane, et qu'il avait envoyés en Europe sans avoir pris soin de les étiqueter séparément'.

Etant domnéce l'insuffisance de nos comnaissances pelatives aux premiers états des Coléoptères, il est probablo que beancoup rle cas du méme grenre doivent passer inaperçus dans cet ordre d'Insectes, et que, dans les familles très homogènes, des individus en apparence identiques mais

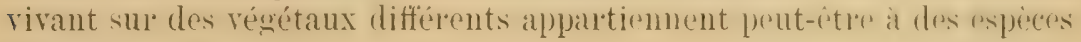
distinctes.

Tous les Insectes à métamorphoses complètes peuvent présenter les mêmes phénomènes et, s'ils sont beaucoup plus rares chez les Ametabola, cest que. (chez cenx-ri. le passige de l'état lanvaire a l'etat parfait so faisant graduellement d'une fircon insensible, la lave et l'imago sont soumis à peu près aux mêmes adaptations.

Au point de rue pratiqur. il nest pas toujours bien commode de décider si deux formes ressemblantes sont des espèces poecilogoniques ou

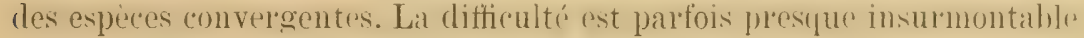
lorsque l'on ne connait pas la bionomie des types en discussion.

Dans certains cas, cependant, la distinction est facile et le doute n'est pas permis.

Lorsque, par exemple, les espèces qui par une lente sélection sont derenues converentes appartiennent à des genles suffismment éloigness. les caracteres anatomiques profonds ret les caractires embryogriniques ne sont pas altérés par la convergence au point d'être méconnaissables. La ressemblance n'est que superficielle, et si les anciens naturalistes ont pu être trompés par l'aspect similaire des adultes examinés superficiellement, l'erreur n'est plus possible aujourd'hui. Le mimétisme si parfait cependant des Leptalis et des Ithomia, celui des Papilio paradoxa et d'Euplar midamus, l'imitation des Danaïdes ou des Acræides par diverses espèces de Papilio ou de Diadema n'en imposent plus à aucun entomologiste. Parmi les Papillons indigenes la resirmblance. d'ailleur: moins exacte, de Dichonia aprilina L. et Moma orion Esp. est encore un exemple du même genre ${ }^{2}$.

${ }^{1}$ Сн. Oвerthür : Etudes de Lépidoptérologie comparée, fasc.1, p. 12 note, mars 1904. - Voir aussi, à l'égard de ces Gynccia: Sepp, Papillons de Surinam, Pl. CXLIX et texte correspondant.

2 Dans un article, Sur quelques cas de faux mimétisme, publié dans le Naturaliste du 15 février 1894, M. le professeur Plateau regarde comme faux le mimétisme de ces deux Noctuelles, car les dates d'apparition des deux espèces sont si 
Mème lorspue la convergence des adultes a lien chez des especes congéneres, ce qui nous prive en grande partie des indications de lanatomie comparée on pent encore dans bien des cas reconnaitre l'origine nettement distincte des deux especes et établir que leur ressemblance est due ¿l l'action du milieu sur l'inago. C'est ce qui a lieu par exemple dans les cas d'isotypie. Quelque voisines que soient des formes telles que Pieris brassicæe et Pieris rapx, les divers Euplæa, etc., le fait que ces espèces fréquentent les mêmes localités sans se confondre et sans se croiser indique clairement que les différences larvaires qu'elles présentent dans des conditions de milieu identiques sont des différences phylogéniques anciennes.

La chose est encore bien plus évidente lor'squ il s'agit d'Hyménoptères parasites isotypes dont les larves ont habité dans un même hôte ou de parasites des végétaux dont les larves ont vécu côte à côte sur la même plante. Hormomyia capráa Bremi se distingue à peine à l'état adulte d'une autre Creidomie (Rhabdopharya sp.) vivant égalenent sur le Salix capraxa et dont la galle a été décrite par Schlechtenda sous le 11"392 de ses "Gallbildungen " (Zoocecidien, p. 40, 1591). Mais les larves de ces denx Diptires restent distinctes ot produisent des galles très différentcs sur les feuilles du même arbi'e. Il n'y a donc nul doute que la ressemblance des adultes est une simple convergence et n'indique pas une parenté immédiate ${ }^{1}$.

diffêrentes, dit notre collègue, qu'il faudrait de véritables perturbations dans les saisons pour les rencontrer à la même époque de l'année. Nous ne pouvons accepter cette manière de voir; les conditions que M. Platead réclame pour le mimétisme vrai sont beaucoup trop étroites. Il suffit de lire les travaux de WALLACE pour comprendre que, même actuellement, une espèce imitatrice n'a pas nécessairement la distribution géographique de l'espèce mimée, l'une ou l'autre pouvant disparaitre par extinction ou apparaitre par introduction d'une façon indépendante dans certaines localités. Il faut, dans les questions de ce genre, envisager les espèces en question non seulement dans l'espace, mais dans le temps, le mimétisme pouvant survivre aux causes qui l'ont produit. En ce qui concerne les deux Noctuelles qui nous occupent, il est facile de répondre à l'objection de M. Plateau. Moma orion parait, en plaine, dans le mois de juin, mais dans les régions montagneuses il est plus précoce. Quant à Dichonia aprilina, Bruand l'a obtenu d'éclosion printanière et Berce émet la supposition très vraisemblable que, en Suède, où l'hiver arrive de bonne heure, les éclosions n'ont lieu qu'au mois d'avril, ce qui justifierait le nom donné par Linsé (roir Berce, Faune entomol. de France, Noctuelles, ${ }^{\theta}$ part., p. 52). D'ailleurs le mimétisme des $M$. orion et $D$. aprilina est manifestement un mimétisme indirect; ces deux Papillons ressemblent aux Lichens, et le dessin fondamental, très constant chez les diverses Noctuelles, vient aider à la convergence.

Un cas opposé à celui de la convergence est celui de la divergence des adultes chez des espèces ayant des larves semblables. Les exemples de ce genre, innombrables chez les Coléoptères et surtout chez les Hyménoptères et les Diptères, peuvent aussi se 
Lorsqu'au lieu de vivie dans un même milieu les larves ont un habitat différent, la question devient beaucoup plus délicate à l'ésoudle.

GứNÉE (58), dans un remarquable mémoire Sur quelques Bombyx enropéens, a considéré comme especes distinctes deux types, Bombyx spartii et Bombys callunte Palen, qui représentent l'un une forme méridionale, l'autre une forme septentrionale de $L$. yuercus L. Si l'on examine une collection un peu étendue de B. quercus, on voit facilement qu'il est a peu prè impossible do séparer lrs adultes de ces trois especes, certaines formes de $B$. quercus passant manifestement, comme Gúxise et Bellier de la Chavignerie le recomnaissaient déjà, soit à $B$. spartii soit à B. callumie.

Guénée a découvert que la chenille jeune de $B$. callunæ diffère de colle de $B$. querus, mais que les divergences ne tardent pas it diminuer après les premières mues et finissent même par disparaître.

On pourrait être tenté de voir dans cette dissemblance des larves à l'état jeune une jreure do la séparation primitive des deux méces. Mais l'habitat différent suffit. il mesemble, it expliquer cette divergener: l'une des deux formes, sans doute $B$. collunde, a gardé la livrée de la promière larve ancestrale qui s'est altérée chéz $B$. quercus et $B$. spartii, et ces espèces doivent vaisemblablement ètre considérécs comme des formes precilogoniques fixées et légèrement modifiées à l'état adulte.

Il en est de même, pensons-nous, pour Eriogaster larestris L., Erio-

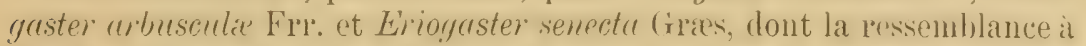
l'etat adulte est tres grande, bien que les chenilles divergent considérablement.

On hésite davantage à se prononcer lorsqu'à la différence de nourriture vient sajouter (quelquautre particularité éthologique (le nombre des cénérations par exemple). Je ('rois cependant qu il faut rncore considérer. comme un cas de poecilngonio l'exemple de Leioptilus curphoductylus IIh). et de sa variété buphthrimi Hfun. yui diffe'ent par la rhenille et par la chrysalide, et dont l'un vit sur Inula conyza et n'a qu'une génération tamdis que l'autre se nomrit de Buphthulmum sorlicifolimm et parait deux fois dans l'année ${ }^{1}$.

Prut-etre aussi faut-il considérer comme porilogonique saisonnier le Charaxes jasius [GIARD (04)].

rencontrer chez les Lépidoptères: "Il m'est éclos, dit de Graslin, dix à douze variétés d'Agrotis ripæ si différentes entre elles que j'aurais été fort embarrassé pour les déterminer si je ne les avais élerés de la même chenille. „ Les Orthosia instabilis et 0 . gracilis sont dans le même cas. Les Jochaera alni d'Europe, et J. funeralis, d'Amérique, différents à l'état adulte, ont la même chenille. De pareils faits rendent plus compréhensible les exemples de pœcilogonie énumérés ci-dessus.

${ }^{1}$ C. Hormann. Illustrierte Zeitsch, f. Entom. Bd. III, p. 341. 1898.

VIe CoNGr. INT. ZooL,, 1904. 
Nous avons d'ailleurs des preuves directes et indiscutables de l'existrnce de la pœeilogonie chez les Insectes.

Siebold (37) avait déjà signalé, sans préciser le nom de l'espèce, la viviparité de certains Ephémères. Les recherches ultérieures de CaLori (48), de rausard (96) et de Hermons (97) ont prouvé que le vulgaire Chloropsis dipter Latreille ovipare dans le nord de son habitat, (Berlin, France septentrionale) devient vivipare dans le midi de la France et en Italie. ("est donc un exemple de poecilogonie géographique en sens inverse de ceux que nous avons vu exister chez les Echinodermes et plusieurs autres groupes d'animaux marins. Peut-être aussi, dans certains points de l'habitat de Chloposis s'agit-il, comme l'a suggéré Hernoss, d'une poecilogonie saisonnière.

Dans deux mémoires très importants, publiés en russe, et dont nous devons l'analyse à notre savant collègue $\mathrm{C}$.- R. von Osten-SACKen, l'entomologiste lusse Portohinsky a démontré que la vulgaire Musca corvina présente deux formes larvaires distinctes dans les diverses parties de son vaste habitat. Dans le nord de la Russie. cette Mouche coprophage pond généralement vingt-quatre oufs de taille moyenne, d'où sol'tent des larves qui présentent deux phases très nettes d'évolution. En Crimée et dans le sud de la Russie, où les Insectes coprophages sont plus abondants et par suite la concurrence vitale plus intense, le même Diptère ne pond plus qu'un œuf très volumineux, dont la métamorphose très rapide et condensée rappelle celle des Pupipares, la larve arrivant presque d'emblée à sa dernière phase évolutive.

Ainsi comme le dit Oster-SAcken :

" 'The wonderful power of adaptation of these lar'væ to their environment in a certain measure, destroys the parallelism which we naturally expect to exist between the systematic characters of larva and imago... Distantly related species belonging to different genera issues from larve almost indistinguishable flom each other. Again closely related and almost indistinguishable imagos, speeies of the same genus differ in their oviposition (size and number of eggs) and their larva follow a different law of development (as to the degree of maturity the larva reaches within the body of the mother, the number of stages of development it passes through). ")

Des faits analogues sont d'ailleurs commus chez divers Lépidopteres et notamment chez le Ver à soie du Mûrier (Sericaria mori). Dans le sud de l'Europe, cette espece fournit une race intéressante, dite Trevoltini, qui, non seulement donne plusieurs genérations annuelles, mais se distingue du type en ceque les chenilles ne subissent que trois mues au lieu de 
quatre. Cultivée dans le nord, cette race no tarde pas à reprendre les caractires ordinaires de l'espexe (la soende ou la troisieme année) ainsi que l'a constaté Rouinet.

Comme la chenille de Sericaria mori varie peu en grandissant, la particularité physiolngique de la suppression d'une mue ne frappe guèe l'œeil; mais dans d'autres cas, les modifications morphologiques sont plus appalentes.

Th. Goossens (70) a signalé plusieurs exemples parfaitement nets de pœecilogonie géographique chez les Lépidoptères.

La chenille de Deilephila euphorbix ne présente pas dans l'Ardèche et dans le département du Var le pointillé jaune ordinaire, et les taches rosées sont remplacées par des taches jaune pâle. La chenille d'Heliothis marginata, blonde ou verte, dans le nord, est le plus souvent d'un brun presque noir en Provence. Dans le midi de la France, la partie dorsale de la chenille de Zygrna fausta est presque toujours fauve; à Paris cette partic est vert-d'eau.

Que dans les cas de ce genle, les adultes des deux variétés pœcilogoniques arrivent à différer quelque peu aux deux extrémités de leur habitat, et l'on ne manquera pas d'établir deux espèces que les divergences larvaires paraîtront rendıe tout à fait légitimes.

C'est ce qui est arrivé bien certainement pour un grand nombre d'espèces dites vicariantes ou représentatives de l'ancien et du nouveau continent: Trixna psi et Trixna occidentalis par exemple. La comparaison des Iconographics de Guenée et d'A Bвотт est très instructive à cet égard. (Voir aussi 'Tutr, British Noctux, V, Introduction, p. XVI, 1892.)

Parfois même, la variation des adultes portant exclusivement sur les caractères anatomiques de l'armature génitale, les formes poecilogoniquès, tout en gardant une grande ressemblance à l'état adulte, ne pourront plus être croisées, et cet état d'amixic facilitera plus tard la divergence des deux espèces, même si elles restent en contact en divers points de leur habitat. C'est ainsi que, d'après Grote et Surth, Agrotis haruspica et Agrotis rubifera sont des formes américaines représentatives des types curopéens: A. augur et A. rubi ilont elles diffèrent seulement par l'armature géuitale mâle.

Au même ol'dre d’idées se rattache l'étude des variétés génitales de certains Orthoptères, des Lépidoptères Hesperidæe du genre Nisoniades et des diverses espèces d'Homoptères du genre Typhlocyba du groupe de T. rosx, hippocastani, Douglasi, etc.

J'ai signalé ailleurs la ressemblance extraordinaire de ces Cicadelles qui vivent sur les mêmes végétaux et aux mêmes époques mais dans un état d'amixie forcée. Les parasites de ces Typhlocyou (Diptères et Hymé- 
noptipes) eitant identiques. il est difficile de voir dans cette ressemblance un mimétisme direct ${ }^{1}$.

Mais. des yue les moditications do l'appareil génital interviennent, on pout se demander si elles nont pas précédé et déterminé la poecilogonie au lieu ditre causées par elle ou produites apres elle. Cette question est difficilement résoluhle aujourd'hui et rentre dans les noubreux problèmes que liomaxes a posés dans son travail sur la sélection physiologique.

() uant aux causes prochaines de la pocilogonie polytaxique (le plus souvent ditaxique) des larves des nombreux Lépidoptères, il est difficile d'en domner des à présent me explication lamarekieme. de me suis contenté d'en esquisser naguire une tentative d'interprétation darwinienne en rattachant cette pecilogonie au mimétisme évolutif et défensif": mais il est clair que nous devons nous efforcel' d'aller plus loin aujourd'hui. Létude des pigments, telle que l'a entreprise M. von Lrisdex, jettera sans doute quelque lumière sur ce sujet délicat.

I)ans plusieurs circoustances. la poecilogonic paraît due, comme nous l'avons dit ci-dessus, à la nourriture différente des larves. Si certaines chenilles sont modifiés directement par la plante nourriciere. comme cela est connu chez un grand nombre d'espèces (Eupithecia, etc.), on comprend aussi que quelques-unes se soient adaptées définitivement à une plante déterminée et aient gradé d'une facon permanente une livéée

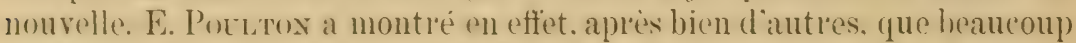
de chenilles se laissent mourir de faim, plutot que de toucher it une nourriture dont leur race a perdu l'hahitude. Peut-ètre est-ce à une poecilogonie nomriciere quil faut attribure les liveres différentes des chenilles de Cucullin revbusci et C'. serophluluriat par exemple. Noctuelles dont la ressemblance ì létat adulte ne sexplicue guere facilement par la convergence.

Enfin certains cas de ressemblance chez des Insectes dont les larves sont diffélentes mais vivent dans les mèmes localités, parfois mème sur les memes plantes. sont difficiles à interpretele daus l'une on l'autre des alternatives, converuence ou pocilogonie. Nous citerons entre autres cas énigmatiques ceux de Lithosia complana et $\dot{L}$. hrideola, et celui de Deilephila euphorbia et $D$. niccea.

Tunicata. - Les Tuniciers, comme beaucoup d'animaux à larves pelagiques, presentent dassez grandes variations dans leur ontugénie. mine chez une espece domnée. ut la fixation de la larve urodele dépend beaucoup de l'état du milieu aquatique où elle est placée."

'A. Giard. Sur la castration parasitaire des Typhlocyba par une larve d'Hyménoptère (Aphelopus melalencos Dalm), C. R. de l'Ac. des Sc., 8 juillet et 4 nov. 1889.

2A. Grard. Recherches sur les Synascidies. Archiv. de Zool. expér. et gén., T. I, p. 561 note. 1872 . 
Un fait dune grando impoltance ot rui na pas suffisamment attire

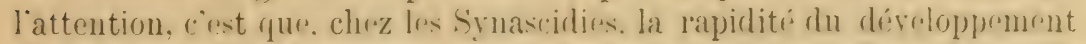

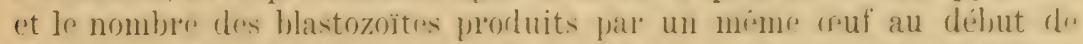
l'évolution dépend tress souvont, dans unre lange mesule. des conditions

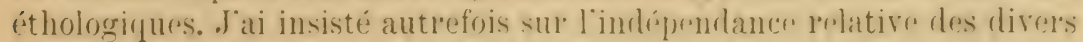

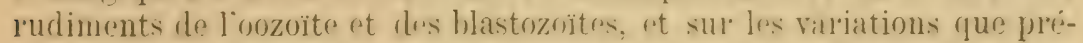

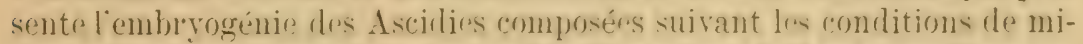
liru ot les réserves nutritives mises a la di-position do lombryon. Ians ses

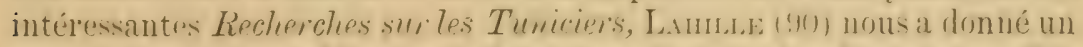
nouvel rexmple fort démonstratif de ress variations. Le Lepotorlimem Lor-

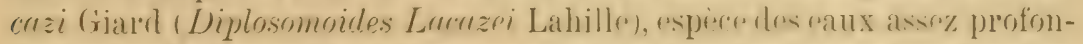

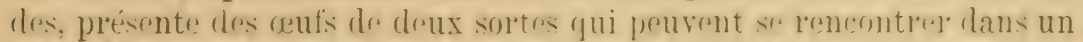
meme cormus. Les uns, pauves en vitellus nutritif, Iomment de jolitrs larves dont la queue se résolbe de très bonne heure et qui n'ont pas encope bourgeonne lo troisiene jour apros la nairane au moment ril relles se fixent. Les autres, riches en deutoplasme, produisent des larves qui

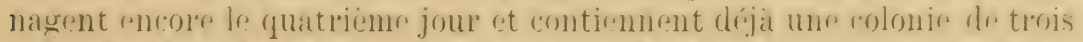

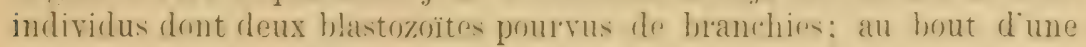
douzaine d'heures, apres la fixation on a une colonie d'une dizaine de blastozoïtes.

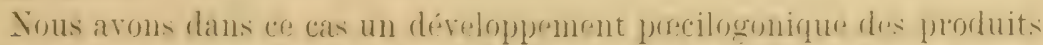

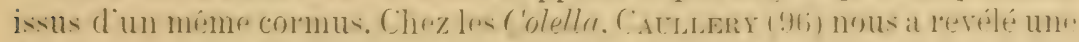

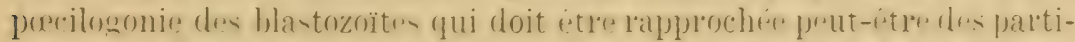

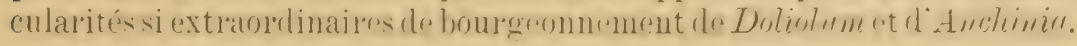

Te r tebl'a ta. -- C'est Enrexbaum (96) qui, le premiel', à ma connaissance, signala un cas de pocilogonie chez les Poissons. En étudiant le

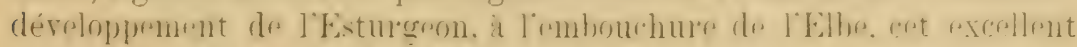

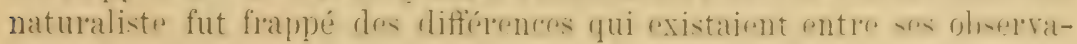
tions et celles de J.-B. Rrder faites sur le même Poisson en Amélique.

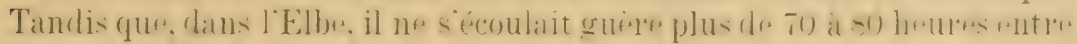
le moment de la fécondation et celui de l'éclosion des larves, Rrver in-

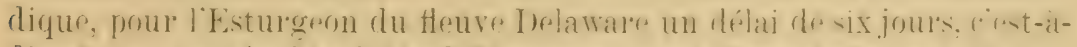

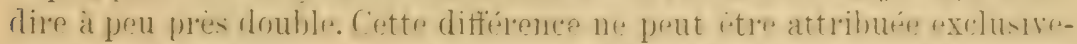
ment à la température, car les larves observées par Rrver mesuraient

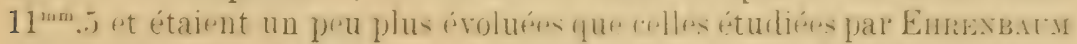
qui avaient $9^{\mathrm{mm}}, 3$ de longueur. Les plus jeunes larves de l'Elbe présen-

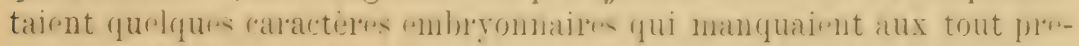
miers stades tigurés par Rroer. Ceux-ci ressemblaient au stade 2 des planches d'Eurexbaum, cor'respondant aux larves de $11^{\mathrm{mm}}$ dans le Delaware.

Ehrexbacur a lui-même rapproché ces faits de ceux que nous avons sionalés linas et moj chez Puliemonetes zorions et qui constituent un dres 
meillous exemples de purilogonie. Il est probable que des recherches ultérierues montreront que l'érolution coxnogénétique des Esturgeons du Delaware est due à une salure moins intense des eaux du fleuve américain comparées à celles de l'Elbe.

()uelyue trmps apres. Hexcke (gs) décourrit des faits analogues chez un autre Poisson it tres vaste dispersion, le Hareng (Chpen hurempus). Le jeune Ifareng des baux sammâtres de la schley appartenant a la race de printemps (Friihjahrshering) sort de l'ouf dans un état d'évolution r'emarquablement plus avancé que la larve du Hareng d'eau salée de la bate d'Echerforder qui appartient il la race dautomne (Herbsthering).

Il importe de remarquer que, outre le facteur salure, nous voyons intervenir ici la saison.

Des obselvations de Bruno Hover (97), de InhoF (96) et surtout de A. Fedoensen (95), il semble bien r'ésulter que l'Anguille est aussi un Poisson poecilogonique susceptible de donner en mer une larve pélagique Leptocephale comme l'ont démontré surtout les travaux de Grassi et CALANDruccio, ou de se développer plus ou moins cœenogénétiquement en eau saumâtr'e ou en eau douce. D'après Fedoersex, l'Anguille du nord serait même devenue en certains points strictly fiesh water species, différ'ente de l'Anguille migratrice par des caractères bien définis.

I)ans mes dragages anx îles Glenaus et au large de Concalneau, il mest arrivé sourent de lecueillir de jeunes Congres déja parfaitement transformés et diune taille notablement inférieure aux Leptoceplualus Morrisii que je trouvais abondamment, non loin de là, dans l'anse de Porzou. Il est diallun's manifeste que des modifications. mêne très légèes, dans les conditions éthologiques peuvent déterminer rapidenent des changements mol'phologiques dans l'ontogénie de diver's Poissons.

A Wimereux, en avril-mai, le filet fin permet de se procurer en abondance des laves de Plies (Pleuronectes platessa) longues de 10 à $12^{\text {mm }}$, encore parfaitement simetriques et transparentes. Tramsportées en aquatrium, ces larves se couchent sur le côté et commencent à se pigmenter dès le lendemain, tout en continuant d'ailieurs à évoluer très normalement.

D'une façon générale, je puis d'ailleur's confirmer les vues émises il y a déjà longtemps par Wheldox: beaucoup de larves nageuses peuvent prolonger plus ou moins longtemps leur existence pélagique, quelquefois même acquérir une maturité progénétique et néoténique sous ret état, et, inversement, beancoup de ces larves peuvent aussi se fixer plus rapidement que de coutume si elles sont placées brusquement dans une eau dormanter on dans des conditions nouvelles de temperature, doxygénation. etc.

Les Batraciens sont, comme les Trématodes, un groupe de choix pour leitule expérimentale de la precilogonie. La néoténie, normate chez l'Axo- 
Intl, accidentello chez les l'ritons, a domné lireu ì des mémoirex nombromx et importants qui ont fait ressortir la plasticité embryonnaire de ces Urodèles.

Les mémorables expériences de Marie von Crauvis sur la Salamandre

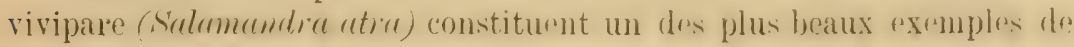
pocilogonio expérimentale.

Ces recherches ont été reprises récemment par Kanswerer (04) qui a démontré en outre que lo dévoloppenent palingénéticupe de Sulcomandia maculosa pouvait être plus ou moins abrégé par l'action des milieux

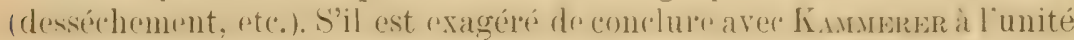

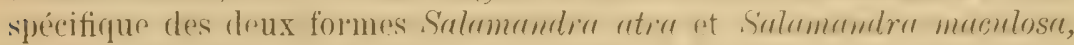

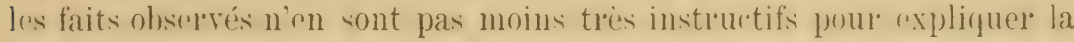
for'mation des deux espèces aux dépens d'un type ancestral commun.

Chez les Anoures. Hertwr: a réussi a transforme l'ouf holoblastique de la Grenouille en un ouf méroblastique en condensant le jaune au moyen d'un centrifugeur.

Les particularités si diverses d'iurubation due mous offient beaucoup de Batraciens des regions chaudes, premettent do complendro comment les caractères embryogéniques ont pu se modifier et différ'el chez des genres très voisins et mêne chez les espèces d'un même genre.

Nototrema marsupiatum du Pérou et de l'Equateur, porte un très

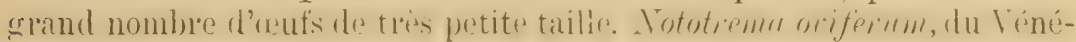
zuéla, ne porte que 30 cufs environ, de grandes dimensions. Il y a done

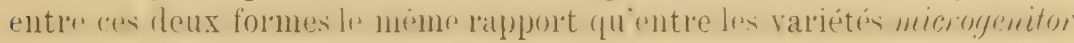
et macrogenitor de Palæmonetes varians.

Hylodes martinicensis présente une embryogénie plus ou moins condensée selon la sécheresse ou l'humidité des diver'ses îles qu'elle habite dans les Antilles. On trouve d'autres exemples fort intéressants dans le résumé très complet et très suggestif que Lilian SAxrsox a donné de la question dans American Naturalist (sept. 1900).

La viviparité du Lacerta vivipara et celle de la Vipèle, qui n'est qu'une

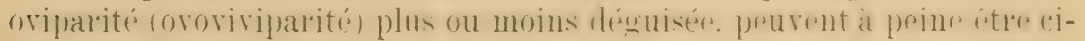
tées comme des cas de poecilogonic, mais il existe chez les Reptiles Ophi-

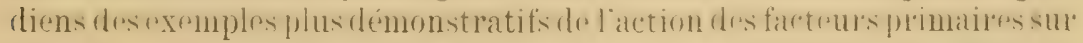
l'évolution. Les serpents du genre Epicrates pondent, suivant les cir-

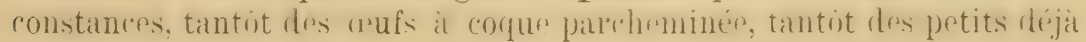
complètement formés.

On peut aussi l'approcher de la pocilogonie les faits intéressants de polymorphisme des reufs (he\% plusicus ()iseaux. Lus unfs rle certains Sylvidae et Turdidx ont une tendance au polychroisme ditaxique.

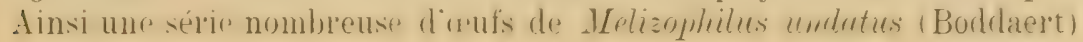


peut être facilement divisée en deux groupes dont l'un incline ver's l'olive, l'autre ver's le rougeâtre (il s'agit de la couleur des mouchetures). La couleur olive des nufs d'Oiseaux a souvent une teudance à viler au bleu (Rossignols. Faisans, Pluviers, Mouettes). Chez Cisticolu schannicula, che\% Lrin troile, chez le Coucou, le polymorphisme est encore plus accentué ${ }^{1}$.

A coté de la precilogonie éthologique (géographique ou saisonnière). on pent aussi observer, chez certains animaux, une purcilogonie sexuelle qui prarfois se manifeste déjà dans la production des a'uf́s. Les u’ufs mâles et femelles de quelques Dinophilus (D. aputivis), de certains Rotifères, etc., les embryons mâles à évolution spéciale des Rotifères, de la Bonellie. des Epicarides. (Eutomiscus, etc.) sont des exemples classiques de cette poreilogonie dont l'étude est tres importante au point de vue de la question si obscure de la détermination du sexe 2 .

Une très curieuse et très instructive variété de pocilogonie sexuelle s'olserve dans le développement de certains castrats d'un sexe déterminé comparés aux individus normaux du sexe opposé; par exemple dans l'évolution convergente du plumage chez lo jeune Faisan mâle et chez la vieille Poule Faisane à ovaires avortés.

"Le même changement doit s'opérer de part et d'autre, dit Isidor'e (ieofrroy-S'-Hiatre. puisque le point de départ est le méme et que la vieille femelle ot le jeune mâle tendent vel's le même but. Jais le changement se fait chez l'une et chez lautre trè inégalement vite à l'une il faut plusieur's années, à l'autre une seule année suffit. En outre, l'or'dre selon lequel sopère le changement n'est pas non plus exactement le même. Il suffira de comparer les jeunes mâles conservés dans tous les musées avec les détails que jai domnés sur les vieilles femelles, pour s'aperceroir que dans l'un et lautre cas. le changement s'opere d'une manière différente. Il n'est jamais possihle de dire d'une vieille ponle Faisane chez laquelle le changement a commencé. qu'elle a exactement le plumage d'un jeune Faisan mâle de tel ou tel âge. C"est donc par deux roies différentes que la nature dans l'un et l'autre cas marche rep's un r'ésultat semblable. " (Essai de zoologie générale, p. 507-505, 1841.)

Le morphogéniste peut tirel les déductions les plus importantes de létude des développements porcilogoniques provoqués par les actions lentes mais profondes de la castration parasitaire.

Dans ce travail, nous n'avons envisagé que les animaux, mais il selait facile de prouver qu'il existe chez les régétaux des faits analogues au développement pereilogonique consideré sous ses formes diverses. Nous

${ }^{1}$ Kortel. Naumannia, p. 137. 1858. Lunel. Bul. Soc. Ornith. Suisse, p. 9. 1865.

${ }^{2}$ Chez divers Lépidoptères (Sphinx, Zygènes, etc.), on observe des caractères diftérents de la chenille dans les deux sexes. 
nous contenterons de citer seulement divers mémoires de Huти (95) de Kuckuck et de Satvageau (99) très suggestifs à cet égard.

Outre l'intérêt qu'ils juésentent 'n eux-mênues, les faits que nous avons rappelés ci-rlessus nous paraissent acquérir par leur groupement une valeur scientifique plus grande, et les vues d'rusemblr qu'ils suggèront favoriseront, je pense, les progrès de l'embryogénie.

La poecilogonie, celasaute aux yeux, nous montre de la faron la plus nette et avec la précision de réritables expériences réalivéce par la nature, par quels processus s'est opéré le passage de l'emlnyogénie dilatée (paliugénier) à l'embryogénie condensée (comogéni(1) dans les groupes tris nombleux où ces deux modes de reproduction se rencontrent côte à côte chez des genles voisins, parfois méme chez des especes voisines. ('ortaines espèces precilogoniques ne mous offrent-elles pas en quelque solte it volonté tantot le premier mode évolutif, et tantôt le second, suivant les circonstances et les conditions de milieu où elles sont placées?

Dans un mémoire des plus intéressants $(03)$ et qui est un modèle d'observations ethologiques almirablement conduites R. LActernors a montré le lien très étroit qui unit les variations saisonnières de certains Rotifères (Anuræa cochlearis) à l'existence chez cette espèce d'une pocilogonie parthénogénétique.

Ces variations saisonnières, auxquelles LAuTerborn donne le nom de cyclomorphoses, s'expliquent facilement chez les animaux qui comme Anurxa ou comme nombre de Cladocères présentent, à côté de la reproduction sexuée, une série de générations monogoniques qui transmettent intécralement (sans l'action diminuante dre l'amphimixie) toutes les modifications lamarckiennes acquises ou augmentées par chaque génépation successive sous l'influence des variations du milieu.

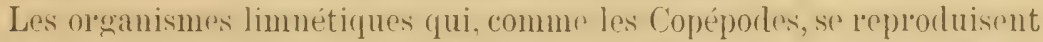
sans schizogonie et sans parthénogénés valont hien localenent mais non avece périodicité saisonnière quoi qüils puissent présenter comme nous l'avons vu (chez Leptodor" par (exemple) un développement poreilogonique.

Au point de vue taxonomique, l'importance de la pocilogonie est très grande également. Qu une des variétés d'origine pureilogonique issues d'une méme espèce viemne à prósenter une modification, si légère soitelle, de l'état adulte, les classificateurs ne mancueront pas, comme nous l'avons dit, d'en faire une espèce nouvelle. En raisonnant d'après les idcées génélalement acceptées aujourd'hui, on justifierait ainsi cettre crération: "Sans doute, dirait-on, les deux formes sont très voisines à l'état adulte, mais les differences 'mbryogéniquessutfisent pour nécessiter une distinction spécitique. "Et. en fait, une foule d'espères sutomologicunes sont établirs sur des considérations de cette nature. (Par exemple dans les genres Melitra, Deilephita, Cucullia, etc.) 
Sans doute il ne ser'a pas toujours facile de distinguer si des espèces voisines sont issues de races porilogoniques ou si elles proviennent de formes convergentes à l'état adulte mais ayant des larves originairement distinctes. Je n'ai pas cherché à dissimuler cette difficulté; je crois cependant, comme je l'ai dit plus haut, que, dans beaucoup de cas, l'expél'ience pourra nous aider à sortir d'embarras.

En offiet, la fécondité ou l'infécondité des espèces considérées dans leurs croisements inter se pourra souvent permettre de r'econnaître si, dans un cas donné, il y a convergence ou pœcilogonie.

Chez les espexes d'origine precilogonique, la différenciation des adultes portant sur des caractères quelconques ot généralement indépendants du système génital, la fécondité sera le plus souvent conservéc à un certain degré dans les croisements. Chez les espèces convergentes, au contraire, la différenciation spécifique étant établie depuis longtemps et bien antérieurement à la convergence, les croisements seront stériles et sollvent même impossibles. J'ai constaté, par exemple, que les diverses espèces de Typhlocyba de la section de $T$. Ros , si merveilleusement convergentes qu'on les distingue difficilement même à la loupe, sont entre elles dans un état d'amixie forcée. l'appareil copulateur présentant des différences énormes chez des formes qui paraissent identiques et qui vivent souvent côte à côte sans jamais pouvoir se croiser ( $T$. Rosæ, T. himporostrmi, T. Douglasi, etc.). La comparaison des formes precilogoniques et des formes convergent's nous explique ainsi, dans une certaine mesure, les différences qui existent au point de vue de la fécondité des croisements de diverses espèces sauvages, différences qui ont vivement, et à juste titre, préoccupé Darivin et Romanes.

Dans tout ce qui précède, j'ai apporté, je crois, pas mal de données nouvelles mais je me suis borné le plus souvent à rapporter des faits déjà connus, dont quelques-uns n'avaient pas, ce me semble, attiré l'attention d'une façon suffisante, et j'ai cherché à dégager de ces faits des points de vue nouveaux.

En créant le mot de "Pœecilogonie " pour désigner tout un ensemble de phénomenes évolutifs, en apparence assez dissemblables, jai parfaitement conseince de n'avoir pas supprimé du mème coup toutes les difficultés de la question. Maisil y a déjà un progrès réalisé quand des problemes, méme non résolus, sont rattachés à un autre problème considéré jus(pur-là comme distinct. et uos uxplications seientifiques ne sont géuéralement pas autre chose.

On l'a dit folt justement: "L'exemple si mémorable de l'illustre NewTux, nous invite a considerel un phénomene naturel comme suftisamment (xplirue. des que nous pourons le synthétiser. cest-à-dire (littéralement) le poser en compagnie de plusieur's autres w. (Raoul Baron, 1888.) 


\section{INDEX BIBLIOGRAPHIQUE}

00. AppeltöF. Studien über Actinien-Entwicklung. Bergens Museums Aarbog, $\mathrm{n}^{0}$ 1, p. 4, note 1, 1900.

89. Boss, J. Kleinere Garcinolog. Mittheil. über d.ungleiche Entwickl.d. Salzw. z. der Suisswasserform von Palcemonetes varians. Zool. Jahrb. f. System., Bd. IV, p. 793-805, pl. XXXIII. Voir aussi : Vidensk Heddel fra natur, Foren i Kjobenhavn.

03. Boettger, 0. Ueber den wissenschaftlichen Wert der Schneclien-und Muschelschelen. Ber. Senckenb. Nat. Ges., p. 177-187, 1903.

03. Boulenger, G.-A. On the mursing habit of a South-American frog. Proc. Zool. Soc. London 1903, II, pp. 115-116.

03. Breslau, E. Die Sommer- und Wintereier der Rhabdocolen des süssen Wassers und ihre biologische Bedeutung. VerhandIgn. deutsch. Zool. Ges., pp. 126-139, 1903.

92. Brooks, W. H. et Herrick, F. H. The embryolog!y and metumorphosis of the Macrour John Hopkins University Circulars, vol. XI, no 97, Baltimore, avril 1892, pl). 67-68.

18. Calon, L. Sulla generazione viviqura della Chloe dipter'u. L. Annale delle Sc. natur. (2), vol. 9, Bologne 1848. Traduit et annoté par Jocy. Bull. Soc. d'études de Sc. Nat. de Nimes V, no 4,1877 . Cf. Archiv f. Nat., Jahrg. 45, Bd. 2, p. 66, 1879.

01. Carlgrex, 0. Die Brutpllege der Actinarien. Biol. Centralbl. XXI, pp.468484 (13 Text Fig.), 1901.

96. Caudueny, M. Sur les Colella et le polymorphisme de lpurs bourgeons. C. R. Acad. Si.., t. LXXII.

98. Caúlemi, M. et Mesnil, F. Les formes épitolies el lévolution des Cirraluliens. Ann. de l'Université de Lyon, fasc. XXXIX, 1898, voir aussi: International Congress of Zoology, Cambridge, p. 220, 1898.

96. Causard, M. Sur une Ephémère vivipare. C. R. Acad. Sc., t. CXXIII, p. 703 - $708,1896$.

92. Chun, C. Dis Dissogonie, eine neue Form der geschlechtl. Zengung. Festschr. f. Leuckart, Leipzig 1892.

12. Cuubs, J.-A. Actinix, with an. Account of their peculiar Broor Chamber's. (Rep. Coll. Nat. Hist. "Southern Cross" p. 294, London, 1902.

00. Conte, A. De l'influence du milien nutritif sur le developpement des Nematodes libies. C. R. Soc. de Biologie. Séance du $2 \partial$ avril 1900.

04. CuÉnot, 1. Contribution à la faune du bassin d'Arcachon (III. Doridiens). Bull. Soc. scient. Arcachon, p. 13 et 14, 1904. 
99. Coutrène, H. Les Alpheida, morphologie externe et interne, formes larvaires, bionomie. Ann. Sc. Nat. Zool. (sér. 8), t. IX, Ch. V, p. 414 et suiv.

96. Ehrenbaum, E. Beilräge zur Naturgeschichte einiger Elbfische. Wissensch. Meeresuntersuch. Biol. Anst. Helgoland, N. F. I, p. 71-72, 1896.

73̈. Encolani, J. B. Osservazioni elmintologiche sulla dimorfobios, nei Nematodi. Mem. Acad. Bologne, V, pp. 391-441, pl. Rendic. 1874-75, pp. $73-80$.

9:3. Feddersen, A. Ueber das Laichen des Aales in Süsswasser. Zeitschr. f. Fischerei u. deren Hilfwissenschaften, pp. 1506-167. 189دั.

7'. Gard, A. Sur l'éthologie de Sacculina carcini. C. R. Acad. Sciences, t. LXXIX, p. 241. Séance du 27 juillet 1874.

89. - De l'influence de l'éthologie de l'adulte sur l'ontogénie de Palomonetes varians Leach. C. R. de la Soc. de Biologie, $9^{\text {me }}$ sér., I, p: 326 , 1889 .

78. - Particularités de reproduction de certains Echinodermes en rapport avec l'éthologie de ces animaux. Bull. Scient. du Dép. du Nord, t. X, p. 296.

91. - Sur le bourgeonnement des larves d'Astellium spongiforme G. et sur la pæcilogonie chez les Ascidies composées. C. R. Acad. Sc., t. CXII, p. 301. Séance du 2 février.

92. - Nouvelles remarques sur la precilogonie. C. R. Acad. Sciences, t. GXIV, p. 1549 . Séance du 27 juin.

94. - Convergence et pocilogonie chez les Insectes. Ann. Soc. Entomol. de France, t. LXIII, pp. 128, 137. 1894.

98. - Sur l'éthologie de Campanularia calyculata Hinchis : Stolonisation et Allogonie. C. R. de la Soc. de Biologie, p. 17-20, 8 janv.

04. - Y a-t-il pxcilogonie saisomière chez Charaxes Jasiens. Bull. soc. entom. Fr., 10 facs. pp. 43-45. 1904.

96. Grassi. Ulteriori studi sullo Sviluppo dell'Anguilla e sul Grangi. Atti Accad. Lincei. Rend. (3), vol. 5̆, sem. I, p. 241.

96. Grassi et Calandruccio. Reproduction and metamorphoses of the common Eel (Anguilla vulgaris). Proceed. R. S., vol. LX, p. 260, Dec. 1896 et Q. J. Micr. Sc., p. 371. Nov. 1896.

71. Goosens, H. Des variations chez les chenilles. Ann. Soc. entom. Fr. 1871. Voir aussi sur le même sujet les observations de Mach Lachlan, Maurice Girard et J. Fallou, Ann. Soc. entom. Fr. 1867, p. 323 et suiv. Ces diverses publications ont été trop oubliées par les auteurs qui se sont occupés récemment de ces questions si importantes, et généralement si peu étudiées.

58. GuExéE. Monographie des Bombyx Européens du groupe de B. Quercus Ann. Soc. ent. Fr., pp. 43อ̈-442. 18308. 
04. Hargitt, Chas.-W. Notes on some Hydromedusce from the Bay of Naples. Mitth. Zool. Stat. zu Neapel, Bd. 16, p. 574 et 3777. 190\%.

98. Hencke, T. Naturgeschichte des Herings. Th. I, Text I. Hälfte, p. 20, fig. 1. 1898.

88. Hernck, F. H. The abbrev. melam. of Alpheus and its relation to the conditions of life; habils, color, variations etc. of Alpheus. J. Hopk. Univers. Circulars VII, no 63, pp. 34-35.

97. Hermons, R. Ueber den Nachweis der Viviparitüt bei Eintagefliegen. Zool. Anz., Nr. 533, XX, p. 205. 1897.

00. Hichson, S. J. Marine investigations in South Africa. Cape Town, p. 84 en note. 1900.

97. Hofer, Bruno. Welche Aussicht hat der Versuch zur Einbürgerung des Aales im Donangebiet? Allg. Fischereizeitschr. XXII. Jhrg., Nr. 23, p. 445ั-449. 1897. Analỹsé dans Zool. Centralbl. VI, no 3, p. 102.

98. Нuтн, E. Heteromericarpie und ähnliche Erscheinungen der Fruchthildung. Abhandl. u. Verträge aus d. Ges. d. Naturwiss. IV. 1895.

96. Імноғ. Biolog. Centralblatt XVI, p. 431.

04. Kammerer, Paul. Beitrag zur Erhentnis der Verwandtschaftsverhälnisse von Salamondra atra und maculosa. Arch. Entw. mech., Bd. XVII, Heft 2, p. 10\%, 1 Pl. 1904.

83. Kowalevsky, A. et Marion, A.-J. Documents pour l'histoire embryogénique des Alcyonaires. Ann. du Musée d'Hist. Nat. de Marseille, Zool., t. I, Mém. no 4 .

90. Lahille, F. Recherches sur les Tumiciers des Côtes de France. Toulouse 1890.

03. Lauterbons, R. Der Formenlireis von Anuraca cochlearis II. Tcil. Die cyclische aler temporale Variation von Anurva cochlearis. Verhandl. Naturhist. med.Ver. Heidelberg, N. F. Bd. 7, Hft. 4, p. 529-621. 1903.

04. Ludwig, H. Brutpflege bei Echinodermen. Aug. Weissmann's Festschrift. Zoologische Jahrbüicher, supplément VII, P. 683. 1904.

04. - Notiz über Brutptlege bei Echinodermen. Zoolog. Anzeiger, p. 423.

78. Manion, A.-J. Deux jour's de dragages dans le Golfe l'Alger. Rev. de Sc. Nat. de Montpellier, t. VII, $\mathrm{n}^{0}$ 2, p. 137-164, pl. 6, 7, 8 et 9.

87. Osten Sacken, R. von. On M. Portschinslit's publications on the larree of Muscide. Berlin. Entomolog. Zeitschr., Bd. XXXI, Heft I, pp. 17-28. 1887.

90. Rrden, J. A. The Sturgeon and Sturgeon industries of the eastern Cioast of the U.S. with an account of experiments bearing upon Sturgeon culture. Bull. of the U. S. Fish Commission, vol. VIII for 1888. Washington, p. 231-328, 1890. 
00. Sasipsor, Lilian V. Unusual modes of breeding and development among Anura. Amer. Nat., vol. 34, Sept. 1900, pp. 687-7.18̈. Ce mémoire contient une excellente bibliographie du sujet.

44. Sars, M. Mémoire sur le développement des Astéries. Ann. Sc. nat. (3), Zoologie II, p. 190, pl. 13 A.

37. Siebold, Th. von. Innere Beobachlungen über die Spermatozon der wirbellosen Thiere. Archiv f. Anat. und Phys. Jahrg. 1837.

04. Sunnotn, H. Nenere Arbeiten über die Morphologie und Biologie der Gastropoden: Zool. Centralbl., XI, p. 752. 1904.

99. Saurageau, C. Les Cutleriacées et leur alternance de génération. Ann. sc. Nat., sér. 8, vol. 10. déc. 1899.

76. Thonson, C. Wyville. Notice of some peculiarities in the mode of propagation of cerlain Echinoderms of the Southern Sea. Journ. Linn. Soc. Zool., vol. XIII, pp. 5̈s̆-79.

04. Trouessint, E.-L. Sur la coexistence de deux formes d'Hypopes dans une même espèce chez les Acariens du genre Trichotarsus. C. R. Soc. de Biol., t. LVI, 13 fasc., p. 234. 1904.

04. - Deuxième note sur les hypopes du genre Trichotarsus. C. R. Soc. de Biol., t. LVI, p. 363 . 27 févr. 1904.

00. Wiedensueim. Brutpflege bei niederen Wirbeltieren. Biol. Centralbl., Bd. 20,19011 .

01. Woltereck, R. Ueber awei Entwiclilungstypen der Polygordius Larve. Verhandl. d. V. Intern. Zoologen Congress zu Berlin, pp. 729-736. 1901. Voir aussi : Zoologica, Heft 34, Trochophoru-Studien I; 1902.

72. ZeLuen, E. Untersuchungen über die Entwicklung von Polystomum integervimum. Zeitschr. f. wiss. Zool., XXII. Bd. 1872.

76. - Weitere Beiträge zu Kemutnis der Polystomen. Zeitschr. f. wiss. Zoologie, XXVI, pp. 238-27k, pl. XVII et XVIII. 1876. 


\title{
Die Frage der Fortpflanzungsperiodizität des Palolowurmes im Lichte der allgemeinen Biologie der Chætopoden.
}

\author{
Von G. BRUNELLI und II. SCHOENER (Roma).
}

Die Frage der Fortpttanzungsperionlizitait des Palolowurmes hat zur Aufstellung nicht weniger Hypothesen Voranlassung gegroben, da solche oft die Vorbedingung für eine richtige I) eutung und Verwertung des volhandenen Tatsachenuaterials bilden, andererseits anch für die Bereichepung desisolben wertvolle Fingerzeigr gohen kömmen und esim Allgeneinen Aufwahe des Biologen ist, zwischen scheinhar vereinzelt dastehenden Firscheinungen das verkutipfende Band zu suchen. Es mögre uns deshall, aus dem spekulativen Verfiluren, das wir eingeschlagen, und dem Viele nicht allzuwohlwollend gegenüher stehen, kein Vorwurf gemacht werden, musomehr als. sritclem Friedränser dic Palolofrace fül "beim gegenwältigen Stande der Biologie vollkommen rätselliaft w erklärte, niemand behaupten kitun. dass in del Aufklärung dessellon bis zum heutigen Tage ein Schritt rorwärts getan worden sei. Wir hathen uns gefragt: Wird den Forderungen riner biologischen Erklärumgsmethode genügt. wemm man sich darauf beschränkt in der Botrachtmo einer Einzelercheimung die Ausbildung eines hochentwickelten In-tinktes verfolgen zu wollon, olne den Zusammenhang dieser Erscheinung mit der allgemeineren o)twanisation der Gruppe, welcher der betreffende Organismus angehört, im A uge zu behalten: ['nserer [oberzenewng nach liegt un allen hisherigen Hypothesen üher den Palolo dex gemeinsime. showerwiegendo Fehler zu Grunde. die Erscheinungen jenes Anueliden als einzigatige aufzufassen. ihnen ganz spezitische Lrsachen, wireine direlite Finwirkung von Lichtund Warmestrahlen, von Salggehalt. Druck und Temperatur des Walssers, von atmosphärischer Elektrizität u.s.w. unterzuschieben, und sie somit anc dom Zusammenhane mit dor Biologie nicht nur dor Annolidrun im Allgemeinen, sondern sellst du ihm nächststehenden filuppen und Gattungen zu reissen. Im Uebriger stützen sich unsere Vermutungen auf die wesentlichen hisher hekannten Tatsachen und heansprurehen nichts weiter als eine Nachprüfung auf Grund fernerer Beobachtungen und Versuche. 
Die Arbeiten üher die Regeneration der Ammeliden seit Boxwet (1745) his auf den heutigen Tag sind das Ergebnis zahlereher. weitgehender 1.ntersuchungen. die jedoch vom hiologischen Gesiehtspunkt aus einer gremeinsamen Orientierung ermangeln. Wir wollen hier nicht so sehr auf dir Frophnise dor künstlichen Verstümmelungen. welche bei jenen Tieren im Grunde nur ein starkes Regenerationsrermögen und einen heachtenswerten Einflus: des Nervenswitems in den Vorwängen der Regenepation splbst heweisen. als vielmehr auf die Betrachtung der in dep Natur spontan sich abspielenden Wriedererzeugungs- und Fortptlanzungserscheinungen das Hauptoewicht legen. Da der Palolowurm nach Eni.ERs pine ganz besontere Form der Epitolie aufweist und seine Periodizitait mit der Lostrennung eines hestimmten Teiles-des sou. epitolen Individuums - in Verbindung steht, können wir nicht umhin, zuerst auf die allgemeine Vatur der Fpitoliv und ihres Verhältnisses zur Schizogamie näher einzugehen. umsomehr als beim Palolowurm beide Formen sich gewissermassen repeinigt zeigen. Lm die hesonderen Erscheinungen des Paloln eventuell aus ihrer stammesgeschichtlichen Entwiclelung heraus beleuchten zu können, müssen wir ferner noch auf ihren Zusammenhang mit den allgemeineren Vorgingen der Autotomie und ungeschlechtlichen Propagation zurückliommen.

An der Wundstelle eines velwundeten Anneliden kann sich eine Gewebeknospe hilden. Welcher alsklann ein neues Schwanzstück seinen Lrsprung verdankt, so hei Autolytus smittie (Madancras und bei Podurie oldscura (Axurews): dieser Torgang kam an jeder heliebigen hörperstelle stattfiuden. ist bei Podarlie z. B. sehr häutig und tritt auch zufällig und unahhängig von der sogenannten Schizogamie bei den Hesioniden und bri den ()lignchaten (Axurews) auf. Wemn das Tier vollstandig in zwei Stücke zerbricht. kann eine einseitige Regeneration stattfinden, wie dies häufig bei künstlich’n Verstümmelungen der Fall ist, oder eine doppelte d. h. beiderseitige. sowohl der vorderen als der hinteren Extremi-

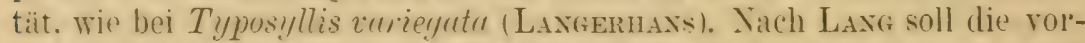
dere leichter rewenerieren als die hintere. Endlich zeigen uns, wie auch Perniter und Gravier elwähnem. mamnigfache Frscheinungen die un-

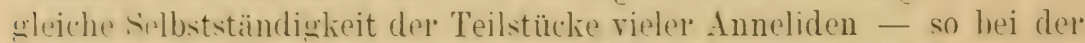
spontane'n, ungeschlechtlichen Teilung ('tenodritida'. Syllidar. Filugrama.

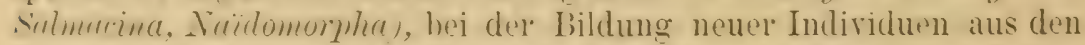
Trilun zulailig zerstuclielter (Lumbriculus. Euares) und bei der einfachen Regeneration ver'lorener Körperteile (Polychæten, Oligochæeten).

LANG und v. Kexiec (18SS) haben schon versucht, die Fihigkeit der musechlechtlichen Fortptlanzung von rinem hochanswehildeten Rege-

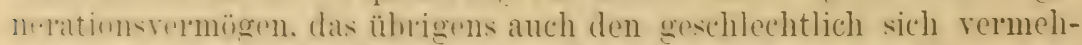

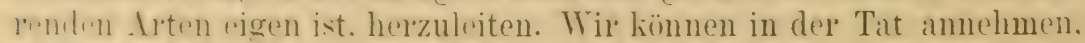
dik die liegenerationsel'scheinumgen zuerst rine rinfache Reaktion des 


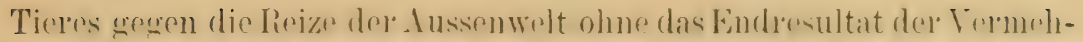
rung darstellen: was anfanges dop blosion Erlattung des Individumus diente, ist durch grarlweise Anpasinge dor Erhaltung der Alt zu Gute gekommen und dies auf zwei verschiedenen Wegen: auf dem einer einfachen, ungeschlechtlichen Schizogenese und einer mit geschlecht-

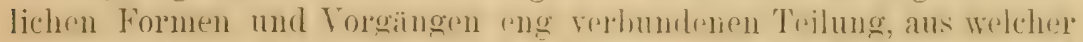

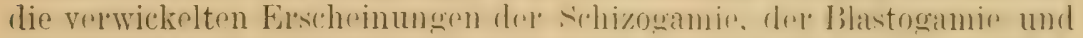

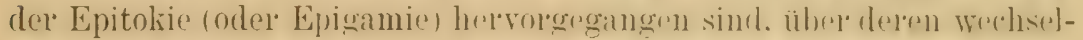
seitiges Verhältnis selı verschiedene Ansichten herrschen.

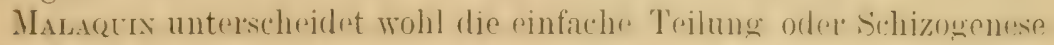

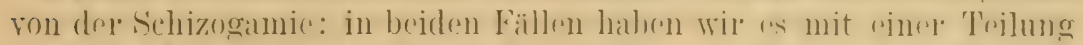

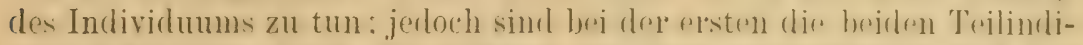

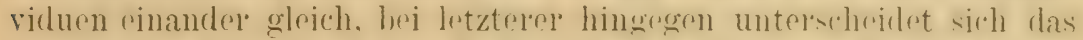
ahgetrennte Individum vom Muttertier, as enthält vom Zeitpunkt sminer 'Trenumg an die (irschlechtepondukte, hildet keine nemen ans und

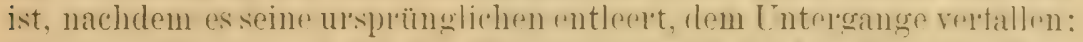

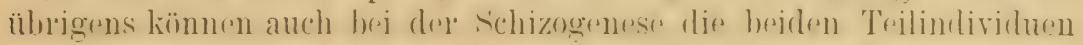
sich nicht nur durch abermalige einfache Teilung, sondern auch geschlechtlich fortphanzen. Kleinenbert, Eisig und Andere leiten die Schizogamie der Syllideen von dere Ephokie ab. In Falle der Blastogamio entstrht das geschlechtliche Individum nicht dureh einen Teilunesalit, wie hei der Schizoganie, sondern durch einen mit riner Kinospenbildung veloleichbaren Voroane: man kann sogal olie Blastogamio als rino lor-

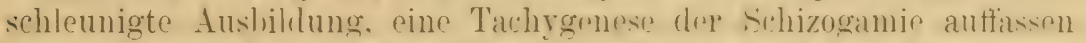

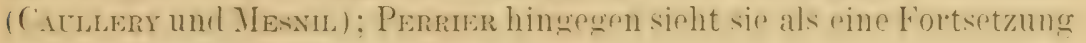
embryogenetischer Vorgänge an.

Bei der Epitokie endlich nimmt das ganze Indivirluum, nach MAIAQuin's Ausdruck, einen geschlechtlichen Charakter' an, d. h. deutlicher: man hat in derselben Art atoke (ungeschlechtliche) und epitoke (geschlechtliche) - ja zuweilen abermals unter einander verschiedene epi-

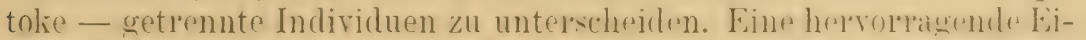

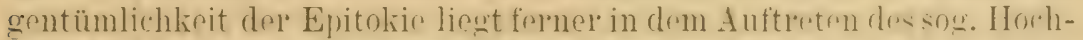
zeitskleides heim epitoken Individum. in dem z. L. die wohlhelianutru Formen der Gattung Heteronereïs prangen: hierbei ist gleich zu bemerken, dass mit der auffallenden Entwicklung der Paraporlirn und Bor-

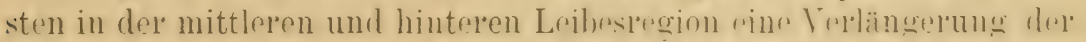
Fühler und L'mwandlung der Angen Hand in Hand geht : anch hilden dic Paraporlien einen dorsalen Ast aus. Teuredings hat Protor repsucht. d.n Zusammenhang zwischen der Kentenhildung der schizogamen Syllidern und der Epitoki dar\%ulegen: er stützt sich dabri anf die Entwiclilung

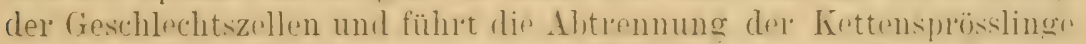
auf einen durell die Ansammlung jener Zellen herrorgerufenen Verren-

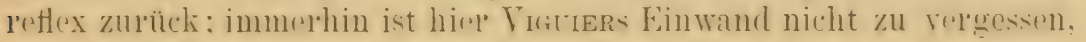


diass dieser Iroflex nur für die Fälle, in denen die Bruchtlïche ummittelbar vor das erste greschlechtsreife segment zu liegen kommt, eine befriedigende Eiklärung bietet, wïhrend die Tremnung sehr oft auch an anderen Stellen erfolgt (Claparìe, Ehlers, de Saint-Joseph, Malaquin, Viguier). Viguier macht überdies noch darauf aufmerksam, dass die hette vou surösslingen mit dem Muttertier vermittelst eines sehwachen sticles zusimmmenhängt, der erst allmählig durch die Bewegungen der

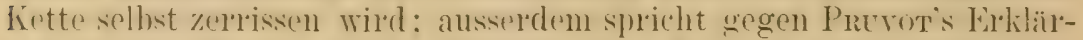
mug dir yon P'ERmen betonte Tatsache, dass hettenhildung auch hei den Naidomorphen S'iusswasserlumbricinen vorkommt, die keine Epitokie aufweisen: endlich ist zu hemerken. dass (nach Vigraer) Lavaernass die

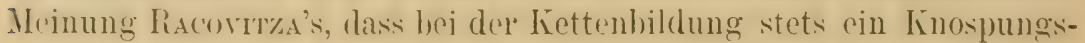
vorgang im Spiele sei, nicht bestätigt hat.

Zur Begründung des stammesgeschichtlichen Zusammenhanges. den wir zwischen Schizogamie, Blastogamie und Eppitokie annehmen möchten, dient uns der Lmstand. dass wir diese drei Fortptlanzungsformen in den verschiodenen Amolidengruppen sellst innerhalh der (irenzen ciner einzigen Art auf die rerschiedenste Wroise mit einander verlinüpft finden. Es gibt Fälle, in denen nur Schizogamie (Syllina) - andere, in denen nur Blastogamie herrscht (Autolytina), wieder andere in denen I3hastogamie mit Shehogamie vereint vorkommt (Autolytus): nach dem von Eisig beobachteten Falle des Notomastus (Clistomastus) scheint Schizogamie auch bei den Capitelliden vorzukommen.

Die Epitokie tritt in ihrer typischen Gestalt bei den Nereïden auf, bei denen zum ersten Male die von EHLens als "epitoke» von Chaparìde als " epigame " bezeichnete Form wahrgenommen und als "Heteronereis" beschrieben wurde; sie kommt ausser bei den Nereïden auch bei den Eunicidm, Sylliten, Hesioniden. Phyllodociden, dilyeeriden und hei einem Cirratuliden (Dodecaceria concharum) vor. Auch sie kann entweder ausschliesslich herrschen (Exogonine, viele Hesionidenarten, Nereidx, Phyllodocidie), in anderen Fällen zur Blastogamie oder zur Sichizogamie ofler gar zu heiden zusammen hinzutreten futolytus lon(yeferiens): hei Erongue gemmifern ist die häutigste Fortpflanzungsweise die Epitokie, welche in dieser Art die einzig bekannte war, bis MaLAgets in derselluen anch das Vorkommen von sehizogamie nachwies. Ier Umstand, dass bei Autolytus longeferiens zur gewohnheitsgemässen Shlizogamie zuweilen Epitokie und umgekehrt zur Epitokie von Eitogrone yemmiferen zuwrilen sehizogamir hinzutritt, hestirlit uns in dem cidanlien, dass hoide Fortptlanzungsformen in imnigem Zusammenhang strhen. Wir wissen. dass bej dere Kettenlbildung zuweilen die Sprösslinge cinen eigenen liopf besitzen, wie bei Autolytus; in anderen Fällen hin-

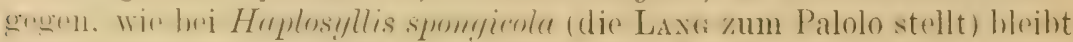
der losgetrennte, geschlechtliche Teil ohne Kopf; wenn wir nun, wie 
Perier bemerkt, annehmen, dass der sozusagen epitoke, nïmlich der kopflose Teil sich nicht mehr vom ungeschlechtlichen trennt, so sind wil von der Schizogamie zur Epitokie übergegangen, d. h. diese wüıde als ein Fall von Schizogamie mit Bildung eines einzigen, kopflosen Sproisslings und unterhliebener Lostremung des letzteren vom Inttertier zu betrachten sein. Untel diesem Gesichtspunkt möchten wir als-

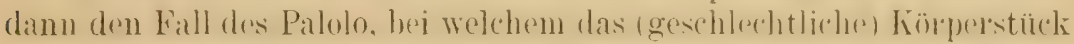
sich rom ungeschlechtlichen lostrennt als "Schizoepigamie ) (resp. -epitokie) bezeichnen; wir berufen uns hierbei auf EHLER's Worte : "Die Ausbildung des Palolo stellt eine ron allen bisher bekannten Fol'men abweichende Form der Epitokie dar, denn in der A r't tritt eine atoke und epitoke Form, am Individ u um eine atoke und epitoke Förperstrecke auf ${ }^{\natural}$. »)

Schizoganie, Blastogamir und Epitokir strllen offenhall höhere Ent-

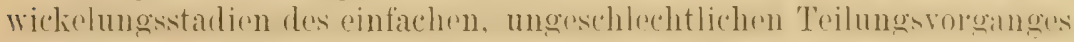
dar: diesere steht in engstem Zusammenhang mit eiuen starken lirgonerationsvermögen, das seinerseits durch oft und lange wiederholte Verstümmelungen hervorgerufen und stetig erhöht worden ist. Schon Andere, wie Lang und von Kénnes, haben die Fortptlanzung durch 'T'eilung und Knospung mit den Erscheinungen der Autotomie in Zusammenhang gebracht. Graro gebührt vor' allem das Verdienst, den Begriff der Autotomic bedeutend weiter aufgefasst zu haben, als sein Vorgänger, wie RigGenbach bemerlit; überdies giebt Giard (Controver'ses transformistes, Paris 1904) mit Recht seiner Verwunderung dar'iber Ausdruck,

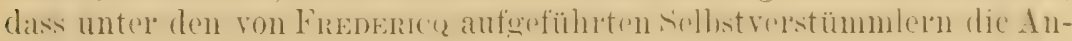
neliden fehlen. Sowohl Fredericr. del: zuerst die Aufmerksamkeit auf die Autotomie gelenkt hat, als auch von Kexwes und viele Andere sind der Meinung, dass der früheste Ursprung der Autotomie in den Reizwirkungen der Aussenwelt zu suchen sei, und dass die Vorgänge der Selbstverstümmelung auf dem Wege der Anpassung sich himreichend vervolikommnet haben, um auch ohne Wiederholung jener Reize auftreten zu können. RigaEnBach ordnet die zahlreichen Fälle spontanel Verstümmelung, die sich bei den Anneliden beobachten lassen in drei Gruppen: "In Verstümmelungen die in keiner Beziehung zu der Velmehrung stehen, in Verstümmelungen, die zur ungeschlechtlichen Propagation, zur Teilumg führen, und in Verstümmelungen, die als T'eilelscheinungen der sexuellen Fortpflanzung zu betrachten sind. » Diesen

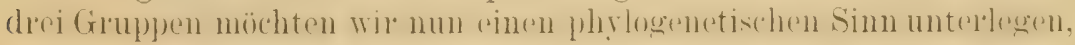
insofern, als der Organismus anfangs die Reize der Aussenwelt rein

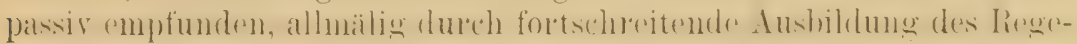
nerationsvermögens beantwortet hat und auf Grund des letzteren zu

${ }^{1}$ Ueherdies treten nach EHLERs beim Palolo noch Individuen auf, die als Uebergangsformen zwischen den atoken und den sog. epitoken Individuen dastehen. 
jenel Autotomie gestheitten ist, die nur gradweise eine sozusagen freiwilligr werdon konnte, und deren Tresurung folglich nicht wie Frenenere annimmt. in Scelbsterhaltungstrieb liegen kann. Im besonderen Falle der Anneliden mö̈hton wir nun für die Entstehungsgeschichte der Fortptianzungsantotomic auf dic Einwirkungen del anorgan ischen Welt den weitaus grössten Wert legen.

Aus der Kohlen-Jura- und Kreideformation sind uns Abdrücke ausgestorbener Nereildenarten erhalten, die an Grösse, kaum hinter den lentigrn zurüclistrhen. Auf die Frage nach der Entstehung einer hedentenden hiegnentzahl kïnnte man antworten, dass ein Organismus, der ühey rine gewisse Art und Weise des Wachstums rerfügt, dieselle so) langer ausnutyt. bis diu Aussenwelt das verlorene Gleichgewicht wieder herastellen sich bestreht und ihn durch noue Einwirliungen in die üherschritt(men I)imensionsgrenzen zurückweist. Dies wurde das Fnd-

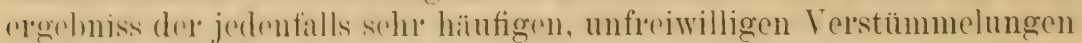

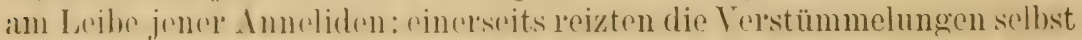

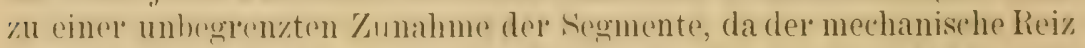
als trophischere wirkt ${ }^{1}$, andersoits führten sie bei manchen Arten auch zur uneseschlechtlichen 'Teilung, da der lange und häufig ron aussen her wirkende Verstümmelungsirejz srine allnälige Reantwortung im inneren Vorventerz find, auf dem dor Automatisums des Teilumgsprocesses beruht.

Nun ist zu heachten, dass oinige jotzt lebende Annolidenfolmen, bei wolehen sich rinfarhe Trilums odere deren höhere, verwicheltere l'hasen zoigen, oluenfalls oine sehre grosse segmentenzahl aufweisen: einige Syl-

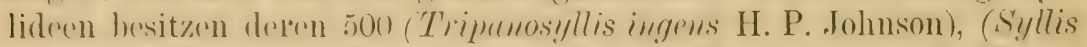
longissma (Bravior). oinge Arten der (Battung Eumice bis zu 900 (Eunire Fimberyi Ghlops). Wir wissen ausserem. dass nur wenige Polychaten.

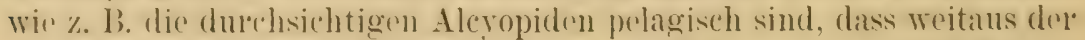
erösste Teil diesere T'iere die Küusten und zwall felsige odel steinige Küusten bevoryut, wo die Reibung der anbramdenden Wogen, der mitbewegten (iestrinsfragmente, das steigen und Fallen der Flut in hohem . Maasse eine perstimmelnde. Wirkung ausüben mussten. Fs liegt auf der Hand, dass

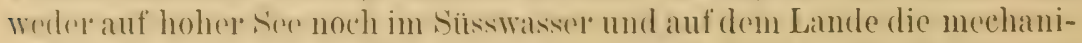

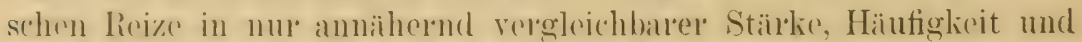
Mannigfaltigleit wirksan sein können wie vornehmlich im folsigen

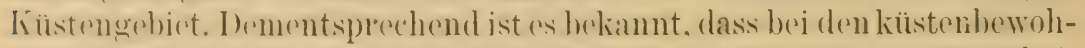
menden marinen chatopoden die verwickielsten Fortptlanzungserscheimungen auftreten, dass diese hei den süsswasserformen sehr reducirt sind

1 Wir erinnern an das von GIARD anerkannte Gesetz ron Lessosa, nach dem die Ausbildung des Regenerationsrermögens der Häutigkeit des die Regeneration herausfordernden Reizes proportional ist. 


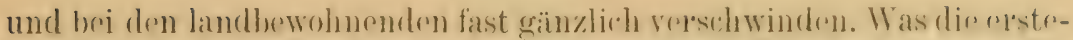

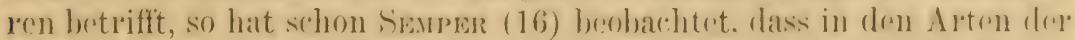

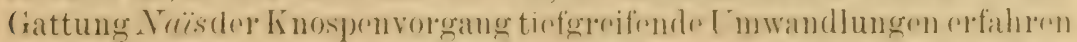
lat; wir glauben auch desshalb nicht die Knospenbildungen von Lambriculus, Nräs, Cheretogrester, - wir linasisbarn hei Ausfïhrung gewissel vor Kexwer'scher Gedanken es thut, - als Zwischenstufen in der

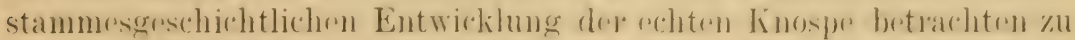

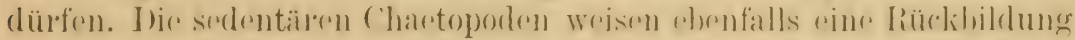
der Fortpflanzungserscheinungen auf: es ist dies von Sars und Oscai Schmot (Filograna), von Huxley (Protula), von Ciaramède (Salmacina)

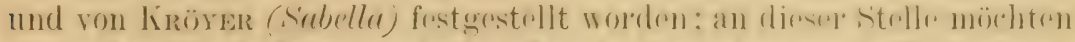
wir zudem die Anfmerksamkeit auf eine sehe interesante Enteleckung

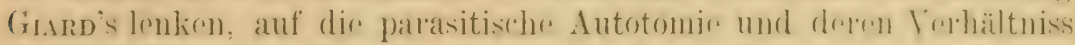
zur reproductiven Autotomie der Anneliden: danach sollen gewisse

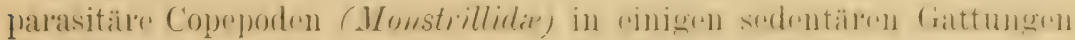

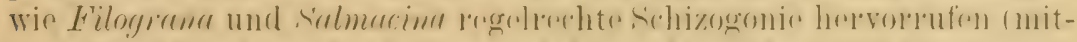

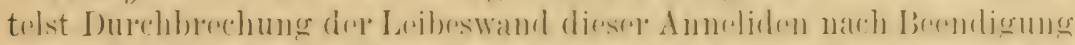

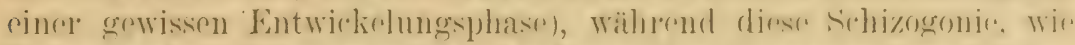

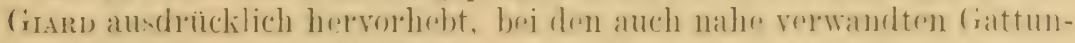
gen nicht beobachtet worden ist.

Der Umstand, dass die pseudo-sedentären d. h. riff'bewohnenden (nicht eigene Röhren bewohmendeni), Polychaeten verwickeltere, viel-

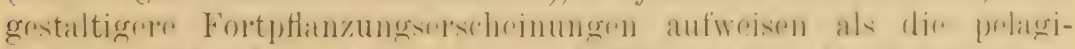

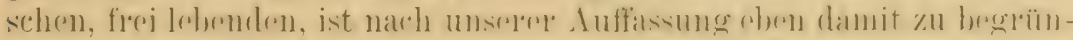
den, dass die grade im unmittelbaren Bereich der wassel'umspülten

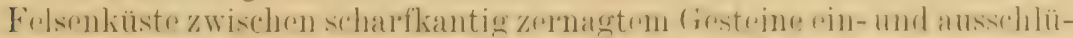

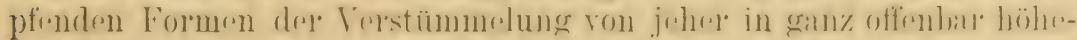

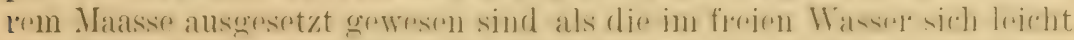

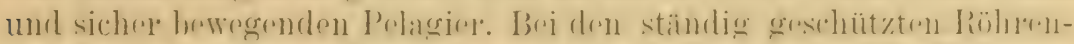

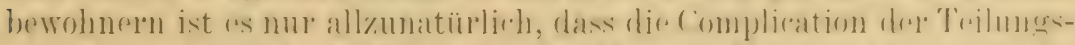

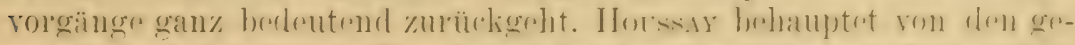

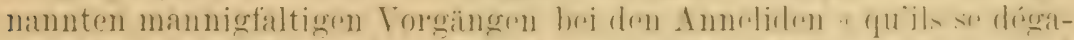

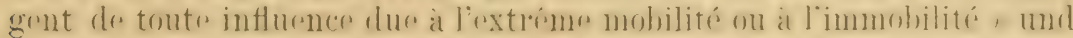
stützt sich hierbei auf das Verhalten von Dodecuceria concharum Oerst.

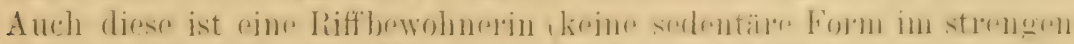

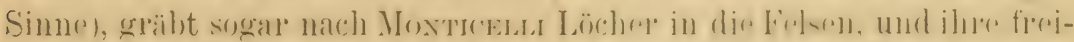

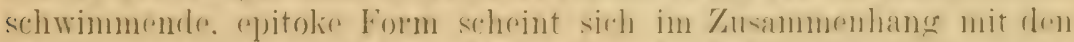

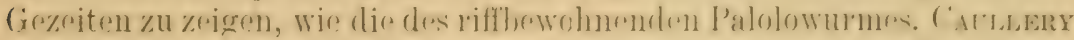

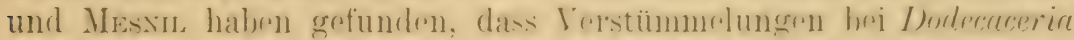

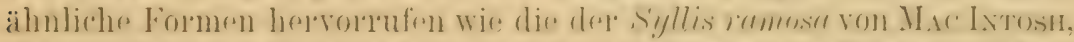

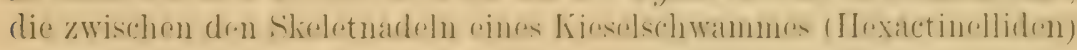
lebend vielleicht von diesen ihre Wundreize empfängt (Viguner).

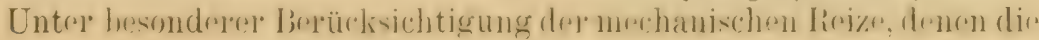


rifflewohnenden ('hactoporlen in hesonderem Daasse ausgesetzt sind, möchten wir nun mit IIülfe einiger specieller Betrachtungen über Epitokie eine mehr allgemeingültige Errklälung der Fortptlanzungsperiodicitit des Palolowurmes sowie ihm verwandter Alten zu geben versuchen:

Der Palolowurm (Eunice viridis Gr.) ist eine nach EnLERs der Eunice siciliensis Gr. verwandte Eunicide und lebt längs den Küsten der Samoa-Fiji-Tongat- und Gilbertinseln in Lörhern und Tiöhren der Poritrsfelsen ("Palolosteine ) nach Krräser). Die Abweichung seiner Fortjiflanzungsormen von denen der typischen Epitokie ist unter dem Namen "Schizorpitolice "hereits erwilhnt worden. Die sog. epitolien Segmente am sehizon)itoken Individum unterschejilen sich von den anderen num durch die weringere Breite und grösire Länge und dadurch, dass sie - nach EhLers - mit sog. "Bauchaugen » (kreisrunden, dunkeln, ventralen Flecken) versehen sind. Zwei Mal im Jahre und zwar in den Monaten Oktober und November steigt die epitoke losgetrennte hintere Körpperstrecke ( ( Palolo ) im strengen Sinne) in mächtigen Schaaren an

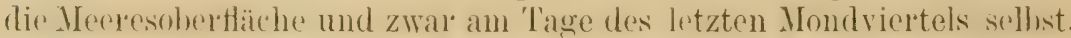

Die zur Erkläıung dieser und ähnlicher auffälliger Erscheinungen aufgestellten Hypothesen und Analogieen sind im Wesentlichen folgende:

1. Wirkung der Sonnenstrahlung (insbesondere Wärmestrahlung).

2. „) des Mondlichtes.

3. Negativer Geotiopismus.

4. Wechsel der Temperatur und des Salzgehaltes des Wasser's (bei Formen, die im Grenzgebiet zwischen Salz- und Süsswasser - z. B. nächst den Flussmündungen - leben).

5. Wechsel des Wasserdruckes bei Ebbe und Flut.

6. Analogie mit der Blüteperiodicität im Pflanzenreich.

7. „ " cinigen physiologischen und pathologischen Erscheinungen im Gebiete der übrigen 'Tierwelt.

S. Atavismus.

9. Einwirkung der Mondphasen auf den elektrischen Zustand der Atmosphäre.

Dagegen wäle einzuwenden :

1. Dass nach Frieduänders Beobachtungen die Palōloschwäıme sich oft schon lange vor Sonnenaufgang -

2. Auch in völlig mondscheinlosen Nächten zeigen -

3. Dass Geotropismus in keinem erwiesenen Zusammenhang mit der Mondesperiodicität steht -

4. und 5. Dass Versuche über das Auftreten der Epitokie beim Palolo 
und verwandten Formen (nach OSAwA) ausserhalb ihrer natülichen Umgebung, in cinem (iofïss mit Wassel dessen 'Temperatur, Salzowalt und Druck nach Belieben verändert werden konnte, zu keinem befriedigenden Ergebniss geführt haben.

6. Was die Analogie mit der Blüteperiodicität betrifft, welche FrIEDLÄNDER mit Bezugnahme auf eine Angabe von Sommper andeutet, nach welcher das Aufblühen riner Pflanzenart in selu auswedehnten (rehietren am gleichen 'Tage einsetzt, möchten wir' daran erinnern, dass für die Pflanzen - aber auch nur für diese — die Existenz bestimmter Wärmeconstanten für verschiedene ihrer phrsiologischen Esscheinumgen wic für die Entfaltung der Blätter, der ersten Blüten, das Reifen del Früchte, das Abfallen der Blätter nachgewiesen ist - derartige, eine strenge Poriodicitat bedingende Wärmeconstanten ontspringen den innigen, ganz eigenartigen Band, welches das Pflanzenleben an die Sonnenstrahlung fesselt und haben nur für dieses bewiesene und entscheidende Bedeutung. Wollten wir uns von der blossen äusseren Erscheinung trügen lassen, so würde die alte "Flora-Uhr"), nach welcher" bestimmte Blüten gar zu bestimmten Stunden des Tages sich öffinen und schliessen, zu noch grösserer. Verwunderung berechtigen.

7. Kann und will die Analogie mit del Periodicität einiger pliysiologischer und pathologischer Erscheinungen im Thiresich natülich keine Erkläl'ung für die hiel speciell betrachteten Fälle bieten; sie deutet unserer Ansicht nach jedoch an, dass diese in die Reihe derjenigen Erscheinungen gehören, deren regelnuässige Wiederliehr in mmittrlharem Zusammenhang mit der Physiologie des Nervensystems steht und auf diese Abhängigkeit, die uns im Gebiete der Biologie von grosser Bedeutung erscheint, möchten wir am Schlusse zurücklkommen.

S. Erklären das Wort "A tavismus " oder ihm gleichbedeutende Ausdrücke in solcher Allgemeinheit recht wenig; der Gedanke an Atavis-

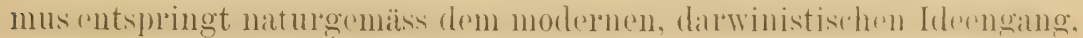
aber wo auch nicht entfernt angedeutet wird, worin dieser Atavismus bestehen könnte und wo der Palolo gleichsam als Unicum in Betracht gezogen wird, kann von rinrm Erklitungsversuche im biologischen sinne kaum die Rerle sein.

9. Kommen wir auf Armuenius' Hypothese, welcher im Verein mit Eкwoux entdeckt haben soll, dass der Mond einen Einfluss auf den elektrischen Zustand der Atmósphär $r^{\circ}$ ausübt. Nach Arrhenius würde demnach der Mond nicht nur auf dem Wege der Gravitation und Beleuchtung, sondern auch auf dem Wege der atmosphärischen Elektricität periodische Reize auszuüben im Stande sein. Arrhensus gibt zu, dass keine Brobachtungen ühere don (iang dere Luftelelitricitit in den Palologegrilden vorliegen, sagt aber": "Es ist wahrscheinlich, dass die Luftelektricität da ein Maximum in del Nähe der Zeit besitzt, wenn der Mond am 
tiefsten unter dem Horizont steht. » Dagegen lassen sich schon mach

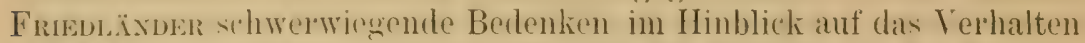
dos Atlantischen Palolo rrhehen. für den die Verhältnisse der Mondesdeklination umgekehrt liegen sollen wie für den Pacifischen.

Um nun die Erscheinungen bei einigen anderen Anneliden, die eine ähnliche Provodicitit aufweisen wio der Palolo und deren Entrdeckung wir nebst anderen ' OsAwA und A. G. MAYER, verdanken, etwas näher zu beleuchten und einige verallgemeinernde Schlüsse daraus ziehen zu könmen, lassen wir eine kurze Beschreibung derselben folgen :

Osawa berichtet in den Verhandlungen des V. Internat. zoolog. Congresses zu Berlin (1901) über zwei von ihm in den japanischen Meeren beobachtete Anneliden u. a. folgendes:

Beide Arten leben im Küstenschlamm. Eine ist nach Dr. A. Jzuka Nereis versicolor, die andere, im Volksmund "Itome », ist von demselben Cerato-

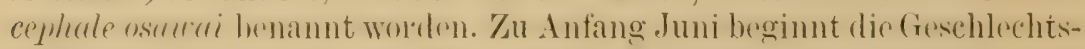
l'eife der" "Itome » und das Tiel" wächst bis Oktober auf $12-20 \mathrm{~cm}$. Länge aus. Dic Epitokir scheint im septrmber zu beginnen, im Olitober bis November beendet zu sein. Der viel breitere vordere Teil, "Batzi » genannt, strigt in der 2. Hälfte Olitoher und in der 1. Hälite Norember. am 1. oder 2. Tage nach Neu-oder Vollmond (1-4 Tage nach Neu- und Vollmond nach A. JzukA), Abends zwischen 6 und 7 Uhr, wenn die Abendflut wirdere zu fallen breinnt, in diehten Schaaren an die Meresobertlitehe. Sie zeigen sich nicht bei 'l'age. Von dem Palolo unterscheidet sich der' "Batzi") vornehmlich dadurch, dass er den vorderen, Palolo hingegen den hinteren Mbsehnitt am noch ungetrilten Annelisen darstellt: zweitens dass batzi rine lalb), Pillolo rine ganzmonatlieho Fortptlanzungsperiode hat: drittens dass Batzi nach den Springfluten, Palolo in den Nippzeiten erscheint ${ }^{2}$.

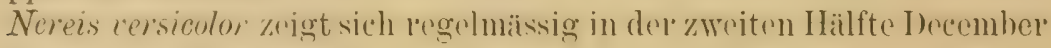
und ersten Hälfte danuar.

Finen dritten Anmeliden (Eumice fucutr, Ehlers der sich zur Zeit der

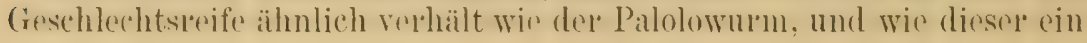

'Siche in Woodworth's Bericht: Powell, Codrington, Brown, Seeman, Rummurs und CoLis über ähnliche Erscheinungen bei den Banksinseln, Neu-Irland, Molukken und den Neu-Hebriten.

${ }^{2} \mathrm{Zu}$ bemerken ist besouders, dass am schwärmenden " Batzi " oft noch die hintere Körperstrecke hängen bleibt, was damit zu erklären wäre, dass sich bei dem im Uferschlamm lebenden Ceratocephale die mechanische Wirkung der Gezeiten weniger scharf fühlbar macht als beim Pacifischen und Atlantischen Palolo. A. JzukA weist auf einen bemerkenswerten Parallelismus zwischen dem Erscheinen des dichtesten Schwarmes und der höchsten Springzeit wïhrend der betreffenden Monate hin. 


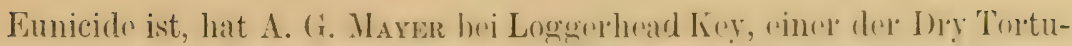
gas-Inseln hei Florida, cnterelit: "Fhenfalls zur Zoit des letztrin Mondviertels ${ }^{1}$ beobachtet man (nach RigGeNisACH), wie der Wurm lange vol Sonnenaufgang an dere Merersobertliche rescheint. Wem das Licht der. Morgensonne den Wasserspiegen bescheint, so wereden die reifon filinder des Wurmes von heftigen liontraktionen lufallen. Dir Leibeswand reisst. Sperma und Eier treten durch weite Risse aus. Manchmal reissen die

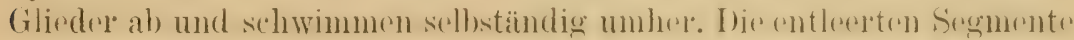
sind äusserst br üchig; wenn sie nur mit der Hand berührt werden, zerfallen sie sofort. Nach dem Schwäl'men sinken die Wüumer wieder zu Boden ».

Endlich möchten wir an dieser Stelle eine Nereïde nicht uner'wähnt lassen, die ein italienischer Marinearzt, Dr. R. Marantonio, im vergan-

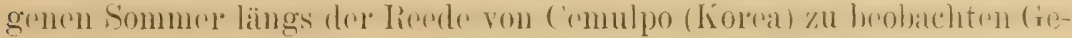
legenheit hatte, und von der er uns die wenigen mitgebrachten Exemplare freundlichst zur Vorfügumg stellte. Aus dro bergerügten Aufzerchnungen hiologischen Inhaltes ist es resiehtlich, dass diese Form für dio

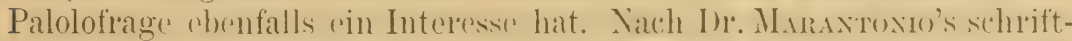
lichen Angaben wurden die betreffonden Fixemplare (10. Angust 190:3) aus rinem ansehnliche'n Schwarm ïhnlicher', dureh dic ehen andringende

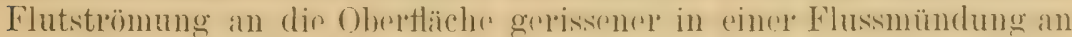

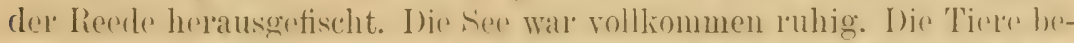
wegten sich mit der grössten Lublnatigheit mol lïmpften mit Erfolg wogen die Strömung an. In einem Gefäss mit Seewasser, in welches sie gebracht wurden, verendeten sie nach 2-3 Stunden, nachdem sie sich un-

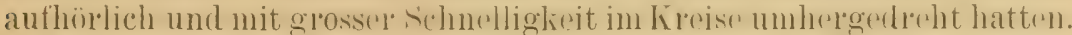

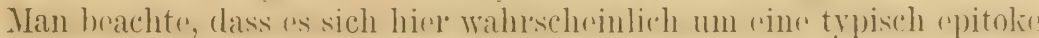

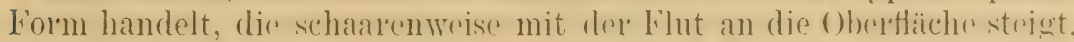

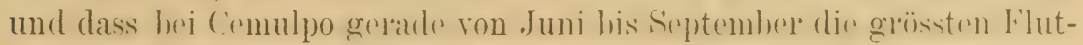
höhen des Jahres herrschen ${ }^{2}$.

Was den Palolo betrifft, so ist man früher der Ansicht gewesen, er steige aus den Tiefen des Meeres empor - der veraltete Ausdruck

${ }^{1}$ Im Juni und Juli nach A. G. Mayer.

${ }^{2}$ Ganz kürzlich berichtet MAc InTosh über weitere interessante Fälle des Schwärmens bei verschiedenen anderen Anneliden, nämlich bei Nereis Dumerilii And. et Ed. in der Castlebay und bei Nereis longissima, deren ein Schwarm 1865 von Hearder im Plymouth Sound an der Meeresoberfläche beobachtet und auf Millionen von Individuen geschätzt worden ist. Aenliche Beobachtungen liegen nach MIAC InTosH ron VerriL an Nectonereis megalops vor; ebenso an Nereis irritabilis Webster, welche im August an der virginischen Küste schwärmt.

Ferner über den "Wawo" (Lysidice sp.) von Amboyna siehe R. Honst, Nature, vol. 69 (1904), p. 582 . 


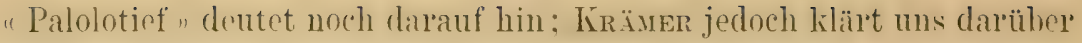
aut. dass die Portifelsen oder -bruchstüclie (Palolosteine) in deren Höhlungern der Palolowurm zu schlüpfen pthegt, sich auf den Riften so nahe unter der Wassertläche befinden, dass man sie zur Ebbezeit mit den Händen greifen kann und sie oft auch trocken liegen. Woopwortu bestitigt dius eingehend: "Dies (l etztes Mondviertel ()ktober und Novenber) ist die Zeit der niedrigsten oder Springzeit, wenn das Rifflach an seichten Plätzen unberleckt ist orler nur bei Niedrigwasse $r^{\circ}$ gewaschen wird.

Aus der Betrachtung aller dieser Fälle geht in erster Linie die Bedeutumg der Grapiton als bestimmenden Factors dor Fortphtanzungsperiodicität dieser 'Tiere hervor, welche in den verschiedenen Gegenden höchst wahrscheinlich in Folge hesonderer, localer, mareographischer Verhailtnisse wehselt: anderseits zeigt sich bei diesen Tieren eine groske Zerbrechlichkeit d. h. ein hochentwickeltes Selbstzerstückelungsvermögen, das sich, wie bereits Andere hervorgehoben, auch ohne dass der äussere Reiz, aus dem es hervol'gegangen, in seiner ursprünglichen Intensität wirksam sei - wir möchten sagen in der durch eine auch leichte Andeutung zu gelegener Zeit erweckbaren Erinnerung an die Wirkungen jener Intensität betätigen kann, wie es bei vielen Instinkten geschieht. Als solcher " erinnernder Reiz » tritt heute für viele liüstenhewohnende Anneliden das Steigen und Fallen der Flut auf, sio löst die Lostremnung dere epitoken Kïnperstrecke am schizoepitolen Individum ans (Batzi, Palolo) oder verulsacht das Emporsteigen des ganzen, typisch epitoken Individuums an die Oberfiäche des Meeres; in beiden Fällen ruft sie die eine oder das andere mittelst der von der Küste abdrängenden Gezeitenströmungen zu einer Periode atavischer Lebensweise ins freie Wasser hinaus und in beiden Fällen wird dazu ein atavisches, d. h. pelagisches (dem normalen Kleide der rein pelagischen Formen ähnliches) Hochzeitskleid angelegt.

Als höchster Grad der Emancipation won den Reizen der Aussenwelt gelten uns alsdam gewisse Erscheinungsformen der Epitokie, bei welchen selbst die den Fortptlanzungsmechanismus a uslösenden Ursachen der Aussenwelt entruickt zu sein und ausschliesslich dem Oreanismus innezuwohnen scheinen.

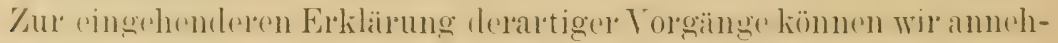
men, dass während der bedeutenden morphologischen Umwandlungen,

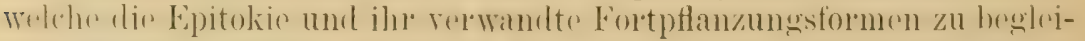
ten pHlegen, das Nervensystem im Zusammenhang mit der Reife der Ge-

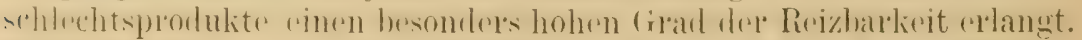
Wron wil num zugeben, dass die Epitolie ihren äusseren Anstoss in dem mechanischen Wirken der Gezeiten hat und dies natülich innerhalb

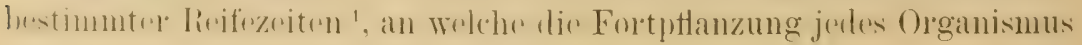




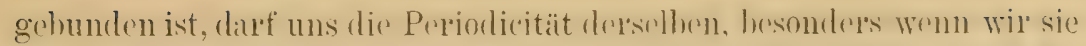

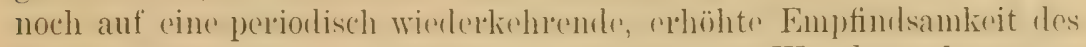
Nelvensystems mit zurückführen nicht allzuseh' Wunder nehmen; es ist wohl vorauszusehen, dass ähnliche Erscheinungen mit der Zeit bei einer stets wachsenden Zahl von Annelidenarten auftauchen werden; schon bei den wenigen helannten Fällen hat sich rine, wiegesagt, hö̈hst wahrscheinlich an locale Neeres- und Küustenverhältnisse gebundene Verschiebung der Epitokiezeiten gezeigt, und wer weiss, ob auch deren

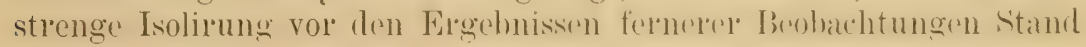
halten wird.

Werfen wir zuallerletzt, um die angedeutete periodische Zunahme ner-

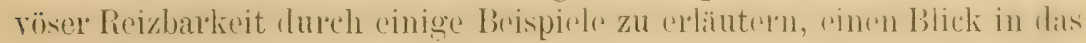
(iebiet dere Psychologie und Pathologie, in welchem sich spercioll nervöse

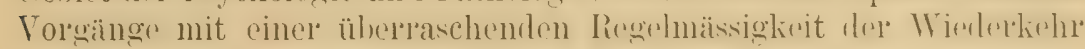
abspielen; wir halten natürlich daran fest, dass diese keine irgendwie

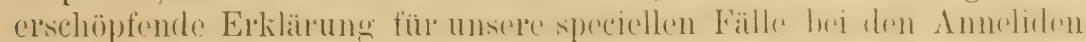

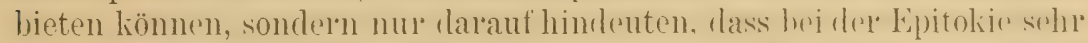
wohl eine sexuelle Nerven kr is is im Spiele sein kann. Ein ganz neues und sehr interessantes Werk von H. Swoboda "Die Perioden des mensch-

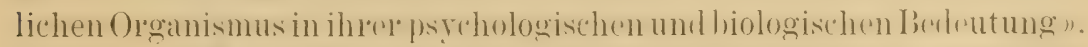

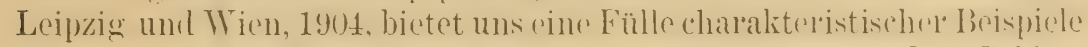
derartiger, periodisch wiederkehrender Nervenkr'isen aus dem Gebiete der menschlichen Physiologie.

Der Organismus lebt, nach SwoвоDA, " in Wellenbewegungen ». So sehr man über die Auslegung dieser sicher nicht leicht zu elklälenden Vorgänge zu streiten berechtigt ist, hat nach unserem Dafürhalten der Biologe schon ein Interesse daran, die blosse Tatsache festzu-

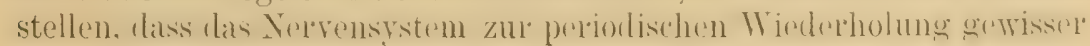
Lebensäusserungen neigt, nach welchen im Organismus eine latente Orientirung vorliegt. Man möge es uns darum nicht verargen, wenn wir in der unbefangenen Absicht, die Frage des Palolo in das Gebiet allgemein biologischer Betrachtungen zu ziehen, an scheinhat lin'mlingendes gestreift haben.

Zuscmmenfussend: S'chizogamie, Ilastoganio umul bipitolio stammen

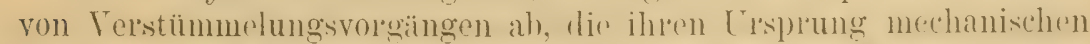

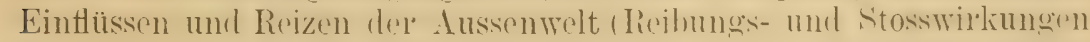

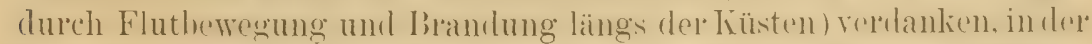

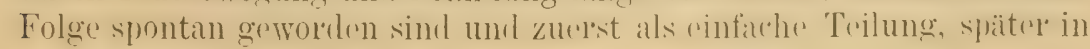

1 Siehe Woodworth: " Diese Frühlingszeit (Oktober und November) ist bekannt als die Zeit der Reife und sexulen Lehendigkeit auf den ganzen Pacifischen Inseln»- «dies gilt im Allgemeinen auch für die übrige Rifffauna » u. s. w. 
Grstalt der genannten verwickelteren Fortphtanzungsformen der Terbreitung ller Alt sich angepasst haben. Derartige im grössten Maasstahe del anorganisehen Welt entspringende Reize haben vorwiegend die pseudoserlentïren Küustenlıwohner betroffen. Beim Palolo und den ihm biologisch verwandten Anneliden erinnert rler periodische, mechanische Einfluss der Gezeiten an einen mechanischen Reiz, der in ihrer Stammesgeschichte oine hervorragende Rolle gespielt hat. Das Nervensystem velfiillt vinele durch die (ieschlechtspoife und -motalolie physiologisch begründharen Fytokiekrise und hewirkt das Freiwerden der epitoken Form (sei es als 'Teilstück orler als Indiviluum). So ist es verständlich, wie die Fortptlanzungsperioden einerseits von der Reife der Geschlechtsproducte, andererseits vom Gange bestimmter Gezeiten abhängig sein kïnnen, und wio siorlementsprechend für verschiedene Arten von einander abweichen ja vielleicht für (lie gleiche Art an velschierlenen Orten verschieden sind aher stets mit hestimmten Phasen des Monrles ihren ursprünglichen Zusammenhang noch bewahlen.

So erscheint es uns möglich, aus allgemein biologischen Betrachtungen heraus ciniges Licht auf die seltsamen Erscheinumgen auch des Palolowurmes zu werfen, die sich - aus jedem derartigen Zusammonhang gerissen - in den geheimnissrollen Schleier der Eingeborenenlegende hüllen.

\section{LITTERATURVERZEICHNIS :}

Andnews, E. A. Some abnormal A mnelides, Quart. Journ. of Micr. Sc. 1894.

Arruenus, S. Die Eimwirkung liosmischer Einflüsse auf physiologische Verhälnisse. Skand. Arch. f. Physiol., Bd. 8, S. 367. 1898.

BonNet, C. Traité d'Insectologie ou obserciations sur quelques espèces de Ver's d'eau donce qui, coupés en morceaux, deviennent autrant d'animaux. complets. Oeuvres d'Histoire naturelle et de Philosophie de Charles BoNver. Neufchatel, 174 .

Camerano, L. La vita di M. Lessona. Acad. R. di Torino. 1896.

Caullerr, M. et Mesnil, F. Sar un cas de ramification chez une Amélide (Dodecaceria concharum Oerst.). Zool. Anz., Bd. 20. 1897.

Clapanède, E. Les Amnélides Chélopodes du Golfe de Naples. Mém. de l’Inst. Genevois. 1869.

Colurix, A. Ueber den Palolowurm. Anhang zu A. Kräser. Ueber den Bau der Korallemiffe. Kiel u. Leipzig, 1897.

De Sant-Josepri. Les Amuelides polychètes des côtes de Dinard. Ann. des Sc. nat. $189 \%$. 
Ehlens, E. Die Borstenwürmer. 1864-68.

- Veber Palolo (Éunice virilis Gr.). Nachr. der K. Ges. d. Wissensch. zu Göttingen; mat. phys. Ǩlasse. 1898.

- Ueber atlantischen Palolo. Nachr. der K. Ges. d. Wissensch. zu Göttingen, math.-phys. Kl. H. 4. 1800.

Eisig, H. Zur Entwichehnesgeschichte der Capitelliden. Mitt. d. Zool. Station, Neapel. 1887.

Ekuolu, N. und Arruevius, S. Ueber den Einfluss des Mondes auf den elelitrischen Zustand der Erile. Bihang till Sv. Vet.-Akad., Bd. 19-20, Nr. 8, 6. 1893, 1894.

Frederice, L. Sur l'autotomie ou mutilation par voie reflexe comme moyen de défense chez les amimaux. Arch. de Zool. expér., T. 1, Sér. 2, p.413426. 1882 .

Friedländer, B. Ueber den sogenanuten Palolowurm. Biol. Centrbl., Bd. 18. 1898.

- Nochmals der Pulolo unil die Frage nach unbeliamnten liosmischen Einfliissen auf physiolorjische Vorgünge. Biol. Centrbl., Bd. 19. 1899.

- Herrn Alfred Goldsborough Mayer's Entdeclung eines "Allantischen Palolo » ete. Biol. Centrbl., Bd. 21.1901.

- Zur Geschichte der Palolofrage. Zool, Anz., Bd. 27. 1904.

Giard, A. Sur le parasitisme placentaire des Monstrillidae. C. R. de la Soc de Biologie. 6 février 1897.

- Sur l'aitotomie parasitaire el ses rapports avec l'autotomie gonophorique et la schizogonie. C. R. de la Soc. de Biologie. 1er mai, 1897.

- Controverses transformistes. Paris, 1904.

Honst, R. Wawo and Palolo Worms. Nature, Vol. 69, p. 582. 1904.

Houssar, F. La forme et la vie. Essui de la mithode mécanique en Zoologie. Paris, 1900.

Huxley, T. H. Edinburgh New philosophical Journal. 1830 .̈.

Jzuki, A. Observations on the Japanese Palslo (Ceratocephale osawai n. sp.). Journ. Coll. of Sc. Imp. univ. Tokyo, Vol. 17, Art. 11, 2 pl. 1903.

Kexsed, J. von. Ueber Teilung und Knospung der T'iere. Festrede. Dorpat, 1887.

Kräuer, A. Palolountersuchungen im Olitober und November 1898 in Samoa. Biol. Centralbl., Bl. 19. 1899.

Kröyer, H. Uversigt over videnskabelige selskab Forhandlingar. 1850.

LANG A. Ueber den Einfluss der festsilzenden Lebensweise anf die Tiere und über den Ursprung der ungeschlechtlichen Fortpflanzung durch T'eilung und Knospung. Jena, 1888.

- Beitrüge zu einer Trophocöltheorie. Jena, 1903.

Langerhans, P. Ueber einige cunarische Anneliden. Nova acta der K. Leop. Carol. Ak. 1881 .

Lessona, M. Sulla riprouluzione delle parti in molti animali. Atti Soc. ital. Se. nat., Vol: 11. Milano, 1868. 
Mac Ixtosh, W. C. Report on the scientific results of the royage of H. MI. S. Challenger. Zool., vol. 12. 1885.

- On the Pacific, Atlantic and Japanese Palolo. Notes from the Gathy Mar. Lab. St. Andrews. Ann. and Mag. of Nat. Hist. (7) Vol.15. $190 \%$.

Malaguix, A. Recherches sur les Syllidiens, Lille, 1893.

- Epigamie el Schizogamie chez les A muélides. Zool. Anz., Bd. 19. 1896.

- Le parasilisme évolutif des Monstrillides (Crustacés copépodes). Arch. Zool. (3), Paris. 1901.

Mayer, A. G. An Allantic "Palolo" (Staurocephalus qregaricus). Bull. Mus. comp. Zool., Harvard Coll. Vol. 36. 1900.

- The Atlantic Palolo (Euniee fucata). Mus. of the Brooklyn Inst. of Arts and Sciences. Sc. Bull. Vol. 1., No. 3, 1 pl. 1902.

Montricelur, F. S. Sulla fauna di Porto-Torres (Sardegna). Boll. Soc., Nat. Napoli. 1895.

Osawa, K. Ueber die japunischen Palolo. Verhandl. d. V. Internat. Zool. Congress in Berlin (1901). Jena, 1902.

Perrier, E. Sur lorigine des formations stoloniales chez les Syllidiens. C. R. Acad. des Sc. 1902.

Perrier, E. et Gravier, C. La tuchygénèse. Ann. des Sc. nat. Zool., Tome 16. Paris, 1902.

Pruvor, G. Sur la réyénération des parties amputées comparée à la stolonisation normale chez les Syllidés. Congr. de l'Assoc. Française. Limoges, 1890 .

- Sur lévolution des formations stoloniales chez les Syllidiens. Ac.-des Sc. 1902 .

Rrggexbaci, E. Die Selbstverstümmelung der Tiere. Ergeb. d. Anat. u. Entwickelungsgesch., Bd. 12 (1902). Wiesbaden. 1903.

SArs, N. Faume literalis Norregia. 1846.

Scnмmт, 0. Neue Beiträge zur Naturgeschichte der Wïrmer. 1848.

Semper, C. Die Verwondlschaflsbeziehungen der gegliederten Tiere. Arb. d. Zool. Zoot. Inst., in Würzburg, Vol. 3. 1876-77.

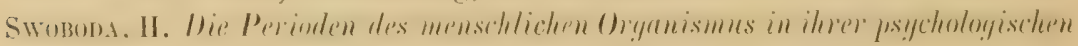
und biologischen Bedeutuny. Leipzig u. Wien, 1904.

Viguien. C. Sur la valeur morphologique de la lête des Amélides. Amn. des Sc. nat. 1902.

Woodwonth, W. Mc M. Vorläufiger. Bericht über den Palolowurm. Abdruck aus: Die Samoainseln. Entwurf einer Monographie etc. von Dr. A. Krïmer, Bd. II, p. 399-403. Stutgart, 1903. 


\title{
Recherches de statistique sur la descendance des Pigeons voyageurs.
}

\author{
Par le $\mathbf{D}^{\mathrm{r}}$ G. LOISEL (Paris).
}

Le ministère de la Guerre ayant bien voulu mettre à notre disposition le colombier militaire de Vaugirard, à Paris, nous avons pu étudier les l'egistres où les accouplements, les pontes et les élevages sont inscrits très exactement par les personnes spécialement chargées de ces soins; nous avons pu étudier ainsi près de 4000 pontes correspondantes aux années 1893 à 1903 et envisager plusieur's points intéressant la descendance des Pigeons voyageur's.

I. - La mature des sexes de chaque poute.

On sait que l'ovaire des Pigeons ver'se à peu près au même moment, dans l'oviducte, deux neuf's qui ne seront pondus qu'à un ou deux jours d'intervalle; ces œuf's écloront après 19 ou 20 jours d'incubation.

Sur 63 couvées ayant fourni chacune deux oufs dont les sexes ont pu être déterminés ${ }^{1}$, nous avons eu :

34 fois un mâle et une femelle;

14 ) deux mâles:

15 ») deux femelles.

Ces résultats concordent parfaitement avec ceux obtenus par Cúnot en $1900^{2}$; ils vont donc à l'encontre de la tradition répandue depuis Aristote, parmi les éleveurs et quelques savants, tradition qui veut que chaque ponte donne habituellement naissance à un couple; c'est-à-dire que l'un des œuf's pondus est mâle, l'autre femelle.

\section{II. - Le mremier beuf poudu est-il un maile?}

Si l'on considère maintenant les 34 pontes pleécédentes qui ont donné des sexes différents, nous trouvons que, dans 21 fois, c'est l'œuf mâle qui a été pondu le premiel' et 13 fois l'œuf femelle.

${ }^{1}$ Ce nombre relativement minime de 63 s'explique parce que le colombier de Vaugirard envoie à d'autres colombiers la plupart de ses jeunes Pigeons avant d'avoir pu déterminer leur sexe.

"Cú́not. La distribution des sexes dans les pontes des Pigeons, C. R. de l'Assoc. pour l'avancement des sciences, II, 756. 1900. 
C'eci semble donc contirmel juscul'ici cette autre idée, répandue également depuis Aristote, que le premier reuf pondu domnait géméralement naissance à un Pigeon mâle. Mais dans 240 autres cas, où je n'ai pu déterminer que le sexe de la première ponte, jai obtenu presque autant de fois iles fomelles (119) que des mâles (121). D’un autre côté, daus 1 lifiutres cas encol’e où je n'ai connu que le sexe de la deuxième ponte, j'ai trouvé plus de mâles (86) que de femelles (80).

Nous concluerons done que la croyance que le premier œuf pondu est toujour's mâle n'est pas plus justifiée que l'idée de la bisexualité des pontes. Et nous nous rencontrons encore là avec Cúxót.

III. - Les enfants héritent-ils plus du plumage du père que de celui de la mère?

C'est une opinion qui se trouve répandue, en effet, parmi les éleveur's et que Darwin confirme quelque part, croyons-nous. Voyons donc ce que fournissent, à ce sujet, les données que nous avons pu recueillir.

Il est tout d'abord à noter que les petits des Pigeons héritent, en général, du plumage des parents, quand ces parents ont un plumage semblable. Ainsi, sur 1o66 jeunes provenant de parents ayant un plumage écaillé, 153 senlement avaient un plumage différent '. Sul 39 petits plovenant de parents à plumage blen, 32 ont eu la mème eoloration et $T$ seulement un plumage différent (écaillé). Enfin des 18 jemnes provenant de parents rouges, 11 ont été rouges, 6 écaillés et 1 gris.

Si nous prenons maintenant les jeunes provenant do couples différant l'un de l'autre par' la couleur du plumage, nous trouvons que sur' 591 cas, 299 avaient pr'is le plumage du père et 294 celui de la mère. L'excès de 5 à l'avantage du père, ne permet pas, il me semble, de conclure comme le font les éleveruss à l’influence prépondérante du mâle. Du r'este, l'étude de cas particuliers montre que la question est autre.

Ainsi, sur $45 \mathrm{~S}$ ('nfants provenant de parents à plumage respectif écaillé et bleu :

228 avaient le plumage du père;

$\begin{aligned} 220 \text { ") " un plumage nouveau. } & \\ 10 & \text { " }\end{aligned}$

Nous trouvons donc toujours une légère prédominance du côté mâle.

${ }^{1}$ Je ne prends ici que les écaillés purs. Je laisse de côté les écaillés jabotés, noirs, clairs, à plumes blanches, etc.

Voici comment se décomposent les 153 plumages autres que l'écaillé : le bleu était représenté à lui seul 115 fois, le rouge 12 fois, le bronzé.7, le mosaïque et le bariolé (qui ne sont que des rariétés de plumage écaillé) 5 fois chacun, le mẹnier 4 fois, le noir 3 fois, le marron et le blane chacun une fois. 
Mais, en réalite. derux cas sont ì distinguer ici : ou hien le piere est écaillé et la mère bleue, ou bien c'est le contraire.

Dans le premier cas (père écaillé, mère bleue), sur 218 enfants :

135 ont le plumage du père (écaillé);

77 » „) de la mère (bleue);

6 » un plumage nouveau.

Dans le second cas (père bleu, mère écaillée), sur 240 cas :

93 ont le plumage du père (bleu);

143 » „ de la mère (écaillé);

4 ) un plumage nouveau.

Il y aurait donc, dans le premier cas, forte prédominance mâle et dans le second, forte prédominance femelle.

La réalité est que la prédlominance est au plumage écaillé qui, dans le premier cas, est porté par le père, dans le second, par la mère.

Les mêmes conclusions résultent de l'étude de 4501 enfants provenant de parents à plumage respectif écaillé et rouge. Ici :

198 avaient le plumage du père;

200 》 \# de la mère:

53 ont un plumage nouveau.

En décomposant cet exemple, on trouve pour le premier cas (père écaillé, mère rouge) :

229 enfants dont :

95 avaient le plumage du père:

103 » de la mère;

31 ) un plumage nouveau.

Le second cas (père rouge, mère écaillée) comprend 222 enfants, dont:

103 avaient le plumage du père;

97 » $)$ de la mère;

22 un plumage nouveau.

Là, encol’e, dans ce second exemple, la prépondérance suit la nature du plumage et non le sexe. Ici la piomentation écaillé so trouve nettement dominée par la pigmentation rouge.

IV. - Les lois de Mendel s'appliquent-elles à l'hérédité de la coloration du plumage chez les Pigeons voyageurs?

Nous venons de voir que, dans les croisements, le plumage écaillé domine, dans la descendance. le plumago blen et est dominé lui-memé par le plumage rouge.

Nous sommes naturellement amenés à nous demander si ces phénomènes rentrent dans les lois de Mendes.

Grogor Mexnes supposa. en 1s6i?. yur les untés fécondés (ou les graines, 
car Mendel a exclusivement expérimenté sur les plantes) renferment, en puissance ou sous forme de particules representatives, les caractères des parents. Il supposa ensuite que les particules porteuses de ces caracteres, accolés dans l'ouf parent, peuvent se disjoindre pour passer différemment chez chaque descendant

Ainsi, si l'ou croise deux individus se distinguant nettement par deux caractères nettement tranchés, tels qu'une Souris grise et une Souris alhinos, tous les descendants de ce couple ne porteront qu'un seul caractère: dans le cas donné, ils seront tous gr’is et alor's le caractère gr ris sera appelé dominant par rapport au caractère albinos qui sera appelé dom iné.

Les particules représentatives de ce dernier caractère n'auront cependant pas disparu. En effet, si on accouple ces descendants gris entre eux, on voit la deuxiène génération se composer de petites Souris albinos aussi bien que grises. Mais. pour Mranes, le rapport du nombre entre ces deux sortes de Souris serait toujour's celui de 3 à 1 . Ainsi, sur les Souris de cette seconde génération, 3 seront grises et une seule albinos.

Ces données ayant été vérifiées sur un grand nombre d'espèces animales ou végétales, on pouvait espérer les retrouver chez nos Pigeons. Or, sur 516 enfants viables provenant de couples écaillés et rouges ${ }^{1}$ :

229 avaient un plumage rouge;

$\begin{array}{rlll}210 & \text { » } & \text { écaillé: } \\ 67 & \text { » } & \text { nouveau. }\end{array}$

Sur 566 enfants viables provenant de couples bleus et écaillés ${ }^{2}$ :

350 avaient un plumage écaillé;

$\begin{array}{rlll}211 & \text { \# } & \text { bleu; } \\ 5 & \# & \text { nouveau. }\end{array}$

Sur 219 enfants viables provenant de couples bleus et rouges ${ }^{3}$ :

79 avaient un plumage rouge;

29 " " bleu :

111 ) nouveau (dont 80 écaillés).

Sur 131 enfants viables provenant de couples argentés et écaillés i :

58 avaient un plumage argenté;

43 ) $)$ écaillé;

30 " " nouveau (dont 28 bleus).

${ }^{1}$ Ces couples ont donné en plus, dans l'espace de dix ans (en ne tenant compte que des deux premières courées) 30 œufs qui ont été cassés, abandonnés, ou dont les jeunes sont morts de très bonne heure.

2 Comme ci-dessus, il faut encore noter 77 œufs dont je n'ai pu avoir la descendance.

s En plus 26 œufs avortés.

- En plus 11 cufs avortés. 
Sur 98 enfants viables provenant de couples argentés et bleus ${ }^{1}$ :

39 avaient un plumage argenté;

35 » $)$ bleu;

24 " \) nouveau (dont 21 écaillés).

Enfin, dans 13 cas. des Pigeons noirs ont été acenuplés avec des Pigeons d'autre couleur (écaillés, louges, bleus ou blanes). Sur : 44 petits viables, 14 avaient un plumage noir. les 20 autres un plumag' diffélent. Le mélange noir et blanc, par exemple, qui répond exactement aux conditions de caractères antagonistes étudiéos par Mrxwes. a donné sur 7 petits : un noir pur, deux écaillés bariolés, un argenté, un noir vol blanc, un écaillé et un blanc.

Toutes ces donnees nous montrent donc qu'on ne saurait parler ici de prédominance diun caractere de pigmentation quelconque au sens mencléléen du mot.

Nous avons vu pourtant que, dans les accouplements bleus et écaillés, cette dernière pigmentation avait montré une prédominance marouée dans la descendance. Mais si nous avons affaire ici à un véritahle caractère prédominant, la descendance doit donnel les " de jemnes écaillés. ()r. nous avous vu qu'il n'en était pas amsi, puisque sur 1066 jeunes provenant de couples écaillés et écaillés, nous avons obtenu 913 écaillés au lieu du nombre 798 qu'exigerait la loi de Mendel.

D'un autre côté, les pigmentations bleues unies ensemble ne se comportent pas comme des véritables calactères dominés: nous avons vu en effet que sur 39 jeunes provenant de père et mère bleus, 7 avaient eu un plumage différent de celui de leurs parents.

Yous pourrions faire les mêmes réflexions pour la pigmentation rouge que nous avous vu prédominer égalenent, dans Jes alcouplements rouges et écaillés.

Nous devons donc conclure que les lois de Mendel ne sont pas applicables à l'hérédité de la pigmentation chez les Pigeons voyageurs.

V. Les lois de Galton ou de Pearson s'appliquent-elles à l'hérédité de la coloration du plumage chez les Pigeons voyageur's?

Lon sait que pour Gartor les outís fícondés lenferment non seulement les caractères des parents, mais encore ceux des ancêtres; et que la répartition de ces héritages chez les enfants se fait de la façon suivante:

Dans un groupe d'enfants déterminé les $\% / 3$ hériteraient des parents $(1 / 3$ de la mère et $1 / 3$ du père) et l'autre tier's des ancêtres.

L'on sait également que PEARson, après avoir combattu les lois de GAL-

${ }^{1}$ En plus 8 oufs avortés. 
Ton, y levint plus tard, mais pour les modifiel de la facon suivante: les parents contribueraient seulement par moitié dans l'héritage total, les quatre grands parents pour un quart et tous les $2 n$ ancêtres du $n^{\text {ième }}$ degré pour $\frac{1}{2 n}$ du total héréditaire.

A première vue ces lois semblent devoir s'appliquer à nos faits. Nous avons vu plus haut, en effet, que la plus grande partie des petits des $\mathrm{Pi}$ geons heritrut en général du phomagr de leurs parents; et nous pourons peruser qur ceux de ces petits qui ont un plumage différent sont ceux qui héritent des ancêtres. Ceci est très admissible et les résultats que nous avons obtenus ne peuvent que confirmer les idées régnantes sur l'atavisme, mais ce que nous ne rencontrons pas, c'est la rigueur mathématique donnée par Galton et Pearson.

Ainsi, daus le mélange écaillé et rouge, le chiffre 67 que nous supposerons avec GALTon représenter l'influence ancestrale est beaucoup trop faible, puisque le tier's de 516 est 172. Le désaccord est encore plus grand avec Pearson puisque cet auteur attribue une moitié de l'héritage (et non plus le tier's) à l'influence reéunie de tous les ancêtres.

Du reste, voici, en résumé, d'après nos recher'ches, quelle selait la part de l'influence de l'atavisme en comptant cette influence au moyen des plumages nouveaux apparaissant dans les couvées ${ }^{1}$ :

\begin{tabular}{|c|c|c|c|}
\hline L'atavisme compterait poü & $50 \%$ & es & bleu-rouge \\
\hline 》) & $38 \%$ & ) & rouge-louge \\
\hline ) & $17 \%$ & ") & bleu-bleu \\
\hline ) & $14 \%$ & ) & écaillé-écaillé \\
\hline ) & $12 \%$ & » & écaillé-rouge \\
\hline b) & $0,88 \%$ & ) & écaillé-bleu. \\
\hline
\end{tabular}

Certes nous n'avons pu tenir compte, dans nos statistiques, des mortalités de jeunes Pigenss ou d'embryom, mais, comme nous l'avons vu plus haut, ces mortalités sont relativement peu nombreuses.

Du reste, les écarts sont ici trop considérables pour qu'on puisse vraiment en faire soltir une loi et surtout une formule mathématique quelconque.

\section{Etude de quelques cas particuliers.}

Dans ces conditions, nous avons voulu prendre le problème d'une autre façon. Au lieu de considérer des moyennes qui donnent toujours quelque chose de schématique et par conséquent ne répondent pas réel-

1 Il est à remarquer que rien ne prouve que tous ces plumagrs nouveaux soient dus ì un retour ancestral; si nous le supposons, c'est pour donner le plus de chances possible aux lois que nous critiquons. 
lement à la vie de la nature, nous avons étudié la descendance de couples déterminés.

Nous nous sommes d'abor'd demandé si la descendance était toujour's la même au cour's de la vie d'un couple. Et nous avons vu qu'il en était généralement ainsi quand les parents ont la même couleur et la même

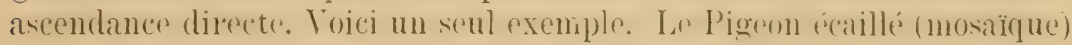
$n^{\circ} 350$, accouplée avec la Pigeonne écaillée noire, $n^{\circ} 523$, ayant tous les deux des parents écaillés, a domé constamment, pendant quatre années consécutives, des petits au plumage écaillé.

Par contre, lor'sque les parents ont un plumage différent, la descendance varie d'aunée en année. Pour ne pas augmenter outre mesure ce travail préliminaire, je ne donnerai également ici qu'un seul exemple.

Le Pigeon écaillé $\mathrm{n}^{\circ} 969$ (parents: écaillé et bleue) accouplé avec la Pigeonne argentée $\mathrm{n}^{\circ} 1551$ (parents : argenté et écaillée) a donné en 1900 4 petits écaillés, en 1901, un écaillé, un bleu, un rouge et un argenté, en 1902 deux argentés et un écaillé.

Considérons maintenant des suites de généalogies déterminées et là encore; ne prenons qu'un seul cas relativement simple:

Grands-parents paternels: rouge et écaillée.

$$
\text { ") maternels : rouge et écaillée. }
$$

Parents: rouge et écaillée.

'Tloois petits-enfants : écaillé, écaillée, rouge.

Le petit-enfant écaillé accouplé avec une femelle rouge donne 6 arrièrepetits-enfants: 4 écaillés, 2 rouges.

Un des arrière-petits-enfants écaillés, uni avec une femelle écaillée, donne 10 arrière-arrière-petits-enfants : 8 écaillés, 2 bleus.

Voilà donc tout un groupe familial qui a eu pour point de départ deux colorations sculement, la rouge et l'écaillée; tous les nembres de ce

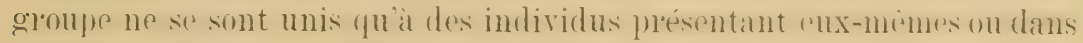
leur's ascendants les mémes colorations rouge et écaillée 1 . Et cependant, à la cinquième génération, nous voyons apparaître une coloration nouvelle, la bleue, alors que la coloration rouge disparaît.

\section{Résumé.}

Les résultats de la dernière partie de notre travail peuvent se résumer ainsi :

1" (quand on aceouple deux l'igeons ayant le mêmr plumagr. on obtient $85 \%$ de jeunes possédant un plumage identique à celui des parents ${ }^{2}$.

${ }^{1}$ Les femelles qui ont été unies avec un des petits-enfants et un des arrière-petitsenfants, n'ont elles-mêmes que des colorations écaillées ou rouges eu remontant dans leurs ascendants jusqu'à la quatrième génération.

${ }^{2}$ Il est à remarquer que, dans ce cas, l'on a obtenu à peu près les mêmes pour- 
$2^{\circ}$ Quand on accouple deux Pigeons ayant un plumage différent, la descendance varie, suivant la nature des accouplements, dans les proportions suivantes:

a. Le mélange écaillé-bleu donne:

$61 \%$ d'écaillés,

$37 \%$ de bleus,

$0,88 \%$ de plumages nouveaux.

b. Le mélange écaillé-rouge donne :

$42 \%$ d'écaillés,

$44 \%$ de rouges,

$12 \%$ de plumages nouveaux.

c. Le mélange bleu-rouge donne:

$13 \%$ de bleus,

$36 \%$ de rouges,

$50 \%$ de plumages nouveaux.

d. Le mélange argenté-bleu donne:

$36 \%$ de bleus;

$40 \%$ d'argentés,

$24 \%$ de plumages nouveaux.

e. Le mélange argenté-écaillé donne:

$33 \%$ d'écaillés,

$44 \%$ d'argentés,

$23 \%$ de plumages nouveaux.

Nous voyons donc certains plumages dominer. tels que l'écaillé sur le bleu et lo rouge sur l'écaillé. Mais la discussion de ces chiffres nous a montré que, ni les lois de Mendel, ni celles de Galton et de Pearson ne peuvent s'appliquer réellement à l'hérédité de la coloration du plumage chez les Pigeons.

$3^{\circ}$ Pour un même couple, la descendance reste, en général, la même charue année, ut cela, au moins pendant quatre années consécutives quand les parents et les grands-parents ont un plumage semblable.

t. La descendance diffère au contraire chaque année quand les ascendants ont des plumages différents.

centages chez les animaux de ferme: Chevaux anglais pur-sangs, ${ }^{-85}, 6 \%$; pur-sangs et demi-sangs anglo-arabes, $83 \%$; bœufs Durham, 83,33\%. (Marcel VAcher. Transmission de la couleur chez les animaux de la ferme. L'Agriculture nouvelle, 1905. XV. 192, 212. 
50 En suivant la généalogie de couples déterminés pendant un l’ortain nombre de générations. on voit parfois des plumages nouveaux apparaître sans qu'on puisse retrouver ees plumages dans les ascendants, en remontant jusqu'à la $4^{\text {me }}$ et la $8^{\text {mo }}$ génération.

En somme tous ces faits concordent pour montrel que, chez les Pigeons, les caractères de pigmentation du plumage ne sont pas déterminés dans l'œuf fécondé.

Les petits Pigeons n’héritent pas d'une pigmentation donnée, mais d'un état physiologique particulier transmis par les ascendants; de même, les médecins nous appreunent que les enfants de tuberculeux n'héritent pas de la tuberculose, mais d'un organisme facilement tuberculisable.

Cet héritage ne se transmet pas sous forme do particules représentatives immuahles et en quelque sorte immortelles, mais sous forme d'une constitution physiologique pouvant être modifiée dans le courant de la vie des parents comme aussi sans doute dans le courant de la vie embryonnaire des enfants. Ceci nous fait revenir en somme à l'étude des facteur's de l'évolution, facteurs qui ne seront bien connus qu'en considérant le plus grand nombre possible de cas particuliers. Voici, à titre d'exemple, un de ces cas :

En 1893, le Pigeon écaillé n 496 (parents: écaillé et écaillée) est accouplé avec la Pigeonne écaillée $n^{\circ} 286$ (parents : écaillé noir et écailléo). I)ans l'espace de quatre années, ce couple domme une descendance de $1+$ individus portant tous le plumage écaillé des parents (9) écaillés + j) écaillés noils).

A u bout de ce temps, en $15 ! 77$, la femelle $n^{\circ} 286$ ayant disparu, on la remplace par une autre femelle, $n^{\circ}: 373$, âgée de cinq ans et de couleur bleue. Or, ce nouvel accouplement donne en deux ans l'abord fi petits bleus et à la deuxième couvée de 1898, ㄹ petits écaillés (un écaillé et un écaillé plumes blanches).

Il semble donc, à première vue, que nous ayons là une application de la loi de Mendel ; on peut croire en effet que la coloration bleue est venue, lors du deuxième accouplement, dominer d'abord la coloration écaillée.

Mais, si on recherche quelle était la descendance de la femelle bleue $n^{\circ} 373$ avant son accouplement avec le mâle écaillé $n^{\circ} 496$, on voit que cette femelle avait été accouplée pendant les trois années avec un mâle louge, $n^{\circ} 410$. Or, pendant tout ce temps, cette femelle a eu 11 enfants dont aucun n'avait le plumage hleu (fi avaient le plumage louge et jo le plumage écaillé). Done ici la loi de Mendes se trouve completement en défaut: ou hien il faut dire que le bleu est dominant pour l'écaillé et dominé par le rouge, mais ceci se trouve encore infirmé par la suite de l'observation.

Une étude attentive de ce cas montre qu'on ne saurait davantage 
invoquer lâge l'elatif des deux conjoints. En effet. lors de son second accouplement en 1897, la femelle bleue trouve un mâle plus âgé qu'elle et donne la descendance que l'on sait. En 1899, ce vieux mâle dispraraît pour être remplacé par un jeune mâle âgé de 11 mois et de couleur rouge: cependant la femolle continue à domner des enfants bleus à coté d'autres enfants rouges.

On est donc obligé de s'arrêter à cette idée. que létat phrsiologique de l'ovaire de la femelle bleue a dû se modifier à partir de 1897; et cela, sous lintluence de facteurs que nous fera commaître sans rloute une étude poursuivie des phénomènes fle la sexualité considérés dans leurs rapports avec les autres fonctions de l'or'ganisme.

\title{
Zur Wahrung des Prioritätsgesetzes in der Nomenclatur gegenüber dem sogenannten Vorrecht des ersten sichtenden Autors.
}

\author{
Von 0. KLEINSCHMIDT' (Volkmaritz).
}

Dass auf den VI. internationalen Zoologen-Congress keine Sektion fül Nomenklatur eingerichtet war, sehe ich als einen Fortschritt in mehrfacher Hinsicht an, unter ander'm auch als ein Anzeichen, dass in allen wesentlichen Punkten eine Einigung erzielt ist. Ich will daher um so weniger als Störenfreded auftreten, auch keineswegs eine Aenderung der Regeln bei der Kommission für Nomenlilatur heantragen. Ich will lediglich in Nachstehendem zeigen, dass man der überaus dankenswerten Arheit der Nomenklatur-Kommission auf dem liongress dankbar zustimmen kann und doch deshalb sich nicht sklavisch an alle Einzelheiten zu binden braucht, denu die sich einbülogernde Praxis ist schliesslich das Ausschlaggebende.

So 'ntspricht zum Beispiel dor Satz: "Fs ist nicht zulässig, den Variutïts- oder Subspecies-Namen ohne den Artnamen zu gebranchen, "durehaus meiner Xaturauffassung und ist mir überaus sympathisch. Da aber bei vielen binzelfälen die Ansichten. (b) cine Species oder Subspecies vorliegt, geteilt sind, lässt sich hierüber niemandem eine Vorschrift machell. Ich sellst hemenne sogar subspecies in derersten Beschreibung meist binär, weil dieser binär reduzierte Name neutral ist und jeder 
späteren Auffissung einen korrelit gebildeten Nanen üborliofert zur Einfügung in das wechselnde trinäıe System.

Dies ist aber nur als Commentar gemeint. Es ist gut wenn der betreffende Passus stehen bleibt. Besser ein bestimmtes Wort als \%. B. das schwankende "Eigennamen liöme"n mit grossen Anfangshuchstaben gerschrieben werden ». Da man in zahlreichen Fällen nicht wissen kann, ob ein Eigenname vorliegt oder nicht, sollte die längst (wenigstens in del ()rnithologie) eingebürgerte schreihwerse mit lileimem Anfangshuchstaben empfohlen werden.

Solch ein unbestimmtes Schwanken zeigt sich nun auch in den Regeln ühes die Priorität bei (ammïhernd) gleidhritig veröffentlichten Namen.

Hier soll nach der deutschen und englischen Fassung der Regeln der die Gruppe zuerst revidierende Autor auf alle Fälle massgebend sein,

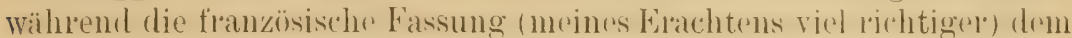
an erster Stelle im Originalwerk genannten Namen den Vorzug gibt.

Wenn übrigens in allen Fällen der' sichtende Autol' gelten soll, so sind, da in allen Fällen die Gruppe einmal früher oder später revidier't wil'd, die drei volanstehenden Regeln zwecklos. Sie können höchstens als Ratschläge für den ersten sichtenden Autor gelten, der sich aber nicht darum zu kümmer'n braucht. Ich meine, es gibt im Grunde nur eine Nomenclaturegel, das Gesetz der absoluten Priorität (ab 1758). Dieses verlangt, dass der in einem Buch räumlich weiter vor'n stehende Name, der also auch zeitlich friiher gelesen wird, auf alle Fälle dem späteren Namen vorgezogen werde. Bei neueren Publikationen wird man leicht feststellen können, ob z. B. zwei auf den 1. Januar datierte Zeitschriften, in denen dieselbe Art unter verschiedenen Namen neu beschrieben ist. wirlich gloichzeitig versandt worden sind. Meist wird die

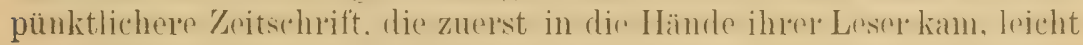
zu el'mitteln sein, denn nicht die Priorität des Manuscripts oder des Drucks, sondern die der wirklichen Publikation ist die logische und praktisch massgebende. Für ältere Publikationen ist der el'ste revidierende Autor lediglich, so lange kein besserer Gr'und vorliegt, der glaubwärdigste Zerge dirser Prioritat. In mö̈glicherweise ein noch frouheres Revisol gefunden welden kann, sollte man solehe Vamen zweifelhafter Prioritat mit zwei Autorennamen bezeichnen (z. B. N fide X).

Leider wird der " erste sichtende Autor" " vielfach auch zur Deutung von Namen herangezogen. Ireren Diagnose fraglich ist. Namen, die nicht sicher gedentet werden lïmnen. sollte man mit Fragezeichen in die Srmonymik stellen und his zu ilner sichreen Deutung vom (robrauch ausschliesen : demu die wissenschaftliche Klatheit steht üher allem. Theshalb kann ich auch einen nur " angedeuteten " Namen, wenn der Name selbst nicht genannt ist, nicht als veröffentlicht ansehen.

Gruppenrevision und die Einschaltung von subgenusnamen sind (re- 
danken die sich eng mit der von mir angewandten Formenring-Nomenklatur berühren und deshalb habe ich diese verwandten Begriffe andrerseits sogar mit grosser Freude begrüsst. Ich will hier hemerken, dass die von mir vorgeschlagene neue Systematik durchaus die einfachste Linvésche Nomenlikatur lionserviert, aber erst richtig beurteilt werden kann, wenn dic ersten Lieferungen meiner geplanten grossen Zoographie vorliegen.

\section{Ueber die Genesis und die Entwicklung der Geselligkeit im Tierreiche.}

Von Dr. W. von WAGNER (Moskau).

Dio Gesellschaft entwickelt sirh aus der Familie und legt den Grund zum Staate.

So lautete die fast während des ganzen XIX. Jahrhunderts fest eingebürgerte Formel für die Entscheidung der Frage über dic Genesis und die Entwicklung der Geselligkeit im Tierreiche und dieser Formel lag als unerschütterliches Aroument ein Material zu Grunde, welches dem Leben der sogenannten "sozialen » Insekten entnommen war : " das gesellige Leben der Bienen, Ameisen und Termiten beginnt mit der Familie, welche sich anfangs zu einer Gesellschaft und darauf zu einem Staate entwickelt - hei den Bienen nach dem Vorloilde einer Monarehie, bei den Ameisen als Republik. )

Nachdem Bochowan, Mac-Lennax, Taylor, M. Kowalevski, Grosse, Montin und eine Menge anderer Forscher, welche die Uranfïnge der menschlichen Gesellschaft zu ihrem Studium machten, die Tatsache festEestollt hatten. dass nicht die Familie, sondern der Herdenzustand mit mhestimmter Gemeinschaft der Geschlechter den Koim der menschlichen (iesellschaft bildet, wïhrend die Familie ein Prodult des Zerfalles diess Herdenlebens war, erlitt dir ursprüngliche Formel für die Entshohidung der Frage üher die (renesis und die Entwicklung der Geselligkeit im 'Ticreiche einige Variationen, welche jedoch an ihrem Wesen nichts änderten. Das Zusammenleben der sogenannten "sozialen " 
Insckten wurde nicht molur als Gesellschaft und nicht als Statat aufgefasst, sondern als eine Helole, welcher das Familienelement, bis zur Mutterliehe einschliesslich (Rıвот, u. a. m.). vollständiu abgesprorhen wurde.

Ueber diese Verbesserung hinaus ist nicht gengangen worden und die Antwort auf dio Frage übel die Genesis der Geselligkeit ist auch in gegenwärtiger Zeit dieselbe gebliehen, in dem Simne, dass das Zusammenleben der Bienen, Ameisen und Termiten als der nächstliegende Tebergang von dem menschlichen Staate zu der Tierwolt angesehen wird: ein Unterschied hesteht hierbei nur darin, dass nach der Ansicht der Finen dieses Zusammenlehen vergrösserte Familien, nach dre Ansicht von Anderen - Gesellschaften und Staaten, nach Ansicht Dritter endlich Herden repräsentiert.

Das Sonderlare einer derartigen Ordnung der Iningr. wobei die Evolution der sozialen Fähigkeiten, welche von Allen als eine der höchststehenden Formen der Evolution angesehen wird, im Widerspruche steht mit der Evolution im Tierreiche überhaupt, so dass die Bienen und Ameisen nicht nur über den am hörhsten entwickelten Säugetieren. sondern (nach Ansicht vicler Autor॰n) auch üher manchen Menschenrassen zu stehen liommen, hat schon längst die Anfmerksamkeit denkender Naturforscher auf sich gelenkt; alle Versuche. diesem Widerspruche zu entgehen, gringen jedoch nicht über theoretische Erörterungen darüher hinaus, dass zum Beispiel die Entwicklung der Organisation nicht immer mit der Entwicklung der sozialen Instinlite gleichen Sehritt hält. Ind dies ist ganz verständlich: theoretische Erörterungen allgemeinen Charakter's kömnen wohl als Hinweis dafür dienen, welche Richtung die Forschung einzuschlagen hat, nicht aber als Nittel für die Fntscheidung streitiger Fragen in der Naturkunde.

Man muss die Tatsachen, welche diesen Widersprüchen zu Grunde liegen, revidi e re n und dieselben nicht, wie dies lisher in den meisten Fällen geschehen ist, ad hominem, sonde $\mathrm{l}^{\mathrm{n}}$ objektiv, d. h. vermittelst der Evolutionsmethode, hewerten. Diese Aufgabe ist es num, welche ich im Auge hatte, als ich vor neun .Jahren das studium des Zusammenlebens bei den Hummeln (und zum Teile auch hei anderen sog. "sozialen ") Insekten) hegann; die Ergelnnisse meiner Studien hale ich in einer Arbeit "Psycho-biologische Studien üher Hummeln, im Zusammenhauge mit der Frage über die Gruesis der ferselligheit der Tiere n niedergelegt, deren Résumé die vorliegende Mitteilung hildet, soweit die im Titel berührte Frage ins Spiel kommt.

Die Beantwortung dieser Frage zerfällt offenbar in zwei Teile, und zwar: :

Erstens in die Antwort auf die Frage: was stellt in Wirklichkeit das Zusammenlehen der sog. "sozialen » Insekten vor und kamn dasselbe 
als rin Glied auf dem Wege jener Evolution der Geselliglieit aufgefasst werden, welche in dem Auftreten der ersten Menschenhorde auf dem Erdball gipfelte?

$Z_{\text {we ite n }}$ - wenn dies nicht der Fall ist, welchen Verlauf hat dann diese Evolution der Geselligkeit genommen und welchen Platz nimmt das " Zusammenleben " der geselligen Insekten in dieser Evolution ein?

In Beantwortung der er'sten dieser Fragen kann ich schon jetzt, ohne zu entscheiden, was dieses: Zusammenlehen eigentlich darstellt, aussiagen. dass die 'Tatsachen es unmöglich machen, diese's Zusammenlehen bei den Insekten als Familie, als Gesellschaft, als Stat, oder endlich als Helde aufzufassen; die zweite Frage will ich einstweilen nur insofern beantworten, dass das Zusammen leben bei den Insekten keinen Platzin der Evolutionsreihe der Geselligkeit e innimm t. Ich begreife natürlich vollauf, dass eine solche Behauptung, welche mit den Ansichten, soviel mir bekannt ist, ausnahmslos aller diese Frage behandelnden Forscher in direktem Widerspruche steht, mich nicht zu Erörterungen allein, sondern auch zur Angabe von Tatsachen rerpttichtet, welche ich denn auch in meiner etwa 20 Druckbogen unfassenden Arheit anführe: in dem vorliegenden Berichte kann ich selbstverständlich infolge Zeitmangels weniger üher die Tatsachen als über die von mir aus diesen gezogenen Schlussfolgerungen Nitteilung machen. Zu diesen letzteren will ich drum auch nummehr überwehen.

Meine erste These besteht darin, dass das "Zusammenleben" der Hummeln (und anderer (sozialer") Insekten) keine Familie darstellt.

Dieser Satz wird durch folgende Beweisgründe festgestellt:

A. Dieses "Zusammenleben " entbehrt der mor phologischen Einheit, welche die "Gemeinschaft " der Glieder" ausmacht und unbedingt für jede, a uf einem sozialen Instinkte begründete hiologische ()ronisation, erforderlich ist. I) Unterschied zwischen den Gliederu einer solehen Organisation darf ehen in deren biologiseher Bedeutung nicht weiter gehen. als die : erschiedonheiten, welche die Männchen von den Wribchen trennen; indessen ist bei einigen gesellig lebenden Insekten der Unterschied zwischen den ihre Gemeinschaft ausmachenden (iliedorn so weitgehend, dass diese letzteren, wenn sie nicht zusammen walel, hïtten, von den Naturforschern nicht nur in verschicdene Gattungen, sonder'n sogar in verschiedene Familien hätten untergebracht weriten müssen.

B. Ausser dem Fehlen der morphologischen Einheit, welches an sich schon senügt hïte, um den Gedanken an eine Auffassung des Zusamnenlelons bei den sogenamnten "sozialen " Insekten als Familis aller" Grundlage zu beraulen. wird der Satz noch dureh die Tatsache unterstïtzt. dass dir Instinkte, welche als del deutlichste Ausdruck des Fa- 
milienlebens angesehen werden, bei den sogenannten « sozialen » Insekten nicht nur nicht schärfer und bestimmter, sonder'n sogar weniger anschaulich zu Tage treten, als bei den solitären Hymenopteren.

Ich will an dieser Stelle auf drei Gruppen von Erscheinungen des sogenannten mütterlichen Instinktes hinweisen.

$1^{\circ}$ Erscheinungen, welche die Eiablage begleiten,

$2^{\circ}$ das Füttern der Brut.

:" die aut die Verteidigung der Nachkommenschaft und auf die Sicherstellung ihrer Fntwicklung gerichteten Instinkte.

Alle diese Erscheinung sind, wie dies aus den von mir gesammelten Daten hervorgeht, bei den sogen. "sozialen " Insecten entweder in keiner Weise verschieden von dem, was wir bei den solitären Insecten sehen, oder aber sie stehen gerade in derjenigen Hinsicht, in welcher sie diese letzteren übertreffen sollten, hinter ihnen zurück.

Ferner behaupte ich, dass das "Zusammenleben » der H ummeln weder einer Herde, noch einer Gesellschatt und noch weniger cinem State entspricht. Diese Behauptung wird durch drei Kategorien von Beweisgründen festgestellt:

A. Durch das Fehlen der morphologischen Einheit, welche die eine Zusammenschaarung, eine Herde oder eine Gesellschaft zusammensetzenden Individuen auszeichnet, einerlei auf welcher Entwicklungsstufe diese biologischen Organisationen auch stehen mögen.

Ich will hier nur bemerken, dass die Wichtigkeit dieses Merkmales, abgesehen von anderen Umständen, schon aus der 'Thatsache hervorgeht, dass unter den. Merkmalen der Geselligkeit (als biologische Erscheinung), während des gesammten V'rlaufes ihrer langsamen Entwicklung auf der ansteigenden Stufenleiter des Thierreichs, eine molpholowische Infferenzierung der (ilieder der (iesellschaft in Ku:anmenhange mit ihren Functionen, wie wir dies bei den Bienen, Wespen und Termiten sehen, nicht zu finden ist.

Die wahre Geselligkeit erweist sich als um so vollkommener, je vielseitiger und vollständige die Instincte der die Geneinschaft zusammensetzenden Individuen sind und je mehr Vortheil ein jedes der letzteren von dem geselligen Leben hat; bei der Geselligkeit der sogen. "sozialen " Insecten sehen wir gerade das Gegentheil hiervon : sie erweist sich als um so volkommener, je mehr ihre einzelnen Glieder an Individualität verlieren und je mehr deren Instincte durch spezielle Functionen, welche für die Artvon Vortheil, für das Individuum dagegen von Nachtheil sind, verändert und eingeschrinkt werden.

B. Dadureh, dass den einzelnen Individuen, "zusammenlebender» Insecten, Fähigkeiten a uch zur elementarsten Nachahmungabgehen, das heisst derat primitive Fähigkeiten, wie sie nicht nur für die Organisation einer 
Gesellschaft, sondern selbst für die einfachste Form des Zusammenlebens in Herden eine notwendige Vorbedingung bilden.

Zwei Drittel der von mir gesammelten und in meiner Arbeit mitgetheilten Daten, hestätigen dic Richtigkeit dieser Schlussfolgerung; sie alle stellen fest, dass die Thätigkeit der Hummeln (und zweifellos auch der anderen sogen. "sozialen " Insecten), wenn man von den Elementen des Giedächtnisses bezüglich der Auffindung des Nestes und der Beute absieht, durchgehends eine instinctive, d. h. vererbte Thätigkeit darstellt.

Es übrigt nunmehr zu beweisen,

C. dass die Individuen, welche das "Zusammenleben" der sogen. "sozialen" Insecten zusammensetzen, nicht i m Standesind, sich untereinander durch solche Mittel zu verständigen, welche ihrer Form und ihrer psychologischen Bedeutung nach denjenigen. Mitteln analog sind, wie sie den Vertretern der in Herden lebenden Thiere und anderer höchster Formen des Zusammenlebens im Thierreiche zu Gebote stehen.

Sind nun aber diese Individuen im Stande, sich gegenseitig auf eine solehe Art und Weise irgend welehe Mittheilung zu machen, welche keinerlei Beziehungen hat zu der Sprache der höheren Thiere: Auf diese Frage antworte ich mit der gleichen Ueberzeugung - ja, sie sind dazu befïhigt, aber als Sprachorgane dienen ihnen der Geruchs- und der Tastsinn, und die Psychologie dieser Sprache trägt einen ganz anderen Character, als die Psychologie einer jeden durch das Gehör aufgenommenen Sprache; entgegen der Ansicht der Autoren, stellt diese Sprache nicht nur nichts dar, was die gesellig lebenden Insecten in irgend welchor Weise besonder's auszeichnen und dieselben direct der höchsten For'm der Geselligkeit - derjenigen des. Menschen - nähern würde, sonder’n sie geht um kein Jota üher Dasjenige hinaus, was wir in derselben Klasse hei den solitïren Insecten sehen und bleibt sogar hinter einigen dieser letzteren zurück.

Die Sprache des Ger uches dient bei den Hummeln als Mittel zur Lösung hauptsächlich zweier Aufgaben: a) in der Frage nach dor Lage des neuen Nestes bei einer Uebersiedlung (durch wen und wie dieser nene Platz hestimmt wird) und $b$ ) in der' Frage des Erkennens iler Nestgenossen und fremder Hummeln.

a) Die Entscheidung der Frage über den Platz, an welchem das Nest bei der Uebersiedlung angelegt werden soll.

Ein Schwärmen kommt bei den Hummeln nicht vor, allein es kommen, wenn auch ä usser'st selten, Fälle vor, wo sie zusammen mit dem 
Wribchen in ein anderes Nest übersiedeln; solche Lehersiedlung('n worden stets " zu Fusse » und niemals im Fluge ausgeführt. Die Ursache dieser Fracheinung liegt natürlich in jenen Eigenschaften des Geruchsvelmögens der Hummeln, welehe heweisen, dass während die Bien'n, durch den Geruchssinn und die von den "Kameradinnen ") in der Luft zurückgelassenen Spuren geleitet, nicht Gefahr laufen vom Wrge abzukommen, die Hummeln ein solches (feluchsvermögen nicht hesitzen und nur dann Fühlung unter pinander behalten, wenn sie die Möglichlieit haben, sich von dem tactilen Geruchssinne leiten zu lassen.

Das Weibchen der Hummeln hesitzt die Fähigkeit pine Spur zu hinterlassen, welche den Arbeiterinnen als Wroisung für die Ausführung ihror Arheit dient. gerade wie die von den Ameisen hinterlassene Spur für das Nachfolgen der" "Kameraden ».

Erkennen wir solche Erscheinungen als ein Element der "Sprache » an, so wird die Sprache des Hummelweihchens von diesen letzteren mit Hilfe des Abdomens niedergeschrieben und von den Arbeiterinnen mit Hilfe der Fühler gelesen ".

6) Ueber das Erkennen von Individuen aus dem eigenen und a us fremden Nestern.

I)ie Hummeln sind befähigt, velmittelst der "Sprache des (reruches v ausser nach dem Orte des Nestbaues auch danach zu fragen, ob eine im Neste angetroftene Artgenossin zu den Ihrigen oder zu cinem fremden Teste gehört, und auf diese Frage eine mehr oder weniger bestimmte Antwort zu erhalten. Die Tatsache, dass ein persönliches Erlennen weder bei den Bienen, noch bei den Wrespen und Anoisen stattfindet, scheint in nenerer Zeit augenscheinlich von allen Forscher'n als unzweifollatt festgestellt angesehen zu werden, während natürlich gleichzeitig zugr(2geben wirl, dass dasjenige Erkennen, zu welchem diese Insekten fïhig sind, stwas durchaus Anderes ist, als das Erkennen bei den hölıeren 'Tier'en.

Diese Fähigkeit bezeichnot BeTHe bei den Birmen und Ameisen bekanntlich als Chimioreftex, indem er ihr jeden Psychismus abspricht und sie bui den Organen des Geruches unterbringt. Durch dieses Geruchsvermögen einerseits und andererseits durch die Eigenschaft, welche ein jeder einzelne Stock oder ein jedes einzelne Test einer jeden Art dieser Insekten besitzt, einen bestimmten Geruch von sich zu geben - wird denn auch bei der Berührung mit den Fühlern ihrer (renosen und fremder Nesthewohner seitens del Individuen ainel gegehenen Familie ein Chimiorettex hervorgerufen. Die Ausschridung dieses Geruches, oder

${ }^{1}$ Darüber, welche Wirkungen von den Weibchen ausgeübt werden und in welcher Weise die Arbeiterinnen darauf reagieren, gebe ich in meiner Arbeit über die Biologie der Hummeln ausführliche Auskunft. 
nach BETHE - einer besonderen chemischen Substanz - ist eine Folge des Stoffiwechsels im Organismus.

Was die Hummeln betrifft, so sind bei ihnen die Beziehungen der Weibchen verschiedener Familien zu einander unveränderlich und bestiandig: rine Begegnung zwischen ihnen im Neste hat rinen hartnäcligen und tötlichen Kampf zur Folge.

Die Fähigkeit, ihre Genossinnenvon fremden Individuen derselben Artzu unterseheiden. ist bei den Arbeiterinnen der Hummeln viel schwächer ausqubildet als bei den Wribchen und sie ist nicht nur bei den verschiedenen Nestern, sondern auch bei den Individuen einer und derselben Familie in verschieden hohem Grade ausgebildet.

Beobachtungen und Versuche ergeben, dass das Erkennen der Hummeln untereinander und das Unterscheiden der Bewohner des eigenen von denjenigen emes fremden Nestes ausichliesslich durch den taktilen Geruchssinn bewerkstelligt wird; ohne direkte Berührung können sie Fremde von den Ihrigen selbst dann nicht unterscheiden, wenn sie sich dicht nobeneinander halten: dieses Geruchsormögen entsehoidet dabei nur die eine Frage, und gibt nur die eine Antwort: "Etwas zu uns gehöriges n) alles was nicht zu ihnen gehört - ist etwas Fremdes, was durch seino Wirkung anf die Hummeln bei diesen letzteren auch cine putsprechende Reaktion herrvorruft: "ein fremdes Nest". "das Glied eines fremden Nestes », u. s. w. Unter dem Begriffe " des Ihrigen » verstehen die Hummeln alles, was den Geruch ihres Nestes besitzt, ohne dass sie auch nur die geringste Vorstellung von allen ülrrgen Eigenschaften des betreffenden Gegenstandes haben.

Woher kommt nun dieser für ein jedes gegebene Nest spezifische Gel'uch und von wem wird diese Substanz ausgeschieden? Auf den ersten Blick sprechen, wenigstens in Bezug auf die Hummeln. virle Custände zul Gunsten der Auffasiung von Berne, dass diese "Substanz " von einem joden der Indivilurn pines joden Nestes von gesellig lehenden Insekten ausgeschieden wird und eine Folge des Stoftwechsels in deren Organismus darstellt.

Genauere Untersuchungen beweisen jedoch, dass der Gedanke dieses Forschers nach zwei Richtungen hin einer Korrektur bedarf.

Erstens ist der Stoff, welcher den Glieder'n eines Nestes als Mittel dient, einander zu erkennen, keine besondere Substanz in Gestalt eines Produktes des Stoffweehsels. Zweitens hesitzen nicht alle Individuen die Fähigkeit, diesen Stoff auszuscheiden, sondern ausschliesslich nur die Weibchen.

Es hleibt nummehr noch diejenige "Sprache » der" "sozialen " Insekten zu

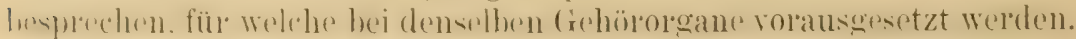
Ich beginne damit, die Ansichten der For'scher mitzuteilen, welche sich 
mit dieser Frage heschätigt hahen; dieselhen kionnen in Kürze wie folgt zusammengefasst werden :

Die Hummeln sind taub - wie die Bienen und die Ameisen; dies ist eine festgestellte 'Tatsache, welche keinem Zweifel unterliegt - sagen die Einen.

Die Hummeln haben eine Sprache, vermittelst welcher sie sich gegenseitig auf Distanz Witteilungen machen kïnn'n - auch dies ist ein' Tatsache, welche keinem Zweifel unterliegt - behaupten die Anderen.

Aus meinen eigenen Beobachtungen geht Folgendes hervor:

Ein Gehörorgan ist bei den Hummeln in der Tat vorhanden, allein dasselhe gibt nicht nur keine Veranlassung fül jene Erwägungen ülwo die hohe "Psychik » der gesellig lebenden Insekten, auf welche noch Wund in Bezug auf deren "Sprache" hingewiesen hat, sonder"n es gibt auch nicht die geringste Teranlassung, diose Inschten aus der Rieihe dor solitären Insekten auszuscheiden.

Die von mir gewonnenen Daten liefern den Beweis, dass bei den Hummeln die Flügel als Gehörorgan dienen (durch Kionsonnanz) und dass i h $l^{\circ}$ Ge hör eigentlich ein G e fühl a u $\mathrm{D}$ is tan $\mathrm{z}$ ist; vermittelst ihrer Flügel können die Hummeln ilıre Genossinnon ausschliesslich nur von eincr drohenden Gefahr in Kenntnis setzen und von nichts Anderem.

Diesor Umstand findet seine Erkbärung darin, dass die Hummeln zwa die Fähigkrit besitzen, Töne von sohr verschiedene Höhe und Klangfarbe zu produzieren, allein nur a f einen einzigen dieser Töne zu reagieren im Stande sind.

Es gibt sehr viele Nuancen in dem von den Flügeln der Hummeln hervorgehrachten Tone, welehe nutweder infolge dro Lage des Körpers zu der Linie dor Torwärtsheregung oder aber dadurh rentstehen, dass die Hummel aufwärts oder abwïts fliegt oder endlich infolge grosserer orler geringerer Erresung ete. Fast alle diese Nuancen kann ich richtig unterscheiden und erliennen : lie Hummeln selbst unterseheiden sie dagegen gar nicht und beachten sie in keiner' Weise.

Das gesammte Wörterbuch del " Hummolsprache ) beschränkt sich demnach auf drei “Wörter»).

\section{Wort}

1. "Gefahr "

2. Anweisung des Arbeitsplatzes

3. "Die Unsrigen " oder * Fremde »
Organ, welches das Wort Organ der Aufnahme ausspricht

$\begin{array}{ll}\text { Flügel } & \text { Flügel } \\ \text { Abdomen } & \text { Fühler } \\ \text { jeder Gegen- } & \text { Fühler }\end{array}$

Ein jeder Gegenstand des Nestes

In psychologiseher Hinsicht unterseheidet sich diese Sprache in liviner Treise von den mannigfaltigen Finudehtungen bei den solitianen Insecten. 
während einige dieser Einrichtungen bei diesen letzteren, wie zum Beispiel die Oroane der Erzeugung und der Aufinahme ron Tönen bei den Orthopteren, natürlich noch vollkommener erscheinen.

Indem ich die hier angeführten Thatsachen in Betracht ziehe, d. h. den Polymorphismus der Glieder eines Nestes, das Fehlen des mütterlichen Instinctes. die röllige Enfähigkeit zum Nachahmen und zur Verständigung untel einander durch solche Mittel, welche diese Fähigkeit bei der Herde und der Gesellschaft characterisieren, gelange ich zu der Annahme, dass das Zusammenlehen bei den sogenannten "sozialen "Insect'n weder einer Familie, noch einer Herde. noch einer Gevellschaft und noch weniger einem Staate entspricht.

Was stellt denn nun eigentlich dieses Zusammenleben bei den Insecten dar?

Es stellt eine spezielle Form der Symbiose mit dem Character eines deutlich a usgesprochenen Parasitis mus d a l:.

Die Gründe, auf welche ich diesen Grundsatz basiere, sind die folgenden.

$1^{\circ}$ Den Ausgangspunkt in der, Phylogenie der Bienen bilden, wie dies wissensehaftlich erwiesen ist, Folmen, welche durch parasitische Instincte und durch Parthenogenese characterisiert sind u. zw. die Gattungen Sphecodes und Halictus.

Was die Sphecodes-Arten betrifft, so sind diese Bienen nach der Ansicht von P. Marchal, Ferton, Alfken, Breitenbach, v. Butrel-Reepen u. a. m. entweder echte Parasiten, oder aber Formen, welchr im Begriffe stehen Parasiten zu werden.

Was die Gattung Halictus betrifft, deren Vertreter die untersten Stufen in der Phylogenie der gesellig lebenden Insecten eimmehmen und von einigen Fol'schern (wic z. B. r. Butrez-Respex) den Hummeln nahegestellt werden, so können wir auf Grund der Beobachtungen von Fabre $^{1}$ und Friese das Vorkommen von Parthenogenese bei dieser (rattung, ungeachtet der von Perez in seinem Werlichen "Les Aheilles " gemachten Einwände, als eine festgestellte Thatsache betrachten.

Fine " Geselligheit ". Welche kluch Parthenogeneso berlingt wird und derselben ihre Entstehung verdankt, stellt schon aus diesem Grunde allein eine Erscheinung dar, welche mit der wahren Geselligkeit, in welcher die Parthenogenese kejue liolle spielt, nicht identisch ist, sondren sich scharl von derselhen unterscheidet. Diese Erwïgungen geben nir umsomehr das Recht auf der gründlichen Verschiedenheit zwischen

${ }^{1}$ J. H. Fabre. Etudes sur la parthénogénèse des Halictus. Annales d. Sc. Natur., 9) sér. 
der Geselligkeit im wahlen Simno dieses Wortos und derjenigen Elscheinung zu bestohen. Welcho die auf Parthenogenes begründete sngenaunte Geselligkeit der Hymenopteren darbietet, als der Character der Parthenogenese hior nicht oinen Progress sondern einen liegress bedeutet.

$2^{\circ}$ Die Fluchtbarkeit der Weibchen beiden sogenannten "sozialen ) Insecten, welche bei freilebenden Formen eine ganz ungewohnte Esscheinung, für Parasiten dagegen sehr characteristisch ist. bildet rin neues Argument zu (runsten mreincr Auffastumg ron der Natur der Geselligkeit bei den Insecten.

Wir finden bei keiner einzigen solitälen Biene eine ähnliche El'scheinung wie diese Fruchtbarkeit, ebenso we wir ane solehe hei keinem Vertreter der wahren Geselligkeit im ganzen Thier'eiche finden, und eine solche Fruchtbarkeit erscheint bei den Insecten als das characteristischste Merkmal des Parasitismus.

Die biologische Bedentung der Erscheinung der Ablage riner grossen Anzahl von Eiern bei den Hummeln ist im Wesentlichen dieselbe wie diejenige der Ablage einer grossen Anzahl von Eiern hei den Parasiten : hier wie dort liegt die Bedeutung in der Nothwendigkeit vieler Opfer, um das Leben Weniger zu erhalten.

$3^{\circ}$ Die Instincte bei den sog. "sozialen "Insecten erweisen sich im Vergleiche mit denjenigen der solitälen Insecten als reduziert.

Zu solchen reduzierten Instincten gehört bei den Hummelweibchen natürlich auch das Benehmen bei einer das Nest und die Familie bedrohenden Gefahr: niemals greifen sie den Feind an und verteidigen sich sogar nur in seltenen Fïllen. (xwöhnlich verstedien sich die Wribchen rasch zwischen den Waben und verlassen dieselben nur, wenn sie auch hier beumruhigt werden, um sich im Monse des Nestes zu verkiriechen.

Die jungen (wie übrigens auch die alten) Weibchen fliegen, wenn sie das Nest verlassen, viel öfter und "gründlicher » vol demselben umher, um sich dessen Lage genau einzuprägen, als die Arbeiterinnen dieses thun, und dennoch haben sie mehr Mühe, ihr Nest bei der Heimkehr wiederzufinden, als die Arbeiterinnen: es ist dies eben, wie es scheint, nicht ihre spezielle Beschäftigung.

$4^{\circ}$ Das Nelvensystem erscheint im Vergleiche mit den solitären Bienen reduziert, und im Zusammenhange hiermit sind auch einige morphologische Merkmale reduziert.

Allerdings beträgt das Gewicht des Gehirnes in Vergleiche zu dem Gewichte des ganzen Körpers bei den solitïren Bienen ${ }^{1} / 400$, bei den Arbeiterinnen der Hummeln dagegen $1 / 1$, allein man darf' nicht vergessen, dass wir bei der Feststellung des Verhältnisses zwischen dem Ge- 
wichte des Gehirnes bei den sogen. "sozialen 》 und demjenigen der solitären Insecten keinesfalls das Gehirn der Arbeiterinnen, sondern dasjenige dor Männche'n und Weibchen helanziehen müssen, um genaue Resultate zu orzielen. Verwenden wir jedoch zu einer solchen Vergleichung das Mittel aus den Gehirnen aller drei Formen, so wird das oben "rwähnte Verhältniss folgende Abänderung erleiden: während das Fewicht des Gehirnes hei den solitären Wespen $1 / 400$ beträgt. wird dasjenige der sozialen Bienen $1 / 4000$ betragen, d. h. wir haben es dann mit eiriel ungeheuren lieduction gerade derjenigen Organe zu thun, nach weichen die Höhe der psychischen Entwicklung der Art bemessen wird.

Mit der Reduction der Instincte bemerken wir gleichzeitig, wie immer in den Erscheinungen des Parasitismus, auch eine Reduction der morphologischen Merkmale.

So bestehen bei der Bienenkönigin die zusammengesetzten Augen aus nur 8-9000 Einzelaugen, während deren normale Zahl 12-13000 beträgt. Die Königin besitzt keine Drüsen zur Ausscheidung von Wachs; an ihren Beinen befinden sich wedex Bürsten noch Körbchen. Statt dieser Organe, welche lee einigen ihrer froilehenden frefährten vorhanden sind, hesitzt sie, wie ein echter Parasit, ('nor'm entwickelte Eierstöcle' und es ist bei ihr ein neues Organ, das Receptaculum seminis, aufgetreten, welches sie zu einem an Hermaphroditen erimnernden Wesen gemacht hat: nachdem sie einmal in ihrem Lehen samen empfangen hat, mit welchem ihr leceptaculum gefüllt wurde, hefruchtet die Königin im Verlaufe von 3-4 Jahren die durch ihren Eileiter hindurchgohenden Eier selhst. Schr lehrreich und elenfalls nicht nach Gebühr gewüroligt ist die Thatsache, dass der [tntersehied in den morphologischen Eigenthümlichkeiten zwischen den Wribchen und Arbeiterimnen im Simne der Reduction von Merkmalen un in hedeutender ist, je vollkommener die Geselligkeit bei diesen gesellip lebenden Insecten entwickelt ist, cin Cmstand. welchel uns wieder'um auf den Gedanken an Parasitismus führt.

Ine Erscheinung dere verchiedenen Kasten selbst stellt ein unzweifolhaftes Product der regressiven Metamorphose dar, denn

a) sind die Organe des Geschlechtsapparates bei den Arbeiterinnen der Hummeln, Wespen und Bienen reduziert, worin diese Reduction auch bestehen möge, in welcher Lebensperiode sie auch auftritt und wozu sie schliesslich auch führt;

b) sind auch einige morphologische Merkmale reduziert, welche zum Theil mit dem Geschlechtsapparate im Zusammenhange stehen, zum Theile gan\% unahhängig von demselhen sind. jedoch für ein Lehen, wie es das Weibchen führen kann, eine Arbeiterin aber nicht zu führen im Stande ist, nothwendig erscheinen ;

c) ist eine gewisse Anzahl von Instincten reduziert; welche mit der 
geschlechtlichon Sphäle der Thätigkeit hrei den Wrabchen im Zusammenhange steht, bei den Arbeiterinnen dagegen mehl oder weniger vollständig verschwunden ist;

d) werden endlich selhst morphologische und psychologische Merkmale del Weibchen und Männchen mach Massswabe dessen reduziert, jo mehr ihre Abhängigleit von den Arbeitrinmen zunimmt. oder mit and'ren Worten, je tiefgehender ihr Parasitismus wird. Das Weibchen der Hummeln übertrifft die Arbeiterinnen noch in virlen Bezichungen und diese letzteren sind nicht sowohl für das Weibchen nothwendig, als dazu, damit die jungen Männchen und Wribchen zum Beginne des Winters ausschlüpfen können; bei den gesellig hebenden Bienen und Wespen hingegen stellen die Weilochen bereits rein parasitische und stark regressive Formen dar.

Endlich stellt eine Erscheinung, auf welche hartnäckig als auf cin Merkmal hoch entwickelter Geselligkeit bei den sogenannten. " sozialen » Insecten hingewiesen wird, nämlich " die Arbeitstheilung unter den Gliedern der Gemeinschaft ", durchaus kejne Arbeitstheilung dar und hat keinerlei Beziehung zu einer derartigen Vertheilung in oiner echten Gesellschaft.

Dasjenige, was wir bei diesen Insecten sehen, ist keine 'Theilung' der Arbeit unter Gliedern einer Gesellschatt, sondern eine Vertheilung der psychologischen Functionen.

Welch' eine wahre Geselligkeit im Thiereiche wir auch in's Auge fassen mögen, in der genannten Ausdehnung ihrer Entwicklung; von den ersten Momenten dor Entstehung bis zu den höchsten Formen des Zu11sammenlebens der Menschen, - nirgends begegnen wir und lïmmen wir anf

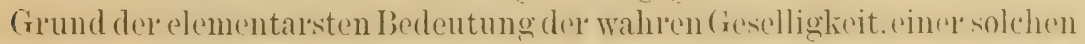
Organisation rieser lotzteren legegenen. Welehe duleh molphologisehe Eigenthümlichleiten der sie zusammensetzonden Glieder bedingt wäre (diejenigen Merkmale ausgenommen, welche das Männchen vom Weibchen unterschesiden), während hei den wesellig lebenden Insecten die Vertheilung der Arbeit eine directe Folge entsprechender Abändrungen der Organisation ist. Indem die Autoren von der Arbeitstheilung in den Insectrustaten sprechen, verogesen sir aus irgend welchem Grounde, dass sie nicht daroübre reden, worüller man allein bei Besprechung der Geselligkeit reden kiann, d. h. nicht über die Männchen und Wreihehen, aus welchon solche (rescllschafton zusammengesetzt sein müssen, sondern über solche Producte derselben, welche durch Parthenogenese entstanden sind und zum Parasitismus geführt haben, d. h. über die Kasten.

Aus allem diesem geht von selbst hervor, dass dasjenige, was fü die höchste Form der Geselligkeit im Tierreiche gehaltrn wird, wie sie angeblich nur bei den Hymenopteren und bei dom Menschen angrotroften 
wird, nicht nur nicht die höchste, sondern überhaupt keinerlei Form einer Geselligkeit in der wahren und einzig möglichen Bedeutung dieses Irortes darstellt; es stellt vielmehr eine selbständige Form del Svmbiose (eine hiologische Organisation) dar, mit scharf ausgesprochenen Ierlimalen des Parasitismus, oder, mit anderen Worten: dasjenige. was man bei den sogenannten "sozialen" Hymenopteren als Geselligkeit hezeichnet, ist eine spezielle $\mathrm{Ar}^{\circ} \mathrm{t}$ von Par a itismus, welcher sich in der Familie eines oded mehreres unter einander blutsverwandter Weilchen auf dem Boden der Parthenogenese entwickelt hat.

Es hleiht noch eine letzte Frage zu entscheiden: wenn das Zusammenleben der Insekten weder einer Familie, noch einer Herde, noch einer Gesellschaft sonder'n einer speziellen Form der Symbiose entspricht. in welcher Ibeziehung strht daun dieses Zusammenloben zu der Evolution der Geselligkeit im Tierleiche?

Zur Entrcheidung diesel Flage wird man in Verbindung mit meinem Urteil darüber, was das "Zusammenleben" bei den sogenamnten "sozialen Insekten") darstellt, die Lehre von dem Individuellen in der Biologie von Neuem durchsehen müssen.

Die Prüung der diesen Gegenstand betreffenden Daten haben mich zu der Ucher'zongung geführt, dass diese Individualitäten in er'ster Linie in zwei wrosse, prinzipiell von einander rel'schiedene Gruppen zerfallen; zu der einen dieser Gruppen rechne ich die Organ ismen, wie hoch auch die Stufe ihrer Complieation sein möge. Der enge Zusammenhang, in welehem dic einzelnen Teile bri diesem Tyjus von Individualitäten untereinander stehen, sowie das Vorhandensein eines Sensorium's, wie Spexcer denjenigen Teil des Organismus bereichnet. in welchem sich das Bewusstsein konzentriert - sind ihre grundlegenden Merkmale.

Zu der anderen Gruppor biologischer Individualititen rochne ich die A ssociationen von Organ ismen überhaupt (einerlei in welehen gegenseitigen beziehungen dirse letzteren zu cinander stehen mögen) und die Gesellschaften im Speziellen.

Die räumliche 'Trennung der einzelnen Bestandteile, sovie das Vorhandensein eines Senson ium's, dienen gleichzeitig als die chankiteristischsten Merkmale, wie auch als Eigentümlichkeiten, welche diesen Typus von dem ersten Typus von Individualitäten unterscheiden.

Die Versuche, welche gemacht worden sind, Im den radikalen und tiefgehenden Unterschied zwischen diesen beiden Typen (behufs Annäherung der (resellschaft mit den morphologischen () ganismen) zu besei-

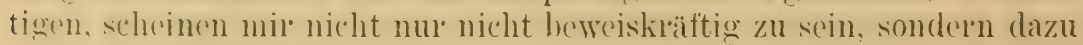
beizutragen, diesen Unterschied nur noch mehr hervortreten zu lassen.

Aus dem Obengesagten geht hervor, dass ich übcr die erste dieser beidem (rruppen. d. h. ülere die Gruppe dere Individualitäten der morpholozrischen ()reanismen hier nicht zu reden brauche, da alle Týpen dieser 
Individualititen mit der uns beschäftigenden Frage nicht das Gelingste zul tun haben.

Was nun die Gruppe der Individualitäten bi olog is che r O r g a n isation en betrifft, so werden wir uns mit ihl wohl zu beschäftigen haben, da so vir mir bekannt ist. in der einschlägigen Litteratur nicht von ihr die Rede ist und da eine genaue Feststellung derselben für die Entseheidung dor zu behandelnden Frage aine ungeheure Berleutung hat.

Ich beginne damit, dass ich in diesel Gruppe drei parallele, unabhängig von einander zur Entwicklung gelangte und trotz bisweilen seho enger Berührung unahhängig von einander bestehende Ro ihe n unterscheide. welche ihre Geschichte, ihre specifischen Züge und ihre, nur ihnen allein eigentümlichen Eigenheiten besitzen.

Diese Reihen mit ibren hauptsächlichsten Glienlorn sind die folgenden :

$$
\text { Biologische Organisationen. }
$$

\section{I-te Reihe}

a) der Kommensalismus

b) der Mutualismus

c) der Parasitismus

d) die biologische Organisation der sogenannten "sozialen" Insekten

\section{II-te Reihe}

a) das Paar

b) die Familie
III-te Reihe
(i) die Zusammenschaarung
b) die Herde
A. die Horde
B. die Gesellschaft
C. der Staat

Ich habe bereits betont, dass eine jede der von mir angegowenen Reihen von biologischen ()rganisationen durch Eigenheiten charaliterisiert ist, welche ihr allein eigentümlich sind. Die hauptsächlichsten derselben hestehen in Folgendem: Die erste Reihe unterscheidet sich dadureh von den beiden anderen, dass die biologischen ()rganisationen dieser Reihe stets zwischen T'ieren mit verschiedenen morphologischen In rekmalen zu Stande kommen, wobei die Eigentümlichkeiten. welehe diese Untersehiede bedingen, zwar schr bedentend (wie die Unterschiede zwischen den besonteren Kennzeichen der verschiedenen lílassen oder selbst Phylen), aber auch sehr geringfügig sein können, stets aber diejenigen Eigenheiten übertreffen, durch welche sich die Männchen von den Weibchen ein und derselben Art unterscheiden.

Diese Reihe, welche ich, um die Aufstellung neuer Bezeichnungen zu vermeiden, die symbiotische Reihe biologischer Organisation en nenne, wird noch dadurch charakterisiert, 1. dass der Nutzen soleher ()rganisationen meistens nicht nur einseitie ist, sondern sogar auf hosten der einen von den heteiligten Seiten erreicht wird, und 2. dass 
die gesammte Tïtigkeit der Tiere dieser Reihe sich auf solitäre Instinkte beschränkt, wobei hier weder altru istische Emotionen, noch Anlage zu Traditionen, noch Fähigkeiten zur Nachahmung zur Beobachtung kommen.

Die zweite Reihe, welche am Passendsten als geschlechtliche hi ologische organisatione n hezeichnot werden könnte, kommt, im Gegensatze zu der ersten Reihe, stets zwischen Individuen ein und derselben Art zu Stande, deren morphologische Eigenheiten nicht über die Eigenheiten, welche das Männchen rom Weilichen unterscheiden, hinausgehen. Diese Organisation ist für alle Individuen der gegebenen Art und des gegebenen Bündnisses stets von Tutzen. Dieser Organisation liegt der geschlechtliche Instinkt in Verhindung mit altruistischen Emotionen zu Grunde, wozu bei den höheren Tieren noch die Befähigung zu Traditionen und zur Nachahmung hinzukommt.

Die dritte Reihe, für welche die Bezeichnung als gruppenförmige oder sozialebiologische organ isationen am Nächsten liegt, unterscheidet sich von der ersten Reihe 1. dadurch, dass sie zwischen Individuen zu Stande kommt, deren morphologische Eigentümlichkeiten n i e m a ls über diejenigen h i n a usg e he n, welche das Männchen rom Weilochen unterscheiden, und 2. dadurch, dass diese Organisation. möge sie auf einer noch so tiefen Entwicklungsstufe stehen, stets für alle Individuen der gegehenen Art und der gegebenen Vereinigung von Nutzen ist, endlich 3. dadurch, dass diesen biologischen Organisationen die Befähigung zur Nachahmung und, hei den höchsten Typen des Tierreiches, auch zur Tradition zu Grunde liegt.

Von der zweiten Reihe unterscheidet sich diese biologische Organisation dadurch, dass sie sowohl für ihre Genesis wie auch für ihre Evolution der geschlechtlichen Instinkte nicht bedarf; infolgedessen kann die Gruppenorganisation hei Tieren ganz fehlen, während die Bildung der Familie, die typischste Organisation, stattfindet. und umgekehrt: die Gesellschaft kann entwickelt sein, wïhrend die Familie, selbst in ihrer primitivsten Form, fehlt.

Ausserdem unterscheidet sich die dritte Reihe von der zweiten noch dardurch, dass ihr ein Instinct zu Grunde liegt, welchen man am passendsten als den Gruppeninstinct oder sozialen Instinct bezeichnen könnte.

Wir treffen diesen Instinct bereits auf der ersten Stufe dieser Reihe von biologischen ()rganisationen an. als welche ich die Znsammenschaarungen von Thieren auffasse.

I ieses ist der liestand der Individualitäten in den biologischen Organ isationen und in deren Reihen.

I als vorgeschlagene Schema giebt uns eine ganz bestimmte Antwort auf clie aufgeworfene Frage: in welcher Beziehung steht das Zusammen- 
leben der sogenannten "sozialen " Insecten zu der Fvolution der (reselligkeit im 'Thierreiche? - Hierauf giebt es nur die eine Antwort: dieses Zusammenleben steht ingar keiner Beziehung zu dieser Evolution, mit welcher es überhaupt gar nichts zu thun hat.

Nachdem wir auf diese Weise diejenige Stelle bestimmt hahen, wo die Soziologie sich an die Biologie anschliesst, erlangen wir nummehr die Möglichkeit, nicht nur alte Verirrungen, welehe durch oine unrichtige Qualification der (ieselligleit hei den sogenannten "sozialen s Insecten entstanden sind, zu beseitigen, sondern ausserdem noch den Zusammen-

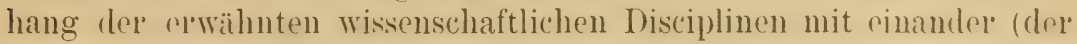
Soziologie mit der Biologie) festzustellen.

Dieser Zusammenhang wude bisher nur im (iebiete der allgemeinen biologischen Gesetze erllickt, welche in gleicher Weise sowohl die biologischen Organisationen als auch die morphologischen ()ronisationen legieren, d. h. der Gesetze über' die Ver'theilung de l' Functionen, die natürliche Auslese und den Kampf um's Dasein, von welchen bisher in Fragen der biologischen Soziologie ausschliestich die Rede wal.

Jetzt können wir diesen Zusammenhang nicht nur innerhalh des engeren Gebietes der alle Reihen von Individualitaten der biologischon Organisation umfassenden biologischen (resetze feststellen, sondern wir kïnmen auch, was noch viel wichtiger ist, don Zusammenhang der (r'wähnten wissenschaftlichen Disziplinen im Gebiete der nïchstliegenden speziellen Gesetze fixieren, welche nur auf die in Rede stehende (IIIte) Reihe der biologischen Organisationen eine Anwendung finden; hierher. gehören zum Beispiel die Gesetze über die Nachahmungen und Traditi on en, als Factoren der Geselligkeit.

Ihre Bedentung und Rolle in der (ienesis und Evolution der mensehlichen Gesellschaft begann erst im Verlaufe des letzten Dezenniums gebührend gewürdigt zu werden ${ }^{2}$, withrend sie bei den gesellig lehenden Thieren. wenn man von den fragmentarisehen Bemerkungen Moratis's, Hutsox's und weniger anderer absioht, noch heute ilues Erforschers har'en.

${ }^{1}$ G. Tarde. Les lois de l'imitation. Paris, 1895. 


\title{
Sur une nouvelle espèce du genre Sipunculus.
}

\author{
Par M.-A. HÉRUBEL (Paris).
}

Avec 1 figure dans le texte.

1I. Ch. Gravier a bien voulu me confier les Géphyriens quil a recueillis au couls's de sa mission dans la Mer Rouge. Ie ne veux aujourd'hui m'occuper que d'un Siponcle très curieux de cette collection (S'imunculus Gravieri nov. sp.) (lont je vais donner une description complète.

L'animal a été trouvé près de la ville d'Obock, à l'extrémité d'une digue, au niveau des plus basses ea $u x$, dans du sable vaseux, fin, mais semi-consistant, avec des Bulanoylossus et une espèee de Crustacé non encore déterminée. Des herbier's abondants sont dans le voisinage. L'espèce y est rare.

L'aspect extérieur, sauf pour les tentacules, est très semblable à celui de S. mudus Linné (papilleśs en V'sur l'introvert. quadrillage de la peau, irisation, etc.). La taille moyenne est de $19^{\mathrm{mm}} .5$; le diamctre d'environ $1^{\mathrm{mm}}$; l'introvert ne mesure que $\mathbf{1}^{\mathrm{cm}}, 5$. Il y a quatre muscles rétracteurs de longueur égale (2"m.8): les deux dorsaux sont plus développés que les ventraux. Chacun des quatre prend naissance aux dépens de trois bandes musculaires longitudinales du tégument. On compte en tout 34 de ces bandes. Je n'ai pas trouvé de canaux cutanés.

La bouche est axiale, tout entouréc de tentacules digitiformes innombrables. Ils s'insèrent sur l'anneau péripharyngien de Pozi creusé dans la masse musculaire de l'extrémité antériempe de l'introvert. Remarquons ('n passant que, comme cette masse, d'unc part, perd en largeur ce qu'elle gigno en hauteur, l'origine supérieurro des quatre rétracteur's est très audesious de la couronne tentaculaire; et, comme. d'autre part. Ir cerveau est, chez tout Sipunculicle, fiché dans l’angle formé par lés rétracteur's dorsaux, il est, lui aussi, situé tres bas. L’appareil digestif ne nous retiendra pas: il est pourvu de brides musculo-conjonctives rayomnantes, d'un muscle de la spire; le rectum n'a pas de cocum. Le système nerveux ne présente pats non plus de particularités. I)isons seulement que le corveau ne porte pas de houppes vibratiles et que le cordon ventral se termine par un renflement ganglionnaire.

Tous cescaractènes śloignent peu, en somme, du type normal du genre. Mais rn voici d'autres tout a fait aberants: ils intéresient le canal tentaculaile de Por., l'appareil génital et les néphridies. 


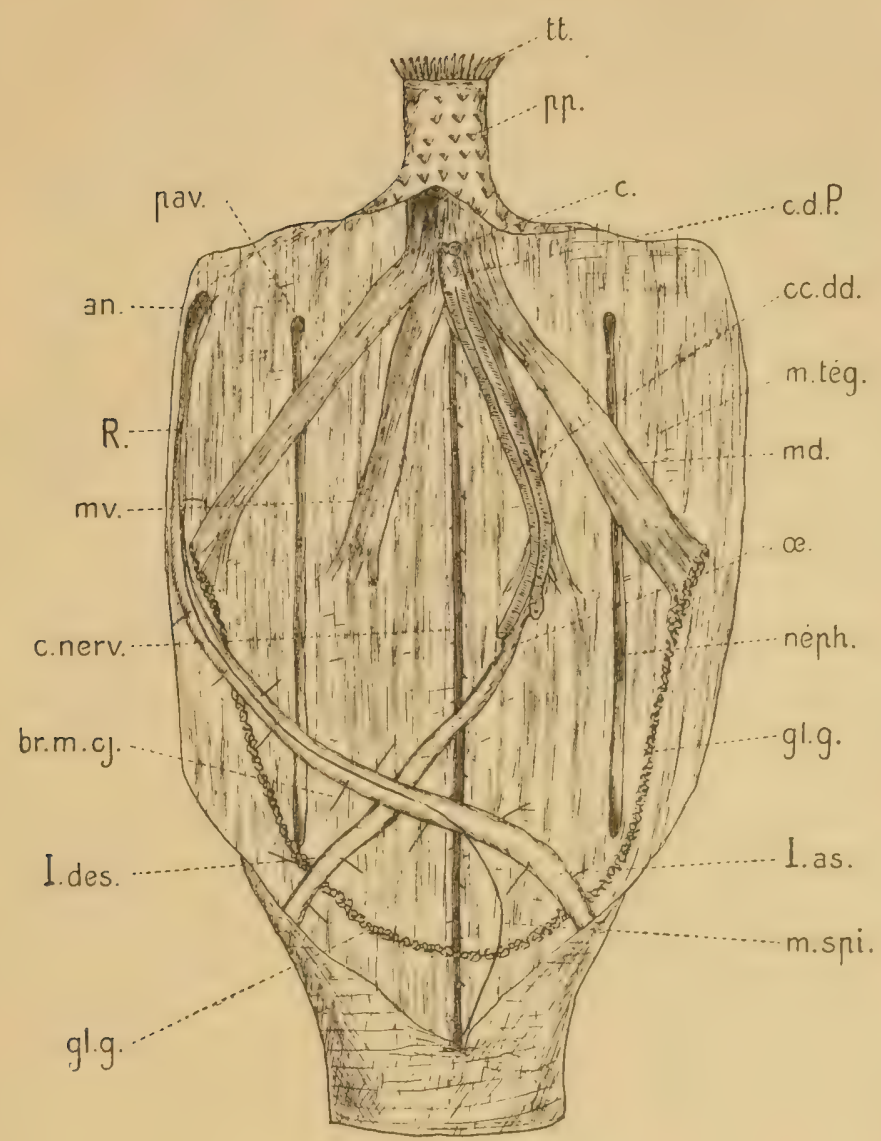

L'animal est fendu dorsalement. Le tiers supérieur seul a été représenté.

tt., tentacule, - pp., papilles de l'introvert, - m. tég., muscles tégumentaires, $-\mathrm{m}$. d., muscle rétracteur dorsal, $-\mathrm{m}$. v., muscle rétracteur ventral, - - ., œsophage, $-\mathrm{I}$. des., intestin descendant, - I. as., intestin ascendant, $-R$., rectum, - an., anus, - m. spi., muscle de la spire, - br. m. cj., brides musculo-conjonctives, - Cd. P., canal de Poli médian et dorsal, - cc. dd., les deux coecums dorsaux, - C., cerveau, - c. nerv., cordon nerveux ventral, - gl. g., glande génitale, néph., néphridie, - pav., pavillon néphridien. 
En général, il y a deux canaux tentaculaires, un dor`sal et un ventral, comme chez S.mulus, ou bien un seul dorsal, comme chez Phascolosoma. L'appareil tentaculaire de S. Grarieri, lui, est construit sur un autre plan. Il commence par un canal dorsal unique et médian. Mais, après son passage sous le cerveau, il se bifurque en deux branches latérales dorsales qui courent le long de l'cesophage et laissent la face rentrale de celui-ci completement libre. La branche gauche est plus dévelopjée que la branche droite. Leur surface externe est hérissée de digitations très courtes et très minces. Enfin, l'csophage ot les deux branches sont enserrées par' les deux rétracteurs dorsaux: le tout est réuni par un mésentère très dense.

L'appareil génital décrit une grande anse à concavité tournée ver's l'extrémité supérieure de l'animal. Cette anse est fixée par ses deux bouts à la base des rétracteurs dorsaux. De là, elle gagne attachée au tégument la face ventrale, dépasse les néphridies, pourtant très longues, et passe, comme une sorte de pont, au-dessus du cordon nerveux. Chez les animaux étudiés, qui avaient été capturés en mars, les oufs n'étaient pas encore mûrs, mais ils étaient tout à fait détachés de la glande formatrice.

Les 2 néphridies mesurent $3^{\mathrm{cm}}$ de long. Je n'en ai jamais vu de semblables chez les Sipunculides. Elles sont attachées, sur presque toute leur étendue, au tégument. Au pavillon, de nature musculo-conjonctrve et fortement cilié, succède un canal étroit, qui, à mesure qu'il descend, s'élargit et bientot devient glandulaire. Il aboutit dans le cul de sac terminal de la néphridie. De ce même cul de sac part' un second canal glandulaire, intimement accolé au premicr par sa face dorsale et accolé au tégument par sa face ventrale. Il remonte jusque sous le pavillon et là s'ouvre au dehors par le porc excréteur entouré d'un sphincter. En un mot, cette néphridie est un tube ouvert a ux de ux bouts et recourbé sur luimême en une sorte de $\mathrm{U}$.

Recherchel les affinités de cet être est chose sans doute difficile. Mais, le fait que ses tentacules sont digitiformes, tend à le rapprecher d'une certaine catégorie de Siponcles. Nous le placerons done à la suite de ceuxci, juste après Sipunculus mundanus Sel. et Bülow.

En parlant de la néphridie, j'ai dit que c'était un tube ouvert aux deux bouts et j'ai souligné ces mots. En effet, cette disposition est le propre des Anuélides en général. Je me contente de signaler ce rapprochement, sans insister davantage. 


\title{
Note sur la Zoologie médicale mexicaine.
}

\author{
Par le Dr J. SANCHEZ (Hexico).
}

Deux faits, observés récemment dans la République mexicaine, ont montré l'importance des relations qui existent entre la Zoologie et la Médecine. Ils seront certainement de nature à intéresser l'assemblée à laquelle je les présente en ma qualité de Délégué cłu Gouvernement mexicain it la VI $\mathrm{I}^{\mathrm{mm}}$ session du Congrès International de Zoologie ì lierne.

Les deux faits en question sont:

$1^{\circ}$ L'extinction presque complète de la fièvre jaune.

$2^{\circ}$ L'extinction complète de la peste bubonique.

Ces deux résultats ont spécialement pour base des données fournies par la Zoologie.

La fière janne. - Lor'sque l'illustre HuмвoLdт parcourut l'Amérique, comme il le raconte dans son "Voyage aux Régions equinoxiales du nouveau continent». il apprit de la bouche des indigeness de certaines régions, que les maladies paludérnness se transmettaient par les piquires envenimés des Moustiques qui se trouvent r'n grande quantité dans crs régions.

Récemment, on a décourert que le Doustique nommé Steyomyiu fusciata transmet, à l'homme bien portant, le germe de la fièvre jaune en le piquant après avoir absorbé le sang d'un individu malade. Ces faits amenent ì la conclusion que, pour empecher la propagation de cette terrible maladie. l'essentiel est d'empecher la multiplication ot la propagation des agents qui semblent seuls pouvoir la produire, c'est-à-dire les Moustigues connus sous le nom de s'tegommin frisriatu. Si l'on olutient ce résultat, on peut logiquement affirmer que la maladie commencera à diminuer et arrivera plus tard à disparaître complètement.

Les conditions biologiques de cet Insecte sont les suivantes.

Ses larves, comme celles de tous les Diptères, naissent et se développent dans l'eau et y vivent jusqu'à l'âge adulte (imago). Contrairement à ce que l'on supposait, les Moustiques de l'espèce Stegomyia fasciata passent leur état larvaire dans l'eau claire, tandis que les autres espèces préfèrent généralement les eaux impures et stagnantes. Mais les larves 
ont toujour's besoin de respirer l'air atmosphérique, puisque leur respiration est aérienne. Leur respiration se fait au moyen de trachées, en appliquant à la surface de l'eau un tube spécial dont elles sont pourvues, tandis que les Poissons et les autres animaux qui vivent toujours plongés dans l'eau respirent au moyen de branchies, profitant de la sorte de l'ail" qu'elle contient.

S'il était possible d'empêcher d'une facon quelconque les larves des Moustigues d'arriver' a la surface de l'eau, elles mourraient toutes en peu de temps par asphyxie. Tel est le résultat auquel on cherchait à arriver, et qui a été obtenu en versant sur l'eau de l'huile minérale ou pétrole cn quantité suffisante pour former une légère couche superficielle. Ce même procédé peut servil aussi pour les récipients que l'on emploie dans les maisons. L'eau étant, de cette manière, isolée de tout contact amosphérique, les larves qu'elle contient meurent aussitot et, par ce procédé très simple, on évite leur multiplication.

En ce qui concerne la propagation de la maladie, on l'évite en isolant les malades attaqués de fièro jaume et en les logeant dans des appartements dont les portes et les fenitres sont pourvues de toiles métalliques assez fines pour permettre à l'air de passer, tout en empèchant les Moustiques mon contaminés d'entrer et cerux qui ont sucé le sang des malades, de sortir.

Voilà en résumé ce qui a été découvert et mis en pratique par les différentes commissions que le Gouvernement de l'Amérique du Nord a envoyées pour étudier la fièvre jaune à la Havane et dans d'autres r'égions. C'est donc grâce à cette importante et curieuse découverte, qu'il est déjà possible de délivie's l'humanité du terrible fléau qui, pendant plusieur's années, a coûté la vie à des milliers de personnes.

Dans la République mexicaine, ainsi que dans d'autres pays où ce fléau règne sous forme cndémique ou épidémique, on adopte pour se préserver de la fièvre qui est connue vulgairement sous le nom de vomissement noir, des procédés identiques à ceux que l'on vient d'indiquer. De cette manière, on a presque obtenu la disparition de la maladie dans quelques régions (Vra-Cruz, Tehuantrpec), et sa disparition complete dans d'antres (Laredo, ete., ete.). Nous pourons donc nous attendre comme l'amnonçait à l'Académie nationale de Médecine de Nexico, l'illustre Président du Conseil supérieur de Santé, le Dr E. Liciaga, à obtenir dans quelques années le résultat complet qui est si vivement désiré par les Mexicains.

La peste bubonique. - Cette terrible maladie, qui visite presque toutes les contrées du monde, est connue depuis longtemps. Etant donné sa forme épilémique. alle produit une panique qui ressemble beaucoup a celle que produit le Cholera morbus et autres maladies contagieuses qui 
éprouvent si cluellement l'humanité. Il est rare qu'elle fasse son apparition dans la Républigue mexicaine, mais l'année dernière, cropendant, elle apparût dans le Port de Nazatlan, importée par un vaisseau ayant à bord des malades renant de San-Francisco de Californie, oì selon les rapports du Conseil supérieur de Santé, elle rógne endémiquement depuis quelque? temps.

Cette espèce de typhus, dont le symptôme principal, dès que la fièvre pal'aît, est celui des tumemrs inflammatoires suppuratives, est causé par les Rats, et le germe se transmet par leur sang aux Puces qui vivent sur eux. Les Puces, à leur tour, contaminent l'homme, surtout quand les Rats meurent et qu'elles les abandonnnent pour se loger ailleurs. Les Rats étant beauroup plus gros que les Moustigues, leur dostruction est bion plus facile, surtout depuis que l'on comnât les différents germes transmissibles de l'un à l'autre et ploduisant la mor't.

Parmi les moyens adoptés à Mazatlan pendant l'épidémie de peste bubonique, le principal fut la destruction des rongeurs. Une prime fut accoldée aux persomnes qui présenteraient des cadaves de Rats. On se servit également des moyens dont la science dispose aujourd'hui.

Dans les cas que je viens de citer, la Zoologie a été un puissant auxiliaire de la Médecine et l'on pour'rait encore mentionner plusieurs cas analogues, comme l'a montré le Dr Branchard de Paris dans son intéressante conférence à la séance d'inauguration du Congrès Zoologicyue te Belne.

On comprend chaque jour davantage l'importance de l'étude de l'histoire naturelle dans les Ecoles de Médecine. Il est heureux, pour nous Mexicains, que le Inecteur de notre Feole Nationale de Nédecine soit en mème trmps Président du Conseil supérieur de Santé. Grâce à son autorité et à ses conseils, l'illustre Président qui gouver’ne le Mexique saura accordel à ces études la protection dont elles ont lesoin, comme il l'a toujours fait pour tout ce qui est "Ordre et Progrès ». 


\title{
Ueber den Parasitismus der Larven von Hypostena seti- ventris Macquart (Diptera) im Innern der Larven von Tettix bipunctatus Linn. (Orthoptera).
}

\author{
Von W. N. RODZIANKO (Poltawa).
}

Es wurde mehr'mals angezeigt, dass Tettix Schronki Fieher die Lar've von Tettix bipmetatus Linn. (T. temuicornis .). Sahlherg) ist. So sagt darüber L. R. Mertr-Dür (indem er an den Resultaten der Beobachtungen A. Yerstx's sich hält) in seiner systematischen Uebersicht der schweizerischen Geradtlügler ${ }^{1}$. C. BRunner von WatTEnwy bemerkt auch, dass nach Ph. Chr. ZelLen's Angahe (veröffentlicht im Jahre 1\$56) T. Schranlii nichts anderes ist, als die Larve von T. bipunctatus ${ }^{2}$.

Vor einigen Jahren, während meines Aufenthaltes in der Stadt Lubny (Gouvernement Poltawa, Süd-Russland). machte ich den Versuch. den T. bipunctutus durch Zucht aus seiner Larve (d. h. aus dem T. Schrenti) zu erhalten.

Für dieson Zweck sammelte ich im Frühjahre (im März) eine Anzahl von $T$. Schranki; dann brachte ich diese Larven in ein Glas und ernährte dieselben mit den Blïttern verschiedener Pflanzen. hauptsiichlich mit denen der Gräser (Graminex). Die Zucht des T. bipunctutus aus seiner Larve ist nach meiner Frfahrung gar nicht schwer. Seinerzeit veröffentlichte ich die Frgehnisse meiner Beobachtungen über die Verwandlung des T. bipunctatus, sowie üher seine Sitten ${ }^{3}$. In meiner Vitteilung fehlt aher die Angabe, dass ich gleichizeitig auch den Parasitismus der Larven "'iner schwärzlichen oder dunkelgrauen Tachima-Art in den Larven von T. bipunctatus (d. h. im Inner'n des T. Schranki) beobachtete.

${ }^{1}$ L. R. MEYER-Dür. Ein Blick über die schweizerische Orthopteren-Fauna. Neue Denkschriften der Allg. Schweizerischen Gesellschaft für die gesammten Naturwissenschaften, Bd. XVII, pag. 22. 1860.

${ }^{2}$ C. Brunner von Wattenwyi. Prodromus der europäischen Orthopteren, pag. 237. Leipzig, 1882.

${ }^{3}$ W. N. Rodzianko. Bemerkungen über die Orthopteren. "Verhandlungen ("Trudy ") der naturforschenden Gesellschaft zu Charkow ", Band XXII. Charkow, 1889 (in russischer Sprache). 
Der verstorbene r'ussische Entomologe IV. A. JARoschewsky in Charliow beschäftigte sich damals mit der Erforschung der Dipteren-Fauna des europäischen Russlands ' el hestimmtr die von mil el'zogene Fliegre als Hypostena setiventris Macquart.

Nach meinen Beobachtungen lebt im Inne'n jorler befallenen Lave von $T$. bipunctatus nur eine Larve von $H$. setiventris.

Die parasitische Made schlüptt im Frühling aus dem Loibe ihres Wirthes aus und verwandelt sich damn in ein längliches brames Tönnchen (Puparium), dessen Wand, wie bekannt, weitel nichts als die erhärtete Madenhaut ist: in diesere Hülle liegt wie in cinem Cocon die zarte Puppe der Fliege.

Tachdem die Lalre des schmarotzenden Zweiflüglers die Lalve von Tettix bipunctutus verlassen hat, geht die letztgemannte Larve zu Grunciu; niemals verwandelt sie sich in ein vollkommenes Insekt.

Die Dauer des Puppenstandes bei $H$. setiventris ist kurz.

Ich habe bemerkt, dass die Larve von $T$. bipunctatus, die in ihrem Leibe die parasitische Fliegen-Made hesitzt. sich durch den monströsen (gekrümmten) Vorderrücken (Pronotum) unterscheidet.

Die Larven von Hypostena setiventris kommen nur in den Larven von $T$. bipunctatus vor, niemals im Innern der geschlechtsreifen Insekten dieser Art.

Der Parasitismus der Maden vieler Tachinc- Irten in den Raupen verschiedener Schmetterlinge ist rine gemeine und allbekimnte Tatsache, die der Gegenstand mehrerer Beobachtungen war.

Der Parasitismus der Maden der genannten F'liegen im Innern der Larven der Insekten anderer Ordnungen und unter andern der Geradflügler (Orthoptera) wurde weniger beohadet und ist auch weniger hekiannt. Es freut mich, dass ich jetzt in der Lage hin, noch rtwas" hezüglich dieser Erscheinung zu publizieren.

' W. A. Jaroschewsky. Verzeichnis der Dipteren Charkows und seiner Umgebung, mit den Angaben ïber ihre geographische Verbreitung im europäischen Russland. 8 Teile. "Verhandlungen ("Trudy ") der naturforschenden Gesellschaft zu Charkow ", Bände X-XX, Charkow, 1876-1887 (in russischer Sprache).

2 W. N. Rodzianko. Ueber den Parasitismus der Larven von Roselia antiqua Meigen im Innern der Larven von Forficula Tomis Kolenati. "Horæ Societatis Entomologicæe Rossicæ ». T. XXXI, St. Petersburg, 1897 (russisch mit einem Résumé in deutscher Sprache). 


\title{
Salmon Migration.
}

\author{
Bỹ J. W. Wiluis BUND (London).
}

With 3 Tables.

In the course of the last five years observation on the movements of Salmon in the Severn disclose a state of things not in accoldance with the irloas generally received of the life history of the Salmon. Therefore they are deselving the most careful consideration both from a biological and a commercial point of view.

It should be premised that in $18 \cdot 10$ a large quantity of water $45.400,00()$ Litres a day was taken from the Severn to supply Liverpool. This and a series of dry seasons prevented the fish from ascending the river until the middle of Xovember. so the spawning season instead of, as formerly, extending from October to March has bern cut down to the time between November and January practically at least a third. 'The result has been a series of bad spawning seasons. This led to the falling off in the takes between 1896 and 1900 of salmon. The modes of capture have practically remained the same but the number of fish caught decliued from 18,000 to 8,500 . Each year the number of fish spawning has also decreased.

Some other noticeable features should be mentioned :

a) 'The Smolts descending appear' to be getting fewer and fewer.

b) The Grilse have almost wholly disappeared.

c) The large fish for which the Severn used to be famous have also disappeared.

(l) Arerage weigth of the Salmon caught remains about the same. only varying 907 grammes in 10 years.

It is imposible to give any figmes as to the actual number of (a) spawning fish (b) decreasing number of Smolts. but all the Watcher's agree that the numbre of "redds " they can count are fewer than formerly and that no Salmon can be seen on Fords where they always used to be. It is also agreed that the number of descending Smolts appear to be much smaller while the number of those aceidentally caught are eretainly less than formerly. From these facts it is not an unfair inference that the number of spawning Salunon hats fallon of and therefore the number of deseending Smolts has done the same. 
The next point, that the Grilso have practically loft the river, is certain. It is proved by the fact that the average weight of the fish has only varied 907 grammes and has gone up in the rrar of largest take instrard of decreasing as it always used to do with the largest take. All the fishermen agree that they have caught no Grilse. The fishmongers also say they get no Grike from the Severn; what fish they cot ane large, mature fish. This point may therefore be regarded as established.

The large fish, that is, fish over 15,875 grammes have disappeared. A record of those caught has been kept for some year's. For the last five years s is the highest number. Table III shows the number for 10 years.

\section{TABLE 1}

Number of Salmon caught in the Severn in the 10 year's 1894-1903.

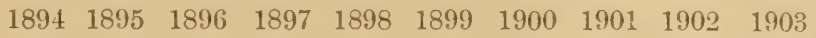

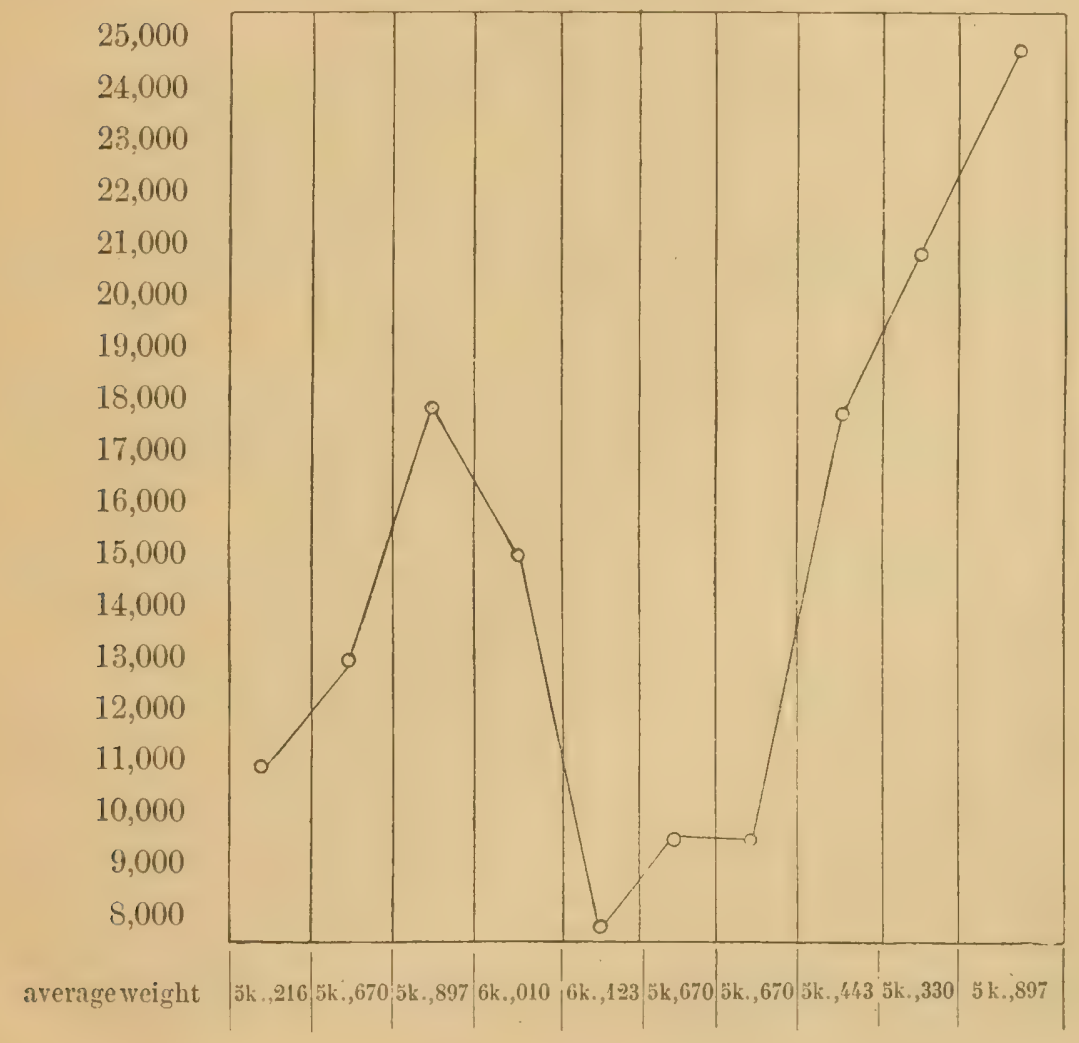




\section{TABLE II}

Weight of Salmon caught in Severn for the 10 years 1894-1903. $\begin{array}{llllllllll}1894 & 1895 & 1896 & 1897 & 1898 & 1899 & 1900 & 1901 & 1902 & 1903\end{array}$

$6 \mathrm{k}^{\circ}, 123$

$6 \mathrm{k}^{\circ}, 010$

$5 k^{\circ}, 897$

$5 \mathrm{k}^{\circ}, 783$

$5 \mathrm{k}^{\circ}, 670$

$5 \mathrm{k}^{\circ}, 556$

$5 \mathrm{k}^{\circ} .443$

$5 \mathrm{k}^{\circ}, 330$

$5 \mathrm{k}^{\circ}, 216$

Fish caught

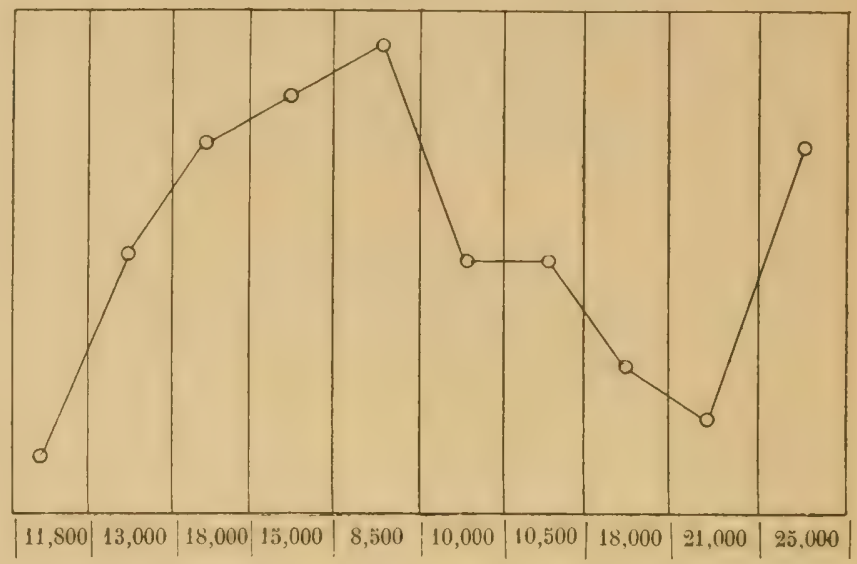

The Severn Salmon are therefore now confined to merlium sized fish 4536 grammes to 9072 grammes fish. that have migrated twice or three times from the sea. The points on the migration for consideration therefore are:

1. What becomes of the fish bred in the Severn on their first return to fresh water from the sea: as Grilse they do not come up the Serern, do they go up any other river or do they for some reason spend their Grilse life in the sea instead of returning to fresh water?

2. Why do the older fish and not the Grilse ascend the Severn if they have, as is generally supposed, been hack to the fresh water as Grilse?

Some vely important questions arise on these facts (1) what has become of the Severn Grilse? Do the fish for some reason miss the Grilse stage altogether and only return as mature fish. This is possible but it would be an unheard of fact in Salmon history: or do the Severn bred grilse return to some other river. 'This seems the more likely explanation but it is very difficult to say to what river they return as during the time the Grilse have been absent from the Severn it does not appear from the evidencer available that in any of the river's Howing into the Severn Sea there has been a larger number of ( irilse than usual. They may go further afield but so far no river has been heard of with an abnormal number of 
Grilse during the time the Serern (irilse have fallen of. All one can say with certainty is, that for some reason the Grilse have left the River Severn.

\section{TABLE III}

Number of Salmon over $15 \mathrm{k}^{\circ}, 875$ taken in Serern letween 1s94-1903.

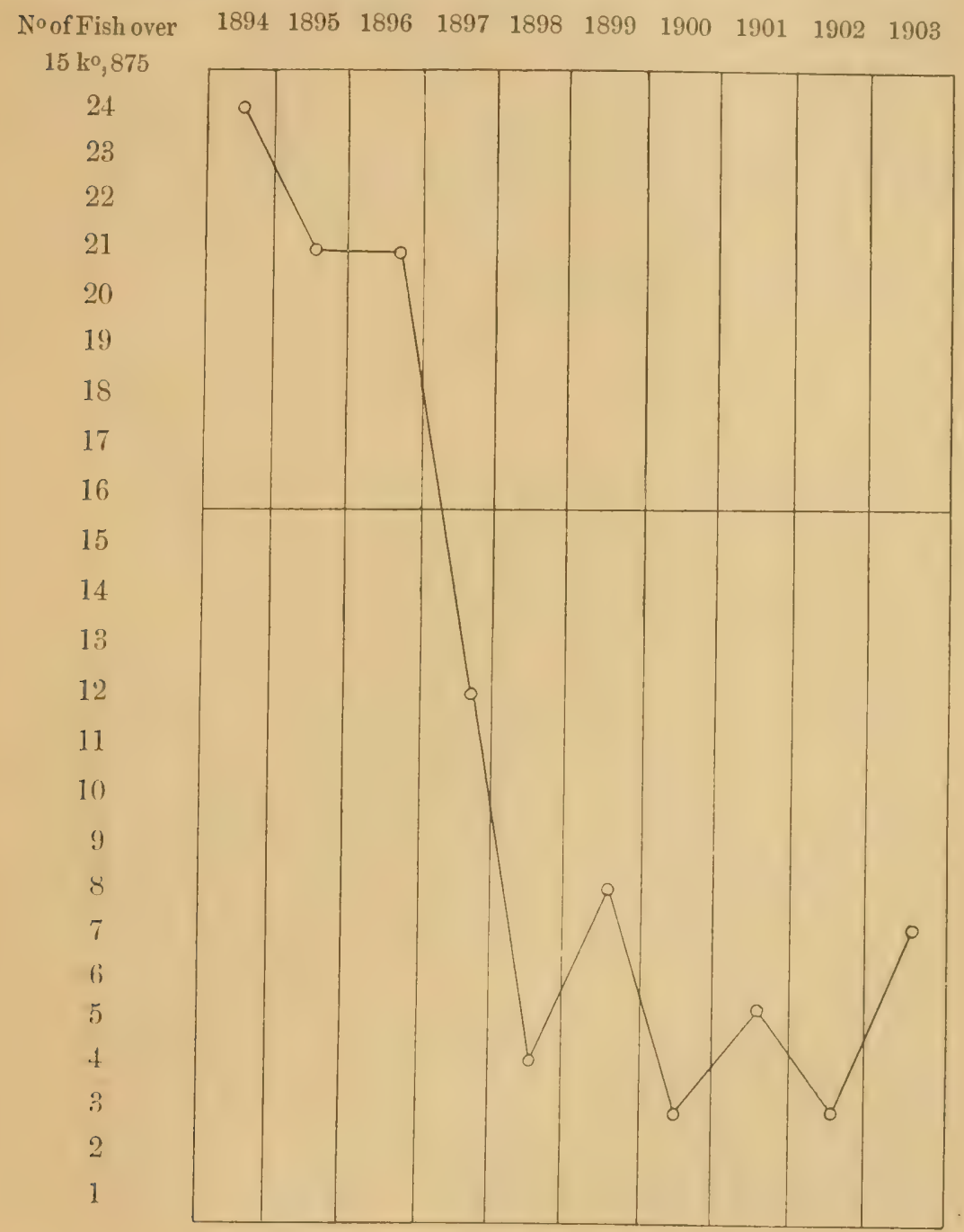


If any answer, could be given to this question it might be possible to answer the next. Why do only mature fish resort to the Severn? The number so resorting are in excess of the number bred in the river. Why do they come and what is the inducement?

That the fish caught in the Severn are all nearly of a size will be seen from Table II which gives the average weight of the Fish during the last 10 years. It will be noticed it has kept fairly constant only varying 907 grammes while the takes have varied from 25,000 to 8,000 , thus showing that the fish that are caught are mostly of the same size, mature fish. of about the same age.

The disappearance of large fish over 15,875 grammes is shown in Tahle III. Here again it is rlfticult to give any satisfactory explanation. The important point however is that Salmon appear only to frequent the Severn as mature fish. If this is so, and so far as one can judge it appear's to be so, it will modify to a very great extent the principles on which Salmon preservation and cultivation are based.

\title{
Sur un Cestode du Rhombus maximus.
}

\author{
Par le $\mathrm{D}^{\mathrm{r}} \mathrm{J}$. IVERUS (Lovisa).
}

En examinant le contenu des intestins de Turbots du Golfe de Finlande, dans le but de déterminer leur genre de nourriture, j’ai été bien surpuris de trouver des Cestodes hien développés dans un Rhombus maximus de $0^{\mathrm{ko}}, 9$. Ayant fait une incision dans l'estomac, j'en vis sol'tir l'extrémité d'un Cestode. Le contenu de l'estomac fut mis dans de l'alcool à $80 \%$. Il renfermait deux Acerina cermu, l'une de 10,2 cm., l'autre de 11 cm. et de nombreux fragments d'un Poisson de la même espece. Ce fait montrerait que les Arerina avaient constitué la nourriture principale du 'T'urlot.

L'estomac renfermait, en outre, des Cestodes. En les examinant, je reconnus qu'il s'agissait du Botriocephalus cordutus. Nalheureusement, su' trois exemplaires, un seul était complet.

L' exempla ire $\mathrm{n}^{\circ} 1$, complet, mesurait $38,4 \mathrm{~cm}$. de long sur $4,3 \mathrm{~mm}$. de large. Le seolex, fixé a l'ouverture supérieure du cardia, avait $1,5 \mathrm{~mm}$. (t les ventouses étaiunt lién développées. Les progłottis, qui mesuraient $3,7 \mathrm{~cm}$. de long, se trouvaient placés en ligne droite à la suite du scolex. 
L'exemplaire $\mathrm{n}^{\circ} 2$ avait $16 \mathrm{~cm}$. de long sur $3,5 \mathrm{~mm}$. de large. Les proglottis mesuraient $3,5 \mathrm{~cm}$. et le scolex $1,3 \mathrm{~mm}$. Cet exemplaire était placé au-dessous du précédent.

L' exe m pla ir e $n^{\circ} 3$ avait à peine $3 \mathrm{~cm}$. de long sur $3,5 \mathrm{~mm}$. de large. Il était placé au-dessous des deux autres.

Il n'est pas difficile de comprendre comment ces Cestodes ont pu s'établir dans un Poisson de mer, lor'squ'on connait son gemre de nourriture. L'Acerina cernua qui vit dans les eaux douces et salées est un Poisson très vorace, qui mange tout ce qu'il trouve. Il est donc naturel qu'il avale des ceufs de Cestodes, surtout dans une contrée comme la Finlande où il est rare que les Vertébrés ne soient pas infectés de Vers intestinaux. Leur grande extension, dans ce pays, est due surtout à la malpropreté et à l'habitude que l'on a de manger beaucoup de Poissons qui ne sont pas cuits, mais seulement salés ou séchés.

\title{
Some Notes on the Cetacea of the Irish Atlantic Coast.
}

\author{
By Prof. R. J. ANDERSON (Galway).
}

With 4 Plates.

The following Cetaceans were stranded during the last few years Balænoptera rostrata, Globiocephalus melas, Grampus griseus (iissoumus), Mesoplodon Hectori Gray, and Lagonorhychus. Several other genera have been met with Delphis and Plocæna, skeletons of Orca (in whole or part), Megaptera, Balænoptera musculus, Mesoplodon bidens.

Mesoplodon Hectori Gray, is one of the most interesting that has come to us. A young specimen was stranded some year's ago near Galway. We obtained an almost complete skeleton and have it mounted. A second specimen was stranded on one of the Aran Islands last winter, we obtained the head of this latter. 'The length of the first specimen was $4^{\mathrm{m}}, 25$ and that of the second $6^{\mathrm{m}}, 37$. The girth of the latter at its thickest part $3^{\mathrm{m}}, 64$.

'This whale was originally describer from a young surecimen and thought to be a Ziphius because of the pair of small teeth near the apex of the mandihle. Sir Willam Terser showed that the genus Ziphins differs from Mesoplodon in the character's of the tympanic and nasal bones and the nasopremaxillary region. 
Our furst specimen did suggest relationships with Ziphins Cuvieri ${ }^{1}$. Dr. ScharfF, however, who examined the drawings and read the deseription said that the specimen was Mesoplodon Hectori Gray. The nasal bones of the larger skull are of the same character as the smaller and separate. So we regard the diagnosis as confirmed. 'The skull of the second is $1^{\mathrm{m}}, 05$ long, $54 \mathrm{~cm}$. wide behind the rostrum. $47 \mathrm{~cm}$. in height to the occipital summit. The apex of the beak is $68 \mathrm{~cm}$. from the anterior part of the frontals. 'The skull is very asymmetric. The internarial septum is $295^{\text {man }}$ from the right side and $225^{\mathrm{mm}}$ from the left side. The premaxillæe bound the nares at thesides. That of the left side is $5 \mathrm{~cm}$. wide at the lower part of the nares, whilst the right is $11 \mathrm{~cm}$. wide at the anterior limit of nares. The walls of the rostral groove are thick, much thicker than in the first found specimen which had thin and brittle outer walls. The vomer presents a hollow surface in the floor of the groove.

The left nasal is much smaller than the right and the internasal suture runs outwards, downwards and forwards.

A groove runs along the outer side of each maxilla for $20 \mathrm{~cm}$. and along the upper margins of the lower jaw for $41 \mathrm{~cm}$.

A large socket is situated on each side of the apex of the lower jaw, each is $18^{\mathrm{mm}}$ from before back by $13^{\mathrm{mm}}$ from side to side, for the two teeth. The teeth are conical or double conical, the fang of each is truncated (see Irish Naturalist, June 190t); botin teeth are concealed by the mucous membrane.

The condyles of the occipital bone are $6 \mathrm{~mm}$. apart in front and $76^{\mathrm{mm}}$ behind.

The premaxillat which reach far back in the middle line of the palate ale separated from each other for $110^{\mathrm{mm}}$ by the vomer. The premaxillæ form the apex of the beak also and extend backwards for a distance of $15 \mathrm{~cm}$. (beneath) the maxilla externally.

The palatines, as was pointed out, reach the middle line behind the romer. The palato-maxillary suture rums forwards, inwards and backwards, and hounds anteriorly a surface that is $4 \mathrm{~cm}$. broarl at the middle line and the same breadth at the bend forwards.

The maxille make an acute angle between the palatines. The cranial cavity could not be satisfactorily measured. It appears to be $19 \mathrm{~cm}$. from before back $35 \mathrm{~cm}$. from side to side and $17^{\mathrm{cm}}, 5$ in height.

The skull in the smaller specimen is less asymmetric than the present example, which is half as large again as the first.

The sterum of the first has four pieces separated by three foramina, placed at the joints, the foramina heing in the middle line as usual. The

${ }^{1}$ The nasal bones are conjoined in Ziphius. There are differences in the trmpanic and naso-premaxillary bones. 
antero-posterior diameters of these pieces are of one (1) $14 \mathrm{~cm}$. middle line (2). $10 \mathrm{~cm}$. to $4^{\mathrm{cm}}, 7$ (3). $8^{\mathrm{cm}}, 5$ to $9^{\mathrm{cm}}, 7$ (4). The breadths are (1). 14 cm. to $8^{\mathrm{cm}}, 5(2) .11 \mathrm{~cm}$. to $8^{\mathrm{cm}}, 5(3) .5^{\mathrm{cm}}, 5$ to $13 \mathrm{~cm}$. (4). $18 \mathrm{~cm}$. sides to 5 $\mathrm{cm}$. The lower four cervical vertebræ are free. The skull of the first was figured in the Irish Naturalist. The outhine sketch, which appeared in the paper on the Premaxilla in the Berlin Thansactions, was figured side by side with a piece of the skull of Orca gladiator, which was also cast on the Western Shore.

The second specimen is that of the skull (lowel jaw was wanting) of Grampus griseus, which was stranderl near the city of lialway the young offspring seem to have got on shore first and the dam afterwards. We were not able to get the entire animal and the skull wants the mamlible.

Prof. Thompson got what remained of the young specimen.

The skull is $6 \mathrm{~cm}$. long, $33 \mathrm{~cm}$. wide at the broadest part.

The premaxillæ are $35^{\mathrm{cm}}, 5$ left, $35 \mathrm{~cm}$. right long from fl'ont to back and reach to the tip of the beak, each has a foramen one quarter of an inch in diameter at its apex and other foramina further back.

There are no teeth in the upper jaw. A groove reaches along each side of the upper jaw for a distance of $115^{\mathrm{nm}}$ from the apex. The intermaxille show for $6 \mathrm{~cm}$. in the middle line of the palate. The posterior end of the palatine surface formed by these bones is pointed. The maxillæ reach back far between the palatines reaching almost to the pterygoids.

The vomer does not appear between the maxillæ in any part of the palate.

The palatine of the right side does not seem to reach the middle line. The difficulty of determining the condition is due to anchylosis. The ohliteration of the ptergeopalatine suture on the other side renders the exact boundary lines of the bones where they touch difficult to follow. The pterygoids meet mesially behind the palatines.

The occipital condyles are $63^{\mathrm{mm}}$ apart behind and $2 \mathrm{~cm}$. apart in front.

The intermaxillary groove has the vomer lining its floor. It is $3 \mathrm{~cm}$. deep behind and $1 \mathrm{~cm}$. in front. The breadth is $17^{\mathrm{mm}}$ in front and $7^{\mathrm{mm}}$ behind.

The left nasal is rather more than half the breadth of the right one. The breadth of the right Intermaxilla is 6 cm. and of the left $4^{m}, 5$ at anterior part of superior nares.

The cranial cavity is $16^{\mathrm{cm}}, 5$ from beforc back, $23^{\mathrm{cm}}, 5$ from side to side, and $16^{\mathrm{cm}}, 5$ from above down ${ }^{1}$. The premaxilla shut out almost completely the maxilla from the superior narial orifice. The grey Grampus approaches ylobiorephalus in the shape of the skull but in the edentulous

${ }^{1}$ Dimensions of brain cavity in a large skull of Hyperoodon $23 \mathrm{~cm}, 5$ long $\times 17 \mathrm{~cm}, 5$ high $\times 33 \mathrm{~cm}$. wide. 
condition of the upper jaw lesembles Mesopludon Hectori in which the only teeth present at the apex of the lower jaw are concealed by mucous membrane. The lower jaw has usually $2-6$ teeth on each side in front. An adult specimen of this Cetacean has never been stranded. before the present example, in Ireland, so far as one is guided by records, a somewhat uncertain criterion. Small wroups have been noted from elsewhere.

Globiocephalus melas Trail. is somewhat rare on the West Coast of Ireland although a shoal of 300 were killed on the Coast of Kerry in 1844. There are several records of their visiting other parts of Ireland. I formed one of a shooting party that tried to get some specimens from a group that visited the North and North East of lreland some years ago. One was secured for dissection and some interesting points noted in connection with the peritoneum. The specimen now noted for comparison was evidently a stray member of a school which had wandered away from the place where the herd was being hunted. It had received a spear thrust, or an harpoon wound in the side a few feet in front of the tail. It was $6^{\mathrm{m}}, 41 \mathrm{long}$, greyish white in colour. The colour was due to a thin pellicle of epiclermis which came away leaving the skin black beneath. The globular projecting fat-formed forehead overhung the duck-like beak more than the drawing of Mure's typical specimen would suggest. Seven teeth only appeared on each side. 'There are however ten sockets in the skull. An edentulous condition seems common in old specimens, sockets, however, appeared after the skull had been cleaned, on each side of the upper jaw and eight sockets on each side of the lower jaw.

The maxille bound the narial openings as Owen pointed out, and this takes place anteriorly.

The intermaxillary bones form the apex of the upper jaw, but show oniy for a short distance in the anterior part of the palate. Widening gradually as they are traced back in the palate, the measure across is $5^{\mathrm{cm}}, 3\left(2^{\mathrm{cm}}, 6\right)$. This is opposite the second tooth, farther back the surface gets rapidly narrow and the intermaxillary bones do not show beyond a point $8 \mathrm{~cm}$. behind the apex of the jaw. It is very different on the upper surface where these bones form plates that lie on the maxillæ.

'The teeth in the upper jaw are set in distinct cup-like cases which appear as if inserted in the upper jaw. The lining ossific deposit in the lower jaw rises only a short distance round the teeth at the base and does not stand out from the bone as happens in the upper jaw. This tendency to extension of the ossification suggests a step toward the formation of composite teeth.

The palatines meet in the middle line of the palate and separate the pteryouds from on another on the palate surface and from the median line.

The reptilian characters of the teeth and lower jaw, - the position of 
the occipital condyles and the condition of the premaxille, which present an appearance somewhat like the palatine portion of the vomer in some Crocodilia, and have a position like that of the dumb-ledl hone in Ornithorhynchus - may be here alluded to. FLower mentioned that the external bony nares of the Eilephant, especially the romo Elephant, were in position like similar openings in Cetacea. A comparison of the Mesoplodon and Cetacean skulls seems to bear out the suggestions of FLower and others that Cetacea and Sirenia are redated to the Ingulates or rathere to the Elephantine group, "albeit very far back » (Lyoekкer). 'The formation and mucous membrane of the mouth and the edentalous jaws suggest a resemblance to the Bovidæ. The large cerebral cavity reminds one of the cranial cavity in Proboscidea ${ }^{1}$. The anterior mares look up and are far back in the proboscidean and this holds decidrelly tor Mesophlodon. The rostrum is greatly developed in the latter (in the adult state). In the Elephant and Dugong however this structure, though short, is thick and solid and gets always a special description. The incisor teeth demand a larger supporting bone mass, and it is possible that the disappearance of these teeth may nave led to elongation of the snout by a bony deposit. The figures given of a fossil Elepliantine skull preserved in the Museum d'Histoire naturelle at Paris seem to show that some ancient proboscidean groups had trunks intermediate in size between Elephants and Tapirs and one of these which had two tusks in the lower jaw may be taken as one of the nearest relatives, in the Elephantine group, to the ancestor's of Mesoplodon. It is probable (possible) that the factors that produced the primitive form of trunk in an Elephant produced a cylindrical rostral groove in a Mesoplodon. The miniature tusks in the lower jaw of the latter look like the shrunken tusks in lower jaw of Deinotherium. The Dugong seems more closely connected with the parentage of the Elephant.- The incisons, memaxillae aborted masals, and rostruin all point to relationships with Elephants and whales, besides the placentation, the position of Mamme and the succession of the molar teeth high up, in the forms. The premaxillæ in these three groups reach high up). (the frontals in Ineongs or Elephante). and the maxillie although less, massive in Mesoplodon for want of tectl are in Orce and Globiocephalus strong, whilst in the latter the extension of the tooth cases upwards or downwards shows how a composite tooth may lead to separate and smaller teeth. The large maxillae in the Proboscideans (Elephants) are due to the formation and extension of the great composite molars. This leads to a shrinkage of the alisphenoids and indirectly to shortening of the parietals, below and in front, for the squamosals

1 KüKenthats brain weights of Cetacea may be compared with that of Elephant.

2 WoOdward and ANDrews. 
reach the frontals. If at an rarly period the osteololasts transfered their action to the beak. the shrinking teeth could supply the lime salts. The flattening out of the premaxilla and the manufacture of the rostral cylinder, follow maturally from the altered conditions. The appearance of the romer in the palate and in the Hoor of the rostral crlinder is significant in pointing to a still earlier reptilian connection.

The following is the letter announcing the arrival of the Mesoplodon.

I)ar Sir. A large fish covered with a kind of hairy fur, is reported to low washed ashore at South Aran. its dimensions ale length $6^{m}, 56$, wirth $3^{\mathrm{m}}, 75$, will you please inform me of its name and oblige $)$.

'Two Species of $\boldsymbol{M}$. bidens (Ziphius Sowerbyi) were found on the west Coast of Ireland many years ago.

An account of the siull of Mesoplodon Layardi is given by Sir William Turner ${ }^{1}$ who refers to the observations of Sil William Fuower, Dr Hector, Prof. Moseley and Dr. von Has's. The measurements of the skull in some specimens are given and compared with those of a Ziplizus of New Zealand and one from Shethand and the skull in Mesoplodon srucerbyi. The length of the adult Mesoplodom Layorili (xamined was $1^{\text {m. }}$. ().28, that of the skull of the young specimen $0^{\mathrm{m}}, 635$. The descriptions had reference to one complete skull and a part of a skeleton, a skull with out the lower jaw and a rostrum. The skull figured is so like the skulls in the Galway Museum, that one can have little difficulty in recognising the rolationship. The descriptive particulars given concerning the arlult skull correspond in their main feature with those which have heen noted with regard to Lesoplodon Hertori Gray. Sir William Tersen mentions that in the absence of a knowledge of the sex of the individual, it is impossible to draw conclusions with reference to the specific value of the teeth, and has pointed out the generic value of the tympanic bone in enabling one to distinguish between Ziphius and Mesoplodon.

Mr. Moseleys notes are given. One animal had (large tusks ) in the lower jaw. 'The density of the bone in the second specimen is referred to, the first specimen measured $5^{\mathrm{m}}-5^{\mathrm{m}}, 625$ and the second seems to have been $5^{\mathrm{m}}, 62$. Both specimens were from the cape of Good Hope, a thirel much smaller $+2 \cdot 2^{\mathrm{m}}, 7$ came from the Fast Falliand Islands. A previously found skull had been described by Dr. Gray and Prof. Owex. Slinetomsare mentioned from New Zealand. All the Mesmplodon Layordi species are southern.

The mesorostral hone was absent in the third specimen of the first lot lyeause the specimen was immature the lengths given for the mature and yomme specimems of Mesoplodon Layardi correspond at least the for-

${ }^{1}$ W. Turner. Report on the Bones of Cetacea collected during the voyage of HIIS Chalenger. London, 1880. 
mer) to the leneth of Mesoplodom Hectori (iray. ()ne small Mesoplodon Hectori, was rather longel than the smallel' one of M. Layard. The beak of the adult Mesoplodon Layardi, was very much larger than that of the young specimen (twice the Size). The mesorostral bone was fused to the premaxillæ, near their tips in the adult, so that it was not possible to indicate the amount of the beak apex that was formed by the premaxillæ. The premaxillæ formed the beak in the young specimen. I give the coudition with the premaxilla and mesorostral home mited in Lesoplodon Hecturi. In Mesoplodon Luyurdi (juv) an elongated mesolostral furrow was seren and a bal of cartilage, in this furow, which disappeared after maceration, the eburnated bone takes its place afterwards, but no mesorostral bone seems to exist in the young. The growth of this bone is associated with elongation of the beak, this bone becomes fused with the vomer, the maxille are more spongy. 'The surface of section through the beak is compared to a heraldic lozenge, the inferior angle of which was rounded. The vomer appear's between the pterygoids. In Mesoplodon Layserli the two pterygoids articulated with rach other mesially for $10^{\mathrm{cm}}, 2$ from the base of each plate in the adult skull, but these diverged and allowed first the vomer, and then the two superior maxillaries, to appear between them. In the younger skull the mesial articulation, between the two pterygoids was more complete, for the pterygoid intervened at the anterior part. (Turner. Report on Cetacea of Challenger).

The extracted tooth in Mesoplodon Layardi presented an alveolar end that was jagged at the border and closed. 'The length of the extracted tooth was $35^{\mathrm{cm}}, 8,16^{\mathrm{cm}}, 5$ of which had been included in the alveolus. In Mesoptorlon Hectori the teeth were short. conical, and concealed hemeath the nucous membrane. The pulp cavity existed beneath the proximal end of each small tusk-like tooth. Enamel, dentine and cement were found in the teeth of Layardi. Sections of the teeth of HEcror's Mesoplodon were not examined. In the quotation given by Prof. Turner it will be seen that the teeth are referred to as tusks. A figure of the jaw is given by the courtesy of Prof. WELDON of the Oxford University Museum. Sir W. Turner described the teeth in the Challenger Rep.

In giving an estimate of the value of the parietal when comparing Mammalian groups it may be woll to mention that Jeckes, who examined several fœetal skulls says that the condition usual prevailed in the foetal Dolphin, a foctal Narwhal and a fotal Whale and the parietals are separated by the interparietal where the former bones approximate. The parietals in the Elephant resemble apparenty those of the foetal certare rather than those of the adult, and comparison of the bones in Platanista with the parjetals in a young Elephant is suggestive ${ }^{1}$. The points

1. KRAUSE's Festschrift. Internat. Monatssch., Band XXI. 
that have been made by Andrews seem to show that the Sirenia have several characters that suggest an approximation to the Proboscidian type. The ablominal testes, zonary placentation, non deciduate placentation, Pectoral mamma, bifid apex of the heart, usual absence of foramen in lower end of humerus, similarity in form of molar teeth etc. and the treth being pushed forwards in advancing age (Summary by LroekKER in Knowledge). 'The recent investigations in the early tertiary Proboscidia seem to show a nearer approach of the Proboscidia to the Cetacea than many thought. The lower jaw of Tetrabelodon as figured by Woonward in Knowledge is much more like that of Mesoplodon Hectori than is the jaw of other Elephants. But the great capacity of the skull cavity seems to point also to an alliance of earlier types, although one can never expect to prove an absolute affinity. The great size of the brain in the Elephants and in the Whales also is not without significance.

The outline drawings made from Mesoplodon and Orca are in Irish Natur. 1900.

The photographs were made by D1. White of the Royal College of Surgeons Museum, Dublin, Miss M. K. Anorews of Belfast, Mr. Rominson of Oxford by permission of Prof. Weldon and Mr. Sloan Milus of Galway.

Prof. Ray Lankester of London, formerly of Oxford who is quoted by Turxer. has helpred me to get a photograph of Ziphius cavirostrus, which may make this paper more complete.

I am indebted to the writings of the gentlemen named in this paper.

Plof. T'urner says ${ }^{1}$ : In Mesoplodon Layardi the sphenoid takes but a rery small part in the formation of the temporal tossa. The parietal forms the greater part of its floor and in the young skull could be foll owed as a distinct bone situated between the supra oceipital and the frontal to the vertex, where it was united hy swnostosis to its fellow. In the alult, though the outline of the parietal in the temporal fossa could be readily seen, no part could be traced beyond the fossa to the vertex, for it was overlapped by the growth of the supra occipital, so that only the thin edge of the frontal bone appeared in the interval between the supra occipital and the superior-maxillary. 'The vertex part of the frontal articulated in $M$. Layardi as in $M$. Sowerby with the maxillæ, premaxilla and nasals anteriorly. 'The frontal formed the roof of the orbit and had a large post frontal and a small prefrontal process. The malar consisted anteriorly of a flattened piece which articulated with the superior maxilla and lachrymal, a posterior zygomatic bar that reached beneath the orbit and articulated with the zygomatic part of the temporal. The lachrymal articulated with the prentital part of frontal, the superiol maxillary and the malar in $M$. Luyardi and $M$. Sowerby.

'Challenger Report, p. 9. 

EXPLANATION OF PIATE I

Fig. 1. Mesoplodon hectori Skull. Lower surface.

Fig. 2. " " Lateral surface.

Fig. 3. " " 

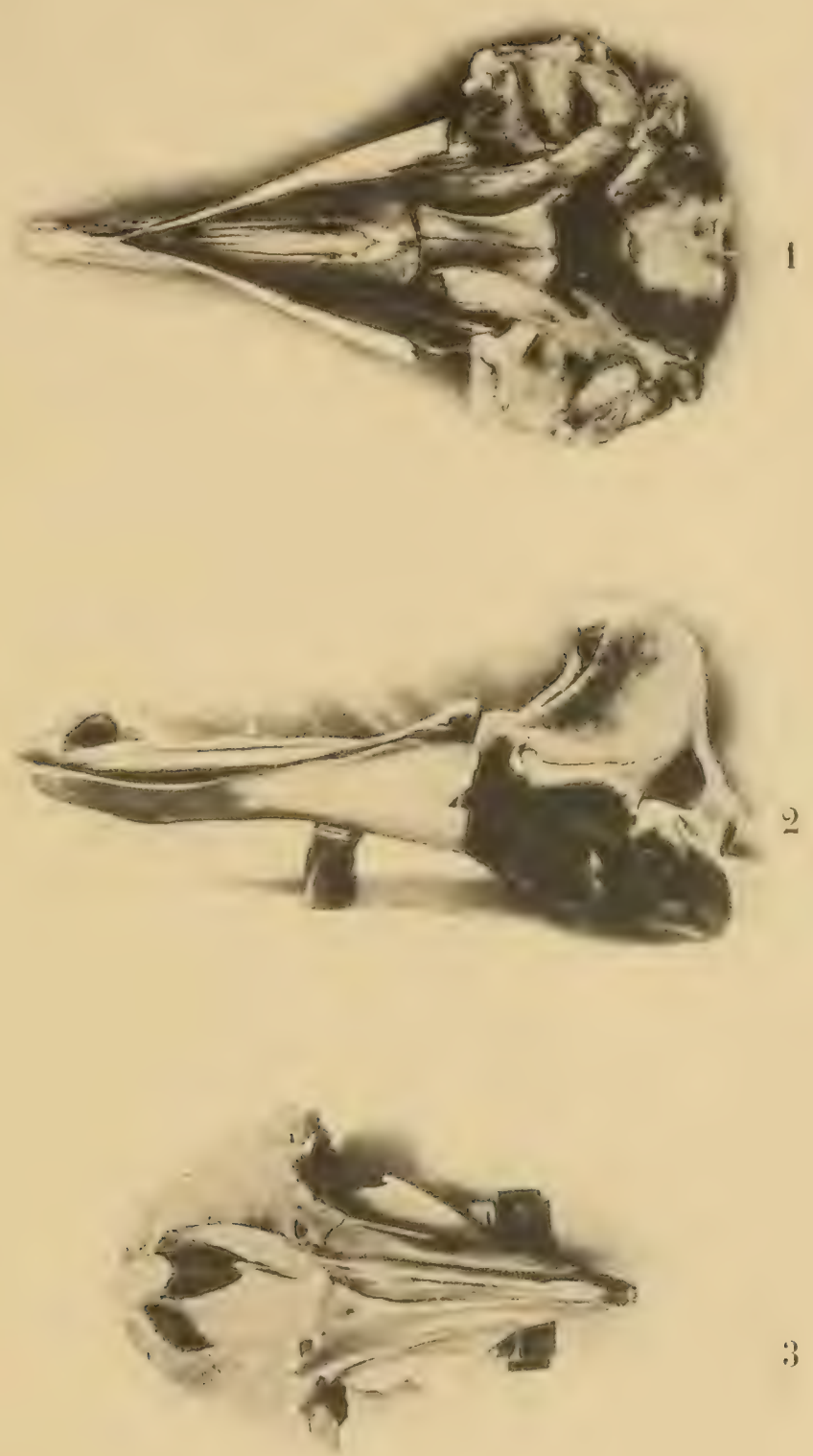




\section{EXPLANATION OF PLATE II}

Fig. 1. Mesoplodon bidens. Skull. Lowersurface and mandible palate) (Nat. mus.). Fig. 2. " " Upper surface showing nasals (Nat. mus.). Fig. 3. Mesoplodon Sowerbyji (bidens) adult. Skull Lateral view. 

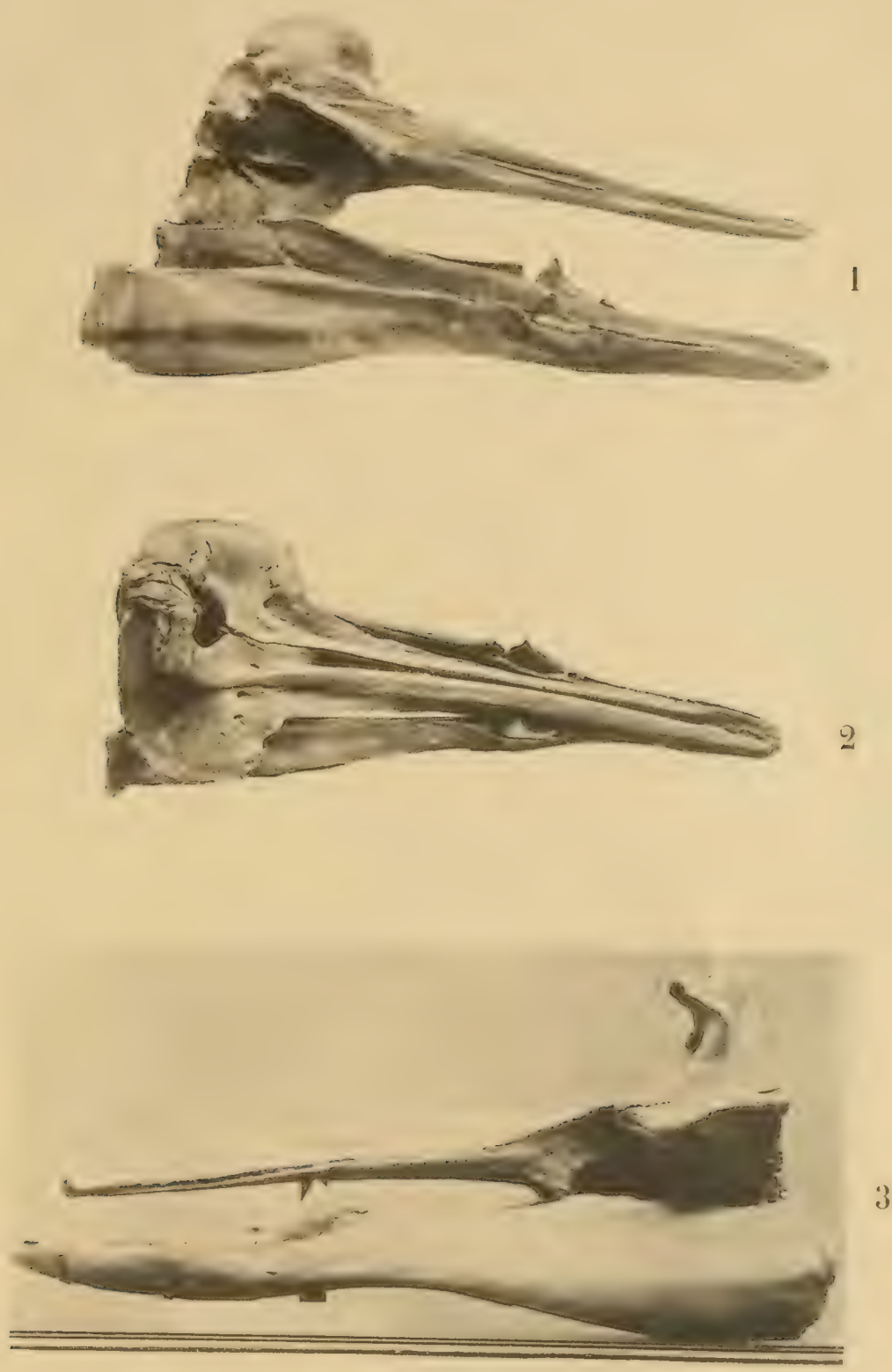

R. J. ANDERSON 


EXPLANATION OF PLATE III

Fig. 1. Mesoplodon Layardi Skull. Lateral view. (Oxon). Fig. 2.

"Lower surface.

Fig. 3. " " Mandible. 

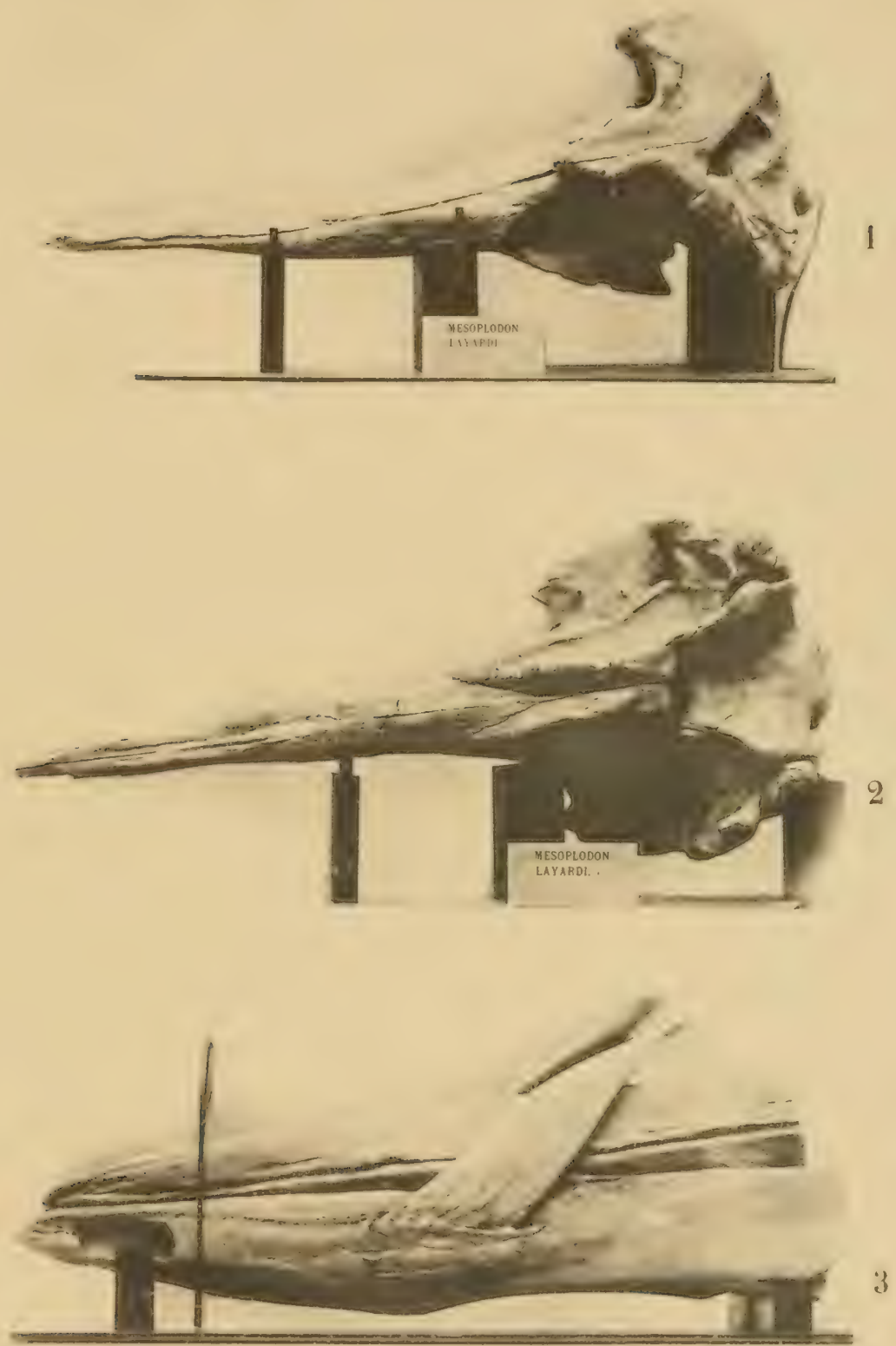

R. J. ANDERSON 




\section{EXPLANATION OF PLATE IV}

Fig. 1. Grampus griseus Skull. Upper surface.

Fig. 2. " " Lower surface.

Fig. 3. Phoccena (Globiceplalus) melas. Skull Upper surface.

Fig. 4. " ” ” " Lower surface. 

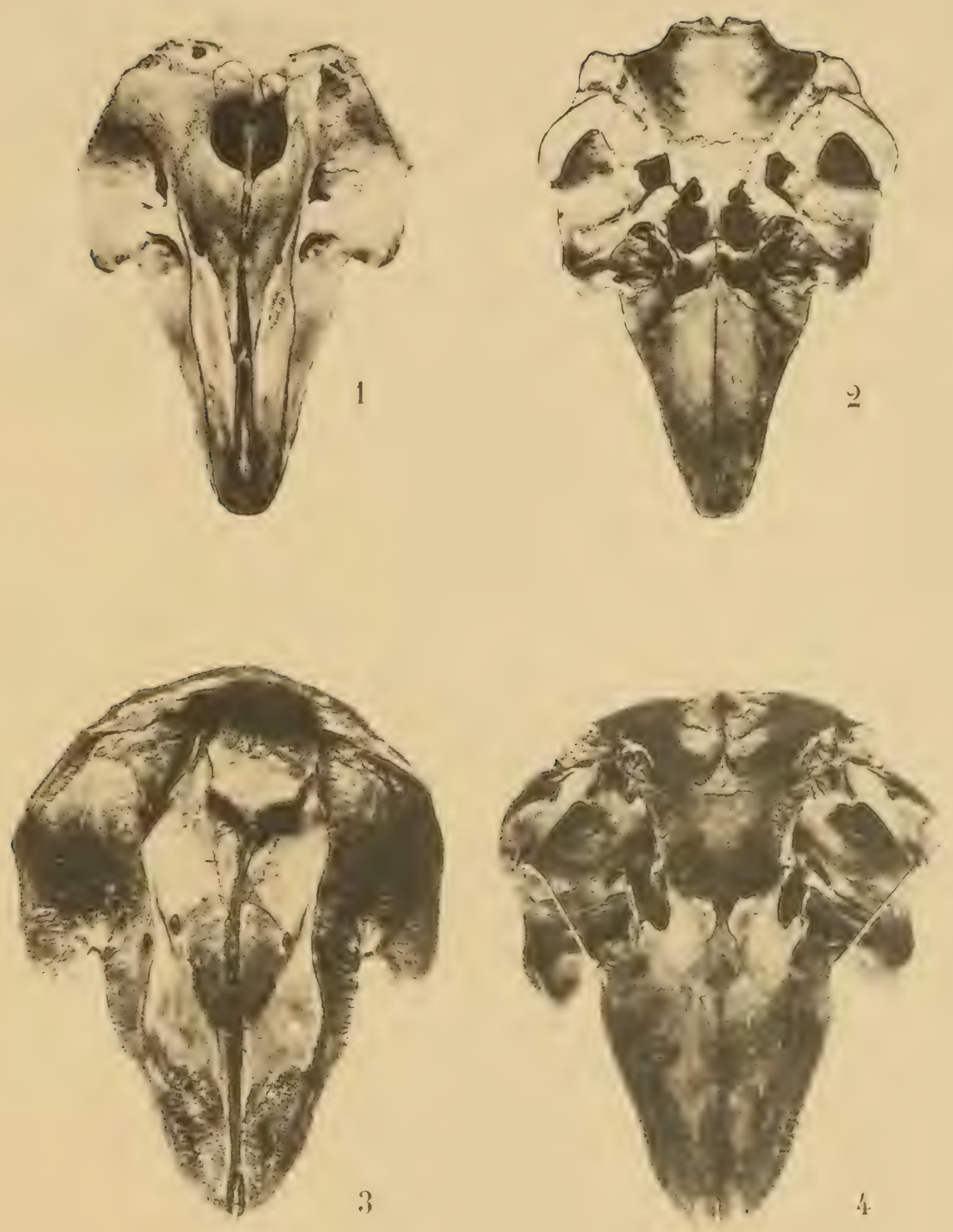

R. J. ANDERSON 

The tympanic is bilobed in Mesoplodon Layardi and not in Ziphius. (Delphis and Berardius have the same).

$$
\text { Longest diametel of petrous brearlth }
$$

$\begin{array}{rll}\text { In Ziphius Cavirosta } & 6^{\mathrm{cm}}, 2 & 3^{\mathrm{cm}}, 5 \\ \text { Adult Mesoplodon Layardi } & 4^{\mathrm{cm}}, 5 & 3^{\mathrm{cm}}, 2\end{array}$

Internal meatus of petrous, single in young Mesoplodon, divided into two in adult Mesoplodon and Ziphius.

In Mesoplodom petrous, mastoid and tympanic difforentiated lut mastoid and tympanic fused, and separated by a constriction. 

ANNEXE II

\section{RÉSUMÉ EN FRANÇAIS DU MÉMOIRE REMIS PAR M. HEYMONS A LA COMMISSION INTERNATIONALE DES PRIX'}

\section{Sur les premières phases du développement de Galeodes caspius.}

Par le Prof. R. HEYIIONS (Hamover-Miinden).

Les œufs de Galeodes sont sphériques, leur diamètre est d'environ $4^{\mathrm{mm}}$. La segmentation suit en général lo type de lat segmentation superficielle, si bien connue chez les Arthroporles.

L'œuf est entouré d'une fine cuticule. L'intérieur' est rempli de grands globules vitellins sphériques entre lesquels ne se trouve que peu de substance protoplasmique. Celle-ci se lencontre on plus glandra quantitio la péripherie de l'ceuf. Dans les premieres phases du dépeloppenentent que j’ai observées, j'ai vu, à la périphérie de l'œuf, quelques grandes cellules dispersées montrant un protoplasme homogène et un petit noyau contenant un réseau distinct de chromatine. Ces collules dérivent sans doute du noyan de segmentation primare entouré déjai dr protoplasume. Bien qu'il soit probable que ces cellules sont en communication entre "lles par des prolongements protoplasmiques, je nai pourtant pas résisi à observer ce fait. On voit souvent que les noyaux de ces cellules se divisent par mitose et que, dans les phases plus avancées, se for'ment ça et là de petits groupes de cellules. Ce n'est qu'un peu plus tard que l'augmentation des cellules donne naissance à une couche continue, qu'on peut nommer blastoder'me. Cette couche est formée de cellules très aplaties qui contiennent maintenant dans leur protoplasme de petits globules d'une substance graisseuse, lesquels proviennent du vitellus nutritif.

1 Note préliminaire présentée à la Commission internationale des prix à Berne. Voir plus haut, p. 131. 
Dans la phase où les îles blastodermiques sont encore isolées, on remarque déjà que quelques cellules de forme plus ronde, pénetrent dans le vitellus en se détachant de la surface. On trouve même parfois des cellules encole en connexion avec les cellules blastodermiques par un fil protoplasmique très ténu. Bien que l'on ne sache pas s'il s'agit d'une immigration de cellules ou, au contraire, d'une ascension vers la surface d'éléments. je crois cependant que l'on doit. saus restriction. donner la préférence à la première altermative. A mon avis, il se produit une immigration des cellules hastodermiques superficielles dans une direction centriprete. ver's le vitellus, immigration qui a lieu particulièrement dans les endroits oì les cellules blastodermiques forment. par suite de divisions actives, des groupes.

Des phénomènes précédemment décrits, il résulte que l'on trouve maintenant des cellules isolées dans le vitellus. On peut les appeler cellules vitellines. ('os dernières apparaissent. des les premières phases, aux points les plus différents, sous la couche cellulaire superficielle. On peut donc dire qu'ils s'agit d’une immigration multipolaire des éléments vitellins.

Histologiquement, il n'y a aucune différence. an commencement, entre les cellules blastodermiques restées à la surface de l'œuf et les cellules vitellines. Ce n'est que plus tard que les noyaux des cellules vitellines sagrandissent jusquà devenir plus grands que ceux de toutes les antres cellules de l'embryon.

Avec la formation du blastoderme ot des cellules vitellines, se terminent les phénomènes que l'on peut comparer à la segmentation des autres Arachnoïdes. Chez Guleodes, il n'est donc pas question d'une segmentation totale, c'est-à-dire d'une segmentation du vitellus. La formation de pyramides vitellines, telles qu'elless ont été décrites chez quelques Araignées, et dernièrement encole chez lo Telyphomls par Sommewrosen. fait défaut non senlement maintenant, mais aussi pendant tout le cours du développement ultérieur.

Avant meme' que le déreloppement d'une couche blastodermique continue soit fini, de nouveaux amas cellulaires apparaissent. Comme premier signe d'ébatuche embryonnaire, on apereoit d'abord. en un endroit de la surface de l'opuf. une petite tache sphérique grisatre. (On peut appeler l'endroit où se montre cette tache embryonnaire: pôle végétatif. Si.on sectionne cette tache, on voit qu'elle est constituée par une accumulation de cellules blastodermiques formant une petite protubérance sphérique, qui pénètre dans le vitellus. J appelle cette protubérance coniqur. cône embryonnaire ou cumulus primitivus (= cumulus genitalis). Chez des œuf's plus vieux, la petite tache sphérique se trouve remplacée par' une masse blanchâtre, plus élargie. Ce n'est que par l'examen de séries de compes que nous pouvons avoir des ichaircissoments sul les phénomenes se produisant à cet endroit. 
On remarque que, maintenant, les cellules immigrent en plus grand nombre, et, qu'en même temps, elles se multiplient beaucoup. En outre, à la place d'une seule protubérance conique, il en existe, à présent, deux, projetées dans le vitellus. On trouve, en effet, à cette phase, deux centres de proliferation localises, qui tous doux sont pros l'un de lautre au pole végétatif et qui, tous deux, fournissent des éléments nouveaux prenant part à la formation de ces deux amas coniques.

Le plus gros des deux cônes de cellules cor'respond, sans aucun doute, au cumulus primitivus décrit plus haut, cal ses cellules forment une accumulation cellulaire ronde et gloluliforme, diun aspect rntiemenent semblable à celui des stades précédents. Cependant, cet amas de cellules s'est encore accru et a pénétré plus avant dans le vitellus. Un peu en avant de cet amas de cellules appartenant au cumulus primitivus, s'est formé un deuxième cône de cellules, plus petit et plus aplati, que j'appelle cumulus embryonalis et qui a produit l'accroissement de la tache grisâtre apparue extérieurement à la surface de l'œuf. Le cumulus primitivus et le cumulus embryonalis sont séparés l'un de l'autre par une zone étroite dans laquelle il ne s'est produit aucune immigration de cellules ou du moins aucune qui vaille la peine d'être mentionnée.

Je n'ai pu relever' des traces d'une invagination ni dans l'amas antérieur (cumulus embryonalis). ni dans l'anas postéricur primail' ut plus grand (cumulus primitivus). Je suis porté à regarder comme un produit artificiel mone petite dépression en forme de renfoncement. que lon lomarque tams le cumblus primitivus sur une le mes prejarations. Мais. comme mes préparations se rapportent seulement à quelques phases peu nombreuses, il est possible que le moment de l'invagination m'ait échappé. Je tiens cependant pour tres raisemblable que. pendant la formation des "ones embryonmaires, me puissante immigration de collules se produise. qui s'achève sans invagination particulière.

Quant aux différences histologiques, elles sont sans importance. Les cellules migratrices se distinguent à peine de celles qui sont restées encore à la surface. Quelques cellules voisines du vitellus contiennent parfois, dans leur plasma, de petits globules graisseux. Chez la plupart des cellules, au moment de l'immigration, cette formation fait défaut, mais on trouve souvent les noyaux de ces cellules en voie de division.

L'amas sphérique de cellules fourni par le cumulus primitivus, après avoir pénétré dans le vitellus, se compose en général d'éléments clairs, riches en plasma et de noyaux pauvres en chromatine. Mais cette particularité n'est pas très caractéristique, car il n'existe pas une limite exacte entre ces cellules claires et les cellules périphériques.

A une époque plus avancée du développement, il se produit une fusion de deux centres d'immigration, sans doute par' suite de l'extension que prend le cumulus embryonalis. Si l'amas sphérique de cellules qui 
est plus grand ot provient du cumulus primitivus ne survivait pas, on ne pourrait savoir qu"il existait deux centres distincts d'immigration dans les phases précédentes.

Les cellules dérivées du cumulus embryonalis produisent la couche entomésodramique de l'embryon de Galeodes, et les éléments de cette couchr s'étendent de plus 'm plus en avant. Je ne puis malheureusement pas trancher la question de savoir si la formation de l'entomésoder'me est exclusirement duc au cumulus embryonalis ou si des cellules entomésodermiques se détachent encor'e un peu plus tard des régions antérieures de l'embryon. Ce qui reste de cellules à la partie supérieure, après la séparation de l'entomésoderme, forme l'ectoderme.

En résumé, pour ce qui concerne la formation de la couche embryonnaire profonde. il faut distinguer, chez Galeodes raspius, les phénomènes suivants :

$1^{\circ}$ La formation d'un amas cellulaire sphérique, provenant du cumulus primitivus et situé à l'extrémité postérieure de l'embryon.

2. La formation d'une couche entomésodermique provenant du cumulus embryonalis.

L'amas dr cellules formé par le cone primitif garde, comme on la déja dit, son indépendance pendant quelque temps. Alors même qüil sit encore a lextat do handelettes embryommaires en roie de segmentation, il a la forme d'une accumulation cellulaire sphérique, située sous l'extrémité postérienre de l'ébanche embryonnaire encore non segmentée. Ce n'est que dans le cours ultérjeur du développement de ces bandelettes ('mh) 'yonnaires. que l'amas cellulaire disparaît, pour mêlel' sés éléments à ceux des autres cellules de l'embryon.

On ne pent constater de différences histologiques entre les ćléments de l'amas cellulaire provenant dn cumulus primitivus et les autres cellules do l'embryon (cellules mésodermiques). A partir du moment où cet amas cellulaire disparait, il est derenu impossible do distinguel les cellules détachées du cumulus primitivus des autres cellules do l'embryon et de connaître leur sort ultérieur.

J'ai appelé le cumulus primitivus: cumulus genitalis. Bien que l'obscrvation ne permettr pas, chez Graleodes cuspins, d'attribuer d'emblér aux cellules provenant du cumulus primitivus le caractère de cellules génitales, je clois cependant, à la suite d'une comparaison avec d'autres Arthropodes, pouvoic justifier suffisamment cette appellation.

Dans des travaux précédents, j'ai montré que chez les Arthropodes, l'éhauche desorganes génitaux apparait généralement à l'extrémité postérieure de l'embryon. J'ai pu, chez différents Insectes, constater ce fait avec toute la certitude désirable, parce que leur's cellules génitales présrutaient, de tres homme heure, des differences histologiques permettant 
de les distinguer des autres cellules de l'embryon, depuis les premieres jusqu'aux dernières phases du développement embryonnaire.

Dans tous ces cas où, par suite d'une différenciation histologique précoce, les circonstances étaient favorables, on a pu découvrir que l'immigration des cellules se produisait à la partie postérieure de l'embryon. Les cellules génitales se forment, chez les Insectes, ou par immigration solide, comme chez Galeodes, ou au moyen d'un petit renfoncement en

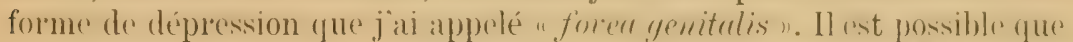

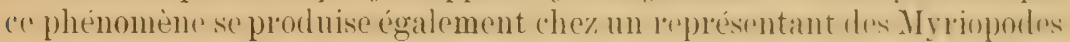
(Scolopendra), cal il est probable que l'ébauche génitale se forme chez

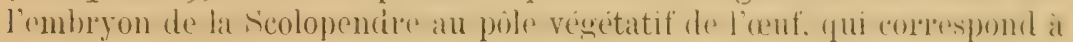
la partie postéricure de l'embryon. Les observations faites jusqu'à présent confirment les mêmes faits chez les Arachnoüdes. Nous devons à

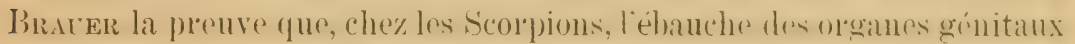
se trouve à l'extrémité postérieure de l'embryon. FavneK a obtenu des l'ésultats semblables chez les Phalangides.

Les récentes observations, si intéressantes, de Scnumewrsci sur le T'elyphonus caudatus, se rapprochent beaucoup des résultats que j'ai ob-

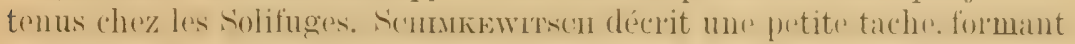
un cône, située postérieurement et une autre grosse tache, située un peu plus en avant. Ces deux taches se confondant le long de la ligne médiane; il en résulte un aplatissement du cône. Schimкwirsch se demande « ob die ühor dem Primitivhügen liegenden Zellen nicht die fronitalanlage?

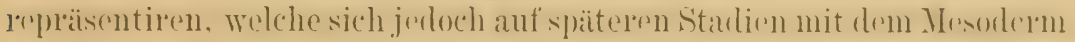
veleinigt und von den Zellen dieses IBlattes nicht mehr zu untershesiden ist (wenigstens mit den uns zur Untersuchung zu Gebote stehenden Mitteln) $)$.

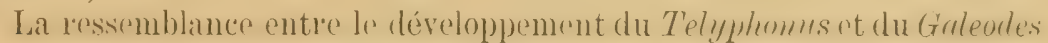
est apparente. Schniкewitsch, sous le nom de cône primitif, semble comprendre un amas de cellules qui fait saillie au-dessus de la surface de l'œuf, dans une direction centrifuge, tandis que, chez Galeodes, cet amas de collules pénetre à l’intérieme du vitullus daus unc dirertion centripete. Je ne doute cependant pas que le cumulus primitivus, chez ces deux formes n'ait la même importance, et que la grosse tache antérieure

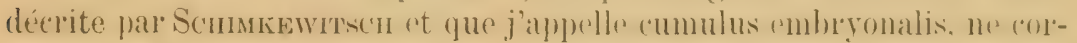

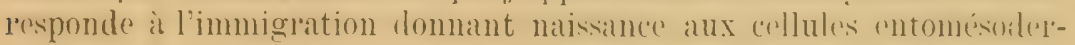
miques.

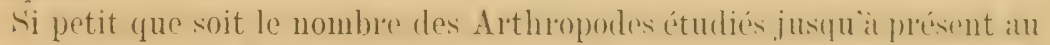
point de vue de la formation primaire de l'éloauche desologanes cénitatux

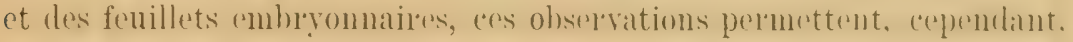
de l'econnaitre les traits fondamentaux et de constater ce fait, que chez Galeodes ot probablement choz quelques autres Arachnoüdes il semble y avoir deux centres embryonnaires distincts. 
L'indépendance longtemps conservée, chez Galeodes, par les cellules immigrantes du cumulus primitivus permet de reconnaître facilement que ces cellules ne concourent pas à l'ébauche des véritahles feuillets embryonnaires. Ces cellules ne peuvent produire des cellules vitellines, car, même en faisant abstraction de l'apparition plus précoce des cellules vitellines, il est impossible de r'emarquer, plus tard, à l'extrémité de l'emhryon et dans le voisinage do la bandelette embryonnaire, une angmentation du nombre des cellules vitellines.

La seule explication qui reste est donc celle-ci : les vellules qui ont formé le cumulus primitivus sont véritablement celles qui, plus tard, serviront à la reproduction. C'est seulement de cette manière que je peux expliquer l'apparition de deux ceutres d'immigration distincts (cumulus primitivus = genitalis et cumulus embryonalis) et être d'accord avec les observations faites sur d'autres formes plus favorables.

Ces observations tendraient à montrer qu'il existe, chez Galeodes, une séparation fondamentale entre l'ébauch" des cellules des organes génitaux et l'ébauche des cellules de l'embryon. Cette séparation serait d'autant plus curieuse que, d'après toutes nos expériences sur les autres Arthropodes terrestres (Myriopodes, Insectes), le détachement des cellules génitales a lisu en connexion avec les cellules somatiques, au méme endroit, c'est-à-diı'e au pôle végétatif de l'œuf.

Mais. malgré cette particularité frappante, je ne crois pas qu'une différence plus essentielle se manifeste. On ne doit pas oublier que cente séparation n'est pas très significative. car les cumulus embryonalis et primitivus sont voisins l'un de l'autre chez (tuleodes rt, à en juger par la description de Schmomitsch, ces deux centres se trouvent très près l'un de l'autre chez Telyphomes. Les deux cumuli se contondent certainement plus tard et les promicres cellules ébauchées (cellules génitales) pénètrent ensuite dans l'intérieur sous forme d'un amas sphérique bien distinct. Je tiens pour très vraisemblable qu'après la conjonction des deux cumuli et l'immigration des cellules génitales. la formation do quelqurs cellules somatiques a lieu non seulement à la place du cumulus embryonalis, mais aussi à la place du cumulus primitivus (= genitalis).

Par conséquent, le trait le plus caractéristique, chez Galeodes comme chez quelques autres Arachnoïdes, consisterait simplement en ce que la formation des cellules génitales n'est pas encore terminée, lorsque la formation dos cellules somatiques commence fléjà assez énergiquement dans la meme couche hastodermique. Saturellement. la formation des cellules somatiques ne peut pas commencer en même temps et au même point que celle des cellules génitales: par conséquent, cllo commence un peu plus en avant et produit là le cumulus embryonalis. Puis, après la séparation des cellules génitales, elle s'étend aussi sur la région du cumulus primitivus (ou genitalis), se réunissant avec ce centre de l'immi- 
gration dont les collules (genitales dejat immigrés forment maintronnt l'amas sphérique mentionné plus haut.

En résume. la rhose la plus essentiolle dans la formation des collules en question se manifeste, chez tous les Arthroporles chez lesquels on a,

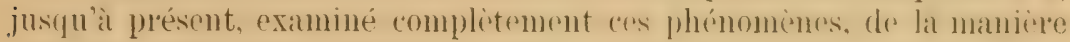
suivante. L'ébauche des cellules génitales, qu’elle soit en premiel lieu unie ou séparée des cellules sonutiques. prend son origine dans la partic la plus postérieur'e de l'ébauche de l'embryon.

Si maintenant, en terminant, nous portons encole une fois notre attention sur les collules somatigues deriveres du cumulus embryonalis, nous remarquous - eoume nous lavons déji indiqué plus haut - quivlles drvienment en partie cellules de l'entodrime. en partie ("ellules du mésoderme. Pendant la phase de l'immigration que nous avons décrite, on ne peut reconmaître de différences histologiques entre cos drux sortes de cellules qui sont encore entièrement non différenciées.

Les diffél’ences n'apparaissent que dans les phases ultérieures du développement. Les cellules qui ont immigré les premières et qui ont le plus profondément pénétré dans lintérienr forment, il la partie supérieure du vitellus, un épithélium unique : la couche entodermique. Les cellules ayant immigré plus tarol et qui restent entre l'entoderme et la couche superficielle (ectoderme) forment le mésoderme. 



\section{ANNEXE III}

\section{RËGLEMENTS DES PRIX}

\section{I. - PRIX DE S. M. L'EMPEREUR ALEXANDRE III}

Prix décerné par la Société Impériale des amis des Sciences naturelles, d'Anthropologie et d'Ethnographie de Moscou, en mémoire des Congris

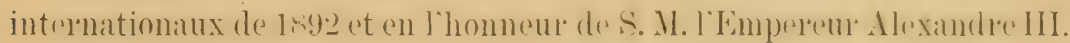

\section{RĖGL,LMIENT.}

Artricle premer. - Le Comité d'organisation des Congrès internationaux d'Anthropologie. d'Archéologir préhistorique et de Zoologrie. réunis à Moscou en 1892, remet à la Société Impériale des amis des

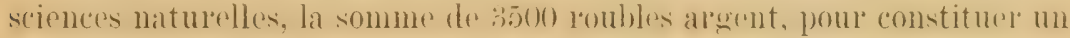
capital perpétuel en souvenir de ces deux Congrès internationaux et de

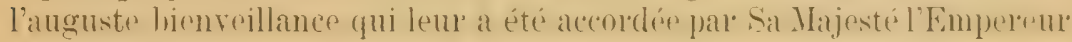
Alexandre III.

ART. 2. - Les intérêts de ce capital seront affectés à la création d'un prix en l'honneur de S. M. l'Empereur' Alexandre III. Ce prix sera attrilué alternativement an ('ongrés d'Anthropologie et d'Archéologie bréhistorique et au Congrès de Zoologie.

ArT. 3. - La quotité du prix est égale au revenu du capital pendant deux ans. Au cas où il s'écoulerait plus de deux années entı’e deux Con-

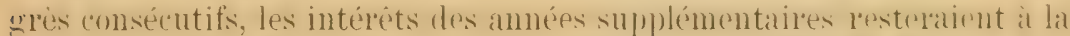

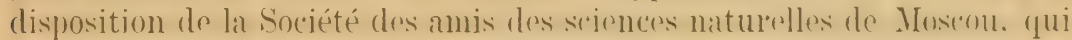

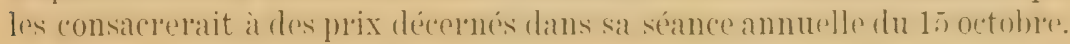

Ant. 4. - Si l'un des deux Congrès cesse d'exister, la part qui lui rerient daprès les articles ci-dessus sera attribuén a la société Imperdial. des amis des sciences naturelles, qui la consacrera également ì des prix distribués dans sa séance anmuelle. 
ART. ว̆. - Le prix attribué au Congrès d'Anthropologie et d'Archéologif préhistorique est décerné par une Commission spéciale nommée à cet effet par le Conseil permanent de ce Congrès. Le prix attribué au Congrès dr Zoologie est également décrené par une Commission spéciale, nommée à cet effet par le Conseil pel'manent de ce Congrès.

ArT. 6. - Les prix peuvent consister en médailles ou en sommes d'argent.

Art. 7. - Ils selont décernés en séance solennelle pendant la session du Congrès.

Art. 8. - Le programme des prix sera élaboré par le Conseil permanent de chacun des deux Congrès.

ArT. 9. - Ce Conseil permanent est en outre chargé de centraliser les travaux présentés, de désignel lessavants ou les Commissions à l'examen desquels ils seront soumis et qui devront déposer un rapport écrit.

Art. 10. - Tout savant est admis au concours, à la condition qu'il nippartienne pas au pays dans lequel doit avoir lieu la prochaine session du Congrès.

ArT. 11. - Le président du Congrès notifie immédiatement au président de la Société Imperiale des amis des sciences naturelles de Moscou le nom de la personne à laquelle le prix a été décerné.

\section{II. - PRIX DE S. M. L'EMPEREUR NICOLAS II}

Prix décerné par la Société Impériale des amis des sciences naturelles de Moscou en mémoire des Congrès internationaux de 1892 et en l'honneur de S. A. I. le Grand-Duc héritier Nicolas Alexandrovitch.

\section{Rìgiearent.}

Article premer. - Le Comité d'organisation des Congrès internatiouaux d'Anthropologie et d'Archéologie préhistorique et de Zoologie, réunis à Moscou en 1s!2. lemet à la Société Impériale des amis des sciences naturelles la somme de 2000 roubles argent, pour constituer un cápital prepétuel en souvenir du Congrès inteluational de Zoologie de 189:2 et de l'auguste bienveillance qui lui a été accoldée par' S. A. I. le GrandDuc héritier Nicolas Alexandrovitch. 
ArT. 2. - Les intérêts de ce capital seront affectés à la création d'un prix en l'honneur de S. A. I. le Grand-Duc héritier Nicolas Alexandrovitch. Ce prix sera attribué au Congrès de Zoologie.

Arт. 3. - La quotité du prix est égale au revenu du capital pendant deux ans. Au cas où il s'écoulerait plus de deux années entre deux Conareses consécutifs, les intérets des amnées supplémentaires resteraient it la disposition de la Société des amis des sciences naturedles de Moscou. qui les ensacrerait à des prix décermés dans sa séaner anmulle du 15 octolnere.

Art. 4. - Si le Congrès cesse d'exister, la part qui lui revient l'après les articles ci-dessus sera attribuée à la Société Impériale des amis des scremres naturelles, qui la consacrera également à des prix distribués dans sa séance annuelle.

ArT. 5. - Le prix décerné pal le Congrès de Zoologie est décerné pal une Commission spéciale nommér à cet rffet par le Conseil permanent de ce Congrès.

Arт. 6. - Les prix peuvent consister en médailles ou en sommes d'argent.

Arт. 7. - Ils selont décernés en séance solennelle pendant la session du Congrès.

Art. 8. - Le programme des prix sera élaboré par le Conseil permanent du Congrès.

ArT. 9. - Ce Conseil permanent est en outre chargé de centraliser' les travaux présentés, de désignel les savants ou les commissions à l'examen desquels ils seront soumis et qui devront déposer un rapport écrit.

ART. 10. - Tout savant est admis au concoul's, à la condition 'qu'il n'appartienne pas au pays dans lequel doit avoir lien la prochaine sescion du Congrès.

ArT. 11. - Le président du Cougrès notifie inmédiatement au prósident de la Société Impéviale des amis des sciences naturelles, le nom de la personne à laquelle le prix a été décermé. 



\section{TABLE ALPHABÉTIQUE DES AUTEURS ET ORATEURS}

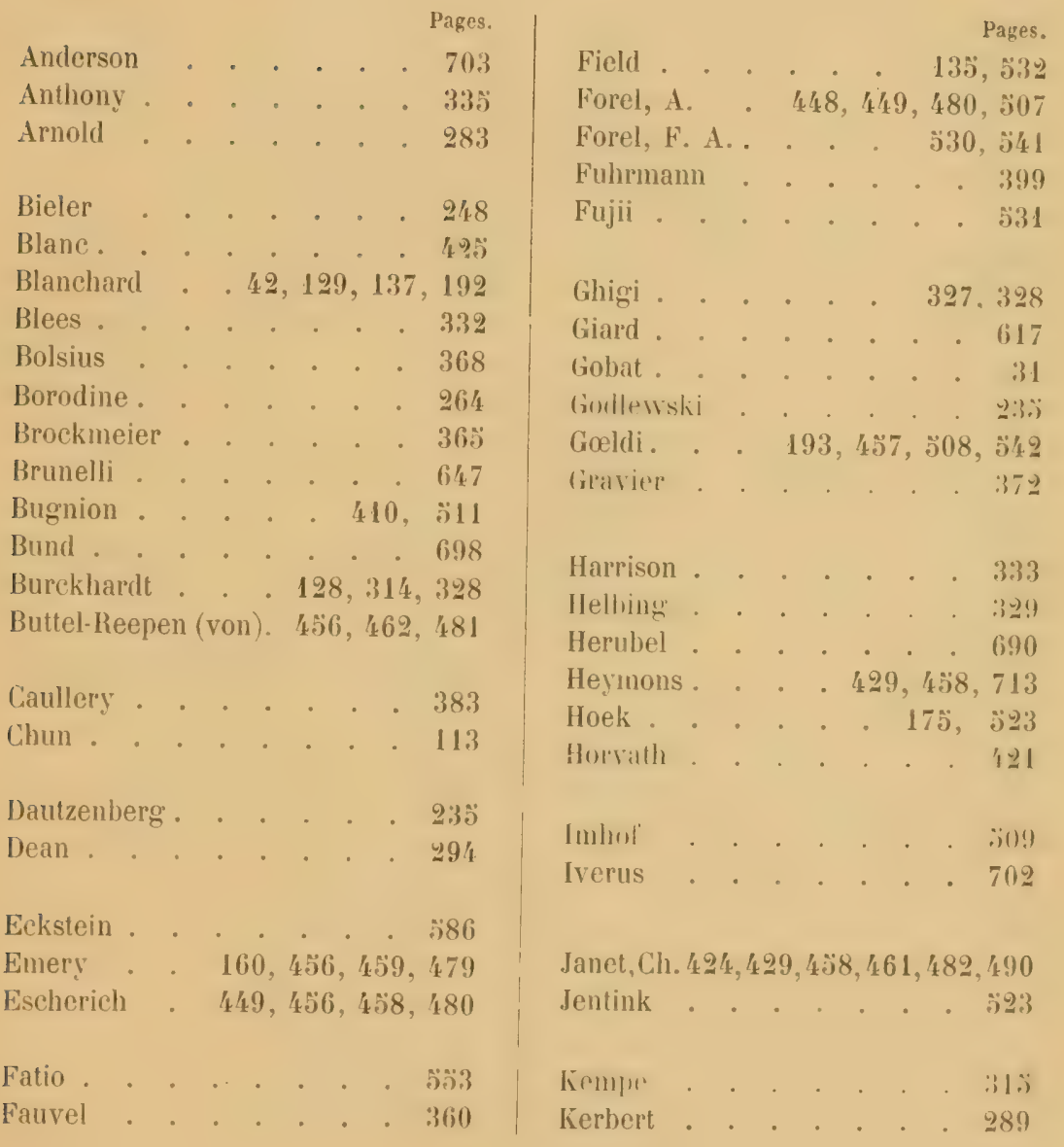


Pages.

Pages.

KJeinschmidt 138, 212,573, อ866,672

Kohler . . . . . . 610

Korotnefl" . . . . . . 384

Kronecker . . . . 497

Lang . . . . . . 5 5ว

Linden (von) . . . . 491

Loisel . . . . . 663

Lönnberg . . : 323, 326

Loos . . . . . . 228

Naas . . . . . . . 238

Whely. . . . . . 280

Irriam . . . . . . 247

Mertens . . . : . . 950

Mesnil . . . . 383, 384

Mever : : . . . . 367

Minot . : : : :42, 2200

Monticelli . . : 400,402

Osborli : . : : : 86,282

Palacki . . . . . . . $\quad 239$

Rodzianko . . . . . 696

Roux . . . . . 3.j2

Salensky . . $67,338,377,381$

Sanchez . . . . . . . 6993

Sarasin . . . . . 147

Schaudinn . . . . . 292.;

Schmidt . . . . . $\$ 361$

Schoner . . . . . lifi

Schuilz . . . . . . 38 '

Scott . . . . . 2't1

Sergent, Et. . . . . . . $38^{\prime}$

Serent, Ed. . . . . . : is'

Simroth . . . 346, 388

Spemain : : : : : . . 233

Spiess . . . . . . 391

Standfuss . . . . . . 4 4 !

Stiles . . . . . . 129

Stingelin . . . . . . $\quad 333$

Strasser . . : . 3333

Studer $\cdot \quad: \quad \therefore 32,192$

Tornier . . . . 282, 327

Pellegrin . . : 330,608

Pelseneer : . : 343, 5อ๊0

Perrier . . . . . . 3है

Petren . . . 213. 496

Pictet. . . . 497, 498

Pieron . . . . . . 482

Pizon . . . 4 404, 410

Plitro. . . . . 2013, , ;29?

Vaney . . . . . . 6111

Vejdorsky . . . . . 292't

Wagner (von) . . . . 6iz't

Wasmann 436, 449, 456, 491, כ̈s'1

Wijhe (van). . . . 319

Wiolterstortf . . . 29\%, 9.78

Popull . . . . . . 1 . 110

Poptล . . . . . . วัอั9

Yung . . . . . . . $9 ! 9$ 


\section{TABLE DES MATIẺRES}

Comité permanent . . . . . . . . . . . . . . til

Commission internationale des prix. . . . . . . . . . . 1111

Commission internationale de nomenclature . . . . . . . . $1 \mathrm{x}$

Commission internationale du Concilium bibliographicum. . . . . $x$

Liste des lauréals des Congrès internationaux de Zoologie. . . . . . xI

Liste des Congrès internationaux de Zoologie . . . . . . . . xul

Comite suisse diormanisalim . . . . . . . . . . . . 1

Rièglement . . . . . . . . . . . . . . . 1

Programme . . . . . . . . . . . . . $1 \mathrm{i}$

Délégués officiels des Gouvernements . . . . . . . . . . 8

Délégués des Universités, Académies, Musées et Sociétés savantes . . ! !

Membres d'honneur . . . . . . . . . . . . 1?

Liste des membres du Congrès par ordre alphabétique . . . . . 1:3

Liste des membres du Congrès par ordre géographique. . . . . . . . 23

Première assemblée générale. . . . . . . . . . . 31

Discours de M. Goßat, Conseiller d'litat du Canton de Berne . . . . 31

Discours de M. Th. Studer, Président du Congrès. . . . . . 32

Discours de II. E. Pennten, Président du Comité permanent des Congrès internationaux de Zoologie . . . . . . . . . . . 3 . .

Fomination des Présidents, Vice-présidents et Secrétaires des Assemblées générales et des Séances de sections . . . . . . . . . !

Propositions relatives à la réunion de la prochaine session du Congrìs . 4l

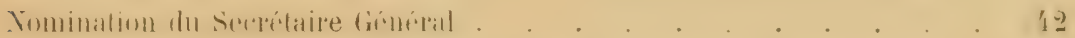

Proposition de l'Association francaise pour l'avancement des sciences. 12

BL.ıchand, R. Zoologie et médecine . . . . . . . . 1ㅡㅡ

LAxG, A. Alexander Moritzi, ein schweizerischer Vorlüufer Danwss. . ;i; 
Deuxième Assemblée Générale .

SAlensky, IV. Ueber die Hauptresultate der Erforschung des im Jahre 1901 am Ufer der Beresowska entdeckten männlichen Mammutkadavers .

OsBons, H. F. Ten years progress in the Mammalian Palieontology of North America

Chus, C. Die vertikale Verbreitung des Marinen Planktons

Brrckitant, R. legt dem Kongress das I. Heft der "Zoologischen Amnalen " vor . . . . . . . . . . . . 128

\section{Troisième Assemblée Générale .}

Nomination de II. le prof. A. Agassiz comme Président de la prochaine session du Congrès

Blaxchand, R., Rapport sur le Prix de S. M. I'Empereur Alexandre III

Blaxchard, R., Rapport sur le Prix de S. M. l'Empereur Nicolas II . .

Blanchard, R., Rapport sur une proposition émanant de la section de zoologie de l'Association franģaise pour l'avancement des sciences.

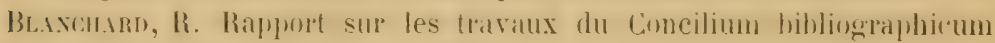
pendant les années 190 ! à 1904 .

Fíecd, H. Etat du Concilium bibliographicum pendant les années 1901 à 1904

Vou proposé par la Commission de nomenclature .

KLeinschumd, 0. Bericht über den Beschluss des V. internationalen Zoologenkongresses betreffend Schutz bedrohter Tierarten .

Samasix. F. Tiergengraphisches. Biologisches und Anthropologisches aus Celebes.

EMERT, C. Ethologie, phy̆logénie el classification . . . . . . . 160

Quatrième Assemblée Générale.

Новк, P. P. C. Ziele und Wege der internationalen Meeresforschung.

BLanchano, R. Rapport sur le vœu de II. O. Kílinschand, présenté à la troisième Assemblée générale du Congrès

GoELDr, E. A. Stryomyin fasciatu, der das Gelbfieber ïbertragende Mosquito und der gegenwärtige Stand der Kenntnisse ïber die Ursache dieser Krankheit 
PLate, L. Die Mutationstheorie im Lichte zoologischer Tatsachen.

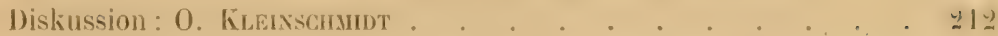

Petensen, IV. Leber die Berleutumg der Generationsorgane für die Entstehung der Arten.

Vejnowsky. F. Demonstration des Bakterienkernes Diskussion : Prof'. Schiudixy

Mror, Ch. S. Die Verinderung der tierischen Zelle Wïhrend der. Verjüngung und der. Veraltung.

Looss, A. Die Wanderung der Ancylostomm- und Strong!yloides-Larven von der Haut mach dem Darm.

Spemaxx, II. Ueber Linsenbildung nach experimenteller Entfernung der primären Linsenbildungszellen

D.utzenbere, $\boldsymbol{1}^{3}$. Nomenclature générique . . . . . . . .

Godewski, E. Der Einfluss des Zentralnervensystems auf die Regeneration bei Tritonen

Mus, 0. Entwickjungsmechanische Studien an Schwämmen. . . . 2:33

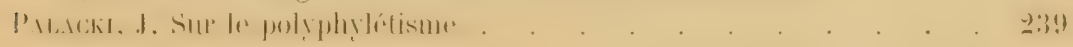

\section{Ðِme Section VERTÉBRÉS (Systématique) .}

ScotT, W.-B. The Mammalian fauna of the Santa-Cruz beds of Patagonia

עerimam, J.-C. A new group of marine Reptiles from the Triassic of Calilornia .

BiEı,E1, S. SUr un Our's nain des Alpes grisonnes (Uisus formicarius)

Inentexs, A. Vom Biber an der Elbe

Wotdenstome, W. Triton Blasii und die Mexues'schen Regehn .

WoztenstonfF, W. Zwergformen der palïarktischen Urodelen

Borodre, N. Les Clupéidées de la ller Caspienne . . . . . .

Osions, H.-F. Evolution of the horse. Recent discoveries and studies

Mínely, L. vox. Ueber den phyletischen Verhand der Spular-irten .

Tonsien, G. Entstehen und Bedentung der Hauptlarbkleidmuster der Replilien

AnxoLn, J. Zur Biologie des Kaspischen Finte, Chupen caspin Eichw.

Kenbent, C. Leber die Eier und Larven von Megalobatiachus maximus schl.

Dean, B. Some embryological evidence as to the position of Chimiren

Yuxr, E. Je l'influence de l'alimentation sur la longueur de l'intestin. Expériences sur les larves de Rana esculenta . . . . . . . . 297

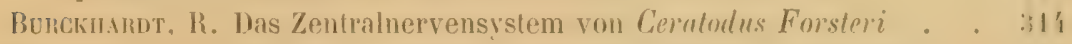

Kinpe, H.-A.-E. Beiträge zu einer Entwicklungstheorie des Hymen . . 31:; 
Wrue, E. vax. Ueber die Entwicklung des Kopfskeletts bei Selachiern .

Lönnerg, E. Demonstration eines Fuetus von westafrikanischen Elefanten, Elephas cyclotis Matschie.

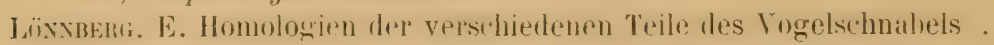

Torsier, G. Experimentelle Ergebnisse iiber Hydropswasserkopfbildung fünf- und mehrbeiniger Individuen

Gunir. A. Dimostrazome di preparati sullo sviluppo dei denti nel Bulistes cupriscus . . . . . . . . . . . . . .

Ghigi. A. Dimostrazione di preparati sopra una nuova forma di epitrichio nelle penne embrionali di Fulica atru . . . . . . . . . 328

Burckinat, R. Rekonstruktionsbilder fossiler Wirbeltiere . . . . 3פ 39

Helbixg, H. Beiträge zur Anatomie und Systematik der Liemargiden. . 3 399

Pellegrin, J. L'incubation buccale chez le Tilapia galilixa Artedi - . 330

BLEs, E.-J. On the hatching of anuran tadpoles and the function of KuppFEn's Stirnknospe.

Harrison, R.-G. Neue Versuche und Beobachtungen über die Entwickelung der peripheren Nerven der Wirbeltiere . . . . . .

Strasser, H. Die Pneumatisation der Yogelknochen . . . . . . 33:

\section{4'1"' Section. INVERTÉBRÉS (à l'exclusion des Arthropodes)}

Asthosx, R. L'acquisition de la forme arrondie chez les Mollusques acéphales dimyaires fixés en position pleurothétique.

Salexskx, W. Ueber den Bau des Prototrochs der Echiuruslarven . .

Pelseneer, P. Le mode de nutrition des embryons chez l'urpma lapillus

Simroth, H. Ueber den Ursprung der Cephalopoden . . . . . . 340

FaUver, P. Les Otocystes du Branchiomma resiculosum Mont. . . . 360

Brockmeier, H. Beobachtungen an Land- und Süsswasserschnecken. . 336.;

MEYER, E. Theoretische Betrachtungen über die ersten Anfänge des ambulacralen Wassergefüsssystems der Echinodermen

BoLsius, H. Le sperme de la Hacmenteria costata, du spermatophore à l'oviducte

Gravier, C. Sur un nouveau genre de Syllidien, Alluaudella nov. gen., madagascariensis nov. sp.

Sildexst. IV. Caher dir: Bildung des Hesohlastes hei den Echiuruslarven Salensk 1 , W. Zur Morphologie der Cardialorgane der Appendicularien .

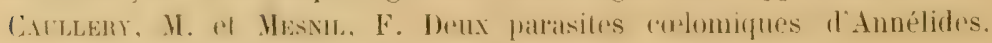
1. Pelmatosphara polycirri. 2. Syheractinomyron stolei . . . Mesvil, F. Le Protozoaire du bouton d'Orient . . . . . . . .

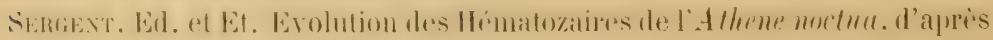
F. Schuduis, Recherches expérimentales . . . . . . . 384

Konotxefr. A. Note sur la Dolchinie . . . . . . . . . . 389 
Spress, C. Sur la structure intime du tube digestif d'A ulastoma yne Norf.-

Tand.

Funmaxy, O. Getrennt-geschlechtliche Cestoden . . . . . . . 399

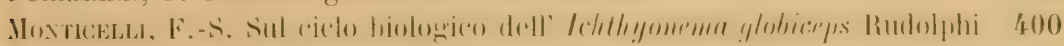

Nonticelit, F -S. Il gruppo delle Temnocefale . . . . . 4 402

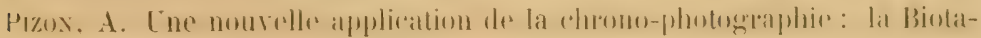
chygraphic .

Pizox, A. Nouvelles observations sur le mécanisme de la circulation che\% les Tunicicr's . . . . . . . 410

Bugrion, E. et Poporf, X. La spermatogénèse du Lombrie (L. agricolii).

Honvath, G. Sur les cornicules ou nectaires des Aphidicns . . . . 421

Discussion : M. JANET . . . . . . . . . . 124

BLAnc, H. Un Caprellidé dans le lac Léman . . . . . . . . $490 \hat{0}$

Discussion : M. JANET . . . . . . . . . . 429

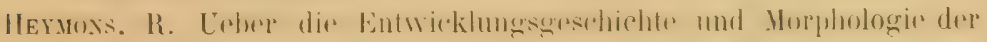
Solifugen

Wasmaxx, E. Die phylogenetische Umbildung ostindischer Ameisengäste in Termitengäste

Diskussion : (1) FunEt.

E. Wasmins . . . . . . . . 14?

Dr Escherich . . . . . . . . . . . . . . . . . . . . . . . .

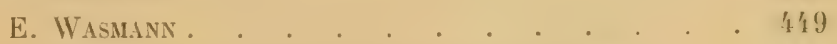

Fonel, A. Einige neue biologische Beobachtungen üher Ameisen . . 14?

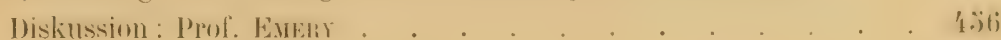

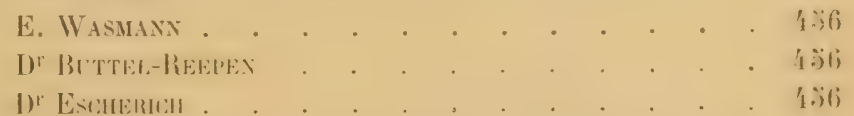

GoELDr, E. Beobachtungen über die erste Anlage einer neuen liolonie von

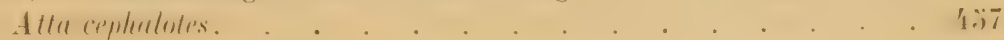

Diskussion : Dr. Eschenich. . . . . . . . . . 4.)8

II. JANET — . 4.88

R. HeymoNs . . . . . . . . 4:38

EverY, C. Sur l'origine des fourmilières . . . . . . . . . 4399

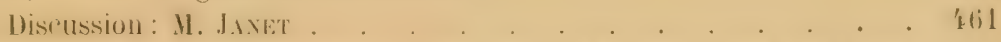

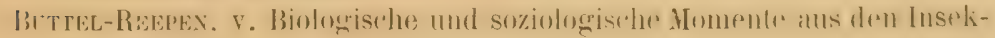

tenstaaten . . . . . . . . . 1692

Diskussion : Prof. EMeri . . . . . . . . . 47!

Prof. Standfuss . . . . . . . . . . . . 47 . . .

D ${ }^{\mathrm{r}}$ Eschenter . . . . . . . . . . . . . 181 
Ppul. Fontit

...+ .480

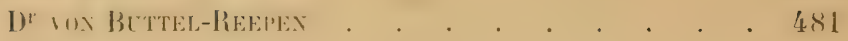

Ch. JANet . . . . . . . . . . 182

Prérox; H. Contribution à l'étude du problème de la reconnaissance chez les Fourmis

Discussion :

$$
\begin{aligned}
& \text { Ch. JANET. } \\
& \text { E. WASMANX }
\end{aligned}
$$

Lrsdex, M. vox. Ueber den Einfluss der Sauerstoffentziehumor wïhrend des Puppentebens auf die Gestaltung der Schmetterlinge . . $\$ 291$

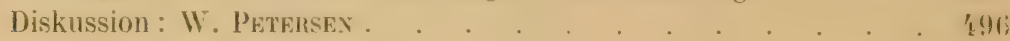

Prof. Kronecker . . . . . . . . . . . 4997

А. Рістет . . . . . . . . . . . . 4 . . . . .

Pictet, A. Des variations des Papillons provenant des changements d'alimentation de leurs chenilles et de l'humidité. . . . . . . 198 Discussion : Prof. Fonel .. . . . . . . . . . . . . . . .

GotLd, E. Myrmecologische Mitteilung das Wachsen des Pilzgartens bei Atta cephalotes betreflend. . . . . . . . . . . .

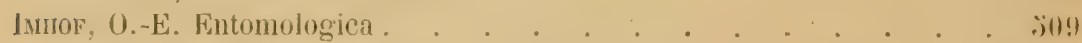

Bugriox, E. Les oufs pédiculés et la tarière de Rhyssaf persuusorin . . \$ill

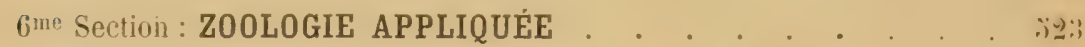

Jentuxi, F.-A. Das Ideal eines naturhistorischen Huseums . . . . , :2::

PLATE, L. Demonstration eines Schaumikroskopes für offentliche Museen :iæ!

Foris, F.-A. La pèche sur les fauberts . . . . . . . . . 733!

Fus, K. Kleinere Beitrige zur Mikrotechnik . . . . . . . . 8331

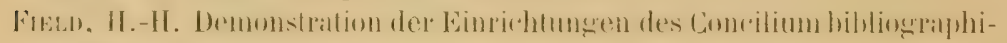
(')

7 me Section : Z0OGÉOGRAPHIE .

Stingelix, Th. Unser heutiges Wissen über die Systematik und die geographische Verhreitung der Cladoceren . . . . . . . . ;3:

Fonel, F.-A. Les Mouettes du Léman . . . . . . . . . . . .'141

Gorddr, E. Nova zoologica aus der Amazonas-Region. Neue Wirbeltiere . :i't2

Pelseneer, P. La ligne de Weber, limite zoologique de l'Asie et de l'Australie

Discussion : J. Roux • . . . . . . . . . . . .

Fitio, V. Principales lignes de passages des Oiseaux à travers la Suisse

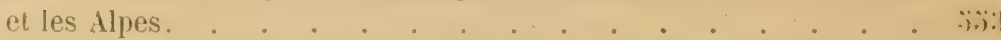

Poptı, C. Ueber die Entwicklung der Fisch-Fauna von Nittel-Borneo :3.i!

Schmint, P. Ueber die Verbreitung der Fische im nördlichen Stillen Ozean und die damit zusammenhängenden zoogeographischen Probleme 
Kuexschuтt, 0. Ueber Erfolge und Ziele zoogeographischer Forschungen, insbesondere über die Zeichnung der Vogelfedern und Schmetterlingslliigel

Diskussion :
E. Wassman
1. Sicill $1 \%$
II. Eckstein
O. Ḱleinschitd .

Siмnoтir, H. Zur Natur- und Entstehungsereschichte der Suidalpen

PEtcegnix, J. La faune ichtyologique du lac T'chad el du Chari

Koenden, R. et VAnex, C. Holothuries abissales recueillies par l'" Investigator " dans l'Océan indien

\section{MYYESE}

I. Travaux remis au Congreis et qui nomt pras été lus duns les siances

Bruxell, G. u. Schoexen, H. Die Frage der Fortpflanzungsperiodizität des Palolowurmes im Lichte der allgemeinen Biologie der Chietoporlen.

L.olsel, G. Recherches de statistique sur la descendance des Pigeons royawints

KLexscmint, 0. Zur' Wahrung des Prioritïtscesetzes in der Nomenclatur gegenïber dem sogenannten Vorrecht des ersten sichtenden Autors

Wagxen, W. vox. Ueber die Genesis und die Entwicklung der Geselligkeit im Tierreiche.

İ́nuber, M.-A. Sur une nouvelle espèce du genre Sipunculus

Sixchez, J. Note sur la Zoologie médicale mexicaine.

Ronziasko, W.-N. Leber den Parasitismus der Larven von Hypostena setirentris Macquart (Diptera) im Innern der Larven von Tellix bipunctatus L. (Orthoptera)

Buxd, W. Salmon Migration

II. Résumé en francais du mémoire remis par 11 . Lleymons ì la Commission internationale des prix

Hermoss, R. Sur les premières phases du déreloppement de Guleodes caspius

III. Règlements des mix . . . . . . . . . . . . . 诖1

Tahle alphabétique des auteurs . . . . . . . . . . . . . $7:$.

Table des matieres 





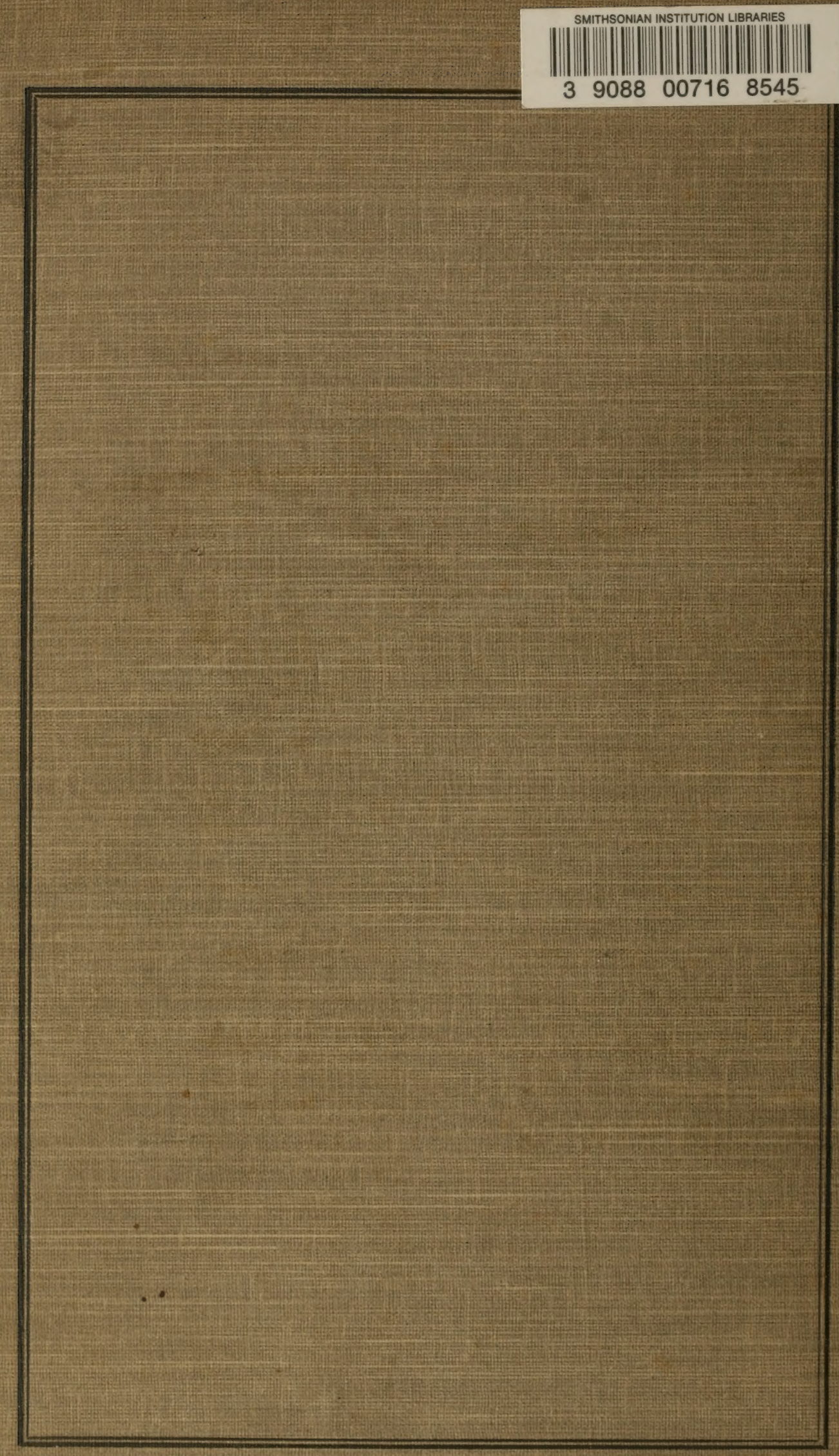

